\title{
Tank Waste Remediation System Multi-Year Work Plan
}

Date Published

September 1994

Prepared for the U.S. Department of Energy Office of Environmental Restoration and Waste Management

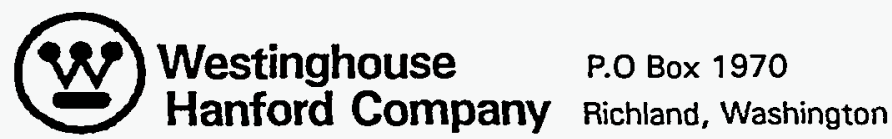

Hanford Operations and Engineering Contractor for the

U.S. Department of Energy under Contract DE-AC06-87RL10930

Approved for Public Release 


\section{LEGAL DISCLAIMER}

This repori was prepared as an account of work sponsored by an agency of the United States Government. Neither the United States Government nor any agency thereof, nor any of their employees, nor any of their contractors, subcontractors or their employees, makes any warranty, express or implied, or assumes any legal liability or responsibility for the accuracy, completeness, or any third party's use or the results of such use of any information, apparatus, product, or process disclosed, or represents that its use would not iniringe privately owned rights. Reference herein to any specific commercial product, process, or service by trade name, trademark, manufacturer, or otherwise, does not necessarily constitute or imply its endorsement, recommendation, or favoring by the United States Government or any agency thereof or its contractors or subcontractors. The views and opinions of authors expressed herein do not necessarily state or reflect those of the United States Government or any agency thereof.

This report has been reproduced from the best available copy. Available in paper copy and microfiche.

Available to the U.S. Department of Energy

and its contractors from

Office of Scientific and Technical Information

P.O. Box 62

Oak Ridge, TN 37831

(615) $576-8401$

Avallable to the public from the U.S. Department of Commerce National Technical Information Service

5285 Port Royal Road

Springfield, VA 22161

(703) $487-4650$

Printed in the United States of America

DISCLM-1 CHP (1-91) 


\section{DISCLAIMER}

Portions of this document may be illegible in electronic image products. Images are produced from the best available original document. 


\section{RELEASE AUTHORIZATION}

Document Number: WHC-SP-1101, Vo7ume 1 - 5

Document Title: Tank Waste Remediation System Multi-Year Work Plan

Release Date: $\quad$ September 29, 1994

$* * * * * * * * * * * * *$

This document was reviewed following the procedures described in WHC-CM-3-4 and is:

APPROVED FOR PUBLIC RELEASE

***************

WHC Information Release Administration Specialist:

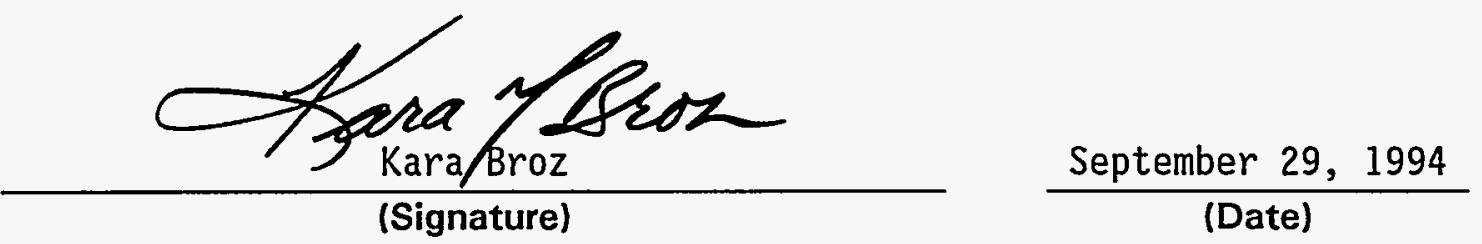


TANK WASTE REMEDIATION SYSTEM - WBS 1.1

MULTI YEAR WORK PLAN APPROVAL SHEET

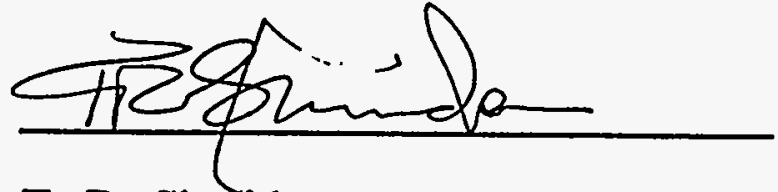

T. R. Sheridan, Acting Program Manager

Tank Waste Remediation System

Department of Energy

Richland Operations Office

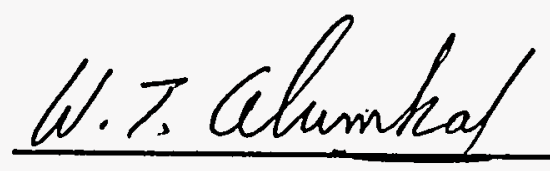

W. T. Alumkal, Executive Vice President Tank Waste Remediation System Westinghouse Hanford Company

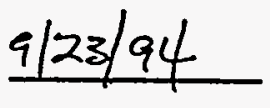

Date 
WHC-SP-1101

This page intentionally left blank. 


\section{EXECUTIVE SUMMARY}

The Tank Waste Remediation System (TWRS) Multi-Year Work Plan (MYWP) documents the detailed total Program baseline and was constructed to guide Program execution. The TWRS MYWP is one of two elements that comprise the TWRS Program Management Plan (see document hierarchy in Section 7.2.1.1.6, Program Management and Administration Assumptions). The TWRS MYWP fulfills the Hanford Sitę Management System requirement for a Multi-Year Program Plan and a Fiscal-Year Work Plan.

The MYWP addresses program vision, mission, objectives, strategy, functions and requirements, risks, decisions, assumptions, constraints, structure, logic, schedule, resource requirements, and waste generation and disposition. Sections 1 through 6 , Section 8 , and the appendixes provide programwide information. Section 7 includes a subsection for each of the nine program elements that comprise the TWRS Program.

The foundation of any program baseline is base planning data (e.g., defendable product definition, logic, schedules, cost estimates, and bases of estimates). The TWRS Program continues to improve base data. As data improve, so will program element planning, integration between program elements, integration outside of the TWRS Program, and the overall quality of the TWRS MYWP.

The MYWP establishes the TWRS baseline objectives to store, treat, and immobilize highly radioactive Hanford waste in an environmentally sound, safe, and cost-effective manner. The TWRS Program will complete the baseline mission in 2040 and will incur costs totalling approximately 40 billion dollars. The primary legal driver is the Hanford Federal Facility Agreement and Consent Order $^{1}$. Other drivers include all local, State, and Federal laws and regulations.

The summary strategy is to meet the above objectives by using a robust systems engineering effort, placing the highest possible priority on safety and environmental protection; encouraging "out sourcing" of the work to the extent practical; and managing significant but limited resources to move toward final disposition of tank wastes, while openly communicating with all interested stakeholders.

The TWRS Program baseline established by this MYWP assumes that funding will be available to support an expanding effort with annual total funding growth rates ranging from four to sixteen percent. This baseline projects peak annual funding levels of approximately $\$ 1.3$ billion in the 2001 to 2005 time frame. Annual funding levels would then begin to gradually decline beginning in the 2006 to 2010 time frame.

The TWRS Program baseline established by this MYWP assumes all tank waste, including the cesium and strontium capsules, will be retrieved for treatment and disposal.

${ }^{1}$ Hanford Federal Facility Agreement and Consent Order, Fourth Amendment, Washington State Department of Ecology, U.S. Environmental Protection Agency, and U.S. Department of Energy, Olympia, Washington (January 1994). 
Program risks include technical uncertainties and the associated potential impacts on projected resource requirements, institutional uncertainties and the associated potential impacts on overall schedule, and funding uncertainties.

The appropriate technical work is underway to support decision making, reduce uncertainty, and improve base data. The MYWP will be updated annually to take advantage of improving base data. 


\section{CONTENTS}

1.0 VISION AND MISSION $\ldots \ldots \ldots \ldots \ldots \ldots \ldots \ldots \ldots \ldots \ldots \ldots \ldots \ldots \ldots \ldots$

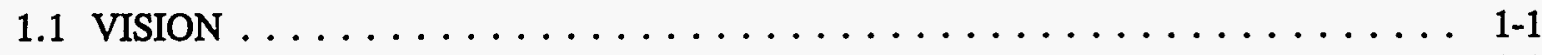

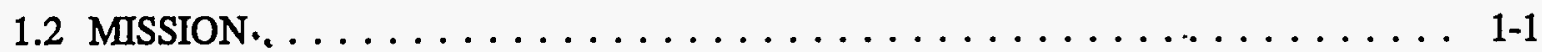

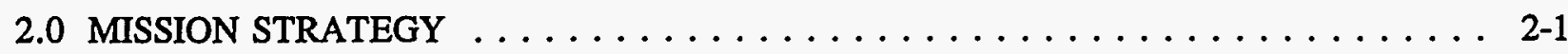

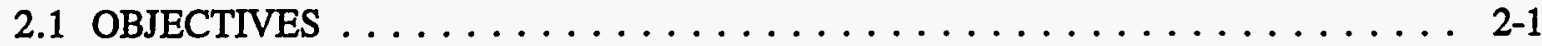

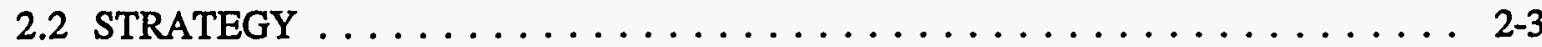

2.3 FUNCTIONS AND REQUIREMENTS $\ldots \ldots \ldots \ldots \ldots \ldots \ldots \ldots \ldots 2-4$

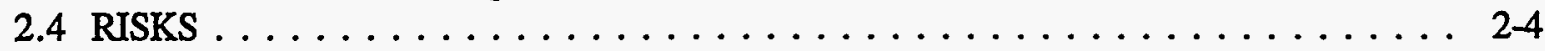

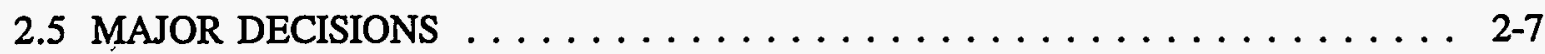

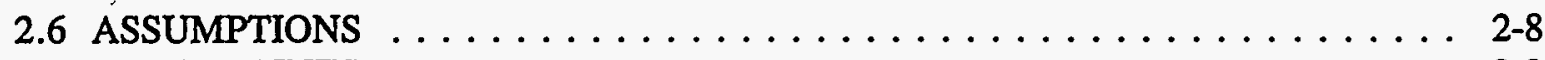

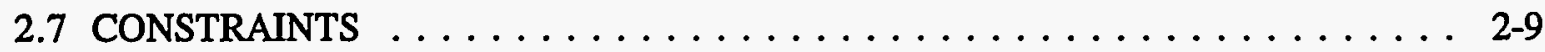

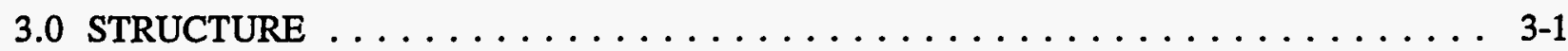

3.1 WORK BREAKDOWN STRUCTURE $\ldots \ldots \ldots \ldots \ldots \ldots \ldots \ldots \ldots \ldots, 3-1$

3.2 ORGANIZATION AND RESPONSIBILITIES $\ldots \ldots \ldots \ldots \ldots \ldots \ldots \ldots$.

4.0 LOGIC AND SCHEDULES $\ldots \ldots \ldots \ldots \ldots \ldots \ldots \ldots \ldots \ldots \ldots \ldots \ldots$ 4-1

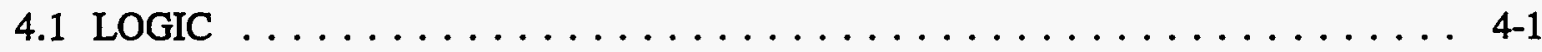

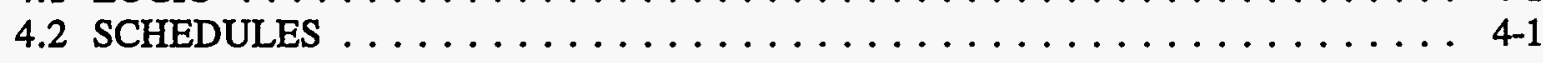

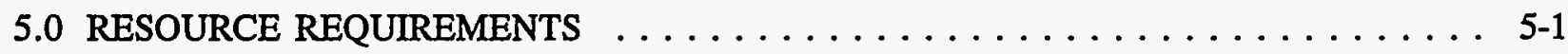

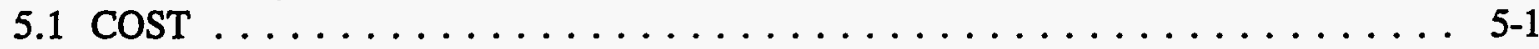

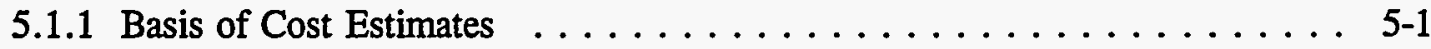

5.1.2 Total Program Cost by Year by Program Element ........... 5-1

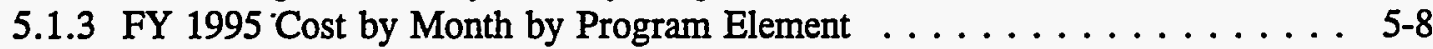

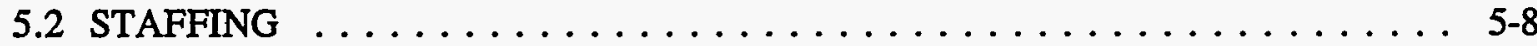

5.2.1 Basis of Staffing Projections $\ldots \ldots \ldots \ldots \ldots \ldots \ldots \ldots \ldots \ldots \ldots$

5.2.2 Total Full-Time Equivalents by Year by Program

Element by Major Participant through $2000 \ldots \ldots \ldots \ldots \ldots \ldots$. $5-8$

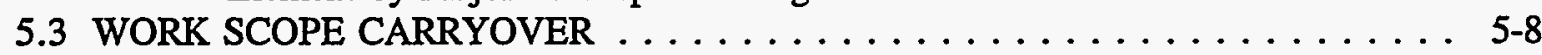

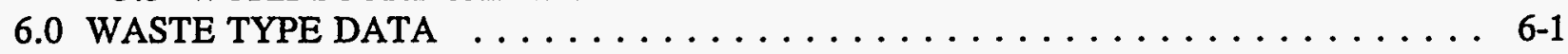




\section{CONTENTS (continued)}

7.0 PROGRAM ELEMENT BASELINES $\ldots \ldots \ldots \ldots \ldots \ldots \ldots \ldots \ldots \ldots \ldots \ldots$

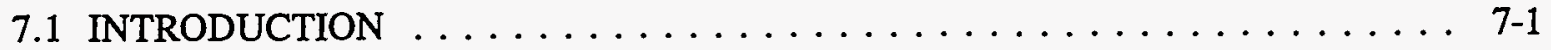

7.2 PROGRAM MANAGEMENT AND ADMINISTRATION BASELINE . . . . . 7.2-1

7.2 .1 Technical Bases . . . . . . . . . . . . . . . . . 7.2-1

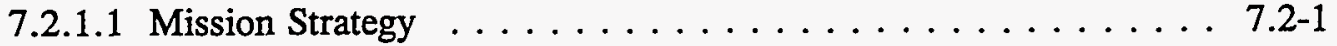

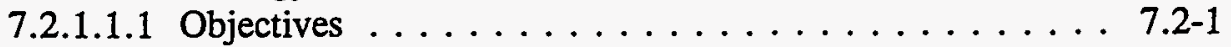

7.2.1.1.2 Strategy . . . . . . . . . . . . . . 7.2-1

7.2.1.1.3 Functions and Requirements . . . . . . . . . . 7.2-1

7.2.1.1.4 Risks . . . . . . . . . . . . . . . . 7.2-2

7.2.1.1.5 Major Decisions . . . . . . . . . . 7.2-2

7.2.1.1.6 Assumptions . . . . . . . . . . . 7.2-3

7.2.1.1.7 Constraints . . . . . . . . . . . 7.2-3

7.2.1.2 Structure ......................... 7.2-3

7.2.1.2.1 Work Breakdown Structure . . . . . . . . 7.2-4

7.2.1.2.2 Organization and Responsibility . . . . . . . . 7.2-4

7.2.1.3 FY 1995 Work to be Performed . . . . . . . . . . . . . 7.2-5

7.2.1.4 FY 1996 - FY 1997 Work to be Performed . . . . . . . . . . . 7.2-7

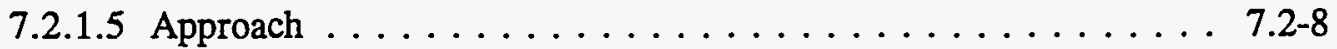

7.2.1.6 Drivers and Deliverables . . . . . . . . . . . . . 7.2-9

7.2 .2 Schedule . . . . . . . . . . . . . . . . . . . 7.2-10

7.2.2.1 Program Management and Administration

Summary Schedule . . . . . . . . . . . . . . . . . . 7.2-10

7.2.2.2 Program Management and Administration Expanded Baseline

(FY 1995-FY 1997) . . . . . . . . . . . . . . . . . . 7.2-10

7.2 .3 Resource Requirements . . . . . . . . . . . . . . . . . . 7.2-10

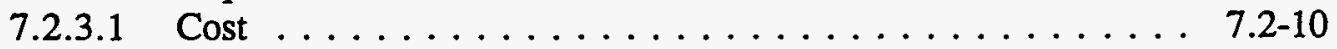

7.2.3.1.1 Basis of Cost Estimates . . . . . . . . . . . 7.2-10

7.2.3.1.2 Total Program Element Cost by Year

by Fund Type by Activity and Cost Account) . . . . . . . . 7.2-11

7.2.3.1.3 FY 1995 Cost by Month by Fund Type

by Activity and Cost Account . . . . . . . . . . 7.2-11

7.2.3.2 Staffing . . . . . . . . . . . . . . . . 7.2-11

7.2.3.2.1 Basis of Staffing Projections . . . . . . . . . 7.2-11

7.2.3.2.2 Total Full-Time Equivalents by Year by Activity

by Major Participant (Direct and Indirect) . . . . . . . . 7.2-11

7.2.3.2.3 FY 1995 through FY 1997 Direct

Full-Time Equivalents by 88 Labor Categories . . . . . . 7. 7.2-11

7.2.3.3 Work Scope Carryover . . . . . . . . . . . . . . 7.2-11

7.2 .4 Waste Type Data . . . . . . . . . . . . . . . . . . 7.2-12

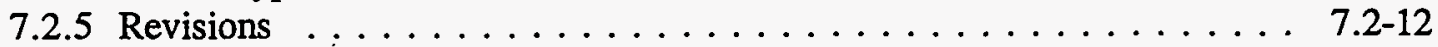




\section{CONTENTS (continued)}

7.3 TANK FARM OPERATIONS AND MAINTENANCE BASELINE . . . . . . . . 7.3-1

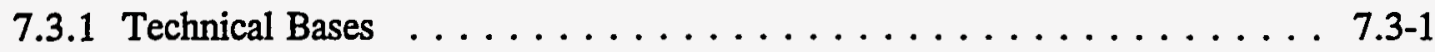

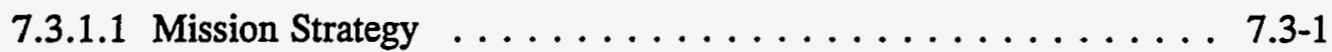

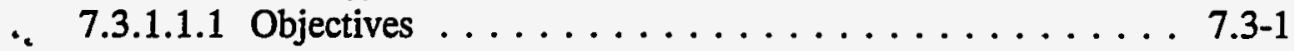

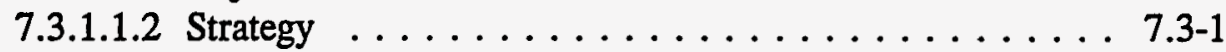

7.3.1.1.3 Functions and Requirements ............ 7.3-2

7.3.1.1.4 Risks . . . . . . . . . . . . . . 7.3-3

7.3.1.1.5 Major Decisions $\ldots \ldots \ldots \ldots \ldots \ldots \ldots . \ldots \ldots$. . . . . . . .

7.3.1.1.6 Assumptions . . . . . . . . . . . . . 7.3-4

7.3.1.1.7 Constraints . . . . . . . . . . . . 7.3-5

7.3.1.2 Structure $\ldots \ldots \ldots \ldots \ldots \ldots \ldots \ldots \ldots . \ldots \ldots$. . . . . . . . . .

7.3.1.2.1 Work Breakdown Structure . . . . . . . . 7.3-6

7.3.1.2.2 Organization and Responsibility . . . . . . . 7.3-6

7.3.1.3 FY 1995 Work to be Performed . . . . . . . . . . . . . 7.3-6

7.3.1.4 FY 1996 - FY 1997 Work to be Performed . . . . . . . . . . . 7.3-11

7.3.1.5 Approach . . . . . . . . . . . . . . . 7.3-15

7.3.1.6 Drivers and Deliverables .............. 7.3-17

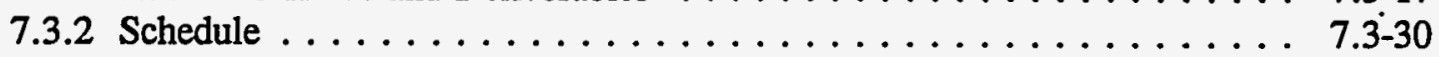

7.3.2.1 Tank Farm Operations and Maintenance

Summary Schedule . ............... 7.3-30

7.3.2.2 Tank Farm Operations and Maintenance Expanded Baseline

(FY 1995-FY 1997) . . . . . . . . . . . . . . . 7.3-30

7.3.3 Resource Requirements . . . . . . . . . . . . . . 7.3-30

7.3.3.1 Cost $\ldots \ldots \ldots \ldots \ldots \ldots \ldots \ldots \ldots \ldots \ldots \ldots \ldots \ldots \ldots \ldots \ldots \ldots .3-30$

7.3.3.1.1 Basis of Cost Estimates ............ 7.3-30

7.3.3.1.2 Total Program Element Cost by Year by Fund Type by Activity and Cost Account) $\ldots \ldots \ldots$ 7.3-30

7.3.3.1.3 FY 1995 Cost by Month by Fund Type by Activity and Cost Account $\ldots \ldots \ldots \ldots \ldots \ldots$ 7.3-31

7.3.3.2 Staffing $\ldots \ldots \ldots \ldots \ldots \ldots \ldots \ldots \ldots \ldots \ldots \ldots \ldots \ldots \ldots \ldots .31$

7.3.3.2.1 Basis of Staffing Projections . . . . . . . . .

7.3.3.2.2 Total Full-Time Equivalents by Year by Activity by Major Participant (Direct and Indirect) . . . . . . . 7.3-31

7.3.3.2.3 FY 1995 through FY 1997 Direct

Full-Time Equivalents by 88 Labor Categories . . . . . . 7.3-31

7.3.3.3 Work Scope Carryover . . . . . . . . . . . . . 7.3-31

7.3.4 Waste Type Data . . . . . . . . . . . . . . . . . . .

7.3.5 Revisions $\ldots \ldots \ldots \ldots \ldots \ldots \ldots \ldots \ldots \ldots \ldots \ldots \ldots \ldots \ldots \ldots \ldots \ldots .3 .32$ 


\section{CONTENTS (continued)}

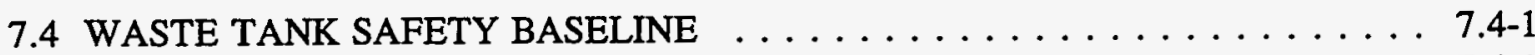

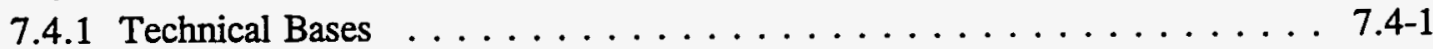

7.4.1.1 Mission Strategy . . . . . . . . . . . . . . 7.4-1

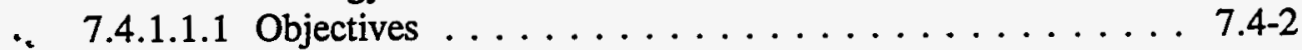

7.4.1.1.2 Strategy . . . . . . . . . . . . . 7.4-2

7.4.1.1.3 Functions and Requirements . . . . . . . . 7.4-2

7.4.1.1.4 Risks . . . . . . . . . . . . . 7.4-3

7.4.1.1.5 Major Decisions $\ldots \ldots \ldots \ldots \ldots \ldots \ldots . \ldots \ldots$

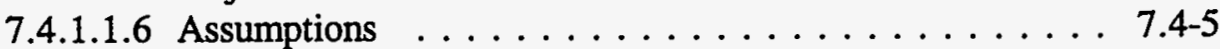

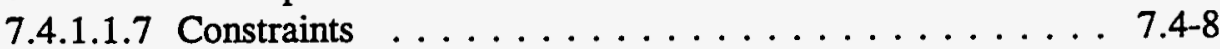

7.4.1.2 Structure ... . . . . . . . . . . . . . 7.4-8

7.4.1.2.1 Work Breakdown Structure . . . . . . . . . 7.4-8

7.4.1.2.2 Organization and Responsibility ... . . . . . 7.4-8

7.4.1.3 FY 1995 Work to be Performed . . . . . . . . . . . . . 7.4-10

7.4.1.4 FY 1996 - FY 1997 Work to be Performed . . . . . . . . . . 7.4-10

7.4.1.5 Approach . . . . . . . . . . . . . . . 7.4-10

7.4.1.6 Drivers and Deliverables . . . . . . . . . . . . 7.4-11

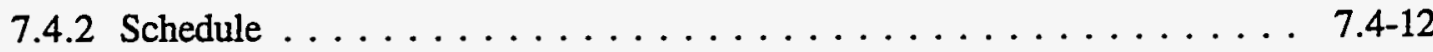

7.4.2.1 Waste Tank Safety Summary Schedule . . . . . . . . . . . 7.4-12

7.4.2.2 Waste Tank Safety Expanded Baseline

(FY 1995 - FY 1997) . . . . . . . . . . . . . . . . . . . 7.4-12

7.4 .3 Resource Requirements . . . . . . . . . . . . . . . 7.4-12

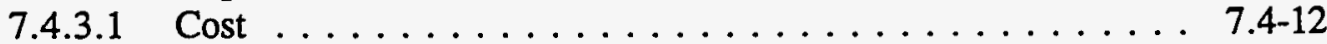

7.4.3.1.1 Basis of Cost Estimates . . . . . . . . . . 7.4-13

7.4.3.1.2 Total Program Element Cost by Year

by Fund Type by Activity and Cost Account) . . . . . . 7.4-13

7.4.3.1.3 FY 1995 Cost by Month by Fund Type

by Activity and Cost Account . . . . . . . . . . 7.4-13

7.4.3.2 Staffing .................... 7.4-13

7.4.3.2.1 Basis of Staffing Projections . . . . . . . . . 7.4-13

7.4.3.2.2 Total Full-Time Equivalents by Year by Activity by Major Participant (Direct and Indirect) . . . . . . . 7.4-14

7.4.3.2.3 FY 1995 through FY 1997 Direct

Full-Time Equivalents by 88 Labor Categories . . . . . . . 7.4-14

7.4.3.3 Work Scope Carryover . . . . . . . . . . . . . . . 7.4-14

7.4 .4 Waste Type Data . . . . . . . . . . . . . . . . . . . . . 7.4-14

7.4 .5 Revisions ...................... $7.4-14$ 


\section{CONTENTS (continued)}

7.5 TANK FARM UPGRADES BASELINE $\ldots \ldots \ldots \ldots \ldots \ldots \ldots \ldots \ldots \ldots$. $\ldots \ldots \ldots$

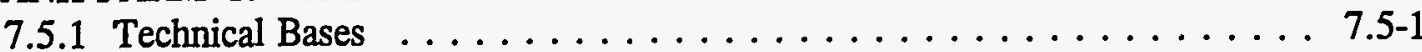

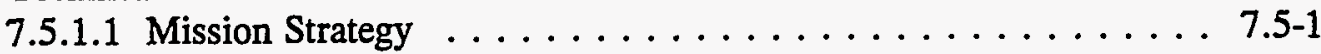

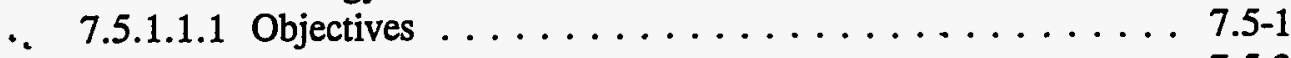

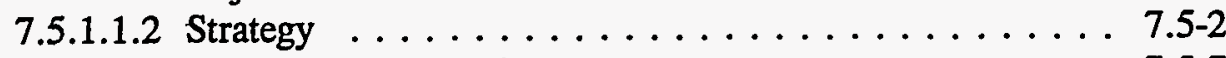

7.5.1.1.3 Functions and Requirements . . . . . . . . 7.5-7

7.5.1.1.4 Risks . . . . . . . . . . . . . . . 7.5-9

7.5.1.1.5 Major Decisions $\ldots \ldots \ldots \ldots \ldots \ldots \ldots \ldots . \ldots \ldots$. . . . . . .

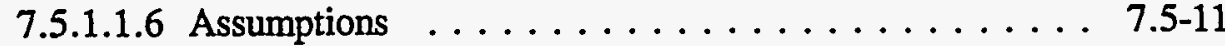

7.5.1.1.7 Constraints . . . . . . . . . . . 7.5-16

7.5.1.2 Structure .................... 7.5-19

7.5.1.2.1 Work Breakdown Structure . . . . . . . . . 7.5-19

7.5.1.2.2 Organization and Responsibility . . . . . . . . 7.5-20

7.5.1.3 FY 1995 Work to be Performed . . . . . . . . . . . . . . 7.5-20

7.5.1.4 FY 1996 - FY 1997 Work to be Performed . . . . . . . . . . . 7.5-28

7.5.1.5 Approach . . . . . . . . . . . . . . . . 7.5-33

7.5.1.6 Drivers and Deliverables ... . . . . . . . . 7.5-37

7.5 .2 Schedule . . . . . . . . . . . . . . . . . . . 7.5-41

7.5.2.1 Tank Farms Upgrades Summary Schedule . . . . . . . . . 7.5-41

7.5.2.2 Tank Farms Upgrades Expanded Baseline

(FY 1995 - FY 1997) . . . . . . . . . . . . . . . . . . . . 7.5-42

7.5 .3 Resource Requirements . . . . . . . . . . . . . . . . . 7.5-42

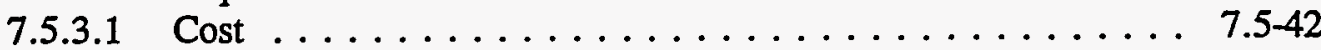

7.5.3.1.1 Basis of Cost Estimates . . . . . . . . . . 7.5-42

7.5.3.1.2 Total Program Element Cost by Year

by Fund Type by Activity and Cost Account) . . . . . . 7.5-42

7.5.3.1.3 FY 1995 Cost by Month by Fund Type

by Activity and Cost Account ............. 7.5-43

7.5.3.2 Staffing . . . . . . . . . . . . . . . 7.5-43

7.5.3.2.1 Basis of Staffing Projections . . . . . . . . . . 7.5-43

7.5.3.2.2 Total Full-Time Equivalents by Year by Activity

by Major Participant (Direct and Indirect) . . . . . . . . 7.5-43

7.5.3.2.3 FY 1995 through FY 1997 Direct Full-Time

Equivalents by 88 Labor Categories . . . . . . . . 7.5-43

7.5.3.3 Work Scope Carryover . . . . . . . . . . . . . 7.5-43

7.5 .4 Waste Type Data . . . . . . . . . . . . . . . . . . 7.5-44

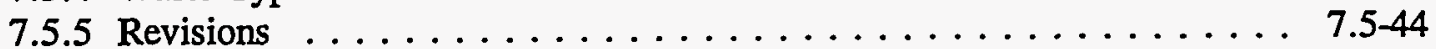




\section{CONTENTS (continued)}

7.6 CHARACTERIZATION BASELINE $\ldots \ldots \ldots \ldots \ldots \ldots \ldots \ldots \ldots \ldots .6 .6 \ldots \ldots$

7.6.1 Technical Bases . . . . . . . . . . . . . . . . . . . .

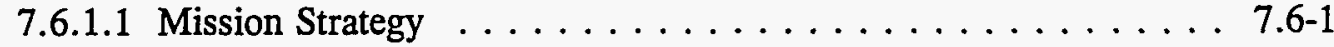

. 7.6.1.1.1 Objectives .................... 7.6-1

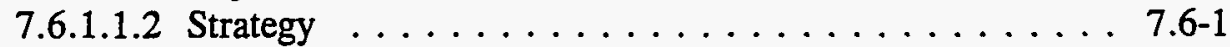

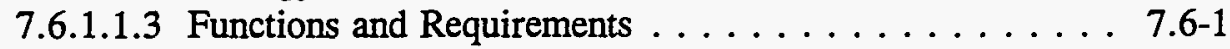

7.6.1.1.4 Risks . . . . . . . . . . . . . . . .6-2

7.6.1.1.5 Major Decisions . . . . . . . . . . . . 7.6-2

7.6.1.1.6 Assumptions $\ldots \ldots \ldots \ldots \ldots \ldots \ldots \ldots \ldots$ 7.6-2

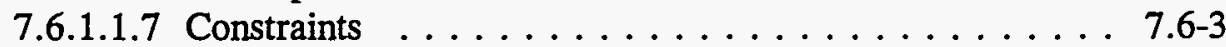

7.6.1.2 Structure $\ldots \ldots \ldots \ldots \ldots \ldots \ldots \ldots \ldots \ldots \ldots$ 7.6-3

7.6.1.2.1 Work Breakdown Structure . . . . . . . . . 7.6-3

7.6.1.2.2 Organization and Responsibility $\ldots \ldots \ldots \ldots \ldots$ 7.6-3

7.6.1.3 FY 1995 Work to be Performed . . . . . . . . . . . . 7.6-4

7.6.1.4 FY 1996 - FY 1997 Work to be Performed . . . . . . . . . 7.6-10

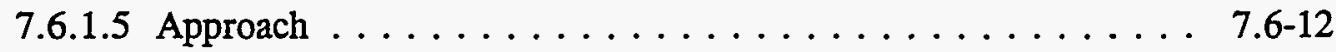

7.6.1.6 Drivers and Deliverables $\ldots \ldots \ldots \ldots \ldots \ldots \ldots \ldots$ 7.6-17

7.6 .2 Schedule . . . . . . . . . . . . . . . . . . 7.6-20

7.6.2.1 Characterization Summary Schedule . . . . . . . . 7.6-20

7.6.2.2 Characterization Expanded Baseline

(FY 1995 - FY 1997) . . . . . . . . . . . . . . . . 7.6-21

7.6.3 Resource Requirements . . . . . . . . . . . . . . . . 7.6-21

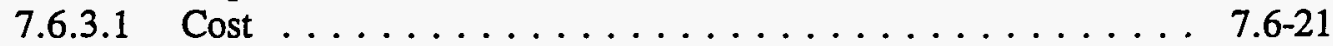

7.6.3.1.1 Basis of Cost Estimates . . . . . . . . . . . 7.6-21

7.6.3.1.2 Total Program Element Cost by Year

by Fund Type by Activity and Cost Account) $\ldots \ldots \ldots$ 7.6-21

7.6.3.1.3 FY 1995 Cost by Month by Fund Type

by Activity and Cost Account $\ldots \ldots \ldots \ldots \ldots$ 7.6-21

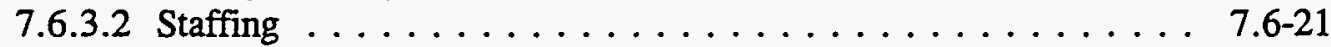

7.6.3.2.1 Basis of Staffing Projections . . . . . . . . . 7.6-21

7.6.3.2.2 Total Full-Time Equivalents by Year by Activity

by Major Participant (Direct and Indirect) $\ldots \ldots \ldots \ldots$ 7.6-22

7.6.3.2.3 FY 1995 through FY 1997 Direct Full-Time

Equivalents by 88 Labor Categories $\ldots \ldots \ldots \ldots \ldots$ 7.6-22

7.6.3.3 Work Scope Carryover . . . . . . . . . . . . . . 7.6-22

7.6 .4 Waste Type Data . . . . . . . . . . . . . . . . . . .

7.6.5 Revisions . . . . . . . . . . . . . . . . . . . . 


\section{CONTENTS (continued)}

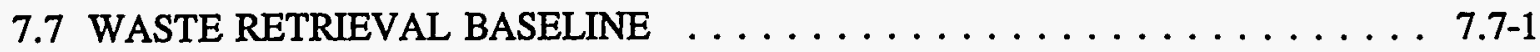

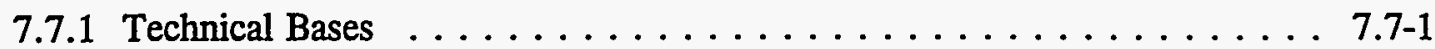

7.7.1.1 Mission Strategy $\ldots \ldots \ldots \ldots \ldots \ldots \ldots \ldots . \ldots \ldots$. . . . . . . . . . . .

. 7.7.1.1.1 Objectives $\ldots \ldots \ldots \ldots \ldots \ldots \ldots \ldots \ldots$. . . . . . .

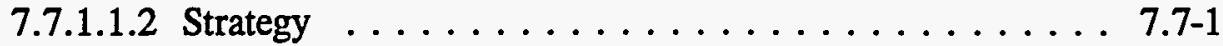

7.7.1.1.3 Functions and Requirements . . . . . . . . . 7.7-1

7.7.1.1.4 Risks . . . . . . . . . . . . . 7.7-2

7.7.1.1.5 Major Decisions $\ldots \ldots \ldots \ldots \ldots \ldots \ldots \ldots \ldots$ 7.7-2

7.7.1.1.6 Assumptions $\ldots \ldots \ldots \ldots \ldots \ldots \ldots \ldots \ldots$. . . . . . . . . . . .

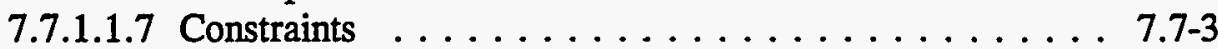

7.7.1.2 Structure . . . . . . . . . . . . . . . 7.7-3

7.7.1.2.1 Work Breakdown Structure ........... 7.7-3

7.7.1.2.2 Organization and Responsibility $\ldots \ldots \ldots \ldots . \ldots .7 .7 .4$

7.7.1.3 FY 1995 Work to be Performed . . . . . . . . . . . . . . 7.7-4

7.7.1.4 FY 1996 - FY 1997 Work to be Performed . . . . . . . . . . . 7.7-8

7.7.1.5 Approach . . . . . . . . . . . . . . . 7.7-12

7.7.1.6 Drivers and Deliverables . . . . . . . . . . . 7.7-13

7.7.2 Schedule . . . . . . . . . . . . . . . . . . 7.7-13

7.7.2.1 Waste Retrieval Summary Schedule . . . . . . . . . . 7.7-13

7.7.2.2 Waste Retrieval Expanded Baseline

(FY 1995 - FY 1997) . . . . . . . . . . . . . . . . 7.7-13

7.7.3 Resource Requirements . . . . . . . . . . . . . . . . 7.7-13

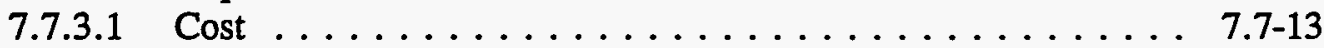

7.7.3.1.1 Basis of Cost Estimates . . . . . . . . . . 7.7-13

7.7.3.1.2 Total Program Element Cost by Year by Fund Type by Activity and Cost Account) . . . . . . . . . 7.7-13

7.7.3.1.3 FY 1995 Cost by Month by Fund Type by Activity and Cost Account . . . . . . . . 7.7-13

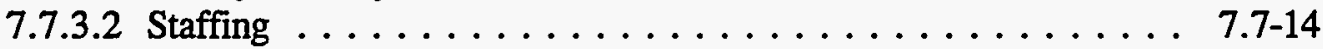

7.7.3.2.1 Basis of Staffing Projections . . . . . . . . . 7.7-14

7.7.3.2.2 Total Full-Time Equivalents by Year by Activity by Major Participant (Direct and Indirect) . . . . . . . . 7.7-14

7.7.3.2.3 FY 1995 through FY 1997 Direct Full-Time Equivalents by 88 Labor Categories ... . . . . . . . . . . 7-7-14

7.7.3.3 Work Scope Carryover . . . . . . . . . . . . . 7-7-14

7.7 .4 Waste Type Data . . . . . . . . . . . . . . . . . . 7-7-15

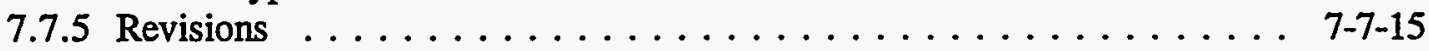


WHC-SP-1101

CONTENTS (continued)

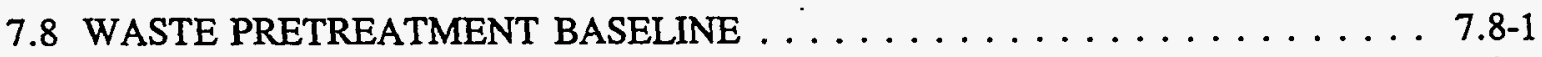

7.8.1 Technical Bases . . . . . . . . . . . . . . . . . 7.8-1

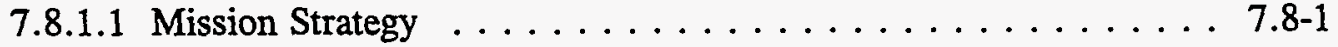

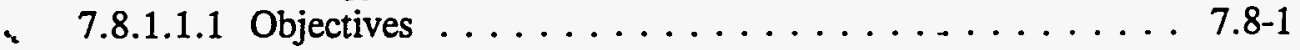

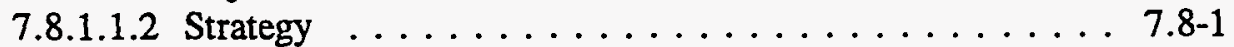

7.8.1.1.3 Functions and Requirements . . . . . . . . . 7.8-4

7.8.1.1.4 Risks . . . . . . . . . . . . . . 7.8-4

7.8.1.1.5 Major Decisions . . . . . . . . . . . 7.8-7

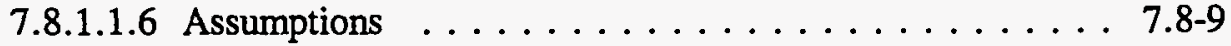

7.8.1.1.7 Constraints . . . . . . . . . . . 7.8-11

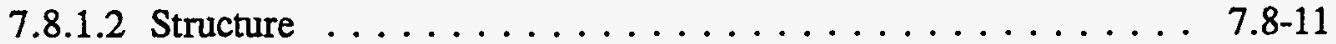

7.8.1.2.1 Work Breakdown Structure . . . . . . . . 7.8-11

7.8.1.2.2 Organization and Responsibility . . . . . . . 7.8-11

7.8.1.3 FY 1995 Work to be Performed . . . . . . . . . . . . . 7.8-13

7.8.1.4 FY 1996 - FY 1997 Work to be Performed . . . . . . . . . . . 7.8-15

7.8.1.5 Approach . . . . . . . . . . . . . . . 7.8-18

7.8.1.6 Drivers and Deliverables . . . . . . . . . . . . 7.8-19

7.8 .2 Schedule . . . . . . . . . . . . . . . . . . . 7.8-22

7.8.2.1 Waste Pretreatment Summary Schedule . . . . . . . . . 7.8-22

7.8.2.2 Waste Pretreatment Expanded Baseline

(FY 1995 - FY 1997) . . . . . . . . . . . . . . . . 7.8-22

7.8 .3 Resource Requirements . . . . . . . . . . . . . . . . 7.8-22

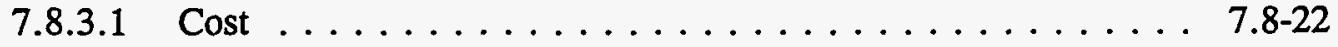

7.8.3.1.1 Basis of Cost Estimates . . . . . . . . . . 7.8-22

7.8.3.1.2 Total Program Element Cost by Year

by Fund Type by Activity and Cost Account) $\ldots \ldots \ldots$ 7.8-23

7.8.3.1.3 FY 1995 Cost by Month by Fund Type

by Activity and Cost Account . . . . . . . . . 7.8-23

7.8.3.2 Staffing . . . . . . . . . . . . . . . . 7.8-23

7.8.3.2.1 Basis of Staffing Projections . . . . . . . . . . 7.8-23

7.8.3.2.2 Total Full-Time Equivalents by Year by Activity by Major Participant (Direct and Indirect) . . . . . . . 7.8-23

7.8.3.2.3 FY 1995 through FY 1997 Direct

Full-Time Equivalents by 88 Labor Categories . . . . . . . . 7.8-24

7.8.3.3 Work Scope Carryover . . . . . . . . . . . . . . 7.8-24

7.8 .4 Waste Type Data . . . . . . . . . . . . . . . . . . . 7.8-25

7.8 .5 Revisions . . . . . . . . . . . . . . . 7.8-26 


\section{CONTENTS (continued)}

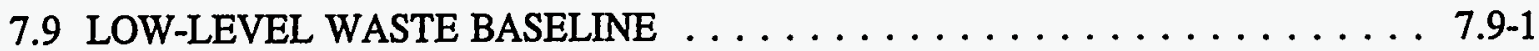

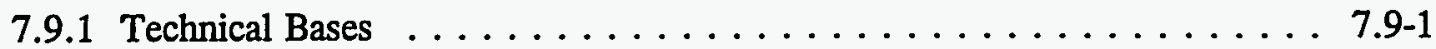

7.9.1.1 Mission Strategy $\ldots \ldots \ldots \ldots \ldots \ldots \ldots \ldots . \ldots \ldots$. . . . . . . . . . .

. 7.9.1.1.1 Objectives $\ldots \ldots \ldots \ldots \ldots \ldots \ldots \ldots \ldots \ldots . \ldots \ldots$ 7.9.1

7.9.1.1.2 Strategy $\ldots \ldots \ldots \ldots \ldots \ldots \ldots \ldots . \ldots \ldots$. . . . . . . . . . . .

7.9.1.1.3 Functions and Requirements . . . . . . . . . 7.9-2

7.9.1.1.4 Risks . . . . . . . . . . . . . . . . 7.9-3

7.9.1.1.5 Major Decisions $\ldots \ldots \ldots \ldots \ldots \ldots \ldots . \ldots \ldots$. . . . . . . . . . .

7.9.1.1.6 Assumptions $\ldots \ldots \ldots \ldots \ldots \ldots \ldots \ldots . \ldots \ldots$

7.9.1.1.7 Constraints . . . . . . . . . . . . 7.9-7

7.9.1.2 Structure . . . . . . . . . . . . . . . . 7.9-7

7.9.1.2.1 Work Breakdown Structure . . . . . . . . . . 7.9-8

7.9.1.2.2 Organization and Responsibility $\ldots \ldots \ldots \ldots \ldots$. . . . . . . . . . . .

7.9.1.3 FY 1995 Work to be Performed . . . . . . . . . . . . . . . 7.9-9

7.9.1.4 FY 1996 - FY 1997 Work to be Performed . . . . . . . . . . . 7.9-15

7.9.1.5 Approach . . . . . . . . . . . . . . . 7.9-19

7.9.1.6 Drivers and Deliverables $\ldots \ldots \ldots \ldots \ldots \ldots . \ldots \ldots$. . . . . . . . . . . . . .

7.9 .2 Schedule . . . . . . . . . . . . . . . . . . . . 7.9-22

7.9.2.1 Low-Level Waste Summary Schedule . . . . . . . . . . . 7.9-22

7.9.2.2 Low-Level Waste Expanded Baseline

(FY 1995 - FY 1997) . . . . . . . . . . . . . . . . 7.9-22

7.9.3 Resource Requirements . . . . . . . . . . . . . . . . . 7.9-22

7.9.3.1 Cost . . . . . . . . . . . . . . . . . 7.9-22

7.9.3.1.1 Basis of Cost Estimates . . . . . . . . . 7.9-22

7.9.3.1.2 Total Program Element Cost by Year

by Fund Type by Activity and Cost Account) $\ldots \ldots \ldots 7.9-23$

7.9.3.1.3 FY 1995 Cost by Month by Fund Type

by Activity and Cost Account . . . . . . . . . 7.9-23

7.9.3.2 Staffing . . . . . . . . . . . . . . . 7.9-23

7.9.3.2.1 Basis of Staffing Projections . . . . . . . . . 7.9-23

7.9.3.2.2 Total Full-Time Equivalents by Year by Activity by Major Participant (Direct and Indirect) . . . . . . 7.9-23

7.9.3.2.3 FY 1995 through FY 1997 Direct

Full-Time Equivalents by 88 Labor Categories . . . . . . . . 7.9-23

7.9.3.3 Work Scope Carryover . . . . . . . . . . . . . . . 7.9-24

7.9 .4 Waste Type Data . . . . . . . . . . . . . . . . . . . 7.9-24

7.9 .5 Revisions . . . . . . . . . . . . . . . . . 7.9-24 
WHC-SP-1101

\section{CONTENTS (continued)}

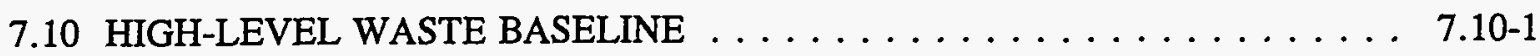

7.10 .1 Technical Bases . . . . . . . . . . . . . . . . . . . . . . . . . .

7.10.1.1 Mission Strategy . . . . . . . . . . . . . 7.10-1

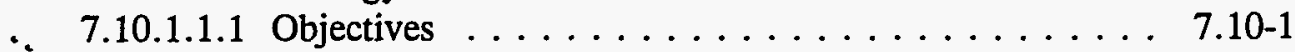

7.10.1.1.2 Strategy . . . . . . . . . . . . . . 7.10-1

7.10.1.1.3 Functions and Requirements . . . . . . . . . 7.10-1

7.10.1.1.4 Risks . . . . . . . . . . . . . . 7.10-2

7.10.1.1.5 Major Decisions $\ldots \ldots \ldots \ldots \ldots \ldots . \ldots \ldots$. . . . . . . . . . . . . . . . . . . .

7.10.1.1.6 Assumptions . . . . . . . . . . . . 7.10-7

7.10.1.1.7 Constraints . . . . . . . . . . . . 7.10-8

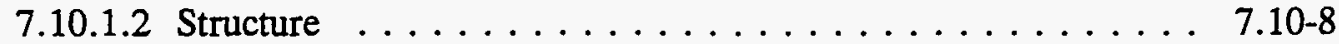

7.10.1.2.1 Work Breakdown Structure . . . . . . . . . 7.10-8

7.10.1.2.2 Organization and Responsibility . . . . . . . . . 7.10-8

7.10.1.3 FY 1995 Work to be Performed . . . . . . . . . . . . . . . . 7.10-9

7.10.1.4 FY 1996 - FY 1997 Work to be Performed . . . . . . . . . . . 7.10-10

7.10.1.5 Approach . . . . . . . . . . . . . . . . 7.10-10

7.10.1.6 Drivers and Deliverables ... . . . . . . . . . 7.10-11

7.10 .2 Schedule . . . . . . . . . . . . . . . . . . 7.10-12

7.10.2.1 High-Level Waste Summary Schedule . . . . . . . . . . 7.10-12

7.10.2.2 High-Level Waste Expanded Baseline

(FY 1995 - FY 1997) . . . . . . . . . . . . . . . . . 7.10-12

7.10 .3 Resource Requirements . . . . . . . . . . . . . . . 7.10-12

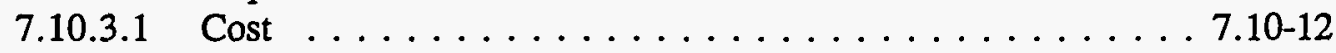

7.10.3.1.1 Basis of Cost Estimates . . . . . . . . . . 7.10-12

7.10.3.1.2 Total Program Element Cost by Year

by Fund Type by Activity and Cost Account) . . . . . . 7.10-13

7.10.3.1.3 FY 1995 Cost by Month by Fund Type

by Activity and Cost Account . . . . . . . . . 7.10-13

7.10.3.2 Staffing . . . . . . . . . . . . . . . 7.10-13

7.10.3.2.1 Basis of Staffing Projections . . . . . . . . . 7.10-13

7.10.3.2.2 Total Full-Time Equivalents by Year by Activity

by Major Participant (Direct and Indirect) . . . . . . . 7.10-13

7.10.3.2.3 FY 1995 through FY 1997 Direct

Full-Time Equivalents by 88 Labor Categories . . . . . . . 7.10-13

7.10.3.3 Work Scope Carryover . . . . . . . . . . . . . . . . 7.10-13

7.10 .4 Waste Type Data . . . . . . . . . . . . . . . . . 7.10-14

7.10 .5 Revisions . . . . . . . . . . . . . . . 7.10-14

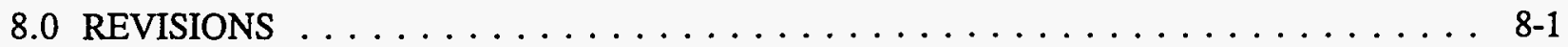

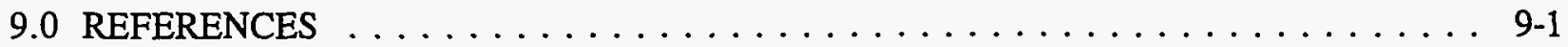




\section{CONTENTS (continued)}

\section{APPENDIXES}

VOLUME

A WBS INDEX AND DICTIONARY $\ldots \ldots \ldots \ldots \ldots \ldots \ldots \ldots \ldots$

B RESPONSIBILITY ASSIGNMENT MATRIX $\ldots \ldots \ldots \ldots \ldots \ldots \ldots$

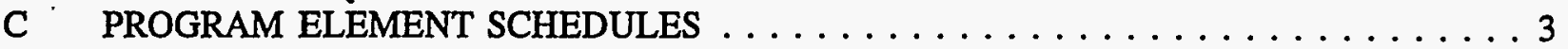

D MILESTONE LOG AND DESCRIPTION SHEETS $\ldots \ldots \ldots \ldots \ldots \ldots \ldots \ldots \ldots \ldots$

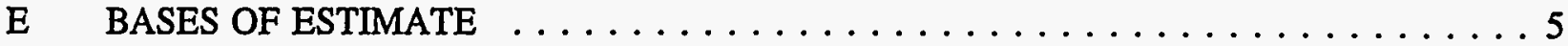

F MAJOR DECISIONS LOGIC/SCHEDULE $\ldots \ldots \ldots \ldots \ldots \ldots \ldots \ldots \ldots \ldots$

G INTEGRATED OPERATIONS SCHEDULE $\ldots \ldots \ldots \ldots \ldots \ldots \ldots \ldots$

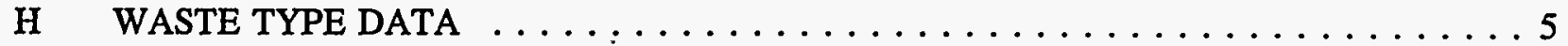

I PROGRAM MASTER BASELINE SCHEDULE $\ldots \ldots \ldots \ldots \ldots \ldots \ldots$ 
WHC-SP-1101

This page intentionally left blank.

xviii 
WHC-SP-1101

\section{LIST OF FIGURES}

2-1 Tank Waste Remediation System Program Logic . . . . . . . . . . . . . . . 2-2

2-2 Tank Waste Remediation System Program Summary Work Breakdown Structure . . . . 2-5

7.2-1 Risk of Inadequate Resource Availability in FY $1995 \ldots \ldots \ldots \ldots \ldots \ldots$. . . . . . F F.2-1

7.2-2 TWRS Program Management System Documẹnt Hierarchy $\ldots \ldots \ldots \ldots$. . . . . F7.2-3

7.2-3 Program Management and Administration Work Breakdown Structure . . . . . . . F7.2-4

7.2-4 Program Management and Administration Summary Schedule . . . . . . . . . . F7.2-5

7.2-5 The Baseline Pyramid $\ldots \ldots \ldots \ldots \ldots \ldots \ldots \ldots \ldots \ldots \ldots$ F7.2-6

7.3-1 Tank Farm Operations and Maintenance Work Breakdown Structure . . . . . . . . F7.3-1

7.3-2 Tank Farm Operations and Maintenance Summary Schedule . . . . . . . . . . F7.3-2

7.4-1 Organization Chart $\ldots \ldots \ldots \ldots \ldots \ldots \ldots \ldots \ldots \ldots \ldots \ldots \ldots \ldots$ F7.4-1

7.4-2 Waste Tank Safety Work Breakdown Structure . . . . . . . . . . . . . F7.4-2

7.4-3 Waste Tank Safety Responsibility Assignment Matrix . . . . . . . . . . . . . F7.4-3

7.4-4 Waste Tank Safety Work to be Performed FY 1995 through FY 1997 . . . . . . . . . F7.4-4

7.4-5 Waste Tank Safety Approach $\ldots \ldots \ldots \ldots \ldots \ldots \ldots \ldots \ldots \ldots$ F7.4-5

7.4-6 Waste Tank Safety Summary Schedule . . . . . . . . . . . . . . F7.4-6

7.4-7 Waste Tank Safety Cost $\ldots \ldots \ldots \ldots \ldots \ldots \ldots \ldots \ldots \ldots \ldots$ F . . . . . . . .

7.4-8 Waste Tank Safety Staffing $\ldots \ldots \ldots \ldots \ldots \ldots \ldots \ldots \ldots \ldots \ldots$ F . . . . . .

7.5-1 Tank Farm Upgrades Work Breakdown Structure . . . . . . . . . . . . . F7.5-1

7.5-2 Tank Farm Upgrades Summary Schedule . . . . . . . . . . . . . F F.5-3

7.6-1 Characterization Work Breakdown Structure . . . . . . . . . . . F7.6-1

7.6-2 Characterization Summary Schedule . . . . . . . . . . . . . . F7.6-2

7.7-1 Waste Retrieval Work Breakdown Structure . . . . . . . . . . . F7.7-1

7.7-2 Waste Retrieval Summary Schedule $\ldots \ldots \ldots \ldots \ldots \ldots \ldots \ldots \ldots$ F . . . . . . . .

7.8-1 TWRS Function Tree $\ldots \ldots \ldots \ldots \ldots \ldots \ldots \ldots \ldots \ldots \ldots$ F7.8 
WHC-SP-1101

LIST OF FIGURES (continued)

7.8-2 Waste Pretreatment Work Breakdown Structure . . . . . . . . . . . . . F7.8-2

7.8-3 Waste Pretreatment Summary Schedule $\ldots \ldots \ldots \ldots \ldots \ldots \ldots \ldots \ldots$ F7.8 $\ldots \ldots$

7.9-1 Low-Level Waste Work Breakdown Structure . . . . . . . . . . . . . . . . . F7.9-1

7.9-2 Low-Level Waste Summary Schedule . . . . . . . . . . . . . . . . F7.9-3

7.10-1 High-Level Waste Work Breakdown Structure . . . . . . . . . . . . . . F7.10-1

7.10-2 High-Level Waste Summary Schedule $\ldots \ldots \ldots \ldots \ldots \ldots \ldots$. . . . . . . F7.10-2 
WHC-SP-1101

\section{LIST OF TABLES}

2-1 Tri-Party Agreement Major Milestones for the Tank Waste Remediation

System Program . . . . . . . . . . . . . . . . . . 2-2

2-2 Top-Level Requirements Defining Tank Waste Remediation System

Technical Scope ........................ 2-5

4-1 Tank Waste Remediation System Summary Schedule . . . . . . . . . . . . . . 4-3

5-1 Total Program Cost by Year by Program Element $(\$ K) \ldots \ldots \ldots \ldots \ldots$

5-2 FY 1995 Cost by Month by Program Element . . . . . . . . . . . . . . . . 5-9

5-3 Total Full-Time Equivalents by Year by Program Element by Major

Participant through FY $2000 \ldots \ldots \ldots \ldots \ldots \ldots \ldots$. . . . . . . . . . . .

5-4 Tank Waste Remediation System Work Scope Carryover . . . . . . . . . . . . 5-11

7.2-1 Total Program Element Cost Baseline by Year $\ldots \ldots \ldots \ldots \ldots \ldots . \ldots \ldots$. . . . . . . .

7.2-2 FY 1995 Cost by Month by Fund Type by Activity and Cost Account . . . . . . 7.2-2

7.2-3 Total Full-Time Equivalents by Year by Activity by Major Participant

(Direct and Indirect( Including all Subcontractors) $\ldots \ldots \ldots \ldots \ldots . \ldots . . \ldots .2-3$

7.2-4 FY 1995 through 1997 Direct Westinghouse Hanford Company Full-Time

Equivalents by 88 Labor Categories . . . . . . . . . . . . . . 7.2-4

7.3-1 Total Program Element Cost Baseline by Year $\ldots \ldots \ldots \ldots \ldots \ldots$. . . . . . . .

7.3-2 FY 1995 Cost by Month by Fund Type by Activity and Cost Account . . . . . . . 7.3-2

7.3-3 Total Full-Time Equivalents by Year by Activity by Major Participant

(Direct and Indirect) (Including all Subcontractors) . . . . . . . . . . . 7.3-3

7.3-4 FY 1995 through 1997 Direct Westinghouse Hanford Company Full-Time

Equivalents by 88 Labor Categories $\ldots \ldots \ldots \ldots \ldots \ldots \ldots \ldots \ldots \ldots . . \ldots \ldots$

7.4-1 Total Program Element Cost Baseline by Year $\ldots \ldots \ldots \ldots \ldots \ldots$. . . . . . .

7.4-2 FY 1995 Cost by Month by Fund Type by Activity and Cost Account $\ldots \ldots$. . . 7.4-2

7.4-3 Total Full-Time Equivalents by Year by Activity by Major Participant

(Direct and Indirect) (Including all Subcontractors) . . . . . . . . . . 7.4-3

7.4-4 FY 1995 through 1997 Direct Westinghouse Hanford Company Full-Time

Equivalents by 88 Labor Categories $\ldots \ldots \ldots \ldots \ldots \ldots \ldots \ldots \ldots \ldots$ 
WHC-SP-1101

\section{LIST OF TABLES (continued)}

7.5-1 TWRS Organizational Breakdown $\ldots \ldots \ldots \ldots \ldots \ldots \ldots \ldots \ldots \ldots \ldots \ldots$

7.5-2 FY 1995 - 1997 Deliverables and Milestones for Project W-314 . . . . . . . . 7.5-2

7.5-3 Total Program Element C̣ost Baseline by Year $\ldots \ldots \ldots \ldots \ldots$. . . . . . . . .

7.5-4 FY 1995 Cost by Month by Fund Type by Activity and Cost Account $\ldots \ldots$. . . . 7.5-4

7.5-5 Total Full-Time Equivalents by Year by Activity by Major Participant

(Direct and Indirect) (Including all Subcontractors) . . . . . . . . . . 7.5-5

7.5-6 FY 1995 through 1997 Direct Westinghouse Hanford Company Full-Time

Equivalents by 88 Labor Categories . . . . . . . . . . . $6 \ldots \ldots$

7.6-1 Total Program Element Cost Baseline by Year $\ldots \ldots \ldots \ldots \ldots . \ldots \ldots$. . . . . .

7.6-2 FY 1995 Cost by Month by Fund Type by Activity and Cost Account $\ldots \ldots$. . . . 7.6-2

7.6-3 Total Full-Time Equivalents by Year by Activity by Major Participant

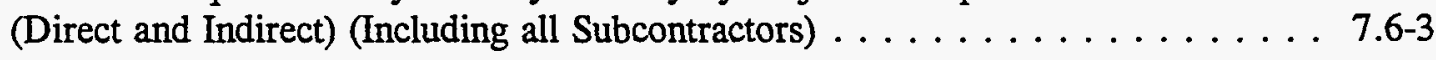

7.6-4 FY 1995 through 1997 Direct Westinghouse Hanford Company Full-Time

Equivalents by 88 Labor Categories . . . . . . . . . . . . . 7.6-4

7.7-1 Total Program Element Cost Baseline by Year $\ldots \ldots \ldots \ldots \ldots \ldots \ldots$. $\ldots \ldots$

7.7-2 FY 1995 Cost by Month by Fund Type by Activity and Cost Account . . . . . . 7.7-2

7.7-3 Total Full-Time Equivalents by Year by Activity by Major Participant

(Direct and Indirect) (Including all Subcontractors) . . . . . . . . . . 7.7-3

7.7-4 FY 1995 through 1997 Direct Westinghouse Hanford Company Full-Time

Equivalents by 88 Labor Categories . . . . . . . . . . . . 7.7-4

7.8-1 Total Program Element Cost Baseline by Year $\ldots \ldots \ldots \ldots \ldots \ldots . . \ldots \ldots$

7.8-2 FY 1995 Cost by Month by Fund Type by Activity and Cost Account $\ldots \ldots$. . . . 7.8-2

7.8-3 Total Full-Time Equivalents by Year by Activity by Major Participant

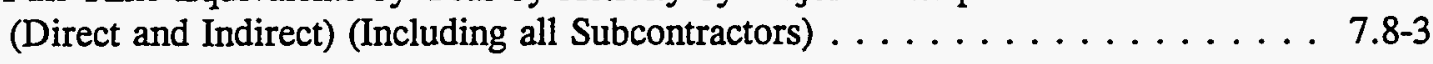

7.8-4 FY 1995 through 1997 Direct Westinghouse Hanford Company Full-Time

Equivalents by 88 Labor Categories . . . . . . . . . . . . 7.8-4

7.9-1 Total Program Element Cost Baseline by Year $\ldots \ldots \ldots \ldots \ldots \ldots . \ldots . . \ldots$. . . . .

7.9-2 FY 1995 Cost by Month by Fund Type by Activity and Cost Account . . . . . . 7.9-2 


\section{LIST OF TABLE (continued)}

7.9-3 Total Full-Time Equivalents by Year by Activity by Major Participant (Direct and Indirect) (Including all Subcontractors) . . . . . . . . . . . . 7.9-3

7.9-4 FY 1995 through 1997 Direct Westinghouse Hanford Company

Full-Time Equivalents by 88 Labor Categories . . . . . . . . . . . 7.9-4

7.10-1 Total Program Element Cost Baseline by Year . . . . . . . . . . . . 7.10-1

7.10-2 FY 1995 Cost by Month by Fund Type by Activity and Cost Account . . . . . . . 7.10-2

7.10-3 Total Full-Time Equivalents by Year by Activity by Major Participant

(Direct and Indirect) (Including all Subcontractors) . . . . . . . . . . . . 7.10-3

7.10-4 FY 1995 through 1997 Direct Westinghouse Hanford Company Full-Time

Equivalents by 88 Labor Categories . . . . . . . . . . . . . . . 7.10-4 


\section{WHC-SP-1101}

This page intentionally left blank.

xxiv 


\section{LIST OF TERMS}

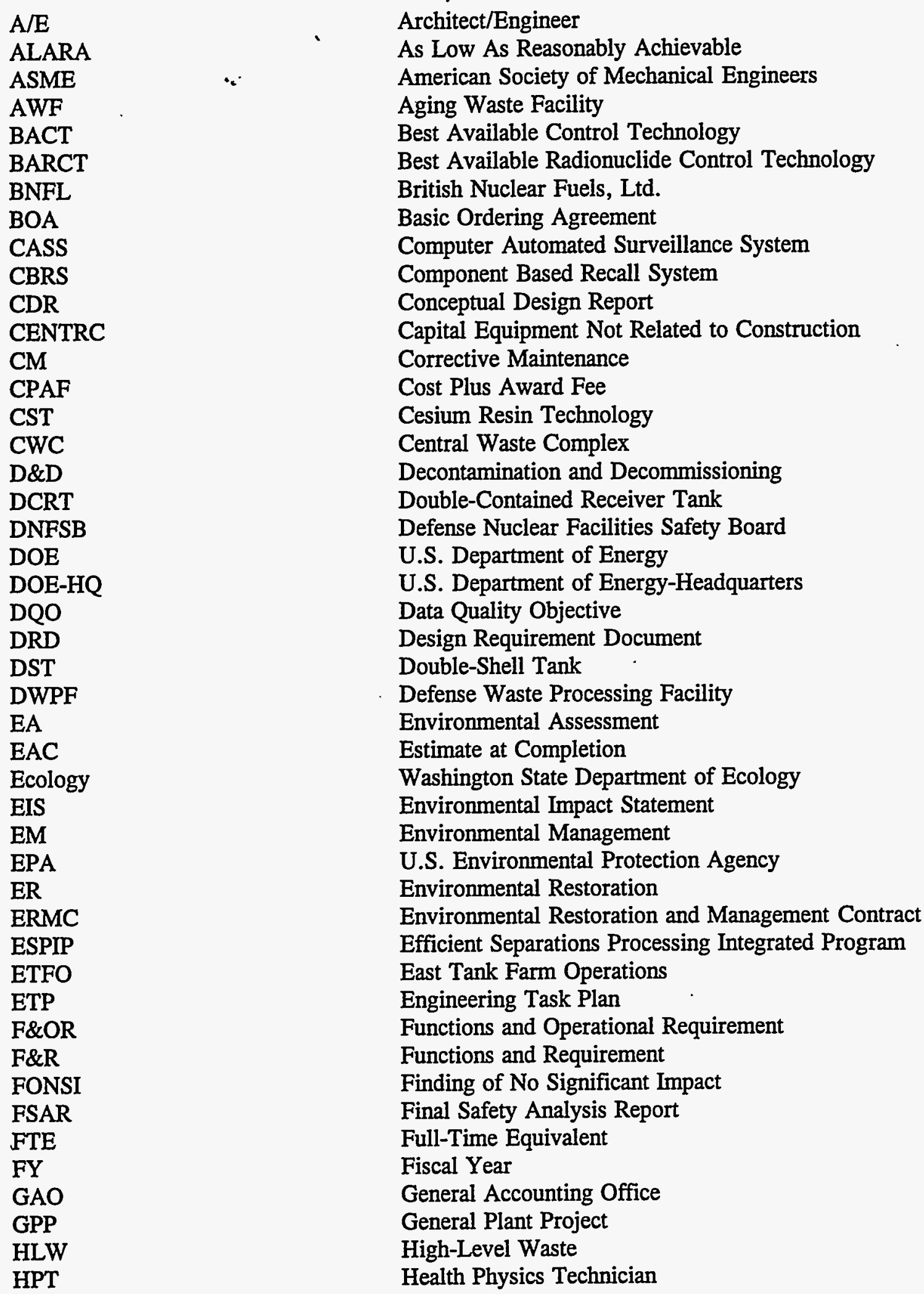




\section{LIST OF TERMS (continued)}

HTCE

HVAC

I\&C

ICF KH

ICP

ILLW

INEL

IOSR

IPM

ISB

ISEL

IX

JMN

LDUA

LERF

LETF

LIMS

LLCE

LLMW

LLW

LOI

LOW

M\&O

MCS

MPS

MSA

MWTF

MYWP

NCAW

NCR

NEPA

NOAS

NRC

O\&M

OCRWM

OSD

OSR

OTP

PA

PMP

PNL

PSAR

PSE

QA

QC

RAM
Historical Tank Contents Estimate

Heating, Ventilating, and Air Cond́ditioning

Instrumentation and Control

ICF Kaiser Hanford

Ion Coupled Plasma

Immobilized Low-Level Waste

Idaho National Engineering Laboratory

Interim Operational Safety Requirements

Initial Pretreatment Module

Interim Safety Basis

Interim Safety Equipment List

ion exchange

Justification of Mission Need

Light Duty Utility Arm

Liquid Effluent Retention Facility

Liquid Effluent Treatment Facility

Laboratory Information Management System

Long-Length Contaminated Equipment

Low-Level Mixed Waste

Low-Level Waste

Letter of Instruction

Liquid Observation Well

Management and Operating

Management Control System

Multi-Purpose Canister

Major System Acquisition

Multi-Function Waste Tank Facility

Multi-Year Program Plan

Neutralized Current Acid Waste

Non-Conformance Report

National Environmental Policy Act

Noxious Odor Advisory System

U.S. Nuclear Regulatory Commission

Operations and Maintenance

Office of Civilian Radioactive Waste Management

Operating Specification Document

Operating Safety Requirement

Operational Test Procedure

Performance Assessment

Project Management Plan

Pacific Northwest Laboratory

Preliminary Safety Analysis Report

Preliminary Safety Evaluation

Quality Assurance

Quality Control

Responsibility Assignment Matrix 


\section{LIST OF TERMS (continued)}

RCRA

RD\&D

RL

RMW

ROD

SACS

SDD

S/RID

SSC

SST

TCAP

TCD

TCR

TDPO

TEC

TMACS

TPC

Tri-Party Agreement

TRU

TSD

TWAP

TWRS

USQ

USTID

UT

WAC

WBS

WHC

WR

WTFO

WTSP

WVR
Resource Conservation and Recovery Act

Research, Development, and Demonstration

U.S. Department of Energy-Richland Operations Office

Radioactive Mixed Waste

Record of Decision

Surveillance Analysis Computer System

System Design System

Standards/Requirements Identification Document

Structures, Systems, and Components

Single-Shell Tank

Tank Characterization Advisory Panel

Tank Characterization Database

Tank Characterization Report

Technology Development Program Office

Total Estimated Cost

Tank Monitoring and Control System

Total Project Cost

Hanford Federal Facility Agreement and Consent Order

Transuranic

Treatment, Storage, and Disposal

Tank Waste Analysis Plan

Tank Waste Remediation System

Unreviewed Safety Question

Underground Storage Tank Integrated Demonstration

Ultrasonic Testing

Washington Administrative Code

Work Breakdown Structure

Westinghouse Hanford Company

Waste Retrieval

West Tank Farm Operations

Waste Tank Safety Program

Waste Volume Reduction 
WHC-SP-1101

This page intentionally left blank.

xxviii 


\subsection{VISION AND MISSION}

The Tank Waste Remediation System (TWRS) Program is described by two elements, the Program Vision and the Program Mission. The Program Vision defines the desired end state of the Program along with its primary. policies. The Program Mission is a statement of the Program's purpose. The vision and mission combine to provide the envelope within which the Program Strategy is developed.

\subsection{VISION}

The TWRS Program Vision represents the desired end state for the remediation of tank wastes, as expressed by the stakeholders. The TWRS Program Vision is to clean up the Hanford tank wastes by the year 2028 and complete shipment of waste capsules to an offsite geologic repository by the year 2040. At the end of the Program, the TWRS facilities and systems will be ready for turnover to the agency responsible for the decommissioning, decontamination, and disposition processes. This vision will be achieved through the safe retrieval, treatment, and immobilization of the wastes in the tanks and the eventual disposal of the high-level wastes in a geologic repository.

\subsection{MISSION}

The TWRS Program Mission represents the stakeholders' desires for implementing the TWRS Program Vision. The mission of the TWRS Program is to store, treat, and immobilize the highly radioactive Hanford Site waste in a safe, environmentally sound, and cost-effective manner. Highly radioactive Hanford waste is defined as current and future tank waste and the cesium/strontium capsules.

To achieve this mission the TWRS Program will be dedicated to the minimization of radiological impacts to onsite and offsite personnel and the environment and the safe operation of the Site and TWRS facilities. The Program will be managed to minimize or eliminate the inherent risks by providing a safer work place, improve the management system so the Program is under managerial and financial control, become more outcome oriented, and develop a stronger partnership with all Program interested parties (e.g., U. S. Department of Energy [DOE], regulatory agencies, and other stakeholders). 
WHC-SP-1101

This page intentionally left blank. 


\subsection{MISSION STRATEGY}

The proposed process flow for the TWRS Program is depicted on Figure 2-1. This proposed flow forms the basis for the Program Mission Strategy. This section describes the connection of the various elements that make up the Mission Strategy: objectives, strategy, functions and requirements, risks, decisions, assumptions, and constraints.

The Mission Strategy is designed to integrate the major Program Objectives into a cohesive whole to ensure that these objectives are reached in the most cost-effective manner. The Program Objectives provide the framework for the Program Strategy, which in turn provides sufficient detail to ensure that all the Program's Functions have been accounted for and that the associated requirements have been considered and met.

Program-wide integration and planning have identified a number of Program uncertainties. To reduce or eliminate these uncertainties, certain Program decisions must be made. To make these decisions requires making some assumptions. Assumptions cover areas where there is presently a lack or shortage of information and provide the bridges necessary to make informed decisions. Program-wide integration has also identified a number of Program constraints. Constraints range from programmatic through the full gamut of technical issues. Typical constraints include funding, technological development, and inherent limits to technical processes.

These preceding subjects are addressed at a summary level in the following subsections. Specific details for each program element are contained in the appropriate subsection of Section 7.

\subsection{OBJECTIVES}

The seven main objectives of the TWRS Program are listed below.

1. Operate all facilities and store existing and future wastes in a safe and environmentally sound manner.

2. Provide the technologies necessary to achieve the Program's mission in a timely and costeffective manner.

3. Process the wastes in keeping with established environmental, safety, and budgetary guidelines.

4. Continually improve the Program's involvement with the public.

5. Enhance the Program's management and technical staff capabilities.

6. Develop an effective risk management and decision making process.

7. Ensure continued compliance to all applicable Federal, State, and local regulations.

These Program objectives form the nucleus around which the Program's strategy is developed. 
Figure 2-1. Tank Waste Remediation System Program Logic.

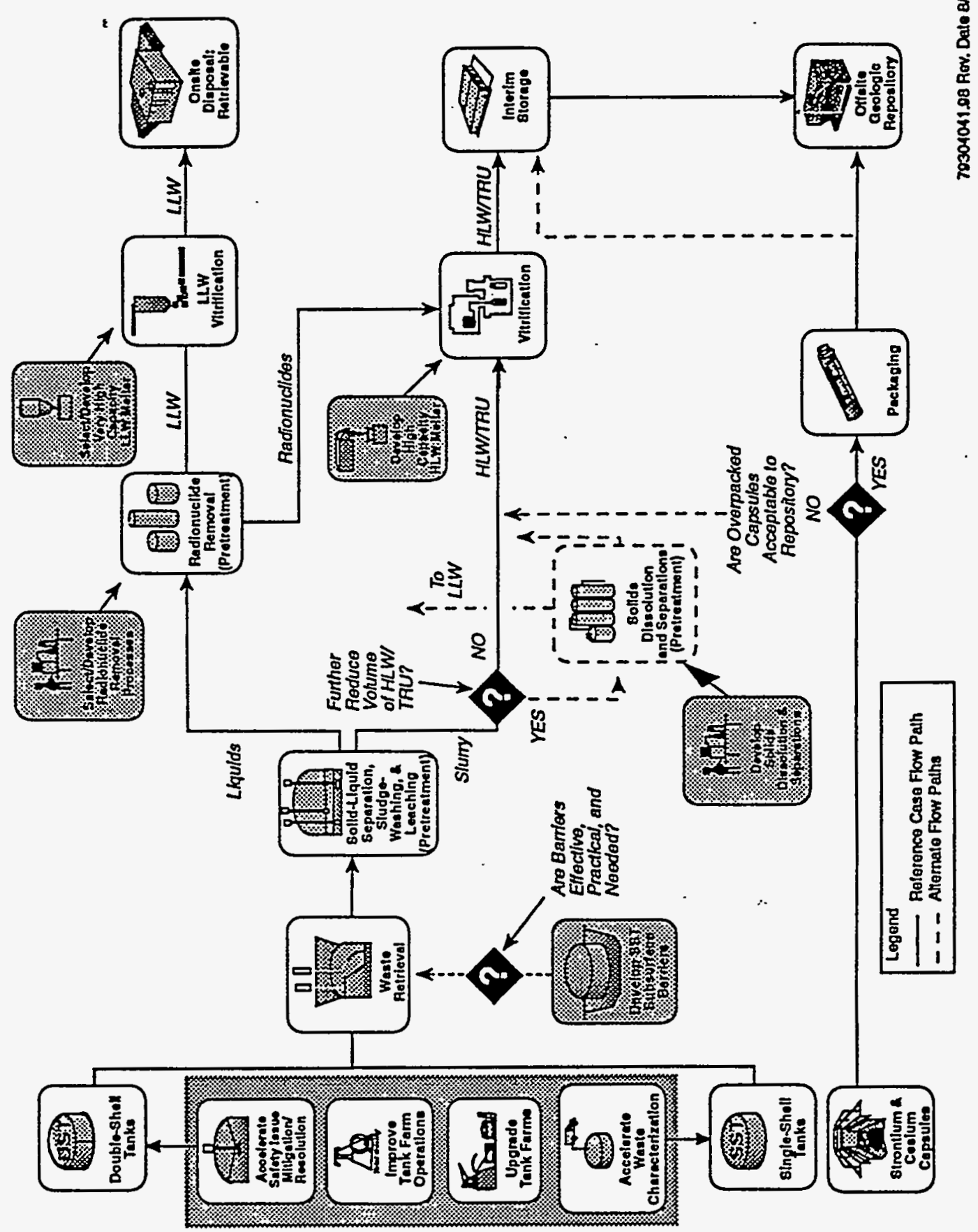


A summary follows of each program element's objectives, compiled from the program element descriptions in Section 7.

1. Program Management and Administration. Improve management and control capabilities.

2. Tank Farm Operations and Maintenance. Provide safe operations of tanks; establish a final safety bases in fiscal year (FY) 2001; and prepare tanks for closure.and turnover.

3. Waste Tank Safety. Mitigate and solve waste tank safety issues.

4. Tank Farm Upgrades. Provide enhancements for the safe operations of the tanks; replace deficient equipment and instrumentation.

5. Characterization. Provide tank waste characterization data in a timely and cost-effective manner.

6. Waste Retrieval. Remove wastes from aging single-shell tanks and transfer to double-shell tanks; mobilize sludges in double-shell tanks for in-tank treatment or transfer to treatment facilities; and retrieve wastes from miscellaneous underground tanks.

7. Waste Pretreatment. Separate tank wastes into appropriate streams for low-level waste and high-level waste; construct pretreatment facility.

8. Low-Level Waste. Vitrify all low-level wastes by the end of 2028; maintain Grout Facility in standby condition.

9. High-Level Waste. Vitrify all high-level waste, and store the immobilized waste until shipped to a Federal geologic repository.

\subsection{STRATEGY}

The TWRS Program strategy is the selected path for achieving the Program's objectives and for converting the Program's functions into reality. The Program's strategy consists of the following three main elements: technical strategy, management strategy, and acquisition strategy. The Program's strategy is consistent with the Secretary of Energy's complex-wide strategy as well as the more focused Hanford Mission Plan. The TWRS Program's strategy is incorporated in Program approaches, policies, and processes, and is reflected in the Program baseline. Figure 2-1 provides a schematic process flow which demonstrates the Program's strategy.

A summary follows of each program element's strategy, compiled from the program element descriptions in Section 7.

1. Program Management and Administration. Use systems engineering to define and develop the Program's baseline; take actions to improve the data supporting the Program's baseline.

2. Operations and Maintenance. Improve conduct of operations; expand evaporation capability; expedite closure of single-shell tanks; and shut down and turn over all tank farm facilities. 
3. Waste Tank Safety. Improve the safety mitigation and solution process; accelerate implementation through streamlining the review process; improve the management of the safety issue tanks; and incorporate improved technology development and applied engineering practices.

4. Tank Farm Upgrades. Improve the tank farm infrastructure through implementation of planned projeets (e.g., W-030, W-058, W-028, and W-188); and improve the technical integration and planning processes.

5. Characterization. Establish and implement the Data Quality Objectives process; increase sampling and analytical laboratory capabilities.

6. Waste Retrieval. Utilize known technical solutions to the maximum extent possible (e.g., Savannah River mixer pumps); develop and implement new technologies as required.

7. Waste Pretreatment. Develop and implement a phased program for the design and construction of pretreatment facilities; minimize the volume of the high-level waste fraction; and maximize the radionuclides inventory of the high-level waste fraction.

8. Low-Level Waste. Utilize commercially available melter and other key processing technologies as much as possible; complete the vitrification of most low-level waste within 3 years of the completion of single-shell tank retrieval.

9. High-Level Waste. Vitrify the high-level waste; and place the vitrified waste in stainless steel canisters.

\subsection{FUNCTIONS AND REQUIREMENTS}

The upper-level functions and requirements for the TWRS Program have been defined using a systems engineering approach. This systems engineering approach will continue to be used to provide ever more detailed levels of functions and requirements down to operational activities. A detailed exhibit of the Program's functions and requirements is contained in Tank Waste Remediation System Functions and Requirements (DOE/RL-92-60).

The Program's functions and requirements are mapped to the Program's work breakdown structure (WBS), thereby providing yet another crosscut of the Program. The TWRS Program Summary WBS is shown on Figure 2-2. The TWRS Program WBS Dictionary is provided in Appendix A.

\subsection{RISKS}

Several significant risks currently exist in the TWRS Program as a result of program uncertainties. Uncertainties range from technical issues to resource estimates to schedules and include interfaces with other government entities.

Technical uncertainties are typified by the lack of knowledge concerning the tank waste characteristics and the implications that has on safe tank operations as well as the processing of the wastes for immobilization and disposal. 


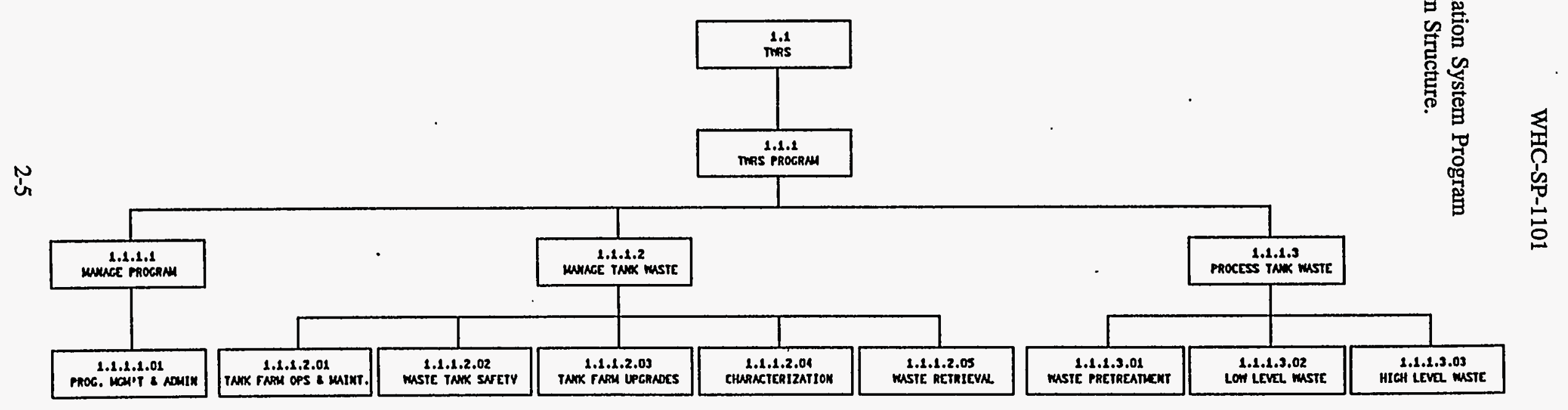

ORCPL USWEM / 09/24/98 
The primary uncertainties having to do with resource estimates revolve around the lack of present day knowledge on the exact processes and facilities that will be required to accomplish the Program's mission as well as when much of this work will be done. The further out in the future work is done, the more uncertain any estimate of required resources will be.

The schedule uncertainties are really a reflection of the inexactitude of estimates of resources required for future actions. As. knowledge of the Program is increased, the preciseness of estimates will improve, which will lead in turn to less uncertainty in the Program's schedules.

Interfaces with other government entities entail uncertainty because these other entities need not consult with the DOE before changing requirements applicable to the TWRS Program. An example of an ongoing interface uncertainty is the Program's dependence on the U.S. Nuclear Regulatory Commission to make a.ruling on the waste type classification to be used on any low-level waste generated by the processing of high-level waste.

Some of these uncertainties are pointed up on Figure 2-1 by the large questions marks contained on the figure.

A summary follows of each program element's uncertainties/risks, compiled from the program element descriptions in Section 7.

1. Program Management and Administration. Uncertainty as to the TWRS Program baseline documentation requirements, uncertainty associated with estimating resource requirements and developing schedules for presently fuzzily defined activities.

2. Tank Farm Operations and Maintenance. Uncertainty as to future demands for tank storage space; uncertainty as to the need for cross site transfer lines.

3. Waste Tank Safety. Uncertainty as to the characteristics and composition of the wastes in the tanks, uncertainty of the ability to achieve the level of technical competence required within available funding and schedule constraints.

4. Tank Farm Upgrades. Uncertainty as to future usefulness of installed electronic instrumentation and controls; uncertainty as to funds available for implementing upgrade projects; and uncertainties as to the documentation required by the systems engineering process.

5. Characterization. Uncertainty as to availability of access to tanks.

6. Waste Retrieval. Uncertainty as to ability to resolve tank safety questions sufficiently to allow retrieval; uncertainty as to closure requirements and if a single retrieval system can meet those closure requirements; uncertainty as to ability to complete retrieval of all singleshell tanks by the end of 2018; and uncertainty as to the need to remove technetium.

7. Waste Pretreatment. Lack of a Performance Assessment for near-surface dispoal of lowlevel waste; lack of a definitive limit on the amount of Hanford high-level waste, destined for geologic disposal, considered to be "reasonable." 
8. Low-Level Waste. Uncertainty as to the resource requirements developed in concert with the Hanford Federal Facility Agreement and Consent Order (Tri-Party Agreement) negotiations; uncertainties as to the availability of sufficient funds to implement the necessary actions to comply with Tri-Party Agreement milestones; and uncertainty as to the ability of commercially available melters to meet the program requirements.

9. High-Level Waste. Uncertainty as to the adequacy of TWRS Program integration of requirements and constraints; uncertainty as to the specifications for melter feed and repository acceptance.

\subsection{MAJOR DECISIONS}

Decisions are required to accomplish each step of the Program's strategy, to mitigate each Program uncertainty, and to establish each Program assumption. Some of the Program's major decision points are highlighted on Figure 2-1, and schedule for actions necessary to make all known major decisions is contained in Appendix F.

A summary follows of each program element's major decisions, compiled from the program element descriptions in Section 7.

1. Program Management and Administration. Determine disposition of Hanford tank waste (Decision 4.2); completion of the Environmental Impact Statement is required to make Decision 4.2.

2. Tank Farm Operations and Maintenance. Determine tank waste management strategy (Decision 4.2.1); determine double-shell storage space required to support TWRS activities (Decision 4.2.1.1.a); determine single-shell tank storage concept (Decision 4.2.1.1.b); determine waste concentration mechanism (Decision 4.2.1.5); determine excess facilities interface requirement (Decision 4.2.3.5); determine recycle/reuse concept (Decision 4.2.3.6)

3. Waste Tank Safety. Determine the functional responsibility for miscellaneous underground storage tanks.

4. Tank Farm Upgrades. Determine the need and priority for the Tank Farm Upgrades Program; pursue Project W-314 as a single project rather than four separate projects.

5. Characterization. Use historical tank waste characterization information where available.

6. Waste Retrieval. Determine waste transfer mechanism(s); determine excess facilities interface requirements.

7. Waste Pretreatment. Determine waste separations process; select supernate pretreatment facility concept.

8. Low-Level Waste. Determine low-level waste form and processes, determine whether immobilized low-level waste can be disposed of in the 200 Areas; determine if the Nuclear Regulatory Commission supports disposal of SST incidental waste onsite; and determine immobilized low-level waste disposal configuration. 
9. High-Level Waste. Determine acceptability of alternative containers for the Federal geologic repository; determine disposition of cesium and strontium capsules; determine if transuranic (TRU) and high-level wastes can be processed in same facility; determine high-level waste vitrification facility concept; and determine the disposal container configuration.

\subsection{ASSUMPTIONS}

Assumption are necessary to bridge gaps in Program knowledge. Assumptions associated with technology, regulations, required resources, and future conditions must be made if any reasonable planning, long-range or short-range, is to be made. The TWRS Program's major assumptions have to do with programmatic and technical knowledge gaps.

The following are TWRS Program major programmatic assumptions.

1. The mission of the TWRS Program will not be appreciably changed.

2. The funding necessary to accomplish the mission will be made available in a timely manner.

3. The safety and health of the public and workers will continue to be the highest priority.

The following are TWRS Program major technical assumptions.

1. The technologies necessary to achieve the Program's mission will be available when needed at a reasonable cost.

2. All tank waste, including the cesium and strontium capsules, will be retrieved for treatment and disposal.

3. The tank waste will be separated into high-level and low-level activity fractions.

4. Glass will be the immobilization medium for both high-level and low-level waste fractions.

5. Tank safety issues will be resolved and not impede the treatment and disposal of the wastes.

A summary follows of each program element's assumptions, compiled from the program element descriptions in Section 7.

1. Program Management and Administration. Program Management and Administration will achieve its objectives; the Environmental Impact Statement will be developed within 24 months, as directed by the Secretary of Energy; and the Program's baseline will not be changed as a result of future Management and Operating (M\&O) contract negotiations.

2. Tank Farm Operations and Maintenance. A fifth shift will be established; on-shift engineers will be provided; interim stabilization of the single-shell tanks will be complete by September 2000; six new double-shell tanks will be constructed; and safety issues will be resolved to allow single-shell pumping. 
3. Waste Tank Safety. No new major safety issues will be identified that will require a diversion of resources; the structural integrity of the waste tanks will not be found wanting; safety issues will be mitigated or resolved; and the contents of the Watch List tanks will be characterized by September 1995.

4. Tank Farm Upgrades. Systems engineering will validate the need for proposed upgrades to the tank farmsi the final safety basis of the waste tanks will not appreciably change from that presently defined.

5. Characterization. Tank safety concerns will not prevent sampling of the tanks; an adequate number of tank access spots meeting the requirements of the Data Quality Objectives program will be available; and historical information on tank waste contents will provide suitable information on the key analytes.

6. Waste Retrieval. Waste retrieval will be required for all single-shell tanks and the identified miscellaneous underground storage tanks; two mixer pumps will successfully mobilize all sludge in each double-shell tank; and sluicing will not provide all the capabilities to retrieve the single-shell tank waste in conformance with the Tri-Party Agreement objectives.

7. Waste Pretreatment. Current characterization information is sufficient as a basis for pretreatment planning; sludge washing, leaching, and blending will be adequate to achieve an acceptably low high-level waste volume and at the same time meet high-level waste feed composition requirements.

8. Low-Level Waste. Vitrified low-level waste can be disposed of in the 200 area; the Nuclear Regulatory Commission will concur with the U.S. Department of Energy plans for treatment and disposal of the single-shell tank waste; other program elements will proceed or a schedule that supports Low-Level Waste activities; and low-level waste vitrification will be complete by 2022 .

9. High-Level Waste. The high-level waste vitrification plant will start hot operations in 2009 .

\subsection{CONSTRAINTS}

Major constraints to the TWRS Program include milestones contained in the Tri-Party Agreement; the projected availability of funding; and regulatory requirements. A listing of Tri-Party Agreement milestones is shown on Table 2-1, and a listing of top-level Program requirements is presented in Table 2-2. A more detailed listing and description of Program constraints is contained in Tank Waste Remediation System Functions and Requirements (DOE/RL-92-60).

A summary follows of each program element's constraints, compiled from the program element descriptions in Section 7.

1. Program Management and Administration. Procurement by the U.S. Department of Energy of the Environmental Impact Statement contractor.

2. Tank Farm Operations and Maintenance. The Tri-Party Agreement schedule milestones and the present approved funding profile. 
3. Waste Tank Safety. Evaluation and definition of safety issues.

4. Tank Farm Upgrades. The present approved funding profile, acquisition regulations, and lack of sufficient staff to manage or procure work.

5. Characterization. Tri-Party Agreement milestones; safety consideration as to accessibility of tanks.

6. Waste Retrieval. Tri-Party Agreement milestones; safety considerations as to accessibility of tanks; lack of an overall integrated TWRS physical model.

7. Waste Pretreatment. Lack of an established Performance Assessment document; lack of an established limit on the volume of high-level waste to be sent to the Federal geologic repository.

8. Low-Level Waste. Regulatory constraints, in particular the 40 Code of Federal Regulations (CFR) 200 series of regulations.

9. High-Level Waste. The ability to successfully start up the high-level waste vitrification plant with only limited test data from which to develop a design. 
Table 2-1. Tri-Party Agreement Major Milestones for the Tank Waste Remediation System Program.

\begin{tabular}{|c|c|}
\hline 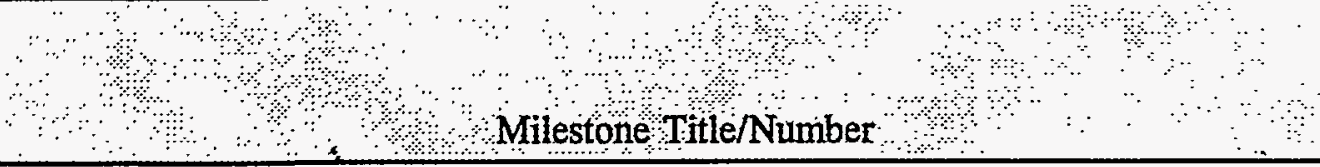 & $\begin{array}{l}\text { Scheduled } \\
\text { Completion } \\
\because \text { Date }\end{array}$ \\
\hline Mitigate/resolve tank safety issues / M-40-00 & $9 / 2001$ \\
\hline Complete single-shell tank interim stabilization / M-41-00 & $9 / 2000$ \\
\hline Provide additional double-shell tank capacity / M-42-00 & $12 / 1998$ \\
\hline Complete tank farm upgrades / M-43-00 & $6 / 2005$ \\
\hline $\begin{array}{l}\text { Tank Waste Characterization / M- } 44-00 \\
\text { - Issue Tank Characterization Reports for all single- and double-shell tanks } \\
\text { (177) }\end{array}$ & 9/1999 \\
\hline $\begin{array}{l}\text { Complete closure of all single-shell tank farms }(M-45-00) \\
\text { - Complete evaluation and demonstration testing of small scale sub-surface } \\
\text { barriers } \\
\text { - Initiate full-scale demonstration of waste retrieval } \\
\text { - } \quad \text { Initiate tank waste retrieval from one single-shell tank } \\
\text { - } \quad \text { Complete waste retrieval from all single-shell tanks }\end{array}$ & $\begin{array}{l}9 / 2024 \\
9 / 1997 \\
10 / 1997 \\
12 / 2003 \\
9 / 2018\end{array}$ \\
\hline Double-shell tank space evaluation (M-46-00A) & $\begin{array}{l}9 / 1994 \\
\text { (annually } \\
\text { thereafter) }\end{array}$ \\
\hline $\begin{array}{cc}\text { Complete pretreatment processing of Hanford tank waste (M-50-00) } \\
\text { - } & \text { Start construction of Low-Level Waste Pretreatment Facility } \\
\text { - } & \text { Start hot operations of Low-Level Waste Pretreatment Facility } \\
\text { - } & \text { Start hot operation of High-Level Waste Pretreatment Facility }\end{array}$ & $\begin{array}{l}12 / 2028 \\
11 / 1998 \\
12 / 2004 \\
6 / 2008\end{array}$ \\
\hline $\begin{array}{c}\text { Complete vitrification of Hanford high-level tank waste }(M-51-00) \\
\text { - Initiate construction of the High-Level Waste Vitrification Facility } \\
\text { - } \quad \text { Initiate hot operations of the High-Level Waste Vitrification Facility }\end{array}$ & $\begin{array}{l}12 / 2028 \\
6 / 2002 \\
12 / 2009\end{array}$ \\
\hline $\begin{array}{cl}\text { Complete vitrification of Hanford low-level tank waste }(\mathrm{M}-60-00) \\
\text { - } & \text { Select reference melter } \\
\text { - } & \text { Initiate construction of the Low-Level Waste Vitrification Facility } \\
\text { - } & \text { Initiate hot operations of the Low-Level Waste Vitrification Facility }\end{array}$ & $\begin{array}{l}12 / 2028 \\
6 / 1996 \\
12 / 1997 \\
6 / 2005\end{array}$ \\
\hline
\end{tabular}




\begin{tabular}{|c|c|c|}
\hline \multicolumn{3}{|c|}{$\begin{array}{l}\text { Table 2-2 } \\
\text { Top-Level Requirements Defining TWRS Technical Scope }\end{array}$} \\
\hline $\begin{array}{l}\text { FUNCTION OR } \\
\text { INPUT / OUTPUT }\end{array}$ & REQUIREMENT & SOURCE DOCUMENT \\
\hline REMEDIATE TANK WASTE & $\begin{array}{l}\text { "...the exposure of members of the public to radiation } \\
\text { sources as a consequence of all routine DOE activities shall } \\
\text { not cause, in a year, an effective dose equivalent greater } \\
\text { than } 100 \text { mrem." }\end{array}$ & $\begin{array}{l}\text { DOE Order 5400.5, Radiation Protection of the Public and } \\
\text { the Environment, Chapter I, 1.a }\end{array}$ \\
\hline REMEDIATE TANK WASTE & $\begin{array}{l}\text { "...the exposure of members of the public to radioactive } \\
\text { materials released to the atmosphere as a consequence of } \\
\text { routine DOE activities shall not cause members of the public } \\
\text { to receive, in a year, an effective dose equivalent greater } \\
\text { than } 10 \text { mrem." }\end{array}$ & $\begin{array}{l}\text { DOE Order 5400.5, Radiation Protection of the Public and } \\
\text { the Environment, Chapter II, 1.b }\end{array}$ \\
\hline REMEDIATE TANK WASTE & $\begin{array}{l}\text { "...provide a level of protection for persons consuming } \\
\text { water from a public drinking supply... these systems shall } \\
\text { not cause persons consuming the water to receive an } \\
\text { effective dose equivalent greater than } 4 \text { mrem in a year." }\end{array}$ & $\begin{array}{l}\text { DOE Order 5400.5, Radiation Protection of the Public and } \\
\text { the Environment, Chapter II, 1.d }\end{array}$ \\
\hline REMEDIATE TANK WASTE & $\begin{array}{l}\text { Federal Facility Compliance Agreement establishing plans, } \\
\text { procedures and schedules for CERCLA and RCRA } \\
\text { implementation at the Hanford Site. }\end{array}$ & $\begin{array}{l}\text { EPA Docket Number 1089-03-04-120: Hanford Federal } \\
\text { Facility Agreement and Consent Order }\end{array}$ \\
\hline REMEDIATE TANK WASTE & $\begin{array}{l}\text { "The high-level fraction of existing double-shell tank wastes } \\
\text { would be disposed of in a geological repository according to } \\
\text { the reference alternative (Section 3.3.3.1), with the } \\
\text { low-activity fraction being converted to a cementious grout." } \\
\text { "Single-shell tank wastes would continue to be stored until } \\
\text { sufficient information is available to support a future } \\
\text { decision for geological, in-place stabilization or other } \\
\text { disposal method." }\end{array}$ & $\begin{array}{l}52 \text { FR 12449: Final Environmental Impact Statement for } \\
\text { the Disposal of Hanford Defense High-Level, Transuranic } \\
\text { and Tank Waste, Hanford Site, Richland Washington, } \\
\text { Record of Decision. } \\
\text { Section 3.3.5.1 Preferred Alternative for Existing Tank } \\
\text { Waste }\end{array}$ \\
\hline
\end{tabular}




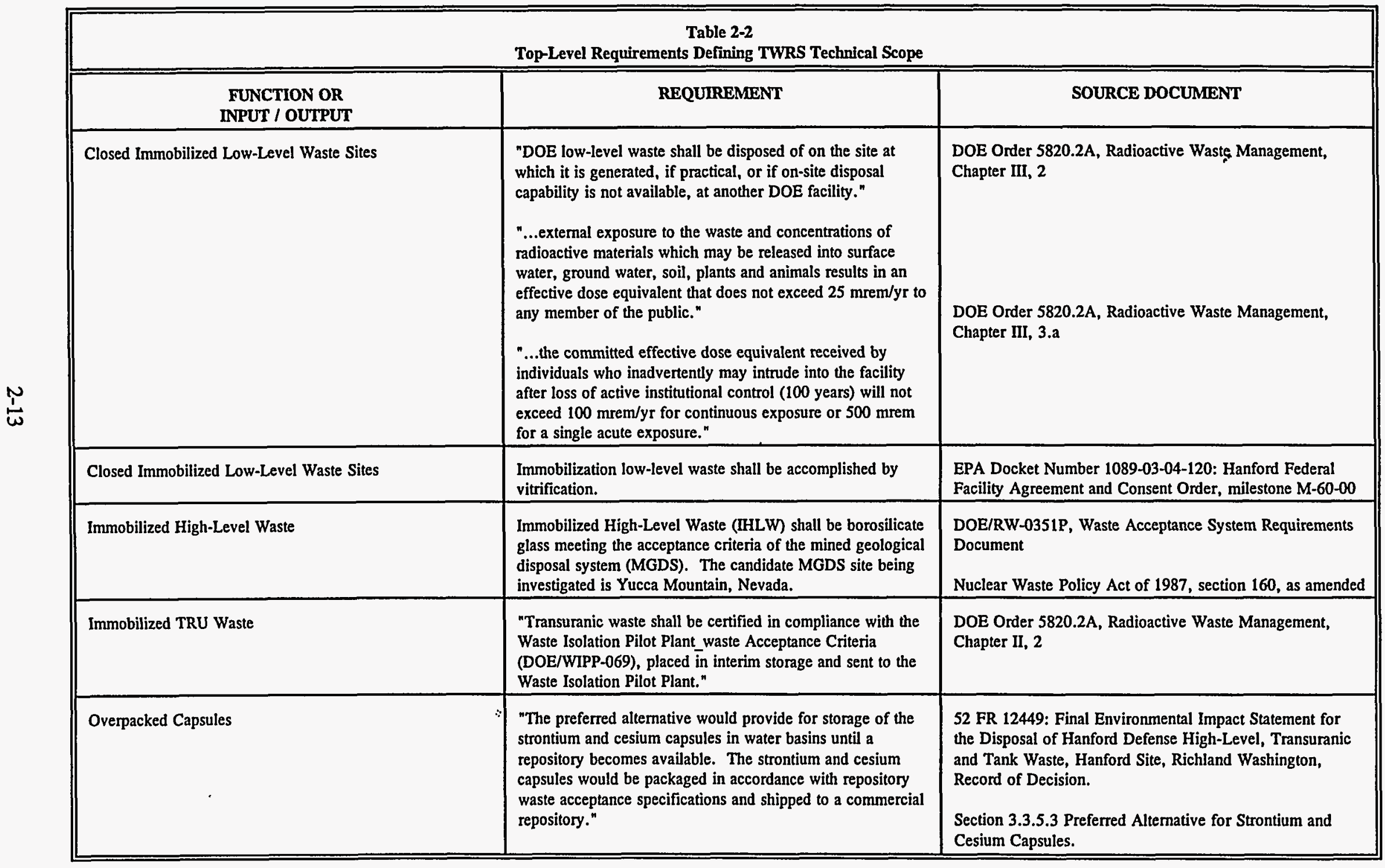


WHC-SP-1101

This page intentionally left blank. 


\subsection{STRUCTURE}

The TWRS Program is structured in both a functional and physical configuration. This structure is reflected in the TWRS Program WBS, which is necessary to provide critical tools for planning and communication and enhanced cost performance. This organization of program elements distinguishes between existing and planned facilities, institutional and programmatic interfaces, and technical and administrative requirements. The TWRS Program structure is continually under review and revision based on evolving systems engineering analyses and program requirements. Significant changes in the WBS are anticipated over the 40-year life of this Program.

\subsection{WORK BREAKDOWN STRUCTURE}

The structure illustrated in Figure 2-2, Tank Waste Remediation System Program Summary Work Breakdown Structure, shows the logical division of program elements (Level IV). This structure also illustrates the top-level Program functions determined through the application of systems engineering.

The WBS for each program element is included in the appropriate subsections of Section 7, Program Element Baselines. Each program element WBS is broken down to the level necessary to provide effective management of the work. The near-term planning window for any given 3-year period is summarized in the program element's WBS at Level VI. Summarization to higher levels of the TWRS Program Summary WBS are provided as required.

\subsection{ORGANIZATION AND RESPONSIBILITIES}

Organizational responsibilities are determined for all levels of the TWRS Program WBS. The Westinghouse Hanford Company (WHC) responsibilities flow through line management starting at the President Office level. The day-to-day management of the TWRS Program is vested with the WHC TWRS Program Office at Level III of the WBS. TWRS program element managers have responsibility for the management of their work scope at Level IV of the WBS and below. The DOERichland Operations Office (RL) responsibilities for oversight of these program areas flow through the RL Site Managers Office to the appropriate line managers or their designated representatives. The RL TWRS Program Office is closely aligned with the WHC TWRS Program Office. 
WHC-SP-1101

This page intentionally left blank. 


\subsection{LOGIC AND SCHEDULES}

The logical progression of work for the TWRS Program comes from the integration of critical activities within the many program elements and the desired end state of major physical facilities. A program of this size and duration must address numerous requirements and constraints, which occur at many levels. This lggical progression of work is then transformed into more detailed plans and schedules.

\subsection{LOGIC}

The Tri-Party Agreement milestones and TWRS Program functions and requirements are the principal drivers for the Program logic. The TWRS Program Hanford Site Tank Waste Remediation System Technical Strategy (1994) was developed to address these major drivers at the integrated Program level. This technical strategy is illustrated in Figure 2-1. Program element logics were also developed to address these and other activity specific requirements. These program element logics have been incorporated into program element schedules. The program element schedules have been integrated into an overall TWRS Program Master Schedule (refer to Appendix I).

The program element logics used initially are on file for record purposes. However, the logics that represent the baseline in any given program element are now an integral part of the schedule database. Logics and critical path information are drawn exclusively from this database.

\subsection{SCHEDULES}

The TWRS Program schedule, a critical path method network, reflects the technical strategy contained in the TWRS Program technical strategy and conforms to the constraints contained in the Tri-Party Agreement. The Program's schedule integrates the activities of each of the elements and interfaces between these elements as well as the major interfaces with other Hanford Site programs and external organizations, such as regulatory agencies. The Program's schedule uses the resource information developed by the activity-based estimating efforts to transform the logic into a schedule. The information necessary to construct or reproduce the schedule is contained in an integrated database. Graphical representation of the overall Program is illustrated in the Figure 4-1, Tank Waste Remediation System Program Summary Schedule. More detailed schedules for the program elements are included in Appendix C, Program Element Schedules. The milestones on these schedules are further detailed by the information included in Appendix D, Milestone Log and Milestone Description Sheets.

Two additional schedules are included because of their significance to the overall TWRS Program. The first schedule illustrates the work required to make all known major decisions and is included as Appendix F, Major Decisions Logic/Schedule. This schedule cuts across the total Program to examine planned engineering analyses needed to confirm the strategy alternatives that form the baseline. All activities/milestones on this schedule are already a part of the integrated schedule database and appear on the appropriate program element schedules. The second schedule addresses the integration of tank operations with key program elements such as Waste Tank Safety, Characterization, Tank Farm Upgrades, and Waste Retrieval. This cross-cut schedule is included as Appendix G, Integrated Operations Schedule. This cross-cut schedule illustrates the importance of interactive (i.e., integration) planning among the various program elements. 
WHC-SP-1101

This page intentionally left blank. 


\section{Tank Waste Remediation System Program Sumr}

$T \cdot W \cdot R \cdot S$

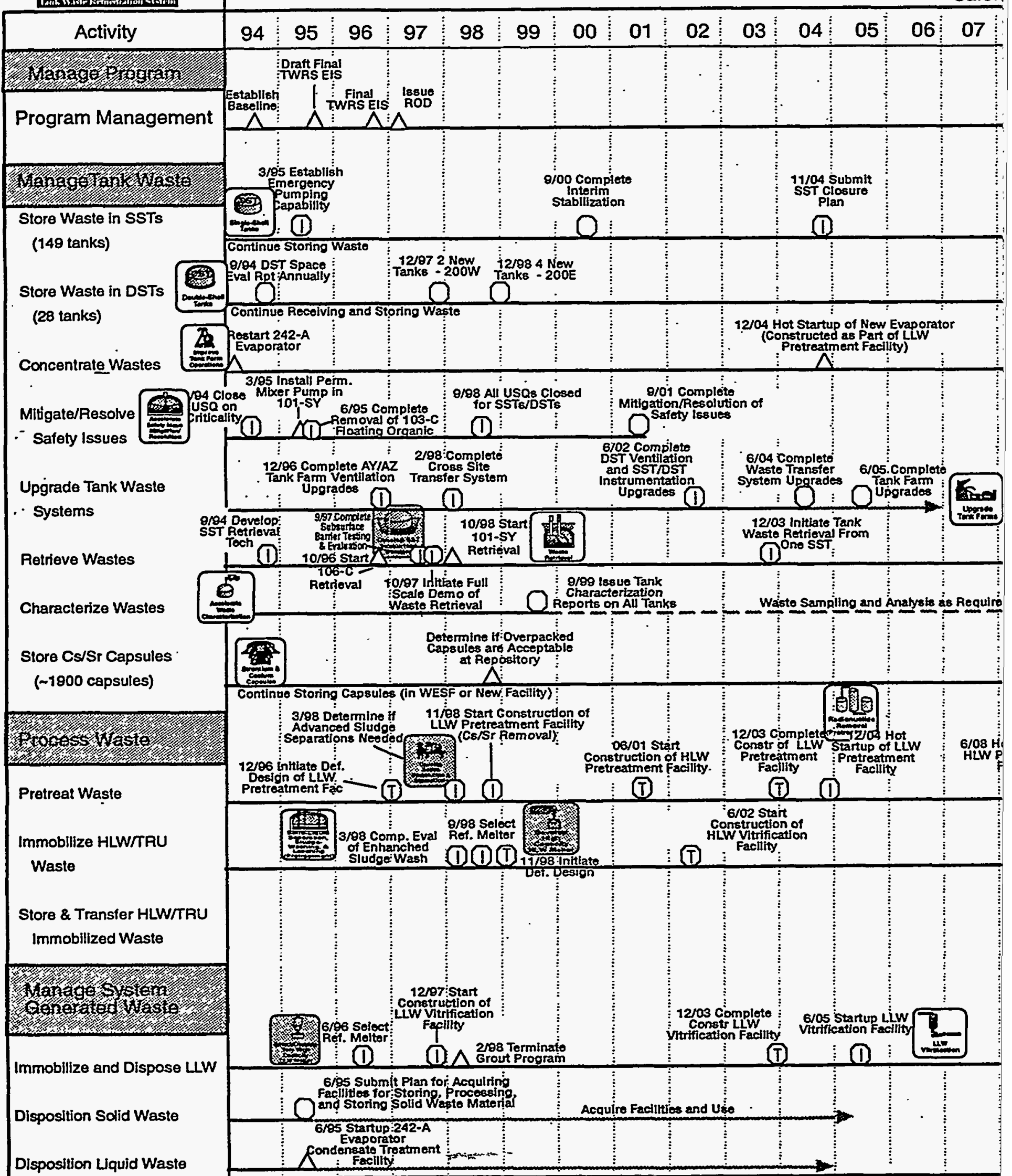

(T) Tr-Party Agroement TWRS Major Milestono (T) Tr-Party Agreement TWRS Target Milestone

(1) Tri-Party Agreement TWRS Interim Mllestone $\triangle$ Non-TPA Milestones 
Figure 4-1.

ary Schedule For Information Only Draft Tank Waste Remediation System Program Summary Schedule. ar Years

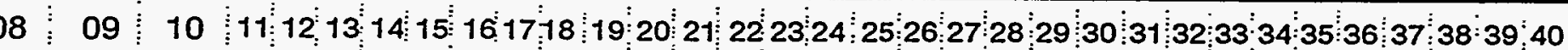

3/12 Initiate SST

From SSTB $\vdots \vdots \quad \vdots \quad 9 / 24$

$:(T)$

$$
1
$$
12228 Comploto

$12 / 09$ Startup HLW
Vitrfication Facility

Ext?




\subsection{RESOURCE REQUIREMENTS}

The cost bases (resource requirements) provide a quantification of the resources needed to accomplish the technical bases of the baseline. The cost bases of the TWRS Program were developed using the guidelines and precepts provided in EM-30's Cost and Schedule Estimating Guide and in conjunction with the Program's scheduling efforts.

The TWRS Program's cost estimate is a compilation of the cost estimate made for each activity in the Program's schedule (i.e., activity-based cost estimating). These activities encompass the full scope of the TWRS Program. The cost estimate for each activity is, for the most part, an extension and summation of the basic resource estimates prepared for each activity. Various methodologies were used in the preparation of the estimates, but the methodology chosen was appropriate for each activity dependent on the information available.

The activity resource estimates were prepared by subject matter experts. These experts broke direct manpower requirements down into 88 labor categories through the year 1997. Additional differentiation was supplied in the resultant cost estimates by providing both direct and indirect costs, three categories of funds, four cost types, and inclusion of the WBS as an identifier.

These rough-order-of-magnitude cost estimates are expected to change as systems engineering analyses proceed and more cost-effective alternatives are determined. The TWRS Program life-cycle costs identified in Table 5-1 will be updated annually as MYWP revisions are scheduled.

\subsection{COST}

\subsubsection{Basis of Cost Estimates}

The TWRS Program cost estimates were developed using the guidelines and precepts provided in EM-30's Cost and Schedule Estimating Guide and in conjunction with the Program's scheduling efforts. The TWRS Program's cost estimate is a compilation of the cost estimates made for each activity in the Program's schedule, consistent with the activity-based cost estimating approach. Summary-level activities were estimated to produce an estimate for the full scope over the life of the Program (i.e., life-cycle costs). More detailed estimates were performed for a greater number of activities in the near-term 3-year planning cycle.

The activity resource estimates were prepared by subject matter experts, who focused on quantities and rates to the extent that these were available. The details of these activity estimates are supported by worksheets and other documentation. The cost estimating methodology and additional discussion regarding bases of estimates are included in Appendix E, Bases of Estimate.

\subsubsection{Total Program Cost by Year by Program Element}

Table 5-1 establishes the baseline cost for the TWRS Program. The baseline cost is exhibited in annual costs for FY 1994 through FY 2000 and 5-year totals for FY 2001 through completion down to the program element level (Level IV). 


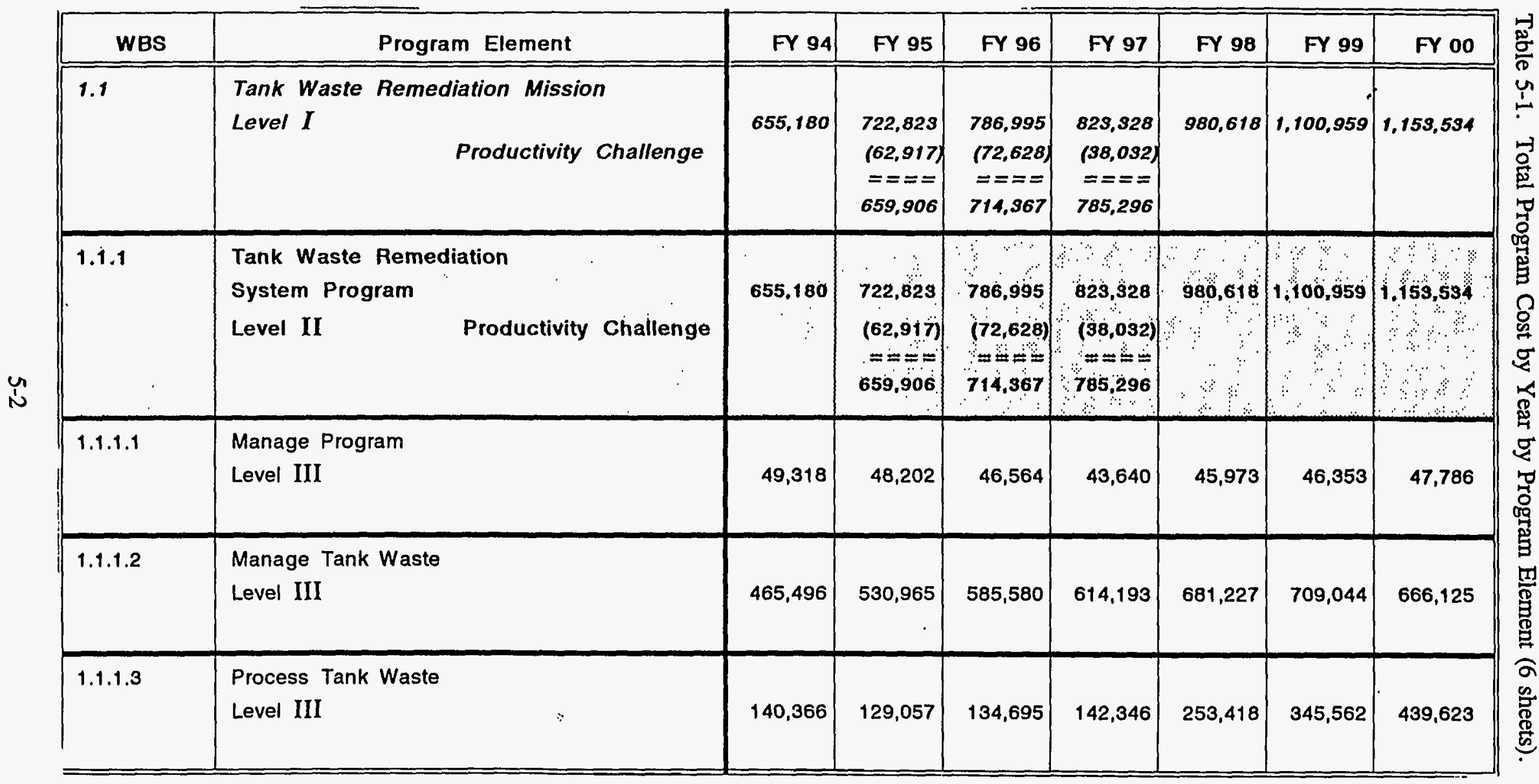




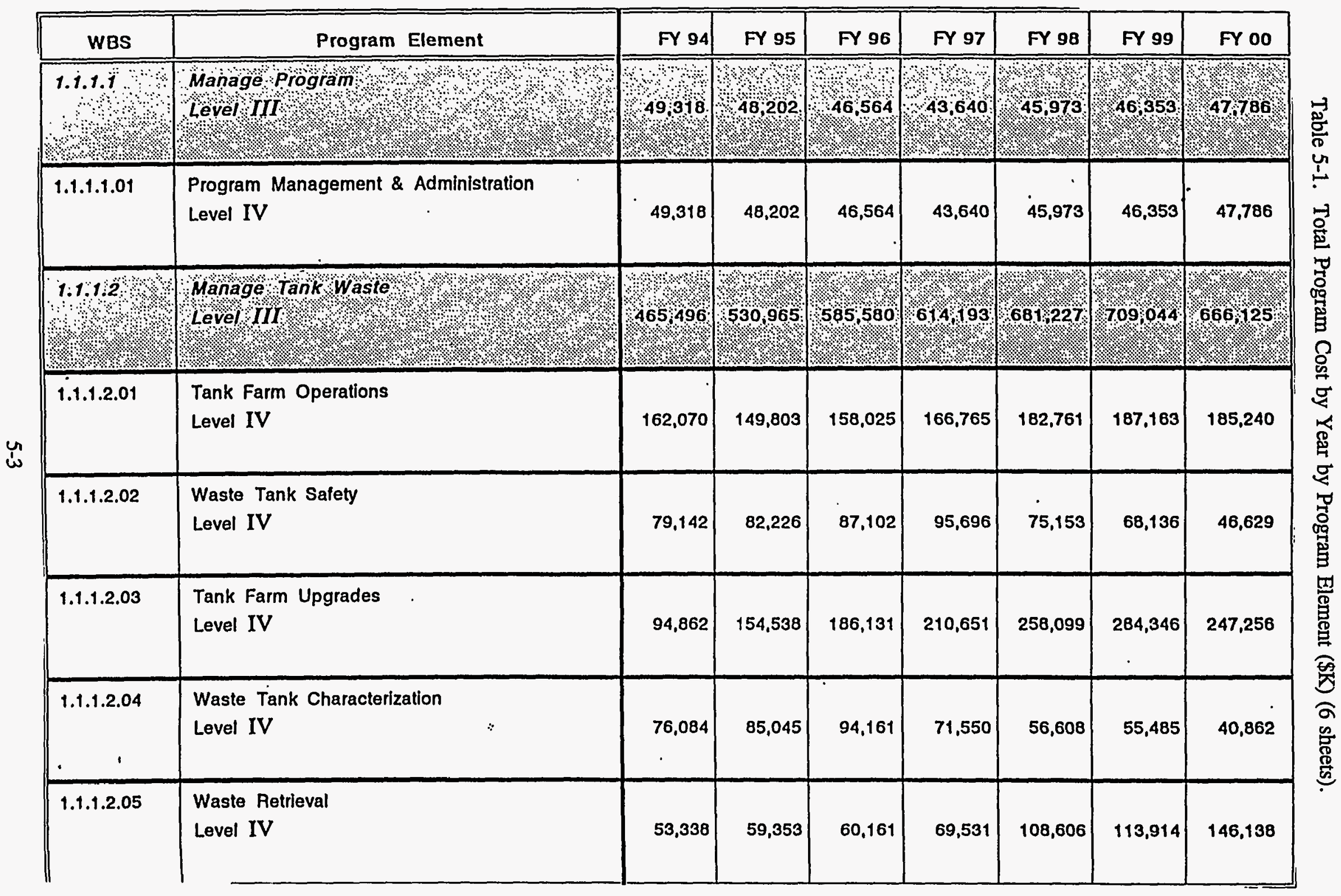


Table 5-1. Total Program Cost by Year by Program Element (\$K) (6 sheets).

\begin{tabular}{|c|c|c|c|c|c|}
\hline$\frac{8}{8}$ & 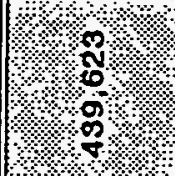 & 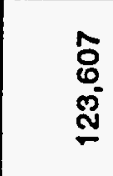 & 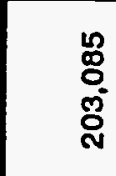 & 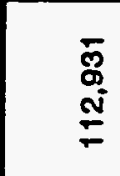 & 0 \\
\hline $\begin{array}{l}8 \\
8 \\
\check{L}\end{array}$ & 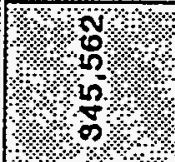 & 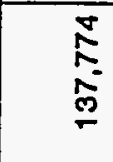 & $\begin{array}{l}\hat{0} \\
\stackrel{0}{0} \\
\stackrel{D}{\square}\end{array}$ & $\begin{array}{l}\overline{0} \\
\infty \\
\infty\end{array}$ & 0 \\
\hline 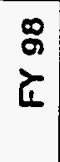 & 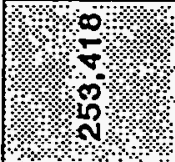 & \begin{tabular}{l} 
D \\
N \\
N \\
\multirow{2}{N}{}
\end{tabular} & $\frac{\bar{N}}{\bar{N}}$ & $\begin{array}{l}\text { gू } \\
\Phi \\
\text { oू }\end{array}$ & 0 \\
\hline$\hat{a}$ & Plo & $\begin{array}{l}8 \\
8 \\
\frac{8}{\pi}\end{array}$ & $\begin{array}{l}\text { पू } \\
\text { ஜू } \\
\text { षे }\end{array}$ & 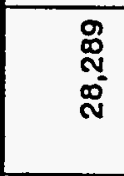 & $\frac{\frac{g}{S}}{\frac{D}{N}}$ \\
\hline $\begin{array}{l}0 \\
\vdots \\
2\end{array}$ & $\begin{array}{ll} & 6 \\
& 6 \\
& \\
& \end{array}$ & $\begin{array}{l}8 \\
0 \\
0 \\
0\end{array}$ & $\begin{array}{l}\infty \\
\frac{\infty}{N} \\
\frac{0}{\sigma}\end{array}$ & $\begin{array}{l}\mathbb{8} \\
\mathbb{0} \\
\text { N } \\
\text { N }\end{array}$ & 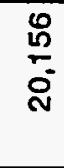 \\
\hline $\begin{array}{l}n \\
\downarrow\end{array}$ & +ैo & 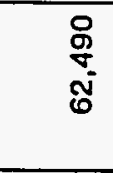 & $\begin{array}{l}0 \\
\mathbb{U} \\
0 \\
\sigma^{-} \\
\forall\end{array}$ & $\begin{array}{l}\bar{\nabla} \\
0 \\
\sigma^{-}\end{array}$ & 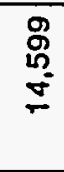 \\
\hline $\begin{array}{l}5 \\
\text { ธे }\end{array}$ & 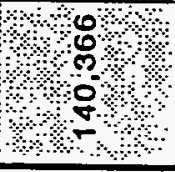 & 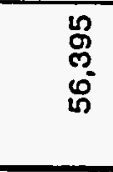 & $\begin{array}{l}\text { Jे } \\
\text { ల } \\
\text { J }\end{array}$ & 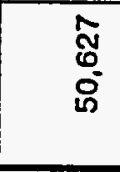 & 0 \\
\hline 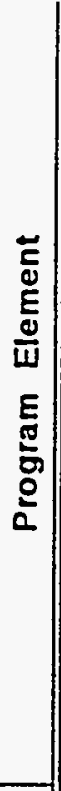 & 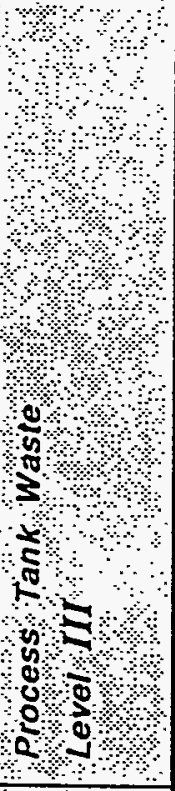 & 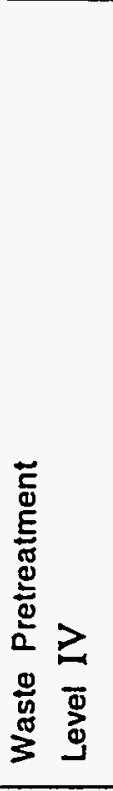 & 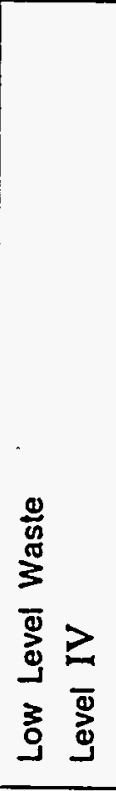 & 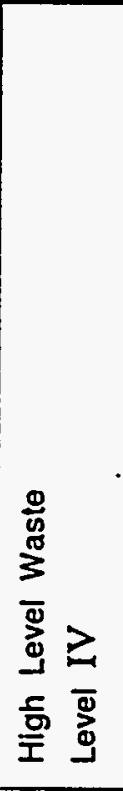 & 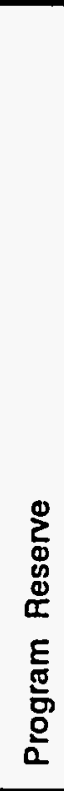 \\
\hline$\sum_{3}^{\infty}$ & 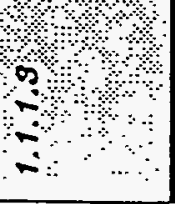 & 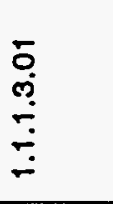 & 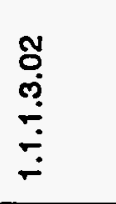 & $\begin{array}{l}\stackrel{8}{\circ} \\
\stackrel{\circ}{\circ} \\
\check{-}\end{array}$ & \\
\hline
\end{tabular}




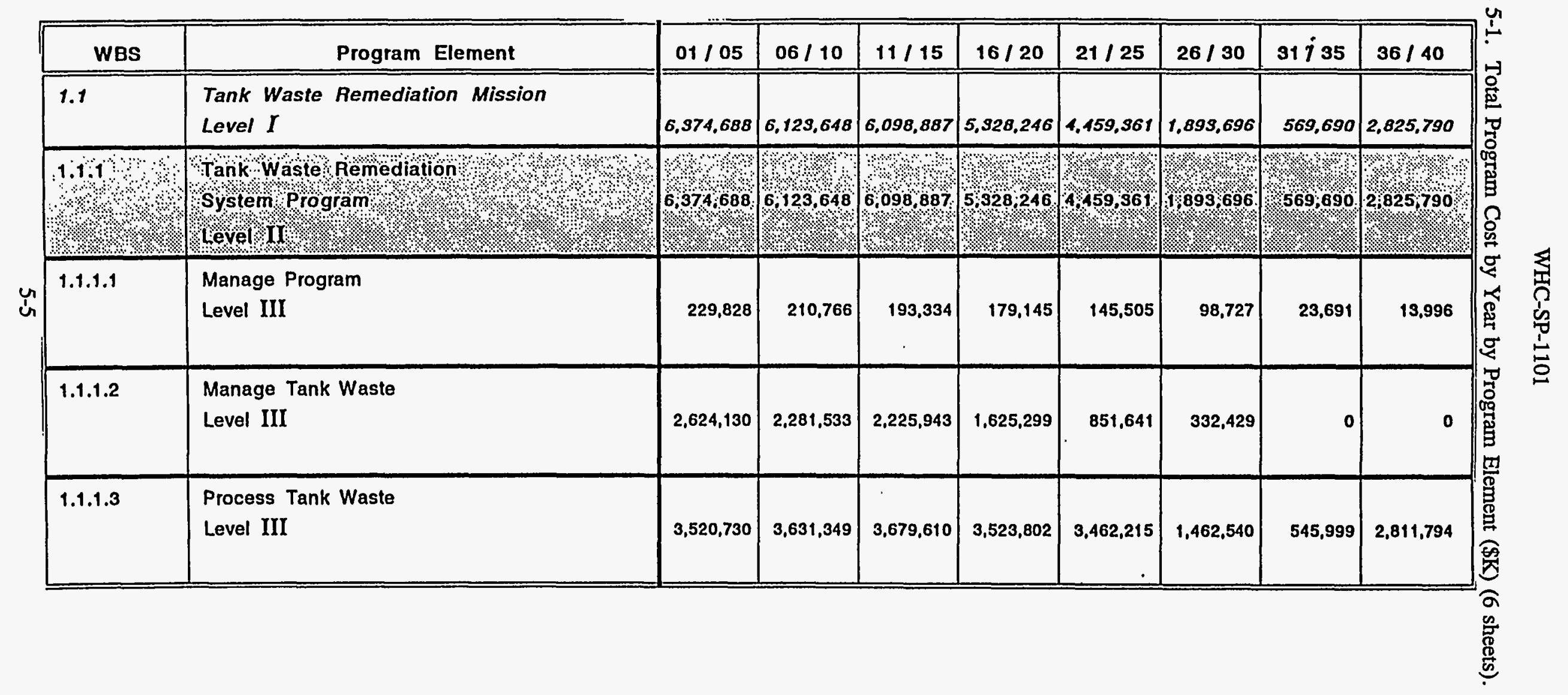




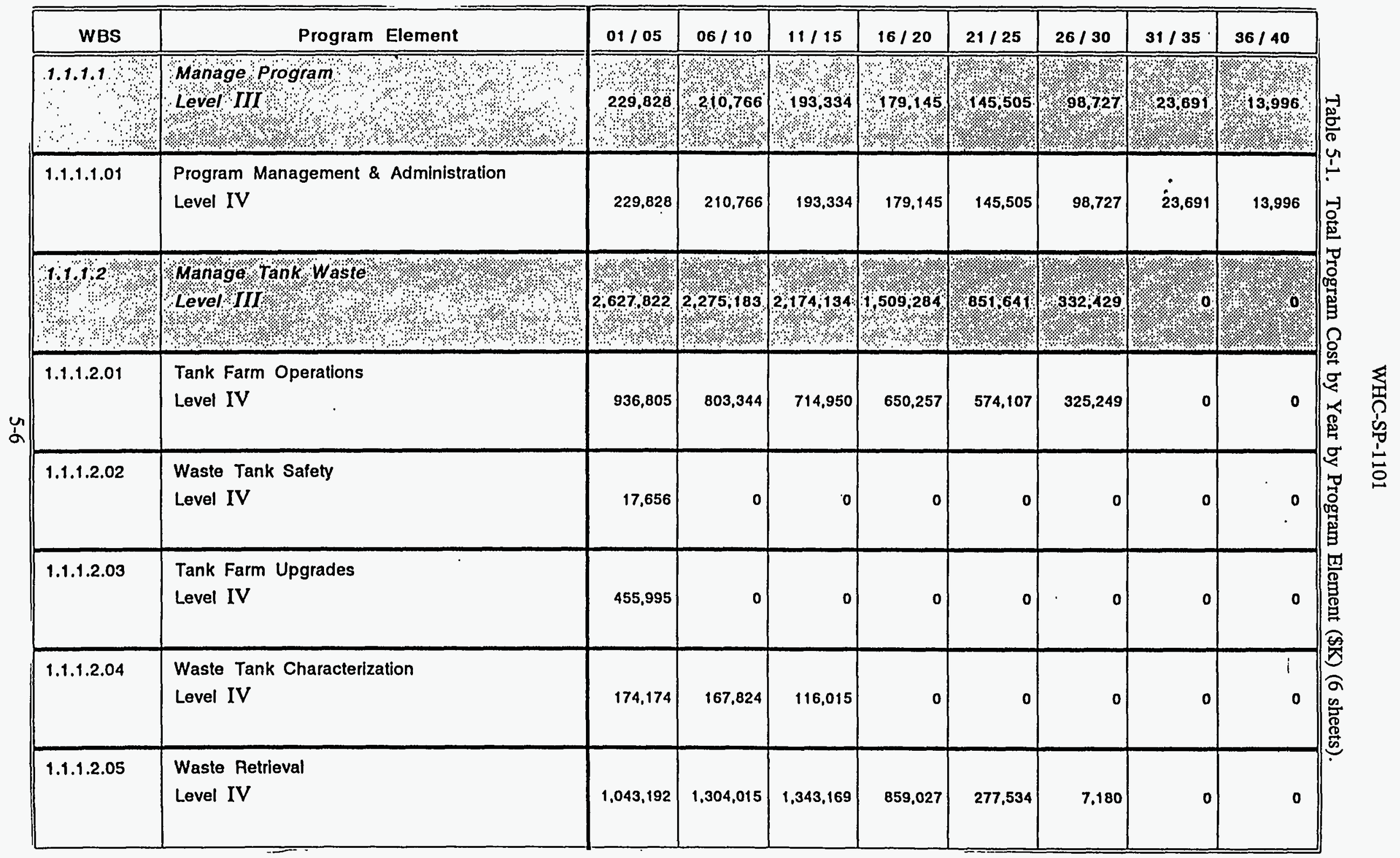


WHC-SP-1101

Table 5-1. Total Program Cost by Year by Program Element (\$K) (6 sheets).

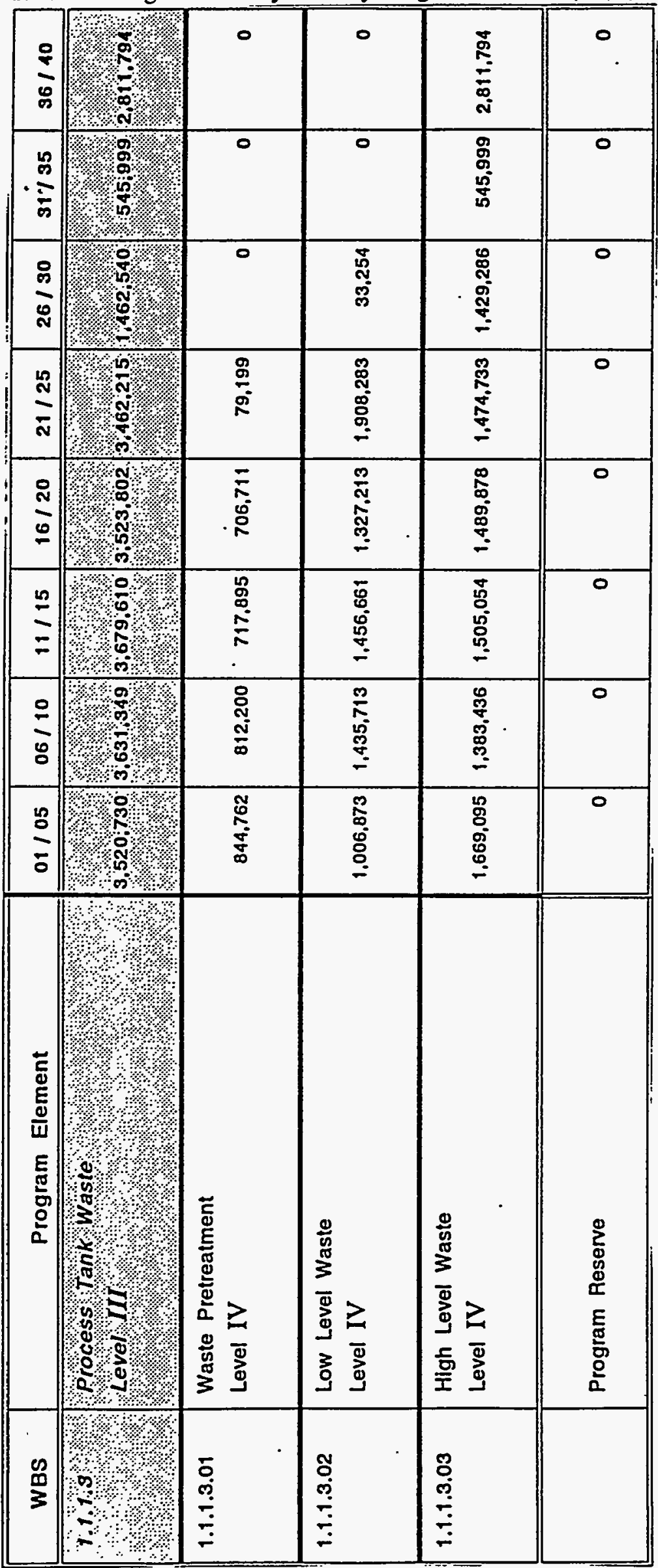




\subsubsection{FY 1995 Cost by Month by Program Element}

Future updates of the MYWP will include Table 5-2, which will establish the month-by-month cost baseline for the current fiscal year.

\subsection{STAFFING}

\subsubsection{Basis of Staffing Projections}

Manpower projections are determined by compiling the labor resource requirements at the lowest level of planned activities, converting the manhours into Full-Time Equivalents (FTEs), and summarizing the information at the appropriate level of the TWRS Program WBS. An FTE is equivalent to 1,812 hours in any given fiscal year. The labor manhour estimates are included in the Estimating Work Sheets supporting each Activity Planning Form. Near-term plans divide the labor into 88 distinct categories. Intermediate planning groups labor into nine categories, and the longrange planning identifies three categories. Manpower projections are also summarized by major Program participants. The planning of labor resources is reviewed and refined with each annual revision of the TWRS MYWP.

The labor resource projections are first summarized at Level VI of the TWRS WBS. Collection at this level supports the preparation and review of the performance measurement baseline at the Cost Account level. The information is further summarized to Level IV, which is the TWRS program element level. The summary at this level supports the annual preparation and submission of the Activity Data Sheets. These labor resources are contained in the Primivera ${ }^{1}$ schedule/resource database.

\subsubsection{Total Full-Time Equivalents by Year by Program Element by Major Participant through FY 2000}

Future updates of the MYWP will include Table 5-3, which exhibits total FTEs by Year by Program Element by Major Participant through 2000.

\subsection{WORK SCOPE CARRYOVER}

The TWRS work scope carryover is identified on Table 5-4 by each program element. The work scope is broken into two parts. The first part is the carryover amounts that were identified at MidYear and includes approximately \$10M worth of work scope that was formally deferred into FY 1995 as part of the Mid-Year Review. The second part of the carryover incorporates any changes to the original estimates and identifies any additional items that may be carried into FY 1995 . These carryover amounts are best estimates at this time and will be reevaluated for accuracy and validity at the start of FY 1995.

\footnotetext{
${ }^{1}$ Primavera is a registered trademark of Primavera Systems, Inc., Bala Cynwyd, PA.
} 


\section{WHC-SP-1101}

Table 5-2. FY 1995 Cost by Month by Program Element

Future updates of the MYWP will include Table 5-2, which will establish the month-by-month cost baseline for the current fiscal year. 
Table 5-3. Total Full-Time Equivalents by Year by Program Element by Major Participant through FY 2000

Future updates of the MYWP will include Table 5-3, which exhibits total FTEs by Year by Program Element by Major Participant through 2000. 


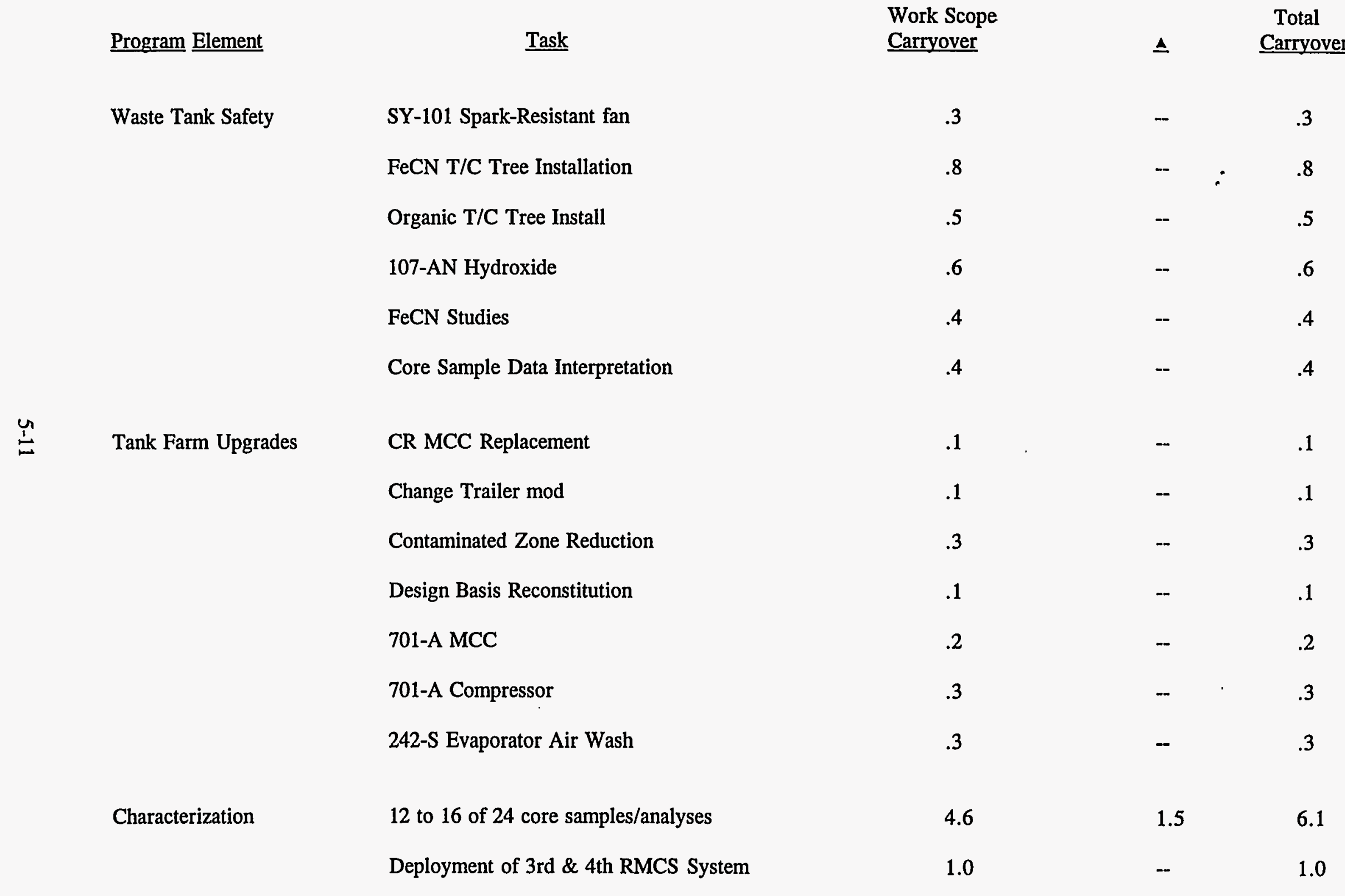


Program Element

Characterization

(Cont'd)

Waste Retrieval

虽
Task

325-A Hot Cell

Sample performance evaluation (PNL)

SST Retrieval Tech Proj Def.

Long Reach Arm Project Conceptual Design

Subsurface barrier procurement/construction

W-320-106C Sluicing

W-211 DST Tank Retrieval System

W-211 DST Uniformity/Slurry Mobilization Test

SST Test \& Training Facility Requirements

AZ-101 decontamination pump

Calcine Dissolution Vendor

Transportation Study - Sandia

325 Lab Shutdown

LANL Organic Support
Mid-Year Review

Work Scope

Carryover

.5

.7

.3

.6

1.5

1.0

.2

.3

.4

$$
.1
$$

.4

.2

.5

.5
Total

$\triangle \quad$ Carryover

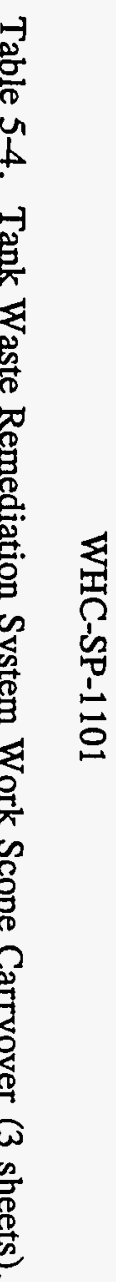


Low-Level Waste

Melter testing \& simulant procurement for melter selec. and misc. support

Research Glass Quality

TDPO Melter Support

Tank Farm Operations and Management

Program Management and Administration

High Level Waste
UT testing

S-Farm

Vent \& Balance

LDUA

Program Management - Review of the General Management System documents and supporting Data.

$\mathrm{QA}$ - Review of the documents associated w/ the Quality Management Plan, Management System Description and Annexes.

PNL - System engineering decision analysis support to prioritize technology development activities.

Stirred Melter/Pilot Scale Melter Testing \& Rheology
Lab Analysis Task

\begin{tabular}{lll}
.2 & - & .2 \\
.4 & .1 & .5 \\
.3 & - & .3 \\
.2 & - & .2 \\
.3 & - & .3 \\
.5 & -- & .5 \\
.2 & - & .2 \\
-- & .2 & .2 \\
- & .2 & .2 \\
- & & .2 \\
\hline$\underline{24.1}$ & .2 & $\underline{2.8}$ \\
\hline
\end{tabular}

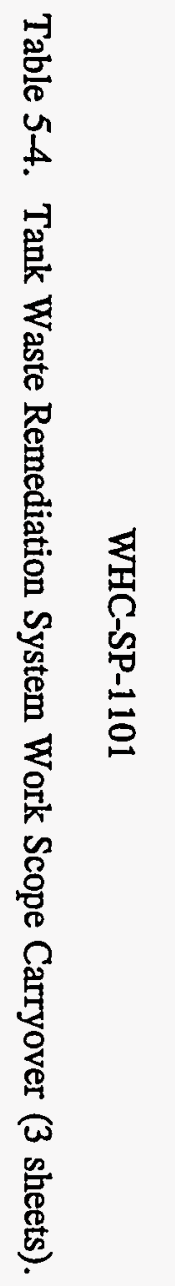

TOTAL 
WHC-SP-1101

This page intentionally left blank. 


\subsection{WASTE TYPE DATA}

Data on the different waste types encountered on the TWRS Program are collected and summarized to fulfill the Hanford Site requirement for long-range strategic planning. The data include the projected quantities of different waste types (e.g., liquids, solids, and soils), which are contaminated and uncontaminated, and are categorized with regard to level of radioactivity. Categories such as highlevel waste, low-level waste, transuranic waste, and others are important in determining plans for dealing with the projected volumes and/or masses. The waste type data are collected by program element by year for the 3-year planning window and then in total for FY 1998 through 2025. These data identify key functions required to generate, receive, transfer, and store the different waste types. Plans for performing these functions are also summarized in conjunction with the projections. Issues regarding any aspect of these principal waste functions are identified for resolution.

The waste projection data and associated planning activities are prepared by each program element on standard forms used by all Hanford Site programs. The waste type data from each program element are summarized for the TWRS Program in Appendix H, Waste Type Data. These data are expected to change significantly from one planning cycle to another until an integrated process flow diagram has been defined and approved as a baseline [TWRS Program Process Flow Diagram(s)] In the meantime, the function of maintaining a strict accountability of the critical waste types on hand is carried out by the Tank Farm Operations and Maintenance Program Element. The tank waste data are officially reported in the Tank Farm Surveillance and Waste Status Summary Report for (Month) 1994 (WHC-EP-0182-XX). 
WHC-SP-1101

This page intentionally left blank. 


\subsection{PROGRAM ELEMENT BASELINES}

\subsection{INTRODUCTION}

This section of the MYWP documents the multi-year baseline for each of the nine program elements that comprise the TWRS Program. Strategy, structure, work to be performed, approach, drivers and deliverables, schedule, resource requirements, waste type data, and baseline revisions are addressed. In some cases, baseline information is provided in the section using text, graphics, and tables as appropriate. In other cases, information is provided through a reference to one of the appendices to the MYWP or to a separate document. The program element sections are organized with graphics and tables appearing at the end of the baseline narrative. 
WHC-SP-1101

This page intentionally left blank. 


\subsection{PROGRAM MANAGEMENT AND ADMINISTRATION BASELINE}

The Program Management and Administration (PM\&A) Program Element baseline consists of the technical bases, schedule, resource requirements, and waste type data for FY 1995 through completion of the mission. The major emphasis is on deliverables, schedule, and resource requirements for FY 1995, 1996, and 1997.

\subsubsection{Technical Bases}

This section provides the mission strategy; work breakdown structure and organization responsibility; work to be performed in FY 1995, 1996 and 1997; technical approach; and drivers and deliverables.

\subsubsection{Mission Strategy}

\subsection{Objectives}

The overall objectives of the PM\&A Program Element are to continue to improve and deploy TWRS baselining and management control capabilities and effectively manage compliance with environmental requirements and, in so doing, improve Program credibility. More specific objectives are documented in the Level IV, V, and VI WBS dictionary sheets found in Appendix A.

\subsection{Strategy}

The above objectives will be met through the use of a robust systems engineering effort. Systems engineering will be coupled with an effort to develop and gain approval of an initial multi-year program baseline. Actions will be identified and taken to move toward more disciplined baseline management. Actions will be identified and taken to continually improve the data that support the multi-year baseline. Formal change control will be used to revise the baseline to take advantage of improved baseline support data. The Environmental Impact Statement (EIS) process will be utilized to formally make and document important decisions associated with processing and dispositioning Hanford tank waste.

\subsection{Functions and Requirements}

The PM\&A Program Element provides the resources necessary to produce and maintain the Tank Waste Remediation System Functions and Requirements document (DOE/RL-92-60) as the first step in the systems engineering process. By presenting a comprehensive description of the top levels of functions, requirements, and architectural concepts necessary to accomplish the TWRS mission, the document provides a firm foundation for establishing the TWRS functional requirements baseline and for allowing the systems engineering process to continue. 
WHC-SP-1101

\subsection{Risks}

The PM\&A Program Element has identified three summary-level risks specifically linked to meeting the objectives identified in Section 7.2.1.1.1 above. These risks are listed below.

1. Uncertainty associated with TWRS Program baseline and management control documentation requirements. See Assumption 1 in Section 7.2.1.1.6.

Mitigation: DOE approval of the MYWP will baseline the TWRS document hierarchy. Changes to the hierarchy will require formal change control actions including justification, consideration of cost and schedule impacts, and provision of additional resources or reduction of current resources as appropriate.

2. Inadequate resource availability to implement the PM\&A Program Element strategy documented in Section 7.2.1.1.2 above. See Assumption 2 in Section 7.2.1.1.6. Refer to Figure 7.2-1 for specific information associated with this risk in FY 1995.

Mitigation: PM\&A deliverables, task durations, and resource requirements will be documented in Sections 7.2.1.6, 7.2.2 and 7.2.3. DOE approval of the MYWP will baseline deliverables, task duration, and resource requirements; thereby providing approval to incur costs, adequate human resources, and adequate time to produce the deliverables identified in Section 7.2.1.6. Changes that impact resource availability will require formal change control actions including justification. See Figure 7.2-1 for specific information associated with the mitigation of this risk in FY 1995.

3. Uncertainty associated with the predicted duration for the various tasks that comprise the EIS schedule. See Assumption 3 in Section 7.2.1.1.6.

Mitigation: The EIS schedule will be baselined in Section 7.2.2. Progress against the baseline schedule will be measured and corrective action will be taken as appropriate per the reporting requirements, processes, and procedures documented in the T.WRS Program Management System Description and Annexes (DOE/RL-93-0106) and the WHC TWRS Management Plan.

\subsection{Major Decisions}

All major TWRS Program decisions and the tasks required to be completed to make the decisions are identified in Appendix F, Major Decisions Logic/Schedule. Decision 4.2, Determine Disposition of Hanford Tank Waste, is the responsibility of the PM\&A Program Element. As indicated in Appendix F, completion of the EIS is required to make decision 4.2. Major Decision 4.2 is required to meet the objectives of the Waste Retrieval, Waste Pretreatment, Low-Level Waste and High-Level Waste Program Elements. Sections 7.2.1.3, 7.2.1.4, and 7.2.2 and supporting data document the work to be done to make Decision 4.2. Although PM\&A is responsible for making a major decision, no major decisions are required to meet the PM\&A objectives identified in Section 7.2.1.1.1. 


\subsection{Assumptions}

The PM\&A Program Element has identified five summary-level assumptions specifically linked to meeting the objectives identified in Section 7.2.1.1.1 above. These assumptions are listed below.

1. TWRS baseline and management control documentation will be as depicted on the document hierarchy represented on Figure 7.2-2.

2. PM\&A objectives will be met; and specific PM\&A deliverables will be provided as described in Appendix D, Milestone Log and Description Sheets, using the resources baselined in Section 7.2.3.

3. The EIS schedule can be completed in 24 months (accelerated from 36 months) in accordance with direction from the Secretary of Energy to shorten DOE EIS schedules.

4. The Hanford Dangerous Waste Permit will be approved in FY 1995.

5. No impacts to the baseline documented in the approved TWRS MYWP will result from future Maintenance and Operations contract renegotiations and/or revisions.

Other more detailed assumptions (WBS Level VII and below) are documented on TWRS Program Activity Planning Forms.

\subsection{Constraints}

The PM\&A Program Element has identified three summary-level constraints specifically linked to meeting the objectives identified in Section 7.2.1.1.1 above. These constraints are listed below:

1. Receipt of baseline support data from each of the other eight program elements that comprise the TWRS Program such that PM\&A deliverables can be produced per the schedule documented in Section 7.2.2

2. Pre-validation reviews, validation reviews, and subsequent actions associated with the Energy Systems Acquisition Advisory Board occurring as scheduled in Section 7.2.2

3. DOE procurement of the EIS contractor to support the schedule documented in Section 7.2.2.

Additional constraints (at lower levels of detail) are depicted in the schedules discussed in Section 7.2.2 and provided in Appendix C.

\subsubsection{Structure}

The PM\&A Program Element is the focal point for the management of the overall TWRS Program. This program element represents the WHC TWRS Program Office with all internal and external customers and maintains all integrated program-level products that make up the baseline. This program element is also structured to accommodate numerous support areas that cut across all or many different TWRS Program elements and provide continuity over the life of the Program. This program element addresses the top-level systems engineering requirement to "Manage Program." 


\subsection{Work Breakdown Structure}

The PM\&A Program Element is further broken down into activities entitled Formulation, Execution, Evaluation, and Program Management Systems. This breakdown resulted from the application of systems engineering analysis to the repetitive program management functional drivers such as the Congressional budget cycle and the DOE requirements. A further breakdown to the levels required to manage discrete tasks.js shown in Figure 7.2-3, Program Management and Administration Work Breakdown Structure.

The Formulation Activity includes all program management and administration activities, program strategic planning, baseline development, and systems engineering activities at all levels. The Execution Activity covers the Program Administration activities at the total Program level; program management activities of the Pacific Northwest Laboratory Technology Development Program Office; program management and control of all TWRS Program projects; the Program-level support for regulatory compliance and permitting, including coordination of the preparation of the TWRS EIS; and numerous support activities including environmental, safety, and health; quality assurance; qualification and training; information resources management; acquisition management; reengineering; and communications and building management. The Evaluation Activity includes analyses of the Program from both an administrative and technical point of view. The technical evaluations will be performed by organizations other than the systems engineering organizations formulating the TWRS Program. This activity also includes DOE support through the Customer Support Service Contract. The Program Management Systems Activity was identified to provide enhanced program management initiatives, tools, and products.

\subsection{Organization and Responsibility}

The PM\&A Program Element is responsible for the overall formulation, execution, and continuous evaluation of performance for the TWRS Program. The activities within PM\&A are focused on the total integration of the Program. Eight other program elements exist to manage specific logical divisions of the overall TWRS Program. Each of these program elements is covered in a separate section of this document. The roles and responsibilities of those organizations performing through the resources of the PM\&A Program Element are described below.

The Formulation Activity is performed primarily by three organizations. The Program Management and Integration organization has the lead responsibility for planning and total Program integration and for developing and controlling the TWRS Program baseline. The Baseline Development and Management Systems organization, a part of the overall Business Systems organization, has the responsibility for developing and maintaining all of the programmatic and administrative products required by the TWRS Program. The development of the technical portion of the total Program baseline through the application of systems engineering is the responsibility of the Strategic Planning organization. This organization also serves as a primary interface with external organizations on technical issues and coordinates public involvement activities for the TWRS Program as a whole.

The Execution Activity has a diversity of organizational contributors. The Business Management organization handles the working products for day-to-day financial and administrative management. This organization also handles the communications and facilities administration tasks for two major office complexes. Program management of a fully integrated technology development activity is the responsibility of the Pacific Northwest Laboratory Technology Development Program Office organization. The management and integration of all TWRS Projects is the responsibility of the Central Projects Systems organization. The TWRS Projects are fully integrated into the TWRS 
program elements for which they were justified, but are managed by a dedicated project organization. The Waste Tanks Environmental Compliance Officer (and organization) is responsible for total TWRS Program regulatory compliance and permitting activities, including the coordination of the preparation of the TWRS EIS. The functional areas of Quality Assurance, Information Resources Management, and Acquisition Management are the responsibility of full-time matrixed managers assigned to the TWRS Program from their respective WHC organizations. The responsibility for reengineering initiatives resides with the Reengineering organization. This organization reports to the Executive Vice President and Director of TWRS.

The evaluation activity is the responsibility of two primary organizations, with support from a third. The Business Management organization has the primary responsibility for evaluation of routine program management and financial data. The responsibility for performance assessment against the baseline and periodic reporting rests with this organization. The evaluation of specific technical aspects of the Program is a shared responsibility. The Program Management and Integration organization is responsible for evaluating specific areas related to either programmatic or technical performance. The Engineering Systems Support organization is responsible for evaluating specific technical performance primarily in the engineering areas. The Customer Support Services Contractor, MACTEC, is responsible for supporting the DOE TWRS Program Office in any area required.

The Program Management Systems activity was identified to cover enhancement of the overall program management and administrative activities across the entire TWRS Program including production of the WHC TWRS Management Plan. The Business Management Organization is the primary performing organization for this activity.

Program-wide information on the responsibilities for specific activities and tasks is included in Appendix B, Responsibility Assignment Matrix.

\subsubsection{FY 1995 Work to be Performed}

All four activities that comprise the PM\&A Program Element will be active in FY 1995. In general, systems engineering, planning, scheduling, estimating, baselining, management systems documentation, execution and evaluation, EIS, and permit efforts will be conducted to support the objectives and strategy summarized in Section 7.2.1.1. Activity-level work statements are provided below. It should be noted that more detailed, cost account, task and subtask scope statements are documented in Level VI WBS Dictionary Sheets (refer to Appendix A), and Level VII and VIII Activity Planning Forms.

\section{Formulation (WBS 1.1.1.1.01.01)}

- Provide overall guidance to the TWRS Program in the area of planning, scheduling, cost estimating, and baselining.

- Improve cost baseline data by establishing a multi-organizational team to conduct cost engineering; completing a cost estimating status/recommendation paper; scheduling and conducting cost estimates; initiating construction of a cost database; writing a cost engineering/estimating system description. 
- Improve technical baseline data by ensuring that the issues and enabling assumptions in the baseline documents (and supporting documents) are addressed and resolved through the required associated analyses. Obtain approval of the Tank Waste Remediation System Functions and Requirements document (DOE/RL-92-60). Begin the development of other documents required by the TWRS Systems Engineering Management Plan and the TWRS Systems Engineering Working Plan.

:

- Prepare the Technical Requirements Specification documents for the three functional areas: Manage Waste; Process Waste; and Manage Systems Generated Waste and Excess Facilities. Incorporate trade studies for the FY 1994 and FY 1995 activities. Begin the preparation of the Design Requirements documents for each of the projects.

- Establish a risk management approach and perform risk assessments on the priority risk areas. Provide training and tools to implement this discipline within the TWRS Program. Assess and provide alternatives to the TWRS Program Strategy with emphasis on cost-effective use of authorized budgets.

- Perform baseline verifications, at both the Program level and the Project level, to ensure that the products satisfy the respective functions and requirements. Perform Program and Project reviews to ensure that action items and open issues related to systems engineering functions and interfaces are properly dispositioned.

- Improve schedule baseline data by making use of improved planning information developed through cost engineering and systems engineering work summarized above.

- Improve TWRS baseline validity and credibility by completing a TWRS RL multi-year baseline internal validation effort, completing a DOE-HQ EM-36 pre-validation review, completing a DOE-HQ FM validation review, and submitting an updated MYWP for DOE approval.

\section{Execution (WBS 1.1.1.1.01.02)}

- $\quad$ Provide overall TWRS Program management services and operate management control and program administration systems.

- $\quad$ Provide overall TWRS coordination and funding for preparation of the TWRS EIS and data packages needed by the EIS contractor to support submission of the EIS to DOE-HQ.

- $\quad$ Provide environmental permit, communications, quality assurance, safety and health, qualification and training, information management, and acquisition support to the TWRS Program.

- Provide necessary expertise, integration, and coordination of techniques to identify and pursue work process improvements.

\section{Evaluation (WBS 1.1.1.1.01.03)}

- Issue program analysis procedures, schedule and conduct selected program analyses, and report results to program management.

- $\quad$ Collect and report data related to performance against approved baselines. 
- $\quad$ Provide customer support including review of incoming documents, preparation of briefing material.

- Schedule and conduct reviews of engineering documentation to ascertain level of compliance with DOE Orders, industry standards and codes, WHC engineering procedures.

\section{Program Management Systems (WBS 1.1.1.1.01.04)}

- Complete program management/management systems documentation including submittal of a WHC TWRS Management Plan to RL.

- $\quad$ Prepare a change management system status/recommendation white paper.

\subsubsection{FY 1996 - FY 1997 Work To Be Performed}

All four activities that comprise the PM\&A Program Element will be active in the FY 1996 FY 1997 time frame. In general, systems engineering, planning, scheduling, estimating, baselining, management systems documentation, execution and evaluation, EIS, and permit efforts will be conducted to support the objectives and strategy summarized in Section 7.2.1.1. Activity-level work statements are provided below. It should be noted that more detailed, cost account, task and subtask scope statements are documented in Level VI WBS Dictionary Sheets (refer to Appendix A) and Level VII and VIII Activity Planning Forms.

\section{Formulation (WBS 1.1.1.1.01.01)}

- Provide overall guidance to the TWRS Program in the area of planning, scheduling, cost estimating, and baselining.

- Improve cost baseline data by scheduling and conducting cost engineering and estimating tasks and continuing to improve the cost data base initially established in FY 1995.

- Improve technical baseline data by driving the definition of functions and requirements to successively lower levels. Continue to assess the engineering analyses for impacts to the strategies and enabling assumptions. Continue to assess the risks and provide recommendations on technical alternatives which provide more cost-effective program baseline. Continue to preform Program and Project reviews to verify compliance with established functions and satisfactory resolution to action items and open issues in this area. Additional information on this work scope and deliverables can be found in Appendix A, WBS Index and Dictionary; Appendix C, Program Element Schedules; and in Appendix D, Milestone Log and Descriptions Sheets.

- Improve schedule baseline data by making use of improved planning information developed through cost engineering and systems engineering work summarized above.

- Improve TWRS baseline validity and credibility by annually completing a TWRS RL multi-year baseline internal validation effort; completing a DOE-HQ EM-36 pre-validation review; completing a DOE-HQ FM validation review; and submitting an updated MYWP for DOE approval. 


\section{Execution (WBS 1.1.1.1.01.02)}

- $\quad$ Provide overall TWRS Program management services and operate management control and program administration systems.

- $\quad$ Provide overall TWRS coordination and funding for preparation of the TWRS EIS and data packages needed by the EIS contractor to support completion of the EIS and issue of the Record of Decision no later than October 1997.

- Provide environmental permit, communications, quality assurance, safety and health, qualification and training, information management, and acquisition support to the TWRS Program.

- Continue to provide expertise, integration, and coordination of techniques to identify and pursue work process improvements.

\section{Evaluation (WBS 1.1.1.1.01.03)}

- Schedule and conduct selected program analyses and report results to program management.

- Collect and report data related to performance against approved baselines.

- Provide customer support including review of incoming documents, preparation of briefing material.

- Schedule and conduct reviews of engineering documentation to ascertain level of compliance with DOE Orders, industry standards and codes, and WHC engineering procedures.

\section{Program Management Systems (WBS 1.1.1.1.01.04)}

- Review and update program management/management systems documentation including updating the WHC TWRS Management Plan to RL.

\subsubsection{Approach}

\section{Formulation (WBS 1.1.1.1.01.01)}

The Formulation Activity within the PM\&A Program Element will utilize existing Automated Data Processing (ADP) technologies to complete the work identified in Sections 7.2.1.3 and 7.2.1.4; however, additional application capability is required. Additional capability will be needed principally in the Cost Engineering tasks. Additional capability will be provided through a moderate increase in WHC TWRS Program Office staff as well as three sources which are new to the PM\&A Program Element. These sources include ICF Kaiser Hanford Project Cost Estimating Department and their subcontractors; the existing support contract with Los Alamos Technical Associates (LATA), which includes access to several other subcontractors (e.g., Project Time and Cost, Inc.); and the WHC Planning and Integration Basic Ordering Agreement (BOA). 
The overall approach to execution of the work scope within the Formulation Activity is to plan, schedule, and fund work to improve TWRS technical, cost, and schedule data on a continual basis for the next several years using distinct scope statements and deliverables. This overall approach is coupled with the planned update of the baseline "package" (e.g., MYWP) on an annual basis.

\section{Execution (WBS 1.1.1.1.01.02)}

*

The Execution Activity within the PM\&A Program Element will utilize existing ADP technologies to complete the work identified in Sections 7.2.1.3 and 7.2.1.4.

The overall approach to execution of the work scope within the Execution Activity is to operate the existing management control and program administration systems using data improvements provided by the Formulation Activity and system improvements recommended by the Evaluation Activity and provided by the Formulation and Program Management Systems Activities.

\section{Evaluation (WBS 1.1.1.1.01.03)}

The Evaluation Activity within the PM\&A Program Element will utilize existing WHC and RL staff to complete the work identified in Sections 7.2.1.3 and 7.2.1.4.

The overall approach to execution of the work scope within the Evaluation Activity in the near term is to establish and implement program analysis procedures to provide WHC directed program evaluation capability.

\section{Program Management Systems (WBS 1.1.1.1.01.04)}

The Program Management Systems Activity within the PM\&A Program Element will need additional application capability in the Program Documentation Development Cost Account (1.1.1.1.01.04.01). Additional capability will be provided through two sources that are new to the PM\&A Program Element. These sources include the existing support contract with LATA, which includes access to several other subcontractors (e.g., Project Time and Cost, Inc.), and the WHC Planning and Integration Basic Ordering Agreement (BOA).

The overall approach to execution of the work scope within the Program Management Systems Activity in the near term is to establish and implement program management systems documentation and in the ensuing years upgrade and/or revise management systems documentation as required by TWRS stakeholders.

\subsubsection{Drivers and Deliverables}

PM\&A drivers and deliverables are documented in Level IV, V, and VI WBS Dictionary Sheets (refer to Appendix A), Level VII and VIII Activity Planning Forms, and Milestone Description Sheets (refer to Appendix D). 
WHC-SP-1101

\subsubsection{Schedule}

\subsubsection{Program Management and Administration Summary Schedule}

As depicted in Figure 7.2-4, Program Management and Administration Summary Schedule, PM\&A workload is at its heaviest in the near to medium term with some of the decision making, planning, and baselining efforts Jeveling off and concluding in the later stages of the TWRS Program schedule. Examples include conclusion of EIS support and the eventual completion of the efforts associated with systems engineering. During the later stages of the overall TWRS schedule, the Execution and Evaluation Activities within PM\&A will be the principal efforts.

\subsubsection{Program Management and Administration Expanded Baseline (FY 1995 - FY 1997)}

As depicted in the PM\&A Program Element Schedule in Appendix C, the FY 1995 portion of the schedule is dominated by improving the data that are the foundation for the TWRS baseline during the first half of the year and utilizing the second half of the year to package, validate, and gain approval of the updated baseline while base data are continually improved. Figure 7.2-5, The Baseline Pyramid, graphically portrays the TWRS baseline pyramid and the baseline process and its relationship to the packaging requirement. Packaging or production and approval of an updated MYWP are assumed to be an annual requirement to support the Federal budget cycle and the Hanford Site Management System. Management system documentation will be improved throughout the year.

During FY 1996 and FY 1997, the base data continue to be improved and baseline capability enhancements and products pursued in FY 1995 (e.g., the TWRS cost estimating database and approval of the TWRS Functions and Requirements document) begin to be utilized. The TWRS Record of Decision (ROD) is scheduled to be issued in early FY 1997.

More detailed schedule information is provided in Appendix C, Program Element Schedules.

\subsubsection{Resources Requirements}

\subsubsection{Cost}

\subsection{Basis of Cost Estimates}

Cost estimates for the work scope represented by the PM\&A Program Element are improving. Better, more defendable estimates are needed, however, as the overall TWRS baseline matures. At present, the bases for cost estimates are the product definitions addressed in Section 7.2.1 as well as the WBS Dictionary Sheets (Appendix A) and the associated Activity Planning Forms. Also providing a basis for estimates are the assumptions documented in Section 7.2.1.1.6 and the Activity Planning Forms, as well as the logic construction and task identification represented in Section 7.2.2. Several sources of cost data are currently available to the PM\&A Program Element: Industry standard (such as the recent Hanford Site Environmental Restoration Project baselining experience); TWRS cost history; and Hanford cost history. A TWRS cost database is needed, and its development and use are planned in this baseline.

For more information on basis of estimates see Appendix E. For more detailed basis of cost estimates, see backup TWRS Cost Estimate Sheets. 


\subsection{Total Program Element Cost by Year by Fund Type by Activity and Cost Account}

Table 7.2-1 establishes the baseline cost for this program element. The baseline cost is exhibited in annual costs for FY 1994 through FY 2000, and 5-year totals for FY 2001 through program element completion. Where the data are available, these baseline costs are exhibited at the cost account level with fund type totals provided. Cost data are then rolled up to the activity and program element levels. At present, the program element cost baseline is the program element cost totals exhibited on this table less a productivity challenge for FY 1995 through FY 1997.

\subsection{FY 1995 Cost by Month by Fund Type by Activity and Cost Account}

Future MYWP updates will include Table 7.2-2, which establishes the month-by-month cost baseline for FY 1995 for this program element. The FY 1995 cost baseline is exhibited at the cost account level with fund type totals provided. Cost data are then rolled up to the activity and program element levels. Also exhibited is the total cost baseline for the program element for FY 1995. At present, the program element cost baseline for FY 1995 for this program element is the total provided on this table less a productivity challenge for FY 1995.

\subsubsection{Staffing}

\subsection{Basis of Staffing Projection}

Staffing projections for the PM\&A Program Element are based on the same product definition, assumption, logic construction, and task identification summarized in Section 7.2.3.1.1. FY 1995 cost engineering work will begin to improve the tools available to TWRS program element planners to match specific tasks with the appropriate staff levels and qualifications. The overall thrust of PM\&A staff planning, however, is to "out source" or utilize subcontractors rather than Management \& Operating contractor employees to the extent practical.

\subsection{Total Full-Time Equivalents by Year by Activity by Major Participant (Direct and Indirect)}

Future MYWP updates will include Table 7.2-3, which establishes total staffing projections for this program element. Included in the table are FY 1994 staffing data as well as projections by year for FY 1995 through FY 2000. The projections in this table include all direct and indirect Full-Time Equivalents (FTEs), including all subcontractor personnel. Where data are available, projections are exhibited at the activity level with major program participant totals provided.

\subsection{FY 1995 through FY 1997 Direct Full-Time Equivalents by 88 Labor Categories}

Future MYWP updates will include Table 7.2-4, which establishes direct WHC staffing projections for this program element. Projections do not include subcontractor support and are provided by 88 labor categories for FY 1995 through FY 1997.

\subsubsection{Work Scope Carryover}

There is no significant work scope carryover in the PM\&A Program Element. 


\subsubsection{Waste Type Data}

The PM\&A Program Element is comprised of program management and administrative activities and does not generate or receive any wastes requiring direct funding to disposition. TWRS waste type data are provided in Appendix $\mathrm{H}$.

\subsubsection{Revisions}

In future updates of the MYWP, this section will include a summary of the revision that has occurred in this program element since the last issue of the MYWP. 
Figure 7.2-1. Risk of Inadequate Resource Availability in FY 1995.

(2 Sheets)

RISK

\section{SYSTEMS ENGINEERING}

1. Authorized $\$ 10889 \mathrm{~K}$ against a need of $\$ 11230 \mathrm{~K}$. Some minor adjustments in schedule may be required. DOE-RL staff believe that another $\$ 1500 \mathrm{~K}$ are necessary to satisfy all DNSFB requirements.

2. Technical Strategic Analysis $(\$ 906 \mathrm{~K})$ is new scope and unfunded. This activity was formally established during the reorganization which established the Strategic Planning and Systems Engineering function.

3. Public involvement is funded at $\$ 173 \mathrm{~K}$ against a request of $\$ 1007 \mathrm{~K}$.

\section{PROGRAM ADMINISTRATION}

1 Authorized \$1184K against a request of $\$ 3772 \mathrm{~K}$. All major systems upgrades to the business management system are new scope above the Program Master Schedule (PMS).

\section{PROJECT BUSINESS MANAGEMENT/SITE INTEGRATION}

1. Authorized $\$ 3572 \mathrm{~K}$ against a request of $\$ 3730 \mathrm{~K}$. The need for the added $\$ 158 \mathrm{~K}$ results from work scope carryover from

FY 1994 into FY 1995. Lack of the added funding could preclude completion of the Site Integration Plan.

\section{MITIGATION}

Program management will review activities throughout the year and make adjustments as funds become available to fund the highest priority activities.

Program management will review activities throughout the year and make adjustments as funds become available to fund the highest priority activities.

This activity was funded through G\&A in FY 1994. It must be funded though G\&A in FY 1995 until a mechanism to transfer funds from cost savings though rate changes can be implemented.

Program management will review activities throughout the year and make adjustments as funds become available to fund the highest priority activities.

If carryover funding is not available, Project Business Management will be expected to adjust their activities to address the shortfall in funding. 


\section{QUALITY ASSURANCE}

1. Development of the Quality Assurance Management Plan (QAMP) to comply with 10 CFR 183.120 is new scope for the baseline and unfunded.

Noncompliance,can have legal implications.

2. The QA assessment program ( $\$ 1025 \mathrm{~K})$ is not funded by this Program Element. In FY 1994, this activity was funded by the QA overhead. The lack of independent overview is noncompliant with DOE orders.

3. DOE-RL (GSSC and Support) authorized $\$ 5334 \mathrm{~K}$ against a stated need of $\$ 8800 \mathrm{~K}$. The shortfall of $\$ 3466 \mathrm{~K}$ affects ability to perform Independent Cost Estimates (ICE), Validation Reviews, benchmark development, and pay facility rent.

\section{PROGRAM DOCUMENTATION} DEVELOPMENT

1. Since all program management documentation was to have been completed in FY 1994, any new work is classified as new scope and is currently unfunded. The scope of the work will not be known until thirty days after the Management System Description is issued as an approved Document.
Since this new activity is a must, it is being considered as an item to be funded by the TWRS overhead.

This activity was funded in FY 1994 by the QA overhead. It must be funded by the same source until a mechanism can be established to recover savings from reduced rates and funds transferred to the appropriate program element to fund the new work scope.

This is scope above that defined in the Program Master Schedule. Program Management (DOE-RL and WHC) will review overall priorities of these activities against activities currently funded and make appropriate adjustments.

Funding for this activity will be determined after the scope is defined and understood. 


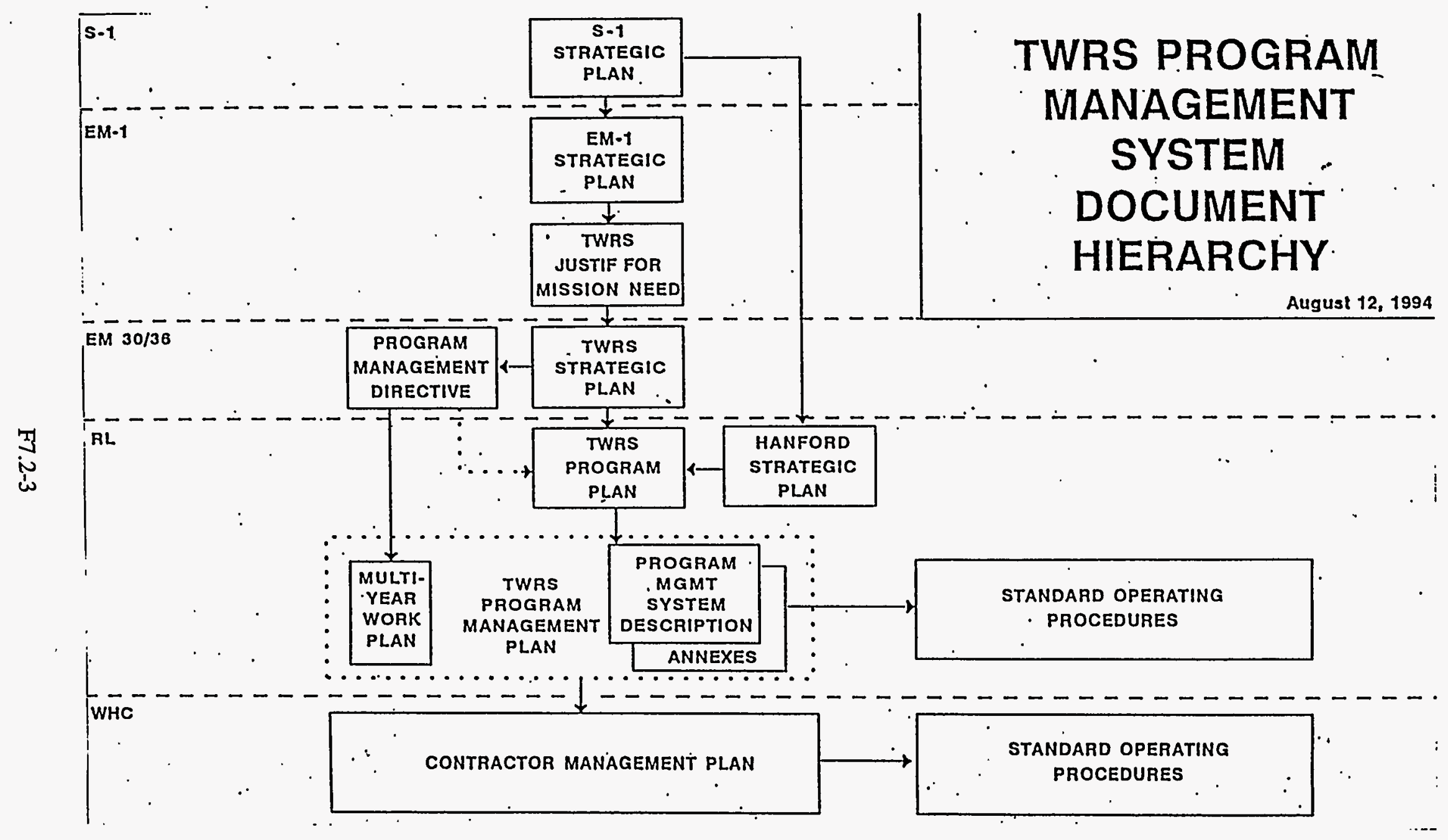




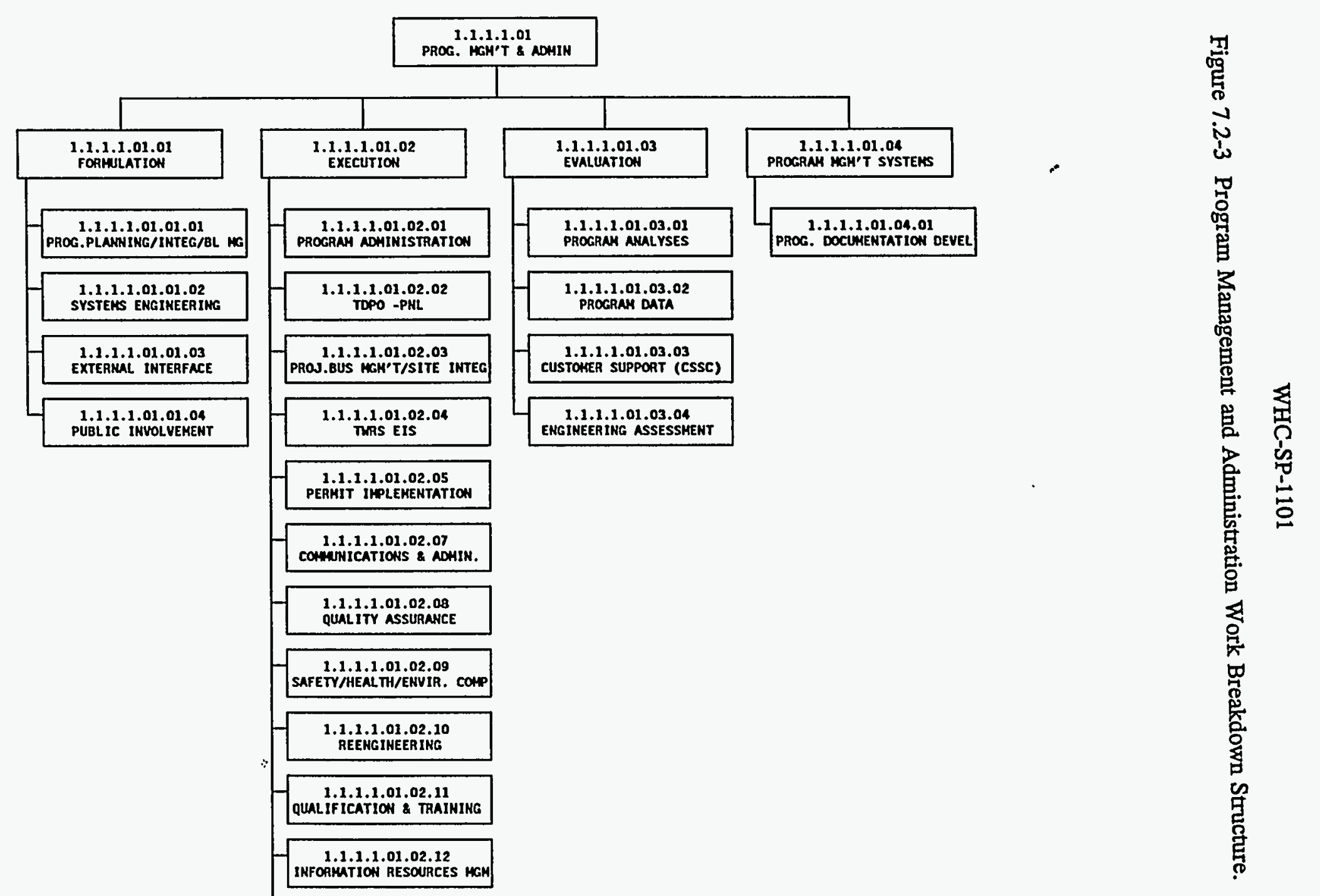




\subsection{Program Management and Administration Summary Schedule}

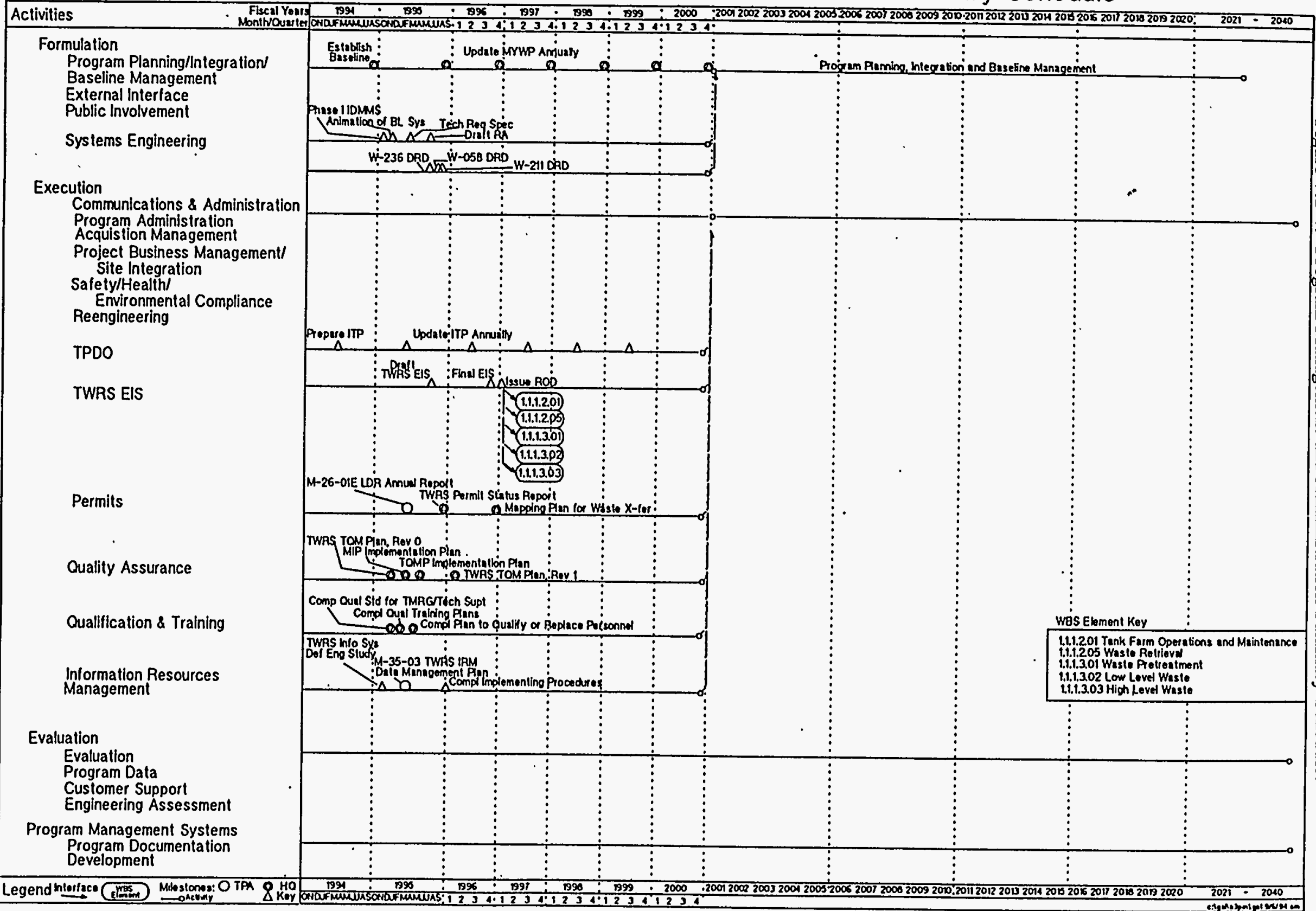


Figure 7.2-5. The Baseline Pyramid.

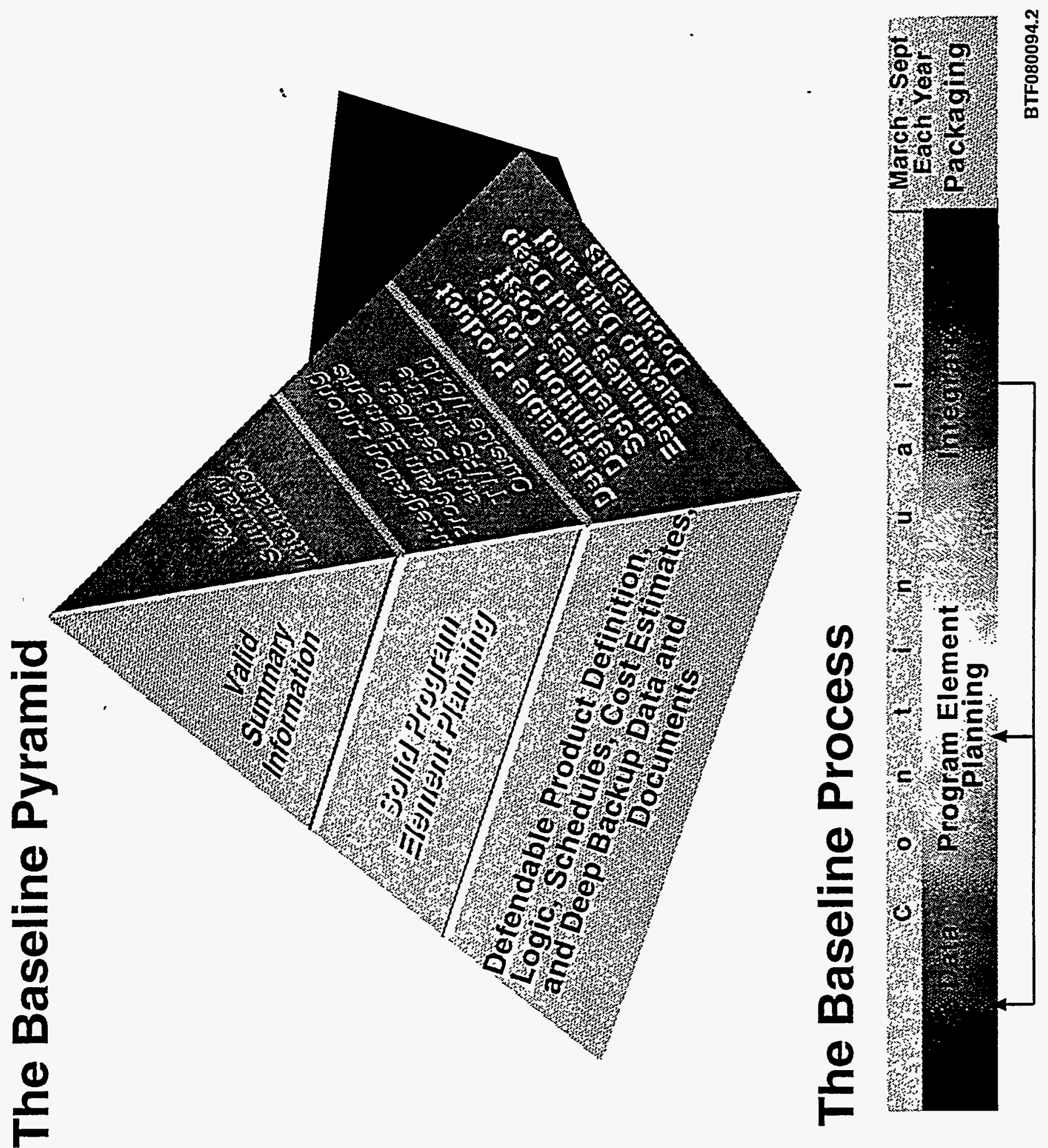


TABLE 7.2-1

TOTAL PROGRAM ELEMENT [ R1 ]

COST BASELINE - BY YEAR

(K\$)

\begin{tabular}{|c|c|c|c|c|c|c|c|c|c|}
\hline WBS / LEVEL & ACTIVITY TITLE / ADS & $\begin{array}{l}\text { FUND } \\
\text { TYPE }\end{array}$ & $\begin{array}{l}\text { TOTAL } \\
\text { FY94 }\end{array}$ & FY95 & FY96 & FY97 & FY98 & FY99 & FYO0 \\
\hline \begin{tabular}{c}
$1.1: 1.101$ \\
Level 10 \\
\hdashline \\
$\vdots$
\end{tabular} & $\begin{array}{r}\text { Program Management \& } \\
\text { Administration } \\
\text { TOS 1200-0 TAL TARGET }\end{array}$ & OE & $\begin{array}{r}4 \\
49,319 \\
\vdots \\
49,319 \\
4\end{array}$ & $\begin{array}{r}48,202 \\
-48,202\end{array}$ & $\begin{array}{r}46,564 \\
46,564\end{array}$ & $\begin{array}{r}4040 \\
430 \\
43640\end{array}$ & $\begin{array}{r}45,973 \\
4 \\
45,973 \\
4\end{array}$ & $\begin{array}{r}4,8 \\
46,353 \\
4 \\
46,353\end{array}$ & $\begin{array}{l}48,786 \\
4 \\
4 \\
47,786\end{array}$ \\
\hline & $\begin{array}{l}\text { Delta to TARGET. } \\
\text { (See notes at end of Table.) }\end{array}$ & $O E$ & NA & 0 & (1) & 0 & & & \\
\hline $\begin{array}{l}1.1 .1 .1 .01 .01 \\
\text { Level } \mathrm{V}\end{array}$ & $\frac{\text { Formulation }}{\text { ADs } 1200-0-A A}$ & OE & NA & 14,445 & 15,061 & 15,544 & $\mathbf{0}$ & $\mathbf{0}$ & $\mathbf{0}$ \\
\hline $\begin{array}{l}\text { 1.1.1.1.01.01.01 } \\
\text { Level VI }\end{array}$ & $\begin{array}{l}\text { Prog. Planning / Integration } \\
\text { \& Baseline Mgmt. }\end{array}$ & OE & & 2,883 & 3,238 & 3,336 & & & \\
\hline $\begin{array}{l}\text { 1.1.1.1.01.01.02 } \\
\text { Level VI }\end{array}$ & Systems Engineering & OE & & 10,889 & 11,216 & 11,552 & & & \\
\hline $\begin{array}{l}\text { 1.1.1.1.01.01.03 } \\
\text { Level VI }\end{array}$ & External Interface & OE & & 500 & 412 & 424 & & & \\
\hline $\begin{array}{l}\text { 1.1.1.1.01.01.04 } \\
\text { Level VI }\end{array}$ & Public Involvement & OE & & 173 & 195 & 232 & & & \\
\hline $\begin{array}{l}1.1 .1 .1 .01 .02 \\
\text { Level } \mathrm{v}\end{array}$ & $\frac{\text { Execution }}{A D S 1200-0-A B}$ & OE & NA & 26,605 & 24,388 & 20,930 & $\mathbf{0}$ & $\mathbf{0}$ & $\mathbf{0}$ \\
\hline $\begin{array}{l}\text { 1.1.1.1.01.02.01 } \\
\text { Level VI }\end{array}$ & Program Administration & OE & & 1.879 & 2,694 & 1,468 & & & \\
\hline
\end{tabular}


TABLE 7.2-1

TOTAL PROGRAM ELEMENT

COST BASELINE - BY YEAR

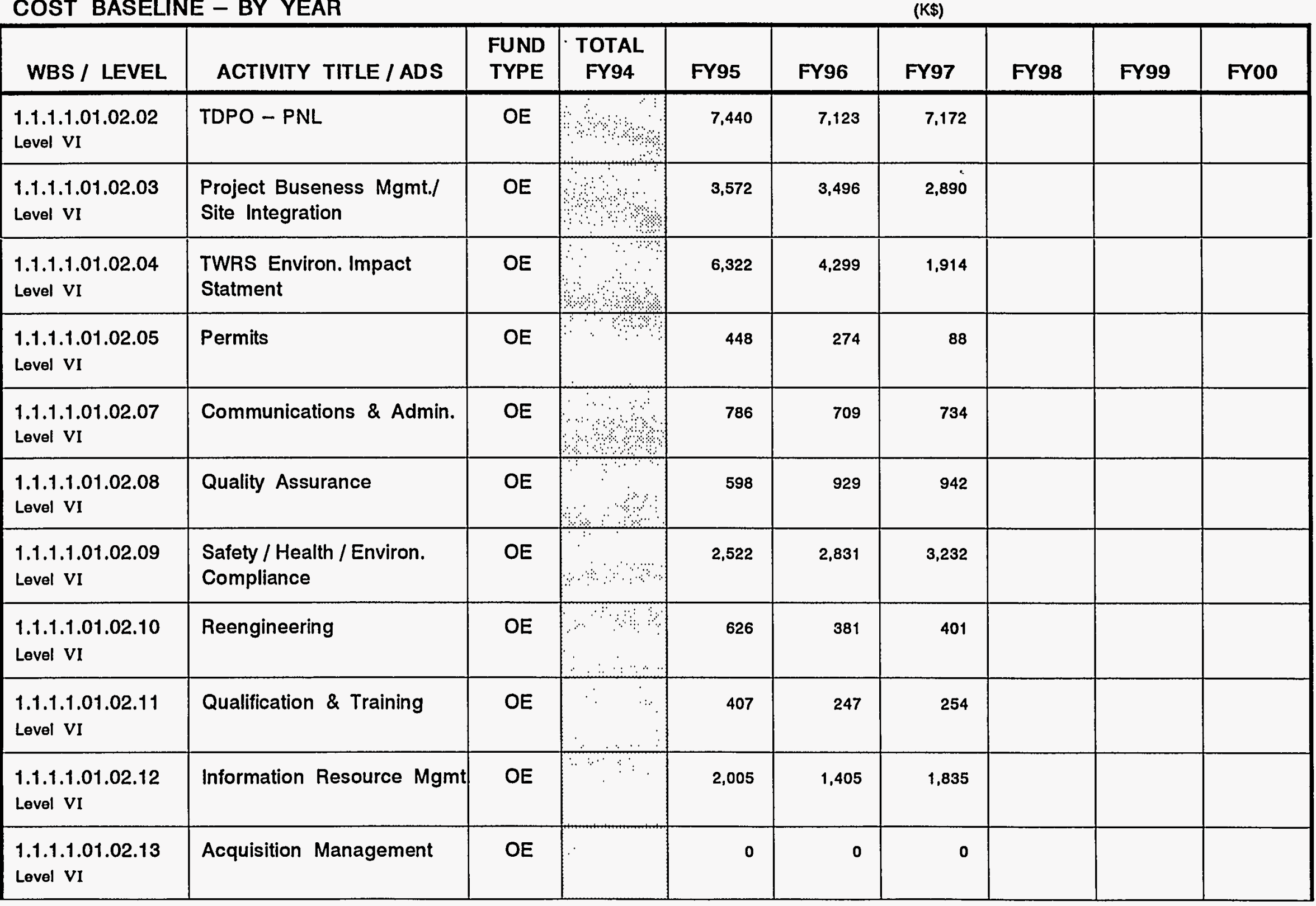

\section{[ R1 ]}


TABLE 7.2-1

TOTAL PROGRAM ELEMENT

COST BASELINE - BY YEAR

\begin{tabular}{|c|c|c|c|c|c|c|c|c|c|}
\hline WBS / LEVEL & ACTIVITY TITLE / ADS & $\begin{array}{l}\text { FUND } \\
\text { TYPE }\end{array}$ & $\begin{array}{l}\text { TOTAL } \\
\text { FY94 }\end{array}$ & FY95 & FY96 & FY97 & FY98 & FY99 & FYOO \\
\hline $\begin{array}{l}\text { 1.1.1.1.01.03 } \\
\text { Level } \mathrm{v}\end{array}$ & $\frac{\text { Evaluation }}{\text { ADS } 1200-0-A C}$ & OE & NA & 7,152 & 7,116 & 7,166 & $\mathbf{0}$ & $\mathbf{0}$ & $\mathbf{0}$ \\
\hline $\begin{array}{l}1.1 .1 .1 .01 .03 .01 \\
\text { Level VI }\end{array}$ & Program Analyses & OE & & 53 & 0 & 0 & & & \\
\hline $\begin{array}{l}1.1 .1 .1 .01 .03 .02 \\
\text { Level VI }\end{array}$ & Program Data & OE & & 1,640 & 1,690 & 1,740 & & & \\
\hline $\begin{array}{l}\text { 1.1.1.1.01.03.03 } \\
\text { Level VI }\end{array}$ & Customer Support (CSSC) & OE & $\begin{array}{ll} & \\
3 & \vdots \\
\vdots\end{array}$ & 5,334 & 5,426 & 5,426 & & & \\
\hline $\begin{array}{l}\text { 1.1.1.1.01.03.04 } \\
\text { Level VI }\end{array}$ & Engineering Assessment & OE & ২। & 125 & 0 & 0 & & & \\
\hline $\begin{array}{l}1.1 .1 .1 .01 .04 \\
\text { Level VI }\end{array}$ & $\begin{array}{l}\text { Program Mgmt. System } \\
\text { ADS } 1200-0-A D\end{array}$ & OE & NA & $\mathbf{0}$ & $\mathbf{0}$ & $\mathbf{0}$ & $\mathbf{0}$ & $\mathbf{0}$ & $\mathbf{0}$ \\
\hline $\begin{array}{l}1.1 .1 .1 .01 .04 .01 \\
\text { Level VI }\end{array}$ & $\begin{array}{l}\text { Program Documentation } \\
\text { Development }\end{array}$ & OE & & 0 & 0 & 0 & & & \\
\hline
\end{tabular}

\section{[ R1 ]}


TABLE 7.2-1

TOTAL PROGRAM ELEMENT COST BASELINE - BY YEAR

\section{[ R1 ]}

\begin{tabular}{|c|c|c|c|c|c|c|c|c|c|c|}
\hline WBS / LEVEL & ACTIVITY TITLE / ADS & $\begin{array}{l}\text { FUND } \\
\text { TYPE }\end{array}$ & $\begin{array}{l}\text { FY01 I } \\
\text { FY05 }\end{array}$ & $\begin{array}{l}\text { FY06 I } \\
\text { FY10 }\end{array}$ & $\begin{array}{l}\text { FY11 I } \\
\text { FY15 }\end{array}$ & $\begin{array}{l}\text { FY16 I } \\
\text { FY20 }\end{array}$ & $\begin{array}{l}\text { FY21 I } \\
\text { FY25 }\end{array}$ & $\begin{array}{l}\text { FY26 I } \\
\text { FY30 }\end{array}$ & $\begin{array}{l}\text { FY31 I } \\
\text { FY35 }\end{array}$ & $\begin{array}{l}\text { FY36 I } \\
\text { FY40 }\end{array}$ \\
\hline 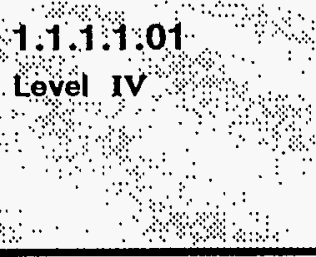 & $\frac{\text { Program Management \& }}{\text { Administration }}$ & & $\begin{array}{l}229,828 \\
2,9,6 \\
229,828\end{array}$ & $\begin{array}{r}7 \\
2107666 \\
210,766\end{array}$ & 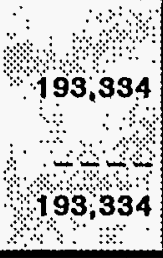 & $\begin{array}{r}0 \\
179,145 \\
179,145\end{array}$ & 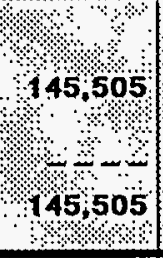 & $\begin{array}{c}0.9 \\
98,727 \\
98,727\end{array}$ & 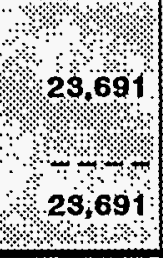 & 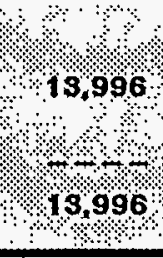 \\
\hline & $\begin{array}{l}\text { Delta Required to } \\
\text { Balance P3 to TARGET }\end{array}$ & $O E$ & & & & & & & & \\
\hline $\begin{array}{l}\text { 1.1.1.1.01.01 } \\
\text { Level V }\end{array}$ & $\frac{\text { Formulation }}{\text { ADS } 1200-0-M}$ & OE & 0 & 0 & 0 & o & 0 & 0 & 0 & 0 \\
\hline $\begin{array}{l}\text { 1.1.1.1.01.01.01 } \\
\text { Level VI }\end{array}$ & $\begin{array}{l}\text { Prog. Planning / Integration } \\
\text { \& Baseline Mgmt. }\end{array}$ & OE & & & & & & & & \\
\hline $\begin{array}{l}\text { 1.1.1.1.01.01.02 } \\
\text { Level VI }\end{array}$ & Systems Engineering & $O E$ & & & & & & & & \\
\hline $\begin{array}{l}\text { 1.1.1.1.01.01.03 } \\
\text { Level VI }\end{array}$ & External Interface & $\mathrm{OE}$ & & & & & & & & \\
\hline $\begin{array}{l}\text { 1.1.1.1.01.01.04 } \\
\text { Level VI }\end{array}$ & Public Involvement & $\mathrm{OE}$ & & & & & & & & \\
\hline $\begin{array}{l}\text { 1.1.1.1.01.02 } \\
\text { Level v }\end{array}$ & $\frac{\text { Execution }}{\text { ADS } 1200-0-A B}$ & $\mathrm{OE}$ & 0 & 0 & o & 0 & 0 & o & 0 & 0 \\
\hline $\begin{array}{l}\text { 1.1.1.1.01.02.01 } \\
\text { Level VI }\end{array}$ & Program Administration & $\mathrm{OE}$ & & & & & & & & \\
\hline
\end{tabular}


TABLE 7.2-1

\begin{tabular}{|c|c|c|c|c|c|c|c|c|c|c|}
\hline WBS / LEVEL & ACTIVITY TITLE / ADS & $\begin{array}{l}\text { FUND } \\
\text { TYPE }\end{array}$ & $\begin{array}{l}\text { FY01 I } \\
\text { FY05 }\end{array}$ & $\begin{array}{l}\text { FY06 I } \\
\text { FY10 }\end{array}$ & $\begin{array}{l}\text { FY11 I } \\
\text { FY15 }\end{array}$ & $\begin{array}{l}\text { FY16 I } \\
\text { FY20 }\end{array}$ & $\begin{array}{l}\text { FY21 I } \\
\text { FY25 }\end{array}$ & $\begin{array}{l}\text { FY26 I } \\
\text { FY30 }\end{array}$ & $\begin{array}{l}\text { FY31 I } \\
\text { FY35 }\end{array}$ & $\begin{array}{l}\text { FY36 I } \\
\text { FY40 }\end{array}$ \\
\hline $\begin{array}{l}\text { 1.1.1.1.01.02.02 } \\
\text { Level VI }\end{array}$ & TDPO - PNL & OE & & & & & & & & \\
\hline $\begin{array}{l}\text { 1.1.1.1.01.02.03 } \\
\text { Level VI }\end{array}$ & $\begin{array}{l}\text { Project Buseness Mgmt./ } \\
\text { Site Integration }\end{array}$ & OE & & & & & & & & \\
\hline $\begin{array}{l}\text { 1.1.1.1.01.02.04 } \\
\text { Level VI }\end{array}$ & $\begin{array}{l}\text { TWRS Environ. Impact } \\
\text { Statment }\end{array}$ & OE & & & & & & & & \\
\hline $\begin{array}{l}\text { 1.1.1.1.01.02.05 } \\
\text { Level VI }\end{array}$ & Permits & OE & & & & & & & & \\
\hline $\begin{array}{l}\text { 1.1.1.1.01.02.07 } \\
\text { Level VI }\end{array}$ & Communications \& Admin. & OE & & & & & & & & \\
\hline $\begin{array}{l}\text { 1.1.1.1.01.02.08 } \\
\text { Level VI }\end{array}$ & Quality Assurance & OE & & & & & & & & \\
\hline $\begin{array}{l}\text { 1.1.1.1.01.02.09 } \\
\text { Level VI }\end{array}$ & $\begin{array}{l}\text { Safety / Health / Environ. } \\
\text { Compliance }\end{array}$ & OE & & & & & & & & \\
\hline $\begin{array}{l}\text { 1.1.1.1.01.02.10 } \\
\text { Level VI }\end{array}$ & Reengineering & $\mathrm{OE}$ & & & & & & & & \\
\hline $\begin{array}{l}\text { 1.1.1.1.01.02.11 } \\
\text { Level VI }\end{array}$ & Qualification \& Training & $O E$ & & & & & & & & \\
\hline $\begin{array}{l}\text { 1.1.1.1.01.02.12 } \\
\text { Level VI }\end{array}$ & Information Resource Mgmt & OE & & & & & & & & \\
\hline $\begin{array}{l}\text { 1.1.1.1.01.02.13 } \\
\text { Level VI }\end{array}$ & Acquisition Management & OE & & & & & & & & \\
\hline
\end{tabular}


TABLE 7.2-1

TOTAL PROGRAM ELEMENT

COST BASELINE - BY YEAR

[ R1 ]

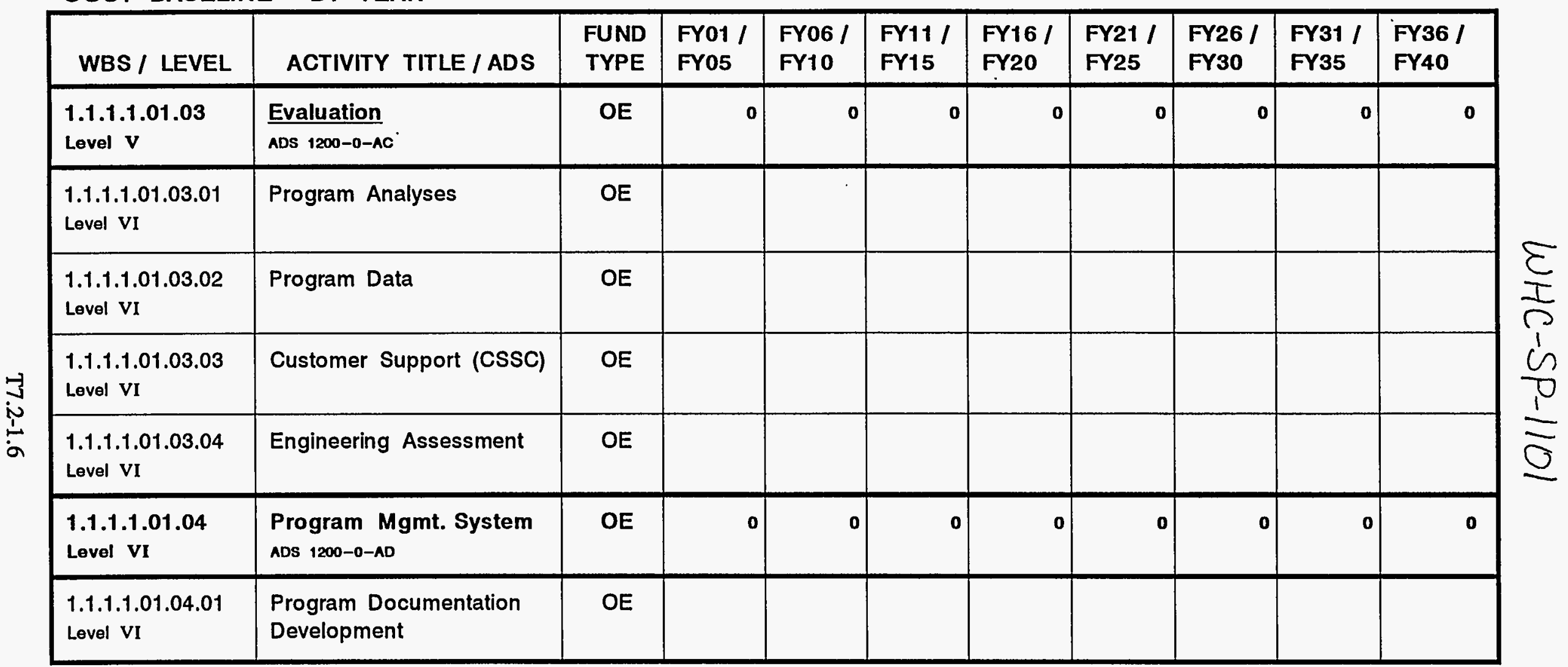




\section{NOTES \\ TOTAL PROGRAM ELEMENT COST BASELINE - BY YEAR}

The cost exhibit tables in each of the Program Element Baselines (Sections 7.2 through 7.10) identify the program element target funding by year by fund type. The tables also provide details of the baseline allocation of funding down to activity levels (level V) and cost account levels (level VI) of the WBS for Fiscal Year 1995 through 1997. The details exhibited on these tables are taken from the baseline WBS Dictionary Sheets. Deltas exist between the details and the annual target totals. For Fiscal Years 1996 and 1997 the deltas will be addressed in the future, following the completion of the resolution of expected differences between current Fiscal Year 1995 baseline funding and expected actual Fiscal Year 1995 funding. Deltas for Fiscal Year 1995 are noted below by program element:

PROGRAM MANAGEMENT AND ADMINISTRATION:

No deltas.

\section{TANK FARM OPERATIONS AND MAINTENANCE:}

No overall delta. This baseline requires revised fund type mix.

\section{WASTE TANK SAFETY:}

Delta is due to need to revise an overhead pricing rate in the P3 system.

\section{TANK FARM UPGRADES:}

Delta is due to differences between Budget Authority (BA) in the target and Budget Obligation (B0) in the P3 system.

\section{CHARACTERIZATION:}

No significant deltas.

\section{WASTE RETRIEVAL:}

Delta is primarily due to need to resolve issues associated with expense funding support to capital projects and differences between Budget Authority (BA) in the target and Budget Obligation (BO) in the $\mathrm{P} 3$ system.

\section{WASTE PRETREATMENT:}

Delta is due to the sum of the detail cost estimates being lower than the target total as a result of reestimation of resource requirements to complete baseline scope.

\section{LOW LEVEL WASTE:}

Delta is primarily due to carryover expense funded workscope on melter testing addressed in June 9, 1994 RL letter.

$$
77.2-1.7
$$


WHC-SP-1101

\section{HIGH LEVEL WASTE:}

Delta is due to carryover of capital funding, PNL expense funding carryover, and ADS corrections submitted but not reflected in target totals. 
Table 7.2-2. FY 1995 Cost by Month by Fund Type by Activity and Cost Account

Future MYWP updates will include Table 7.2-2, which establishes the month-by-month cost baseline for FY 1995 for this program element. The FY 1995 cost baseline is exhibited at the cost account level with fund type totals provided. Cost data are then rolled up to the activity and program element levels. Also exhibited is the total cost baseline for the program element for FY 1995. At present, the program element cost baseline for FY 1995 for this program element is the total provided on this table less a productivity challenge for FY 1995. 
Table 7-2-3. Total Full-Time Equivalents by Year by Activity by Major Participant (Direct and Indirect) (Including all Subcontractors)

Future MYWP updates will include Table 7.2-3, which establishes total staffing projections for this program element. Included in the table are FY 1994 staffing data as well as projections by year for FY 1995 through FY 2000. The projections in this table include all direct and indirect Full-Time Equivalents (FTEs), including all subcontractor personnel. Where data are available, projections are exhibited at the activity level with major program participant totals provided. 
Table 7.2-4. FY 1995 and FY 1996 Direct Westinghouse Hanford Company Full-Time Equivalents by 88 Labor Categories

Future MYWP updates will include Table 7.2-4, which establishes direct WHC staffing projections for this program element. Projections do not include subcontractor support and are provided by 88 labor categories for FY 1995 through FY 1997. 


\subsection{TANK FARM OPERATIONS AND MAINTENANCE BASELINE}

The Tank Farm Operations and Maintenance (O\&M) Program Element baseline consists of the technical bases, schedule, resource requirements, and waste type data for FY 1995 through completion of the mission. The major emphasis is on deliverables, schedule, and resource requirements for FY 1995, 1996, and 1997.

$\therefore$

\subsubsection{Technical Bases}

This section provides the mission strategy; work breakdown structure and organization responsibility; work to be performed in FY 1995, 1996 and 1997; technical approach; and drivers and deliverables.

\subsubsection{Mission Strategy}

\subsection{Objectives}

The O\&M Program Element supports the operations of all waste tank storage, concentration, and transfer facilities. Continued safe operation of these facilities requires near- and long-term upgrades in safety bases and hardware restoration. Planning anticipates achieving a final safety bases in FY 2001 following numerous upgrades, Unreviewed Safety Question (USQ) resolution, and tank characterization.

Major end-item deliverables include, but are not limited to, the following: maintain the safe operating configuration within tank farms; operate the 242-A Evaporator, achieving a yearly waste volume reduction goal; complete $M-41-00$, single-shell tank (SST) interim stabilization; complete $M-46-00$, double-shell tank (DST) space evaluation; release waste tanks for retrieval and eventual turnover for closure; and turnover all tank farm facilities to decontamination and decommissioning for closure.

Complete interim stabilization and intrusion prevention for SSTs with pumpable liquid (except 241-C-106). Prepare for emergency pumping leaking SSTs and pump leaking SSTs. Procure Light Duty Utility Arm (LDUA) and obtain and maintain ability to operate LDUA. Install liquid observation wells (LOWs) in all SSTs with sufficient material to function. Deliver functional LOW monitoring systems.

The O\&M Program Element has become "standards-driven" and committed to continuous improvement. TWRS Plant has adopted standards for Conduct of Operations against which performance will be measured. Operating crews are staffed for five shifts to better support training requirements for nuclear process operators. Training will be comprehensive and formal and will use auditable certification examinations. Configuration control of all waste tank operation facilities will be maintained to modern standards through a commitment by line management and formal procedures to manage updates to drawings, documents, and labels.

\subsection{Strategy}

The strategy for operating and maintaining storage, transfer, and concentration functions for tank waste is based on four elements: significantly improved conduct of operations, evaporation capability, expedited closure of existing SSTs, and the shutdown and turnover for decontamination and decommissioning of all tank farm facilities. 
Managing tank waste safely in a cost-effective manner requires operating to modern standards for nuclear facilities. The strategy to upgrade conduct of operations in the tank farms has four components. Success in managing tank waste depends on changing to a "standards based" culture; operations and maintenance actions are based on (and traceable to) specific regulations, standards, and good practices. Tank farm facilities must be upgraded and maintained to an acceptable material condition. This includes accurate as-built drawings, labeling of equipment, proper operating and maintenance procedures, and a complete safety basis. Operations personnel must be upgraded in terms of experience, training, and numbers so that facilities can be properly operated and maintained. A credible and effective training and qualification process is required to maintain a disciplined, knowledgeable operating staff and supporting infrastructure. Implementation of a "fifth" shift will ensure continuing training for operating personnel. In the period from 1995 to 2000 , operations will build to a stable operating staff and improved infrastructure. Beyond 2000, mature safe operations will be established and maintained; however, attention to conduct of operations issues will be necessary for the entire life cycle of the tank farms.

Tank volume cannot be managed effectively without evaporation capability. Liquid waste to be stored will increase due to stabilization of waste stored in the SSTs and the final "cleanout" of former facilities at the Hanford Site. Evaporation will reduce the waste volume and allow operations to manage available DST space. The 242-A Evaporator facilities will perform this function.

The SSTs have aged beyond their useful life. In addition, these underground tanks cannot be properly monitored to ensure integrity as required by the State of Washington. A high priority is placed on interim stabilization of these tanks and then on closure. Closure will require transferring the interim stabilized waste from SSTs to available DST storage. The Waste Retrieval Program Element will determine the technology to be used; operations personnel will be responsible for conducting field activities. Closure of SSTs will be completed in 2018.

The final phase in the life cycle of the tank farms is the closure of all remaining facilities under the control of operations. As the last of the tank waste is processed into glass, unneeded DSTs will be cleaned out and prepared for turnover for decontamination and decommissioning. West Area facilities will be closed first; the last of the East Area tanks will close in 2028. Timely closure and turnover of unneeded facilities will reduce operating costs in this period.

\subsection{Functions and Requirements}

The functional product of O\&M is management of existing and generated tank farm waste and facilities until waste is removed from all tanks for final disposal processing and facilities have been turned over to the Environmental Restoration (ER) Decontamination \& Decommissioning (D\&D) Program. This program element is currently scheduled to end in the year 2028. The D\&D turnover deliverable standard will be as established by EM-40 in the Office of Environmental Restoration Decontamination and Decommissioning Guidance Document. Standards to which waste must be removed from the waste tanks will be established by final closure plans developed by the Waste Retrieval Program Element.

Technical requirements are to receive, contain, and monitor radioactive liquid wastes from Hanford facilities in an environmentally safe and cost-effective manner. This will continue until all waste is received and processed for final disposal. This element includes performance of routine surveillance, operations, maintenance, and stabilization activities necessary for safe and compliant underground waste storage and waste concentration activities. Continued safe operation of these facilities requires hardware restoration and both near- and long-term upgrades in the safety bases. Planning anticipates 
achieving a final safety bases in the beginning of FY 2001 following numerous upgrades, USQ resolution, and tank characterization. This will achieve a significantly improved infrastructure, allowing a mature safe operation for the planned life cycle (through 2028) of tank farms.

The SSTs (149) have aged beyond their useful life. In addition, these underground waste storage tanks cannot be properly monitored to ensure integrity as required by the State of Washington. Pumpable liquid is being removed from these tanks (interim stabilization) with retrieval operations and final closure to follow. This program element will complete interim stabilization activities by the year 2000 allowing Retrieval activities (4.2.1.3) to be performed. This activity is of low programmatic risk, 107 of 149 SSTs have been interim stabilized and numerous safety studies have been or are nearing completion to allow pumping of Watch List SSTs.

The final phase in the life cycle of the tank farms is the closure of all remaining facilities under the control of operations. As the last of the tank waste is processed into glass, unneeded waste tanks will be cleaned out and prepared for turnover to ER for D\&D. The 242-A Evaporator will be the first "major" facility earmarked for turnover (initial work on turnover will start in the year 2007), followed by farms in West Area and finally East Area. Timely closure and turnover of unneeded facilities will reduce operating costs in the Tank Farm life cycle. This final phase of Tank Farms has the highest uncertainty factor from a planning standpoint. To date, a major tank farm facility has not been prepared for turnover to ER for D\&D activities. Planning is preliminary and will be better defined following preparation of study and detail cost estimates. Activity for turnover preparations is not scheduled to begin until FY 1997. On a smaller scale, the first tank farm facilities scheduled for turnover to ER for D\&D will be the 242-T and 242-S Evaporators.

\subsection{Risks}

There are two risk elements that dominate the O\&M Program Element. The first is related to the management of storage space and the need for a new cross-site transfer line. The second is associated with achieving a stable disciplined operating organization.

A method to transfer liquid waste from the West to the East Area is needed to ensure completion of SST stabilization in West Area. Existing cross site transfer lines (2) are non-compliant with State of Washington regulations and are not active. If these lines can not be used to transfer waste from the West Area to the 242-A Evaporator in the East Area, then the Interim Stabilization West Area SSTs will be impacted until a compliant cross site transfer line can be completed. This-will delay completion of some Tri-Party Agreement milestones. In addition, Evaporator operation may also be temporary impacted by any delays in unrestricted operation of the 200 Area Liquid Effluent Treatment Facility (LETF), now scheduled to begin operation in June 1995. If planned Evaporator operations have to be curtailed because LETF can not process liquids from the Evaporator, space for liquids from SSTs may not be available in time to meet the current Tri-Party Agreement timetable.

Considerable progress has been made in strengthening Conduct of Operations in Tank Farms. The corner stone of this improvement has been the implementation of a 5th shift coupled with upgraded personnel and rigorous training/certification program. If funding is not sufficient to maintain these improvements, then rigor of operations will regress to unacceptable levels. A falloff in operation performance could affect successful completion of the entire TWRS Program, in that storage and retrieval goals would be impacted. 


\subsection{Major Decisions}

The key decisions for the TWRS Program are described in Tank Waste Remediation System Program Decisions and Risk Assessment, WHC-EP-0786 DRAFT (July 20, 1994). This document provides information on each key decision. Each decision is described, alternative solutions discussed and information available or necessary for evaluating alternative solutions identified.

The key decisions supported by the O\&M Program Element are found in EP-0786, Table 2-1, Decisions 4.2.1, 4.2.1.1.a, 4.2.1.1.b, 4.2.1.5, 4.2.3.5, and 4.2.3.6.

\subsection{Assumptions}

The assumptions on which planning for this program element has been based are describe below. The program sensitivity to most of these assumptions is low over the next 2 to 3 years. However, the longer term sensitivity increases rapidly after that time.

1. Implement 5th shift (Training Shift). The training shift is required to maintain the identified qualifications of the operators while minimizing the overtime required and maximizing the effectiveness of the training. Failure to staff the 5 th shift would result in missed training and increased overtime costs.

2. Emergency pumping will be required. There will be one new "assumed leaking" SST annually until interim stabilization is complete..

3. Interim Stabilization of SSTs will be complete by September 2000. Failure to interim stabilize the SSTs on an aggressive schedule increases the risk of additional leaking tanks, causing environmental insult. Space will be available in DSTs for SST liquids. A cross site transfer will be made.

4. Retrieval will be initiated by December 2003. Failure to initiate retrieval on schedule would compromise the schedule for getting out of the SSTs and turning them over to EM-40 for D\&D. Delays in the schedule would increase the cost of operating the tank farms.

5. Evaporator operations will be used to manage/optimize DST liquid volume. The evaporator is required to achieve the Waste Volume Reduction (WVR) required to avoid the need for additional DST storage tanks at a greatly increased cost.

6. Four new DSTs will be built in 200 East Area and two new DSTs will be built in 200 West Area. Delay in the construction of the new DSTs will cause delays in the retrieval schedule and delays in the eventual removal of waste from all tanks. This would cause an increase in cost.

7. Surveillance/maintenance costs for a retired facility will be transferred to $\mathbf{E M - 4 0}$ when custody of the facility is transferred to EM-40. Additional staff and funding would be required to retain the responsibility to support maintenance and surveillance beyond the planned date of turnover to EM-40. 
8. The Grout facilities surveillance and maintenance costs will be assumed by operations beginning in FY 1997 under the Retired Facilities Activity. The Retired Facilities Activity receives initial funding in FY 1997 and assumes responsibility for those facilities no longer required for operation, preparing them for turnover to EM-40.

9. Liquids from SST retrieval and new liquid waste will go to DSTs. The SSTs are beyond design life and the risk of leaking is unacceptable. All pumpable liquid is to be removed from the SSTs by 2000 , and all new liquid waste is to be added to the DSTs. Evaporation will be used to achieve the WVR needed to ensure adequate DST space for projected new waste and retrieval volumes.

10. The 242-A Evaporator will cease operations in 2007. This assumption is based on the Pretreatment Modules incorporating evaporation in their design. A major upgrade would be required to operate $242-\mathrm{A}$ beyond 2007.

11. Low-Level Waste Pretreatment Facility will have evaporation capability to replace the 242-A function. Major upgrades to the $242-A$ facility will be required to operate beyond 2007.

12. Safety issues will be resolved to allow SST pumping in accordance with Tri-Party Agreement interim milestones. Flammable gas monitoring equipment will be installed on flammable gas Watch List SSTs before interim stabilization work is scheduled to start on these tanks. Liquid grab samples are taken and laboratory analysis completed prior to scheduled start of pumping.

13. There will be improved capability for in-tank surveillance and measurement. LDUA will be delivered and tested on schedule. Operation of LDUA for other programs is funded by the requesting program.

14. LOW installations will be complete in FY 1998. LOW material is mild steel, and installation is with current lancing procedures. There will be no major changes in safety or environmental requirements or approvals. Core sampling will be completed on schedule to support LOW installation.

\subsection{Constraints}

The major constraints on the O\&M Program Element are time, cost, and the regulatory requirements.

1. Time Constraints. The O\&M Program Element has been defined in the Baseline schedule with corresponding deliverables.

2. Cost Constraints. The O\&M Program Element duration continues well into the next century. The program element is characterized by significant technical and programmatic uncertainties, which will lead to fluctuating costs in the out years.

- The program element life cycle is currently estimated at $\$ 4.31$ Billion in FY 1994 dollars. 
- The 3-year program element cost (FY 1995 through FY 1997) is based on meeting or exceeding all scheduled commitments within the funding of $\$ 492$ million, which has been requested.

- $\quad$ The 7-year program element cost (FY 1995 through FY 2001) is based on meeting or exceeding all scheduled commitments within the funding of $\$ 1.14$ billion, which has been requested.

\subsubsection{Structure}

This program element is structured to effectively provide program management and direction to TWRS Operations. In order to accomplish this, the element has been designed to provide Administrative and Technical Support, Compliance and Corrective Action management, East and West Tank Farm and Evaporator operations management and engineering support, and support to the Interim Stabilization of the SSTs. Beginning in FY 1997, this program element will be expanded to include management and support to turnover of retired facilities to EM-40. The WBS providing this management and support is discussed below.

\subsection{Work Breakdown Structure}

The O\&M Program Element is composed of seven activities, of which one, Retired Facilities and Turnover Support, is not funded until FY 1997. The WBS is shown in Figure 7.3-1.

\subsection{Organization and Responsibility}

Programmatic direction for this effort shall in all cases and at all times be provided by the RL TWRS Office. A lead RL staff member will be appointed to oversee work in a specific activity and, in some cases, specific cost accounts. This individual is responsible for ensuring that sufficient clear guidance and direction is at all times available to the contractor to perform the requested work. THE CONTRACTOR SHALL NOT FOLLOW GUIDANCE OR DIRECTION PROVIDED BY ANY OTHER RL OFFICE. The contractor will refer any conflicts between the RL TWRS office guidance and guidance offered by another RL office to the RL TWRS office for resolution.

\subsubsection{FY 1995 Work to be Performed}

FY 1995 work scope includes all direct and indirect work required to operate and maintain the plant systems necessary for the safe storage and transfer of wastes at the SST and DST farms. This includes operation of the 242-A Evaporator for concentrating low-heat-generating liquid wastes and performing SST stabilization activities.

\section{Technical Integration and Planning (WBS 1.1.1.2.01.01)}

The Technical Integration and Planning activity provides the support needed to achieve and maintain the continued safe receipt, storage, and monitoring of radioactive mixed wastes in tank farms. This WBS element consists of numerous activities in FY 1995. These activities include the following:

1. Program element and baseline management support

2. Utilities and services including electricity, steam/water, laundry services, and vehicle/equipment maintenance 
3. Planning and administration support. Deliverables will include revising the Administration manual and updating emergency preparedness manuals/plans.

4. Tank farm training that focuses on initial and continuing training for operations, maintenance, and support staff. The major deliverable will be completing the recertification of incumbent tank operators (supervisors) using newly developed training material.

5. Quality Assurance (QA)/Quality Control (QC) support, confirming the attainment of quality through independent verification

\section{Configuration management support}

7. Tank farm security, which in FY 1995 will complete upgrades identified as "high risk" by the just completed Tank Farms Vulnerability Assessment

8. Tank farms operations procedures that will review/revise approximately 180 operational procedures to support continued tank farm operations including routines and transfers

9. Nuclear safety oversight function to ensure that operations are performed within the established safety envelope

10. Computer Automated Surveillance System (CASS)/Tank Monitoring and Control System (TMACS)/Surveillance Analysis Computer System (SACS) maintenance and development with TMACS software release 6.0 and 7.0 and CASS release 3.0 planned in FY 1995.

\section{Tank Farm Compliance and Corrective Activities (WBS 1.1.1.2.01.02)}

FY 1995 work scope will include the activities required to attain and/or ensure continued compliance with regulatory requirements. Specific activities in FY 1995 will include the following:

1. DST integrity assessments. This work will focus on completing procurement and testing of the ultrasonic testing (UT) device used for inspections in the DST annulus. One DST annulus UT inspection is scheduled to be completed in FY 1995.

2. Permitting and regulatory compliance with chapter revisions for the DST Part B RCRA permit application and the combining of the 242-A Evaporator, Liquid Effluent Retention Facility (LERF), and the LETF permit application into one application

3. Environmental engineering including surface contaminátion reduction, and waste minimization/pollution prevention

4. Radioactive/hazardous solid waste shipping support, which will prepare/ship approximately $35,000 \mathrm{ft}^{3}$ of low-level waste (LLW) and 4,000 $\mathrm{ft}^{3}$ of radioactive mixed waste (RMW) to the Central Waste Complex (CWC)/burial grounds for final disposal

5. Radioactive and hazardous solid waste characterization, which performs analytical analysis and required documentation for shipping of solid waste. FY 1995 efforts include solid waste delisting of tank farm waste streams and special solid waste disposal projects

6. Criticality engineering support for maintaining the established criticality safety bases 
7. DST emergency pumping preparations, which will issue the design drawings and procurement document for needed equipment to expedite transfers

8. Implementation of OSRs. Phase 2 and 3 of the interim OSRs will be implemented, and Phase 4 through 6 will be developed.

9. Safety documentation upgrades will continue. Significant efforts will be spent on incorporating comments and revising the tank farms Accident Analysis as well as the Interim Safety Bases (ISB) document. In addition, aging waste facility (AWF)/DST chapter writing will continue.

10. Compliance management and As Low As Reasonably Achievable (ALARA) support will be provided. This will include initial development of an integrated compliance management module.

11. Industrial Health and Safety support, including baseline hazard assessments of 16 farms and 5 facilities and chemical vapor monitoring

12. Radiological control completing RADCON manual compliance reviews, emergency drills in RADCON, and developing/implementing radiological work permit training.

\section{Tank Farm East Storage Facilities (WBS 1.1.1.2.01.03)}

Specific FY 1995 work scope will include the following.

1. Provide Preventative and Corrective Maintenance support in accordance with the priorities established by Operations and the Integrated Schedule. Activities include support staff for maintenance, planning, scheduling, technical services, material services, ventilation and balance services, fabrications services, non-destructive examination services, Component Based Recall System (CBRS) procedure and database support services, and maintenance program development.

2. Perform receipt, storage, transfer, and surveillance of wastes in Tank Farm East Storage Facilities. These activities include Routine Surveillance, Facility Operations, Industrial Hygiene and Safety support, Engineering and Analysis support and Preventative and Corrective Maintenance necessary for the safe and compliant underground waste storage.

3. Provide administrative, technical, and field-related work activities within the limits of established specifications, standards, budgets, and procedural guidelines, and ensure compliance to policies and procedures or as directed by RL. Additionally, adequate staffing needs are identified and implemented to support completion of scheduled milestones and enditem deliverables. All work activities will be conducted in support of the overall safe and cost-effective operation of the tank farm facilities. The primary support functions include Facility Operations, Health Physics, Conduct of Operations, Herbicide Spraying, Crane and Rigging and Custodial Services, and miscellaneous Site Services support. 
4. Provide Engineering and Analysis support for Tank Farm East Storage Facilities. This includes technical support for preventative/corrective maintenance, facility modifications, repairs, configuration control, work packages, Non-Conformance Reports (NCRs), procedure development/revisions, waste transfer compatibility assessments, OSR/OSD reviews, CBRS system, occurrence reports, system design descriptions/technical basis document development, and maintenance of essential drawings.

\section{Tank Farm West Storage Facilities (WBS 1.1.1.2.01.04)}

Specific FY 1995 work scope will include the following.

1. Provide Preventative and Corrective Maintenance support in accordance with the priorities established by Operations and the integrated schedule. Activities include support staff for maintenance, planning, scheduling, technical services, material services, ventilation and balance services, fabrications services, non-destructive examination services, CBRS procedure and database support services, and maintenance program development.

2. Perform receipt, storage, transfer and surveillance of wastes in Tank Farm West Storage Facilities. These activities include Routine Surveillance, Facility Operations, Industrial Hygiene and Safety support, Engineering and Analysis support, and Preventative and Corrective Maintenance necessary for safe and compliant underground waste storage.

3. Provide administrative, technical, and field-related work activities within the limits of established specifications, standards, budgets, and procedural guidelines, and ensure compliance to policies and procedures or as directed by RL. Additionally, adequate staffing needs are identified and implemented to support completion of scheduled milestones and enditem deliverables. All work activities will be conducted in support of the overall safe and cost-effective operation of the tank farm facilities.

4. Provide Engineering and Analysis support for Tank Farm West Storage Facilities. This includes technical support for preventative/corrective maintenance, facility modifications, repairs, configuration control, work packages, NCRs, procedure development/revisions, waste transfer compatibility assessments, OSR/OSD reviews, CBRS system, occurrence reports, system design descriptions/technical basis document development, and maintenance of essential drawings.

\section{Tank Farm SST Program (WBS 1.1.1.2.01.05)}

Specific FY 1995 work scope will include the following.

1. Complete interim stabilization of five SSTs.

2. Complete emergency pumping of tank 241-T-111.

3. Prepare to interim stabilize one non-Watch List and three organic Watch List tanks in 241-U farm.

4. Partially complete interim stabilization preparation for seven non-Watch List tanks in 241-S tank farm. 
WHC-SP-1101

5. Partially complete interim stabilization preparations for four flammable gas Watch List tanks in 241-U tank farm.

6. Partially complete interim stabilization preparations for one flammable gas Watch List tank in 241-A farm and one flammable gas Watch List tank in 241-AX farm.

7. Complete safety studies for interim stabilization of Watch List SSTs.

8. Complete intrusion prevention for seven SSTs.

9. Complete emergency pumping procedures for remaining SSTs with pumpable liquid.

10. Prepare for emergency pumping of SSTs.

11. Procure high-level liquid waste cask and design loading facility.

12. Provide technical studies to support SST interim stabilization and emergency pumping improvements.

13. Complete emergency pumping of tank 241-T-111.

14. Emergency pump additional leaking SSTs, as required.

15. Install 12 LOWs.

16. Restore capability to measure interstitial liquid level in tank 241-SX-104.

17. Provide technical support for existing drywell vans until spectral gamma logging system is available.

18. Provide a fully functional interstitial liquid level monitoring system for 4 new LOW vans.

19. Provide operations and cognizant engineering support for design and procurement of LDUA.

20. Complete the Operational Readiness Review package for LDUA.

21. Prepare training plans and procedures for operation of LDUA.

22. Complete the safety and environmental reviews for LDUA.

23. Participate in preparation and conducting of the acceptance test procedure for LDUA.

24. Participate in the cold demonstration of LDUA with EM-50.

Tank Farm Evaporator/AW and AP Tank Farm Operations (WBS 1.1.1.2.01.06)

Specific FY 1995 work scope will include the following.

1. The 242-A Evaporator will complete Campaign 94-2 and run Campaign 95-1 during FY 1995. There will be an outage in preparation for 95-1. All technical, administrative, and field support for the two campaigns and outage will be provided. 
2. All surveillance routine activities required to ensure that the facilities operate within the approved safety and environmental envelopes will be performed.

3. The identified Corrective and Preventive Maintenance activities will be performed on schedule in accordance with priorities established by Operations and within the Integrated Schedule.

4. A 5th shift for training will be implemented.

5. All design and engineering support to plant modifications and maintenance, technical input to operation and transfer procedures, and safety analysis documents will be provided.

6. Process control plans, post-campaign reports, and sample schedules will be developed.

7. Data quality objectives will be implemented to reduce Resource Conservation and Recovery Act (RCRA) required process control samples, reducing cost during FY 1995.

8. Engineering support of operations will be provided as required around the clock.

\section{Tank Farm Facilities and Turnover Support (WBS 1.1.1.2.01.07)}

A minimal planning effort will be conducted to develop the WBS structure, estimate resources, and define strategy for the activity in preparation for FY 1997 receipt of funding.

\subsubsection{FY 1996 - FY 1997 Work to be Performed}

FY 1996 through FY 1997 work scope will include all direct and indirect work required to operate and maintain the plant systems necessary for the safe storage and transfer of wastes at the SST and DST farms. This includes operation of the 242-A Evaporator for concentrating low-heat-generating liquid wastes and performing SST stabilization activities.

Beginning in FY 1997, funded activities will be directed at identifying and preparing retired facilities for turnover to $\mathrm{EM}-40$.

\subsection{Technical Integration and Planning (WBS 1.1.1.2.01.01)}

The Technical Integration and Planning activity provides the support needed to achieve and continue safe operations and maintenance in tank farm facilities. This WBS element consists of many

activities. In FY 1996 and FY 1997, the work scope will include the following:

1. Program element and baseline management support

2. Utilities and services including electricity, steam, water, laundry services, and vehicle/equipment maintenance

3. Planning and administration support. Deliverables will include reviewing/revising administration procedures and updating emergency preparedness manuals/plans as required.

4. The major deliverable for tank farm training, which focuses on initial and continuing training for operations, maintenance, and support staff, will be completing development of a support staff certification program and implementing the newly developed program. 
WHC-SP-1101

5. QA/QC support, confirming the attainment of quality through independent verification

6. Configuration management support

7. Tank farm security, which in FY 1996 through FY 1997 will complete upgrades identified as "medium/low risk" by the Tank Farms Vulnerability Assessment and implement improved access control.improvements

8. Tank farms operations procedures, which will review/revise approximately 200 operational procedures to support continued tank farm operations including routines and transfers

9. Nuclear safety oversight function to ensure that operations are performed within the established safety envelope

10. CASS/TMACS/SACS maintenance and development, with two TMACS software releases and retirement of the CASS system in FY 1997.

\section{Tank Farm Compliance and Corrective Activities (WBS 1.1.1.2.01.02)}

Work scope will include the activities required to attain and/or ensure continued compliance with regulatory requirements. Specific activities in FY 1996 through FY 1997 will include the following:

1. Completing initial DST integrity assessments and issuing Integrity Assessment Reports by farm

2. Permitting and regulatory compliance with issuance in FY 1997 of a revised DST Part B RCRA permit application and completion of the combining 242-A Evaporator, LERF, and LETF permit application into one application

3. Environmental engineering, including continuing maintenance in the surface contamination reduction program and full implementation of a waste minimization/pollution prevention program

4. Radioactive/hazardous solid waste shipping support, which will per year prepare/ship approximately $20,000 \mathrm{ft}^{3}$ of LLW and $2,000 \mathrm{ft}^{3}$ of $\mathrm{RMW}$ to the CWC/burial grounds for final disposal

5. Radioactive and hazardous solid waste characterization, which performs analytical analysis and required documentation for shipping of solid waste, and continuing special solid waste disposal projects

6. Criticality engineering support for maintaining the established criticality safety bases

7. DST emergency pumping preparations, which will complete implementation of a plan including equipment procurement for responding to an emergency DST leaker within the prescribed (regulatory) 24 hour response time

8. Implementation of OSRs. Phase 4 through 6 will be implemented. 
9. Safety documentation upgrades will continue with a draft final AWF/DST safety analysis report prepared

10. Compliance management and ALARA support will be provided. This will include completing development and implementation of a integrated compliance management module.

11. Industrial Health and Safety support, including continuing hazard assessments of tank farms and identified facilities and chemical vapor monitoring

12. Radiological control continuing RADCON manual compliance reviews, emergency drills in RADCON, and radiological work permit training.

\section{Tank Farm East Storage Facilities (WBS 1:1.1.2.01.03)}

Specific activities in FY 1996 through FY 1997 will include the following:

1. Provide Preventative and Corrective Maintenance support in accordance with the priorities established by Operations and the Integrated Schedule. Activities include support staff for maintenance, planning, scheduling, technical services, material services, ventilation and balance services, fabrications services, non-destructive examination services, CBRS procedure and database support services and maintenance program development.

2. Perform receipt, storage, transfer, and surveillance of wastes in Tank Farm East Storage Facilities. These activities include Routine Surveillance, Facility Operations, Industrial Hygiene and Safety support, Engineering and Analysis support, and Preventative and Corrective Maintenance necessary for the safe and compliant underground waste storage.

3. Provide administrative, technical, and field-related work activities within the limits of established specifications, standards, budgets and procedural guidelines, and ensure compliance to policies and procedures or as directed by RL. Additionally, adequate staffing needs are identified and implemented to support completion of scheduled milestones and enditem deliverables. All work activities will be conducted in support of the overall safe and cost-effective operation of the tank farm facilities. The primary support functions include Facility Operations, Health Physics, Conduct of Operations, Herbicide Spraying, Crane and Rigging and Custodial Services, and miscellaneous Site Services support.

4. Provide Engineering and Analysis support for Tank Farm East Storage Facilities. This includes technical support for preventative/corrective maintenance, facility modifications, repairs, configuration control, work packages, NCRs, procedure development/revisions, waste transfer compatibility assessments, OSR/OSD reviews, CBRS system, occurrence reports, system design descriptions/technical basis document development, and maintenance of essential drawings. 
Tank Farm West Storage Facilities (WBS 1.1.1.2.01.04)

Specific activities in FY 1996 through FY 1997 will include the following:

1. Provide Preventative and Corrective Maintenance support in accordance with the priorities established by Operations and the Integrated Schedule. Activities include support staff for maintenance, planning, scheduling, technical services, material services, ventilation and balance services, fabrications services, non-destructive examination services, CBRS procedure and database support services, and maintenance program development.

2. Perform receipt, storage, transfer, and surveillance of wastes in Tank Farm West Storage Facilities. These activities include Routine Surveillance, Facility Operations, Industrial Hygiene and Safety support, Engineering and Analysis support and Preventative and Corrective Maintenance necessary for the safe and compliant underground waste storage.

3. Provide administrative, technical, and field-related work activities within the limits of established specifications, standards, budgets, and procedural guidelines, and ensure compliance to policies and procedures or as directed by RL. Additionally, adequate staffing needs are identified and implemented to support completion of scheduled milestones and enditem deliverables. All work activities will be conducted in support of the overall safe and cost-effective operation of the tank farm facilities. The primary support functions include Facility Operations, Health Physics, Conduct of Operations, Herbicide Spraying, Crane and Rigging and Custodial Services, and miscellaneous Site Services support.

4. Provide Engineering and Analysis support for Tank Farm West Storage Facilities. This includes technical support for preventative/corrective maintenance, facility modifications, repairs, configuration control, work packages, NCRs, procedure development/revisions, waste transfer compatibility assessments, OSR/OSD reviews, CBRS system, occurrence reports, system design descriptions/technical basis document development, and maintenance of essential drawings.

\section{Tank Farm SST Program (WBS 1.1.1.2.01.05)}

Specific activities in FY 1996 through FY 1997 will include the following:

1. Complete interim stabilization of one non-Watch List tank in 241-U farm (Tri-Party Agreement milestone M-41-08-T01) and three organic Watch List tanks in 241-U farm (TriParty Agreement milestone M-41-13-T01).

2. Complete interim stabilization preparations and start interim stabilization of seven non-Watch List tanks in 241-S farm (Tri-Party Agreement milestone M-41-09), four flammable gas Watch List tanks in 241-U farm (Tri-Party Agreement milestone M-41-11), two flammable gas tanks in 241-A and 241-AX farms (Tri-Party Agreement milestone M-41-10), four ferrocyanide Watch List tanks in 241-BX and 241 -BY farms (Tri-Party Agreement milestone M-41-12), seven flammable gas tanks in 241-S and 241-SX farms (Tri-Party Agreement milestone M-41-14), and two organic Watch List tanks in 241-S and 241-SX farms (Tri-Party Agreement milestone M-41-15); and complete interim stabilization of 11 of these tanks (TriParty Agreement milestones M-41-09-T01 and M-41-11-T01). Complete installation of 30 LOWs. Complete the field testing of the LDUA. 


\section{Tank Farm Evaporator/AW and AP Tank Farm Operations (WBS 1.1.1.2.01.06)}

The plan for FY 1996 and FY 1997 is to perform one to two evaporation campaigns each year, with preparatory operational and maintenance activities performed between each campaign. All required surveillances, corrective maintenance, and preventive maintenance tasks will be completed.

Engineering and Analysis will provide all technical support for the campaigns, outages, and ongoing operational and safety. requirements. The Washington State Department of Ecology/U.S.

Environmental Protection Agency-approved 242-A and DST Part B Permits will be issued by September 30, 1997.

\section{Tank Farm Facilities and Turnover Support (WBS 1.1.1.2.01.07)}

During FY 1996, the planning will continue to perform the detailed planning in preparation for receipt of funds in FY 1997. In FY 1997 the engineering and field work required prior to turnover of retired facilities to EM-40 will begin.

\subsubsection{Approach}

The bases for O\&M are the safe receipt, storage, and monitoring of the liquid radioactive wastes from Hanford facilities until equipment and processes for cleanup and disposal are implemented. Because the 149 SSTs have aged beyond their useful life and cannot be properly monitored to ensure integrity as required by the State of Washington, these tanks will have pumpable liquid removed, with retrieval and final closure following.

Continued safe operation of these facilities requires near- and long-term upgrades in safety bases and hardware restoration. Planning anticipates achieving a final safety basis in the beginning of FY 2001, following numerous upgrades, USQ resolution, and tank characterization. These actions will achieve a significantly improved infrastructure allowing a mature, safe operation for the planned life cycle (through 2028) of tank farms.

\section{Technical Integration and Planning (WBS 1.1.1.2.01.01)}

Provide the technical management and planning support needed to continue safe and compliant operations and maintenance in tank farm facilities. This element will continue the day-to-day interaction ensuring continued support in near-term and long-range goals and objectives. Overall, the element will provide the foundation of which operations and maintenance field activities are performed.

\section{Tank Farm Compliance and Corrective Activities (WBS 1.1.1.2.01.02)}

Provide the technical management and planning support needed to continue safe and compliant operations and maintenance in tank farm facilities. This element will continue the day-to-day interaction ensuring continued support in near-term and long-range goals and objectives. Overall, the element will provide the foundation of which operations and maintenance field activities are performed. 
Provide Preventative and Corrective Maintenance support in accordance with the priorities as established by Operations and the integrated schedule.

Provide Facility Operations support for the receipt, storage, and transfer operations of 200 East Tank Farms Operations (ETEO). Conduct all activities pertaining to operation of a permitted treatment, storage, and disposal (TSD) facility within the boundary of current ISBs and in a manner that ensures compliance with all applicable Federal, State, and local environmental regulations. Provide routine surveillance to 200 East Tank Farms (DST/SST farms). Includes facility data acquisition/analysis, LOW/drywell van operation, SST photo/video mission, and Health Physics surveys/checks. Supporting organizations include Facility Operations, and Health Physics.

The major activities of ETFO include corrective and preventative maintenance; waste transfers; monitoring and data acquisition; administrative and technical expertise to 241-A, AX, AN, AY, AZ, BX, BY, B, and C-Complex; waste transfer/routing piping and associated catch tanks, purge water facilities, ponds, cribs, ditches, and the 204-/244-AR facilities. This entails maintenance and development of technical basis for the receipt, storage, and transfer operations of East Tank Storage Facilities.

\section{Tank Farm West Storage Facilities (WBS 1.1.1.2.01.04)}

Provide Preventative and Corrective Maintenance support in accordance with the priorities as established by Operations and the Integrated Schedule.

Provide Facility Operations support for the receipt, storage, and transfer operations of 200 West Tank Farms Operations (WTFO). Conduct all activities pertaining to operation of a permitted TSD facility within the boundary of current ISBs and in a manner that ensures compliance with all applicable Federal, State, and local environmental regulations. Provide routine surveillance to 200 West Tank Farms (DST/SST farms). Includes facility data acquisition/analysis, LOW/drywell van operation, SST photo/video mission, and Health Physics surveys/checks. Supporting organizations include Facility Operations and Health Physics.

The major activities of WTFO include corrective and preventative maintenance; waste transfers; monitoring and data acquisition; administrative and technical expertise to 241-S, SX, SY, U, T, TX, TY, T-Complex; waste transfer/routing piping and associated catch tanks, purge water facilities, ponds, cribs, ditches, and the 242-T/242-S facilities. This entails maintenance and development of technical basis for the receipt, storage, and transfer operations of West Tank Storage Facilities.

\section{Tank Farm SST Program (WBS 1.1.1.2.01.05)}

Completion of SST interim stabilization and intrusion prevention is based on definition in the Tri-Party Agreement. Complete installation of the LOWs. 


\section{Tank Farm Evaporator/AW and AP Tank Farm Operations (WBS 1.1.1.2.01.06)}

The 242-A Evaporator was upgraded in 1992/1993 and restarted in FY 1994. It will be operated as needed until 2007, concentrating diluted low-heat-generating radioactive liquids in order to minimize tank space requirements. It is planned that it will run two evaporation campaigns per year, but the actual number of runs will depend on the amount of waste generated each year and the actual schedule of other activities, such as retrieval.

\section{Tank Farm Facilities and Turnover Support (WBS 1.1.1.2.01.07)}

All waste will be removed from tanks, pipes, and equipment prior to turnover to EM-40 for final decontamination and disposal. To the extent possible, equipment and technology used for waste removal for treatment will be employed to prepare the retired facilities for turnover to EM-40. Schedules will be developed to permit the Management and Operating contractor and the ER contractor to effectively coordinate resources and planning, thus minimizing funding requirements.

\subsubsection{Drivers and Deliverables}

The safe receipt, storage, and monitoring of radioactive and RMWs is required by Federal and State Environmental and Health and Safety regulations, as well as DOE Orders. The major deliverables of the O\&M Program Element are operation of the 242-A Evaporator until 2007, interim stabilization of all SST storage tanks by 2000 , and safe storage and monitoring of the waste in the tanks until all waste is removed and the facilities have been turned over to ER (2028).

\section{Technical Integration and Planning (WBS 1.1.1.2.01.01)}

This element is required to support the continued safe operation and maintenance of the tank farm facilities through the year 2027. The element provides the base (i.e. utilities/services, QA, training) for which to perform work scope described in the other elements.

\section{Tank Farm Compliance and Corrective Activities (WBS 1.1.1.2.01.02)}

The primary driver of the element and program is the Washington Administrative Code (WAC) Chapter 4, pages 173-303, "Dangerous Waste Regulations." This regulation implements Chapter 70.105 RCW, the Hazardous Waste Management Act of 1976 as amended in 1980 and 1983; implements, in part, Chapter 70.105A RCW; and Subtitle C of Public Law 94-580, the Resource Conservation and Recovery Act, which the legislature has empowered the department to implement. The purposes of this regulation are to (1) designate those solid wastes that are dangerous or extremely hazardous to the public health and environment; (2) provide for surveillance and monitoring of dangerous and extremely hazardous wastes until they are detoxified, reclaimed, neutralized, or disposed of safely; (3) provide the form and rules necessary to establish a system of manifesting, tracking, reporting, monitoring, recordkeeping, sampling, and labeling dangerous and extremely hazardous wastes; (4) establish the siting, design, operation, closure, post closure, financial, and monitoring requirements for the transfer, treatment, storage, and disposal facilities required for dangerous and extremely hazardous waste; (5) establish design, operation, and monitoring requirements for managing the State's (Hanford) extremely hazardous waste disposal facility; (6) establish and administer a program for permitting dangerous and extremely hazardous waste management facilities; and (7) encourage recycling, reuse, reclamation, and recovery to the maximum extent possible. 


\section{Tank Farm East Storage Facilities (WBS 1.1.1.2.01.03)}

1. Drivers:

Department of Energy Established:

- Standards/Requirements Identification Documents (S/RIDs)

Tank Waste Remediation System Functions and Requirements:

- $\quad$ Section 2, Table F4.2.1.1, item V, paragraph B

- $\quad$ Section 3, Table A4.2.1.1, item VIII, paragraph C

WAC 173-303, "Dangerous Waste Requirements"

2. Deliverables: (Tank Farm East Storage Facilities)

- $\quad$ Plan, schedule, and perform all required Preventative Maintenance (PM) work packages for the A, AN, AX, AY, AZ, B, BX, BY and C 200 East Tank Farms in accordance with East Operations' priorities.

- $\quad$ Review the current PM work packages for applicability and reduce the number of scheduled PM work packages where available at a rate of 2 per quarter and provide documentation on the packages removed from the performance schedule.

- $\quad$ Provide monthly progress reports identifying PMs scheduled and completed, and craft production rates for PMs completed.

- Eliminate the 200 East Tank Farm preventative maintenance work package backlog through elimination of unneeded work packages and increased productivity in completion of needed work packages.

- Write and approve 24 new Vent and Balance procedures to increase productivity of maintenance and operations personnel.

- Perform three quarterly and one annual cathodic protection surveys at $\mathrm{A}, \mathrm{AN}, \mathrm{AX}$, and AZ 200 East Tank Farms and make necessary system adjustments. Procure pulse generators and wave form analyzer for survey use by the second quarter.

- Group PMs with other packages by farm to reduce meeting, suit-ups, transportation, entry into tank farms, and support requirements. Success in grouping packages will result in increases in work package completions per month.

- $\quad$ Provide CBRS database updates and procedure revisions on a timely basis to prevent performance of superseded CBRS procedures. 
- $\quad$ Plan, schedule, and perform all required work packages to repair FICs, manual tapes, CASS alarms, exhausters, compressors, lighting and electrical systems, in-tank instrumentation, and for summer and winter preparation in the $A, A N, A X, A Y, A Z$, B, BX, BY, and C 200 East Tank Farms in accordance with the priorities established by East Tank Farm Operations. Increase the monthly Corrective Maintenance (CM) work package completion rate from 125 to 140 .

Provide monthly progress reports identifying CMs scheduled and completed, and craft production rates for the CMs completed.

- Group CMs with other packages by tank farm or facility to reduce suit-up, transportation, tank farm entry, and support requirements to increase productivity of the crafts in the field.

- Design, fabricate, and install new waste tank pump storage stands to satisfy a safety concern over the high probability of tip-overs with the current stands.

- $\quad$ Provide component labelling, master equipment list updates, and drawing revisions for 200 East Tank Farms beyond the scope funded by the 1N3 Upgrades Program.

- Develop a Maintenance Work Plan for quality and productivity improvement, which will address what will be done, who will do it, when they will do it, and what the expected results will be.

- $\quad$ Perform a maintenance self assessment, prepare and internally approve a Maintenance Improvement Plan for Waste Tanks Maintenance.

- $\quad$ Remove failed and degraded FIC level measuring devices; purchase and install ENRAF level measuring instruments.

- $\quad$ Rebuild eight air compressors in East Tank Farms to assure more reliable air system operation.

- $\quad$ Complete selected work packages to restore operating conditions in the AN Tank Farm.

- Employ heavy duty drivers/equipment and laborers to remove contaminated soil, gravel, and paving to reduce surface contamination which will result in reduced personnel radiation exposures and eliminate some of the suit-up and air pack requirements.

- $\quad$ Provide support for modifications to fire systems at 271-CR and in A Tank Farm.

- $\quad$ Remove and dispose of all East Tank Farm RAMPS.

- $\quad$ Reduce the East Tank Farm Corrective Maintenance work package backlog by 25 percent from approximately 700 to 525 by review of work packages for applicability and through increased work package productivity. 
WHC-SP-1101

- $\quad$ Reduce the work package Plan/Schedule/Field Work cycle by approximately 20 percent from the current 110-day duration by implementing planning standardizations and grouping of work packages.

- Incorporate the East Tank Farm Operations Safety Equipment List into the Master Equipment list database and meet or exceed an availability rate of 95 percent for safety equipment through prompt identification of problems and work package execution.

- $\quad$ Provide short-term [POD, POW, and POM) scheduling services, technical service reviews of procedures and work packages, material ordering, receiving and staging, carpenter/light and heavy driver/other craft support, vent and balance craft support, shop fabrication craft and material support, and non-destructive examination support.

\section{$\underline{\text { Administration }}$}

- $\quad$ Self-Assessment S/RIDs support - provide support to 18 teams.

- $\quad$ Ensure that all Off-Normal/Unusual Occurrence Reports (Notification, 10-day, Finals) generated in East Tank Farms are submitted in accordance with established procedural time constraints.

- Manage Facility Operations and Routine Surveillance Cost Accounts. Provide monthly status to Program Office/RL.

- Issue/revise FY 1996 multi-year plan by August 30, 1995.

- $\quad$ Track and maintain ETFO personnel training in compliance with the Tank Farms Dangerous Waste Training Plan and WHC Radiation Area Management (WRAM) access requirements.

- $\quad$ Secure funding and issue requisition for the procurement of $\mathrm{CO} 2$ mobile decontamination unit by March 31, 1995.

\section{$\underline{\text { Transfers }}$}

- Conduct 45 internal (double-contained receiver tanks [DCRTs], catch tank, tank-totank) waste transfers allowing sufficient tank space to accommodate contents of largest pumpable liquid inventory of any SST in East Area.

- 20 catch tank transfers

- $\quad 10$ DCRT transfers

- $\quad 15$ miscellaneous tank-to-tank

- Support 13 external (222-S, 300 Area, T-Plant) High-Level Waste (HLW)/LLW from other Hanford Site facilities in operation or cleanup phases to avoid noncompliance or operation interruptions at those facilities. 
WHC-SP-1101

Restore Cross-Site Transfer Capability

- Support the development of work plans/packages to restore cross-site transfer capability.

- $\quad$ Conduct all jumper changes necessary to support the transfer deliverables.

Field Activities

- $\quad$ Complete all FY 1995 scheduled Operational Test Procedures (OTPs).

Technical Support

- Complete Phase III Interim Operational Safety Requirements (IOSR) implementation.

- $\quad$ Provide quarterly Rad Con Manual field assessments.

- $\quad$ Provide quarterly Conduct of Operations assessments.

\section{Conduct of Operations}

- Conduct monthly inspections of ETFO facilities.

\section{Health Physics}

NOTE: All deliverables herein will be supported by Health Physics.

- $\quad$ ETFO to obtain full compliance with new DOE Radiological Control Manual in FY 1995.

- Health Physics shall be in full compliance with all Articles of the DOE Radiological Control Manual, Rev. O, by March 31, 1995.

- Health Physics shall complete all annual Radiological Control Manual compliance reviews by December 31, 1995.

\section{Herbicide Control and Custodial Services}

- $\quad$ Complete herbicide spraying per the FY 1995 herbicide spray schedule.

- Issue FY 1996 herbicide schedule by September 30, 1995.

- $\quad$ Custodial Services - LOE.

\section{$\underline{\text { Routine Surveillance }}$}

- Obtain daily and quarterly liquid level readings of DST/SST/catch tanks in accordance to OSD-T-151-00031.

- $\quad$ Prepare/provide engineering analysis of liquid levels, drywells, LOW, temperatures, pressures and other DST/SST data within specified frequencies to assure detection of tank leaks and unsafe or non-compliant conditions of waste in (83) HLW tanks. 
WHC-SP-1101

- Obtain the ability to photograph the interior of any SST within 7 working days of the need to do so.

\section{$\underline{\text { Technical Support }}$}

- Issue Surveillance Alert List monthly per WHC-IP-0842.

- Issue Surveillance Discrepancy Reports as necessary when anomalies occur per WHC-IP-0842.

- $\quad$ Provide data analysis within 24 hours.

\section{Data Analysis}

- Issue two tank evaporation analysis.

- Issue sensitivity plots for future tank evaporation analysis.

- Issue 8-monthly system performance assessments for six systems.

- Issue East Tank Farm Annual System Assessment Reports for six systems.

\section{Surveillance Operations}

- $\quad$ Obtain drywells, LOW, temperatures, pressures, and other DST/SST data within specified frequencies to ensure detection of tank leaks and unsafe or non-compliant conditions of waste in (83) HLW tanks.

\section{In-Tank Photography/Pit Inspections}

- $\quad$ Complete 11 in-tank photography missions.

- $\quad$ Complete 20 pit inventory and/or inspection videos.

\section{Health Physics}

- $\quad$ Provide Operations/Health Physics support to obtain all required DST/SST data within specified frequencies.

- Health Physics will perform required radiological surveys according to established frequencies as stated in current task descriptions, maintaining a 95 percent completion rate for scheduled radiological surveys.

- Health Physics will issue Monthly Radiological Status Report.

- Health Physics will maintain and update Tank Farm status maps.

\section{Corrective Maintenance}

- $\quad$ Provide engineering documentation for 30 work packages per quarter. 
- The disposition of 50 NCRs.

\section{Operating Documents}

- Development, revision, review and approval of 60 operating procedures supporting east tank farm systems.

Waste Transfer Support to 45 internal tank farm transfers and 13 transfers from generators

- Compatibility assessments as required by the compatibility program document to support the transfer and receipt of tank waste

- Technical input to the development and issuance of transfer procedures.

\section{Preventative Maintenance}

- Technical reviews of preventative maintenance work packages and data sheets

- Complete the review and revision of OSR/OSD CBRS data and input forms

- $\quad$ Review of CBRS data for non-OSR/OSD instrumentation

- $\quad$ Resolution of CAR 92-1

- Issuance of annual corrosion program review.

\section{System Evaluation}

- Issue 8 monthly system performance assessments for six systems.

- Issue East Tank Farm Annual System Assessment Report for six systems.

\section{Shift Engineering Coverage}

- $\quad$ Provided on an on-call basis.

\section{Response to Occurrences/Abnormal Operation}

- Issue Occurrence Reports as needed.

- $\quad$ Provide corrective actions in a timely manner.

Review and modify the spare parts inventory for east tank farm systems

- Review OSR/OSD instruments and provide input to the spare parts program.

\section{Technical Basis Documentation development}

- $\quad$ Develop four System Design Systems (SDDs) first half of FY 1995.

- $\quad$ Develop four SDDs second half of FY 1995.

- $\quad$ Review and validate equipment tracking system.

- $\quad$ Develop DST leak detection technical basis. 
WHC-SP-1101

Maintain Cognizant Engineering training requirements and professional development associated with areas of responsibilities

- Maintain training requirements for Tank Farm Dangerous Waste Training Plan.

Co-locate East Systems Engineering personnel near the facility

$\because$

- Relocate East Systems Engineering Group to Operations complex.

Provide management of the cost account

- $\quad$ Provide monthly status to RL lead for this cost account.

- Develop FY 1996 budget.

Tank Farm West Storage Facilities (WBS 1.1.1.2.01.04)

1. Drivers:

Department of Energy Established:

- $\quad$ Standards/Requirements Identification Documents (S/RIDs)

Tank Waste Remediation System Functions and Requirements

- $\quad$ Section 2, Table F4.2.1.1, item V, paragraph B

- $\quad$ Section 3, Table A4.2.1.1, item VIII, paragraph C

WAC 173-303, "Dangerous Waste Requirements"

2. Deliverables: (Tank Farm West Storage Facilities)

- $\quad$ Plan, schedule, and perform all required PM work packages for the U, T, TX, TY, S, SX, and SY 200 West Tank Farms in accordance with West Tank Farms Operations' priorities.

- $\quad$ Review the current PM work packages for applicability and reduce the number of scheduled PM work packages where available at a rate of 2 per quarter and provide documentation on the packages removed from the performance schedule.

- $\quad$ Provide monthly progress reports identifying PMs scheduled and completed, and craft production rates for the PMs completed.

- Eliminate the 200 West Tank Farm preventative maintenance work package backlog through elimination of unneeded work packages and increased productivity in completion of needed work packages.

- $\quad$ Perform three quarterly and one annual cathodic protection surveys at U, TX, and S 200 West Tank Farms and make necessary system adjustments. 
- Group PMs with other packages by farm to reduce suit-ups, transportation, tank farm entry and support requirements. Success in grouping packages will result in increases in the craft productivity rate.

- $\quad$ Provide CBRS database updates and procedure revisions on a timely basis to prevent performance of superseded CBRS procedures.

- Plan, schedule, and perform all required work packages to repair FICs, manual tapes, CASS alarms, exhausters, compressors, lighting and electrical systems, in-tank instrumentation, and for summer and winter preparation in the U, T, TX, TY, S, SX, and SY 200 West Tank Farms in accordance with the priorities established by West Tank Farms Operations. Increase the monthly CM work package completion rate from 162 to 190 .

- $\quad$ Provide monthly progress reports identifying CMs scheduled and completed, and craft production rates for the CMs completed.

- Group CM work packages with other CM and PM work packages by tank farm to reduce suit-up, transportation, and support requirements to increase the productivity of crafts in the field.

- $\quad$ Provide component labelling, master equipment list updates, and drawing revisions for 200 West Tank Farms beyond the scope funded by the 1N3 Upgrades Program.

- $\quad$ Remove all failed or degraded FIC level measuring devices; procure and install ENRAF level measuring instruments.

- Rebuild four air compressors in West Tank Farms to assure more reliable air system operations.

- $\quad$ Provide upgrades for the P-28 exhauster.

- $\quad$ Support modification of fire systems at 241-T and 2707-SX.

- $\quad$ Restore the 241-SX sludge cooler north and south fans.

- $\quad$ Remove the gantry crane at 241-SX Tank Farm.

- $\quad$ Stabilize and shotcrete 242-S.

- Install 272-S connex lighting and electrical outlets.

- $\quad$ Repair 241-S yard lighting.

- $\quad$ Remove and dispose of all West Tank Farm RAMPS.

- Employ heavy-duty drivers and equipment and laborers to remove contaminated soil, gravel, and paving to reduce surface contamination. This reduction will reduce personnel radiation exposure and lessen suit-up and air pack requirements. 
- $\quad$ Reduce the West Tank Farm Corrective Maintenance work package backlog by 25 percent from approximately 400 to 300 by review of work packages for applicability and through increased work package productivity.

- $\quad$ Reduce the work package Plan/Schedule/Field Work cycle by approximately 20 percent from the current 110-day duration by standardized planning implementation and work package grouping by area.

- Incorporate the West Tank Farm Safety Equipment List as defined by Operations into the Master Equipment List database and meet or exceed a 95 percent availability rate for safety equipment through prompt problem identification and work package execution.

- $\quad$ Provide POD, POW, and POM short-term scheduling services, technical service procedure and work package reviews, material ordering, receipt and staging for field work, carpenter/driver/other craft support, vent and balance support, shop fabrication support, and non-destructive examination services.

\section{Administration}

- Ensure that all Off-Normal/Unusual Occurrence Reports (Notification, 10-day, Final) generated in West Tank Farms are submitted in accordance with established procedural time constraints.

- $\quad$ Provide support to 18 teams during their assessment of West Area.

- Manage Facility Operations and Routine Surveillance Cost Accounts. Provide monthly status to Program Office/RL.

- Issue/revise FY 1996 multi-year plan by August 30, 1995.

- $\quad$ Track and maintain WTFO personnel training in compliance with the Tank Farms Dangerous Waste Training Plan and WRAM access requirements.

- Secure funding and issue requisition for procurement of a RADROVER radiation monitoring vehicle by March 31, 1994.

\section{Waste Transfers}

- Support five external (PFP) HLW/LLW from other Hanford Site facilities in operation or cleanup phases to avoid interruptions in operations caused by compliance waste storage time constraints.

\section{$\underline{\text { Restore Cross-site Transfer Capability }}$}

- $\quad$ Restore Cross-site Transfer Capability

- $\quad$ Sample and pump related catch tanks

- $\quad$ Pressure test cross-site transfer line

- Develop and approve procedures

- Support CBRS/PMs of related instrumentation. 
Field Activities

- The deliverable for corrective/preventive maintenance is covered in the maintenance CAPs.

- Complete acceptance OTPs for 12 major maintenance upgrades and projects.

- Perform seasonal equipment and facility surveillances and preparations.

\section{Removal of Waste}

- Removal of equipment classified as waste

- P-29 exhauster

- $\quad$ 241-S duct work and two skid mounted exhausters

- $\quad$ Slurry distributor at 241-SY

- Vacuum truck

- SX-Farm water tank.

\section{Technical Support}

- $\quad$ Complete phase III Interim Operational Safety Requirement (IOSR) implementation

- Transfer custody of 101-SY mixer pump operations to WTFO

- Write and approve 80 procedures for WTFO.

Conduct of Operations

- $\quad$ Conduct monthly inspections of WTFO facilities

- Support implementation of the new DOE Radiological Control Manual.

\section{Health Physics}

- TWRHP shall complete all annual Radiological Control Manual compliance reviews, by December 31, 1994.

- $\quad$ TWRHP shall be in full compliance with all of the DOE Radiological Control Manual, Rev. O, by March 31, 1995.

- Support the Surface Contamination Area zone reduction program.

\section{Pesticide Control and Custodial Services}

- Perform pesticide control services as requested by WTFO

- $\quad$ Custodial Services - LOE.

\section{Project Management}

- This task will provide overall management of the WTFO facilities, which includes management of the above activities. 
Routine Surveillance

- Obtain daily and quarterly liquid level readings of DST/SST/catch tanks in accordance to OSD-T-151-00031

- Monitor and acquire daily surveillance data on 200 West ventilation systems in support of Waste Tank Storage tanks (in accordance with OSD-T-151-00007, OSD-T-151-00011, and OSD-T-151-00013)

- Obtain weekly breather filter data (in accordance with OSD-T-151-00013)

- $\quad$ Obtain dome surveys (in accordance with OSD-T-151-00013)

- Obtain swab riser readings as requested

- Perform investigations related to tank temperature/level/pressures/anomalies and intrusions as requested.

Surveillance Operations

- Obtain drywell, LOW other DST/SST data within specified frequencies to assure detection of tank leaks and unsafe or non-compliant conditions of waste in (87) TX tanks.

\section{In-Tank Photography/Pit Inspections}

- Obtain in-tank photographs from 13 SSTs

- Complete 11 pit inventory and/or inspection videos.

\section{Health Physics}

- Perform required radiological surveys according to established frequencies, as stated in current task descriptions, maintaining a 95 percent completion rate for scheduled radiological surveys.

- Write/revise task descriptions as necessary.

- $\quad$ Provide Health Physics support to routine surveillance milestones identified by TWRS.

- $\quad$ Maintain and update Tank Farm status maps.

\section{Engineering \& Analysis (Tank Farms West Storage Facilities)}

- $\quad$ Schedules, Estimates at Completion, mid-year changes and budgets

- Letter reporting status of training completed

- $\quad$ Letter stating that four engineers have been Certified Phase II

- $\quad$ Phase III Certification Packages

- Letter stating Certification of 3 Phase III

- Applicable Engineering Change Notices and Interface with Configuration Control 
- $\quad$ Provide technical input to procedures

- Updated CBRS

- Support corrective maintenance and modification activities

- Support preventative maintenance and calibration activities

- $\quad$ Timely responses to Technical Requests

- Provide technical support to waste transfers

- Applicable Process Memos

- Review/Comment Records on H-14 Drawings

- West Storage Engineering Responsibility Bounding Document

- West Storage Engineering Performance Guidelines

- $\quad 50$ Updated Tank Top Drawings

- $\quad$ Assessment/Surveillance/NCR Responses

- $\quad$ Reports stating number NCRs Dispositioned

- $\quad$ Quarterly Reports of 200-W System Performance

- $\quad$ Engineering Study for Steam Elimination

- $\quad \operatorname{Design}(\mathrm{s})$ for Steam Elimination

- $\quad 50$ Updated Critical Support Drawings

- Input Gathered from System Walkdowns

- $\quad$ Applicable Process Tests

- $\quad$ Engineering Study for Compressed Air Elimination

- Design(s) for compressed Air Elimination

- $\quad$ Revised Essential Drawing List

Tank Farm SST Program (WBS 1.1.2.01.05)

The primary drivers for this activity are:

- Tri-Party Agreement, Fourth Amendment, January 1994

- Washington Administrative Code (WAC) Chapter 4 173-303, Dangerous Waste Regulations

- Standards/Requirements Identification Documents

The major end-item deliverables are:

- $\quad$ Tri-Party Agreement Milestone M-41-00, complete SST Interim Stabilization. Complete Interim Stabilization activities and intrusion prevention for all SSTs except 241-C-106.

- $\quad$ Removal of pumpable liquid from leaking SSTs.

- Complete installation of LOWs in SSTs. (Proposed Tri-Party Agreement Milestone M-43-09)

- Deliver functional LOW monitoring systems.

- $\quad$ Provide a functional LDUA system. 
WHC-SP-1101

Tank Farm Evaporator/AW and AP Tank Farm Operations (WBS 1.1.1.2.01.06)

The 242-A Evaporator is required to operate to maintain the ability to accept waste transfers from facilities during their operation and terminal cleanout. WVR is much cheaper and environmentally preferable to building additional DSTs.

\section{Tank Farm Facilities.and Turnover Support (WBS 1.1.1.2.01.07)}

Following removal of waste from the SSTs and DSTs and end of useful life of facilities such as the evaporators, underground pipes, tanks, and equipment, additional preparations are required before they can be released to ER for D\&D. Engineering, planning, and field work will be performed to safely release the facilities and equipment to ER.

\subsubsection{Schedule}

\subsubsection{Tank Farm Operations and Maintenance Summary Schedule}

The Tank Farm Operations and Maintenance Summary Schedule is shown on Figure 7.3-2.

\subsubsection{Tank Farm Operations and Maintenance Expanded Baseline (FY 1995 - FY 1997)}

More detailed schedule information for FY 1995 through FY 1997 is provided in Appendix C, Program Element Schedules.

\subsubsection{Resources Requirements}

\subsubsection{Cost}

\subsection{Basis of Cost Estimates}

Cost estimates for the work scope represented by the O\&M program element are improving. Better, more defendable estimates are needed; however, as the overall TWRS baseline matures. At present, the basis for cost estimates are the product definitions addressed in section 7.3.1 as well as the WBS Dictionary Sheets (Appendix A) and the associated Activity Planning Forms. Also providing a basis for estimates are the assumptions documented in section 7.3.1.1.6 and the Activity Planning Forms as well as the logic construction and task identification represented in Section 7.3.2.

For more information on basis of estimates see Appendix E. For more detailed basis of cost estimates see backup TWRS Cost Estimate Sheets.

\subsection{Total Program Element Cost by Year by Fund Type by Activity and Cost Account}

Table 7.3-1 establishes the baseline cost for this program element. The baseline cost is exhibited in annual costs for FY 1994 through FY 2000 and 5-year totals for FY 2001 through program element completion. Where the data are available, these baseline costs are exhibited at the cost account level with fund type totals provided. Cost data are then rolled up to the activity and program element levels. At present, the program element cost baseline is the program element cost totals exhibited on this table less a productivity challenge for FY 1995. 


\subsection{FY 1995 Cost by Month by Fund Type by Activity and Cost Account}

Future MYWP updates will include Table 7.3-2, which establishes the month by month cost baseline for FY 1995 for this program element. The FY 1995 cost baseline is exhibited at the cost account level with fund type totals provided. Cost data are then rolled up to the activity and program element levels. Also exhibited is the total cost baseline for the program element for FY 1995. At present, the program element cost baseline for FY 1995 for this program element is the total-provided on this table less a productivity challenge for FY 1995.

\subsubsection{Staffing}

\subsection{Basis of Staffing Projections}

Staffing projections for the O\&M Program Element are based on the same product definition, assumption, logic construction, and task identification summarized in section 7.3.3.1.1. FY 1995 cost engineering work will begin to improve the tools available to TWRS program element planners to match specific tasks with the appropriate staff levels and qualifications. The overall thrust of Tank Farm Operations and Maintenance staff planning, however, is to "out source" or utilize subcontractors rather than Management and Operating contractor employees to the extent practical.

\subsection{Total Full-Time Equivalents by Year by Activity by Major Participant (Direct and Indirect)}

Future MYWP updates will include Table 7.3-3, which establishes total staffing projections for this program element. Included in the table are FY 1994 staffing data as well as projections by year for FY 1995 through FY 2000. The projections in this table include all direct and indirect FTEs, including all subcontractor personnel. Where data are available, projections are exhibited at the activity level with major program participant totals provided.

\subsection{FY 1995 through FY 1997 Direct Full-Time Equivalents by 88 Labor Categories}

Future MYWP updates will include Table 7.3-4, which establishes direct WHC staffing projections for this program element. Projections do not include subcontractor support and are provided by 88 labor categories for FY 1995 through FY 1997.

\subsubsection{Work Scope Carryover}

There are work scope carryovers identified, mostly driven by funding issues. The items that are being carried over into FY 1995 have been identified in the TWRS Mid-Year Change Request TWR-94-042. The key items include:

- Take the 3 rd evaporator campaign samples

- $\quad$ Complete 6 System Design Descriptions for the Evaporator

- Issue System Status Report Plan for the Evaporator

- $\quad$ Perform DST Integrity Assessment

- Prepare DST Emergency Pumping Plan

- Implement Intrusion Investigation Plan

- $\quad$ Complete development of Waste Volume Projections System Upgrades

- $\quad$ Dispose of portable exhausters and duct work

- Issue annual report of East System performance 


\section{WHC-SP-1101}

- Issue annual report of West System performance

- Complete implementation of surveillance improvements

- Issue four System Design Descriptions for West

- $\quad$ Restore SX and A Farm laterals.

\subsubsection{Waste Type Data}

Waste type data are provided in Appendix $\mathrm{H}$.

\subsubsection{Revisions}

In future updates of the MYWP, this section will include a summary of the revision that has occurred in this program element since the last issue of the MYWP. 


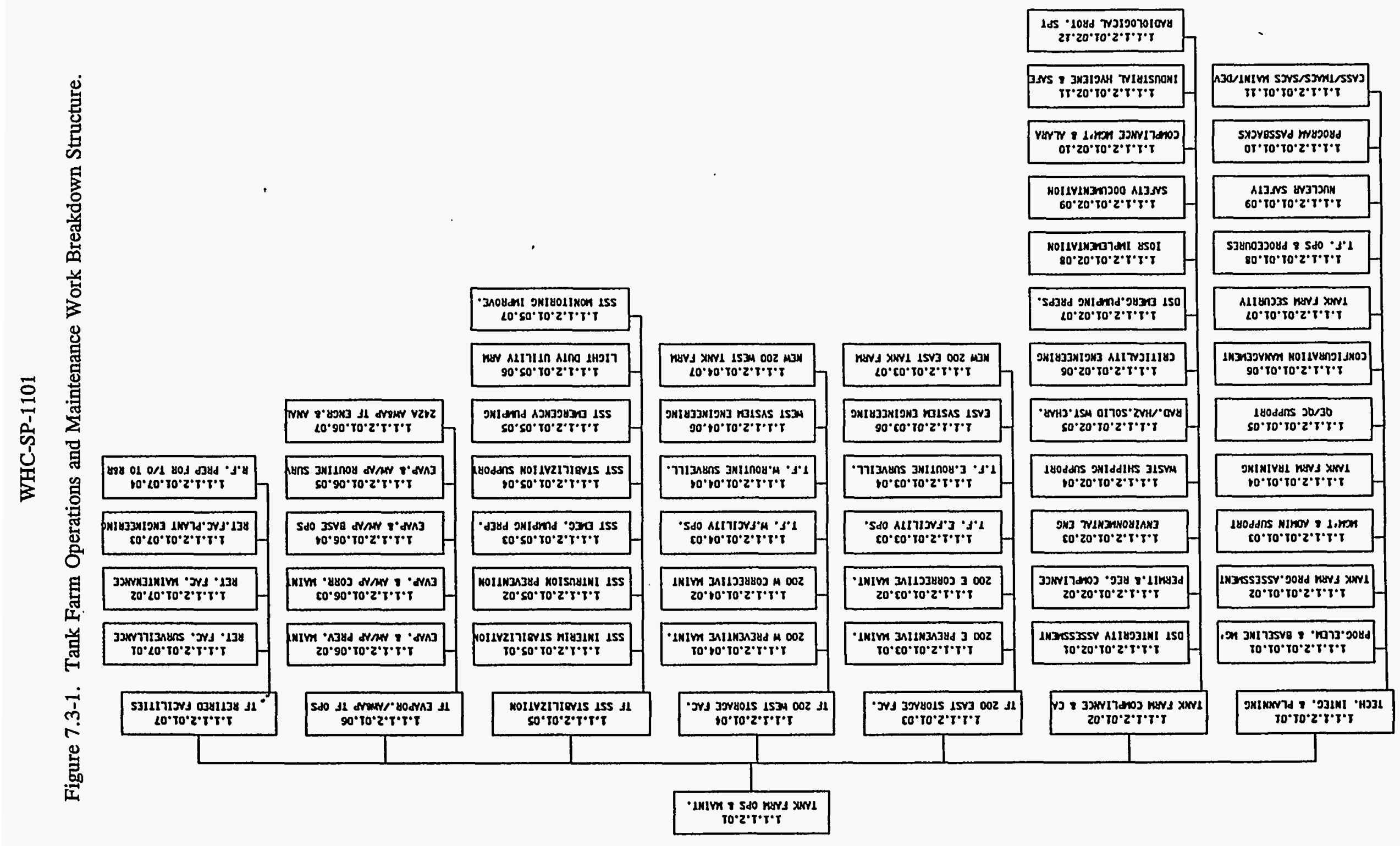


1.1.1.2.01 Tank Farm Operations and Maintenance Summary Schedule

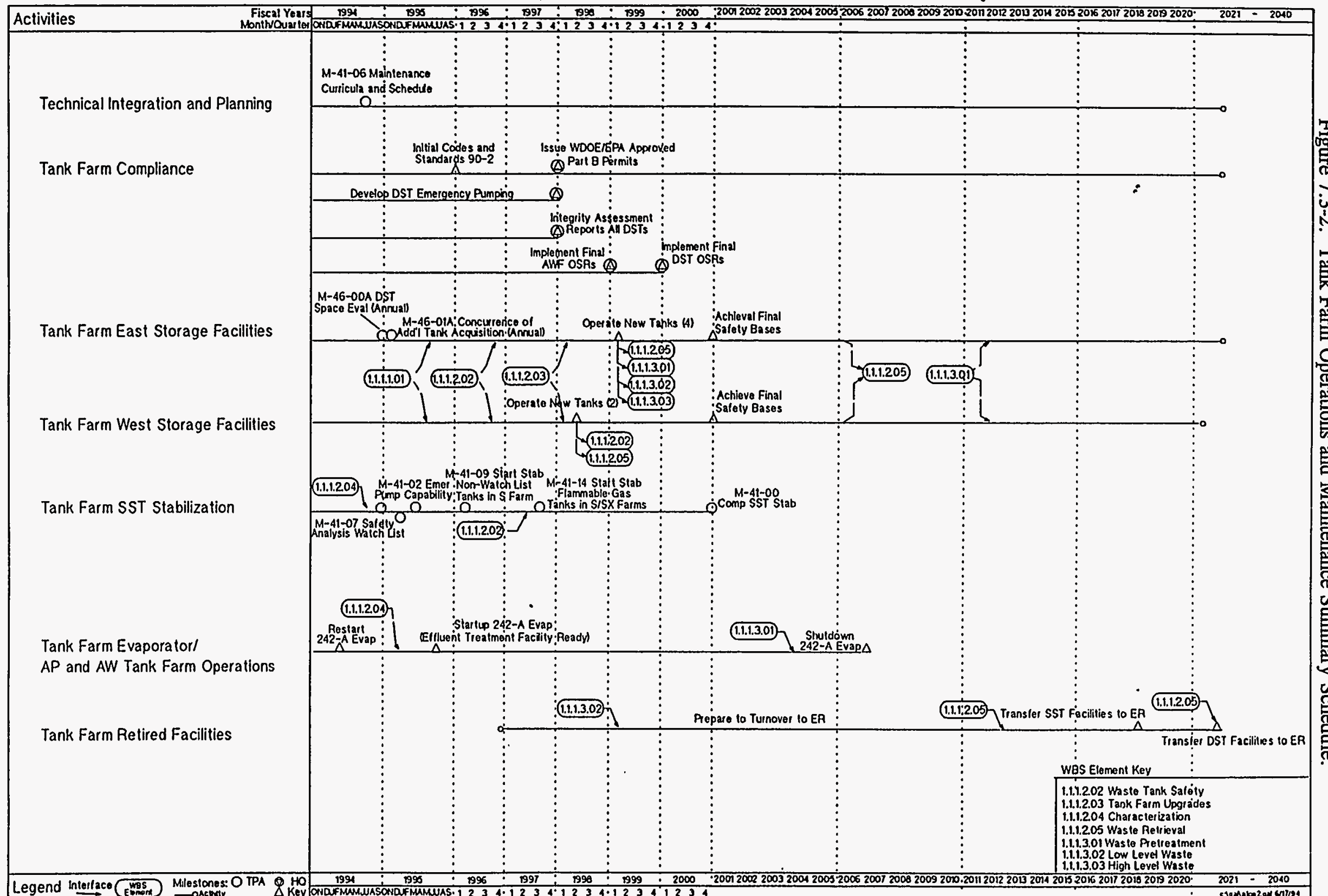


TABLE 7.3-1

TOTAL PROGRAM ELEMENT COST BASELINE - BY YEAR

\begin{tabular}{|c|c|c|c|c|c|c|c|c|c|}
\hline WBS / LEVEL & ACTIVITY TITLE / ADS & $\begin{array}{l}\text { FUND } \\
\text { TYPE }\end{array}$ & $\begin{array}{l}\text { TOTAL } \\
\text { FY94 }\end{array}$ & FY95 & FY96 & FY97 & FY98 & FY99 & FY00 \\
\hline 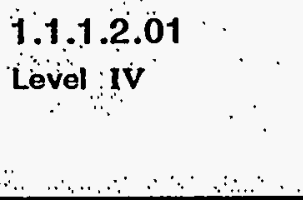 & $\begin{array}{c}\text { Tank Farm Operations } \\
\text { \& Maintenance } \\
\text { TOTAL TARGET }\end{array}$ & $\underset{C}{\mathrm{OE}}$ & $\begin{array}{r}156,525 \\
5,545 \\
162,070 \\
\end{array}$ & $\begin{array}{r}146,756 \\
3,047 \\
149,803\end{array}$ & $\begin{array}{l}155,089 \\
2,936 \\
158,025\end{array}$ & $\begin{array}{r}163,908 \\
2,857 \\
-2=-765\end{array}$ & $\begin{array}{r}179,966 \\
2,795 \\
182761\end{array}$ & $\begin{array}{r}184,376 \\
2787 \\
187,163\end{array}$ & $\begin{array}{r}182,443 \\
2,797 \\
185,240\end{array}$ \\
\hline & $\begin{array}{l}\text { Delta to TARGET. } \\
\text { (See notes at end of Table.) } \\
\text { TOTAL }\end{array}$ & $\begin{array}{l}O E \\
C / E\end{array}$ & $\mathbf{N A}$ & $===\frac{(807)}{807}$ & $\begin{array}{c}(4,083) \\
2,046 \\
==== \\
(2,037)\end{array}$ & $\begin{array}{r}1,648 \\
2,657 \\
==== \\
4,305\end{array}$ & & & \\
\hline $\begin{array}{l}1.1 .1 .2 .01 .01 \\
\text { Level } \mathrm{V}\end{array}$ & $\begin{array}{l}\text { Technical Integration } \\
\frac{\text { and Planning }}{\text { ADS } 1100-0-N D} \\
\quad \text { Sub Total }\end{array}$ & $\begin{array}{l}\text { OE } \\
C / E\end{array}$ & NA & $\begin{array}{r}25,138 \\
0 \\
--- \\
25,138\end{array}$ & $\begin{array}{r}28,687 \\
200 \\
---- \\
28,887\end{array}$ & $\begin{array}{r}30,670 \\
200 \\
---- \\
30,870\end{array}$ & $\begin{array}{r}0 \\
0 \\
--- \\
0\end{array}$ & $\begin{array}{r}0 \\
0 \\
--- \\
0\end{array}$ & $\begin{array}{r}0 \\
0 \\
--- \\
0\end{array}$ \\
\hline $\begin{array}{l}\text { 1.1.1.2.01.01.01 } \\
\text { Level VI }\end{array}$ & $\begin{array}{l}\text { Program Element \& } \\
\text { Baseline Management } \\
\qquad \text { Total }\end{array}$ & $\begin{array}{l}O E \\
C / E\end{array}$ & & $\begin{array}{r}3,327 \\
0 \\
-- \\
3,327\end{array}$ & $\begin{array}{r}3,597 \\
0 \\
---- \\
3,597\end{array}$ & $\begin{array}{r}3,705 \\
0 \\
---- \\
3,705\end{array}$ & $\begin{array}{r}---- \\
0\end{array}$ & ---- & $\begin{array}{r}---- \\
0\end{array}$ \\
\hline $\begin{array}{l}\text { 1.1.1.2.01.01.02 } \\
\text { Level VI }\end{array}$ & $\begin{array}{l}\text { Tank Farm Program } \\
\text { Assessment } \\
\\
\end{array}$ & $\begin{array}{l}O E \\
C / E\end{array}$ & & $\begin{array}{r}6,721 \\
0 \\
--- \\
6,721\end{array}$ & $\begin{array}{r}7,112 \\
0 \\
---- \\
7,112\end{array}$ & $\begin{array}{r}7.208 \\
0 \\
---- \\
7.208\end{array}$ & $\begin{array}{r}---- \\
0\end{array}$ & $\begin{array}{r}---- \\
0\end{array}$ & $\begin{array}{r}--- \\
0\end{array}$ \\
\hline $\begin{array}{l}1.1 .1 .2 .01 .01 .03 \\
\text { Level VI }\end{array}$ & Total & $\begin{array}{l}\text { OE } \\
\text { C/E }\end{array}$ & & $\begin{array}{r}1,930 \\
0 \\
--- \\
1,930\end{array}$ & $\begin{array}{r}2,072 \\
0 \\
---- \\
2,072\end{array}$ & $\begin{array}{r}2,137 \\
0 \\
---- \\
2,137\end{array}$ & $\begin{array}{r}--- \\
0\end{array}$ & $\begin{array}{r}---- \\
0\end{array}$ & $-\cdots$ \\
\hline
\end{tabular}

[ R1 ]

(K\$) 
TABLE $7.3-1$

TOTAL PROGRAM ELEMENT

[ R1 ]

COST BASELINE - BY YEAR

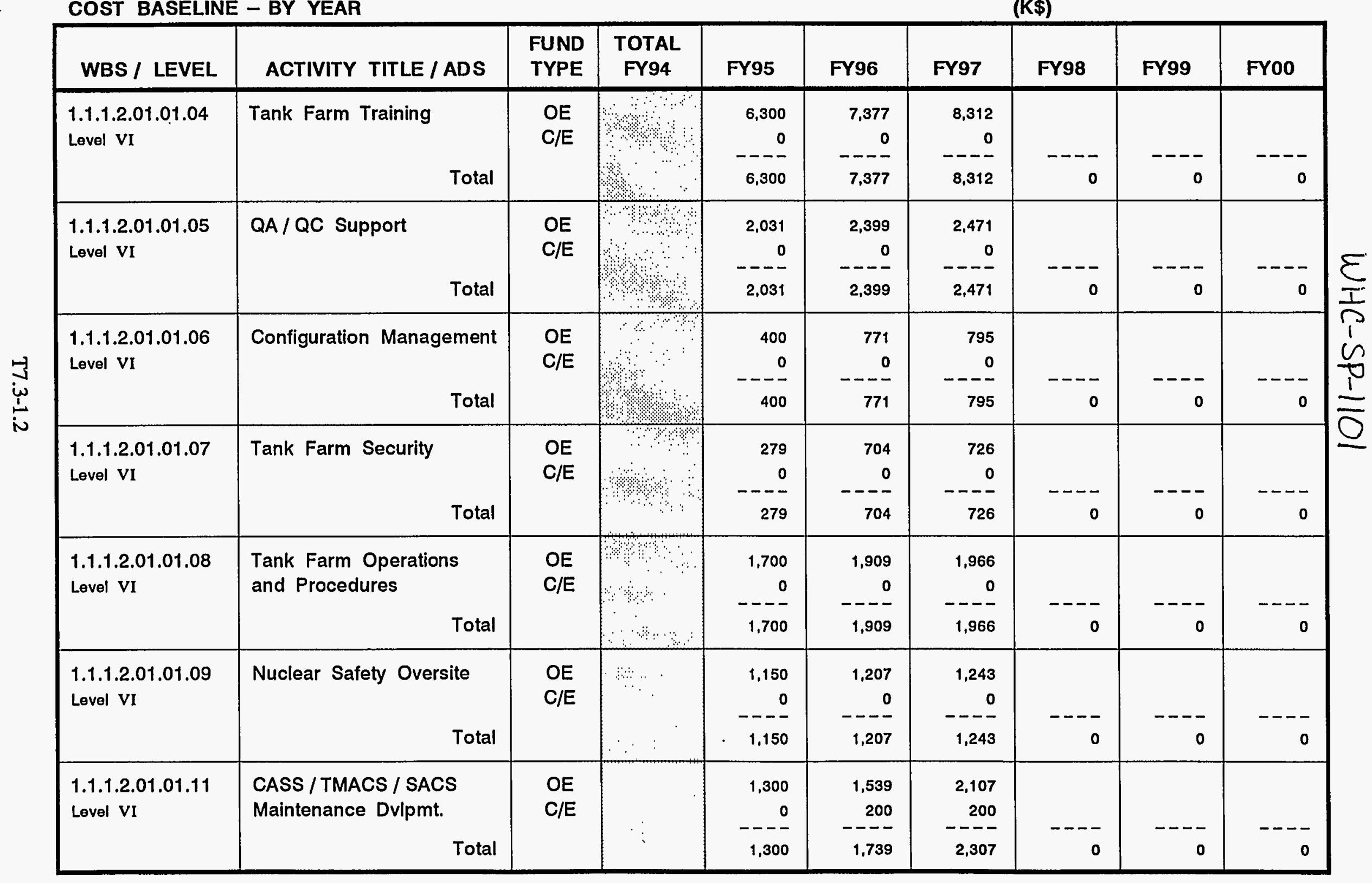


TABLE $\quad 7.3-1$

TOTAL PROGRAM ELEMENT

COST BASELINE - BY YEAR

[ R1 ]

\begin{tabular}{|c|c|c|c|c|c|c|c|c|c|}
\hline WBS / LEVEL & ACTIVITY TITLE / ADS & $\begin{array}{l}\text { FUND } \\
\text { TYPE }\end{array}$ & $\begin{array}{l}\text { TOTAL } \\
\text { FY94 }\end{array}$ & FY95 & FY96 & FY97 & FY98 & FY99 & FYOO \\
\hline $\begin{array}{l}\text { 1.1.1.2.01.02 } \\
\text { Level } \mathrm{v}\end{array}$ & $\frac{\frac{\text { Tank Farm Compliance }}{\text { \& Corrective Actions }}}{\frac{\text { ADS } 1100-0-\mathrm{NC} \quad \text { Sub Total }}{}}$ & $\begin{array}{l}O E \\
C / E\end{array}$ & NA & $\begin{array}{r}32,269 \\
1,000 \\
---- \\
33,269\end{array}$ & $\begin{array}{r}33,012 \\
0 \\
---- \\
33,012\end{array}$ & $\begin{array}{r}39,666 \\
0 \\
---- \\
33,666\end{array}$ & $--\frac{0}{0}$ & $--\frac{0}{0}$ & $\begin{array}{r}0 \\
-- \\
-0\end{array}$ \\
\hline $\begin{array}{l}1.1 .1 .2 .01 .02 .01 \\
\text { Level VI }\end{array}$ & $\begin{array}{l}\text { Dbl. Shell Tank Integrity } \\
\text { Assessment } \\
\qquad \\
\qquad \text { Total }\end{array}$ & $\begin{array}{l}O E \\
C / E\end{array}$ & & $\begin{array}{r}1,747 \\
1,000 \\
--- \\
2,747\end{array}$ & $\begin{array}{r}1,827 \\
0 \\
---\frac{1}{1,827}\end{array}$ & $\begin{array}{r}1,628 \\
0 \\
---- \\
1,628\end{array}$ & --- & $\overline{0}$ & -- \\
\hline $\begin{array}{l}\text { 1.1.1.2.01.02.02 } \\
\text { Level VI }\end{array}$ & $\begin{array}{l}\text { Permits and Regulatory } \\
\text { Compliance } \\
\qquad \text { Total }\end{array}$ & $\begin{array}{l}O E \\
C / E\end{array}$ & ?.: & $\begin{array}{r}2,310 \\
0 \\
---310\end{array}$ & $\begin{array}{r}2,781 \\
---- \\
2,781\end{array}$ & $\begin{array}{r}2,834 \\
0 \\
---\frac{1}{2,834}\end{array}$ & --- & $---\frac{-}{0}$ & $---\frac{-}{0}$ \\
\hline $\begin{array}{l}\text { 1.1.1.2.01.02.03 } \\
\text { Level VI }\end{array}$ & $\begin{array}{l}\text { Environmental Engin. } \\
\qquad \text { Total }\end{array}$ & $\begin{array}{l}O E \\
C / E\end{array}$ & अै: & $\begin{array}{r}4,915 \\
0 \\
---\frac{1}{4,915}\end{array}$ & $\begin{array}{r}5,494 \\
0 \\
---- \\
5,494\end{array}$ & $\begin{array}{r}5,629 \\
0 \\
---- \\
5,629\end{array}$ & --- & $---\frac{-}{0}$ & 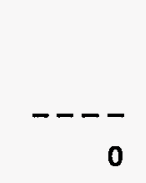 \\
\hline $\begin{array}{l}1.1 .1 .2 .01 .02 .04 \\
\text { Level VI }\end{array}$ & $\begin{array}{l}\text { Waste Shipping } \\
\text { Support }\end{array}$ & $\begin{array}{l}O E \\
C / E\end{array}$ & $\begin{array}{r}\text { औ } \\
3 \\
3 \\
\end{array}$ & $\begin{array}{r}7,630 \\
0 \\
---- \\
7,630\end{array}$ & $\begin{array}{r}7,734 \\
0 \\
---- \\
7,734\end{array}$ & $\begin{array}{r}7,966 \\
0 \\
---- \\
7,966\end{array}$ & $---\frac{-}{0}$ & $\begin{array}{r}--- \\
0\end{array}$ & $\begin{array}{r}---- \\
0\end{array}$ \\
\hline $\begin{array}{l}1.1 .1 .2 .01 .02 .05 \\
\text { Level VI }\end{array}$ & $\begin{array}{l}\text { Radioactive / Hazardous } \\
\text { Solid Waste } \\
\text { Characterization } \\
\quad \text { Total }\end{array}$ & $\begin{array}{l}O E \\
C / E\end{array}$ & 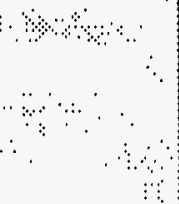 & $\begin{array}{r}3,317 \\
0 \\
---- \\
3,317\end{array}$ & $\begin{array}{r}3,883 \\
0 \\
--- \\
3,883\end{array}$ & $\begin{array}{r}3,146 \\
0 \\
---- \\
3,146\end{array}$ & ---- & $-\cdots$ & $\begin{array}{r}--- \\
0\end{array}$ \\
\hline $\begin{array}{l}1.1 .1 .2 .01 .02 .06 \\
\text { Level VI }\end{array}$ & Criticality Engin. & $\begin{array}{l}O E \\
C / E\end{array}$ & $:$ & $\begin{array}{r}400 \\
--0 \\
-400\end{array}$ & $\begin{array}{r}276 \\
0 \\
---- \\
276\end{array}$ & $\begin{array}{r}284 \\
0 \\
--- \\
284\end{array}$ & $---\frac{-}{0}$ & $---\frac{-}{0}$ & --- \\
\hline
\end{tabular}


TABLE $7.3-1$

TOTAL PROGRAM ELEMENT

[ R1 ]

COST BASELINE - BY YEAR

\begin{tabular}{|c|c|c|c|c|c|c|c|c|c|}
\hline WBS / LEVEL & ACTIVITY TITLE / ADS & $\begin{array}{l}\text { FUND } \\
\text { TYPE }\end{array}$ & $\begin{array}{l}\text { TOTAL } \\
\text { FY94 }\end{array}$ & FY95 & FY96 & FY97 & FY98 & FY99 & FYOO \\
\hline $\begin{array}{l}\text { 1.1.1.2.01.02.07 } \\
\text { Level VI }\end{array}$ & $\begin{array}{l}\text { Dbl. Shell Tank Emerg. } \\
\text { Pumping Preparation } \\
\text { Total }\end{array}$ & $\begin{array}{l}\text { OE } \\
C / E\end{array}$ & & $\begin{array}{r}300 \\
--- \\
-300\end{array}$ & $\begin{array}{r}255 \\
--- \\
255\end{array}$ & $\begin{array}{r}269 \\
0 \\
--- \\
269\end{array}$ & $\begin{array}{r}---- \\
0\end{array}$ & $\begin{array}{r}---- \\
0\end{array}$ & --- \\
\hline $\begin{array}{l}\text { 1.1.1.2.01.02.08 } \\
\text { Level VI }\end{array}$ & $\begin{array}{l}\text { IOSR Implementation } \\
\text { Total }\end{array}$ & $\begin{array}{l}O E \\
C / E\end{array}$ & & $\begin{array}{r}2,948 \\
0 \\
---- \\
2,948\end{array}$ & $\begin{array}{r}500 \\
0 \\
---- \\
500\end{array}$ & $\begin{array}{r}1,175 \\
0 \\
---- \\
1,175\end{array}$ & $\begin{array}{r}---- \\
0\end{array}$ & $\begin{array}{r}--- \\
0\end{array}$ & 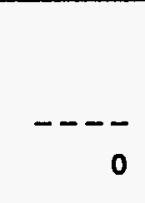 \\
\hline $\begin{array}{l}\text { 1.1.1.2.01.02.09 } \\
\text { Level VI }\end{array}$ & $\begin{array}{l}\text { Safety Documentation } \\
\qquad \text { Total }\end{array}$ & $\begin{array}{l}\text { OE } \\
\text { C/E }\end{array}$ & & $\begin{array}{r}3,455 \\
0 \\
---- \\
3,455\end{array}$ & $\begin{array}{r}3,964 \\
0 \\
---- \\
3,964\end{array}$ & $\begin{array}{r}4,084 \\
0 \\
--0 \\
4,084\end{array}$ & $\begin{array}{r}---- \\
0\end{array}$ & $\begin{array}{r}---- \\
0\end{array}$ & ---- \\
\hline $\begin{array}{l}1.1 .1 .2 .01 .02 .10 \\
\text { Level VI }\end{array}$ & $\begin{array}{l}\text { Compliance Mgmt. } \\
\text { \& ALARA } \\
\\
\\
\text { Total }\end{array}$ & $\begin{array}{l}O E \\
C / E\end{array}$ & & $\begin{array}{r}3,200 \\
0 \\
---- \\
3,200\end{array}$ & $\begin{array}{r}3,479 \\
0 \\
---- \\
3,479\end{array}$ & $\begin{array}{r}3,618 \\
0 \\
--- \\
3,618\end{array}$ & $\begin{array}{r}---- \\
0\end{array}$ & $\begin{array}{r}---- \\
0\end{array}$ & $\begin{array}{r}--- \\
0\end{array}$ \\
\hline $\begin{array}{l}1.1 .1 .2 .01 .02 .11 \\
\text { Level VI }\end{array}$ & $x^{2}$ & $\begin{array}{l}\text { OE } \\
C / E\end{array}$ & \begin{tabular}{ccc}
0 \\
\hdashline \\
4
\end{tabular} & $\begin{array}{r}867 \\
0 \\
---- \\
867\end{array}$ & $\begin{array}{r}1,367 \\
0 \\
--- \\
1,367\end{array}$ & $\begin{array}{r}1,567 \\
0 \\
--- \\
1,567\end{array}$ & $---\overline{0}$ & $\frac{---}{0}$ & $\begin{array}{r}---\overline{0} \\
0\end{array}$ \\
\hline $\begin{array}{l}\text { 1.1.1.2.01.02.12 } \\
\text { Level VI }\end{array}$ & $\begin{array}{l}\text { Radiological Control } \\
\qquad \text { Total }\end{array}$ & $\begin{array}{l}\text { OE } \\
C / E\end{array}$ & 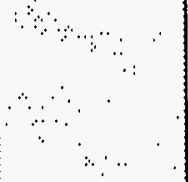 & $\begin{array}{r}1,180 \\
0 \\
---- \\
1.180\end{array}$ & $\begin{array}{r}1,452 \\
0 \\
---- \\
1,452\end{array}$ & $\begin{array}{r}1,466 \\
0 \\
---- \\
1,466\end{array}$ & $\begin{array}{r}---- \\
0\end{array}$ & $\begin{array}{r}--- \\
0\end{array}$ & --- \\
\hline
\end{tabular}


TABLE $7.3-1$

TOTAL PROGRAM ELEMENT

COST BASELINE - BY YEAR

\begin{tabular}{|c|c|c|c|c|c|c|c|c|c|}
\hline WBS / LEVEL & ACTIVITY TITLE / ADS & $\begin{array}{l}\text { FUND } \\
\text { TYPE }\end{array}$ & $\begin{array}{l}\text { TOTAL } \\
\text { FY94 }\end{array}$ & FY95 & FY96 & FY97 & FY98 & FY99 & FY00 \\
\hline $\begin{array}{l}\text { 1.1.1.2.01.03 } \\
\text { Level } \mathrm{v}\end{array}$ & $\begin{array}{l}\frac{\text { Tank Farm } 200 \text { East }}{\text { Storage Facility }} \\
\text { ADs } 1100-0-N F \quad \text { Sub Total }\end{array}$ & $\begin{array}{l}\text { OE } \\
C / E\end{array}$ & NA & $\begin{array}{r}34,950 \\
700 \\
--0 \\
35,650\end{array}$ & $\begin{array}{r}38,060 \\
0 \\
---0 \\
98,060\end{array}$ & $\begin{array}{r}38,034 \\
0 \\
---0 \\
38,034\end{array}$ & $\begin{array}{r}0 \\
0 \\
---\end{array}$ & $\begin{array}{r}0 \\
0 \\
---\frac{1}{0}\end{array}$ & $\begin{array}{r}0 \\
0 \\
0\end{array}$ \\
\hline $\begin{array}{l}1.1 .1 .2 .01 .03 .01 \\
\text { Level VI }\end{array}$ & $\begin{array}{l}200 \text { E Preventive } \\
\text { Maintenance }\end{array}$ & $\begin{array}{l}\text { OE } \\
C / E\end{array}$ & & $\begin{array}{r}1,870 \\
0 \\
--- \\
1,870\end{array}$ & $\begin{array}{r}1,926 \\
0 \\
---- \\
1,926\end{array}$ & $\begin{array}{r}1,930 \\
0 \\
--- \\
1,930\end{array}$ & $\begin{array}{r}---- \\
0\end{array}$ & $\begin{array}{r}---- \\
0\end{array}$ & $\begin{array}{r}---- \\
0\end{array}$ \\
\hline $\begin{array}{l}1.1 .1 .2 .01 .03 .02 \\
\text { Level VI }\end{array}$ & $\begin{array}{l}200 \mathrm{E} \text { Corrective } \\
\text { Maintenance } \\
\text { Total }\end{array}$ & $\begin{array}{l}O E \\
C / E\end{array}$ & +3 & $\begin{array}{r}11,030 \\
0 \\
11,030\end{array}$ & $\begin{array}{r}12,490 \\
0 \\
--2 \\
12,490\end{array}$ & $\begin{array}{r}12,495 \\
0 \\
--- \\
12,495\end{array}$ & $\begin{array}{r}---- \\
0\end{array}$ & $\begin{array}{r}---- \\
0\end{array}$ & $---\overline{0}$ \\
\hline $\begin{array}{l}1.1 .1 .2 .01 .03 .03 \\
\text { Level VI }\end{array}$ & $\begin{array}{l}\text { Tank Farm East Facility } \\
\text { Operations } \\
\text { Total }\end{array}$ & $\begin{array}{l}O E \\
C / E\end{array}$ & $\begin{array}{lll}\because & \cdots \\
\vdots & \ddots\end{array}$ & $\begin{array}{r}11,720 \\
700 \\
---- \\
12,420\end{array}$ & $\begin{array}{r}12,077 \\
0 \\
---- \\
12,077\end{array}$ & $\begin{array}{r}12,080 \\
0 \\
---- \\
12,080\end{array}$ & $\begin{array}{r}---- \\
0\end{array}$ & $---\overline{0}$ & $---\frac{-}{0}$ \\
\hline $\begin{array}{l}1.1 .1 .2 .01 .03 .04 \\
\text { Level VI }\end{array}$ & $\begin{array}{l}\text { Tank Farm East Routine } \\
\text { Surveillance } \\
\text { Total }\end{array}$ & $\begin{array}{l}O E \\
C / E\end{array}$ & $\begin{array}{c}\because \quad \cdot \% \\
\quad \because\end{array}$ & $\begin{array}{r}7,400 \\
0 \\
--- \\
7,400\end{array}$ & $\begin{array}{r}7,622 \\
0 \\
---- \\
7,622\end{array}$ & $\begin{array}{r}7,625 \\
0 \\
---- \\
7,625\end{array}$ & $\begin{array}{r}---- \\
0\end{array}$ & --- & $---\overline{0}$ \\
\hline $\begin{array}{l}\text { 1.1.1.2.01.03.06 } \\
\text { Level VI }\end{array}$ & $\begin{array}{l}\text { East Systems } \\
\text { Engineering } \\
\end{array}$ & $\begin{array}{l}\text { OE } \\
C / E\end{array}$ & $\therefore \quad:$ & $\begin{array}{r}2,930 \\
0 \\
--- \\
2,930\end{array}$ & $\begin{array}{r}3,945 \\
0 \\
---- \\
3,945\end{array}$ & $\begin{array}{r}3,904 \\
0 \\
--- \\
3,904\end{array}$ & $-\frac{--}{0}$ & $---\overline{0}$ & ---- \\
\hline
\end{tabular}

\section{[ R1 ]}


TABLE $\quad 7.3-1$

TOTAL PROGRAM ELEMENT

COST BASELINE - BY YEAR

\begin{tabular}{|c|c|c|c|c|c|c|c|c|c|}
\hline WBS / LEVEL & ACTIVITY TITLE / ADS & $\begin{array}{l}\text { FUND } \\
\text { TYPE }\end{array}$ & $\begin{array}{l}\text { TOTAL } \\
\text { FY94 }\end{array}$ & FY95 & FY96 & FY97 & FY98 & FY99 & FYOO \\
\hline $\begin{array}{l}1.1 .1 .2 .01 .03 .07 \\
\text { Level VI }\end{array}$ & $\begin{array}{r}\text { New } 200 \text { E Tank Farm } \\
\text { Total }\end{array}$ & $\begin{array}{l}O E \\
C / E\end{array}$ & & $\begin{array}{r}0 \\
0 \\
--- \\
0\end{array}$ & $\begin{array}{r}0 \\
0 \\
-- \\
0\end{array}$ & $--\frac{0}{0}$ & $-\cdots$ & --- & $-\cdots$ \\
\hline $\begin{array}{l}1.1 .1 .2 .01 .03 .08 \\
\text { Level vi }\end{array}$ & $\begin{array}{l}\text { East Tank Farm } \\
\text { Improvements }\end{array}$ & $\begin{array}{l}O E \\
C / E\end{array}$ & & $\begin{array}{r}0 \\
0 \\
-- \\
0\end{array}$ & $\begin{array}{r}0 \\
0 \\
-- \\
0\end{array}$ & $\begin{array}{r}0 \\
0 \\
-0\end{array}$ & $--\frac{-}{0}$ & $---\frac{-}{0}$ & $---\frac{-}{0}$ \\
\hline $\begin{array}{l}1.1 .1 .2 .01 .04 \\
\text { Level } \mathrm{v}\end{array}$ & $\begin{array}{l}\frac{\text { Tank Farm } 200 \text { West }}{\text { Storage Facility }} \\
\text { ADS } 1100-0-\text { NG Sub Total }\end{array}$ & $\begin{array}{l}\text { OE } \\
\text { C/E }\end{array}$ & NA & $\begin{array}{r}26,630 \\
400 \\
--- \\
27,030\end{array}$ & $\begin{array}{r}28.415 \\
0 \\
---1 \\
28,415\end{array}$ & $\begin{array}{r}30,020 \\
0 \\
-30,020\end{array}$ & $--\frac{0}{0}$ & $--\frac{0}{0}$ & $\begin{array}{r}\mathbf{0} \\
--\frac{\mathbf{0}}{\mathbf{0}}\end{array}$ \\
\hline $\begin{array}{l}1.1 .1 .2 .01 .04 .01 \\
\text { Level VI }\end{array}$ & $\begin{array}{l}200 \mathrm{~W} \text { Preventive } \\
\text { Maintenance } \\
\end{array}$ & $\begin{array}{l}O E \\
C / E\end{array}$ & & $\begin{array}{r}1,260 \\
0 \\
---- \\
1,260\end{array}$ & $\begin{array}{r}1,498 \\
0 \\
--- \\
1,498\end{array}$ & $\begin{array}{r}1,505 \\
0 \\
--- \\
1,505\end{array}$ & --- & $--\frac{-}{0}$ & $---\overline{0}$ \\
\hline $\begin{array}{l}1.1 .1 .2 .01 .04 .02 \\
\text { Level VI }\end{array}$ & $\begin{array}{l}200 \mathrm{~W} \text { Corrective } \\
\text { Maintenance } \\
\end{array}$ & $\begin{array}{l}O E \\
C / E\end{array}$ & & $\begin{array}{r}7,700 \\
0 \\
--- \\
7,700\end{array}$ & $\begin{array}{r}7,927 \\
0 \\
--- \\
7,927\end{array}$ & $\begin{array}{r}7,993 \\
0 \\
---- \\
7,993\end{array}$ & --- & $\begin{array}{r}--- \\
0\end{array}$ & --- \\
\hline $\begin{array}{l}\text { 1.1.1.2.01.04.03 } \\
\text { Level VI }\end{array}$ & $\begin{array}{l}\text { Tank Farm West Facility } \\
\text { Operations } \\
\text { Total }\end{array}$ & $\begin{array}{l}O E \\
C / E\end{array}$ & 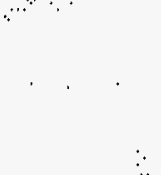 & $\begin{array}{r}9,300 \\
400 \\
--0 \\
9,700\end{array}$ & $\begin{array}{r}9,584 \\
0 \\
---- \\
9,584\end{array}$ & $\begin{array}{r}9,872 \\
0 \\
--- \\
9,872\end{array}$ & $---\frac{-}{0}$ & $---\overline{0}$ & ---- \\
\hline
\end{tabular}

\section{[ R1 ]}


TABLE 7.3-1

TOTAL PROGRAM ELEMENT

COST BASELINE - BY YEAR

\begin{tabular}{|c|c|c|c|c|c|c|c|c|c|}
\hline WBS / LEVEL & ACTIVITY TITLE / ADS & $\begin{array}{l}\text { FUND } \\
\text { TYPE }\end{array}$ & $\begin{array}{l}\text { TOTAL } \\
\text { FY94 }\end{array}$ & FY95 & FY96 & FY97 & FY98 & FY99 & FY00 \\
\hline $\begin{array}{l}\text { 1.1.1.2.01.04.04 } \\
\text { Level VI }\end{array}$ & $\begin{array}{l}\text { Tank Farm West Routine } \\
\text { Surveillance } \\
\text { Total }\end{array}$ & $\begin{array}{l}O E \\
C / E\end{array}$ & נa & $\begin{array}{r}5,800 \\
0 \\
--- \\
5,800\end{array}$ & $\begin{array}{r}6,037 \\
0 \\
--- \\
6,037\end{array}$ & $\begin{array}{r}6,202 \\
0 \\
---202\end{array}$ & --- & --- & $---\frac{-}{0}$ \\
\hline $\begin{array}{l}1.1 .1 .2 .01 .04 .06 \\
\text { Level VI }\end{array}$ & $\begin{array}{l}\text { West Systems } \\
\text { Engineering } \\
\\
\text { Total }\end{array}$ & $\begin{array}{l}O E \\
C / E\end{array}$ & 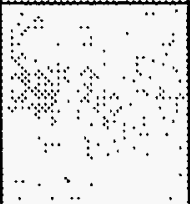 & $\begin{array}{r}2,570 \\
0 \\
--- \\
2,570\end{array}$ & $\begin{array}{r}3,369 \\
0 \\
---- \\
3,369\end{array}$ & $\begin{array}{r}4.448 \\
---0 \\
4,448\end{array}$ & $---\frac{}{0}$ & $\begin{array}{r}--- \\
0\end{array}$ & -- \\
\hline $\begin{array}{l}1.1 .1 .2 .01 .04 .07 \\
\text { Level VI }\end{array}$ & $\begin{array}{l}\text { New } 200 \text { West } \\
\text { Tank Farm } \\
\end{array}$ & $\begin{array}{l}O E \\
C / E\end{array}$ & अ? & $\begin{array}{r}0 \\
0 \\
--0\end{array}$ & $\begin{array}{r}0 \\
0 \\
-- \\
0\end{array}$ & $--\frac{0}{0}$ & $-\frac{-}{0}$ & --- & --- \\
\hline $\begin{array}{l}\text { 1.1.1.2.01.04.08 } \\
\text { Level VI }\end{array}$ & $\begin{array}{l}\text { West Tank Farm } \\
\text { Improvements } \\
\end{array}$ & $\begin{array}{l}O E \\
C / E\end{array}$ & ४ & $\begin{array}{r}0 \\
0 \\
-- \\
0\end{array}$ & $\begin{array}{r}0 \\
0 \\
-0\end{array}$ & $\begin{array}{r}0 \\
-0 \\
-0\end{array}$ & $---\frac{-}{0}$ & --- & --- \\
\hline $\begin{array}{l}\text { 1.1.1.2.01.05 } \\
\text { Level } v\end{array}$ & 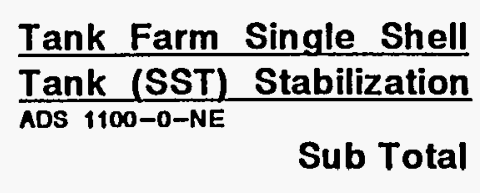 & $\begin{array}{l}O E \\
C / E\end{array}$ & NA & $\begin{array}{r}12,973 \\
140 \\
-13,113\end{array}$ & $\begin{array}{r}15,726 \\
690 \\
-16,416\end{array}$ & $\begin{array}{r}14,384 \\
0 \\
---9 \\
14,384\end{array}$ & $---\frac{0}{0}$ & $\begin{array}{r}0 \\
-0 \\
-0\end{array}$ & $\begin{array}{r}0 \\
0 \\
-- \\
0\end{array}$ \\
\hline $\begin{array}{l}1.1 .1 .2 .01 .05 .01 \\
\text { Level VI }\end{array}$ & $\begin{array}{l}\text { SST Internal } \\
\text { Stabilization }\end{array}$ & $\begin{array}{l}O E \\
C / E\end{array}$ & 3 & $\begin{array}{r}7,127 \\
0 \\
---- \\
7,127\end{array}$ & $\begin{array}{r}7,719 \\
0 \\
---- \\
7,719\end{array}$ & $\begin{array}{r}6,621 \\
0 \\
--- \\
6,621\end{array}$ & $---\frac{-}{0}$ & --- & $---\frac{}{0}$ \\
\hline
\end{tabular}

\section{[ R1 ]}

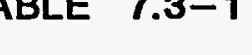


TABLE $7.3-1$

TOTAL PROGRAM ELEMENT

COST BASELINE - BY YEAR

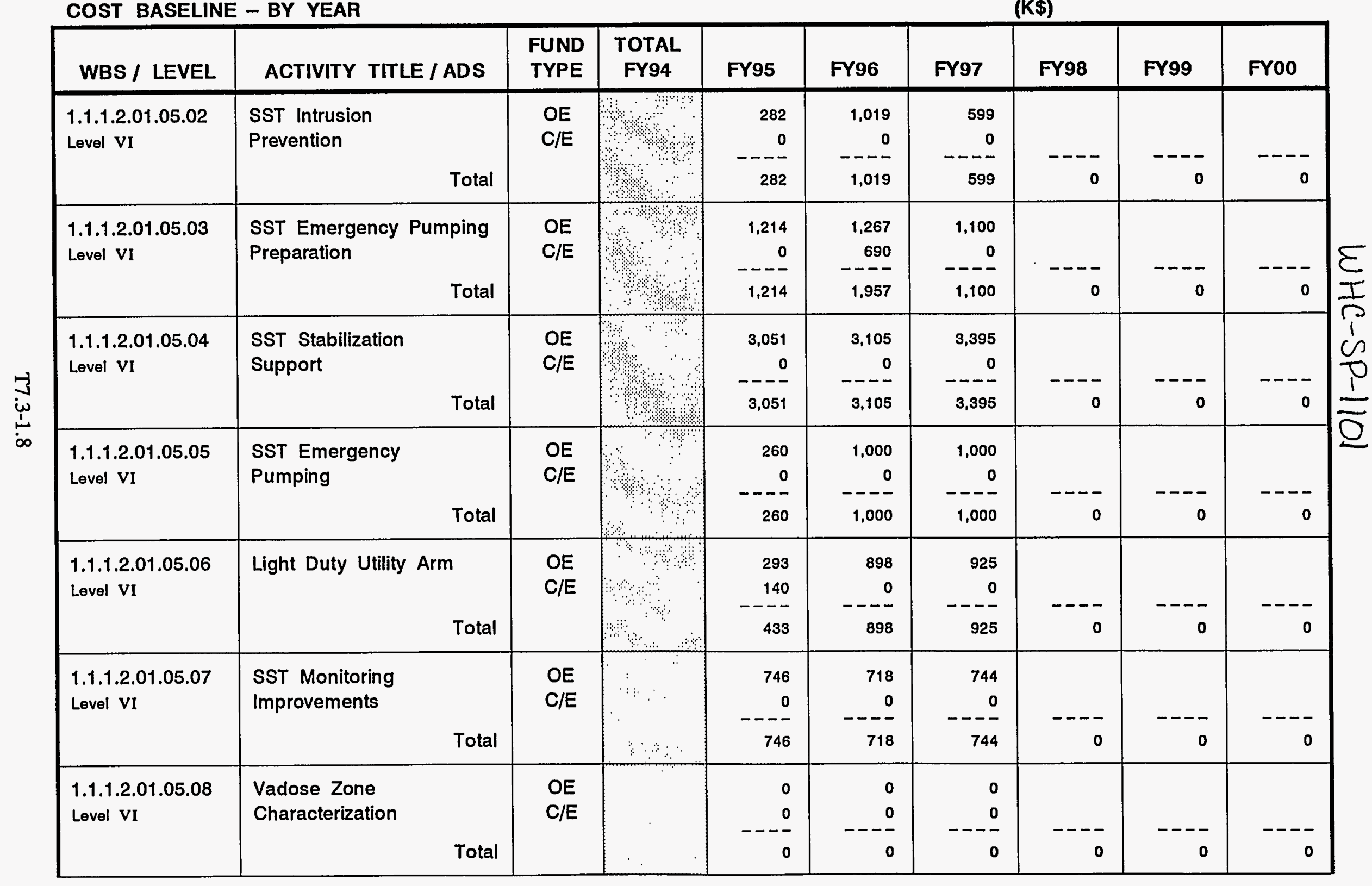

\section{[ R1 ]}


TABLE $7.3-1$

TOTAL PROGRAM ELEMENT COST BASELINE - BY YEAR

\begin{tabular}{|c|c|c|c|c|c|c|c|c|c|}
\hline WBS / LEVEL & ACTIVITY TITLE / ADS & $\begin{array}{l}\text { FUND } \\
\text { TYPE }\end{array}$ & $\begin{array}{c}\text { TOTAL } \\
\text { FY94 }\end{array}$ & FY95 & FY96 & FY97 & FY98 & FY99 & FY00 \\
\hline $\begin{array}{l}1.1 .1 .2 .01 .06 \\
\text { Level V }\end{array}$ & $\begin{array}{l}\text { Tank Farm Evaporator } \\
\text { AW / AP Prevent. Maint. } \\
\text { ADS 1100-0-NA Sub Total }\end{array}$ & $\begin{array}{l}\text { OE } \\
C / E\end{array}$ & NA & $\begin{array}{r}15,603 \\
0 \\
---- \\
15,603\end{array}$ & $\begin{array}{r}15,272 \\
0 \\
---- \\
15,272\end{array}$ & $\begin{array}{r}15,486 \\
0 \\
---- \\
15,486\end{array}$ & $\begin{array}{r}0 \\
0 \\
---\end{array}$ & $\begin{array}{r}0 \\
0 \\
---\end{array}$ & $\begin{array}{r}0 \\
0 \\
---\end{array}$ \\
\hline $\begin{array}{l}\text { 1.1.1.2.01.06.02 } \\
\text { Level VI }\end{array}$ & $\begin{array}{l}\text { Evaporator \& AW / AP } \\
\text { Preventative Maintenance } \\
\text { Total }\end{array}$ & $\begin{array}{l}\text { OE } \\
\text { C/E }\end{array}$ & 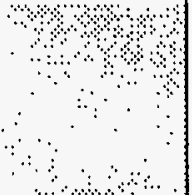 & $\begin{array}{r}673 \\
0 \\
---- \\
673\end{array}$ & $\begin{array}{r}692 \\
0 \\
--- \\
692\end{array}$ & $\begin{array}{r}700 \\
0 \\
--- \\
700\end{array}$ & $\begin{array}{r}---- \\
0\end{array}$ & $\begin{array}{r}---- \\
0\end{array}$ & $\begin{array}{r}--- \\
0\end{array}$ \\
\hline $\begin{array}{l}\text { 1.1.1.2.01.06.03 } \\
\text { Level VI }\end{array}$ & $\begin{array}{l}\text { Evaporator \& AW / AP } \\
\text { Corrective Maintenance } \\
\qquad \text { Total }\end{array}$ & $\begin{array}{l}\text { OE } \\
\text { C/E }\end{array}$ & 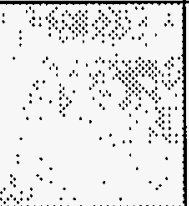 & $\begin{array}{r}3,584 \\
0 \\
--- \\
3,584\end{array}$ & $\begin{array}{r}3,600 \\
0 \\
-- \\
3,600\end{array}$ & $\begin{array}{r}3,686 \\
0 \\
---- \\
3,686\end{array}$ & $\begin{array}{r}---- \\
0\end{array}$ & $\begin{array}{r}---- \\
0\end{array}$ & $---\frac{-}{0}$ \\
\hline $\begin{array}{l}\text { 1.1.1.2.01.06.04 } \\
\text { Level VI }\end{array}$ & $\begin{array}{l}\text { Evaporator \& AW / AP } \\
\text { Base Operations } \\
\qquad \text { Total }\end{array}$ & $\begin{array}{l}\text { OE } \\
C / E\end{array}$ & & $\begin{array}{r}3,825 \\
0 \\
---- \\
3,825\end{array}$ & $\begin{array}{r}3,488 \\
0 \\
--- \\
3,488\end{array}$ & $\begin{array}{r}3,600 \\
0 \\
--- \\
3,600\end{array}$ & $\begin{array}{r}--- \\
0\end{array}$ & $\begin{array}{r}--- \\
0\end{array}$ & $\begin{array}{r}---- \\
0\end{array}$ \\
\hline $\begin{array}{l}\text { 1.1.1.2.01.06.05 } \\
\text { Level VI }\end{array}$ & $\begin{array}{l}\text { Evaporator \& AW / AP } \\
\text { Routine Surveillance } \\
\qquad \text { Total }\end{array}$ & $\begin{array}{l}O E \\
C / E\end{array}$ & 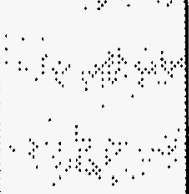 & $\begin{array}{r}2,421 \\
0 \\
---- \\
2,421\end{array}$ & $\begin{array}{r}2,492 \\
0 \\
--- \\
2,492\end{array}$ & $\begin{array}{r}2,500 \\
0 \\
--- \\
2,500\end{array}$ & $\begin{array}{r}---- \\
0\end{array}$ & $\begin{array}{r}---- \\
0\end{array}$ & $\begin{array}{r}---- \\
0\end{array}$ \\
\hline $\begin{array}{l}1.1 .1 .2 .01 .06 .06 \\
\text { Level VI }\end{array}$ & $\begin{array}{l}\text { Outage / Restart Planning } \\
\text { Total }\end{array}$ & $\begin{array}{l}O E \\
C / E\end{array}$ & & $\begin{array}{r}0 \\
0 \\
- \\
0\end{array}$ & $\begin{array}{r}0 \\
0 \\
-0\end{array}$ & $\begin{array}{r}0 \\
0 \\
-- \\
0\end{array}$ & $\begin{array}{r}--- \\
0\end{array}$ & $\begin{array}{r}---- \\
0\end{array}$ & $\begin{array}{r}---- \\
0\end{array}$ \\
\hline $\begin{array}{l}1.1 .1 .2 .01 .06 .07 \\
\text { Level VI }\end{array}$ & $\begin{array}{r}\text { 242A AW \& AP Tank Farm } \\
\text { Engineering \& Analysis } \\
\text { Total }\end{array}$ & $\begin{array}{l}\text { OE } \\
C / E\end{array}$ & $\begin{array}{l}\cdot \quad \begin{array}{l} \\
\ddots\end{array} \\
\therefore \quad\end{array}$ & $\begin{array}{r}5,100 \\
0 \\
--- \\
5,100\end{array}$ & $\begin{array}{r}5,000 \\
0 \\
--- \\
5,000\end{array}$ & $\begin{array}{r}5,000 \\
0 \\
--- \\
5,000\end{array}$ & $\begin{array}{r}---- \\
0\end{array}$ & $\begin{array}{r}--- \\
0\end{array}$ & $\begin{array}{r}--- \\
0\end{array}$ \\
\hline
\end{tabular}

\section{[ R1 ]}

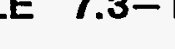


TABLE $\quad 7.3-1$

TOTAL PROGRAM ELEMENT COST BASELINE - BY YEAR

[ R1 ]

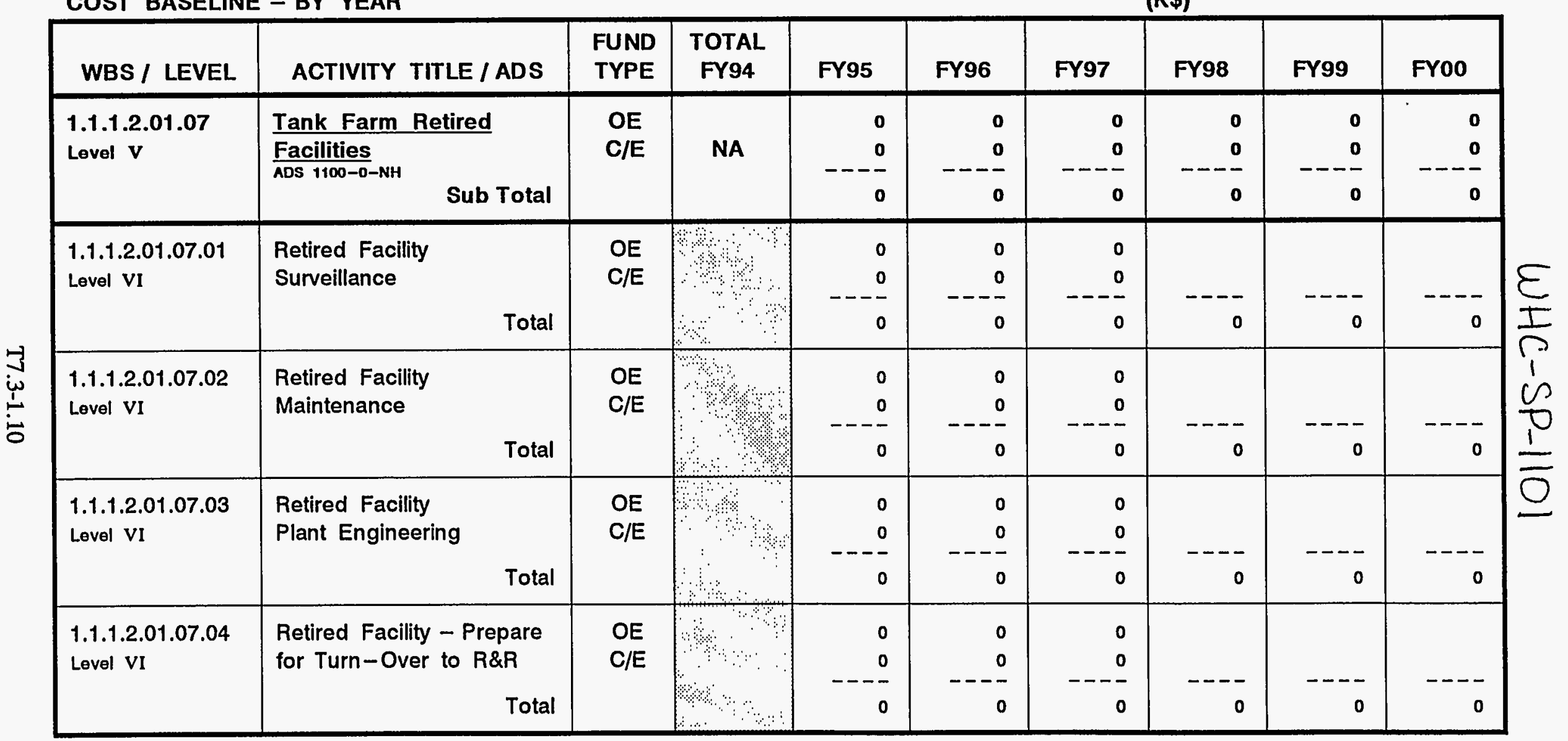


TABLE 7.3-1

TOTAL PROGRAM ELEMENT COST BASELINE - BY YEAR
[R1]

(K\$)

\begin{tabular}{|c|c|c|c|c|c|c|c|c|c|c|}
\hline WBS / LEVEL & ACTIVITY TITLE / ADS & $\begin{array}{l}\text { FUND } \\
\text { TYPE }\end{array}$ & $\begin{array}{l}\text { FY01 / } \\
\text { FY05 }\end{array}$ & $\begin{array}{l}\text { FY06 / } \\
\text { FY10 }\end{array}$ & $\begin{array}{l}\text { FY11 / } \\
\text { FY15 }\end{array}$ & $\begin{array}{l}\text { FY16 / } \\
\text { FY20 }\end{array}$ & $\begin{array}{l}\text { FY21 I } \\
\text { FY25 }\end{array}$ & $\begin{array}{l}\text { FY26 / } \\
\text { FY30 }\end{array}$ & $\begin{array}{l}\text { FY31 / } \\
\text { FY35 }\end{array}$ & $\begin{array}{l}\text { FY36 / } \\
\text { FY40 }\end{array}$ \\
\hline $\begin{array}{lll}1.1 .1 .2 .01 & \\
\text { Level 1v } & 1 \\
1 & & \\
& & \end{array}$ & $\frac{\text { Tank Farm Operations }}{\& \text { Maintenance }}$ & \begin{tabular}{l} 
OE \\
C/E \\
\hdashline \\
$<$
\end{tabular} & $\begin{array}{rr}0 \\
936,805\end{array}$ & $\begin{array}{r}0 \\
-80 \\
-803,344 \\
\end{array}$ & $\begin{array}{r}0 \\
0 \\
-114,950\end{array}$ & $\begin{array}{r}0 \\
650,257 \\
0\end{array}$ & $\begin{array}{r}0 \\
300 \\
574,107\end{array}$ & $\begin{array}{rr}3 & 0 \\
3 & 0 \\
-325,249\end{array}$ & $\begin{array}{r}0 \\
-0 \\
0\end{array}$ & $\begin{array}{l}0 \\
8 \\
8\end{array}$ \\
\hline & $\begin{array}{l}\text { Delta Required to } \\
\text { Balance to TARGET } \\
\text { Budget. } \\
\text { TOTAL }\end{array}$ & $\begin{array}{l}O E \\
C / E\end{array}$ & & & & & & & & \\
\hline $\begin{array}{l}\text { 1.1.1.2.01.01 } \\
\text { Level } \mathrm{v}\end{array}$ & $\begin{array}{l}\text { Technical Integration } \\
\frac{\text { and Planning }}{\text { ADS } 1100-0-N D} \\
\quad \text { Sub Total }\end{array}$ & $\begin{array}{l}O E \\
C / E\end{array}$ & $\begin{array}{r}0 \\
0 \\
-- \\
0\end{array}$ & $-\begin{array}{r}0 \\
0 \\
-0\end{array}$ & $-\begin{array}{r}0 \\
0 \\
-0\end{array}$ & $--\frac{0}{0}$ & $\begin{array}{r}0 \\
0 \\
-0\end{array}$ & $\begin{array}{r}\mathbf{0} \\
\mathbf{0} \\
-0 \\
0\end{array}$ & $\begin{array}{r}\mathbf{0} \\
--0 \\
\mathbf{0}\end{array}$ & $--\frac{0}{0}$ \\
\hline $\begin{array}{l}\text { 1.1.1.2.01.01.01 } \\
\text { Level VI }\end{array}$ & $\begin{array}{l}\text { Program Element \& } \\
\text { Baseline Management } \\
\qquad \text { Total }\end{array}$ & $\begin{array}{l}O E \\
C / E\end{array}$ & $\begin{array}{r}---- \\
0\end{array}$ & $\overline{0}$ & $\begin{array}{r}---- \\
0\end{array}$ & $\begin{array}{r}--- \\
0\end{array}$ & -1 & 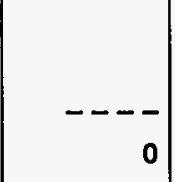 & ---- & --- \\
\hline $\begin{array}{l}\text { 1.1.1.2.01.01.02 } \\
\text { Level VI }\end{array}$ & $\begin{array}{l}\text { Tank Farm Program } \\
\text { Assessment } \\
\\
\end{array}$ & $\begin{array}{l}O E \\
C / E\end{array}$ & --- & $\begin{array}{r}---- \\
0\end{array}$ & -- & $\begin{array}{r}---- \\
0\end{array}$ & - & ---- & $\begin{array}{r}---- \\
0\end{array}$ & ---- \\
\hline $\begin{array}{l}1.1 .1 .2 .01 .01 .03 \\
\text { Level VI }\end{array}$ & Administration & $\begin{array}{l}\text { OE } \\
C / E\end{array}$ & - & -- & - & - & - & $\begin{array}{r}---- \\
0\end{array}$ & $\begin{array}{r}---- \\
0\end{array}$ & $---\frac{-}{0}$ \\
\hline
\end{tabular}


TABLE 7.3-1

TOTAL PROGRAM ELEMENT COST BASELINE - BY YEAR

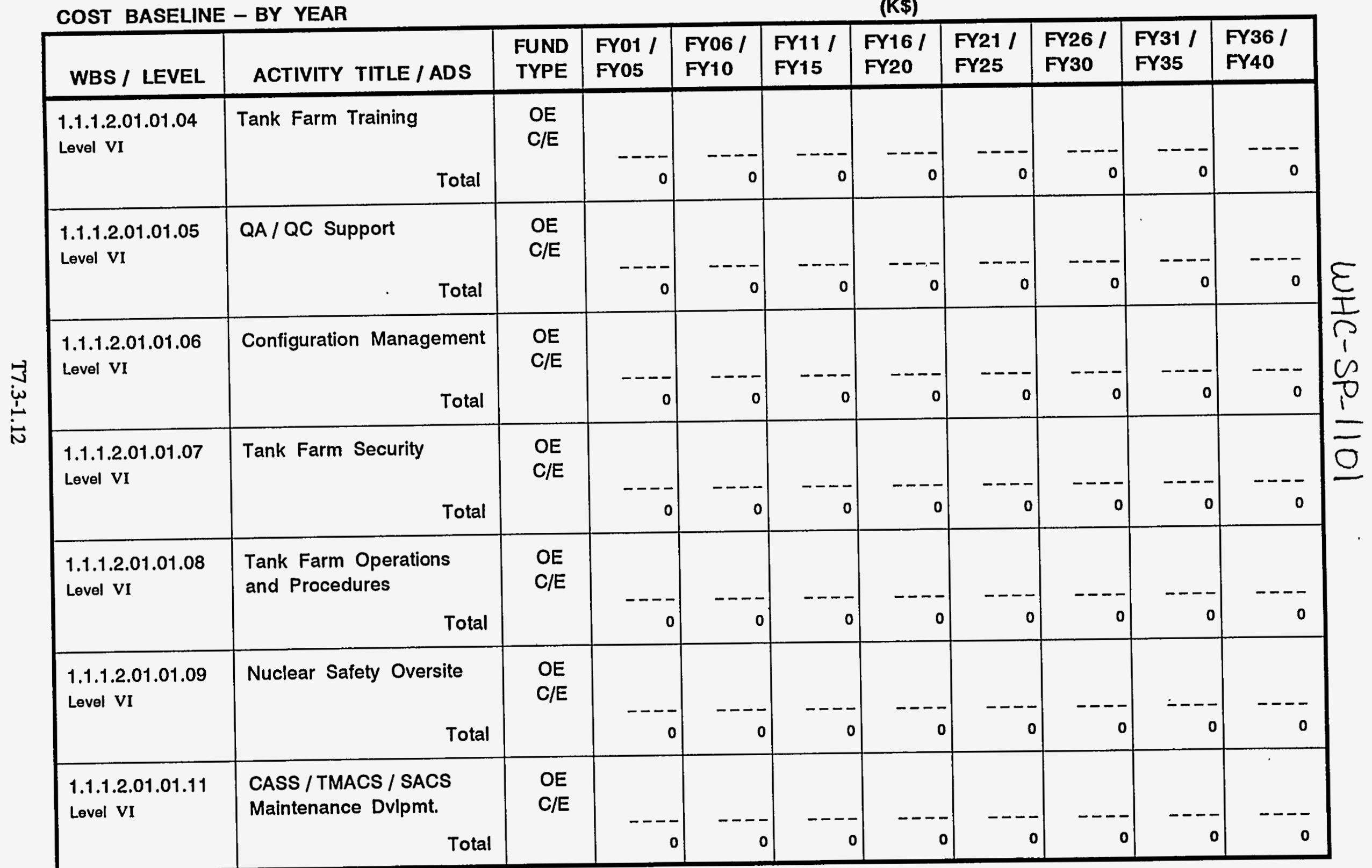

[ R1] 
TABLE $7.3-1$

TOTAL PROGRAM ELEMENT COST BASELINE - BY YEAR

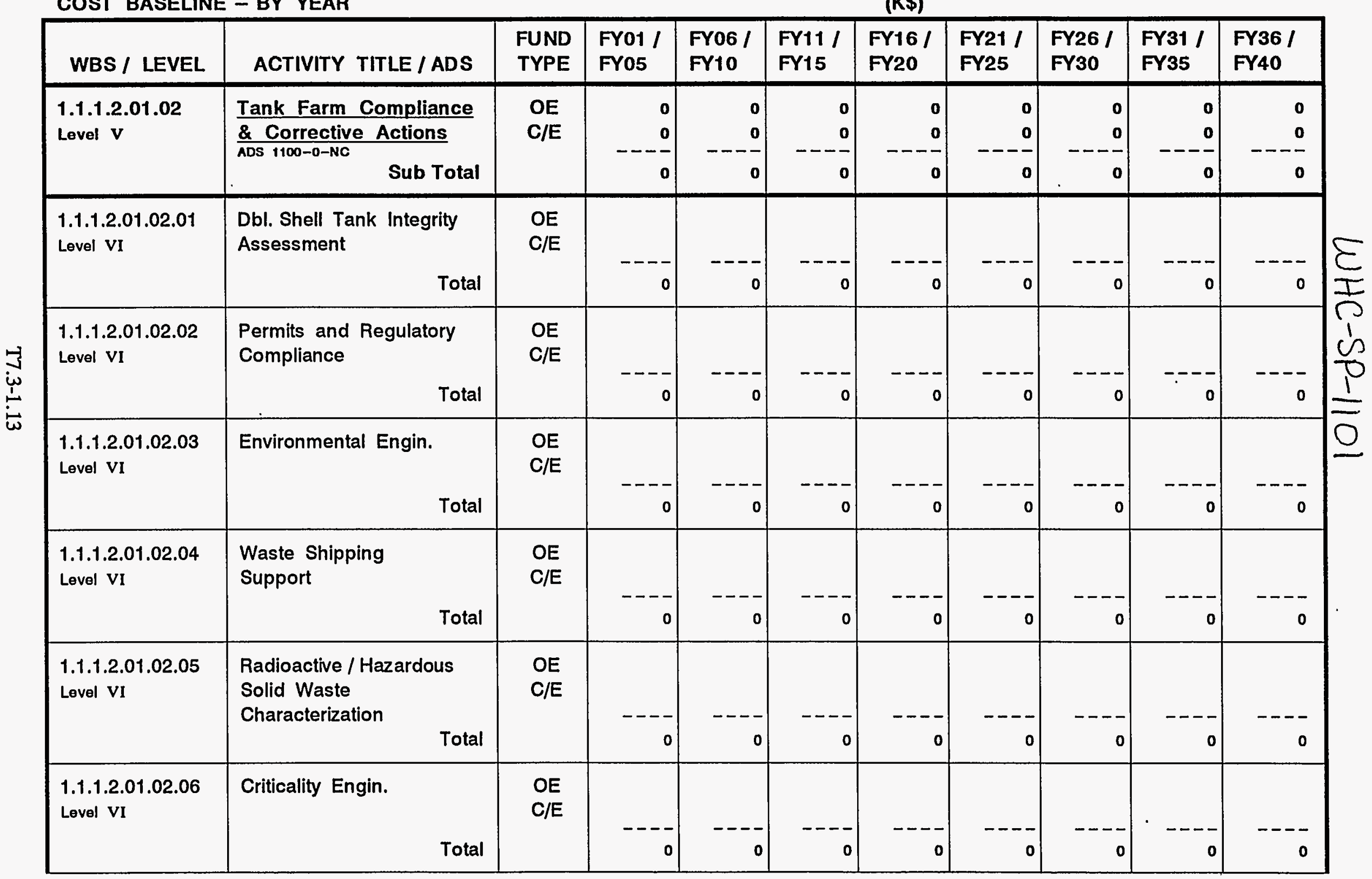

[R1] 
TABLE $7.3-1$

TOTAL PROGRAM ELEMENT

COST BASELINE - BY YEAR

\begin{tabular}{|c|c|c|c|c|c|c|c|c|c|c|}
\hline WBS / LEVEL & ACTIVITY TITLE / ADS & $\begin{array}{l}\text { FUND } \\
\text { TYPE }\end{array}$ & $\begin{array}{l}\text { FY01 I } \\
\text { FY05 }\end{array}$ & $\begin{array}{l}\text { FY06 I } \\
\text { FY10 }\end{array}$ & $\begin{array}{l}\text { FY11 I } \\
\text { FY15 }\end{array}$ & $\begin{array}{l}\text { FY16 / } \\
\text { FY20 }\end{array}$ & $\begin{array}{l}\text { FY21 / } \\
\text { FY25 }\end{array}$ & $\begin{array}{l}\text { FY26 I } \\
\text { FY30 }\end{array}$ & $\begin{array}{l}\text { FY31 / } \\
\text { FY35 }\end{array}$ & $\begin{array}{l}\text { FY36 / } \\
\text { FY40 }\end{array}$ \\
\hline $\begin{array}{l}\text { 1.1.1.2.01.02.07 } \\
\text { Level VI }\end{array}$ & $\begin{array}{l}\text { Dbl. Shell Tank Emerg. } \\
\text { Pumping Preparation } \\
\qquad \text { Total }\end{array}$ & $\begin{array}{l}O E \\
C / E\end{array}$ & ---- & $\begin{array}{r}---- \\
0\end{array}$ & $\begin{array}{r}--- \\
0\end{array}$ & $\begin{array}{r}---- \\
0\end{array}$ & ---- & $\begin{array}{r}---- \\
0\end{array}$ & ---- & --- \\
\hline $\begin{array}{l}1.1 .1 .2 .01 .02 .08 \\
\text { Level VI }\end{array}$ & $\begin{array}{l}\text { IOSR Implementation } \\
\text { Total }\end{array}$ & $\begin{array}{l}O E \\
C / E\end{array}$ & ---- & ---- & ---- & ---- & $---\frac{-}{0}$ & ---- & $---\frac{-}{0}$ & $-\frac{-}{0}$ \\
\hline $\begin{array}{l}\text { 1.1.1.2.01.02.09 } \\
\text { Level VI }\end{array}$ & $\begin{array}{l}\text { Safety Documentation } \\
\text { Total }\end{array}$ & $\begin{array}{l}O E \\
C / E\end{array}$ & ---- & ---- & ---- & ---- & $\begin{array}{r}--- \\
0\end{array}$ & ---- & 0 & --- \\
\hline $\begin{array}{l}\text { 1.1.1.2.01.02.10 } \\
\text { Level VI }\end{array}$ & $\begin{array}{l}\text { Compliance Mgmt. } \\
\text { \& ALARA } \\
\\
\text { Total }\end{array}$ & $\begin{array}{l}O E \\
C / E\end{array}$ & --- & ---- & ---- & ---- & ---- & ---- & ---- & 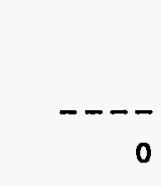 \\
\hline $\begin{array}{l}\text { 1.1.1.2.01.02.11 } \\
\text { Level VI }\end{array}$ & $\begin{array}{l}\text { Industrial Hygiene } \\
\qquad \text { Total }\end{array}$ & $\begin{array}{l}O E \\
C / E\end{array}$ & $\begin{array}{r}---1 \\
0\end{array}$ & $\begin{array}{r}--- \\
0\end{array}$ & $\begin{array}{r}---- \\
0\end{array}$ & ---- & $-\cdots$ & $\begin{array}{r}---- \\
0\end{array}$ & $\begin{array}{r}--- \\
0\end{array}$ & $\frac{---\overline{0}}{0}$ \\
\hline $\begin{array}{l}\text { 1.1.1.2.01.02.12 } \\
\text { Level VI }\end{array}$ & $\begin{array}{l}\text { Radiological Control } \\
\qquad \text { Total }\end{array}$ & $\begin{array}{l}O E \\
C / E\end{array}$ & - & ---- & ---- & 0 & --- & ---- & ---- & $---\frac{-}{0}$ \\
\hline
\end{tabular}


TABLE $7.3-1$

TOTAL PROGRAM ELEMENT COST BASELINE - BY YEAR

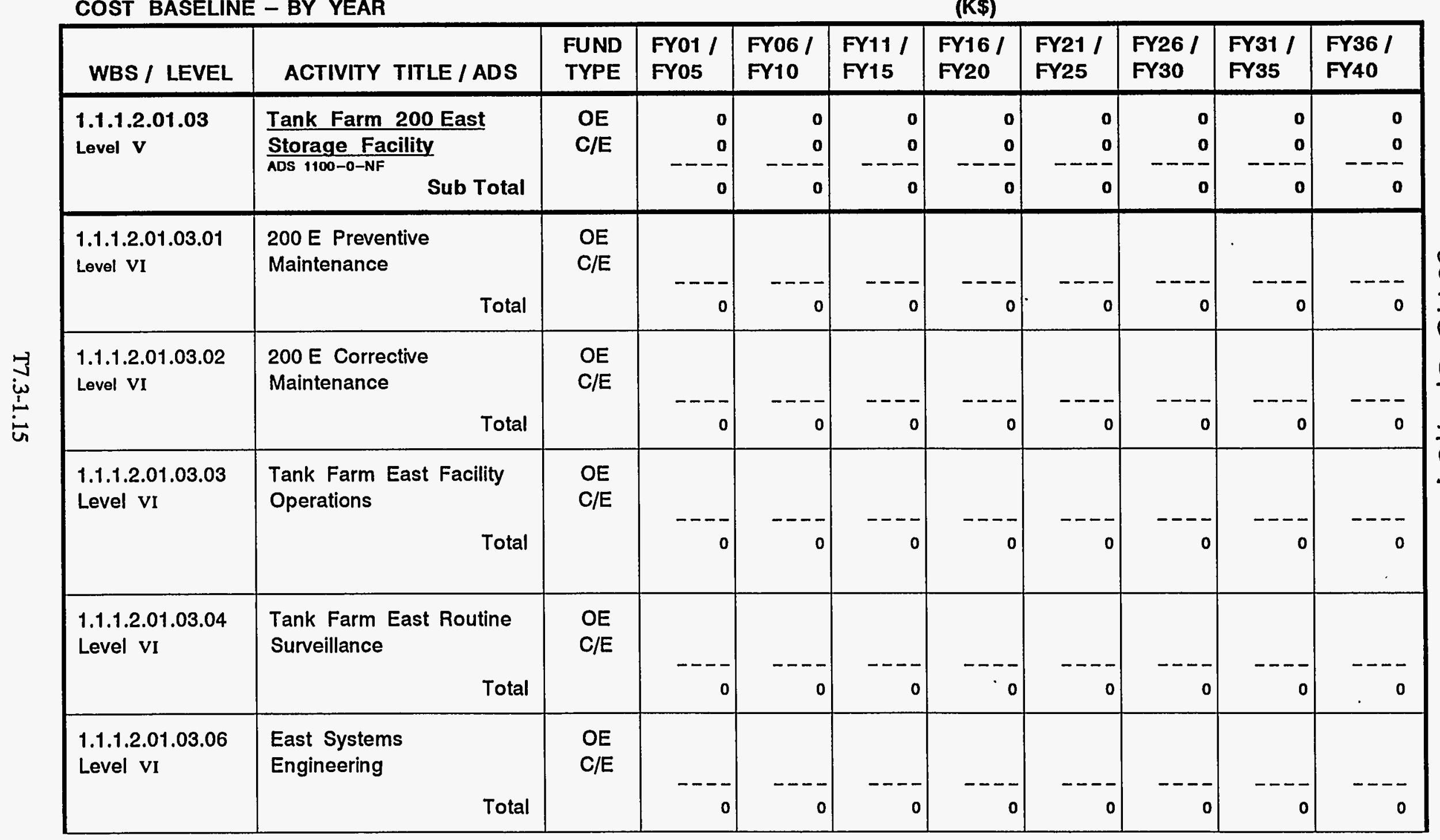

\section{[ R1 ]}


TABLE 7.3-1

TOTAL PROGRAM ELEMENT

[ R1 ]

COST BASELINE - BY YEAR

(K\$)

\begin{tabular}{|c|c|c|c|c|c|c|c|c|c|c|}
\hline WBS / LEVEL & ACTIVITY TITLE / ADS & $\begin{array}{l}\text { FUND } \\
\text { TYPE }\end{array}$ & $\begin{array}{l}\text { FY01 I } \\
\text { FY05 }\end{array}$ & $\begin{array}{l}\text { FY06 / } \\
\text { FY10 }\end{array}$ & $\begin{array}{l}\text { FY11 I } \\
\text { FY15 }\end{array}$ & $\begin{array}{l}\text { FY16 / } \\
\text { FY20 }\end{array}$ & $\begin{array}{l}\text { FY21 / } \\
\text { FY25 }\end{array}$ & $\begin{array}{l}\text { FY26 / } \\
\text { FY30 }\end{array}$ & $\begin{array}{l}\text { FY31 / } \\
\text { FY35 }\end{array}$ & $\begin{array}{l}\text { FY36 / } \\
\text { FY40 }\end{array}$ \\
\hline $\begin{array}{l}1.1 .1 .2 .01 .03 .07 \\
\text { Level vI }\end{array}$ & $\begin{array}{r}\text { New } 200 \text { E Tank Farm } \\
\text { Total }\end{array}$ & $\begin{array}{l}O E \\
C / E\end{array}$ & ---- & ---- & $\begin{array}{r}---- \\
0\end{array}$ & $-\cdots$ & --- & $---\frac{-}{0}$ & ---- & --- \\
\hline $\begin{array}{l}\text { 1.1.1.2.01.03.08 } \\
\text { Level vI }\end{array}$ & $\begin{array}{l}\text { East Tank Farm } \\
\text { Improvements } \\
\\
\text { Total }\end{array}$ & $\begin{array}{l}O E \\
\text { C/E }\end{array}$ & --- & ---- & --- & ---- & -- & $-\cdots$ & - & $---\overline{0}$ \\
\hline $\begin{array}{l}1.1 .1 .2 .01 .04 \\
\text { Level } \mathrm{V}\end{array}$ & $\begin{array}{l}\frac{\text { Tank Farm } 200 \text { West }}{\text { Storage Facility }} \\
\text { ADS } 1100-0-\text { NG Sub Total }\end{array}$ & $\begin{array}{l}O E \\
C / E\end{array}$ & $\begin{array}{r}\mathbf{0} \\
-- \\
-0\end{array}$ & $\begin{array}{r}0 \\
0 \\
-0\end{array}$ & $-\begin{array}{r}0 \\
-0 \\
-0\end{array}$ & $\begin{array}{r}0 \\
0 \\
-- \\
0\end{array}$ & $\begin{array}{r}\mathbf{0} \\
---\frac{0}{0}\end{array}$ & $\begin{array}{r}\mathbf{0} \\
\mathbf{0} \\
-0 \\
\mathbf{0}\end{array}$ & $--\begin{array}{r}0 \\
0 \\
0\end{array}$ & $-\frac{0}{0}$ \\
\hline $\begin{array}{l}\text { 1.1.1.2.01.04.01 } \\
\text { Level VI }\end{array}$ & $\begin{array}{l}200 \mathrm{~W} \text { Preventive } \\
\text { Maintenance } \\
\\
\text { Total }\end{array}$ & $\begin{array}{l}O E \\
C / E\end{array}$ & ---- & $\begin{array}{r}--- \\
0\end{array}$ & $---\frac{-}{0}$ & $\begin{array}{r}---- \\
0\end{array}$ & -- & -- & $---\frac{-}{0}$ & $---\frac{-}{0}$ \\
\hline $\begin{array}{l}\text { 1.1.1.2.01.04.02 } \\
\text { Level VI }\end{array}$ & $\begin{array}{l}200 \mathrm{~W} \text { Corrective } \\
\text { Maintenance } \\
\\
\text { Total }\end{array}$ & $\begin{array}{l}O E \\
C / E\end{array}$ & $-\cdots$ & ---- & --- & ---- & --- & $-\cdots$ & $\begin{array}{r}---- \\
0\end{array}$ & --- \\
\hline $\begin{array}{l}\text { 1.1.1.2.01.04.03 } \\
\text { Level VI }\end{array}$ & $\begin{array}{l}\text { Tank Farm West Facility } \\
\text { Operations } \\
\text { Total }\end{array}$ & $\begin{array}{l}O E \\
C / E\end{array}$ & -- & $---\frac{-}{0}$ & o & $---\frac{-}{0}$ & $---\frac{-}{0}$ & - & $---\frac{-}{0}$ & $---\frac{-}{0}$ \\
\hline
\end{tabular}


TABLE $\quad 7.3-1$

TOTAL PROGRAM ELEMENT COST BASELINE - BY YEAR

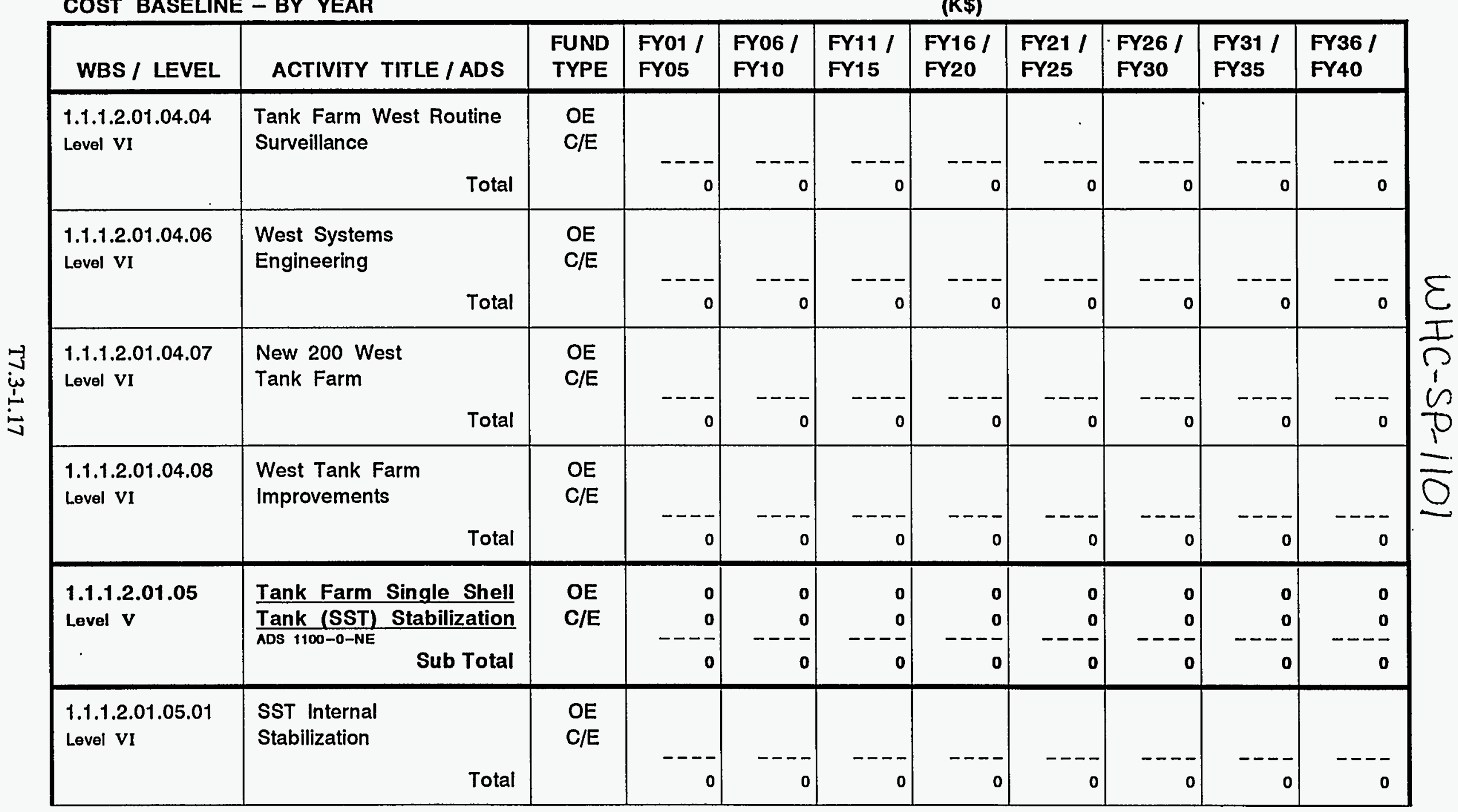

\section{[ R1 ]}


TABLE $7.3-1$

TOTAL PROGRAM ELEMENT COST BASELINE - BY YEAR

\begin{tabular}{|c|c|c|c|c|c|c|c|c|c|c|}
\hline WBS / LEVEL & ACTIVITY TITLE / ADS & $\begin{array}{l}\text { FUND } \\
\text { TYPE }\end{array}$ & $\begin{array}{l}\text { FY01 I } \\
\text { FY05 }\end{array}$ & $\begin{array}{l}\text { FY06 I } \\
\text { FY10 }\end{array}$ & $\begin{array}{l}\text { FY11 / } \\
\text { FY15 }\end{array}$ & $\begin{array}{l}\text { FY16 / } \\
\text { FY20 }\end{array}$ & $\begin{array}{l}\text { FY21 / } \\
\text { FY25 }\end{array}$ & $\begin{array}{l}\text { FY26 / } \\
\text { FY30 }\end{array}$ & $\begin{array}{l}\text { FY31 / } \\
\text { FY35 }\end{array}$ & $\begin{array}{l}\text { FY36 / } \\
\text { FY40 }\end{array}$ \\
\hline $\begin{array}{l}\text { 1.1.1.2.01.05.02 } \\
\text { Level VI }\end{array}$ & $\begin{array}{l}\text { SST Intrusion } \\
\text { Prevention }\end{array}$ & $\begin{array}{l}O E \\
C / E\end{array}$ & ---- & ---- & ---- & ---- & ---- & --- & ---- & --- \\
\hline $\begin{array}{l}\text { 1.1.1.2.01.05.03 } \\
\text { Level VI }\end{array}$ & $\begin{array}{l}\text { SST Emergency Pumping } \\
\text { Preparation } \\
\\
\text { Total }\end{array}$ & $\begin{array}{l}O E \\
C / E\end{array}$ & --- & $\begin{array}{r}---- \\
0\end{array}$ & --- & 0. & $---\frac{1}{0}$ & ---- & ---- & 0 \\
\hline $\begin{array}{l}\text { 1.1.1.2.01.05.04 } \\
\text { Level VI }\end{array}$ & $\begin{array}{l}\text { SST Stabilization } \\
\text { Support } \\
\end{array}$ & $\begin{array}{l}O E \\
C / E\end{array}$ & --- & $\begin{array}{r}---- \\
0\end{array}$ & $\begin{array}{r}--- \\
0\end{array}$ & ---- & $\begin{array}{r}---- \\
0\end{array}$ & ---- & ---- & $---\frac{-}{0}$ \\
\hline $\begin{array}{l}\text { 1.1.1.2.01.05.05 } \\
\text { Level VI }\end{array}$ & $\begin{array}{l}\text { SST Emergency } \\
\text { Pumping } \\
\\
\end{array}$ & $\begin{array}{l}O E \\
C / E\end{array}$ & --- & ---- & ---- & ---- & 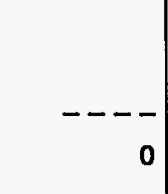 & ---- & ---- & ---- \\
\hline $\begin{array}{l}\text { 1.1.1.2.01.05.06 } \\
\text { Level VI }\end{array}$ & $\begin{array}{l}\text { Light Duty Utility Arm } \\
\qquad \text { Total }\end{array}$ & $\begin{array}{l}\text { OE } \\
C / E\end{array}$ & --- & ---- & ---- & $\begin{array}{r}--- \\
0\end{array}$ & $\begin{array}{r}--- \\
0\end{array}$ & ---- & $--\frac{-}{0}$ & 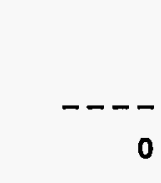 \\
\hline $\begin{array}{l}\text { 1.1.1.2.01.05.07 } \\
\text { Level VI }\end{array}$ & $\begin{array}{l}\text { SST Monitoring } \\
\text { Improvements } \\
\end{array}$ & $\begin{array}{l}O E \\
C / E\end{array}$ & --- & $\begin{array}{r}---- \\
0\end{array}$ & ---- & $---\overline{0}$ & $\begin{array}{r}--- \\
0\end{array}$ & ---- & $---\frac{-}{0}$ & $---\frac{-}{0}$ \\
\hline $\begin{array}{l}\text { 1.1.1.2.01.05.08 } \\
\text { Level VI }\end{array}$ & $\begin{array}{l}\text { Vadose Zone } \\
\text { Characterization } \\
\\
\text { Total }\end{array}$ & $\begin{array}{l}O E \\
C / E\end{array}$ & --- & ---- & $---\frac{-}{0}$ & ---- & $\begin{array}{r}--- \\
0\end{array}$ & --- & $---\frac{}{0}$ & -- \\
\hline
\end{tabular}

[ R1 ] 
TABLE 7.3-1

TOTAL PROGRAM ELEMENT

COST BASELINE - BY YEAR

\begin{tabular}{|c|c|c|c|c|c|c|c|c|c|c|}
\hline WBS / LEVEL & ACTIVITY TITLE / ADS & $\begin{array}{l}\text { FUND } \\
\text { TYPE }\end{array}$ & $\begin{array}{l}\text { FY01 / } \\
\text { FY05 }\end{array}$ & $\begin{array}{l}\text { FY06 I } \\
\text { FY10 }\end{array}$ & $\begin{array}{l}\text { FY11 / } \\
\text { FY15 }\end{array}$ & $\begin{array}{l}\text { FY16 / } \\
\text { FY20 }\end{array}$ & $\begin{array}{l}\text { FY21 / } \\
\text { FY25 }\end{array}$ & $\begin{array}{l}\text { FY26 / } \\
\text { FY30 }\end{array}$ & $\begin{array}{l}\text { FY31 / } \\
\text { FY35 }\end{array}$ & $\begin{array}{l}\text { FY36 / } \\
\text { FY40 }\end{array}$ \\
\hline $\begin{array}{l}\text { 1.1.1.2.01.06 } \\
\text { Level } v\end{array}$ & $\begin{array}{l}\text { Tank Farm Evaporator / } \\
\text { AW / AP Prevent. Maint. } \\
\text { ADS } 1100-0-N A \quad \text { Sụb Total }\end{array}$ & $\begin{array}{l}O E \\
C / E\end{array}$ & $\begin{array}{r}0 \\
-0 \\
-0\end{array}$ & $\begin{array}{r}\mathbf{0} \\
-- \\
\mathbf{0}\end{array}$ & $\begin{array}{r}\mathbf{0} \\
-- \\
-0 \\
0\end{array}$ & $\begin{array}{r}0 \\
0 \\
-0 \\
0\end{array}$ & $---\frac{0}{0}$ & $\begin{array}{r}\mathbf{0} \\
\mathbf{0} \\
-- \\
\mathbf{0}\end{array}$ & $\begin{array}{r}0 \\
0 \\
-- \\
0\end{array}$ & $\begin{array}{r}0 \\
--- \\
0\end{array}$ \\
\hline $\begin{array}{l}\text { 1.1.1.2.01.06.02 } \\
\text { Level VI }\end{array}$ & $\begin{array}{l}\text { Evaporator \& AW / AP } \\
\text { Preventative Maintenance } \\
\qquad \text { Total }\end{array}$ & $\begin{array}{l}\text { OE } \\
C / E\end{array}$ & $---\frac{-}{0}$ & --- & $---\overline{0}$ & ---- & - & -- & -- & $---\frac{-}{0}$ \\
\hline $\begin{array}{l}\text { 1.1.1.2.01.06.03 } \\
\text { Level VI }\end{array}$ & $\begin{array}{l}\text { Evaporator \& AW / AP } \\
\text { Corrective Maintenance } \\
\qquad \text { Total }\end{array}$ & $\begin{array}{l}O E \\
C / E\end{array}$ & $---\frac{-}{0}$ & --- & $---\overline{0}$ & ---- & 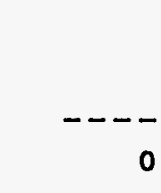 & $---\frac{-}{0}$ & $---\overline{0}$ & $---\overline{0}$ \\
\hline $\begin{array}{l}\text { 1.1.1.2.01.06.04 } \\
\text { Level VI }\end{array}$ & $\begin{array}{l}\text { Evaporator \& AW / AP } \\
\text { Base Operations } \\
\qquad \text { Total }\end{array}$ & $\begin{array}{l}\text { OE } \\
\text { C/E }\end{array}$ & $---\frac{-}{0}$ & --- & 0 & ---- & --- & $---\frac{-}{0}$ & $---\frac{-}{0}$ & $---\frac{-}{0}$ \\
\hline $\begin{array}{l}\text { 1.1.1.2.01.06.05 } \\
\text { Level VI }\end{array}$ & $\begin{array}{l}\text { Evaporator \& AW / AP } \\
\text { Routine Surveillance } \\
\qquad \text { Total }\end{array}$ & $\begin{array}{l}O E \\
C / E\end{array}$ & $\cdots$ & --- & -- & $\begin{array}{r}---- \\
0\end{array}$ & -- & $\begin{array}{r}--- \\
0\end{array}$ & ---- & ---- \\
\hline $\begin{array}{l}\text { 1.1.1.2.01.06.06 } \\
\text { Level VI }\end{array}$ & $\begin{array}{l}\text { Outage / Restart Planning } \\
\text { Total }\end{array}$ & $\begin{array}{l}O E \\
C / E\end{array}$ & -- & --- & $\cdots$ & $-\cdots$ & $\overline{0}$ & ---- & $\begin{array}{r}--- \\
0\end{array}$ & $---\frac{}{0}$ \\
\hline $\begin{array}{l}\text { 1.1.1.2.01.06.07 } \\
\text { Level VI }\end{array}$ & $\begin{array}{l}\text { 242A AW \& AP Tank Farm } \\
\text { Engineering \& Analysis } \\
\text { Total }\end{array}$ & $\begin{array}{l}\text { OE } \\
\text { C/E }\end{array}$ & - & -- & - & 0 & ---- & ---- & ---- & ---- \\
\hline
\end{tabular}

\section{[ R1 ]}


TABLE $7.3-1$

TOTAL PROGRAM ELEMENT

COST BASELINE - BY YEAR

\begin{tabular}{|c|c|c|c|c|c|c|c|c|c|c|}
\hline WBS / LEVEL & ACTIVITY TITLE / ADS & $\begin{array}{l}\text { FUND } \\
\text { TYPE }\end{array}$ & $\begin{array}{l}\text { FY01 / } \\
\text { FY05 }\end{array}$ & $\begin{array}{l}\text { FY06 I } \\
\text { FY10 }\end{array}$ & $\begin{array}{l}\text { FY11 / } \\
\text { FY15 }\end{array}$ & $\begin{array}{l}\text { FY16 / } \\
\text { FY20 }\end{array}$ & $\begin{array}{l}\text { FY21 / } \\
\text { FY25 }\end{array}$ & $\begin{array}{l}\text { FY26 I } \\
\text { FY30 }\end{array}$ & $\begin{array}{l}\text { FY31 / } \\
\text { FY35 }\end{array}$ & $\begin{array}{l}\text { FY36 I } \\
\text { FY40 }\end{array}$ \\
\hline $\begin{array}{l}\text { 1.1.1.2.01.07 } \\
\text { Level } v\end{array}$ & $\frac{\text { Tank Farm Retired }}{\frac{\text { Facilities }}{\text { ADS } 1100-0-N H}}$ & $\begin{array}{l}O E \\
C / E\end{array}$ & $\begin{array}{r}0 \\
0 \\
--1 \\
0\end{array}$ & $\begin{array}{r}0 \\
0 \\
-0\end{array}$ & $\begin{array}{r}0 \\
0 \\
--1 \\
0\end{array}$ & $\begin{array}{r}0 \\
-0 \\
0\end{array}$ & $\begin{array}{r}\mathbf{0} \\
\mathbf{0} \\
-0 \\
0\end{array}$ & $\begin{array}{r}0 \\
0 \\
-0\end{array}$ & $\begin{array}{r}0 \\
0 \\
-0 \\
0\end{array}$ & $---\frac{0}{0}$ \\
\hline $\begin{array}{l}\text { 1.1.1.2.01.07.01 } \\
\text { Level VI }\end{array}$ & $\begin{array}{l}\text { Retired Facility } \\
\text { Surveillance } \\
\\
\end{array}$ & $\begin{array}{l}\text { OE } \\
\text { C/E }\end{array}$ & ---- & 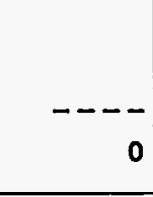 & ---- & --- & ---- & ---- & --- & 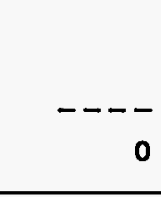 \\
\hline $\begin{array}{l}1.1 .1 .2 .01 .07 .02 \\
\text { Level VI }\end{array}$ & $\begin{array}{l}\text { Retired Facility } \\
\text { Maintenance }\end{array}$ & $\begin{array}{l}O E \\
C / E\end{array}$ & ---- & --- & ---- & --- & ---- & $\begin{array}{r}--- \\
0\end{array}$ & ---- & --- \\
\hline $\begin{array}{l}\text { 1.1.1.2.01.07.03 } \\
\text { Level VI }\end{array}$ & $\begin{array}{l}\text { Retired Facility } \\
\text { Plant Engineering } \\
\\
\quad \text { Total }\end{array}$ & $\begin{array}{l}O E \\
C / E\end{array}$ & ---- & -- & ---- & - & --- & 0 & -- & -- \\
\hline $\begin{array}{l}1.1 .1 .2 .01 .07 .04 \\
\text { Level VI }\end{array}$ & $\begin{array}{l}\text { Retired Facility - Prepare } \\
\text { for Turn-Over to R\&R } \\
\text { Total }\end{array}$ & $\begin{array}{l}O E \\
C / E\end{array}$ & ---- & - & $\begin{array}{r}--- \\
0\end{array}$ & $---\overline{0}$ & $\begin{array}{r}--- \\
0\end{array}$ & --- & $\begin{array}{r}---- \\
0\end{array}$ & --- \\
\hline
\end{tabular}

\section{[ R1 ]}


WHC-SP-1101

\section{NOTES \\ TOTAL PROGRAM ELEMENT COST BASELINE - BY YEAR}

The cost exhibit tables in each of the Program Element Baselines (Sections 7.2 through 7.10) identify the program element target funding by year by fund type. The tables also provide details of the baseline allocation of funding down to activity levels (level V) and cost account levels (level VI) of the WBS for Fiscal Year 1995 through 1997. The details exhibited on these tables are taken from the baseline WBS Dictionary Sheets. Deltas exist between the details and the annual target totals. For Fiscal Years 1996 and 1997 the deltas will be addressed in the future, following the completion of the resolution of expected differences between current Fiscal Year 1995 baseline funding and expected actual Fiscal Year 1995 funding. Deltas for Fiscal Year 1995 are noted below by program element:

\section{PROGRAM MANAGEMENT AND ADMINISTRATION:}

No deltas.

\section{TANK FARM OPERATIONS AND MAINTENANCE:}

No overall delta. This baseline requires revised fund type mix.

\section{WASTE TANK SAFETY:}

Delta is due to need to revise an overhead pricing rate in the P3 system.

\section{TANK FARM UPGRADES:}

Delta is due to differences between Budget Authority (BA) in the target and Budget Obligation (B0) in the P3 system.

\section{CHARACTERIZATION:}

No significant deltas.

\section{WASTE RETRIEVAL:}

Delta is primarily due to need to resolve issues associated with expense funding support to capital projects and differences between Budget Authority (BA) in the target and Budget Obligation (B0) in the P3 system.

\section{WASTE PRETREATMENT:}

Delta is due to the sum of the detail cost estimates being lower than the target total as a result of reestimation of resource requirements to complete baseline scope.

\section{LOW LEVEL WASTE:}

Delta is primarily due to carryover expense funded workscope on melter testing addressed in June 9, 1994 RL letter.

$$
.77 .3-1.21
$$


WHC-SP-1101

\section{HIGH LEVEL WASTE:}

Delta is due to carryover of capital funding, PNL expense funding carryover, and ADS corrections submitted but not reflected in target totals.

$T 7.3-1.22$ 
Table 7.3-2. FY 1995 Cost by Month by Fund Type by Activity and Cost Account.

Future MYWP updates will include Table 7.3-2, which establishes the month by month cost baseline for FY 1995 for this program element. The FY 1995 cost baseline is exhibited at the cost account level with fund type totals provided. Cost data are then rolled up to the activity and program element levels. Also exhibited is the total cost baseline for the program element for FY 1995. At present, the program element cost baseline for FY 1995 for this program element is the total provided on this table less a productivity challenge for FY 1995. 
Table 7.3-3. Total FTEs by Year by Activity by Major Participant (Direct and Indirect) (Including all Subcontractors).

Future MYWP updates will include Table 7.3-3, which establishes total staffing projections for this program element. Included in the table are FY 1994 staffing data as well as projections by year for FY 1995 through FY 2000. The projections in this table include all direct and indirect FTEs, including all subcontractor personnel. Where data are available, projections are exhibited at the activity level with major program participant totals provided. 
Table 7.3-4. FY 1995 and FY 1996 Direct Westinghouse Hanford Company FTEs by 88 Labor Categories.

Future MYWP updates will include Table 7.3-4, which establishes direct WHC staffing projections for this program element. Projections do not include subcontractor support and are provided by 88 labor categories for FY 1995 through FY 1997. 


\subsection{Waste Tank Safety Baseline}

The Waste Tank Safety Program (WTSP) baseline consists of the technical basis; schedule; resource requirements; and waste type data for FY 1995 through completion of the mission. The major emphasis on this MYWP is on the technical basis, milestones and deliverables, resource loaded schedules, and resource requirements for periods of FY 1995 to FY 1997.

The WTSP was established in mid-1990 to address the hazards associated with storage of radioactive mixed waste in the large underground radioactive waste storage double-shell tanks (DST) and single-shell tanks (SST) at the Hanford Site.

Functional areas of responsibility are:

1. Technical Integration and Planning

2. Hydrogen Mitigation

3. Waste Tank Ferrocyanide Safety Program

4. Waste Tank Flammable Gas Safety Program

5. Waste Tank Organic Safety Program

6. Waste Tank High-Heat Safety Program

7. Miscellaneous Underground Storage Tanks

8. Waste Tank Vapor Resolution

9. Waste Tank Nuclear Criticality

10. Other Waste Tank Safety Issues (inactive)

11. Waste Tank Hydroxide Control

See Figure 7.4-1 for respective Organization Charts.

\subsubsection{Technical Basis}

This section provides the mission strategy; program element structure; work to be performed in FY 1995; work to be performed in FY 1996 and 1997; approach; and drivers and deliverables.

The U.S. Department of Energy's (DOE's) most pressing safety problem nationwide is resolution of the safety concerns and system deficiencies relating to the Hanford Site waste tanks. As a result of past processes and aging of waste within approximately 56 Watch List tanks, safety concerns have arisen over the potential for release of high-level waste (HLW) from the tanks to the environment.

The six most serious safety concerns are the potential for high flammable gas concentrations in 25 tanks, potentially explosive mixtures of ferrocyanide in 20 tanks, potential for runaway organic-nitrate reactions in twenty tanks, high heat generation in SST 241-C-106, tank vapor releases, and potential for nuclear criticality in all high-level radioactive waste tanks.

\subsubsection{Mission Strategy}

This section provides the program objectives; program strategy; technical functions and requirements; program risk; major decisions; assumptions; and constraints. 


\subsection{Objectives}

The WTSP will meet the applicable functions and requirements and will deliver the products and end items necessary to achieve the Tank Waste Remediation System (TWRS) Program mission. The purpose of the WTSP is to mitigate and/or resolve the waste tank safety issues at the Hanford Site and the objectives are:

1. Serve as a focal point to identify and resolve selected high-priority waste tank safety issues

2. Ensure safe storage of waste during the interim period until retrieval or disposal operations begin.

\subsection{Strategy}

The WTSP will successfully mitigate and/or resolve tank safety issues in-tank to the fullest extent practical, and will effectively implement the following overall strategy:

1. Evaluate the in-tank hazard, develop criteria to define the safety issue, evaluate adequacy of safety basis, close any Unreviewed Safety Questions (USQs), monitor the hazard, ensure interim safe storage conditions exist, mitigate the hazard (if necessary), and resolve the safety issue

2. Accelerate implementation schedules by streamlining the review and approval processes and developing safety initiatives to quantify the work requirements; and

3. Assess technologies and applied engineering strategies for applicability.

\subsection{Functions and Requirements}

Technical functions and requirements (F\&R) have been established for the WTSP. The F\&R requirements lie within Level 4 of the functional hierarchy of TWRS. The functional definition of this respective level is: "Contain and monitor SST waste, waste in miscellaneous tanks, and cesium and strontium capsules; receive, contain and monitor DST waste and in-process waste; and define and initiate actions for mitigation / resolution of safety issues".

The F\&R levels are:

1. Level 0 is "Clean Up Hanford" (0);

2. Level 1 is "Remedy Unsafe and Unacceptable Conditions" (4);

3. Level 2 is "Remediate Tank Waste" (4.2);

4. Level 3 is "Manage Tank Waste" (4.2.1); and

5. Level 4 is "Store Waste" (4.2.1.1).

This program element supports the 4.2.1.1 "Store Waste" function by defining and initiating actions for mitigation/resolution of safety issues with the potential for offsite release during storage. This element does not address resolution of safety issues for or during disposal.

This program element provides data requirements and objectives to the 4.2.1.2 "Characterize Waste" function; and 4.2.1.2 provides characterization reports necessary to evaluate and resolve safety issues. 
.This program element provides waste data to the 4.2.1.3 "Retrieve Waste" function and ensures that resolution of safety issues does not alter the waste in a manner that will adversely affect retrieval operations; and 4.2.1.3 retrieves tank waste as part of the actions necessary to resolve safety issues.

This program element shares waste data with the 4.2.2.1 "Pretreat Waste" function and ensures that resolution of safety issues does not alter the waste in a manner that will adversely affect pretreatment operations.

This program element installs monitoring and mitigation equipment in the Watch List waste tanks and provides solid waste in the form of contaminated equipment removed from the tanks to the 4.2.3.4 "Disposition Solid Waste" function; and the 4.2.3.4 function disposes of the solid waste.

See Tank Waste Remediation System Functions and Requirements, DOE/RL-92-60 Rev. 1, dated March 28, 1994: Section 2.0 Tank Waste Remediation System Function and Requirement Descriptions, Table 2-1 Function Definitions and Table F4.2.1.1 Store Waste (Item V Function Requirements, Paragraphs A and B); and Section 3 Tank Waste Remediation System Architecture, Table A4.2.1.1 Waste Storage System (Item VIII Requirements Satisfied, Paragraphs B and C).

\subsection{Risk}

Risk management for the WTSP is composed of identifying, assessing, and mitigating technical and programmatic risks. Technical risks for the WTSP are caused by uncertainties in the characteristics and composition of stored tank waste. Programmatic risks involve achieving the required level of technical excellence within available funding and schedule constraints and managing the WTSP to properly consider values of public interest groups, Tribes, local and regional governments, and State agencies; institutional influences; and the merging of new facilities with existing operations and facilities, both within the TWRS and sitewide.

Risks are not generally independent of each other. As risks are identified, the impact of one type of risk on other types of risks will be determined. Identified risks will be described in detail and include both technical and programmatic risks.

\section{Technical Risks}

1. Assumptions that underlie technical analyses

2. Degree to which selected technologies are mature for a given WTSP application

3. Range of uncertainty surrounding quantitative values used in analyses and models

4. Depth, breadth, and completeness of analyses used to support the technical bases

5. The extent that mixing and/or dilution will be required to successfully mitigate the five double-shell flammable gas-containing Watch List tanks (besides tank 241-SY-101) has not yet been determined

6. For the twenty organic/nitrate-containing tanks (other than tank 241-C-103), none are assumed to require pretreatment. This is largely based on the similarity of these wastes to the wastes in the ferrocyanide tanks 
7. The chemistry and thermal hydraulics of the waste contents in tank 241-C-106 are not understood, thus raising the uncertainty of work completion

8. The evaluation and definition effort conducted to-date for organic tanks has been limited

9. A certain level of moisture may need to be assured during storage, retrieval, and treatment of ferrocyanide-containing wastes to prevent the possibility of exothermic reactions; and

10. If aging of the waste is proven as the solution to organic- and ferrocyanide-containing tanks through the studies described in this plan, the uncertainty will be reduced.

\section{Programmatic Risks}

1. Operational records are incomplete, thus making data analysis and the removal of tanks from the Watch List, difficult

2. Chemical analysis of in-tank contents is lacking key information, thus making data interpretation difficult

3. The regulatory acceptability of mitigation strategies is not certain, especially if more stringent environmental and safety regulations are implemented in the future. Thus the impact of increased safety and hazardous waste requirements on the time and cost to mitigate the tanks is uncertain (e.g., disposal costs for pumps and equipment);

4. Although integration of field work activities is in progress, the availability of tank risers may impact installation of mitigating equipment and tank monitoring instrumentation

5. The methods required to mitigate or resolve waste tank safety issues have not yet been fully developed. For example, the proposed in tank dilution for tank 241-SY-101 may not be adequate, and additional DST space for the removal and treatment of waste from some tanks may be requife

6. The technical risks associated with tank 241-C-106 will challenge funding levels and milestone dates associated with the High Heat Program

7. The process and procedure for removing tanks from the Watch List has not been fully established

8. Unscheduled events may impact scheduled work

9. Availability of an adequate number of qualified resources in the time frame required

10. Depth of planning, including maturity of cost estimates

11. Perception of stakeholder and institutional oversight agencies regarding progress and the effective use of available resources

12. Funding stability 
13. Stability of technical and programmatic strategy.

\subsection{Major Decisions}

There is one specific major decision associated with the WTSP. This decision is, "Determine Functional Responsibility for Miscellaneous Underground Storage Tanks."

There are approximately 50 miscellaneous underground storage tanks located in the 200 Area of the Hanford Site which potentially contain waste similar to other TWRS tank waste. These miscellaneous underground storage tanks were previously used in association with processing of defense wastes. A decision is needed whether the remediation of the waste within these tanks is or is not within the WTSP scope. A Class 1 Change Request will document this decision.

\subsection{Assumptions}

The assumptions listed below are for the current planning cycle and are based on current knowledge. Assumptions will be revised as necessary and as new information becomes available for future planning cycles.

Key assumptions to the WTSP are as follows.

1. All the major waste tank safety issues have been identified, approved plans are in place for resolving these issues, and no new major safety issue and/or concerns will be identified that will require a diversion of resources.

2. Safety issues will be mitigated and/or resolved. Resolution of safety issues for some tanks may be delayed if out-of-tank treatment is required.

3. Activities to resolve waste tank safety issues shall be based on continued in-situ safe storage of the waste until the waste is retrieved for treatment and/or disposal.

4. Safe interim storage of waste shall support continued operations, waste retrieval, characterization, pretreatment, vitrification of high-level and low-level waste fractions, and other cleanup activities.

5. The program element funds developmental instrumentation, modular ventilation exhauster, mitigation upgrades, and operation/maintenance of installed mitigation equipment, as required, for all Watch List tanks.

6. SSTs and DSTs requiring characterization to address safety issues shall be identified in a timely manner. This program element will fund specific analyses to interpret the characterization data as the data relate to resolution of the issues.

7. All Watch List tanks will be sampled to categorize waste tanks as a Watch List tank or as a safe tank.

8. In general, no new breakthroughs in technology are considered to be a prerequisite to the safety issues resolution, however, lab research may be necessary to resolve the issues. 
9. Technology applications shall be identified, and research, development, demonstration, test and evaluation funds shall be available as needs are identified.

10. There shall be no major discoveries related to structural integrity of waste tanks that require an accelerated approach to close a safety issue.

11. Priorities shall be established consistent with the legal requirements of protecting human health, safety and the environment.

12. The high heat safety issue of tank $241-\mathrm{C}-106$ will be resolved by transferring the waste to a double-shell tank that has better leak resistance and heat dissipation capacity.

13. The Retrieval and Disposal Programs shall fund development of the technical basis necessary to support the safety considerations of the waste under conditions other than storage as it relates to these safety issues.

Specific assumptions by Activity are as follows.

\section{Technical Integration and Planning (1.1.1.2.02.01)}

1. Tri-Party Agreement Safety Issue Overview Milestone M-40-00, "Mitigate/Resolve Tank Safety Issues for High Priority Watch List Tanks, 9/2001," assumes that mitigation activities, if applicable, will be implemented in all Watch List tanks to ensure safe storage of waste during the interim period until the safety issue can be resolved (e.g., retrieval for treatment and/or disposal operations begin).

2. All tank waste retrieval costs, including understanding the safety impacts of changing the waste conditions, will be paid by the Waste Retrieval Program.

3. More Watch List tanks may be identified as a result of characterization and historical review. These potential increased costs have not been factored into the current planning cycle.

\section{Hydrogen Mitigation (1.1.1.2.02.02)}

1. All six DSTs on the Watch List (241-SY-101, 241-SY-103, 241-AW-101, 241-AN-103, 241-AN-104, and 241-AN-105) will have mixer pumps installed to mitigate the waste hazard until the waste has been retrieved.

2. The retrieval operation of the six DSTs will be initiated in the 1999 time frame.

\section{Ferrocyanide Safety Program (1.1.1.2.02.03)}

1. Four ferrocyanide tanks (241-BX-110, 241-BX-111, 241-BY-101, and 241-T-101) were removed from the ferrocyanide Watch List in July 1993, and the USQ for these tanks was closed in March 1994. It is assumed that two other tanks (241-BX-102 and 241-BX-106) will be removed from the ferrocyanide Watch List by FY 1995.

2. The remaining 18 ferrocyanide tanks on the ferrocyanide Watch List will not require retrieval or pretreatment of the waste to enable the ferrocyanide safety issue to be resolved. 
3. If needed to resolve the safety issue for any of the ferrocyanide tanks, moisture monitoring and control will be installed.

\section{Flammable Gas Program (1.1.1.2.02.04)}

1. All six flammable gas DSTs on the Watch List (241-SY-101, 241-SY-103, 241-AW-101, 241-AN-103, 241-AN-104, and 241-AN-105) will be retrieved to enable the safety issue to be resolved.

2. No single-shell flammable gas tank will be retrieved to enable the flammable gas safety issue to be resolved for those tanks.

\section{Organic Safety Program (1.1.1.2.02.05)}

1. Nineteen organic-containing tanks (241-C-103 not included) will not require retrieval or pretreatment to enable resolution of the organic safety issue.

2. Moisture monitoring and control will be installed in organic tanks requiring mitigating actions.

3. The organic liquid layer floating on top of the waste in tank 241-C-103 may be pumped out of the tank to resolve the flammability issue related to that layer as well as potentially the noxious vapor safety issue.

4. Disposal costs for the organic liquid will be funded by the Tank Farm Operations and Maintenance Program.

\section{High-Heat Safety (1.1.1.2.02.06)}

1. The single-shell high-heat tank 241-C-106 will be retrieved to resolve the high-heat tank safety issue.

\section{Miscellaneous Underground Storage Tanks (1.1.1.2.02.07)}

1. There are only 50 miscellaneous underground storage tanks.

2. A Class 1 Change Request will be approved by RL to provide funding to the WTSP to identify and evaluate the priority of safety issues associated with the miscellaneous underground storage tanks.

\section{Vapor Issue Resolution (1.1.1.2.02.08)}

1. Tank vapor sampling in support of the Noxious Odor Advisory System (NOAS) will be funded by the Tank Farm Operations and Maintenance Program.

2. Sampling and analysis of tank vapors will be funded by the Characterization Program. 


\section{Nuclear Criticality (1.1.1.2.02.09)}

1. Passive and active neutron detection equipment will be installed on selected tanks to better understand the quantity and distribution of fissile material in order to resolve the criticality safety issue.

2. Additional analytical data obtained through the characterization program will be evaluated to assure continued subcriticality and refine criticality safety analyses.

\section{Other Safety Issues (1.1.1.2.02.10)}

Currently not active.

\section{Hydroxide Control (1.1.1.2.02.11)}

1. Tanks $241-\mathrm{AN}-107,241-\mathrm{AZ}-102$, and $241-\mathrm{AN}-102$ are the only tanks requiring hydroxide controls.

\subsection{Constraints}

The primary constraint within the WTSP is the evaluation and definition of safety issues. This type of effort leaves one to establish assumptions within an ever changing environment, thus leaving many challenges for work plan implementation.

\subsubsection{Structure}

The WTSP work breakdown structure (WBS), responsibility assignment matrix (RAM), and the organization and responsibility are provided in this section.

\subsection{Work Breakdown Structure}

Refer to Figure 7.4-2 for the WTSP Work Breakdown Structure.

\subsection{Organization and Responsibility}

The WTSP is responsible for providing TWRS with leadership and management direction in order to ensure the successful resolution of waste tank safety issues. This role requires work by functional work groups and work by interfacing work groups.

Refer to Figure 7.4-1 for Organization Charts of the WTSP, TWRS Program Office, and TWRS. Refer to Figure 7.4-3 for the FY 1995 WTSP Responsibility Assignment Matrix.

\section{Functional Organizations of Responsibility}

The functional work groups follow.

1. Technical Integration and Planning. Provides a leadership role in the development and implementation of work plans, budgets, and reviews. 
2. Hydrogen Mitigation. Provides a leadership role in the development and implementation of work plans to mitigate hazards associated with flammable gas tanks.

3. Waste Tank Ferrocyanide Safety Program. Provides a leadership role in the development and implementation of work plans to mitigate and/or resolve the safety issue associated with tanks containing ferrocyanide waste.

4. Waste Tank Flammable Gas Safety Program. Provides a leadership role in the development and implementation of work plans to mitigate and/or resolve the safety issue associated with tanks containing flammable gases.

5. Waste Tank Organic Safety Program. Provides a leadership role in the development and implementation of work plans to mitigate and/or resolve the safety issue associated with tanks containing organic.

6. Waste Tank High-Heat Safety Program. Provides a leadership role in the development and implementation of work plans to mitigate and/or resolve the high heat safety issue associated with tank 241-C-106.

7. Miscellaneous Underground Storage Tanks. Provides a leadership role in the development and implementation of work plans to mitigate and/or resolve the concerns associated with 50 miscellaneous underground storage tanks.

8. Waste Tank Vapor Resolution. Provides a leadership role in the development and implementation of work plans to mitigate and/or resolve the tank vapor issues.

9. Waste Tank Nuclear Criticality. Provides a leadership role in the development and implementation of work plans to mitigate and/or resolve concerns associated with a possible in-tank criticality.

10. Other Waste Tank Safety Issues. Currently inactive and provides no function.

11. Waste Tank Hydroxide Control. Provides a leadership role in the development and implementation of work plans to mitigate and/or resolve concerns associated with insufficient hydroxide concentration in several tanks.

12. Safety Initiatives Management. Provides a leadership role in the development and implementation of work plans to complete TWRS safety initiatives on or ahead of schedule.

\section{Interfacing Organizations of Responsibility}

The work groups that interface with the WTSP follow.

1. Tank Farm Operations and Maintenance provides field work support, operation of equipment, monitoring of waste tank parameters, and required surveillance checks.

2. Characterization provides actual waste sample material for process development, waste characterization data from ongoing tank sampling, and characterization information from historical data review. 
3. Tank Farm Upgrades maintains the infrastructure (pipe lines, diversion boxes, pump pits, Project 236-A, New Tank Farm, etc.) needed to support in-tank processing and operation.

4. Waste Retrieval provides the retrieval of tank waste.

5. Low-Level Waste and High-Level Waste provide disposal of low-level and high-level waste.

6. Pretreatment provides the pretreatment of tank waste for subsequent storage and/or disposal.

7. Program Management and Administration provides overall integration of the TWRS program elements in preparing Multi-Year Program Plans, Multi-Year Work Plans, budget submittal, monthly reviews, and quarterly reviews.

8. Engineering and Projects provide project management for capital construction work efforts, manages the onsite construction forces, procurement, construction contractors for expense and capital construction activities, safety analysis and regulations, and NEPA services to support all WTSP work.

9. WHC's Process Engineering and Systems Engineering provides for engineering support for trade studies, systems definition, functions and requirements (F\&Rs), engineering evaluations, testing, and demonstrations.

10. The Washington State Department of Ecology (Ecology), the U.S. Environmental Protection Agency (EPA), stakeholder, advisory panels, and the Department of Energy (DOE) provide recommendations, comments and inputs to the WTSP as to work scope, priorities, and strategies.

11. The U.S. Department of Energy provides overall programmatic guidance, and approves decision making documents and recommendations prepared by WHC.

12. Los Alamos National Laboratory, Oak Ridge National Laboratory, and Pacific National Laboratory provide scientific analyses and studies, provides recommendations to WHC, and equipment development tasks for the WTSP.

\subsubsection{Work to be Performed in FY 1995}

Refer to Figure 7.4-4 for this information.

\subsubsection{Work to be Performed in FY 1996 - FY 1997}

Refer to Figure 7.4-4 for this information.

\subsubsection{Approach}

This section provides a summary of the technical approach, method of performance and acquisition strategy for achieving the WTSP objectives. Safety issue resolutions will be completed in a priority order. Tanks with the higher risk will be evaluated and mitigated and/or resolved first. 
Approach to work is completed in logic blocks called functions. A function is a simple statement that defines what type of work must done in a specific logic path. Functions form the basis for identifying work to be accomplished within the Waste Tank Safety Program.

Refer to Figure 7.4-5 for a block logic diagram. The work requirements to resolve the safety issue are planned, scheduled, and implemented by the following major functions:

1. Evaluate and Define Safety Issue. This function establishes the work to be done based on historical data, sampling and analysis, simulant waste studies, laboratory tests, monitoring data, mathematical modeling, and sensitivity analysis. It defines safe conditions for operation and decides on the appropriate path to resolve the safety issues. In addition, it includes the choice of the "no-action" option if it is determined no safety issue exists for a single waste type or a group of similar wastes or tanks.

2. Close Unreviewed Safety Question (USQ). This function establishes the technical bases for closure of the USQs. The safety envelope will be redefined, operating procedures modified, control limits established, and documentation completed for recommendation of USQ closure.

3. Mitigate Condition. This function develops corrective actions for safety issues that address possible treatment to reduce the severity of the safety issues. Work includes development, design, fabrication, installation, testing, and demonstration of the equipment for moisture control, mixing wastes, heating and diluting wastes, and vapor treatment.

4. Store and Monitor. This function provides monitoring systems to ensure safe storage until the safety issue is resolved. Work includes developing, procuring, and installing additional equipment to continuously monitor tank parameters for temperature, pressure, gas concentration, neutron flux, and surface level.

5. Resolve Safety Issue. This function implements corrective actions to eliminate the hazard and resolve the safety issue.

Each of these major functions have supporting functions of some combination of monitoring, mathematical analyses, laboratory studies, and in-tank testing.

\subsubsection{Drivers and Deliverables}

This section provides the drivers and deliverables.

\section{Drivers:}

- Hanford Federal Facility Agreement and Consent Order, Tri-Party Agreement Fourth Amendment, Washington State Department of Ecology, U.S. Environmental Protection Agency, and U.S. Department of Energy, Olympia, Washington, January, 1994, Milestones M-40-00, "Mitigate/Resolve Tank Safety Issues for High Priority Watch List Tanks by 2001"

- Tank Waste Remediation System Functions and Requirements, DOE/RL-92-60, Rev 1, March 1994. 
2. Deliverables:

- Develop plans to close USQs/resolve safety issues

- $\quad$ Upgrade monitoring equipment

- Temperature monitoring

- Gas monitoring

- Moisture monitoring

- Liquid level and/or surface level monitoring

- Neutron monitoring

- Install mitigation pumps

- $\quad$ Close Unreviewed Safety Questions

- Ferrocyanide

- Criticality

- Organic Layer/Flammability

- Flammable Gas

- $\quad$ Resolve safety issues.

For specific deliverables, refer to the respective WBS Dictionary Sheets (Appendix A) and Milestone Description Sheets (Appendix D).

\subsubsection{Schedule}

This section provides the program element schedule; FY 1995 schedule and logic; and FY 1996 1997 schedule and logic.

\subsubsection{Waste Tank Safety Summary Schedule}

Refer to Figure 7.4-6.

\subsubsection{Waste Tank Safety Expanded Baseline (FY 1995 - FY 1997)}

Refer to Appendix C for this information.

\subsubsection{Resource Requirements}

The WTSP cost, staffing, and FY 1994 work scope carryover are provided in this section.

\subsubsection{Cost}

The cost base for the WTSP is based on the past four year history of costs required to effectively manage this program. Empirical cost data exists for field work support, technical studies, and construction activities. In addition, construction estimates exist for major capital activities. All cost 
estimates have been validated by the performing organization and by the respective management function responsible for the work activity.

\subsection{Basis of Cost Estimates}

The basis of cost estimates for WTSP is based on the past four year history of costs for resource quantity and type. The resource usage profile is based on an estimate of the apportionment of these resources to the work scope within the cost accounts based on FY 1994 resource usage and WTSP Program Office changes planned for in FY 1995 - FY 1997.

\subsection{Total Program Element Cost by Year by Fund Type by Activity and Cost Account)}

Table 7.4-1 establishes the baseline cost for this program element. The baseline cost is exhibited in annual costs for FY 1994 through FY 2000, and 5-year totals for FY 2001 through program element completion. Where the data are available, these baseline costs are exhibited at the cost account level with fund type totals provided. Cost data are then rolled up to the activity and program element levels. At present, the program element cost baseline is the program element cost totals exhibited on this table less a productivity challenge for FY 1995 through FY 1997. Also, refer to Figure 7.4-7 for this information.

\subsection{FY 1995 Cost by Month by Fund Type by Activity by Cost Account}

Future MYWP updates will include Table 7.4-2, which establishes the month-by-month cost baseline for FY 1995 for this program element. The FY 1995 cost baseline is exhibited at the cost account level with fund type totals provided. Cost data are then rolled up to the activity and program element levels. Also exhibited is the total cost baseline for the program element for FY 1995. At present, the program element cost baseline for FY 1995 for this program element is the total provided on this table less a productivity challenge for FY 1995. Also refer to Figure 7.4-7 for this information.

\subsubsection{Staffing}

The staffing base for the WTSP is based on the past four year history of manpower required to effectively manage this program. Empirical staffing data exists for field work support, technical studies, and construction activities. In addition, construction estimates exist for major capital activities. All staffing estimates have been validated by the performing organization and by the respective management function responsible for the work activity.

\subsection{Basis of Staffing.Projection}

The basis of staffing estimates for WTSP is based on the past four year history of manpower quantity and type. The resource usage profile is based on an estimate of the apportionment of these resources to the work scope within the cost accounts based on FY 1994 resource usage and WTSP Program office changes planned for in FY 1995 - FY 1997. 
WHC-SP-1101

\subsection{Total Full-Time Equivalents by Year by Activity by Major Participant (Direct and Indirect)}

Future MYWP updates will include Table 7.2-3, which establishes total staffing projections for this program element. Included in the table are FY 1994 staffing data as well as projections by year for FY 1995 through FY 2000. The projections in this table include all direct and indirect Full-Time Equivalents (FTEs), including all subcontractor personnel. Where data are available, projections are exhibited at the activity level with major program participant totals provided. Also, refer to Figure 7.4-8 for this information.

\subsection{FY 1995 through FY 1997 Direct Full-Time Equivalents by 88 Labor Categories}

Future MYWP updates will include Table 7.2-4, which establishes direct WHC staffing projections for this program element. Projections do not include subcontractor support and are provided by 88 labor categories for FY 1995 through FY 1997. Also, refer to Figure 7.4-8 for this information.

\subsubsection{Work Scope Carryover}

Completion of several pieces of work in FY 1994 were impacted due to tank riser availability issues, and the 325 laboratory shutdown event. The work scope carryover into FY 1995 is as follows: 1) Installation of thermocouple trees in ferrocyanide tanks; 2) Installation of thermocouple trees in organic tanks; 3) Field work in support of hydroxide control of tank 241-AN-107; 4) Laboratory studies of ferrocyanide waste samples; 5) Core sample data interpretation of samples from ferrocyanide tanks; and 6) Installation of ventilation and monitoring equipment in flammable gas tanks.

\subsubsection{Waste Type Data}

Small quantities of tank waste will be supplied to laboratories for process development testing, waste samples, and decontaminating solutions. The waste will be returned to the underground waste tanks at the completion of work activities where it will be stored until it is retrieved.

In addition, equipment will be removed from tank waste and decontaminated. Decontamination procedures will remove sufficient contaminated material to permit disposal.

Refer to Appendix $\mathrm{H}$ for this information.

\subsubsection{Revisions}

In future updates of the MYWP, this section will include a summary of the revision that has occurred in this program element since the last issue of the MYWP. 


\section{WHC-SP-1101 \\ Tank Waste Remediation System}

Figure 7.4-1. Organization Chart.
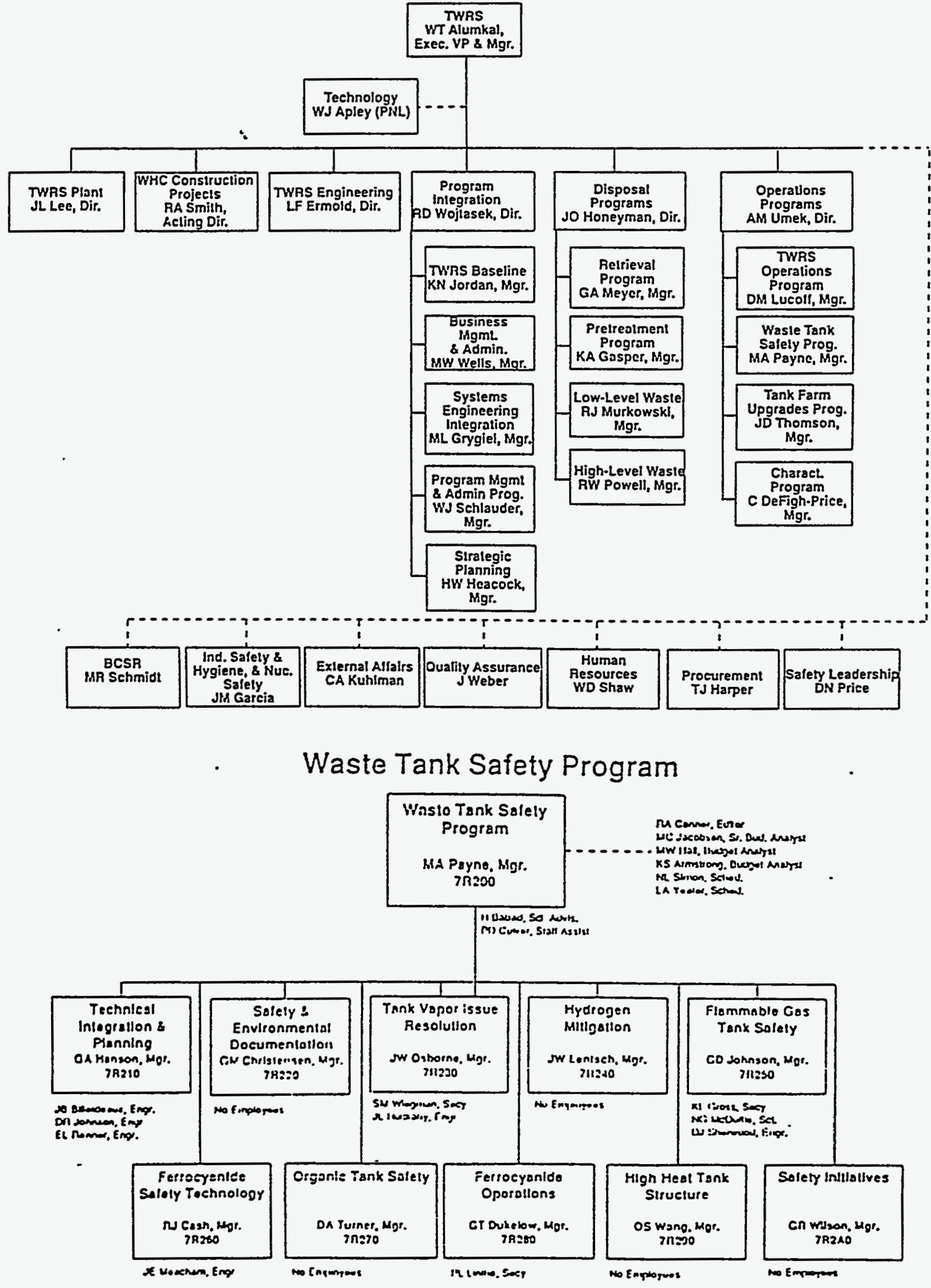

F7.4-1 

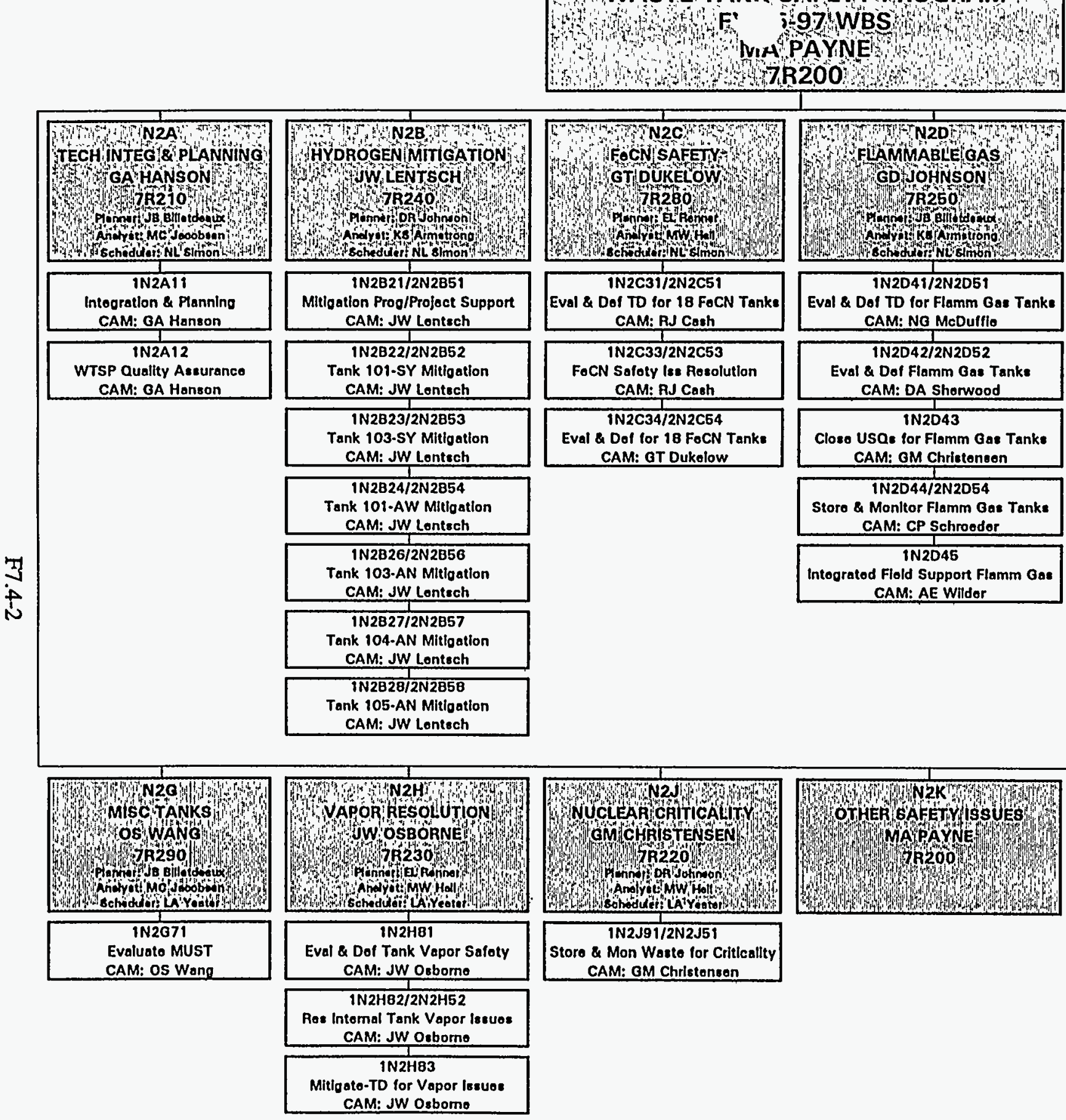

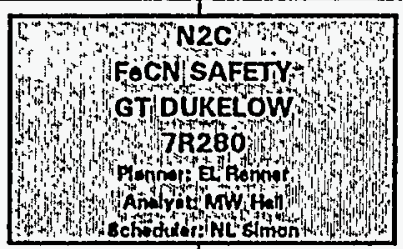

1N2C31/2N2C51

Eval \& Dof TD for 18 FoCN Tanke CAM: RJ Cash

1N2C33/2N2C53

FoCN Saloty les Resolution CAM: RJ Gash

\section{IN2C34/2N2C54}

Eval \& Dof for 18 FoCN Tank CAM: GT Dukelow
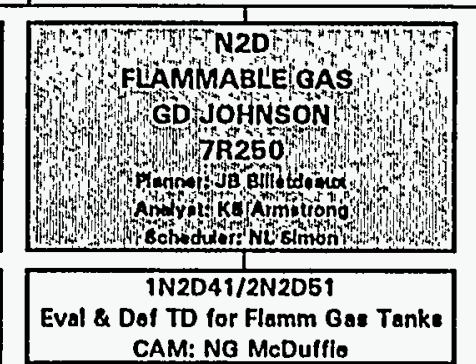
CAM: NG MaDulflo

\section{N2D42/2N2D52
Eval \& Dol Fiamm Gas Tanks} CAM: DA Shorwood

IN2D43
Closo USQE for Flamm Gas Tanks
CAM: GM Chrietoneon

CAM: OM Christoneon

1N2D44/2N2D54
Storo \& Monltor Flamm Gas Tanke CAM: CP Schroedor

\section{Intogratod Fiold Support Flamm Gos} CAM: AE Wildar
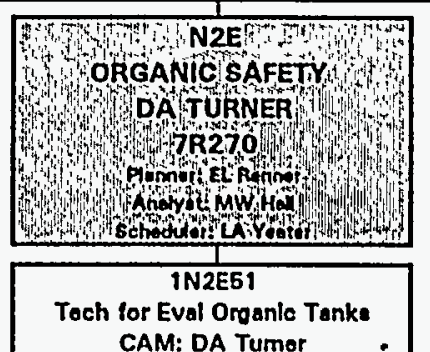
CAM: DA Tumer

\section{IN2E62}

Eval \& Dof Organle Tank
CAM: DA Tumer IN2E54/2N2E5D

In.Tank Ros of Organic Tanks CAM: GT Dukolow

\section{N2E65 \\ Res of USO for Oroanic Tank} CAM: DA Tumor

\section{Ros Saloty fesuo Organic Tank} CAM: DA Tumor

IN2E57/2N2E5G

Pump \& Dlup C-103 Organle Layor CAM: GT Dukolow

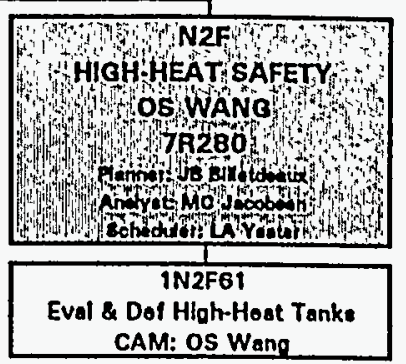

彳亍

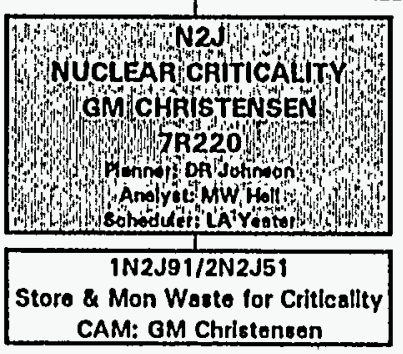

CAM: OM Christonsen
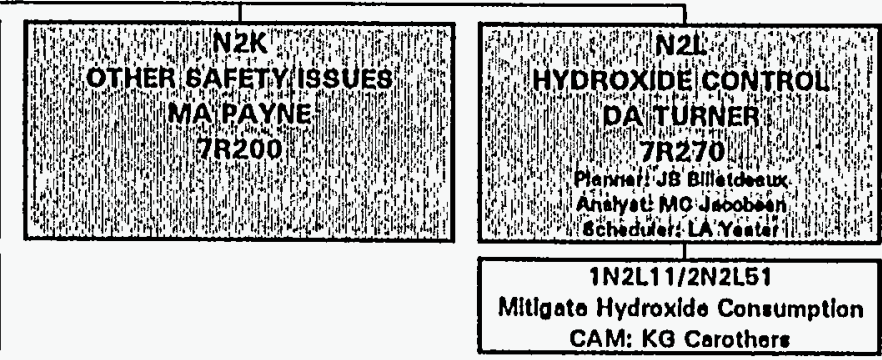

M: JW Osbome 
Figure 7.4-3. Waste Tank Safety Responsibility Assignment Matrix.

MBS DESCRIPT HHC RESP RL RESP

1.1.1.2.02........MASTE TANK SAFETY............ PAYME.......R. E. GERTON 1.1.1.2.02.01..... KT TECH INTEG \& PLANHING...6.A. HAHSOH....... F. CHRISTENSEN 1.1.1.2.02.01.01.. IHTEGRATIOH \& PLAHHIHG.....6.A. HAKSON....... 1.1.1.2.02.01.02.. . ST SAFETY QA................ HAKSOH....... 1.1.1.2.02.02..... HT HYDROGEH MITIGATION.....J.H. LENTSCH...... 1.1.1.2.02.02.01.. MITGATION PROG/PROJ MGH'T..J.H. LEKTSCH...... 1.1.1.2.02.02.02..101-SY MITIGATIOH............. LENTSCH...... 1.1.1.2.02.02.03..103-SY MITIGATIOH............. LENTSCH...... 1.1.1.2.02.02.04..101-AK MITIGATIOH............. LEHTSCH...... 1.1.1.2.02.02.05..104-AH MITIGATION............. LENTSCH...... 1.1.1.2.02.02.06..103-AN MITIGATION............. LENTSCH...... 1.1.1.2.02.02.07..105-AH MITIGATIOH............. LENTSCH...... 1.1.1.2.02.03...... FT FERROCYAHIDE SAF. PROG..6.T. DUKELOH...... H.F. HENDRICKSOH 1.1.1.2.02.03.01..EVAL \& DEFIHE TECH DEV.....R.J. CASH........ 1.1.1.2.02.03.02.. NUMBER PREVIOUSLY USED ................. 1.1.1.2.02.03.03. FERROCY SAFETY ISSUE RESO..R.J. CASH........ 1.1.1.2.02.03.04..EVAL \& DEFINE FOR 18 FeCH..G.T. DUKELOH...... 1.1.1.2.02.04.....UT FLAM. GAS SAFE.PROG........ JOHMSOH...... GRAY/ROSENWALD 1.1.1.2.02.04.01..EVAL \& DEFIME TECH DEV......... MCDUFFIE..... 1.1.1.2.02.04.02..EYAL \& DEFIKE FLAM GAS TK..D.J. SHERHOOD..... 1.1.1.2.02.04.03..CLOSE USQS FOR FLAH GAS T..G.M. CHRISTENSEN. 1.1.1.2.02.04.04..STORE \& MOHITOR F.GAS TKS..C.P. SCHROEDER.... 1.1.1.2.02.05...... ORT ORGANIC SAFE. PROG.......... TURNER....... S.0. BRANCH 1.1.1.2.02.05.01.. TECH FOR EVAL ORGAMIC TKS..D.A. TURNER...... 1.1.1.2.02.05.02..EVAL \& DEFIHE ORGAHIC TKS..D.A. TURHER....... 1.1.1.2.02.05.03..PUMP \& DISP C-103 ORG LAY..G.T. DUKELOH...... 1.1.1.2.02.05.04..IH-TK RES OF ORG.H/O TH'T..G.T. DUKELOH...... 1.1.1.2.02.05.05..RESOL.OF USQ FRO ORG.TKS...D.A. TURNER....... 1.1.1.2.02.05.06. .RESOLVE SAFETY ISSUES 0.T..D.A. TURNER....... 1.1.1.2.02.06.....WT HIGH-HEAT SAFE. PROG....0.5. HANG............. HARHOOD 1.1.1.2.02.06.01.. EVAL \& DEFIHE HI HEAT TKS..0.5. HANG........ 1.1.1.2.02.07 ..... MISC. UWDERGROUND STOR.TKS..0.5. HANG........ 1.1.1.2.02.07.01..EVAL.MISC.UNDERGR. TANKS...0.5. HANG........ 1.1.1.2.02.08..... HT VAPOR RESOLUTION........... OSBORNE...... S.0. BRANCH 1.1.1.2.02.08.01.. EVAL \& DEFIHE FOR TK VAP...J.H. OSBORNE...... 1.1.1.2.02.08.02..RES.INTER. TANK VAP.1SSUES..J. . . OSBORNE...... 1.1.1.2.02.08.03.. MITIGATE TECH DEV FOR VAP..J.H. OSBORNE...... 1.1.1.2.02.09..... KT NUCLEAR CRITICALITY...... G.M. CHRISTEHSEH.. K. COADY 1.1.1.2.02.09.01..STORE \& MON. HASTE FOR CR..6.M CHRISTENSEH... 1.1.1.2.02.10..... OTHER HST TK SAFE. ISSUES.. H.A. PAYHE........ 1.1.1.2.02.11......KST TK HYOROXIDE CONTROL...D.A. TURHER....... 1.1.1.2.02.11.01.. MITIGATE HYOROX. CONSUMP...K.G. CAROTHERS.... 
Waste Tank Safety Program

Fiscal Year 1995 Planning

(Program \$000 - Escalated)

A. TECHNICAL INTEGRATION AND PLANNING (Hanson)

1. Integration and Planning (Hanson)

a. Baseline Planning (Hanson)
(1) WTSP PTanning (Hanson)
(2) PRA (PNL/Fassbender)
(3) Financial Analysts (Lenseigne)
(4) Program Schedulers (Black)
(5) Program BOA (Hanson)

7
$i$
$i$
5

b. Management and Integration (Payne)
(1) WTSP Management (Payne)
(2) TPA/SI Management (Wilson)
(3) PNL Program Management (PNL/Bean)
(4) Technical Editor Support (BCSR/Conner)
(5) Secretarial Support (Payne)
(6) Temporary Support (Payne)
c. Advisory Support (Payne)
(1) External Advisory Committee (Wodrich)
(2) Environmental Interface (Landon)

Sub-Total

\begin{tabular}{lll} 
FY95 & FY96 & FY97 \\
EXP/CAP & EXP/CAP & EXP/CAP \\
\hline
\end{tabular}

Sub-Total

$2412 / 0$

$359 / 0$
$518 / 0$

$241 / 0$

$701 / 0$

$493 / 0$

$100 / 0$

$370 / 0$

$532 / 0$

$249 / 0$

(3) TWRS Quality Oversight (Hogan)

(6) Fab Shop QC (Scott)

$722 / 0$

$508 / 0$

$103 / 0$

$381 / 0$

$549 / 0$

$256 / 0$

$744 / 0$

$523 / 0$

$106 / 0$

$2484 / 0$

$2559 / 0$

ACTIVITY TOTAL

$7719 / 500 \quad 7150 / 515$

$8426 / 531$ 
Waste Tank Safety Program

Fiscal Year 1995 Planning

(Program \$000 - Escalated)

\section{B. WASTE TANK HYDROGEN MITIGATION}

1. Mitigation Program/Project Support

a. Program Management

b. Project Management

c. PNL Engineering Services (PNL)

(1) PNL Dilution Support

d. LANL Engineering Services (LANL)

PNL Total
(1) DACS 2A SY Farm
(2) DACS 2B 101-AW
(3) DACS 2C AN Farm
(4) Gas Monitoring
(5) SA/TECH SPT 101-SY
(6) SA/TACH SPT 103-SY
a. Dilution Support
(7) SA/TACH SPT 101-AW
(8) SA/TACH SPT 104-AN
(9) SA/TACH SPT 105-AN
(10) SA/TACH SPT 103-AN

(LANL Total)

Sub-Total

\section{101-SY Mitigation}
a. Mixer Pump Operations
b. DACS Maintenance \& Operation
c. Gas Monitoring Maintenance \& Operation
d. Instrumentation
(1) Void/Viscometer Testing
(2) Transport container
e. Test Pump Removal Prep

Sub-Total

$\begin{array}{lll}\text { FY95 } & \text { FY96 } & \text { FY97 } \\ \text { EXP/CAP } & \text { EXP/CAP } & \text { EXP/CAP }\end{array}$

$\begin{array}{ccc}843 / 0 & 571 / 0 & 882 / 0 \\ 918 / 0 & 737 / 0 & 974 / 0 \\ 2017 / 260 & 1890 / 57 & 2777 / 64 \\ 591 / 0 & 309 / 0 & 371 / 0 \\ 2608 / 260 & 2199 / 57 & 3148 / 64\end{array}$

$\begin{array}{ccl}108 / 4165 & 515 / 1002 & 265 / 750 \\ 0 / 0 & 0 / 1357 & 381 / 181 \\ 0 / 0 & / 0 & 616 / 597 \\ 400 / 0 & 309 / 0 & 318 / 0 \\ 518 / 0 & 309 / 0 & 309 / 0 \\ 2200 / 0 & / 0 & 531 / 0 \\ 440 / 0 & 284 / 0 & 0 / 0 \\ 0 / 0 & 1231 / 0 & 154 / 0 \\ 0 / 0 & 0 / 0 & 564 / 0 \\ 0 / 0 & 515 / 0 & 488 / 0 \\ 0 / 0 & 0 / 0 & 0 / 0 \\ 3666 / 4165 & 3163 / 2359 & 3626 / 1528 \\ & & \\ 8035 / 4425 & 6670 / 2416 & 8630 / 1592\end{array}$

$656 / 0$

$885 / 150$

$962 / 100$

$694 / 103 \quad 1020 / 106$

$517 / 0$

$25 / 172$

$218 / 0$

$3263 / 422$

$694 / 103$

$1020 / 106$ 


\section{Waste Tank Safety Program \\ Fiscal Year 1995 Planning \\ (Program \$000 - Escalated)}

103-sY Mitigation
a. Design Pump
b. Procure and Fab Pump
c. Install Pump
d. DACS 2A System
e. DACS Maintenance \& Ops
f. Gas Characterization System (GCS)
g. Instrumentation (Void/Viscometer)
h. Mixer Pump Operations
i. Removal/Disposal System
j. In-Tank Dilution Test
(1) Sampling
(2) Material

Sub-Total

\begin{tabular}{lll} 
FY95 & FY96 & FY97 \\
EXP/CAP & EXP/CAP & EXP/CAP \\
\hline
\end{tabular}

$\begin{array}{ccc}/ 3480 & 0 / 1092 & \\ 0 / 2222 & 249 / 55 & \\ 0 / 169 & 261 / 682 & \\ 313 / 1713 & 344 / 458 & \\ 0 / 0 & 440 / 103 & 666 / 0 \\ / 1035 & 96 / 82 & 170 / 0 \\ 516 / 0 & 535 / 0 & \\ 0 / 0 & 282 / 0 & \\ 301 / 721 & & \\ 1277 / 0 & 1099 / 0 & 85 / 0 \\ 586 / 0 & 0 / 0 & 0 / 0 \\ 2993 / 9340 & 3306 / 2472 & 921 / 0\end{array}$

4. 101-AW Mitigation
a. Design Pump
b. Procure and Fab Pump
c. Install Pump
d. DACS 2B System
e. DACS 2B Maintenance \& Ops
f. Gas Characterization System (GCS)
g. Instrumentation
(1) Void/Viscometer
h. Mixer Pump Operations
j. Removal/Disposal System
(1) Removal
(2) Fences \& Roads

\begin{tabular}{ccc}
$0 / 0$ & $0 / 2453$ & \multicolumn{1}{c}{$0 / 0$} \\
$0 / 0$ & $0 / 1221$ & \\
& $0 / 0$ & $995 / 915$ \\
$0 /$ & $0 / 2130$ & $691 / 0$ \\
& $0 / 0$ & $618 / 78$ \\
$00 / 338$ & $0 / 301$ & \\
$519 / 0$ & $535 / 0$ & \\
& $67 / 0$ & $173 / 0$ \\
$0 / 0$ & $0 / 0$ & \\
& & $210 / 675$ \\
$519 / 338$ & $535 / 6105$ & $2687 / 1668$
\end{tabular}

Sub-Total

$519 / 338$ 
Waste Tank Safety Program

Fiscal Year 1995 Planning

(Program \$000 - Escalated)

7
7
-1
1
-5
-1

FY95

EXP/CAP

FY96

FY97

5. 104-AN Mitigation

a. Design Pump

b. Procure and Fab Pump

c. Install Pump

d. DACS 2C System

e. DACS 2C Maintenance \& Ops

f. Gas Characterization System (GCS)

g. Instrumentation (Void/Viscometer)

h. Mixer Pump Operations

i. Removal/Disposal System
(1) Removal
(2) Fences \& Roads

$\begin{array}{ccc}0 / 0 & 0 / 657 & 0 / 3059 \\ & 0 / 1197 & 0 / 1126 \\ & 74 / 25 & 355 / 1056 \\ & 0 / 0 & 739 / 599 \\ 0 / 0 & & 396 / 129 \\ & & 0 / 502 \\ & 532 / 0 & 548 / 0 \\ & & 171 / 0 \\ & & \\ & & \\ & & \\ & & \end{array}$

Sub-Total

$0 / 0$

$606 / 1879 \quad 2209 / 6471$

6. 103-AN Mitigation

a. Design Pump

b. Procure and Fab Pump

c. Install Pump

d. Gas Characterization System (GSC)

e. Instrumentation (Void/Viscometer)

f. Mixer Pump Operations

g. Removal/Disposal System

(1) Removal

(2) Fences \& Roads

$0 / 0 \quad 0 / 0$

$0 / 0 \quad 0 / 0$

$\begin{array}{lll}0 / 0 & 0 / 0 & 0 / 0 \\ & 0 / 0 & 0 / 0\end{array}$

$548 / 0$

$0 / 0$

$0 / 0$

$191 / 0$

Sub-Total

$0 / 0$

$0 / 0 \quad 739 / 0$


Waste Tank Safety Program

Fiscal Year 1995 Planning

(Program \$000 - Escalated)

7. 105-AN Mitigation
a. Design Pump
b. Procure and Fab Pump
c. Install Pump
d. Gas Characterization System (GCS)
e. Instrumentation (Void/Viscometer)
f. Mixer Pump Operations
g. Removal/Disposal System
(1) Removal
(2) Fences \& Roads

Sub-Total

ACTIVITY TOTAL

$\begin{array}{lll}\text { FY95 } & \text { FY96 } & \text { FY97 } \\ \text { EXP/CAP } & \text { EXP/CAP } & \text { EXP/CAP }\end{array}$

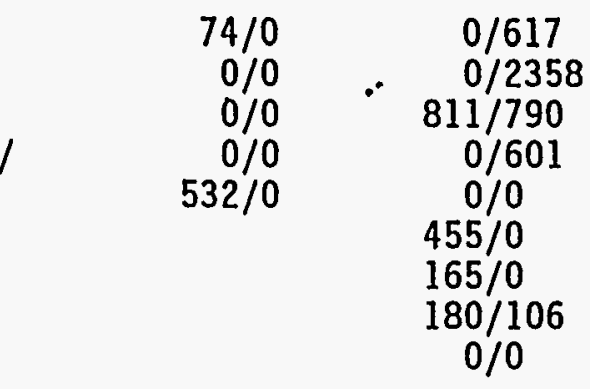

$0 / 0$

$606 /$

$1611 / 4472$ 
Waste Tank Safety Program

Fiscal Year 1995 Planning

(Program \$000 - Escalated)

$\begin{array}{lll}\text { FY95 } & \text { FY96 } & \text { FY97 } \\ \text { EXP/CAP } & \text { EXP/CAP } & \text { EXP/CAP }\end{array}$

C. WASTE TANK FERROCYANIDE SAFETY PROGRAM

1. Evaluate \& Define Technology Development for 18 FeCN Tanks (Cash)
a. FeCN Simulated Waste Studies (Fauske)
$377 / 0$
b. Mossbauer Spectrometer (Lockrem)
c. Project Management (PNL/Schiefelbein)
$48 / 22$
Aging Waste (PNL/Lilga)
$225 / 0$
e. Cesium Uptake (PNL/Burgeson)
$535 / 25$
Special Studies/Consultation (PNL/Schiefelbein)
$65 / 0$
Compare Real Waste w/Simulated Waste (PNL/Scheele) $160 / 30$
Infrared/ICP Speciation Transfer (PNL/Bryan)
Cesium Nickel Ferrocyanide Solubility (PNL/Rai)
$65 / 0$
Sludge Moisture Modeling (PNL/Simmons)
$65 / 0$
$135 / 0$
DNFSB/CAP Management (Cash)
$132 / 0$
In-Situ Relative Humidity Instrumentation (Schroeder) $116 / 29$
Infrared Speciation Transfer (Winkelman)
$192 / 53$
$\mathrm{n}$. Scanning Electron Microscope Investigation Support (Lockrem)365/0

Sub-Total

$2706 / 159$

$0 / 0$

$0 / 0$

2. Evaluate \& Define for 18 FeCN Tanks (Dukelow)

a. Engineering Document Control (SV Cook)

b. CAP Management FeCN Tanks (Dukelow)

c. Installation of TC Trees/Hookup TMACS (Dukelow)

d. Pressure Monitoring Design \& Installation (Blanchard)

e. Surface Moisture Measuring (CPAC) (Winkelman)

f. Neutron Probe Moisture Monitor LOW Engineering (Toffer)

g. Neutron Probe Moisture Monitoring LOW Install (Toffer)

h. Infrared Scan Device Modification \& Scans (Moore)

i. ISB Amendment (Grigsby)

j. Electromagnetic Induction Probe (Toffer)

$\begin{array}{cll}15 / 0 & 15 / 0 & 16 / 0 \\ 86 / 0 & 88 / 0 & 91 / 0 \\ 1013 / 184 & & \\ 446 / 1020 & 227 / 429 & 266 / 193 \\ 323 / 165 & 345 / 244 & 233 / 251 \\ 542 / 180 & 202 / 0 & 208 / 0 \\ 396 / 893 & 391 / 757 & 341 / 0 \\ & 130 / 231 & 148 / 109 \\ 42 / 0 & & \\ 336 / 283 & 119 / 547 & 196 / 566 \\ 3199 / 2725 & 1517 / 2208 & 1498 / 1120\end{array}$

Sub-Total

$3199 / 2725 \quad 1517 / 2208$ 
Waste Tank Safety Program

Fiscal Year 1995 Planning

(Program \$000 - Escalated)

3. Ferrocyanide Safety Issue Resolved (Cash)

a. CAP Management FeCN Tank Safety (Cash)

b. Update Safety Documentation (Grigsby)

c. Resolve SI for 4 C Farm Tanks (Grigsby)

d. Tech Support - SI Resolution (Hunter)

e. Resolve SI for 14 FeCN Tanks (Grigsby)

f. Core Sample/Data Interpretation (Haller)

g. Fauske Contract - SI Resolution (Cash)

Sub-Total

ACTIVITY TOTAL

\begin{tabular}{llll}
$\begin{array}{l}\text { FY95 } \\
\text { EXP/CAP }\end{array}$ & $\begin{array}{l}\text { FY96 } \\
\text { EXP/CAP }\end{array}$ & & $\begin{array}{l}\text { FY97 } \\
\text { EXP/CAP }\end{array}$ \\
\cline { 1 - 2 } & & & \\
$222 / 0$ & $269 / 0$ & & $277 / 0$ \\
$114 / 0$ & $113 / 0$ & & $117 / 0$ \\
$211 / 0$ & $51 / 0$ & & \\
$388 / 0$ & $398 / 0$ & $376 / 0$ \\
$660 / 0$ & $204 / 0$ & $210 / 0$ \\
& $643 / 0$ & $222 / 0$ \\
$1594 / 0$ & $259 / 0$ & $372 / 0$ \\
$7499 / 2884$ & $3452 / 2208$ & $3073 / 1120$
\end{tabular}

7
-3
-1
$\frac{1}{3}$ 


\section{WASTE TANK FLAMMABLE GAS SAFETY PROGRAM}

1. Eval \& Define Technology Dev for Flam Gas

a. Program Management

(1) Program/CAP Management (McDuffie)

(2) Project Management (PNL/Brothers)

b. Gas Retention Mechanisms

(1) Phase Equilibria Studies - Gases (PNL/Pederson/Liu)

(2) Gas Reaction Studies (PNL/Pederson)

(3) Gas Bubble Retention Studies (PNL/UT/Gauglitz)

c. Waste Behavior Modeling

(1) Waste Tank Physics Modeling (PNL/Trent)

(2) Thermal/Hydraulic Analysis (Ogden \& Contracts)

(3) Gas Release Mechanism/Waste Modeling (LANL)

d. Gas Generation Mechanisms

(1) 222-S Gas Generation Tests (Jewett)

(2) 222-S Analytical Support (Marshall)

(3) Gas Generation Studies (PNL/GIT/Pederson)

e. Gas Reaction Studies

(1) USBM Tests for Mixtures/Detonation Sensitv (LANL)

(2) Benchmark USBM Experiments/Modeling (LANL)

f. Organic Characterization

(1) Organic Analysis and Methods Dev (PNL/Campbel1)

FY95
EXP/CAP

EXP/CAP

FY96

EXP/CAP

FY97

EXP/CAP

Sub-Total

$\begin{array}{ccc}107 / 0 & 110 / 0 & 114 / 0 \\ 300 / 0 & 330 / 0 & 318 / 0 \\ & & \\ 215 / 60 & 196 / 26 & 202 / 0 \\ 216 / 25 & 196 / 0 & 95 / 0 \\ 368 / 20 & 232 / 0 & 95 / 0 \\ & & \\ 326 / 50 & 515 / 154 & 584 / 106 \\ 422 / 0 & 446 / 0 & 470 / 0 \\ 0 / 0 & 515 / 0 & \\ 234 / 0 & 0 / 0 & \\ 331 / 0 & 343 / 0 & 282 / 0 \\ 116 / 0 & 371 / 21 & \\ 502 / 75 & & \\ 368 / 0 & & \\ 0 / 0 & 436 / 0 & 106 / 0 \\ 238 / 0 & 232 / 0 & \\ 3743 / 230 & 3920 / 201 & 2267 / 106\end{array}$

2. Eval \& Define Flammable Gas Tanks

a. Vapor Space Monitoring (M-40-10)
(1) Program/CAP Management (Sherwood)
(2) Vapor Sampling (Zuroff)
(3) Vapor Sample Analysis W/Mass Spec (PNL/M.Goheen)
(4) Vapor Sample Data Analysis (Kirch)

$\begin{array}{lll}107 / 0 & 110 / 0 & 113 / 0 \\ 107 / 0 & 110 / 0 & 114 / 0 \\ 337 / 0 & 412 / 52 & 239 / 53 \\ 161 / 0 & 276 / 0 & 340 / 0\end{array}$


Waste Tank Safety Program

Fiscal Year 1995 Planning

(Program \$000 - Escalated) b. Retained Gas Evaluation

(1) Develop Retained Gas Sampler System (Bauer)

(2) RGS Sample Analys is (Jewett)

(3) 222-S Analytical Support (Marshall)

(4) RGS Data Analysis (Kirch)

(5) Retained Gas Sampler Safety Assessement (LANL)

(6) Retained Gas Sampler
c. Waste Behavior Evaluation
(1) Waste Behavior Data Evaluation (PNL/Schienbein)
(2) DQOs/Technical Support (Sherwood)
(3) Auger/Core Sample Data Analys is (Kirch)
(4) Radioactive Waste Sample Analysis (PNL/Tingey)
(5) Tank Behavior Interpretive Reports (Kirch)
(6) Data Anatysis (LANL)

Sub-Total

3. Close USQs for Flammable Gas Tanks

a. Close SY Farm USQ (SI 21)

(1) USQ Closure SY Farm (Fein)

USQ Closure

Hazard Evaluation

Safety Issue Resolution

LANL Oversight

(2) DST Bounding Analys is (LANL)

b. Close Remaining USQs (M-40-09)

(1) Program/CAP Management (Christensen)

(2) Safety Documentation (Fein)

USQ Closure

Hazard Evaluation

Safety Issue Resolution

LANL Oversight

$\begin{array}{lll}\text { FY95 } & \text { FY96 } & \text { FY97 } \\ \text { EXP/CAP } & \text { EXP/CAP } & \text { EXP/CAP }\end{array}$

$\begin{array}{cll}501 / 0 & 129 / 0 & \\ 208 / 0 & & \\ 15 / 0 & & 159 / 0 \\ 0 / 0 & 134 / 0 & \\ 132 / 0 & & \\ 101 / 20 & & \\ 188 / 0 & 464 / 52 & 690 / 212 \\ 25 / 0 & & 182 / 0 \\ 140 / 0 & 170 / 0 & 212 / 106 \\ 0 / 0 & 293 / 128 & 200 / 0 \\ 130 / 28 & 200 / 0 & \\ 264 / 0 & & \\ & & \\ 2416 / 48 & 2298 / 232 & 2249 / 371\end{array}$

$187 / 0$

$250 / 0$

$\begin{array}{rlr}0 / 0 & 110 / 0 & 114 / 0 \\ 187 / 0 & 650 / 0 & 547 / 0\end{array}$


(3) Environmental Support/EA (Engelmann)

(4) SST Bounding Analysis (LANL)

Sub-Total

\begin{tabular}{lcccc} 
FY95 & & FY96 & & FY97 \\
EXP/CAP & & EXP/CAP & & EXP/CAP \\
\cline { 1 - 2 } $0 / 0$ & & $15 / 0$ & & $16 / 0$ \\
$250 / 0$ & $824 / 0$ & $637 / 0$ \\
$874 / 0$ & $1599 / 0$ & $1314 / 0$
\end{tabular}

$102 / 0$

$46 / 0$

$176 / 198$

$236 / 647$

$140 / 0$

d. Program Management

(1) Program/CAP Management (Johnson)

(2) Engineering Management Support (Popie]arczyk)

(3) Operations Management Support (Dunford)

e. Gas Monitor Upgrades

(1) SST Gas Monitors Follow on (Witt/KEH)

(2) Gas Monitoring Support (LANL)

(3) Gas Monitor Follow On Support (Bauer)

(4) Fab 6 Spare Gas Monitors (KEH)

f. Temperature Upgrades
(1) MITs for DSTs and 101-A (BIanchard)
(2) Temperature Trees for SSTs (Moore)
(3) Monitoring Upgrades Support (Schroeder)

(4) Validation Probes for MITs (LANL)

g. Video System Upgrades

(1) Video Cameras for DSTs (Moore)

(2) Portable Camera Systems for SSTs (Moore)

h. Surface Level Upgrades

(1) Surface Level Upgrades for 16 Tanks (Moore)

$\begin{array}{ccc}107 / 0 & 110 / 0 & 114 / 0 \\ 59 / 0 & 61 / 0 & 62 / 0 \\ 59 / 0 & 61 / 0 & 63 / 0 \\ & & \\ & 0 / 164 & \\ 135 / 0 & 309 / 0 & 159 / 0 \\ 100 / 0 & & \\ 0 / 552 & & \\ 165 / 0 & & \\ 0 / 261 & 267 / 660 & 276 / 680 \\ 155 / 0 & 266 / 0 & 276 / 0 \\ 0 / 300 & & \\ 125 / 2024 & & \\ 0 / 586 & 0 / 377 & \\ & & \end{array}$

$59 / 277$ 
Waste Tank Safety Program

Fiscal Year 1995 Planning

(Program \$000 - Escalated)

i. Ventilation Upgrades

(1) AW Farm Vent Upgrades (Reed)

(2) Facility Systems Support (Jenkins)

(3) Fabrication Services (Hay)

(4) AN Farm Vent Upgrades (Reed)

(5) Portable Exhauster for SSTs (Jenkins)

j. Ammonia Upgrades

Permitting Support (Engelmann)

(1) Ammonia Monitors AW \& AN Farms (Bauer)

k. Misc Tank Farm Equipment \& Upgrades

(1) Multi-Port Risers \& Elec Upgrds (Popielarczyk)

1. Equipment Disposal

(1) Waste Disposal of 01d Exhausters (Geier)

(2) Equipment Removal, Storage \& Disposal

Sub-Total

$\begin{array}{lll}\text { FY95 } & \text { FY96 } & \text { FY97 } \\ \text { EXP/CAP } & \text { EXP/CAP } & \text { EXP/CAP }\end{array}$

$\begin{array}{lll}100 / 598 & 149 / 880 & \\ 90 / 0 & & \\ 35 / 0 & 360 / 2010 & 446 / 1035 \\ 50 / 549 & & \\ 50 / 0 & & \end{array}$

$172 / 153$

\begin{tabular}{cll}
$0 / 0$ & \multicolumn{1}{c}{$178 / 1382$} & \multicolumn{1}{c}{$217 / 1424$} \\
& $2678 / 0$ & $3395 / 0$ \\
& $1081 / 0$ & $1167 / 0$ \\
$2161 / 6145$ & $5521 / 5473$ & $6175 / 3139$
\end{tabular}

5. Integrated Field Support for Flammable Gas Tanks

a. Integrated Field Support (Wilder)
(1) West Area Operations (Wicks)
(2) Maintenance (Legg)
(3) West Area Planning (Jennings)
(4) HPT Support (Lueck)
(5) IH Support (Held)
(6) East Area Operations ( $\mathrm{Ni}$ )
(7) East Area Planning (Kimbrough)
(8) Test Engineering (Reed)
(9) AW Farm Operations (Ross)
(10) Major Maintenance (Bryant)

$175 / 0$
$250 / 0$
$100 / 0$
$203 / 0$
$86 / 0$
$225 / 0$
$150 / 0$
$555 / 0$
$100 / 0$
$53 / 0$

Sub-Total

$1897 / 0$

$2074 / 0$

$1735 / 0$

ACTIVITY TOTAL

$11091 / 6423 \quad 15412 / 5906 \quad 13740 / 3616$ 


\section{E. WASTE TANK ORGANIC SAFETY PROGRAM}

1. Technology for Evaluating Organic Tanks (Turner)

a. Project Management (PNL/Bean)

b. Organic Analytical Methods Development (PNL/Campbe11)

c. Waste Aging Studies (PNL/Camaioni)

d. Organic Concentrations Mechanisms (PNL/Gerber)

e. Organic Laydown Model (PNL/Gerber)

f. Waste Energetics (PNL/Scheele)

h. SST Waste Carbon and Mo isture Study (PNL/Toth)

i. Analysis Tank T-111 Core Sample (PNL/Scheele)

j. Special Studies (PNL/Bean)

k. Organic Analytical Chemistry Support (PNL/Campbe11)

1. Waste Sample Analysis (Work Order) (PNL/Bean)

Sub-Total PNL

m. Waste Energetics w/Simulants (FAI) (Grigsby)

n. Waste Moisture W/Simulants (FAI) (Grigsby)

o. Basis Safe Ops SSTs - Organic/Nitrate (Grigsby)

p. Basis Safe Ops SSTs - Organic Layer (Grigsby)

q. Basis Safe Ops SSTs - Absorbed Organic (Grigsby)

$r$. Organic Tanks Safety Criteria (Grigsby)

s. Organics Solubility Study (Turner)

Sub-Total WHC

$\begin{array}{lll}\text { FY95 } & \text { FY96 } & \text { FY97 } \\ \text { EXP/CAP } & \text { EXP/CAP } & \text { EXP/CAP }\end{array}$

$\begin{array}{lrr}300 / 115 & 278 / 0 & \\ 450 / 0 & 464 / 0 & 287 / 0 \\ 270 / 0 & 278 / 0 & 143 / 0 \\ 225 / 0 & 232 / 0 & 96 / 0 \\ 180 / 0 & 93 / 0 & 48 / 0 \\ 450 / 155 & 464 / 0 & 239 / 0 \\ 180 / 0 & 93 / 0 & 48 / 0 \\ 290 / 0 & & \\ 180 / 0 & 185 / 0 & 48 / 0 \\ 180 / 200 & 185 / 0 & 96 / 0 \\ 101 / 0 & 246 / 0 & 253 / 0 \\ 2806 / 470 & 2518 / 0 & 1497 / 0 \\ 400 / 0 & 412 / 0 & 212 / 0 \\ 100 / 0 & 103 / 0 & 54 / 0 \\ 299 / 0 & 515 / 0 & 212 / 0 \\ 100 / 0 & & \\ 102 / 0 & 52 / 0 & \\ 100 / 0 & 103 / 0 & 106 / 0 \\ 75 / 0 & 77 / 0 & 82 / 0 \\ 1176 / 0 & 1262 / 0 & 666 / 0 \\ 3982 / 470 & 3779 / 0 & 2161 / 0\end{array}$

Sub-Total 
2. Evaluate and Define Organic Tanks (Turner)
a. CAP Management (Turner)
b. Program Plan (Turner)
c. Organic Tanks Watch List (Grigsby)
d. Core Data Interpretation (Kirch)

Sub-Total

$\begin{array}{lll}\text { FY95 } & \text { FY96 } & \text { FY97 } \\ \text { EXP/CAP } & \text { EXP/CAP } & \text { EXP/CAP }\end{array}$

$\begin{array}{ccc}412 / 0 & 231 / 0 & 238 / 0 \\ 50 / 0 & 52 / 0 & 53 / 0 \\ 25 / 0 & 26 / 0 & 26 / 0 \\ 100 / 0 & 103 / 0 & 106 / 0 \\ 588 / 0 & 411 / 0 & 424 / 0 \\ & & \\ & & \\ & 97 / 0 & 212 / 0 \\ 0 / 0 & 97 / 0 & 212 / 0 \\ & & \\ 21 / 0 & 22 / 0 & 23 / 0 \\ 0 / 0 & 819 / 328 & 843 / 338 \\ 0 / 0 & 257 / 3736 & 265 / 133 \\ 268 / 184 & 1114 / 276 & 1000 / 285 \\ & 2000 / 5529 & 5608 / 15501 \\ 90 / 0 & 93 / 0 & \\ 379 / 184 & 4305 / 9869 & 7739 / 16257\end{array}$

Sub-Total

Resolve USQ for Organic Tanks (Turner)

a. USQ Closure Documentation (Grigsby)

Sub-Total

4. In-Tank Resolution of Organic Tanks w/o Treatment (Dukelow)
a. CAP Management (Dukelow)
b. Install TC Trees/TMACS (Dukelow)
c. Pressure Monitoring Installation (Blanchard)
d. Moisture Monitor Engineering \& (LOW) Instal1 (Toffer)
e. Moisture Monitoring/Control Demonstration (Jenkins)
f. Safety Evaluation/LOA (Grigsby)

$379 / 184 \quad 4305 / 9869 \quad 7739 / 16257$ 
Waste Tank Safety Program

Fiscal Year 1995 Planning

(Program \$000 - Escalated)

5. Pump \& Dispose C-103 Organic Layer/Resolve Issue (Dukelow)

a. Organic Receiver Tank (C. Hanson)

b. Separations Equipment Fabrication (Hay)

c. Separations Equipment Install/0perations (Leliefeld/Klos)

d. Organic Storage Support (Ni)

e. Laboratory Analys is (Dukelow) (PNL work order)

f. Engineering Support (C. Hanson)

g. Separations Equipment Burial (C. Hanson)

h. Safety \& Permitting (Grigsby)

i. Treatment System Design/Fabrication (C. Hanson/Leliefeld)

j. Treatment System Instal1/0perations (K1os)

k. Treatment System Burial

$\begin{array}{lll}\text { FY95 } & \text { FY96 } & \text { FY97 } \\ \text { EXP/CAP } & \text { EXP/CAP } & \text { EXP/CAP }\end{array}$

Subtotal

6. Resolve Safety Issue Organic Tanks (Turner)

a. Moisture Monitoring/Control Engineering (Turner)

b. Safety Issue Closure Documentation (Grigsby)

$0 / 214$

$400 / 0$

$901 / 0$

$135 / 0$

$424 / 0$

$635 / 0$

$0 / 400$

$400 / 0$

$2896 / 613$

$676 / 0$

$676 / 0$

$676 / 0$

$8521 / 1267$

$\begin{array}{cc}154 / 0 & \\ 371 / 0 & 287 / 0 \\ & \\ 360 / 0 & 179 / 0 \\ 515 / 0 & 318 / 0 \\ 253 / 0 & \\ 1051 / 0 & 53 / 0 \\ 309 / 0 & 318 / 0 \\ 82 / 103 & 0 / 106\end{array}$

$3095 / 103 \quad 1155 / 106$

Sub-Total

ACTIVITY TOTAL

$\begin{array}{rr}103 / 0 & 0 / 0 \\ 106 / 0 & 109 / 0 \\ 208 / 0 & 109 / 0\end{array}$




\section{Waste Tank Safety Program Fiscal Year 1995 Planning (Program $\$ 000$ - Escalated)}

$\begin{array}{lll}\text { FY95 } & \text { FY96 } & \text { FY97 } \\ \text { EXP/CAP } & \text { EXP/CAP } & \text { EXP/CAP }\end{array}$

F. WASTE TANK HIGH HEAT SAFETY PROGRAM (Wang)

1. Evaluate and Define High Heat Tanks (Wang)

a. Conduct 106-C Process Test (TPA M-40-05, SI 2x) (Wang)
(l) Program/CAP Management (Wang)
(2) Plant Engineering Support (Dodd)
(3) Operations Support (Ni)
(4) Video Surveillance/Equipment (Smet)
(5) Contingency Action Planning (Jenkins)
(6) Thermal Model/Analysis (McCall)
(7) Safety \& Environmental Support (Hinckley)
(8) Core Sample Data Interpretation (PNL/Eyler)
(9) Investigate 106-C Anomalies (Wang)
$64 / 0$
$45 / 0$
$107 / 0$
$100 / 0$
$47 / 0$
$41 / 0$
$90 / 0$
$260 / 0$

b. Resolve High-Heat Safety Issue (Wang)
(1) Program/CAP Management (Wang)
(2) Contingency Action Planning (Jenkins)
(3) 105-C Process Test Support (Engelman)
(4) Safety Issue Resolution (Hinckley)

EXP/CAP

EXP/CAP

EXP/CAP

Sub-Total

$808 / 0$

ACTIVITY TOTAL

$808 / 0$

$55 / 0$

$174 / 0$

$110 / 0$

$57 / 0$

$122 / 0$

$85 / 0$

$44 / 0$

$339 / 0$

$308 / 0$

$339 / 0$

$308 / 0$ 


\section{G. MISCELLANEOUS UNDERGROUND STORAGE TANKS (Wang)}

1. Evaluate Misc Underground Tanks (Wang)

a. MUST Hazards and Safety Analyses (Wang)

$\begin{array}{lr}\text { (1) Program/CAP Management (Wang) } & 54 / 0 \\ \text { (2) Identify Tanks (KEH) } & 79 / 0\end{array}$

(3) Evaluate and Define (Powers)

$79 / 0$
$136 / 0$

Obtain Historical Data/Info

Identify Potential Safety Issues

Technical Support

(4) Effect of Geometry on Criticality (Chiao)

$68 / 0$

(5) Safety Analysis (Smith/Jason Assoc-Almodavar)

$264 / 0$

Perform Discovery USQ Screening

Perform Addit Prelim Hazard Analysis

Complete USQ Evaluation

DQO Review/Prepare Necessary Safety Docmntn

(6) Review Safety Eval \& Risk Assmt (UW-McCormack)

$50 / 0$

(7) Establish Controls (Kennedy/Foster) 79/0

Establish Immediate Controls

Establish Addit Contrls, if Needed

b. Vapor Sampling (Mahon)

(1) Vapor Sampling (Mahon)

(2) Vapor Sample Analys is (PNL)

c. Safety Screening Sampling (Zuroff)

(1) Auger/Scoop Sampling (Zuroff)

(2) Auger/Scoop Sample Analysis (Jewett) 
Waste Tank Safety Program

Fiscal Year 1995 Planning

(Program $\$ 000$ - Escalated)

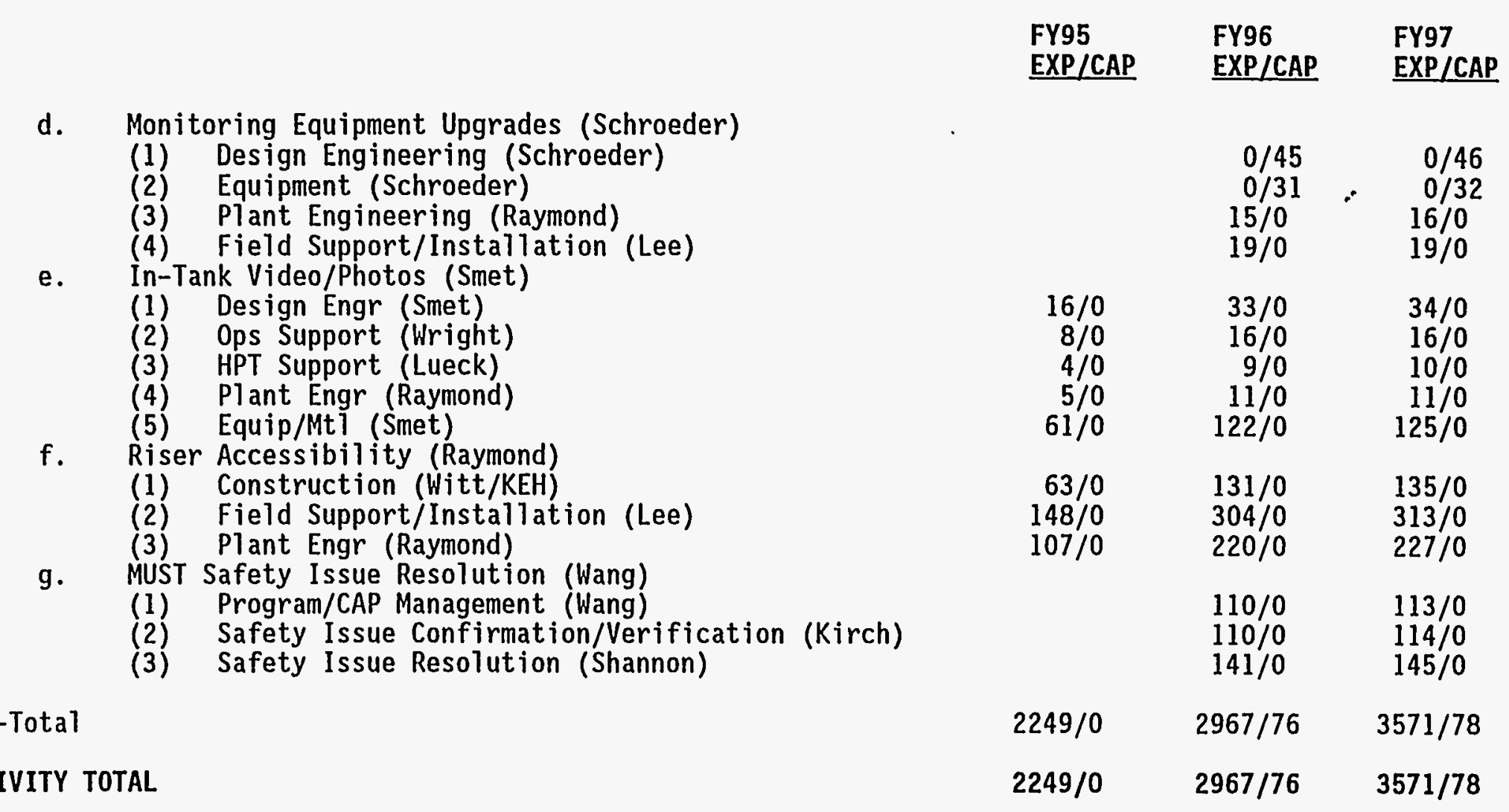

\section{ACTIVITY TOTAL}


Waste Tank Safety Program

Fiscal Year 1995 Planning

(Program $\$ 000$ - Escalated)

$\begin{array}{lll}\text { FY95 } & \text { FY96 } & \text { FY97 } \\ \text { EXP/CAP } & \text { EXP/CAP } & \text { EXP/CAP }\end{array}$

H. WASTE TANK VAPOR RESOLUTION

1. Mitigate - Technology Development for Tank Vapor Safety (Osborne) a. Vapor Flow Monitoring Device (Moore)

Sub-Total

$40 / 0$

$40 / 0$

$0 / 0$

$0 / 0$

2. Resolve Internal Tank Vapor Issues (Osborne)
a. Execute Recommendations from EEA (Design baseline)(Moore)
b. Fabricate, Install Vapor Treatment System (Moore)
c. Vapor Flow Monitoring Device (Engrg support)(Moore)
d. Vapor Flow Monitor Characterization Analysis (Moore)
$98 / 0$
$368 / 603$
$40 / 0$
$30 / 0$

Sub-Total

$536 / 603$

$0 / 0$

$0 / 0$

3. Evaluate \& Define for Tank Vapor Safety (Osborne)

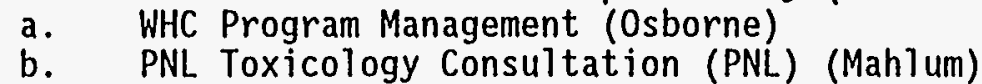

c. Weather Station Ops/Maint/Calibration (PNL) (Allwine)

d. Atmospheric Pathways (PNL) (Allwine)

\section{$458 / 0$}

$325 / 0$

$55 / 0$

$120 / 0$

$351 / 0$

$258 / 0$

$57 / 0$

$309 / 0$

Sub-Total

$958 / 0$

$974 / 0$

$0 / 0$

ACTIVITY TOTAL

$1534 / 603$

$974 / 0$

$0 / 0$ 
Waste Tank Safety Program

Fiscal Year 1995 Planning

(Program \$000 - Escalated)

I. N/A

J. WASTE TANK NUCLEAR CRITICALITY

1. Store and Monitor Waste for Criticality

a. Nuclear Criticality Integration

(1) Program Mgmt/Safety Issue Resolution

(2) Maintenance of Data Base

(3) Material/Supplies/Travel/Computer

Sub-Total

$\begin{array}{lll}\text { FY95 } & \text { FY96 } & \text { FY97 }\end{array}$

EXP/CAP EXP/CAP EXP/CAP

b. Neutron Detection/Evaluation
(1) Passive Neutron Detection
(2) Active Neutron Detection Source
(3) LOW Installation \& Measurements
(4) Surveillance/Data Acquisition
(5) Contracts (LANL/ORNL)

Sub-Total

c. Criticality Detection/Evaluation
(1) Noise Analysis
(2) Pulsed System Studies
(3) Criticality Event Characterization
(4) Contract (LANL/ORNL)

Sub-Total

ACTIVITY TOTAL

K. $\quad N / A$

$\begin{array}{rrr}107 / 0 & 109 / 0 & 111 / 0 \\ 68 / 0 & 35 / 0 & 36 / 0 \\ 24 / 0 & 25 / 0 & 27 / 0 \\ 199 / 0 & 169 / 0 & 174 / 0\end{array}$

$\begin{array}{ccc}284 / 0 & 311 / 0 & 299 / 0 \\ 0 / 248 & 0 / 112 & \\ & 200 / 300 & 212 / 318 \\ 170 / 62 & 100 / 0 & 115 / 0 \\ 116 / 0 & 119 / 0 & 82 / 0\end{array}$

$570 / 310 \quad 730 / 412 \quad 708 / 318$

$\begin{array}{ccc}210 / 0 & 245 / 0 & 205 / 0 \\ & 0 / 77 & 0 / 317 \\ 105 / 0 & 78 / 0 & 125 / 0 \\ 116 / 0 & 119 / 0 & 82 / 0 \\ 431 / 0 & 442 / 77 & 412 / 317 \\ 1200 / 310 & 1341 / 489 & 1294 / 635\end{array}$


Waste Tank Safety Program

Fiscal Year 1995 Planning

(Program \$000 - Escalated)

$\begin{array}{lll}\text { FY95 } & \text { FY96 } & \text { FY97 } \\ \text { EXP/CAP } & \text { EXP/CAP } & \text { EXP/CAP }\end{array}$

L. WASTE TANK HYDROXIDE CONTROL (Turner)

1. Mitigate Hydroxide Consumption (Carothers)

a. Mitigate 107-AN Hydroxide (Carothers)

(1) 107-AN Lab Process/Sample Analysis (Jewett/Marshall) 113/0

(2) 107-AN DQO (Raymond) $\quad 43 / 0$

$\begin{array}{lll}\text { (3) 107-AN Procedures/Trng/RR (Reed/Fox/Grantham) } & 128 / 0 \\ \text { (4) 107-AN Monitoring Instr (Moore/Smet/Bauer) } & 107 / 534\end{array}$

$\begin{array}{lll}\text { (4) 107-AN Monitoring Insitr (Moore/Smet/Bauer) } & 107 / 534 \\ \text { (5) 107-AN Instal1 Mixing System (Ni/Mohre/Lueck) } & 294 / 0\end{array}$

(6) 107-AN Caustic Addit Op (Ni/Mohre/Raymond) 70/0

(7) 107-AN Conduct Mix Ops/Eval Perf (Ni/Mohre/Raymond) 215/0

(8) 107-AN Disposal Cask (Raymond) 246/0

7
2
1
1
-2
0
0

b. Mitigate 102-AZ Hydroxide (Carothers)

(1) 102-AZ Grab Sampling (Klos) 21/0

$56 / 0$

$57 / 0$

$108 / 0$

(2) 102-AZ Lab Process/Sample Analysis (Jewett/Marshall)

(3) 102-AZ Engr Eval of Mit Alternatives (Raymond)

(4) 102-AZ DQ0 (Raymond)

(5) 102-AZ Design Caustic Sys (Raymond/Jenkins/Towne)

(6) 102-AZ Install Caustic Sys (Mohre/Ni/Lueck)

(7) 102-AZ Conduct Caustic Addition Op (Ni/Raymond/Mohre)

c. Mitigate 102-AN Hydroxide (Carothers)
(1) 102-AN Grab Sampling (KTos)
(2) 102-AN Lab Process/Sample Analysis (Jewett/Marshal1)
(3) 102-AN Design/Install Corrosion Probe (Raymond)
(4) 102-AN Engr Eval of Mit Alternatives (Raymond)
(5) 102-AN Procure Mixer Pump (Jenkins)
(6) 102-AN Design Caustic Sys (Jenkins/Towne/Raymond)
(7) 102-AN DQ0 (Raymond)
(8) 102-AN Install Caustic Sys (Mohre/Ni/Lueck/Raymond)

$\begin{array}{ccr}68 / 0 & 35 / 0 & \\ & 158 / 0 & \\ & 44 / 0 & \\ & 173 / 0 & \\ & 495 / 437 & 307 / 0 \\ & & 57 / 0 \\ & & 742 / 0 \\ 1382 / 534 & 1569 / 437 & 1518 / 0 \\ 1382 / 534 & 1569 / 437 & 1518 / 0\end{array}$

Sub-Total

$1382 / 534$
$44 / 0$
$44 / 0$
$115 / 0$
$113 / 0$

$142 / 0$

$105 / 0$

ACTIVITY TOTAL

$1518 / 0$ 
WHC-SP-1101

Figure 7.4-5 Approach.

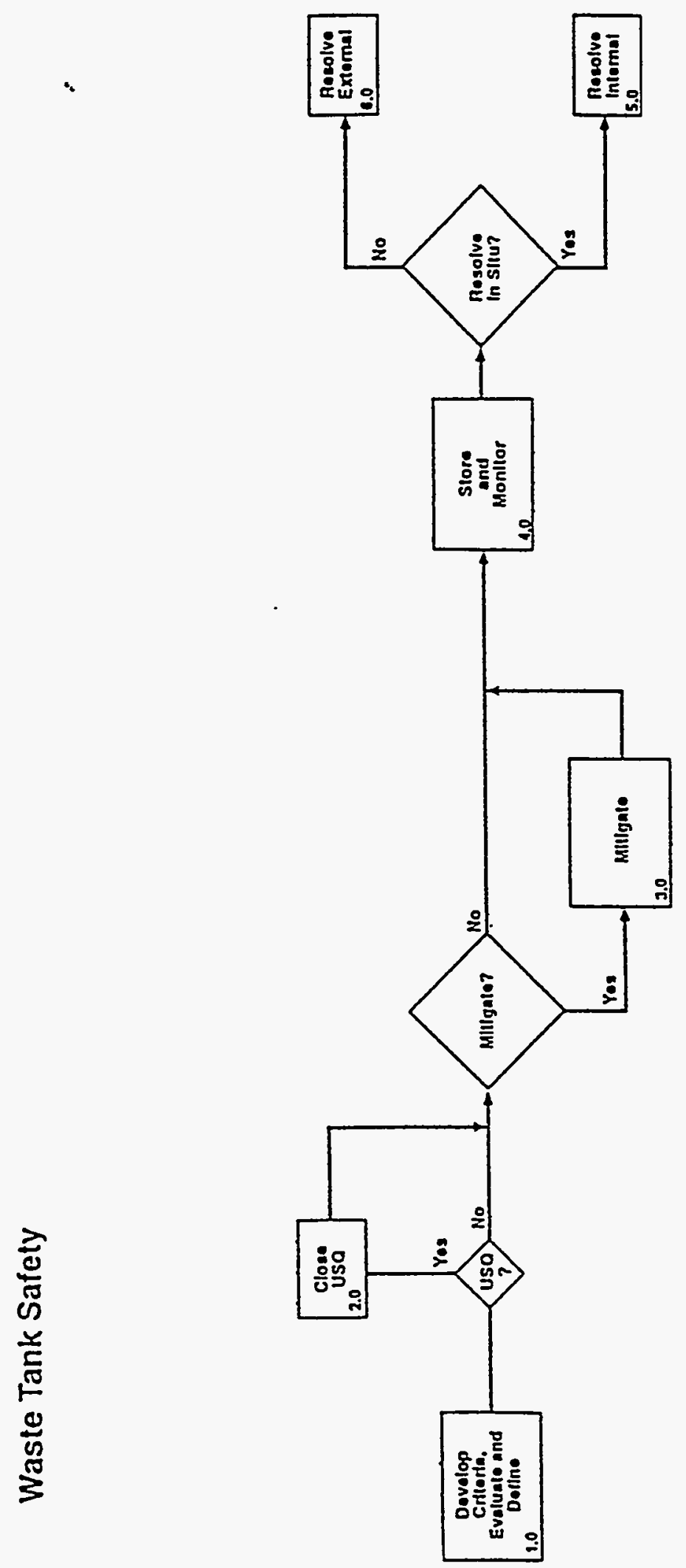

F7.4-5 
Figure 7.4-6.

1.1.1.2.02 Waste Tank Safety Summary Schedule

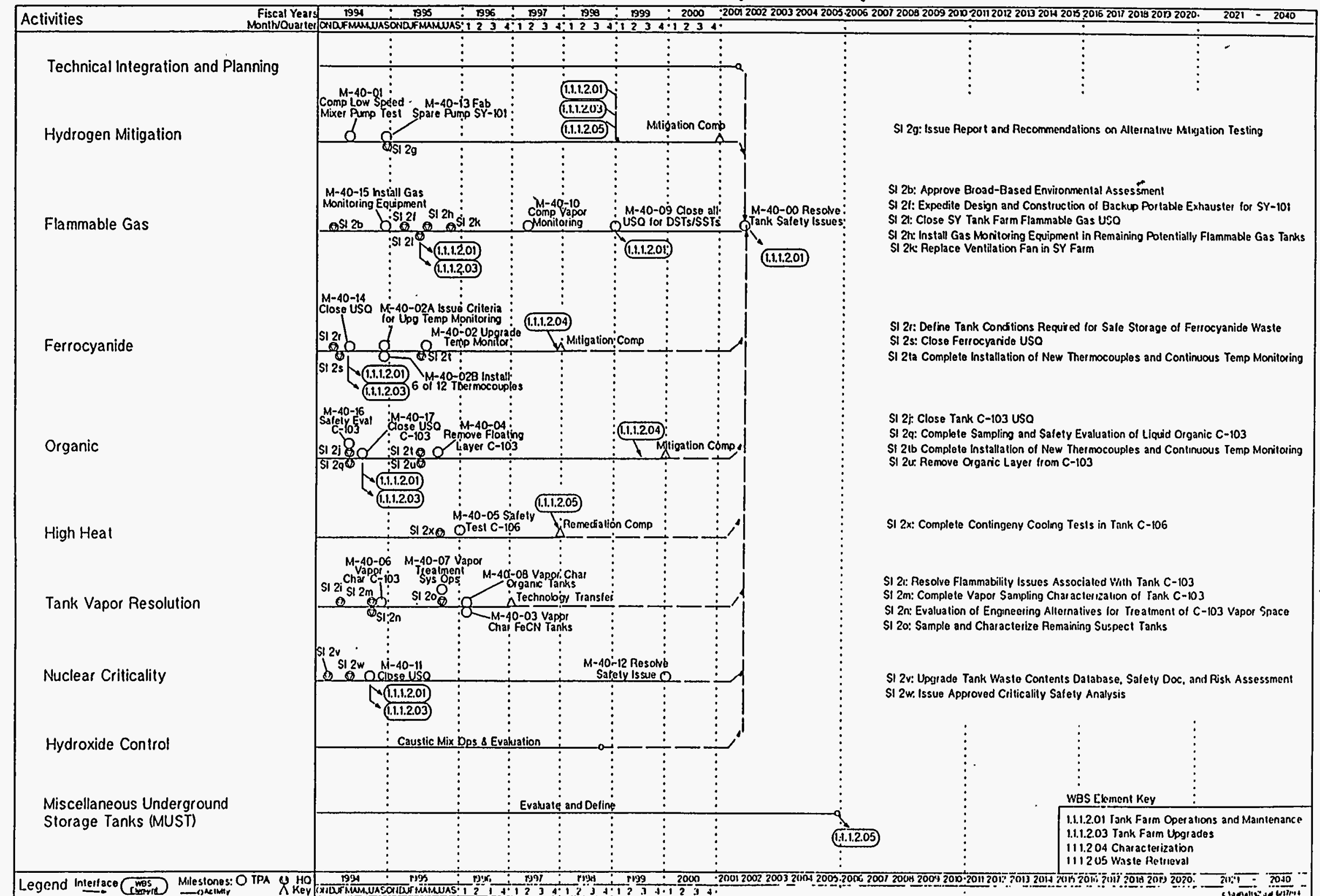




\section{WHC-SP-1101}

Figure 7.4-7. Waste Tank Safety Cost.

ESTIHJHOUSE HAYFORD COMPAYY

EPORT PATE 15SEPMA RUH HO. 415 $16: 27$
PRIKAVERA PROJECT PLAWHER

COST LOADING REPORT

WASTE TANK SAFETY PROERAY

START DATE 010CT92 FIN DATE 30SEPO5

data date oloct93 page mo. 1

SST LUADIHS - SUYHAYY BY YEAR - EXPEHSE TOTAL USAGE FOR YEAR

\begin{tabular}{|c|c|c|c|c|}
\hline & & FY & FY & FY \\
\hline$\therefore T$ ID & DESC & 1995 & 1995 & 1997 \\
\hline 11 & 8 & 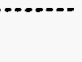 & .... & 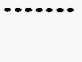 \\
\hline .24110402 & BASELIHE PLAHHIHG FY95 & 2566.5 & 56.6 & \\
\hline ¿2A110AO4 & ISSUE FIKAL WTS PROGRAYATIC RISK ASSHT (PHL) & 478.0 & & \\
\hline .24130408 & BASELINE PLAHMIHG FY95 & & 2391.0 & \\
\hline ¿2A110A12 & BASELIHE PLAYHIHG FY97 & & & 3524.0 \\
\hline :2A110BO4 & MAUAGEHEKT \& IHTEGRATION FY95 & 1832.9 & & \\
\hline :2A110B0B & HARAGEKENT \& IHTEGRATIOH FY96 & & 1890.4 & \\
\hline .2k110812 & HAMAGEMENT \& INTEGRATION FY97 & & & 1947.2 \\
\hline :2AIIOEOO & SYSTEHS EHGR \& RISK MAYAGEREHT & 62.3 & & \\
\hline :2Al10K04 & ADVISORY SUPPORT FYS5 & 373.5 & & \\
\hline :24110K06 & ADVISORY SUPPORT FY96 & & 384.7 & \\
\hline :24110K08 & ADVISORY SUPPORT FY97 & & & 396.3 \\
\hline TOTAL & All & 5313.2 & 4722.7 & 5857.5 \\
\hline
\end{tabular}

$\$ 12$.

NZA120AO4 WASTE TANK SAFETY OA FY95 NU120406 NASTE TAKK SAFETY OA FY96 Ni2A120408 NASTE TAKK SAFETY OA FY97

TOTAL $\quad$ A12

821 .

II2B21OAO4 PROGRAY MAYAGEMENT FY95 N2B21OAO6 PROCPAH MAULGEKEMT FY96 128' PROCRAH MAMAGEREMT FYG7

H28. PROUECT HAKAGEHEMT FY95

R2B21UDUG PRDSECT HARAGEKENT FY95

N28210808 PROUECT MAHSGEKENT FY97

H28210DO4 PML HYOROGEN HITIGATION PROU KOMT

H28210006 PML TAKK SPECIFIC HITIGATIOH

H26210008 PML COAPUTATIONAL HOOELINS BASELIHE

H2B210009 PKL ISSU TEST PLAH DILUTIOH TESTIHS 103-SY

N2B210D11 DATA HAUGGEMENT BASELIHE

N2B210012 DATA ARLLYSIS BASELIHE

N2B210014 PHL JHSTRLWENTATION DEVELOPHEKT

K28210015 LTR REPT VOID/VIDSCOMETER OHARACTER 101-SY

128210017 PKL DILUTIOH STDIES

N2B210DIB PHL DILUTIOH STUDIES

Hi2B210D19 PHL EHGINEERIHG SERVICES FY9S

IRB210D2O PML EHGIMEERING SERVICES FY97

H2B210EOO SAFETY AYALYSIS UPDATE SY-101 (LAYL) 518.0

M2B210E02 LAYL BUILD DACS 2A SYS FOR SY EARH

M2B210E06 LAYL SAFETY AHALYSIS 103-SY/IK-TAKK DILUTION 2200.0

M2B210E1O LAN TECHNICAL SUPPORT 103-SY

N2B2IOE12 LAYL GAS HONITORING SUPPCRT

IIZ210E14 IN-TANK DILUTIOH SUPPORT (LAHIL)

M2B21OE16 IH-TANK DILUTIOH SUPPORT (LAHL)

H2B210E2I I5SUE TANK I01-AN SA (LAKL)

N2B210E24 LARL DACS 2A SYS FOR SY FARY MHIMTERANCE

N28210E25 LAHL 101-SY TECHMICAL, SUPPORT

H2B210E27 GAS HONITORIHG SUPPORT (LAHL)

H2B210E29 I5SUE TANK 105-AY SA (LAHL)

H2B21OE3O LAHL TECHWICAL SUPPORT IOL-AY

H2B21OE31 LAML DACS 2C FOR AM FARM MAIKTERAHCE

H2B210E32 LAUL TECHNICAL SUPPORT 104-AN

N2B210E34 LAML DACS $2 A$ SYS FOR SY FAPH MAIKTEHANCE

\$2B210E35 TECHNICAL SUPPORT 105-AH (LAML)

H2B210E35 LAHL DACS 2B SYS FOR SY FARY MAIHTEHAHCE

H2B210E38 LAYL 101.5Y TECHNICAL SUPPORT

H2E' GAS HONITORIHG SUPPORT (LAML)

2411.6

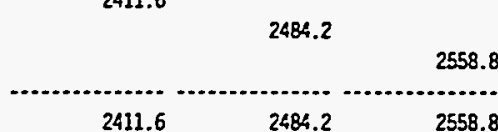

843.5

570.5

917.6

882.0

737.2

973.6

355.0

677.0

323.0

591.0

202.0

190.0

200.0

70.0

309.0

1890.1

371.4

2776.6

530.5

400.0

440.0
284.3

1230.9

515.0

309.0

309.0

488.1

117.9

616.4

564.5

265.3

515.0
380.9

308.8

318.3 


\section{WHC-SP-1101}

ESTIHGHOUSE HANFORD COMPANY

EPORT DATE 15SEPQA RUN HO. 415 $16: 27$
PRIKAVERA PROUECT PLAYHER

COST LOADIHG REPCRT
WASTE TANK SAFETY PROGRAM

START DATE DIOCT92 FIN DATE 3OSEP05

DATA DATE OLOCT93 PAGE ND. 2

OST LOADIHG - SLYHARY BY YEAR - EXPEHSE TOTAL USAGE FOR YEAR

\begin{tabular}{|c|c|c|}
\hline & $F Y$ & FY \\
\hline ET ID DESC & 1995 & 1996 \\
\hline
\end{tabular}

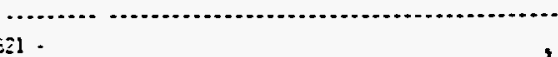

TOTAL $\quad 821$

22

Z2B220E00 DACS MAINTEKANCE \& OPERATION FY95 ¿26220F00 GAS HONITORING MAINT \& OPERATION FY95

Z2B220FO4 GAS HONITORIHS RAINT \& OPERATION FYSG

2E220F08 GAS HOWITORING KAIHT \& OPERATION FY97

26220610 1HSTRU:EKTATION TRAYSPORT CONTAINER FY95

¿23220616 VISCOEETER 101-SY SUPPCRT

:22220G18 VOIDKETER 101-SY SUPPORT

Z25220H0O MIXER PLAP OPERATIONS FY95

26220K00 TEST PUKP REKOVAL PREP

TOTA1 B22

23.

26230 COO WORK PLAY/WORK PKG 103-5Y 228230LO4 TRAINING/READINESS REVIEW

128230000 DACS 2A SYSTEM HOTI

128230002 DACS $2 A$ SYSTEK PROC \& FAB 103-SY

228230500 DACS MAIHTENANCE \& OPS 103-5Y

226230504 DACS MAIHTENANCE \& OPS 103.5Y

126230F04 GAS CHARACTERIZATION SYSTEM (GCS)103-SY

¿26230F08 GAS CHARACTERIZATION SYSTEH (GCS) 103-SY

2E230500 VISCOHETER 103-SY SUPPORT

:26230504 VOIDFETER 103-SY SUPPORT

Z2BE30EOB VOIOKETER 103-SY TESTIHG SUPT

¿26230ज10 VISCOETTER 103.5Y TESTING SUPT

¿26230000 REHOVAL/OISPOSEL SYSTEM 103.SY

22B230K00 IN-TAWK DILUTION TEST PREP 103-SY

W26230K04 IN-TAKK DILUTIOW SAYPLING I03.SY

22B230K08 IN-TAKX DILUTION SAYPLIHG 103-5Y

TOTAL E23

624

HZB240COD INSTALL PUMP 101-AH

H26240C02 CONPL 101-AH IHSTALL READINESS REY

N26240CO8 COAPLETE IN TANK TESTIHS AN-101

K2B240DOA DACS 2B SYSTEM 101-AH

KZE240EO4 DACS 28 OPERATIONS 101-AS

K2B240EOB DACS 28 MAIKTERAMCE \& OPS 101-AM

N2B240GO2 VOIDEETER 101-AW INST, SUPPORT

N2B240505 VISCONETER 101-AW INSTL SUPT

N2824060B VOIDKETER 101-AN INSTL SUPPORT

M26240G10 VISCOHETER IO1-AK INSTL SUPT

W2B24OHOS MIXER PUMP OPERATIOKS 101-AK

N26240J06 REKOYAL/OISPOSAL SYSTEH 101-AH

TOTA

B24

626

23260C04 INSTALL PLAP 103-AN

N2B260C1O CONPL PUAP AK-103 READINESS REVIEN

N2B260F00 GCS MATERIAL PURCHASES AH-103

N2B260500 VISCONETER 103-AN SUPPORT

K2B260GO4 VOIDHETER 103-AN SUPPORT
8035.2

6669.9

8594.1

FY

884.5

961.9

693.5

1020.5

25.2

149.2

367.2

654.7

218.1

3260.7

695.3

1020.5

239.2

313.4

552.6

344.1

$96.5 \quad 665.7$

367.2

$\begin{array}{ll}105.5 & 47.1\end{array}$

$257.5 \quad 128.8$

301.3

1862.9

1098.6

85.2

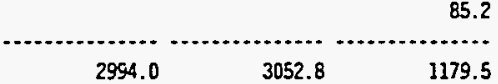

881.8

76.1

37.5

691.8

210.2

147.8

407.7

371.3

152.3

$207.9 \quad 179.9$

120.7

210.3

$\begin{array}{lll}519.1 & 360.2 & 2816.0\end{array}$

158.3

389.6
$357.8 \quad 82.7$ 
WHC-SP-1101

\begin{tabular}{|c|c|c|c|c|}
\hline \multicolumn{2}{|c|}{-ESTIHGHOUSE HAHFORD COAPANY } & $\begin{array}{l}\text { PRIKAYERA PROUECT PLAYKER } \\
\text { COST LOADING REPORT }\end{array}$ & & $\begin{array}{l}\text { WASTE TAYK SAF } \\
\text { START DATE } 010 \\
\text { DATA DATE O1O }\end{array}$ \\
\hline \multicolumn{2}{|c|}{ ESS, IIIHS - SUAFARY BY YEAR - EXPEHSE } & \multicolumn{2}{|l|}{ TOTAL USAGE FOR YEAR } & \\
\hline$\therefore 0 \pi 10$ & DESC & $\begin{array}{c}\mathbf{F Y} \\
1995\end{array}$ & $\begin{array}{l}\mathrm{FY} \\
1996\end{array}$ & $\begin{array}{c}\text { FY } \\
1997\end{array}$ \\
\hline$\Xi 20^{\circ}$ & & 2 & & \\
\hline 1:2B260H06 & MIXER PUHP OPERATIONS & & & \\
\hline Si2B2600J00 & REHOYAL/DISPOSAL SYSTEM 103-AN & & & 191.4 \\
\hline TOTAL & B26 & & & 739.3 \\
\hline \multicolumn{5}{|l|}{ E27 - } \\
\hline 1.28270C00 & INSTALL PREPARATIOH PUMP 104-AH & - & 73.8 & \\
\hline $1: 28270<02$ & IHSTALL PREPARATIOH PUAP 104-AH & & & 214.7 \\
\hline 1i28270<04 & COHPL TAYX 104-AH PLAP INSTALL RR & & & 76.1 \\
\hline Ri28270CO6 & IHSTALL TAYK 104-AY MIT PUMP & & & 64.4 \\
\hline 1:28270006 & OACS 2C SYSTEH HGT & & & 739.3 \\
\hline K28270E00 & DACS 2C MAINTEHANCE \& OPS & & & 395.7 \\
\hline$N 28270600$ & VISCOKETER 104-AH SUPPORT & & 153.7 & \\
\hline 1228270604 & VOIDKETER 104-AN SUPPORT & & 378.2 & \\
\hline N28270606 & VISCOKETER 104-AN SUPPCRT & & & 158.3 \\
\hline 1:28270608 & VOIDKETER 104-AN SUPPORT & & & 389.6 \\
\hline 128280+100 & HIXER PUAP OPERATIONS & & & 170.7 \\
\hline TOTAL & 827 & & 605.8 & 2208.9 \\
\hline \multicolumn{5}{|l|}{ B28 - } \\
\hline K28280CO4 & IHSTALL PUHP 205-AY & & & 811.4 \\
\hline N2B280608 & VOIDAETER 105-AN SUPPORT & & & 268.6 \\
\hline N28280510 & VISCOHETER 105-AN SUPPORT & & & 186.5 \\
\hline H2B? an & HIXER PUMP OPERATIOHS & & & 265.4 \\
\hline N2: & REHOVAL/DISPOSAL SYSTEH & & & 179.7 \\
\hline OTAL & 828 & & & 1611.5 \\
\hline
\end{tabular}

c31 .

N2C310R0A FECH SIHLLATED MASTE STUDIES (FAUSKE) N2C310005 PML MAULGEMENT

N2C310007 PML AGIHG WASTE STDOIES

H2C310015 PPL CESTUH UPTAKE

RZCOIODIB PML SPECIAL STUDIES/COUSULTATJOH

R2C310D19 PM COHPARE REAL WASTE H/SIMULATED HASTE

MZCSIOD21 IHFRARED/ICP SPECIATIOH TRAUSFER (PHL)

Ni2C310D24 PML CESIUH HICKEL FECN SOLUBILITY

H2C310028 SLLOSE HOISTURE HOOELING

MRC310EO4 DHFSB/CAP MAUAGEKENT

NIZC310S02 HOSSBAUER SPECTROMETER

N2C31OHOA IH-SITU RELATIVE HUMITITY IMSTRLMENTATION

N2C31OJOA IMFRARED SPECIATION TRANSFER

N2C31OLOD SCANHIHG ELECTRON MICROSCOPE INVESTG SUPT

TOTAL COI

c33.

H2C330205 CAP MAULGEHERT FECH TAHK SAFETY FY95 H2C330L24 CAP MANAGEHENT FECH TANK SAFETY FY96 Hi2C330430 CAP MAVAGEKENT FECH TANK SAFETY FY97 12C330B04 UPDATE SAFETY DOCNAENTATIOH FY95 H2C33OBOG UPDATE SAFETY DOCUMENTATION FY96 HICC330BOB UPDATE SAFETY DOCUMENTATION FY97 H2C330CDO TRANSHIT DOC TO RESOLVE SI C FAPH TAMKS HiZC3 30 COB DOE REVIEW/COMERT - HAC DISPOSITIOH R2C330014 TEOHNICAL SUPPORT SI RESOLUTION FY95 HZCPN.'G TECHNICAL SUPPORT SI RESOLUTION FY96

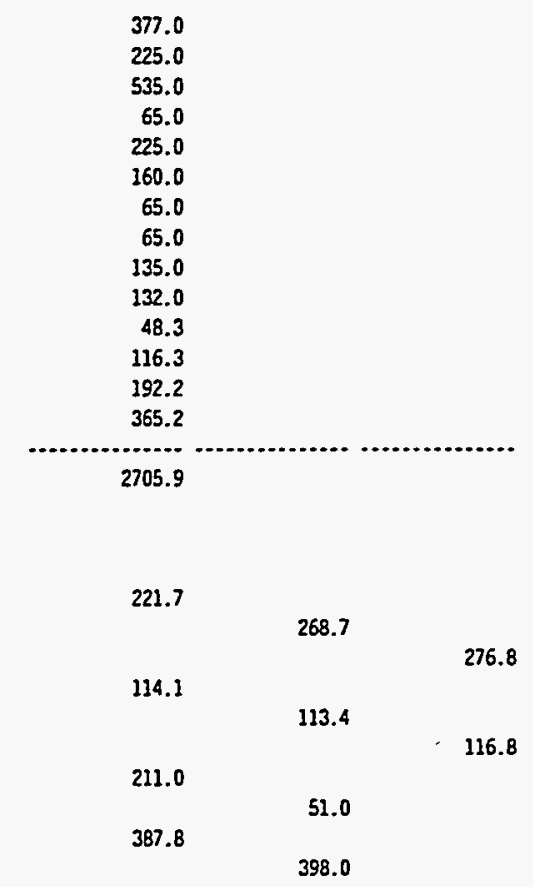




\section{WHC-SP-1101}

¿STIHEOOSE HAMFORD COYPANY

EPORT DATE 15SEP94 RUN NO. 415 $16: 27$
PRIMAVERA PROUECT PLANHER

COST LOADIHS REPCRT

WASTE TAKK SAFETY PROCRAH

START DATE 01OCT92 FIH DATE 30 SEP05

OST LOADING - SUMHARY BY YEAR - EXPERSE

TOTAL USAGE FOR YEAR

DATA DATE O1OCT93 PAGE HO.

FY FY FY

$1995 \quad 1996 \quad 1997$

TT ID DESC

33

2 C330018 TEONICAL SUPPORT SI RESQUUTION FY97 2C330E06 PREPARE SAFETY SUPT/SI RESOUUTION DOCOMENTS 2C330EOB TRANS DOC RESOLVE FECN SI ALL TANKS

2C330FDO CORE SAMPLE/DATA INTERPRETATIO

2C330F2O CORE SAYPLE/OATA IKTERPRETATIOH

2C330F30 CORE SAYPLE/DATA IHTERPRETATIOI

2C330G00 FAUSKE CONTRACT - SI RESOLUTION FY96

2C330504 FAUSKE CONTRACT - SI RESOLUTION FY97

DTAL C C

4.

20340A04 CAP MALAGEVENT FECH TANKS FY9S

2C340A06 CAP MAMAGEKENT FECH TAKKS FY96

2C340A08 CAP MALAGEYENT FECH TANKS FY97

2C340314 IISTALLATION OF TC TREES/HOOXUPS THACS

2C340C12 NEUTRON PROBE MOISTURE HONITOR LON EHGR

2C340C20 KEUTRON PROBE WOISTURE HONITOR LOU EHCR

2C340C30 NEUTRON PROBE WOISTLPE HONITOR LOU EHGR

2C340D02 SURFACE WOISTURE WEASIRIHG

2C340020 SURFACE HOISTURE REASURIHG (CPAC) FY96

20340030 SURFACE MOISTIRE MEASURIHG (CPAC) FY97

2C340E06 EHGINEERIHG DOCAMENT CONTRO

2C340E08 ENGINEERIHS DOCUNENT CONTROL FY9S

2C340E10 EHGIHEERIHÂ DOCAMEKT CONTRO FY97

2C340F10 PRESSIRE HONITORING DESIGN \& ENGT FECN TANKS

2C340F14 PRESSLRE HOHITORING EHGINEERIHG \& INSTALLATION

2C340F18 PRESSLRE HONITORIHS INSTALLATION FECN TAHKS

$2 C 340500$ ISSU STATUS REPT ON LON INSTALL ACTIVITIES

2C34OGZO NEUTRON PROBE WOISTURE HONITOR LON INSTALL

2C340G30 NEUTRON PROBE WOISTURE HONITOR LOA IISTALL

2C34OHOA IR SCAN DEVICE MOO \& SCANS

2C34OHRO IR SCAN DEVICE HOO \& SCAMS

2C340J00 ELECTROMAGNETIC IHOUCTIOH (EKIP)

2C34OU2O ELECTROAGGETIC INOUCTION (EMIP)

2C340J30 070CTROAGGIETIC INDUCTION (EMIP)

2C340KOO ISB ANENDNENT

DTAL C34

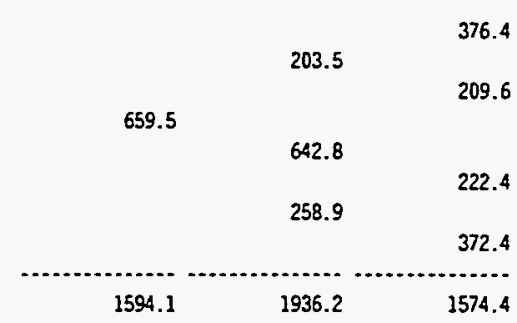

85.6

88.2

1013.3

542.4

202.2

323.1

344.5

14.6

232.8

15.1

445.7

227.0

396.2

390.7

$129.8 \quad 341.1$

147.8

336.4

118.8

41.7

3199.0

1516.1

1498.2

4

20410A0O VASTE BEHAVIOR HOOELIHG FY95

20410A05 WASTE BEHAVIOR YOOLLIHS FY96

2D410A08 MASTE BEHAVIOR HOOELIHAS FY97

20410810 PRDGRAY MAYAGEKEKT FY95

20410B11 PROGRAY MAVAGEMEKT FY96

20410812 PROCRAM MANAGEMENT FY97

20410COD GAS GEMERATION KEOHANISHS FY95

20210020 GAS GENERATJON HECHANISHS FY9

20410C30 GAS GERERATION MECHANISMS FY9

20410E00 GAS RETENTION RECHAHISHS FYOS

20410E05 GAS RETENTION KECHANISHS FY96

20110:08 GAS RETENTION KEOHAHISHS FY97

ZDS1OHDO GAS REACTION STUDIES FY9S

2041OHZO GAS REACTION STLOIES FY96

ZOA1OH3O GAS REACTION STLOIES FY97

ZOA1ONOO ORGAHIC CHARACTERIZATIOH

IDDA10JOS DEV \& TRAYSFER OF METHCOS ORGANIC ANALYSIS

$\begin{array}{lll}982.0 & 1476.2 & 1053.7 \\ 407.0 & & \\ & 439.8 & 431.8 \\ 948.8 & & 282.5 \\ 799.0 & & \\ & 613.6 & 392.6 \\ 368.0 & & \\ & & \\ 238.0 & & \end{array}$

231.8 
WHC-SP-1101

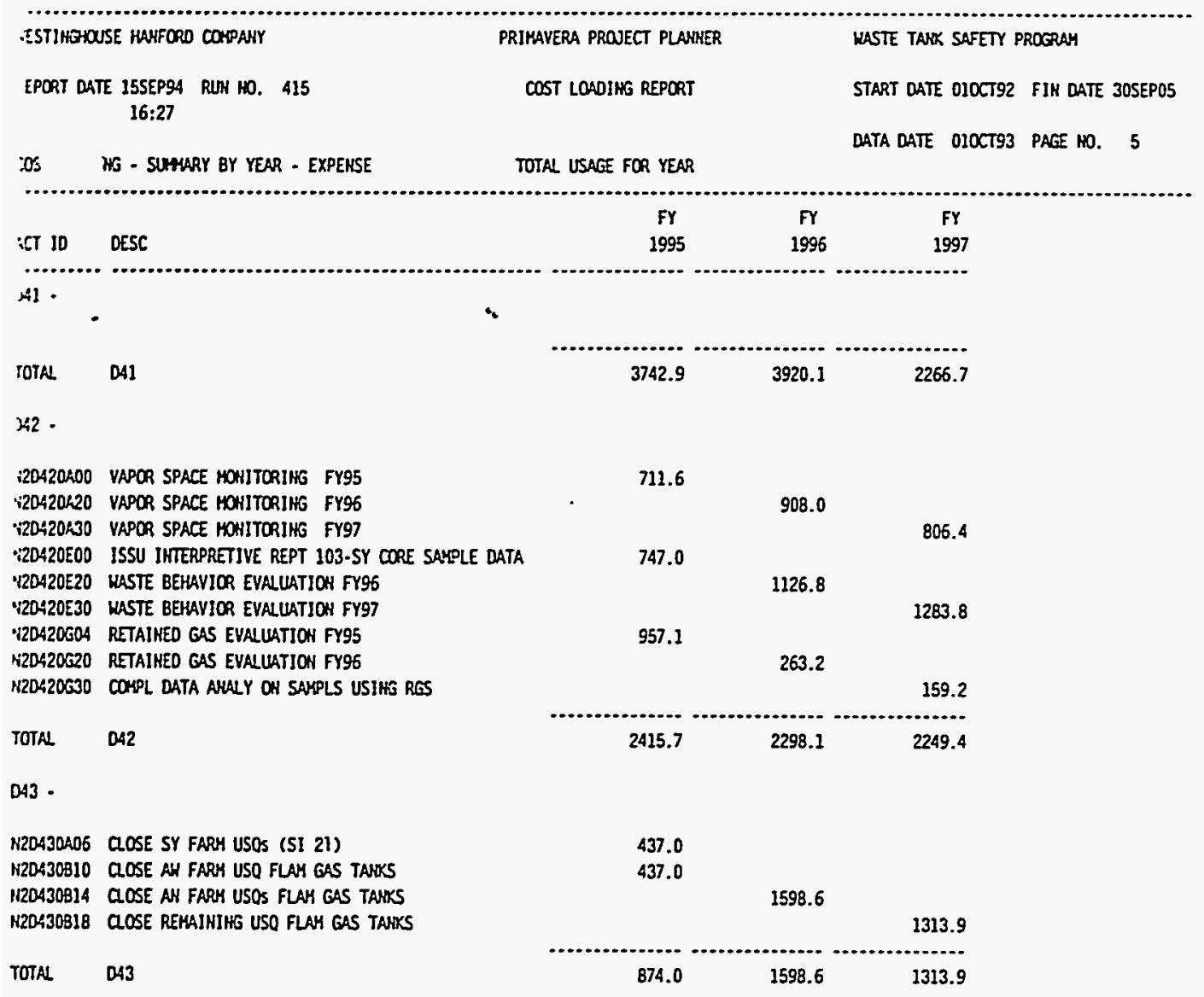

D44.

120

PROCRAY HANAGEHEHT FY95

N2D44UKU6 PRDGRAS MAHAGEMENT FY96

N2D44CA10 PROGRAY MAULGEYENT FY97

N2D440B12 SY FARY SPARK RESISANT FAN (SI 2K) $\quad 46.0$

H2D44OC12 EXPEDITE DESG/CONSTRU BACXUP PORTB EXH SY-101

N2D440002 VIDEO SYSTEK UPGRADES FY95

M2D440E10 TEMPERATURE UPGRADS FY95

N2D44OE2O TEMPERATURE UPGRADES FY96

H20440E30 TEHPERATURE UPGRADES FY97

H2D440FO2 SURFACE LEVEL UPGRADES FY95

N20440G02 AH FARH VENT UPGRAOES FY95

H2O4A0614 AU FAPY VENT UPGRADES

N20440.620 AH FARH VEKT UPGRADES

N20440G24 AN FARH VENT UPGRADES

N20440G30 PORTABLE EXHAUSTER FOR SSTS

N2O44OHOS GAS HONITOR UPGRADES FY95

N2O44OH1O GAS HONITOR UPGRADES FY95

H2O44OH14 GAS HOHITOR UPGRADES FY97

M2OA4OKO2 IHSTL AMHONIA HONITORS 241-AH/AH FARHS

N20440L02 HISC TAKK FARH EOUIPHENT UPGRADES

H2O44OLO6 MISC TAKK FARH EOUIPKENT UPGRADES

N2O44OKOO IHSTALL DST/SST GAS MONITORS (SI 2h)

N2O44ONOO EQUIPHEAT OISPOSAL FY96

N2DA4OHO4 EOUJPMENT DISPOSAL FY97

TOTAL $\quad 044$

DA5 .

231.9

238.9

532.5

551.7

58.9

225.3

149.3

360.5

100.0

445.6

309.0

159.2

172.4

178.2

551.7

217.5

\begin{tabular}{rrrr} 
& 551.7 & & \\
& & 3759.4 & \\
& & & 4562.0 \\
\hline 2161.4 & 5520.8 & 6174.7
\end{tabular}

1896.9

2074.0

1735.7 
WHC-SP-1101

WASTE TAKK SAFETY PROGRAM

ESTIHGOUSE HAHFORD COPAYY

PRIMAVERA PROJECT PLANWER

START DATE 010CT92 FIN DATE 3OSEP05

इPORT DATE 15SEP94 RUN W. 415

COST LOADIHG REPORT

DATA DATE OHOCT93 PAGE NO. 6

XST LOAOJHS - SUITARY BY YEAR - EXPENSE

TOTAL USAGE FOR YEAR

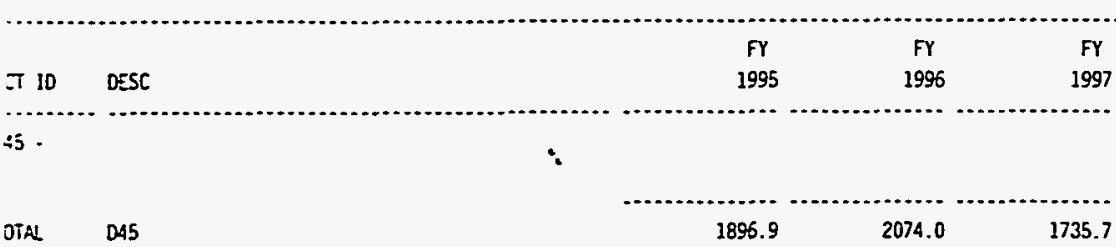

51.

2E51042 PHL PROJECT MAMAGEHENT

?E5IOA4 PHL PROUECT MANAGEREKT

ZE510A6 PNL PROJECT HAYACENENT

2E51082 ORGAHIC WASTE ERERETTICS

2E51034 ORGANIC WASTE EHERTETIICS

?E51036 CRGAHIC WASTE ENEREETICS

ZESIOC2 WASTE ERERGETICS WNSIMULANTS - FAI

2E510С4 WASTE EMERGETICS WISIMLANTS - FAI

2E510C6 WASTE ENERGETICS INSIMULANTS - FAI

2E51002 ORGANIC ANAL TICAL HETHOOS OEVELOPMENT

2E51004 ORGAHIC ARAL VTICAL HETHOOS DEVELOPNENT

?E51005 ORGAHIC ARALYTICAL HETIDOS DEVELOPRENT

?E5IOE2 ORGANIC WASTE AGING STDOIES

?E5IOE4 ORGAHIC WASTE AGIHE STUDIES

?E510E6 ORGANIC WASTE AGING STUDIES

?E510:2 ORGANIC CONCENTRATION RECHANISHS

?E51OF4 ORSANIC CONCENTRATION HECHANISHS

?E510F6 ORGANIC CONCEMTRATIOH MECHAHISUS

?E51OES ORGANIC ALAL TITICAL CHEMISTRY SUPPCRT

?E5IOGA ORGAHIC ANALYTICAL CHEMISTRY SUPPORT

?E510G6 ORGANIC AULLYTICAL CHEMISTRY SUPPORT

?E51OHR SST ORGANIC CAREOH \& MOISTURE STIDY

?ES1OH4 SST ORGAHIC CARBON \& MOISTIRE STOYY

?ESIOHIG SST ORGAHIC CARBOH \& MOISTURE STOYY

EESIOJ2 AYLLYSIS OF TANK T.111 CORE SAMPLE ANALYYSIS

?E51OK2 SPECIAL STDIES

?E510K4 SPECIAL STODIES

?ESIOK6 SPECIAL STDDIES

TE51OL2 ORGANICS SOLUILITY STDY

IE510L4 ORGAMICS SOLBILITY STIDY

EESIOL6 ORGANICS SOLUBILITY STOYY

TE51OK2 WASTE SAYPLE AYHYYSIS (WO)

?ESIOHA WASTE SAMPLE AKALYSIS (WO)

EE510H6 WASTE SAMPLE ANALYSIS (WO)

'E5ION2 CRGANIC LAYDONN HDOLL (PNL)

iEsIOHA DEGANIC LATDONN HDOLL (PHL)

E5HONG ORGANIC LAYDOWN HOOEL (PHL)

IES1OP2 WASTE MOISTURE WISIKULANTS - FAI

IE510P4 WASTE MOISTURE WISIMULANTS - FAI

:E51OP6 WASTE HOISTURE MSIMLANTS - FAI

!ESIOR2 BASIS FOR SAFE OPS SSTS - ORGAHIC/WITRATE

IESIOR4 BASIS FOR SAFE OPS SSTS - ORGANIC/NITRATE

IESIOR6 BASIS FOR SAFE OPS SSTS - ORGANIC/WITRATE

IESIOT2 BASIS FOR SAFE OPS SSTS - ORGANIC LAYER

IESIOU2 BASIS FOR SAFE OPS SSTS - ABSORBED ORGANIC

!ESIOUA BASIS FOR SAFE OPS SSTS - ABSOREED ORGAHIC

!Es10V2

155104

:E510V6

ORGAYICS TAKKS SAFETY CRITERIA

ORGANICS TAHXS SAFETY CRITERIA

2074.0

1735.7

ThA

E51

300.0

278.1

450.0

285.5

400.2

412.2

450.0

463.5

270.0

278.1

225.0

231.8

180.0

185.4

180.0

92.7

290.0

180.0

185.4

75.0

77.3

100.8

245.9

180.0

92.7

100.0

47.7

103.0

299.4

514.8

101.9

100.0

51.5

103.0

106.1

3982.3

3778.9

2161.1

2 .

412.4

230.9 
WHC-SP-1101

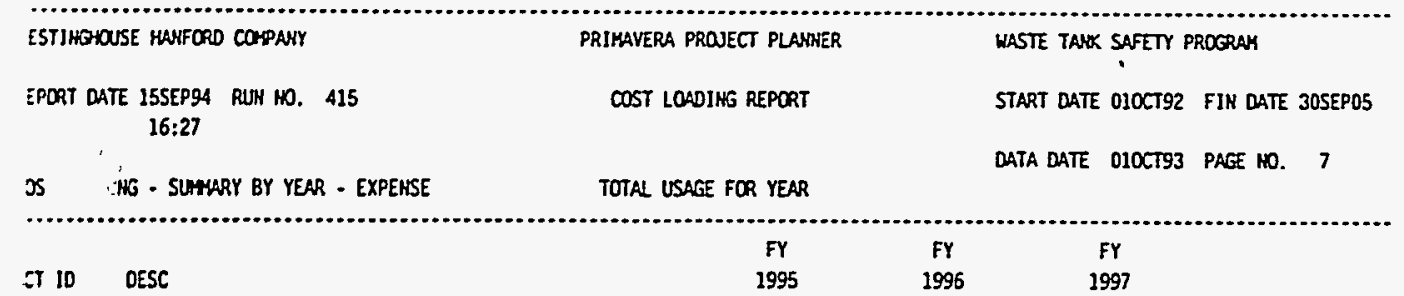

52 -

.2E52082 PROGRAY PLAN FOR ORGANIC SAFETY PROCRAY

IZE520BA PROGRAY PLAH FOR ORGAHIC SAFETY PROERAY

i2E520BG PROCRAY PLAH FOR ORGAHIC SAFETY PROCRAH

.2E520C2 ORGAHIC TAKKS WATOH LIST

ZZE520C4 ORGAHIC KATCH LIST TAKKS

2E520C6 ORGAHIC WATCH LIST TAKKS

I2E520E2 CORE DATA IKTERPRETH

;2E520E4 CORE OATA INTERPRETH

I2E520E6 CORE OATA IMTERPRETN

'OTAL $\quad E 52$

\begin{tabular}{|c|c|c|}
\hline \multicolumn{3}{|l|}{50.2} \\
\hline \multicolumn{3}{|c|}{51.7} \\
\hline & & 53.3 \\
\hline \multicolumn{3}{|l|}{24.9} \\
\hline & 25.7 & \\
\hline & & 26.4 \\
\hline \multicolumn{3}{|l|}{100.1} \\
\hline & 103.1 & \\
\hline & & 106.2 \\
\hline ana & $\ldots . .$. & ....... \\
\hline 587.6 & 411.3 & 423.7 \\
\hline
\end{tabular}

.54 .

¿2E54012 CAP MANAGEKEKT

V2E54014 CAP MAMAGEMENT

V2E54016 CAP KAVAGEMENT

I2ESAOBA INSTALL TC TREES/THACS

V2ESAOBS INSTALL TC TREES/TTRCS

N2ESAOCA PRESSURE MONITORIHG IHSTALLATIO

N2E54OC6 PRESSURE HONITCRIHG IHSTALLATION

N2E540D2 HISTURE HOHITOR EHG \& (LOW) IHSTALL

N2E5ACOA HOIST HOHITOR EHE \& LON INSTALLATIOW

H2E5AODG HOISTURE HOHITOR \& (LON) IHSTALL

N2E540E4 HOISTURE HOHITOR/CONTROL

N2E540E6 HOISTURE HOHITOR/COHTROL OEHOHSTRATION

N2E54OF2 SAFETY EVRLUATIOH/LOA

HZE' SAFETY EVALLUTIOH/LOA

TOTAL $\quad E 54$

54

21.4

\begin{tabular}{|c|c|c|}
\hline & 22.0 & \\
\hline & & 22.7 \\
\hline & 818.7 & \\
\hline & & 843.3 \\
\hline & 257.4 & \\
\hline & & 265.1 \\
\hline 267.8 & & \\
\hline & 1114.2 & \\
\hline & & 1000.6 \\
\hline & 2000.2 & 5607.8 \\
\hline 90.0 & & \\
\hline & 92.7 & \\
\hline 379.2 & 4305.1 & 7739.4 \\
\hline
\end{tabular}

ES5 -

H2E55OAA USQ CLOSURE DOCNENTATIOH

M2E550A6 USQ COOSURE DOCOMENTATIOH

TOTAL $E 55$

96.6

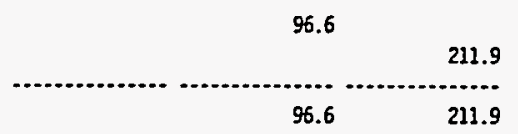

676.1

HOISTURE HONITORIHS/CONTROL EHS. hOISTURE HOHITORIHS/CONTROL EHG. SAFETY ISSUE CLOSURE DOCOMERTATIOI SAFETY ISSUE CLOSURE DOCUMENTATION

E56

\begin{tabular}{|c|c|c|}
\hline \multirow{2}{*}{070.1} & 102.6 & \\
\hline & 105.6 & \\
\hline & & 108.8 \\
\hline 676.1 & 208.2 & 108.8 \\
\hline
\end{tabular}

SEPARATIONS EOUIPHENT FABRICATION SEPARATIOHS EQUIPHEKT IHSTALL/OPER SEPARATIONS EQUIPHENT IHSTALL/OPER SEPARATIONS \& ORGANIC STORAGE SUPPORT SEPARATIONS \& ORGAHIC STORAGE SUPPORT SEPARATIONS \& ORGAHIC STORAGE SUPPORT UABORATORY AMALYSIS

EHSIHEERIHG SUPPORT

EHGIHEERIHG SUPPORT

EHGIHEERIHG SUPPORT

FAB SEPARATIONS EOUIPKENT BURIAL CONTAIHERS

SEPARATIOWS EOUIPMENT BURIAL/STORAGE

SAFETY \& PERHITTIHS

$\begin{array}{lll}400.1 & & \\ 901.0 & 153.8 & \\ 135.3 & 370.6 & 287.0 \\ & & \\ 424.4 & & \\ 635.0 & 360.4 & 178.9 \\ & 514.6 & 318.3 \\ & 253.4 & \end{array}$


WHC-SP-1101

\begin{tabular}{|c|c|c|}
\hline ESTIHFTOUSE HAKFORD COMPANY & PRIMAYERA PROJECT PLAMHER & KASTE TAMK SAFETY PROGRAM \\
\hline $\begin{array}{c}\text { EPORT DATE 15SEPS4 RUN HO. } 415 \\
16: 27\end{array}$ & COST LOADIHG REPCRT & START DATE O1OCT92 FIN DATE 30SEP05 \\
\hline OST LOAOIAS - SUTAARY BY YEAR - EXPENSE & TOTAL USAGE FOR YEAR & DATA DATE O10CT93 PAGE NO. \\
\hline
\end{tabular}

\begin{tabular}{|c|c|c|c|c|}
\hline T ID & DESC & $\begin{array}{c}F Y \\
1995\end{array}$ & $\begin{array}{c}F Y \\
1996\end{array}$ & $\begin{array}{c}F Y \\
1997\end{array}$ \\
\hline & : & & & \\
\hline 57022 & TREATMENT SYSTEM DESIGY & 400.1 & & \\
\hline i2E57024 & TREATRENT SYSTEM FABRICATIOW & & 1050.7 & \\
\hline $2 E 57016$ & TREATRENT SYSTEM FIKALIZATION & & & 52.9 \\
\hline $2 E 570 \times 4$ & TREATRENT SYSTEM INSTALL/OPERATIOHS & & 309.0 & \\
\hline i2E570K6 & TREATEENT SYSTEM INSTALL/OPERATIONS & & & 318.2 \\
\hline $2 E 570<4$ & TREAT.SYSTEH BURINL CANTAINERS-EHG DSH/EOUIP & & 82.4 & \\
\hline & E57 & 2896.0 & 3095.0 & 1155.3 \\
\hline b104] & INVESTIGATE C-105 AMOTALIES & 259.9 & & \\
\hline 1042 & CONOUCT 106-C PROCESS TEST (SI-2X) & 412.2 & & \\
\hline 1043 & DOCAREMT 106-C PROCESS TEST (TPA K-40-05) & 135.5 & & \\
\hline 3034 & RESOLVE HIGH-HEAT SAFETY ISSUE & & 339.3 & \\
\hline & RESQ_VE HIQH-HEAT SAFETY ISSUE & - & & 307.8 \\
\hline & $\mathrm{F} 61$ & 807.6 & 339.3 & 307.8 \\
\hline
\end{tabular}

(7) -

226710A3 MUST HAZARDS \& SAFETY AMAL YSES

12G71062 HUST HAZARDS \& SAFETY AVLLYSES

:2G71032 VAPOR SAPPLIHG

V2G71034 VAPOR SAMPLIHE OF 5 MUSTS

I2671035 VAPOR SAMPLIHG OF 10 RUSTS

IZG710C2 SAFETY SCREENING SAYPLIHE

ZZG71OC4 SAFETY SOREENIHE SAMPLIHG

Z2G710C6 SAFETY SCREENINO SAMPLIHG

¿26710094 MONITORIMG EOUIPHEMT UPGRADES

¿2G71005 HONITORING EQUIPYENT UPGRADES

V2G710E2 IH-TAKK VIDEO/PHOTOS

I2G71OEA IN-TAKK VIDEO/PHOTOS

Z2G710E6 IH-TANK VIDEO/PHOTOS

I2G7IOF2 RISER ACCESSIBILIT

I2G710F4 RISER ACCESSIBILIT

IZG710F6 RISER ACCESSIBILITY

H2G7IOGA MUST SAFETY ISSUE RESOUUTION

I2G710GS MUST SAFETY ISSUE RESOLUTION

TOTAL G71

526.6

203.1

346.5

662.5

760.0

$\begin{array}{ll} & \\ & 682.5 \\ .7 & 1609.4\end{array}$

181

TZHBIOA] TANK VAPOR PROGRAM MANAGEHENT

VZHBIOAA TAKK VAPOR PROGRAY HAMUGEKENT

V2HBIOBZ PHL TOXICO.OOY CONSULTATION

L2H810SA PNL TOXICOLOGY CONSULTANTION

IZHBIOCZ WEATHER STATION OPS/MAINT/CALIBRATION (PHL)

IZHB1OC4 WEATTER STATION OPS/MAINT/CALIBRATION (PHL)

I2HB1002 ATMOSPHERIC PATHAAYS (PNL)

¿ZH81004 ATHOSPHERIC PATHWAYS (PKL)

HB1

1062.7

$34.3 \quad 1609.4$

94.7

35.3

190.5

$318.3 \quad 196.3$

$655.5 \quad 675.2$

$361.3 \quad 372.2$

$\begin{array}{lll}2249.3 & 2966.9 & 3570.9\end{array}$

458.0

257.5

$55.0 \quad 56.7$

$\quad 309.0$

$958.0 \quad 974.3$

$\begin{array}{lr}\text { VAPOR FLOU HONITOR OUARACTERIZATION AYALYSIS } & 30.0 \\ \text { VAPOR FLON HOHITORIHS DEVICE (ENG SUPPT) } & 40.0 \\ \text { EXECOTE RECOMENDATJOUS FROM EEA (DSH BASELINE) } & 98.0 \\ \text { FABRICATE. INSTALL VAPOR TREATIENT SYSTEM } & 368.1\end{array}$

30.0

40.0
98.0

368.1 
WHC-SP-1101

\begin{tabular}{|c|c|c|}
\hline :STJHGHOUSE HAYFORD COKPANY & PRIMAVERA PRONECT PLANMER & WASTE TASK SAFETY PROERAY \\
\hline $\begin{array}{l}\text { PORT DATE 15SEP94 RUWH HO. } 415 \\
16: 27\end{array}$ & COST LOADING REPORT & START OATE 010CT92 FIN DATE 30 SEP05 \\
\hline G - SUHFARY BY YEAR - EXPENSE & TOTAL USAGE FOR YEAR & DATA DATE 010CT93 PAGE NO. \\
\hline
\end{tabular}

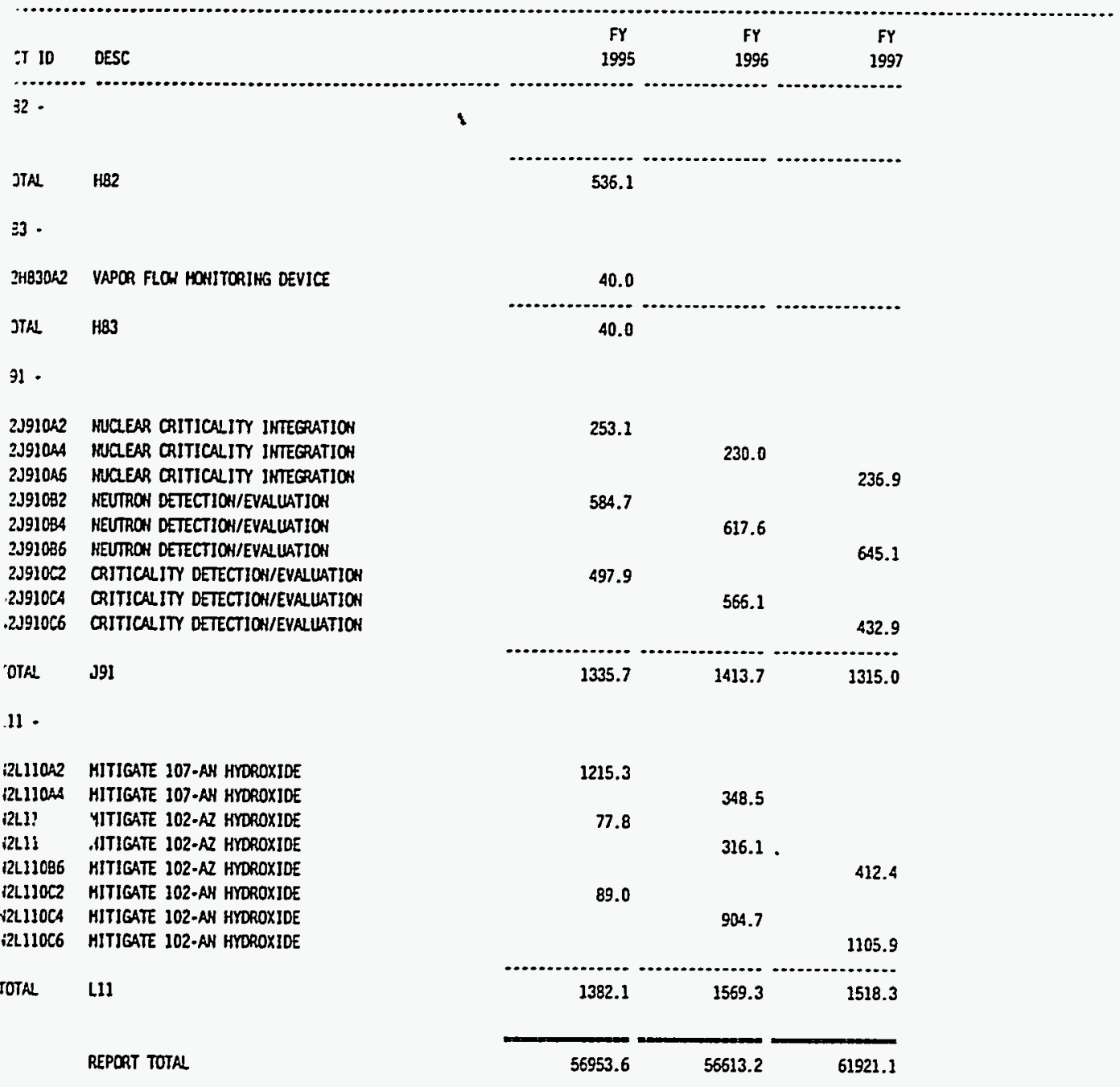


WHC-SP-1101

\begin{tabular}{|c|c|c|}
\hline -ESTIHGHOUSE HAMFORD COYPANY & PRIMAYERA PROJECT PLAYNER & HASTE TAHK SAFETY PROGRAH \\
\hline $\begin{array}{l}\text { 3EPORT DATE 155EP94 RUN HO. } 414 \\
16: 25\end{array}$ & COST LOADING REPORT & START DATE O1OCTS2 FJH DATE 30 OSEPOS \\
\hline IHG - SUHAARY BY YEAR - CAPITAL & TOTAL USAGE FOR YEAR & DATA DATE O10CTG3 PAGE $\mathrm{NO}$ \\
\hline
\end{tabular}

\begin{tabular}{|c|c|c|c|c|}
\hline :CT ID & DESC & $\begin{array}{c}\text { FY } \\
1995\end{array}$ & $\begin{array}{c}F \gamma \\
1996\end{array}$ & $\begin{array}{c}\text { FY } \\
1997\end{array}$ \\
\hline 324 . & & & & \\
\hline Y28240E04 & DACS $2 B$ OPERATIOHS 101-AN & & & 77.6 \\
\hline iZB240F00 & CAS COARACTER SYS MATERIAL - 101-AW & 337.9 & & \\
\hline P2B240FO4 & FAB \& IHSTALL GCS 101-AM & & 300.5 & \\
\hline 'i28240J08 & EHGIKEERIHS \& FEHCE MDOIFICATIOAS & & & 675.4 \\
\hline TOTAL & B24 & 337.9 & 6105.4 & 1668.1 \\
\hline
\end{tabular}

326 .

N2B260A1O DESIGH IHSTALLATJOH SUPT 103-AN

N2B260CO4 IHSTALL PUAP 103.AN

N2B260F00 GCS MATERIAL PURCHLSES AN-103

N2B260FO4 FAB \& INSTALL (GCS)

M28260F10 GAS OALRACTERIZATION SYSTEM (GCS) 103-AN

H28260JO4 REEOVAL/DISPOSAL SYSTEM 103-AM

TOTAL $\quad B 26$

327 .

N2B270A00 DESIGH PLATP 104-AN

H2B270AO4 DESIGH PUMP 104.AH

I2B280AO4 DESIGH SUPPORT PUAP 105 AH

$12 B 270 B 00$ PROCORE \& FAB PLMP 104-AH

N2B270B06 FAB \& ASSEKRLE PUTP 104-AN

N2B270COO INSTALL PREPARATIOH PUNP 104-AM

N2B27MFOZ IKSTALL PREPARATION PLKP 104-AL

N2E INSTALL TANK 104-AN MIT PUSP

H26. DACS $2 C$ SYSTEM HON

H2B270EOD DACS $2 C$ MAIHTERENCE \& OPS

N2B270FOO GAS CHURACTERIZATION SYSTEM (GCS)

TOTAL B27

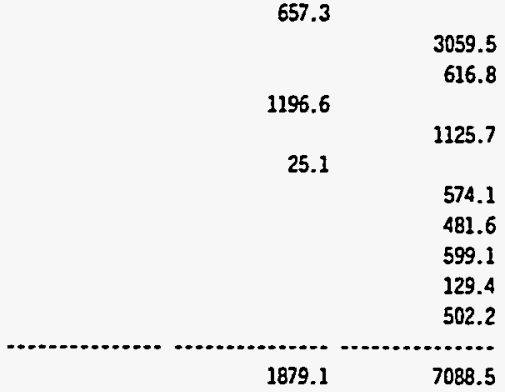

828

128280800 PROCORE PUMP 105-A

K2B280BO4 FAB PUAP 105.AN

N2B2BOCO4 INSTALL PUHP 105-AH

MEB2BOCOB COMPL TAKK 105-AH PUMP IHSTALL REVIEH

H2B2BOFOO GSS OURACTERIZATIOH SYSTEM (GCS)

RZB28OFO4 GAS CHARACTERIZATION SYSTEH (GCS)

HZB280SOO REHOVAL/DISPOSAL SYSTEM

TOTAL B28

1232.6

1125.7

600.0

189.6

309.6

291.8

106.1

3855.4

COII.

MZC310DO7 PNL AGIMG WASTE STOIES

N2C310D19 PHL COKPARE REAL HESTE H/SIHULATED KASTE

N2C310GO2 HOSSBAUER SPECTROMETER

M2C31OHOS IH.SITU RELATIVE HUMITITY INSTRLMENTATION

H2C31ONO4 IMERARED SPECIATIOW TRAYSFER

TOTAL COI

C34.

M2C34OB14 IHSTALLATIOH OF TC TREES/HOOKUPS THACS H2C340:12 MEUTROH PROBE MDISTURE HOHITOR LOH ENGR H2C3AODO2 SURFACE HOISTURE HEASURIHG

H2C3AMA? SO SURFACE MOISTURE MEASURIHG (CPAC) FY96

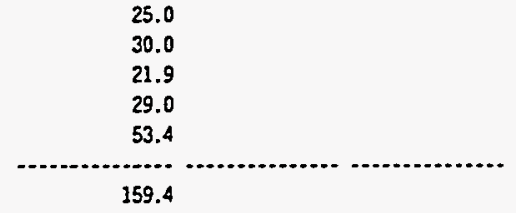

184.2

179.7

164.9 


\section{WHC-SP-1101}

PRIMAVERA PROJECT PLAMHER

COST LOADIKG REPORT
WASTE TAYK SAFETY PROGRAY

START DATE O1OCT92 FIK DATE 30SEP05

DATA DATE O1OCT93 PAGE NO. 3

.2ST LOADIHG - SUAARY BY YEAR - CAPITAL

TOTAL USAGE FOR YEAR

\begin{tabular}{|c|c|c|c|c|}
\hline & & $F Y$ & FY & FY \\
\hline CT ID & DESC & 1995 & 1996 & 1997 \\
\hline & : & & & \\
\hline 20340030 & SURFACE MOISTURE MEASURIHS (CPAC) FY97 & & & 251.3 \\
\hline $2 C 340=10$ & PRESSLRE HONITORIHG DESIQN \& EHGR FECH TAKKS & 1020.0 & & \\
\hline $2 C 340 F 14$ & PRESSLRE HONITORIHS EMGIHEERIHG \& INSTALLLATIOH & & 429.3 & \\
\hline $2 C 340=18$ & PRESSURE MONITORIHS IHSTALLATION FECN TAKXS & & & 193.4 \\
\hline 20340600 & ISSU STATUS REPT OH LOU INSTALL ACTIVITIES & 893.5 & & \\
\hline 20340520 & MEUTRON PROBE MOISTURE MONITOR LON IMSTALL & & 756.7 & \\
\hline $2 \mathrm{C} 340 \mathrm{HOS}$ & IR SCAN DEVICE HOO \& SCANS & & 230.6 & \\
\hline $2 C 340 \div 20$ & IR SCAN DEVICE HDO \& SCANS & & & 109.3 \\
\hline 26340000 & ELECTRONAGETIC INDUCTION (EMIP) & 282.6 & & \\
\hline $2 \mathrm{C} 340 \mathrm{2} 20$ & ELECTROHAGNETIC INDUCTION (EMIP) & & 547.3 & \\
\hline $2 C 340430$ & O7OCTRONAGETIC INDUCTION (EMIP) & & & 565.9 \\
\hline OTAL & C34 & 2724.9 & 2207.7 & 1119.9 \\
\hline
\end{tabular}

al.

2DA1OADO WASTE BEHAYIOR MOOELING FY9S

2D41OADS WASTE BEHAVIOR HOOELIHG FY96

20410A0B WASTE BEHAVIOR HOOELIHE FY97

$20410 C 00$ GAS GENERATIOH MEOUAKISYS FY95

20410220 GAS GENERATION MECHAHISHS FY96

$20410 E 00$ GAS RETERTION REOAANISHS FY95

20410E06 GAS RETERTIOH MECHANISHS FY96

OTAL DA1

$42-$

20420420 VAPDR SPACE HONITORIHG FY96 20420430 VAPOR SPACE MONITORINE FYG7

20420520 WASTE BEHAVIOR EVALLLTION FY96

20:20:30 WASTE BEHAVIOR EVALUATION FY97

20420GO4 RETAIHED GOS EVALUATIOW FY95

OTAL $\quad$ D42

50.0

154.5

$\begin{array}{ccc} & 154.5 & \\ 75.0 & & 106.1 \\ 105.0 & 20.6 & \\ & & \\ & 25.8 & \\ 230.0 & 200.9 & 106.1\end{array}$

51.5

180.2

53.1

318.3

20.0

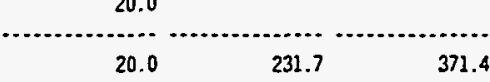

44

20440002 VIDEO SYSTEN UPGRADSS FY95

ZD440D20 VIDEO SYSTEM UPGRADES FY96

20440E10 TEKPERATLRE UPGRADES FY95

20440E20 TEMPERATIRE UPGRADS FY95

20440530 TEMPERATRE UPGRADSS FY97

20440F02 SURFACE LEVEL UPGRADES FY95

20440002 AN FARY VERT UPGRADES FY95

20440G14 AN FAPY VENT UPGRADES

$20440: 20$ AN FARY VENT UPGRADES

20440524 AN FARY VENT UPGRADES

20440530 PORTABLE EXHLUSTER FOR SSTS

20440HD5 GAS MONITOR UPGRADES FY95

2044OHIO GAS MONITOR UPGRADES FY9S

ZDA4OKG2 INST ATONIA MONITORS 241-AH/AN FAPHS

20440:02 MISC TANX FARK EOUIPHENT UPGRADES

20440LOS MISC TANX FARH EOUIPHENT UPGRADES

2044OMOO INSTALL DST/SST GAS HONITORS (SI $2 \mathrm{~h}$ )

OTAL

044

5!

2ESIOA2 PKL PROJECT MARAGENERT

2E51032 ORGANIC WASTE ENERGETICS

377.0

659.7

679.6

276.9

597.8

879.6

2010.6

1035.5

549.0

551.7

163.9

152.5

1382.3

1423.9

845.3

$\begin{array}{lll}6144.8 & 5473.1 & 3139.0\end{array}$

115.0

155.0 


\section{WHC-SP-1101}

¿STIHJHOUSE HAYFOFO COMPANY

PRIKAVERA PROUECT PLAKHER

COST LOADIHG REPORT
WASTE TAKK SAFETY PROGRAY

START DATE O1OCT92 FIH DATE 30SEP05

O. IH - SUHAARY BY YEAR - CAPITAL

TOTAL USAGE FOR YEAR

\begin{tabular}{|c|c|c|c|c|}
\hline & & FY & FY & FY \\
\hline$\therefore$ T ID & DESC & 1995 & 1996 & 1997 \\
\hline 51 & өкан, & & & \\
\hline .2E510S2 & OREAHIC AUALYTICAL CHEMISTRY SUPPORT & 200.0 & & \\
\hline TDTAL & E51 & 470.0 & & \\
\hline
\end{tabular}

DTRL E5I

470.0

$\$ 54$.

:2E540BA IHSTALL TC TREES/TMACS

:2E540B6 INSTALL TC TREES/TMACS

IZESAOC4 PRESSLRE HONITORIHG INSTALLATION

NZESSOCG PRESSURE HONITORINO INSTALLATIOH

MZES4O02 HOISTRRE HOHITOR EHE \& (LOH) IHSTALL

MZESAODA HOIST HOHITOR EHS \& LON IHSTALLATIOH

Hi2ESAODG HOISTURE HOWITOR \& (LON) JHSTALL

H2ESAOE4 HOISTRE HOHITOR/COUTRO

N2E540E6 HOISTRE HONITOR/CONTROL OEHOHSTRATJOH

TOTRL E5A

327.9

$3736.1 \quad 337.8$

132.8

183.6

$275.8 \quad 285.4$

5529.4

15501.2

$\begin{array}{llr} & & \\ 183.6 & 9869.3 & 16257.3\end{array}$

213.5

399.8

DESIGH SEPARATIOHS EQUIPHENT BURIAL CONTAINERS TREAT.SYSTEH BURIAL CANTAIMERS-ENG DSH/EQUIP

TREATMEKT SYSTEK BURIAL CONTAIH. EQUIP

E57

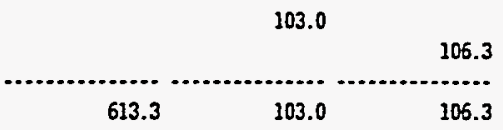

671

M2G7IOUA HONITORIHS EOUIPMERT UPGRADES

H2G710D6 MONITORIHS EQUIPMEKT UPGRADES

TOTAL $\quad 671$

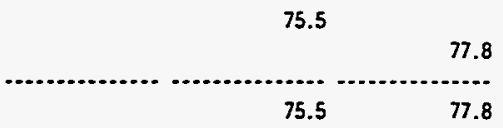

HB2

H2H82002 FABRICATE, INSTALL VAPOR TREATMENT SYSTEM

TOTAL $\quad H 82$

602.5

J91

H2J910B2 HEUTROH DETECTIOW/EVALUATIOW

N2J910B4 HEUTRON DETECTION/EVALUATION

N2J91086 HEUTRON DETECTIOW/EVALUATIOW

H2J910C4 CRITICALITY DETECTION/EVALLATION

N2JSIOC6 CRITICALITY DETECTION/EVALLATIOH

TOTAL J91

L11.

Hi2LIIOL2 MITIGATE 107-AH HYDROXIDE

H2LIIOCA HITIGATE 102-AH HYOROXIDE

TOTAL , LII

11

437.3

$534.4 \quad 437.3$

REPORT TOTAL

27018.1

32579.0

36654.8

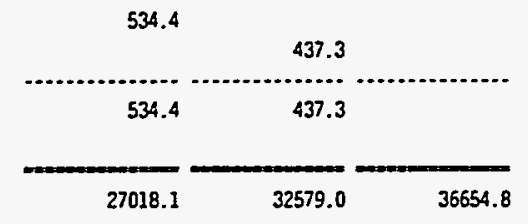

F $7.4-7.13$ 


\section{WHC-SP-1101}

:A2 EXEEYSE FYgS

TECHNICAL INTEGRATIOH \& PLANHING

:EPOتTi JATE 15SEPg

$$
11: 33
$$

HORK PACXAGE TOTALS

START DATE 010CT92 FIN DATE O7JULDO

:REPARED SY NL SIMON 373-2587

-TS FRDERAY MANAGEKENT \& OA

\begin{tabular}{|c|c|}
\hline $\begin{array}{c}\text { ailit } \\
10\end{array}$ & OESCKIPTION \\
\hline & \\
\hline
\end{tabular}

OA

:27:Ci:2 EASELINE PLANING FY95

\begin{tabular}{|c|c|c|c|c|}
\hline \multirow[t]{7}{*}{2500300794} & 29SEPQS & IK2A110A01 L 7א210010 & 3812 & 107017 \\
\hline & & IK2A110A02 0 TK21021C & 1300 & 1301698 \\
\hline & & 1N2K110A03 L 7M610010 & $\Delta 983$ & 294296 \\
\hline & & IN24110406 L 7CK00010 & 3624 & 214033 \\
\hline & & $1 \times 24110401<7 \% 210010$ & 6795 & $40 \mathrm{j} 313$ \\
\hline & & JK2A110AOS L 7MA00000 & 453 & 10473 \\
\hline & & 1N2h110AOS L 7M400010 & 4983 & 294295 \\
\hline $250030 \leq T 94$ & 2OSEPOS & JN2A110A05 O $7 \AA 21030 C$ & 478 & 478000 \\
\hline
\end{tabular}

DAUIOSR MAMAGEFENT \& INTEGRATION FYSS

250 030:T94 29SEPS5 1K2\&110301 L 7\$200010 IN2A110B03 0 7R2003DC IN2A]10BO4 L 67021010 1К2A110B05 L 7K200000 1K2A110B06 L 7P2000CO JH2AIIOBOI L 7K200010 1K2A110BOI $47 \$ 200 j 40$ 1K2A:10801 H 7R200190 1Ki2A?10801 O 7R200270 $1 N 2 A 110 B C 207 \mathrm{k} 2004 C 0$ IN2A110BOL O 7R2005PO 1K2A110801 L 7\$200010 1N24110B02 L 7R2A0010

BLDGET BUDEET OUANTITY COST

OUR START FINISH ACCOUNT

c..........

$24428 \quad 3101124$ BHijXiL WUISORY SUPPORT FYOS

250 030TTg4 2OSEPSS IK2AI1OKOI O 7E900210 1К24110K03 L 88?00010

\begin{tabular}{rr}
280 & 341600 \\
540 & 31892 \\
\hline 820 & 373492 \\
& \\
44030 & 5307494
\end{tabular}

F7.4-7.14 
WHC-SP-1101

$\because$ EXPENSE FY95

TECHNICA INTEgRaTION \& PLANNING

:VGET DLTE 15SEPSA

$31: 38$

MORK PACXAGE TOTALS

START DATE 010CT92 FIN DATE 07JUL0O

:EFARED SY NIL SIHOW 373-2587

- PROGRLY MALEERENT \& OA

- inlit DESchiption

$: 2$

DUR START FINISH ACCOWT

DATA DATE OMOCT93 PAGE nO. I

.$i$

2

:AIIOROS SASELINE PLANMINE FY96

251 020โT95 30SEP96 JN2A]10ADI $\perp$ 7R210010

IN2A110A02 O 7R21021C

]N2A130A03 L 7M610010

IN2A110A06 L 7CX00010

IN2A110A01 $\llcorner$ 7R210010

IN2A110AD4 $L$ 7M400000

1N2A110A04 L 7M400010

BUDGET

BUDETT

OUATITT COST

:

GE

2E:1OBOE MALEGENENT \& INTEGSATION FY95

251 020CT95 30SEP96 1N2A110B01 $L$ 7R200010 IN2A110BO2 \& 7R2A0O10 JK2A110B03 O 7R2003DC IN2A110B04 L 67021010 IN2A110B05 ᄂ 7R200000 INZAIIOBO6 L 7R200040 IN2A119BOI $\llcorner 7 R 200010$ IN2A110BO1 M 7R200140 JK2A110BO1 M 7R200190 IN2A110BO1 O 7R2002TO INZA110BO1 0 7R2OO4CO 1N24110BO1 0 7R2005PO ]N2A110BO1 L 75200010

\begin{tabular}{rr}
1812 & 110224 \\
1000 & 1030000 \\
4983 & 303116 \\
3624 & 220448 \\
6795 & 413340 \\
453 & 10790 \\
4963 & 303116 \\
\hline 23650 & 2391034
\end{tabular}

$23650 \quad 2391034$ IAHOKOS NUISOAY SLPPOAT FYOE

\begin{tabular}{|c|c|c|c|c|}
\hline \multirow[t]{3}{*}{2510200795} & 30SEP95 & $\begin{array}{l}\text { 3N2A110K01 } 0 \text { 7E900210 } \\
\text { IN2A310K03 } \angle \quad 65100010\end{array}$ & $\begin{array}{l}280 \\
540\end{array}$ & $\begin{array}{r}351 \& 48 \\
32 E 54\end{array}$ \\
\hline & & & 820 & 384702 \\
\hline & & & & \\
\hline
\end{tabular}




\section{WHC-SP-1101}

:1.2 EXFEISE FY96

:EPOAT DATE 15SEP94

$11: 38$

:FT BY ML SIMON 373.2567

- ah mavagerent \& oa

\section{ETIVITY OESCRIPTION}

10

$\div ! 2$

Oh
TECANICAL INTEGGATIOH I PLANNING

MORK PACKAGE TOTALS

START DATE O1OCT92 FIK DATE 07JULOO

DATA DATE O1OCT93 PATE ND. 2

ORIG EARLY EARIY COST RESOURCE BUDET BUDGET

DLR START FINISH ACCOUKT

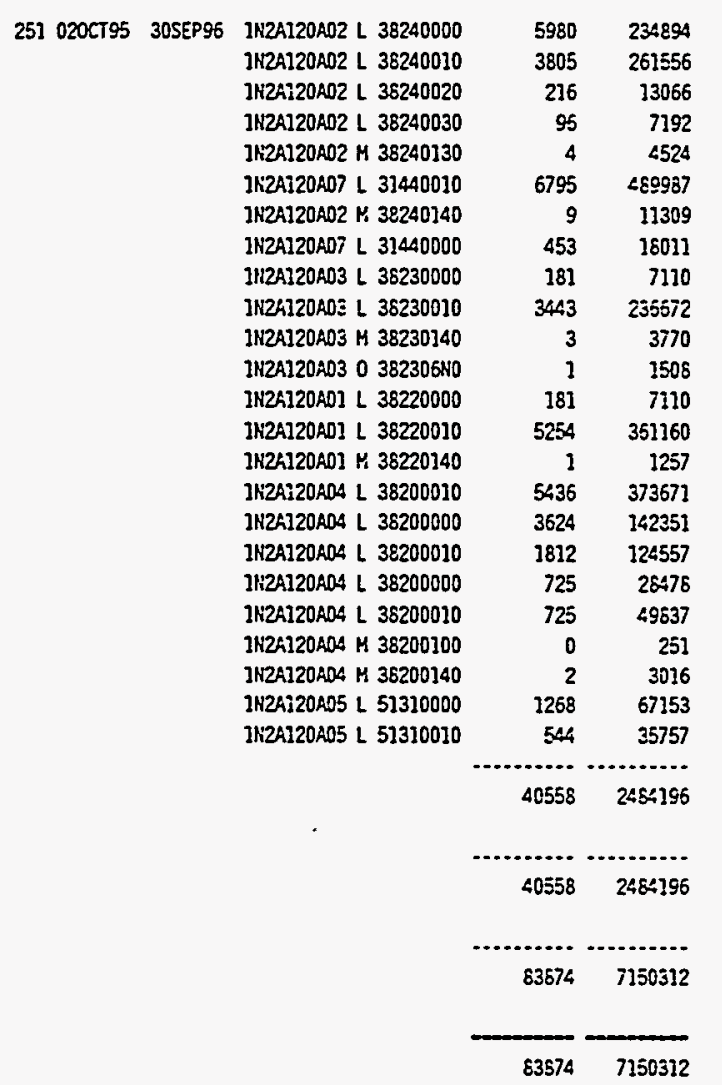


WHC-SP-1101

\begin{tabular}{|c|c|c|c|c|c|c|}
\hline$\because$ EYDERSE FYOY & & & TECH & NICAL INTE & GRATIOA \& & PLANKING \\
\hline $\begin{array}{r}\text { EPOST DRIE 15SEPQ4 } \\
11-41\end{array}$ & HORK PACKAGE TOTALS & & START DATE & E $010 C T 92$ & FIN DATE & 07Ju 00 \\
\hline $\begin{array}{l}\text { SEPARED BY HL SIHOH 373-2567 } \\
\text {-S PROSRAY MANAGEFENT } 8 \text { OA }\end{array}$ & & & DATA DATE & 010 Tा93 & PAGE NO. & 1 \\
\hline $\begin{array}{l}:=\text { IVITY DESCRIPTION } \\
10\end{array}$ & $\begin{array}{l}\text { ORIG EARLY } \\
\text { DUR START }\end{array}$ & $\begin{array}{l}\text { COST } \\
\text { ACCOUNT }\end{array}$ & RESOLRCE & $\begin{array}{l}\text { BUDGET } \\
\text { OUANTITY }\end{array}$ & $\begin{array}{l}\text { BUDGET } \\
\text { COST }\end{array}$ & \\
\hline
\end{tabular}

$\because: i$

OA

1.3:110A12 BASELINE PLANNING FY97

hZA]10B12 MANAGEHENT \& INTEGRATION FY9?

or

RPHIOKOS AOVISORY SLPPORT FY97

\begin{tabular}{|c|c|c|c|c|}
\hline \multirow[t]{8}{*}{$251010 C T 96$} & $305=297$ & 1K2A110ADI L 7R210010 & 1812 & 112540 \\
\hline & & 1K2A110A02 O 7R21021C & 2000 & 2122000 \\
\hline & & IK2A110A03 L 7M510010 & 4983 & 312235 \\
\hline & & 1K2A110A06 L 7CK00010 & 3624 & 227080 \\
\hline & & IK2A110A0I L 7R210010 & 6795 & 425775 \\
\hline & & 1K24110A04 L 7M400000 & 453 & 11112 \\
\hline & & IN2AI10A04 L 7M400010 & 4963 & 312235 \\
\hline & & & 24650 & 3523976 \\
\hline
\end{tabular}

251 010CT96 30S:Е $\$ 7$ 1K2A110BO1 L 7R200010 1K2AI10BO2 L 7K2A0010 IN2Ri10BO3 O 7R2003DC 1K2A110BO4 L 67021010 1K2A130805 L 7R200000 1K2A110306 L 7R200040 1K2:? 10B01 L 7R200010 1K2A110Bo1 M 7K200140 1N2AijOBO1 H 7R200190 1H2A110501 O 7R2002TO 1K2A110BOI O TR2004CO 1K2A110BO1 O 7F2006PO IKi2A110801 L 7R200010

\begin{tabular}{rr}
1820 & 114041 \\
1620 & 114041 \\
800 & 848800 \\
1620 & 66758 \\
7272 & 178362 \\
2273 & 46642 \\
1820 & 114041 \\
40 & 51777 \\
100 & 129442 \\
20 & 25888 \\
35 & 45305 \\
120 & 155330 \\
906 & 56770 \\
\hline $1 . . \ldots$ & $\ldots . .$. \\
18846 & 1947218
\end{tabular}

251 010CT95 325E天ET lW2A110K01 0 7E900210 1K2:310K03 L 88100010

$\begin{array}{rrr}280 & 362438 \\ 540 & 33842 \\ \ldots \ldots . .2 & \\ 820 & 395279 \\ & \\ & \\ 44316 & 5867473\end{array}$


WHC-SP-1101

:Hi? E)=EHSE FYgY

TECHNICAL INTEGRATION 6 PLANHINS

:EFR̆: DRTE IESEPQ4

$11: 41$

MORK PACKAE TOTALS

START DATE O10CT92 FIN DATE OJJULOO

IY HL SIHOH 373.2587

DATA DATE O1OCT93 PAGE NO. 2

ATS AY MAMRESFENT \& OA

ORIG EARLY EARLY COST RES RESOURCE BUDGET BUDGET
SEIIV:TY DESCKIFTION

FIMISH ACCOUNT

QUANTITY COST

$\therefore 12$

on

VIZAL2LKOE WASTE TAHK SAFETY OA FYG7

251 010CT95 30SEP97 1K2A120A02 L 36240000 1K2A120A02 L 36240010 IH2A120A02 L 36240020 ]N2A120A02 L 38240030 IN2A120A02 H 38240130 1K2A]20A07 L 3]440010 7N2A]20A02 M 38240140 IN2A120A07 L 31440000 1W2A120A03 L 35230000 1N2A120A03 L 36230010 1\%2A120A03 M 38230140 1K2A120AD3 O 362306NO IN2A120A01 L 38220000 1K2A120A01 L 36220010 1K2A120A01 $\mathrm{r} 38220140$ IK2A120A04 L 38200010 11:2A120A04 L 38200000 1H2R120A04 L 36200010 IN2A120ROA L 38200000 I1124120A04 L 36200010 2N24120404 M 36200100 1H2A120ADE M 36200140 IH2A120A05 L 51310000 112A120A05 L 51310010

\begin{tabular}{|c|c|}
\hline 5980 & 241951 \\
\hline 3805 & 269394 \\
\hline 216 & 13459 \\
\hline 95 & 7409 \\
\hline 4 & 4650 \\
\hline 6795 & 504733 \\
\hline 9 & 11650 \\
\hline 453 & 18555 \\
\hline 181 & 7323 \\
\hline 3443 & 243764 \\
\hline 3 & 3883 \\
\hline 1 & 1553 \\
\hline 181 & 7323 \\
\hline 5254 & 371983 \\
\hline 1 & 1294 \\
\hline 5436 & 384869 \\
\hline 3624 & 146627 \\
\hline 1812 & 128290 \\
\hline 725 & 29334 \\
\hline 725 & 51330 \\
\hline 0 & 259 \\
\hline 2 & 3107 \\
\hline 1268 & 69169 \\
\hline 544 & 36834 \\
\hline 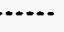 & 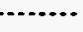 \\
\hline 40558 & 2558753 \\
\hline ....... & ............ \\
\hline 40558 & 2558753 \\
\hline ...... & ............ \\
\hline 84874 & 8426225 \\
\hline 84874 & 8426226 \\
\hline
\end{tabular}




\section{WHC-SP-1101}

ZXFEUSE FY95

or

EN1OLOG PROGRAH MANAGEKENT FYGS

03

?E210BO4 PROJECT MAMAGEMEIIT FY95

of

25210D34 PIL HYDROEEN MITIGATION PRD HONT

2E2: CDJ6 PHL TANK SPECIFIC MITIGATION

282J000S PIIL COMPUTATIOHLL MDDELING BESELIME

.25210009 PHL ISSU TEST PLAN DILUTION TESTING 103-SY

,23210DII DATA MANAGEMENT BASELINE

:2B2ION1? DRTA AIALYSIS BASELIHE

:2E: , PHL INSTRUENTATION DEVELOPRENT

.2B' LTR REPT VOID/VIOSCOKETER CHAFACTER 101-SY

of

"2E210:00 SAEETY ANALYSIS UPDATE SY-101 (LANL)

:2B210E02 LAKL BUILO DACS 2A SYS FOR SY FARM

HZE2J0E06 LANL SAETYY ANALYSIS 103-SY/IN-TANK DILUTION

HEE2JOEI2 LAIL GAS HOHITORING SUPPORT

K2E210EI4 IH-TAHK DILUTION SUPPORT (LANE)

622

H2E220E00 DACS MAIHTENANCE \& OPERATION FY95
250 030CTS4 295EP95 IN2E:10AOI L 7R240C10 $1812 \quad 107017$

1N252IOA01 O 7R2403RO $103 \quad 109601$

1N2E?10A03 L 80210010 $2718 \quad 198849$

1N2E310A04 L 7ES10010 $3624 \quad 214033$

IHi25210A05 L 7E870010 $3624 \quad 214033$

$13881 \quad 643534$

250 030CT94 295EPS5 IN2E:0301 L 7E620010 $1812 \quad 107017$

llizE:10501 \ 7E620010 $9060 \quad 535084$

$1 K 25 \div 10302 \perp 60220010 \quad 1812 \quad 132566$

$1 K 2 E 210302$ L $80220000 \quad 4530 \quad 142967$

17214917633

250 C30CTS4 295EPS5 1K25Z;0D01 O 7R2403D: $355 \quad 355000$

250 030CT94 295EP95 1N25210001 O 7R24030C $677 \quad 677000$

250 030CT94 20SEPOS 1W2EZ10D01 O 7R2403DE $323 \quad 323000$

250 030CT94 295EPS5 1K2EZ10201 O 7R24030C $591 \quad 591000$

250 030CTA4 20SEPS5 1N2E210D91 O 7R24C3DC $202 \quad 202000$

250 030CT94 20SEP95 1K2EZ10001 0 7R.24030C $\quad 190 \quad 190000$

250 030CT94 295EPES 1N2E2103010 7R24C3DC $200 \quad 200000$

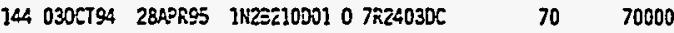

26082608000

250 030CT94 2OSEPOS 1KZEZ:0EC5 0 7K24021C 250 030CT94 2OSEPOS 1NZEZIJEO1 0 7R24021C 231 030T794 0ISEPS5 1K2EZ:2E06 0 7R24021C 250 030CT94 29SEP95 1K2E2:0504 0 7R24021C 250 C30CT94 295EP95 INZE2ICEO4 O 7R24021C

\begin{tabular}{|c|c|}
\hline 518 & 518000 \\
\hline 108 & 108000 \\
\hline 2200 & 2200000 \\
\hline 400 & 400000 \\
\hline 440 & 440000 \\
\hline 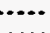 & ;....... \\
\hline 3666 & 3665000 \\
\hline
\end{tabular}

2500306794 20SEPS5 JK25220501 L 60210910 $7248 \quad 530264$ IN2E220EO2 L 6D520000 $3524 \quad 199356$ IN2EZ2REC3 \& 7C930050 $3624 \quad 154854$ 
WHC-SP-1101

$\therefore$ EXPENSE FY9S

HMOROEEM MITIGATIOH

:P0FT DATE 15SEPQ4

12:22

MORK PACKAGE TOTALS

START DATE O1OCT92 FIN DATE O7JULO

-E' YY IIL SIHON 373.2567

DATA DATE O1OCT93 PAGE NO. 3

is :1TIGATICH

:IIVITY DESCRIPTIOH

ORIG EARLY EAR Y COST

RESOURCE BUDGET BUDEET

ID

DUR START FINISH ACCOUNT OUANTIT COST

$\vdots 2$

on. $\vdots 23$

OD

:Z2230D00 DACS 2A SYSTEH MGT

$O G$

N22230600 VISCOYETER 103-SY SUPPORT

M2E2SUSUA WOIDYETER 103-SY SUPPORT
250 030CT94 20SEPS5 IN28230001 L 80210010 JN25230DO1 0 8D2103RO ]N2B230002 L 7CH20010

\begin{tabular}{rr}
2643 & 193362 \\
92 & 97612 \\
380 & 22443 \\
\hline 3115 & 313417
\end{tabular}

E3 O1NOV94 O34ARES IN26230G01 L 8D210010 ]N25230502 L 7CH30010 IN2B230503 L 7CC50010 1N23230502 L 7CC50060 1N26230504 \& 33340060 1N28230004 L 33340010 $1 N 20230505$ L 7 C930010 JN26230G0S L 7C930050 IN23230505 L 7C9303\%0

106 030-7T94 07ref55 1N252303J1 L 80210010 IN2B230501 L 60210000 $1 \% 28230502$ L 7 CH30010 $1 N 28230503 \perp 7 C C 60010$ $1 \times 28230003\lfloor 7 C 660000$ ]К23230304 \& 33340060 1К25230जग4 L 33340010 $1 \kappa 23230605$ L 7C930010 1N26230c05 \& 7C930060 $1 \Re 262306050769303 R 0$
$2802 \quad 218093$
$45575 \quad 3262462$

$\ldots$


WHC-SP-1101

R2 EXFENSE FYOS

HYOROSEN MITIGATJON

EFOTI DRTE 15SEPSA

$12: 22$

¿EPARED BY NL SIHON $373-2587$

DROEEN MITIGATIOH

oivitr DESCRIPTIOA

iD

RESOLRE BUDGET BUDGET

MORK PACOAGE TOTALS

START DATE DIOCT92 FIN DATE OJJU.00

DATA DATE DIOTT93 PAGE NO. 4

0.

2E230J00 REYOVAL/DISPCSAL SYSTEM 103-SY

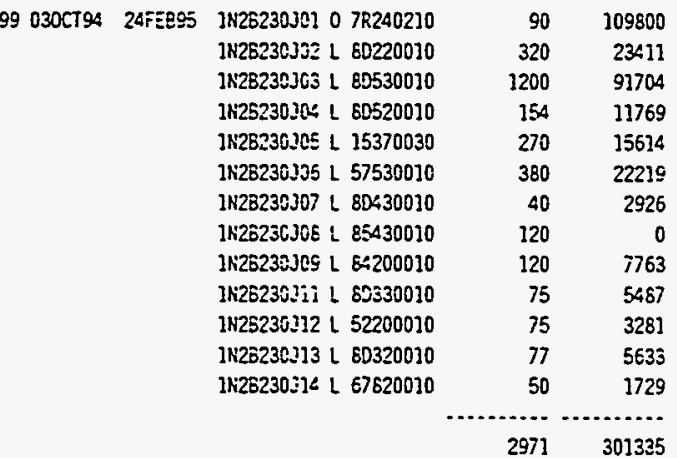

oK

22330100 1K-TANK DILUTION TEST PREP 103.SY

250 030CT94 2OSEPSS 1N2B230KCI L TE620010 $1 N 26230 K 01$ \% 7E620100 IN2623CKOI L TE620210 IN28230KO2 L 7C900060

\begin{tabular}{rr}
14496 & 856134 \\
480 & 585600 \\
250 & 305000 \\
2718 & 116140 \\
$\ldots \ldots .$. & $\ldots \ldots \ldots$ \\
17944 & 1862874 \\
& \\
\hline 27256 & 2994030
\end{tabular}

388 C30CT94 30JtN95 1N23240501 L 80210010 $1 \mathrm{i} 28240552 \mathrm{~L}$ 7CH:300]0 1 1K28240502 L 7CC60010 1N23240533 L 7CC60000 IN2B24053: L 33340050 1N2B240334 L 33340010 1N2B240055 L 7 C930010 IN2B240:AS L 7C930060 $1 \mathrm{~N} 2 \mathrm{P} 2403 ; 507 \mathrm{CS} 303 \mathrm{R} 0$

166 02NOVg4 30ILNGS 1N262403i! L E 210010 IN2B240501 \& 80210000 1א26240332 L 7CH30010 ]N26240333 L 7CC60010 ]พ220240003 L 7CC60060 IN28240304 L $\$ 3340060$ 1N26240504 L 33340010 IN26240S? I 7 CS30010 JN28240G05 L 7C930060 1N28240305 L $7 C 9303 R 0$

$\begin{array}{rr}435 & 31625 \\ 145 & 8564 \\ 70 & 4134 \\ 70 & 1618 \\ 104 & 5147 \\ 37 & 2241 \\ 37 & 2185 \\ 70 & 2991 \\ 84 & 89124 \\ 378 & 27654 \\ 189 & 5965 \\ 189 & 11162 \\ 210 & 12403 \\ 210 & 8973 \\ 314 & 15540 \\ 111 & 6722 \\ 111 & 6556 \\ 210 & 8973 \\ 252 & 267372\end{array}$




\section{WHC-SP-1101}

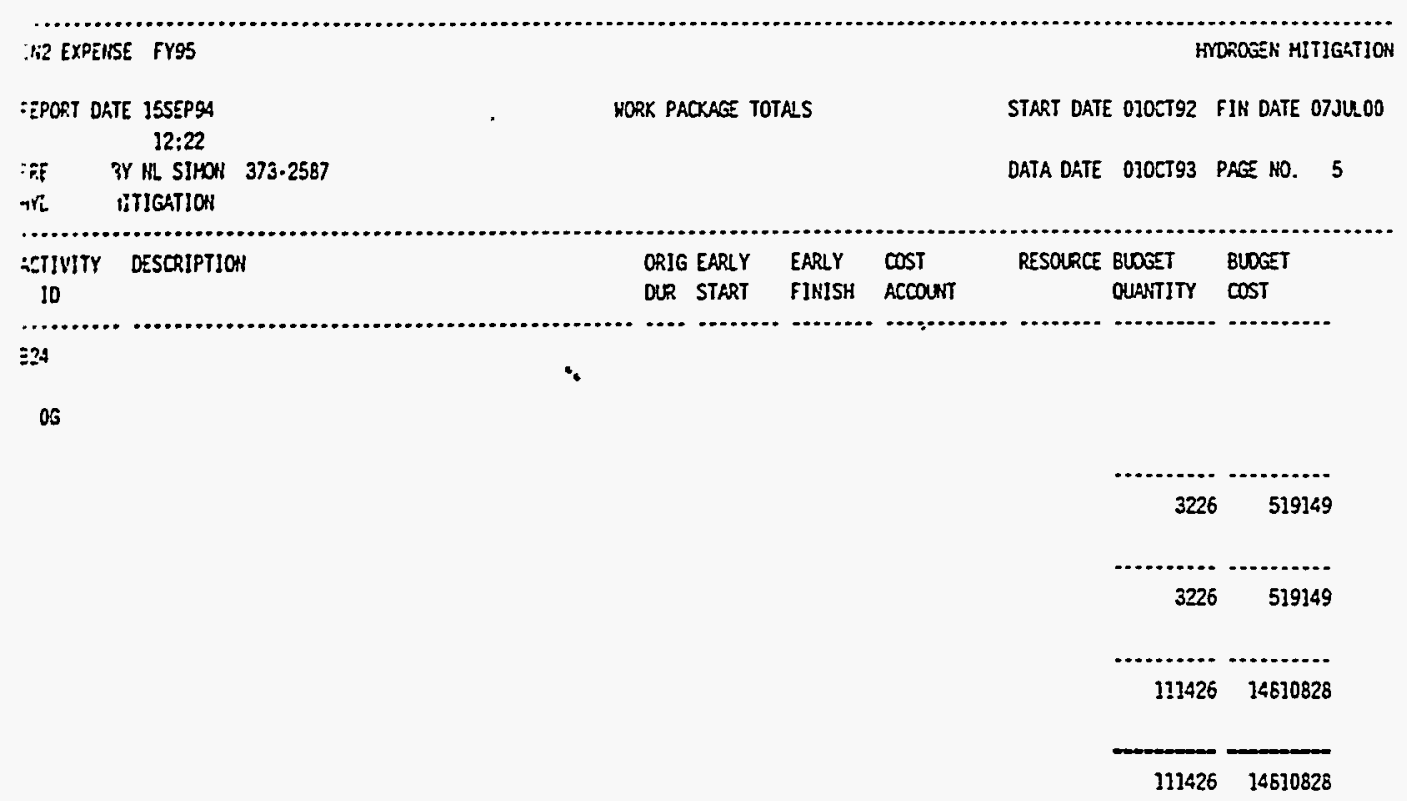




\section{WHC-SP-1101}

$\because$ IPJTAL FY9S

ミニテAT DATE ISSEPQA RLW NO. 358 $12 \cdot 48$

:EVARED BY NL SIMON 373-2567

-cles: Hame: $\mathrm{N2O3}$

ОOËN HITIGRTIOA

EARLY
START

02

Z=10005 PRL COPUIATIONAL MODELING BASELINE

MORK PACAKGE TOTALS

START DATE O1OCT92 FIN DATE O7JU:O0

DATA OATE OLOCT93 PAGE NO. I

$\because$

ב:10:02 LAKL BUILC DACS 2A SYS FOR SY FARM

\begin{tabular}{|c|c|c|c|c|c|}
\hline 030ст94 & 29SEPS5 & 2N28510D01 & $7 \pi 24030 \mathrm{C}$ & $\begin{array}{l}260 \\
\ldots \ldots \ldots \\
260\end{array}$ & $\begin{array}{l}260000 \\
\ldots \ldots \ldots \\
260000\end{array}$ \\
\hline 030टT94 & 29SEPSS & $2 \mathrm{~K} 22510 \mathrm{Cl}$ & $7 R 24021 C$ & $\begin{array}{l}4165 \\
\ldots 165 \\
\ldots \ldots . . \\
4425\end{array}$ & $\begin{array}{l}4165000 \\
4165000\end{array}$ \\
\hline $030 \approx 794$ & 29SEPSS & $2 \times 22520=0 ?$ & SD21010C & $\begin{array}{l}150 \\
\ldots \ldots \ldots \\
150\end{array}$ & $\begin{array}{l}150000 \\
150000\end{array}$ \\
\hline 030乞T9: & 29SEPSS & $2128520 F 01$ & $8021010 \mathrm{C}$ & $\begin{array}{l}100 \\
\ldots \ldots \ldots \\
100\end{array}$ & $\begin{array}{l}100000 \\
100000\end{array}$ \\
\hline 23NOV94 & 25APR95 & $\begin{array}{l}2 k 23520 G 21 \\
2 k 2 E 520022\end{array}$ & $\begin{array}{l}85100010 \\
52700060\end{array}$ & $\begin{array}{l}1270 \\
2127 \\
\ldots \ldots \ldots . . . \\
3397 \\
\ldots \ldots \ldots \\
3647\end{array}$ & $\begin{array}{l}75514 \\
96842 \\
\ldots \ldots \ldots \\
172357 \\
\ldots . . . \\
422357\end{array}$ \\
\hline 0300794 & 3ONOYSS & $\begin{array}{l}2 \mathrm{~N} 22 \mathrm{E} 530 \mathrm{AOI} \\
2 \mathrm{~K} 2 \mathrm{~B} 530 \mathrm{AOZ} \\
2 \mathrm{~K} 2 \mathrm{2} 530 \mathrm{AO1}\end{array}$ & $\begin{array}{l}7 E 620010 \\
1 E 510010 \\
7 E 6202 T 0\end{array}$ & $\begin{array}{l}3220 \\
501 \\
10\end{array}$ & $\begin{array}{l}190173 \\
25035 \\
12200\end{array}$ \\
\hline 310टT94 & 27FEB95 & $\begin{array}{l}2 \mathrm{~N} 25530 \mathrm{AO} 1 \\
2 \mathrm{~N} 2 \mathrm{E} 530 \mathrm{AC2} \\
2 \mathrm{~N} 25530 \mathrm{AOI}\end{array}$ & $\begin{array}{l}7 E 620010 \\
1 E 510010 \\
7 E 620270\end{array}$ & $\begin{array}{l}3220 \\
501 \\
10\end{array}$ & $\begin{array}{l}190173 \\
25035 \\
12200\end{array}$ \\
\hline с30ст94 & 10EEES & 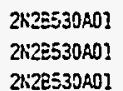 & $\begin{array}{l}7 \pm 620010 \\
18510010 \\
7 E 6202 T 0\end{array}$ & $\begin{array}{l}3220 \\
501 \\
10\end{array}$ & $\begin{array}{l}190173 \\
25035 \\
12200\end{array}$ \\
\hline
\end{tabular}

:32200500 DACS MEITTENANEE G OPERATION FYOS

of

:Z5220E00 GAS HONITORING MAINT \& OPERATION FYSS

$0 *$

:2E:2UG10 INSTRUNENTATION TEANSPORT CONTAINER FY95

12200 


\section{WHC-SP-1101}

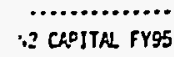

WORK PACAKGE TOTALS

START DATE 010CT92 FIN DATE 07JULOO
$12: 48$
EEI TL SIHOH 373-2587
- गु. ne: $\mathrm{H2O3}$
DROGEN MITJGATIOH

DATA DATE 010CT93 PAGE NO. 2

\begin{tabular}{|c|c|c|c|c|c|c|c|}
\hline$\because 10$ & ACT DES & $\begin{array}{l}\text { EARLY } \\
\text { START }\end{array}$ & $\begin{array}{l}\text { EARLY } \\
\text { FINISH }\end{array}$ & $\begin{array}{l}\cos t \\
\text { Accosurt }\end{array}$ & RESOURCE & $\begin{array}{l}\text { BUDGET } \\
\text { OUANTITY }\end{array}$ & $\begin{array}{l}\text { BUDGET } \\
\text { COST }\end{array}$ \\
\hline 3 & & & & & & & \\
\hline Of & & & & & & & \\
\hline $2 \Xi 230 A 12$ & ENGIHEERIHG DESIG SUPPORT & 0300794 & 295EPS5 & $\begin{array}{l}2 \mathrm{~N} 23530 \mathrm{ROI} \\
2 \mathrm{~K} 23530 \mathrm{RO1}\end{array}$ & $\begin{array}{l}7 E 6203 R C \\
7 E 63021 C\end{array}$ & $\begin{array}{l}2600 \\
250\end{array}$ & $\begin{array}{l}2600000 \\
250000\end{array}$ \\
\hline & & & & & & 14043 & \\
\hline
\end{tabular}

OB

2B230BO0 ANARD BID FOR PUAP MANLFACTURE SY-103 . $2 E 230 S 02$ FAB \& POUR CVK BLKS/FAS LOAD FRAME

oc

:2223OCO2 PUMP INSTALLATIOH SUPPORT

OD

YZE230D00 DAOS 2A SYSTEM MOT

os

LES230J00 REMOVAL/DISPOSAL SYSTEM 103-SY

\begin{tabular}{|c|c|c|c|c|c|}
\hline 13FEB95 & 27FEBS & $212253050 i$ & $7 \$ 240100$ & 300 & 366000 \\
\hline 14MARQS & 20SEPSS & 2K2E5รŪS01 & 78240380 & 1700 & 1803700 \\
\hline & & & & & 2169700 \\
\hline
\end{tabular}

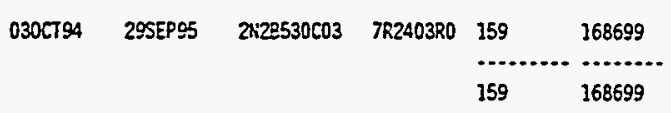

O30LT94 295EPS5 2N22530001 $80210010 \quad 3231 \quad 236380$

2KZE530D01 8D2103RO $864 \quad 937924$ $2 \pi 25330001 \quad 80210100 \quad 300 \quad 365000$ $\begin{array}{llll}2 \times 2 E 530001 & 80210010 & 471 & 34458\end{array}$ $\begin{array}{llll}2 \times 23530002 & 52700060 & 3036 & 136229\end{array}$

$7922 \quad 1712991$

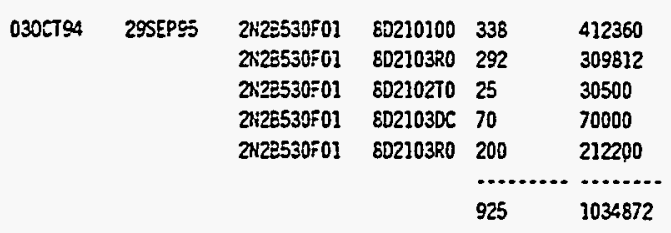

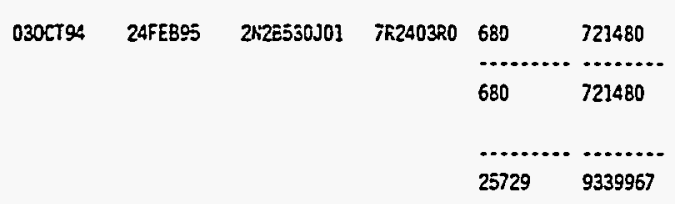


WHC-SP-1101

$\because$ XEITA FYgS

:I:3A: DRTE 15SEPGS RUN NO. 258 12:48

$\because::$ :AะD BY NL SIMON 373-2587

$\because$ Iest Name. N203

- FEN MitIGATIOH

\begin{tabular}{|c|c|c|c|c|c|c|}
\hline$\because 2$ & $\begin{array}{l}\text { EARLY } \\
\text { START }\end{array}$ & $\begin{array}{l}\text { EARLY } \\
\text { FINISH }\end{array}$ & $\begin{array}{l}\cos T \\
\text { ACcount }\end{array}$ & RESOURCE & $\begin{array}{l}\text { BUDGET } \\
\text { QUANTITY }\end{array}$ & $\begin{array}{l}\text { BUDGET } \\
\text { COST }\end{array}$ \\
\hline
\end{tabular}

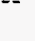

$i$

DEZZS:00 GAS CHAEATER SYS MATERIAL - 101-AN

\begin{tabular}{|c|c|c|c|c|c|}
\hline \multirow[t]{5}{*}{ 030ст94 } & 29SEPQS & 2N28540F01 & 65210110 & 277 & 337940 \\
\hline & & & & 277 & 337940 \\
\hline & & & & 277 & 337940 \\
\hline & & & & & 14525263 \\
\hline & & & & 34078 & 14525263 \\
\hline
\end{tabular}


WHC-SP-1101

$\therefore$ EXPENSE FY96

इPORT DATE ISSEPQ4

13:09

FETAREO EY NL SIHON 373-2587

- DAOEER M:TIGATION

\begin{tabular}{|c|c|c|}
\hline $\begin{array}{c}\therefore=\text { TIVIT } \\
10\end{array}$ & DESCRIPTIOH & \\
\hline$\Xi 23$ & & \\
\hline oc & & \\
\hline$: 25230500$ & WORK PLAW/KORK PKG & $303.5 Y$ \\
\hline 1.25230C0L & TRAINING/READIHESS & \\
\hline
\end{tabular}

¿2SZZOCOL TRAINIHS/READINESS REVIEH

RZB230002 DACS 2A SYSTEM PROE \& FAB 103-SY

OE

R:2B230E00 DACS MAINTENANCE \& OPS 103-SY

of

NZE230FOS GAS CHARACTERITATION SYSTEM (GS)LOZ-SY

06
HYROGEN MITIGATION

WORK PACKAOE TOTALS

START DRTE OIOCT92 FIN DATE O7JUROO

DATA DATE O1OCT93 PAGE NO. 2

$\begin{array}{lllrl}\text { ORIG EARLY } & \text { EARLY } & \text { COST } & \text { RESOURCE BUDEET } & \text { BUDEET } \\ \text { DUR STAET } & \text { FINISH } & \text { ACCOWT } & \text { OUANTITY COST }\end{array}$

$\ldots$

$\begin{array}{lllrl}\text { ORIG EARLY } & \text { EARLY } & \text { COST } & \text { RESOURCE BUNGET } & \text { BUDEET } \\ \text { DUR STAFT } & \text { FINISH } & \text { ACCOWN } & \text { QUANTITY COST }\end{array}$

$:$

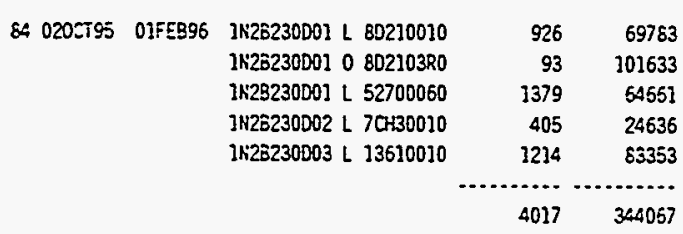

157 O2FEะS5 30SEP95 IK2E230E01 L 80210010 3350 252456 $1 \kappa 26230502$ L $80620000 \quad 1283 \quad 73654$ $\begin{array}{rrr}1 N 28230503 \perp 7 C 951060 & 2556 & 114<19\end{array}$

$7199 \quad 440529$

251 C20ニTS5 30SEP95 IK23230EOI L 80210010

\begin{tabular}{rr}
1280 & 96461 \\
\hline 1280 & 96461
\end{tabular}

251 020ะT:5 30SEP96 1Ki26230S01 L 80210010 11:26230502 L 7CH30010 $1 \% 26230003$ L 7 CC60010 1K26230603 L 7CC6000 1125230604 L 33340060 IN25230604 L 33340010 1H2e230605 L 7C930010 1א2B230305 L 7C930065 1K25230505 0 7C9303?0

\begin{tabular}{|c|c|c|c|c|}
\hline \multirow[t]{2}{*}{640206795} & OAJAN96 & $1 \mathrm{~N} 26230 \mathrm{CO}] \mathrm{L} 7 \mathrm{CH} \mathrm{KOD} 10$ & 2573 & 156516 \\
\hline & & $1 \mathrm{~K} 26230 \mathrm{CO}$ L $7 \mathrm{C} 900010$ & 1359 & 62668 \\
\hline \multirow[t]{6}{*}{102 05JAK35 } & 29MAY96 & $1 \mathrm{~K} 28230 \mathrm{CO4} \mathrm{L} 333300010$ & 478 & 29818 \\
\hline & & IK26230C05 L 52200010 & 84! & 37867 \\
\hline & & $1 \mathrm{~K} 25230 \mathrm{CO} 1 \mathrm{~L}$ 7CH:30010 & 2174 & 132244 \\
\hline & & $1425230 C 02 \mathrm{~L} 7 \mathrm{C900010}$ & 4595 & 279514 \\
\hline & & $1 \% 28230005 \& 7 N 000010$ & 1203 & 73178 \\
\hline & & & 13223 & 791625 \\
\hline
\end{tabular}


WHC-SP-1101

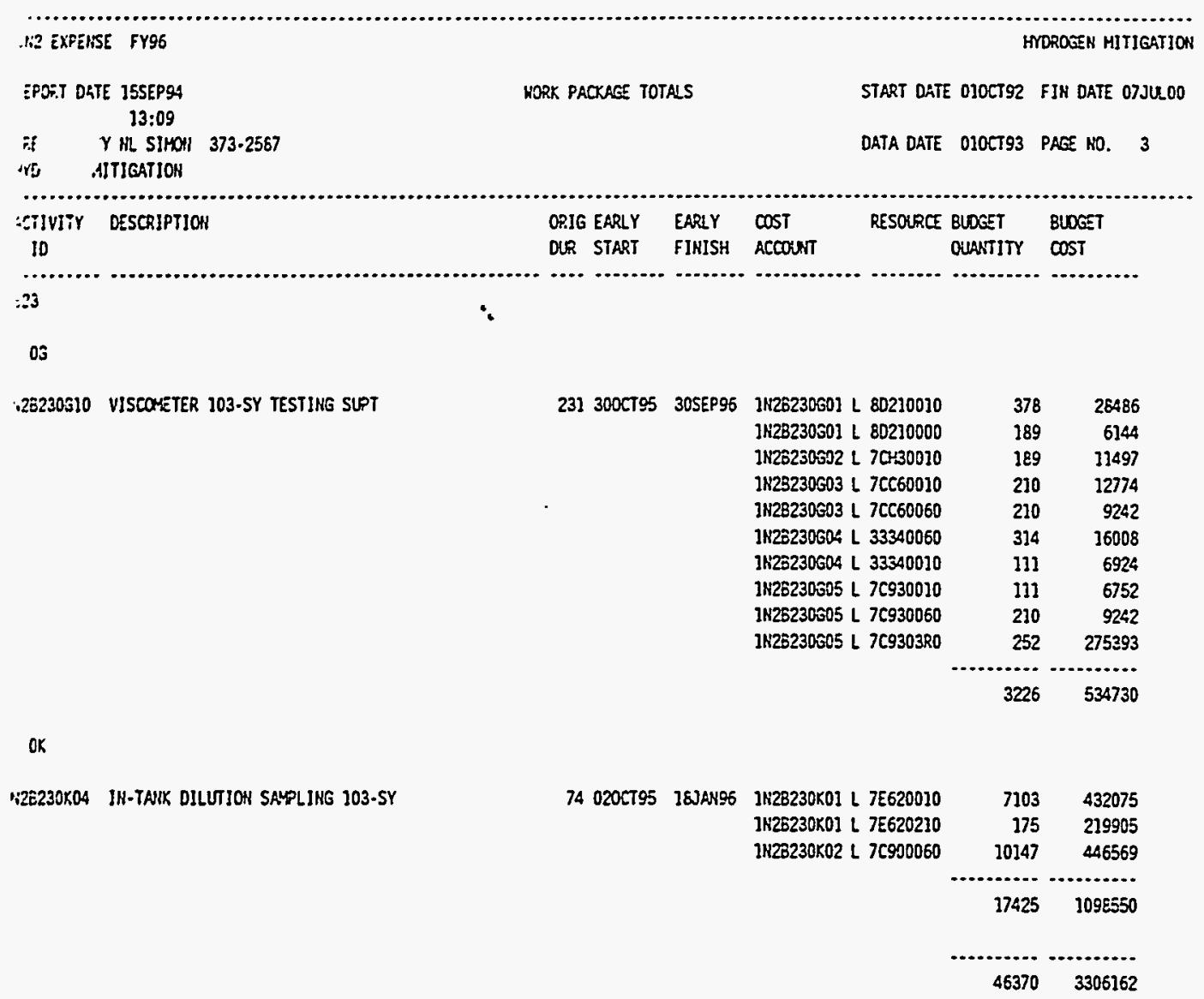

324

$a$

M2E240GDE VOIOAETER 101-AN INSTL SUPPORT

:2E240510 VISCONETER 101-AN INSTL SUPT

\begin{tabular}{|c|c|c|c|c|}
\hline \multirow[t]{9}{*}{$251020 C T 95$} & 30SEP95 & 1N2224031 L 80210010 & 435 & 32762 \\
\hline & & 1N2B240G02 L. 7CH30010 & 345 & 8820 \\
\hline & & 1K28240203 L 7CC60010 & 70 & 4253 \\
\hline & & $1 \% 26240303 \mathrm{~L} \mathrm{7CC60000}$ & 70 & 1657 \\
\hline & & IN25240504 L 33340060 & 104 & 5302 \\
\hline & & $21426240504 L 33340010$ & 37 & 2308 \\
\hline & & $1 N 28240605 \& 7 C 930010$ & 37 & $225 !$ \\
\hline & & 1K228240005 L 7C930060 & 70 & 3081 \\
\hline & & $1 \mathrm{~N} 2864060507 \mathrm{C} 930380$ & 84 & 91798 \\
\hline \multirow[t]{11}{*}{156 C5FEB96 } & 30SEP95 & $11 \% 28240601 \div 80210010$ & 376 & 26486 \\
\hline & & $1 \mathrm{~N} 26260501<80210000$ & 189 & 6144 \\
\hline & & $1 \mathrm{~N} 25240502 \perp 70430010$ & 189 & 11497 \\
\hline & & ]N23240303 L 7CC60010 & 230 & 12774 \\
\hline & & 1א2E240503 $L 7 \mathrm{CC60060}$ & 210 & 9242 \\
\hline & & 1128240304 L 33340060 & 314 & 16008 \\
\hline & & 1א2E240G04 L 33340010 & 111 & 6924 \\
\hline & & 1N26240G05 L 7C930010 & mII & 6752 \\
\hline & & 1K26240G05 L 7C930060 & 210 & 9242 \\
\hline & & 1N25240G05 L 7C9303R0 & 252 & 275393 \\
\hline & & & & \\
\hline
\end{tabular}




\section{WHC-SP-1101}

\section{$\therefore$ CAPITAL FY96}

:FORT DATE 155EP94 RUH HO. 251

MORK PACAKGE TOTALS

START DATE 010CT92 FIN DATE 07JU.00

$\because \quad$ Y ML SIHON 373.2567

rC, sme: $\mathrm{H} 2 \mathrm{OO}$

TOROSEN MITIGATION

\begin{tabular}{|c|c|c|c|c|c|c|c|}
\hline$=10$ & ACT DES & $\begin{array}{l}\text { EARLY } \\
\text { START }\end{array}$ & $\begin{array}{l}\text { EARLY } \\
\text { FINISH }\end{array}$ & $\begin{array}{l}\cos T \\
\text { ACCoONT }\end{array}$ & RESOURCE & $\begin{array}{l}\text { BUDGET } \\
\text { OUANTITY }\end{array}$ & $\begin{array}{l}\text { BUDGET } \\
\text { COST }\end{array}$ \\
\hline \multicolumn{8}{|l|}{$.2 !$} \\
\hline \multicolumn{8}{|l|}{$O D$} \\
\hline .2E210019 & PML EHGINEERING SERVICES FYG5 & 0200795 & 30SEP96 & 2426610001 & $7 R 2403 D C$ & 55 & 56650 \\
\hline & & & & & & 55 & 56650 \\
\hline
\end{tabular}

OE

[2E2JOEO4 LAML INSTALL DACS 2A SYS FOR SY FARY MZE210E24 LAVI DAES 2A SYS FOR SY FARY HAINTENAMCE K35210E26 LAML BUILD DACS $2 B$ SYSTEM 101-AS

\begin{tabular}{|c|c|c|c|c|c|}
\hline 020:T95 & O1FEB9S & $2 \times 23510 \equiv 01$ & $7 R 24022 \mathrm{C}$ & 165 & 169950 \\
\hline 020CT95 & 30SEP96 & $2428510=01$ & $7 R 24021 C$ & 808 & 832240 \\
\hline \multirow[t]{3}{*}{$020<795$} & 30SEP96 & 2N28610E02 & $7 \mathrm{R} 24021 \mathrm{C}$ & 1318 & 1357540 \\
\hline & & & & 2291 & 2359730 \\
\hline & & & & 2346 & 2416380 \\
\hline
\end{tabular}

522

of

Ni2E2ZOFO4 GLS MONITORING MAINT \& OPERATIOH FY96

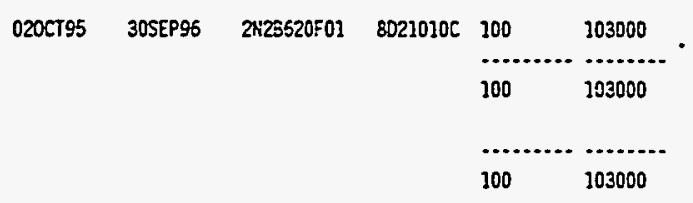

223

OA

R23230A2O ENGINEERIHE DESIGH SUPPORT

\begin{tabular}{|c|c|c|c|c|c|}
\hline \multirow[t]{6}{*}{ 020टT:5 5} & \multirow[t]{6}{*}{ 30SEP96 } & 2NZ2530A01 & 7E6203R0 & 555 & 605521 \\
\hline & & 2N2EE3DACI & $7 E 620110$ & 50 & $62 \varepsilon 30$ \\
\hline & & 2 N23530A01 & 18510010 & 2718 & 139895 \\
\hline & & 2N2BE30A02 & 12510010 & 308 & 15853 \\
\hline & & 2K28530A02 & 18510060 & 6342 & 266745 \\
\hline & & & & 9973 & 3093843 \\
\hline
\end{tabular}

OB

H2S230304 ASSEMBLE FAB PUMP 103.SY

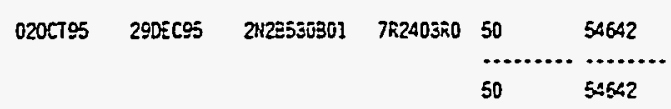

oc

H2E230CO4 TRAINIMG/READINESS REVIEY 


Zg2tzZt
zortsot

0S669t
ZIL

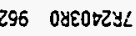

s9L Jototzyi

LGEOサLSZNZ

L080ל EZWZ

G6d3SOE

görivzo

95 ariso

9600HSO

96ะsจ? $82 \varepsilon s i$

6ESSEL BLZE 0900TSBI ZOTOt98ZKZ

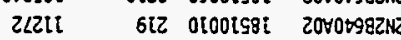

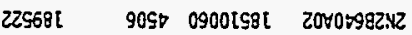

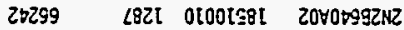

$959 \angle 92$ ELZ OLZOZ93L LOFOF9EZNZ

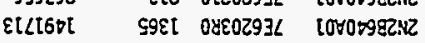

ootolz
S6ajSOE $\$ 610020$

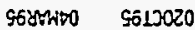

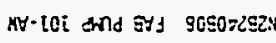

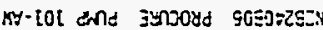

so

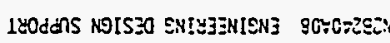

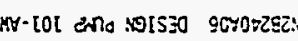

$8602 \angle わ 2 \quad 6999$

$29618 \quad$ SL

$\begin{array}{ccc}29618 & \\ 2 L & \end{array}$

Z96โ8 SL O\&EOLZOS

OOOEOT $00 \mathrm{t}$

n..................

ooosot

oot jototzos

I050ع9g2N2

$96 \mathrm{~d} 3508$

$9683 \pm 20$

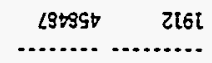

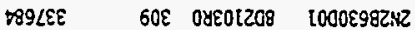

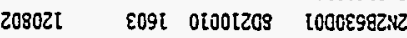

96:3t

†2789 659E

\section{0} $\because$

1503 MIINATO

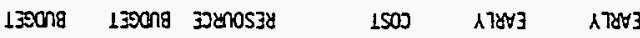

2 ON IOHd E6LOLO 31YQ YIYO

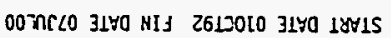

STY101 30XYOYd XECA

$S=0$ I $\mathrm{Cl}:$

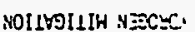
EOz.Y : awen:zal:.

LSSZ-ELE YYWIS TN A8 CEYT: $\rightarrow \mathrm{il}: \mathrm{zl}$

Ist ON my odisst 3170 : 
WHC-SP-1101

\section{IR. CAPITAL FY96}

EPOAT DATE 15SEPMA RUN NO. 151 12:14

af 'Y ML SIHOH 373.2587

ro. dame: $\mathrm{N2O3}$

- MOROËH MITIGATIOH

ECT ID ACT DES

$\vdots 24$

OD

.26240006 DACS 2B SYSTEM 101-AH

or

H:2E240FO4 FAB \& IISTALL GOS 101-AN

E27

OA

Ni22270A0O DESIGN PUPP 104-AN

0

1126270BO0 PROCURE \& FAB PUPP 104-AH

oc

II2B270COO INSTALL PREPARATIOH PLAP 104-AN
MORK PAEAKGE TOTALS

START DATE O1OTT92 FIN DATE OJJUROO

DATA DATE OJOCT93 PAGE HO. 3

$\begin{array}{llll}\text { EARLY } & \text { EARLY } & \text { COST } & \text { RESOURCE BUDETT } \\ \text { START } & \text { FINISH } & \text { ACCOUNT } & \\ \text { QUANTITY COST }\end{array}$

\begin{tabular}{|c|c|c|c|c|}
\hline \multirow[t]{5}{*}{200795} & $2 \times 28740001$ & 80210010 & 5268 & 395996 \\
\hline & 2426740001 & 80210000 & 1516 & 59038 \\
\hline & $2 N 28740001$ & 80210320 & 1167 & 1297189 \\
\hline & $2 \mathrm{~N} 28740001$ & 80210100 & 300 & 376980 \\
\hline & & & 8571 & 2130204 \\
\hline
\end{tabular}

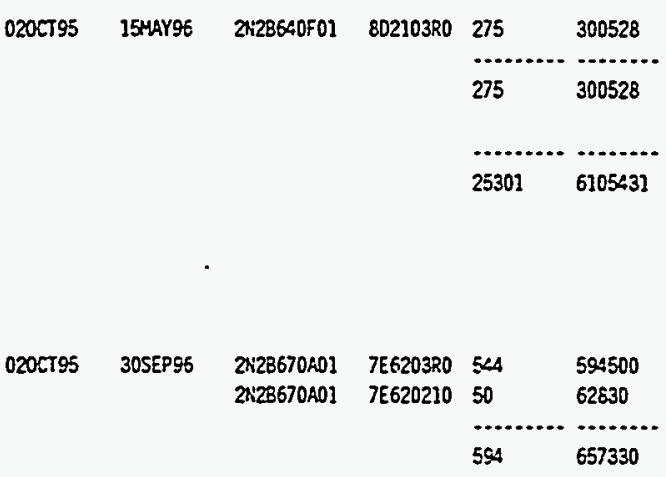

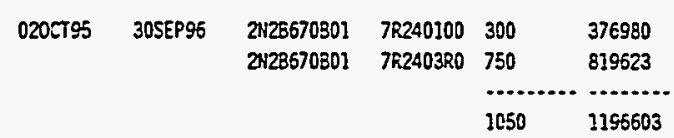

020CT95 305EP96 2K2B670COI 7CH303RO $23 \quad 25135$

$23 \quad 25135$

$1657 \quad 1879057$

.....................

$45083 \quad 12975976$

$\overline{45083 \quad} \overline{12975976}$

F7.4-7.35 
WHC-SP-1101

$\because$ EXPENSE FY97

HYOROGEN MITIGATION

$:=0: T$ DATE ISSEPQ4

12:12

$:=2:=0$ SY NL SIRON $373-2587$

- SINUESR MITIGATION

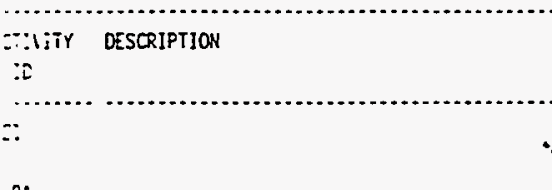

If

YE210ADS PROGRAY MAYAGERENT FYG7

251 010CT95 3CSEPG7 1K2B210A01 \& 7R240010 $1612 \quad 113540$

1א26210A02 L 50440010 $1812 \quad 103284$

1N2B210A03 $\perp 80210010 \quad 2718 \quad 210971$

IN2621040 $17 E 310010 \quad 3624 \quad 227080$

1א26210205 L 7E870010 $3624 \quad 227080$

$13590 \quad 881955$

CE

2E210E0S PROJECT MANAGEMENT FY97

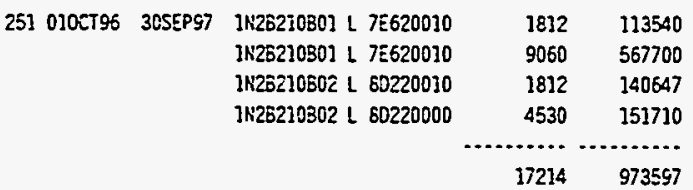

Co

2520018 PNL DILUTION STUDIES

2Sล:0J20 PNi ENGINEERING SERVICES FY97

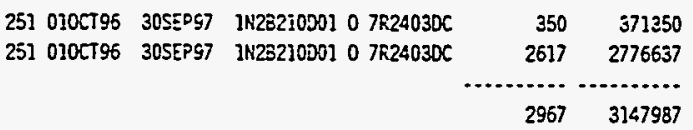

0

2E:IOE10 LAML TECHAICAL SUPPONTT 103-SY

$2 E-\mathrm{IUE} 29$ ISSUE TANK 105-AN SA (LANL)

2E210E30 LANL TECHNICAL SUPPORT 101-AV

ZEZIOE31 LANL DACS $2 C$ FOF AN FARM MAJNTENANCE

252I0:32 LANL TECANICAL SUPPOETT 104-AN

ZE2IOE 34 LANL DAOS 2A SYS FOA SY FARH MAINTENANCE

GE:jCE 36 LANL DACS $2 B$ SVS FOR SY FARY MAINTENANCE

IS210E36 LANL 101-SY TECHNICAL SUPPORT

3E210:39 GAS HONITORING SUPPORT (LANL)

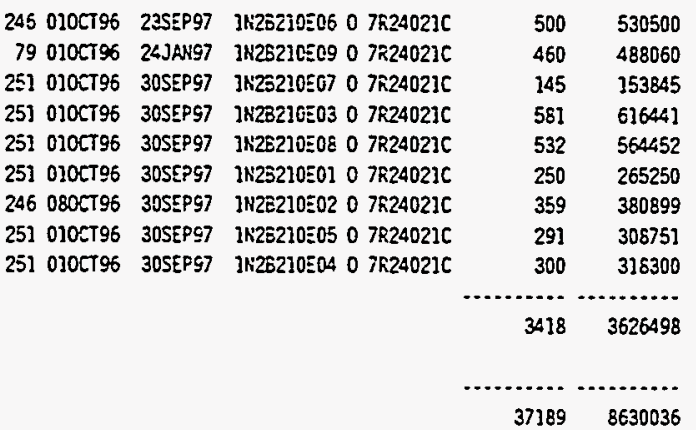

2

of

ZEZ2G:OB GAS MONHTORING MAINT \& OPELATION FYG7

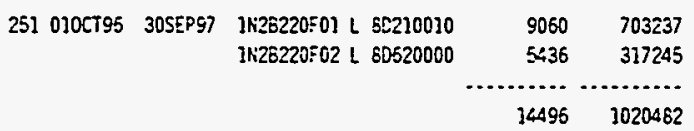

$F 7.4-7.36$ 


\section{WHC-SP-1101}

1.2 EXFELISE FY97
HMROELN MITIGATIOH

:POTI DATE 15SEP94 HORK PACKAGE TOTALS START DATE O1OCT92 FIN DATE O7JULOD

$\begin{array}{lll}=\quad \text { BY HIL SINON } 373.2587 & \text { DATA DATE OIOCT93 PAE NO. } 2\end{array}$

-il MITIGATIOH

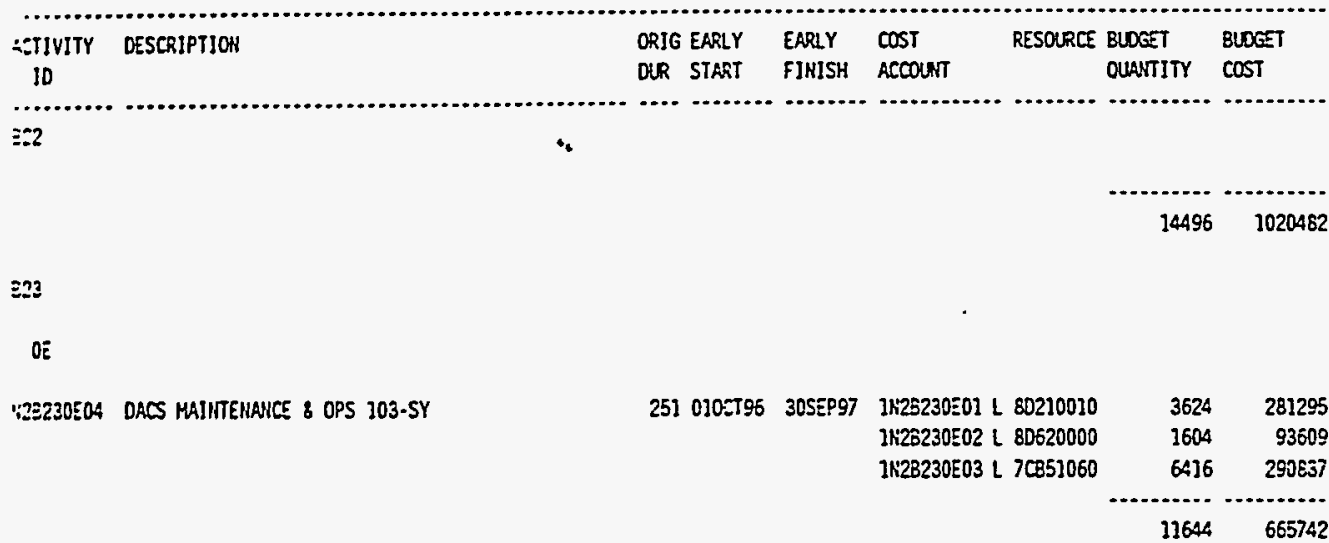

of

Mi25230F08 GAS CHARACTERIZATION SYSTEM (GS) 103-SY

ox

N26230K0B IN-TANK DILUTION SAMPLIHG 103-SY

N:29240COO INSTALL PLPP 101-AH

N25240CO2 COPL 101-AN INSTALL READIMESS REV HZ25240COB CONLLTE IN TANK TESTING AH-101

ob

N28240004 DRCS 2B SYSTEM 101-AH
251 010TT95 30SEP97 ]1125230K0] L 7E620010

\begin{tabular}{rrr}
1259 & 85155 \\
\hline 1359 & 85155 \\
& \\
\hline 15193 & 920884
\end{tabular}

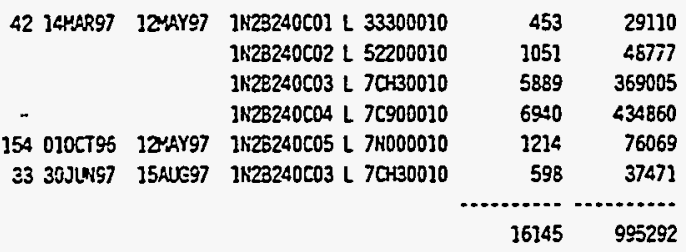

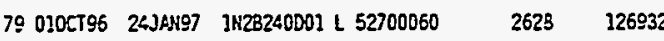
3N23240D01 L $80210010 \quad 3799 \quad 294878$ Jฬ25240002 L $13510010 \quad 1577 \quad 111541$ $1 \kappa 25240003$ L 7CH30010 $1523 \quad 9543$ I $1 \mathrm{H} 25240001 \perp 602103 R 0 \quad 55 \quad 63040$

$9583 \quad 691823$ 
WHC-SP-1101

$\because Z$ EXPENSE FYG7

HYOROGEN MITIGATION

:ニデ D DATE 15SEPQ⿻

WORK PACKAO TOTALS

START OATE O1OCT92 FIN DATE OTJU.DO

ZEFARED BY WL SIHON 373.2587

DAOSEK MITIGATION

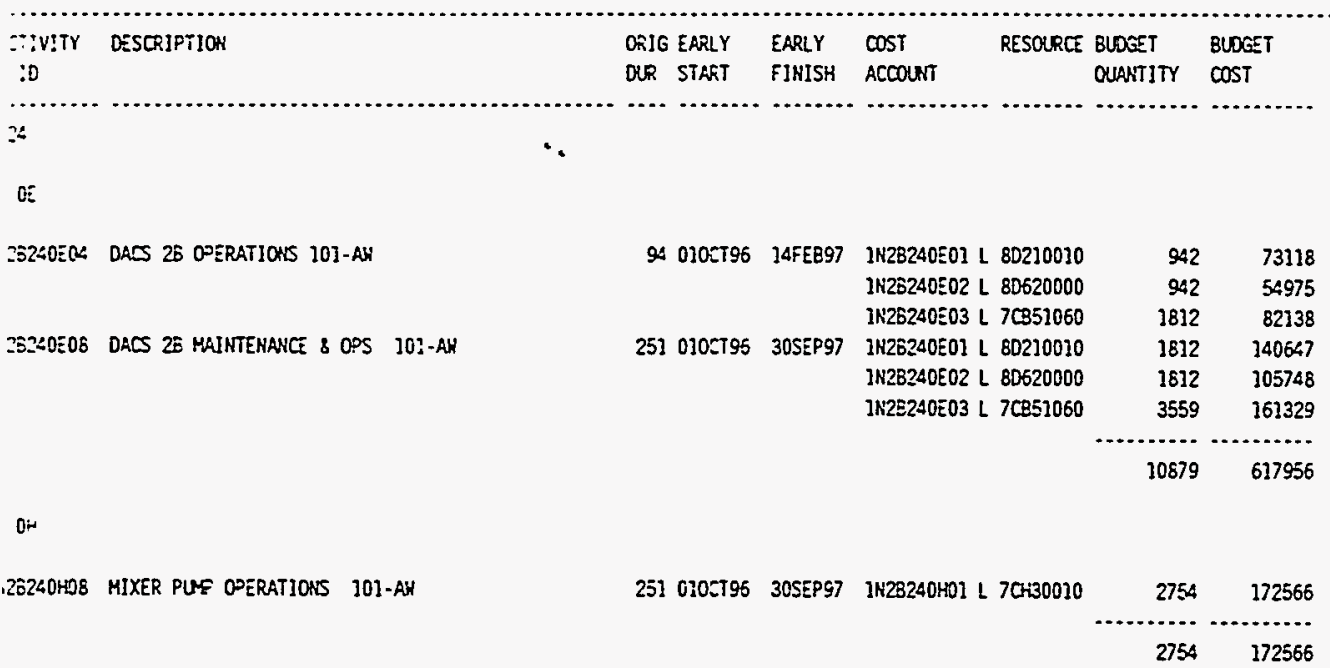

oJ

Z2524CJ06 REMOVAL/DISPOSAL SYSTEM 101-AH

$2510102 T 95$ 30SEP97 JN28240J02 60220010 $1 \times 28240303$ L 80530010 IN28240J04 L 80520010 ]К2B240J05 L 15370030 1Кi26240J06 ᄂ 57530010 1N28240J01 $07 R 240210$ 1ผ220240J07 L 80330010 1К28240J08 L 80320010 IN25240J09 L 67820010 1К28240.110 L 84100010

$320 \quad 24838$

$460 \quad 37297$

$229 \quad 18567$

$270 \quad 16567$

$730 \quad 45252$

$20 \quad 25888$

$75 \quad 5822$

$150 \quad 11643$

$\begin{array}{rr}150 & 5502 \\ 300 & 18927\end{array}$

$2704 \quad 210334$

-.....................

$42065 \quad 2687971$

$: 25$

05

Z23260300 VISCOETER 103-AN SLPPOET

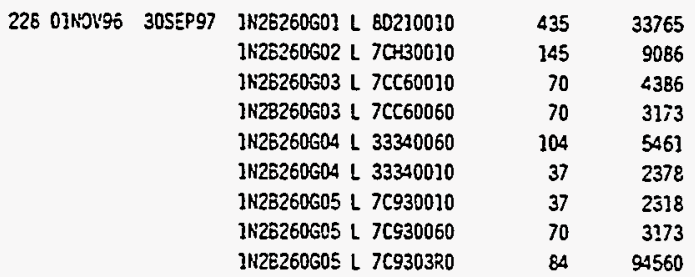

$F 7.4-7.38$ 


\section{WHC-SP-1101}

$\because 2$ SE:TA- FY97

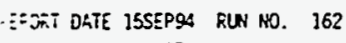

$$
\text { 13:37 }
$$

MORK PACAKGE TOTALS

START DATE OJOCT92 FIH DATE OJJULO

:EFAEDO EY NL SIMAN 373-2587

-ojesi kame: 1203

-DKDEEN MITIGATIOH

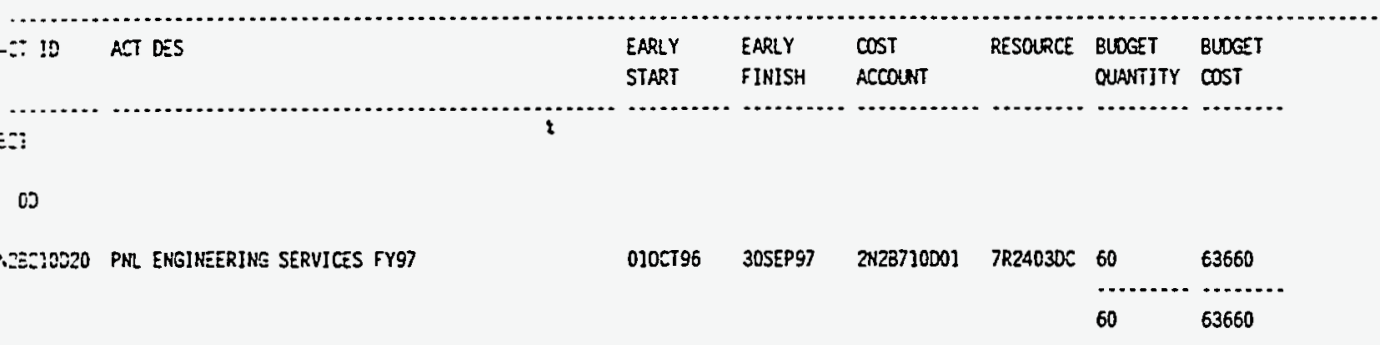

QE

2323iYE23 LAML BUILD DACS $2 C$ SYSTEM AN FAFM

125210E31 LANL DACS $2 C$ FOR AN FARY MAINTENANCE

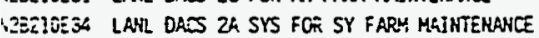

25210E36 LANL DACS 23 SYS FOR SY FAPM HAINTENANEE

\$22

YZE2ะSCOO INSTALL PLAP 10]-AL

SZ\$24GCOB COPLETE IN TANK TESTINS AK-101

CE

NZEZAOEOA DACS 23 OPERATIONS 30?-AW

R2EZZ:JJS ENGINEERING \& FENLE MDJTIFICATIONS
DATA DATE 010CT93 PAGE NO. 1

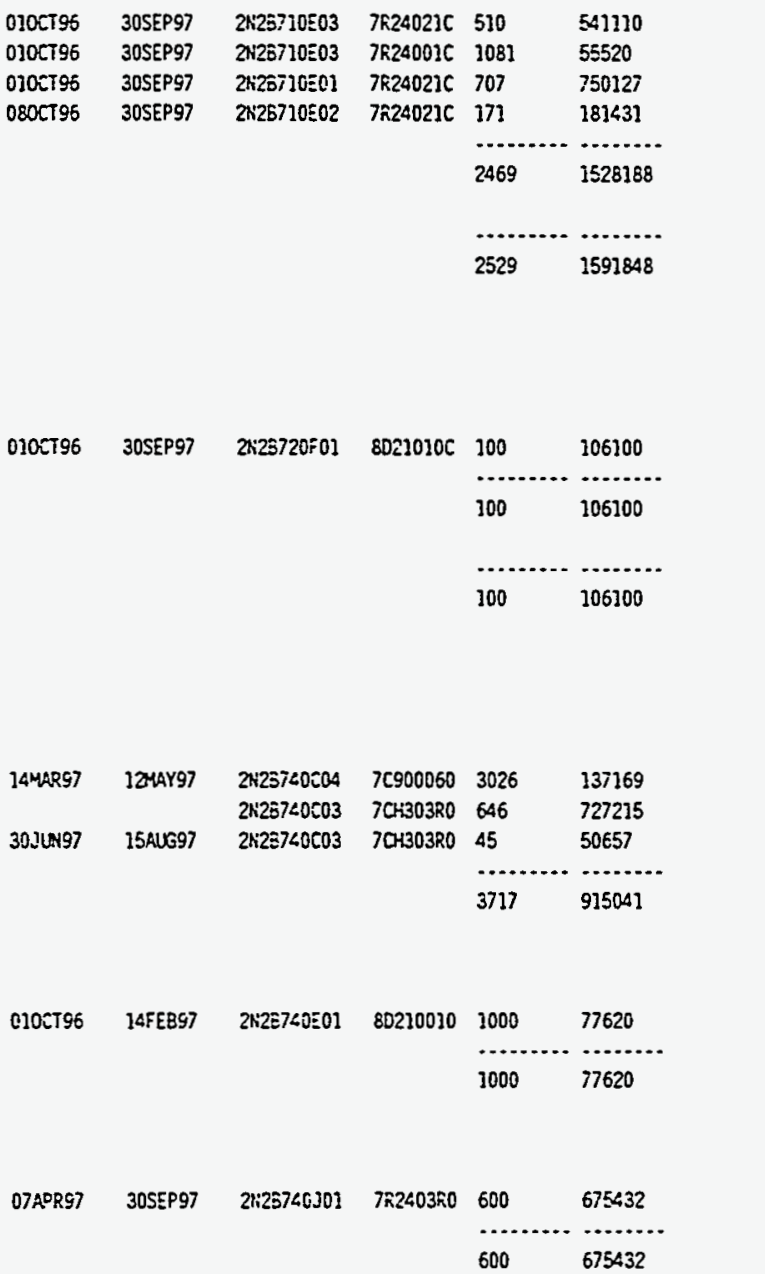


$\therefore 2$ CAPITAL FYG7

:POKT DATE 15SEP94 RUN NO 162 $13: 37$

$\Rightarrow \quad$ IY NL SIHON 373.2587

c. Jne: 11203

DDROSEN MITIGATION

IT ID ACT DES

HORK PACAKGE TOTALS

START DATE 010CT92 FIN DATE 07JULOO

24

START FINISH ACCOUNT QUANTITY COST

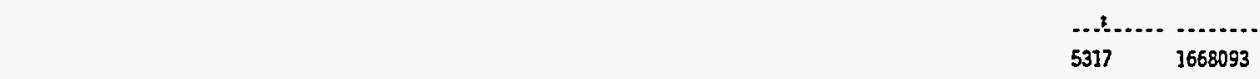

37

of

20270A04 DESIGN PUAP 104-AN

:26260R04 DESIGN SUPPORT PUPP 105 AN

\begin{tabular}{|c|c|c|c|c|c|}
\hline \multirow[t]{8}{*}{$010 \mathrm{CT}: 5$} & \multirow[t]{8}{*}{ 30SEP97 } & $2 \mathrm{~N} 26770 \mathrm{ADl}$ & $7 \in 620010$ & 2881 & 180523 \\
\hline & & 2125770202 & 18510060 & 3624 & 156992 \\
\hline & & $2 \times 28770 \mathrm{AD1}$ & 7E6203R0 & 1904 & 2143371 \\
\hline & & $2128770 \mathrm{AD1}$ & $7 E 620210$ & 250 & 323605 \\
\hline & & $2 \times 25770 \mathrm{AO1}$ & 7E5202T0 & 30 & 38833 \\
\hline & & $2 \times 25770 \mathrm{A01}$ & $7=620100$ & 20 & 25888 \\
\hline & & $2 \times 25770 A 02$ & 18510010 & 1812 & 96072 \\
\hline & & $2 \times 26770401$ & $7 E 620010$ & 1504 & 94241 \\
\hline \multirow[t]{6}{*}{010 t795 } & \multirow[t]{6}{*}{ 27JuKi97 } & $2 \times 28780 A 01$ & $7 E 620010$ & 3099 & 194183 \\
\hline & & 2स26780A02 & 18510010 & 1812 & 95072 \\
\hline & & $21283780 A 02$ & 18510060 & 6342 & 274735 \\
\hline & & 2N26780R01 & $7 E 620100$ & 20 & $25 E 68$ \\
\hline & & $2 \times 25780401$ & 7E620210 & 20 & 25888 \\
\hline & & & & & $30 / 6293$ \\
\hline
\end{tabular}

OB

K2B27nRMG FAB \& ASSERBLE PUPP 104-AN

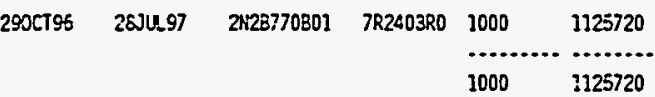

OC

K.:B270CO2 INSTALL PREPARATION PUP 104-AN H2B270CO6 JISTALL TANK 104-AN MIT PLPP

\begin{tabular}{|c|c|c|c|c|c|}
\hline \multirow{4}{*}{$\begin{array}{l}\text { O10\&T95 } \\
\text { OAAUES7 }\end{array}$} & 30SEP97 & $2 \mathrm{~N} 253770 \mathrm{COI}$ & $7 R 2403 R 0$ & 510 & 574117 \\
\hline & 30SEP97 & $2 \mathrm{~N} 2 \mathrm{~B} 370 \mathrm{CO}$ & $7 R 2403 R 0$ & 306 & 344470 \\
\hline & & $2 \times 26,770 \mathrm{CO} 2$ & 70900050 & 3026 & 137169 \\
\hline & & & & 3642 & 1055756 \\
\hline
\end{tabular}

OD

N2B270006 DACS $2 C$ SYSTEM KGTT

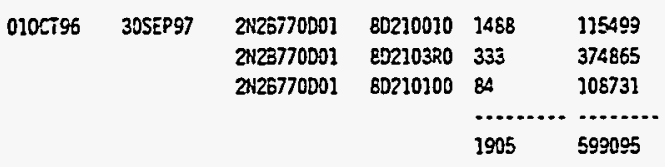

0

Ii2B270EOD DACS $2 C$ MAINTEMANCE \& OfS

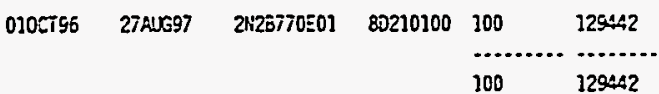




\section{WHC-SP-1101}

h2

ih2 EXEEHSE FY95

UTS FERROCYANIDE PROGRAM

-EPORT DATE I5SEP94

HORK PACKAGE TOTALS

START DATE 010CT92 FIN DATE O7JULOD

$\because \quad$ TY KL SILN $373-2567$

DATA DATE O1OCT93 PAGE NO. 1

. . CYANIDE SAFETY PROGRAM

OETIVITY DESCRIPTIOH

10 DUR START FIMISH ACCONT OUAATITY COST

:1

OA

IIZCZ1OAOA FECH SIHULATED NASTE STUDIES (FALSKE)

250 030CT94 29SEPS5 1K2C3104DI O 7F260210 $290 \quad 353800$ JN2C310A21 O 7R2604CO $19 \quad 23180$

$309 \quad 376980$

$O D$

Y2C3JODOS PILL MANAGEMENT

YI2C310DO7 PIIL AGIHG NASTE STUDIES

$1.2 C 310015$ PNL CESIUH UPTAKE

1:2C310D1B PHL SPECIAL STUDIES/CONSULTATION

1:2C310D19 PIIL COPARE REAL KASTE W/SIMILATED NASTE

VIC310D21 INFRARED/ICP SPECIATION TRAISFER (PNL)

V:2C310024 PML CESIUY NICKEL FECN SOLLBILITY

R2C310D28 SLUDGE MOISTURE HODELIHE

DUR START FIMISH ACCOUNT

-

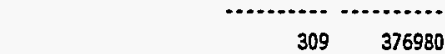

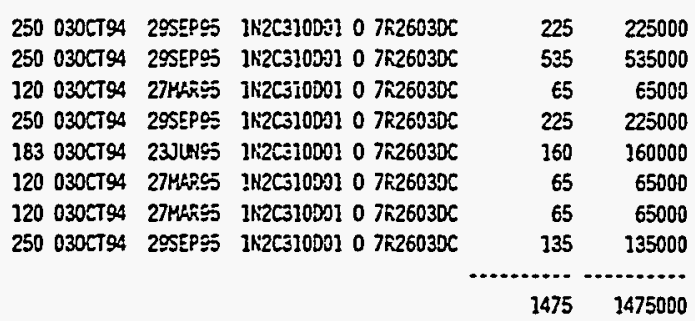

OE

W2CZ1OEO4 DNFSB/CAP MAHAGEHEMT

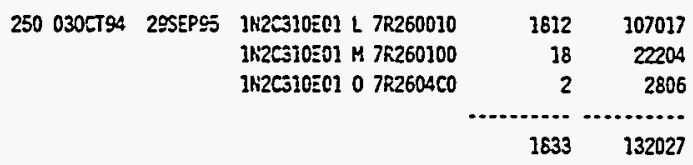

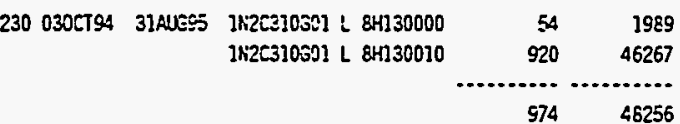

N2C310HO4 IN-SITU RELATIVE HMITITY INSTRLMENTATION

250 030CT94 29SEPSS IK2C310HEI L TEA10000 1K2C210HO1 L TEA10000 IK2CE]0:DI L TEA10010 JH2CSIOKDI L TEA1OOJO 1K2CE10HDI Y 7EA1O100 IN2C310:4S1 O 7EA104CO

\begin{tabular}{rr}
1200 & 27744 \\
255 & 5896 \\
165 & 9745 \\
770 & 45476 \\
19 & 22570 \\
4 & 4880 \\
\hline 2413 & 116311
\end{tabular}


WHC-SP-1101

$\because$ EY=ENSE FYGS

UTS FERROCYANIDE PROERAY

EFOR: DATE 15SEPQ4

11:03

:EPAFED BY NL SIHON 373.2587

-S FERROTYANIDE SAFETY PROGRAY

nestion

$\because 2$

$\cdots$

3

c.

2CEICJO4 INFRARED SPECIATION TRANSFER

MORK PACKAGE TOTALS

DATA DATE OLOCTI3 PAGE NO. 2

$\begin{array}{llll}\text { ORIG EARLY EARLY } & \text { COST } & \text { RESOIRCE BUDETT } & \text { BUDGET } \\ \text { DUR START } & \text { FINISH } & \text { ACCONT } & \text { QUANTITY COST }\end{array}$

\begin{tabular}{|c|c|c|c|c|}
\hline \multirow[t]{8}{*}{250 030СT94 } & 295EP95 & $1 \% 2 C 310 J 01 ~ L 60532010$ & 200 & 14832 \\
\hline & & $1 K 2 C 310301$ L 80532010 & 400 & 29664 \\
\hline & & $1 N 2 C 310 J 01 \perp 8 E 400000$ & 180 & 5584 \\
\hline & & $1 \times 2 C 310 J 01$ L 8E400010 & 180 & 10285 \\
\hline & & $1 \times 2 C 310301$ L $8 E 400010$ & 1812 & 103538 \\
\hline & & IN2C310JO1 M 8E400100 & 14 & 17446 \\
\hline & & IN2C310J01 0 854002TO & 8 & 9760 \\
\hline & & $1 H 2 C 310 J 010$ 8:4004CO & 1 & 1098 \\
\hline
\end{tabular}

ol

N2C310,00 SCANING ELECTRON MICKOSCONE INUESTG SUPT

\begin{tabular}{|c|c|c|c|c|}
\hline \multirow[t]{10}{*}{250 030टт94 } & \multirow[t]{10}{*}{ 29SEPQS } & $3 \mathrm{H}_{2} \mathrm{C} 310 \mathrm{0} 1 \perp 85920000$ & 362 & 13332 \\
\hline & & 1N2C310!01 L 85920010 & 3262 & 164046 \\
\hline & & IHi2C310LO1 L 8E920010 & 362 & 18205 \\
\hline & & 1K2C310LO1 H 85920100 & 59 & 71980 \\
\hline & & IN2C310LOI O EE920210 & 57 & 69540 \\
\hline & & 1K2ะ310L01 O BE9202TO & 12 & 14640 \\
\hline & & $1 \times 2 C 310 L 010859204 C 0$ & 11 & 13420 \\
\hline & & & $\cdots+$. & (n....... \\
\hline & & & 4125 & 365163 \\
\hline & & & 13923 & 2705943 \\
\hline
\end{tabular}

$\cos$

OA

N2C330A0S CAP MANAOERENT FECN TANK SAEETY FYOS

250 030СT94 29SËP9S 1N2CS30A0I L 7K260010 1Ni2C330A01 $07 \mathrm{~F} 260210$ IN2C33OACI 0 7R2504CO

\begin{tabular}{rr}
1812 & 107017 \\
88 & 107360 \\
6 & 7320 \\
\hline 1906 & 221697
\end{tabular}

NZCZ3QSOA DPOATE SEETY DOCUNENTATION FYSS 


\section{WHC-SP-1101}

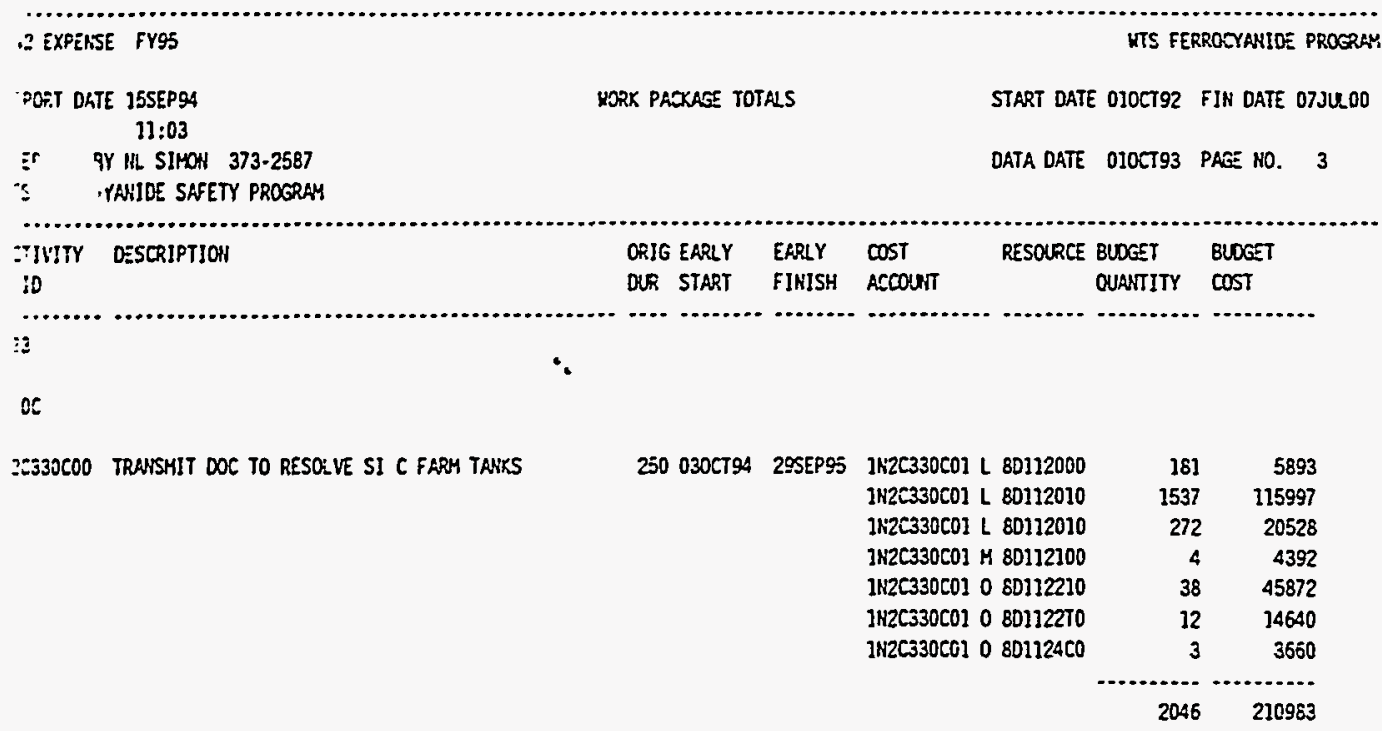

OD

ZE330014 TECHNICAL SUPPORT SI RESOLUTION FYGS

of

:2C330F00 CORE SAYPLE/DATA INTERPRETATION
250 0300794 295EP95 1K2C330001 L 80570000 IN2C330001 L 80570000 $1 K 2 C 330001$ L $8 D 570010$ Jא2C330001 L 80670010 ]พ2C330001 M 80570100 $1 \mathrm{~K} 2 \mathrm{C230001} 0 \mathrm{806702T0}$ 1H2C330001 0 806704CO 1N2C330DS2 L 8A40000 3N2C330002 L 8A400000 IN2C330002 L BA400010 1אi2C330DG2 L 8A400010 IN2C330002 H 84400100 1К2C330002 $O$ 844004CO IN2C330002 L 84400010 1N2C330002 L 84400010

\begin{tabular}{|c|c|}
\hline 906 & 42292 \\
\hline 181 & 8449 \\
\hline 2718 & 195725 \\
\hline 181 & 13034 \\
\hline 16 & 22204 \\
\hline 12 & 14640 \\
\hline 2 & 2806 \\
\hline 80 & 3021 \\
\hline 450 & 16092 \\
\hline 320 & 19914 \\
\hline 80 & 4978 \\
\hline 11 & 13298 \\
\hline 0 & 573 \\
\hline 80 & 4978 \\
\hline 400 & 24892 \\
\hline 5440 & 387795 \\
\hline
\end{tabular}

250 030CT94 20S\$D05 IN2C330FOI L 7E720010 $\quad 600 \quad 35436$ $1 \kappa 2 C 330 F 01\llcorner 7 E 720000 \quad 800 \quad 18496$ 1N2C330:01 L 7E720010 $\quad 1750 \quad 103355$ IN2C330F01 L 7E720010 $4900 \quad 289394$ JH2C330F01 M 7E720100 $11 \quad 13420$ $1 K 2 C 330=0107 E 720210 \quad 115 \quad 140300$

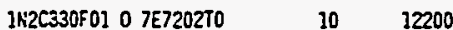
1N2C330:01 07 7E7204CO 9 1N2C330F02 L TE360010 -.........................

$6795 \quad 659505$

$19728 \quad 1594106$ 


\section{WHC-SP-1101}

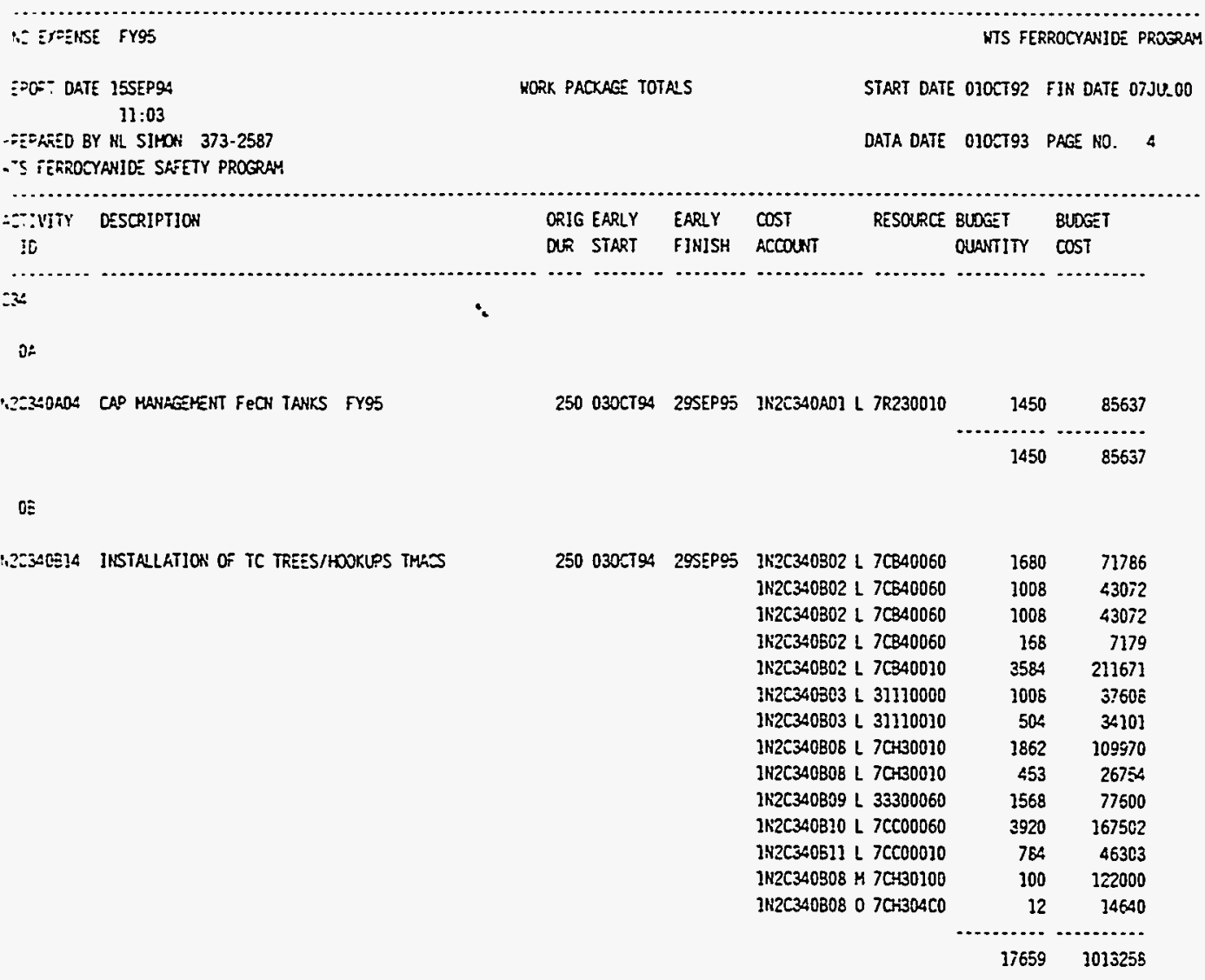

F7.4-7.48 


\section{WHC-SP-1101}

: EXFETSE FY

'is EXFEHSE FYgS

HORK PACKASE TOTALS

START DATE O10ET92 FIN DATE O7JULOO

: $=$ ST: DATE 255EPS4

11:03

DATA DATE O10ET93 PAEE NO. 5

$\because \quad$ इY KL SIMON 373.2587

$\therefore \quad$ ENANIDE SAEETY PROGRAY

TIVITY DESCRIPTHOH

DUR START FINISH ACCOUNT QURNTITY COST

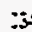

0

.2C3:AE12 MEUTRON PROBE MOISTURE MOHITOR LON ENBR

231 C30CT94 015EPE5 1K2C340C02 L 7EA30010

1N2C340C04 L 52720060

1K2C340504 L 52720050

ik2C340CO5 L 84200060

1)i2C340C03 L 8A400010

1N2C340C03 L 8A400010

1K2C340COE L 80340010

$1 \times 22340201$ L 80520000

$1 \times 2 C 340 C 01 \perp 80520000$

$1 N 2 C 340 C 01 \perp 80520000$

$1 \times 2 i 340 \mathrm{COl} \perp 80520000$

1 1*2C340CO1 L 80520000

1א2E340CO1 L 80520010

1K2C3:0COI \& 80520010

1א2C340C0] L 85520040

1א22340C0] L 80520050

IN2C340C01 M 80520100

IN2C340C01 M 80520100

IN2C340COI M 8D520150

1N2C340CO1 H 80520150

$112 C 340 C 01$ M 80520190

1N2C340C01 080520210

1K2C340CO1 0 8D520210

$1 \mathrm{k} 2 \mathrm{C340C01} 080520210$

2א2C340C01 O 8D5202T0

1K2C340C01 0 805202T0

1K2C340C0] L 8D5204CO

1K2C340C01 L 8D5204C0

IN2C340COI L 8D5204PO

11i2C340CO) ᄂ 855206PO

OUANTITY COST

1K2C340C01 L 8D5206PO

$196 \quad 8924$

$76 \quad 3460$

$415 \quad 28008$

231 $\quad 14375$

$246 \quad 15309$

$260 \quad 19022$

$78 \quad 2572$

$523 \quad 17243$

$208 \quad 6858$

374 12331

$2095 \quad 69072$

$347 \quad 26518$

$608 \quad 46463$

$490 \quad 12054$

$116 \quad 6583$

Q4 114070

$9 \quad 10980$

$7 \quad 7930$

34148

67320

$20 \quad 24400$

21952

89150

$3 \quad 3050$

$3 \quad 3050$

$14 \quad 17080$

$1 \quad 1342$

22684

$\begin{array}{rr}1 & 1342 \\ 2 & \end{array}$

$7169 \quad 542357$

OS

HIZEZOODD2 SLRFACE HOISTURE KEASUF.IHE

HICSESEOS ERGINEERIHS DOCUNENT CONTROL
250 030CT94 29SEPGS TK2C340001 L 80832010 ItizC340001 0 80832210 1li:2C340001 0 808324CO li:2C340002 L 8E400000

\begin{tabular}{rr}
2900 & 215064 \\
42 & 51240 \\
3 & 3660 \\
1712 & 53106 \\
\hline 4657 & 323070
\end{tabular}

250 030Cig4 295Epos ?1:2C340501 L 80730010

\begin{tabular}{rr}
200 & 14632 \\
\hline 200 & 14652
\end{tabular}


WHC-SP-1101

1.2 EXPEHSE FY95

EPORT DATE 15SEPSA

$$
11: 03
$$

3T 3 ML SIHON $373-2587$

TYA TALEE SAFETY PROGRAY

SIVITY DESCRIPTION

15

$\ddot{3}$

QS

ok

:2E340KOO ISB AFENDMENT
MORK PACKAGE TOTALS

START DATE O1OCT92 FIN DATE 07JU OO

DATA DATE OJOCT93 PAGE NO. 7

ORIG EARLY EARLY COST RESOLRCE BUDEET BUDGET .

OUR START FINISH ACCOUNT

OUANTITY COST

..................

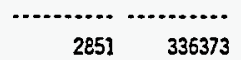

251 030СT9: 020CTe5 1N2C340K01 L $80112010-500$

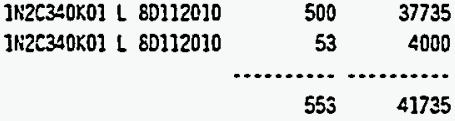

$48261 \quad 3198981$

$81912 \quad 7499029$

$61912 \quad 7499029$ 
WHC-SP-1101

$\because$ EXEENSE FY96

:=ST: DKTE 15SEPG 11:04

WORK PACKAGE TOTALS

START DATE 010CT92 FIN DATE 07JULOO

:EFEED BY K: SIMON $373-2587$

DATA DATE OHOCT93 PAGE NO. 1

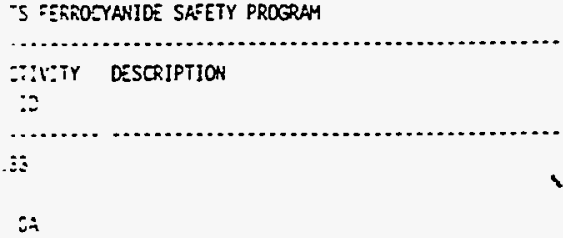

Z3330:24 CAP MAHAEЕENT FEN TANK SAEETY FY95

ORIG EARLY EARLY COST RESOURCE BUOGET BUDEET

DUR START FINISH ACCOUTT QUANTITY COST

251 020CT95 30SEP95 IKI2C330R0] L 7R260010 IK2C330ADI L 7R260010 1K2C330A01 O 7R260210 IK2C33OADI 0 7R2604CO

\begin{tabular}{rr}
1812 & 110224 \\
1812 & 110224 \\
35 & 45238 \\
2 & 3016 \\
\hline 3662 & 268701
\end{tabular}

251 020СTS5 30SEP96 1K:2330301 L 80112000 1K2 3330801 L 80112010 $1 \kappa 2 \div 330301$ L 80112010 1K2C330BO1 M 8D112100 1\%:2ะ30BO1 0 801124CO

\begin{tabular}{rr}
180 & 6037 \\
180 & 13991 \\
1120 & 67058 \\
4 & 5026 \\
1 & 1257 \\
\hline 1485 & 113369
\end{tabular}

83 020CT95 31JANG6 IK2C330C01 L 80112000 ]K2C330C01 L 8D112010 $1 \mathrm{~K} 2 \mathrm{Cs} 30 \mathrm{CO} \mathrm{L} 80112010$

\begin{tabular}{rr}
44 & 1476 \\
576 & 44772 \\
61 & 4742 \\
\hline$\ldots \ldots . .$. & \\
681 & 50990
\end{tabular}

251 020CT95 30SEPG6 1K2 2330001 L 80570000 $1 \mathrm{~K} 2 \approx 330001 \perp 80570000$ 1K2E330001 L 80570010 $1 \kappa 26330001 \perp 80670010$ IN25330001 н 80670100 $1 \mathrm{~K} 25330001080570210$ 1K2:330001 0 8D6704C0 IK2C330D02 L 8A400000 1K2:330002 L BFA00010 $1 \mathrm{~K} 25330002 \mathrm{~L} 8 A 400010$ 3K2ะ330002 O \&A4004CO ]K2:530003 L 8A400010 1\%:2Е30003 L 84400010 1K2:330002 H $8 A 400100$ 1122330002 L 84400000

\begin{tabular}{|c|c|}
\hline 905 & 43560 \\
\hline 181 & 8702 \\
\hline 2683 & 198998 \\
\hline 181 & 13425 \\
\hline 18 & 22870 \\
\hline 12 & 15079 \\
\hline 2 & 2890 \\
\hline 450 & 17501 \\
\hline 80 & 5128 \\
\hline 320 & 20512 \\
\hline 1 & 1759 \\
\hline 80 & 5128 \\
\hline 400 & 25640 \\
\hline 11 & 13697 \\
\hline 80 & 3111 \\
\hline 5406 & \\
\hline
\end{tabular}


WHC-SP-1101

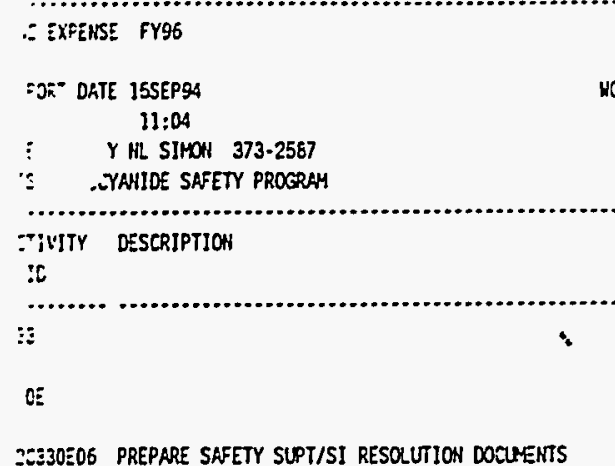

בE330:06 PREPARE SAFETY SUPT/SI RESOLUTION DOCUNENTS

WORK PACKAGE TOTALS START DATE O1OCT92 FIN DATE O7JULOO

YTS FERPOCAMIDE PROCPAY

ORIG EARLY EARLY COST RESOURLE BUDGET BUDSET

DUR START FINISH ACCOUTT OUANTITY COST

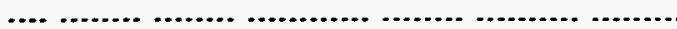

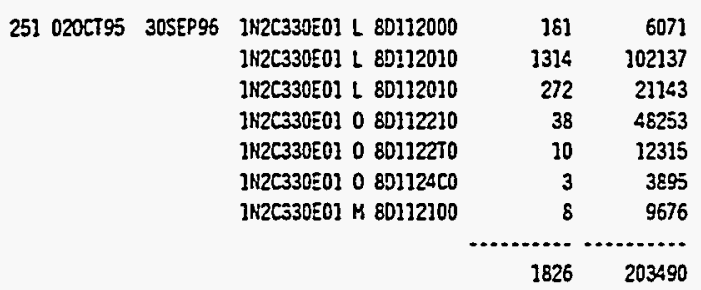

of

.2C:30F20 CORE SAMPLE/DATA INTERPRETATION

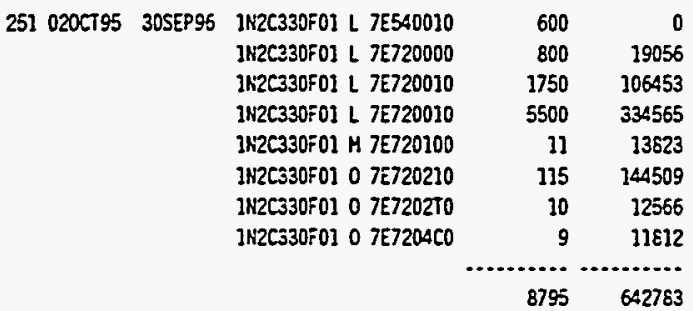

251 020CT95 30SEP95 $162 C 330501078260210 \quad 193 \quad 242524$

\begin{tabular}{|c|c|}
\hline 100400 & 13 \\
\hline
\end{tabular}

$206 \quad 258860$

$22062 \quad 1936194$

F2CF_DADG CAP MAMLGEMENT FECN TAMKS FY96

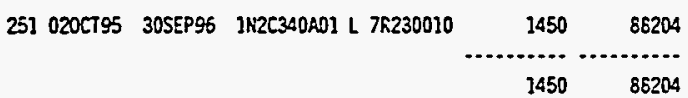


WHC-SP-1101

$\because$ EXPENSE FYgS

:PJTE DATE 15SEPQC

$11-04$

:EPAEED BY ML SIMON 373-2587

- S FERROCYANIDE SAEETY PROERAY

$\begin{aligned} & \text { ORIG EARLY EARLY COST } \\ & \text { DLR STAST FINISH ACCOUT }\end{aligned}$
IS

0

.2C340:20 NEUTRON PROSE MOISTLRE MONITOR LON ENGR

03

2T300020 SLRFACE ROISTLRE MEASURIKTO (CPAC) FYG6

oE

RC3:OSOS EHIIHEEFIK DOCUENT CONTROL FY96

oi

.2C340F:2 FRESSIKE MDNTTORING ENGINEEPING \& INSTALLATION IN2C340503 L 7C500060 IN2C340F02 \& 7EA40000 1N2C340F01 L 7EA40000 IN2C340FOI L 7EAAODIO IN2C340FOI L TEA4001O 1K2C340F01 L 7EA40010

\begin{tabular}{|c|c|c|c|}
\hline \multirow[t]{11}{*}{2510202795} & 1N2C340C02 L 7EA30010 & 611 & 37167 \\
\hline & $1 N 2 C 340 C 0] \perp 80520000$ & 523 & 17761 \\
\hline & $1 \% 2 C 340201$ L 82520000 & 1720 & 58411 \\
\hline & $3 k 2 C 340 C 01$ L 60520010 & 506 & 39827 \\
\hline & $1 N 2 C 340 C 01080520150$ & 5 & 6283 \\
\hline & IN2C340CO1 L 8D5202LO & 1 & 1257 \\
\hline & $1 \mathrm{~N} 2 \mathrm{C} 340 \mathrm{CO}\rangle \mathrm{L}$ \52720060 & 195 & 9190 \\
\hline & 1N2C340C07 L 52720060 & 415 & 19459 \\
\hline & 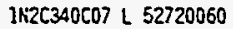 & 76 & 3564 \\
\hline & $1 \mathrm{H} 2 \mathrm{C340C03} \mathrm{L} 8 \mathrm{AA} 00010$ & 144 & 9230 \\
\hline & & 4197 & 202150 \\
\hline
\end{tabular}

251 020CT95 30SEP96 IK2C340001 L 80832010 ]H2C340001 L 52720060 $1 \mathrm{N2C3400010} \mathrm{80832210}$ IN2C340D01 0 8DS324CO

\begin{tabular}{rr}
2831 & 215260 \\
1475 & 69163 \\
44 & 55290 \\
3 & 3770 \\
\hline 4353 & 344483
\end{tabular}

251 020CT95 30SEP96 1N2C340EO1 L 80730010

$\begin{array}{rr}200 & 15072 \\ 200 & 15072\end{array}$

\begin{tabular}{rr}
580 & 25526 \\
415 & 18264 \\
330 & 7861 \\
320 & 7622 \\
170 & 10341 \\
920 & 55964 \\
1667 & 101404 \\
\hline $4 .$. &......... \\
\hline 402 & 226981
\end{tabular}


WHC-SP-1101

1.2 EXFENSE FYg6

EFSET DATE 15SEPgA

11:04

$=\quad$ IY NL SIHON 373.2587

IS STYMIDE SAEETY PROSRAM

ETIWT DESCRIPTIOH

(1)

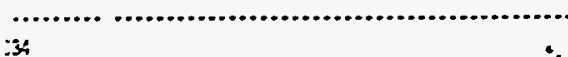

OG

.2C34GEZO HEUTRON PROSE HOISTLRE MONITOA LOH INSTALL
MORK PACLAGE TOTALS

OUR START FINISH ACCOUNT
START DATE 010CT92 FIN DATE O7JU.00

DATA DATE O10CT93 PAGE NO. 4
OUANTITY COST

$251020 C 7953055 P 96 \quad$ 1N2C340602 L 7C500050

OH

12C34OH2S IR SCAN DEVICE HOD \& SCANS

oJ

N2C240:20 ELECTROHANETIC IHDUCTION (EMIP) 1\%2C340502 L 76500050 1N2C340G02 L 75500050 ]N2C340501 L 80520000 $1 \times 2 C 340501<80520000$ 1N2C340501 L 80520000 11:26340601 L 80520010 IN2C340601 L 80520010 [N2C34000] L 80520010 1N2C340501 L 80520010 IN2C340501 L 80520040 1H2C340G01 L 80520040 1N2C340G01 L BO520040 1N2C340601 L 80520050 ]N2C340601 H 80520100 1א22C340GD] H 80520150 1N2C3405D1 M 80520150 2N2C340501 H 80520190 2N2C340501 O 80520210 1N2C340G01 O 805202T0 2N2C340501 $0805204 C 0$ 1 H2C340501 O 805204PO 1N2C34CGO1 0 80520040 1\$2C340G01 0 8D5206PO

\begin{tabular}{|c|c|c|}
\hline & 135 & 5985 \\
\hline & 951 & 42308 \\
\hline & 272 & 11971 \\
\hline & 8 & 255 \\
\hline & 262 & 8898 \\
\hline & 374 & 12701 \\
\hline & 195 & 15427 \\
\hline & 588 & 46281 \\
\hline & 347 & 27312 \\
\hline & 785 & 61761 \\
\hline & 876 & 22215 \\
\hline & 264 & 6695 \\
\hline & 10 & 254 \\
\hline & 87 & 5066 \\
\hline & 30 & 37698 \\
\hline & 7 & 8796 \\
\hline & 1 & 1257 \\
\hline & 8 & 10053 \\
\hline & 4 & 5026 \\
\hline . & 12 & 15079 \\
\hline & 5 & 6283 \\
\hline & 9 & 11309 \\
\hline & 12 & 15079 \\
\hline & 10 & 12943 \\
\hline & 5263 & 390654 \\
\hline
\end{tabular}

190 O2JAN96 30SEP95 IN2C340HOI L 7EA30010

\begin{tabular}{rr}
2133 & 129750 \\
\hline 2133 & 129750
\end{tabular}

251 020CTSS 30SEP96 IH2C340J01 ᄂ 52720060 1N2C340J01 \& 80520000 IW2C340J0I L 60520000 ]N2C340J01 L 80520010 IN2C340J01 L 80520010 1H2C340J01 \& 80520040 IN2C340J01 L 80520040 1K2C340J01 L 80520060 2N2C340301 H 80520100 1N2C340J01 M 8D520150 2N2C340J01 0 8D5202LO 1K2C340J01 O 805202T0 1N2C340J01 O 805204PO 1K2C340J01 O 805204CO

$\begin{array}{rr}437 & 20491 \\ 8 & 255 \\ 151 & 5128 \\ 97 & 7625 \\ 521 & 41008 \\ 10 & 254 \\ 264 & 6595 \\ 437 & 0 \\ 10 & 12556 \\ 6 & 7540 \\ 2 & 2513 \\ 7 & 8168 \\ 3 & 4021 \\ 2 & 2513\end{array}$




\section{WHC-SP-1101}

$\because$ J)ENSE FY9S

$\because:=:$ DATE 15SEPgA

11:0s

:Z= FED BY NC SIHON 373-2587

$\square$ IEEROCYANIDE SAEETY PROGRAY

::iiY DESCRIPTIOA

$:$

$\because$

$\therefore$

$\because$
WTS FERROCYANIDE PROGRAM

NORK PACKAGE TOTALS START DATE O10CTS2 FIN DATE OTJU.00 DATA DATE OHOCTS3 PATE NO. 5

ORIG EARLY EARLY COST

DUR SIART FINISH ACCOUNT
BUDEET BUDEST

QUANTITY COST

\begin{tabular}{|c|c|}
\hline 1954 & 116786 \\
\hline 23953 & 2516080 \\
\hline 46014 & \\
\hline 46014 & 3452274 \\
\hline
\end{tabular}


WHC-SP-1101

1.2 EXPENSE FYG7

:PST:T OATE 15SEPS

MORK PADAGE TOTALS

START DATE OMOCT92 FIN DATE O7JU,00

$\therefore \quad$ BY NL SIMON 373.2557

$\therefore \quad$ LYANIOE SAEETY PROGRAY

DATA DATE OHOCT93 PAES NO. I

:IVITY DESCRIPTION ORIG EARLY EARLY COST RES RESOLCE BUDEET BUDETT

10

DUR START FINISH ACCOUNT

QUANTITY COST

of

.2T330R30 CAP HAHREEHENT FEQT TANK SAEETY FY97

251 010CT96 30SEPS7 IK2C330A01 L 7R260010

IKi2:330AG1 L 7R260010

IH2C330A01 0 7R260210

IK2C330A01 0 7R2604CO

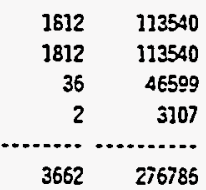

Q8

IZCZ3GEOS UPDATE SAETY DJCUENTATION FYG7

251010 T95 30SEFG7 J1i2C330901 L 80112000 IKi2C330901 \& 8D112010 $1 K 2 C 330201 \perp 80112010$ ]K2 330801 н 80112100 2K2C33DSOI 0 801124CO

\begin{tabular}{rr}
180 & 6219 \\
180 & 14413 \\
1120 & 89678 \\
4 & 5176 \\
1 & 1294 \\
\hline 1485 & 116762
\end{tabular}

$O D$

K2Eב30DI8 TECHIICA SUPPORT SI RESOLUTIO: FY97

HZCZ3GEOB TRAHS DOE RESOLVE FECN SI ALL TANKS

$124010 \mathrm{CT95}$ 3144897 IK2E330501 L 80112000 $181 \quad 6254$ 1N2C330E01 L 80112010 $1314 \quad 105212$ I1:2Cะ30501 L 80112010 $272 \quad 21779$ 1K2द330501 $080112210 \quad 38 \quad 49706$ 1li2Eะ30E01 $089112270 \quad 10 \quad 12685$ 1K2:330501 0 801124CO $3 \quad 4013$ IK2:330501 M 8DI12100

]ก2 $330001\llcorner 80570000$ $1 \mathrm{~K} 2[330001$ L 80570000 JK2C:30DO1 M 8D570100 IK2C 330001 L $8 D 570010$ 1K2โ330D01 0 8D5702TO 1K2CE30001 O $805704 C 0$ $1 \mathrm{~N}^{2}=30002 \mathrm{~L}$ 84400010 1 1\%2Cะ30002 L 8A400000 $1{ }^{\prime} 2$ CS30002 L $8 A 400010$ $1 \times 2 C 530002 L 8 A 400000$ IK2C330D01 L 8A400010 $1 \times 2 C 330002 \mathrm{M} 84400100$ 1K'2C330002 $O$ 8A4004CO

376427

$\begin{array}{rrr}8 & 9967 \\ 1626 & 209515\end{array}$


WHC-SP-1101

$\because$ :XPENSE FYG7

:QSTT OATE 15SEPSA

HORK PACXAGE TOTALS

START DATE O3OET92 FIN DATE O7JULOO

FAFED BY ML SIHON 373.2587

- FERROCYANIOE SAFETY PROGRAM

DATA DATE 010TT93 PAEE NO. 2

\begin{tabular}{|c|c|}
\hline $\begin{array}{l}\text { :IVity } \\
: \Sigma\end{array}$ & DESCRIFTION \\
\hline
\end{tabular}

$\vdots$

2:

$=: 33 \% 30$ CORE SAPLE/DATA INTERPRETATION

ORIG EARLY EARLY COST

DIR START FIN!SH ACCOUTT

RESOURCE BUDGET BUDEET

OUANTITY COST

\begin{tabular}{|c|c|c|c|c|}
\hline $187010 \tilde{T 95}$ & 27JUNG7 & IN2C330F02 \& 7E610010 & 60 & 3700 \\
\hline & & ]N2C330F01 L 7E720000 & 60 & \\
\hline & & $1 N 2 C 330 F 01 \perp 7 E 720010$ & 400 & \\
\hline & & $1 \mathrm{H} 2 \mathrm{C} 330 \mathrm{~F} 01$ L $7 E 720010$ & 2865 & 1795 \\
\hline & & 1N2C330F01 H 7E720100 & 5 & \\
\hline & & IN2C330F01 C 7E7202TO & 4 & \\
\hline & & JK2С330F01 0 7E7204CO & 1 & \\
\hline
\end{tabular}

Do

aC330604 FALSKE CONTRACT - SI RESOLUTION FYST

251010796 30SEF97 1N2C330G01 0 7R269210 $\quad 270 \quad 348976$ $1 \times 2 C 330601$ O $7 \AA 2604 C 0$
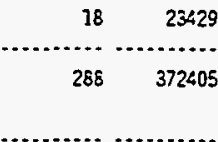

$15582 \quad 1574386$

\author{
34
}

OA

2:300ADB CAD MANGGEMENT FECN TANKS FYG7

05

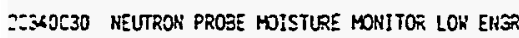

OD

2ES־0030 SIFEACE MDISTURE MEASURING (CPAE) FYGT

\begin{tabular}{|c|c|c|c|c|}
\hline & & \multirow[t]{2}{*}{$1 \times 2 C 33050107,2604 C 0$} & 18 & 23429 \\
\hline & & & 288 & 372405 \\
\hline & & & 15582 & 1574386 \\
\hline \multirow[t]{2}{*}{2510105795} & \multirow[t]{2}{*}{ 30SEP97 } & \multirow[t]{2}{*}{$1 K 2 C 340 A 01$ L 7R230010 } & 1450 & 90857 \\
\hline & & & 1450 & 90857 \\
\hline \multirow[t]{11}{*}{251 010ст96 } & \multirow[t]{11}{*}{ 30SEP97 } & $1 K 2 C 340 C 02$ L 75A30010 & 611 & $382 E 5$ \\
\hline & & $1 \mathrm{~N} 2 \mathrm{C} 340 \mathrm{CO} 1\llcorner 80520000$ & 523 & 18295 \\
\hline & & 1ki2C340C01 L 80520000 & 1720 & 60166 \\
\hline & & IN2C340COI L 80520010 & 506 & 41026 \\
\hline & & $2 \mathrm{~N} 2 \mathrm{C} 340 \mathrm{CO}$ o 80520150 & 5 & 6472 \\
\hline & & $112(340 C 0] \perp 80520210$ & 1 & 1294 \\
\hline & & $2 \mathrm{~N} 2 \mathrm{C} 340 \mathrm{CO} 07$ L 52720060 & 196 & 9467 \\
\hline & & $3 N 2 C 340 C 07$ L 52720050 & 415 & 20045 \\
\hline & & $1 N 2 C 340 C 07$ L 52720060 & 76 & 3571 \\
\hline & & $1 K 2 C 340 \operatorname{Cos} L 84400010$ & 144 & 9507 \\
\hline & & & 4197 & 202227 \\
\hline \multirow[t]{4}{*}{$251010 c i 96$} & \multirow[t]{4}{*}{ 30SEP97 } & $1 \mathrm{~N} 2 C 340001$ L 80832010 & 1855 & 145970 \\
\hline & & $1 \% 2 C 34000]$ L 52720060 & 956 & 46658 \\
\hline & & $1 \times 2 C 340001080832210$ & 29 & 37538 \\
\hline & & $1 \mathrm{~N} 2 \mathrm{C} 3400010 \mathrm{8DS324C0}$ & 2 & 2589 \\
\hline
\end{tabular}


WHC-SP-1101

$\therefore$ E.FENSE FY97

$\because \because$ DATE 15SEPYE HORK PACKAOE TOTALS STAET DATE OIOTT92 FIN DATE O7JLROO

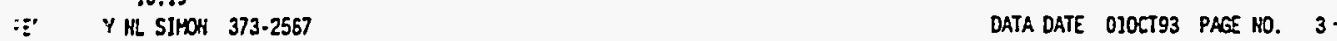

¿ . YANIOE SAETY PROGRAY

:-VITY DESCKIPTION

$\because j$

DUR STAKT FINISH ACCOUNT QUANTITY COST

泟

3

$\hat{\imath}$

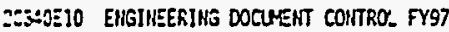

if

9EZOJFI8 PRESSLRE HOHITORING IMSTALLATION FECN TANIKS

li3C340330 MEUTRON PROBE HOISTURE MONITOR LOH INSTHLL
D...

...........

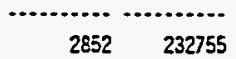

$251010 C T 96$ 30SEF97 1K2C340501 L 8D730010

$\begin{array}{rrr}200 & 15524 \\ 200 & 15524\end{array}$

$188010 \div 795 \quad 30 J 4 x \cdot 97$ 1K2C340F01 L 7C840050 IN2C340F0I $\perp 7$ CE40060 $500 \quad 22665$ IN2C340FO1 L TEA40000 $400 \quad 9812$ IN2C340FO1 L 7EA40000 $\quad 150 \quad 3680$ IN2C340FOI L TEA40010 $200 \quad 12532$ IN2C340F01 L 7EA40010 $1100 \quad 68926$ IN2C34OFOI L TEAADOIO 2000 125320

$4850 \quad 265600$

$251010 C T 95 \quad 3055057 \quad$ ]N2C340502 L 7C500050 4351 IN2C340602 L 7C500050 $272 \quad 12330$ IN2C340602 L 7C500050 $\quad 336 \quad 6165$ 1K2C340501 L 80520000 151 IN2C340G01 L $80520000 \quad 262 \quad 9165$ IN2C540GO] ᄂ $60520010 \quad 785 \quad 6362]$ IN2C340501 L 80520010 $405 \quad 32837$ JW2C340G0] L $80520010 \quad 588 \quad 47675$ 1N2C340501 L 80520010 $195 \quad 15892$ 1N2C340G01 L $80520040 \quad 876 \quad 22890$ JN2C340501 L 80520040 $\quad 198 \quad 5174$ IN2C340501 L 80520050 $67 \quad 5218$ 1N2C340501 H 8D520100 $6 \quad 10355$ IN2C340G01 $\mathrm{K} 80520150 \quad 5 \quad 6472$ IN2C340GOI M EC520150 $\quad 1 \quad 1294$ JN2C340501 M 80520190 8035 IN2C340501 $080520210 \quad 2 \quad 2589$ JH2C340501 0 805202TO 5 $1 N 2 C 3406010805204 C 0 \quad 120$ IN2C340601 0 805204PO 77 IN2C $340501087520540012 \quad 12$ 1N2C340GOI $08 D 52055^{\circ} \quad 5 \quad 6472$ IN2C340501 0 805206PO 
WHC-SP-1101

$\because$ EYEENSE FYg?

WTS FERROCYANIDE PROZRAY

EPNT DATE J5SEPQ4

$10: 19$

HORK PACKAGE TOTALS

START DATE OJOCTS? FIN DATE O7JU_OO

ZEPARED BY HL SIHOW $373-2587$

-S FERROCYANIDE SAEETY PROGRAM

DATA DATE O1OCT93 PAGE NO. 4

TIVITY DESCRIPTIOA

:

ORIG EARLY EARLY COST

RESOLRCE BUDEET BUDETT

DUR START FINISH ACCOUNT

QUANTITY COST

$\mathrm{OH}$

TEZCH:20 IR SCAN DEVICE HOD \& SCANS

187 010CT96 27JUN97 JK2C340HDI $\downarrow$ 7EA30010 $525 \quad 32897$

]H 2 C340+101 $\perp$ 7EA30010 $254 \quad 15916$

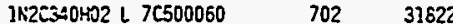

$1 \% 2 C 340 \% 03$ L $33300050 \quad 303 \quad 15911$

$1 \mathrm{~N} 2 \mathrm{C} 340 \mathrm{HO4} L 31 \mathrm{~N} 20000 \quad 309 \quad 10517$

1K2C340H04 L 7CBO0060 $468 \quad 21214$

1 1R2C340:45 0 7R280440

$15 \quad 19416$

$2575 \quad 147792$

oj

2C340J30 OTOCTROMAFETIC INDUCTION (EMIF)

\begin{tabular}{|c|c|c|c|c|}
\hline \multirow{18}{*}{\multicolumn{2}{|c|}{251 010СT95 30SEP97 }} & $2 N 2 C 340301<80520000$ & 113 & 3953 \\
\hline & & $11: 2 C 350301$ L 80520010 & 347 & 28135 \\
\hline & & $11 \mathrm{2C} 340,01<80520040$ & 132 & 3449 \\
\hline & & $1 k 2 C 340301$ L 52720050 & 327 & 15794 \\
\hline & & $1 k 2 C 340301<52720060$ & 327 & 15794 \\
\hline & & $1 \mathrm{~N} 263 \div 0 \mathrm{J01}$ M 80520100 & 5 & 6472 \\
\hline & & 1K2C380J01 M 80520150 & 3 & 3883 \\
\hline & & $1 k 2 C 340 J 010$ 8c5202LO & 1 & 1294 \\
\hline & & $1 \% 2 C 3403010805204 C 0$ & 49 & 63168 \\
\hline & & $1 \% 2 C 3401010805206 P 0$ & 1 & 1294 \\
\hline & & $1 K 2 \approx 340 \mathrm{~J} 01 \mathrm{~L} 80520010$ & 654 & 53026 \\
\hline & & & 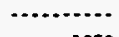 & (n)........ \\
\hline & & & 1959 & 195263 \\
\hline & & & ...... & $\cdots$ \\
\hline & & & 23055 & 1498166 \\
\hline & & & ........... & ......... \\
\hline & & & 38637 & 3072553 \\
\hline & & & 38637 & 3072553 \\
\hline
\end{tabular}


WHC-SP-1101

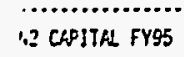

EFGFT DATE I5SEP94 RUW NO. 142 11:20

:E' , TY HL SIHON 373-2587

re, me: 4203

.IS FEKKOCYAMIOE SAFETY PROGRAM

\begin{tabular}{|c|c|c|c|c|c|c|c|}
\hline$\therefore 10$ & ACT DES & $\begin{array}{l}\text { EARLY } \\
\text { START }\end{array}$ & $\begin{array}{l}\text { EARLY } \\
\text { FINISH }\end{array}$ & $\begin{array}{l}\cos T \\
\text { ACCOUNT }\end{array}$ & RESQURCE & $\begin{array}{l}\text { BLDGET } \\
\text { OUANTITY }\end{array}$ & $\begin{array}{l}\text { BUDGET } \\
\text { COST }\end{array}$ \\
\hline \multicolumn{8}{|l|}{ II } \\
\hline \multicolumn{8}{|l|}{ oc } \\
\hline$: 26310007$ & PHL AGING NASTE STLDIES & 0300794 & 295EP95 & $2 \times 2 C 510001$ & $7 \% 26030 \mathrm{C}$ & 25 & 25000 \\
\hline i2C310019 & PHL COAPARE REAL WASTE W/SIHLLATED XASTE & $030 c 794$ & 23JWHS5 & $2 \times 26510001$ & $7826030 \mathrm{C}$ & 30 & 30000 \\
\hline & & & & & & 55 & 55000 \\
\hline
\end{tabular}

os

:2E310502 MOSSBAUER SPECTROEETER

YORK PACAKEE TOTALS

START DATE O1OCT92 FIN DATE O7JU_.0O

DATA DATE DIOCT93 PAGE NO. 1

$55 \quad 55000$

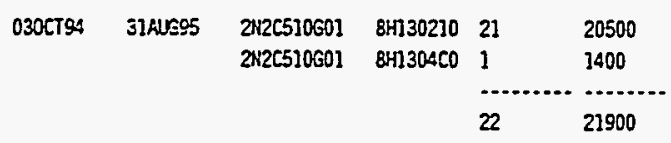

$\mathrm{OH}$

HZCSIOHOS IN-SITU RELATIVE HMITITY INSTRLMENTATIOH

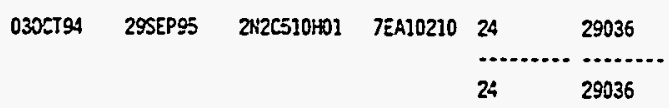

os

1:2C31CJOA IMFRARED SPECIATJON TRANSFER

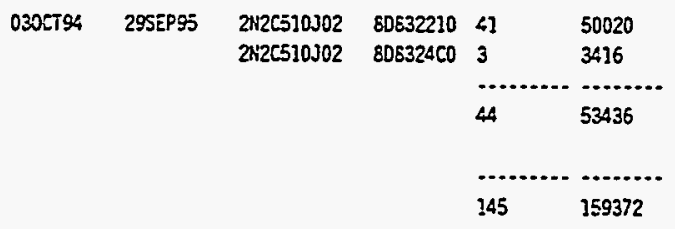

034

05

N2C340614 INSTALLATIOH OF TC TREES/HOOKUPS TMACS

\begin{tabular}{|c|c|c|c|c|c|}
\hline \multirow[t]{12}{*}{ 0305T94 } & 29SEPSS & $2 \mathrm{H} 2 \mathrm{C5} 50 \mathrm{BO4}$ & 52120010 & 46 & 4024 \\
\hline & & $2 \times 2 C 540504$ & 52120010 & 46 & 4024 \\
\hline & & $2 \times 2 C 540304$ & 52120050 & 360 & 23063 \\
\hline & & 2N2C54OBO4 & 52120050 & 360 & 23083 \\
\hline & & $2 \times 2 C 540 B O 4$ & 52120110 & 19 & 23180 \\
\hline & & $2 \times 2 C 540304$ & $521204 C 0$ & 3 & 3660 \\
\hline & & $2 \times 2 \angle 540 B C 5$ & 57570010 & 560 & 32743 \\
\hline & & $2 \times 2 C 540 B 05$ & 57570100 & 5 & 6100 \\
\hline & & 2N2C540305 & 57570400 & 1 & 1220 \\
\hline & & $2 N 2 C 540305$ & 7EA10010 & 550 & 33074 \\
\hline & & $2 \times 26540312$ & $7 \mathrm{R} 280 \div 40$ & 25 & 30012 \\
\hline & & & & 1985 & 184203 \\
\hline
\end{tabular}


WHC-SP-1101

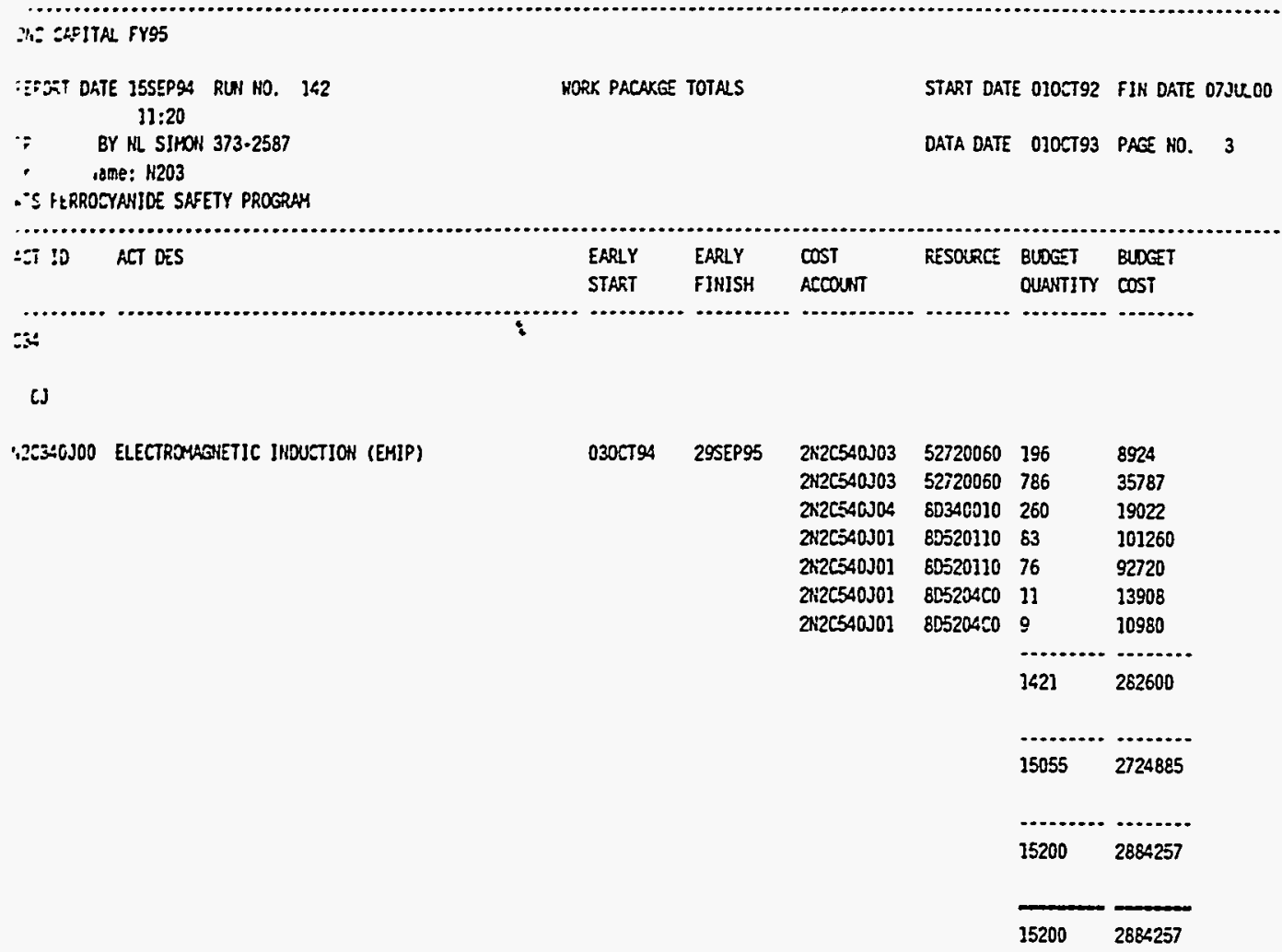

F 7.4-7.63 
WHC-SP-1101

$\because$ CAEITAL FY95

SDOET DRTE LSSEP94 RUN NO. 132 HORK PACAKGE TOTALS START DATE O1OCT92 FIN DATE OTJUL00

TERARED BY KL SIHON 373-2587

- t. lact kiane: $\mathrm{N2O3}$

DATA DATE O1OCTS3 PAGE NO. 1

.TS FERPOSYANIDE SAEETY PROGRAY

\begin{tabular}{|c|c|c|c|c|c|c|c|}
\hline$=10$ & ACT DES & $\begin{array}{l}\text { EAREY } \\
\text { START }\end{array}$ & $\begin{array}{l}\text { EARLY } \\
\text { FINISH }\end{array}$ & $\begin{array}{l}\text { Cost } \\
\text { Accoust }\end{array}$ & RESOLRCE & $\begin{array}{l}\text { BUDEET } \\
\text { QUANTITY }\end{array}$ & $\begin{array}{l}\text { BUDEET } \\
\text { COST }\end{array}$ \\
\hline $\begin{array}{l}=1 \\
00\end{array}$ & $\because$ & & & & & & \\
\hline 340020 & SLRFACE MOISTURE KEASURINS (CPAC) FY96 & 0200795 & 30SEP96 & $2 \times 2<640001$ & 80832010 & 602 & 45987 \\
\hline & & & & $2 \times i 2 \div 640001$ & 80832110 & 66 & 82936 \\
\hline & & & & $2 \pi i 2=649001$ & $805324 \mathrm{CO}$ & 4 & 5026 \\
\hline & & & & $2 \times 2 C 640032$ & 52100060 & 554 & 36592 \\
\hline & & & & $2 \pi 2 \div 540052$ & 52100060 & 554 & 36592 \\
\hline & & & & $2 \times i 2 C 6500 \hat{2} 2$ & 52100060 & 554 & 36592 \\
\hline & & & & & & 2334 & 243724 \\
\hline of & & & & & & & \\
\hline 20340514 & PRESSLRE HOHITORING ENEINEEFING \& INSTALLATION & $020 \approx T 95$ & 30SEP95 & $2 \times 2<650 \div 01$ & 7EAA0010 & 1300 & 79079 \\
\hline & & & & $2 \pi i 2 c 6<0 \% 02$ & 7EAA0100 & 63 & 79166 \\
\hline & & & & $2 \times 2<5<0=01$ & 7EA403RO & 240 & 262279 \\
\hline & & & & $2 \pi i 2 \div 5<0=01$ & 7EAAOACO & 7 & 8796 \\
\hline & & & & & & (n.......... & …...... \\
\hline & & & & & & 1610 & 429320 \\
\hline $0 \overline{3}$ & & & & & & & \\
\hline 340620 & MEUTRON PROSE MDISTURE MOAITON LON INSTALL & 0200795 & 30SEPS5 & 21245<030E & 52720060 & 2622 & 122946 \\
\hline & & & & $2 k i 2 c 5<9306$ & 52720060 & 2622 & 122946 \\
\hline & & & & $2 \mathrm{r} 25540 \Omega 5]$ & 80520000 & 2279 & 77395 \\
\hline & & & & 2120540301 & 80520010 & 1521 & 119718 \\
\hline & & & & $2 \times 2 \div 5 \div 0501$ & 80520040 & 132 & 3348 \\
\hline & & & & $2 \times 2 \div 540501$ & 80520110 & 15 & 18849 \\
\hline & & & & $2 \times 2 \div 540 \geq 01$ & 80520110 & 200 & 251320 \\
\hline & & & & 2N2CECGG & $805204 C 0$ & 32 & 40211 \\
\hline & & & & & & ........... & $\ldots \ldots$ \\
\hline & & & & & & 9423 & 756732 \\
\hline $\mathrm{OH}$ & & & & & & & \\
\hline $340 \mathrm{HOA}$ & IR SCAN OEVICE HOD \& SCANS & 02JANG5 & 3OSEFSE & $2125540+101$ & 7EA30010 & 423 & 25731 \\
\hline & & & & 21:2C6애DI & 7ER30110 & 39 & 49007 \\
\hline & & & & $2 x 22540+207$ & 52100060 & 292 & 19287 \\
\hline & & & & $2 \pi \cdot 2 c 5=0407$ & 52100060 & 292 & 19287 \\
\hline & & & & $2 \times 225=0407$ & 52100060 & 292 & 19267 \\
\hline & & & & $212235 \div 3 \div 21$ & 7EA30110 & 55 & 50113 \\
\hline & & & & $2 \times 2<5<0 \div 30 ?$ & 7EA30110 & 16 & 20106 \\
\hline & & & & $2 \pi 2 C 54043 \mathrm{i}$ & $75 \mathrm{~A} 304 \mathrm{CO}$ & 2 & 2513 \\
\hline & & & & 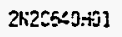 & $7 E A 304 C 0$ & 5 & $62 \varepsilon 3$ \\
\hline & & & & & & 1416 & 230513 \\
\hline
\end{tabular}

21:2EGEOHDI 7ER30110 $39 \quad 49007$

$\begin{array}{llll}52100060 & 292 & 19287\end{array}$

2X2C54040i 75A304CO 2

$1416 \quad 230513$

F $7.4-7.64$ 
WHC-SP-1101

$\because$ CAPITAL FY96

:FJFT DATE 15SEP94 RUW HO, 132 HORK PACAKGE TOTALS START DATE O1OCT92 FIK DATE O7JU.OD

10:35

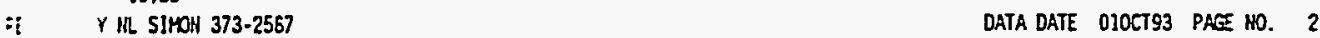

C. ,ane: $\mathrm{N203}$

-S FERROCYANIDE SAFETY PROGRAY

IT ID ACT DES EARLY EARLY COST RESOLRCE BLDEET BUDGET

START FINISH ACCOUNT QUUATITY COST

3

os

$.2[340 \mathrm{20}$ ELECTRQARGNETIC INDJUTION (EMIP)

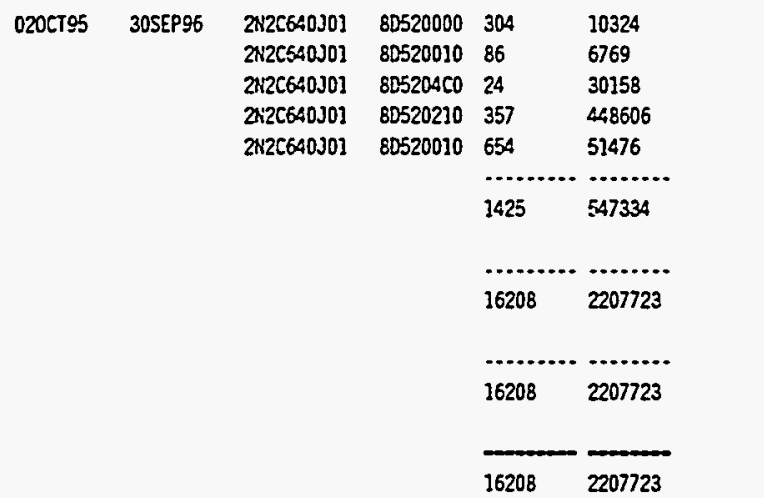


WHC-SP-1101

$\because$ : 2 : IIN FY97

::3TT DRTE J5SEPGA RUN NO. 1333 WORK PACAKGE TOTALS START DATE O1OCT92 FIN DATE 07JU.00

10.37

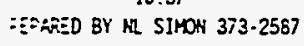

$\because \because$ eet Hame: 1203

- S FERROCYANIDE SAFETY PROGRAY

\begin{tabular}{|c|c|c|c|c|c|c|c|}
\hline 10 & $A C T D S S$ & $\begin{array}{l}\text { EARLY } \\
\text { START }\end{array}$ & $\begin{array}{l}\text { EARLY } \\
\text { FINISH }\end{array}$ & $\begin{array}{l}\cos T \\
\text { Account }\end{array}$ & RESOLRCE & $\begin{array}{l}\text { BUDGET } \\
\text { QUANTITY }\end{array}$ & $\begin{array}{l}\text { BUOGET } \\
\text { COST }\end{array}$ \\
\hline
\end{tabular}

o

0

23340530 SLRFACE MOISTLRE YEASLRING (CPAC) FY97

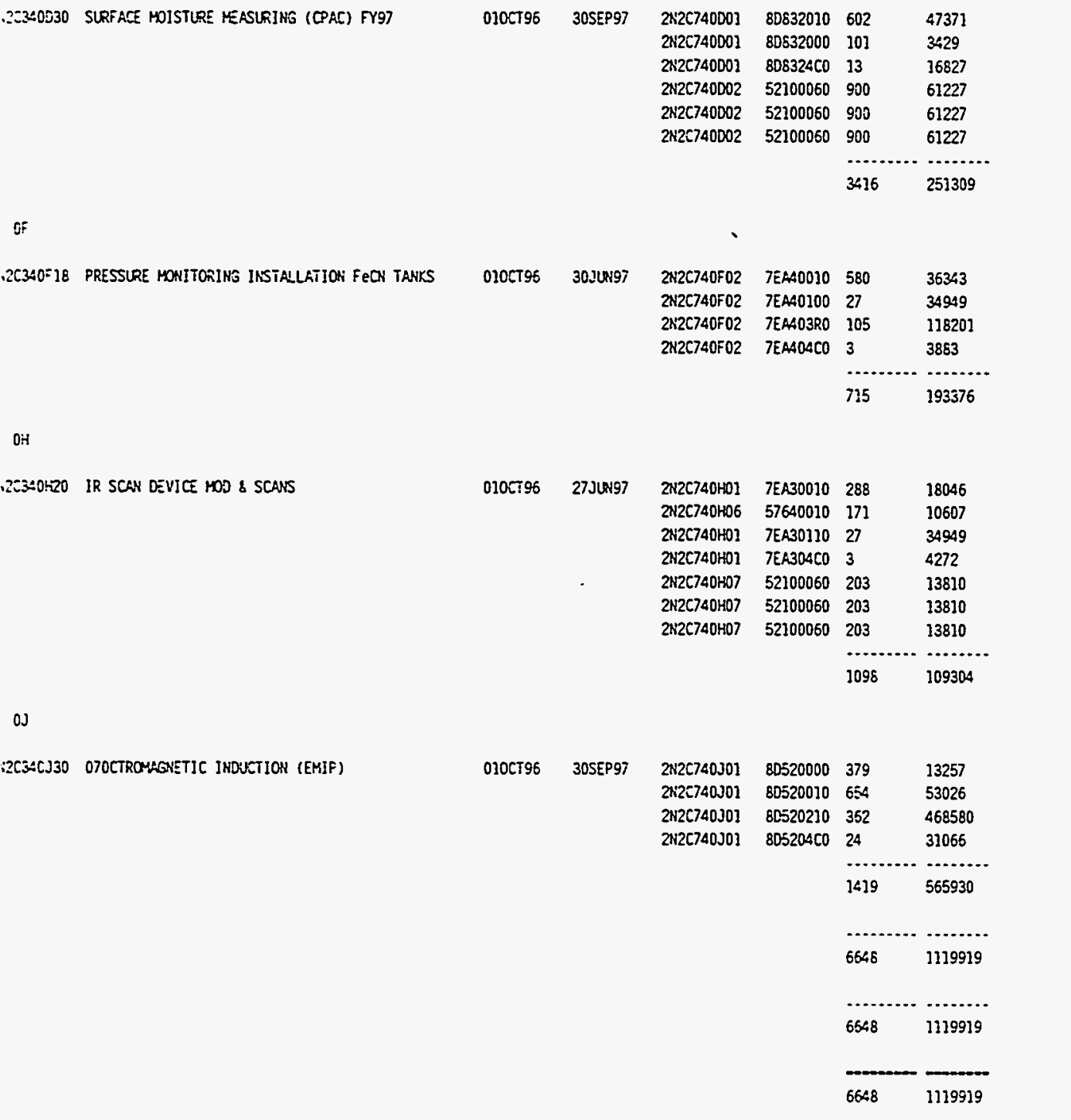

$\mathrm{OH}$

20340420 IR SCAN DEVICE HOJ \& SCANS

0.J

:2C3ACJ30 O7OCTROMLGETIC INDUETION (EMIF)
DATA DATE O1OCT93 PAGE ND. 1 of

2C340:18 PRESSURE MONITORING INSTALLATION FECN TAMKS
WORK PACAKE TOTALS 


\section{9'L-Hㄴ.}
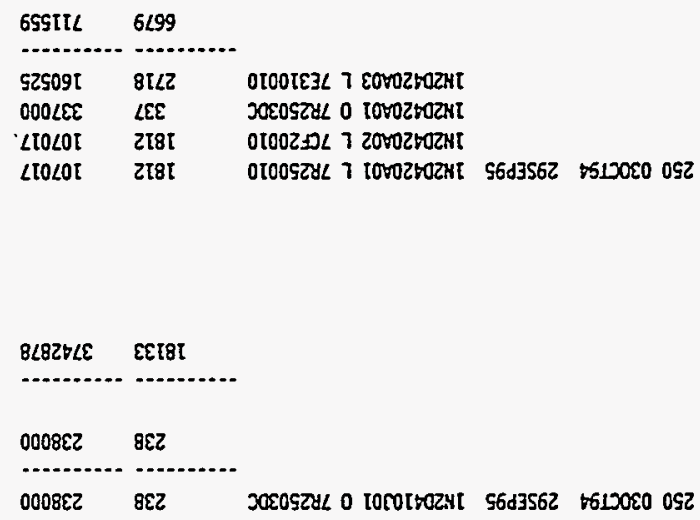

S6RJ SHLCOLINOH 3OYdS BOAY OOVOZSOZK

vo

210

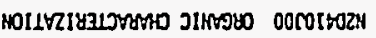

co

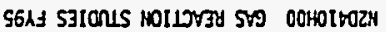

\section{HO}

S5AJ SHSIWGOZH HOLLHבLZY S\$O 0030tHOZN

$$
10
$$<smiles></smiles> 
WHC-SP-1101

OX.......

DORT OATE 15SEPQ4

$14: 11$

:PARED BY LA YEATER 373-1903

"S FLAMHBLE GAS SAFETY PROGRAH

Civitr DESCRIPTIOA

ID

DIG EARLY EARLY COST

DUR START FINISH ACCOUNT

RESORCE BUDGET BUDGET

START DATE 010CT92 FIN DATE 30SEP05

LORK PACKAGE TOTALS

OATA DATE OJOCT93 PAGE HO. 2
2

OE

D220E00 ISSU INTERPRETIVE REPT 103-SY CORE SAMPLE DATA

20420S0S RETAIHEO GAS EVALLRTIOH FY9S

OA

2DA30A05 CLOSE SY FARH USOS (SI 21)

03

20430310 COOSE AM FARM USO FLAM GAS TAHKS

Da

20440A02 PROGRAY KAMAGEMENT FY95

(1)

20440312 SY FARM SPARK RESISAHT FAN (SI 2k)
250 030CT94 295EP95 1N20420E02 L 7E310010 $4572 \quad 270022$ 1K20420EO1 O TR2503DC $\quad 188 \quad 188000$ 1H20420E01 $\perp$ 7R25021C $264 \quad 264000$ IN20420E03 L TR250010 $423 \quad 24982$

$5447 \quad 747005$

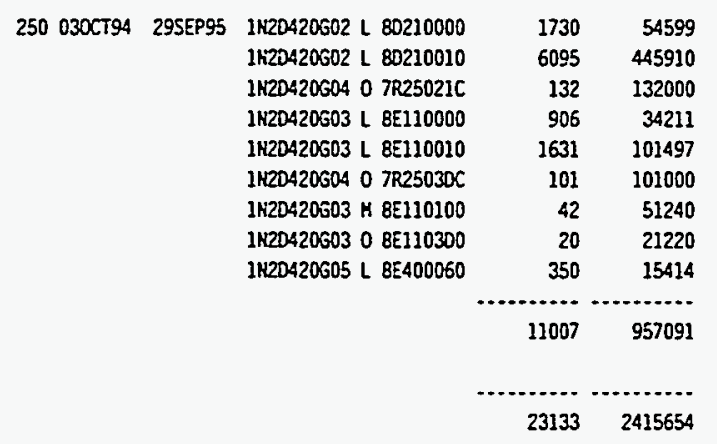

$124030 C T 94$ 31MAR95 IN20430403 L 7R25021C $250 \quad 250000$ 1N20430A02 L $80114000 \quad 544 \quad 17713$ IN2DA30A02 ᄂ 80114010 $2243 \quad 169279$

$3037 \quad 435992$

42 05JUL95 31AUG95 IN20430301 O 7R25021C $1 N 20430303$ L 80114000 1N20430303 L 80114010

\begin{tabular}{rr}
250 & 250000 \\
544 & 17713 \\
2243 & 169279 \\
\hline 3037 & 436992 \\
\hline & \\
\hline 6074 & 873984
\end{tabular}

250 030CT94 295EP95 1N2D440A01 L 7R250010 1 1N20440A02 L TEA00010 IK2D440A03 L 7CH00010

$1812 \quad 107017$

$1000 \quad 59060$

1000.5960.

$3812 \quad 225137$

230 030C794 31AUG95 1N20440801 ᄂ 7CH30010 $779 \quad 46008$ 
WHC-SP-1101

H2 EXPEKSE

EPORT DATE 15SEP94

$14: 11$

HOPX PACKAGE TOTALS START DATE 010CT92 FIN DATE 30SEP05

R BY LA YEATER 373-1903 Data DATE O10CT93 PAGE hO. 3

.7 ABLE GAS SAFETY PROCRAM

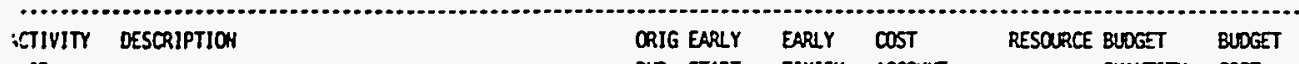

ID OUR START FIHISH ACCONTT QUANTITY COST

44

OB

$779 \quad 46008$

oc

:2D44OC12 EXPEDITE DESG/COHSTRU BACKUP PORTB EXH SY-101

$1727 \quad 101997$

$1727 \quad 101997$

OD

H2D440DO2 VIDEO SYSTEH UPGRADS FY95

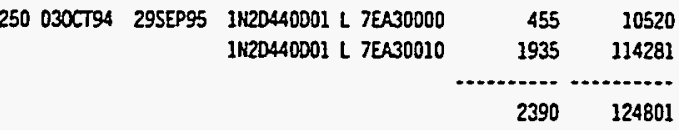

OE

N2OA40E1O TEKPERATURE UPGADES FY95

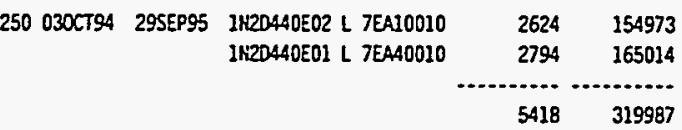

of

Hir , SURFACE LEVEL UPGRADS FY95

250 030CT94 29SEP95 IHLD440F01 L 7EA30010 $\quad 997 \quad 58883$

OG

M20440502 AH FARY VEYT UPGRADSS FY95

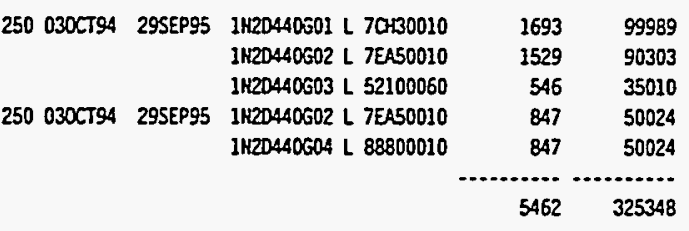

of

M2D44OHOG GAS HONITOR UPGRADES FY95

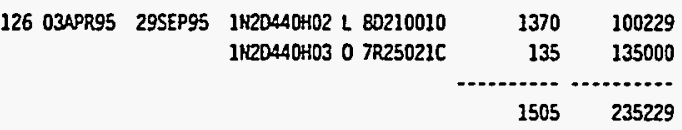

OK

N2D44OKO2 IHSTL AHONIA MONITORS 241-AHIAH FARHS

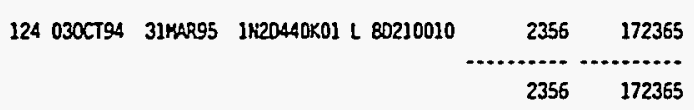

os

N20440HOO IHSTALL DST/SST GAS HOHITORS (SI 2h) 
WHC-SP-1101

2 CAPITAL

SPORT DATE 15SEP94 RUN HO. 382

$08: 28$

REPARED BY LA YEATER 373-1903

roject Hane: $\mathrm{K202}$

.TS FLATHABLE GAS SAFETY PROGRAY

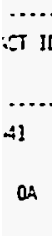

oc

20410CO0 GAS GEHERATION MECHANISYS FY95

OE

20410E00 GAS RETENTIOH MEOHANISHS FY95

Z20420E00 WASTE BEHAVIOR EYALLATION FY95

as

120420504 RETAINED GAS EVALLATION FYgS
WORK PACAKGE TOTALS START DATE 010CT92 FIN DATE 3OSEPO5

DATA DATE OLOCTS3 PAGE NO. 1

EARLY EARLY COST RESORCE BUDGET BLDGET

START FIHISH ACCOUNT

...........

\begin{tabular}{|c|c|c|c|c|c|}
\hline $030 C 794$ & 29SEP95 & $2 \mathrm{~N} 20510 \mathrm{AD2}$ & $7 R 25030 \mathrm{C}$ & 50 & 50000 \\
\hline & & & & 50 & 50000 \\
\hline
\end{tabular}

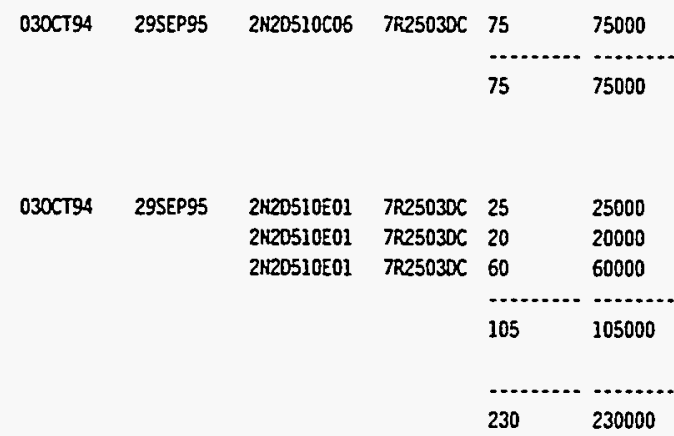


1.2 CAPITAL

EPORT DATE 15SEP94 RUN HO. 382 $08: 28$

RE YY LA YEATER 373-1903

rC ine: $\mathrm{H2O2}$

TS FLAHABLE GAS SAFETY PROGRAY

\begin{tabular}{|c|c|c|c|c|c|c|c|}
\hline 10 & ACT DES & $\begin{array}{l}\text { EARLY } \\
\text { START }\end{array}$ & $\begin{array}{l}\text { EARLY } \\
\text { FIHISH }\end{array}$ & $\begin{array}{l}\cos T \\
\text { Account }\end{array}$ & RESOURCE & $\begin{array}{l}\text { BUDGET } \\
\text { QUANTITY }\end{array}$ & $\begin{array}{l}\text { BLOGET } \\
\text { Cost }\end{array}$ \\
\hline
\end{tabular}

$\because 4$

00

20240DD2 VIDEO SYSTEH UPGRADS FY95

WORK PACAKGE TOTALS

START DATE O10CT92 FIN DATE 3OSEPO5

\begin{tabular}{|c|c|c|c|c|c|}
\hline 030Ст94 & 29SEP95 & $2 \mathrm{H} 20540011$ & 31111010 & 80 & 5413 \\
\hline & & $2 \mathrm{~N} 20540013$ & 31440010 & 160 & 11202 \\
\hline & & 2 2N20540016 & 31440010 & 112 & 7841 \\
\hline & & $2 \mathrm{~N} 2054001 \mathrm{I}$ & 31111010 & 24 & 1624 \\
\hline & & $2 \mathrm{~N} 20540012$ & 38220010 & 160 & 10577 \\
\hline & & $2 \mathrm{H} 20540012$ & 38220010 & 112 & 7474 \\
\hline & & 2H2D5400007 & 52000010 & 255 & 23316 \\
\hline & & $2 \mathrm{~K} 20540005$ & 52700010 & 5160 & 320488 \\
\hline & & $2 \times 20540014$ & 52700010 & 720 & 44719 \\
\hline & & $2 \times 20540005$ & 57500010 & 567 & 33152 \\
\hline & & 2420540005 & 57500010 & 1600 & 93552 \\
\hline & & $2 \mathrm{H} 20540006$ & 76900010 & 900 & 53154 \\
\hline & & $2 \mathrm{~N} 20540003$ & 7CF80000 & 385 & 8901 \\
\hline & & $2 \mathrm{~N} 205400003$ & 7CF 80000 & 230 & 5318 \\
\hline & & $2 \mathrm{~N} 20540003$ & 7CF80010 & 3665 & 216455 \\
\hline & & 2N205400003 & $7 C F 80010$ & 1890 & 111623 \\
\hline & & 2N2B540DOB & 70430010 & 790 & 46657 \\
\hline & & $2 \mathrm{~N} 20540001$ & TEA30000 & 560 & 12947 \\
\hline & & $2 \mathrm{H} 20540001$ & 7EA30000 & 120 & 2774 \\
\hline & & $2 \mathrm{~K} 20540001$ & 7EA30010 & 2860 & 168912 \\
\hline & & $2 \mathrm{~N} 205400001$ & 7EA30010 & 1665 & $\mathbf{9 8 3 3 5}$ \\
\hline & & $2 \mathrm{H} 20540014$ & 7E640000 & 140 & 3237 \\
\hline & & $2 \hbar 20540014$ & $7 E 640010$ & 955 & 56402 \\
\hline & & $2 \mathrm{H} 20540002$ & 7EA30110 & 709 & 864980 \\
\hline & & 2N20540002 & $75 A 30110$ & 165 & 201300 \\
\hline & & 2N20540010 & 88800000 & 60 & 1398 \\
\hline & & 2N20540010 & 88800000 & 130 & 3029 \\
\hline & & $2 N 20540010$ & 88800010 & 415 & 24510 \\
\hline & & 2N20540010 & 88800010 & 275 & 16242 \\
\hline & & $2 \times 20540009$ & 80110000 & 40 & 1302 \\
\hline & & $2 \mathrm{~K} 205400009$ & 80110000 & 80 & 2605 \\
\hline & & 2420540009 & 80110010 & 450 & 33962 \\
\hline & & 2H2D540009 & 80110010 & 250 & 18868 \\
\hline & & $2 \mathrm{H} 205400004$ & 80200010 & 100 & 7316 \\
\hline & & $2 \mathrm{H} 205400004$ & 80200010 & 1240 & 90718 \\
\hline & & & & & (n......... \\
\hline & & & & 27025 & 2610402 \\
\hline 030СТ94 & 29SEP95 & 2120540E03 & 7EA30110 & 214 & 261080 \\
\hline & & 2H20540EO4 & 7R25021C & 300 & 300000 \\
\hline & & & & $\cdots$ & (2...... \\
\hline & & & & 514 & 561080 \\
\hline 030CT94 & 29SEP95 & 2H20540F03 & 7EA30110 & 227 & 276940 \\
\hline & & & & $\cdots$ & ....... \\
\hline & & & & 227 & 276940 \\
\hline 030СT94 & 29SEP95 & 2N20540601 & 70430110 & 490 & 597800 \\
\hline ОЗОСТ94 & 29SEP95 & $2 \mathrm{H} 20540 \mathrm{O} 02$ & TEA50110 & 450 & 549000 \\
\hline & & & & 940 & 1146800 \\
\hline
\end{tabular}

OE

H2D440E10 TEMPERATURE UPGRADES FY95

of

K20440FC2 SURFACE LEVEL UPGRADES FY95

06

K2D440G02 AU FARH VENT UPGRADES FY95 1120440G30 PORTABLE EXHANSTER FOR SSTS
DATA DATE O1OCT93 PAGE MO. 2

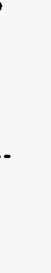


WHC-SP-1101

H: CAPITAL

-EPOKT OATE 15SEPY4 RUA MO. $382 \quad$ WORK PACAKGE TOTALS START DATE OIOCT92 FIN DATE 30SEP05 08:28

DREPARED BY LA YEATER 373-1903

roject Name: $\mathrm{K} 202$

DATA DATE 010CT93 PAGE hO. 3

TS FLAYABLE GAS SAFETY PROCRAM

\begin{tabular}{|c|c|c|c|c|c|c|}
\hline ACT DES & $\begin{array}{l}\text { EARLY } \\
\text { START }\end{array}$ & $\begin{array}{l}\text { EARLY } \\
\text { FINISH }\end{array}$ & $\begin{array}{l}\cos T \\
\text { ACcount T }\end{array}$ & RESORCE & $\begin{array}{l}\text { BUDGET } \\
\text { QUAATITI }\end{array}$ & $\begin{array}{l}\text { BUDGET } \\
\text { COST }\end{array}$ \\
\hline
\end{tabular}

$\mathrm{OH}$

204AOHOE GAS HOHITOR UPGRADES FY95

03APR95 29SEP95 2K20540H01 7F5103RO $520 \quad 551720$

$520 \quad 551720$

ox

:20\$4OKO2 INSTL AMOHIA HONITORS 241-AK/AN FARMS

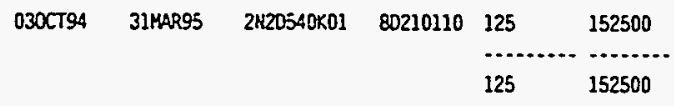

on

I2OA4OHOO INSTALL DST/SST GAS HONITORS (SI 2h)

\begin{tabular}{|c|c|c|c|c|c|}
\hline \multirow[t]{5}{*}{ 030СT94 } & 31HAR95 & $\begin{array}{l}2 \mathrm{~N} 20540 \% 01 \\
2 \mathrm{M} 20540 \% 102 \\
2 \mathrm{~N} 20540 \mathrm{M01}\end{array}$ & $\begin{array}{l}7 F 510010 \\
80210110 \\
7 F 5103 R 0\end{array}$ & $\begin{array}{l}906 \\
162 \\
560\end{array}$ & $\begin{array}{l}53508 \\
197640 \\
594160\end{array}$ \\
\hline & & & & 1628 & 845308 \\
\hline & & & & & 6144751 \\
\hline & & & & 31252 & \\
\hline & & & & 31252 & \\
\hline
\end{tabular}


WHC-SP-1101

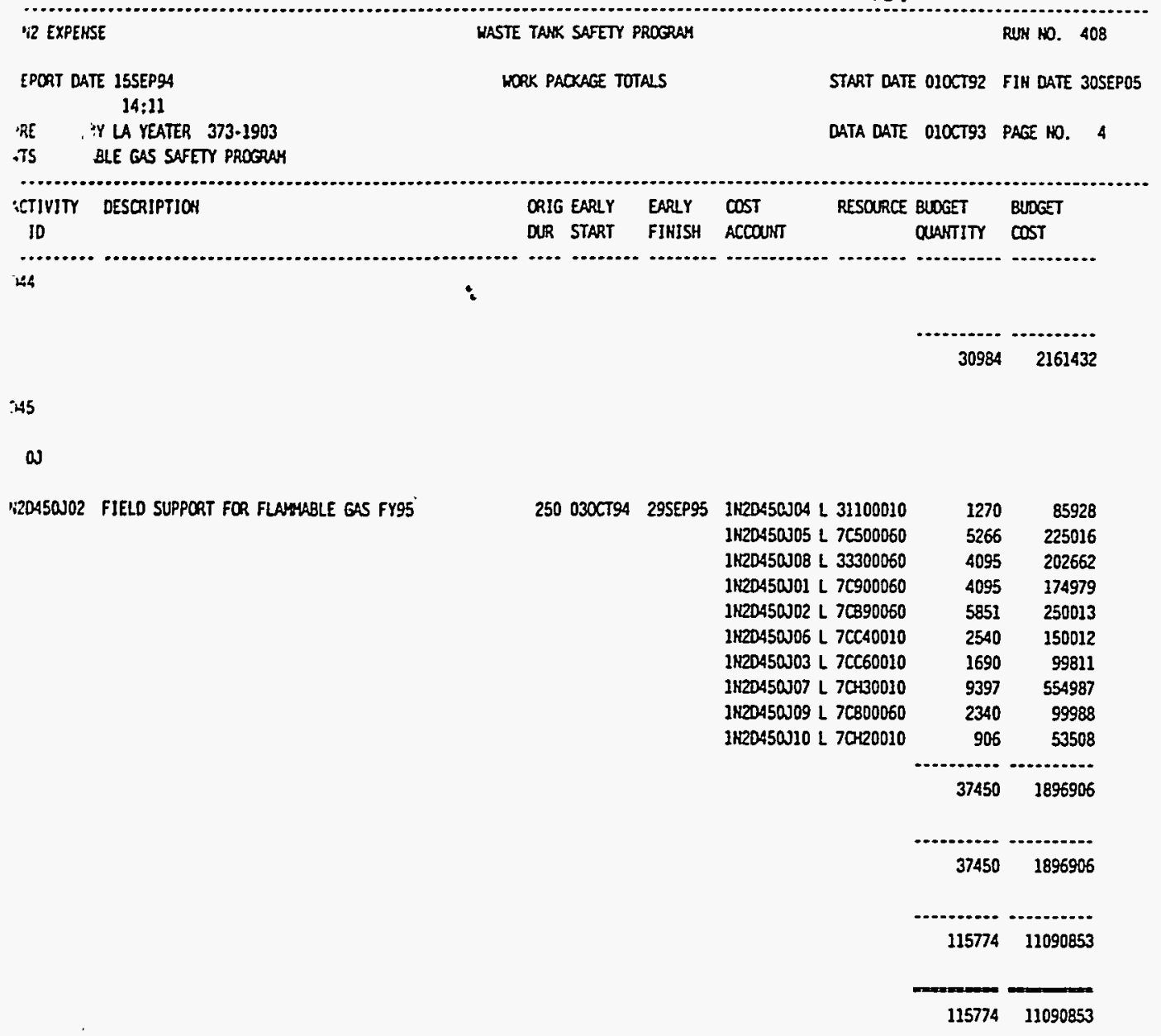


WHC-SP-1101

2 EXPENSE
WASTE TANK SAFETY PROGRAY

WORK PACKAGE TOTALS
RUN No. 386

START DATE O10CT92 FIN DATE 30SEP05

DATA DATE O1OCT93 PAGE MO. 1

$$
\text { 09:03 }
$$

IEPARED BY LA YEATER 373-1903

-S FLAMTABLE GAS SAFETY PROGRAM

TIVITY DESCRIPTIOH

10

ORIG EARLY EARLY COST RESOURCE BUDGET BUDGET

OR START FINISH ACCOWNT

QuANTIT COST

$\therefore$

of

20:10A06 WASTE BEHAVIOR HOOELIHE FY96

DS

2D:10311 PROGRAM MANAGERERT FY9S

$\alpha$

20410C20 GS GEMERATION KECHAHISMS FY96

$0=$

?DIOEO6 GAS RETEKTIOH REOHANISHS FY96

$\mathrm{OH}$

DOH1OH2O GAS REACTION STUDIES FY96

w

?DAlOSO4 DEV \& TRAYSFER OF METHOOS ORGANIC AYALYSIS
251 020CT95 30SEP96 IH2OA1OAO2 L 7E880000 1K20410A02 L 7E880010 IN20410A02 $07 E 880210$ 1H20410403 O 7R25021C IN2D410A03 0 TR25030C

\begin{tabular}{rrr}
1330 & 31681 \\
10 & 4686 & 285049 \\
10 & 103 & 129430 \\
$1 C$ & 500 & 515000 \\
\hline $1 . .2$ & 500 & 515000 \\
& 7119 & 1476160
\end{tabular}

251 020CT95 30SEP96 1N2D410BO1 O 7R2503RC 1K20410801 L 7R250010

\begin{tabular}{rrr}
320 & 329600 \\
1812 & 110224 \\
\hline$\ldots . .2132$ & 439824
\end{tabular}

$251020 \mathrm{CT95}$ 30SEP95 1N20410CO6 O 7R25030C 1N2D410CO1 L 8E110000 1K20410CO1 \& 8E110010 IHED410COI H $8 E 110100$

\begin{tabular}{rrr}
360 & 370800 \\
4866 & 189239 \\
1905 & 122111 \\
25 & 31415 \\
\hline 7156 & 713564
\end{tabular}

251 020CT95 3OSEPFS 1KROA10EO1 O 7R2503DC IN2DA10EOI 0 7R2503DC 1N2DA10E01 $07 R 25030 C$

$225 \quad 231750$

$190 \quad 195700$

$190 \quad 195700$

$605 \quad 623150$

251 020CT95 30SEP9S 1N20410HO2 O 7R25021C

\begin{tabular}{rr}
423 & 435690 \\
\hline 423 & 435690
\end{tabular}

12

OA

DS20A20 VAPOR SPACE MONITORIHS FY96
251 020CT95 3OSEP9S IN2D410.J01 O 7R25030C

\begin{tabular}{rrr}
225 & 231750 \\
225 & 231750 \\
& \\
& \\
\hline 17660 & 3920138
\end{tabular}

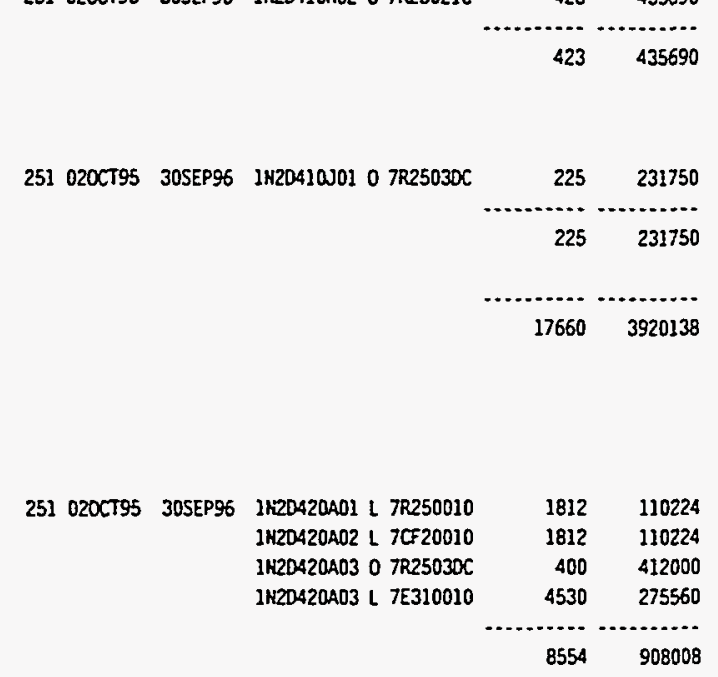


WHC-SP-1101

$\begin{array}{lll}\text { N.2 EXPEHSE } & \text { RASTE TANK SAFETY PROGRAM } & \text { RUM NO. } 386\end{array}$

EPDRT DATE LSSEP94

HORK PAOCAGE TOTALS

START DATE O1OCT92 FIN DATE 30SEPO5

R $3 Y$ LA YEATER $373-1903$

DATA DATE DLOCT93 PAEE HO. 2

TS ABLE GAS SAEETY PROCRAY

ETIVITY DESCRIPTIOH ORIG EARLY EARLY COST RESORCE BUDGET BUDGET

10 DUR START FIMISH ACCONTT OUASTITY COST

42

OE

:20420E20 WASTE BEHAVIOR EVALUATION FY96

251 020CT95 30SEP96 1H20420E02 L 7E310000 $1 \mathrm{~N} 20420 E 03$ L $7 E 310010$ 1H20420E01 07250300 1H2D420E03 O $7 R 25030 \mathrm{C}$

\begin{tabular}{rr}
973 & 23177 \\
5715 & 347643 \\
450 & 463500 \\
284 & 292520 \\
\hline $7 . .$. &... \\
\hline 7422 & 1126840
\end{tabular}

as

H2DA20G20 RETALHED GAS EVALLATIOH FY96

08

k2

COOSE AY FAPY USOS FLAM GAS TAMSS

N2D440A06 PROGRAS MALAGEMENT FY96

H2DA40E2O TEKPERATURE UPGRADES FY96

06

H2D440514 RH FARY VERT UPGRADES N20440520 AN FARY VERT UPGRADES
251 020CT95 305EP96 1N20420602 L 80210000 1H20420501 L 7E310000 1N20420502 L 80210010 1H20420601 L 7E310010 1H20420601 L 7E310000 1K20420501 L 7E310010

\begin{tabular}{rr}
433 & 14077 \\
433 & 10314 \\
1524 & 114849 \\
1524 & 92705 \\
762 & 18151 \\
216 & 13139 \\
\hline $4 . .$. & $.3 . .$. \\
\hline 4892 & 263235 \\
\hline
\end{tabular}

$20868 \quad 2298083$

251 020CT95 30SEP96 1N20430301 L 7R250010 1 N20430301 O 7R25021C I 1 200430BO2 L 88800010 1 1\%20430803 L 80114000 $1 K 20430303$ L 80114010

\begin{tabular}{rr}
1812 & 110224 \\
800 & 824000 \\
254 & 15453 \\
2111 & 70803 \\
7437 & 578078 \\
\hline 12414 & 1598558 \\
\hline & \\
\hline 12414 & 1598558 \\
\hline
\end{tabular}

251 020CT95 30SEP95 IK20440401 L 7R250010 IHPOA40A02 I TEADOO1O IN20440A03 L 7CHO0O1O

$1812 \quad 110224$

$1000 \quad 60830$

$1000 \quad 60830$

$3812 \quad 231884$

251 020CT95 305EP95 INZO440E02 L 7EA10010 4377 266253

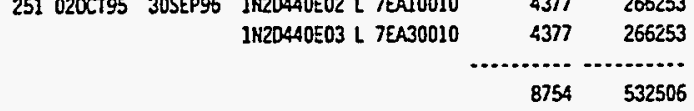

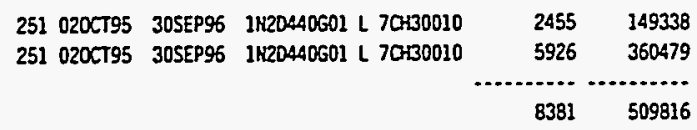




\section{WHC-SP-1101}

$$
\text { i2 EXPENSE }
$$

SPORT DATE 15SEP94

$$
\text { 09:03 }
$$

REPARED BY LA YEATER 373-1903

\begin{tabular}{|c|c|}
\hline $\begin{array}{l}\text { otivitr } \\
10\end{array}$ & DESCRIPTIOH \\
\hline 04 & \\
\hline 2040410 & GAS HONITOR UPGRADES FY96 \\
\hline $\mathrm{OL}$ & \\
\hline 20440102 & MISC TAMK FARM EQUIPHERT UPGRADES \\
\hline ON & \\
\hline & EQUIPMENT DISPOSAL FY96 \\
\hline
\end{tabular}

is FLAYMBLLE GAS SAFETY PROGRAY

20S40NOO EQUIPYENT DISPOSAL FY96

45

w

?0450106 FIELD SUPPORT FOR FLAYMABLE GAS FY96
WASTE TAKK SAFETY PROGRAM

RUK ผ0. 386

WORK PACKAGE TOTALS

START DATE O1OCT92 FIN DATE 3OSEPO5

DATA DATE 010CT93 PAGE NO. 3

ORIG EARLY EARLY COST

RESORCE BUDGET BLOEET

DUR START FINISH ACCOUNT

QUANTITY COST

251 020CT95 30SEP95 1H20440HO1 O TR25021C

$\begin{array}{rr}300 & 309000 \\ 300 & 309000\end{array}$

251 02OCT95 3OSEP9S INLO44OLO1 L TEADO01O

\begin{tabular}{rr}
2929 & 178171 \\
\hline 2929 & 178171
\end{tabular}

251 020CT95 30SEP95 1R20440H02 L 7C900050

$24573 \quad 1081458$

IN20440NO1 L 7R250010

$44023 \quad 2677919$

$68595 \quad 3759377$

$92772 \quad 5520754$

$251020 C T 95$ 305EP96 1 1K20450JOA L $31100010 \quad 1270 \quad 88506$ 1K20450J05 L 7C500060 $5851 \quad 257503$ 1H2D450J08 L $33300060 \quad 4095 \quad 208763$ IH2045010I L 7C900060 $5851 \quad 257503$ IM20450102 L 7C890060 $7489 \quad 329591$ IN20450106 L 7CCA0010 $2540 \quad 154508$ 1N20450J03 ᄂ 7CC60010 $2540 \quad 154508$ 1H20450JO7 L 70130010 $10244 \quad 623143$ $39880 \quad 2074024$

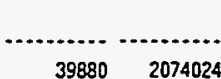

$183594 \quad 15411557$

$183594 \quad 15411557$ 


\section{Lし-tiL」}

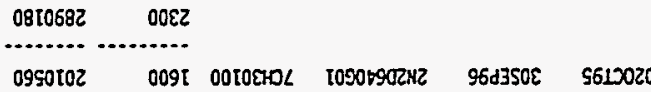

$0296 \angle 8 \quad 00 L$ OOTOEHDL TOSOFSCZHZ $96 \mathrm{~d} 350 \mathrm{~S}$ S6130ZO

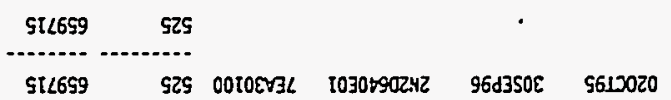

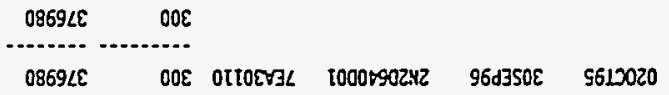

OSLIEZ \$ZZ

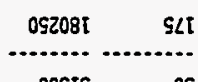

05 Jacoszal t030z902HZ

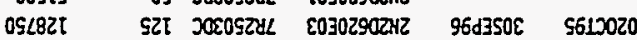

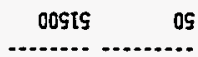

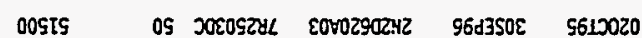

$0 \$ 8002 \quad 56$ T.

$\operatorname{0sLS2} \quad \varsigma 2$

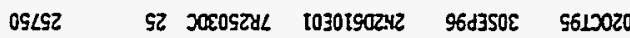

$00902 \quad 02$

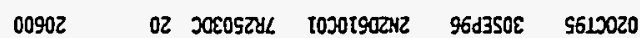

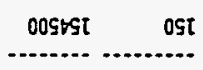

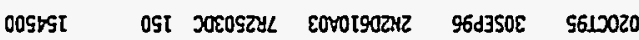

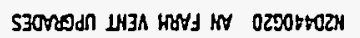

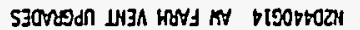

90

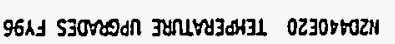

30

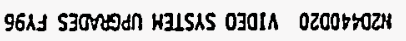

00

$6+0$

g6RJ MOLLกTHA \&OLABH39 3LSWM OZ30ZคOZ!

30

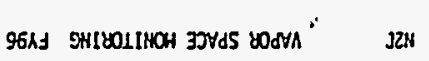

yo

$2+0$

961J SHSINHOZN MOLLZLZY SW 9030T6OZN

30

96AJ SHSIMMOZH MOLLVIZN39 SW OZJOTHZL

30

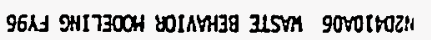

yo

Ir

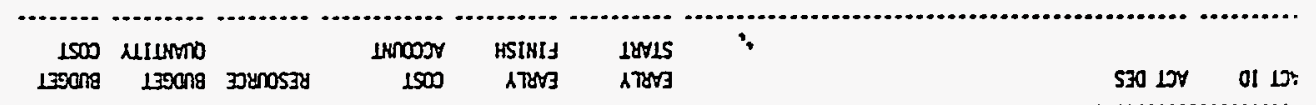

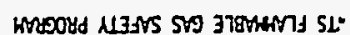

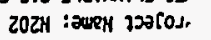

I ON 397d EsLDoto 3lto $\forall 1 \% 0$

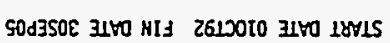

STY101 3xxard XoOA

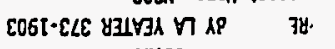
0z:80

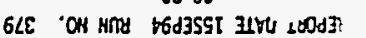


WHC-SP-1101

ST2 CAPITAL

EEPOKT DATE 15SEPYA RUM HO. 379

08:20

WORK PACAKGE TOTALS

START DATE 010CT92 FIN DATE 30SEP05

PREPARED BY LA YEATER 373-1903

Froject kame: 1202

.TS FLAHHABLE GAS SAFETY PROGRAY

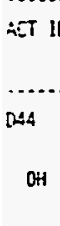

DT 10 ATT DE

START

EARLY

$\cos T$

RESOURCE BUDGET BUDGET

DATA DATE DHOCT93 PAGE hO. 2

R2044OH1O GAS HOHITOR UPGRADES FY96

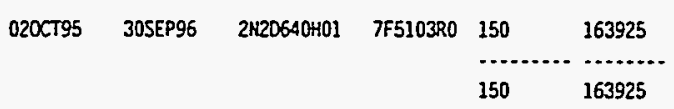

$a$

N2D440L02 HISC TAMK FARY EOUIPMENT UPGRADES

\begin{tabular}{|c|c|c|c|c|c|}
\hline \multirow[t]{4}{*}{ 020CT95 } & 30SEP96 & $2 \mathrm{N20640L01}$ & TEA00100 & $\begin{array}{l}1100 \\
\ldots \\
1100\end{array}$ & $\begin{array}{l}1382260 \\
1382260\end{array}$ \\
\hline & & & & & 5473060 \\
\hline & & & & & 5905660 \\
\hline & & & & 4795 & 5905660 \\
\hline
\end{tabular}

F7.4-7.77 


\section{WHC-SP-1101}

$$
.2 \text { EXPEHSE }
$$

:PORT RATE 25SEPQ4

08:31

2EF. II LA YEATER 373.1903

IS FLAYABLLE GAS SAFETY PROGRAY

TIVITY DESCRIPTION

ID

4

OA

2041OAOB WASTE BEHAVICR HOOELIHG FY97

,

$O B$

20410312 PROCRAH MALAGEYENT FY97

OC

N20410C3O GAS GEHERATIOH HECHAHISHS FY97

GAS RETENTIOH NECHANISMS FY97

of

M2D4IOHBO GAS REACTION STUDIES FY97

D42

$O A$

N2D420R30 VAPOR SPACE HONITORIHG FY97

OE

N2D420E30 HASTE BEHAVIOR EVALLUTION FY97
WASTE TAYK SAFETY PRDGRAY

RIN NO. 383

WORK PACQAGE TOTALS

START DATE DIOCT92 FIK DATE 3OSEPOS

DATA DATE DIOCT93 PAGE MO. 1
251 010СT95 30SEP97 1K2D410402 L 7E880000 IK20410402 L 7E880010 IN20410A02 $07 E 880210$ IN20410403 O TR25030C

\begin{tabular}{|c|c|}
\hline 105 & 135914 \\
\hline 550 & 583550 \\
\hline
\end{tabular}

$6819 \quad 1053691$

251 O10CT95 305EP97 IN20410801 O 7R2503DC $300 \quad 318300$ $\begin{array}{rrr}1 H 20410801 \text { L } 7 R 250010 & 1812 & 113540 \\ & \ldots \ldots \ldots \ldots & \\ & 2112 & 431840\end{array}$

251 010CT96 305EP97 1N20410CO1 ᄂ 8E110000 1H20410CO1 \& 8E110010 IK2D410COI H 8E110100

\begin{tabular}{rr}
3893 & 155954 \\
1524 & 100614 \\
20 & 25888 \\
\hline 5437 & 282456
\end{tabular}

$251010 C T 96$ 30SEP97 1N20410EO1 O 7R25030C IH2DA10EOI 0 7R25030C IKROA10EO1 0 7R25030C

\begin{tabular}{rr}
90 & 95490 \\
190 & 201590 \\
90 & 95490 \\
\hline 370 & 392570
\end{tabular}

251 010CT96 3OSEPS7 1K2041OHO2 O 7R25021C

$\begin{array}{rrr} & 100 & 106100 \\ 100 & 106100 \\ & & \\ 14838 & 2266557\end{array}$

251 010CT96 30SEP97 1K2D420A01 L 7R250010 $1812 \quad 113540$ 3R20420102 L 7CF20010 $1812 \quad 113540$ IK20420AD3 O 7R25030C $225 \quad 238725$ IN20420A03 L 7E310010 $5436 \quad 340620$ $\begin{array}{rr}5436 & 340620 \\ 9285 & 806425\end{array}$

251 010CT96 305EP97 2N20420E02 L 7E310000 $973 \quad 23868$ 1 1H20420E02 L 7E310010 $5715 \quad 358102$ $1 \mathrm{~N} 20420 \mathrm{EO} O \mathrm{TR} 25030 \mathrm{C} \quad 200 \quad 212200$ 1 1N20420E01 $07 R 25030 C \quad 650 \quad 689650$

$7538 \quad 1283820$ 
WHC-SP-1101

$\therefore$ CAPITAL

- PORT DATE 15SEP94 RUY MO. 378

08:19

ZEPARED BY LA YEATER 373-1903

-oject Hane: $\mathrm{hzO2}$

-I FLAYHABLE GAS SAFETY PRDGRAY

IT ACT DES

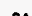

20410A0B WASTE BEHAYIOR HOOELIHG FY97

20420130 VAPOR SPACE HOHITORIHG FY97

OE

20420E30 HASTE BEHAYIOR EVALLATION FY97

of

20440530 TEMPERATURE UPGRADES FY97

DG

:20440624 AN FARH VENT UPGRADES

OL

20440LO6 MISC TANK FARH EQUIPMENT UPGRADES
WORK PACAKGE TOTALS

START DATE O10CT92 FIN DATE 30SEP05

DATA DATE O1OCT93 PAGE HO. 1

\begin{tabular}{|c|c|c|c|c|c|}
\hline \multirow[t]{5}{*}{ 010ст96 } & 30SEP97 & $2 \mathrm{H} 20710 \mathrm{AOC3}$ & $7 R 25030 \mathrm{C}$ & 200 & 106100 \\
\hline & & & & a.......... & ............ \\
\hline & & & & 100 & 106100 \\
\hline & & & & ............ & . \\
\hline & & & & 100 & 106100 \\
\hline
\end{tabular}

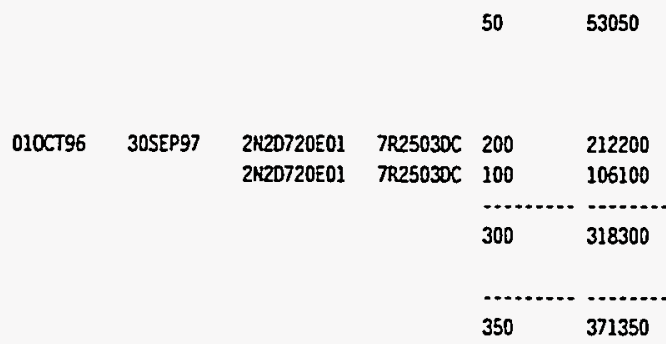

010CT96 30SEP97 2N20740EOI TEA30110 $525 \quad 679571$ $525 \quad 679571$

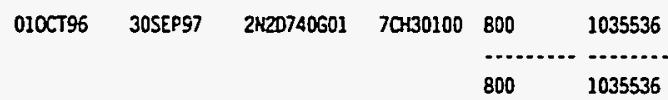

0106796 305EP97 2N20740Z01 7EA00100 $1100 \quad 1423862$

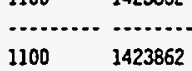

$2425 \quad 3138969$

-......... -.......

$2875 \quad 3616419$

$2875 \quad 3616419$ 


\section{WHC-SP-1101}

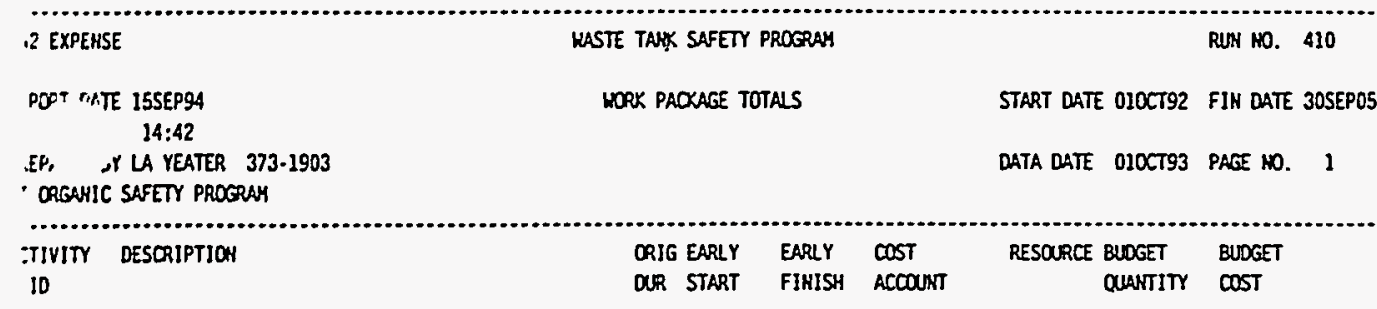

$\ddot{0}$

of

2E5IOA2 PML PROUECT MARUGERENT

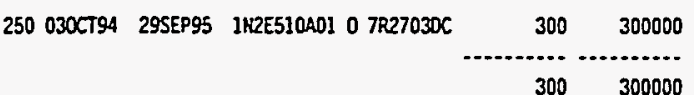

$O B$

$2 Z 510 B 2$ ORGANIC YASTE EHERGETICS

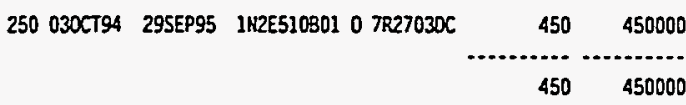

$a c$

$2 E 510 C 2$ WASTE EHERGETICS MISIHQLATTS - FAI

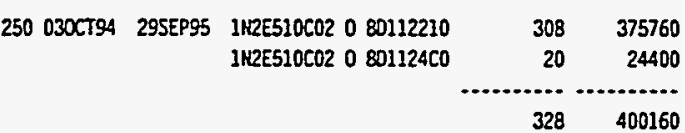

$O D$

I2E51002 ORGANIC ANALTTICAL METHCOS DEVELOPHENT

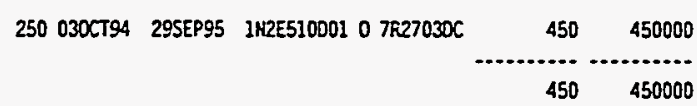

OE

42E5. ORGANIC HASTE AGING STUDIES

250 030CT94 295EP95 1HRE510E01 0 7R27030C $\quad 270 \quad 270000$

250 030CT94 29SEP95 IHZE510F01 0 7R27030C $\quad 225 \quad 225000$

225225000

o.

RiZE510G2 ORGANIC AHALYTICAL CHEHISTRY SUPPORT

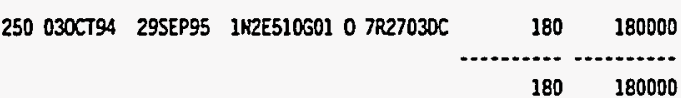

o:

MZE51OH2 SST ORGAMIC CARBOH \& HOISTURE STUDY

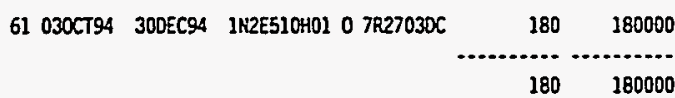

$\boldsymbol{\omega}$

H2E51OU2 ARALYSIS OF TANX T-111 CORE SAYPLE ARALYSIS

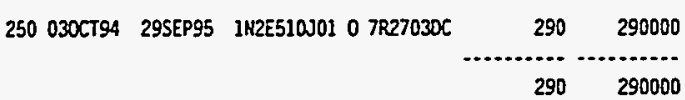

ox

K2E510K2 SPECIAL STUOIES

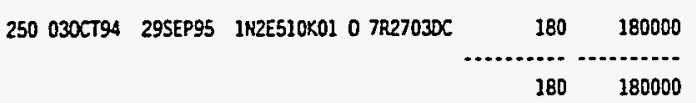

\section{$F 7.4-7.82$}


WHC-SP-1101

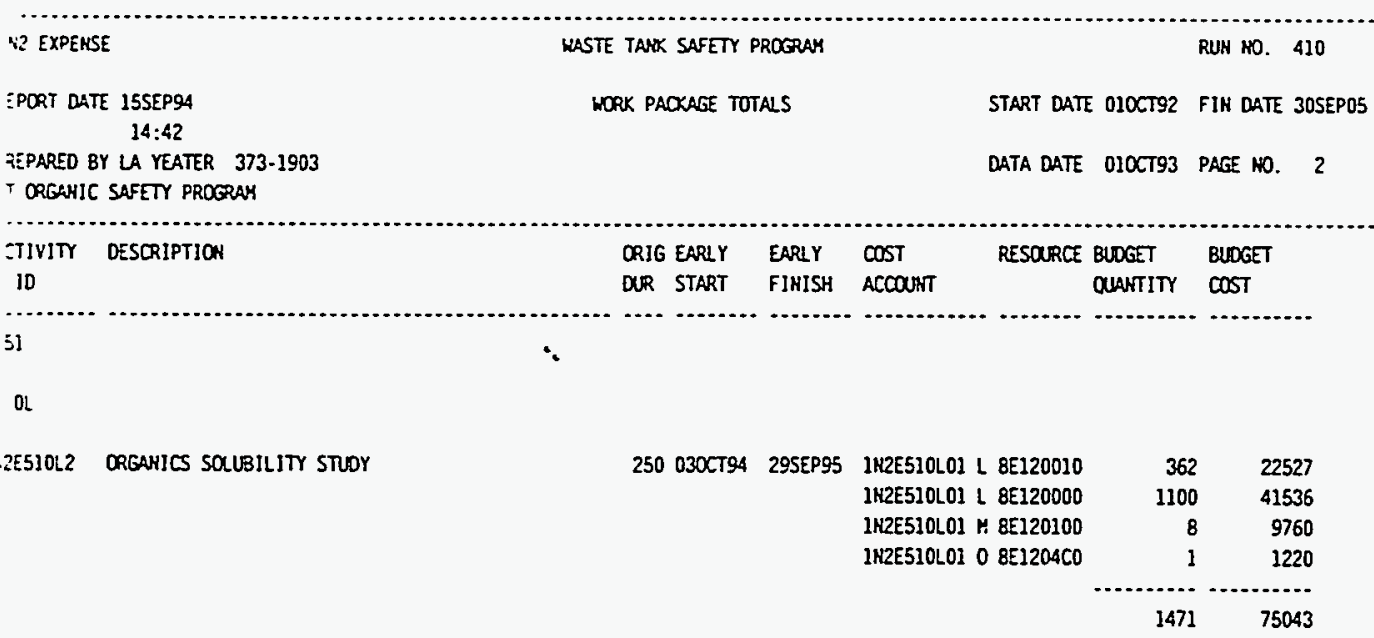

on

2E510K2 WASTE SAMPLE ARALYSIS (WO)

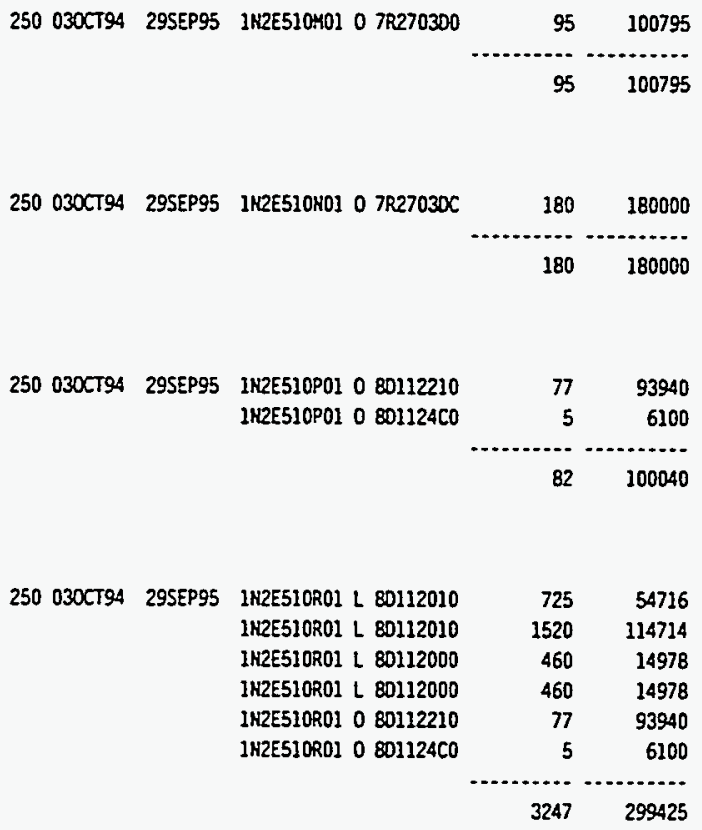

ON

2E510N2 ORGAHIC LAYDONN MOOEL (PHL)

oP

2E510P2 WASTE HOISTURE WISIMJLANTS - FAI

OR

2E510R2 BASIS FOR SAFE OPS SSTS - ORGAHIC/HITTATE

OT

ZESIOT2 BASIS FOR SAFE OPS SSTS - ORGAYIC LAYER

ov

ZEE510U2 BASIS FOR SAFE OPS SSTS - ABSORBED CRGANIC

ov

ZE5!OV2 ORGANICS TAMKS SAFETY CRITERIA 


\section{WHC-SP-1101}

\section{$\because$ EXPENSE}

WASTE TAKK SAFETY PROGRAY

RUN NO. 410

DPRT DATE 15SEP94

14:42

WORK PACKAGE TOTALS

START DATE 010CT92 FIN DATE 3OSEPO5

IEPARED BY LA YEATER 373-1903

- organic safetr PROGaAa

DATA DATE OHOCT93 PAGE NO. 4

GIVITY DESCRIPTIOA

ID

ORIG EARLY EARLY COST

DUR START FINISH ACCONTT

RESORCE BUDGET BUDEET

C.... ................. .........

OUANTIT COST

3

or

2E540F2 SAFETY EVALUATIOH/LOA

250 030CT94 29SEP95 1M2E540F01 L 80112010

\begin{tabular}{rr}
1192 & 89950 \\
$\ldots \ldots . .$. & $8 . .$. \\
1192 & 89960 \\
& \\
\hline$\ldots \ldots .$. & \\
5096 & 379177
\end{tabular}

55

of

EE55042 HOISTURE HONITORIHE/COHTRO EHG.

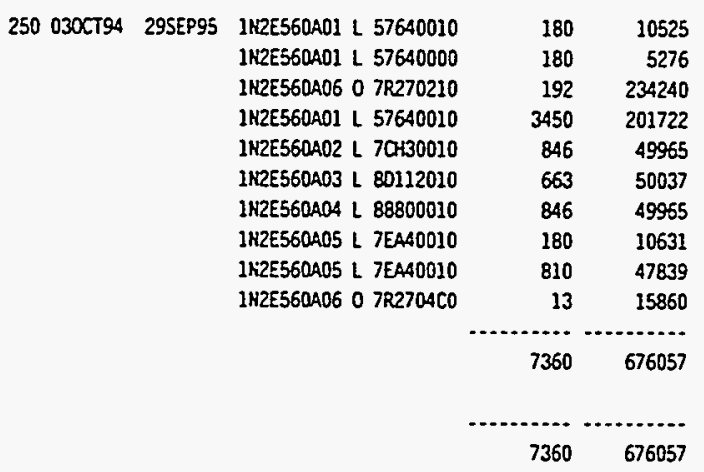

2E57032 SEPARATIONS EOUIPMENT FABRICHTION

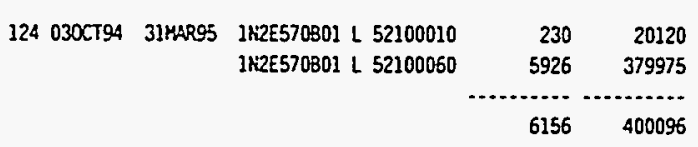

oc

IZE57OCI SEPARATIONS EQUIPYENT INSTALL'OPER

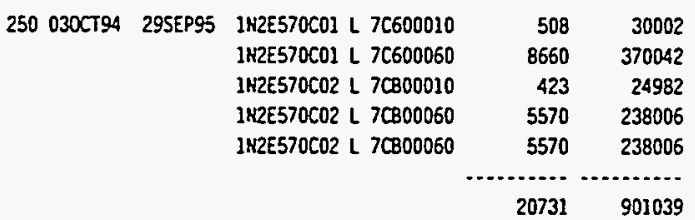

or

IZE57002 SEPARATIONS \& ORGANIC STORAGE SUPPORT

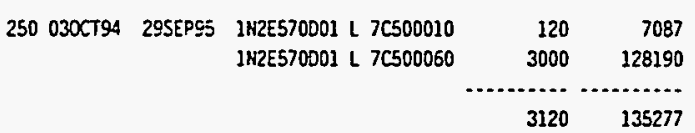

of

ZZE57OEZ LABORATORY ANRL YSIS

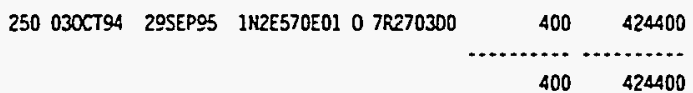


WHC-SP-1101

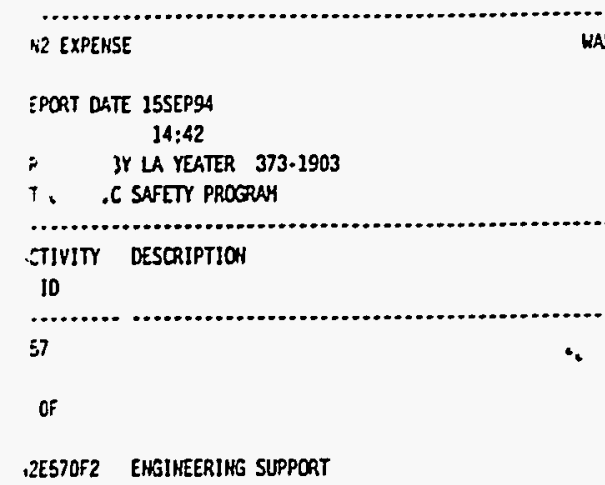

WASTE TANK SAFETY PROGRAM RUN NO. 410

WORK PACKAGE TTTALS START DATE O1OCT92 FIN DATE $305 E P 05$

DATA DATE 010CT93 PAGE hO. 5

ORIG EARLY EARLY COST RESOURCE BUDGT BUDEET

DUR START FIHISH ACCONT TOLATITY COST

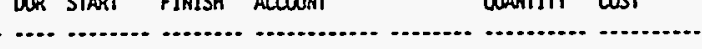

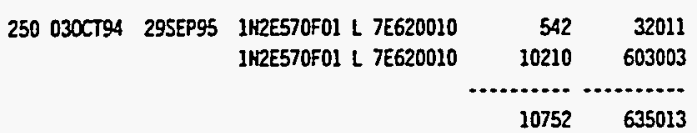

$\omega$

¿2E570I2 TREATMENT SYSTEM DESIG

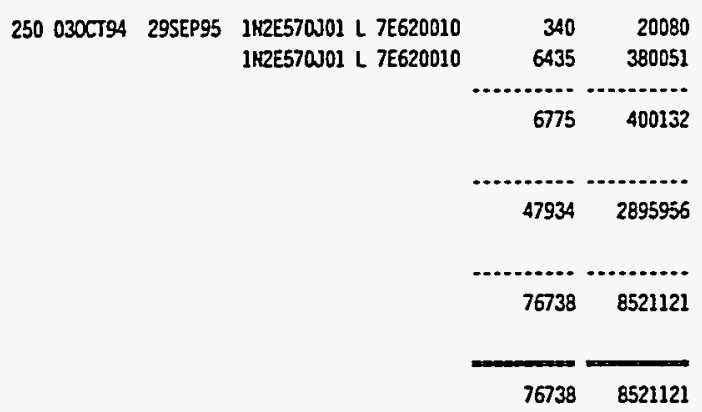

$F 7.4-7.86$ 
WHC-SP-1101

12 CAPITAL

इPORT DATE ISSEPQA RUM HO. 381 08:26

WORK PACAKGE TOTALS

START DATE O1OCT92 FIN DATE 30 SEPOS

EEPARED BY LA YEATER 373-1903

- oject hane: 1202

- ORGAHIC SAFETY PROGRAY

IT 10 ACT DES

EARIY Y COST

DATA DATE O1OCT93 PAGE NO. 1

gt,

$\vdots 1$

02

2E510^2 PKL PRQUECT MAVAGEMENT

03

.2E5IOB2 ORGAHIC WASTE ENERGETICS

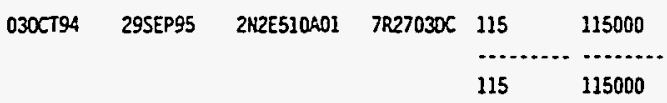

030CT94 29SEP95 2H2E510801 7R27030C $\begin{array}{lll}155 & 155000 \\ & & \ldots \ldots \ldots \ldots \ldots\end{array}$

os

i2E51052 OREAHIC AKALYTICAL CHEKISTRY SUPPORT

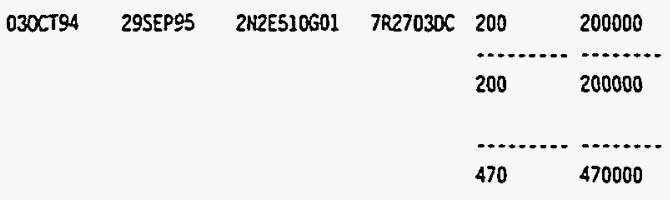

54

00

2E54002 MOISTURE HONITOR ENG \& (LON) INSTALL

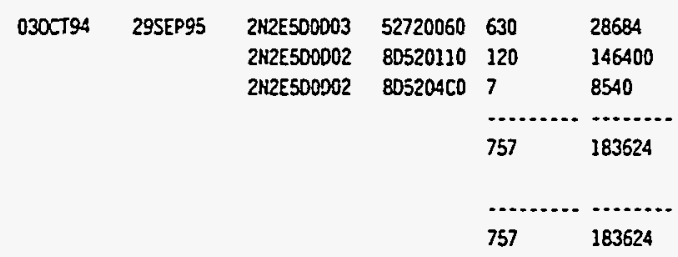

57

of

2E57012 ORGANIC RECEIVER TANK

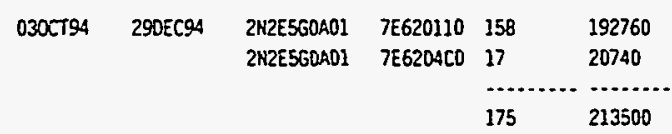

05

2557

03JA495 30JUH95 2N2E56OHOI

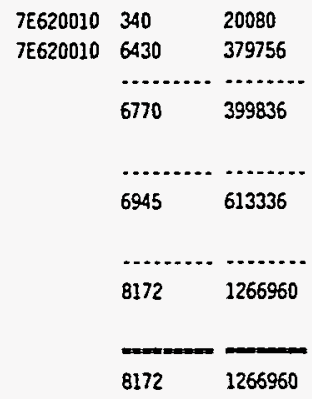

F 7.4-7.87 
WHC-SP-1101

2 EXPEMSE
UASTE TAHK SAFETY PROGRAM

RUM HO. 411

इPDRT RATE 15SEPg4

14:43

MORK PACKAGE TOTALS

START DATE O1OCT92 FIH DATE 3OSEPO5

RE, SY LA YEATER 373.1903

DATA DATE OLOCT93 PACE NO. 1

$T$ ORSAKIC SAFETY PRDGRAY

$\begin{aligned} & \text { GTIVIT } \\ & \text { ID DESCRIPTIOH }\end{aligned}$
$\ldots 1$

OB

22EE1OBA ORGANIC WASTE EMERGETICS

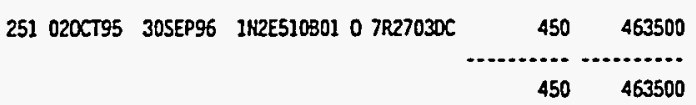

oc

I2E510C4 HASTE EMERGTIICS MSIMLLATS - FAI

$\begin{array}{rrrrr}251 \text { 020CT95 30SEP96 } & \text { IN2E510C02 } 080112210 & 308 & 387033 \\ & \text { IK2E510C02 } 0801124 C 0 & 20 & 25132 \\ & & & 328 & 412165\end{array}$

$\infty$

HZE51004 ORGANIC AHRLYTICAL METHOOS DEVELOPHEKT

OE

H2E: ORGANIC KASTE AGING STUDIES

of

H2ES10F4 ORGAHIC CONCEHTRATION MECHANISHS

os

HZE5IOGA ORGANIC AYLLYTICAL CHEHISTRY SUPPORT

$\mathrm{OH}$

N2E5ICHA SST OREANIC CARBOW \& MOISTURE STUOY

ok

N2E5IOK4 SPECIAL STODIES

$a$

R2E510L4 ORGAMICS SOLUEILITY STUOY

251 020CT95 30SEP96 1KZE510K01 O 7R27030C

$\begin{array}{rr}180 & 185400 \\ 180 & 185400\end{array}$

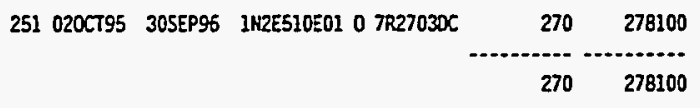

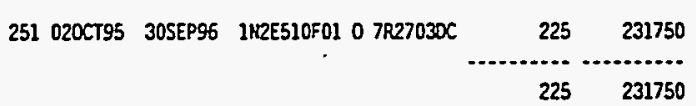

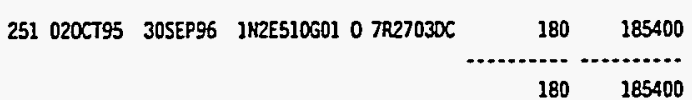

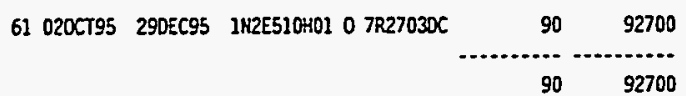

251 020CT95 30SEP96 IN2E510L01 L 8E120010 INEE510LO1 L EE120000
1M2E510LO1 K EE120100 INZE51OLOI O 8E1204CO

$F 7.4-7.88$ 
WHC-SP-1101

$\therefore$ EXPENSE WASTE TANK SAFETY PROGRAY

WORK PACKAGE TOTALS
RUN NO. 411

START DATE 010CT92 FIN DATE 30SEPO5

$14: 43$

EPARED BY LA YEATER 373-1903

- ORGAHIC SAFETY PROGRAY

DATA DATE D1OCT93 PAGE NO. 4

ID
ID

jo

00

3ESA004 MOIST HONITOR EHE \& LON INSTALLATION

251 020CT95 30SEP9S IH2E540002 L 80520000

IH2E540002 L 80520010

IH2E540002 L 80520000

IH2E540002 K 60520100

IH'E5 540002 \& 80520140

IN2E540002 H 80520150

1H2E540D02 A 80520190

1H2ES40002 O 80520210

1H2E540002 0 ODS202LO

1H2E540002 O BD520640

IN2E540002 L 805202TO

IN2E540002 L 805204CO

1K2E540002 L 805204PO

1H2E540D02 L 805205HO

IN2E540002 L 805206PO

IH2E540D04 L 80340010

1H2E540005 L 80510010

1N2E540006 L 80530010

1H2E540007 L 80530010

1H2E54000B \& 52100060

IN2E540008 L 52100060

1H2E54000B L 52100060

IN2E540009 L 7C500050

1H2E540009 L 7C500060

IN2E540009 L $7 C 500050$

1N2E540D10 L 7C900060

1N2E540D10 L 7C900050

1N2E540010 L 7C900060

583

$68 \quad 2309$

$1826 \quad 143724$

$202 \quad 6860$

$10 \quad 12566$

1257

$5 \quad 6283$

$100 \quad 125660$

$48 \quad 2513$

$8 \quad 9425$

$9 \quad 11309$

$1 \quad 1257$

$12 \quad 15079$

$1 \quad 1257$

$219 \quad 16504$

$250 \quad 21850$

$501 \quad 43787$

$730 \quad 57458$

$2092 \quad 138177$

$2092 \quad 138177$

$2092 \quad 138177$

$583 \quad 25658$

$583 \quad 25658$

$583 \quad 25658$

$583 \quad 25658$

$583 \quad 25658$

$583 \quad 25658$

$13772 \quad 1114175$

DE

2ESTOE4 MOISTURE MONITOR/CONTRO

251 020CT95 30SEP96 IN2E540501 \& 7EA50010 $2540 \quad 154508$

IN2E540E02 \& $52100010 \quad 172 \quad 15499$

IN2E540E02 L 52100060 $4445 \quad 293592$

IN2E540EO1 O 7EA5D210 230 289018

IN2E540EO1 O 7EA504CO $\quad 16 \quad 20106$

IN2E540E01 L TEA50010 $5080 \quad 309016$

IN2E540E02 L $52100010 \quad 1430 \quad 128857$

IN2E540E02 H $52100100 \quad 182 \quad 228701$

IN2E540EO2 O 521004CO $23 \quad 28902$

IN2E540E02 L $52100050 \quad 2730 \quad 180317$

IN2E540501 0 7EA50210 $58 \quad 72883$

IN2E540EO1 O 7EA504CO 4 S 5026

IN2E540EO4 L 7CBA0010 $\quad 169 \quad 10280$

IN2E540EO4 L 7CB40050 $936 \quad 41193$

1N2E540EO1 L 7EA50010 $423 \quad 25731$

IH2E540E04 L 7CB40050 $725 \quad 31907$

1N2E540E04 L 7CB40050 $1474 \quad 64871$

1N2E540EO1 L TEA50010 $525 \quad 31936$

IN2E540EO5 L $48003060 \quad 890 \quad 29690$

1N2E540EO4 L 7CBA0050 445 19584

IN2E540501 L 7EA50010 305 18553

$22802 \quad 2000172$ 
WHC-SP-1101

$\begin{array}{lll}\text { R2 EXPEHSE } & \text { RASTE TAHK SAFETY PROGRAM } & \text { RUN HO. } 411 \\ \text { EPORT DATE 15SEPG4 } & \text { HORK PACKAGE TOTALS } & \text { START DATE O1OCT92 FIN DATE } 305 E P 05\end{array}$

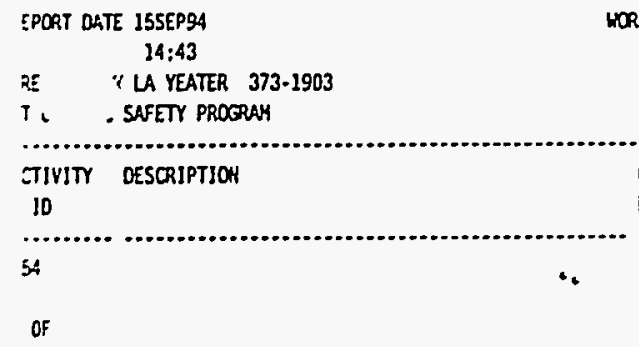

WORK PACAAE TOTALS

DATA DATE O10CT93 PAGE HO. 5

ORIG EARLY EARLY COST RESORCE BUDGET BUDSET

OUR START FINISH ACCONIT QUANTITY COST

0

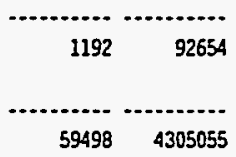

.55

OA

'ZEE5SOAA USQ CQOSURE DOCMMEMTATION

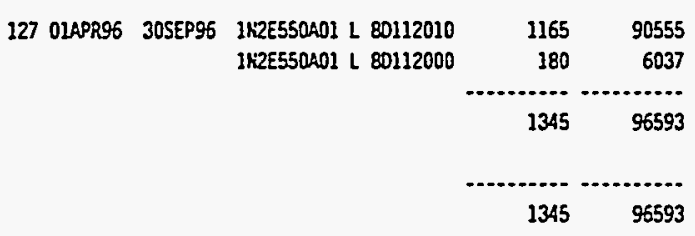

E56

aA

N2E5GOA4 HOISTURE HOHITORIHG/CONTROL EHG.

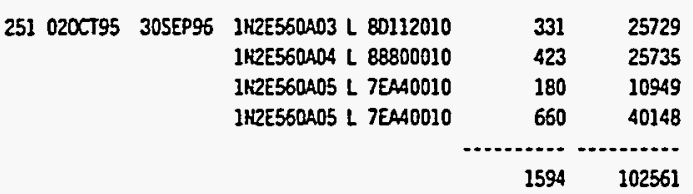

OB

H2E56OBA SAFETY ISSUE CCOSURE DOCMAENTATIOH

127 OLAPR96 30SEP96 IN2E560301 L 80112010 1K2E550301 L 80112000

\begin{tabular}{rr}
1320 & 102604 \\
90 & 3019 \\
\hline 1410 & 105622 \\
\hline 3004 & 208183
\end{tabular}

E57

$o c$

"12E57OCA SEPAPATJOHS EQUIPMENT INSTALL/OPER

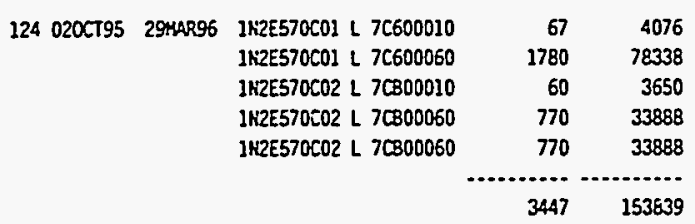

OD

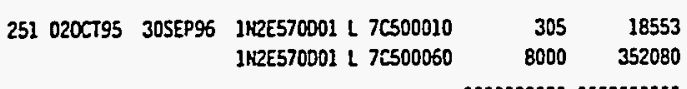

$8305 \quad 370633$ 


\section{thb L-tiL-}

\begin{tabular}{|c|c|c|c|}
\hline LLEбZSS & $08 S 22$ & & \\
\hline 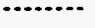 & $\cdots$ & & \\
\hline 005825 & 0008 & 09000IzS & $2000053 Z K 2$ \\
\hline 69Z6टZt & teIT & $08 \varepsilon 0<2 \mathrm{~d} L$ & SOZOOSZZNZ \\
\hline 005829 & OOS & oEzolzd & S0300532N2 \\
\hline โT890T & 58 & OJPOLCZSL & S0300S3ZKZ \\
\hline $0 S b 2+6$ & OSL & OTZOLCYL & S0J00S3ZNZ \\
\hline 08ZS00t & 008 & otroLCHL & S030CSJZNZ \\
\hline 968000 & $8 b 2 L$ & $0100 \angle 2 Y \angle$ & S030053ZNZ \\
\hline$\square Z \varepsilon 98$ & $\$ 29 \varepsilon$ & $0000<28 \angle$ & S0300S32N2 \\
\hline$\angle$ LI888 & $68 \varepsilon$ & ottosyl & t0300S32NZ \\
\hline OEB८29 & OS & OJPOg:BL & t0300@3Z2HZ \\
\hline
\end{tabular}

08829 os ojtogral
grd3SOE S610020

\begin{tabular}{|c|c|c|}
\hline $8 E 89 L 2$ & ottt & \\
\hline$\angle 16 \iota$ & 9 & $0250 z 50$ \\
\hline Eป169 & SS & 06702508 \\
\hline 09992I & oot & ottozscos \\
\hline 2IE8L & $\varepsilon \geqslant 2$ & $0 t 002808$ \\
\hline टहEge & 287 & oto0zsog \\
\hline $5059 t$ & 612 & OTOOН 208 \\
\hline
\end{tabular}

$\begin{array}{rr}\text { trIgele } & 0826 \\ \text { SZOEE } & 005 \\ \text { 8ZS00T } & 08\end{array}$

S†Z6E9I

osbzr6

008829

$8+6022$

ट9tes

I6SLL

हโร乙

IโโรZ

IELรZ
09000125

08 0EZOLZZL

OOGI O\&EOLZZL

OSL OTZOLCXL

oos ottolzdL

TZ9E OTOOLZCL

zा8T 0000LZY

IL Orcostzl

2 0JTOt3L

8t 0otontzl

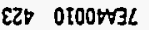

200007932HZ

2000 t932N2

20005932nz

ITOOF932NZ

2000ต93242

t000193242 9odasor

5612020 $9030+932 \times 2$ SOJOF9ZZZR 50J0+932स c0J0t932HZ S0J0493ZHZ SOJ0+93ZnZ s020+93ZHZ †0J06932AZ $60306932 \mathrm{NZ}$ rosotgzzhz HOJO+932NZ 96dJSOE 9512020
TOYLMOS/8OLINOH JURLSIOH B3OTS3ZM

30

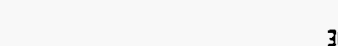

S3EN

00

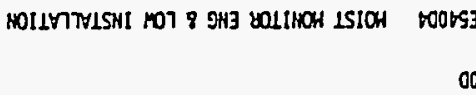

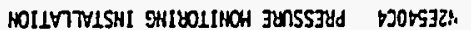

20

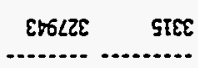

EISZ 2 0370LSLS LT80t932NZ

EรDOL 8 OLTOLSLS LI8O+932HZ

L9995 It6 OTOOLSLS LIEOb9JZNZ

Ee9gs te6 otootrsL Z0806932NZ

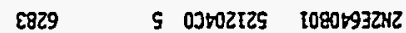

IIZOB ZE OtTOZLZS T080793ZNZ

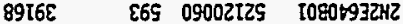

89t6E E65 0900ZtzS t0807932nz

$602 L \quad 08$ 0t00ZtZS t0807932HZ

$602 L \quad 08$ 0t00ZTZS TO8OT9ZZNZ

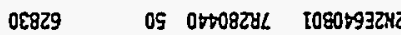

96d3SOE ŞLLOZO

STHL/SJ3Y1 J1 TTLSHI

80

$\leftarrow$

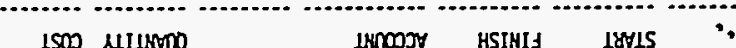

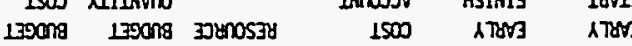

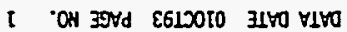

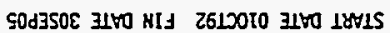

STH101 39xord xaA

S30 LY $011:$

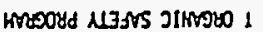
JOLH :200R 32010 .

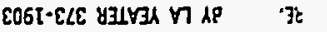
9bitt

ztक OH kny bad3sst alsis c00d: 
WHC-SP-1101

$\checkmark$ EXPERSE

:PORT DATE 15SEP94

=EPARED BY LA YEATER 373-1903

- organil safetr PRDgray

TIVIT DESCRIPTION
ID
$\vdots 1$
$0 \mathrm{C}$

ol

O4.

TE510NS WASTE SAKPLE ARALYSIS (WO)

ON

:2E5IONG ORGAHIC LAYDOAN HOOEL (PHL)

OP

Z2E510PG WASTE HOISTURE WSIMULANTS - FAI

OR

:2E510R6 BASIS FOR SAFE OPS SSTS - ORGAMIC/NITTRATE

ov

iZESIOV6 CRGAHICS TAHKS SAFETY CRITERIA

IZE520A5 CAP MANAGEMENT

OB

Y2E52036 PROGRAY PLAN FOR ORGANIC SAFETY PROGRAY
WASTE TANK SAFETY PROGRAK

WORK PAOKAGE TOTALS

START DATE O10CT92 FIH DATE 30SEPOS

DATA DATE OIOCT93 PAGE HO. 2

ORIG EARLY EARLY COST RESQRCE BUDEET BUDGET
DUR START FINISH ACCONTT

DUR START FINISH ACCOUNT

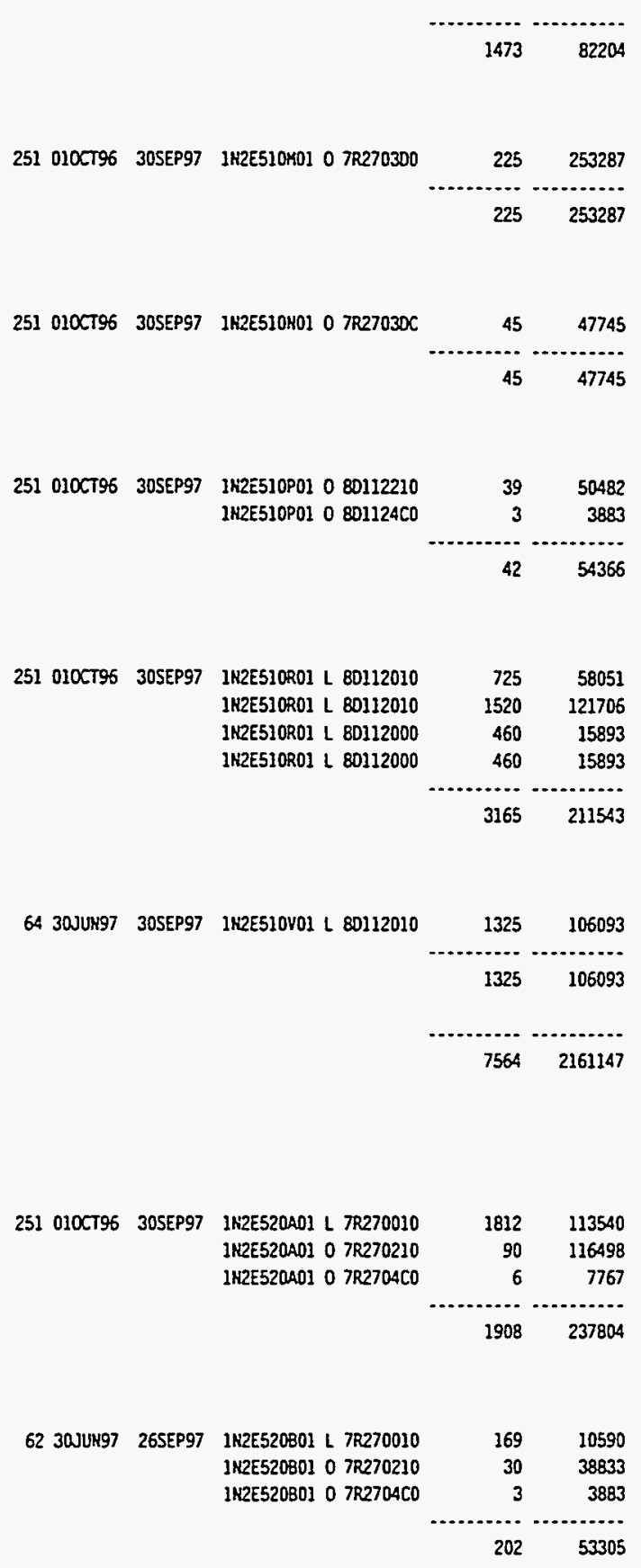

$F 7.4-7.97$ 


\section{WHC-SP-1101}

\section{to: EXPENSE}

EPORT DATE 155EP94

$08: 58$

Fi - IY LA YEATER 373.1903

1. ¿ SAFETV PRDGRAY

GitvIT DESCRIPTION

I0

5E

52

$0=$

:2E520C6 ORGAHIC MATCH LIST TAMKS

oE

i2E520E6 CORE DATA INTERPRETH

\$54

01

N2E5A016 CAP MARGGEHERT

DB

N2E

IHSTALL TC TREES/TRHCS

oc

N2ESAOCG PRESSURE HONITORING INSTALLATIOW
WASTE TAMK SAFETY PROGRAS

WORK PACLAGE TOTALS

RUN NO. 384

START DATE O10CT92 FIH DATE 30SEPO5

data date oloct93 page no. 3

ORIG EARLY EARLY COST RESOURCE BLDEET BUDGET

DUR START FIHISH ACCONT

QLANTITY COST

62 010CT96 310EC96 1K2E520CO1 L 80112010

$\begin{array}{rr}330 & 26423 \\ 330 & 26423\end{array}$

251 010CT96 30SEP97 1M2E520EO1 L 7E310010 IN2E520EOI L 7E310010 IN2E520EOI $\llcorner$ 7E310000
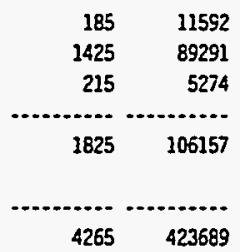

$4265 \quad 423589$

251 010CT96 30SEP97 IN2E540101 L 7R280010

\begin{tabular}{rr}
362 & 22683 \\
\hline 362 & 22683
\end{tabular}

251 010CT96 30SEP97 1K2E540309 L 33300050 1K2E540821 L 7CCO0060 IKRE540821 L 7CCODO1O IK2E540B22 L 70840060 IH2E540B22 L 7CBA0060 IH2E540822 L 7CB40060 1KRE540822 L 7CB40050 IK2E540322 L 7 CB4001O INRE540B25 \& 31110000 IK2E540825 L 31110010 1H2E540B18 L 7CH30010 IN2E540818 L 7CH30010

$15961 \quad 843274$

251 010CT95 30SEP97 1H2E5AOCO1 L 7EA4001O IKIESSACOI L 7EA4001O IHZESAOCOI L TEA4ODOD 1H2ES40CO2 L 7CB40060 1H2ESAOCO3 L 7C500060 IHRESAOCO2 L 7EA4OODO IHIESAOCO4 L 7EA4OOLO

$931 \quad 58336$ 1693106083 $324 \quad 7948$ $585 \quad 26518$ $585 \quad 26518$ $1081 \quad 26517$ $210 \quad 13159$

$5409 \quad 265079$ 
WHC-SP-1101

$\checkmark 2$ EXPENSE WASTE TANK SAFETY PROGRAY

RUN NO. 384

SPORT DATE 15SEP9.

WORK PACKAGE TOTALS

START DATE OIOCT92 FIN DATE 30SEP05

25PAREO BY LA YEATER 373-1903

- orgahic Safetr progray

DATA DATE O1OCT93 PAGE NO. 4

TIVITY OESCRIPTIOH

I0

ORIG EARLY EARLY COST

DUR START FINISH ACCOUNT

RESOURCE BUDGET BUDGET

QLARTITY COST

10

2E54005 HOISTURE MONITOR \& (LON) INSTALL

251 010CT96 305EP97 1N2E540002 L 80520000 IHEE540D02 L 80520010 IH2E540DO2 L 80520000 1H2ES40D02 $\times 80520100$ IN2E540D02 M 80520140 IH2E540002 K 80520150 IN2E540002 H 80520190 IN2E540002 O 80520210 IN2E540002 O 805202LO IN2E540002 O 80520640 1H2E540DO2 L 805202TO INRE540002 L 805204CO 1N2E540002 L 805204PO 1K2E540002 L $805206 \mathrm{NO}$ IN2E540002 L 805206PO 1 N2E540004 L 80340010 1K2E540007 L 80530010 IN2E540006 L 80530010 1H2E540008 L 52100060 1H2E540008 L 52100060 1K2E540008 L 52100060 1\%2E540009 ᄂ 7C500060 1K2E540009 \& 7C500060 1Ni2E540009 L 7C500060 1N2E540010 L 7 C900060 1N2E540010 L 7C900060 IHi2E540010 L 7C900060 1K2E540002 O $8052030 \mathrm{C}$

\begin{tabular}{rr}
68 & 2379 \\
1461 & 118458 \\
262 & 9165 \\
10 & 12944 \\
1 & 1294 \\
4 & 5178 \\
5 & 6472 \\
197 & 255001 \\
2 & 2589 \\
48 & 62132 \\
8 & 9708 \\
33 & 42328 \\
1 & 1294 \\
12 & 15533 \\
1 & 1294 \\
273 & 21190 \\
262 & 21243 \\
236 & 21247 \\
1015 & 69050 \\
1015 & 69050 \\
1015 & 69050 \\
583 & 26427 \\
583 & 26427 \\
583 & 26427 \\
583 & 26427 \\
583 & 26427 \\
583 & 26427 \\
24 & 25464 \\
\hline$\ldots . . . . . .$. \\
9450 & 1000629
\end{tabular}

OE

2E540E6 HOISTURE HOHITOR/CONTROL DEMONSTRATIOW

251 010CT96 30SEP97 1N2E540E05 L 48003060 IN2E540E04 L 7 CBA0060 IN2E540EO1 L 7EA50010 IN2E540505 L 48003060 1N2E540E04 L 7CB40060 IN2E540EO5 L TEA50010 IN2E540EO4 0 7R2703RC

\begin{tabular}{rrr}
\hline 10 & 423 & 26505 \\
\hline$\ldots . . .2$ & 5389880 \\
\hline 9983 & 5607774
\end{tabular}

$41165 \quad 7739439$

35

of

$2 E 55046$ USO CLOSURE DOOMERTATION

128 31HAR97 305EP97 3H2E550A01 L 80112010 IH2E550AOI L 80112000 1K2E550A01 O 80112210 IHZES5OAOI 0 801124CO

$\begin{array}{rr}1165 & 93282 \\ 360 & 12438 \\ 77 & 99670 \\ 5 & 6472 \\ \ldots \ldots . . .2 . . . . \\ 1607 & 211862\end{array}$

1607 


\section{WHC-SP-1101}

\section{$\therefore$ EXPERSE}

PDRT DATE 155EP94

OB:58

if Y LA YEATER 373.1903

-l . SAFETY PROGRAY

TIVITY DESCRIPTIOH

ID

....

35

$O B$

2E550BG SAFETY ISSUE CLOSURE DOCAHENTATION

57

00

.2E570D6 SEPARATIOHS \& ORGAHIC STORAGE SUPPORT

of

M2E570F6 ENGIMEERIHG SUPPORT

os

12

SEPAAATIOHS EQUIPHEIT BURLAL/STORAGE

$\omega$

N2E570J6 TREATKENT SYSTEM FIMLLIZATIOH

OK

N2E570K6 TREATMENT SYSTEH INSTALL/OPERATIOHS
WASTE TAHK SAFETY PROGRAY

WORK PACKAGE TOTALS

START DATE O1OCT92 FIN DATE 305EP05

DATA DATE O1OCT93 PAGE NO. 5

ORIG EARLY EARLY COST

RESORCE BUOGET BUOGET

OUR START FIHISH ACCOWT

QLANTITY COST

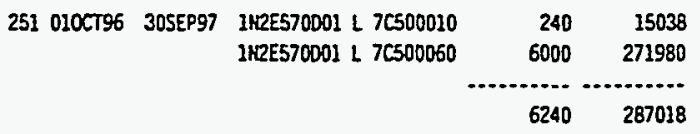

251 010CT95 30SEP97 1K2E57OF01 L 7E620010 305 19111 1HZE570F01 L 7E620010 $\quad 2550 \quad 159783$

$2855 \quad 178894$

63 OIJULS7 30SEP97 IK2E57OHO1 L 7E620010 $254 \quad 15916$ 1K2E570\%01 ᄂ 7E620010 4825 302335

$5079 \quad 318250$

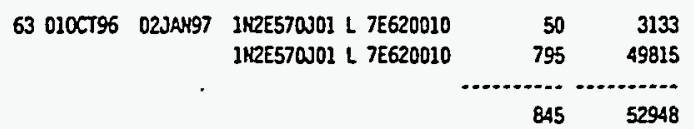

187 010CT96 27JUK97 1H2E570KO1 L 7CB00010 1NZE57OKOI L TCB00060 1N2E570K02 L 76600010 IN2E570K02 L 7C600060

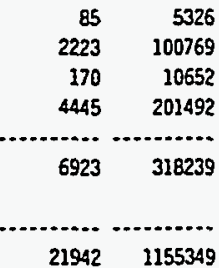

$77953 \quad 11800289$ 


\section{WHC-SP-1101}

SE CAPITAL

EPORT ORTE 15SEPSA RUN WO. 377 $08: 17$

RE 'Y LA YEATER 373-1903

rO. LAR: N2O2

.T OREAHIC SAFETY PROGRAM

: 7 ID ACT DES
WOPK PACAKGE TOTALS

START DATE D1OCT92 FIK DATE 305EPO5

DATA DATE D10CT93 PAGE ho. 2
EARLY EARLY

FINISH $\cos T$

ACCONT
RESOURCE BUDGET BUDGET

QuAstITY COST

$21091 \quad 16363532$ 
WHC-SP-1101

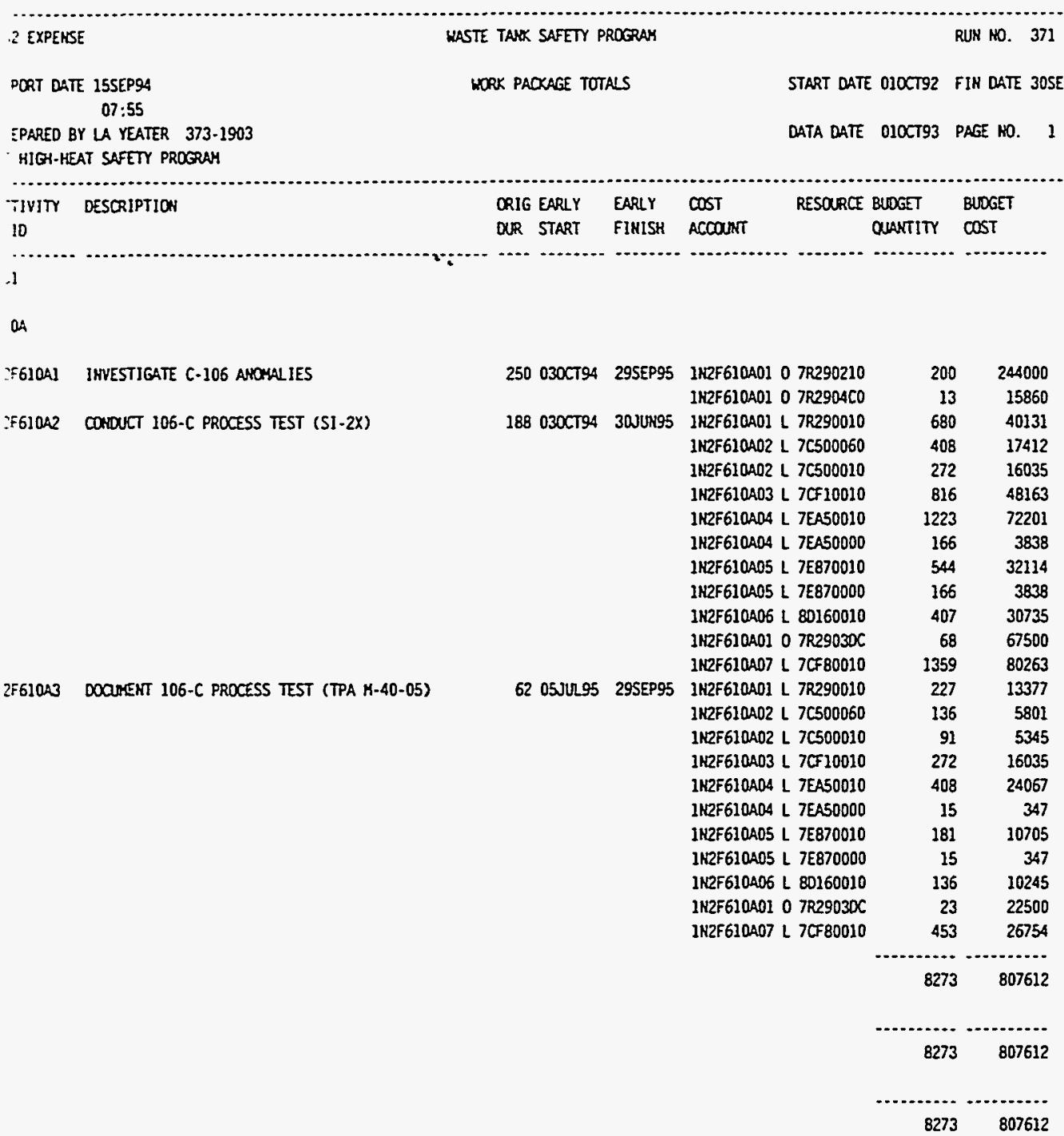


WHC-SP-1101

\section{.2 EXPENLE}

PORT NATE 155EP94

$07: 58$

It I LA YEATER 373.1903

- HIGH-HEAT SAFETY PRDGRAH

Tivitr Description

iD

i)

03

?FG10BA RESQVVE HIGH-HEAT SAFETY ISSUE

\section{HASTE TASK SAFETY PROGRAM}

WORK PACXAGE TOTALS

ORIG EARLY EARLY COST

DUR START FIHISH ACCONT
RUN MO. 372

START DATE O1OCT92 FIH DATE 30SEP05

DATA DATE OLCTI3 PAGE NO. 1

RESORRE BUDGET BUDGET

QUaNTIT COST

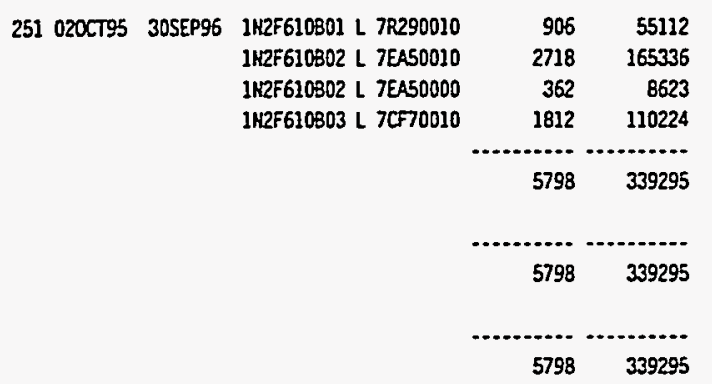

F7.4-7.104 
WHC-SP-1101

KASTE TAHK SAFETY PROGRAH $\quad$ RUN HO. 371

:EPORT DATE 15SEPY4

WORK PACKAGE TOTALS

START DATE D1OCT92 FIN DATE 30SEPO5

PRE $\quad Y$ LA YEATER 373.1903

datA DATE O10CT93 PAGE No. 2

.T h. A ADERGRONOO STORAGE TAKKS

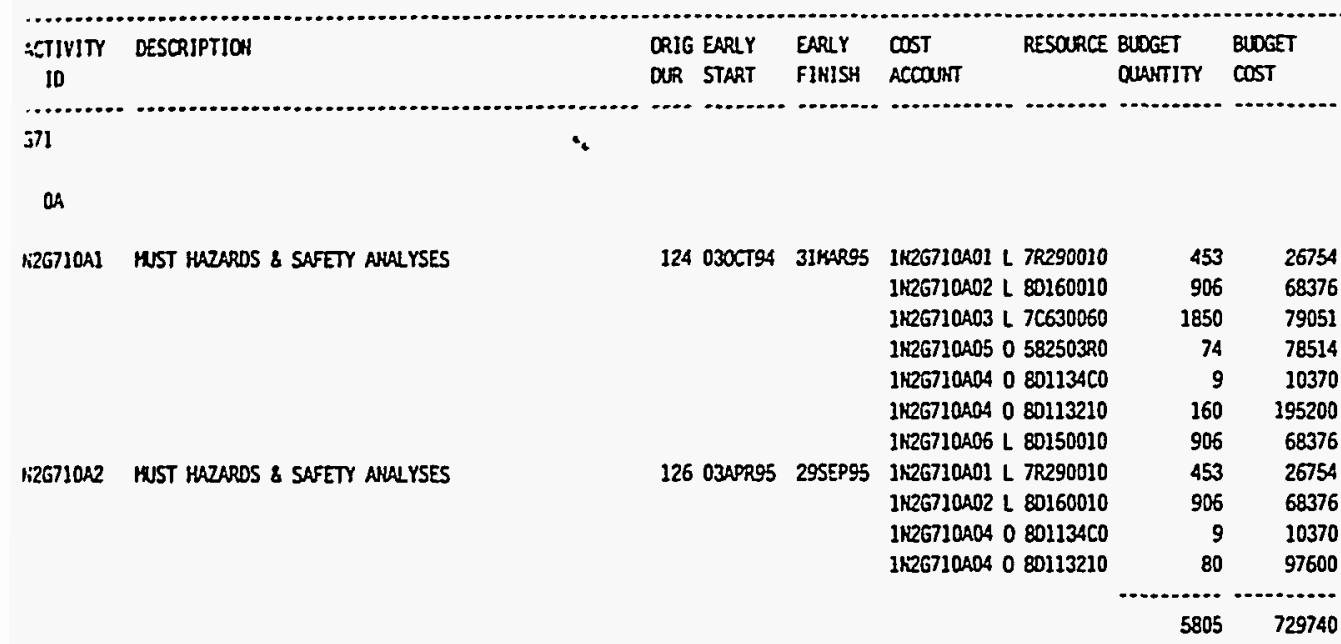

09

12071082 VAPOR SAYPLIHS

126 03APR95 29SEP95 1N2G710301 L BE910010 1 112G710301 L 8E910000 1 1K2G710302 O 72290306

\begin{tabular}{rr}
362 & 18205 \\
362 & 13332 \\
315 & 315000 \\
\hline 1039 & 346537
\end{tabular}

oc

H2G7 P. S SAFETY SCREENIHG SAKPLIHG

126 03APR95 295EP95 1K2G710C01 L 7CF20010

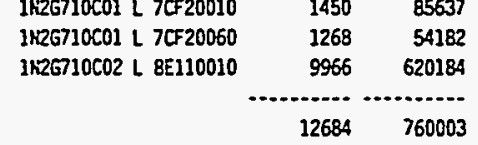

OE

N2G710E2 IH-TANK VIDEO/PHOTOS

126 O3APR95 29SEP95 1H2G710E01 $L$ 7CF80010 2R26710E02 L 7C550060 1K2G710E03 L 33300050 IN2G710EO4 ᄂ 7CF00010 1K2E710EO1 K 7CF80100 1H2G710EO1 0 7CFBO4CO

\begin{tabular}{rr}
272 & 16064 \\
182 & 7777 \\
91 & 4504 \\
91 & 5374 \\
45 & 54900 \\
5 & 6100 \\
\hline 686 & 94719
\end{tabular}

126 03APR95 29SEP95 1N2G710F01 O 7F5103RO INES710F02 L 7 7C000060 2K26710F02 \& 7C000010 1N2G710F03 L 7CF00010

\begin{tabular}{rr}
60 & 63650 \\
1450 & 61959 \\
1450 & 85637 \\
1812 & 107017 \\
\hline .2. & 318272 \\
\hline 4772 &
\end{tabular}

$24986 \quad 2249272$

$24986 \quad 2249272$ 
WHC-SP-1101

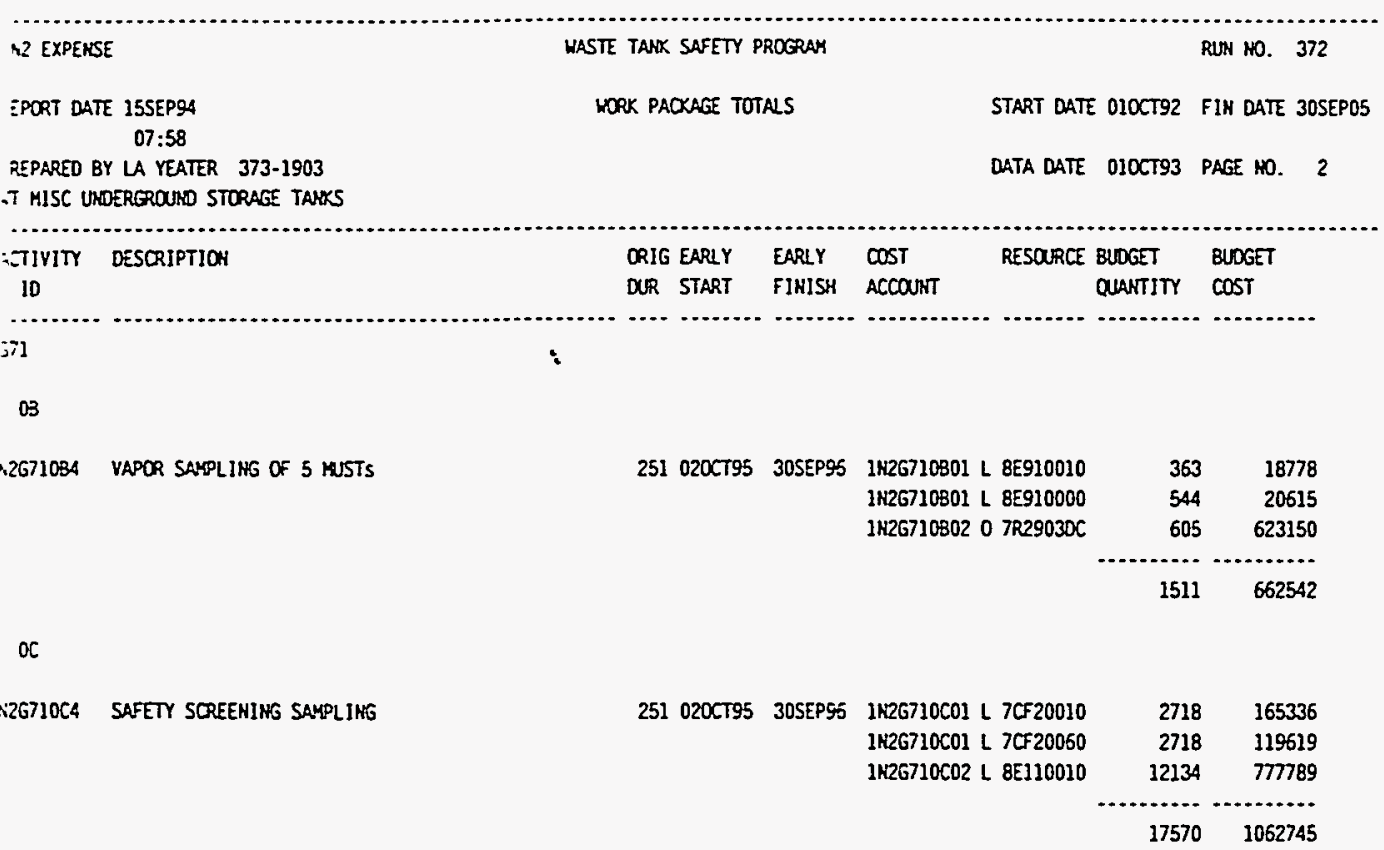

$\infty$

R2GI1004 MONITORING EQUIPHEAT UPGRADES

251 020CT95 305EP95 $3 \mathrm{H} 26710502$ \& 7CF00010 $181 \quad 11010$ $1 \mathrm{~K} 26710002 \& 7$ CF00000 $\quad 181 \quad 4311$ $1 \mathrm{~N} 26710003\llcorner 76000060 \quad 181 \quad 7966$ 1 K2G710003 \& 7C000010 $\quad 181 \quad 11010$

$724 \quad 34298$

OE

N2G710E4 IN-TAKK VIDEO/PHOTOS

$251020 C T 95305 E P 95 \quad 1 \mathrm{~K} 26710 E 01\llcorner$ 7CF80010 $\quad 544 \quad 33092$ 1K26710502 L 7C550060 $363 \quad 15954$ $1 \% 26710 E 03$ L $33300060 \quad 181 \quad 9227$ 1W2G710E04 L 7CF00010 $181 \quad 11010$ 1K2G710E01 H 7CF80100 88 110581 1N2G710EO1 0 7CF804CO $9 \quad 10681$

$1365 \quad 190545$

of

N2G710F4 RISER ACCESSIBJLITY

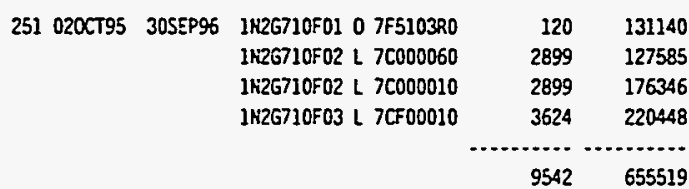

$a$

N2G710GA HUST SAFETY ISSUE RESOLUTION 
WHC-SP-1101

H. CAPITAL

EPORT DRTE 15SEP94 RUN HO. 374 $08 ; 04$

SY LA YEATER 373.1903

roject Hame; $\mathrm{H2O2}$

.T HISC UHDERGROUKD STORAGE TAYKS

CT ID ACT DES

..........

;7!

00

12671004 HOHITORING EOUIPHEKT UPGRADES
LOAK PACAKGE TOTALS

START DATE OIOCT92 FIH DATE 30SEP05

DATA DATE DIOCT93 PAGE HO. I

FIHISH ACCOUNT

QUANTITY COST

QUANTITY COST

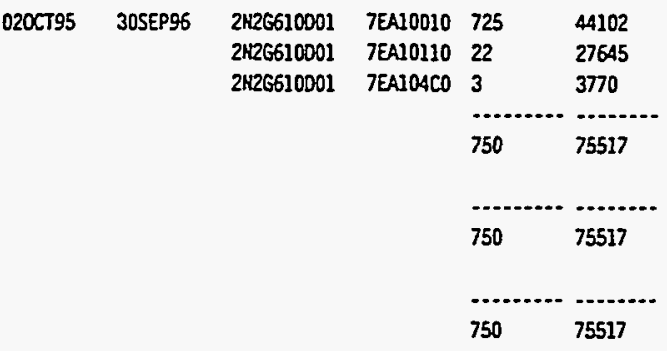


WHC-SP-1101

\begin{tabular}{|c|c|c|c|c|c|}
\hline$\because$ EXPENSE & WASTE TAYK SAFETY PROGRAM & & & & RUN NO. 373 \\
\hline $\begin{array}{l}\text { EDORT DATE 15SEP94 } \\
\text { 08:01 }\end{array}$ & WORK PACKAGE TOTALS & & START DATE & E 010 TT92 & FIN DATE 30SEP05 \\
\hline $\begin{array}{l}\text { FEPARED BY LA YEATER 373-1903 } \\
- \text { MISC UNDERGROULD STORAGE TAYKS }\end{array}$ & & & DATA DATE & $010 C 793$ & PAGE NO. \\
\hline $\begin{array}{l}\text { TIVITY DESCRIPTION } \\
10\end{array}$ & $\begin{array}{l}\text { ORIG EARLY } \\
\text { OUR START }\end{array}$ & $\begin{array}{l}\operatorname{CosT} \\
\text { ACCOUNT }\end{array}$ & RESOURCE & $\begin{array}{l}\text { BLOGET } \\
\text { QLANTITY }\end{array}$ & $\begin{array}{l}\text { BUDGET } \\
\text { COST }\end{array}$ \\
\hline
\end{tabular}

$\because$

03

3G71086 VAPOR SAKPLING OF 10 HUSTS

SAFETY SCREEHIMG SAMPLING

HOWITORIHS EQUIPYENT UPGRADES

oE

2G710E6 IN-TAHK VIDEO/PHOTOS

OF

?G710F6 RISER ACCESSIBILITY

?G71056 MUST SAFETY ISSUE RESOUUTION
$251010 C 796$ 30SEP97 1N2G710801 L 8E910010 1N26710B01 L 8E910000 1K2G710802 O 7R2903DC

\begin{tabular}{rr}
363 & 19339 \\
544 & 21240 \\
605 & 641905 \\
\hline 1511 & 682484
\end{tabular}

251 010CT95 305EP97 1K2G710C01 L 7CF20010 1 2 26710CO1 L 7CF20060 1K26710C02 L 85110010

$2718 \quad 170310$

$2718 \quad 123207$

$19932 \quad 1315911$

$25368 \quad 1609427$

251 010CT96 30SEP97 1N26710002 L 7CF00010 1N2G710002 L 7CF00000 1K26710003 L 7 7C000060 $1 \mathrm{k} 26710003\llcorner 70000010$

$181 \quad 11341$

$181 \quad 4440$

$181 \quad 8205$

$181 \quad 11341$

$724 \quad 35328$

$251010 C T 96$ 305EP97 1N2G710E01 L 7CF80010 1M26710E02 L 7C550050 1K26710E03 L 33300050 1N2G710E04 L 7CF00010 1H2G710EO1 M 7CF80100 IN2G710E01 0 7CFBOACO

\begin{tabular}{rr}
544 & 34087 \\
363 & 16432 \\
381 & 9504 \\
181 & 11341 \\
88 & 113909 \\
9 & 11003 \\
\hline 1365 & 196276
\end{tabular}

$251010 C T 963055 P 97 \quad$ 1N26710F01 O 7F5103R0 1K26710F02 L 7C000050 1\%26710F02 L 7C000010 1K2G710F03 L 7CF00010

\begin{tabular}{rr}
120 & 135086 \\
2899 & 131412 \\
2899 & 181651 \\
3624 & 227080 \\
\hline$\ldots .$. & $\ldots . .$. \\
\hline 9542 & 675229 \\
\hline
\end{tabular}

\begin{tabular}{|c|c|c|c|c|}
\hline \multirow{3}{*}{\multicolumn{2}{|c|}{251 O10СT96 30SEP97 }} & $\begin{array}{l}\text { JN2G710G01 L 7R290010 } \\
\text { IN2G710503 L } 80110010 \\
\text { JN2G710502 L 7E310010 }\end{array}$ & $\begin{array}{l}1812 \\
1812 \\
1812\end{array}$ & $\begin{array}{l}113540 \\
145087 \\
113540\end{array}$ \\
\hline & & & 5436 & 372167 \\
\hline & & & 43946 & 3570912 \\
\hline & & & 43946 & 3570912 \\
\hline
\end{tabular}


WHC-SP-1101

\section{B COPITAL}

PQST DLTE 15SEP94 RUN HO. 376

IE) SY LA YEATER 373-1903

HORK PACAKGE TOTALS

START DATE O1OCT92 FIH DATE 30SEPOS

-oject Hane: $\mathrm{H2O2}$

; MISE UNOERGRONTD STORAGE TAMSS

\begin{tabular}{|c|c|c|c|c|c|c|}
\hline סו דת & ACT DES & $\begin{array}{l}\text { EARLY } \\
\text { START }\end{array}$ & $\begin{array}{l}\text { EARLY } \\
\text { FINISH }\end{array}$ & $\begin{array}{l}\cos T \\
\text { ACCONT }\end{array}$ & RESORCE & $\begin{array}{ll}\text { BLOEET } & \text { BLOSET } \\
\text { QLAMTITY COST }\end{array}$ \\
\hline
\end{tabular}

$\cdots$

os

2671035 HONITORIHS EQUIPKENT UPGRADES

\begin{tabular}{|c|c|c|c|c|c|}
\hline \multirow[t]{4}{*}{ 010CT96 } & 30SEP97 & $\begin{array}{l}\text { 2N2G7100001 } \\
\text { 2स2G710001 } \\
\text { 2N2G710001 }\end{array}$ & $\begin{array}{l}\text { TEALOO10 } \\
\text { TEALO110 } \\
\text { 7EA1OACO }\end{array}$ & $\begin{array}{l}725 \\
22 \\
3\end{array}$ & $\begin{array}{l}45429 \\
28477 \\
3883\end{array}$ \\
\hline & & & & 750 & 77789 \\
\hline & & & & 750 & 77789 \\
\hline & & & & $\ldots$ & 77789 \\
\hline
\end{tabular}


WHC-SP-1101

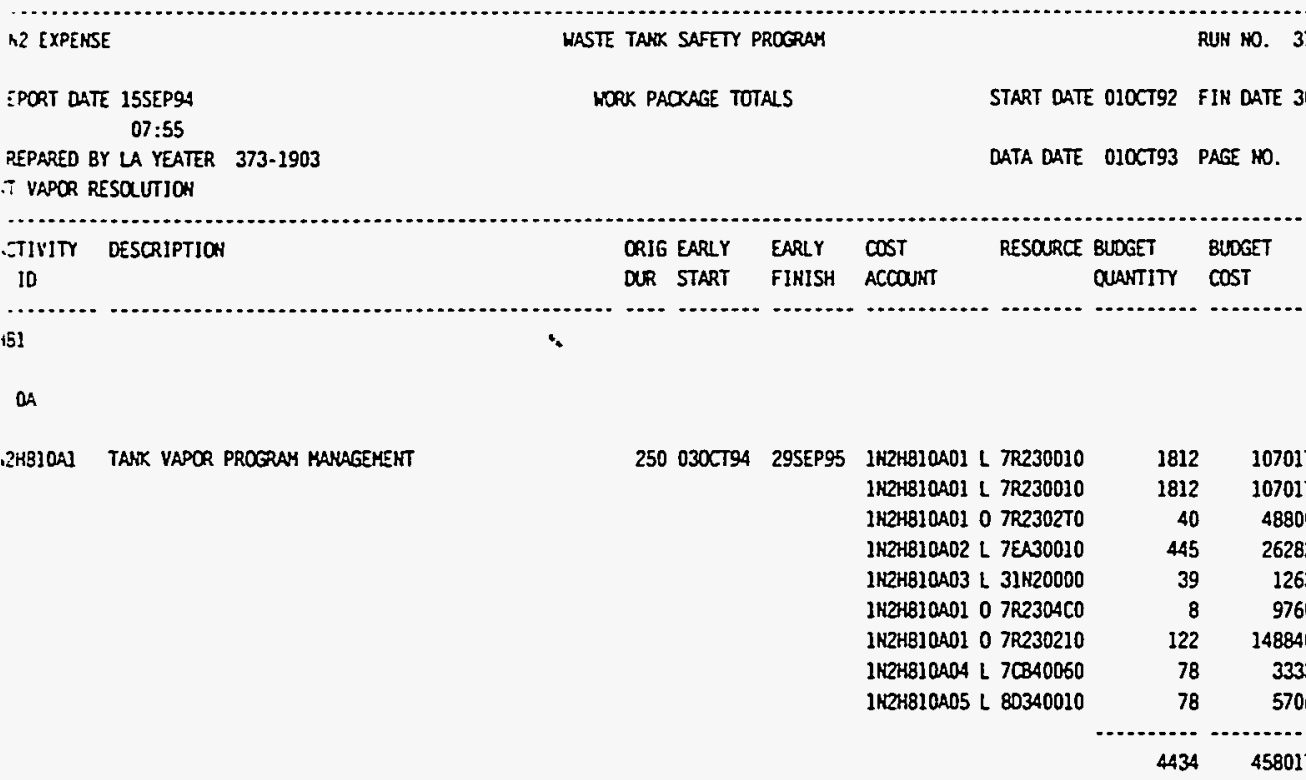

08

Z2HB1032 PHL TOXICOLOGY CONSULTATIOH

OC

:2HB1OC2 WEATHER STATIOH OPS/MAIKT/CALIBRATION (PML)

00

¿2H81002 ATROSPHERIC PATHWAYS (PML)

182

OB

HZHB20B2 VAPOR FLON MONITORIHE DEVICE (ENG SUPPT)

or

KZHB2OC2 EXECUTE RECOMERDATIONS FRON EEA (DSH BASELIHE)

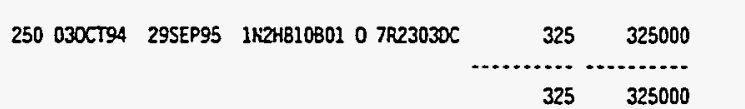

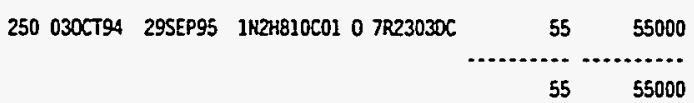

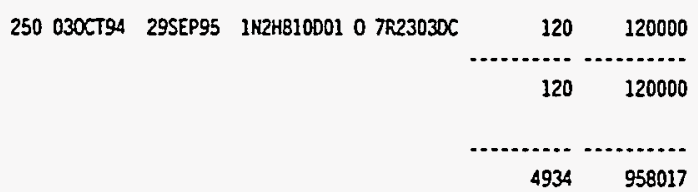

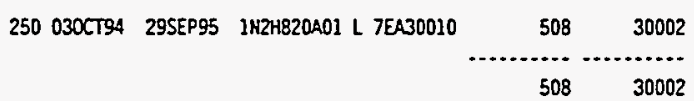

250 030CT94 29SEP9S IN2H820B01 L 7EA30010 $\quad 677 \quad r 39984$

$61030 C T 94$ 300EC94 IN2HB2OCO1 L 7EA30010 B54 50437 1 1\%2H820C02 L 88300010 $280 \quad 16537$ 1स2H820C03 L 80350010 $178 \quad 13022$ 1 1\%2R820C04 L $57570010 \quad 308 \quad 18009$ 


\section{WHC-SP-1101}

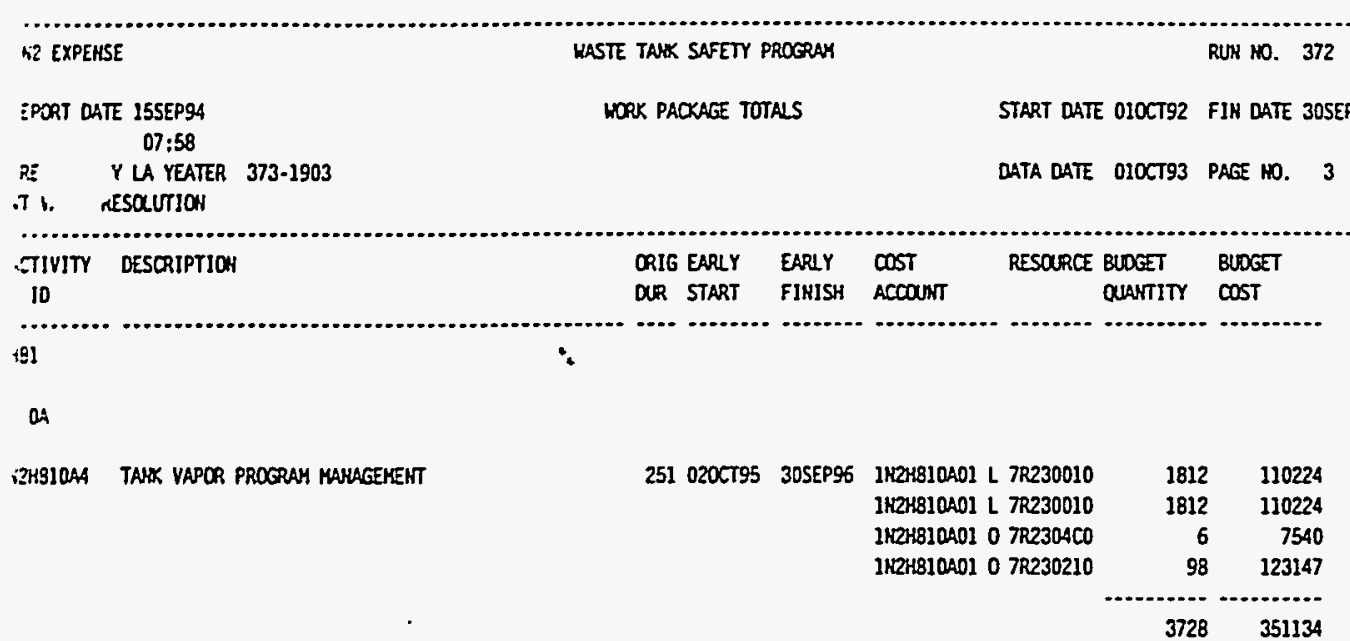

03

PZHB1084 PHL TOXICOLOOY CONSULTANTION

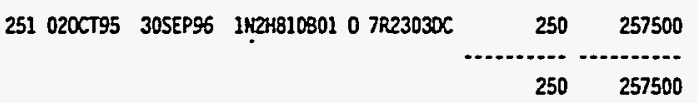

oc

KZHBIOCA WEATHER STATIOH OPS/HAIHT/CALIERATIOH (PHL)

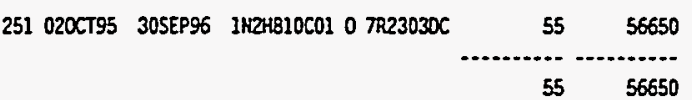

on

N2H81004 ATHOSPHERIC PATHAYS (PNL)

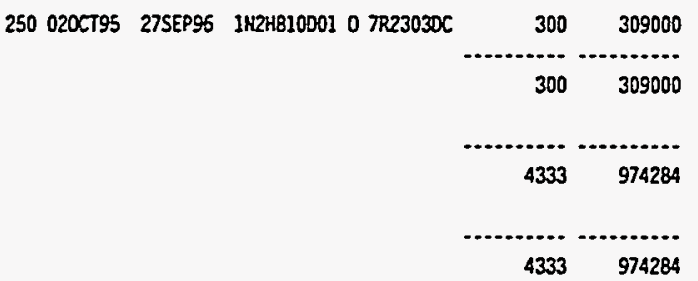




\section{WHC-SP-1101}

H2 CAPITAL

EPORT DATE 155EP94 RUN NO. 375 08:06

SY LA YEATER 373-1903

'ROJ. . Hane: H202

-T HUCLEAR CRITJCALITY

ICT IO ACT DES

...

j9!

09

M2J910B2 MEUTROH DETECTIOH/EVALLATIOH
WORK PACAKGE TOTALS

START DATE O1OCT92 FIN DATE 3OSEPOS

DATA DATE O1OCT93 PAGE MO. 2

EARLY EARLY COST RESORLE BUDGET BUDEET

START FIHISH ACCOUT

OHANTITY COST

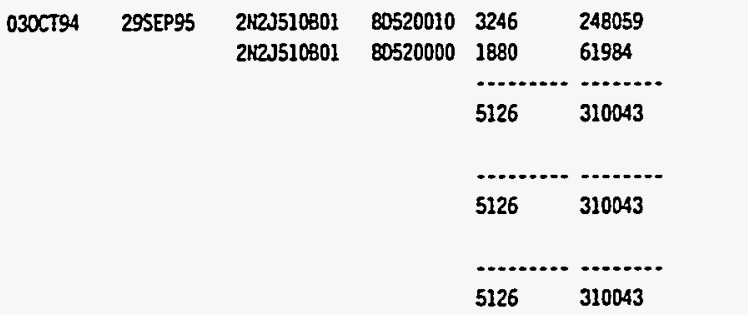


WHC-SP-1101

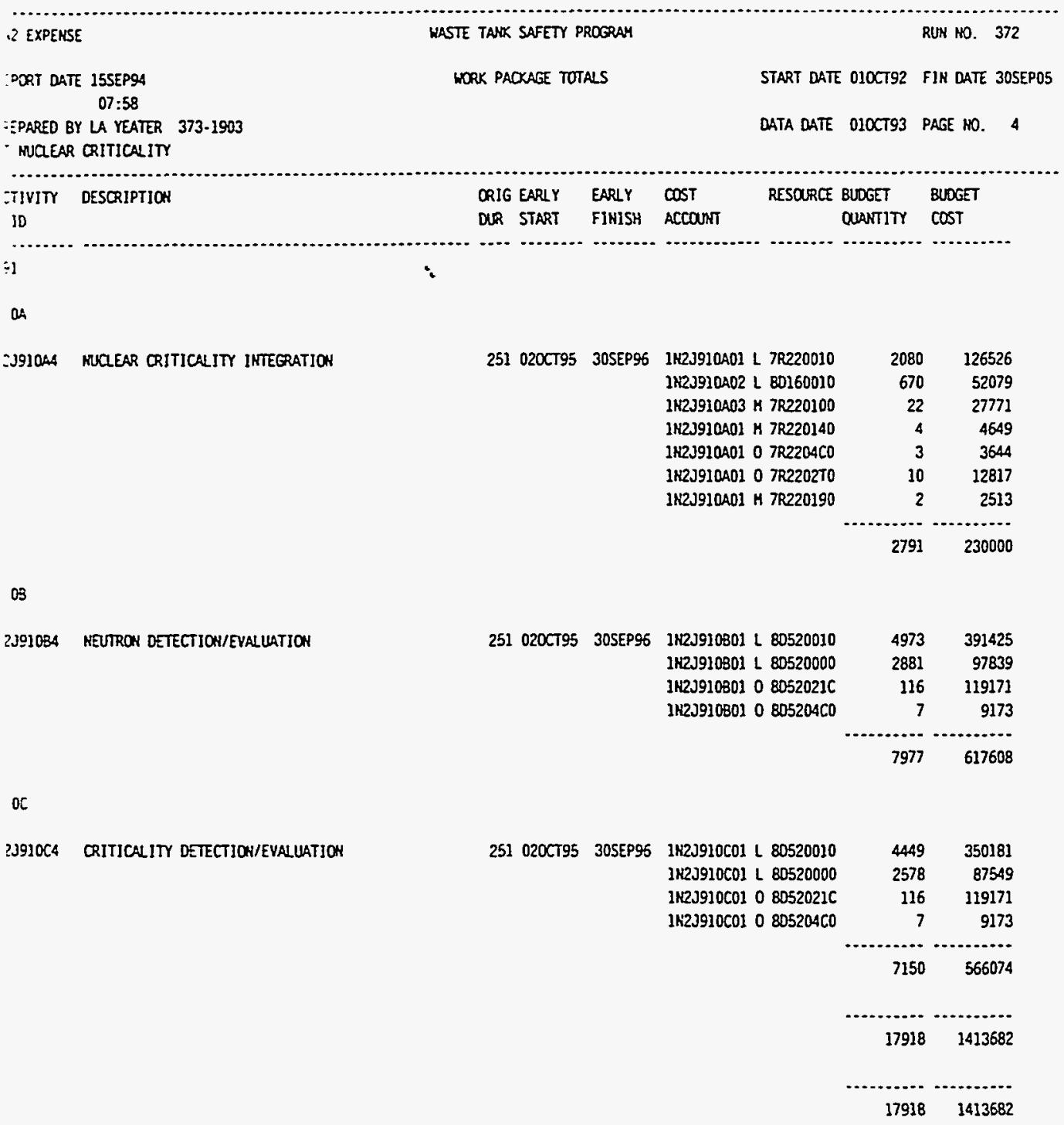




\section{WHC-SP-1101}

H2 CAPITAL

EPORT DATE 15SEPS4 RUH 10. 374 HORK PACAKGE TOTALS START DATE O10CT92 FIN DATE 30SEP05

R. IY LA YEATER 373-1903 DATA DATE O1OCT93 PAGE NO. 2

rC. me: $\mathrm{N} 202$

$T$ MUAEAR CRITICALITY

ST ID ACT DES

START FIHISH ACCONT W QLANTITY COST

$\ddot{1}$

OS

i2J9IOBA MEUTROH DETECTIOH/EVALLATIOH

$\begin{array}{llllll}020 C T 95 & 30 S E P 96 & 2 K 2 J 610801 & 80520010 & 4188 & 329637 \\ & & 2 K 23610801 & 80520000 & 2427 & 82421 \\ & & & \ldots \ldots \ldots . . . & \ldots . . . . . \\ & & & 6615 & 412058\end{array}$

oc

\$2JGIOCA CRITICALITY DETECTIOH/EVALLATION

\begin{tabular}{|c|c|c|c|c|c|}
\hline \multirow[t]{4}{*}{ 020CT95 } & 30SEP96 & $\begin{array}{l}2 \mathrm{~N} 2 \mathrm{J610C01} \\
2 \mathrm{H} 2 \mathrm{~J} 610 \mathrm{CO}\end{array}$ & $\begin{array}{l}80520010 \\
80520000\end{array}$ & $\begin{array}{l}785 \\
462\end{array}$ & $\begin{array}{l}61787 \\
15690\end{array}$ \\
\hline & & & & 1247 & 77477 \\
\hline & & & & 7862 & 489535 \\
\hline & & & & 7862 & 489535 \\
\hline
\end{tabular}

$F 7.4-7.118$ 
WHC-SP-1101

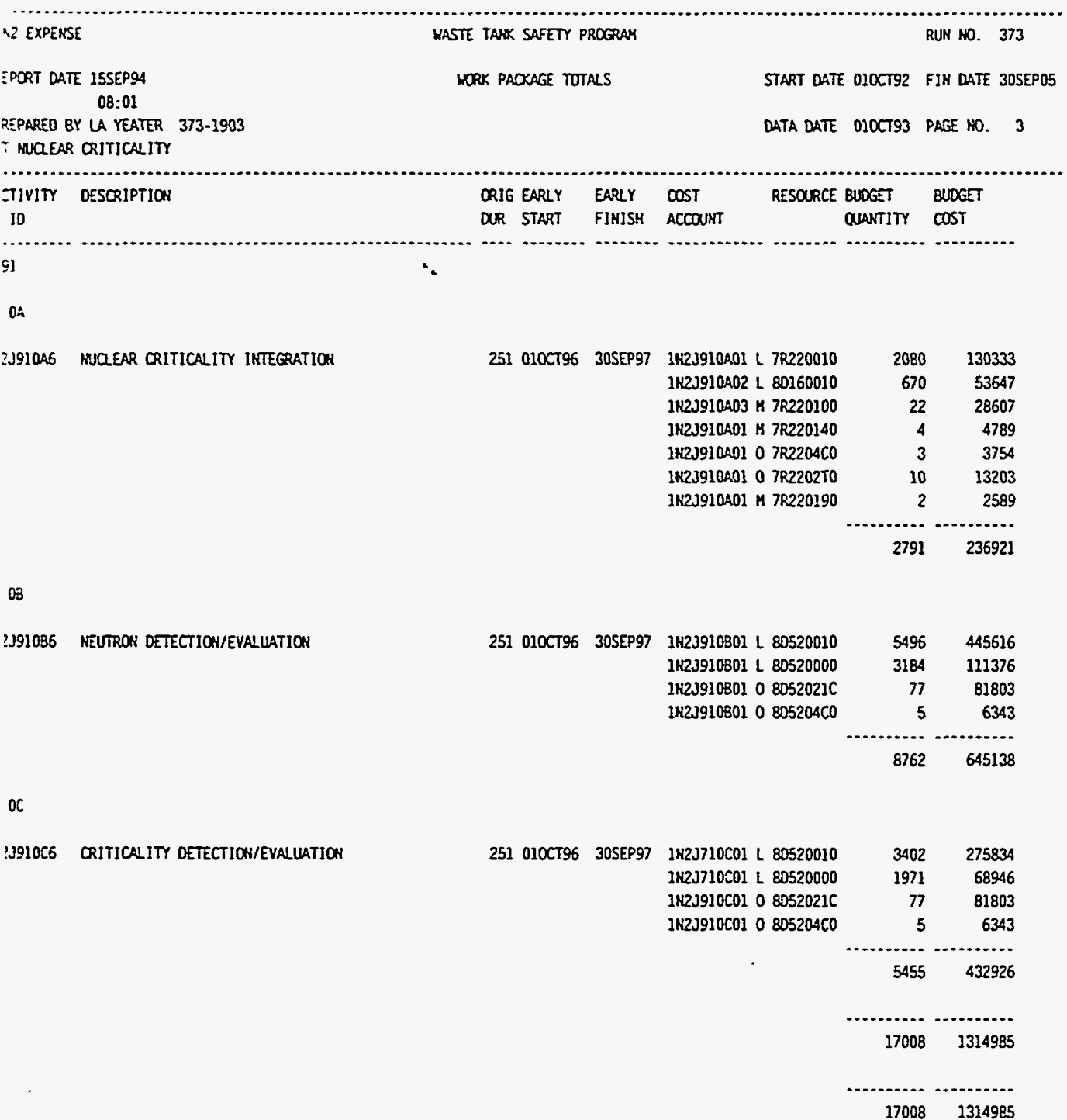

$17008 \quad 1314985$ 


\section{WHC-SP-1101}

\section{$\therefore$ CAPITAS}

:PORT DATE 15SEP94 RUN HO. 376

aE OB:08

-OJ. NE: $\mathrm{K2O2}$

i HUEAR CRITICALJTT

IT ID ACT DES

EARLY
START

WORK PACAKGE TOTALS

START DATE O10CT92 FIN DATE $305 E P 05$

DATA DATE O10CT93 PAGE hO. 2

$\because$

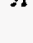

OB

:I91096 HEUTROH DETECTIOH/EVALLATIOH

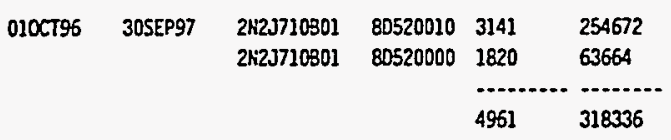

OC

.2J910:6 CRITICALITY DETECTIOH/EVALLATION

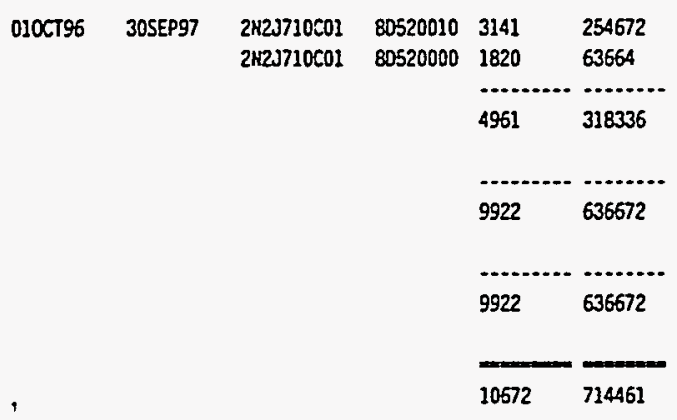


WHC-SP-1101

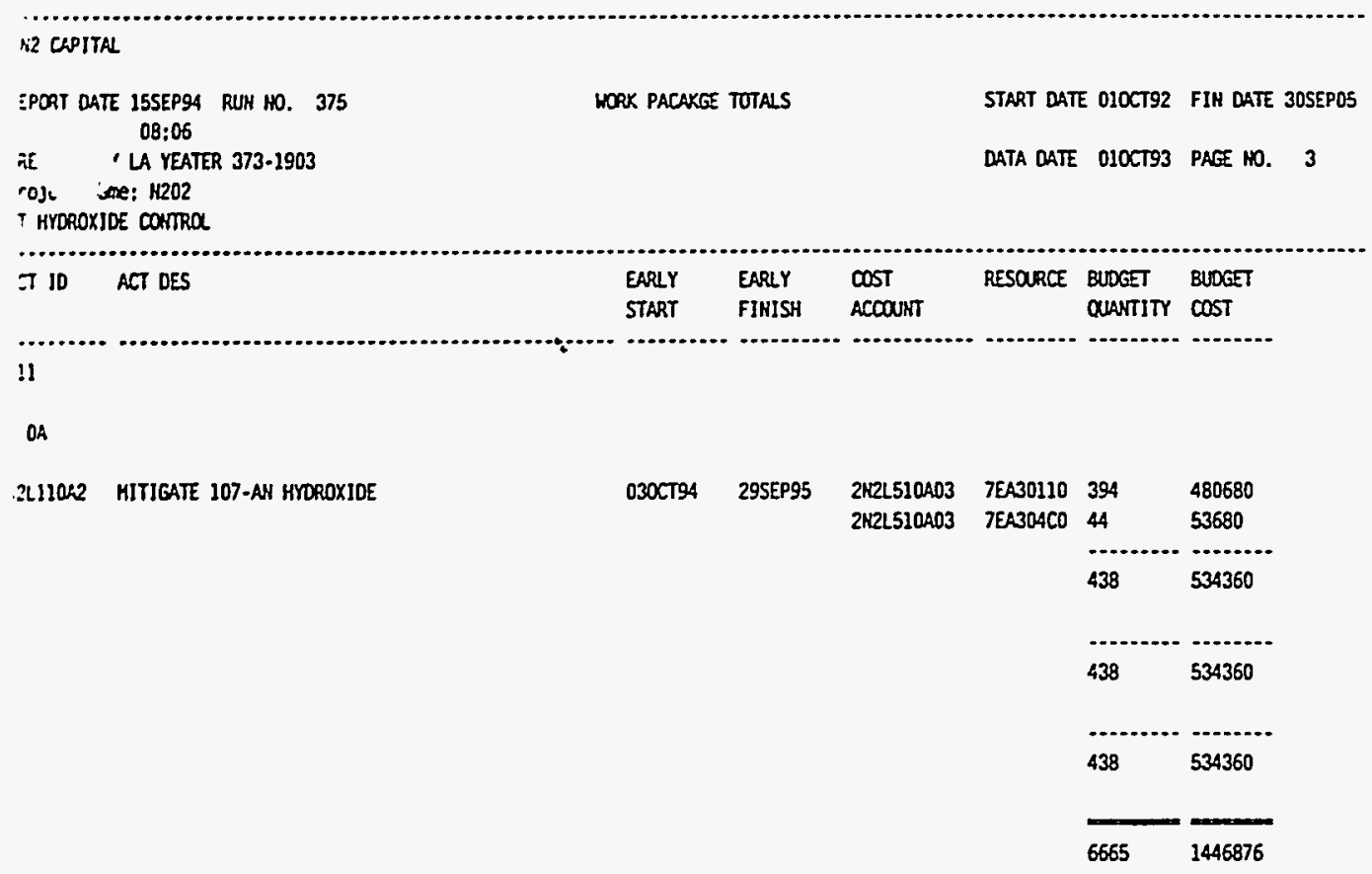


WHC-SP-1101

i2 EXPEHSE

:PORT OATE 15SEP94

08:01

IE I LA YEATER 373-1903

h. IOE COUTRO

TIVITY DESCRIPTIOH

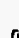

?L11086 HITIGATE 202-A2 HYOROXIDE

2L.11OC6 HITIGATE 102.AN HYOROXIDE
HASTE TANK SAFETY PROGRAY

WORK PACXAGE TOTALS

START DATE 010CT92 FIN DATE 30SEPO5

DATA DATE O10CT93 PAGE MO. 4

ORIG EARLY EARLY COST

DRR START FINISH ACCOUNT

RESOURCE BUDGET BUDGET

QUANTITY COST

$\bullet$

\begin{tabular}{|c|c|c|c|c|}
\hline \multirow[t]{5}{*}{$251010 C 796$} & 30SEP97 & IH2L110301 L 7C600010 & 906 & 56770 \\
\hline & & 1HZLIIOBO2 L 7CF00010 & 906 & 56770 \\
\hline & & 1K2L110BO1 L 7C500010 & 1087 & 68111 \\
\hline & & 1H2L110B04 L 7C500060 & 2717 & 123162 \\
\hline & & IHZL.110B05 L BE110010 & 1630 & 107613 \\
\hline
\end{tabular}

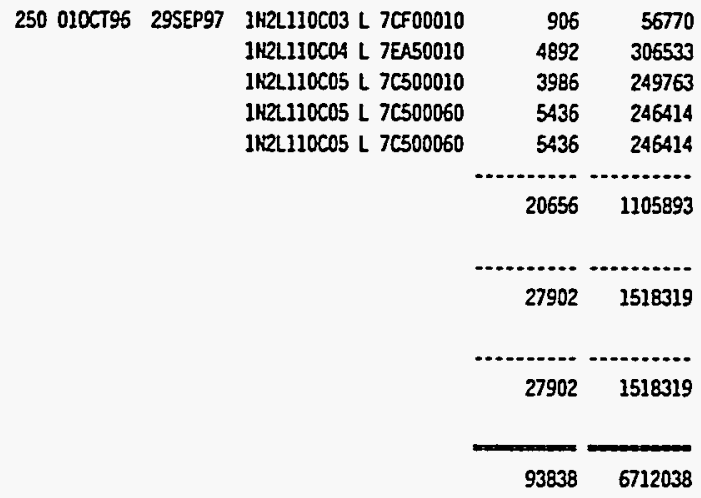

$F 7.4-7.124$ 
Figure 7.4-8. Waste Tank Safety Staffing.

TOTAL USAGE FOR YEAR

TE - TOTAL YEARLY SUHFARY

$\begin{array}{ccccccccc} & & \text { FY } & \text { FY } & \text { FY } & \text { FY } & \text { FY } & \text { FY } & \text { FY } \\ \text { CT ID DESC } & \text { 1995 } & 1996 & 1997 & 1998 & 1999 & 2000 & 2001 & \text { TOTAL }\end{array}$

11.

I2AJJOAD2 BASELIME PLANHIHG FY95

2AIJOA08 BASELINE PLANHIHG FY96

i2A11OAL2 BASELIHE PLAWHIHG FYG7

SOAd01 Perf Kaste Tank Safety Prograw Kanagenent

I2A110304 MANAGEKERT \& IKTEGPATION FY95

.2AJ10308 MAYGGEKEHT \& INTEGPATION FY96

:2AJ10812 MARGGEKEHT \& IHTEGRATIOH FY97

I2AJ1OKO4 ADVISORY SUPPORT FY95

I2AI1OKO6 ADYISORY SUPPORT FY96

i2A11OKOB ADVISORY SUPPORT FY97

TOTAL All

$\$ 12$.

Y2A120ROA WASTE TAKK SAFETY OA FY95

V2A12DADS WASTE TAKK SAFETY OA FY96

V2A120AOB WASTE TAKK SAEET OA FY97

550A120 Waste Tank Safety Quality Assurance

TOTAL A12

321 .

N2B210AO4 PRDGRAY HARAGEMENT FY95

H2B21040S PRDCRAY HAHAGEHEHT FY96

N28' PROCRAK MAYLGEKEFT FY9?

N2B. PROAECT HAHAGERENT FY9S

N2B210805 PRONECT MAUACEHEKT FY96

N2B21OBOB PROYECT HARAGEHEKT FY97

N2B210E31 LAHL DACS 2C FOR AN FAPH KAINTERAYCE

TOTAL B2I

B22.

HZR220E00 DACS MAIHTEKHAHCE \& OPERATION FY95

H2B220FOO GAS HOWITORIHG HAIHT \& OPERATIOH FY95

NZB220FO4 GAS HOHITORING HAINT \& OPERATION FY95

N2B220FOB GAS HOHITORING HAINT \& OPERATION FY97

H2B220510 IMSTRUMENTATIOH TRAYSPORT COHTAIHER FY95

H2B220616 VISCONETER 101-SY SUPPORT

HIZB220G1B VOIDHETER 101-SY SUPPORT

Ni2B220522 IHSTALL CAMERA 101-5Y

H2B220HDO MIXER PUMP OPERATIONS FY95

H2B220K00 TEST PUNP REHOVAL PREP

TOTAL 822

823 .

N28230405 AS BUILT PIT PUMP \& RISER 103-SY

H2B230406 SITE ELECTRICLL/COVER BLOOX DESIGH

HZB230ADB PREP PROC SPEC/RFP/REVIEN BIDS

H2B23012O EHGINEERING DESIGH SUPPORT

12B230COO HORK PLAH/LORK PKS 103.SY

H2B230CO4 TRAIHIMG/READIHESS REVIEM

H2B230000 DACS 2A SYSTEM MGHT

H2B230002 DACS 2A SYSTEM PROC \& FAB 103-SY

H2B230EOO DACS MAJHTELANCE \& OPS 103-5Y

H2B23NFO4 DACS HAINTEHAYCE \& OPS 103.5Y

H2P

GAS CAARACTERIZATJON SYSTEH (GCS)103-5Y

\begin{tabular}{|c|c|c|c|c|c|c|c|}
\hline \multirow[t]{3}{*}{12.5} & & & & & & & 12.5 \\
\hline & 12.5 & & & & & & 12.5 \\
\hline & & 12.5 & & & & & 12.5 \\
\hline \multirow{4}{*}{9.8} & & & 18.9 & 18.9 & 18.9 & 18.8 & 75.6 \\
\hline & & & & & & & 9.8 \\
\hline & 9.8 & & & & & & 9.8 \\
\hline & & 9.8 & & & & & 9.8 \\
\hline \multirow[t]{3}{*}{0.3} & & & & & & & 0.3 \\
\hline & 0.3 & & & & & & 0.3 \\
\hline & & 0.3 & & & & & 0.3 \\
\hline 22.5 & & & 18.9 & 18.9 & 18.9 & 18.8 & 1433 \\
\hline
\end{tabular}

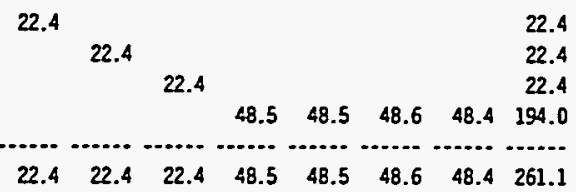

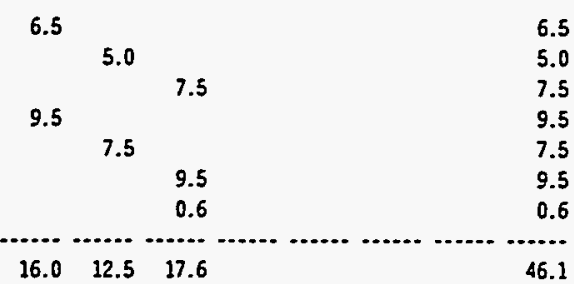

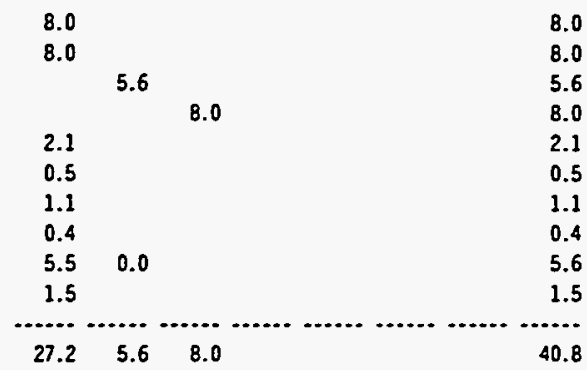

$\begin{array}{llll}2.1 & & & 2.1 \\ 2.1 & & & 2.1 \\ 2.1 & & & 2.1 \\ & 5.2 & & 5.2 \\ & 2.2 & & 2.2 \\ & 6.8 & & 6.8 \\ 5.4 & & & 5.4 \\ & 3.1 & & 3.1 \\ & 3.0 & 0.9 & 4.0 \\ & & 6.4 & 6.4 \\ & 0.7 & & 0.7\end{array}$

$F 7.4-8.1$ 
WHC-SP-1101

ESTIMGHOUSE HARFORD COMPAHY

EPORT DATE 15SEP94 RUN NO. 417 $16: 46$
PRIKAVERA PROJECT PLANEER

RESOURCE LOADINE REPORT

TOTAL USAGE FOR YEAR

$T$ - TOTAL YEARLY SLHAARY

FY

$\begin{array}{ccccccc}\text { FY } & \text { FY } & \text { FY } & \text { FY } & \text { FY } & \text { FY } & \text { FY } \\ 1995 & 1990 & 1997 & 1998 & 1999 & 2000 & 2001 \\ \text { TOTAL }\end{array}$

ST DE OESC

$\begin{array}{lllllll}1995 & 1996 & 1997 & 1998 & 1999 & 2000 & 2001 \text { TOTAL }\end{array}$

$\cdots$

.23

.2B230F08 GAS OHARACTERIZATION SYSTEM (GCS) 103-SY :2B230500 VISCORETER 103-SY SUPPORT

:2B230504 VOIDHETER 103-5Y SUPPORT

¿2B230508 VOIDEETER 103-SY TESTING SUPT

:2B230G10 VISCONETER 103-SY TESTING SUPT

¿2B230J00 REMOVAL/OISPOSAL SYSTEK 103-SY

i2B230K00 IN-TANK DILUTION TEST PREP 103-ST

¿26230K04 IN-TAKK DILUTION SAMPLIHG 103-SY

¿2B230K0B IN-TAMX DILUTION SAMPLING 103-SY

TTAL B23

824 -

23240A06 DESIGH PUYP 101-AN

IZB240408 ENGINEERING DESIGN SUPPORT

:2B240COO INSTALL PUPP 101-AN

I2B240CO2 COPL I01-AN INSTALL READINESS REV

IZB240COB COPLLETE IN TAAK TESTIHG AH-101

Y2B240004 DACS 2B SYSTEM 101-AN

Z2B240005 DACS 2B SYSTEM 101-AN

¿2B240E04 DACS $2 B$ OPERATIOHS 101-AW

U2B240EOB DACS 28 MAINTENANCE \& OPS 101.AN

¿2B240502 VOIORETER 101-AN INST SUPPCRT

VZB240506 VISCONETER 101-AY INSTL SUPT

U2B240G08 VOIOHETER 101-AN INST SUPPORT

228240610 VISCONETER 101-AU IHST SUPT

I2B24OHOB MIXER PLAP OPERATIONS 101-AN

ZB240006 REHOYN/DISPOSAL SYSTEK 101-AN

TOTAL

B24

\begin{tabular}{|c|c|c|c|}
\hline & \multicolumn{2}{|r|}{12} & \multirow{2}{*}{$\begin{array}{l}1.2 \\
0.6\end{array}$} \\
\hline & & & \\
\hline 1.1 & & & 1.1 \\
\hline & 0.4 & 0.2 & 0.5 \\
\hline & 0.8 & 0.4 & 1.2 \\
\hline 1.6 & & & 1.6 \\
\hline 9.6 & & & 9.6 \\
\hline & 9.6 & & 9.6 \\
\hline & & 0.8 & 0.8 \\
\hline 24.4 & 31.7 & 9.9 & 66.0 \\
\hline
\end{tabular}

326 -

H2B260A3O DESIGN INSTALLATIOH SUPT 103-AN

N2B260CO4 INSTALL PUAP 103-AN

12B260C10 COAPL PUAP AN-103 READINESS REVIEN

YZB260F10 GAS OHARACTERIZATIOH SYSTEM (GCS) 103-AY

N2B260500 VISCOKETER 103-AN SUPPORT

YEB260504 VOIDKETER 103-AN SUPPORT

N2B260HDS KIXER PUMP OPERATIOHS

N2B260J00 REMOVAL/DISPOSAL SYSTEM 103-AY

TOTAL

B26

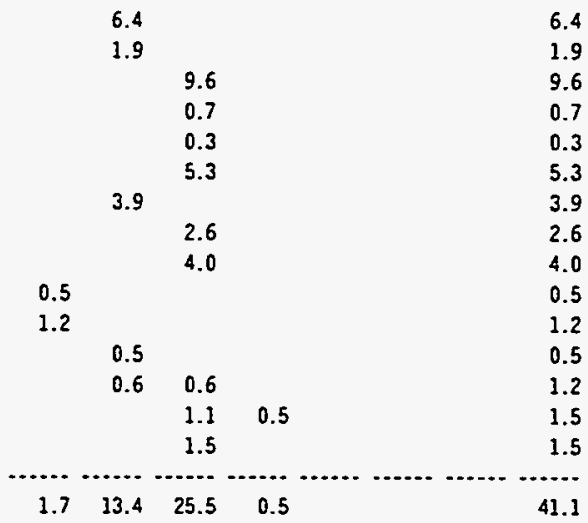

F2B270404 DESIGH PUAP 104-AS

N2B280404 DESIGH SUPPORT PUYP 105 AH

K2B27OCDO INSTALL PREPARATIOH PUMP 104-AA

K2B270CO2 INSTALL PREPARATION PUMPP 104-AN

HZB270COA COAPL TANK 104-AN PUMP INSTALL RR

NZZ27OCOS INSTALL TAKK 104-AN MIT PUMP

NQB270006 DACS $2 C$ SYSTEH HOTT

NZB270EOO DACS 2C MAINTEMANCE \& OPS

N2B270G00 VISCONETER 104-AN SUPPORT

KZB270604 VOIDWETER 104-AH SUPPORT

N2B270505 VISCONETER 104-AN SUPPORT

KZBZ70G08 VOIDHETER 104-AN SUPPCRT

W2B27OHOD MIXER PUMP OPERATIOHS

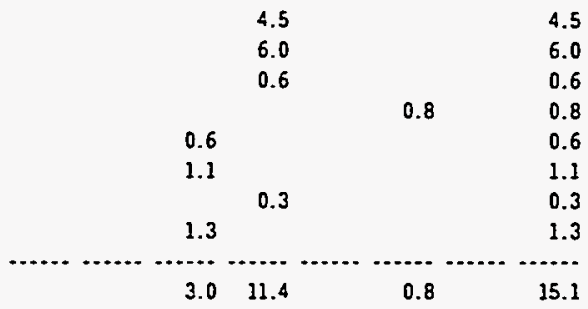

$\begin{array}{cccc} & 5.4 & 5.4 \\ & 6.2 & 6.2 \\ 0.7 & & 0.7 \\ & 1.9 & 1.9 \\ & 0.7 & 0.7 \\ & 2.3 & 2.3 \\ & 7.0 & 7.0 \\ & 3.4 & 3.4 \\ 0.6 & & 0.6 \\ 1.1 & & 1.1 \\ & 0.6 & 0.6 \\ & 1.1 & 1.1 \\ & 1.5 & 1.5\end{array}$

F7 4-8.2 
WHC-SP-1101

.ISTIHEHOUSE HAHFORD COAPAHY

EPORT DATE 15SEP94 RUK 10.417

$16: 46$
PRIMAVERA PRONECT PLALINER

RESOURCE LOADIHG REPORT

TE \& YEARLY SUWARY

TOTAL USAGE FOR YEAR

TOTAL B2B

31 .

H2C310EO4 DHFSB/CAP MALAGEREHT

N2C310602 MOSSBAUER SPECTROMETER

HICCOIOHO4 IH-SITU RELATIVE HUNITITY IHSTRLKENTATION

HOCOJOJOS JHFRARED SPECIATIOH TRALSFER

H2C31OLOD SCANHIHG ELECTROH MICROSCOPE IHVESTG SUPT

TDIAL COI

C33 -

H2C. CAP MAUAGEVEHT FECH TAKK SAFETY FY9S

H2C330430 CAP MAULGEMEMT FEON TAKX SAFETY FY97

H2C330BO4 UPDATE SAFETY DOCAKENTATIOH FY95

N2C330BOS UPDATE SAFETY DOCUNEKTATIOH FY96

N2C33OBOB UPQATE SAFETK DOCNERTATIOH FY97

Y2C330COD TRAHSHIT DOC TO RESOLVE SI C FARH TANKS

N2C330COB DOE REVIEW/CONERT - WHC DISPOSITION

H2C330014 TEONIICAL SUPPCRT SI RESOLUTIOW FY95

H2C330016 TEOHICAL SUPPORT SI RESOUUTOH FY96

H2C330018 TEOHIICAL SUPPORT SI RESOLUTION FY97

N2C330E06 PREPARE SAEETY SUPTISI RESOLUTIOH DOORTENTS

F2C330EOB TRAYS DOC RESOLVE FECN SI ALL TAYKS

NQC33OFOO CORE SAYPLE/DATA INTERPRETATIOW

H2C330F2O CORE SAYPLE/LATA INTERPRETATIOW

K2C330F30 CORE SAYPLE/DATA IHTERPRETATION

TOTAL COS

C34.

R2C34QAD4 CAP MAMAGERENT FECH TAMKS FY95 HRC340AOS CAP RAHAGERENT FECH TAHKS FY96 N2CZAOADB CAP MAUAGEHENT FECH TAKKS FY97 H2C3A0B14 IHSTALLATION OF TC TREES/HOOKUPS THACS HRC3AOC12 KEUTROH PROBE HOISTURE HONITOR LON EHET H2C340C2O HEUTROH PROBE MOISTLRE HONITOR LON EHSR K2C34OC3O NEUTRON PROBE HOISTLRE HOHITOR LON EHZR H2C340002 SURFACE WOISTURE HEASURIHG H2C340020 SURFACE MOISTURE MEASURIHS (CPAC) FY9S HRC340D30 SLRFACE MOISTURE MEASURIHG (CPAC) FY97 H2C3AOEOG EHGIHEERING DOCAMENT COHRO

R2C340EOB EHGIHEERIHG DOCUNENT COITROL FY96 M2C34OE1O EHGIHEERIHL DOCAREHT CONTRO FY97 K2C34OF1O PRESSURE MONITORIHG DESIGH \& EMGR FECH TAYKS

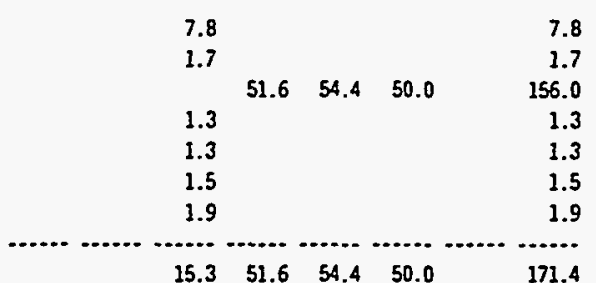

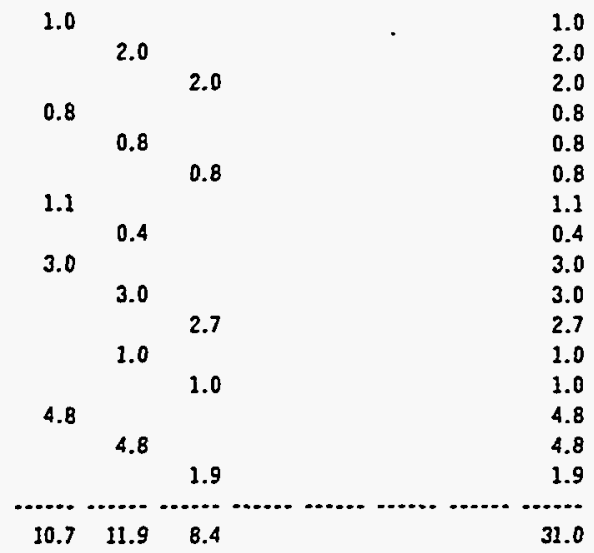

0.8

$\begin{array}{lll}0.8 & 0.8\end{array}$

$\begin{array}{lll}10.7 & 0.8 & 0.8\end{array}$

$\begin{array}{lr}5.1 & 10.7 \\ & 5.1\end{array}$

$2.3 \quad 2.3$

$\begin{array}{lll} & 2.3 & 2.3 \\ 3.5 & & 3.5\end{array}$

3.6

$\begin{array}{lll}0.4 & 3.4 & 3.4\end{array}$

0.10 .1

0.10 .1

6.9

6.9

$F 7.4 \cdot 8.3$ 
WHC-SP-1101

ESTIHGHOUSE HAHFCRD COMPAYY

PRIKAVERA PROJECT PLANAER

SPORT DATE 15SEP94 RUN MO. 417

RESOURCE LOADING REPORT

$16: 46$

$\pi$ ' I YEARLY SUMARY

TOTAL USAGE FOR YEAR

\begin{tabular}{|c|c|c|c|c|c|c|c|c|}
\hline & & FY & FY & FY & FY & FY & FY & FY \\
\hline$\pi$ ID & DESC & 1995 & 1996 & 1997 & 1998 & 1999 & 2000 & 2001 TOTAL \\
\hline 44. & & & & & & & & \\
\hline 500400 & Store \& Monitor_flamable Gas Tanks & & & & 27.4 & 12.2 & & 39.6 \\
\hline$\checkmark 20440 E 10$ & TEHPERATIRE UPGRADES FY95 & 3.0 & & & & & & 3.0 \\
\hline$\cdot 20440 E 20$ & TERPERATRE UPGRAES FY96 & & 5.1 & & & & & 5.1 \\
\hline 'i20440E30 & TEKPERATURE UPGRADES FY97 & & & 5.1 & & & & 5.1 \\
\hline :20440F02 & SURFACE LEVEL UPCRAOES FY95 & 0.6 & & & & & & 0.6 \\
\hline 120440502 & AH FARH VENT UPGRADES FY95 & 2.1 & & & & & & 2.1 \\
\hline 20440514 & AN FARH VEHT UPGRADES & & 1.4 & & & & & 1.4 \\
\hline 'i20440G20 & AH FARS VEKT UPGRADES & & 3.3 & & & & & 3.3 \\
\hline $1: 20440524$ & AN FARY VENT UPGRADES & & & 3.9 & & & & 3.9 \\
\hline 120440630 & PORTABLE EXHAUSTER FCR SSTS & 0.9 & & & & & & 0.9 \\
\hline$\$ 20440 H 05$ & GAS HOHITOR UPGRAES FY9S & 0.8 & & & & & & 0.8 \\
\hline F20440H124 & GAS HOHITOR UPGRADES FY97 & & & 0.1 & & & & 0.1 \\
\hline $120440 \times 02$ & IHSTL AHDWIA MONITORS 241-AH/AH FARHS & 1.3 & & & & & & 1.3 \\
\hline 120440102 & MISC TAKX FARH EOUIPHEKT UPGRADES & & 1.6 & & & & & 1.6 \\
\hline H2044OLO6 & MISC TAKK FARH EQUIPHEHT UPGPADES & & & 1.9 & & & & 1.9 \\
\hline H2D44OHDO & IHSTALL DST/SST GAS HONITORS (SI $2 \mathrm{~h}$ ) & 4.0 & & & & & & 4.0 \\
\hline $1120440 N O 0$ & EQUIPHENT DISPOSAL FY96 & & 37.9 & & & & & 37.9 \\
\hline H20440N04 & EQUIPYEKT DISPOSAL FY97 & & & 44.1 & & & & 44.1 \\
\hline TOTAL & D44 & 31.9 & 51.3 & 57.3 & 27.4 & 12.2 & & 180.1 \\
\hline
\end{tabular}

045 .

H2DA50JO2 FIELD SUPPORT FOR FLAMAALLE GAS FY95 H20450106 FIELD SUPPORT FOR FLAYHABLE GAS FY96 I2OA50UIO FIELD SUPPORT FOR FLAYLABLE GAS FY97

TO' 045

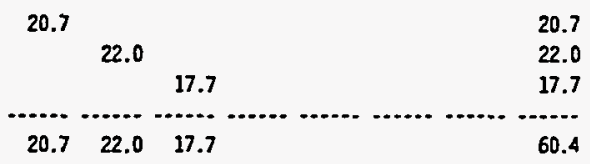

E51 *

SSOE110 Eval \& Define Tech Dev for Org Tanks

FZESIOL2 ORGANICS SOLUBILITY STIDY

H2E5IOL4 ORGAHICS SOLUILITY STUDY

H2E5IOL6 ORGAHICS SOLLBILITY STUDY

N2E51OR2 BASIS FOR SAFE OPS S5TS - ORGANICINITRATE

N2E510R4 BASIS FOR SAFE OPS SSTS - ORGAHIC/NITRATE

HIE5IOR6 BASIS FOR SAFE OPS SSTS - ORGAMICIMITRATE

N2E510T2 BASIS FOR SAFE OPS SSTS - ORGAUIC LAYER

H2E51OU2 BASIS FOR SAFE OPS SSTS - ABSORBED ORGANIC

N2ESIOUA BASIS FOR SAFE OPS SSTS - ABSORBED ORGAHIC

N2E51OV2 ORGAYICS TAKKS SAFETY CRITERIA

H2E51OVA ORGAHICS TANKS SAEETY CRITERIA

H2E5IOV6 ORGAHICS TAHISS SAFETY CRITERIA

TOTAL E5

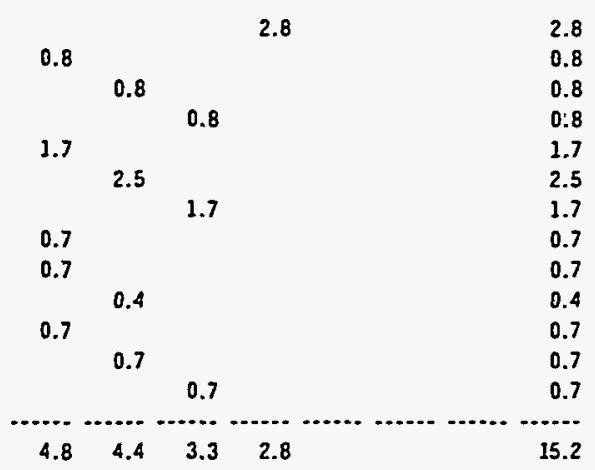

E52 -

N2E52OA2 CAP HANAGERENT

MZE52OAA CAP MARAGEMEHT

N2E520A6 CAP MAYAGEMEKT

NEE520B2 PROGRAY PLAH FOR ORGAHIC SAFETY PROGRAM

N2E520B4 PROGRAY PLAN FOR ORGAHIC SAFETY PROGRAY

N2E520BG PROGPAH PLAH FOR ORGAHIC SAFETY FROGPAM

H2E520C2 ORGANIC TAHXS MATCH LIST

N2E52OCA ORGAHIC WATCH LIST TAKKS

N2E520C6 OAGAHIC WATCH LIST TAKKS

H2E520E2 CORE DATA IHTERPRETI

N2E520E4 CORE OATA IHTERPRETH

MRE520E6 CORE OATA IITERPRET

$\begin{array}{llllll}1.0 & & & & 1.0 \\ & 1.0 & & 1.0 \\ & & 1.0 & 1.0 \\ & & & & 0.1 \\ & 0.1 & & 0.1 & 0.1 \\ & & & & 0.1 \\ & & & & & 0.2 \\ & & 0.2 & 0.2 \\ 1.0 & & & & 0.2 \\ & & 1.0 & & 1.0 & 1.0 \\ & & 1.0 & 1.0\end{array}$

$\mp 7.4-8.5$ 
WHC-SP-1101

\begin{tabular}{|c|c|c|c|c|c|c|c|c|c|}
\hline 10 & DESC & $\begin{array}{c}F Y \\
1995\end{array}$ & $\begin{array}{c}\text { FY } \\
1996\end{array}$ & $\begin{array}{c}\text { FY } \\
1997\end{array}$ & $\begin{array}{c}F Y \\
1998\end{array}$ & $\begin{array}{c}\text { FY } \\
1999\end{array}$ & $\begin{array}{c}F Y \\
2000\end{array}$ & $\begin{array}{c}F Y \\
2001\end{array}$ & TOTAL \\
\hline & : & & & & & & & & \\
\hline & E52 & 2.3 & 2.3 & 2.3 & 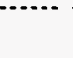 & $\cdots+\cdots$ & ... & $\cdots$ & 6.8 \\
\hline 54012 & CAP MAMAGEKEKT & 0.2 & & & & & & & 0.2 \\
\hline 54014 & CAP MAMAGEMEHT & & 0.2 & & & & & & 0.2 \\
\hline 54016 & CAP MAHAGEMEKT & & & 0.2 & & & & & 0.2 \\
\hline 254034 & IMSTALL TC TREES/THACS & & 10.6 & & & & & & 10.6 \\
\hline 54036 & INSTALL TC TREES/TMACS & & & 10.6 & & & & & 10.6 \\
\hline $540 C 4$ & PRESSLRE IDNITORING INSTALLATION & & 6.5 & & & & & & 6.5 \\
\hline ESA0C6 & PRESSURE MONITORIHG IKSTALLATIOH & & & 3.2 & & & & & 3.2 \\
\hline 54002 & HOISTURE HONITOR EHG \& (LOW) INSTALL & 2.3 & & & & & & & 2.3 \\
\hline 54004 & FIST MOHITOR EHG \& LON IHSTALLATION & & 8.0 & & & & & & 8.0 \\
\hline 54005 & FOISTURE WONITOR \& (LON) INSTALL & & & 5.6 & & & & & 5.6 \\
\hline 500 & Resolve intern - Organic Tanks w/o Treatment & & & & 65.5 & 58.5 & & & 124.1 \\
\hline S40E4 & MOISTURE MOHITOR/CONTR & & 22.7 & & & & & & 22.7 \\
\hline SA0E6 6 & MOISTURE HONITOR/CONTRO DEMOHSTRATIOH & & & 2.7 & & & & & 2.7 \\
\hline $40 \div 2$ & SAFETY EVALLATION/LOA & 0.7 & & & & & & & 0.7 \\
\hline $40=4$ & SAFETY EVALUATIOH/LOA & & 0.7 & & & & & & 0.7 \\
\hline & E54 & 3.1 & 48.7 & 22.3 & 65.5 & 58.5 & & & 198.1 \\
\hline
\end{tabular}

55 .

i2E5SOA4 USO QLOSURE DOCMEETTATIOH

ZES5OAS USO CLOSURE DOCANENTATIOH

E55

FOISTURE HOHITORIHG/CONTRO ENG.

HOISTURE HOHITORIHG/CONTRO EHG.

SAFETY ISSUE QOSURE DOCMMENTATION

SAFETY ISSUE QOSLRE DOCUMENTATION

E56

SEPARATIOUS EQUIPHENT FABRICATIOH SEPARATIONS EQUIPHENT INSTALL/OPER SEPARATIOUS EQUIPMENT INSTALL/OPER SEPARATIOAS \& ORGANIC STORAGE SUPPCRT SEPARATIOHS \& CRGAMIC STORAGE SUPPORT SEPARATIOHS \& ORGANIC STORAGE SUPPORT ENGIMEERIHG SUPPORT

ENGINEERIHG SUPPORT

ENGINEERIHG SUPPORT

DESIGH SEPARATIONS EOUIPMENT BURIAL CONTAIMERS

FAB SEPARATIONS EOUIPHENT BURIAL CONTAIMERS

SEPARATIOLS EOUIPMENT BURIAL/STORAGE

SAFETY \& PERHITIING

TREATEETT SYSTEM DESIGW

TREATEET SYSTEM FABRICATION

TREATEENT SYSTEM FIKALIZATION

TREATENT SYSTEM IHSTALL/OPERATIOWS

TREATEET SYSTEM INSTALL/OPERATIONS

TREAT.SYSTEK BURIAL CANTAIHERS-ENG DSN/EQUIP

TREATHENT SYSTEM BURIAL CONTAIN. EQUIP
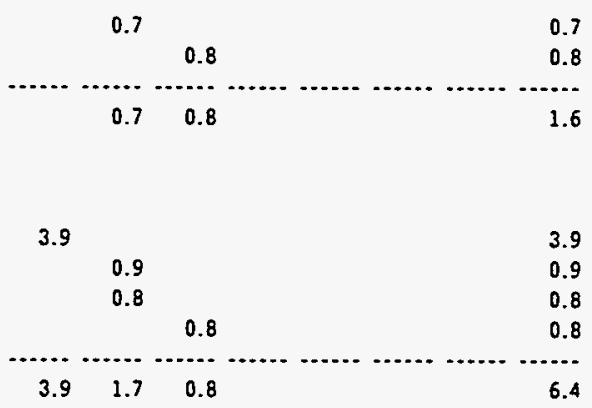

$\begin{array}{rrrr}3.4 & & & 3.4 \\ 11.4 & & & 11.4 \\ & 1.9 & & 1.9 \\ 1.7 & & & 1.7 \\ & 4.6 & & 4.6 \\ & & 3.4 & 3.4 \\ 5.9 & & & 5.9 \\ & 3.3 & & 3.3 \\ & & 1.6 & 1.6 \\ 3.7 & & & 3.7 \\ & 4.7 & & 4.7 \\ & & 2.8 & 2.8 \\ & 1.8 & & 1.8 \\ 3.7 & & & 3.7 \\ & 11.7 & & 11.7 \\ & & 0.5 & 0.5 \\ & 3.8 & & 3.8 \\ & & 3.8 & 3.8 \\ & 1.7 & & 1.7 \\ & & 0.8 & 0.8\end{array}$


WHC-SP-1101

\begin{tabular}{|c|c|c|}
\hline DESTIMG OUSE HAMFORD & COAPAHY & PRIKAVERA PROUECT PLAKHER \\
\hline
\end{tabular}

$16: 46$

7. IAL YEARLY SUYWARY

TOTAL USAGE FOR YEAR

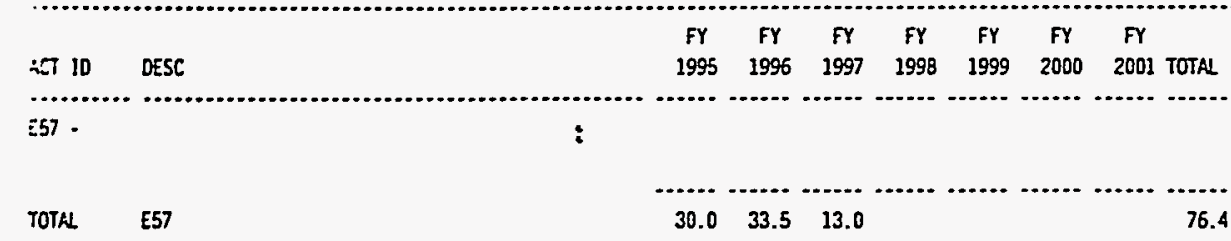

F61.

H2F61042 COHOUCT 106-C PROCESS TEST (S1-2X)

H2F610A3 DODNENT 106-C PROCESS TEST (TPA H-40-05)

N2F610BA RESQCVE HIGH.HEAT SAFETY ISSUE

Hi2F610B6 RESOUE HIGH.HEAT SAFETY ISSUE

TOTAL F6I

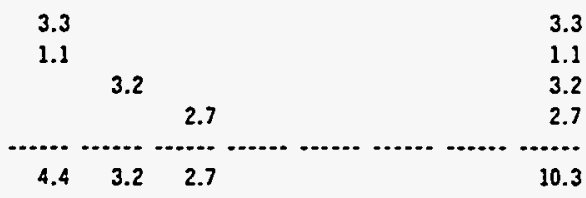

571.

H2G7JOAI HUST HAZARDS \& SAFETY AULLYSES

Hi2G710L2 HUST HAZARDS \& SAFETY AULLYSES

H2G71082 VAPOR SAYPLIHS

N2G710B4 VAPOR SAMPLIHS OF 5 MUSTS

H2O5710B6 VAPOR SAMPLIHS OF 10 RUSTS

N2G710C2 SAFETY SCREEHIHG SAKPLIHG

Hi2G710CA SAFETY SCREENIHG SAYPLIHT

H2G710C6 SAFETY SCREEHIHG SAYPLIHG

H2G71004 HOHITORIHG EQUIPHENT UPGRADES

H2G71006 HOWITORIHG EOUIPMEHT UPGRADES

H2G710E2 IH-TANX VIDEO/PHOTOS

H2G710E4 IH-TAKX VIDEO/PHOTOS

H2G7INF 6 IH-TAKK VIDEO/PHOTOS

H2 RISER ACCESSIBILITY

H2. RISER ACCESSIBILITY

IIZG7IOFG RISER ACCESSIBILITY

N2G710G4 HUST SAFETY ISSUE RESOLUTIOH

N2G710GS HUST SAFETY ISSUE RESOLUTIOH

550G100 Evaluate and Define Kise. Underground Tanks

TOTAL G71

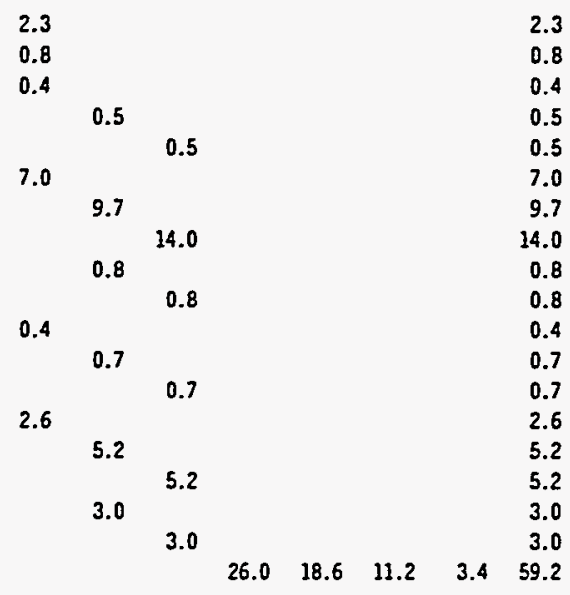

$\begin{array}{llllllll}13.4 & 19.9 & 24.2 & 26.0 & 18.6 & 11.2 & 3.4 & 116.7\end{array}$

HB1 .

N2HBIOAI TANK VAPOR PROCRAY MAHAGEMENT

HEHEIOAA TANK VAPOR PROGRAY MAYAGEMEHT

TOTAL HEI

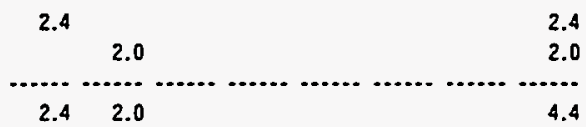

H82 .

N2HB2OA2

H2HB2OB2

N2HE2OC2

К2He2002

TOTAL

VAPOR FLON HONITOR CHARACTERIZATION AMALYSIS

VAPOR FLOA HONITORIHS DEVICE (ENG SUPPT)

EXECUTE RECOHEHDATIOHS FROY EEA (OSH BASELINE)

FABRICATE, INSTALL VAPOR TREATIENT SYSTEH

\begin{tabular}{|c|c|}
\hline 0.3 & 0.3 \\
\hline 0.4 & 0.4 \\
\hline 0.9 & 0.9 \\
\hline 4.1 & 4.1 \\
\hline
\end{tabular}

HE3 •

H2H83OH2 VAPOR FLOH HOHITORIHG DEVICE

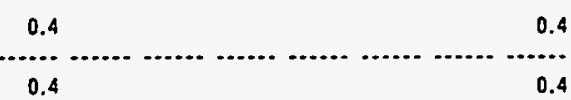

TOTA

HB3

J91 •

H2J9IOA2 MULEAR CRITICALITY INTEGRATIOH H2J910\%4 MULLER CRITICALITY INTEGATIOH

1.7

$1.5-1.7$

$F 7 \cdot 4-8.7$ 
WHC-SP-1101

STIMGROSE HAHFORD COMPAYY

PORT OATE 15SEPYA RUN NO. 417

$16: 46$
PRIMAVERA PROUECT PLANKER

RESORCE LOADIHG REPORT

TUTAL USAGE FCR YEAR

- TOTAL YEARLY SUNAARY

FY

FY FY FY FY FY FY FY

$\begin{array}{llllll}1995 & 1996 & 1997 & 1998 & 1999 & 2000 \\ 2001 & \text { TOTAL }\end{array}$

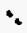

NUEAR CRITICALITY INTEGRATION MEUTRON DETECTION/EVALLATION NEUTRON DETECTION/EVALUATIO NEUTRON DETECTION/EVALLLTION CRITICALITY DETECTION/EVALUATIOH CRITICALITY DETECTIOH/EVALLATIOH CRITICALITY DETECTION/EVALLATION Waste Tank Nuclear Criticality

191

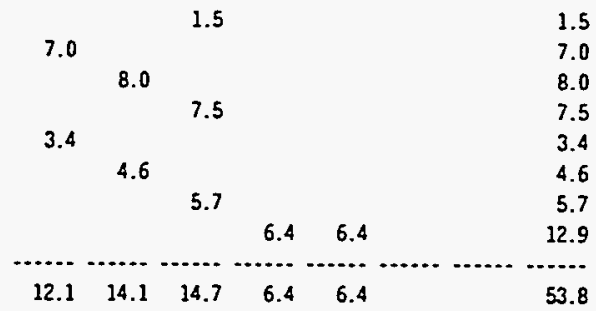

MITIGATE 207-AH HYDROXIOE KITIGATE 107-AN HYOROXIDE MITIGATE 102-AZ HYOROXIDE MITIGATE 102-AZ HYDROXIDE MITIGATE 102-AZ HYRROXIDE MITIGATE 102-AN HYDROXIDE MITIGATE 102-AY MTRROXIOE MITIGATE 102-AN HYOROXIDE Mitigate Hydroxide Consumption

LII

REPQRT TOTAL

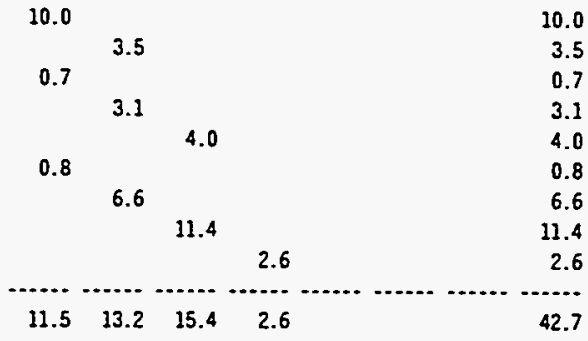

$\begin{array}{llllllll}335.8 & 385.3 & 376.3 & 268.4 & 221.7 & 133.4 & 74.0 & 1795.8\end{array}$ 
TABLE $7.4-1$

TOTAL PROGRAM ELEMENT

COST BASELINE - BY YEAR

\begin{tabular}{|c|c|c|c|c|c|c|c|c|c|}
\hline WBS / LEVEL & ACTIVITY TITLE / ADS & $\begin{array}{l}\text { FUND } \\
\text { TYPE }\end{array}$ & $\begin{array}{l}\text { TOTAL } \\
\text { FY94 }\end{array}$ & FY95 & FY96 & FY97 & FY98 & FY99 & FYOO \\
\hline $\begin{array}{lll}1.1 .2 .02 & \\
\text { Lovel IV } & \\
& & \end{array}$ & $\begin{array}{l}\text { Waste Tank Safety } \\
\text { Aps } \\
\text { TOTAL TARGET }\end{array}$ & OE & $\begin{array}{l}58,642 \\
20,500 \\
-79,142\end{array}$ & $\begin{array}{r}56,742 \\
25,484 \\
-76 \\
62,226\end{array}$ & $\begin{array}{l}56.524 \\
30,579 \\
87,-102\end{array}$ & $\begin{array}{c}61,028 \\
34,668 \\
--- \\
95,696\end{array}$ & $\begin{array}{r}49,124 \\
26,029 \\
-7--75,159\end{array}$ & $\begin{array}{c}49718 \\
24,418 \\
68136\end{array}$ & $\begin{array}{r}34,584 \\
12,045 \\
-46,629\end{array}$ \\
\hline & $\begin{array}{l}\text { Delta to TARGET. } \\
\text { (See notes at end of Table.) } \\
\text { TOTAL }\end{array}$ & $\begin{array}{l}O E \\
C / E\end{array}$ & NA & $\begin{array}{r}(64) \\
(1,564) \\
-(1,628)\end{array}$ & $\begin{array}{r}(24) \\
(2,001) \\
--- \\
(2,025)\end{array}$ & $\begin{array}{c}82 \\
(1,986) \\
--- \\
(1,904)\end{array}$ & ---- & --- & ---- \\
\hline $\begin{array}{l}\text { 1.1.1.2.02.01 } \\
\text { Level } \mathrm{V}\end{array}$ & $\begin{array}{l}\text { Technical Integration } \\
\frac{\text { and Planning }}{A D S 1110-0-\Lambda \Lambda} \\
\text { Sub Total }\end{array}$ & $\begin{array}{l}O E \\
C / E\end{array}$ & NA & $\begin{array}{r}7.718 \\
500 \\
---- \\
8,218\end{array}$ & $\begin{array}{r}7,150 \\
515 \\
--1, \\
7,665\end{array}$ & $\begin{array}{r}8.426 \\
531 \\
---- \\
8,957\end{array}$ & $--\frac{0}{0}$ & $\begin{array}{r}\mathbf{0} \\
0 \\
-0\end{array}$ & $\begin{array}{r}\mathbf{0} \\
-- \\
-\frac{0}{0}\end{array}$ \\
\hline $\begin{array}{l}1.1 .1 .2 .02 .01 .01 \\
\text { Level VI }\end{array}$ & $\begin{array}{l}\text { Integration and Planning } \\
\text { Total }\end{array}$ & $\begin{array}{l}\text { OE } \\
C / E\end{array}$ & & $\begin{array}{r}5,306 \\
500 \\
--- \\
5,806\end{array}$ & $\begin{array}{r}4,666 \\
515 \\
--- \\
5,181\end{array}$ & $\begin{array}{r}5,867 \\
531 \\
--- \\
6,398\end{array}$ & $---\frac{-}{0}$ & $\begin{array}{r}---- \\
0\end{array}$ & $---\frac{-}{0}$ \\
\hline $\begin{array}{l}\text { 1.1.1.2.02.01.02 } \\
\text { Level VI }\end{array}$ & $\begin{array}{r}\text { Waste Tank Safety QA } \\
\text { Total }\end{array}$ & $\begin{array}{l}O E \\
C / E\end{array}$ & & $\begin{array}{r}2,412 \\
0 \\
--- \\
2,412\end{array}$ & $\begin{array}{r}2,484 \\
0 \\
--- \\
2,484\end{array}$ & $\begin{array}{r}2,559 \\
0 \\
--- \\
2,559\end{array}$ & -- & $---\frac{-}{0}$ & --- \\
\hline $\begin{array}{l}\text { 1.1.1.2.02.02 } \\
\text { Level } v\end{array}$ & $\begin{array}{l}\text { Hydrogen Mitigation } \\
\text { SDS } 1110-0-A B \\
\text { Sub Total }\end{array}$ & $\begin{array}{l}O E \\
C / E\end{array}$ & NA & $\begin{array}{l}14,819 \\
14,525 \\
---- \\
29,344\end{array}$ & $\begin{array}{l}11,808 \\
12,975 \\
24,783\end{array}$ & $\begin{array}{l}17,819 \\
14,309 \\
--12 \\
32,128\end{array}$ & $\begin{array}{r}\mathbf{0} \\
--0 \\
-0\end{array}$ & $\begin{array}{r}0 \\
0 \\
-- \\
0\end{array}$ & $\begin{array}{r}0 \\
0 \\
--- \\
0\end{array}$ \\
\hline $\begin{array}{l}\text { 1.1.1.2.02.02.01 } \\
\text { Level VI }\end{array}$ & $\begin{array}{l}\text { Mitigation Program / } \\
\text { Project Management } \\
\text { Total }\end{array}$ & $\begin{array}{l}O E \\
C / E\end{array}$ & & $\begin{array}{r}8,035 \\
4,425 \\
---- \\
12,460\end{array}$ & $\begin{array}{r}6,670 \\
2,416 \\
---- \\
9,086\end{array}$ & $\begin{array}{r}8,630 \\
1.592 \\
---- \\
10,222\end{array}$ & $---\frac{-}{0}$ & $---\frac{-}{0}$ & --- \\
\hline
\end{tabular}


TABLE 7.4-1

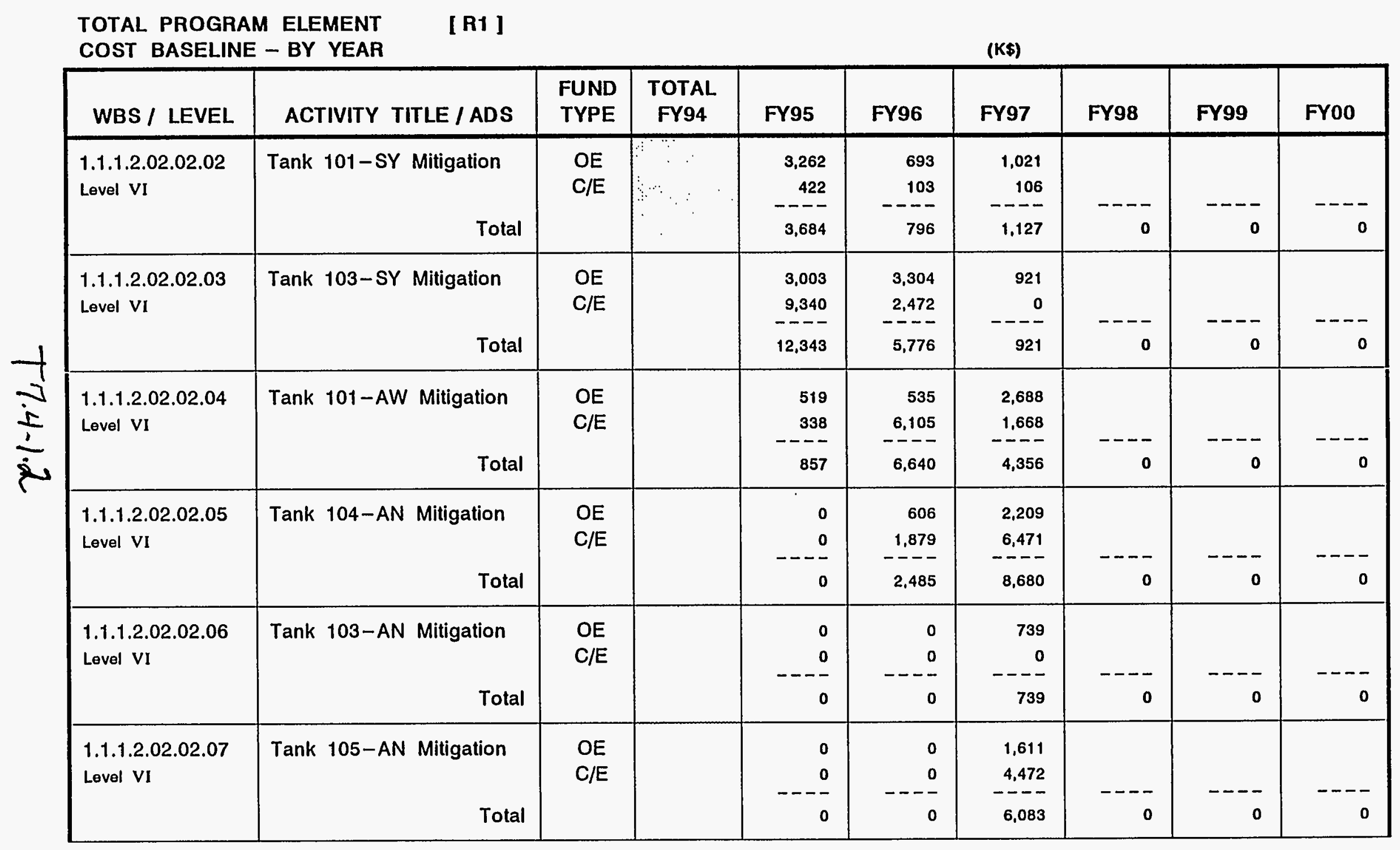


TABLE 7.4-1

TOTAL PROGRAM ELEMENT COST BASELINE - BY YEAR
[R1]

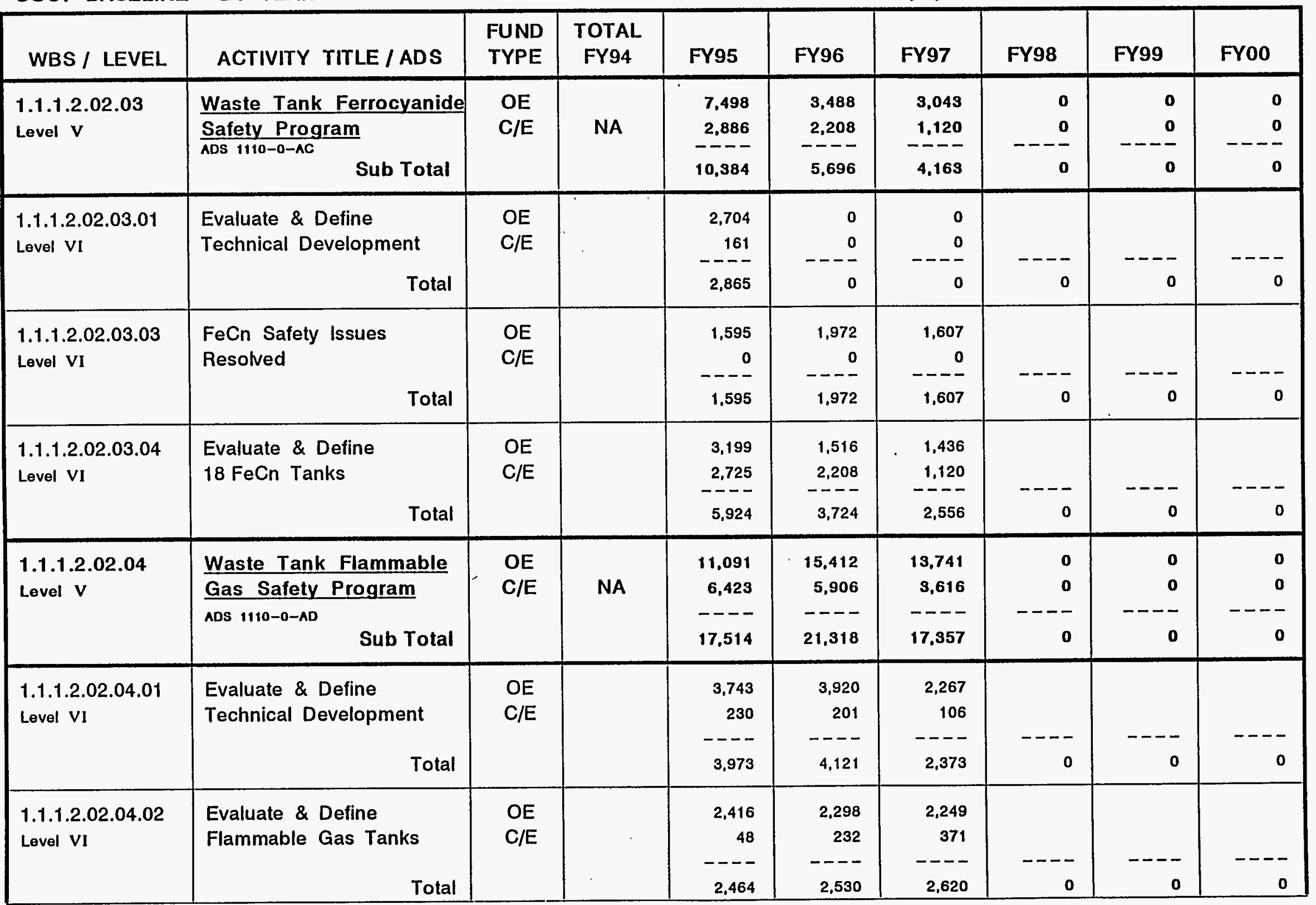


TABLE $\quad$ 7.4-1

TOTAL PROGRAM ELEMENT COST BASELINE - BY YEAR

\begin{tabular}{|c|c|c|c|c|c|c|c|c|c|}
\hline WBS / LEVEL & ACTIVITY TITLE / ADS & $\begin{array}{l}\text { FUND } \\
\text { TYPE }\end{array}$ & $\begin{array}{l}\text { TOTAL } \\
\text { FY94 }\end{array}$ & FY95 & FY96 & FY97 & FY98 & FY99 & FY00 \\
\hline $\begin{array}{l}\text { 1.1.1.2.02.04.03 } \\
\text { Level VI }\end{array}$ & $\begin{array}{l}\text { Close USQs for } \\
\text { Flammable Gas Tanks } \\
\qquad \text { Total }\end{array}$ & $\begin{array}{l}\mathrm{OE} \\
\mathrm{C} / \mathrm{E}\end{array}$ & $\because$ & $\begin{array}{r}874 \\
0 \\
--- \\
874\end{array}$ & $\begin{array}{r}1,599 \\
0 \\
---- \\
1,599\end{array}$ & $\begin{array}{r}1,314 \\
0 \\
---- \\
1,314\end{array}$ & $\begin{array}{r}-- \\
0\end{array}$ & $\begin{array}{r}\cdots \\
0\end{array}$ & $\begin{array}{r}--- \\
0\end{array}$ \\
\hline $\begin{array}{l}1.1 .1 .2 .02 .04 .04 \\
\text { Level VI }\end{array}$ & $\begin{array}{l}\text { Store \& Monitor } \\
\text { Flammable Gas Tank } \\
\qquad \text { Total }\end{array}$ & $\begin{array}{l}O E \\
C / E\end{array}$ & $\because$ & $\begin{array}{r}2,161 \\
6,145 \\
---- \\
8,306\end{array}$ & $\begin{array}{r}5,521 \\
5,473 \\
---- \\
10,994\end{array}$ & $\begin{array}{r}6,175 \\
3,139 \\
---- \\
9,314\end{array}$ & -- & 0 & -- \\
\hline $\begin{array}{l}\text { 1.1.1.2.02.04.05 } \\
\text { Level VI }\end{array}$ & $\begin{array}{l}\text { Integrated Field Support } \\
\text { for Flammable Gas Tanks } \\
\text { Total }\end{array}$ & $\begin{array}{l}O E \\
C / E\end{array}$ & & $\begin{array}{r}1,897 \\
0 \\
---- \\
1,897\end{array}$ & $\begin{array}{r}2,074 \\
0 \\
--- \\
2,074\end{array}$ & $\begin{array}{r}1,736 \\
0 \\
--- \\
1.736\end{array}$ & --- & --- & $\begin{array}{r}--- \\
0\end{array}$ \\
\hline $\begin{array}{l}\text { 1.1.1.2.02.05 } \\
\text { Level } \mathrm{V}\end{array}$ & $\begin{array}{l}\text { Waste Tank Organic } \\
\text { Safety Program } \\
\begin{array}{l}\text { ADS 1110-0-AE } \\
\text { Sub Total }\end{array}\end{array}$ & $\begin{array}{l}O E \\
C / E\end{array}$ & NA & $\begin{array}{r}8,521 \\
1,267 \\
---- \\
9.788\end{array}$ & $\begin{array}{r}11,895 \\
9,972 \\
---- \\
21,867\end{array}$ & $\begin{array}{l}11,800 \\
16,363 \\
---- \\
28,163\end{array}$ & $\begin{array}{r}\mathbf{0} \\
\mathbf{0} \\
-- \\
-0\end{array}$ & $\begin{array}{r}\mathbf{0} \\
\mathbf{0} \\
-- \\
0\end{array}$ & $\begin{array}{r}0 \\
0 \\
--- \\
0\end{array}$ \\
\hline $\begin{array}{l}\text { 1.1.1.2.02.05.01 } \\
\text { Level VI }\end{array}$ & $\begin{array}{l}\text { Technology for Evaluating } \\
\text { Organic Tanks } \\
\text { Total }\end{array}$ & $\begin{array}{l}O E \\
C / E\end{array}$ & & $\begin{array}{r}3,982 \\
470 \\
---- \\
4,452\end{array}$ & $\begin{array}{r}3,779 \\
0 \\
--- \\
3,779\end{array}$ & $\begin{array}{r}2,161 \\
0 \\
--- \\
2,161\end{array}$ & --- & $\begin{array}{r}--- \\
0\end{array}$ & $\begin{array}{r}--- \\
0\end{array}$ \\
\hline $\begin{array}{l}\text { 1.1.1.2.02.05.02 } \\
\text { Level VI }\end{array}$ & $\begin{array}{l}\text { Evaluate \& Define } \\
\text { Organic Tanks } \\
\\
\end{array}$ & $\begin{array}{l}\mathrm{OE} \\
\mathrm{C} / \mathrm{E}\end{array}$ & & $\begin{array}{r}588 \\
0 \\
--- \\
588\end{array}$ & $\begin{array}{r}411 \\
0 \\
--- \\
411\end{array}$ & $\begin{array}{r}424 \\
0 \\
--- \\
424\end{array}$ & --- & $\begin{array}{r}--- \\
0\end{array}$ & --- \\
\hline
\end{tabular}


TABLE $7.4-1$

TOTAL PROGRAM ELEMENT

COST BASELINE - BY YEAR

\begin{tabular}{|c|c|c|c|c|c|c|c|c|c|}
\hline WBS / LEVEL & ACTIVITY TITLE / ADS & $\begin{array}{l}\text { FUND } \\
\text { TYPE }\end{array}$ & $\begin{array}{c}\text { TOTAL } \\
\text { FY94 }\end{array}$ & FY95 & FY96 & FY97 & FY98 & FY99 & FY00 \\
\hline $\begin{array}{l}1.1 .1 .2 .02 .05 .03 \\
\text { Level VI }\end{array}$ & $\begin{array}{c}\text { Pump \& Dispose of Tank } \\
\text { C-103 Organic Layor } \\
\text { Total }\end{array}$ & $\begin{array}{l}O E \\
C / E\end{array}$ & $\because$ & $\begin{array}{r}0 \\
0 \\
-- \\
0\end{array}$ & $\begin{array}{r}0 \\
0 \\
-- \\
-0\end{array}$ & $\begin{array}{r}0 \\
0 \\
-- \\
-0\end{array}$ & --- & 0 & 0 \\
\hline $\begin{array}{l}\text { 1.1.1.2.02.05.04 } \\
\text { Level VI }\end{array}$ & $\begin{array}{l}\text { In-Tank Resolution of } \\
\text { Organic Tanks Without } \\
\text { Treatment } \\
\text { Total }\end{array}$ & $\begin{array}{l}O E \\
\text { C/E }\end{array}$ & $\because$ & $\begin{array}{r}379 \\
184 \\
--- \\
563\end{array}$ & $\begin{array}{r}4,305 \\
9,869 \\
---- \\
14,174\end{array}$ & $\begin{array}{r}7,739 \\
16,257 \\
---- \\
23,996\end{array}$ & --- & --- & $\begin{array}{r}--- \\
0\end{array}$ \\
\hline $\begin{array}{l}\text { 1.1.1.2.02.05.05 } \\
\text { Level VI }\end{array}$ & $\begin{array}{l}\text { Resolve USQ for } \\
\text { Organic Tanks } \\
\\
\text { Total }\end{array}$ & $\begin{array}{l}O E \\
C / E\end{array}$ & : & $\begin{array}{r}0 \\
0 \\
-- \\
0\end{array}$ & $\begin{array}{r}97 \\
0 \\
--- \\
-97\end{array}$ & $\begin{array}{r}212 \\
0 \\
--- \\
212\end{array}$ & --- & 0 & $\begin{array}{r}--- \\
0\end{array}$ \\
\hline $\begin{array}{l}1.1 .1 .2 .02 .05 .06 \\
\text { Level VI }\end{array}$ & $\begin{array}{l}\text { Resolve Safety Issue } \\
\text { Organic Tanks } \\
\qquad \text { Total }\end{array}$ & $\begin{array}{l}\mathrm{OE} \\
\mathrm{C} / \mathrm{E}\end{array}$ & & $\begin{array}{r}676 \\
0 \\
--- \\
676\end{array}$ & $\begin{array}{r}208 \\
0 \\
--- \\
208\end{array}$ & $\begin{array}{r}109 \\
0 \\
--- \\
109\end{array}$ & --- & --- & 0 \\
\hline $\begin{array}{l}\text { 1.1.1.2.02.05.07 } \\
\text { Level VI }\end{array}$ & $\begin{array}{l}\text { Pump and Dispose C-103 } \\
\text { Organic Layer / Resolve } \\
\text { Issue } \\
\qquad \text { Total }\end{array}$ & $\begin{array}{l}O E \\
C / E\end{array}$ & & $\begin{array}{r}2,896 \\
613 \\
---- \\
3,509\end{array}$ & $\begin{array}{r}3,095 \\
103 \\
--- \\
3,198\end{array}$ & $\begin{array}{r}1.155 \\
106 \\
---- \\
1.261\end{array}$ & $\begin{array}{r}--- \\
0\end{array}$ & $\begin{array}{r}--- \\
0\end{array}$ & $\begin{array}{r}---- \\
0\end{array}$ \\
\hline
\end{tabular}

[ R1 ]

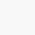


TABLE $\quad 7.4-1$

TOTAL PROGRAM ELEMENT COST BASELINE - BY YEAR

\begin{tabular}{|c|c|c|c|c|c|c|c|c|c|}
\hline WBS / LEVEL & ACTIVITY TITLE / ADS & $\begin{array}{l}\text { FUND } \\
\text { TYPE }\end{array}$ & $\begin{array}{c}\text { TOTAL } \\
\text { FY94 }\end{array}$ & FY95 & FY96 & FY97 & FY98 & FY99 & FY00 \\
\hline $\begin{array}{l}1.1 .1 .2 .02 .06 \\
\text { Level } \mathrm{V}\end{array}$ & $\begin{array}{l}\text { Waste Tank High-Heat } \\
\text { Safety Program } \\
\begin{array}{l}\text { ADS 1110-0-AF } \\
\text { Sub Total }\end{array}\end{array}$ & $\begin{array}{l}O E \\
C / E\end{array}$ & NA & $\begin{array}{r}808 \\
0 \\
--- \\
808\end{array}$ & $\begin{array}{r}339 \\
0 \\
--- \\
339\end{array}$ & $\begin{array}{r}308 \\
0 \\
--- \\
308\end{array}$ & $\begin{array}{r}\mathbf{0} \\
\mathbf{0} \\
-- \\
-0\end{array}$ & $\begin{array}{r}0 \\
0 \\
--- \\
0\end{array}$ & $\begin{array}{r}0 \\
0 \\
--- \\
0\end{array}$ \\
\hline $\begin{array}{l}\text { 1.1.1.2.02.06.01 } \\
\text { Level VI }\end{array}$ & $\begin{array}{l}\text { Evaluate \& Define } \\
\text { High-Heat Tanks } \\
\\
\\
\quad \text { Total }\end{array}$ & $\begin{array}{l}\mathrm{OE} \\
\mathrm{C} / \mathrm{E}\end{array}$ & . & $\begin{array}{r}808 \\
0 \\
--- \\
808\end{array}$ & $\begin{array}{r}339 \\
0 \\
--- \\
339\end{array}$ & $\begin{array}{r}308 \\
0 \\
--- \\
308\end{array}$ & 0 & $---\overline{0}$ & --- \\
\hline $\begin{array}{l}\text { 1.1.1.2.02.07 } \\
\text { Level } \mathrm{V}\end{array}$ & $\begin{array}{l}\frac{\text { Misc. Underground }}{\text { Storage Tanks }} \\
\text { ADs } 1110-0-A G \quad \text { Sub Total }\end{array}$ & $\begin{array}{l}O E \\
C / E\end{array}$ & NA & $\begin{array}{r}2,249 \\
0 \\
--- \\
2,249\end{array}$ & $\begin{array}{r}2,967 \\
76 \\
---- \\
3,043\end{array}$ & $\begin{array}{r}3,571 \\
78 \\
---- \\
3,649\end{array}$ & $\begin{array}{r}\mathbf{0} \\
\mathbf{0} \\
-- \\
\mathbf{0}\end{array}$ & $\begin{array}{r}\mathbf{0} \\
\mathbf{0} \\
--- \\
0\end{array}$ & $\begin{array}{r}0 \\
0 \\
-- \\
0\end{array}$ \\
\hline $\begin{array}{l}\text { 1.1.1.2.02.07.01 } \\
\text { Level VI }\end{array}$ & $\begin{array}{l}\text { Evaluate Misc. } \\
\text { Underground Tanks } \\
\qquad \text { Total }\end{array}$ & $\begin{array}{l}O E \\
C / E\end{array}$ & & $\begin{array}{r}2,249 \\
0 \\
--- \\
2,249\end{array}$ & $\begin{array}{r}2,967 \\
76 \\
--- \\
3,043\end{array}$ & $\begin{array}{r}3,571 \\
78 \\
---- \\
3,649\end{array}$ & 0 & --- & $---\frac{-}{0}$ \\
\hline $\begin{array}{l}\text { 1.1.1.2.02.08 } \\
\text { Level } \mathrm{V}\end{array}$ & 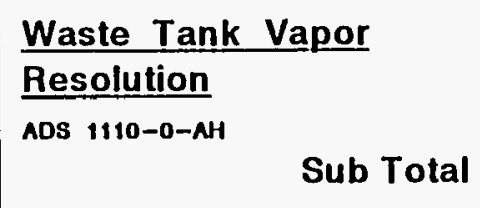 & $\begin{array}{l}O E \\
C / E\end{array}$ & NA & $\begin{array}{r}1,534 \\
603 \\
---- \\
2.137\end{array}$ & $\begin{array}{r}974 \\
0 \\
--- \\
974\end{array}$ & $\begin{array}{r}0 \\
0 \\
--- \\
0\end{array}$ & $\begin{array}{r}\mathbf{0} \\
\mathbf{0} \\
--- \\
0\end{array}$ & $\begin{array}{r}0 \\
0 \\
--- \\
0\end{array}$ & $\begin{array}{r}0 \\
0 \\
--- \\
0\end{array}$ \\
\hline $\begin{array}{l}\text { 1.1.1.2.02.08.01 } \\
\text { Level VI }\end{array}$ & $\begin{array}{l}\text { Evaluate \& Define for } \\
\text { Tank Vapor / Safety } \\
\text { Total }\end{array}$ & $\begin{array}{l}\mathrm{OE} \\
\mathrm{C} / \mathrm{E}\end{array}$ & & $\begin{array}{r}958 \\
0 \\
--- \\
958\end{array}$ & $\begin{array}{r}974 \\
0 \\
--- \\
974\end{array}$ & $\begin{array}{r}0 \\
0 \\
-- \\
-0\end{array}$ & --- & ---- & ---- \\
\hline
\end{tabular}


TABLE $\quad 7.4-1$

TOTAL PROGRAM ELEMENT COST BASELINE - BY YEAR

[ R1 ]

(K\$)

\begin{tabular}{|c|c|c|c|c|c|c|c|c|c|}
\hline WBS / LEVEL & ACTIVITY TITLE / ADS & $\begin{array}{l}\text { FUND } \\
\text { TYPE }\end{array}$ & $\begin{array}{c}\text { TOTAL } \\
\text { FY94 }\end{array}$ & FY95 & FY96 & FY97 & FY98 & FY99 & FY00 \\
\hline $\begin{array}{l}1.1 .1 .2 .02 .08 .02 \\
\text { Level VI }\end{array}$ & $\begin{array}{l}\text { Resolve Internal Tank } \\
\text { Vapor Issues } \\
\qquad \text { Total }\end{array}$ & $\begin{array}{l}O E \\
C / E\end{array}$ & : & $\begin{array}{r}536 \\
603 \\
--- \\
1,139\end{array}$ & $\begin{array}{r}0 \\
0 \\
-- \\
0\end{array}$ & $\begin{array}{r}0 \\
0 \\
-- \\
-0\end{array}$ & --- & --- & --- \\
\hline $\begin{array}{l}\text { 1.1.1.2.02.09 } \\
\text { Level } v\end{array}$ & 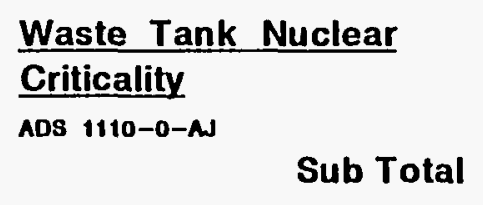 & $\begin{array}{l}O E \\
C / E\end{array}$ & NA & $\begin{array}{r}1,186 \\
310 \\
---- \\
1.496\end{array}$ & $\begin{array}{r}946 \\
490 \\
---- \\
1.436\end{array}$ & $\begin{array}{r}720 \\
637 \\
---- \\
1.957\end{array}$ & $\begin{array}{r}0 \\
0 \\
-- \\
0\end{array}$ & $\begin{array}{r}\mathbf{0} \\
\mathbf{0} \\
-- \\
\mathbf{0}\end{array}$ & $\begin{array}{r}\mathbf{0} \\
\mathbf{0} \\
-- \\
-0\end{array}$ \\
\hline
\end{tabular}


TABLE $\quad 7.4-1$

TOTAL PROGRAM ELEMENT

COST BASELINE - BY YEAR

[ R1 ]

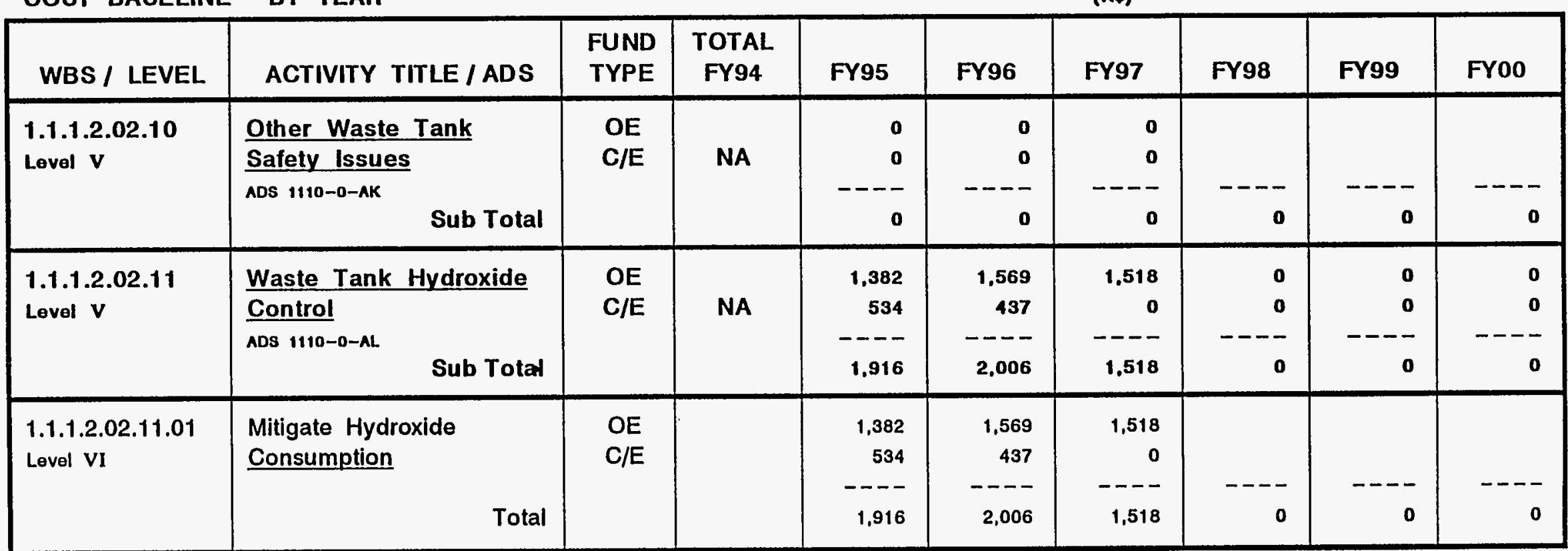


TABLE $7.4-1$

TOTAL PROGRAM ELEMENT COST BASELINE - BY YEAR

\begin{tabular}{|c|c|c|c|c|c|c|c|c|c|c|}
\hline WBS / LEVEL & ACTIVITY TITLE / ADS & $\begin{array}{l}\text { FUND } \\
\text { TYPE }\end{array}$ & $\begin{array}{l}\text { FY01 I } \\
\text { FY05 }\end{array}$ & $\begin{array}{l}\text { FY06 I } \\
\text { FY10 }\end{array}$ & $\begin{array}{l}\text { FY11 I } \\
\text { FY15 }\end{array}$ & $\begin{array}{l}\text { FY16 / } \\
\text { FY20 }\end{array}$ & $\begin{array}{l}\text { FY21 I } \\
\text { FY25 }\end{array}$ & $\begin{array}{l}\text { FY26 I } \\
\text { FY30 }\end{array}$ & $\begin{array}{l}\text { FY31 / } \\
\text { FY35 }\end{array}$ & $\begin{array}{l}\text { FY36 / } \\
\text { FY40 }\end{array}$ \\
\hline $\begin{array}{ll}1.1 .1 .2 .02 \\
\text { Level } \mathrm{rV}\end{array}$ & $\begin{array}{l}\text { Waste Tank Safety } \\
\text { Aos 1110-0 al } \\
\text { SOTAL }\end{array}$ & OE & $\begin{array}{r}0 \\
0 \\
-17.656\end{array}$ & $\begin{array}{r}0 \\
0 \\
-0 \\
\end{array}$ & & 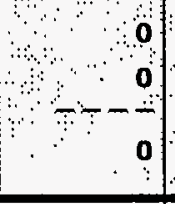 & $\begin{array}{r}0 \\
-10 \\
\end{array}$ & +। & $\left(\begin{array}{rr}0 \\
30 \\
-10 \\
0 & 0 \\
0 & 0\end{array}\right.$ & $\frac{0}{0}$ \\
\hline & $\begin{array}{l}\text { Delta Required to } \\
\text { Balance P3 to TARGET } \\
\text { TOTAL }\end{array}$ & $\begin{array}{l}O E \\
C / E\end{array}$ & & & & & & & -- & \\
\hline $\begin{array}{l}\text { 1.1.1.2.02.01 } \\
\text { Level } \mathrm{V}\end{array}$ & $\begin{array}{l}\frac{\text { Technical Integration }}{\text { and Planninf }} \\
\text { ADS 1110-0-A } \\
\text { Sub Total }\end{array}$ & $\begin{array}{l}O E \\
C / E\end{array}$ & $--\frac{0}{0}$ & $\begin{array}{r}0 \\
0 \\
-- \\
0\end{array}$ & $-\begin{array}{r}0 \\
0 \\
-0\end{array}$ & $\begin{array}{r}0 \\
0 \\
-- \\
0\end{array}$ & $\begin{array}{r}0 \\
0 \\
-- \\
0\end{array}$ & $\begin{array}{r}\mathbf{0} \\
--- \\
0\end{array}$ & $\begin{array}{r}0 \\
0 \\
-0 \\
0\end{array}$ & $--\frac{0}{0}$ \\
\hline $\begin{array}{l}1.1 .1 .2 .02 .01 .01 \\
\text { Level VI }\end{array}$ & $\begin{array}{l}\text { Integration and Planning } \\
\text { Total }\end{array}$ & $\begin{array}{l}O E \\
C / E\end{array}$ & -- & 0 & -- & - & 0 & 0 & 0 & --- \\
\hline $\begin{array}{l}\text { 1.1.1.2.02.01.02 } \\
\text { Level VI }\end{array}$ & $\begin{array}{l}\text { Waste Tank Safety QA } \\
\text { Total }\end{array}$ & $\begin{array}{l}\mathrm{OE} \\
\mathrm{C} / \mathrm{E}\end{array}$ & $\overline{0}$ & -- & $-\overline{0}$ & ---- & & -- & 0 & 0 \\
\hline $\begin{array}{l}\text { 1.1.1.2.02.02 } \\
\text { Level } \mathrm{V}\end{array}$ & $\begin{array}{l}\frac{\text { Hydrogen Mitigation }}{A D S 1110-0-A B} \\
\text { Sub Total }\end{array}$ & $\begin{array}{l}\text { OE } \\
C / E\end{array}$ & -- & $\begin{array}{r}0 \\
0 \\
--- \\
0\end{array}$ & -- & $\begin{array}{r}0 \\
0 \\
-0 \\
0\end{array}$ & $--\frac{0}{0}$ & $-\begin{array}{r}\mathbf{0} \\
--\frac{\mathbf{0}}{\mathbf{0}}\end{array}$ & -- & $\begin{array}{r}\mathbf{0} \\
-0 \\
-0\end{array}$ \\
\hline $\begin{array}{l}\text { 1.1.1.2.02.02.01 } \\
\text { Level VI }\end{array}$ & $\begin{array}{l}\text { Mitigation Program / } \\
\text { Project Management } \\
\\
\text { Total }\end{array}$ & $\begin{array}{l}O E \\
C / E\end{array}$ & 0 & & - & -- & - & -- & -- & --- \\
\hline
\end{tabular}


TABLE $7.4-1$

TOTAL PROGRAM ELEMENT COST BASELINE - BY YEAR

\begin{tabular}{|c|c|c|c|c|c|c|c|c|c|c|}
\hline WBS / LEVEL & ACTIVITY TITLE / ADS & $\begin{array}{l}\text { FUND } \\
\text { TYPE }\end{array}$ & $\begin{array}{l}\text { FY01 / } \\
\text { FY05 }\end{array}$ & $\begin{array}{l}\text { FY06 I } \\
\text { FY10 }\end{array}$ & $\begin{array}{l}\text { FY11 / } \\
\text { FY15 }\end{array}$ & $\begin{array}{l}\text { FY16 / } \\
\text { FY20 }\end{array}$ & $\begin{array}{l}\text { FY21 I } \\
\text { FY25 }\end{array}$ & $\begin{array}{l}\text { FY26 / } \\
\text { FY30 }\end{array}$ & $\begin{array}{l}\text { FY31 / } \\
\text { FY35 }\end{array}$ & $\begin{array}{l}\text { FY36 / } \\
\text { FY40 }\end{array}$ \\
\hline $\begin{array}{l}\text { 1.1.1.2.02.02.02 } \\
\text { Level VI }\end{array}$ & $\begin{array}{r}\text { Tank 101-SY Mitigation } \\
\text { Total }\end{array}$ & $\begin{array}{l}\text { OE } \\
\text { C/E }\end{array}$ & ---- & 0 & $-\frac{-}{0}$ & --- & $\begin{array}{r}--- \\
0\end{array}$ & ---- & $---\frac{-}{0}$ & $\begin{array}{r}0- \\
0\end{array}$ \\
\hline $\begin{array}{l}1.1 .1 .2 .02 .02 .03 \\
\text { Level VI }\end{array}$ & $\begin{array}{r}\text { Tank 103-SY Mitigation } \\
\text { Total }\end{array}$ & $\begin{array}{l}O E \\
C / E\end{array}$ & $\begin{array}{r}--- \\
0\end{array}$ & $\begin{array}{r}-- \\
0\end{array}$ & --- & $---\frac{-}{0}$ & $---\frac{-}{0}$ & $--\frac{-}{0}$ & 0 & $\begin{array}{r}--- \\
0\end{array}$ \\
\hline $\begin{array}{l}1.1 .1 .2 .02 .02 .04 \\
\text { Level VI }\end{array}$ & $\begin{array}{r}\text { Tank 101-AW Mitigation } \\
\text { Total }\end{array}$ & $\begin{array}{l}\text { OE } \\
C / E\end{array}$ & ---- & --- & $\begin{array}{r}---- \\
0\end{array}$ & $---\frac{-}{0}$ & $\begin{array}{r}--- \\
0\end{array}$ & --- & 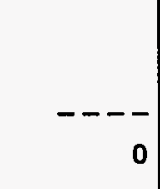 & -- \\
\hline $\begin{array}{l}\text { 1.1.1.2.02.02.05 } \\
\text { Level VI }\end{array}$ & $\begin{array}{l}\text { Tank 104-AN Mitigation } \\
\text { Total }\end{array}$ & $\begin{array}{l}\text { OE } \\
\text { C/E }\end{array}$ & ---- & $\begin{array}{r}--- \\
0\end{array}$ & $\begin{array}{r}--- \\
0\end{array}$ & $---\frac{-}{0}$ & $\begin{array}{r}--- \\
0\end{array}$ & $-\frac{-}{0}$ & $\begin{array}{r}--- \\
0\end{array}$ & --- \\
\hline $\begin{array}{l}\text { 1.1.1.2.02.02.06 } \\
\text { Level VI }\end{array}$ & $\begin{array}{r}\text { Tank 103-AN Mitigation } \\
\text { Total }\end{array}$ & $\begin{array}{l}O E \\
C / E\end{array}$ & -- & -- & ---- & $---\frac{-}{0}$ & $-\frac{-}{0}$ & $-\frac{-}{0}$ & $\begin{array}{r}--- \\
0\end{array}$ & $\begin{array}{r}--- \\
0\end{array}$ \\
\hline $\begin{array}{l}\text { 1.1.1.2.02.02.07 } \\
\text { Level VI }\end{array}$ & $\begin{array}{l}\text { Tank 105-AN Mitigation } \\
\text { Total }\end{array}$ & $\begin{array}{l}O E \\
C / E\end{array}$ & - & - & ---- & $-\cdots-$ & $---\frac{-}{0}$ & $---\frac{-}{0}$ & $---\frac{-}{0}$ & $\begin{array}{r}---- \\
0\end{array}$ \\
\hline
\end{tabular}


TABLE $7.4-1$

TOTAL PROGRAM ELEMENT COST BASELINE - BY YEAR

[ R1 ]

\begin{tabular}{|c|c|c|c|c|c|c|c|c|c|c|}
\hline WBS / LEVEL & ACTIVITY TITLE / ADS & $\begin{array}{l}\text { FUND } \\
\text { TYPE }\end{array}$ & $\begin{array}{l}\text { FY01 I } \\
\text { FY05 }\end{array}$ & $\begin{array}{l}\text { FY06 / } \\
\text { FY10 }\end{array}$ & $\begin{array}{l}\text { FY11 / } \\
\text { FY15 }\end{array}$ & $\begin{array}{l}\text { FY16 I } \\
\text { FY20 }\end{array}$ & $\begin{array}{l}\text { FY21 / } \\
\text { FY25 }\end{array}$ & $\begin{array}{l}\text { FY26 / } \\
\text { FY30 }\end{array}$ & $\begin{array}{l}\text { FY31 / } \\
\text { FY35 }\end{array}$ & $\begin{array}{l}\text { FY36 I } \\
\text { FY40 }\end{array}$ \\
\hline $\begin{array}{l}\text { 1.1.1.2.02.03 } \\
\text { Level } \mathrm{V}\end{array}$ & $\begin{array}{l}\text { Waste Tank Ferrocyanide } \\
\text { Safety Program } \\
\text { ADS } 1110-0-\wedge C \text { Sub Total }\end{array}$ & $\begin{array}{l}O E \\
C / E\end{array}$ & $\begin{array}{r}\mathbf{0} \\
-- \\
-0 \\
0\end{array}$ & $\begin{array}{r}0 \\
0 \\
-0 \\
0\end{array}$ & $\begin{array}{r}\mathbf{0} \\
\mathbf{0} \\
-- \\
0\end{array}$ & $\begin{array}{r}0 \\
0 \\
-0 \\
0\end{array}$ & $\begin{array}{r}\mathbf{0} \\
\mathbf{0} \\
-\mathbf{0}\end{array}$ & $\begin{array}{r}0 \\
0 \\
-0\end{array}$ & $\begin{array}{r}0 \\
0 \\
-0 \\
0\end{array}$ & $\begin{array}{r}\mathbf{0} \\
-0 \\
-0\end{array}$ \\
\hline $\begin{array}{l}\text { 1.1.1.2.02.03.01 } \\
\text { Level VI }\end{array}$ & $\begin{array}{l}\text { Evaluate \& Define } \\
\text { Technical Development } \\
\qquad \text { Total }\end{array}$ & $\begin{array}{l}O E \\
C / E\end{array}$ & ---- & $\begin{array}{r}--- \\
0\end{array}$ & ---- & $---\frac{-}{0}$ & $\begin{array}{r}---- \\
0\end{array}$ & ---- & $\begin{array}{r}--- \\
0\end{array}$ & $---\overline{0}$ \\
\hline $\begin{array}{l}\text { 1.1.1.2.02.03.03 } \\
\text { Level VI }\end{array}$ & $\begin{array}{l}\text { FeCn Safety Issues } \\
\text { Resolved } \\
\qquad \begin{array}{l}\text { Total }\end{array}\end{array}$ & $\begin{array}{l}O E \\
C / E\end{array}$ & $-\cdots$ & & ---- & ---- & - & -- & 0 & $\begin{array}{r}--- \\
0\end{array}$ \\
\hline $\begin{array}{l}\text { 1.1.1.2.02.03.04 } \\
\text { Level VI }\end{array}$ & $\begin{array}{l}\text { Evaluate \& Define } \\
18 \text { FeCn Tanks } \\
\end{array}$ & $\begin{array}{l}O E \\
C / E\end{array}$ & - & --- & - & - & - & - & - & -- \\
\hline $\begin{array}{l}\text { 1.1.1.2.02.04 } \\
\text { Level } \mathrm{v}\end{array}$ & $\begin{array}{c}\text { Waste Tank Flammable } \\
\text { Gas Safety Program } \\
\text { ADS } 1110-0-A D \\
\text { Sub Total }\end{array}$ & $\begin{array}{l}O E \\
C / E\end{array}$ & -- & $\begin{array}{r}\mathbf{0} \\
\mathbf{0} \\
-- \\
-\mathbf{0}\end{array}$ & $\begin{array}{r}0 \\
0 \\
-- \\
0\end{array}$ & $\begin{array}{r}\mathbf{0} \\
\mathbf{0} \\
-- \\
-0\end{array}$ & $\begin{array}{r}0 \\
0 \\
-- \\
-0\end{array}$ & $\begin{array}{r}\mathbf{0} \\
\mathbf{0} \\
-- \\
-0\end{array}$ & $\begin{array}{r}\mathbf{0} \\
\mathbf{0} \\
-- \\
-\mathbf{0}\end{array}$ & $\begin{array}{r}\mathbf{0} \\
\mathbf{0} \\
--- \\
-0\end{array}$ \\
\hline $\begin{array}{l}1.1 .1 .2 .02 .04 .01 \\
\text { Level VI }\end{array}$ & $\begin{array}{l}\text { Evaluate \& Define } \\
\text { Technical Development } \\
\qquad \text { Total }\end{array}$ & $\begin{array}{l}\text { OE } \\
\mathrm{C} / \mathrm{E}\end{array}$ & - & 0 & -- & 0 & -- & - & ---- & ---- \\
\hline $\begin{array}{l}1.1 .1 .2 .02 .04 .02 \\
\text { Level VI }\end{array}$ & $\begin{array}{l}\text { Evaluate \& Define } \\
\text { Flammable Gas Tanks } \\
\\
\text { Total }\end{array}$ & $\begin{array}{l}\mathrm{OE} \\
\mathrm{C} / \mathrm{E}\end{array}$ & $\begin{array}{r}-- \\
0\end{array}$ & 0 & - & $\begin{array}{r}---- \\
0\end{array}$ & $-\cdots--$ & $\begin{array}{r}---- \\
0\end{array}$ & -- & 0 \\
\hline
\end{tabular}


TABLE $7.4-1$

TOTAL PROGRAM ELEMENT COST BASELINE - BY YEAR

[ R1 ]

\begin{tabular}{|c|c|c|c|c|c|c|c|c|c|c|}
\hline WBS / LEVEL & ACTIVITY TITLE / ADS & $\begin{array}{l}\text { FUND } \\
\text { TYPE }\end{array}$ & $\begin{array}{l}\text { FY01 / } \\
\text { FY05 }\end{array}$ & $\begin{array}{l}\text { FY06 / } \\
\text { FY10 }\end{array}$ & $\begin{array}{l}\text { FY11 I } \\
\text { FY15 }\end{array}$ & $\begin{array}{l}\text { FY16 / } \\
\text { FY20 }\end{array}$ & $\begin{array}{l}\text { FY21 i } \\
\text { FY25 }\end{array}$ & $\begin{array}{l}\text { FY26 / } \\
\text { FY30 }\end{array}$ & $\begin{array}{l}\text { FY31 / } \\
\text { FY35 }\end{array}$ & $\begin{array}{l}\text { FY36 / } \\
\text { FY40 }\end{array}$ \\
\hline $\begin{array}{l}1.1 .1 .2 .02 .04 .03 \\
\text { Level VI }\end{array}$ & $\begin{array}{l}\text { Close USQs for } \\
\text { Flammable Gas Tanks } \\
\qquad \text { Total }\end{array}$ & $\begin{array}{l}O E \\
C / E\end{array}$ & $\begin{array}{r}---- \\
0\end{array}$ & & $\begin{array}{r}---- \\
0\end{array}$ & $\begin{array}{r}---- \\
0\end{array}$ & $\begin{array}{r}---- \\
0\end{array}$ & $\begin{array}{r}---- \\
0\end{array}$ & ---- & $\begin{array}{r}--- \\
0\end{array}$ \\
\hline $\begin{array}{l}1.1 .1 .2 .02 .04 .04 \\
\text { Level VI }\end{array}$ & $\begin{array}{l}\text { Store \& Monitor } \\
\text { Flammable Gas Tank } \\
\qquad \text { Total }\end{array}$ & $\begin{array}{l}O E \\
C / E\end{array}$ & $\begin{array}{r}---- \\
0\end{array}$ & $\begin{array}{r}---- \\
0\end{array}$ & $\begin{array}{r}---- \\
0\end{array}$ & $\begin{array}{r}---- \\
0\end{array}$ & ---- & 0 & ---- & --- \\
\hline $\begin{array}{l}\text { 1.1.1.2.02.04.05 } \\
\text { Level VI }\end{array}$ & $\begin{array}{l}\text { Integrated Field Support } \\
\text { for Flammable Gas Tanks } \\
\text { Total }\end{array}$ & $\begin{array}{l}\text { OE } \\
C / E\end{array}$ & - & - & 0 & $\overline{0}$ & -- & 0 & ---- & $\begin{array}{r}--- \\
0\end{array}$ \\
\hline $\begin{array}{l}\text { 1.1.1.2.02.05 } \\
\text { Level } \mathrm{V}\end{array}$ & $\begin{array}{l}\text { Waste Tank Organic } \\
\underline{\text { Safety Program }} \\
\begin{array}{l}\text { ADS } 1110-0-A E \\
\text { Sub Total }\end{array}\end{array}$ & $\begin{array}{l}\text { OE } \\
C / E\end{array}$ & $\begin{array}{r}0 \\
0 \\
-- \\
-0\end{array}$ & $\begin{array}{r}0 \\
0 \\
-- \\
0\end{array}$ & $\begin{array}{r}0 \\
0 \\
-- \\
0\end{array}$ & $\begin{array}{r}\mathbf{0} \\
\mathbf{0} \\
-- \\
-0\end{array}$ & $\begin{array}{r}\mathbf{0} \\
\mathbf{0} \\
-- \\
-0\end{array}$ & $\begin{array}{r}\mathbf{0} \\
\mathbf{0} \\
--- \\
-0\end{array}$ & $\begin{array}{r}0 \\
0 \\
-- \\
-0\end{array}$ & $\begin{array}{r}0 \\
0 \\
--- \\
0\end{array}$ \\
\hline $\begin{array}{l}\text { 1.1.1.2.02.05.01 } \\
\text { Level VI }\end{array}$ & $\begin{array}{l}\text { Technology for Evaluating } \\
\text { Organic Tanks } \\
\text { Total }\end{array}$ & $\begin{array}{l}O E \\
C / E\end{array}$ & $\begin{array}{r}--- \\
0\end{array}$ & -- & $\begin{array}{r}--- \\
0\end{array}$ & $\begin{array}{r}--- \\
0\end{array}$ & --- & $\begin{array}{r}---- \\
0\end{array}$ & ---- & $\begin{array}{r}--\overline{0} \\
0\end{array}$ \\
\hline $\begin{array}{l}\text { 1.1.1.2.02.05.02 } \\
\text { Level VI }\end{array}$ & $\begin{array}{l}\text { Evaluate \& Define } \\
\text { Organic Tanks } \\
\qquad \begin{array}{l}\text { Total }\end{array}\end{array}$ & $\begin{array}{l}O E \\
C / E\end{array}$ & $\overline{0}$ & - & $\begin{array}{r}---- \\
0\end{array}$ & $\cdots$ & $\begin{array}{r}--- \\
0\end{array}$ & $\begin{array}{r}---- \\
0\end{array}$ & $\begin{array}{r}--- \\
0\end{array}$ & $\begin{array}{r}--- \\
0\end{array}$ \\
\hline
\end{tabular}


TABLE 7.4-1

TOTAL PROGRAM ELEMENT

COST BASELINE - BY YEAR

\begin{tabular}{|c|c|c|c|c|c|c|c|c|c|c|}
\hline WBS / LEVEL & ACTIVITY TITLE / ADS & $\begin{array}{l}\text { FUND } \\
\text { TYPE }\end{array}$ & $\begin{array}{l}\text { FY01 I } \\
\text { FY05 }\end{array}$ & $\begin{array}{l}\text { FY06 I } \\
\text { FY10 }\end{array}$ & $\begin{array}{l}\text { FY11 / } \\
\text { FY15 }\end{array}$ & $\begin{array}{l}\text { FY16 I } \\
\text { FY20 }\end{array}$ & $\begin{array}{l}\text { FY21 / } \\
\text { FY25 }\end{array}$ & $\begin{array}{l}\text { FY26 / } \\
\text { FY30 }\end{array}$ & $\begin{array}{l}\text { FY31 / } \\
\text { FY35 }\end{array}$ & $\begin{array}{l}\text { FY36 I } \\
\text { FY40 }\end{array}$ \\
\hline $\begin{array}{l}\text { 1.1.1.2.02.05.03 } \\
\text { Level VI }\end{array}$ & $\begin{array}{l}\text { Pump \& Dispose of Tank } \\
\text { C-103 Organic Layor } \\
\text { Total }\end{array}$ & $\begin{array}{l}O E \\
C / E\end{array}$ & $\begin{array}{r}---- \\
0\end{array}$ & $\begin{array}{r}--- \\
0\end{array}$ & $\begin{array}{r}---- \\
0\end{array}$ & $\begin{array}{r}---- \\
0\end{array}$ & $\begin{array}{r}---- \\
0\end{array}$ & $\begin{array}{r}---- \\
0\end{array}$ & ---- & $\begin{array}{r}--- \\
0\end{array}$ \\
\hline $\begin{array}{l}1.1 .1 .2 .02 .05 .04 \\
\text { Level VI }\end{array}$ & $\begin{array}{l}\text { In-Tank Resolution of } \\
\text { Organic Tanks Without } \\
\text { Treatment } \\
\end{array}$ & $\begin{array}{l}\mathrm{OE} \\
\mathrm{C} / \mathrm{E}\end{array}$ & $\begin{array}{r}--- \\
0\end{array}$ & $\begin{array}{r}--- \\
0\end{array}$ & $\begin{array}{r}--- \\
0\end{array}$ & $\begin{array}{r}--- \\
0\end{array}$ & $\begin{array}{r}--- \\
0\end{array}$ & $\begin{array}{r}--- \\
0\end{array}$ & $\begin{array}{r}--1 \\
0\end{array}$ & $\begin{array}{r}---- \\
0\end{array}$ \\
\hline $\begin{array}{l}\text { 1.1.1.2.02.05.05 } \\
\text { Level VI }\end{array}$ & $\begin{array}{l}\text { Resolve USQ for } \\
\text { Organic Tanks }\end{array}$ & $\begin{array}{l}O E \\
C / E\end{array}$ & -- & $\begin{array}{r}---- \\
0\end{array}$ & -- & $\begin{array}{r}--- \\
0\end{array}$ & --- & --- & $\begin{array}{r}---- \\
0\end{array}$ & --- \\
\hline $\begin{array}{l}\text { 1.1.1.2.02.05.06 } \\
\text { Level VI }\end{array}$ & $\begin{array}{l}\text { Resolve Safety Issue } \\
\text { Organic Tanks } \\
\qquad \text { Total }\end{array}$ & $\begin{array}{l}O E \\
C / E\end{array}$ & $-\cdots$ & $\begin{array}{r}--- \\
0\end{array}$ & - & -- & $\begin{array}{r}--- \\
0\end{array}$ & $\begin{array}{r}--- \\
0\end{array}$ & $\begin{array}{r}--- \\
0\end{array}$ & $\begin{array}{r}--- \\
0\end{array}$ \\
\hline $\begin{array}{l}\text { 1.1.1.2.02.05.07 } \\
\text { Level VI }\end{array}$ & $\begin{array}{l}\text { Pump and Dispose C-103 } \\
\text { Organic Layer / Resolve } \\
\text { Issue } \\
\text { Total }\end{array}$ & $\begin{array}{l}O E \\
C / E\end{array}$ & -- & $\begin{array}{r}--- \\
0\end{array}$ & - & $\ldots$ & $---\frac{-}{0}$ & $---\frac{-}{0}$ & ---- & $-\cdots$ \\
\hline
\end{tabular}


TABLE 7.4-1

TOTAL PROGRAM ELEMENT COST BASELINE - BY YEAR

[ R1 ]

\begin{tabular}{|c|c|c|c|c|c|c|c|c|c|c|}
\hline WBS / LEVEL & ACTIVITY TITLE / ADS & $\begin{array}{l}\text { FUND } \\
\text { TYPE }\end{array}$ & $\begin{array}{l}\text { FY01 / } \\
\text { FY05 }\end{array}$ & $\begin{array}{l}\text { FY06 I } \\
\text { FY10 }\end{array}$ & $\begin{array}{l}\text { FY11 / } \\
\text { FY15 }\end{array}$ & $\begin{array}{l}\text { FY16 I } \\
\text { FY20 }\end{array}$ & $\begin{array}{l}\text { FY21 / } \\
\text { FY25 }\end{array}$ & $\begin{array}{l}\text { FY26 I } \\
\text { FY30 }\end{array}$ & $\begin{array}{l}\text { FY31 I } \\
\text { FY35 }\end{array}$ & $\begin{array}{l}\text { FY36 / } \\
\text { FY40 }\end{array}$ \\
\hline $\begin{array}{l}1.1 .1 .2 .02 .06 \\
\text { Level } v\end{array}$ & $\begin{array}{l}\text { Waste Tank High-Heat } \\
\text { Safety Program } \\
\text { ADs 1110-0-AF } \quad \text { Sub Total }\end{array}$ & $\begin{array}{l}O E \\
C / E\end{array}$ & $\begin{array}{r}0 \\
0 \\
-- \\
0\end{array}$ & $\begin{array}{r}0 \\
0 \\
-- \\
-0\end{array}$ & $\begin{array}{r}0 \\
0 \\
--- \\
0\end{array}$ & $\begin{array}{r}0 \\
0 \\
--- \\
0\end{array}$ & $\begin{array}{r}\mathbf{0} \\
\mathbf{0} \\
--- \\
-0\end{array}$ & $\begin{array}{r}0 \\
0 \\
-- \\
0\end{array}$ & $\begin{array}{r}\mathbf{0} \\
\mathbf{0} \\
-- \\
0\end{array}$ & $\begin{array}{r}0 \\
0 \\
---- \\
0\end{array}$ \\
\hline $\begin{array}{l}\text { 1.1.1.2.02.06.01 } \\
\text { Level VI }\end{array}$ & $\begin{array}{l}\text { Evaluate \& Define } \\
\text { High-Heat Tanks } \\
\\
\\
\text { Total }\end{array}$ & $\begin{array}{l}O E \\
C / E\end{array}$ & $\begin{array}{r}---- \\
0\end{array}$ & -- & $\begin{array}{r}--- \\
0\end{array}$ & --- & -- & --- & ---- & --- \\
\hline $\begin{array}{l}\text { 1.1.1.2.02.07 } \\
\text { Level } \mathrm{v}\end{array}$ & $\begin{array}{l}\frac{\text { Misc. Underground }}{\text { Storage Tanks }} \\
\frac{\text { ADs } 1110-0-\wedge G \quad \text { Sub Total }}{}\end{array}$ & $\begin{array}{l}O E \\
C / E\end{array}$ & $\begin{array}{r}0 \\
0 \\
-- \\
0\end{array}$ & $\begin{array}{r}0 \\
0 \\
-- \\
0\end{array}$ & $\begin{array}{r}0 \\
0 \\
-- \\
0\end{array}$ & $\begin{array}{r}\mathbf{0} \\
\mathbf{0} \\
--- \\
\mathbf{0}\end{array}$ & $\begin{array}{r}0 \\
0 \\
-- \\
-0\end{array}$ & $\begin{array}{r}0 \\
0 \\
--- \\
0\end{array}$ & $\begin{array}{r}0 \\
0 \\
-- \\
-0\end{array}$ & $\begin{array}{r}0 \\
0 \\
--- \\
0\end{array}$ \\
\hline $\begin{array}{l}\text { 1.1.1.2.02.07.01 } \\
\text { Level VI }\end{array}$ & $\begin{array}{l}\text { Evaluate Misc. } \\
\text { Underground Tanks } \\
\qquad \text { Total }\end{array}$ & $\begin{array}{l}O E \\
C / E\end{array}$ & $\begin{array}{r}--- \\
0\end{array}$ & $\begin{array}{r}--- \\
0\end{array}$ & - & -- & -- & - & -- & --- \\
\hline $\begin{array}{l}1.1 .1 .2 .02 .08 \\
\text { Level } \mathrm{V}\end{array}$ & $\begin{array}{l}\frac{\text { Waste Tank Vapor }}{\text { Resolution }} \\
\text { ADS } 1110-0-\wedge H \\
\quad \text { Sub Total }\end{array}$ & $\begin{array}{l}O E \\
C / E\end{array}$ & $\begin{array}{r}0 \\
0 \\
-- \\
0\end{array}$ & $\begin{array}{r}0 \\
0 \\
-- \\
0\end{array}$ & $\begin{array}{r}\mathbf{0} \\
\mathbf{0} \\
-- \\
\mathbf{0}\end{array}$ & $\begin{array}{r}0 \\
0 \\
-- \\
0\end{array}$ & $\begin{array}{r}\mathbf{0} \\
\mathbf{0} \\
-- \\
-0\end{array}$ & $\begin{array}{r}0 \\
0 \\
--- \\
0\end{array}$ & $\begin{array}{r}0 \\
0 \\
--- \\
0\end{array}$ & $\begin{array}{r}0 \\
0 \\
--- \\
0\end{array}$ \\
\hline $\begin{array}{l}\text { 1.1.1.2.02.08.01 } \\
\text { Level VI }\end{array}$ & $\begin{array}{l}\text { Evaluate \& Define for } \\
\text { Tank Vapor / Safety } \\
\\
\text { Total }\end{array}$ & $\begin{array}{l}O E \\
C / E\end{array}$ & $\begin{array}{r}---- \\
0\end{array}$ & $\begin{array}{r}---- \\
0\end{array}$ & -- & - & $\overline{0}$ & - & - & --- \\
\hline
\end{tabular}


TABLE $\quad 7.4-1$

TOTAL PROGRAM ELEMENT COST BASELINE - BY YEAR

\begin{tabular}{|c|c|c|c|c|c|c|c|c|c|c|}
\hline WBS / LEVEL & ACTIVITY TITLE / ADS & $\begin{array}{l}\text { FUND } \\
\text { TYPE }\end{array}$ & $\begin{array}{l}\text { FY01 I } \\
\text { FY05 }\end{array}$ & $\begin{array}{l}\text { FY06 / } \\
\text { FY10 }\end{array}$ & $\begin{array}{l}\text { FY11 I } \\
\text { FY15 }\end{array}$ & $\begin{array}{l}\text { FY16 / } \\
\text { FY20 }\end{array}$ & $\begin{array}{l}\text { FY21 I } \\
\text { FY25 }\end{array}$ & $\begin{array}{l}\text { FY26 / } \\
\text { FY30 }\end{array}$ & $\begin{array}{l}\text { FY31 I } \\
\text { FY35 }\end{array}$ & $\begin{array}{l}\text { FY36 I } \\
\text { FY40 }\end{array}$ \\
\hline $\begin{array}{l}\text { 1.1.1.2.02.08.02 } \\
\text { Level VI }\end{array}$ & $\begin{array}{l}\text { Resolve Internal Tank } \\
\text { Vapor Issues } \\
\text { Total }\end{array}$ & $\begin{array}{l}\text { OE } \\
\text { C/E }\end{array}$ & $-\cdots$ & $\begin{array}{r}---- \\
0\end{array}$ & --- & $\begin{array}{r}---- \\
0\end{array}$ & $\begin{array}{r}---- \\
0\end{array}$ & $\begin{array}{r}---- \\
0\end{array}$ & $\begin{array}{r}--- \\
0\end{array}$ & $\begin{array}{r}---- \\
0\end{array}$ \\
\hline $\begin{array}{l}\text { 1.1.1.2.02.08.03 } \\
\text { Level VI }\end{array}$ & $\begin{array}{r}\text { Mitigate Tech. Dvipmnt. } \\
\text { for Tank Vapor/Safety } \\
\text { Total }\end{array}$ & $\begin{array}{l}\text { OE } \\
\text { C/E }\end{array}$ & $\cdots$ & $\begin{array}{r}--- \\
0\end{array}$ & $\begin{array}{r}---- \\
0\end{array}$ & o & - & --- & --- & $-\frac{-}{0}$ \\
\hline $\begin{array}{l}\text { 1.1.1.2.02.09 } \\
\text { Level } \mathrm{V}\end{array}$ & 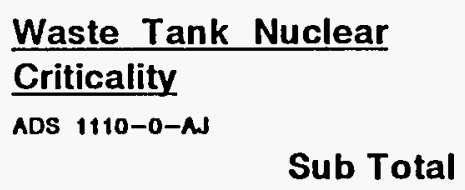 & $\begin{array}{l}O E \\
C / E\end{array}$ & $\begin{array}{r}0 \\
0 \\
-- \\
0\end{array}$ & $\begin{array}{r}0 \\
0 \\
-- \\
0\end{array}$ & $\begin{array}{r}\mathbf{0} \\
\mathbf{0} \\
-- \\
-\mathbf{0}\end{array}$ & $\begin{array}{r}\mathbf{0} \\
\mathbf{0} \\
--- \\
\mathbf{0}\end{array}$ & $\begin{array}{r}0 \\
0 \\
--- \\
0\end{array}$ & $\begin{array}{r}0 \\
0 \\
-- \\
0\end{array}$ & $\begin{array}{r}0 \\
0 \\
-- \\
0\end{array}$ & $\begin{array}{r}0 \\
0 \\
--- \\
0\end{array}$ \\
\hline $\begin{array}{l}\text { 1.1.1.2.02.09.01 } \\
\text { Level VI }\end{array}$ & $\begin{array}{l}\text { Store \& Monitor } \\
\text { Waste for Criticality } \\
\\
\text { Total }\end{array}$ & $\begin{array}{l}O E \\
C / E\end{array}$ & - & - & 0 & $\begin{array}{r}--- \\
.\end{array}$ & -- & 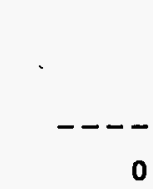 & --- & $\begin{array}{r}---- \\
0\end{array}$ \\
\hline
\end{tabular}

\section{[ R1]}


TABLE $\quad 7.4-1$

\begin{tabular}{|c|c|c|c|c|c|c|c|c|c|c|}
\hline WBS / LEVEL & ACTIVITY TITLE / ADS & $\begin{array}{l}\text { FUND } \\
\text { TYPE }\end{array}$ & $\begin{array}{l}\text { FY01 / } \\
\text { FY05 }\end{array}$ & $\begin{array}{l}\text { FY06 / } \\
\text { FY10 }\end{array}$ & $\begin{array}{l}\text { FY11 I } \\
\text { FY15 }\end{array}$ & $\begin{array}{l}\text { FY16 I } \\
\text { FY20 }\end{array}$ & $\begin{array}{l}\text { FY21 I } \\
\text { FY25 }\end{array}$ & $\begin{array}{l}\text { FY26 / } \\
\text { FY30 }\end{array}$ & $\begin{array}{l}\text { FY31 I } \\
\text { FY35 }\end{array}$ & $\begin{array}{l}\text { FY36 I } \\
\text { FY40 }\end{array}$ \\
\hline $\begin{array}{l}\text { 1.1.1.2.02.10 } \\
\text { Level } \mathrm{V}\end{array}$ & $\begin{array}{l}\text { Other Waste Tank } \\
\text { Safety Issues } \\
\text { ADS 1110-0-AK } \quad \text { Sub Total }\end{array}$ & $\begin{array}{l}O E \\
C / E\end{array}$ & -- & $\begin{array}{r}---\overline{0} \\
0\end{array}$ & $\begin{array}{r}---- \\
0\end{array}$ & $---\frac{-}{0}$ & -- & --- & 0 & $\begin{array}{r}--- \\
0\end{array}$ \\
\hline $\begin{array}{l}\text { 1.1.1.2.02.11 } \\
\text { Level } v\end{array}$ & 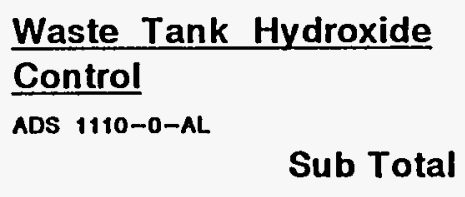 & $\begin{array}{l}O E \\
C / E\end{array}$ & $\begin{array}{r}0 \\
0 \\
--- \\
0\end{array}$ & $\begin{array}{r}0 \\
0 \\
--- \\
0\end{array}$ & $\begin{array}{r}0 \\
0 \\
--- \\
0\end{array}$ & $\begin{array}{r}0 \\
0 \\
-- \\
0\end{array}$ & $\begin{array}{r}0 \\
0 \\
--- \\
0\end{array}$ & $\begin{array}{r}0 \\
0 \\
--- \\
0\end{array}$ & $\begin{array}{r}0 \\
0 \\
---- \\
0\end{array}$ & $\begin{array}{r}0 \\
0 \\
-- \\
0\end{array}$ \\
\hline $\begin{array}{l}\text { 1.1.1.2.02.11.01 } \\
\text { Level VI }\end{array}$ & $\begin{array}{l}\text { Mitigate Hydroxide } \\
\text { Consumption }\end{array}$ & $\begin{array}{l}O E \\
C / E\end{array}$ & -- & -- & $\begin{array}{r}0- \\
0\end{array}$ & --- & $\begin{array}{r}--- \\
0\end{array}$ & $\begin{array}{r}---- \\
0\end{array}$ & --- & $-\cdots$ \\
\hline
\end{tabular}




\section{NOTES \\ TOTAL PROGRAM ELEMENT COST BASELINE - BY YEAR}

The cost exhibit tables in each of the Program Element Baselines (Sections 7.2 through 7.10) identify the program element target funding by year by fund type. The tables also provide details of the baseline allocation of funding down to activity levels (level V) and cost account levels (level VI) of the WBS for Fiscal Year 1995 through 1997. The details exhibited on these tables are taken from the baseline WBS Dictionary Sheets. Deltas exist between the details and the annual target totals. For Fiscal Years 1996 and 1997 the deltas will be addressed in the future, following the completion of the resolution of expected differences between current Fiscal Year 1995 baseline funding and expected actual Fiscal Year 1995 funding. Deltas for Fiscal Year 1995 are noted below by program element:

PROGRAM MANAGEMENT AND ADMINISTRATION:

No deltas.

\section{TANK FARM OPERATIONS AND MAINTENANCE:}

No overall delta. This baseline requires revised fund type mix.

\section{WASTE TANK SAFETY:}

Deita is due to need to revise an overhead pricing rate in the P3 system.

\section{TANK FARM UPGRADES:}

Delta is due to differences between Budget Authority (BA) in the target and Budget Obligation (B0) in the P3 system.

\section{CHARACTERIZATION:}

No significant deltas.

\section{WASTE RETRUEVAL:}

Delta is primarily due to need to resolve issues associated with expense funding support to capital projects and differences between Budget Authority (BA) in the target and Budget Obligation (BO) in the $\mathrm{P} 3$ system.

\section{WASTE PRETREATMENT:}

Delta is due to the sum of the detail cost estimates being lower than the target total as a result of reestimation of resource requirements to complete baseline scope.

\section{LOW LEVEL WASTE:}

Delta is primarily due to carryover expense funded workscope on melter testing addressed in June 9, 1994 RL letter. 


\section{WHC-SP-1101}

\section{HIGH LEVEL WASTE:}

Delta is due to carryover of capital funding, PNL expense funding carryover, and ADS corrections submitted but not reflected in target totals. 
WHC-SP-1101

Table 7.4-2. FY 1995 Cost by Month by Fund Type by Activity and Cost Account

Future MYWP updates will include Table 7.4-2, which establishes the month-by-month cost baseline for FY 1995 for this program element. The FY 1995 cost baseline is exhibited at the cost account level with fund type totals provided. Cost data are then rolled up to the activity and program element levels. Also exhibited is the total cost baseline for the program element for FY 1995. At present, the program element cost baseline for FY 1995 for this program element is the total provided on this table less a productivity challenge for FY 1995. 


\section{WHC-SP-1101}

Table 7.4-3. Total Full-Time Equivalents by Year by Activity by Major Participant (Direct and Indirect) (Including all Subcontractors)

Future MYWP updates will include Table 7.4-3, which establishes total staffing projections for this program element. Included in the table are FY 1994 staffing data as well as projections by year for FY 1995 through FY 2000. The projections in this table include all direct and indirect Full-Time Equivalents (FTEs), including all subcontractor personnel. Where data are available, projections are exhibited at the activity level with major program participant totals provided. 


\section{WHC-SP-1101}

Table 7.4-4. FY 1995 and FY 1996 Direct Westinghouse Hanford Company Full-Time Equivalents by 88 Labor Categories

Future MYWP updates will include Table 7.4-4, which establishes direct WHC staffing projections for this program element. Projections do not include subcontractor support and are provided by 88 labor categories for FY 1995 through FY 1997. 


\subsection{TANK FARM UPGRADES BASELINE}

The Tank Farm Upgrades Program Element baseline consists of the technical bases, schedule, resource requirements, and waste type data for FY 1995 through completion of the mission. The major emphasis is on deliverables, schedule, and resource requirements for the period FY 1995 through FY 1997.

\subsubsection{Technical Basis}

This section provides the mission strategy; work breakdown structure (WBS) and organization responsibility; work to be performed in FY 1995, 1996, and 1997; technical approach; and drivers and deliverables.

\subsubsection{Mission Strategy}

\subsection{Objectives}

The objective of the Tank Farm Upgrades Program Element is to provide specific enhancements to the safe operation of the Tank Farms, pursuant to the store waste (4.2.1.1) and the transfer waste (4.2.1.4) functions, where cost effective. In performing those enhancements, several other functions will be supported: disposition gaseous effluent (4.2.3.2), disposition liquid effluent (4.2.3.3), and disposition solid waste (4.2.3.4). The referenced function numbers are derived from the Tank Waste Remediation System.(TWRS) Functional Hierarchy shown in Figure 2-1 of DOE/RL-92-60, Revision 1.

The selected upgrades are necessary, but may not be sufficient to completely and safely store or transfer waste. The reason only certain systems, equipment, and infrastructure were chosen for upgrades was built primarily around the timing of upgrade completion and remaining service life.

The need for other upgrades not currently in the identified program element scope may result from completion of the Tank Farms Safety Equipment List. Once that list is established and the corresponding safety function of each system is assigned, the performance of each system must be evaluated against its assigned safety functions. Equipment identified as deficient will then be evaluated for upgrade. See Assumptions, Section 7.5.1.1.6, for a more detailed description of safety class and its impact on the Tank Farm Upgrades Program Element.

Programmatic funding constraints also drove the timing of upgrades (refer to Section 7.5.1.1.7, Constraints).

For example, instrumentation systems for single-shell tanks (SSTs) were considered to be included in the scope of Project W-314. However, because W-314 systems will come on line in 2001, and under the current TWRS technical strategy these same SSTs will have all their waste removed by 2018 , it was not cost effective to invest significant capital in improvements to tank systems with service lives of less than 16 years. This assumes that the current level of risk due to the deteriorating condition of the systems will be acceptable during that time. 
WHC-SP-1101

\subsection{Strategy}

Tank Farm upgrades include enhancements to electrical, mechanical, instrumentation, waste storage, and waste transfer systems.

The Tank Farm Upgrades program works closely with Tank Farm Operations and Maintenance so that major policies, work philosophy, training, conduct of operations, facility support activities, and capital projects are coordinated with the Tank Farm Operations Management.

Tank Farm upgrades enhance the safety of facility operations in the interim period until waste is retrieved to meet future mission objectives. The upgrades include a broad spectrum of activities from small, expense-funded component replacement activities; to a very large major systems acquisition (MSA) replacing electrical, instrumentation, and ventilation systems. The MSA also augments other waste transfer projects.

To improve the Tank Farm infrastructure and to contribute to a safe waste storage condition until disposal, the following line item projects are planned: Project W-030, AY/AZ Primary Ventilation System Replacement; Project W-058, Cross-Site Transfer System; Project W-028, Aging Waste Transfer System; Project W-188, Radiological Support Facility; Project W-236A, Multi-Function Waste Tank Facility; and Project W-314, Tank Farm Restoration and Safe Operations MSA.

Expense and General Plant Project (GPP) funded activities include the following categories of work: application of systems engineering to the program, drawing and configuration management improvements, selected procedure improvements, double-shell tank (DST) life extension and qualification, tank instrumentation improvements, electrical safety problem resolution, tank farm lighting, long-length waste disposal infrastructure, and facility operational improvements.

The Upgrades program addresses hardware improvements to Tank Farms in two ways. First, using expense and Capital Equipment Not Related to Construction (CENRTC) funding, components in existing systems are replaced as required. In some cases, component replacement is all that is required to meet the TWRS interim storage mission. Interim storage here is defined as that storage of waste before the waste is retrieved to be processed for disposal (long-term storage).

Second, the Upgrades program will replace entire systems through projects like W-314. Because of the safety function of the equipment, selected components may need to be replaced, on a limited interim basis, to adequately preserve the safety margin until Project W-314 comes on line in 2001 . In those cases only, the Upgrades program will be forced to replace selected components making up part of complete systems that will eventually also be completely replaced. However, the need to do this is rare, and the option of continuing until system replacement with existing components will be thoroughly evaluated prior to component replacement.

Once Project W-314 is complete, the Tank Farms will have adequate DST instrumentation, electrical, ventilation, and transfer systems to meet the interim or safe storage mission as it is currently defined in the Hanford Site TWRS Technical Strategy. However, if this strategy is revised in the future to significantly extend the interim storage time, the design life of W-314 systems must be reevaluated. To reduce costs and free as many funds as possible for retrieval and disposal activities, the design life of W-314 systems will be closely matched to the Technical Strategy, Revision 0. 
Also, to support the safe interim storage mission, the Tank Farm Upgrades Program Element has invested in existing drawing and procedure improvements to aid in the safe operation of the Tank Farms. These improvements are made under the Configuration Management activity.

To support the TWRS Technical Strategy of storing waste in existing DSTs through 2028, the Life Management activity is analyzing and establishing a strategy for out-year management of the DSTs. The existing DSTs are presently at, or approaching, the end of their original design life. The Life Management activity will determine and document whether these tanks, based on their current condition and operating history, can be safely used to support the Technical Strategy.

In FY 1995 and FY 1996, a self assessment of the project and non-project upgrades scope and logic will be conducted starting with four key elements: ventilation, instrumentation and control, electrical, and transfer lines. Assessments will be made of existing plant system condition, aging effects, and actions necessary to provide cost-effective upgrades to support the store tank waste (4.2.1.1) functions and requirements. These first four elements will be carried out concurrently with the design and construction sequence priority study for Project W-314 during FY 1995. This will ensure integration of project and non-project activities through a systems engineering process. Strategies will be developed and implemented for actions resulting from the assessments. Follow-on assessments at the program element level will then be conducted annually for the duration of the program. This will provide the management process necessary to track the evaluation of systems engineering and identify follow-on corrections to the Upgrades program work.

Specifically, each of the following activities are authorized to perform the following work in FY 1995, FY 1996, and FY 1997:

\section{Technical Integration and Planning (1.1.1.2.03.01)}

This activity is in support of the TWRS Mission and is comprised of three segments of work: program management, baseline management, and systems engineering.

The program management work supports the Upgrades program office and is required for the overall management of this TWRS program.

The baseline management effort supports the Upgrades program office and manages the program baselines (cost, schedule, technical) including cost analysis and reporting, schedule and milestone development and reporting, and technical progress and status reporting in support of the program element mission and the overall TWRS Mission. Also, this activity ensures that the program baseline work scope will be done within the budget as authorized by the U.S. Department of Energy (DOE).

The systems engineering effort guides the programmatic efforts in the implementation of systems engineering within the Upgrades program and projects and in the overall support of the TWRS Mission.

\section{Configuration Management (1.1.1.2.03.02)}

The Configuration Management activity is comprised of configuration management and procedure upgrades. 
The Upgrades program strategy for configuration management is to upgrade TWRS Configuration Management to meet the Guide for Operational Configuration Management Program, DOE-STD-1073-93. Only deficiencies in the existing program will be addressed. Existing compliant systems and processes will continue to be used, and will be integrated into the upgrades configuration management program. In addition, the essential and support drawing verification effort and the component labeling effort will continue, as they are essential to an effective configuration management program. The first step in implementing the DOE Standard was to prepare a program plan, finished in FY 1994, to define the appropriate level of implementation for TWRS. The next major step is to develop an implementation engineering task plan that defines the remaining activities and timing for completing the configuration management upgrades.

The strategy for procedure upgrades is to prepare procedures for equipment and systems upgrades by the program sufficient for turnover to Operations. The planning for procedures is being performed in conjunction with the planning for systems and equipment installation to ensure proper integration.

\section{Life Management (1.1.1.2.03.03)}

The Life Management activity will provide information necessary to calculate an expected useful life for the existing DST storage and transfer systems. This activity will also provide information necessary to manage degradation of the existing DST storage and transfer systems in a cost-effective environmentally sound manner. This will maximize flexibility in use of the entire system, thus minimizing replacement costs and less-than-optimal retrieval/disposal strategies. Specific activities include (WHC-SD-WM-PLN-068, Revision 0, Plan for the TWRS Life Management/Aging

Management Program):

- Compliance with codes and standards

- Evaluation of degradation mechanisms under aging conditions

- Interface with Safety Analysis and Configuration Management

- Structural acceptance and service life calculation

- $\quad$ Testing, surveillances, and inspections

- Component screening process

- Mitigation, upgrades, and retirement strategy development.

\section{Multi-Function Waste Tank Facility W-236A (1.1.1.2.03.04)}

The Multi-Function Waste Tank Facility (MWTF) will provide a facility capable of safe, environmentally acceptable tank capacity for wastes from SSTs and DSTs tank safety issues/mitigation and tank remediation/retrieval activities. This project is currently scoped to provide two tanks in the 200 West Area and four tanks in the 200 East Area.

\section{Instrumentation \& Control/Electrical Upgrades (1.1.1.2.03.06)}

Instrumentation \& Control (I\&C)/Electrical Upgrades will provide electrical support and waste monitoring until new facilities are provided, as defined by the Project W-314 MSA. The FY 1995 through FY 1997 tasks will develop I\&C/Electrical elements that can be integrated as part of the overall power distribution and instrumentation monitoring systems being designed by Project W-314. 
In FY 1995, Project W-314 will prepare the basis for requesting, and request Key Decision (KD)-1. The Design Requirements Baseline will be completed as described by the TWRS systems engineering approach and documentation for Projects. The appropriate safety analysis, which will be prepared in FY 1995, is also required for the KD-1 request.

Additionally in FY 1995, W-314 will select and place a contract with an Architect/Engineer (A/E) for definitive design. Advanced Conceptual Design activities will begin with the selected A/E in FY 1995, and be completed in FY 1996. Through the TWRS Environmental Impact Statement (EIS), support to the National Environmental Protection Act (NEPA) documentation and decision-making process will be provided during FY 1995. This will be completed with a Record of Decision (ROD) on the EIS in FY 1996.

A plan for the sequencing of both design and construction will be prepared during FY 1995 . Work to develop characterization information to support design of the ventilation systems will begin in FY 1995 and be completed in FY 1996. As-building of critical drawings to support definitive design will begin in FY 1995 and be completed in FY 1996. Permitting planning and permit support activities will begin in FY 1995 and continue through FY 1996. These activities will be completed in FY 1997 with the timely issuance of all necessary permits, including but not limited to, Clean Air Act permits from the State of Washington. Title I design will begin in FY 1996, upon receipt of KD-1, and will be completed in FY 1997. Title II design will begin in FY 1997 upon receipt of KD-2, and will continue beyond FY 1997.

Completion of the above work will meet Hanford Federal Facility Agreement and Consent Order, (Tri-Party Agreement) milestones series M-43-02, M-43-04, M-43-05, and M-43-06 (as it is presently written). A change request will be prepared in FY 1994 to consolidate all four of these series of Tri-Party Agreement milestones into a single series to reflect the current project management approach of W-314. When Project W-314 is completed, Tank Farms will have the safe, functional, reliable, and compliant instrumentation, ventilation, electrical, and waste transfer systems it needs to store waste in DSTs to meet the mission as described in the TWRS Technical Strategy. These systems will have a service life matched to the mission life as described in the TWRS Technical Strategy.

Not completing this project, or delaying it, results in prolonged periods of operation under the present level of risk. Further, the already worn and difficult-to-maintain equipment ages during the time it takes to build the project, thereby decreasing the present safety margin and adversely increasing risk.

\section{Mechanical Upgrades (1.1.1.2.03.09)}

Mechanical Upgrades will undertake design and construction activities to improve the physical condition of numerous systems in FY 1995, FY 1996, and FY 1997. Areas of primary improvement involve compressed air; heating, ventilating, and air conditioning (HVAC); and waste transfer system upgrades. An additional effort is underway for the development of protocol and hardware for the cradle-to-grave disposal of long-length contaminated equipment removed from the waste tanks.

All mechanical upgrades are considered necessary to support the safe storage mission of TWRS. Many tasks are DOE-identified safety initiatives and some are Tri-Party Agreement milestone related; these will be addressed with the highest priority. 
This project supports the number two priority placed on ventilation upgrade projects by DOEHeadquarters (DOE-HQ), Office of Environmental Management (EM)-30. The project replaces a cracked ventilation header and provides a new ventilation/cooling system for the AY/AZ Tank Farms to meet DOE and American Society of Mechanical Engineers (ASME) requirements. The current ventilation system has exceeded its design life and is unreliable. The new system will extend the useful life of the system, eliminate cooling water discharge to the soil column, minimize risks of single-point critical component failure, and provide seismic and high wind hardening of the critical systems. The project strategy includes meeting all Tri-Party Agreement milestones on or ahead of schedule: start construction before October 31, 1994; complete construction by October 31, 1996; and begin operation by December 31, 1996.

\section{Cross-Site Transfer System, W-058 (1.1.1.2.03.11)}

Project W-058 is being managed and worked in conjunction with Project W-028, Aging Waste Transfer Line. The two projects will share common design features, and will be worked to the same schedule. They were initially validated as separate projects, but as their missions have evolved, it has become advantageous to work them in conjunction with each other. Therefore, even though the budget amounts and schedules are carried separately, both must be reviewed as an integrated system to gain a complete understanding of this activity. This project has received KD-2 and is in Title II design in FY 1994.

Permitting issues, primarily Clean Air Act permits, will continue through FY 1995. A Notice of Construction is scheduled to be sent to DOE Richland Operations Office (RL) in FY 1995, who will then submit it to the Washington State Department of Health. Subsequent environmental support and reviews continue through FY 1996 to support start of construction.

Title II design and the corresponding Preliminary Safety Analysis Report (PSAR) continue in FY 1995, supporting KD-3 in early FY 1996. Construction contractor selection will occur in late FY 1995 and end in early FY 1996. Based on timely receipt of KD-3, construction, corresponding Final Safety Analysis Report (FSAR), and Engineering Inspection will begin in early FY 1996 and continue through early FY 1998. Project reviews, procurement, and support activities will occur to support the above primary project activities.

\section{Aging Waste Transfer Line, W-028 (1.1.1.2.03.12)}

Project W-028 is being managed and worked in conjunction with Project W-058. The two projects will share common design features, and will be worked to the same schedule. They were initially validated as separate projects, but as their missions have evolved it has become advantageous to work them in conjunction with each other. [See Cross-Site Transfer System, W-058, (1.1.1.3.03.11)].

\section{Facility Upgrades (1.1.1.2.03.16)}

Facility Upgrades consists entirely of GPPs for FY 1995, FY 1996, and FY 1997. Facility Upgrades GPPs provide for capital work in support of urgent needs of Tank Farm Operations. Facility Upgrade GPPs scheduled to begin in FY 1995 through FY 1997 include several tank farm lighting upgrades, which will install lighting systems to provide a minimum of 5-foot-candle light within the farms, a Tank Farm Repackaging Facility, Solid Waste Management Facility, East/West Central 
Monitoring Facility, and a Core Sampling and Storage Facility. Engineering studies, functional design criteria, and conceptual designs for all Tank Farm Upgrades Program Element GPPs will be performed in this activity as well as annual Candidate Project Support Sheets and Project Data Sheets.

\section{Radiological Support Facility, W-188 (1.1.1.2.03.17)}

Project W-188, Radiological Support Facility, was proposed to construct compliant change facilities and decontamination showers, personnel access monitoring and control areas, and support offices for Tank Farm personnel. Although the project was identified as an important element in Tank Farm Operations' ability to provide a safe and efficient working environment for the Tank Farm work force, it has not received the necessary funding priority from DOE-HQ to continue as currently scoped.

Based on a July 1994 memorandum from DOE-HQ (EM-30) to RL, which prioritized project funding, it was determined that the need for Project W-188 is not of a critical nature and, therefore, not urgent enough to justify funding given the numerous higher priority projects. Project W-188 ranked 74, with the current funding cutoff at 70 . The seven criteria used for prioritization included: Safety and health risk, compliance with requirements, disruption of EM-30's mission beyond that included in the other criteria, environmental impact, waste minimization, waste management costs, and technology assurance.

The functional design criteria and conceptual design report for W-188 are complete, so deferring work at this point in the project cycle is appropriate until funding priorities change and the ongoing systems engineering effort identifies the need.

A budget of $\$ 15 \mathrm{~K}$ is planned for FY 1995 to develop a position paper on how to disposition scope identified for Project W-188.

\subsection{Function and Requirements}

\section{Functional Basis}

The TWRS Systems Engineering development (DOE/RL-92-60, Revision 1) identifies no separate "upgrades" function. Upgrades derives its purpose from the store waste (4.2.1.1) and transfer waste (4.2.1.4) functions. Upgrades are needed only when the condition of the systems, equipment, or infrastructure (drawings and procedures) is not adequate to meet the functional requirements allocated to Operations.

As many of the systems being upgraded perform multiple functions, as defined in the TWRS Systems Engineering Architecture (DOE/RL-92-60, Revision 1), the Tank Farm Upgrades Program Element must address those functions and their allocated requirements. In particular, many of the systems being upgraded to support the store waste function do so by monitoring and controlling a waste stream (e.g., tank ventilation systems). Further, the field activities to perform the hardware improvements will generate waste, not only from the operations themselves but also from the existing hardware being replaced. Therefore, Tank Farm Upgrades must also meet the requirements allocated to applicable sub-functions to each of the following functions: disposition gaseous effluent (4.2.3.2), disposition liquid effluent (4.2.3.3), and disposition solid waste (4.2.3.4). There may be applications 
of upgrades to disposition excess facilities (4.2.3.5), but this is to be determined as further definition of that function evolves and requirements are reviewed.

Existing deficiencies have been documented in numerous audits, reports, and findings. Hardware deficiencies are caused by many reasons: old age of equipment (well past service life), unavailability of repair parts, poor maintenance, or non-compliant original design. When a deficiency is noted, if its correction is beyond the normal scope of operational plant maintenance, then the improvements become candidates to be upgrades. Candidate upgrades are then scoped, prioritized, and an acquisition strategy outlined, and if high enough in priority to receive limited funds, the work is pursued. If the work does not make it past the priority funding process, it does not become an upgrade.

\section{Program Participation in System Engineering Development}

The Tank Farm Upgrades Program Element has provided staff to support the development of the TWRS Systems Engineering Architecture for both the store waste and transfer waste functions at the TWRS level. In addition to continued support for development of the TWRS architecture and documentation, this activity will provide resources to address and begin creating the systems engineering architecture and basis for the small expense- and capital-funded (non-line item) work funded by the program. This effort will provide the documentation and basis for continuing this work on a categorical basis as the TWRS Systems Engineering Architecture evolves. As each step of the TWRS Systems Engineering Architecture and documentation is developed, the programmatic categorical systems engineering documentation will be reviewed and revised as necessary to be consistent with the TWRS documentation.

Because of the parallel development of the TWRS Systems Engineering architecture, infrastructure, and processes with the development of the W-314 project, several unique approaches had to be taken. TWRS Systems Engineering is in the process of developing functions and requirements. However, at this time, TWRS has developed its functions and requirements only through Level 4. Eventually TWRS will develop and provide optimization down through the level necessary to initiate projects. However, as this is not done today, the project basis will be established by building a credible path from the bottom up. Then, as each step of the TWRS Systems Engineering Architecture is built from the top down, the project-specific basis will be reviewed and revised as necessary to link up with the TWRS architecture.

Pursuant to that goal, Project W-314 has issued a project-specific functions and operations requirements document which establishes the project functions and requirements and traces them up to the existing TWRS Level IV Architecture, WHC-SD-W314-FRD-001. Additionally, the project has begun work on project-specific Mission Analysis, Systems Engineering Management Plan, Interface Control Document (WHC-SD-W314-ICD-001), and Design Requirements Document (DRD). As TWRS Systems Engineering evolves and generates the TWRS level design requirements documents and interface control documents, the Project W-314-specific documents will be reviewed and revised as necessary to mesh with the TWRS documents. As the design requirements baseline is developed for Project W-314, each of the project-specific systems engineering documents mentioned above will be revised and expanded in detail. 


\subsection{Risks}

\section{Technical Risk}

The program, in general, has very low technical risk. There are no emerging technologies being applied. All technologies being selected are proven applications of commercially available technology, using "off-the-shelf" components.

The rapidly evolving nature of electronics and instrumentation/control technology, and funding and project management time constraints make it difficult to select for use any technology in this area. Any technology selected must be available today and be optimal by the time it is installed.

\section{Financial Risk and Funding Mix}

The area of highest risk is financial, as balanced with safety risk. The questions exist both at the TWRS level with how much funding the Tank Farm Upgrades program should receive, and within the Tank Farm Upgrades with what the best mix of expense activities and capital activities is. Both questions reduce to balancing the investment in mitigating near-term risks, with investing limited resources in resolving long-term risks.

At the TWRS level, given limited resources, the crucial question is: How much should be invested in improving the infrastructure of the tank farms, thereby reducing the current level of risk from interim storage, with the investment in infrastructure to dispose of (long-term store) the waste? As the current and future risks have not been quantified (or better, their quantification has not been widely accepted), these judgements remain somewhat subjective.

Similar questions arise at the Upgrades program level. Given the DOE property accounting and capitalization rules, Upgrades is faced with the choice between selective component replacement with expense and capital funds, and system replacement using the major project (line item) management approach. The expense-funded approach tends to be less costly and much quicker than the line item approach. This is balanced with replacing components that are at or beyond their intended service life when constructed, and extending that service to, in many cases, more than double their service life; or, simply replacing the entire system with one designed to meet the interim storage mission life. Further, many of the existing systems are not compliant with current requirements. The question here becomes: Is the benefit of coming into compliance over the interim storage life appropriate to the investment required to achieve that compliance?

The Tank Farm Upgrades Program Element is managing that risk through a number of avenues. First, the engineering studies to determine how the work should be done have involved a number of outside experts in study analysis sessions. These were intensive several-day efforts to kick off the various aspects of Project W-314, using both academic and industrial experts. These sessions ensured that a wide variety of options were reviewed, that cost-effective and technically optimal options would result from the studies.

Second, the Upgrades program has done a great deal of internal planning, including use of offsite consulting and Westinghouse Hanford Company (WHC) forces teamed to assist in planning. The Upgrades program is also performing a self-assessment by a team of WHC experts to ensure that the expense and capital mix and acquisition strategies are appropriate. (See 7.5.1.1.2, Program Strategy) 
Also, the Upgrades program is investing heavily in systems engineering, both within Project W-314 and at the programmatic level. The TWRS Systems Engineering architecture and processes are evolving. In the interim between the present and the full establishment of systems engineering at the TWRS level, Upgrades is developing the application of systems engineering at the program level. Project W-314 is the prototype for developing systems engineering at the project level. Given the constraints of the existing equipment in the field and the TWRS mission, this program-level systems engineering will ensure that all work planned is both necessary and sufficient to meet the safe interim storage mission.

\section{Systems Engineering Parallel Evolution Risk}

The final programmatic risk is both schedule and cost. The Tri-Party Agreement was negotiated at a time when the TWRS Systems Engineering approach was in its early stages of development. Since that time, the impact to the program and to each project has been more fully understood. Significant time and resources have been spent in tracking this evolution and developing the implementation concept.

The current Tri-Party Agreement milestọnes do not reflect the project documentation that the evolving systems engineering paradigm requires. The basic approach to project development is in transition. Project W-314 will be the first TWRS project to develop that implementation and will require more funds and perhaps take longer than the same project would, once the systems engineering process is fully developed and well understood. Given the fixed nature of both the milestones and the budget, this creates both a cost and schedule risk. Further, the risk is accentuated by the direction received during the Tri-Party Agreement negotiations to negotiate milestones as currently scheduled, with no contingency. This has heightened the risk that unknowns or uncertainties due to systems engineering development will become problematic.

\subsection{Major Decisions}

The TWRS Decision Analysis Report (WHC-EP-0617) in Appendix B 5.2.2 refers the reader to the Upgrades Program Plan. The decision analysis assumes that the cost and schedule for upgrades is the same for the various alternative technical strategies. Therefore, it identifies upgrades as being required to support the safe interim storage of waste, regardless of the disposal methodology. Even though the Upgrades Program Plan no longer represents the current strategy, schedule, or funding, the need for upgrades, as captured in the Decision Analysis Report, is unchanged.

Revision 0 of the Hanford Site TWRS Technical Strategy (March 18, 1994), Section IV.A. last bullet, calls for Tank Farm facilities and systems to be restored and upgraded to improve facility operations and surveillance (ensure safe storage and transfer of the wastes).

Within the program, the most significant decision made was to pursue Project W-314 as a single project. This project consolidates the scopes of four former sub-projects into one project. This was done to provide better design integration and to achieve significant efficiency gains during construction. 
WHC-SP-1101

\subsection{Assumptions}

The Need for Upgrades and Systems Engineering Evolution

A central assumption to the program element is that the existing Tank Farm infrastructure must be restored to meet the safe storage mission. Numerous audits, both internal and external, have pointed out the physical and documentation deficiencies of the plant.

Systems engineering at TWRS is not yet fully developed, but it is the fundamental assumption that TWRS systems engineering, once complete, will validate the need to restore Tank Farms to a minimally safe condition to meet the store waste function. For example, if a particular tank ventilation system is operating beyond its service life, spare parts cannot be obtained, and it is noncompliant with current codes. At the outset, it would be assumed that once systems engineering is fully developed it will confirm the need to upgrade the system.

Therefore, the Upgrades program is proceeding with the infrastructure upgrade process, since waiting for systems engineering to be fully developed will prolong the time at risk. This is based on the assumption that systems engineering will confirm the need to do the planned work.

To help manage the risk, the Upgrades Program is participating, both in dedicated staff and funding, in developing the TWRS Systems Engineering Architecture. Further, as each new product comes out of the systems engineering process, Upgrades as a program intends to review planned work and document traceability back to the Systems Engineering Architecture.

\section{Safety Class, Interim Safety Basis, and Final Safety Basis (Technical Safety Requirements)}

Another pivotal assumption made by the Upgrades program deals with safety classification of the Tank Farm systems and hardware.

Tank Farms is currently operating under the Interim Safety Basis (ISB). In that safety basis, several systems are currently classified as Interim Operational Safety Requirements [IOSR]*, as established in the Interim Safety Equipment List (ISEL). The ISEL states:

"The notation [IOSR]* has been designated for those systems, components, and structures important to health, safety, and environmental protection, but, for which current analyses are not sufficient to establish the safety classification. Such items shall be treated as safety class 3 , with the additional designation an Operational Safety Requirements (OSR) related. The additional OSR designation will ensure the priority and funding for the continued operation and maintenance of these items, consistent with OSR requirements IOSR, where applicable).

The [IOSR]* safety classification will be replaced to be consistent with the requirements of [WHC Controlled Manual WHC-CM-1-3, Management Requirements and Procedures, MRP 5.46, "Safety Classification of Systems, Components, and Structures",] as analyses are completed to establish definite safety classifications. In the interim, approval requirements shall include Nuclear Safety and Quality Assurance (SQ), as a minimum, and Environmental Assurance (E) where deemed appropriate." 
The DOE responded to the submittal of the ISEL with a letter (J. H. Anttonen to T. M. Anderson, "Single-Shell Tank Interim Safety Equipment List," dated September 16, 1993), which states:

"Sections 4.3.2 and 4.3.3 of the present version of the ISEL contain numerous inaccuracies. The discussions provided in these sections to justify the safety classifications are not credible."

The letter goes on to say:

"RL is further disturbed by the [IOSR] designation. This designation does not appear in the definitive basis for safety classification, DOE Order 6430.1A, "General Design Criteria". RL notes that this designation also does not appear in WHC procedure MRP 5.46. As such, WHC appears to have failed to provide for such a classification within its own system. RL further believes, there is sufficient information to perform an analyses at the present time, which would provide a basis for determination of safety class for the systems."

The current WHC position concerning safety classification of tank farm instrumentation is reflected in a letter (M. A. Payne to R. E. Gerton, "In-Tank Instrument Safety Classification", dated October 14, 1993), which states:

- $\quad$ For tank number C-106 and 101-SY

WHC will delete the notation [IOSR]* for in-tank monitoring instruments for tank numbers C-106 and 101-SY in the SST ISEL and the DST ISEL respectively, and replace it with SC-1*. The asterisk will refer to a note that states, "The notation SC-1 is founded on conservative assumptions and not necessarily on system safety analysis. Safety class 1 or 2 seismic, environmental, redundancy, separation, power supply, procurement and quality assurance requirements will not be imposed. Liquid level gages, thermocouple trees, tank dome pressure gages, and associated items, are required to meet SC-3 design, procurement, installation, maintenance, and quality assurance requirements. Approval requirements shall include Nuclear Safety and Quality Assurance (SQ), as a minimum, and Environmental Assurance (E) where deemed appropriate. Accident based safety classifications for in-tank monitoring instruments will be determined only after appropriate system safety analyses are performed." The deviation to DOE 6430.1A will discuss the reasons for not imposing SC-1 criteria and will include: (1) instruments that cannot be purchased to Quality Assurance Program Requirements for Nuclear Power Plants (NQA-1) standards, (2) design basis seismic accident criteria, and (3) existing equipment infrastructure does not support SC-1 items.

- For all other tanks

WHC will delete the notation [IOSR]* for all in-tank monitoring instruments and associated items for all other tanks in the SST, DST, and Aging Waste Tank ISELs and replace it with TBD*. The asterisk will refer to a note that states, "Until such time as the TBDs are removed, in-tank monitoring instruments, and associated items, are only required to meet SC-3 design, procurement, installation, maintenance, and quality assurance requirements. Approval requirements shall include Nuclear Safety and Quality Assurance (SQ), as a minimum, and Environmental Assurance (E) where deemed appropriate. Accident based safety classifications for in-tank monitoring instruments will be determined only after appropriate system safety analyses are performed." This is justified due to the waiver being processed to the environmental hazard safety class criteria of MRP 5.46. WHC will revise the ISELs and 
provide copies to RL 90 days after $R L$ approval of the deviation to DOE 6430.1A. WHC will resolve the TBDs by March 1995."

The systems noted as IOSR* will be designated a safety class 1,2 , or 3 upon completion of the safety analyses.

When a system moves from being treated as a safety class 3 to a safety class 1 , the cost of the system more than doubles. When a system moves from being treated as a safety class 3 to a safety class 2 , the general cost of replacing the system can be expected to go up by at least $50 \%$. Therefore, the program total cost is very sensitive to changes in the safety classification from the interim safety basis to the final safety basis (or technical safety requirements).

Current budgeting exercises have been adjusting funds, and therefore, no schedule is available for completion of the final Safety Equipment List. The planned status of that activity can be found in Section 7.3, Tank Farm Operations and Maintenance, Cost Account 1N1C38.

The following table provides a summary of the various systems' safety classification taken from the interim safety basis for systems being replaced by W-314.

\begin{tabular}{|c|c|c|}
\hline System & Aging Waste Tànks & Double Shell Tanks \\
\hline Primary Ventilation & SC 2 & SC 2 \\
\hline Annulus Ventilation & SC 2 & SC 2 \\
\hline Waste Transfer & SC 1 & SC 1 \\
\hline Primary Instrumentation & $\therefore \quad \therefore N / A \quad \therefore$ & IOSR \\
\hline Secondary Tank Instrumentation & $\because \quad \because \mathrm{N} / \mathrm{A}$ औ. & SC 2 \\
\hline Leak Detection Instrumentation & 政 & SC 3 \\
\hline Ventilation System Instrumentation & $\because \mathbf{N} / \mathrm{A}$ & $\mathrm{SC} 2$ \\
\hline Miscellaneous Instrumentation & $\because \quad$ N/A & SC 3 \\
\hline Primary Liquid Level Instrumentation & IOSR & N/A \\
\hline Primary Pressure/Vacuum Instrumentation & IOSR & $\because \vdots$ \\
\hline Primary Leak Detection & SC 2 & $\because \therefore$ \\
\hline Primary Ventilation Radiation Monitor & SC 2 & $\therefore \because$ \\
\hline Secondary Pressure/Vacuum Instrumentation & SC 2 & $N / A \quad \therefore$ \\
\hline Electrical Power Distribution & $\begin{array}{l}\text { IOSR/SC } 2 / S C 3 \\
\text { depending on system } \\
\text { being supplied }\end{array}$ & IOSR \\
\hline
\end{tabular}

The Upgrades program is proceeding with all work, both line-item project and expense/CENRTC hardware upgrades on the existing (interim) safety basis. If the final safety basis changes a system's safety classification from the interim assigned (or assumed) value, the program budget associated with that system (and its corresponding schedule) can be expected to be significantly impacted. 


\section{Technical Integration and Planning (1.1.1.2.03.01)}

This activity is based on the assumption that a program office is both required and necessary for the overall management of the Upgrades program. The program office provides guidance and support for the program plan execution in support of the TWRS Mission. The Upgrades program office develops the work priorities within the program and coordinates the program priorities with the Operations and Maintenance program, Retrieval, Safety, and other programs as required. This coordination ensures cost-effective utilization of TWRS resources to upgrade Tank Farms infrastructure and facilities.

\section{Configuration Management (1.1.1.2.03.02)}

Configuration Management, as required by DOE-STD-1073-93, Guide For Operational Configuration Management Program, with its five program elements and two adjunct programs (see Approach, Section 7.5.1.5), provides a vital function to improve conduct of operations and assures excellence in facility conditions. This is accomplished by providing programmatic assurance that the Tank Farms physical configuration is consistent with its design requirements and documentation and that this consistency is maintained throughout the life cycle of the farm.

The Configuration Management program for Tank Farms and the 242-A Evaporator meets the intent of the following WHC and DOE documents.

- Hanford Strategic Plan, draft July 11, 1994, "Work Force Effectiveness".

- Hanford Site Tank Waste Remediation System Technical Strategy.

- DOE/RL-93-102, Fiscal Year 1995 Hanford Mission Plan, Volume 1, Site Guidance, DRAFT.

Procedure development will be required prior to initiating the upgrade turnover to the plant. Therefore, procedures that have been identified as required for operation (plant operating procedures, operator rounds procedures, alarm response procedures, and operational safety documents) and that have been affected by the upgrade, will be developed. The timing for completing the procedure package will be tied to completing the upgrade installation.

\section{Life Management (1.1.1.2.03.03)}

The TWRS Technical Strategy has assumed that the existing DSTs are fundamentally sound and capable of unrestricted use through the end of the TWRS storage mission. The DST life management strategy will address the existing inter-area transfer system associated with the DSTs (from doublecontained receiver tanks, evaporator and unloading facilities, to DSTs). Life Management will provide physical upgrades, such as purchase of instrumentation and hardware.

Examination of DSTs, DST transfer piping, 242-A Evaporator, and unloading facilities is driven by WAC 173-303-640 and is controlled by WHC-SD-WM-AP-017, Revision 1, Double-Shell Tank Integrity Assessment Program Plan. Implementation of mitigation strategies and suggested engineering practices (e.g., corrosion monitoring and control) are provided by TWRS Operations and Maintenance (O\&M) in accordance with appropriate priority system(s) (WHC-SD-WM-PAP-60, DRAFT, TWRS Corrosion Monitoring Program Plan). 
The TWRS Life Management program will be required for the duration of the store waste and transfer waste functions (assumed to be 40 years).

\section{Multi-Function Waste Tank Facility, W-236A (1.1.1.2.03.04)}

The MWTF Justification of Mission Need (JMN), approved in January 1993, constituted endorsement of the stated project need, technical objectives, and management approach. This consisted of additional waste storage capacity in support of resolution of all safety issues, day-to-day tank farm operations, retrieval, and management of waste in the SSTs. In addition, this was further substantiated in the project plan, which was approved by DOE-HQ in November 1992. The project plan stated, "The MWTF will provide additional high-level waste (HLW) interim confinement (storage) to support resolution/remediation of the tank safety issues and continued retrieval and operations activities."

\section{Instrumentation \& Control/Electrical Upgrades (1.1.1.2.03.06)}

It is assumed requirements for upgrades in the area of electrical support and waste monitoring will continue until future needs are met through the Project W-314 MSA. Current and planned near-term activities will utilize major maintenance, I\&C installation, and Tank Monitor and Control System (TMACS) funding to meet these requirements and provide a bridge to the MSA. The FY 1995 through FY 1997 I\&C/Electrical Upgrades activities are an extension of work currently in progress.

\section{Tank Farm Restoration and Safe Operations MSA, Project W-314 (1.1.1.2.03.07)}

No additional assumptions exist other that those already discussed in this section.

\section{Mechanical Upgrades (1.1.1.2.03.09)}

It is assumed that safety initiatives and Tri-Party Agreement related work are considered highest priority. O\&M, via the integrated schedule, will have the resources and provide the priority to support field work.

\section{Ventilation Upgrades, W-030 (1.1.1.2.03.10)}

It is assumed that Ventilation Upgrades activities will be funded to support the schedule to complete Tri-Party Agreement milestones, and that startup approval will be obtained from State regulators in FY 1996. Operations support (health physics technicians [HPTs], operators, person-in-charge, planners) will be provided to support the construction schedule.

\section{Cross-Site Transfer System, W-058 (1.1.1.2.03.11)}

A critical assumption is that an offsite construction contractor will be able to resolve interface issues with site personnel in a timely manner. As the Conduct of Operations standards have taken significant strides forward, the potential may have been created for more complex interface issues with off-site construction forces, and the necessary site support forces. As the improvements to the Conduct of Operations process are very new, no experience is available with large project construction performed by offsite forces and the interfaces with onsite forces. 
Another critical assumption is the timely approval of the ROD from the combined Project W-236a and W-028/058 Environmental Impact Statement. In the interests of cost effectiveness, it was decided to combine the above projects together into one EIS. Prompt approval of the ROD for this EIS is critical to proceeding with construction. Approval must be received in September 1994. An Environmental Assessment (EA) was originally prepared for this scope of work, and the Finding of No Significant Impact.(FONSI) resulting from that EA was at DOE-HQ for final approval when the decision was made to go back and perform an EIS.

Timely approval of KD-3 is critical to maintaining the schedule and meeting Tri-Party Agreement milestones. Long delays were experienced in the receipt of $\mathrm{KD}-2$, which caused schedule contingency to be eaten up for definitive design, placing Tri-Party Agreement Milestone M-43-07-T01 in severe jeopardy. Delays of a similar nature, now that schedule contingency is gone, will have an even greater effect on meeting the next Tri-Party Agreement Milestone, M-43-07A, "Start Construction", an enforceable Tri-Party Agreement milestone.

\section{Aging Waste Transfer Line, W-028 (1.1.1.2.03.12)}

See Cross-Site Transfer System, W-058 (1.1.1.2.03.11).

\section{Facility Upgrades (1.1.1.2.03.16)}

Facility Upgrades planning is based on the current priorities, which reflect a strong management emphasis on improving the lighting levels in the Tank Farms. As priorities change within Tank Farms, the sequencing of projects will also change. All other facility upgrades will be provided by the O\&M Program.

\section{Radiological Support Facility, W-188 (1.1.1.2.03.17)}

N/A, see Program Strategy (Section 7.5.1.1.2).

\subsection{Constraints}

\section{Funding}

The first constraint to accomplishing Tank Farm upgrades is funding limits. Today, if more funding were made available, the Upgrades Program could be completed sooner and the time at risk from the present condition of the Tank Farms could be reduced.

\section{Systems Engineering Evolution}

Because systems engineering is in the early stages of development, both the program and Project W-314 are investing significant resources building reasonable paths up to the Level 4 Systems Engineering Architecture as it exists today. If the TWRS process were mature, optimal paths for functions and requirements would be built to lower levels. Were that the case, program and project funds could be focused on work and less diluted by pre-building the systems engineering basis from such a high level. 


\section{Acquisition Regulations}

In order, the next lower constraints to Upgrades program implementation are the project management processes detailed in DOE 4700.1, Project Management System, and RLIP-4700.1A, Project Management System. If these constraints were removed, the completion of upgrades could be accomplished both faster and cheaper.

\section{Staff to Manage the Work}

Finally, if all the above constraints were removed or relaxed or if significant new funds were made available, there is some level at which staff would become the constraint. Today, much of the upgrades work is being done by offsite forces. More work could be done offsite, but at some point the onsite force's ability to effectively manage work performed offsite becomes limited. That limit is not yet reached and would not be reached with significant budget relaxation of the.above constraints.

\section{Technical or Logic}

In general terms, no logic or technical constraints to doing upgrades exist. Many more deficiencies have been identified than there are resources to fix them. These deficiencies do not require technology or process development. They are, in general, hardware or configuration documentation in nature and could be resolved faster with greater resource allocation.

Specific constraints are listed below by activity.

Technical Integration and Planning (1.1.1.2.03.01)

The primary constraints within Technical Integration and Planning are funding and resources. The amount of upgrades planned and executed within a given year is primarily driven by these two constraints. However, other constraints may surface on occasion such as the TWRS Administrative Hold, which began in August 1993 and continued for several months. This administrative action was necessary to mitigate the safety issues surrounding the execution of work and conduct of operations within the Tank Farms and the potential impact on worker safety. This action caused a serious delay in the field work activities the Upgrades Program had planned for FY 1994. In addition, facility upgrades resulting from evolutions in other programs will be coordinated with this work to ensure consistency. Theoretically, the Upgrades Program will work itself out of existence by completing the Program Mission of upgrading the Tank Farm plant equipment and infrastructure by the year 2005 .

\section{Configuration Management (1.1.1.2.03.02)}

An implementation plan is required for the successful execution of the configuration management program. This implementation plan will be based on the initial assessments and audits (identified in WHC-SD-WM-CM-007, Configuration Compliance Matrix for the Waste Tank Farms and the 242-A Evaporator of Tank Waste Remediation System), Standards/Requirements Identification Documents (S/RIDs), and existing WHC procedures that are identified in WHC-SD-WM-CM-008.

The constraints have been identified as assumptions in Configuration Management, 1.1.1.2.03.02, specifically, funding and resource availability. Funding will be a constraint if inadequate to prepare the procedure packages for the identified upgrades. Resources will be a constraint if the upgrades are 
time phased in a manner that makes the procedure writer a critical resource and results in procedure preparation as the critical path to achieving turnover of the upgrade project.

\section{Life Management (1.1.1.2.03.03)}

The primary constraints within the Life Management activity are priority and resource availability. Some constraints also exist that are associated with the timeliness of the DST ultrasonic test inspection program and installation of corrosion monitoring and control strategies by TWRS O\&M.

\section{Multi-Function Waste Tank Facility W-236A (1.1.1.2.02.04)}

See Assumptions (Section 7.5.1.1.6).

\section{Instrumentation \& Control/Electrical Upgrades (1.1.1.2.03.06)}

The constraints that define the scope of the Instrumentation \& Control/Electrical Upgrades activities include the need to continue to support high priority operational requirements while interfacing with the schedule and scope of Project W-314. The FY 1995 through FY 1997 tasks will use staff resources and funding of approximately $\$ 7.7 \mathrm{M}$ per year to complete the work.

\section{Tank Farm Restoration and Safe Operations MSA, Project W-314 (1.1.1.2.03.07)}

None other than already discussed in this section.

\section{Mechanical Upgrades (1.1.1.2.03.09)}

The resource-loaded schedule may place limitations on the amount of field work that can be completed. Also, the Davis-Bacon and work turndown processes must be adhered to and will very likely increase fabrication costs of equipment.

Long-length contaminated equipment is developing a standardized generic system for the retrieval, transport, storage, treatment, and disposal of waste equipment. This system is essential to the timely and cost-effective continued operations of Tank Farms and future retrieval activities.

\section{Ventilation Upgrades, W-030 (1.1.1.2.03.10)}

Specific critical constraints of Ventilation Upgrades are limited expense funding, limited health physics technician (HPT)/operations support, and marginal contingency for uncertainties associated with construction. The Tri-Party Agreement milestones define schedule constraints. The critical path to completion of the work is the in-farm construction. Startup approval from state regulators will be required before proceeding with tank tie-ins and suspending the old system.

Cross-Site Transfer System, W-058 (1.1.1.2.03.11)

See Assumptions (Section 7.5.1.1.6).

Aging Waste Transfer Line, W-028 (1.1.1.2.03.12)

See Assumptions (Section 7.5.1.1.6). 


\section{Facility Upgrades (1.1.1.2.03.16)}

In FY 1995, General Plant Projects (GPP) are severely constrained by funding. The August 1994 RL budget submittal reflects \$2.9M GPP Capital as opposed to a \$5.6M Activity Data Sheet (ADS) submittal. Funding for FY 1996 and FY 1997 is assumed to remain at the ADS submittal level.

Radiological Support Facility, W-188 (1.1.1.2.03.17)

N/A, See Program Strategy (Section 7.5.1.1.2).

\subsubsection{Structure}

The Tank Farm Upgrades Program Element provides specific systems and infrastructure for the safe operation of Tank Farms in support of the TWRS mission. This program element is structured into well-defined technically related areas, which ensure proper integration, consistency, and technical strategy.

\subsection{Work Breakdown Structure}

The Tank Farm Upgrades Program Element is composed of ten activities. Their scope is generally described as follows:

- Technical Integration and Planning. Program element management, performance monitoring and reporting, and systems engineering.

- Configuration Management. Drawing field verification and procedure upgrades.

- Life Management. Analysis of the existing DST condition and their ability to support the TWRS Technical Strategy.

- I\&C/Electrical, Mechanical, and Facility Upgrades. Expense/CENRTC- and GPP Capitalfunded activities to improve the hardware condition of the Tank Farms.

- Project W-314. Major Systems Acquisition to replace instrumentation, ventilation, electrical, and transfer systems to support the store waste function.

- Project W-030. Replacement of the AY/AZ primary ventilation system

- Project W-058 Cross-Site Transfer System and Project W-028 Aging Waste Ventilation System. Two projects worked concurrently to provide compliant waste transfer capability.

- Project W-188 Radiological Support Facility. Replace existing temporary change trailers and provide operational support at the Tank Farms.

The Tank Farm Upgrades Work Breakdown Structure (WBS) is shown on Figure 7.5-1. The WBS dictionary is found in Appendix A. 


\subsection{Organization and Responsibility}

\section{Relationship with RL}

Programmatic direction for this effort shall in all cases and at all times be provided by the RL-TWRS office. A lead RL staff member will be appointed to oversee work in a specific account. This individual is responsible for ensuring that sufficient clear guidance and direction is available at all times to the contractor to perform the requested work.

THE CONTRACTOR SHALL NOT FOLLOW GUIDANCE OR DIRECTION PROVIDED BY ANY OTHER RL OFFICE. The contractor will refer any conflicts between the guidance from the RL TWRS office and guidance offered by another RL office to the RL TWRS office for resolution.

The RL lead for this cost account shall be apprised, as soon as reasonably possible, of any impacts to work costs or schedules in this cost account that occur as the fiscal year progresses.

\section{General Responsibilities}

The following general responsibilities were summarized from the TWRS Systems Engineering Work Plan Draft C, May 9, 1994. The Upgrades program office is responsible for ensuring that tasks are scoped, planned, scheduled, and funded. In Operations, the Waste Tanks Upgrade Installation/ Testing organization is responsible for performing major maintenance activities. TWRS Systems Engineering is responsible for developing the TWRS Systems Engineering Infrastructure.

Engineering is responsible for defining projects. Once defined, Projects is responsible for the design and execution of capital-funded projects.

\section{Organization and Cost Account Management}

Cost account managers are responsible for assuring that their assigned scopes of work are carried out on schedule and within budget. Table 7.5-1 depicts the organization breakdown, the Upgrades cost accounts, and the cost account managers. The chart only shows part of the TWRS organization, but it does trace each cost account manager back to the TWRS level.

\subsubsection{FY 1995 Work to be Performed}

The FY 1995 work scope is summarized in this section based on the FY 1995-1997 Multi-Year Work Plan.

\section{Technical Integration and Planning (1.1.1.2.03.01)}

The work to be performed by this activity is comprised of three segments, as shown in Section 7.5.1.1.2. of this plan. These are: (1) program management, (2) baseline management, and (3) systems engineering. This work is necessary to plan, coordinate, and manage the Upgrades program and provide support to the O\&M Program by facilities and infrastructure.

The Program Management segment, in coordination with O\&M, develops priorities for the upgrades necessary to support the Tank Farm restoration and upgrades to the aging facilities and equipment. Also, Program Management ensures that the Upgrades program work is integrated with other Tank Farm programs in support of the overall TWRS Mission. 
Baseline Management is necessary to plan, develop, coordinate, and report on the baseline (cost, schedule, technical) in support of the Upgrades Program Mission for the Multi-Year Work Plan for FY 1995. This work includes developing, monitoring, and reporting of the baseline status on a monthly, quarterly, and annual basis, as required by DOE. The deliverables for this segment of program management are:

- Annual update of the Multi-Year Work Plan (MYWP)

- $\quad$ Annual update of the Activity Data Sheets (ADS)

- Annual update of the Program Validation for Upgrades Program.

The Systems Engineering segment of this activity guides the programmatic strategy and development of the systems engineering approach to the Upgrades program and projects in support of the Defense Nuclear Facilities Safety Board (DNFSB) initiative on the systems engineering approach to program management. This activity supports the programmatic aspect of systems engineering and works closely with the TWRS Systems Engineering group in support of the TWRS Mission. The deliverables for FY 1995 are:

- $\quad$ Prepare a Technical Strategy for Instrumentation Upgrades

- $\quad$ Prepare a Technical Strategy for Ventilation Upgrades

- $\quad$ Prepare a Technical Strategy for Electrical Upgrades

- $\quad$ Prepare a Technical Strategy for Transfer Lines.

The above mentioned strategies will be developed, and updated annually, using both WHC engineering resources and external consultants.

The productivity challenge will be achieved in this activity by utilizing some of the following methods listed below. It should be noted that while this activity plans to utilize these methods, plans may change at the time the work is executed (i.e., who does the work and how the work is done).

- ICF Kaiser Hanford Company (ICF KH) versus WHC versus outsourcing of work

- Strategic alliances with vendors

- $\quad$ Sub-contractor Technology Infusion (STI)

- Upfront review of methods/approach (fund work, not people)

- Equipment standardization (size, type, manufacturer, spares)

- Utilize fixed price contracts and incentive clauses

- $\quad$ Eliminate non-productive capabilities (i.e., seldom used labor types)

- Value Engineering on projects

- Government-Owned Contractor-Operated (GOCO) Savings-Through-Sharing program

- Information exchange and integration (i.e., mixer pumps).

\section{Configuration Management (CM) (1.1.1.2.03.02)}

The work to be performed by the Field Verification activity is to computer-aided design and drafting (CADD) convert, field verify, and release into the site-wide drawing archive system Essential and Support drawings identified in the Tank Farm Essential Drawing Plan, WHC-SD-WM-PC-002. Drawings are field verified in accordance with Engineering Drawing Field Verification program, WHC-SD-WM-WP-072. 
The work to be performed by the CM Database segment of this activity is to initiate a facilitated session with representatives from Configuration Management, Engineering, Maintenance, and others to determine user needs and which data sets are required to establish a CM database. This includes determining requirements for the CM Database and data sets. The CM Database will link component numbers with Engineering Change Notices (ECNs), Master Equipment Lists (MELs), Safety Analysis Reports (SARs), Operational Safety Requirements (OSRs), vendor files, procedures, spare parts, etc.

The work to be performed by the CM Implementation segment of this activity is to issue a Configuration Management Implementation Action Plan that:

- Implements a Configuration Management (CM) program for Tank Farms and the 242-A Evaporator Facility.

- $\quad$ Meets the intent of WHC-SD-WM-CM-008, Configuration Management Plan for Waste Tank Farms and 242-A Evaporator of TWRS, which is based upon DOE-STD-1073-93, Guide For Operation Configuration Management Program.

Also, work to be performed by the CM Implementation segment of this activity is to issue a Supporting Document (SD) that lists identified systems (process, transfer, water, air, etc.) with their boundaries (beginning and end points) for Tank Farms and the 242-A Evaporator.

Work to be performed by the Design Basis Reconstitution segment of this activity is to issue a Design Base Reconstitution (DR) Program Implementation Action Plan that:

- Implements a Design Base Reconstitution (DR) program for Tank Farms and the 242-A Evaporator Facility.

- $\quad$ Meets the intent of WHC-SD-WM-CM-009, Design Reconstitution Program Plan for Waste Tank Farms and the 242-A Evaporator of Tank Waste Remediation System, which is based on DOE-STD-1073-93, Guide for Operation Configuration Management Program.

Other work to be performed under the $\mathrm{CM}$ activity is to prepare or revise all required operating procedures for turnover of upgraded equipment for approved upgrade projects identified in the TWRS baseline. This cost account only funds the actual preparation and approval of the procedures including the preparation/revision of the first procedure draft, the table-top discussion and comment incorporation, writer field verification and comment incorporation, and the approval cycle of the procedure. This cost account does not fund activity or reviews by engineering or oversight organizations required to support the issuance of the procedure.

The following upgrade projects have been identified that will require procedure support for FY 1995: CR Motor Control Center, BX/BY Compressor, SY Diesel Generator, TX/TY Compressor, 701-A Motor Control Center, 701-A Compressor, 242-S Liquid Waste Monitoring System, 241-AP Closed Loop Cooling, 242-S Alarms, 241-T Compressor, 241-U Compressor, and 241-T Alarms. 


\section{Life Management (1.1.1.2.03.03)}

Detailed descriptions of the work to be done over the life of the program can be found in WHC-SD-WM-PLN-068, Rev. 0, Plan for the TWRS Life Management/Aging Management Program. This program plan describes the work to be performed for each of the seven program tasks identified earlier in Section 7.5.1.1.2. In FY 1995, the work will focus on:

- Evaluation of Degradation Mechanisms

- Corrosion program management

- $\quad$ DST crack growth rate experiments

- DST pitting/waterline attack experiments

- Technical reports (hydrogen embrittlement, uniform, microbiologically-induced corrosion, etc.)

- $\quad$ Support facilities assessment

- Mitigation Upgrades and Retirement Strategy Development

- $\quad$ DST Corrosion Control Upgrades - Cathodic Protection System.

\section{Multi-Function Waste Tank Facility, W-236A (1.1.1.2.03.04)}

Work to be performed during FY 1995 is as follows:

- $\quad$ Continue project management

- $\quad$ Continue Title II Design

- Initiate Title III Inspection

- Initiate $\mathrm{KD}-3 \mathrm{~A}$ construction contracts:

- 200 East demolition

- 200 East/West construction trailers

- 200 East civil site preparation

- 200 East administration building

- 200 East/West tank erection

- 200 East/West construction fence

- Initiate $\mathrm{KD}-3 \mathrm{~B}$ construction contracts:

- 200 East/West construction substation and site electrical

- $\quad 200$ East Tank Farm steam and condensation

- 200 West support facilities, tank farm completion and weather enclosure

- Initiate all government-furnished equipment (GFE) procurement

- Complete systems design descriptions 
- Continue the operations/regulatory overview of detailed design

- Continue support of the environmental/safety documentation

- $\quad$ Support outside agency reviews

- Continue preparation of operating documentation procedures

- $\quad$ Continue selected materials testing.

\section{Instrumentation \& Control/Electrical Upgrades (1.1.1.2.03.06)}

The FY 1995 tasks to be performed by the I\&C/Electrical Upgrades include the following:

- Perform electrical assessments and resolve safety issues.

- Upgrade the 242-S Evaporator control room alarm panels.

- Install new ENRAF level monitors on 64 tanks.

- Expand TMACS to the A, AX, and U Tank Farms.

- Automate data collection rounds in C, S, SX, SY, and TY Tank Farms.

- Install, test, and turn over the 701-A Motor Control Center.

\section{Tank Farm Restoration and Safe Operations MSA, Project W-314 (1.1.1.2.03.07)}

Engineering studies and the consolidated functions and operating requirements (F\&OR) for this project have been completed. Work has begun through a Basic Ordering Agreement (BOA) contract with a team of off-site $\mathrm{A} / \mathrm{E}$ personnel to develop the design requirements baseline for the project. The design requirements baseline is now required, under the TWRS systems engineering process, to request KD-1. KD-0 has been requested for design only, and has been withheld pending approval of the JMN by DOE-HQ. There are no open comments against the JMN, and approval has been recommended.

WHC will proceed with pre-concept development work prior to receipt of KD-0, but delays in receipt of KD-0 beyond October 1, 1994, will jeopardize completion of the Tri-Party Agreement milestones for the completion of conceptual design (M-43-02A and M-43-04A, May 1995).

The largest activity for Project W-314 will be to create the design requirements baseline for the project. This baseline is necessary in preparation for requesting KD-1, to be in compliance with the TWRS systems engineering process and documentation, and in taking the next step toward this project development. This design requirements baseline will consist of a number of supporting deliverable documents:

- Further development and expansion of detail in the existing Project Functions and Operational Requirements Document (a revision to the document)

- The concept development package, including drawings, sketches, analyses, calculations, and specifications

- A logistics plan 
- Energy/mass flow sheets

- An expanded and detailed development of the existing Project-Specific Interface Control Document, by revision to the document

- Design specification, a specification, which with proper procurement documentation could be used to select an $\mathrm{A} / \mathrm{E}$ for definitive design

- $\quad$ Trade studies and decision analysis

- Test and Evaluation Plan

- Waste Management Plan

- Cost estimates and schedules for the balance of the project

- WBS for the balance of the project.

A formal design requirements review will also be performed on the design requirements baseline. An A/E will be selected for the definitive design process, a contract will be awarded, and advanced conceptual design will begin in FY 1995. Safety analysis and documentation will be developed and released to accompany the design requirements baseline developed by the BOA team, in support of request for $\mathrm{KD}-1$. This analysis work will be performed by WHC forces. NEPA documentation will be accomplished as a part of the TWRS EIS, and subsequent ROD on the EIS. Permitting plans under the Clean Air Act will also be developed in FY 1995. For a specific and complete list of deliverables, see Section 7.5.1.6.

Specifically not authorized in the activity (and cost account) is the development of Programmatic Systems Engineering documentation. This project will be the prototype project and must interface with the rest of the program-specific systems engineering documentation. Interface and liaison activities from Project W-314 are part of the scope, but creating the program-specific documentation is not. Those activities are a part of the Technical Integration and Planning Activity.

\section{Mechanical Upgrades (1.1.1.2.03.09)}

The FY 1995 tasks to be performed by Mechanical Upgrades include the following:

1. Mechanical Upgrades (N3K95)

- 241-A-701 compressed air system upgrades Install, conduct testing, and turn over

- 241-TX/TY modular compressed air system upgrade Design, install, conduct testing, and turn over

- 241-AP closed loop cooling system Install, conduct acceptance testing, and turn over 
- $\quad$ 242-S Liquid waste minimization

Install effluent elimination measures, conduct testing, and turn over

- $\quad$ BX/BY compressor

Conduct operational testing and turn over

$\because$

- 241-T Compressor

Conduct operational testing and turn over

- 241-U Compressor

Conduct operational testing and turn over

2. Long-Length Contaminated Equipment Disposal (N3K94)

- FY 1995--Develop waste generator characterization plan

- $\quad$ FY 1995--Develop LLCE process path document

- $\quad$ FY 1995-Prepare cold test facility

- Component removal equipment modifications

FY 1995--Design, fabricate, and write operating procedures

- $\quad$ LLCE Packaging Equipment (Containers)

FY 1995--Design, fabricate, develop Safety Analysis Report for packaging

- $\quad$ LLCE Transport system

FY 1995--Procure and test

- $\quad$ Evaluate Regulatory Alternatives

FY 1995--Treatment by generator

- $\quad$ Assess and modify safety documents

FY 1995--Complete

3. Engineered Tank Farm Hardware (N3K97)

- Transfer pump development

FY 1995--Design, fabricate prototype, off-site Acceptance Test Procedure

- Sponsor GOCO pump users technology exchange

- $\quad$ Partner with Washington State University to develop the Adjustable Pump Intake Level Device (APILD) 


\section{Long-Length Waste Equipment Storage Facility (W-373)}

In FY 1995, Upgrades program will provide capital work order funding to assist the Solid Waste Disposal program in the construction of a permitted greater-than-90-day storage pad. This pad will provide enough space for near-term storage needs of long-length contaminated equipment remøved from waste tanks.

5. Long-Length Contaminated Equipment Empty Container Storage Facility (W-374)

In FY 1995, a fenced storage yard will be constructed to provide a regulated storage area for empty LLCE transport/storage containers, retrieval equipment, and transport equipment.

\section{Ventilation Upgrades, W-030 (1.1.1.2.03.10)}

FY 1995 work to be performed by this activity includes completing MICON (monitor and control) system logic diagrams and initiating software development.

A fixed-price construction contract will be awarded and construction initiated for a ventilation building, diesel generator building, control building, evaporative cooling towers, and four recirculation modules.

Construction will be initiated in AY/AZ Tank Farms to lay yard piping for the ventilation and recirculation system, complete the trench system for power and water, install inlet air stations on all four tanks, and set the recirculation modules on concrete pads. Utility and waste disposal support will be provided. A draft Final Safety Analysis Report will be completed and safety questions answered. Startup, ALARA, operational readiness review, and tie-in plans will be prepared; a records system established; and startup/operations procedures initiated. Engineering and inspection will be provided, including field support. Project management will provide integration for startup, scheduling/cost control, and quality assurance.

\section{Cross-Site Transfer System, W-058 (1.1.1.2.03.11)}

FY 1995 work to be performed by this activity includes environmental support and Notice of Construction reviews, as a part of permitting activities. Title II (detailed definitive) design, and its corresponding activities, software development, PSAR amendments, design reviews, and project management will be performed. EIS interface activities, construction contractor selection, and longlead procurement will occur in preparation for construction and KD-3, which authorizes construction. KD-3 support activities occur in late FY 1995.

\section{Aging Waste Transfer Line, W-028 (1.1.1.2.03.12)}

Definitive design, and supporting activities including PSAR Amendments will occur in FY 1995.

See Cross-Site Transfer System W-058 (1.1.1.2.03.11). 


\section{Facility Upgrades (1.1.1.2.03.16)}

Ongoing projects to be completed in FY 1995 include: Project W-322, Replace 242-S Substation; Project W-364, Upgrade 209-E Septic Tank System; Project W-280, Install Lighting System to Provide 5-foot Candle Light Within SY and C Tank Farms. The design work for these three GPPs will be completed in FY 1994.

Design and construction of Project W-414 will begin in FY 1995 to install a lighting system for BX and BY Tank Farms. The design work for Project W-415 will be completed in FY 1995 to install a lighting system for S and SX Tank Farms. Also to be completed in FY 1995 are the FY 1996 Candidate Project Support Sheets, FY 1997 Project Data Sheets, FY 1996 Functional Design Criteria and Conceptual Design Reports, and FY 1997 Engineering Studies.

\section{Radiological Support Facility, W-188 (1.1.1.2.03.17)}

N/A, see Program Strategy (Section 7.5.1.1.2).

\subsubsection{FY 1996 - FY 1997 Work to be Performed}

\section{Technical Integration and Planning (1.1.1.2.03.01)}

The activities in Section 7.5.1.3 are ongoing in subsequent fiscal years and will be continued and improved through lessons learned and productivity improvement. Additional deliverables which have been identified for FY 1996 for Systems Engineering are:

- Prepare a Technical Strategy for Life Extension

- $\quad$ Prepare a Technical Strategy for Configuration Management

Additional deliverables will be developed once the initial systems engineering effort is implemented and specific actions are identified to effect improved integration both within the program and within TWRS.

\section{Configuration Management (1.1.1.2.03.02)}

\section{Field Verification}

Work to be performed for the Field Verification segment of this activity in FY 1996 is to CADD convert, field verify, and release into the site-wide drawing archive system Essential and Support drawings identified in the Tank Farm Essential Drawing Plan, WHC-SD-WM-PC-002. Drawings are field verified in accordance with Engineering Drawing Field Verification Program, WHC-SD-WM-WP-072.

In FY 1997, this activity will conduct an assessment to determine the effectiveness of the program. The Field Verification activity had been tasked with CADD converting and field verifying tank farm Essential and Support drawings listed in WHC-SD-WM-PC-002. 


\section{Database}

Work to be performed for the CM Database requirements segment of this activity in FY 1996 is to develop an action plan derived from the results and recommendations of the facilitated session in FY 1995.

In FY 1997, a working CM Database will be established based on the FY 1995 facilitated session recommendations and the action plan developed in FY 1996.

\section{Implementation}

Work to be performed for CM Implementation segment of this activity in FY 1996 is to issue a Structures, Systems and Components (SSC) List Supporting Document (SD) that:

- $\quad$ Meets the requirements of WHC-SD-WM-CM-008, Configuration Management Plan for Waste Tank Farms and 242-A Evaporator of TWRS.

- Meets the requirements of WHC-SD-WM-CM-009, Design Reconstitution Program Plan for Waste Tank Farms and the 242-A Evaporator of Tank Waste Remediation System.

- $\quad$ Provides a listing of the structures, systems and components (SSC).

- Grades the SSC list by applying the "Grading Criteria" developed in the supporting document from the FY 1995 Milestone Control Number N3C310707M to grade the SSCs.

The FY 1997 scope of work will be performed to the resource loaded schedule of the Implementation Configuration Management (CM) Action Plan developed under FY 1996 Milestone Control Number N3C312506M. This milestone will implement WHC-SD-WM-CM-008, Configuration Management Plan for Waste Tanks and 242-A Evaporator of TWRS.

\section{Design Base Reconstitution Implementation}

Work to be performed for the Design Base Reconstitution segment of this activity for FY 1996 is to issue revisions to the Implementation Action Plans for:

- The Design Base Reconstitution Program Implementation Action Plan developed from the FY 1995 Milestone Control Number N3C312503M.

- The CM Program Implementation Action Plan developed from the FY 1995 Milestone Control Number N3C310130M.

- $\quad$ Resolve any "To Be Determined" (TBD) actions.

The FY 1997 scope of work will be performed to the resource loaded schedule of the Implementation Design Reconstitution (DR) Implementation Action Plan developed under FY 1996 Milestone Control Number N3C312506M. This milestone will implement WHC-SD-WM-CM-009, Design Reconstitution Program Plan for Waste Tank Farms and the 242-A Evaporator of Tank Waste Remediation System. 


\section{Life Management (1.1.1.2.03.03)}

Work to be performed during FY 1996 and FY 1997, will largely be a continuation of the work initiated in FY 1995. This activity can expect more work under the heading of Mitigation Upgrades and Retirement Strategy Development as more information regarding the existing condition of separate tank system components is discovered through examination and analysis.

\section{Multi-Function Waste Tank Facility, W-236A (1.1.1.2.03.04)}

Work to be performed during FY 1996-1997 will largely be a continuation of work initiated in FY 1994 and FY 1995:

- Continue project management

- Complete detailed design

- Continue Title III Design/Acceptance Inspection

- Continue procurement actions

- Continue construction activities

- Initiate construction contracts on:

- 200 East/West jumper fabrication

- 200 East support facility, tank farm completion, and weather enclosure

\section{Instrumentation \& Control/Electrical Upgrades (1.1.1.2.03.06)}

Work to be performed during FY 1996 - FY 1997 includes:

- $\quad$ Resolve remaining TWRS electrical safety issues.

- Install instrument and alarm panel upgrades.

- Install new ENRAF level monitors on remaining tanks.

- Expand TMACS to the remaining tank farms.

- Automate data collection rounds in remaining farms.

- Install, conduct testing, and turn over the 101-SY Diesel Generator.

- Install, conduct testing, and turn over the T-complex alarm upgrade.

- Install, conduct testing, and turn over the 271-CR motor control center.

Tank Farm Restoration and Safe Operations MSA, Project W-314 (1.1.1.2.03.07)

Work to be performed includes completion of advanced conceptual design and as-building of selected drawings in FY 1996. Title I design will begin in FY 1996 and be completed in FY 1997, pending approval of KD-1. Title II design will begin in FY 1997, pending receipt of KD-2. 
WHC-SP-1101

\section{Mechanical Upgrades (1.1.1.2.03.09)}

Work to be performed during FY 1996 and FY 1997 includes:

1. Mechanical Upgrades

- $\quad$ East area steam line upgrades

FY 1997--Design starts

- West area steam line upgrades

FY 1997-Design starts

- East/West change trailer modifications

FY 1996-Complete modifications

2. Long-Length Contaminated Equipment (LLCE) Disposal

- Component removal equipment modifications

FY 1996-Complete fabrication and operating procedures, test and turn over

- Field cut-up equipment

FY 1997--Design

- LLCE Packaging Equipment (Containers)

FY 1996--Complete fabrication and SARPs, test

- LLCE Transport system

FY 1996--Complete testing

- $\quad$ Evaluate Regulatory Alternatives

FY 1996-Additional alternatives

3. Engineered Tank Farm Hardware

- Transfer pump development

FY 1996--Complete fabrication and ATP, perform on-site QTP, begin procurement of plant units

FY 1997--Procure plant units

- Sponsor GOCO pump users technology exchange

\section{Ventilation Upgrades, W-030 (1.1.1.2.03.10)}

The MICON monitor and control system software will be completed in FY 1996. Revisions to the draft FSAR will continue through FY 1996, and in FY 1997, the final FSAR will be issued. In FY 1996, the new stacks and sources will be registered with the state regulators and a notice of startup provided. 
Fixed-price construction will continue and be completed in FY 1996. Construction forces will continue construction activities and complete work in FY 1996, except final tie-ins which will continue through early FY 1997. Operational construction support (HPTs, operator, person-incharge, planners) will continue through FY 1996 and be completed in early FY 1997.

Engineering and inspection will include field support during construction and completion of acceptance test procedure documents in FY 1996. In FY 1997, all documents and as-builts will be completed and turned over to operations.

Startup testing will be initiated in FY 1996 and completed in FY 1997. The operational test procedures, operational readiness reviews, maintenance and operating procedures, and tie-in plan will be completed in FY 1996. Operational tests will continue through FY 1996 and complete in FY-97. In FY 1997, the system will be turned over to operations, and the project will assist with training.

Project management will provide integration for startup, scheduling/cost control, and quality assurance.

\section{Cross-Site Transfer System, W-058 (1.1.1.2.03.11)}

Final safety analysis, engineering inspection, and construction will occur in the FY 1996 and 1997 time frame. Project management and other necessary supporting activities to construction will also occur.

\section{Aging Waste Transfer Line, W-028 (1.1.1.2.03.12)}

See Cross-Site Transfer System, W-058 (Section 1.1.1.2.03.11).

\section{Facility Upgrades (1.1.1.2.03.16)}

Construction of Project W-415 to upgrade the S and SX Tank Farm lighting and design, and construction of T Farm lighting upgrade (Project TBD) are scheduled for FY 1996. Design of the A-complex lighting upgrade (Project TBD) will be completed in FY 1996. Design and construction of the Tank Farm Repackaging Facility at 209 E (Project W-416) and the Solid Waste Management Facility at 209 E (Project TBD) will take place in FY 1996.

Construction of A-complex lighting is scheduled for FY 1997, as well as, design and construction of TX and TY Farm lighting upgrade (Project TBD) and U Farm lighting upgrade (Project TBD). The design and construction of the East/West Central Control Facility Monitoring (Project TBD) and the Core Sampling and Storage Facility (Project TBD) is also scheduled for FY 1997.

\section{Radiological Support Facility, W-188 (1.1.1.2.03.17)}

N/A, see Program Strategy (Section 7.5.1.1.2). 


\subsubsection{Approach}

Technical Integration and Planning (1.1.1.2.03.01)

The Upgrades Program is responsible for developing and implementing an approach for cost-effective upgrades and enhancements to the safe operation of the Tank Farms, pursuant to the store waste function (4.2.1.1) and the transfer waste function (4.2.1.4).

Prioritized upgrades will be performed for systems, equipment, and infrastructure in support of the Tank Farm Operations and Maintenance mission and needs. All upgrades are coordinated with the plant and are carried out on an integrated basis within TWRS for a safe waste storage condition until disposal.

\section{Configuration Management (1.1.1.2.03.02)}

The Tank Farm and Evaporator Configuration Management activities defined in this document including WHC-SD-WM-CM-008, Configuration Management Plan for Waste Tank Farms and the 242-A Evaporator of TWRS, comply with the WHC configuration management requirements established in WHC-CM-1-3, Management Requirements Procedures, MRP N.N, "Configuration Management" (Draft). In addition, the WHC Configuration Management program of WHC-CM-1-3 aligns with the criteria established in DOE-STD-1073-93, while complimenting the requirements of DOE 4700.1, Project Management System, DOE 5700.6C, Quality Assurance, RLIP-4700.1A, Project Management System, and RLID 5000.12, Richland Field Office-Hanford Site Management System.

The intrinsic purpose of configuration management is to provide programmatic assurance that facility documents support facility physical configuration for the life of the facility, increases productivity and efficiency, and reduces project, operating, and maintenance costs.

DOE-STD-1073-93 defines the configuration management program by the five basic program elements of program management, design requirements, document control, change control, and assessments; and the two adjunct recovery programs of design reconstitution and material condition and aging management. The CM model of five elements and two adjunct programs strengthens the necessary technical and administrative controls to establish and maintain a consistent technical relationship among the requirements, physical configuration, and documentation.

The configuration management activities that have been presented in this document endorse DOE-STD-1073-93. They have been tailored specifically to address the technical relationship of requirements, physical configuration, and documentation during the full life cycle of the Tank Farms and 242-A Evaporator of TWRS.

The configuration management activities thus presented are administrative in nature and may require the services of a CM consultant who is conversant with $\mathrm{CM}$ databases. Otherwise, the field verification of drawings, labeling, Master Equipment List, configuration management, and design reconstitution implementation plans are being done by WHC personnel.

The approach to be used in the preparation of procedure packages will be to use logic ties to the start of the project turnover. Start dates and resource requirements will then be managed to achieve the best available productivity. By tying the procedure preparation to the upgrade project logic, resources 
will be focused and a team concept can be utilized to produce a specific product that can be tied to a critical path and managed accordingly. Productivity will also come about when procedures are prepared for similar types of equipment (compressors being upgraded at different tank farms) due to the inherent similarities and reduced learning curve that will be required to initially produce the procedures.

\section{Life Management (1.1.1.2.03.03)}

Life Management activities will be performed by technical experts and panels; contracted services for structural analysis, cathodic protection upgrade design and laboratory experiments; and through interfacing with Safety Analysis and Configuration Management.

\section{Multi-Function Waste Tank Facility, W-236A (1.1.1.2.03.04)}

WHC has established a Project Office to accomplish its work on the MWTF Project in an expeditious and cost-effective manner, with appropriate attention to quality and safety. The Project Office, in its capacity as the Integrating Contractor, is responsible for, and supports the RL Multi-Function Waste Remediation Facility (MRF) Project Office by supplying technical direction, cost and schedule management, control, integration, quality assurance, applied technology, safety/regulatory compliance support, and startup and operations activities. The Project Office manages and integrates the $\mathrm{A} / \mathrm{E}$, Technology, Construction Management, and WHC support organizations.

The work performed by each participant will be coordinated, guided, technically monitored, and reviewed for compliance to requirements by the Project Office as the Integrating Contractor. The Project Office will be responsible for preparing Letters of Instruction (LOI), or other appropriate documentation for the A/E, Construction Management, and other participants. This is further detailed in the Project W-236A, Multi-Function Waste Tank Facility Management Plan, WHC-SD-W236A-MP-001, Rev.0.

WHC, who will ultimately have responsibility for operation of the MWTF, has been assigned the responsibility for successfully managing the project within cost, schedule, and technical baselines. RL has a Cost Plus Award Fee (CPAF) contract (\#DE-AC-06-87RL10930) with WHC. ICF KH, the onsite Engineering/Construction Contractor $(E / C)$, is the $A / E$ and Construction Manager for the MWTF. RL has a Cost Plus Award Fee (CPAF) contract (\#DE-AC-06-87RL10900) with ICF KH. ICF $\mathrm{KH}$ performs constructibility reviews to determine the most efficient and cost-effective method of packaging design into construction packages for subcontracting. To the maximum extent possible, subcontracts will be awarded on a fixed-price competitive bid basis. Small business, disadvantaged business, and labor surplus area concerns will be given appropriate consideration consistent with DOE policy.

Acquisition of certain project equipment will be initiated soon after the start of construction to ensure compliance with schedule baselines. These procurements include standard equipment and engineered equipment. Contracts for these items will be awarded by the $\mathrm{E} / \mathrm{C}$ on the basis of competitive fixedprice bids whenever practicable.

\section{Instrumentation \& Control/Electrical Upgrades (1.1.1.2.03.06)}

TWRS Engineering is the lead organization responsible for developing the technical strategy and assuring individual activities are implementing the strategy. Since the I\&C and Electrical technical 
work must integrate into the overall plan being developed by Project W-314, the engineering staff will remain closely cognizant of the MSA design and provide "lessons learned" feedback to the MSA organization.

\section{Tank Farm Restoration and Safe Operations MSA, Project W-314 (1.1.1.2.03.07)}

$\therefore$

WHC Projects is the lead organization responsible for accomplishing this work, in accordance with the TWRS Draft Systems Engineering Work Plan. In accordance with the Project acquisition strategy, a team of off-site $\mathrm{A} / \mathrm{E}$ personnel under a basic ordering agreement has been retained to develop the Project systems engineering documentation and the Design Requirements Baseline. Both WHC and ICF KH personnel will work closely with the A/E to prepare the baseline.

Advanced Conceptual Design, Title I, and Title II design will be performed by an off-site A/E to be selected through the competitive bid process. Again, WHC and ICF KH personnel will work closely with the $\mathrm{A} / \mathrm{E}$ to assure the design is well managed, cost-effective, and technically adequate. Safety analysis support will be provided by WHC personnel. NEPA documentation will be provided by being included with the TWRS Environmental Impact Statement. As-building of selected drawings to support Title I and II design will be performed by a joint team of $\mathrm{A} / \mathrm{E}$ and WHC personnel. For profiles of the staff totals and skills mixes, see Section 7.5.3.2.

\section{Mechanical Upgrades (1.1.1.2.03.09)}

Mechanical Upgrades is approaching the system upgrades by first writing ETPs to identify and negotiate agreement on cost, scope, schedule, responsibilities, and deliverables for an upgrade task. Next, design basis information is established since much of it is either non-existent or outdated due to after-construction system modifications. Designs for new installations are being developed both by WHC design engineers and by ICF KH. Installation is performed by ICF KH, and testing by a combination of Westinghouse and ICF KH personnel. Turn over to facility operations is accomplished through the "Acceptance for Beneficial Use" process.

Thorough up-front planning increases productivity by eliminating un-needed and redundant work, reducing errors, assuring that the product delivered is the product needed, and assuring optimal use of concurrent engineering.

\section{Ventilation Upgrades, W-030 (1.1.1.2.03.10)}

The organizational responsibilities and baseline controls for management of the project are contained in the Project Management Plan (PMP), WHC-SD-W030-PMP-030. Specific sections have been taken from the PMP to assist the reader of this work plan:

Designs provided by this project shall comply with ALARA principles and with current state, federal, and DOE regulations governing the monitoring, release, and disposal of liquid and gaseous effluents. The W-030 upgrades shall be designed and installed to provide maximum protection to construction and operating personnel while safely maintaining the AY and AZ Tank Farm operations. The technical basis for the work is the Functional Design Criteria (FDC), SD-600-FDC-001, and the Supplemental Definition of Requirements, WHC-SD-W030-RD-001. Approved drawings, specifications, and other design media developed and approved during definitive design supplement the FDC as controlling technical documents. 
The following actions have been specifically taken to increase productivity, increase efficiency, and reduce costs:

- Combined the Project W-320 and this project's fixed-price work scope into one contract to accelerate the procurement schedule and reduce procurement and construction costs.

- Startup activities are centrally coordinated within WHC Construction Projects. Startup activities for this project will be coordinated with the central group. Utilizing the startup administrative procedures developed by Project W-320 will assist this project with its startup plans and reduce cost.

Principal project participants are the following:

WBS Element

1.2 Engineering/Inspection

3.1 Construction Forces

3.2 Fixed Price Construction

3.3 Plant Forces Construction

4.0 Project Management
Method of performance

ICF KH

ICF KH CPAF

Fixed price contractors

WHC

WHC

RL Tank Waste Projects and WHC Tank Farm Upgrades is apprised of any impacts to work costs or schedules that occur via monthly management review meetings. Operations support (HPTs, operator, $\mathrm{PIC}$, planners) is being coordinated via WHC project and operations management. RL will be required to support operational readiness and assist with obtaining state regulator approval for startup.

For profiles of the staff totals and skill mixes, see Section 7.5.3.2.

Cross-Site Transfer System, W-058 (1.1.1.2.03.11)

Design is being performed by a team of WHC and ICF KH personnel, with construction being performed by an off-site contractor. Engineering inspection and site forces support activities will also be performed by WHC and ICF KH personnel.

\section{Aging Waste Transfer Line, W-028 (1.1.1.2.03.12)}

See Cross-Site Transfer System, W-058 (Section 1.1.1.2.03.11).

\section{Facility Upgrades (1.1.1.2.03.16)}

WHC Projects is the lead organization responsible for accomplishing this work. The method of performance for each General Plant Project will be developed during the project planning phase. To the extent practical, construction and procurement will be accomplished by fixed-price contract, and subcontracts will be awarded on the basis of competitive bid.

For profiles of the staff totals and skills mixes, see Section 7.5.3.2.

\section{Radiological Support Facility, W-188 (1.1.1.2.03.17)}

N/A, see Program Strategy (Section 7.5.1.1.2). 


\subsubsection{Drivers and Deliverables}

\section{Technical Integration and Planning (1.1.1.2.03.01)}

The drivers for this activity are centered around DOE Order 4700.1, RLIP 4700.1A, and the WHC Management Control System (MCS). The MCS calls for program and project management which fosters objectives, accountability, stewardship, visibility and performance assessment. Other drivers do drive the Upgrades program, but are not specific to this activity (i.e., Tri-Party Agreement, Safety Initiatives).

The deliverables for this activity were covered in the aforementioned Sections 7.5.1.3, FY 1995 Work to be Performed and 7.5.1.4, FY 1996 - FY 1997 Work to be Performed.

\section{Configuration Management (1.1.1.2.03.02)}

The drivers for this activity are centered around WHC-SD-WM-CM-008, "Configuration Management Plan for Waste Tank Farms and the Evaporator of Tank Waste Remediation System." This CM plan complies with the WHC Configuration Management requirements established in WHC-CM-1-3, Management Requirements and Procedures, MRP N.N, "Configuration Management."

The following deliverables will be provided by the Configuration Management activity:

- FY 1995 Field Verification Activity

- Complete Scope Planning

- Complete Task Planning

- $\quad$ Field Verify Minimum of 150 Essential/Support Drawings

- FY 1996 Field Verification Activity

- $\quad$ Field Verify Minimum of 175 Essential/Support Drawings

- $\quad$ Provide Letter of Completion to DOE-HQ

- FY 1997 Field Verification Activity

- $\quad$ Program Assessment and Ongoing Support

- Issue White Paper

- FY 1995 Configuration Management Database Activity

- $\quad$ Conduct Facilitated Evaluation Session To Determine:

- User Needs

- CM Database Requirements

- Data Set Requirements

- Issue "Results" Supporting Document

- FY 1996 Configuration Management Database Activity

- Issue CM Database "Action Plan" 
- FY 1997 Configuration Management Database Activity

- Develop CM Database

- FY 1995 Configuration Management/Design Reconstitution Implementation

- Develop CM Implementation Action Plan

- $\quad$ Develop CM Systems and Boundaries List

- $\quad$ Develop CM and DR Grading Criteria

- Review CM Implementation Action Plan

- $\quad$ Issue CM Implementation Action Plan

- $\quad$ Review CM Systems and Boundaries List

- $\quad$ Issue CM Systems and Boundaries List

- $\quad$ Review CM and DR Grading Criteria

- $\quad$ Issue CM and DR Grading Criteria

- Develop DR Implementation Action Plan

- Review DR Implementation Action Plan

- Issue DR Implementation Action Plan

- $\quad$ Budget Planning for FY 1996, 1997, 1998

- FY 1996 Configuration Management/Design Reconstitution Implementation

- $\quad$ Review Current SSC SD Documents

- Identify Components To Add To SSC SD

- Develop SSC SD or ECN Existing Systems-Structures Document

- Issue SSC SD

- $\quad$ Update/Revise CM and DR Implementation Action Plan

- $\quad$ Review Updated/Revised CM and DR Implementation Action Plan

- $\quad$ Issue Updated/Revised CM and DR Implementation Action Plan

- $\quad$ Budget Planning for FY 1997, 1998, 1999

- FY 1997 Configuration Management/Design Reconstitution Implementation

- Work CM Implementation Action Plan

- Work DR Implementation Action Plan

- $\quad$ Budget Planning for FY 1998, 1999, 2000

Procedures for upgrades equipment will be prepared using DOE Order 5480.19, Conduct of Operations, standards and have been identified by TWRS Plant Engineering as impacted by the upgrade project. The procedure packages will be deliverables which are finish-to-start logic tied to the start of the upgrade project turnover. No separate milestone will be developed; however, critical path analysis will be used to manage the delivery of the procedure packages to prevent impacting project turnover.

\section{Life Management (1.1.1.2.03.03)}

TWRS Systems Engineering and Tri-Party Agreement productivity improvement goals drive the Life Management activity. 
This activity will provide the following deliverables:

- Corrosion monitoring and control systems

- Analyses of Record for each DST, support facility, and transfer system

- Useful life estimates

Multi-Function Waste Tank Facility, W-236A (1.1.1.2.03.04)

The following major schedule objectives are the primary drivers for the project:

Activity
Preliminary Design (Title I)
Detail Design (Title II)
Construction
200 West Area
200 East Area
Operations Test
200 West Area
200 East Area
Hot Startup
200 West Area
200 East Area

$\underline{\text { Start }}$

January 1993

2nd Qtr FY 1994

4th Qtr FY 1994

3rd Qtr FY 1995

2nd Qtr FY 1997

1st Qtr FY 1999

2nd Qtr FY 1998

1st Qtr FY 2000
Complete

4th Qtr FY 1994

2nd Qtr FY 1996

2nd Qtr FY 1997

1st Qtr FY 1999

2nd Qtr FY 1998

1st Qtr FY 2000

\section{Instrumentation \& Control/Electrical Upgrades (1.1.1.2.03.06)}

Operational and safety considerations produce requirements which define the scope of the I\&C and Electrical Upgrades. In addition, external oversight organizations have a need to review the continuing status of the farms. The data acquisition and information display systems supplied through the I\&C/Electrical Upgrades activities provide consolidated information and analysis results to interested parties. As new instruments and additional monitoring or control requirements are developed, the I\&C/Electrical Upgrades maintains an integrated structure to furnish needed information to requesting organizations.

\section{Tank Farm Restoration and Safe Operations MSA, Project W-314 (1.1.1.2.03.07)}

Refer to Table 7.5-2 for FY 1995 - 1997 Deliverables and Milestones for Project W-314.

\section{Mechanical Upgrades (1.1.1.2.03.09)}

The majority of Mechanical Upgrades work is needed to resolve outstanding safety initiatives and Tri-Party Agreement milestones.

The exception to these drivers is the long-length contaminated equipment disposal initiative. This work will turn disposal of waste equipment removed from tanks from costly case-specific engineered tasks into standard operations. Cost savings are anticipated to be in excess of $\$ 1$ billion over the life of Tank Farms. 


\section{Ventilation Upgrades, W-030 (1.1.1.2.03.10)}

Ventilation Upgrades work is being performed to meet Tri-Party Agreement milestones; eliminate discharges to the soil column; provide a longer reliable operational life; provide a ventilation treatment system that supports retrieval efforts; and provide compliance with current waste storage criteria.

The primary deliverable for this project is a fully operational ventilation system. This project will provide the following:

- A new primary ventilation treatment system. This will include a ventilation building designed to meet moderate hazard design basis accident conditions.

- A new recirculating cooling system for each of the four AY/AZ Farm DSTs.

- A distributed control system which will be controlled from a new control building.

- A new standby power system will be housed in a building designed to meet seismic and high wind design bases accident conditions.

For a complete list of deliverables, see Sections 7.5.1.4 and 7.5.1.5.

\section{Cross-Site Transfer System, W-058 (1.1.1.2.03.11)}

Project W-058 will provide the following deliverables:

\section{$\underline{\text { FY-1995 }}$}

- W-058 Complete Definitive Design (M-43-07-T-01)

- Transmit NOC to WA Department of Health

- Obtain Water and Steam Utilities Concurrence

- Award Transfer Pump Contract

- Issue Title II Design Report for Approval

\section{$\underline{\text { FY-1996 }}$}

- W-058 Start Construction (M-43-07A)

- Obtain KD-3

- Obtain NOC Approval

- Issue letter of award for fixed price construction contract

$\underline{\text { FY-1997 }}$

- W-058 Complete Construction (M-34-07B)

- Issue FSAR for approval 
Aging Waste Transfer Line; W-028 (1.1.1.2.03.12)

Project W-028 will provide the following deliverables:

$\underline{\text { FY-1995 }}$

- Transmit NOC to WA Department of Health

- Obtain Water and Steam Utilities Concurrence

- Issue revised FDC

- Issue revised PSAR

FY-1996

- W-028 Start Construction (M-43-07A)

- Complete Definitive Design Activities

- Issue letter for fixed price construction contract

FY-1997

- W-028 Complete Construction (M-34-07B)

- Issue FSAR for approval

Facility Upgrades (1.1.1.2.03.16)

The drivers for Facility Upgrades are the urgent needs and priorities of Tank Farm Operations. No Tri-Party Agreement milestones are associated with this activity.

Radiological Support Facility, W-188 (1.1.1.2.03.17)

N/A, see Program Strategy (Section 7.5.1.1.2).

\subsubsection{Schedule}

\subsubsection{Tank Farm Upgrades Summary Schedule}

See Figure 7.5-2 for the Tank Farm Upgrades Summary Schedule.

The Upgrades program utilizes resource loaded, logic-driven schedules at the cost account level to determine monthly status and performance assessment for the program. Several work packages and tasks within the cost accounts are developed at a lower level of detail in order to monitor and control the detailed work being performed.

The Life Management program will be in place throughout the duration of the TWRS store waste and transfer waste functions. Development and implementation of life management documentation and corrective actions will be completed by the end of FY 1999 requiring only program review and maintenance activities from FY 2000 through completion of the TWRS store waste (Target 2029) and transfer waste (Target 2039) functions. 
Project W-314 is awaiting approval of KD-0. The Tri-Party Agreement, by direction from RL, was negotiated without any slack or schedule contingency. This means that any slippage in either requesting or receiving KDs can place Tri-Party Agreement interim and target milestones in jeopardy. A feature of the re-negotiated Tri-Party Agreement is that target milestones which become critical path to accomplishing enforceable milestones, also become enforceable. As no schedule contingency was built into the negotiated dates, any early slippage, will place target milestones on critical path and elevate them to enforceable status. Therefore, it is critical that the WHC/RL/DOE-HQ team function in a timely manner to accomplish these milestones.

The key activities for the Cross-Site Transfer System W-058 and Aging Waste Transfer Line W-028 are definitive design, safety analysis, and EIS approval, which are prerequisites to KD-3 approval. Then prompt, timely $\mathrm{KD}-3$ approval is required, along with procurement activities to begin construction.

\subsubsection{Tank Farm Upgrades Expanded Baseline (FY 1995 - FY 1997)}

See Appendix C for the Tank Farm Upgrades Expanded Baseline.

The text to support the schedule and logic ties for FY 1995 - FY 1997 is contained in the supporting Cost Estimating Sheets (CESs), Activity Planning Forms (APFs), and other supporting documents to this work plan.

Logic ties are reviewed within the program, within TWRS, and with all Hanford programs managed by the current Maintenance and Operations Contractor, WHC, to ensure proper logic ties to other programs on the Hanford Site.

\subsubsection{Resource Requirements}

\subsubsection{Cost}

Program costs are based largely on the cost of past program performance, labor, materials, and other costs associated with performing the work.

For more information on basis of estimates, see Appendix E. For more detailed basis of cost estimates, see backup TWRS Cost Estimate Sheets.

\subsection{Basis of Cost Estimate}

\subsection{Total Program Element Cost by Year by Fund Type by Activity and Cost Account}

The budget for each fund type represents a program total for the Upgrades program and was developed using detailed Cost Estimating Sheets and Activity Planning Forms for each year, beginning in FY 1995 and through the life of the program, by fund type. The FY 1995 budgets are in more detail, as cost estimates in the near term are more detailed and precise than those used for estimates in out years.

Table 7.5-3 establishes the baseline cost for this program element. The baseline cost is exhibited in annual costs for FY 1994 through FY 2000, and 5-year totals for FY 2001 through program element completion. Where the data are available, these baseline costs are exhibited at the cost account level 
with fund type totals provided. Cost data are then rolled up to the activity and program element levels. At present, the program element cost baseline is the program element cost totals exhibited on this table less a productivity challenge for FY 1995 through FY 1997.

\subsection{FY 1995 Cost by Month by Fund Type by Activity and Cost Account :}

Future MYWP updates will include Table 7.5-4, which establishes the month-by-month cost baseline for FY 1995 for this program element. The FY 1995 cost baseline is exhibited at the cost account level with fund type totals provided. Cost data are then rolled up to the activity and program element levels. Also exhibited is the total cost baseline for the program element for FY 1995. At present, the program element cost baseline for FY 1995 for this program element is the total provided on this table less a productivity challenge for FY 1995.

\subsubsection{Staffing}

\subsection{Basis of Staffing Projections}

\section{Technical Integration and Planning (1.1.1.2.03.01)}

The staffing basis for the Upgrades program is derived from the schedules, logic, APFs, and cost estimates utilized in developing this Multi-Year Work Plan. These estimates and deliverables have been reviewed with the RL staff prior to submitting this work plan.

\subsection{Total Full-Time Equivalent by Year by Activity by Major Participant (Direct and Indirect)}

Future MYWP updates will include Table 7.5-5, which establishes staffing projections for this program element. Included are FY 1994 staffing data as well as projections by year for FY 1994 through FY 2000. The projections include all direct and indirect FTEs, including all subcontractor personnel. Where data is available, projections are exhibited at the activity level with major program participant totals provided.

\subsection{FY 1995 through FY 1997 Direct Full-time Equivalents by 88 Labor Categories}

Future MYWP updates will include Table 7.5-6, which establishes direct WHC staffing projections for this program element. Projections do not include subcontractor support and are provided by 88 labor categories for FY 1995 through FY 1997.

\subsubsection{FY 1994 Wórk Scope Carryover}

Some activities which were planned in FY 1994 were not completed on schedule and will slip into FY 1995. These activities and the related milestones are reflected in the schedule and are planned for completion in FY 1995. Specific activities and milestones are provided in Appendix $\mathrm{C}$ and Appendix D. 
The following FY 1994 work scope for Project W-030 has been delayed and will be carried over into FY 1995:

- Completion of the MICON monitoring and control system logic diagrams was delayed until December 1994 due to a late start. The logic diagrams started three months late. The engineering respurces needed to start the logic diagrams were allocated to the definitive design effort. Since these diagrams would not affect the overall schedule, this was an acceptable decision and allowed the project team to concentrate on completing definitive design, meeting a Tri-Party Agreement milestone.

\subsubsection{Waste Type Data}

TWRS Waste Type Data are provided in Appendix H.

\subsubsection{Revisions}

A change request will be prepared to remove scope from Project W-314 and transfer that scope and associated funding to the Retrieval program. That scope is the portion of the former sub-project W-314c, Waste Transfer Systems, which supplies waste receiver facilities and transfer lines in direct support of waste retrieval. The portion of the former sub-project W-314c which supports the store waste (4.2.1.1) or transfer waste (4.2.1.4) functions from the TWRS Functions and Requirements DOE/RL-92-60, will remain a part of the W-314 scope.

This realignment of work scope will more closely link the transfer lines with the effort they support, and will allow clearer funding and scheduling linkage.

The I\&C/Electrical Upgrades for FY 1995 through FY 1997 were scoped in the previous years' baseline budget. Future changes will add planning to move the TMACS data acquisition communication lines to a network compatible with the Project W-314 data network.

In future updates of the MYWP, this section will include a summary of the revision that has occurred in this program element since the last issue of the MYWP. 
TANK FARM UPGRADES (PAGE 1 OF 2) WORK BREAKDOWN STRUCTURE

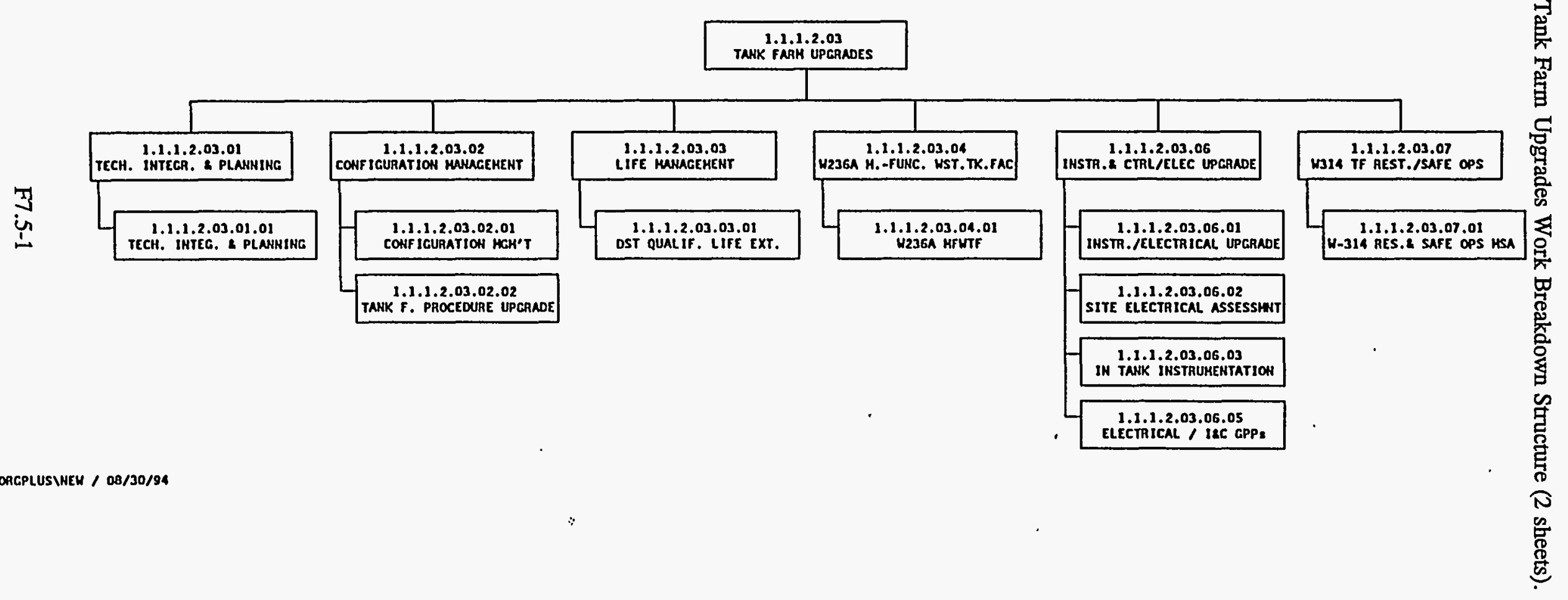


TANK FARM UPGRADES (PAGE 2 OF 2) WORK BREAKDOWN STRUCTURE

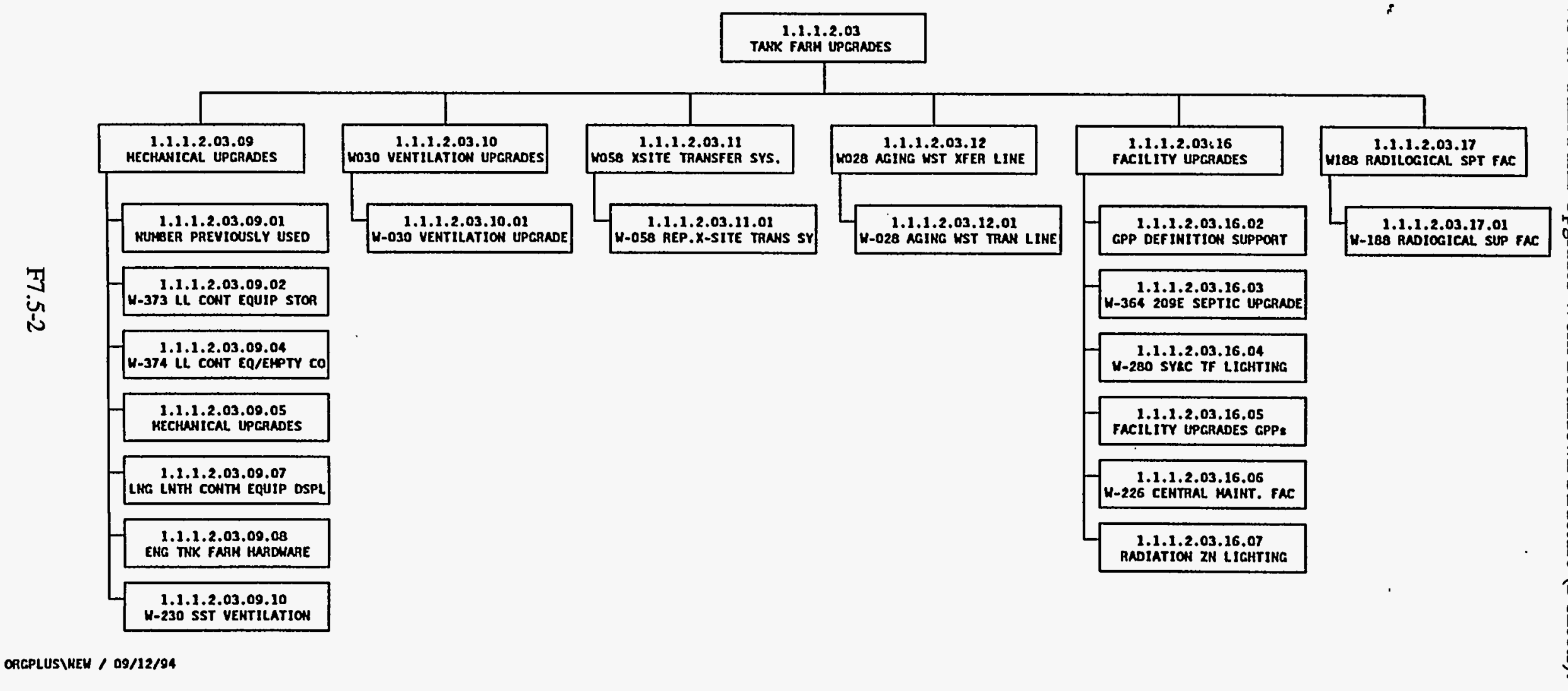


1.1.1.2.03 Tank Farm Upgrades Summary Schedule

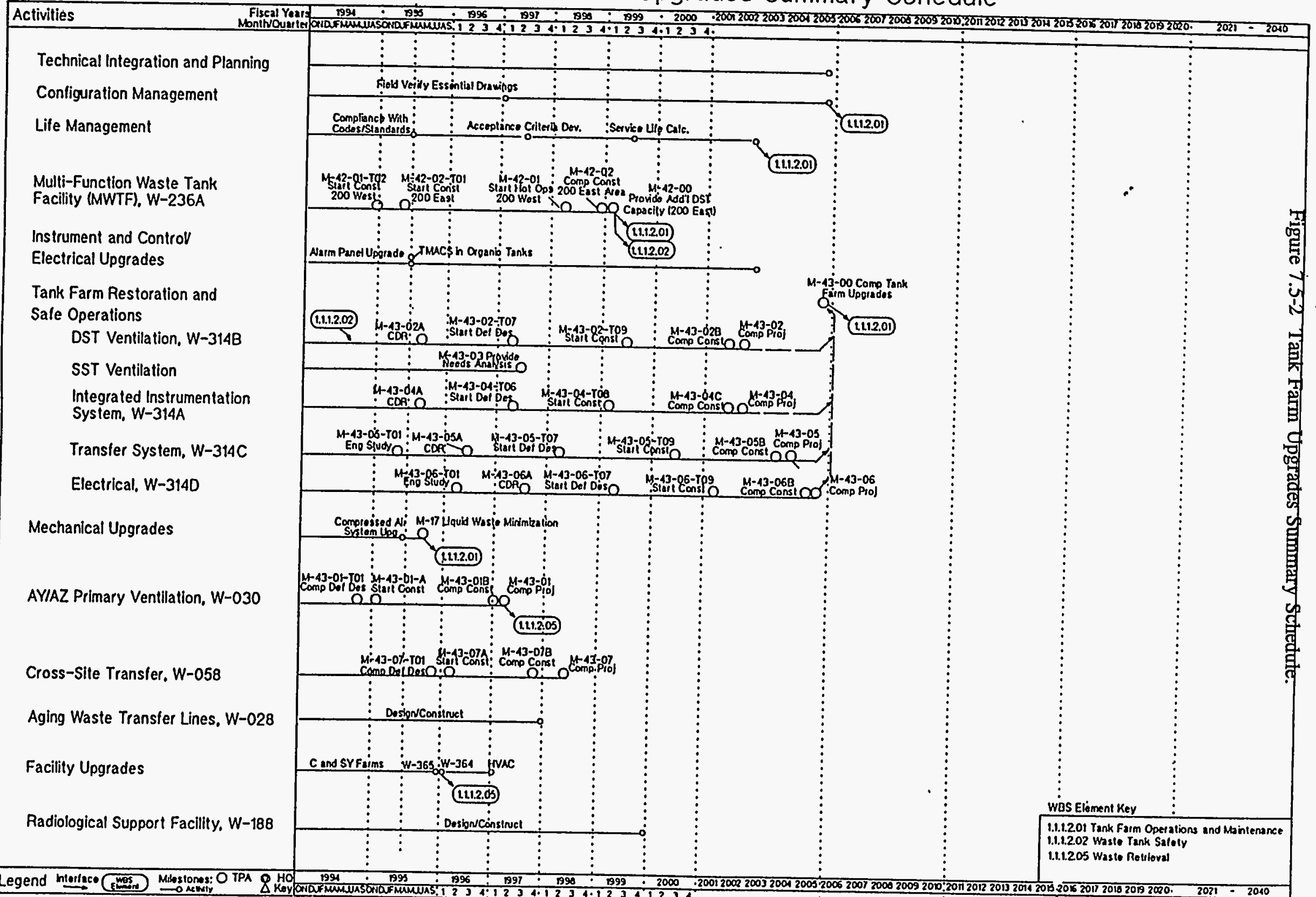




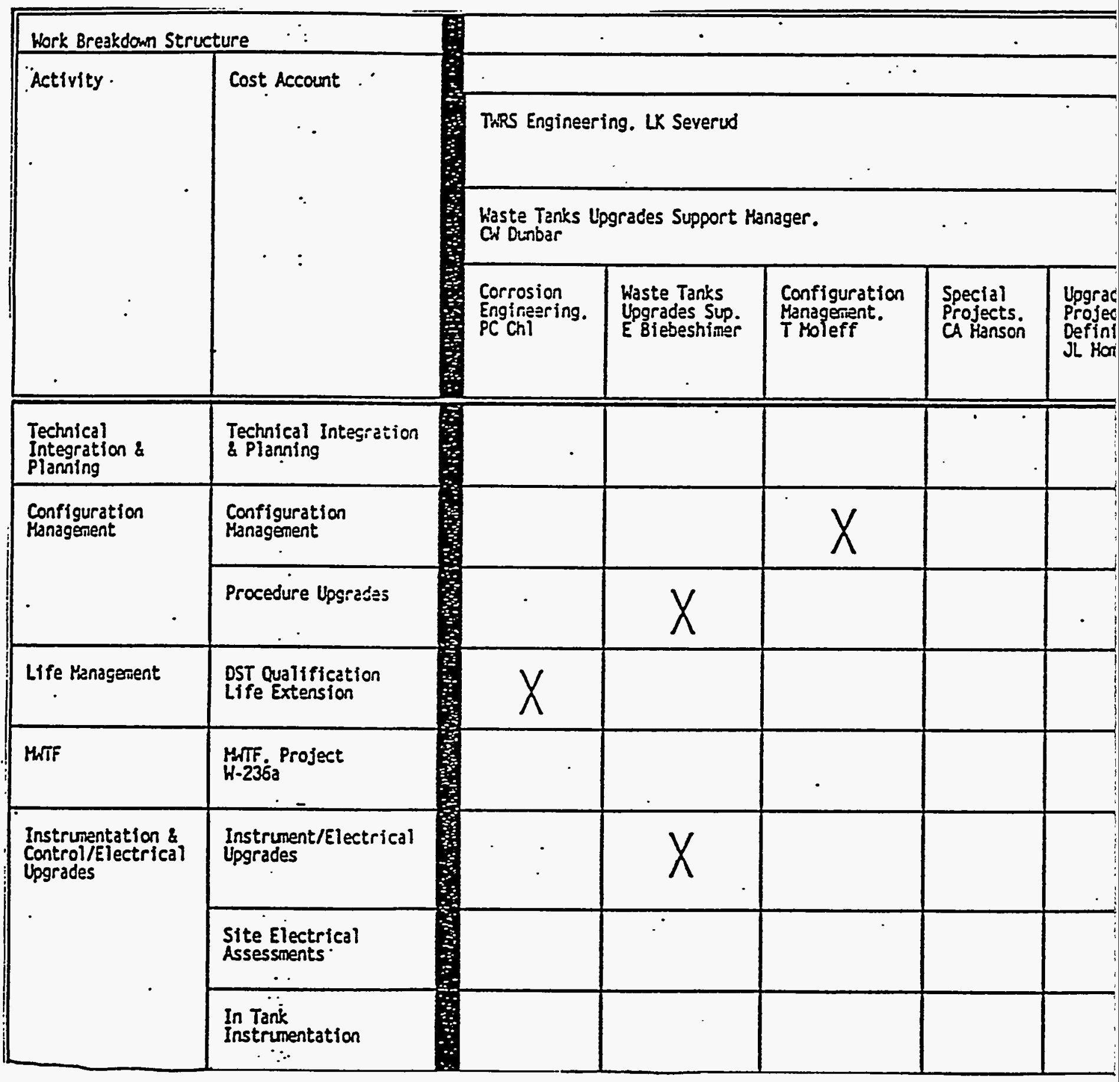


WHC-SP-1101

Table 7.5-1. TWRS Organizational Breakdown (4 sheets)

Construction Projects.

R. A. Smith

Waste Tanks Design

Engineering Manager.

RS Poofelarczyk

\section{Electrical \\ Instrumentation}

Systens.

n.

or Tonne

\& Control.

CP Schroeder

\begin{tabular}{l|l} 
MWTF. Fitz & Tarik Waste Projects. MA Cahill \\
RL Fitz
\end{tabular}

TWRS

Program

Office.

RO Hojtasek

Upgrades

Program.

JD Thomson

I

\begin{tabular}{|c|c|c|c|}
\hline $\begin{array}{l}\text { Tank Farm } \\
\text { Projects. } \\
\text { hid Rutherford }\end{array}$ & $\begin{array}{l}\text { Waste Transfer } \\
\text { Projects. } \\
\text { GL Parsons }\end{array}$ & $\begin{array}{l}\text { General } \\
\text { Support } \\
\text { Projests. }\end{array}$ & $\begin{array}{l}\text { Technical } \\
\text { Integration } \\
\text { \& planning. }\end{array}$ \\
\hline & & & \\
\hline
\end{tabular}

\begin{tabular}{|l|l|l|l|l|l|l|l|}
\hline \hline & & & & & & & $X$ \\
\hline
\end{tabular}

X

$X$ 


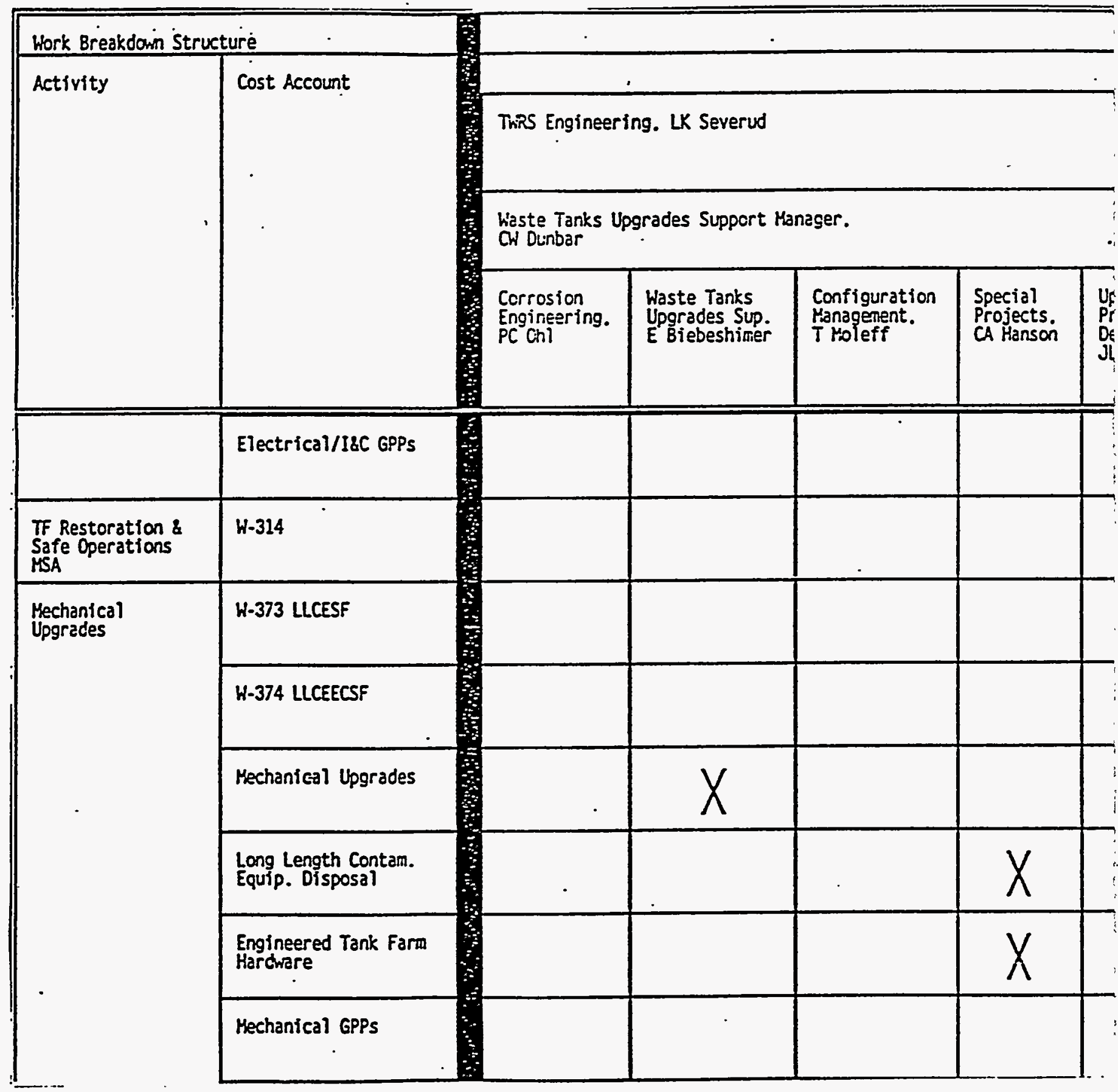


Table 7.5-1. TWRS Organizational Breakdown (4 sheets)

Construction Projects.

R. A. Smith

TWRS

Program

Office.

RD Wojtasek

Waste Tanks Design

Engineering Manager. RS Podielarczýx

\begin{tabular}{l|l} 
Electrical & Instrumentation \\
Systens. & a Control.
\end{tabular}

MNTF.

RL Fritz

Control.

CH Tome CP Schroeder
Tank Waste Projects. MA Cahill

Upgrades

Program.

JD Thonson

\begin{tabular}{|l|l|l|l}
\hline Tank Farm & Waste Transfer & General & Technical
\end{tabular}

Projects. $\quad$ Projects. $\quad$ Support Integration

WW Rutherford GL Parsons

JB Witt EC Ladd
GL

$\mathrm{KA}$ 


\begin{tabular}{|c|c|c|c|c|c|c|}
\hline \multicolumn{2}{|c|}{ Work Breakdown Structure } & \\
\hline \multirow[t]{4}{*}{ Activity } & \multirow{4}{*}{$\begin{array}{c}\text { Cost Account } \\
\therefore \\
\because\end{array}$} & \\
\hline & & \multicolumn{5}{|c|}{ TWRS Engineering. LK Severud } \\
\hline & & \multicolumn{5}{|c|}{$\begin{array}{l}\text { Waste Tanks Upgrades Support Manager. } \\
\text { CW Ounbar }\end{array}$} \\
\hline & & $\begin{array}{l}\text { Corrosion } \\
\text { Engireering. } \\
\text { PC Ohi }\end{array}$ & $\begin{array}{l}\text { Waste Tanks } \\
\text { Upgrades Sup. } \\
\text { E Biebeshimer }\end{array}$ & $\begin{array}{l}\text { Configuration } \\
\text { Manasement. } \\
\text { T Holeff }\end{array}$ & $\begin{array}{l}\text { Special } \\
\text { Projects. } \\
\text { CA Hanson }\end{array}$ & $\begin{array}{l}\text { Upgr } \\
\text { Proj } \\
\text { Defi } \\
J L ~\end{array}$ \\
\hline & W-230 SST Ventilation & & & & & \\
\hline $\begin{array}{l}\text { Ventilation } \\
\text { Upgrades }\end{array}$ & H.030 & & & & & \\
\hline $\begin{array}{l}\text { Cross Site } \\
\text { Transfer Systen }\end{array}$ & W-058 & & & & & \\
\hline $\begin{array}{l}\text { Aging Waste } \\
\text { Transfer Systen }\end{array}$ & H-028: & & & & & \\
\hline \multirow[t]{5}{*}{ Facility Upgrades } & $\begin{array}{l}\text { H-322 } 242.5 \\
\text { Substation }\end{array}$ & & . & & & \\
\hline & W-364 $209 E$ Septic & & & & & \\
\hline & $W-280$ Lighting & & & & & \\
\hline & Facility GPPs & & & & & \\
\hline & & & & & & \\
\hline
\end{tabular}




\begin{tabular}{|c|c|c|c|c|c|c|}
\hline \multicolumn{2}{|c|}{ Work Breakdown Structure } & \multicolumn{5}{|c|}{$\therefore$} \\
\hline \multirow[t]{6}{*}{ Activity } & \multirow{4}{*}{ Cost Account } & \multicolumn{5}{|c|}{$\cdot$} \\
\hline & & \multicolumn{5}{|c|}{ TwiRS Engineering. LK Severud } \\
\hline & & \multicolumn{5}{|c|}{$\begin{array}{l}\text { Kaste Tanks Upgrades Support Manager. } \\
\text { CH Dunbar }\end{array}$} \\
\hline & & $\begin{array}{l}\text { Corrosion } \\
\text { Engineering. } \\
\text { PC Onl }\end{array}$ & $\begin{array}{l}\text { Waste Tanks } \\
\text { Upgrades Sup. } \\
\text { E Biebeshimer }\end{array}$ & $\begin{array}{l}\text { Configuration } \\
\text { Managenent. } \\
\text { T Holeff }\end{array}$ & $\begin{array}{l}\text { Special } \\
\text { Projects. } \\
\text { CA Hanson }\end{array}$ & $\begin{array}{l}\text { Upg } \\
\text { Pro } \\
\text { Def } \\
\text { JL }\end{array}$ \\
\hline & $\begin{array}{l}\text { W-226 Central Maint. } \\
\text { Facflity }\end{array}$ & & & - & & \\
\hline & $\begin{array}{l}\text { Rad Zone Lighting } \\
\text { GPPs }\end{array}$ & & & & & \\
\hline $\begin{array}{l}\text { Radiological } \\
\text { Support Facility }\end{array}$ & K-188 & & & & & \\
\hline
\end{tabular}


WHC-SP-1101

Table 7.5-1. TWRS Organizational Breakdown (4 sheets

Constriction Projects.

R. A. Sinith

TWRS

Program

Office.

RD Wojtasek

Waste Tanks Design

Engineering Manager.

RS PoDielarczyk

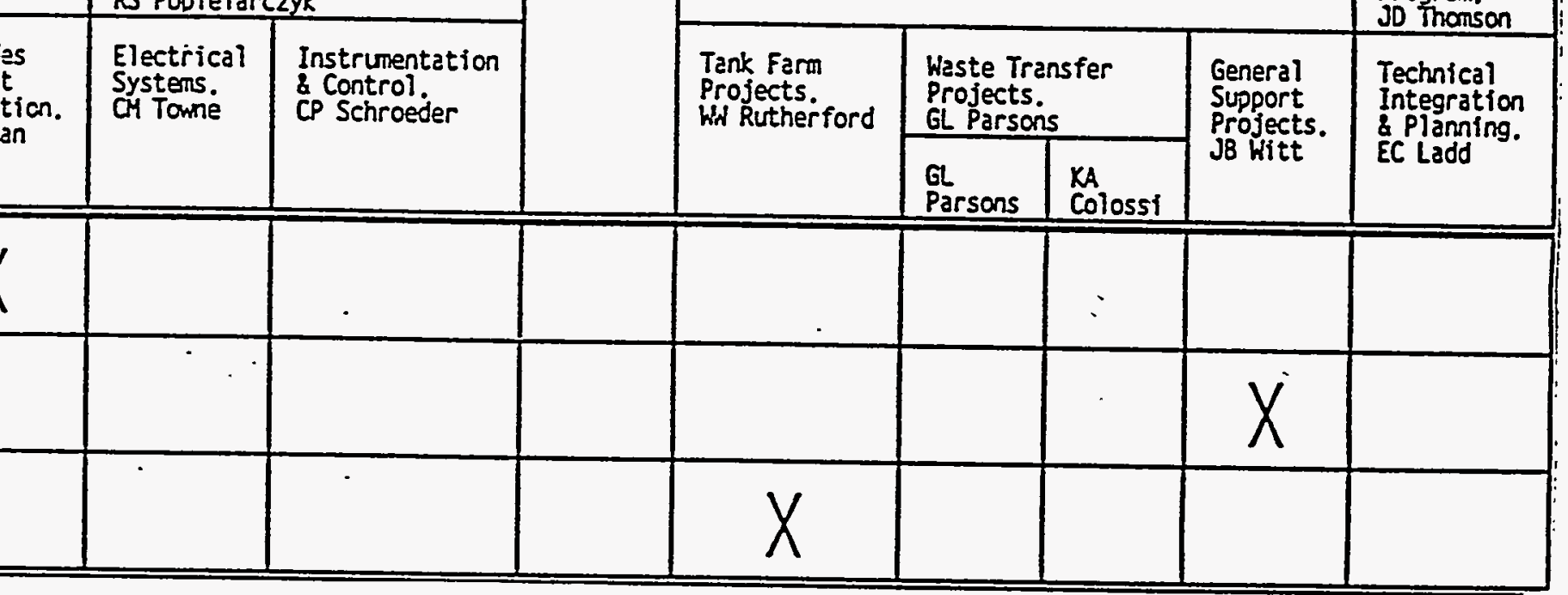




\begin{tabular}{|c|c|c|c|c|c|}
\hline DUE DATE & DESCRIPTION & DELIVERABLE & MILESTONE & MAX. COST & ACCEPTANCE CRITERIA \\
\hline $5 / 31 / 95$ & $\begin{array}{l}W-3140 \cdot C D R \text { complete }(M-43- \\
06 A)\end{array}$ & $x$ & . $x$ & -- & $\begin{array}{l}\text { TPA change request } \\
\text { eliminating this } \mathrm{m} / \mathrm{s} \text { is } \\
\text { pending }\end{array}$ \\
\hline $5 / 30 / 95$ & $\begin{array}{l}\text { Complete } \mathrm{H}-314 \text { Permitting } \\
\text { Plan }\end{array}$ & $x$ & $\cdot$ & $\$ 15 K$ & $\begin{array}{l}\text { WHC approval of plan } \\
\text { documenting permitting } \\
\text { actions req'd to support } \\
\text { W- } 314 \text { construction and } \\
\text { startup }\end{array}$ \\
\hline $5 / 31 / 95$ & $\begin{array}{l}\text { Issue } W-314 \text { QAPP to RL for } \\
\text { approval }\end{array}$ & $x$ & $x$ & $\$ 10 \mathrm{~K}$ & $\begin{array}{l}\text { WHC approved QAPP } \\
\text { formally submitted to } \mathrm{RL} \\
\text { for approval }\end{array}$ \\
\hline $2 / 15 / 95$ & $\begin{array}{l}\text { Approve W-314 Tank Farm } \\
\text { Prioritization Study }\end{array}$ & $x$ & & $\$ 85 K$ & $\begin{array}{l}\text { WHC approval of plan } \\
\text { documenting the criteria } \\
\text { and analysis used to } \\
\text { determine W- } 314 \\
\text { construction sequence }\end{array}$ \\
\hline $.1 / 31 / 95$ & $\begin{array}{l}\text { Issue } W-314 \text { Hazard } \\
\text { Classification }\end{array}$ & $x$ & & $\$ 84 K$ & $\begin{array}{l}\text { WHC approval of Hazard } \\
\text { Classification document } \\
\text { for } \mathrm{W}-314 \text { upgrades }\end{array}$ \\
\hline $3 / 24 / 95$ & $\begin{array}{l}\text { Issue W-314 Prel iminary } \\
\text { Safety Equipment List } \\
\text { (PSEL) }\end{array}$ & $x$ & & $\$ 60 \mathrm{~K}$ & $\begin{array}{l}\text { WHC approval of } W-314 \\
\text { PSEL document }\end{array}$ \\
\hline $4 / 30 / 95$ & $\begin{array}{l}\text { Is sue W-314 Prel iminary } \\
\text { Safety Evaluation (PSE) }\end{array}$ & $x$ & $x$ & $\$ 239 K$ & $\begin{array}{l}\text { WHC approval of } \\
\text { completed PSE for } \mathrm{W}-314 \\
\text { upgrades }\end{array}$ \\
\hline $9 / 30 / 95$ & $\begin{array}{l}\text { Issue } W-314 \text { Construction } \\
\text { Plan/Schedule }\end{array}$ & $x$ & & $\$ 107 K$ & $\begin{array}{l}\text { WHC approval of } \mathrm{W}-314 \\
\text { construction plan and } \\
\text { schedule }\end{array}$ \\
\hline
\end{tabular}




\begin{tabular}{|c|c|c|c|c|c|}
\hline DUE DATE & DESCRIPTION & DELIVERABLE & MILESTONE & MAX. COST & ACCEPTANCE CRITERIA \\
\hline $5 / 31 / 95$ & $\begin{array}{l}\text { Issue Final } \mathrm{W}-314 \text { Project } \\
\text { Plian to RL }\end{array}$ & $x$ & $x$. & $\$ 18 K$ & $\begin{array}{l}\text { WHC approved Project } \\
\text { Plan, prepared in } \\
\text { accordance with DOE } \\
4700.1 \text {, submitted to RL } \\
\text { for approval }\end{array}$ \\
\hline $7 / 31 / 95$ & $\begin{array}{l}\text { Issue } W-314 \text { Project } \\
\text { Management P1an to RL }\end{array}$ & . & $x$ & $\$ 18 \mathrm{~K}$ & $\begin{array}{l}\text { WHC approved Project } \\
\text { Management PIan, } \\
\text { prepared in accordance } \\
\text { with DOE } 4700.1 \text {, } \\
\text { submitted to RL for } \\
\text { approval }\end{array}$ \\
\hline $9 / 1 / 95$ & $\begin{array}{l}\text { Approve/issue } A-E / C M \\
\text { Contract for } W-314 \text { ACD and } \\
\text { Title I Design }\end{array}$ & $x$ & $x$ & $\$ 312 K$ & $\begin{array}{l}\text { Final approval of } W-314 \\
\text { A-E/CM contract by all } \\
\text { required signatories } \\
\end{array}$ \\
\hline $6 / 30 / 95$ & Complete $W-314$ DRB & $x$ & $x$ & $\$ 6111 \mathrm{~K}$ & $\begin{array}{l}\text { RL approval of the } W-314 \\
\text { DRB documentation, } \\
\text { including incorporation } \\
\text { of DRR comments }\end{array}$ \\
\hline $6 / 30 / 95$ & $\begin{array}{l}\text { Receive DOE-HQ Project } \\
\text { Validation }\end{array}$ & . & $x$. & $N / A$ & $\begin{array}{l}\text { Receive formal } \\
\text { notification from DOE-HQ } \\
\text { of validation approval }\end{array}$ \\
\hline $1 / 31 / 96$ & $\begin{array}{l}\text { Start Definitive Design for } \\
W-314 A(M-43-04-T 06)\end{array}$ & . & $x$ & $N / A$ & $\begin{array}{l}\text { Authorize A-E/CM } \\
\text { contractor to begin. W- } \\
314 \text { Title I design work }\end{array}$ \\
\hline $12 / 31 / 95$ & $\begin{array}{l}\text { Issue W-314 Advanced } \\
\text { Conceptual Design Report }\end{array}$ & $x$ & & $\$ 4194 K$ & $\begin{array}{l}\text { WHC approval of } A-E / C M ' s \\
A C D R\end{array}$ \\
\hline $12 / 31 / 95$ & $\begin{array}{l}\text { Issue W-314 Title I Design } \\
\text { Plan }\end{array}$ & $x$ & & $\begin{array}{l}\text { Included } \\
\text { in ACDR }\end{array}$ & $\begin{array}{l}\text { WHC approval of A-E/CM's } \\
\text { Title I design plan and } \\
\text { schedule documentation }\end{array}$ \\
\hline
\end{tabular}




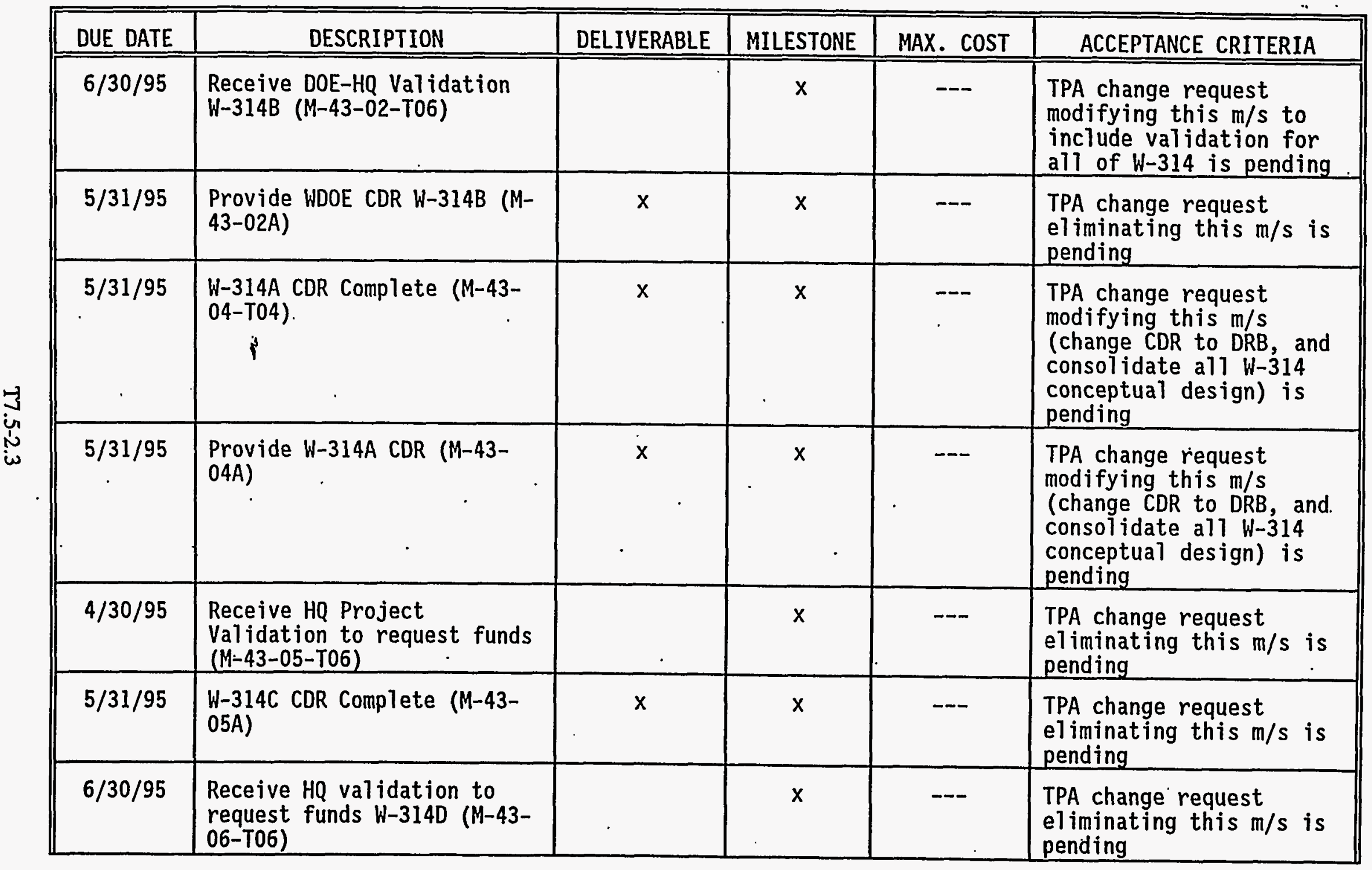




\begin{tabular}{|c|c|c|c|c|c|}
\hline $1 / 31 / 97$ & $\begin{array}{l}\text { Start Definitive Design for } \\
W-314 B(M-43-02-T 07)\end{array}$ & & $x$ & $N / A$ & $\begin{array}{l}\text { TPA change request } \\
\text { eliminating this } \mathrm{m} / \mathrm{s} \text { is } \\
\text { pending }\end{array}$ \\
\hline $2 / 1 / 97$ & Start W-314 Title II Design & & $x$ & $N / A$ & $\begin{array}{l}\text { Authorize A-E/CM } \\
\text { contractor to begin W- } \\
314 \text { Title II design work }\end{array}$ \\
\hline $6 / 30 / 97$ & Issue Final $W-314$ PSAR & $x$ & $x$ & $\$ 2132 K$ & $\begin{array}{l}\text { WHC approval of final W- } \\
314 \text { PSAR documentation }\end{array}$ \\
\hline
\end{tabular}


TABLE 7.5-3

TOTAL PROGRAM ELEMENT COST BASELINE - BY YEAR

\begin{tabular}{|c|c|c|c|c|c|c|c|c|c|}
\hline COST BASELIN & - BY YEAR & & & & & (K\$) & & & \\
\hline WBS / LEVEL & ACTIVITY TITLE / ADS & $\begin{array}{l}\text { FUND } \\
\text { TYPE }\end{array}$ & $\begin{array}{l}\text { TOTAL } \\
\text { FY94 }\end{array}$ & FY95 & FY96 & FY97 & FY98 & FY99 & FYOO \\
\hline \multirow[t]{2}{*}{$\begin{array}{l}\text { 1.1.1.2.03 } \\
\text { Level IV } \\
\quad\end{array}$} & $\begin{array}{l}\text { Tank Farm Upgrades } \\
\text { ADs } 1120-0 \\
\because \because \\
\because \quad \text { TOTAL TARGET }\end{array}$ & $\begin{array}{l}\text { OE } \\
\text { C/E } \\
\text { GPP } \\
\text { LI }^{\prime}\end{array}$ & $\begin{array}{r}38,347 \\
5,155 \\
2,000 \\
49,360 \\
-6--15 \\
94,862\end{array}$ & $\begin{array}{r}37,962 \\
7,277 \\
5,596 \\
103,704 \\
154,539\end{array}$ & $\begin{array}{r}28,279 \\
4,093 \\
6,527 \\
147,231 \\
186,130\end{array}$ & $\begin{array}{r}28,259 \\
6,482 \\
6,822 \\
169,089 \\
210,652\end{array}$ & $\begin{array}{r}30,463 \\
5,927 \\
6,822 \\
214,687 \\
-6,- \\
258,099\end{array}$ & $\begin{array}{r}38,484 \\
5,377 \\
6,822 \\
239,663 \\
284,346\end{array}$ & $\begin{array}{r}27,018 \\
4,206 \\
6,822 \\
209,210 \\
29-2 \\
24,256\end{array}$ \\
\hline & $\begin{array}{l}\text { Delta to TARGET. } \\
\text { (Seo notes at end of Table.) } \\
\text { Total }\end{array}$ & $\begin{array}{l}O E \\
C / E \\
G P P \\
L I\end{array}$ & NA & $\begin{array}{c}(7) \\
0 \\
(980) \\
20,038 \\
==== \\
19,051\end{array}$ & $\begin{array}{r}286 \\
(33) \\
22 \\
1.900 \\
==== \\
2,175\end{array}$ & $\begin{array}{r}134 \\
32 \\
119 \\
(7.254) \\
==== \\
(6,969)\end{array}$ & & & \\
\hline $\begin{array}{l}\text { 1.1.1.2.03.01 } \\
\text { Level } v\end{array}$ & $\begin{array}{l}\text { Technical Integration } \\
\frac{\text { and Planning }}{\text { ADS } 1120-0-O C} \\
\quad \text { Sub Total }\end{array}$ & $\begin{array}{l}\text { OE } \\
C / E\end{array}$ & NA & $\begin{array}{r}2,244 \\
---\frac{0}{2,244}\end{array}$ & $\begin{array}{r}1.711 \\
0 \\
--1.711\end{array}$ & $\begin{array}{r}3,094 \\
0 \\
--- \\
3,094\end{array}$ & $\begin{array}{r}0 \\
0 \\
-- \\
0\end{array}$ & $\begin{array}{r}0 \\
0 \\
-0 \\
0\end{array}$ & $\begin{array}{r}0 \\
-0 \\
-- \\
0\end{array}$ \\
\hline $\begin{array}{l}1.1 .1 .2 .03 .01 .01 \\
\text { Level VI }\end{array}$ & $\begin{array}{l}\text { Technical Integration } \\
\text { and Planning } \\
\qquad \text { Total }\end{array}$ & $\begin{array}{l}O E \\
C / E\end{array}$ & & $\begin{array}{r}2,244 \\
0 \\
--- \\
2,244\end{array}$ & $\begin{array}{r}1.711 \\
0 \\
---- \\
1.711\end{array}$ & $\begin{array}{r}3,094 \\
0 \\
--- \\
3,094\end{array}$ & $-\cdots$ & -- & $---\frac{-}{0}$ \\
\hline $\begin{array}{l}1.1 .1 .2 .03 .02 \\
\text { Level VI }\end{array}$ & 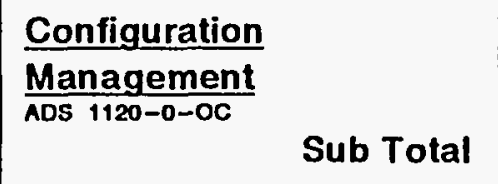 & $\begin{array}{l}O E \\
C / E\end{array}$ & NA & $\begin{array}{r}3,364 \\
0 \\
---\frac{0}{3,364}\end{array}$ & $\begin{array}{r}3,212 \\
0 \\
---\frac{1}{3,212}\end{array}$ & $\begin{array}{r}8,237 \\
0 \\
---2 \\
8,237\end{array}$ & $--\frac{-}{0}$ & $---\overline{0}$ & --- \\
\hline $\begin{array}{l}\text { 1.1.1.2.03.02.01 } \\
\text { Level VI }\end{array}$ & $\begin{array}{l}\text { Configuration } \\
\text { Management }\end{array}$ & $\begin{array}{l}O E \\
C / E\end{array}$ & & $\begin{array}{r}2,325 \\
0 \\
--- \\
2,325\end{array}$ & $\begin{array}{r}3,002 \\
0 \\
--0 \\
3,002\end{array}$ & $\begin{array}{r}8,058 \\
0 \\
--- \\
8,058\end{array}$ & --- & $-\cdots$ & ---- \\
\hline
\end{tabular}

[ R1 ] 
TABLE $\quad 7.5-3$

TOTAL PROGRAM ELEMENT

COST BASELINE - BY YEAR

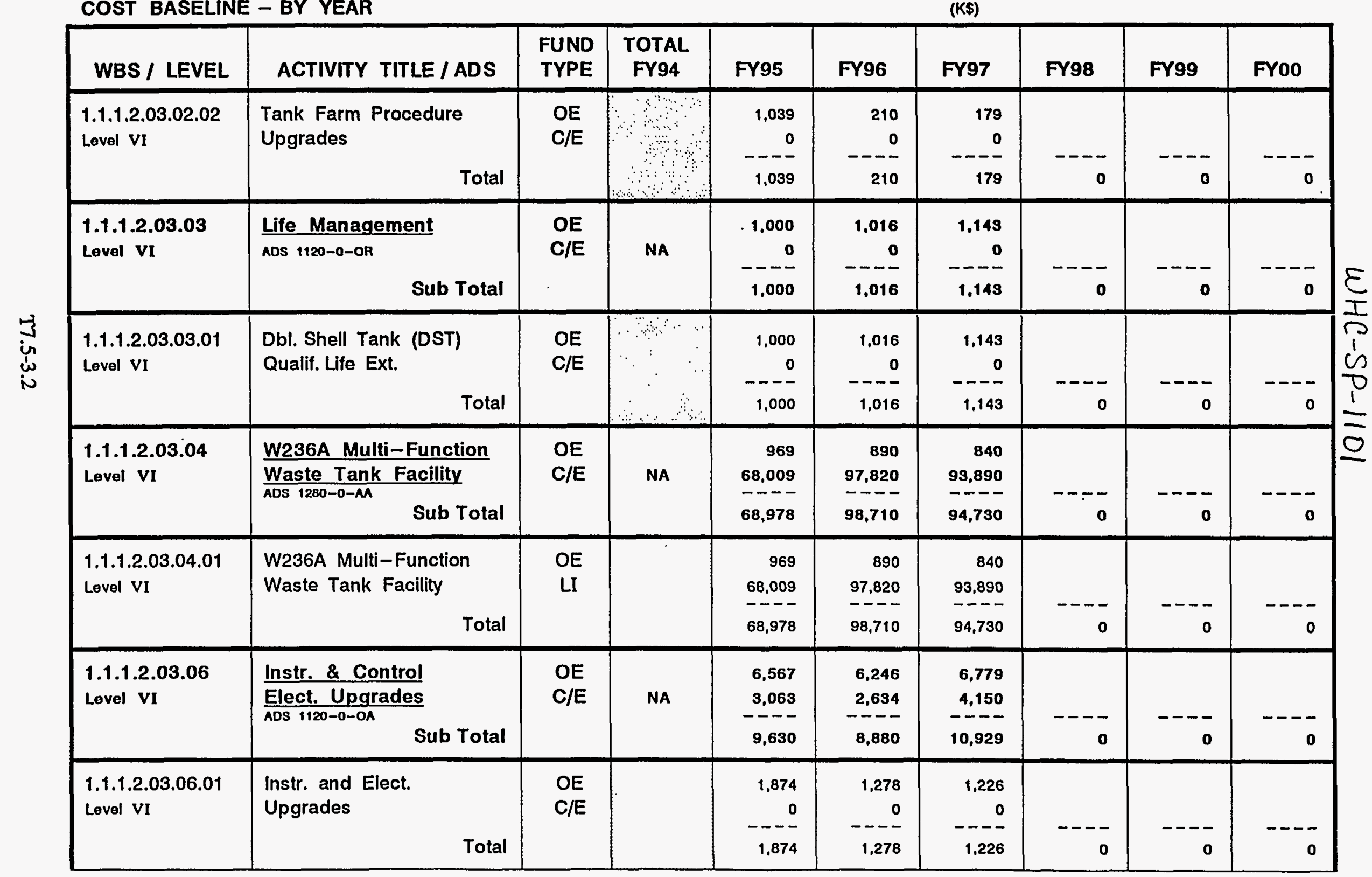

[R1] 
TABLE $7.5-3$

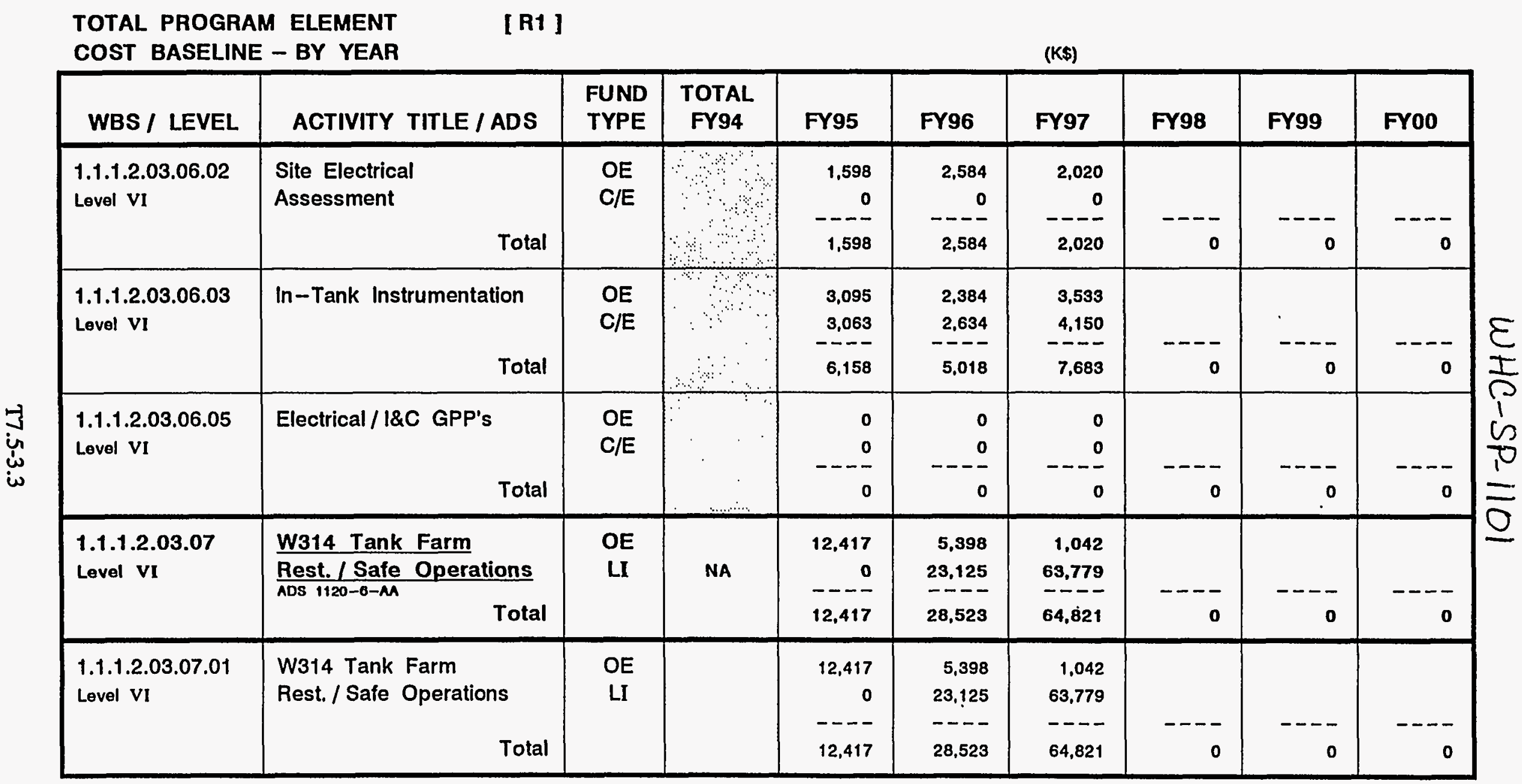


TABLE 7.5-3

TOTAL PROGRAM ELEMENT

COST BASELINE - BY YEAR

\begin{tabular}{|c|c|c|c|c|c|c|c|c|c|}
\hline WBS / LEVEL & ACTIVITY TITLE / ADS & $\begin{array}{l}\text { FUND } \\
\text { TYPE }\end{array}$ & $\begin{array}{c}\text { TOTAL } \\
\text { FY94 }\end{array}$ & FY95 & FY96 & FY97 & FY98 & FY99 & FYOO \\
\hline $\begin{array}{l}\text { 1.1.1.2.03.09 } \\
\text { Lovel VI }\end{array}$ & $\begin{array}{l}\frac{\text { Mechanical Upgrades }}{\text { ADS } 1120-0-0 \mathrm{~A}} \\
\quad \text { Total }\end{array}$ & $\begin{array}{l}\text { OE } \\
\text { C/E } \\
\text { GPP }\end{array}$ & NA & $\begin{array}{r}7,107 \\
4,214 \\
980 \\
-11,321\end{array}$ & $\begin{array}{r}3,905 \\
1,492 \\
0 \\
---\frac{0}{5,397}\end{array}$ & $\begin{array}{r}3.607 \\
2,300 \\
0 \\
---\frac{0}{5,907}\end{array}$ & $---\frac{-}{0}$ & $---\frac{-}{0}$ & $---\frac{-}{0}$ \\
\hline $\begin{array}{l}1.1 .1 .2 .03 .09 .01 \\
\text { Level VI }\end{array}$ & $\begin{array}{l}\text { W372 LL. Contr. Equip. } \\
\text { /T Plant } \\
\\
\text { Total }\end{array}$ & $\begin{array}{l}O E \\
C / E\end{array}$ & $\begin{array}{ll}\therefore \\
\vdots\end{array}$ & $\begin{array}{r}0 \\
0 \\
-- \\
0\end{array}$ & $-\begin{array}{r}0 \\
0 \\
-0\end{array}$ & $\begin{array}{r}0 \\
0 \\
-0\end{array}$ & --- & $--\frac{-}{0}$ & $---\frac{}{0}$ \\
\hline $\begin{array}{l}\text { 1.1.1.2.03.09.02 } \\
\text { Level VI }\end{array}$ & $\begin{array}{l}\text { W372 LL. Contr. Equip. } \\
\text { Storage } \\
\qquad \text { Total }\end{array}$ & $\begin{array}{l}\text { OE } \\
\text { C/E } \\
\text { GPP }\end{array}$ & & $\begin{array}{r}0 \\
0 \\
490 \\
--- \\
490\end{array}$ & $\begin{array}{r}0 \\
0 \\
0 \\
-0\end{array}$ & $\begin{array}{r}0 \\
0 \\
0 \\
-0\end{array}$ & --- & $--\frac{-}{0}$ & $\begin{array}{r}--- \\
0\end{array}$ \\
\hline $\begin{array}{l}\text { 1.1.1.2.03.09.03 } \\
\text { Level VI }\end{array}$ & $\begin{array}{l}\text { W-375 LL. Contr Equip } \\
\text { / Mockup } \\
\\
\text { Total }\end{array}$ & $\begin{array}{l}O E \\
C / E\end{array}$ & & $\begin{array}{r}0 \\
0 \\
--\frac{0}{0}\end{array}$ & $\begin{array}{r}0 \\
0 \\
-- \\
0\end{array}$ & $\begin{array}{r}0 \\
0 \\
-0\end{array}$ & $-\frac{-}{0}$ & $-\frac{-}{0}$ & $\begin{array}{r}--- \\
0\end{array}$ \\
\hline $\begin{array}{l}1.1 .1 .2 .03 .09 .04 \\
\text { Lovel VI }\end{array}$ & $\begin{array}{l}\text { W-374 LL. Contr Equip } \\
\text { Empty Container } \\
\\
\text { Total }\end{array}$ & $\begin{array}{l}\text { OE } \\
\text { C/E } \\
\text { GPP }\end{array}$ & & $\begin{array}{r}0 \\
0 \\
490 \\
--- \\
490\end{array}$ & $\begin{array}{r}0 \\
0 \\
0 \\
-0 \\
0\end{array}$ & $\begin{array}{r}0 \\
0 \\
0 \\
-0\end{array}$ & --- & $--\frac{-}{0}$ & --- \\
\hline $\begin{array}{l}1.1 .1 .2 .03 .09 .05 \\
\text { Level VI }\end{array}$ & $\begin{array}{l}\text { Mechanical Upgrades } \\
\qquad \text { Total }\end{array}$ & $\begin{array}{l}\text { OE } \\
C / E\end{array}$ & & $\begin{array}{r}3,599 \\
69 \\
---- \\
3,668\end{array}$ & $\begin{array}{r}627 \\
0 \\
--- \\
627\end{array}$ & $\begin{array}{r}1.153 \\
0 \\
---1 \\
1.153\end{array}$ & --- & --- & --- \\
\hline
\end{tabular}

\section{[ R1 ]}


TABLE 7.5-3

TOTAL PROGRAM ELEMENT COST BASELINE - BY YEAR

\begin{tabular}{|c|c|c|c|c|c|c|c|c|c|}
\hline \multicolumn{2}{|c|}{ COST BASELINE - BY YEAR } & \multicolumn{8}{|c|}{ (K\$) } \\
\hline WBS / LEVEL & ACTIVITY TITLE / ADS & $\begin{array}{l}\text { FUND } \\
\text { TYPE }\end{array}$ & $\begin{array}{l}\text { TOTAL } \\
\text { FY94 }\end{array}$ & FY95 & FY96 & FY97 & FY98 & FY99 & FY00 \\
\hline $\begin{array}{l}\text { 1.1.1.2.03.09.07 } \\
\text { Level VI }\end{array}$ & $\begin{array}{l}\text { Long Length Control } \\
\text { Equip. Disposal } \\
\\
\text { Total }\end{array}$ & $\begin{array}{l}O E \\
C / E\end{array}$ & 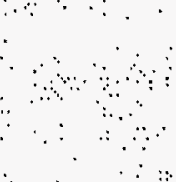 & $\begin{array}{r}3,200 \\
2,590 \\
---- \\
5,790\end{array}$ & $\begin{array}{r}2,469 \\
0 \\
--- \\
2,469\end{array}$ & $\begin{array}{r}934 \\
1,006 \\
-1,940\end{array}$ & $---\frac{}{0}$ & $\begin{array}{r}---- \\
0\end{array}$ & $---\frac{-}{0}$ \\
\hline $\begin{array}{l}\text { 1.1.1.2.03.09.08 } \\
\text { Level VI }\end{array}$ & $\begin{array}{l}\text { Engin. Tank Farm } \\
\text { Hardware }\end{array}$ & $\begin{array}{l}O E \\
C / E\end{array}$ & 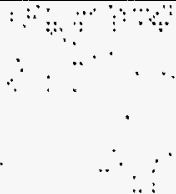 & $\begin{array}{r}308 \\
1,555 \\
---- \\
1,863\end{array}$ & $\begin{array}{r}809 \\
1,492 \\
---- \\
2,301\end{array}$ & $\begin{array}{r}383 \\
1,294 \\
---- \\
1,677\end{array}$ & $\begin{array}{r}---- \\
0\end{array}$ & $\begin{array}{r}--- \\
0\end{array}$ & $\begin{array}{r}---- \\
0\end{array}$ \\
\hline $\begin{array}{l}1.1 .1 .2 .03 .09 .10 \\
\text { Lovel VI }\end{array}$ & $\begin{array}{l}\text { W-230 SST Ventilation } \\
\text { Total }\end{array}$ & $\begin{array}{l}\text { OE } \\
\text { C/E }\end{array}$ &. & $\begin{array}{r}0 \\
0 \\
--- \\
0\end{array}$ & $\begin{array}{r}0 \\
0 \\
--- \\
0\end{array}$ & $\begin{array}{r}1.137 \\
0 \\
-2-- \\
1.137\end{array}$ & $\begin{array}{r}---- \\
0\end{array}$ & $\begin{array}{r}---- \\
0\end{array}$ & --- \\
\hline $\begin{array}{l}\text { 1.1.1.2.03.10 } \\
\text { Level VI }\end{array}$ & $\frac{\text { W030 Ventilation }}{\frac{\text { Upgrades }}{\text { ADS } 1120-2-0 G}}$ & $\begin{array}{l}\text { OE } \\
C / E\end{array}$ & NA & $\begin{array}{r}1,954 \\
0 \\
---- \\
1,954\end{array}$ & $\begin{array}{r}1,950 \\
0 \\
---- \\
1,950\end{array}$ & $\begin{array}{r}594 \\
0 \\
--- \\
594\end{array}$ & $---\frac{-}{0}$ & $---\frac{-}{0}$ & $\frac{---}{0}$ \\
\hline $\begin{array}{l}\text { 1.1.1.2.03.10.01 } \\
\text { Level VI }\end{array}$ & $\begin{array}{l}\text { W-030 Ventilation } \\
\text { Upgrades }\end{array}$ & $\begin{array}{l}\text { OE } \\
C / E\end{array}$ & & $\begin{array}{r}1,954 \\
0 \\
--- \\
1,954\end{array}$ & $\begin{array}{r}1,950 \\
0 \\
---- \\
1,950\end{array}$ & $\begin{array}{r}594 \\
0 \\
--- \\
594\end{array}$ & $\begin{array}{r}---- \\
0\end{array}$ & $\begin{array}{r}---- \\
0\end{array}$ & $\begin{array}{r}---- \\
0\end{array}$ \\
\hline $\begin{array}{l}\text { 1.1.1.2.03.11 } \\
\text { Level VI }\end{array}$ & 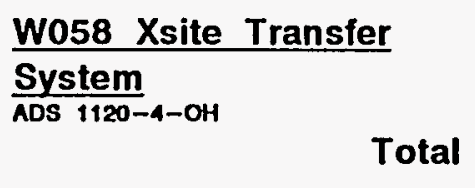 & $\begin{array}{l}\text { OE } \\
\text { LI }\end{array}$ & NA & $\begin{array}{r}841 \\
14,849 \\
---- \\
15,684\end{array}$ & $\begin{array}{r}1,023 \\
19,988 \\
---- \\
20,411\end{array}$ & $\begin{array}{r}540 \\
13,168 \\
---- \\
13,708\end{array}$ & ---- & $\begin{array}{r}--- \\
0\end{array}$ & $\begin{array}{r}---\overline{0} \\
0\end{array}$ \\
\hline $\begin{array}{l}\text { 1.1.1.2.03.11.01 } \\
\text { Level VI }\end{array}$ & $\begin{array}{l}\text { W-058 Xsite Transfer } \\
\text { System } \\
\\
\text { Total }\end{array}$ & $\begin{array}{l}\text { OE } \\
\text { LI }\end{array}$ & & $\begin{array}{r}841 \\
14,843 \\
---- \\
15,684\end{array}$ & $\begin{array}{r}1,023 \\
19,388 \\
---- \\
20,411\end{array}$ & $\begin{array}{r}540 \\
13,168 \\
--- \\
13,708\end{array}$ & $\begin{array}{r}---- \\
0\end{array}$ & $\begin{array}{r}---- \\
0\end{array}$ & $\begin{array}{r}--- \\
0\end{array}$ \\
\hline
\end{tabular}

[R1]

TABLE 7.5 3 
TABLE $7.5-3$

TOTAL PROGRAM ELEMENT COST BASELINE - BY YEAR

\begin{tabular}{|c|c|c|c|c|c|c|c|c|c|}
\hline WBS / LEVEL & ACTIVITY TITLE / ADS & $\begin{array}{l}\text { FUND } \\
\text { TYPE }\end{array}$ & $\begin{array}{l}\text { TOTAL } \\
\text { FY94 }\end{array}$ & FY95 & FY96 & FY97 & FY98 & FY99 & FYOO \\
\hline $\begin{array}{l}\text { 1.1.1.2.03.12 } \\
\text { Level VI }\end{array}$ & $\begin{array}{l}\frac{\text { W028 Aging Waste }}{\text { Xfer Lines }} \\
\frac{\text { ADS } 1120-7-O M}{} \quad \text { Total }\end{array}$ & $\begin{array}{l}\text { OE } \\
\text { LI }\end{array}$ & NA & $\begin{array}{r}502 \\
814 \\
-1.316\end{array}$ & $\begin{array}{r}687 \\
4,998 \\
-5,-- \\
5,685\end{array}$ & $\begin{array}{r}424 \\
3,706 \\
4.130\end{array}$ & $---\overline{0}$ & --- & $---\frac{-}{0}$ \\
\hline $\begin{array}{l}\text { 1.1.1.2.03.12.01 } \\
\text { Level vI }\end{array}$ & $\begin{array}{l}\text { W028 Aging Waste } \\
\text { Xfer Lines } \\
\\
\text { Total }\end{array}$ & $\begin{array}{l}\text { OE } \\
\text { LI }\end{array}$ & & $\begin{array}{r}502 \\
814 \\
---- \\
1,316\end{array}$ & $\begin{array}{r}687 \\
4,998 \\
---- \\
5,685\end{array}$ & $\begin{array}{r}424 \\
3,706 \\
---- \\
4,130\end{array}$ & --- & - & --- \\
\hline $\begin{array}{l}\text { 1.1.1.2.03.16 } \\
\text { Level } \mathrm{V}\end{array}$ & $\begin{array}{l}\text { Facility Upgrades } \\
\text { ADS } 1120-0-0 \mathrm{~A} \\
\text { Sub Total }\end{array}$ & $\begin{array}{l}\text { OE } \\
\text { C/E } \\
\text { GPP }\end{array}$ & NA & $\begin{array}{r}989 \\
0 \\
4.616 \\
5,605\end{array}$ & $\begin{array}{r}1,886 \\
0 \\
6,505 \\
8,391\end{array}$ & $\begin{array}{r}1,801 \\
0 \\
6,703 \\
-0,504 \\
8,504\end{array}$ & $\begin{array}{r}\mathbf{0} \\
\mathbf{0} \\
--- \\
\mathbf{0}\end{array}$ & $\begin{array}{r}0 \\
0 \\
-0\end{array}$ & $\begin{array}{r}0 \\
0 \\
--- \\
0\end{array}$ \\
\hline $\begin{array}{l}1.1 .1 .2 .03 .16 .02 \\
\text { Level VI }\end{array}$ & $\begin{array}{l}\text { W-322 242-S Sub Station } \\
\text { Upgrade } \\
\text { Total }\end{array}$ & $\begin{array}{l}O E \\
C / E\end{array}$ & & $\begin{array}{r}989 \\
0 \\
--- \\
989\end{array}$ & $\begin{array}{r}1,886 \\
0 \\
---- \\
1,886\end{array}$ & $\begin{array}{r}1,801 \\
0 \\
---- \\
1,801\end{array}$ & $-\cdots$ & $-\cdots$ & -- \\
\hline $\begin{array}{l}\text { 1.1.1.2.03.16.03 } \\
\text { Level VI }\end{array}$ & $\begin{array}{l}\text { W364 209E Septic Tank } \\
\text { Upgrade } \\
\qquad \text { Total }\end{array}$ & $\begin{array}{l}\text { OE } \\
\mathrm{C} / \mathrm{E}\end{array}$ & & $\begin{array}{r}0 \\
0 \\
-- \\
-0\end{array}$ & $\begin{array}{r}0 \\
0 \\
-- \\
-0\end{array}$ & $\begin{array}{r}0 \\
0 \\
-- \\
0\end{array}$ & --- & $-\cdots$ & --- \\
\hline $\begin{array}{l}1.1 .1 .2 .03 .16 .04 \\
\text { Level VI }\end{array}$ & $\begin{array}{l}\text { W-280 SY\&C Tank Farm } \\
\text { Lighting } \\
\\
\text { Total }\end{array}$ & $\begin{array}{l}O E \\
C / E\end{array}$ & & $\begin{array}{r}0 \\
0 \\
-- \\
0\end{array}$ & $\begin{array}{r}0 \\
0 \\
-- \\
0\end{array}$ & $\begin{array}{r}0 \\
0 \\
-- \\
-0\end{array}$ & $---\frac{-}{0}$ & 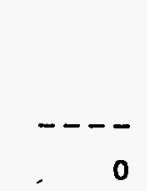 & --- \\
\hline
\end{tabular}

[ R1 ] 
TABLE $7.5-3$

TOTAL PROGRAM ELEMENT COST BASELINE - BY YEAR

\begin{tabular}{|c|c|c|c|c|c|c|c|c|c|}
\hline \multicolumn{3}{|c|}{ COST BASELINE - BY YEAR } & \multicolumn{7}{|c|}{ (K\$) } \\
\hline WBS / LEVEL & ACTIVITY TITLE / ADS & $\begin{array}{l}\text { FUND } \\
\text { TYPE }\end{array}$ & $\begin{array}{c}\text { TOTAL } \\
\text { FY94 }\end{array}$ & FY95 & FY96 & FY97 & FY98 & FY99 & FYOOO \\
\hline $\begin{array}{l}1.1 .1 .2 .03 .16 .05 \\
\text { Level VI }\end{array}$ & $\begin{array}{l}\text { Facility Upgrades } \\
\text { (GPP's) } \\
\\
\end{array}$ & $\begin{array}{l}\text { OE } \\
\text { C/E } \\
\text { GPP }\end{array}$ & $\therefore$ & $\begin{array}{r}0 \\
0 \\
4,616 \\
--- \\
0\end{array}$ & $\begin{array}{r}0 \\
0 \\
6,505 \\
--- \\
0\end{array}$ & $\begin{array}{r}0 \\
0 \\
6.703 \\
--- \\
0\end{array}$ & ---- & --- & --- \\
\hline $\begin{array}{l}\text { 1.1.1.2.03.16.06 } \\
\text { Level VI }\end{array}$ & $\begin{array}{l}\text { W-226 Central Maint. } \\
\text { Facility Upgrade } \\
\qquad \text { Total }\end{array}$ & $\begin{array}{l}O E \\
C / E\end{array}$ & & $\begin{array}{r}0 \\
0 \\
- \\
-0\end{array}$ & $\begin{array}{r}0 \\
0 \\
-- \\
-0\end{array}$ & $\begin{array}{r}0 \\
0 \\
-- \\
0\end{array}$ & --- & --- & $-\cdots$ \\
\hline $\begin{array}{l}\text { 1.1.1.2.03.16.07 } \\
\text { Level VI }\end{array}$ & $\begin{array}{l}\text { Radiation Zone Lighting } \\
\text { Total }\end{array}$ & $\begin{array}{l}O E \\
C / E\end{array}$ & & $\begin{array}{r}0 \\
0 \\
- \\
-0\end{array}$ & $\begin{array}{r}0 \\
0 \\
- \\
-0\end{array}$ & $\begin{array}{r}0 \\
0 \\
-- \\
-0\end{array}$ & --- & $-\cdots$ & $-\cdots$ \\
\hline $\begin{array}{l}1.1 .1 .2 .03 .17 \\
\text { Level } \mathrm{V}\end{array}$ & $\begin{array}{l}\text { W188 Radiological } \\
\text { Support Facility } \\
\text { ADS 1120-1-as Sub Total }\end{array}$ & $\begin{array}{l}\mathrm{OE} \\
\mathrm{C} / \mathrm{E}\end{array}$ & NA & $\begin{array}{r}15 \\
0 \\
-- \\
15\end{array}$ & $\begin{array}{r}69 \\
0 \\
-- \\
69\end{array}$ & $\begin{array}{r}24 \\
1.800 \\
--- \\
1,824\end{array}$ & $\begin{array}{r}0 \\
0 \\
-- \\
-0\end{array}$ & $\begin{array}{r}0 \\
0 \\
-- \\
0\end{array}$ & $\begin{array}{r}0 \\
0 \\
-- \\
-0\end{array}$ \\
\hline $\begin{array}{l}1.1 .1 .2 .01 .17 .01 \\
\text { Level VI }\end{array}$ & $\begin{array}{l}\text { W188 Radiological } \\
\text { Support Facility } \\
\qquad \text { Total }\end{array}$ & $\begin{array}{l}\text { OE } \\
\mathrm{LI}\end{array}$ & & $\begin{array}{r}15 \\
0 \\
-- \\
-15\end{array}$ & $\begin{array}{r}69 \\
0 \\
-- \\
69\end{array}$ & $\begin{array}{r}24 \\
1,800 \\
--2 \\
1,824\end{array}$ & $\begin{array}{r}--- \\
0\end{array}$ & $---\overline{0}$ & --- \\
\hline
\end{tabular}

\section{[ R1 ]}

TABLE $7.5-3$ 
TABLE $7.5-3$

TOTAL PROGRAM ELEMENT COST BASELINE - BY YEAR

\begin{tabular}{|c|c|c|c|c|c|c|c|c|c|c|}
\hline WBS / LEVEL. & ACTIVITY TITLE / ADS & $\begin{array}{l}\text { FUND } \\
\text { TYPE }\end{array}$ & $\begin{array}{l}\text { FY01 / } \\
\text { FY05 }\end{array}$ & $\begin{array}{l}\text { FY06 I } \\
\text { FY10 }\end{array}$ & $\begin{array}{l}\text { FY11 I } \\
\text { FY15 }\end{array}$ & $\begin{array}{l}\text { FY16 / } \\
\text { FY20 }\end{array}$ & $\begin{array}{l}\text { FY21 / } \\
\text { FY25 }\end{array}$ & $\begin{array}{l}\text { FY26 I } \\
\text { FY30 }\end{array}$ & $\begin{array}{l}\text { FY31 I } \\
\text { FY35 }\end{array}$ & $\begin{array}{l}\text { FY36 I } \\
\text { FY40 }\end{array}$ \\
\hline \multirow[t]{2}{*}{ 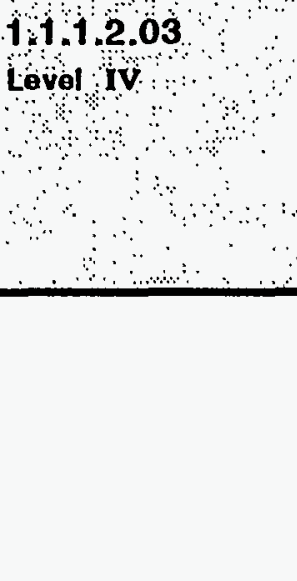 } & 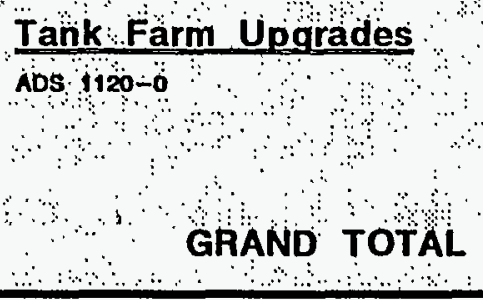 & $\begin{array}{l}\text { OE } \\
\text { C/E } \\
\text { GPP } \\
\text { LI }\end{array}$ & $\begin{array}{r}00 \\
0 \\
0 \\
0 \\
00 \\
455,995\end{array}$ & 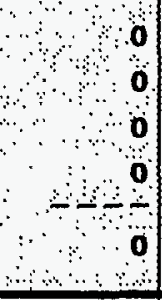 & $\begin{array}{r}0 \\
0 \\
0 \\
0 \\
0 \\
0\end{array}$ & $\begin{array}{r}0 \\
0 \\
0 \\
0 \\
0 \\
0\end{array}$ & 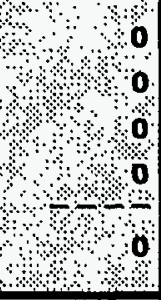 & 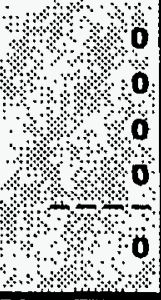 & & \\
\hline & $\begin{array}{l}\text { Delta Required to } \\
\text { Balance to Target } \\
\text { Budget. } \\
\\
\text { Total }\end{array}$ & $\begin{array}{c}O E \\
C / E \\
G P P \\
L I\end{array}$ & & & & & & & & \\
\hline $\begin{array}{l}\text { 1.1.1.2.03.01 } \\
\text { Level } v\end{array}$ & $\begin{array}{l}\text { Technical Integration } \\
\frac{\text { and Planning }}{\text { ADS } 1120-0-O C} \\
\quad \text { Sub Total }\end{array}$ & $\begin{array}{l}\text { OE } \\
\text { C/E }\end{array}$ & $\begin{array}{r}\mathbf{0} \\
--0 \\
-0\end{array}$ & $\begin{array}{r}0 \\
0 \\
-- \\
0\end{array}$ & $\begin{array}{r}0 \\
0 \\
-0\end{array}$ & $\begin{array}{r}\mathbf{0} \\
\mathbf{0} \\
-- \\
\mathbf{0}\end{array}$ & $\begin{array}{r}\mathbf{0} \\
-0 \\
-0\end{array}$ & $\begin{array}{r}\mathbf{0} \\
--- \\
\mathbf{0}\end{array}$ & $\begin{array}{r}\mathbf{0} \\
\mathbf{0} \\
-- \\
\mathbf{0}\end{array}$ & $\begin{array}{r}0 \\
0 \\
-0\end{array}$ \\
\hline $\begin{array}{l}1.1 .1 .2 .03 .01 .01 \\
\text { Level VI }\end{array}$ & $\begin{array}{l}\text { Technical Integration } \\
\text { and Planning } \\
\qquad \text { Total }\end{array}$ & $\begin{array}{l}O E \\
C / E\end{array}$ & --- & 0 & 0 & 0 & -- & - & -- & --- \\
\hline $\begin{array}{l}\text { 1.1.1.2.03.02 } \\
\text { Level VI }\end{array}$ & $\begin{array}{l}\frac{\text { Configuration }}{\text { Management }} \\
\text { ADS 1120-0-OC } \\
\quad \text { Sub Total }\end{array}$ & $\begin{array}{l}O E \\
C / E\end{array}$ & --- & $\begin{array}{r}---- \\
0\end{array}$ & $-\cdots$ & $---\overline{0}$ & ---- & $---\frac{-}{0}$ & $---\frac{-}{0}$ & $-\cdots$ \\
\hline $\begin{array}{l}\text { 1.1.1.2.03.02.01 } \\
\text { Level VI }\end{array}$ & $\begin{array}{l}\text { Configuration } \\
\text { Management }\end{array}$ & $\begin{array}{l}O E \\
C / E\end{array}$ & 0 & 0 & 0 & 0 & $---\frac{-}{0}$ & - & 0 & $-\cdots$ \\
\hline
\end{tabular}

\section{[ R1 ]}


TABLE $7.5-3$

TOTAL PROGRAM ELEMENT COST BASELINE - BY YEAR

[R1 ]

\begin{tabular}{|c|c|c|c|c|c|c|c|c|c|c|}
\hline WBS / LEVEL & ACTIVITY TITLE / ADS & $\begin{array}{l}\text { FUND } \\
\text { TYPE }\end{array}$ & $\begin{array}{l}\text { FY01 / } \\
\text { FY05 }\end{array}$ & $\begin{array}{l}\text { FY06 / } \\
\text { FY10 }\end{array}$ & $\begin{array}{l}\text { FY11 / } \\
\text { FY15 }\end{array}$ & $\begin{array}{l}\text { FY16 / } \\
\text { FY20 }\end{array}$ & $\begin{array}{l}\text { FY21 / } \\
\text { FY25 }\end{array}$ & $\begin{array}{l}\text { FY26 / } \\
\text { FY30 }\end{array}$ & $\begin{array}{l}\text { FY31 / } \\
\text { FY35 }\end{array}$ & $\begin{array}{l}\text { FY36 / } \\
\text { FY40 }\end{array}$ \\
\hline $\begin{array}{l}\text { 1.1.1.2.03.02.02 } \\
\text { Level VI }\end{array}$ & $\begin{array}{l}\text { Tank Farm Procedure } \\
\text { Upgrades } \\
\\
\text { Total }\end{array}$ & $\begin{array}{l}\text { OE } \\
C / E\end{array}$ & -- & --- & --- & ---- & $-\cdots$ & $---\frac{-}{0}$ & ---- & --- \\
\hline $\begin{array}{l}\text { 1.1.1.2.03.03 } \\
\text { Level VI }\end{array}$ & $\begin{array}{l}\text { Life Management } \\
\text { ADS } 1120-0-\text { OR } \\
\text { Sub Total }\end{array}$ & $\begin{array}{l}\text { OE } \\
C / E\end{array}$ & --- & $---\overline{0}$ & --- & ---- & $---\frac{1}{0}$ & $---\frac{-}{0}$ & ---- & $---\frac{-}{0}$ \\
\hline $\begin{array}{l}\text { 1.1.1.2.03.03.01 } \\
\text { Level VI }\end{array}$ & $\begin{array}{l}\text { Dbl. Shell Tank (DST) } \\
\text { Qualif. Life Ext. } \\
\qquad \\
\end{array}$ & $\begin{array}{l}O E \\
C / E\end{array}$ & $-\cdots$ & - & -- & $---\frac{-}{0}$ & $---\frac{-}{0}$ & $\overline{0}$ & - & - \\
\hline $\begin{array}{l}\text { 1.1.1.2.03.04 } \\
\text { Level VI }\end{array}$ & $\begin{array}{l}\text { W236A Multi-Function } \\
\frac{\text { Waste Tank Facility }}{\text { ADS } 1280-0-M} \text { Sub Total }\end{array}$ & $\begin{array}{l}\text { OE } \\
\text { C/E }\end{array}$ & --- & 0 & $\begin{array}{r}0-- \\
0\end{array}$ & $---\frac{-}{0}$ & $---\frac{-}{0}$ & $---\frac{-}{0}$ & $---\frac{-}{0}$ & --- \\
\hline $\begin{array}{l}\text { 1.1.1.2.03.04.01 } \\
\text { Level VI }\end{array}$ & $\begin{array}{l}\text { W236A Multi-Function } \\
\text { Waste Tank Facility } \\
\text { Total }\end{array}$ & $\begin{array}{l}\text { OE } \\
\text { LI }\end{array}$ & -- & 0 & -- & -- & -- & -- & 0 & $---\frac{-}{0}$ \\
\hline $\begin{array}{l}\text { 1.1.1.2.03.06 } \\
\text { Level VI }\end{array}$ & $\begin{array}{l}\frac{\text { Instr. \& Control }}{\text { Elect. Upgrades }} \\
\text { ADS } 1120-0-0 \wedge \quad \text { Sub Total }\end{array}$ & $\begin{array}{l}\text { OE } \\
\text { C/E }\end{array}$ & --- & --- & $---\frac{1}{0}$ & $---\frac{-}{0}$ & $---\frac{1}{0}$ & ---- & $---\frac{1}{0}$ & $---\frac{}{0}$ \\
\hline $\begin{array}{l}\text { 1.1.1.2.03.06.01 } \\
\text { Level VI }\end{array}$ & $\begin{array}{l}\text { Instr. and Elect. } \\
\text { Upgrades } \\
\\
\text { Total }\end{array}$ & $\begin{array}{l}O E \\
C / E\end{array}$ & $\overline{0}$ & 0 & -- & 0 & --- & --- & $\overline{0}$ & $---\frac{-}{0}$ \\
\hline
\end{tabular}


TABLE $7.5-3$

TOTAL PROGRAM ELEMENT COST BASELINE - BY YEAR

\begin{tabular}{|c|c|c|c|c|c|c|c|c|c|c|}
\hline WBS / LEVEL & ACTIVITY TITLE / ADS & $\begin{array}{l}\text { FUND } \\
\text { TYPE }\end{array}$ & $\begin{array}{l}\text { FY01 I } \\
\text { FY05 }\end{array}$ & $\begin{array}{l}\text { FY06 I } \\
\text { FY10 }\end{array}$ & $\begin{array}{l}\text { FY11 I } \\
\text { FY15 }\end{array}$ & $\begin{array}{l}\text { FY16 I } \\
\text { FY20 }\end{array}$ & $\begin{array}{l}\text { FY21 I } \\
\text { FY25 }\end{array}$ & $\begin{array}{l}\text { FY26 I } \\
\text { FY30 }\end{array}$ & $\begin{array}{l}\text { FY31 / } \\
\text { FY35 }\end{array}$ & $\begin{array}{l}\text { FY36 / } \\
\text { FY40 }\end{array}$ \\
\hline $\begin{array}{l}\text { 1.1.1.2.03.06.02 } \\
\text { Level VI }\end{array}$ & $\begin{array}{l}\text { Site Electrical } \\
\text { Assessment } \\
\end{array}$ & $\begin{array}{l}\text { OE } \\
\text { C/E }\end{array}$ & ---- & --- & ---- & ---- & --- & ---- & ---- & --- \\
\hline $\begin{array}{l}\text { 1.1.1.2.03.06.03 } \\
\text { Level VI }\end{array}$ & $\begin{array}{l}\text { In-Tank Instrumentation } \\
\text { Total }\end{array}$ & $\begin{array}{l}O E \\
C / E\end{array}$ & --- & $-\cdots$ & ---- & --- & $---\frac{-}{0}$ & ---- & --- & --- \\
\hline $\begin{array}{l}1.1 .1,2.03 .06 .05 \\
\text { Level VI }\end{array}$ & $\begin{array}{l}\text { Electrical/ I\&C GPP's } \\
\qquad \text { Total }\end{array}$ & $\begin{array}{l}\text { OE } \\
C / E\end{array}$ & --- & ---- & --- & $---\frac{-}{0}$ & ---- & --- & ---- & -- \\
\hline $\begin{array}{l}1.1 .1 .2 .03 .07 \\
\text { Level VI }\end{array}$ & $\frac{\text { W314 Tank Farm }}{\frac{\text { Rest. / Safe Operations }}{\text { ADS 1120-B-M }}}$ & $\begin{array}{l}\text { OE } \\
\text { LI }\end{array}$ & $--\frac{\dot{-}}{0}$ & ---- & $---\overline{0}$ & $---\frac{-}{0}$ & $---\frac{-}{0}$ & $---\frac{-}{0}$ & $---\overline{0}$ & --- \\
\hline $\begin{array}{l}1.1 .1 .2 .03 .07 .01 \\
\text { Level VI }\end{array}$ & $\begin{array}{l}\text { W314 Tank Farm } \\
\text { Rest. / Safe Operations } \\
\qquad \text { Total }\end{array}$ & $\begin{array}{l}\mathrm{OE} \\
\mathrm{LI}\end{array}$ & 0 & -- & -- & - & - & $\begin{array}{r}--- \\
0\end{array}$ & $\begin{array}{r}--- \\
0\end{array}$ & -- \\
\hline
\end{tabular}

\section{[ R1 ]}


TABLE $7.5-3$

TOTAL PROGRAM ELEMENT COST BASELINE - BY YEAR

\begin{tabular}{|c|c|c|c|c|c|c|c|c|c|c|}
\hline WBS / LEVEL & ACTIVITY TITLE / ADS & $\begin{array}{l}\text { FUND } \\
\text { TYPE }\end{array}$ & $\begin{array}{l}\text { FY01 I } \\
\text { FY05 }\end{array}$ & $\begin{array}{l}\text { FY06 / } \\
\text { FY10 }\end{array}$ & $\begin{array}{l}\text { FY11 I } \\
\text { FY15 }\end{array}$ & $\begin{array}{l}\text { FY16 I } \\
\text { FY20 }\end{array}$ & $\begin{array}{l}\text { FY21 I } \\
\text { FY25 }\end{array}$ & $\begin{array}{l}\text { FY26 I } \\
\text { FY30 }\end{array}$ & $\begin{array}{l}\text { FY31 / } \\
\text { FY35 }\end{array}$ & $\begin{array}{l}\text { FY36 / } \\
\text { FY40 }\end{array}$ \\
\hline $\begin{array}{l}\text { 1.1.1.2.03.09 } \\
\text { Lovel VI }\end{array}$ & $\begin{array}{l}\text { Mechanical Upgrades } \\
\text { ADS 1120-0-0A } \\
\text { Total }\end{array}$ & $\begin{array}{c}\text { OE } \\
\text { C/E } \\
\text { GPP }\end{array}$ & $---\frac{-}{0}$ & 0 & $---\frac{-}{0}$ & $---\frac{-}{0}$ & $--\frac{-}{0}$ & $---\frac{-}{0}$ & $---\frac{-}{0}$ & $---\frac{-}{0}$ \\
\hline $\begin{array}{l}\text { 1.1.1.2.03.09.01 } \\
\text { Level VI }\end{array}$ & $\begin{array}{l}\text { W372 LL. Contr. Equip. } \\
\text { /T Plant } \\
\qquad \text { Total }\end{array}$ & $\begin{array}{l}O E \\
C / E\end{array}$ & --- & --- & $-\cdots-$ & ---- & ---- & ---- & ---- & --- \\
\hline $\begin{array}{l}\text { 1.1.1.2.03.09.02 } \\
\text { Level vI }\end{array}$ & $\begin{array}{l}\text { W372 LL. Contr. Equip. } \\
\text { Storage } \\
\qquad \text { Total }\end{array}$ & $\begin{array}{l}\text { OE } \\
\text { C/E } \\
\text { GPP }\end{array}$ & ---- & ---- & ---- & ---- & ---- & $---\frac{-}{0}$ & $\begin{array}{r}-r \\
0\end{array}$ & $---\frac{-}{0}$ \\
\hline $\begin{array}{l}1.1 .1 .2 .03 .09 .03 \\
\text { Level VI }\end{array}$ & $\begin{array}{l}\text { W-375 LL. Contr Equip } \\
\text { / Mockup } \\
\\
\text { Total }\end{array}$ & $\begin{array}{l}O E \\
C / E\end{array}$ & $---\overline{0}$ & ---- & ---- & $---\frac{-}{0}$ & $\begin{array}{r}--- \\
0\end{array}$ & $-\frac{}{0}$ & ---- & $---\frac{-}{0}$ \\
\hline $\begin{array}{l}\text { 1.1.1.2.03.09.04 } \\
\text { Level V1 }\end{array}$ & $\begin{array}{l}\text { W-374 LL. Contr Equip } \\
\text { Empty Container } \\
\\
\text { Total }\end{array}$ & $\begin{array}{l}\text { OE } \\
\text { C/E } \\
\text { GPP }\end{array}$ & $\begin{array}{r}---- \\
0\end{array}$ & -- & ---- & $---\frac{-}{0}$ & ---- & ---- & $\begin{array}{r}---- \\
0\end{array}$ & $-\cdots$ \\
\hline $\begin{array}{l}\text { 1.1.1.2.03.09.05 } \\
\text { Level VI }\end{array}$ & $\begin{array}{l}\text { Mechanical Upgrades } \\
\qquad \text { Total }\end{array}$ & $\begin{array}{l}\text { OE } \\
C / E\end{array}$ & 0 & & 0 & $\overline{0}$ & $---\frac{-}{0}$ & ---- & $-\cdots-$ & $---\frac{-}{0}$ \\
\hline
\end{tabular}

\section{[ R1 ]}


TABLE $7.5-3$

TOTAL PROGRAM ELEMENT COST BASELINE - BY YEAR

[ R1 ]

\begin{tabular}{|c|c|c|c|c|c|c|c|c|c|c|}
\hline WBS / LEVEL & ACTIVITY TITLE / ADS & $\begin{array}{l}\text { FUND } \\
\text { TYPE }\end{array}$ & $\begin{array}{l}\text { FY01 / } \\
\text { FY05 }\end{array}$ & $\begin{array}{l}\text { FY06 I } \\
\text { FY10 }\end{array}$ & $\begin{array}{l}\text { FY11 I } \\
\text { FY15 }\end{array}$ & $\begin{array}{l}\text { FY16 I } \\
\text { FY20 }\end{array}$ & $\begin{array}{l}\text { FY21 / } \\
\text { FY25 }\end{array}$ & $\begin{array}{l}\text { FY26 I } \\
\text { FY30 }\end{array}$ & $\begin{array}{l}\text { FY31 / } \\
\text { FY35 }\end{array}$ & $\begin{array}{l}\text { FY36 / } \\
\text { FY40 }\end{array}$ \\
\hline $\begin{array}{l}\text { 1.1.1.2.03.09.07 } \\
\text { Level VI }\end{array}$ & $\begin{array}{l}\text { Long Length Control } \\
\text { Equip. Disposal } \\
\qquad \begin{array}{l}\text { Total }\end{array}\end{array}$ & $\begin{array}{l}O E \\
C / E\end{array}$ & 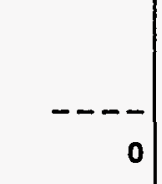 & $---\frac{-}{0}$ & $-\frac{-}{0}$ & $---\frac{1}{0}$ & --- & $---\overline{0}$ & $\begin{array}{r}---- \\
0\end{array}$ & -- \\
\hline $\begin{array}{l}\text { 1.1.1.2.03.09.08 } \\
\text { Level VI }\end{array}$ & $\begin{array}{l}\text { Engin. Tank Farm } \\
\text { Hardware } \\
\\
\quad \text { Total }\end{array}$ & $\begin{array}{l}O E \\
C / E\end{array}$ & $---\overline{0}$ & $-\frac{-}{0}$ & ---- & --- & $---\frac{1}{0}$ & $-\cdots$ & $---\frac{-}{0}$ & $--\frac{-}{0}$ \\
\hline $\begin{array}{l}\text { 1.1.1.2.03.09.10 } \\
\text { Level VI }\end{array}$ & $\begin{array}{l}\text { W-230 SST Ventilation } \\
\text { Total }\end{array}$ & $\begin{array}{l}\text { OE } \\
\text { C/E }\end{array}$ & $---\frac{-}{0}$ & --- & --- & $---\frac{-}{0}$ & -- & $---\frac{-}{0}$ & $---\frac{1}{0}$ & - \\
\hline $\begin{array}{l}1.1 .1 .2 .03 .10 \\
\text { Level VI }\end{array}$ & $\frac{\text { W030 Ventilation }}{\frac{\text { Upgrades }}{\text { ADS } 1120-2-0 G}}$ & $\begin{array}{l}\text { OE } \\
C / E\end{array}$ & $\begin{array}{r}--- \\
0\end{array}$ & --- & $---\frac{-}{0}$ & $---\frac{-}{0}$ & $---\frac{1}{0}$ & $---\frac{-}{0}$ & $---\frac{1}{0}$ & $---\frac{-}{0}$ \\
\hline $\begin{array}{l}1.1 .1 .2 .03 .10 .01 \\
\text { Level vI }\end{array}$ & $\begin{array}{l}\text { W-030 Ventilation } \\
\text { Upgrades }\end{array}$ & $\begin{array}{l}\text { OE } \\
\text { C/E }\end{array}$ & $\begin{array}{r}--- \\
0\end{array}$ & $\begin{array}{r}--- \\
0\end{array}$ & 0 & $\begin{array}{r}--- \\
0\end{array}$ & - & $\begin{array}{r}--- \\
0\end{array}$ & $\begin{array}{r}---- \\
0\end{array}$ & --- \\
\hline $\begin{array}{l}1.1 .1 .2 .03 .11 \\
\text { Level VI }\end{array}$ & $\frac{\text { W058 Xsite Transfer }}{\frac{\text { System }}{\text { ADS 1120-4-OH }}}$ & $\begin{array}{l}\text { OE } \\
\text { LI }\end{array}$ & $---\frac{1}{0}$ & $---\frac{1}{0}$ & $--\frac{-}{0}$ & --- & --- & --- & $---\frac{}{0}$ & --- \\
\hline $\begin{array}{l}\text { 1.1.1.2.03.11.01 } \\
\text { Level vI }\end{array}$ & $\begin{array}{l}\text { W-058 Xsite Transfer } \\
\text { System } \\
\\
\text { Total }\end{array}$ & $\begin{array}{l}\text { OE } \\
\text { LI }\end{array}$ & ---- & $---\frac{-}{0}$ & $---\frac{-}{0}$ & $---\frac{-}{0}$ & ---- & ---- & ---- & --- \\
\hline
\end{tabular}


TABLE $7.5-3$

TOTAL PROGRAM ELEMENT COST BASELINE - BY YEAR

\begin{tabular}{|c|c|c|c|c|c|c|c|c|c|c|}
\hline WBS / LEVEL & ACTIVITY TITLE / ADS & $\begin{array}{l}\text { FUND } \\
\text { TYPE }\end{array}$ & $\begin{array}{l}\text { FY01 / } \\
\text { FY05 }\end{array}$ & $\begin{array}{l}\text { FY06 I } \\
\text { FY10 }\end{array}$ & $\begin{array}{l}\text { FY11/ } \\
\text { FY15 }\end{array}$ & $\begin{array}{l}\text { FY16 / } \\
\text { FY20 }\end{array}$ & $\begin{array}{l}\text { FY21 / } \\
\text { FY25 }\end{array}$ & $\begin{array}{l}\text { FY26 / } \\
\text { FY30 }\end{array}$ & $\begin{array}{l}\text { FY31 / } \\
\text { FY35 }\end{array}$ & $\begin{array}{l}\text { FY36 / } \\
\text { FY40 }\end{array}$ \\
\hline $\begin{array}{l}\text { 1.1.1.2.03.12 } \\
\text { Level VI }\end{array}$ & $\frac{\text { W028 Aging Waste }}{\frac{\text { Xfer Lines }}{\text { ADS } 1120-7-0 \mathrm{M}}}$ & $\begin{array}{l}\text { OE } \\
\text { LI }\end{array}$ & $---\frac{-}{0}$ & --- & $---\frac{1}{0}$ & $---\frac{-}{0}$ & $-\cdots--$ & $---\frac{-}{0}$ & $---\frac{-}{0}$ & $---\frac{-}{0}$ \\
\hline $\begin{array}{l}1.1 .1 .2 .03 .12 .01 \\
\text { Level vi }\end{array}$ & $\begin{array}{l}\text { W028 Aging Waste } \\
\text { Xfer Lines } \\
\\
\end{array}$ & $\begin{array}{l}\mathrm{OE} \\
\mathrm{LI}\end{array}$ & $\begin{array}{r}---- \\
0\end{array}$ & - & $\begin{array}{r}---- \\
0\end{array}$ & $\begin{array}{r}--- \\
0\end{array}$ & $-\cdots$ & 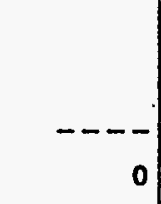 & -- & ---- \\
\hline $\begin{array}{l}1.1 .1 .2 .03 .16 \\
\text { Lovel } v\end{array}$ & $\begin{array}{l}\frac{\text { Facility Upgrades }}{\text { ADS 1120-0-OA }} \\
\text { Sub Total }\end{array}$ & $\begin{array}{l}\text { OE } \\
\text { C/E } \\
\text { GPP }\end{array}$ & $\begin{array}{r}0 \\
0 \\
--- \\
0\end{array}$ & $\begin{array}{r}\mathbf{0} \\
\mathbf{0} \\
--- \\
\mathbf{0}\end{array}$ & $\begin{array}{r}\mathbf{0} \\
\mathbf{0} \\
--- \\
\mathbf{0}\end{array}$ & $\begin{array}{r}\mathbf{0} \\
\mathbf{0} \\
-- \\
\mathbf{0}\end{array}$ & $\begin{array}{r}0 \\
0 \\
--0\end{array}$ & $\begin{array}{r}0 \\
0 \\
-- \\
0\end{array}$ & $\begin{array}{r}0 \\
0 \\
--- \\
0\end{array}$ & $\begin{array}{r}\mathbf{0} \\
\mathbf{0} \\
-- \\
\mathbf{0}\end{array}$ \\
\hline $\begin{array}{l}\text { 1.1.1.2.03.16.02 } \\
\text { Level VI }\end{array}$ & $\begin{array}{l}\text { W-322 242-S Sub Station } \\
\text { Upgrade } \\
\text { Total }\end{array}$ & $\begin{array}{l}\text { OE } \\
\text { C/E }\end{array}$ & $\begin{array}{r}---- \\
0\end{array}$ & -- & ---- & $\begin{array}{r}---- \\
0\end{array}$ & 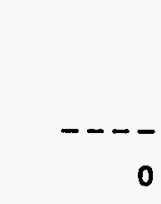 & 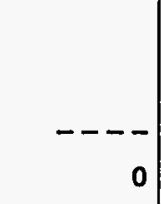 & - & ---- \\
\hline $\begin{array}{l}1.1 .1 .2 .03 .16 .03 \\
\text { Level VI }\end{array}$ & $\begin{array}{l}\text { W364 209E Septic Tank } \\
\text { Upgrade } \\
\qquad \text { Total }\end{array}$ & $\begin{array}{l}O E \\
C / E\end{array}$ & $\begin{array}{r}---- \\
0\end{array}$ & $\begin{array}{r}--- \\
0\end{array}$ & $\begin{array}{r}--- \\
0\end{array}$ & $\begin{array}{r}--- \\
0\end{array}$ & 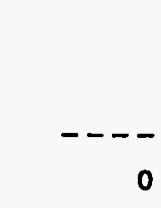 & $\begin{array}{r}--- \\
0\end{array}$ & - & ---- \\
\hline $\begin{array}{l}\text { 1.1.1.2.03.16.04 } \\
\text { Level VI }\end{array}$ & $\begin{array}{l}\text { W-280 SY\&C Tank Farm } \\
\text { Lighting } \\
\text { Total }\end{array}$ & $\begin{array}{l}O E \\
C / E\end{array}$ & --- & -- & --- & ---- & --- & $\begin{array}{r}--- \\
0\end{array}$ & -- & ---- \\
\hline
\end{tabular}

[ R1] 
TABLE $7.5-3$

TOTAL PROGRAM ELEMENT COST BASELINE - BY YEAR

\begin{tabular}{|c|c|c|c|c|c|c|c|c|c|c|}
\hline WBS / LEVEL & ACTIVITY TITLE / ADS & $\begin{array}{l}\text { FUND } \\
\text { TYPE }\end{array}$ & $\begin{array}{l}\text { FY01 I } \\
\text { FY05 }\end{array}$ & $\begin{array}{l}\text { FY06 / } \\
\text { FY10 }\end{array}$ & $\begin{array}{l}\text { FY11 / } \\
\text { FY15 }\end{array}$ & $\begin{array}{l}\text { FY16 / } \\
\text { FY20 }\end{array}$ & $\begin{array}{l}\text { FY21 / } \\
\text { FY25 }\end{array}$ & $\begin{array}{l}\text { FY26 / } \\
\text { FY30 }\end{array}$ & $\begin{array}{l}\text { FY31 I } \\
\text { FY35 }\end{array}$ & $\begin{array}{l}\text { FY36 I } \\
\text { FY40 }\end{array}$ \\
\hline $\begin{array}{l}1.1 .1 .2 .03 .16 .05 \\
\text { Level VI }\end{array}$ & $\begin{array}{l}\text { Facility Upgrades } \\
\text { (GPP's) } \\
\\
\end{array}$ & $\begin{array}{l}\text { OE } \\
\text { C/E } \\
\text { GPP }\end{array}$ & $---\overline{0}$ & $\begin{array}{r}---- \\
0\end{array}$ & $\begin{array}{r}--- \\
0\end{array}$ & $\begin{array}{r}---- \\
0\end{array}$ & $\begin{array}{r}---- \\
0\end{array}$ & --- & $\begin{array}{r}---- \\
0\end{array}$ & --- \\
\hline $\begin{array}{l}\text { 1.1.1.2.03.16.06 } \\
\text { Level VI }\end{array}$ & $\begin{array}{l}\text { W-226 Central Maint. } \\
\text { Facility Upgrade } \\
\qquad \text { Total }\end{array}$ & $\begin{array}{l}O E \\
C / E\end{array}$ & --- & $\begin{array}{r}--- \\
0\end{array}$ & $-\cdots$ & - & $\begin{array}{r}--- \\
0\end{array}$ & - & $\begin{array}{r}--- \\
0\end{array}$ & 0 \\
\hline $\begin{array}{l}\text { 1.1.1.2.03.16.07 } \\
\text { Level VI }\end{array}$ & $\begin{array}{l}\text { Radiation Zone Lighting } \\
\text { Total }\end{array}$ & $\begin{array}{l}O E \\
C / E\end{array}$ & -- & $\begin{array}{r}---- \\
0\end{array}$ & $\begin{array}{r}---- \\
0\end{array}$ & $\begin{array}{r}---- \\
0\end{array}$ & $\begin{array}{r}---- \\
0\end{array}$ & $---\overline{0}$ & --- & ---- \\
\hline $\begin{array}{l}\text { 1.1.1.2.03.17 } \\
\text { Level } \mathrm{V}\end{array}$ & $\begin{array}{l}\frac{\text { W188 Radiological }}{\text { Support Facility }} \\
\text { ADS } 1120-1-0 \text { Sub Total }\end{array}$ & $\begin{array}{l}O E \\
C / E\end{array}$ & $\begin{array}{r}0 \\
0 \\
-- \\
0\end{array}$ & $\begin{array}{r}0 \\
0 \\
--- \\
0\end{array}$ & $\begin{array}{r}0 \\
0 \\
-- \\
0\end{array}$ & $\begin{array}{r}\mathbf{0} \\
\mathbf{0} \\
-- \\
\mathbf{0}\end{array}$ & $\begin{array}{r}0 \\
0 \\
--- \\
0\end{array}$ & $\begin{array}{r}0 \\
0 \\
-- \\
0\end{array}$ & $\begin{array}{r}0 \\
0 \\
-- \\
0\end{array}$ & $\begin{array}{r}0 \\
0 \\
-- \\
0\end{array}$ \\
\hline $\begin{array}{l}1.1 .1 .2 .01 .17 .01 \\
\text { Level VI }\end{array}$ & $\begin{array}{l}\text { W188 Radiological } \\
\text { Support Facility } \\
\\
\text { Total }\end{array}$ & $\begin{array}{l}\mathrm{OE} \\
\mathrm{LI}\end{array}$ & - & -- & - & - & -- & $\begin{array}{r}--- \\
0\end{array}$ & ---- & --- \\
\hline
\end{tabular}

[ R1] 
WHC-SP-1101

\section{NOTES \\ TOTAL PROGRAM ELEMENT COST BASELINE - BY YEAR}

The cost exhibit tables in each of the Program Element Baselines (Sections 7.2 through 7.10) identify the program element target funding by year by fund type. The tables also provide details of the baseline allocation of funding down to activity levels (level V) and cost account levels (level VI) of the WBS for Fiscal Year 1995 through-1997. The details exhibited on these tables are taken from the baseline WBS Dictionary Sheets. Deltas exist between the details and the annual target totals. For Fiscal Years 1996 and 1997 the deltas will be addressed in the future, following the completion of the resolution of expected differences between current Fiscal Year 1995 baseline funding and expected actual Fiscal Year 1995 funding. Deltas for Fiscal Year 1995 are noted below by program element:

\section{PROGRAM MANAGEMENT AND ADMINISTRATION:}

No deltas.

\section{TANK FARM OPERATIONS AND MAINTENANCE:}

No overall delta. This baseline requires revised fund type mix.

\section{WASTE TANK SAFETY:}

Delta is due to need to revise an overhead pricing rate in the P3 system.

\section{TANK FARM UPGRADES:}

Delta is due to differences between Budget Authority (BA) in the target and Budget Obligation (B0) in the P3 system.

\section{CHÄRACTERIZATION:}

No significant deltas.

\section{WASTE RETRIEVAL:}

Delta is primarily due to need to resolve issues associated with expense funding support to capital projects and differences between Budget Authority (BA) in the target and Budget Obligation (B0) in the P3 system.

\section{WASTE PRETREATMENT:}

Delta is due to the sum of the detail cost estimates being lower than the target total as a result of reestimation of resource requirements to complete baseline scope.

\section{LOW LEVEL WASTE:}

Delta is primarily due to carryover expense funded workscope on melter testing addressed in June 9 , 1994 RL letter.

$$
T 7.5-3.15
$$


WHC-SP-1101

\section{HIGH LEVEL WASTE:}

Delta is due to carryover of capital funding, PNL expense funding carryover, and ADS corrections submitted but not reflected in target totals. 
Table 7.5.4. FY 1995 Cost by Month by Fund Type by Activity and Cost Account

Future MYWP updates will include Table 7.5-4, which establishes the month-by-month cost baseline for FY 1995 for this program element. The FY 1995 cost baseline is exhibited at the cost account level with fund type totals provided. Cost data are then rolled up to the activity and program element levels. Also exhibited is the total cost baseline for the program element for FY 1995. At present, the program element cost baseline for FY 1995 for this program element is the total provided on this table less a productivity challenge for FY 1995. 
Table 7.5-5. Total Full-Time Equivalents by Year by Activity by Major Participant (Direct and Indirect) (Including all Subcontractors)

Future MYWP updates will include Table 7.5-5, which establishes total staffing projections for this program element. Included in the table are FY 1994 staffing data as well as projections by year for FY 1995 through FY 2000. The projections in this table include all direct and indirect Full-Time Equivalents (FTEs), including all subcontractor personnel. Where data are available, projections are exhibited at the activity level with major program participant totals provided. 
Table 7.5-6. FY 1995 through FY 1997 Direct Westinghouse Hanford Company Full-Time Equivalents by 88 Labor Categories

Future MYWP updates will include Table 7.5-6, which establishes direct WHC staffing projections for this program element. Projections do not include subcontractor support and are provided by 88 labor categories for FY 1995 through FY 1997. 


\subsection{CHARACTERIZATION BASELINE}

The Characterization Program Element baseline consists of the technical bases, schedule, resource requirements, and waste type data for FY 1995 through completion of the mission. The major emphasis is on deliverables, schedule, and resource requirements for FY 1995, 1996, and 1997.

\subsubsection{Technical Bases}

This section provides the mission strategy; work breakdown structure and organization responsibility; work to be performed in FY 1995, 1996 and 1997; technical approach; and drivers and deliverables.

\subsubsection{Mission Strategy}

\subsection{Objectives}

The Characterization Program's overall objective is to provide, in a timely and cost-effective manner, required characterization data of appropriate quality to TWRS program elements responsible for tank farm process control; tank waste safety issue resolution; and waste treatment, storage, and disposal decisions.

\subsection{Strategy}

The strategy for meeting the Program objectives includes the following. The Data Quality Objectives (DQO) process, as defined by the Environmental Protection Agency, will be utilized to define requirements of characterization data users. Historical processing and analytical data will be used to meet as much of the data requests as possible. Sampling and analytical laboratory capabilities, including use of offsite laboratories, will be increased to supplement historical data. Priority will be given to sampling for safety issue closure and ensuring safe operations in the near term. Tank sampling will be scheduled consistent with systems engineering and planning for all TWRS program elements (e.g., Waste Retrieval, Waste Pretreatment, and Waste Tank Safety).

\subsection{Functions and Requirements}

Systems engineering is being applied to the TWRS Program. This is a structured process to identify and implement a complete, traceable solution to a mission need for complex programs or projects.

Characterize Waste (4.2.1.2) is the function that is the Characterization Program's contribution to completing the TWRS Mission. It involves providing physical, chemical, and radiological characterization information of material stored in waste tanks at TWRS in support of process control; safety issue resolution; treatment, storage, and disposal decisions; or other TWRS needs. Tank waste characterization activities include historical data review and evaluation, determination of what supplemental data are needed to meet DQOs, sample acquisition and transfer to laboratories, analysis of samples, in situ measurements, and data management in a centrally accessible database. 
The Characterize Waste function is divided into the following subfunctions:.

- $\quad$ 4.2.1.2.1, Perform DQO Activities

- 4.2.1.2.2, Prepare Analysis Plans

- $\quad$ 4.2.1.2.3, Acquire Physical Samples for Analysis

- 4.2.1.2.4, Analyze Samples

- $\quad 4.2 .1 .2 .5$, Conduct In Situ Analysis

- 4.2.1.2.6, Manage Data

- 4.2.1.2.7, Evaluate Generator Knowledge and Prepare Tank Characterization Reports.

\subsection{Risks}

Access to the tanks to take samples that best represent the tank's content is a potential impedance to timely characterization of wastes.

\subsection{Major Decisions}

The Defense Nuclear Facilities Safety Board (DNFSB) recognized the importance of obtaining accurate tank waste physical and chemical properties information to resolve safety issues with tank waste storage. A methodology for obtaining tank waste characterization information was evaluated during negotiations of the Hanford Federal Facility Agreement and Consent Order (Tri-Party Agreement). Stakeholder groups, Washington State Department of Ecology, the Yakama Indian Nation, the U.S. Environmental Protection Agency, and the U.S. Department of Energy (DOE) all participated in the formulation and review of proposed revisions to the Tri-Party Agreement. The alternative selected for obtaining tank waste characterization information is, where appropriate, to utilize historical information, obtain and analyze samples of tank waste, and analyze tank waste in situ to obtain necessary information. In response to DNFSB concerns, DOE-Richland Operations Office (RL) developed a plan for obtaining the necessary tank waste physical and chemical properties information (DOE/RL-94-0001).

\subsection{Assumptions}

1. Tank safety concerns will not prevent sampling and characterization. No unforeseen safety concerns arise requiring pause and/or standdown of field sampling or laboratory operation.

2. Two additional push/rotary mode core sampling systems will be deployed in FY 1995 .

3. A consistent level of quality and content will be achieved within the DQOs addressing safety, operations, and disposal issues.

4. An adequate number of tank access spots (risers) will be available to meet requirements in DQOs.

5. Historical information on tank waste contents will provide information on the key analyses and parameters required for disposal programs.

6. Availability of operations crews and laboratory staff is a crucial element. Once all new operations crews are qualified/certified there will be enough crews available to perform all sampling activities. Laboratory capabilities are being expanded by adding hot cells, personnel, and offsite (Idaho National Engineering Laboratory [INEL]) capability. 
WHC-SP-1101

7. It will be possible to verify historical information in order to determine its utility.

\subsection{Constraints}

The scope of the Characterization Program is limited to sampling and characterization of wastes located within underground storage tanks under control of TWRS and is defined by specified needs documented in DQOs.

Certain physical properties change during the sampling process. It these properties need to be measured as they currently exist in the tanks, in-situ measurement methods must be developed.

Tank sampling is currently restricted to locations directly beneath risers and the number of risers that can be used are very limited in most single-shell tanks (SSTs). Access to additional locations will require development of new sampling technology or addition of new risers.

Sample size is currently limited, which may restrict analytical protocols.

\subsubsection{Structure}

\subsection{Work Breakdown Structure}

The Characterization program element is part of the Manage Tank Waste WBS Level III and consists of the following Level V elements: Technical Integration and Planning, Technology Development and Applied Engineering, Field Sampling and Measurement, Analytical Integration, and Data Evaluation and Reporting. A further breakdown to the levels required to manage discrete tasks is shown in Figure 7.6-1. The Characterization Program WBS Dictionary is found in Appendix A.

\subsection{Organization and Responsibility}

The Characterization Program is responsible for managing the tank waste characterization efforts necessary to support safe operations, storage, and disposal of tank wastes. The Characterization Program provides leadership to establish technical bases, identify field sampling needs, evaluate data, recommend actions, provide laboratory analysis guidance, and manage technical activities associated with tank waste characterization, all of which are needed to implement the TWRS Program Mission.

Specific responsibilities of the Characterization Program include the following.

- Ensure the capture, integration, and reporting of known tank waste information, including process history (e.g., transfer records, historical assays); field data taken specifically for characterization; and sampling and analysis data and information.

- Ensure that program element waste characterization requirements for the TWRS Program are formally specified through the DQO process.

- Identify, integrate, and prioritize additional sampling and analysis needs of the TWRS program elements.

- Ensure development of technologies required to satisfy the tank waste sampling and analysis needs of the TWRS program elements. 
- Conduct tank waste historical data review, sampling, analysis, and data reporting responsive to:

- $\quad$ specifications in the Recommendation 93-5 Implementation Plan (DOE/RL-0001)

- $\quad$ prompt resolution of the waste tank safety issues

- the schedule and performance needs for selection, design, and development of processes for waste retrieval, waste classification, and waste pretreatment and vitrification.

- Issue Tank Characterization Reports based on process knowledge, prior characterization data, and recent laboratory data, as defined in Tri-Party Agreement Milestone M-44-00.

The Characterization Program has both internal and external customers. The internal customers, identified as Westinghouse Hanford Company (WHC) organizations and other Hanford Site contractors, need tank characterization data to manage tank waste operations related activities. The external customers are composed mostly of agencies and oversight groups. These groups sometimes focus on the Characterization Program; but, in general, they view characterization as key input to be able to evaluate other TWRS activities, such as retrieval, pretreatment, or disposal.

\subsubsection{FY 1995 Work to be Performed}

The scope of the Characterization Program includes acquisition of tank waste samples and transfer of the samples to laboratories, laboratory analysis of samples, in situ measurements, review of historical data and laboratory results, and the integration and reporting of characterization information.

Characterization information may be in paper and/or electronic form. This information includes, but is not limited to, the following: historical tank contents estimates; Tank Characterization Reports; laboratory analysis results; in situ analysis results; integrated schedules; Tank Waste Analysis Plans; Tank Characterization Plans; Waste Analysis Plans; operational data for characterization; and historical data.

Each TWRS characterization need is validated through formal DQO methodology for analyses required to meet specific Operational Safety Requirement (OSR)/Operating Specification Document (OSD) requirements (e.g., $\mathrm{pH}$ limits/hydroxide limits), as well as end-user program needs from which sampling requirements, schedules that support program decisions, and data specifications are formally documented. Samples taken for development work (not analyses) will be specified in approved test plan(s). These validated needs provide the basis for planning and integration, resource use (i.e., sampling systems, laboratories) and modeling, and prioritization. Systems engineering techniques were used to develop the sampling requirements and schedules for FY 1995 through 1997 with a focus on gathering the right information at the right time. The Characterization Program will provide guidance to other TWRS program elements in the development of DQOs or test plans to address specific issues. Based on the DQOs, guidance will be provided to the Characterization Program for quality assurance/quality control protocols required for sampling and analysis activities. The Tank Characterization Plans will use this information, when available, and provide direction as appropriate. Prior to collection of samples, the Laboratory and the Characterization Program must agree on the data format and validation criteria by approving the Tank Characterization Plan. For OSR/OSD requirements for operational control, letters of instruction referencing the OSR/OSD requirement can substitute for DQO/Tank Characterization Plan requirements. Data format and validation criteria will be specified for each tank in the respective Tank Characterization Plan. 
The Tank Waste Analysis Plan provides direction for sampling activities, which include Part B Permit sampling for double-shell tanks (DSTs) and tank sampling to support 242-A Evaporator campaigns per permit requirements. Sampling and analysis needs for safety screening, safety resolution, disposal characterization needs as specified, compatibility, and grab sampling are defined by the Waste Analysis Plan and DQOs via emergency request to support OSD/OSR requirements. Tank Characterization Plans will cover all types of sampling. Individual tank samples are under the umbrella of the Characterization Program. Blended samples needing analysis are considered process development samples and are not Characterization samples.

The deliverable for data packages or data reports (i.e., laboratory analysis and quality assurance documentation), including validation (if required) and transmittal to the regulators (if required), shall be specified in the Tank Waste Analysis Plans and Tank Characterization Plans.

\section{Technical Integration and Planning (WBS 1.1.1.2.04.01)}

The objective of this activity for FY 1995 is to administratively support the planning, scheduling, and implementation of waste tank sampling and analysis within the Characterization Program and oversee and coordinate the following activities to ensure timely completion of activities, good communication among participants, and effective utilization of funds. Deliverables that will accomplish this support are:

- Financial Planning - prepare budgets and Estimates at Completion (EACs) for this activity.

- Establish milestones and development of a milestone control log.

- Support meeting and presentations concerning stakeholders outside the Characterization Program.

- Provide support to prepare and maintain necessary quality assurance documents that are specifically applicable to the Characterization Program (all activities).

Support TWRS baseline and systems engineering activities.

- $\quad$ Prepare and issue quarterly status reports for DNFSB 93-5

- $\quad$ Support Tri-Party Agreement quarterly unit manager meetings and other special Tri-Party Agreement meetings as requested.

Included in this activity is the budget for the Tank Characterization Advisory Panel (TCAP), which will provide review and recommendations for the Characterization Program. The TCAP will interface with WHC and Pacific Northwest Laboratory (PNL) staff. They will also provide technical recommendations for the various associated areas such as laboratory, retrieval, and pretreatment.

Also included within this activity is budget for PNL Characterization Program management support and SST Riser demonstration support. 
Technology Development and Applied Engineering (WBS 1.1.1.2.04.02)

This activity encompasses all tasks needed to acquire new technology (equipment, instruments, and procedures) to achieve requirements specified in DQO and Tank Characterization Plans. Justification for developing these new technologies falls into three categories - capability to perform sampling or analytical tasks for DQOs that cannot be fulfilled with current technology; substantial improvements in cost effectiveness of sampling or analytical operations; and increased worker safety. In FY 1995, additional efforts will be focussed on confirming the justification of ongoing tasks in this activity by rigorous evaluation of capabilities relative to published DQO reports and projections of future needs by TWRS Programs.

Activity is underway in developing spectroscopic scanning techniques for hot cell analysis of core samples. Original justification for these techniques focussed on safety issues related to the concentration of ferrocyanide compounds in the waste. Subsequent actions in the Safety Program have reduced the need for this type of analysis; however, there are potential time and worker exposure reductions from employing core scanning techniques in the hot cells. Continuation of the Raman spectroscopy, Laser ablation with mass spectroscopy and infrared spectroscopy is planned for FY 1995 contingent on documentation of needs and benefits by the end of FY 1994. (Preparation of this documentation is underway.) Specific actions in FY 1995 are demonstration of scanning analyses on archived waste samples and preparation of conceptual designs for implementation in hot cell arrangements.

Analytical procedures development began in FY 1994 to acquire capability to meet published DQO needs at the onsite laboratories (222-S and 325). That development will continue in FY 1995 , principally for organic complexants, thermal properties, and physical properties. In addition, a rigorous evaluation of currently used procedures and instrumentation will be performed to identify areas for improvement. That evaluation, along with evaluations of new or revised DQO reports, will supply the basis for future analytical procedures development.

Parallel to the hot cell and analytical procedure development, implementation of in situ measurement technologies will be developed. The most likely platform for deploying sensors in waste tanks is the cone penetrometer. Testing and design, which started in FY 1994, will continue. A key uncertainty for this development is the availability of sensors for deployment in a time frame that is beneficial to the TWRS Programs. Physical property measurements are needed by the Retrieval Program; however, those needs are being developed and documented at this time. The cone penetrometer testing and design are being managed in a phased approach with decision points at key intervals to ensure that system design and operability are beneficial to Characterization needs.

In FY 1995 tasks related to improving field sampling methods are consolidated. The major tasks in FY 1995 are development of temperature monitoring for the sampler core bit, development of an instrumented cask to determine the success of individual sampling events at the tank site, evaluation of sampling tools used at Hanford, and evaluation of large volume sampling devices used at other DOE sites for possible applications at Hanford. The bit temperature monitoring task is a continuation of development that began in FY 1994 at Sandia National Laboratories. Prototypes fabricated in FY 1994 and early FY 1995 will be tested and adapted for installation on the core sampling truck systems. A subcontractor has been selected to design and fabricate the instrumented cask. Probable instrumentation includes a load cell and gamma and neutron probes. The sampler performance evaluation will begin in FY 1995 to establish the operating envelope for existing samplers. This evaluation will help identify further needs. The Savannah River Site has used samplers with larger volume capacity than those used at Hanford. TWRS Engineering and Operations staff will evaluate 
the feasibility of using those samplers at Hanford. If they are feasible, the necessary physical and procedural modifications will be made for their use.

\section{Field Sampling and Measurement (WBS 1.1.1.2.04.03)}

The scope of this activity is a composite of all sampling events necessary for the safe collection and transportation of representative physical waste samples (solids, liquids, gasses) from high level waste tanks to an onsite laboratory for analysis. This sampling supports areas of safety issue resolution; process control; treatment, storage, and disposal (TSD) decisions; and other TWRS Program issue resolutions. Physical waste sampling methods include core sampling, auger sampling, vapor sampling, and grab sampling (both supernate and sludge waste types). Sampling activities will be conducted as scheduled in the Characterization Integrated Sampling Schedule and as directed by Tank Characterization Plans. Additionally, the procurement and/or fabrication of all major sampling equipment is included.

Core samples will be collected from tank waste surface to tank bottom in both SSTs and DSTs that have solids greater than 25 inches deep. The method in which the cores are collected is waste type dependent. In general, the rotary mode core sampling system will be used to sample salt cake predominant waste types and the push mode core sampling system on sludges. At the initiation of FY 1995, there will be one push mode only capable core sampling system and one push/rotary capable core sampling system. Two additional push/rotary mode core sampling systems will be deployed prior to FY 1996.

Subactivities required for the core sampling effort include, but are not limited to, tank riser preparation, engineering, sampling operations, planning, scheduling, procedure writing, equipment maintenance, equipment fabrication, equipment decontamination, drafting, packaging support, riser preparation, safety, and quality assurance.

Auger samples will be collected from tank waste surfaces in both SSTs and DSTs and for any tank with less than 25 inches of solid waste. These samples are gathered to support programmatic issue resolutions and occasionally as a prerequisite for push mode core sampling. No auger sampling has been requested after FY 1996. Subactivities required for the auger sampling effort include, but are not limited to, engineering, sampling operations, planning, scheduling, procedure writing, equipment maintenance, equipment fabrication, equipment decontamination, drafting, packaging support, safety, and quality assurance.

Vapor samples will be collected from tank dome spaces in SSTs. Vapor samples are collected as a prerequisite to some core sampling activities and thermocouple tree installations. Additionally, these samples are necessary for the support of vapor safety resolution issues. Subactivities required for the vapor sampling effort include, but are not limited to, engineering, sampling operations, planning, scheduling, procedure writing, equipment maintenance, equipment fabrication, drafting, packaging support, safety, and quality assurance.

Grab supernate and sludge samples will be collected from SSTs and DSTs. Grab samples are collected to provide information in support of tank safety screening, safety resolution, waste compatibility issues, operational control, and pretreatment/retrieval requirements. Subactivities required for the core sampling effort include, but are not limited to, engineering, sampling operations, planning, scheduling, procedure writing, equipment fabrication, equipment decontamination, drafting, packaging support, safety, and quality assurance. 
Activities associated with the PAS-1 transport cask and Neutralized Current Acid Waste (NCAW) transport cask will be pursued in FY 1995. In specific, WHC will follow Nuclear Regulatory Council progress toward the issuance of a Certificate of Compliance amendment for the PAS-1 cask. Secondly, the fabrication of three NCAW casks all be completed. Lastly, the Sample Verification Instrumented will be deployed into field activities, thus providing a means to verify core sampler waste fill.

\section{Analytical Integration (WBS 1.1.1.2.04.04)}

The Analytical Integration activity is responsible for ensuring that all tank samples are analyzed in accordance with analytical requirements defined in DQOs, tank specific Tank Characterization Plans, and Waste Analysis Plans (as part of RCRA Part B for DSTs or Evaporator) or Letters of Instruction to support OSD/OSR compliance. Specific laboratory analysis functions include sample preparation (sample receipt, sample extrusion/breakdown, preparation of subsamples and any required repackaging and shipment of samples for offsite analysis), sample analysis, and data reporting. In addition, the Analytical Integration activity will include vapor sample analyses and data reporting beginning in FY 1995 at Pacific Northwest Laboratory and Oak Ridge National Laboratory.

As required for DST and SST core samples, auger samples, and supernatant samples, letter reports documenting results of selected high-priority safety screening.analyses shall be reported within 45 days of receipt at the laboratory of the last segment from the last core. The 45-day reports for grab and/or auger (noncore) samples will be from the time received at the laboratory. Data reports will be issued per requirements of tank specific Tank Characterization Plans for DST and SST core samples within 216 days of the sampling of the last segment of the last core from the tank and within 176 days for auger samples.

Program management functions for this activity will include program-related laboratory guidance via the Tank Waste Analysis Plan and Tank Characterization Plans, definition of performance requirements, selecting analytical laboratories to conduct the analyses, estimating and planning laboratory capacities and workload, and monitoring analytical progress against schedule.

The laboratory readiness cost account includes a basic lab assessment to maintain the laboratories in a ready-to-serve mode to perform analyses, the sample exchange program to enhance laboratory quality assurance and control, alternative tasks in the event of a stand down, reanalysis of samples, and archiving and disposal of samples. In the event of a stand down, which would result in a reduction in laboratory workload to the point that laboratory readiness cannot be maintained (such as the inability to provide the laboratories with samples), alternative tasks have been defined. Alternative tasks include such activities as housekeeping, cross training, and procedure development/review.

The offsite laboratory cost account includes completion of upgrades to the Idaho National Engineering Laboratory (INEL) for the receipt, analysis, and data reporting on samples in support of the Characterization Program. The work direction for INEL will be defined via tank specific Tank Characterization Plans or letters of instructions, as identified in the statement of work.

The 222-S Laboratory upgrades and Laboratory Information Management System (LIMS) development cost account will provide upgrades to meet analytical capabilities and capacities as requested by the TWRS Characterization Program. This cost account also includes continuing implementation of new LABCORE data management systems. Data management systems will provide the 222-S Laboratory with electronic data transfer of sample analysis data to the Tank Characterization Database (TCD). 
The 325 Laboratory upgrades cost account will provide upgrades to meet analytical capabilities and capacities as requested by the TWRS Characterization Program.

The offsite repackaging and shipping cost account will provide the capability to repackage samples at either the $222-S$ or 325 Laboratory to send offsite to the INÉL laboratory for analysis. This activity is required because it is not possible to ship off site existing sample casks as received from the field sampling program element.

\section{Data Evaluation and Reporting (WBS 1.1.1.2.04.05)}

Activities for Data Evaluation and Reporting will cover the following.

Determine the requirements for characterization as defined by other TWRS program elements and support the definition of a characterization strategy to meet those requirements. Provide documentation of the Characterization Program technical basis, including the necessary analysis of existing data packages and historical data. Support other programs in the development of DQOs defining their needs. Provide statistical support during the development of DQOs and training to DQO teams as needed. Review and update the DQO Strategy and Guidance documents to ensure that they continue to address the needs of the TWRS DQO Program.

Provide an overall plan to obtain the required analytic information (Tank Waste Analysis Plan) as well as specific Tank Characterization Plans to support each tank sampling and analysis event. Complete the compilation and analysis of historical information to provide Historical Tank Contents Estimate reports for the 68 tanks in the northwest and southeast quadrants. Include tank layering models and estimates of the content of each waste layer in the input to these reports. Provide qualitative and quantitative models for the waste layering in tanks. Determine the sampling and analysis requirements to support verification (or modification) of the historic tank layering models. Develop methods for grouping and sorting of tanks to expedite characterization.

Provide Tank Characterization Reports, which combine the historical information with any new sample analysis information, to provide the best current description of waste tank contents. Use existing data packages, statistical methods, and chemical knowledge to develop methods for integrating historical information with new analytic data and determining the resulting confidence levels. Determine the appropriate statistical approach for combining multiple data sources into the reports in a defensible manner.

Enter the appropriate historical, analytical, and Tank Characterization Report information into an online database for ready data accessibility. Ensure characterization information (paper and electronic) is properly archived and readily accessible to users. Inform users regarding data availability. Provide summary information about existing data. Review user needs for the Tank Characterization Database. Maintain the database and add new features to address user needs.

Oversee and coordinate all above activities to ensure timely completion of activities, good communication among participants, and effective utilization of funds.

This activity does not include the actual preparation of DQOs for other programs (which will be supported by the requesting program), the performance of sampling activities, the performance of laboratory analytic activities, or the validation of analytic data. 


\subsubsection{FY 1996 - FY 1997 Work to be Performed}

\section{Technical Integration and Planning (WBS 1.1.1.2.04.01)}

The integration of characterization activities, including program management and administration, planning, and characterization support activities continues in FY 1996 and 1997. Program Management and Administration includes the development of baseline plans, reporting/statusing, and Characterization schedules and budgets as well as program business activities. Support for TCAP and oversight activities, both internal and external, will also be performed.

\section{Technology Development and Applied Engineering (WBS 1.1.1.2.04.02)}

Technology Development and Applied Engineering work scope for FY 1996 and 1997 will be based on the outcome of FY 1995 tasks. Implementation of developed technologies will continue. Those spectroscopic scanning technologies that are shown to be feasible and beneficial to the Characterization mission will be transferred to routine analytical use by preparing appropriate procedures, completing safety and readiness reviews, and conducting training for the laboratory personnel. Deployment of operating systems is anticipated in FY 1997. Based on the preliminary schedule for testing, design, and fabrication of a cone penetrometer system, deployment is planned for FY 1996.

Rigorous evaluations of sampling and analytical practices relative to needs as stated in DQOs and Tank Characterization Plans will continue each year to form the basis for detailed annual planning.

\section{Field Sampling and Measurement (WBS 1.1.1.2.04.03)}

This scope of this activity is a composite of all sampling events necessary for the safe collection and transportation of representative physical waste samples (solids, liquids, gasses) from high-level waste tanks to an onsite laboratory for analysis. This sampling supports areas of safety issue resolution, process control, TSD decisions, and other TWRS Program issue resolutions. Physical waste sampling methods include core sampling, auger sampling, vapor sampling, and grab sampling (both supernate and sludge waste types). Sampling activities will be conducted as prescribed by the Characterization Integrated Sampling Schedule and as directed by Tank Characterization Plans. Additionally, the procurement and/or fabrication of all major sampling equipment is included.

Core samples will be collected from tank waste surface to tank bottom in both SSTs and DSTs. The method in which the cores are collected is waste type dependent. In general, the rotary mode core sampling system will be used to sample salt cake predominant waste types and the push mode core sampling system on sludges. At the initiation of FY 1996 there will be one push mode only capable core sampling system and three push/rotary capable core sampling systems. No push mode core sampling will be required after FY 1996. Subactivities required for the core sampling effort include, but are not limited to, engineering, sampling operations, planning, scheduling, procedure writing, equipment maintenance, equipment fabrication, equipment decontamination, drafting, packaging support, safety, and quality assurance.

Auger samples will be collected from tank waste surfaces in both SSTs and DSTs. These samples are gathered to support programmatic issue resolutions as well as a prerequisite for push mode core sampling. No auger sampling has been requested after FY 1996. Subactivities required for the auger sampling effort include, but are not limited to, engineering, sampling operations, planning, 
scheduling, procedure writing, equipment maintenance, equipment fabrication, equipment decontamination, drafting, packaging support, safety, and quality assurance.

Vapor samples will be collected primarily from tank dome spaces in SSTs. Vapor samples are collected as a prerequisite to core and auger sampling activities and thermocouple tree installations. Additionally, these samples are necessary for the support of vapor safety resolution issues. Subactivities required for the vapor sampling effort include, but are not limited to, engineering, sampling operations, planning, scheduling, procedure writing, equipment maintenance, equipment fabrication, drafting, packaging support, safety, and quality assurance.

Grab supernate and sludge samples will be collected from SSTs and DSTs. Grab samples are collected primarily to provide information in support of tank safety screening, waste compatibility issues, operational control, and pretreatment/retrieval requirements. Subactivities required for the core sampling effort include, but are not limited to, engineering, sampling operations, planning, scheduling, procedure writing, equipment fabrication, equipment decontamination, drafting, packaging support, safety, and quality assurance.

\section{Analytical Integration (WBS 1.1.1.2.04.04)}

At the start of FY 1996, samples will continue to be received from auger, grab, push, and vapor mode sampling operations. The emphasis of laboratory-related work to be performed in FY 1996 through FY 1997, however, will primarily focus on sample analysis and data reporting in support of rotary mode sampling operations (including vapor sampling required to support rotary mode sampling). In addition, it is expected that analytical support may increase in support of Retrieval and Disposal Programs (inclusive of Pretreatment, Low-Level Waste, and High-Level Waste Programs), notably in support of the receipt and repackaging of samples at onsite laboratories for shipment to offsite laboratories to support disposal development work.

\section{Data Evaluation and Reporting (WBS 1.1.1.2.04.05)}

Data Evaluation and Reporting activities for FY 1996 through FY 1997 will include the following.

Review the requirements for characterization as defined by other programs and determine if revisions to the characterization strategy are required to meet those requirements. Update documentation of the Characterization Program technical basis, including the necessary analysis of existing data packages and historical data. Support other programs in the development and revision of DQOs defining their needs. Provide statistical support during the development of DQOs and training to DQO teams as needed. Review and update the DQO Strategy and Guidance documents to ensure that they continue to address the needs of the TWRS DQO Program.

Provide an update to the overall plan to obtain the required analytic information (Tank Waste Analysis Plan) and provide specific Tank Characterization Plans to support each tank sampling and analysis event. Review the Historical Tank Contents Estimate reports for all 177 tanks and revise and update the reports based on new information and model verification results. Apply the quantitative waste layering model to all tanks to improve historical evaluation. Integrate the results of sampling and analysis done to support verification (or modification) of the historic tank layering models. Complete development of methods for grouping of tanks, compare results of sampling and analysis to predicted results and refine methods. 
Provide Tank Characterization Reports, which combine the historical information with any new sample analysis information to provide the best current description of waste tank contents. Apply methods for integrating historical information with new analytic data and determining the resulting confidence levels. Apply the appropriate statistical approach for combining multiple data sources into the reports in a defensible manner.

Enter the appropriate historical, analytical, and Tank Characterization Report information into an online database for ready data accessibility. Ensure that characterization information (paper and electronic) is properly archived and readily accessible to users. Inform users regarding data availability. Provide summary information about existing data. Review user needs for the Tank Characterization Database. Maintain the database and add new features to address user needs. Move the database software and data to a new host computer for simpler maintenance and support.

Oversee and coordinate all above activities to ensure timely completion of activities, good communication among participants, and effective utilization of funds.

This activity does not include the actual preparation of DQOs for other programs (which will be supported by the requesting program), the performance of sampling activities, the performance of laboratory analytic activities, or the validation of analytic data.

\subsubsection{Approach}

The Characterization Program strategy is based on the DQO process to develop and define the data needs. The strategy depends on first determining which data needs can be met with historical information and which require acquisition of new samples. The DQO process supports the development of detailed analytical requirements in cases where new sampling and analysis are necessary. These detailed analytical requirements include the following: the number and type of samples per unit time and per time period, the suite of analyses for field samples, the types of sample matrices, and analytical performance objectives (e.g., detection limit, accuracy, and precision).

In evaluating the data requirements as part of the systems engineering efforts, different program elements require data at different times. This allows the Characterization Program to focus on certain near-term issues and to defer other issues to more opportune times for sampling and analysis. Data gathering needed to support safety issue resolution is being accelerated. The Waste Pretreatment Program Element is likely to be based on a small set of alternative treatments and data can focus on the average composition of a small number of analyses to establish bounding conditions for interfering substances in the waste. All other pretreatment data requirements can wait until the material from several single-shell tanks has been retrieved and blended. Sampling can occur during the transfer steps, which is less complicated than coring. This approach establishes the following four phases:

- Phase I. Define present conditions

- Phase II. Ensure interim safe storage

- Phase III. Characterize disposal feed

- Phase IV. Final waste form qualification.

Defining present conditions (Phase I) focuses on resolving tank safety issues and performing tank screening on all the tanks. Any data (e.g., bounding tank data) required for retrieval and pretreatment design will also be obtained during this phase. Samples are obtained to ensure interim safe storage (Phase II) during transfer from the SSTs to the receiving DSTs or from well mixed interim storage double-shell tanks. These samples are more easily obtained and will be more 
representative of the overall material. This phase is the optimal time for performing any extensive characterization required to support pretreatment and ultimate disposal. Samples supporting data requirements to characterize feed for disposal operations (Phase III) are obtained in the later stages of the program. Samples are obtained during transfers into the feed tanks and while material is in the feed tank.

The final form will be qualified through characterization (Phase IV) to establish that the final disposal forms meet regulatory requirements. Sampling here is done in transfer lines to the final disposal waste form (e.g., high-level waste glass canisters).

The near-term effort involves establishing a priority listing of tanks to be core sampled. This priority list (Tank Farm Sampling Schedule) includes those tanks that will be auger, supernate, vapor, and core sampled. Using the Environmental Protection Agency's DQO process, the Characterization Program is assisting the other TWRS program elements in identifying critical data required for each program to make decisions. The near-term effort is focused primarily on resolving safety issues in compliance with the DNFSBs Recommendation 93-5 and screening tanks to validate earlier decisions to put tanks on the Watch List. Based on the results of the screening analyses, further analyses will be conducted on each tank sampled to address any safety concerns that were identified. Data collected during the near-term effort will form a database that will be used as an input to the DQO processes to define requirements for the long-term effort. The number of samples per tank will be limited by the number of risers that are available and readily accessible. The data from the analyses of cores from each tank will be compared to the requirements stated in the DQO to see if the requirements were met or whether more data will be required. If additional samples are required to meet the DQO requirements, further sampling of the tanks sampled during this period will be deferred until alternatives become available to allow additional sampling, either through new risers or alternate sampling techniques. In addition, as more information is made available through activities such as improved historical data analyses and improved databases and modeling, reassessment of the near-term DQOs will be needed.

The long-term effort involves using additional information as it becomes available to update the DQO and the Tank Characterization Reports. Extensive historical data analyses of the tank farms will be performed during the near-term effort. The historical data include process data, transfer records, and previous characterization data. Near-term data collection efforts will include analyses to verity historical information and provide estimates of confidence in historical results. The historical analysis will be used in part to segregate tanks into groups based on chemical and physical similarities. The strategy will be to sample a few tanks within a group. The optimum number of cores needed to describe the vertical and horizontal homogeneity will be determined using standard statistical models (e.g., the existing tank 241-B-110 statistical model database) and the most appropriate database developed during the near-term effort. The database selected to predict the number of samples from a specific group of tanks will match the group of tanks to be sampled. The data from the first few tanks sampled within a group will be compared to the DQO requirements. Additional samples will be collected, if necessary, to meet the requirements. When the requirements have been met, the next tank within this group will be sampled. The data from the analyses from this tank will be compared statistically to the data from previous tanks to validate the grouping. In addition, the data will be evaluated against the objectives and a determination will be made as to whether additional samples are needed. When the data indicate that the tank matches the grouping and the data quality meets the objectives, the next tank in the series will be sampled for the optimal number of samples and the previous steps will be repeated. The iterative process of sampling or resampling a tank, evaluating the aggregate data, comparing results to the objectives, and collecting more samples and data will be 
repeated until all objectives have been met. The goal is to collect the optimum number of samples to meet the DQO and characterize the tanks to resolve safety issues.

A parallel effort is to establish overall TWRS Program priorities. The priority listing will be revised as the methodology for estimating the number of cores to be taken is improved, and the definition of bounding tanks becomes clearer. The revised priority list will establish the sampling schedule for all types of samples and will be input to the continuing and iterative DQOs processes for each of the program elements.

\section{Technical Integration and Planning (WBS 1.1.1.2.04.01)}

Technical Integration and Planning is the administrative and business support for the Characterization Program. It involves management and administration, planning, and all characterization support functions. It includes, but is not limited to, the development of baseline plans, reporting/statusing, Characterization schedules, and budget preparation and tracking as well as the support for the TCAP and quality assurance.

\section{Technology Development and Applied Engineering (WBS 1.1.1.2.04.02)}

The approach to technology development requires a double focus. First, a thorough examination of the sampling and analytical needs must be completed. This evaluation will identify what new capabilities are needed to satisfy the TWRS Program requirements as documented in DQO reports and will identify areas where substantial improvement in time, cost, or safety are possible. The second focus is locating sources of commercially available new technology that may benefit TWRS tank characterization. The Characterization Program maintains ties with the Office of Technology Development, the National Laboratories, universities, and private industry to stay aware of development activities that can be leveraged to achieve greater benefits at lower costs.

\section{Field Sampling and Measurement (WBS 1.1.1.2.04.03)}

The approach for the sampling activity can be divided into four categories:

1. Solicit potential sampling requirements. The Characterization Program will solicit other TWRS end functions at least annually for potential sampling requirements. Requirements for sampling will be provided to the Characterization Program in the form of DQOs or test plans (for process development samples) as well as program element plans and strategies developed via systems engineering to determine the time to get samples. The DQO will not provide explicit sampling instruction, rather it identifies what types and to what quality the output data from sample analysis needs to be. Using the DQO, a Tank Characterization Plan is then developed to identify explicit sampling instruction. This explicit instruction will ensure that the DQO requirements are satisfactorily met.

2. Schedule and prioritize events. Once all sampling requirements are determined, an integrated sampling schedule will be developed. Sampling activities will be categorized based on the method in which the sample must be gathered. Current sampling methods include full depth core, auger (surfaced up to $25^{\prime \prime}$ deep), grab supernate and sludge and vapor dome space. The sampling events will be prioritized to reflect the urgency of the information needed. With the prioritization and sampling method identified, resources will be allocated as required. 
3. Conduct sampling activity and deliver sample to laboratory. Upon receipt of the integrated sampling schedule, detailed planning of the field activities will be initiated. These activities include, but are not limited to, field walk downs, work package preparation, surveillance preparation, field activity planning/coordination, equipment decontamination, and equipment fabrication. Completion of the sampling activity occurs when the waste sample is released to the laboratory via a chain-of-custody document.

4. Implement in situ data gathering techniques when available (transferred from Technology Development and Applied Engineering). In situ techniques, such as cone penetrometer, will be used to gather key data (e.g., physical properties) or supplement other sampling and analytical techniques.

\section{Analytical Integration (WBS 1.1.1.2.04.04)}

The approach for the Analytical Integration activity can be defined by the following six general DQOs, as identified in the Analytical Integration work breakdown structure. These DQOs are also prioritized as follows in terms of how analytical costs are collected for laboratory analyses.

1. Safety Screening DQO Analyses. As defined by the Safety Screening DQO, analyses results are used to classify the SSTs and DSTs into specific safety categories for safety issues dealing with the presence of ferrocyanide, organics, flammable gases, and criticality. The Safety Screening DQO includes two primary analyses with additional secondary analyses required if primary analyte threshold limits are exceeded. The results of primary analyses are required to be reported within 45 days of receipt of each sample.

2. Safety Resolution Related DQO Analyses. The safety resolution cost account includes sample analysis and data reporting/validation as required to support the organic, ferrocyanide, flammable gas, and high-heat DQOs. A validated data report is required 216 days from the receipt of the last sample from the last core to the laboratory.

3. Compatibility DQO Analyses. The Compatibility DQO cost account includes sample preparation, analysis, and data reporting/validation required to support waste compatibility decisions. The Compatibility DQO will require a data report for each sampling event 136 days from receipt of the last segment of the last core at the laboratory, as identified by approved tank-specific Tank Characterization Plans.

4. Operations Related DQO Analyses. The Operations Related DQO cost account includes the DQOs prepared to meet the Waste Analysis Plan requirements for DSTs or the Evaporator. This cost account includes the sample preparation and analysis data reporting/validation required to support the operations decision criteria. Data reports from each tank also will encompass analysis (usually supernate) to ensure that tanks will continue to be operated within OSR/OSD limits (e.g., $\mathrm{OH}$ and $\mathrm{pH}$ ) sampling event are required 172 days after receipt of the last sample from the last core from the tank.

5. Disposal Related DQO Analyses. The Disposal Related DQO cost account includes the retrieval, pretreatment, low-level waste, and high-level waste DQO requirements. This cost account includes the sample preparation, analysis, and data reporting/validation required to support the disposal related decision criteria. A validated data report is required 216 days from receipt of the last sample from the last core at the laboratory. 
6. Vapor DQO Analyses. The Vapor DQO cost account includes vapor analysis and data reporting for SUMMA ${ }^{1}$ canisters, sorbent tubes, and OSHA versatile samplers (OVSs) at the PNL as well as analysis and data reporting of triple sorbent traps and carbotrap/carbotrap C samplers at the Oak Ridge National Laboratories. Vapor analysis results are required to support decision criteria identified in the DQOs for Generic In-Tank Health and Safety Issue Resolution.

\section{Data Evaluation and Reporting (WBS 1.1.1.2.04.05)}

The work scope falls into four broad categories.

1. Data definition and planning. WHC will lead this activity with support from PNL. Development of strategy and technical basis will be performed with support from outside contractors and consultants, with emphasis on using the needs of other TWRS program elements to drive the strategy. Statistical support from WHC and PNL statisticians will be used to verify the technical basis for planning. DQO development will be the primary responsibility of the TWRS program element requiring the data. The role of this task will be to oversee the DQO activities; provide guidance, strategy documents, and information; and participate in the process to ensure quality product results. This task will provide statistical support for DQO development. Facilitators will be provided separately by MAC Technical Services. The Tank Waste Analysis Plan will be developed following the content specifications identified in Tri-Party Agreement milestone M-44-01 and M-44-02. WHC will lead this activity with support from an outside contractor. The Tank Characterization Plans will be developed to support the sampling schedule as specified by the Field Sampling and Measurement element of the Characterization WBS. These plans will integrate the analytic needs specified in all applicable DQOs to ensure all needs are met cost effectively. Details of the Tank Characterization Plan requirements are also found in Tri-Party Agreement milestone $M-44-01$ and $M-44-02$.

2. Historical Information Development. LANL will complete compilation of historic records regarding tank contents. LANL use records on waste transfers to develop estimates of layers in each tank, combined with information regarding waste types to provide estimates of the chemical and physical properties of each layer. Kaiser Engineers will combine the LANL. information with tank operations information, photographs, and other data to provide the Historic Tank Contents Estimate Reports. LANL will use existing and new sampling information to improve the models and estimates. Statisticians at WHC, PNL and LANL will work together to determine the best methods for using historical data combined with analytic information to increase confidence in characterization results.

3. Characterization Reporting. WHC will work with a subcontractor to provide Tank Characterization Reports (TCRs) in the format that has been agreed to by the Washington State Department of Ecology. The TCRs use the information derived from the historic reports above, combined with the analytic results specified in the Tank Characterization Plans (as noted in Tri-Party Agreement milestone M-44-08). WHC statisticians will support TCR

development. A subcontract will also be placed to determine the appropriate statistical approach for combining multiple data sources into the reports in a defensible manner. The resulting approach will be implemented in TCR analysis.

ISUMMA is a trademark of Molectrics, Incorporated. 
4. Data Dissemination. The historic data, analytic results, and TCRs will be made available in a standard format as part of the TCD. This will be in accordance with Tri-Party Agreement milestone M-44-08. PNL will provide and support the TCD software. WHC will be responsible for entering the data. The new analytic data entered into the LIMS at WHC will be electronically transferrable to the TCD. Data not entered into the LIMS will require hand entry into the TCD. WHC will also maintain (in cooperation with Information Resource Management) archive copies of all relevant reports and provide catalogs to potential users indicating available information.

\subsubsection{Drivers and Deliverables}

\section{Technical Integration and Planning (WBS 1.1.1.2.04.01)}

1. Drivers:

- DOE/RL 94-0001, Recommendation 93-5 Implementation Plan, January 1994

- Tri-Party Agreement Milestone M-44

2. Deliverables:

- $\quad$ Issue DNFSB 93-05 Quarterly Reports

Technology Development and Applied Engineering (WBS 1.1.1.2.04.02)

1. Drivers: There are three drivers for developing new technology or importing technology to the Characterization Program:

- $\quad$ Acquiring capability to satisfy documented data needs

- Substantially improving cost:benefit ratios

- Improving worker operational safety

2. Deliverables: All deliverables for this activity will be directly related to the three drivers listed above.

Field Sampling and Measurement (WBS 1.1.1.2.04.03)

1. Drivers:

- $\quad 40$ CFR 265.196 - Response to Leaks \& Spills and Disposition of Leaking of Unfit-forUse Tank Systems

- WAC 173-303-640 - Tank System

- DOE/RL 94-0001, Recommendation 93-5 Implementation Plan, January 1994

- DOE Order 5820.2A - Radioactive Waste Management

- Hanford Federal Facility Agreement and Consent Order, Fourth Amendment, January 1994 (Milestone M-44) 
- Defense Authorization Act, Public Law 101-520, Section 3137, "Safety Measures for Waste Tanks at Hanford Nuclear Reservation"

2. Deliverables:

- DNFSB 93.5 Commitment 3.12 "Hire train, and qualify four additional rotary mode crews"

- DNFSB 93.5 Commitment 3.18 "Develop means for measuring complete sample recovery. Complete engineering study of alternatives, select method and complete design/fabrication/testing of technique."

- DNFSB 93.5 Commitment 5.14 "Two PAS-1 transfer casks will be ready for use"

- DNFSB 93.5 Commitment 3.13 "Deploy prototype cone penetrometer"

- DNFSB 93.5 Commitment 2.3 "Complete sampling and analysis of all Watch List tanks per the DQOs established in commitments 2.1 and 2.2."

In addition to the above deliverables, sampling activities directly support the completion of Tank Characterization Reports and thus support the completion of Tri-Party Agreement milestone M-44-08.

\section{Analytical Integration (WBS 1.1.1.2.04.04)}

1. Drivers:

- Defense Authorization Act, Public Law 101-510, Section 3137, "Safety Measures for Waste Tanks at Hanford Nuclear Reservation"

- WAC 173-303- Dangerous Waste Regulations

- Hanford Federal Facility Agreement and Consent Order, Fourth Amendment, January 1994 (M-44)

- DOE/RL 94-0001, Recommendation 93-5 Implementation Plan, January 1994

2. Deliverables:

- DNFSB commitment "Complete Sampling and Analysis of all Watch List tanks per the Data Quality Objectives" (9/30/95)

- DNFSB commitment "Complete Sampling and Analysis of all Non Watch List tanks per the Data Quality Objectives" (9/30/96)

- Transmit a 45-day data report for all sampling events based on Safety Screening DQOs as identified in the tank specific Tank Characterization Plans (09/30/95, 09/30/96, 09/30/97) 
- Transmit a 216-day validated data report for all sampling events based on Safety Resolution DQOs as identified in the tank specific Tank Characterization Plans $(09 / 30 / 95,09 / 30 / 96,09 / 30 / 97)$

- Issue Analytical Data Packages from each sampling event within 136 days specific to the Compatibility DQO (09/30/95, 09/30/96)

- Transmit a 172-day validated data report from each sampling event specific to Operation related DQOs (09/30/95, 09/30/96)

- Transmit a 216-day validated data report from each sampling event specific to the Disposal Related DQOs (09/30/95, 09/30/96, 09/30/97)

- DNFSB Commitment 5.12, Complete upgrades of INEL Laboratory to ready-to-serve mode for Characterization support (10/31/94)

- Transmit data reports and complete emergency notification per requirements of Tank Characterization Plan or Letter of Instruction (09/30/95, 09/30/96, 09/30/97)

- Complete DNFSB commitment 5.2, Cleanout and Refurbishing of "A-Cell" (05/01/95)

\section{Data Evaluation and Reporting (WBS 1.1.1.2.04.05)}

1. Drivers:

- Tri-Party Agreement milestones M-44-01 and M-44-02 drive the development of the Tank Waste Analysis Plan and Tank Characterization Plans.

- The Tri-Party Agreement specifies that characterization activities will be specified through the DQO process (M-44).

- DNFSB commitments require the development of a technical basis and statistical methods to determine sample numbers (1.19).

- DNFSB commitment 1.18 drives the delivery of the Historical Tank Contents Estimate (HTCE) reports. Supporting documents are required to develop these reports.

- Modeling and grouping results are needed to support the technical basis for characterization. Historical and modeling information are required as inputs to the Tank Characterization Reports as specified in Tri-Party Agreement milestone M-44-08.

- Tri-Party Agreement milestone M-44-08 specifies the requirements for TCRs.

- Tri-Party Agreement milestone M-44-08 specifies the requirements for TCD information entry.

- The Data Management Improvement Plan specifies activities to increase control of characterization data and make the data more accessible to all users. 
2. Deliverables:

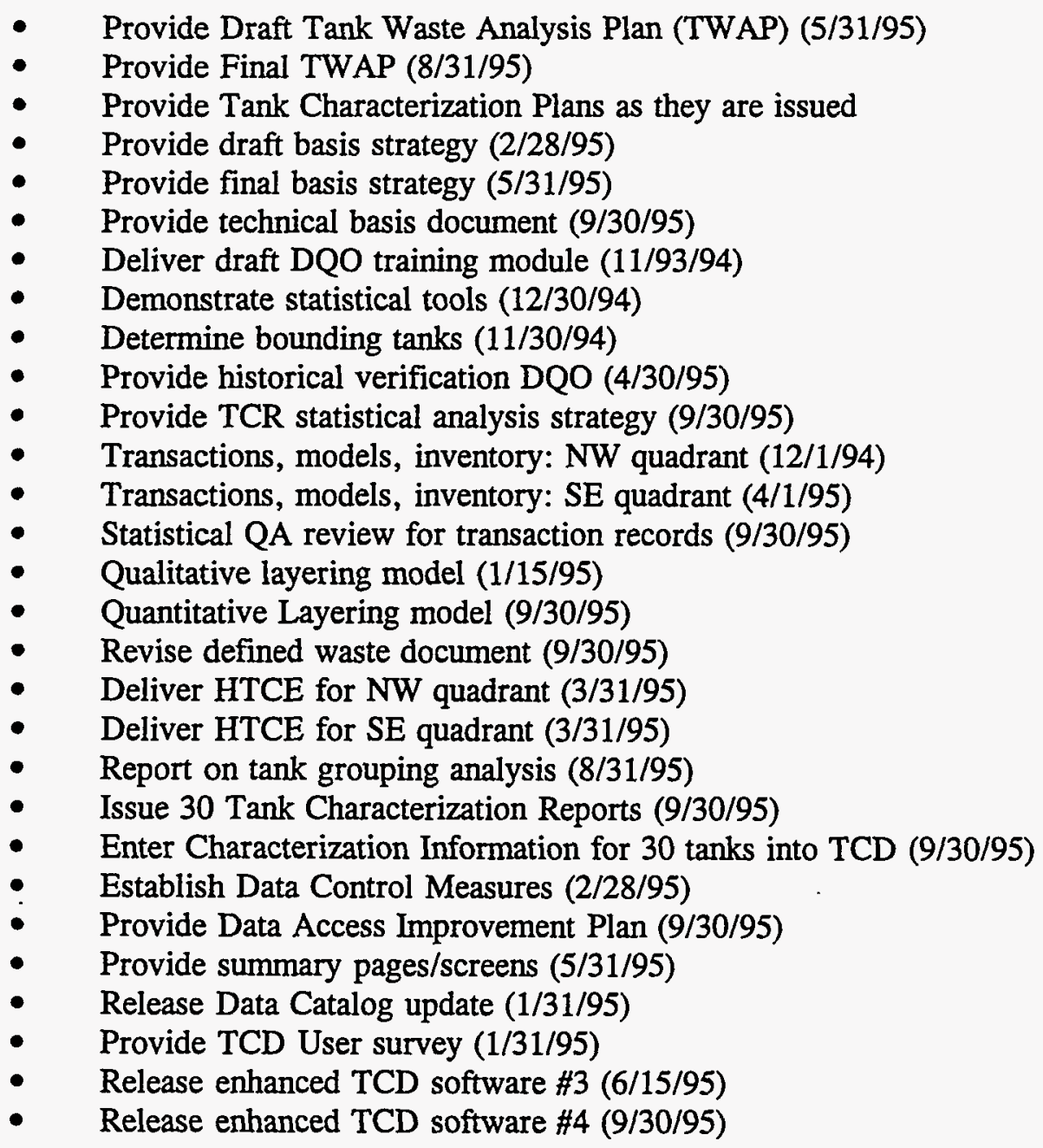

\subsubsection{Schedule}

\subsubsection{Characterization Summary Schedule}

The Characterization Program Element summary schedule is provided in Figure 7.6-2. This schedule accurately portrays in graphic format the summary-level work scope for the life of the Characterization Program. Significant activities and milestones for the Characterization Program are identified from a TWRS Program integrated and controlled database. All Tri-Party Agreement major and selected interim milestones applicable to this program element are included on the summary schedule. The milestone description sheets are located in Appendix D. There is at least one activity for each Level V WBS element. Logical ties within and between other program elements are provided where they offer clarification.

The principal activities within the Characterization Program are those required to develop the capability to sample all of the tanks; perform the sampling activities; and provide the data evaluation, reporting, and database management to maintain the DQOs for the TWRS Program. The Characterization Program is responsible for the completion of Tri-Party Agreement major 
WHC-SP-1101

milestone $\mathrm{M}-44-00$, Issue tank characterization reports for all single- and double-shell tanks to be completed by September 1999.

\subsubsection{Characterization Expanded Baseline (FY 1995 - FY 1997)}

Refer to Appendix C for the Characterization expanded baseline for FY 1995 - FY 1997.

\subsubsection{Resource Requirements}

\subsubsection{Cost}

\subsection{Basis of Cost Estimates}

Cost estimates for the work scope represented by the Characterization Program Element are improving. Cost estimates for Characterization work scope were based on 1994 fiscal year work estimates. At present, the cost estimates are the product definitions found in the Activity Planning Forms, as well as the WBS Dictionary Sheets, and are found in Table 7.6-1. Also, providing a basis for estimates are the assumptions documented in Section 7.6.1.1.6.

\subsection{Total Program Element Cost by Year by Fund Type by Activity and Cost Account)}

Table 7.6-1 establishes the baseline cost for this program element. The baseline cost is exhibited in annual costs for FY 1994 through FY 2000, and 5-year totals for FY 2001 through program element completion. Where the data are available, these baseline costs are exhibited at the cost account level with fund type totals provided. Cost data are then rolled up to the activity and program element levels. At present, the program element cost baseline is the program element cost totals exhibited on this table less a productivity challenge for FY 1995 through FY 1997.

\subsection{FY 1995 Cost by Month by Fund Type by Activity and Cost Account}

Future updates of the MYWP will include Table 7.6-2, which establishes the month-by-month cost baseline for FY 1995 for this program element. The FY 1995 cost baseline is exhibited at the cost account level with fund type totals provided. Cost data are then rolled up to the activity and program element levels. Also exhibited is the total cost baseline for the program element for FY 1995. At present, the program element cost baseline for FY 1995 for this program element is the total provided on this table less a productivity challenge for FY 1995.

\subsubsection{Staffing}

\subsection{Basis of Staffing Projections}

Staffing projections are based on actual staffing to perform identical or similar work in FY 1994. Staff is on board or on contract and is available and trained. Crew sizes for all types of sampling are based on the FY 1994 actual required crew sizes. In addition, time/sampling is based on a WESTIP ${ }^{\text {ns }}$ process that evaluated historic down-time (for maintenance, weather, repairs) on an annual basis. Laboratory staffing requirements are based on actual expected staff time for completing work specified in DQOs issued by August 15, 1994. They are broken down to extrusion/sample prep and by each instrument. Staff projection per instrument (TGA) is based on the previous 2-year's 
laboratory experience at the 222-S and 325 Laboratories. Staff time to prepare TCRs was based on the time required to prepare the last of the 20 TCRs due in FY 1994, after the initial learning curve was past.

\subsection{Total Full-Time Equivalents by Year by Activity by Major Participant (Direct and Indirect)}

Future MYWP updates will include Table 7.6-3, which establishes total staffing projections for this program element. Included in the table are FY 1994 staffing data as well as projections by year for FY 1995 through FY 2000. The projections in this table include all direct and indirect Full-Time Equivalents (FTEs), including all subcontractor personnel. Where data are available, projections are exhibited at the activity level with major program participant totals provided.

\subsection{FY 1995 through FY 1997 Direct Full-Time Equivalents by 88 Labor Categories}

Future MYWP updates will include Table 7.6-4, which establishes direct WHC staffing projections for this program element. Projections do not include subcontractor support and are provided by 88 labor categories for FY 1995 through FY 1997.

\subsubsection{Work Scope Carryover}

1. 325 Building Standdown. Radiological Operations within the 325 Building have been suspended since April 1994. All activities are focused on a potential restart date of October 19,1994 . If restart does occur at that time, only those activities directly related to the 45-day tank characterization samples will begin initially. Other activities will be phased in after that. Anticipated restart for laboratory and hot cell renovation activities is presently approximately November 1, 1994.

2. Renovation of $\mathbf{3 2 5}$ Facility 'A-Cell.' All activities that can be performed without re-entering the hot cells and performing radiological work have been completed. A formal request for carryover of funds has been submitted. A draft Change Request to move the September 30,1994, milestone for completing the cleanout of 'A-Cell' has been prepared.

3. Reconfiguration of $\mathbf{3 2 5}$ Facility 'Special Analytical Laboratory (SAL)' Operating Gallery. Work on this activity has been delayed until FY 1995 due to anticipated manpower limitations after restart of the 325 Laboratory is authorized.

4. Installation of Hot Ion Coupled Plasma (ICP) Instrumentation in 325 Facility 'SAL' Operating Gallery. Drafting for the glove box design has been completed. ICF Kaiser Hanford Company is fabricating a mock-up of the portion of the glovebox to which the ICP will attach; this mock-up will be shipped to the instrument vendor to begin interface design prior to receiving the actual glovebox. All construction-related work on this activity has been delayed until FY 1995 due to anticipated manpower limitations after restart is authorized. 
5. Trucks 3 and 4. The completion of trucks \#3 and \#4 will slip into FY 1995 because changes from the original design forced equipment substitutions for obsolete items. The major alteration was a design modification to accommodate a 6-cylinder gas engine (the drill motor) since the original design, a 4-cylinder motor, is no longer made. Other hardware, certain hydraulic motors, is now obsolete, requiring location of alternate suppliers and corresponding design changes by engineering change notice to accommodate the replacement hardware. Contingency plans are being developed and evaluated.

6. Rotary Core Sampling. X core samples, originally scheduled for completion in FY 1994, have slipped into FY 1995, due to the later-than-planned start of the new rotary truck system.

\subsubsection{Waste Type Data}

\section{Field Sampling and Measurement (WBS 1.1.1.2.04.03)}

Waste generated during field sampling operations is handled in two different methods. Depending on other activities in the farms where sampling is occurring, waste may be accumulated in drums set up for the tank farm or Sampling can establish their own waste drum.

\section{Analytical Integration (WBS 1.1.1.2.04.04)}

Waste types generated by the laboratories in support of the Analytical Integration activity include solid, liquid, mixed, and gaseous wastes. These waste types are produced by both 222-S and 325 Laboratories, which currently receive, extrude, and analyze high-level waste samples from both SSTs and DSTs.

Solid wastes are generated by the laboratories through the handling and analyses of waste tank samples. Solid low-level wastes are packaged at the laboratories for disposal at appropriate low-level waste disposal facilities.

Liquid wastes are generated in the laboratories from the extrusion, preparation, and analysis of waste tank samples. Primary wastes from unused waste tank samples are disposed of in the laboratory hot cells. Secondary wastes are generated at the laboratories from the preparation and analyses of waste tank samples. Ultimately, both primary and secondary liquid waste from the laboratories are returned to double-shell receiving tanks.

Mixed wastes are produced by the laboratories primarily from the analyses of tank samples. Mixed wastes are packaged and stored onsite at mixed waste storage facilities for eventual treatment and disposal.

Gaseous waste is produced at the laboratories (including gaseous waste from hot cells and analytical hoods) and eventually processed through HEPA filters prior to discharge.

With the use of the INEL starting in FY 1995 to support the Analytical Integration activity, primary wastes (i.e., unused waste tank samples) will be returned to Hanford and returned to one of the onsite laboratories, which will in turn be returned to the waste tanks. Secondary waste generated by INEL from the handling and analyses of Hanford waste tank samples will be disposed of at INEL along with other laboratory wastes since this waste can not be separated in the existing INEL laboratory facilities. 


\subsubsection{Revisions}

In future updates of the MYWP, this section will include a summary of the revision that has occurred in this program element since the last issue of the MYWP. 
CHARACTERIZATION

WORK BREAKDOWN STRUCTURE

3

i

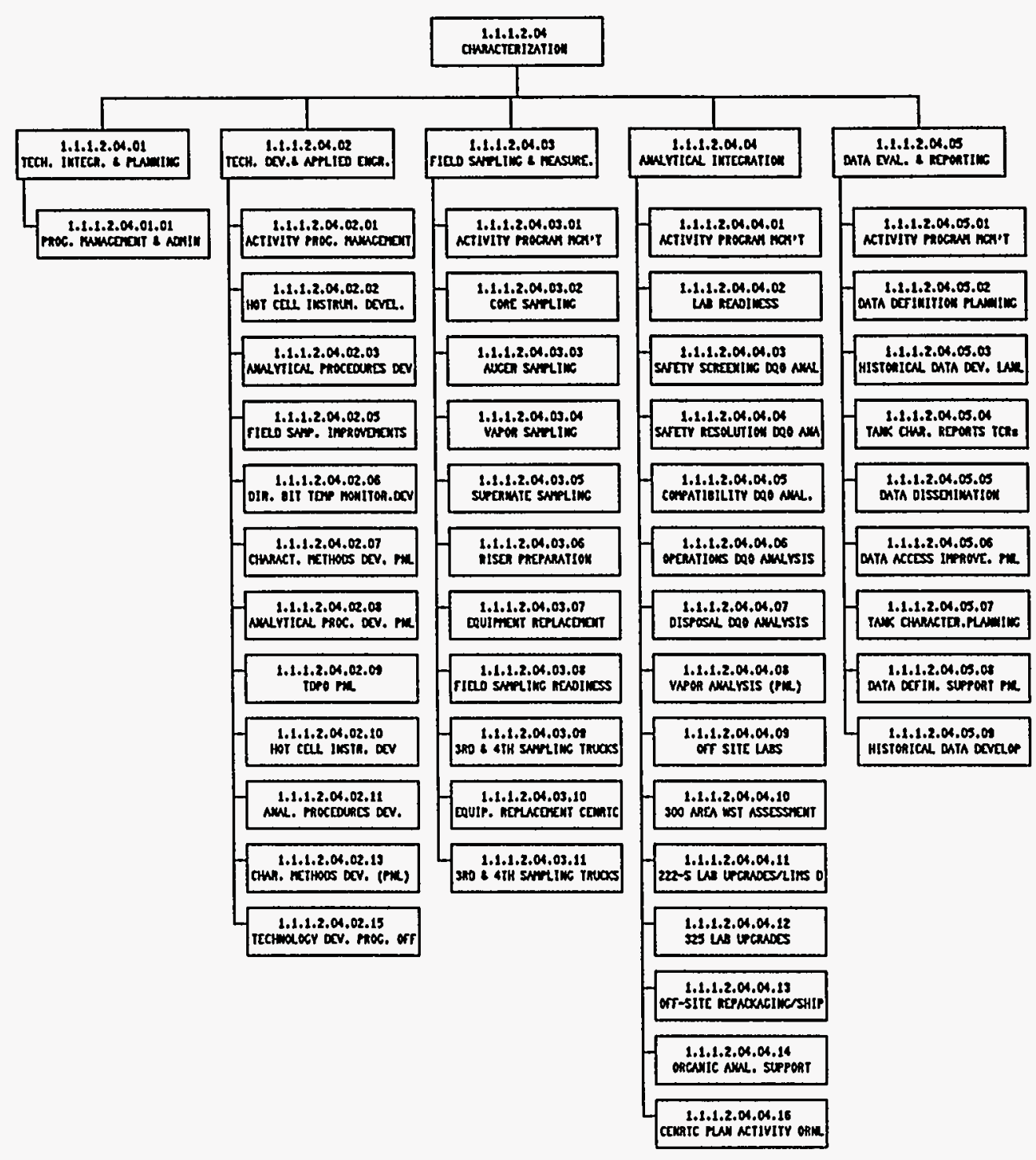


1.1.1.2.04 Waste Tank Characterization Summary Schedule Activities

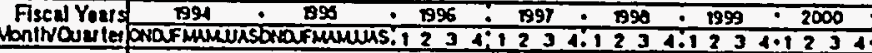

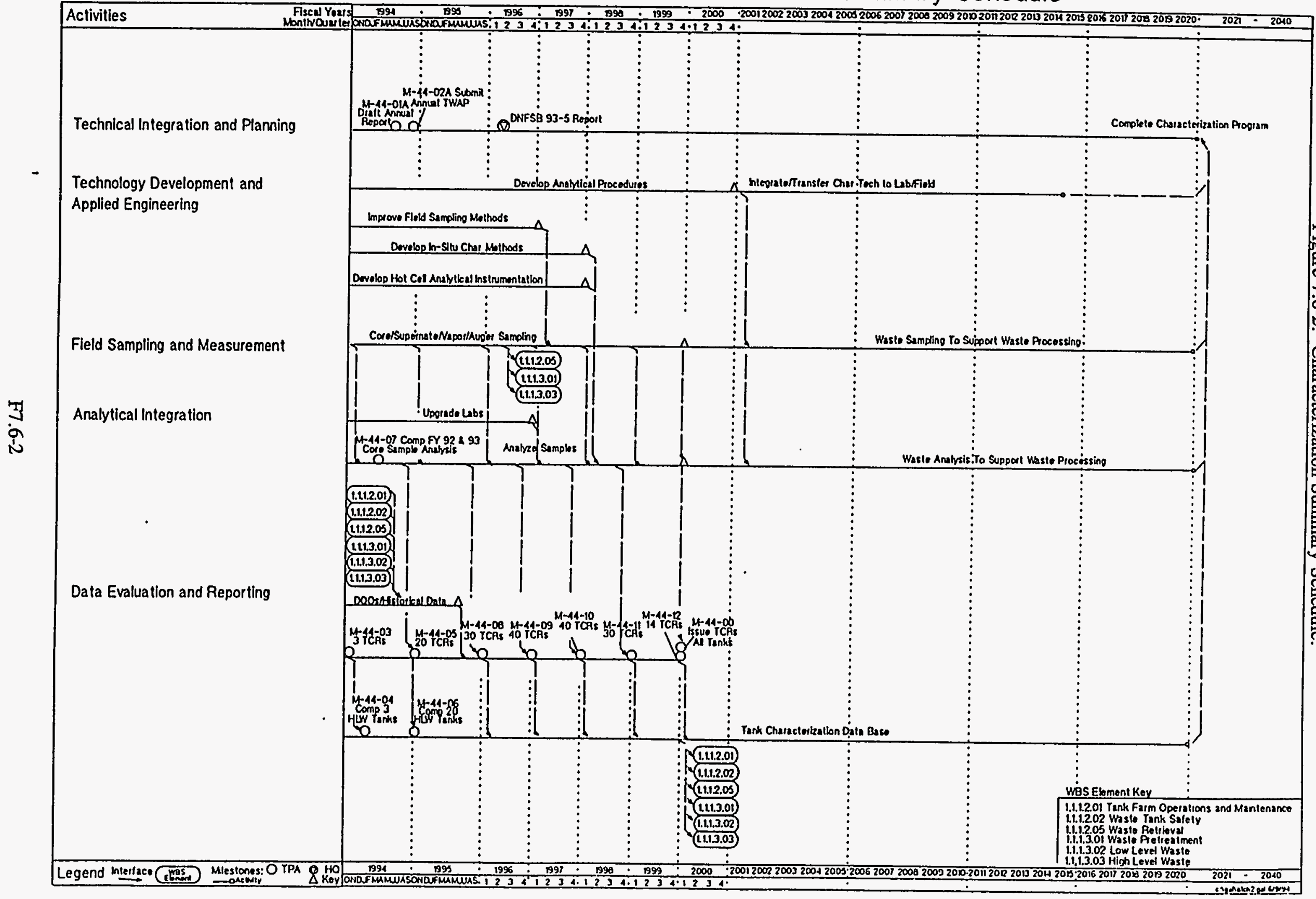


TABLE $7.6-1$

TOTAL PROGRAM ELEMENT COST BASELINE - BY YEAR

\begin{tabular}{|c|c|c|c|c|c|c|c|c|c|}
\hline WBS / LEVEL & ACTIVITY TITLE / ADS & $\begin{array}{l}\text { FUND } \\
\text { TYPE }\end{array}$ & $\begin{array}{l}\text { TOTAL } \\
\text { FY94 }\end{array}$ & FY95 & FY96 & FY97 & FY98 & FY99 & FYOO \\
\hline \multirow[t]{2}{*}{$\begin{array}{l}\text { 1.1.1.2.04 } \\
\text { Level IV } \\
\quad\end{array}$} & $\begin{array}{l}\text { Characterization } \\
\text { ADS } 1130-0 \\
\quad \text { TOTAL TARGET }\end{array}$ & $\underset{\text { OE }}{\dot{O E}}$ & $\begin{array}{r}65,284 \\
10,800 \\
76,084\end{array}$ & $\begin{array}{r}79,176 \\
5,869 \\
-85,045 \\
\end{array}$ & $\begin{array}{r}86,714 \\
7,447 \\
94,161\end{array}$ & $\begin{array}{r}66,187 \\
5,363 \\
-1,550 \\
7\end{array}$ & $\begin{array}{r}52,754 \\
3,854 \\
56,608 \\
\quad 40\end{array}$ & $\begin{array}{r}52,838 \\
2,647 \\
55,485 \\
-40\end{array}$ & $\begin{array}{r}38,545 \\
2,317 \\
40,862\end{array}$ \\
\hline & $\begin{array}{l}\text { Delta to TARGET. } \\
\text { (See notes at end of Tablo.) }\end{array}$ & $\begin{array}{l}\text { OE } \\
\text { C/E }\end{array}$ & NA & $==\begin{array}{c}1 \\
(98) \\
(97)\end{array}$ & $\begin{array}{r}20 \\
1,283 \\
==== \\
1,303\end{array}$ & $\begin{array}{r}300 \\
612 \\
=== \\
912\end{array}$ & . & & \\
\hline $\begin{array}{l}1.1 .1 .2 .04 .01 \\
\text { Level } \mathrm{V}\end{array}$ & $\begin{array}{l}\text { Technical Integration } \\
\frac{\text { and Planning }}{\text { ADS } 1130-0-0 A} \\
\text { Sub Total }\end{array}$ & $\begin{array}{l}\text { OE } \\
\text { C/E }\end{array}$ & NA & $\begin{array}{r}1,600 \\
0 \\
---- \\
1,600\end{array}$ & $\begin{array}{r}1.759 \\
0 \\
---- \\
1.759\end{array}$ & $\begin{array}{r}1.584 \\
0 \\
--- \\
1.584\end{array}$ & $\begin{array}{r}0 \\
0 \\
---\end{array}$ & $\begin{array}{r}0 \\
0 \\
---\end{array}$ & $\begin{array}{r}0 \\
0 \\
---\end{array}$ \\
\hline $\begin{array}{l}1.1 .1 .2 .04 .01 .01 \\
\text { Level VI }\end{array}$ & $\begin{array}{l}\text { Program Management \& } \\
\text { Administration } \\
\\
\text { Total }\end{array}$ & $\begin{array}{l}\text { OE } \\
\text { C/E }\end{array}$ & & $\begin{array}{r}1,600 \\
0 \\
--- \\
1,600\end{array}$ & $\begin{array}{r}1,759 \\
0 \\
---- \\
1,759\end{array}$ & $\begin{array}{r}1,584 \\
0 \\
---- \\
1,584\end{array}$ & --- & $---\frac{}{0}$ & $\frac{--}{0}$ \\
\hline $\begin{array}{l}1.1 .1 .2 .04 .02 \\
\text { Level } \mathrm{V}\end{array}$ & 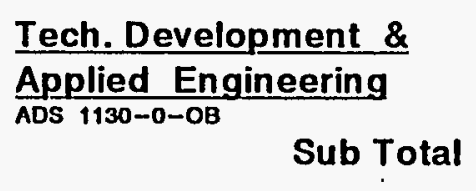 & $\begin{array}{l}\text { OE } \\
C / E\end{array}$ & NA & $\begin{array}{r}4,692 \\
2,321 \\
---- \\
7,013\end{array}$ & $\begin{array}{r}5,608 \\
6,164 \\
---- \\
11,772\end{array}$ & $\begin{array}{r}4.673 \\
4.751 \\
---- \\
9.424\end{array}$ & $\begin{array}{r}0 \\
0 \\
---\end{array}$ & $\begin{array}{r}0 \\
0 \\
---\end{array}$ & $\begin{array}{r}\mathbf{0} \\
\mathbf{0} \\
-- \\
\mathbf{0}\end{array}$ \\
\hline $\begin{array}{l}1.1 .1 .2 .04 .02 .01 \\
\text { Level VI }\end{array}$ & $\begin{array}{l}\text { Activity Program } \\
\text { Management } \\
\end{array}$ & $\begin{array}{l}\text { OE } \\
C / E\end{array}$ & & $\begin{array}{r}205 \\
0 \\
---- \\
205\end{array}$ & $\begin{array}{r}260 \\
0 \\
---- \\
260\end{array}$ & $\begin{array}{r}268 \\
0 \\
---- \\
268\end{array}$ & $\begin{array}{r}---- \\
0\end{array}$ & $\begin{array}{r}--- \\
0\end{array}$ & $---\overline{0}$ \\
\hline $\begin{array}{l}1.1 .1 .2 .04 .02 .02 \\
\text { Level VI }\end{array}$ & $\begin{array}{l}\text { Hot Cell Instrument } \\
\text { Development } \\
\\
\text { Total }\end{array}$ & $\begin{array}{l}\text { OE } \\
C / E\end{array}$ & & $\begin{array}{r}465 \\
177 \\
--- \\
642\end{array}$ & $\begin{array}{r}1.485 \\
182 \\
---- \\
1.667\end{array}$ & $\begin{array}{r}1,067 \\
97 \\
---- \\
1,164\end{array}$ & $---\frac{}{0}$ & $\begin{array}{r}--- \\
0\end{array}$ & --- \\
\hline
\end{tabular}

[ R1 ] 
0
0
0
0
0

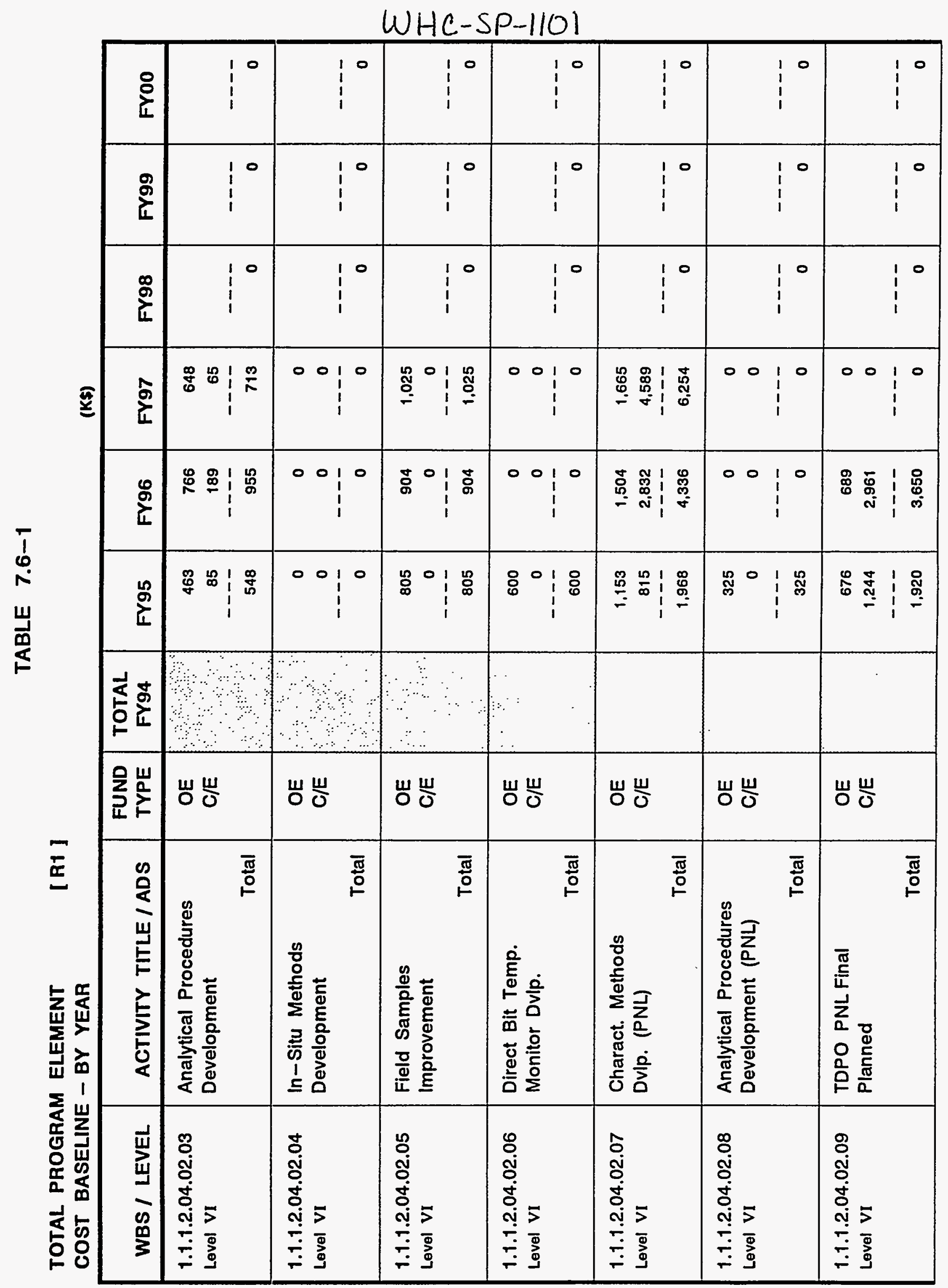


TABLE 7.6-1

TOTAL PROGRAM ELEMENT COST BASELINE - BY YEAR

\section{[ R1 ]}

\begin{tabular}{|c|c|c|c|c|c|c|c|c|c|}
\hline WBS / LEVEL & ACTIVITY TITLE / ADS & $\begin{array}{l}\text { FUND } \\
\text { TYPE }\end{array}$ & $\begin{array}{l}\text { TOTAL } \\
\text { FY94 }\end{array}$ & FY95 & FY96 & FY97 & FY98 & FY99 & FYOO \\
\hline $\begin{array}{l}1.1 .1 .2 .04 .02 .10 \\
\text { Level VI }\end{array}$ & $\begin{array}{l}\text { Hot Cell Instrument } \\
\text { Development } \\
\qquad \begin{array}{l}\text { Total }\end{array}\end{array}$ & $\begin{array}{l}O E \\
C / E\end{array}$ & & $\begin{array}{r}0 \\
0 \\
- \\
-0\end{array}$ & $\begin{array}{r}0 \\
0 \\
-- \\
-0\end{array}$ & $\begin{array}{r}0 \\
0 \\
-- \\
-0\end{array}$ & $-\cdots$ & $---\overline{0}$ & $-\cdots$ \\
\hline $\begin{array}{l}1.1 .1 .2 .04 .02 .11 \\
\text { Level VI }\end{array}$ & $\begin{array}{l}\text { Analysis Procedures } \\
\text { Development } \\
\qquad \text { Total }\end{array}$ & $\begin{array}{l}O E \\
C / E\end{array}$ & & $\begin{array}{r}0 \\
0 \\
-- \\
0\end{array}$ & $\begin{array}{r}0 \\
0 \\
-- \\
-0\end{array}$ & $\begin{array}{r}0 \\
0 \\
-- \\
0\end{array}$ & --- & --- & --- \\
\hline $\begin{array}{l}1.1 .1 .2 .04 .02 .12 \\
\text { Level VI }\end{array}$ & $\begin{array}{l}\text { In-Situ Methods } \\
\text { Development }\end{array}$ & $\begin{array}{l}O E \\
C / E\end{array}$ & ; & $--\frac{0}{0}$ & $\begin{array}{r}0 \\
-0 \\
-0\end{array}$ & $\begin{array}{r}0 \\
---\frac{0}{0}\end{array}$ & -- & $---\frac{-}{0}$ & $---\frac{-}{0}$ \\
\hline $\begin{array}{l}\text { 1.1.1.2.04.02.13 } \\
\text { Level VI }\end{array}$ & $\begin{array}{l}\text { Charact. Methods } \\
\text { Dvip. (PNL) }\end{array}$ & $\begin{array}{l}\mathrm{OE} \\
\mathrm{C} / \mathrm{E}\end{array}$ & & $\begin{array}{r}0 \\
0 \\
-- \\
0\end{array}$ & $\begin{array}{r}0 \\
0 \\
-- \\
-0\end{array}$ & $\begin{array}{r}0 \\
0 \\
-- \\
0\end{array}$ & 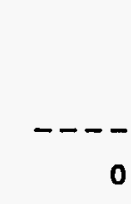 & 0 & -- \\
\hline $\begin{array}{l}\text { 1.1.1.2.04.02.14 } \\
\text { Level VI }\end{array}$ & $\begin{array}{l}\text { Analytical Procedures } \\
\text { Development (LLNL) } \\
\qquad \text { Total }\end{array}$ & $\begin{array}{l}O E \\
C / E\end{array}$ & & $\begin{array}{r}0 \\
0 \\
-- \\
0\end{array}$ & $\begin{array}{r}0 \\
0 \\
-- \\
0\end{array}$ & $\begin{array}{r}0 \\
0 \\
-- \\
0\end{array}$ & -- & --- & --- \\
\hline $\begin{array}{l}\text { 1.1.1.2.04.03 } \\
\text { Level } v\end{array}$ & $\begin{array}{l}\frac{\text { Field Sampling \& }}{\text { Measurement }} \\
\text { ADS } 1130-0-O C \\
\text { Sub Total }\end{array}$ & $\begin{array}{l}O E \\
C / E\end{array}$ & NA & $\begin{array}{r}24,248 \\
3,427 \\
---- \\
27,675\end{array}$ & $\begin{array}{r}26.781 \\
0 \\
---1 \\
26,781\end{array}$ & $\begin{array}{r}24,626 \\
---0 \\
24,626\end{array}$ & $\begin{array}{r}\mathbf{0} \\
-0 \\
--\frac{0}{0}\end{array}$ & $\begin{array}{r}0 \\
0 \\
-- \\
0\end{array}$ & $\begin{array}{r}\mathbf{0} \\
\mathbf{0} \\
-- \\
0\end{array}$ \\
\hline $\begin{array}{l}\text { 1.1.1.2.04.03.01 } \\
\text { Level VI }\end{array}$ & $\begin{array}{l}\text { Activity Program } \\
\text { Management } \\
\\
\text { Total }\end{array}$ & $\begin{array}{l}O E \\
C / E\end{array}$ & & $\begin{array}{r}1,692 \\
0 \\
---- \\
1,692\end{array}$ & $\begin{array}{r}1.774 \\
0 \\
---- \\
1.774\end{array}$ & $\begin{array}{r}1,827 \\
0 \\
--- \\
1,827\end{array}$ & $---\frac{-}{0}$ & $---\frac{-}{0}$ & $---\frac{}{0}$ \\
\hline
\end{tabular}


TABLE $7.6-1$

\begin{tabular}{|c|c|c|c|c|c|c|c|c|c|}
\hline COST BASELIN & - BY YEAR & & & & & (K\$) & & & \\
\hline WBS / LEVEL & ACTIVITY TITLE / ADS & $\begin{array}{l}\text { FUND } \\
\text { TYPE }\end{array}$ & $\begin{array}{l}\text { TOTAL } \\
\text { FY94 }\end{array}$ & FY95 & FY96 & FY97 & FY98 & FY99 & FYO0 \\
\hline $\begin{array}{l}\text { 1.1.1.2.04.03.02 } \\
\text { Level VI }\end{array}$ & 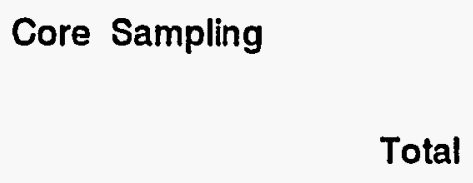 & $\begin{array}{l}O E \\
C / E\end{array}$ & & $\begin{array}{r}12,956 \\
0 \\
---- \\
12,956\end{array}$ & $\begin{array}{r}16,722 \\
0 \\
---- \\
16,722\end{array}$ & $\begin{array}{r}18,162 \\
0 \\
---- \\
18,162\end{array}$ & $---\frac{-}{0}$ & $---\frac{-}{0}$ & $-\cdots$ \\
\hline $\begin{array}{l}\text { 1.1.1.2.04.03.03 } \\
\text { Level VI }\end{array}$ & 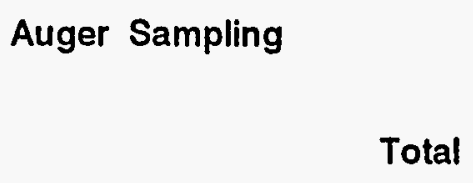 & $\begin{array}{l}O E \\
C / E\end{array}$ & $\because$ & $\begin{array}{r}2,403 \\
0 \\
--- \\
2,403\end{array}$ & $\begin{array}{r}2,436 \\
0 \\
--- \\
2,436\end{array}$ & $--\frac{0}{0}$ & $---\frac{-}{0}$ & --- & $---\frac{-}{0}$ \\
\hline $\begin{array}{l}\text { 1.1.1.2.04.03.04 } \\
\text { Level VI }\end{array}$ & 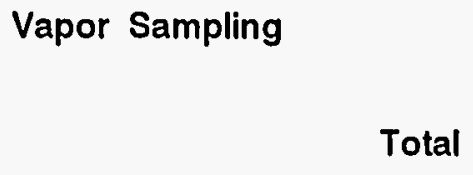 & $\begin{array}{l}O E \\
C / E\end{array}$ & & $\begin{array}{r}3,264 \\
0 \\
---1 \\
3,264\end{array}$ & $\begin{array}{r}3,545 \\
0 \\
--- \\
3,545\end{array}$ & $\begin{array}{r}3,630 \\
0 \\
--- \\
3,630\end{array}$ & $---\frac{-}{0}$ & $-\cdots$ & $---\frac{-}{0}$ \\
\hline $\begin{array}{l}\text { 1.1.1.2.04.03.05 } \\
\text { Level V1 }\end{array}$ & $\begin{array}{l}\text { Supernate Sampling } \\
\qquad \text { Total }\end{array}$ & $\begin{array}{l}O E \\
C / E\end{array}$ & & $\begin{array}{r}1,823 \\
0 \\
--- \\
1,823\end{array}$ & $\begin{array}{r}1,356 \\
0 \\
--- \\
1.356\end{array}$ & $\begin{array}{r}0 \\
0 \\
-- \\
0\end{array}$ & $---\frac{-}{0}$ & $---\frac{-}{0}$ & --- \\
\hline $\begin{array}{l}\text { 1.1.1.2.04.03.06 } \\
\text { Level VI }\end{array}$ & Riser Preparation & $\begin{array}{l}\mathrm{OE} \\
\mathrm{C} / \mathrm{E}\end{array}$ & & $\begin{array}{r}432 \\
0 \\
--- \\
432\end{array}$ & $\begin{array}{r}499 \\
0 \\
--- \\
499\end{array}$ & $\begin{array}{r}514 \\
0 \\
--- \\
514\end{array}$ & --- & $-\frac{-}{0}$ & $-\cdots$ \\
\hline $\begin{array}{l}\text { 1.1.1.2.04.03.07 } \\
\text { Level VI }\end{array}$ & $\begin{array}{l}\text { Equipment Replacement } \\
\text { Total }\end{array}$ & $\begin{array}{l}O E \\
C / E\end{array}$ & & $\begin{array}{r}600 \\
2,826 \\
---- \\
3,426\end{array}$ & $\begin{array}{r}449 \\
0 \\
--- \\
-449\end{array}$ & $\begin{array}{r}493 \\
0 \\
--- \\
493\end{array}$ & $-\frac{-}{0}$ & ---- & ---- \\
\hline $\begin{array}{l}\text { 1.1.1.2.04.03.08 } \\
\text { Level VI }\end{array}$ & $\begin{array}{l}\text { Field Sampling } \\
\text { Readiness } \\
\qquad \begin{array}{l}\text { Total } \\
\end{array}\end{array}$ & $\begin{array}{l}O E \\
C / E\end{array}$ & & $\begin{array}{r}0 \\
0 \\
-- \\
0\end{array}$ & $\begin{array}{r}0 \\
0 \\
-- \\
0\end{array}$ & $\begin{array}{r}0 \\
0 \\
-0\end{array}$ & $---\frac{-}{0}$ & ---- & -- \\
\hline
\end{tabular}


TABLE $7.6-1$

TOTAL PROGRAM ELEMENT COST BASELINE - BY YEAR

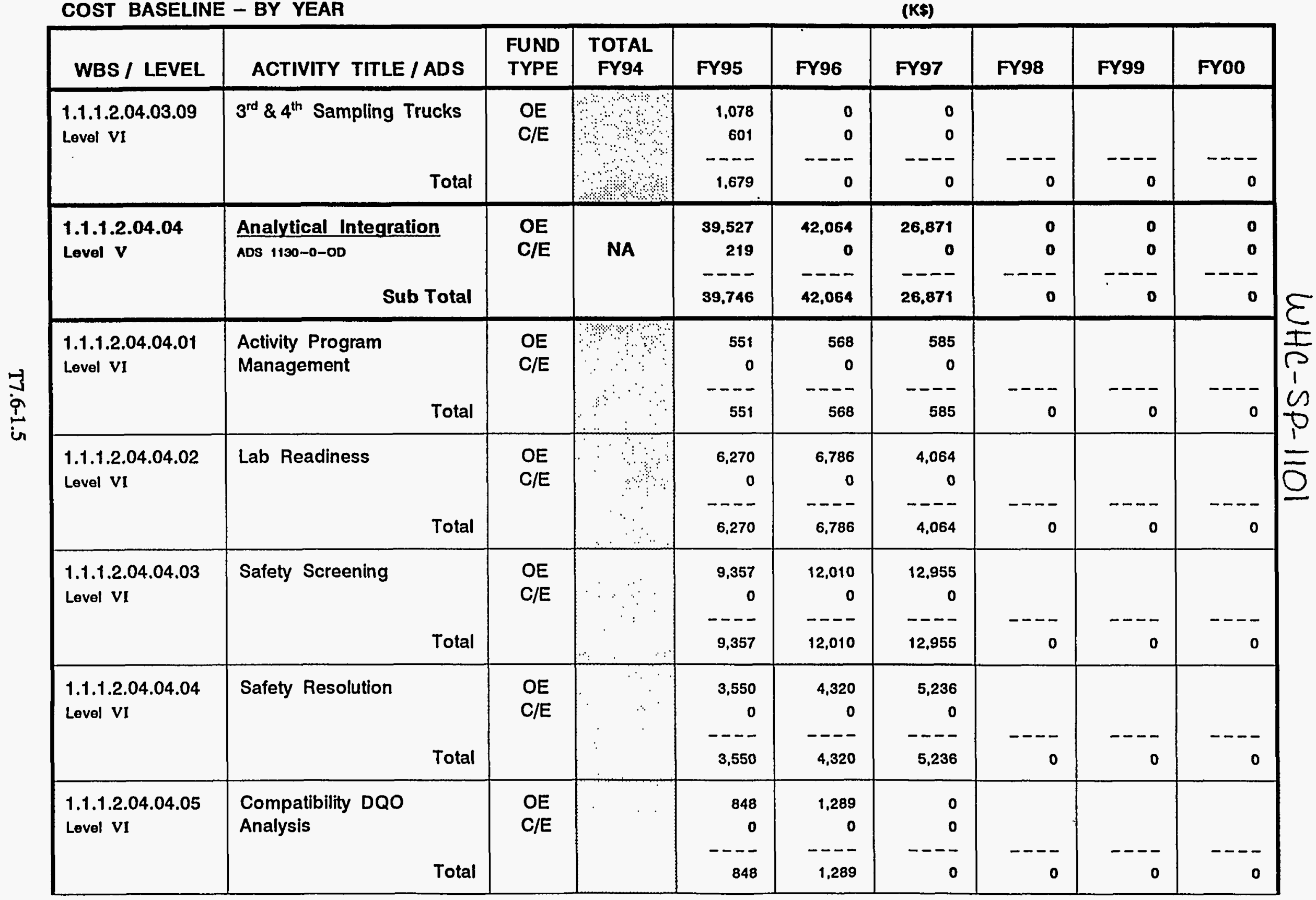

\section{[ R1 ]}


TABLE $7.6-1$

TOTAL PROGRAM ELEMENT

COST BASELINE - BY YEAR
[ R1 ]

\begin{tabular}{|c|c|c|c|c|c|c|c|c|c|}
\hline WBS / LEVEL & ACTIVITY TITLE / ADS & $\begin{array}{l}\text { FUND } \\
\text { TYPE }\end{array}$ & $\begin{array}{l}\text { TOTAL } \\
\text { FY94 }\end{array}$ & FY95 & FY96 & FY97 & FY98 & FY99 & FYoO \\
\hline $\begin{array}{l}\text { 1.1.1.2.04.04.06 } \\
\text { Level VI }\end{array}$ & $\begin{array}{l}\text { Operations DQO } \\
\text { Analysis } \\
\\
\\
\end{array}$ & $\begin{array}{l}\mathrm{OE} \\
\mathrm{C} / \mathrm{E}\end{array}$ & & $\begin{array}{r}2,268 \\
0 \\
-0 \\
2,268\end{array}$ & $\begin{array}{r}2,683 \\
0 \\
-- \\
2,683\end{array}$ & $\begin{array}{r}0 \\
0 \\
-0\end{array}$ & --- & $\cdots$ & - \\
\hline $\begin{array}{l}\text { 1.1.1.2.04.04.07 } \\
\text { Level VI }\end{array}$ & $\begin{array}{l}\text { Disposal DQO } \\
\text { Analysis }\end{array}$ & $\begin{array}{l}O E \\
C / E\end{array}$ & & $\begin{array}{r}3,559 \\
0 \\
--- \\
3,559\end{array}$ & $\begin{array}{r}3,912 \\
0 \\
-3,912\end{array}$ & $\begin{array}{r}4,031 \\
0 \\
--- \\
4,031\end{array}$ & 0 & --- & - \\
\hline $\begin{array}{l}\text { 1.1.1.2.04.04.08 } \\
\text { Level VI }\end{array}$ & $\begin{array}{l}\text { Vapor Analysis Final } \\
\text { Planned (PNL) } \\
\\
\text { Total }\end{array}$ & $\begin{array}{l}O E \\
C / E\end{array}$ & & $\begin{array}{r}1,300 \\
0 \\
-1,300\end{array}$ & $\begin{array}{r}0 \\
0 \\
-- \\
0\end{array}$ & $\begin{array}{r}0 \\
0 \\
- \\
0\end{array}$ & --- & $-\cdots$ & 0 \\
\hline $\begin{array}{l}\text { 1.1.1.2.04.04.09 } \\
\text { Level VI }\end{array}$ & $\begin{array}{l}\text { Off Site Labs - Final } \\
\text { Planned } \\
\qquad \\
\qquad \text { Total }\end{array}$ & $\begin{array}{l}O E \\
C / E\end{array}$ & & $\begin{array}{r}5.150 \\
0 \\
--- \\
5.150\end{array}$ & $\begin{array}{r}4,995 \\
0 \\
--- \\
4,995\end{array}$ & $\begin{array}{r}0 \\
0 \\
- \\
-0\end{array}$ & --- & --- & o \\
\hline $\begin{array}{l}\text { 1.1.1.2.04.04.10 } \\
\text { Level VI }\end{array}$ & $\begin{array}{l}300 \text { Area Waste } \\
\text { Assessment } \\
\\
\text { Total }\end{array}$ & $\begin{array}{l}O E \\
C / E\end{array}$ & & $\begin{array}{r}380 \\
0 \\
--- \\
380\end{array}$ & $\begin{array}{r}0 \\
0 \\
- \\
0\end{array}$ & $\begin{array}{r}0 \\
0 \\
- \\
0\end{array}$ & $-r$ & $-1-$ & -- \\
\hline $\begin{array}{l}\text { 1.1.1.2.04.04.11 } \\
\text { Level VI }\end{array}$ & $\begin{array}{l}\text { 222-S Lab Upgrades } \\
\text { / Lims. } \\
\text { Total }\end{array}$ & $\begin{array}{l}O E \\
C / E\end{array}$ & & $\begin{array}{r}2,631 \\
0 \\
--- \\
2,631\end{array}$ & $\begin{array}{r}2,361 \\
0 \\
--- \\
2,361\end{array}$ & $\begin{array}{r}0 \\
0 \\
- \\
0\end{array}$ & - & --- & o \\
\hline $\begin{array}{l}\text { 1.1.1.2.04.04.12 } \\
\text { Level VI }\end{array}$ & $\begin{array}{l}325 \text { Lab Upgrades } \\
\text { Final Planned } \\
\end{array}$ & $\begin{array}{l}O E \\
C / E\end{array}$ & & $\begin{array}{r}2,735 \\
0 \\
--- \\
2,735\end{array}$ & $\begin{array}{r}2,523 \\
0 \\
-- \\
2,523\end{array}$ & $\begin{array}{r}0 \\
0 \\
-0\end{array}$ & -- & $-{ }_{0}$ & o \\
\hline
\end{tabular}


TABLE 7.6-1

TOTAL PROGRAM ELEMENT

COST BASELINE - BY YEAR

\section{[ R1 ]}

\begin{tabular}{|c|c|c|c|c|c|c|c|c|c|}
\hline WBS / LEVEL & ACTIVITY TITLE / ADS & $\begin{array}{l}\text { FUND } \\
\text { TYPE }\end{array}$ & $\begin{array}{l}\text { TOTAL } \\
\text { FY94 }\end{array}$ & FY95 & FY96 & FY97 & FY98 & FY99 & FYOO \\
\hline $\begin{array}{l}\text { 1.1.1.2.04.04.13 } \\
\text { Level VI }\end{array}$ & $\begin{array}{l}\text { Off-Site Repackaging / } \\
\text { Shipping } \\
\\
\text { Total }\end{array}$ & $\begin{array}{l}\mathrm{OE} \\
\mathrm{C} / \mathrm{E}\end{array}$ & $\begin{array}{l}3 \\
\cdots \\
\cdots\end{array}$ & $\begin{array}{r}428 \\
0 \\
--- \\
428\end{array}$ & $\begin{array}{r}617 \\
0 \\
--- \\
617\end{array}$ & $\begin{array}{r}0 \\
0 \\
-0 \\
0\end{array}$ & --- & ---- & 0 \\
\hline $\begin{array}{l}\text { 1.1.1.2.04.04.14 } \\
\text { Level V1 }\end{array}$ & $\begin{array}{l}\text { Organic Analysis Support } \\
\text { (ORNL) } \\
\text { Total }\end{array}$ & $\begin{array}{l}O E \\
C / E\end{array}$ & & $\begin{array}{r}500 \\
219 \\
--- \\
719\end{array}$ & $\begin{array}{r}0 \\
0 \\
-0\end{array}$ & $\begin{array}{r}0 \\
0 \\
-- \\
0\end{array}$ & --- & --- & --- \\
\hline $\begin{array}{l}\text { 1.1.1.2.04.04.15 } \\
\text { L.evel VI }\end{array}$ & $\begin{array}{l}\text { Vapor Analysis } \\
\text { (PNL) } \\
\end{array}$ & $\begin{array}{l}\mathrm{OE} \\
\mathrm{C} / \mathrm{E}\end{array}$ & & $\begin{array}{r}0 \\
0 \\
- \\
-0\end{array}$ & $\begin{array}{r}0 \\
0 \\
- \\
0\end{array}$ & $\begin{array}{r}0 \\
0 \\
-- \\
0\end{array}$ & --- & --- & --- \\
\hline $\begin{array}{l}\text { 1.1.1.2.04.04.16 } \\
\text { Level VI }\end{array}$ & $\begin{array}{l}\text { ORNL CENRTC Activity } \\
\text { (Enhance Trap Lab.) } \\
\\
\text { Total }\end{array}$ & $\begin{array}{l}O E \\
\mathrm{O} / \mathrm{E}\end{array}$ & & $\begin{array}{r}0 \\
0 \\
- \\
0\end{array}$ & $\begin{array}{r}0 \\
0 \\
- \\
0\end{array}$ & $\begin{array}{r}0 \\
0 \\
-0\end{array}$ & - & -- & - \\
\hline $\begin{array}{l}\text { 1.1.1.2.04.05 } \\
\text { Level } \mathrm{V}\end{array}$ & $\begin{array}{l}\text { Data Evaluation \& } \\
\text { Reporting } \\
\begin{array}{l}\text { ADS } 1130-0-O E \\
\quad \text { Sub Total }\end{array}\end{array}$ & $\begin{array}{l}O E \\
C / E\end{array}$ & NA & $\begin{array}{r}9,108 \\
0 \\
-9 \\
9,108\end{array}$ & $\begin{array}{r}10,482 \\
0 \\
--- \\
10,482\end{array}$ & $\begin{array}{r}8,133 \\
0 \\
--- \\
8,133\end{array}$ & $\begin{array}{r}0 \\
0 \\
-0\end{array}$ & $\begin{array}{r}0 \\
0 \\
-- \\
0\end{array}$ & $\begin{array}{r}0 \\
0 \\
-0 \\
0\end{array}$ \\
\hline $\begin{array}{l}\text { 1.1.1.2.04.05.01 } \\
\text { Level VI }\end{array}$ & $\begin{array}{l}\text { Activity Program } \\
\text { Management } \\
\\
\\
\text { Total }\end{array}$ & $\begin{array}{l}O E \\
C / E\end{array}$ & & $\begin{array}{r}123 \\
0 \\
--- \\
123\end{array}$ & $\begin{array}{r}127 \\
0 \\
-- \\
127\end{array}$ & $\begin{array}{r}131 \\
0 \\
-- \\
131\end{array}$ & -- & 0 & - \\
\hline $\begin{array}{l}\text { 1.1.1.2.04.05.02 } \\
\text { Level VI }\end{array}$ & $\begin{array}{l}\text { Data Definition \& } \\
\text { Planning }\end{array}$ & $\begin{array}{l}O E \\
\mathrm{C} / \mathrm{E}\end{array}$ & & $\begin{array}{r}193 \\
0 \\
-- \\
193\end{array}$ & $\begin{array}{r}252 \\
0 \\
-- \\
252\end{array}$ & $\begin{array}{r}197 \\
0 \\
-- \\
197\end{array}$ & 0 & -- & o \\
\hline
\end{tabular}


TABLE 7.6-1

TOTAL PROGRAM ELEMENT COST BASELINE - BY YEAR

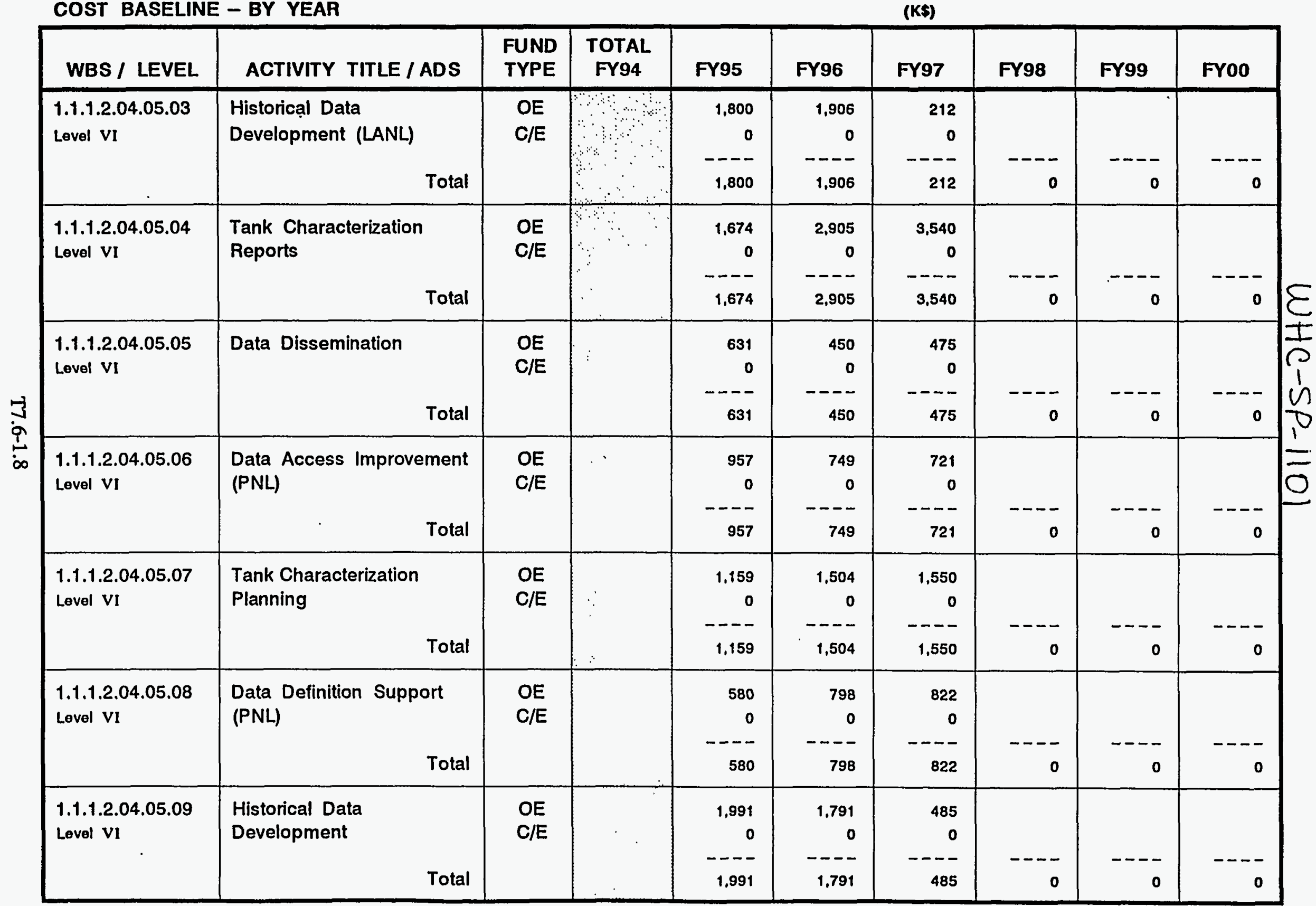


TABLE 7.6-1

TOTAL PROGRAM ELEMENT COST BASELINE - BY YEAR

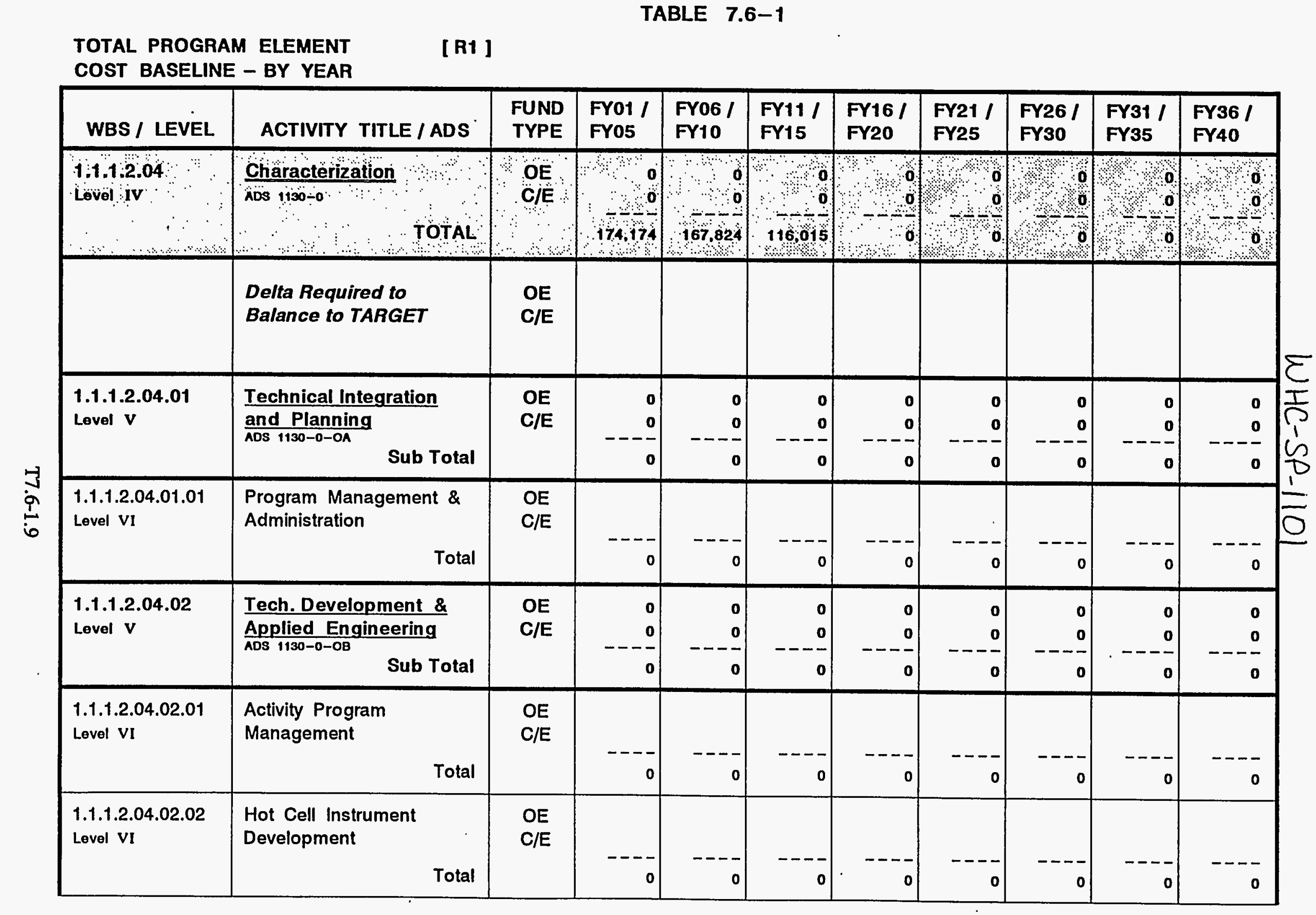

\section{[ R1 ]}


TABLE $7.6-1$

TOTAL PROGRAM ELEMENT

COST BASELINE - BY YEAR

\begin{tabular}{|c|c|c|c|c|c|c|c|c|c|c|}
\hline WBS / LEVEL & ACTIVITY TITLE / ADS & $\begin{array}{l}\text { FUND } \\
\text { TYPE }\end{array}$ & $\begin{array}{l}\text { FY01 / } \\
\text { FY05 }\end{array}$ & $\begin{array}{l}\text { FY06 I } \\
\text { FY10 }\end{array}$ & $\begin{array}{l}\text { FY11 I } \\
\text { FY15 }\end{array}$ & $\begin{array}{l}\text { FY16 / } \\
\text { FY20 }\end{array}$ & $\begin{array}{l}\text { FY21 I } \\
\text { FY25 }\end{array}$ & $\begin{array}{l}\text { FY26 / } \\
\text { FY30 }\end{array}$ & $\begin{array}{l}\text { FY31 / } \\
\text { FY35 }\end{array}$ & $\begin{array}{l}\text { FY36 / } \\
\text { FY40 }\end{array}$ \\
\hline $\begin{array}{l}\text { 1.1.1.2.04.02.03 } \\
\text { Level VI }\end{array}$ & $\begin{array}{l}\text { Analytical Procedures } \\
\text { Development } \\
\qquad \text { Total }\end{array}$ & $\begin{array}{l}O E \\
C / E\end{array}$ & $\begin{array}{r}--- \\
0\end{array}$ & $---\frac{1}{0}$ & ---- & $-\cdots$ & $---\frac{-}{0}$ & $---\frac{-}{0}$ & $\begin{array}{r}---- \\
0\end{array}$ & $--\frac{-}{0}$ \\
\hline $\begin{array}{l}\text { 1.1.1.2.04.02.04 } \\
\text { Level VI }\end{array}$ & $\begin{array}{l}\text { In-Situ Methods } \\
\text { Development }\end{array}$ & $\begin{array}{l}O E \\
C / E\end{array}$ & --- & $-\cdots$ & 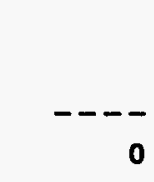 & 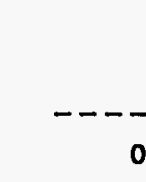 & --- & $---\frac{-}{0}$ & --- & ---- \\
\hline $\begin{array}{l}\text { 1.1.1.2.04.02.05 } \\
\text { Level VI }\end{array}$ & $\begin{array}{l}\text { Field Samples } \\
\text { Improvement }\end{array}$ & $\begin{array}{l}\text { OE } \\
C / E\end{array}$ & 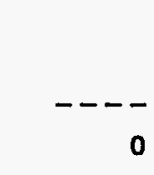 & $--\frac{1}{0}$ & $---\overline{0}$ & $---\frac{1}{0}$ & ---- & --- & $---\frac{-}{0}$ & ---- \\
\hline $\begin{array}{l}\text { 1.1.1.2.04.02.06 } \\
\text { Level VI }\end{array}$ & $\begin{array}{l}\text { Direct Bit Temp. } \\
\text { Monitor Dvlp. }\end{array}$ & $\begin{array}{l}\text { OE } \\
C / E\end{array}$ & $---\overline{0}$ & --- & ---- & $---\frac{1}{0}$ & $\begin{array}{r}--- \\
0\end{array}$ & ---- & ---- & $---\frac{}{0}$ \\
\hline $\begin{array}{l}\text { 1.1.1.2.04.02.07 } \\
\text { Level VI }\end{array}$ & $\begin{array}{l}\text { Charact. Methods } \\
\text { Dvlp. (PNL) }\end{array}$ & $\begin{array}{l}\text { OE } \\
C / E\end{array}$ & --- & $---\frac{-}{0}$ & ---- & $---\frac{1}{0}$ & $\begin{array}{r}---\frac{1}{0} \\
0\end{array}$ & $---\frac{-}{0}$ & $---\frac{-}{0}$ & $---\frac{-}{0}$ \\
\hline $\begin{array}{l}\text { 1.1.1.2.04.02.08 } \\
\text { Level VI }\end{array}$ & $\begin{array}{l}\text { Analytical Procedures } \\
\text { Development (PNL) } \\
\qquad \text { Total }\end{array}$ & $\begin{array}{l}O E \\
C / E\end{array}$ & 0 & $\cdots$ & ---- & --- & $\begin{array}{r}---- \\
0\end{array}$ & $\begin{array}{r}-\cdots- \\
0\end{array}$ & $\begin{array}{r}-\cdots \\
0\end{array}$ & -- \\
\hline $\begin{array}{l}\text { 1.1.1.2.04.02.09 } \\
\text { Level VI }\end{array}$ & $\begin{array}{l}\text { TDPO PNL Final } \\
\text { Planned }\end{array}$ & $\begin{array}{l}O E \\
C / E\end{array}$ & $\begin{array}{r}--- \\
0\end{array}$ & $\begin{array}{r}---- \\
0\end{array}$ & --- & $---\frac{1}{0}$ & $\begin{array}{r}--- \\
0\end{array}$ & --- & $---\frac{-}{0}$ & --- \\
\hline
\end{tabular}

[ R1 ] 
TABLE $7.6-1$

TOTAL PROGRAM ELEMENT

[ R1 ]

COST BASELINE - BY YEAR

\begin{tabular}{|c|c|c|c|c|c|c|c|c|c|c|}
\hline WBS / LEVEL & ACTIVITY TITLE / ADS & $\begin{array}{l}\text { FUND } \\
\text { TYPE }\end{array}$ & $\begin{array}{l}\text { FY01 I } \\
\text { FY05 }\end{array}$ & $\begin{array}{l}\text { FY06 / } \\
\text { FY10 }\end{array}$ & $\begin{array}{l}\text { FY11 / } \\
\text { FY15 }\end{array}$ & $\begin{array}{l}\text { FY16 / } \\
\text { FY20 }\end{array}$ & $\begin{array}{l}\text { FY21 / } \\
\text { FY25 }\end{array}$ & $\begin{array}{l}\text { FY26 / } \\
\text { FY30 }\end{array}$ & $\begin{array}{l}\text { FY31 I } \\
\text { FY35 }\end{array}$ & $\begin{array}{l}\text { FY36 / } \\
\text { FY40 }\end{array}$ \\
\hline $\begin{array}{l}\text { 1.1.1.2.04.02.10 } \\
\text { Level VI }\end{array}$ & $\begin{array}{l}\text { Hot Cell Instrument } \\
\text { Development } \\
\\
\quad \text { Total }\end{array}$ & $\begin{array}{l}O E \\
C / E\end{array}$ & 0 & --- & --- & 0 & --- & --- & --- & 0 \\
\hline $\begin{array}{l}\text { 1.1.1.2.04.02.11 } \\
\text { Level VI }\end{array}$ & $\begin{array}{l}\text { Analysis Procedures } \\
\text { Development } \\
\qquad \text { Total }\end{array}$ & $\begin{array}{l}\text { OE } \\
\mathrm{C} / \mathrm{E}\end{array}$ & 0 & ---- & ---- & ---- & ---- & --- & $-\cdots$ & --- \\
\hline $\begin{array}{l}\text { 1.1.1.2.04.02.12 } \\
\text { Level VI }\end{array}$ & $\begin{array}{l}\text { In-Situ Methods } \\
\text { Development } \\
\end{array}$ & $\begin{array}{l}\mathrm{OE} \\
\mathrm{C} / \mathrm{E}\end{array}$ & $---\frac{-}{0}$ & $---\frac{-}{0}$ & ---- & --- & $---\frac{-}{0}$ & $---\frac{-}{0}$ & --- & $---\frac{-}{0}$ \\
\hline $\begin{array}{l}1.1 .1 .2 .04 .02 .13 \\
\text { Level VI }\end{array}$ & $\begin{array}{l}\text { Charact. Methods } \\
\text { Dvip. (PNL) } \\
\\
\end{array}$ & $\begin{array}{l}O E \\
C / E\end{array}$ & 0 & -- & ---- & 0 & ---- & -- & 0 & --- \\
\hline $\begin{array}{l}1.1 .1 .2 .04 .02 .14 \\
\text { Level VI }\end{array}$ & $\begin{array}{l}\text { Analytical Procedures } \\
\text { Development (LLNL) } \\
\qquad \text { Total }\end{array}$ & $\begin{array}{l}O E \\
C / E\end{array}$ & $\begin{array}{r}--- \\
0\end{array}$ & -- & ---- & --- & $\begin{array}{r}--- \\
0\end{array}$ & 0 & 0 & --- \\
\hline $\begin{array}{l}\text { 1.1.1.2.04.03 } \\
\text { Level } v\end{array}$ & $\begin{array}{l}\frac{\text { Field Sampling \& }}{\frac{\text { Measurement }}{\text { ADS } 1130-0-O C}} \text { Sub Total } \\
\quad \text { Sub }\end{array}$ & $\begin{array}{l}O E \\
C / E\end{array}$ & $\begin{array}{r}\mathbf{0} \\
-- \\
\mathbf{0}\end{array}$ & $\begin{array}{r}0 \\
0 \\
-0\end{array}$ & $\begin{array}{r}\mathbf{0} \\
\mathbf{0} \\
-0 \\
\mathbf{0}\end{array}$ & $\begin{array}{r}\mathbf{0} \\
-- \\
-0 \\
0\end{array}$ & $\begin{array}{r}0 \\
-0 \\
-0\end{array}$ & $\begin{array}{r}0 \\
-0 \\
-0\end{array}$ & $\begin{array}{r}\mathbf{0} \\
\mathbf{0} \\
--\frac{\mathbf{0}}{\mathbf{0}}\end{array}$ & $\begin{array}{r}\mathbf{0} \\
-- \\
-0 \\
0\end{array}$ \\
\hline $\begin{array}{l}\text { 1.1.1.2.04.03.01 } \\
\text { Level VI }\end{array}$ & $\begin{array}{l}\text { Activity Program } \\
\text { Management } \\
\\
\end{array}$ & $\begin{array}{l}O E \\
C / E\end{array}$ & $\begin{array}{r}--- \\
0\end{array}$ & ---- & $---\frac{-}{0}$ & ---- & $---\frac{-}{0}$ & $---\frac{-}{0}$ & $---\frac{-}{0}$ & ---- \\
\hline
\end{tabular}


TABLE 7.6-1

TOTAL PROGRAM ELEMENT COST BASELINE - BY YEAR

\begin{tabular}{|c|c|c|c|c|c|c|c|c|c|c|}
\hline WBS / LEVEL & ACTIVITY TITLE / ADS & $\begin{array}{l}\text { FUND } \\
\text { TYPE }\end{array}$ & $\begin{array}{l}\text { FY01 I } \\
\text { FY05 }\end{array}$ & $\begin{array}{l}\text { FY06 / } \\
\text { FY10 }\end{array}$ & $\begin{array}{l}\text { FY11 / } \\
\text { FY15 }\end{array}$ & $\begin{array}{l}\text { FY16 / } \\
\text { FY20 }\end{array}$ & $\begin{array}{l}\text { FY21 / } \\
\text { FY25 }\end{array}$ & $\begin{array}{l}\text { FY26 I } \\
\text { FY30 }\end{array}$ & $\begin{array}{l}\text { FY31 I } \\
\text { FY35 }\end{array}$ & $\begin{array}{l}\text { FY36 / } \\
\text { FY40 }\end{array}$ \\
\hline $\begin{array}{l}1.1 .1 .2 .04 .03 .02 \\
\text { Level VI }\end{array}$ & $\begin{array}{l}\text { Core Sampling } \\
\qquad \text { Total }\end{array}$ & $\begin{array}{l}O E \\
C / E\end{array}$ & $\begin{array}{r}-- \\
0\end{array}$ & --- & $-\cdots$ & ---- & $-\cdots$ & --- & ---- & $--\frac{1}{0}$ \\
\hline $\begin{array}{l}\text { 1.1.1.2.04.03.03 } \\
\text { Level VI }\end{array}$ & Auger Sampling & $\begin{array}{l}O E \\
C / E\end{array}$ & $\begin{array}{r}---- \\
0\end{array}$ & $\cdots$ & ---- & ---- & ---- & --- & ---- & $-\cdots$ \\
\hline $\begin{array}{l}1.1 .1 .2 .04 .03 .04 \\
\text { Level VI }\end{array}$ & $\begin{array}{l}\text { Vapor Sampling } \\
\qquad \text { Total }\end{array}$ & $\begin{array}{l}O E \\
C / E\end{array}$ & ---- & $---\overline{0}$ & ---- & $\begin{array}{r}--- \\
0\end{array}$ & ---- & $---\frac{-}{0}$ & $-\cdots$ & $---\frac{-}{0}$ \\
\hline $\begin{array}{l}\text { 1.1.1.2.04.03.05 } \\
\text { Level VI }\end{array}$ & $\begin{array}{l}\text { Supernate Sampling } \\
\qquad \text { Total }\end{array}$ & $\begin{array}{l}O E \\
C / E\end{array}$ & $\begin{array}{r}---- \\
0\end{array}$ & $\begin{array}{r}---- \\
0\end{array}$ & ---- & ---- & $\begin{array}{r}--- \\
0\end{array}$ & $-\overline{0}$ & $\begin{array}{r}--- \\
0\end{array}$ & $\begin{array}{r}---- \\
0\end{array}$ \\
\hline $\begin{array}{l}\text { 1.1.1.2.04.03.06 } \\
\text { Level VI }\end{array}$ & $\begin{array}{l}\text { Riser Preparation } \\
\qquad \text { Total }\end{array}$ & $\begin{array}{l}O E \\
C / E\end{array}$ & $-\cdots$ & - & --- & $\begin{array}{r}---- \\
0\end{array}$ & ---- & -- & ---- & -- \\
\hline $\begin{array}{l}\text { 1.1.1.2.04.03.07 } \\
\text { Level VI }\end{array}$ & $\begin{array}{l}\text { Equipment Replacement } \\
\text { Total }\end{array}$ & $\begin{array}{l}O E \\
C / E\end{array}$ & ---- & $\cdots$ & $\begin{array}{r}--- \\
0\end{array}$ & $\begin{array}{r}---- \\
0\end{array}$ & $\begin{array}{r}--- \\
0\end{array}$ & $\begin{array}{r}--- \\
0\end{array}$ & ---- & --- \\
\hline $\begin{array}{l}1.1 .1 .2 .04 .03 .08 \\
\text { Level VI }\end{array}$ & $\begin{array}{l}\text { Field Sampling } \\
\text { Readiness } \\
\qquad \text { Total }\end{array}$ & $\begin{array}{l}O E \\
C / E\end{array}$ & -- & - & $\begin{array}{r}---- \\
0\end{array}$ & ---- & 0 & -- & $-\cdots$ & $-\cdots$ \\
\hline
\end{tabular}

[ R1 ] 
TABLE $7.6-1$

TOTAL PROGRAM ELEMENT

COST BASELINE - BY YEAR

[ R1 ]

\begin{tabular}{|c|c|c|c|c|c|c|c|c|c|c|}
\hline WBS / LEVEL & ACTIVITY TITLE / ADS & $\begin{array}{l}\text { FUND } \\
\text { TYPE }\end{array}$ & $\begin{array}{l}\text { FY01 I } \\
\text { FY05 }\end{array}$ & $\begin{array}{l}\text { FY06 I } \\
\text { FY10 }\end{array}$ & $\begin{array}{l}\text { FY11 I } \\
\text { FY15 }\end{array}$ & $\begin{array}{l}\text { FY16 I } \\
\text { FY20 }\end{array}$ & $\begin{array}{l}\text { FY21 I } \\
\text { FY25 }\end{array}$ & $\begin{array}{l}\text { FY26 I } \\
\text { FY30 }\end{array}$ & $\begin{array}{l}\text { FY31 I } \\
\text { FY35 }\end{array}$ & $\begin{array}{l}\text { FY36 I } \\
\text { FY40 }\end{array}$ \\
\hline $\begin{array}{l}1.1 .1 .2 .04 .03 .09 \\
\text { Level VI }\end{array}$ & $\begin{array}{r}3^{\text {rd }} \& 4^{\text {th }} \text { Sampling Trucks } \\
\text { Total }\end{array}$ & $\begin{array}{l}\text { OE } \\
C / E\end{array}$ & $-\cdots$ & $\begin{array}{r}---- \\
0\end{array}$ & $\begin{array}{r}--- \\
0\end{array}$ & ---- & --- & ---- & ---- & $\begin{array}{r}--1 \\
0\end{array}$ \\
\hline $\begin{array}{l}\text { 1.1.1.2.04.04 } \\
\text { Level } \mathrm{v}\end{array}$ & $\begin{array}{l}\frac{\text { Analytical Integration }}{\text { ADS } 1130-0-00} \\
\text { Sub Total }\end{array}$ & $\begin{array}{l}\text { OE } \\
\text { C/E }\end{array}$ & $\begin{array}{r}0 \\
0 \\
- \\
-0\end{array}$ & $\begin{array}{r}\mathbf{0} \\
\mathbf{0} \\
-- \\
\mathbf{0}\end{array}$ & $\begin{array}{r}0 \\
0 \\
- \\
-0\end{array}$ & $\begin{array}{r}\mathbf{0} \\
\mathbf{0} \\
--- \\
0\end{array}$ & $\begin{array}{r}0 \\
0 \\
-0 \\
0\end{array}$ & $\begin{array}{r}0 \\
0 \\
-- \\
-0\end{array}$ & $\begin{array}{r}0 \\
0 \\
-0 \\
-0\end{array}$ & $\begin{array}{r}0 \\
0 \\
-- \\
0\end{array}$ \\
\hline $\begin{array}{l}\text { 1.1.1.2.04.04.01 } \\
\text { Level VI }\end{array}$ & $\begin{array}{l}\text { Activity Program } \\
\text { Management }\end{array}$ & $\begin{array}{l}O E \\
C / E\end{array}$ & $\begin{array}{r}--- \\
0\end{array}$ & ---- & $\begin{array}{r}---- \\
0\end{array}$ & $\begin{array}{r}---- \\
0\end{array}$ & $\begin{array}{r}--- \\
0\end{array}$ & $\begin{array}{r}--- \\
0\end{array}$ & $\begin{array}{r}---- \\
0\end{array}$ & -- \\
\hline $\begin{array}{l}\text { 1.1.1.2.04.04.02 } \\
\text { Level VI }\end{array}$ & Lab Readiness & $\begin{array}{l}O E \\
C / E\end{array}$ & $\begin{array}{r}--- \\
0\end{array}$ & -- & $\begin{array}{r}--- \\
0\end{array}$ & $\begin{array}{r}--- \\
0\end{array}$ & ---- & $\begin{array}{r}--- \\
0\end{array}$ & $\begin{array}{r}---- \\
0\end{array}$ & --- \\
\hline $\begin{array}{l}\text { 1.1.1.2.04.04.03 } \\
\text { Level VI }\end{array}$ & Safety Screening & $\begin{array}{l}O E \\
C / E\end{array}$ & $\begin{array}{r}--- \\
0\end{array}$ & $-\cdots$ & $\begin{array}{r}--- \\
0\end{array}$ & --- & $\begin{array}{r}---- \\
0\end{array}$ & $\begin{array}{r}---- \\
0\end{array}$ & $\begin{array}{r}---- \\
0\end{array}$ & --- \\
\hline $\begin{array}{l}\text { 1.1.1.2.04.04.04 } \\
\text { Level VI }\end{array}$ & Safety Resolution & $\begin{array}{l}O E \\
C / E\end{array}$ & $\begin{array}{r}--- \\
0\end{array}$ & -- & -- & $\begin{array}{r}---- \\
0\end{array}$ & $\begin{array}{r}--- \\
0\end{array}$ & $\begin{array}{r}---- \\
0\end{array}$ & $\begin{array}{r}--- \\
0\end{array}$ & $-\cdots$ \\
\hline $\begin{array}{l}1.1 .1 .2 .04 .04 .05 \\
\text { Lovel VI }\end{array}$ & $\begin{array}{l}\text { Compatibility DQO } \\
\text { Analysis } \\
\\
\\
\quad \text { Total }\end{array}$ & $\begin{array}{l}O E \\
C / E\end{array}$ & ---- & $\begin{array}{r}---- \\
0\end{array}$ & $\begin{array}{r}--- \\
0\end{array}$ & $\begin{array}{r}--- \\
0\end{array}$ & ---- & ---- & ---- & - \\
\hline
\end{tabular}


○

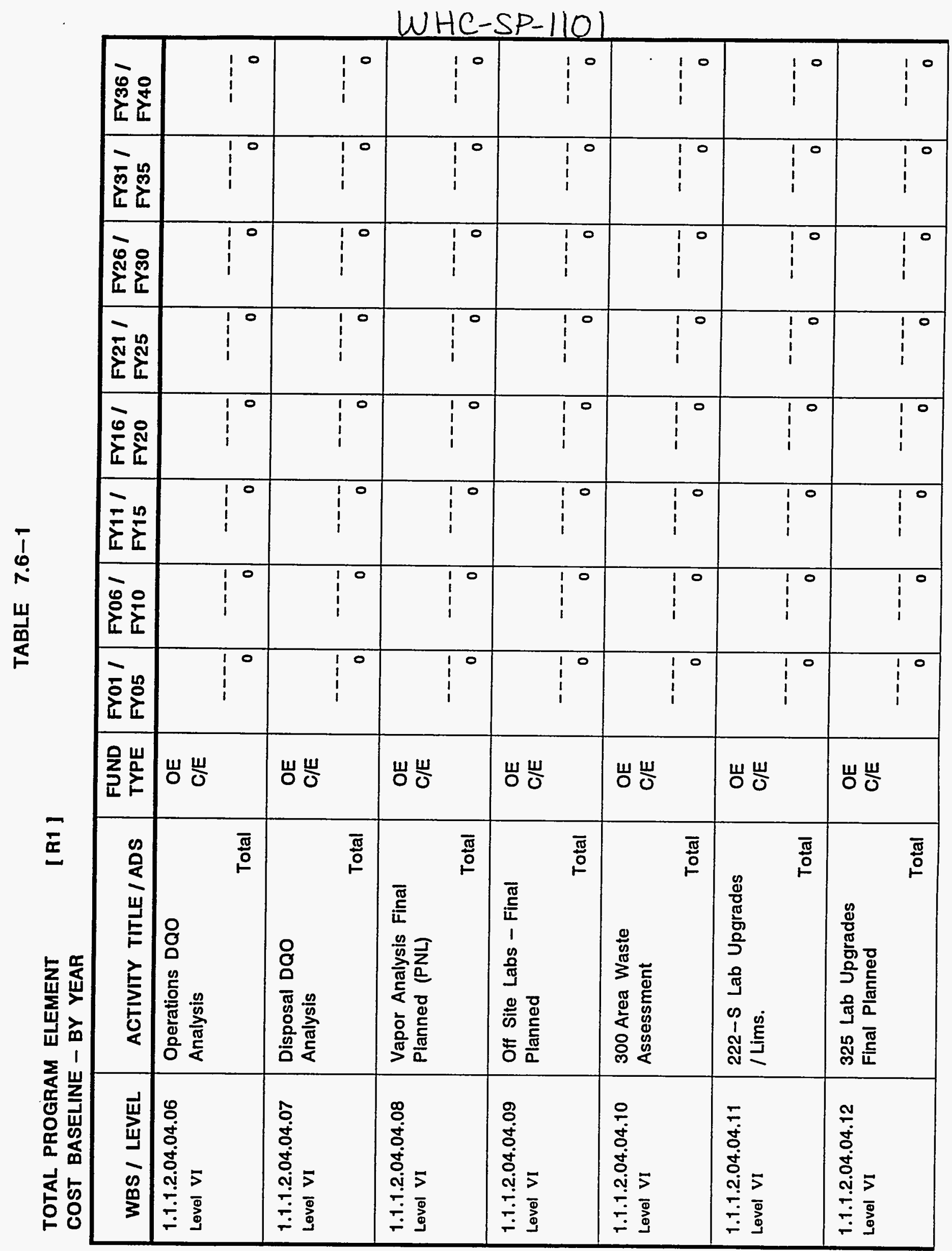


TABLE 7.6-1

TOTAL PROGRAM ELEMENT COST BASELINE - BY YEAR

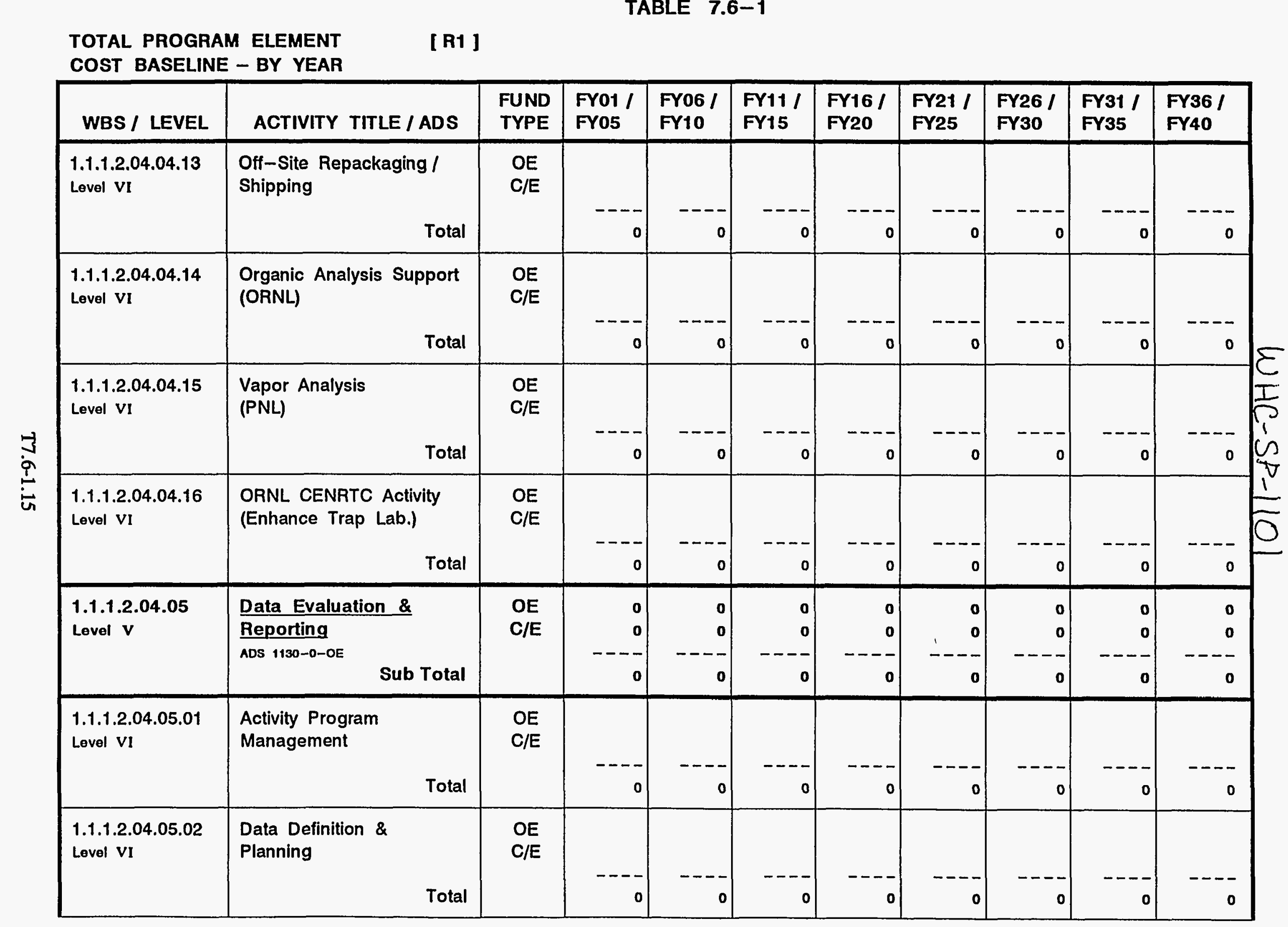

[ R1 ] 
TABLE 7.6-1

TOTAL PROGRAM ELEMENT COST BASELINE - BY YEAR

\begin{tabular}{|c|c|c|c|c|c|c|c|c|c|c|}
\hline WBS / LEVEL & ACTIVITY TITLE / ADS & $\begin{array}{l}\text { FUND } \\
\text { TYPE }\end{array}$ & $\begin{array}{l}\text { FY01 I } \\
\text { FY05 }\end{array}$ & $\begin{array}{l}\text { FY06 I } \\
\text { FY10 }\end{array}$ & $\begin{array}{l}\text { FY11 I } \\
\text { FY15 }\end{array}$ & $\begin{array}{l}\text { FY16 I } \\
\text { FY20 }\end{array}$ & $\begin{array}{l}\text { FY21 I } \\
\text { FY25 }\end{array}$ & $\begin{array}{l}\text { FY26 I } \\
\text { FY30 }\end{array}$ & $\begin{array}{l}\text { FY31 I } \\
\text { FY35 }\end{array}$ & $\begin{array}{l}\text { FY36 I } \\
\text { FY40 }\end{array}$ \\
\hline $\begin{array}{l}1.1 .1 .2 .04 .05 .03 \\
\text { Level VI }\end{array}$ & $\begin{array}{l}\text { Historical Data } \\
\text { Development (LANL) } \\
\qquad \text { Total }\end{array}$ & $\begin{array}{l}O E \\
C / E\end{array}$ & --- & --- & ---- & ---- & --- & ---- & $-\cdots-$ & $---\frac{-}{0}$ \\
\hline $\begin{array}{l}\text { 1.1.1.2.04.05.04 } \\
\text { Level VI }\end{array}$ & $\begin{array}{l}\text { Tank Characterization } \\
\text { Reports } \\
\qquad \text { Total }\end{array}$ & $\begin{array}{l}\text { OE } \\
C / E\end{array}$ & --- & ---- & $\begin{array}{r}--- \\
0\end{array}$ & ---- & ---- & ---- & ---- & $-\cdots$ \\
\hline $\begin{array}{l}\text { 1.1.1.2.04.05.05 } \\
\text { Level VI }\end{array}$ & $\begin{array}{l}\text { Data Dissemination } \\
\qquad \text { Total }\end{array}$ & $\begin{array}{l}O E \\
C / E\end{array}$ & ---- & 0 & 0 & $\begin{array}{r}--- \\
0\end{array}$ & $-\cdots--$ & ---- & ---- & ---- \\
\hline $\begin{array}{l}\text { 1.1.1.2.04.05.06 } \\
\text { Level VI }\end{array}$ & $\begin{array}{l}\text { Data Access Improvement } \\
\text { (PNL) } \\
\qquad \text { Total }\end{array}$ & $\begin{array}{l}O E \\
C / E\end{array}$ & $\begin{array}{r}---- \\
0\end{array}$ & $\begin{array}{r}---- \\
0\end{array}$ & ---- & $\begin{array}{r}--- \\
0\end{array}$ & ---- & ---- & ---- & ---- \\
\hline $\begin{array}{l}\text { 1.1.1.2.04.05.07 } \\
\text { Level VI }\end{array}$ & $\begin{array}{l}\text { Tank Characterization } \\
\text { Planning } \\
\qquad \begin{array}{l}\text { Total }\end{array}\end{array}$ & $\begin{array}{l}O E \\
C / E\end{array}$ & $\begin{array}{r}---- \\
0\end{array}$ & ---- & --- & --- & $\begin{array}{r}--- \\
0\end{array}$ & ---- & $\begin{array}{r}---- \\
0\end{array}$ & --- \\
\hline $\begin{array}{l}\text { 1.1.1.2.04.05.08 } \\
\text { Level VI }\end{array}$ & $\begin{array}{l}\text { Data Definition Support } \\
\text { (PNL) } \\
\text { Total }\end{array}$ & $\begin{array}{l}O E \\
C / E\end{array}$ & ---- & ---- & -- & $\begin{array}{r}--- \\
0\end{array}$ & $\begin{array}{r}---- \\
0\end{array}$ & $\begin{array}{r}---- \\
0\end{array}$ & $\begin{array}{r}---- \\
0\end{array}$ & $-\cdots-$ \\
\hline $\begin{array}{l}\text { 1.1.1.2.04.05.09 } \\
\text { Level VI }\end{array}$ & $\begin{array}{l}\text { Historical Data } \\
\text { Development } \\
\\
\text { Total }\end{array}$ & $\begin{array}{l}O E \\
C / E\end{array}$ & ---- & $\begin{array}{r}0-- \\
0\end{array}$ & ---- & ---- & --- & ---- & $\begin{array}{r}--- \\
0\end{array}$ & -- \\
\hline
\end{tabular}

\section{[ R1]}


WHC-SP-1101

\section{NOTES \\ TOTAL PROGRAM ELEMENT COST BASELINE - BY YEAR}

The cost exhibit tables in each of the Program Element Baselines (Sections 7.2 through 7.10) identify the program element target funding by year by fund type. The tables also provide details of the baseline allocation of funding down to activity levels (level V) and cost account levels (level VI) of the WBS for Fiscal Year 1995 through 1997. The details exhibited on these tables are taken from the baseline WBS Dictionary Sheets. Deltas exist between the details and the annual target totals. For Fiscal Years 1996 and 1997 the deltas will be addressed in the future, following the completion of the resolution of expected differences between current Fiscal Year 1995 baseline funding and expected actual Fiscal Year 1995 funding. Deltas for Fiscal Year 1995 are noted below by program element:

\section{PROGRAM MANAGEMENT AND ADMINISTRATION:}

No deltas.

\section{TANK FARM OPERATIONS AND MAINTENANCE:}

No overall delta. This baseline requires revised fund type mix.

\section{WASTE TANK SAFETY:}

Delta is due to need to revise an overhead pricing rate in the P3 system.

\section{TANK FARM UPGRADES:}

Delta is due to differences between Budget Authority (BA) in the target and Budget Obligation (B0) in the $\mathrm{P} 3$ system.

\section{CHARACTERIZATION:}

No significant deltas.

\section{WASTE RETRIEVAL:}

Delta is primarily due to need to resolve issues associated with expense funding support to capital projects and differences between Budget Authority (BA) in the target and Budget Obligation (B0) in the $\mathrm{P} 3$ system.

\section{WASTE PRETREATMENT:}

Delta is due to the sum of the detail cost estimates being lower than the target total as a result of reestimation of resource requirements to complete baseline scope.

\section{LOW LEVEL WASTE:}

Delta is primarily due to carryover expense funded workscope on melter testing addressed in June 9, 1994 RL letter.

$$
T 7,6-1.17
$$




\section{HIGH LEVEL WASTE:}

Delta is due to carryover of capital funding, PNL expense funding carryover, and ADS corrections submitted but not reflected in target totals.

$$
T 7.6-1.18
$$


- Table 7.6-2. FY 1995 Cost by Month by Fund Type by Activity and Cost Account (\$K)

Future updates of the MYWP will include Table 7.6-2, which establishes the month-by-month cost baseline for FY 1995 for this program element. The FY 1995 cost baseline is exhibited at the cost account level with fund type totals provided. Cost data are then rolled up to the activity and program element levels. Also exhibited is the total cost baseline for the program element for FY-1995. At present, the program element cost baseline for FY 1995 for this program element is the total provided on this table less a productivity challenge for FY 1995. 
Table 7.6-3. Total Full-Time Equivalents by Year by Activity by Major Participant (Direct and Indirect) (Including all Subcontractors)

Future MYWP updates will include Table 7.6-3, which establishes total staffing projections for this program element. Included in the table are FY 1994 staffing data as well as projections by year for FY 1995 through FY 2000. The projections in this table include all direct and indirect Full-Time Equivalents (FTEs), including all subcontractor personnel. Where data are available, projections are exhibited at the activity level with major program participant totals provided. 
WHC-SP-1101

Table 7.6-4. FY 1995 and FY 1996 Direct Westinghouse Hanford Company Full-Time Equivalents by 88 Labor Categories

Future MYWP updates will include Table 7.6-4, which establishes direct WHC staffing projections for this program element. Projections do not include subcontractor support and are provided by 88 labor categories for FY 1995 through FY 1997. 


\subsection{WASTE RETRIEVAL BASELINE}

The Waste Retrieval (WR) Program Element baseline is described through the strategy, the work breakdown structure, the schedule and milestones to be achieved, and the resources required to perform the work. This document provides a focus on a 3-year planning period, FY 1995 through FY 1997.

\subsubsection{Technical Bases}

The program technical bases are derived from the TWRS Mission Analysis and the Functions and Requirements allocated to the function titled "Retrieve Waste."

\subsubsection{Mission Strategy}

\subsection{Objectives}

The WR Program's primary objectives are to support resolution of waste tank safety issues; remove waste from aging single-shell tanks (SSTs) and transfer to double-shell tanks (DSTs) for safe interim storage; mobilize sludge in DSTs for in-tank treatment or transfer; provide for the transfer of wastes for consolidation in DSTs or to pretreatment and immobilization facilities. The program will also provide for retrieval of miscellaneous underground storage tanks and small volumes of retrieved waste for pretreatment and immobilization development testing, and facilitate operational activities of retrieval equipment.

\subsection{Strategy}

The WR program strategy is to begin with known technical solutions; develop enhancements; develop new technologies to improve safety, environmental safeguards, and cost effectiveness; and implement enhancements and new technologies if they demonstrate significant improvements.

This strategy is realized through the use of known technologies such as mixer pumps. which have proven effectiveness in retrieving tank wastes at West Valley and Savannah River facilities, and through a sluicing process that has been successfully demonstrated at the Hanford Site from the 1950 's through the 1970's.

The program anticipates enhancements to both mixer pumps and the sluicing process in areas such as reduced exposures, extended equipment service life, and reuse capability.

The program works actively with the Office of Technology Development Program (EM-50) in areas such as robotics and the development of an arm-based retrieval system. Industry, academia, and national laboratories are involved in developing and evaluating alternatives to the reference retrieval systems.

\subsection{Functions and Requirements}

The WR program is assigned the functional responsibility for "Retrieve Waste" (4.2.1.3), as identified in the TWRS function hierarchy. 


\subsection{Risks}

The WR program has identified the following major areas of risk associated with meeting the program objectives as stated above.

The ability to resolve safety issues through the currently planned deployment of mixer pumps and/or sluicing equipment and the process of mixing through agitation and/or fluid addition may cause conditions that fall outside of the bounding safety limitations on any given tank. In this case, alternates to equipment other than reference systems may be required to be designed, fabricated, installed, and operated to effect retrieval of the waste and resolution of the safety issue.

The requirements for closure of SSTs are not developed at this time and the assumption that a single system will be adequate to retrieve an adequate amount of waste to meet closure requirements is a risk.

The ability to remove waste from SSTs using a single retrieval system carries a high level of uncertainty. While a high level of confidence exists in the capability of sluicing systems to dislodge and convey salt cake and sludges in the SSTs, other waste forms (i.e., solids, debris, and failed equipment) will require alternate means of retrieval. Robotics systems using mechanical arms with a variety of end effectors are currently being developed and tested for use in retrieval of SSTs. It is yet to be determined that this system will be capable of removing sufficient quantities of waste and waste forms to meet the undetermined closure requirements.

Some uncertainty exists in the capability of mixer pumps to adequately mobilize sludges and existing waste heels in DSTs. Simulant development and testing at a one-quarter scale may provide adequate assurance that the mixer pumps will prove successful. Questions regarding the adequacy of simulant use will most likely remain until actual full-scale operations are conducted.

There is a schedule requirement to complete retrieval of SSTs by 2018. Simultaneous deployment and operation of a number of retrieval systems will be necessary to meet this date. The uncertainty associated with the number of systems to be deployed at any given time will be resolved through the development and use of systems models and the process flow sheets.

\subsection{Major Decisions}

All major TWRS program decisions and a description of the tasks required to arrive at the decisions are identified in Appendix F, Major Decisions Logic/Schedule. The following four decisions are the responsibility of the WR program.

- 4.2.1.3 Determine Waste Mobilization (Retrieval) Strategy

- 4.2.1.4 Determine Waste Transfer Mechanism (partial - limited to transfer of tank wastes)

- 4.2.3 Determine Excess Facilities Interface Requirement

- 4.2.3.6 Determine Recycle/Reuse Concept.

The program has developed a detailed decision logic for the mobilization strategy which further identifies decisions, alternates, and studies required to reach the decisions. This work is included in the program element schedules. 


\subsection{Assumptions}

1. The resolution of safety issues will require waste retrieval from all Watch List tanks.

2. The retrieval program will meet the requirements of the Hanford Federal Facility and Consent Order (Tri-Party Agreement).

3. WR will be required for all SSTs and miscellaneous underground storage tanks.

4. Two mixer pumps will successfully mobilize all sludge in each DST.

5. Sluicing is the reference SST retrieval method; additional methods will be required to achieve Tri-Party Agreement objectives.

6. The Hanford Defense Waste Environmental Impact Statement (EIS) and Record of Decision provides sufficient National Environmental Policy Act (NEPA) documentation for the retrieval of DST waste.

7. An environmental assessment is sufficient NEPA documentation for retrieval of the first SST (241-C-106). The TWRS EIS will be necessary for retrieval of the remaining SSTs.

8. WR will perform all activities necessary to transfer DSTs, SSTs, and miscellaneous underground storage tanks to the Environmental Restoration (ER) Program (EM-40) for closure, including the preparation of a closure plan and EIS.

9. Subsurface barriers will be evaluated for use, but will not be included in the baseline work scope.

10. There is no requirement to provide tank waste samples for large-scale pretreatment and immobilization development. System(s) to provide small volumes of retrieved salt cake and sludges are included in the baseline.

Additional details may be found in activity planning forms.

\subsection{Constraints}

The WR program is constrained by the Tri-Party Agreement to meet certain milestones. Safety considerations regarding access to tanks and process restrictions provide a constraint. The program long-range plans are constrained by the lack of a TWRS dynamic model which, along with a current process flow sheet, will provide the bases for a retrieval sequence and provide information for the transport system. Precise knowledge of the content of each tank to be retrieved would permit an optimization of system design.

\subsubsection{Structure}

\subsection{Work Breakdown Structure}

The WR program is one of nine TWRS program elements. The program employs a matrix organization and attempts to close couple the organizational and work breakdown structures. The program has five basic elements: Program Management (Technical Integration and Planning); 
Technology Development and Applied Engineering; Facility Operations; System Definition; and Projects. The WR program element Work Breakdown Structure (WBS) is shown in Figure 7.7-1. A WBS dictionary is included as Appendix A.

\subsection{Organization and Responsibility}

The WR program is organized along four major functions: Programs, Engineering, Projects, and Operations. Responsibilities are assigned to various organizational elements, internal and external to TWRS, for discrete activities identified in the WBS. A responsibility assignment matrix (RAM) is developed to assist in the management and control of the work assigned to WR.

The program office provides direction to the performing organizations in the form of a WBS and a schedule. Each performing organization is responsible for developing a lower level WBS, schedules, and estimates for the work to be performed. Constraints are applied to the proposed schedules and estimates and the plans are established.

Technology Development for the WR program is largely performed by EM-50. The Technology Development Program Office (TDPO) is responsible for establishing the technical requirements for EM-50 activities that relate to WR and monitoring progress against those requirements. The TDPO will also manage the technology development work performed by EM-30 in the WR program.

\subsubsection{FY 1995 Work to be Performed}

\section{Technical Integration and Planning (WBS 1.1.1.2.05.01)}

The routine activities associated with planning and reporting will continue throughout FY 1995. Preparation of work plans and activity data sheets (ADSs) are included in the FY 1995 work scope. A significant portion of the work in FY 1994 was associated with the reporting to ad-hoc requirements and one-of-a-kind reports. These requirements deter from the preparation and development of performance measures and productivity measures, which are fundamental to the success of the program. A waste retrieval strategic plan was issued in draft form in 1994. This document includes the decision logic for program work and will be completed and issued in 1995. Systems Engineering studies at the program level (as identified in 7.7.1.1.5 above) will be completed.

\section{Technology Development and Applied Engineering (WBS 1.1.1.2.05.02)}

Conduct all activities necessary to develop and demonstrate methodologies to retrieve the waste contained in DSTs, SSTs, and the designated miscellaneous underground storage tanks in compliance with tank closure requirements. Focus of the efforts will be on developing fundamental relationships between waste properties and physical performance parameters of waste retrieval equipment/ processes. Identify physical parameters that characterize performance requirements for waste retrieval equipment and processes. Develop simulants that match the selected physical and/or chemical properties selected to characterize the performance of retrieval equipment/processes.

DST technologies in FY 1995 include continuing the test program to demonstrate mixer pump performance, including the ability to mobilize waste in DSTs and to maintain a uniform waste slurry mixture after the waste is mobilized; developing analytical models to provide the bases for predicting performance of waste retrieval equipment for various types of waste; and identifying and/or developing instrumentation systems necessary to monitor and control the retrieval operation. This encompasses defining requirements for retrieval of high-shear strength DST sludges and identifying 
candidate systems to retrieve the high-shear strength sludges, as well as candidate systems to improve the performance, cost, or operational efficiency over existing mixer pump-based systems, as part of the continuing effort to develop/test enhanced and alternate DST retrieval systems.

SST technologies in FY 1995 will identify, develop, and evaluate enhancements to the reference SST retrieval systems to proceed with the recovery of wastes from SSTs that present greater challenges than 241-C-106. The.need for sluicing enhancements will be evaluated to improve sluicing retrieval performance, reduce cost, and reduce tank leakage potential during sluicing operations. Activities to develop and evaluate the feasibility of arm-based retrieval technologies will be performed; these activities include deployment systems, control systems, sensor systems, and evaluation of software modeling packages. Development results will be incorporated into the W-340 project. Alternative retrieval technologies will be assessed by establishing a competitive development of system concepts through an industry fly off concept/development versus concepts established by onsite engineering, versus concepts from other the U.S. Department of Energy (DOE) laboratories and universities.

In FY 1995, an activity will identify the leak detection and monitoring system functions and requirements, evaluate the available technologies, and select the most promising technologies for engineering development/testing. It will also test the selected technologies to confirm their ability to meet the requirements and to provide engineering data needed to evaluate and select a technology and find a design for the leak detection and monitoring system through a series of case studies in which 241-C-106 will be the first case study.

For FY 1995, the principal tasks will be to assist the DOE and Washington State Department of Ecology review of the barrier feasibility assessment and reach a decision on whether or not to proceed with the barrier demonstration test. Once the decision is reached, planning is in place for the design, development, procurement, site modification, construction, installation, and performance testing of small-scale subsurface barriers in order to demonstrate the performance of barriers as a leak confinement system for SSTs. The preparation of test plans, safety analysis, environmental permitting, construction inspection, quality assurance, training, test data analysis, and documentation are also included in this work scope.

\section{Facility Operations (WBS 1.1.1.2.05.03)}

Facility operations will perform miscellaneous work under the project WBS.

\section{System Definition (WBS 1.1.1.2.05.04)}

FY 1995 activities include the following. Prepare engineering trade studies, assessments, project definition, and project-level functions and requirements for initial DST and SST retrieval systems designs and operation. Perform systems engineering assessments of needs and interfaces with other TWRS elements. Assess available technologies, and issue a trade study that provides the bases for the chosen technology. Prepare a test and evaluation plan that outlines the determination of needed testing during project design, construction, and startup. Prepare project documentation required to obtain Key Decision 0.

An activity will be performed to define the tank farm supporting infrastructure requirements necessary to support the retrieval mission and objectives. These requirements will be provided and integrated with the Tank Farms Upgrades Program. The requirements will include electrical power, lighting, compressed air, tank ventilation systems, waste transfer interfaces, and other systems needed to support WR. 
Additional activities planned include the definition and development of the SST retrieval systems training and test requirements to support retrieval of all 149 SSTs. A functions and requirements document will be prepared, facility options will be evaluated against the requirements, trade studies will be performed, and an engineering study will be completed to establish a recommended approach for the SST retrieval training and test facilities.

Also in FY 1995 are the starting activities necessary to define and establish the initial retrieval project. The scope includes the engineering trade studies necessary to bound project scope, establish functional requirements, and complete the engineering study and project documentation necessary to obtain Key Decision O. This project will provide the SST retrieval system maintenance and support retrieval operations for the first tank farm or equivalent number of tanks. This project(s) will provide the maintenance facilities, laydown/setup areas, and support buildings necessary to maintain/support SST retrieval systems for one of the 200 Areas (East or West). The resultant project will include a tank waste transfer vault to accumulate waste in batches prior to transferring the waste to DSTs. It also includes support systems that are necessary to remove the waste from the tanks and support the retrieval operations.

An analysis will be prepared of Federal and State regulations to identify issues requiring additional regulatory definition. Included would be regulations providing sufficient bases for categorizing residual waste in SSTs as to high-level waste, low-level waste, TRU, mixed, and hazardous; promulgation of disposal criteria for each applicable waste type; and definition of jurisdictional authority of Federal and State agencies. Informational exchanges with regulators will be supported, as required. This activity will likely be level of effort based, until issues are resolved.

\section{W-151 101-AZ Retrieval (WBS 1.1.1.2.05.05)}

The effort will continue to provide two 300-horsepower mixer pumps in tank 241-AZ-101 for the purposes of demonstrating sludge mobilization in Hanford's DSTs. This includes the following: project management; technology development specific to this project; conceptual design; definitive design; safety and environmental permitting analysis and documentation; procurement of equipment and materials; modifications to the tanks and their service systems to support installation and operation of the retrieval systems; installation of the retrieval systems; startup preparations, training, and testing; inspection; and quality assurance. Disposal of solid waste generated during the tank modifications and installation of the retrieval hardware is also included.

\section{W-211 Initial Retrieval System (WBS 1.1.1.2.05.00)}

This project provides retrieval systems for the retrieval of radioactive wastes stored in 10 underground DSTs. FY 1995 work will focus on the first phase, which includes tanks 241-SY-101 and 241-SY-103 only, and includes the following: project management; technology development specific to this project; conceptual design; definitive design; safety and environmental permitting analysis and documentation; procurement of equipment and materials; modifications to the tanks and their service systems to support installation and operation of the retrieval systems; installation of the retrieval systems; startup preparations, training, and testing; inspection; and quality assurance. Disposal of solid waste generated during the tank modifications and installation of the retrieval hardware is also included.

\section{Final DST Retrieval System (WBS 1.1.1.2.05.07)}

No work scope is planned for FY 1995. 


\section{W-315 Pilot Scale Retrieval (WBS 1.1.1.2.05.08)}

This activity provides the engineering studies; trade studies; and design, procurement, and testing of the small-volume retrieval systems (provide 25- to 50-liter samples of tank wastes). The scope of this project includes the following: project management; technology development specific to this project; conceptual design; definitive design; safety and environmental permitting analysis and documentation; procurement of equipment and materials; modifications to the tanks and their service systems to support installation and operation of the retrieval systems; installation of the retrieval systems; startup preparations, training, and testing; inspection; and quality assurance.

\section{W-320 241-C-106 Sluicing (WBS 1.1.1.2.05.09)}

Project W-320 will continue in FY 1995 to provide a water sluicing-based retrieval system to remove the high-heat generating sludge from tank $241-\mathrm{C}-106$ to resolve the associated tank safety issue. The equipment provided by this project will also demonstrate and verify the performance of high-volume waste sluicing systems for retrieval of SST waste. The scope of this project includes the following: project management; technology development specific to this project; definitive design; safety and environmental permitting analysis and documentation; procurement of equipment and materials; modifications to the tank and its service systems to support installation and operation of the sluicing retrieval system; installation of the retrieval system; startup preparations, training, and testing; inspection; and quality assurance. This project will also provide transfer lines as required to transport the retrieved wastes to a DST, as well as upgrades to the DST receipt tank as required to receive and store the retrieved sludge from 241-C-106. Disposal of solid waste generated during the tank modifications and installation of the retrieval hardware is also included.

\section{SST Training and Test Facility (WBS 1.1.1.2.05.10)}

All FY 1995 work scope consists of System Definition and is planned as part of WBS 1.1.1.2.05.04.

\section{W-340 Long Reach Arm (WBS 1.1.1.2.05.11)}

All FY 1995 work scope consists of System Definition and is planned as part of WBS 1.1.1.2.05.04.

\section{SST Closure Demonstration (WBS 1.1.1.2.05.12)}

All FY 1995 work scope consists of System Definition and is planned as part of WBS 1.1.1.2.05.04. Baseline effort will not initiate project until FY 1997.

\section{SST Closure (WBS 1.1.1.2.05.13)}

No activities are planned in the FY 1995-1997 planning window.

Miscellaneous Underground Storage Tanks Retrieval Systems (WBS 1.1.1.2.05.14)

No activities are planned in the FY 1995-1997 planning window. 


\subsubsection{FY 1996 - FY 1997 Work to be Performed}

The work scope for FY 1996-1997 continues the efforts to establish and implement technologies to mobilize and remove waste from the underground storage tanks (USTs) and to enable transport of the waste to various facilities for storage, treatment, or disposal. This includes conducting all activities necessary to develop and demonstrate methodologies to retrieve the waste; retrieving the waste contained in DSTs, SSTs, and the designated miscellaneous underground storage tanks in compliance with tank closure requirements; and designing, installing, and operating the equipment required to remove waste from the USTs. Also included are related upgrades and/or replacement of the tank farm infrastructure, and local transfer lines not provided by the Tank Farm Upgrades Program (WBS 1.1.1.2.03) when required to support retrieval.

\section{Technical Integration and Planning (WBS 1.1.1.2.05.01)}

The routine activities associated with planning and reporting will continue throughout FY 1996 and 1997. Preparation of work plans and ADSs are included in this work scope. Updates will be completed to the waste retrieval strategic plan, which will be issued each fiscal year and will identify the strategy to be employed by the systems engineering studies at the program level (as identified in 7.7.1.1.5 above).

\section{Technology Development and Applied Engineering (WBS 1.1.1.2.05.02)}

Activities will continue in FY 1996-1997 to complete development and demonstration of methodologies to retrieve the waste contained in DSTs, SSTs, and the designated miscellaneous underground storage tanks in compliance with tank closure requirements. With the formulation of fundamental relationships between waste properties and physical performance parameters of waste retrieval equipment/processes, finalization of equipment/processes will be done. Performance testing will be done with developed simulants which match the selected physical and/or chemical properties selected to characterize the performance of retrieval equipment/processes.

DST technologies in FY 1996-1997 include completing the test program to demonstrate mixer pump performance, including the ability to mobilize waste in DSTs and the ability to maintain a uniform waste slurry mixture after the waste is mobilized; developing analytical models to provide a bases for predicting performance of waste retrieval equipment for various types of waste; and developing instrumentation systems necessary to monitor and control the retrieval operation. This encompasses defining candidate systems to improve the performance, cost, or operational efficiency over existing mixer pump based systems, as part of the continuing effort to develop/test enhanced and alternate DST retrieval systems.

SST technologies in FY 1996-1997 will continue the identification, development, and evaluation of enhancements to the reference SST retrieval systems. With the FY 1995 needs assessment for sluicing, enhancements will formulate the bases for continued trade studies to assess methods to improve sluicing retrieval performance, reduce cost, and reduce tank leakage potential during sluicing

operations. Activities to develop and evaluate the feasibility of arm-based retrieval technologies will continue; these activities include deployment systems, control systems, sensor systems, and evaluation of software modeling packages. Development results will be incorporated into the proposed W-340 project. Alternative retrieval technologies will continue to be assessed. 
In FY 1996-1997 the leak detection and monitoring system evaluations into the appropriate and available technologies will continue, as well as the selections of the most promising technologies for engineering development/testing. Tests will be conducted to confirm a methods ability to meet the requirements and to provide engineering data needed to evaluate and select a technology and find a design for the leak detection and monitoring system through a series of case studies.

In FY 1996-1997 the principal tasks will be, should the decision be made to proceed with the barrier demonstration test, to start performance testing and complete for other technologies the design, development, procurement, site modification, construction, installation, and performance testing of small-scale subsurface barriers. A September 30, 1997, Tri-Party Agreement milestone, complete evaluation and demonstration testing of small scale subsurface barriers (M-45-07), will be met.

\section{Facility Operations (WBS 1.1.1.2.05.03)}

FY 1996-1997 activities will include the following. Prepare the test plan for the retrieval process test in tank 101-AZ. Determine the test objectives in relation to retrieval program needs, and develop test procedures to achieve the test objectives. Train the personnel that will perform the tests. Perform the operational readiness reviews and other activities necessary to support the testing activity. Correlate the test results with the results from the analytical model development and simulant testing results (development demonstration testing) to validate these programs. Assess the test results to identify additional model development and/or simulant testing necessary to extrapolate results to retrieval of other waste types.

Perform operations necessary to sluice the high-heat-generating sludge from tank 241-C-106. These operations are expected to resolve the high heat safety issue associated with this tank. Activities include preparing procedures and training operating personnel to perform the sluicing retrieval demonstration from tank 241-C-106, completing the operational readiness review, and performing operations and maintenance activities necessary to remove the high-heat sludge from the tank. This activity also includes removal of the retrieval system and decontamination of the hardware once the waste retrieval operations are completed. Disposal of solid waste generated during retrieval operations and maintenance is also included.

\section{System Definition (WBS 1.1.1.2.05.04)}

FY 1996-1997 work scope continues the engineering trade studies, assessments, project definition, and project-level functions and requirements for initial DST and SST retrieval systems designs and operation. Perform systems engineering assessments of needs and interfaces with other TWRS project elements. Complete the assessments of available technologies, and issue all trade studies that provide the bases for the chosen technology. Prepare a test and evaluation plan that outlines the determination of needed testing during project design, construction, and startup. . Prepare project documentation required to obtain Key Decision 0.

Complete the activity to define the tank farm supporting infrastructure requirements necessary to support the retrieval mission and objectives. Establishing the requirements will include electrical power, lighting, compressed air, tank ventilation systems, waste transfer interfaces, and other systems needed to support WR.

Additional activities planned include the definition and development of the SST retrieval systems training and test requirements to support retrieval of all 149 SSTs. From the functions and requirements document prepared in FY 1995, facility options will be evaluated against the 
requirements, trade studies will be performed, and an engineering study will be completed to establish a recommended approach for the SST Retrieval training and test facilities.

The activities necessary to define and establish the initial retrieval project will be completed. The scope includes the engineering trade studies necessary to bound project scope, establish functional requirements, and complete the engineering study and project documentation necessary to obtain Key

Decision O. This project will provide the SST retrieval system maintenance and support retrieval operations for the first tank farm or equivalent number of tanks. This project(s) will provide the maintenance facilities, laydown/setup areas, and support buildings necessary to maintain/support SST retrieval systems for one of the 200 Areas (East or West). The resultant project will include a tank waste transfer vault to accumulate waste in batches prior to transferring the waste to DSTs. It also includes support systems that are necessary to remove the waste from the tanks and support the retrieval operations.

Tank closure requirements assessment will be completed. The annual update to the initial SST Closure Plan and the subsequent annual update will be prepared. Planning will be initiated to conduct closure trade studies and alternatives. Work will be completed on the analysis of Federal and State regulations to identify issues requiring additional regulatory definition. Informational exchanges with regulators will be supported, as required. This activity will likely be level of effort-based, until issues are resolved.

\section{W-151 101-AZ Retrieval (WBS 1.1.1.2.05.05)}

Complete the effort to provide two 300-horsepower mixer pumps in tank 241-AZ-101 for the purposes of demonstrating sludge mobilization in Hanford's DSTs.

\section{W-211 Initial Retrieval System (WBS 1.1.1.2.05.06)}

This project will continue to provide retrieval systems for the retrieval of radioactive wastes stored in 10 underground DSTs, with retrieval system construction for tanks 241-SY-101 and 241-SY-103. The Title II Design for tank 241-AW-101 will start. FY 1996-1997 scope includes the following: project management; technology development specific to this project; conceptual design; definitive design; safety and environmental permitting analysis and documentation; procurement of equipment and materials; modifications to the tanks and their service systems to support installation and operation of the retrieval systems; installation of the retrieval systems; startup preparations, training, and testing; inspection; and quality assurance. Disposal of solid waste generated during the tank modifications and installation of the retrieval hardware is also included.

\section{Final DST Retrieval System (WBS 1.1.1.2.05.07)}

No work scope is planned for FY 1996-1997.

\section{W-315 Pilot Scale Retrieval (WBS 1.1.1.2.05.08)}

The engineering studies; trade studies; and design, procurement, and testing of the small volume retrieval systems (provide 25- to 50-liter samples of tank wastes) will be completed. FY 1996-1997 scope includes the following: project management; definitive design; safety and environmental permitting analysis and documentation; procurement of equipment and materials; modifications to the tanks and their service systems to support installation and operation of the retrieval systems;

installation of the retrieval systems; startup preparations, training, and testing; inspection; and quality assurance 


\section{W-320 241-C-106 Sluicing (WBS 1.1.1.2.05.09)}

Project W-320 will be completed in FY 1996-1997, which is to provide a water sluicing-based retrieval system to remove the high-heat generating sludge from tank 241-C-106 to resolve the associated tank safety issue. A demonstration will be performed, and the performance of high-volume waste sluicing systems for retrieval of SST waste will be verified. The modifications will be completed to the tank and its service systems to support installation and operation of the sluicing retrieval system; installation of the retrieval system; startup preparations, training, and testing; and inspection.

\section{SST Training and Test Facility (WBS 1.1.1.2.05.10)}

All FY 1996-1997 work scope consists of System Definition and is planned as part of WBS 1.1.1.2.05.04.

\section{W-340 Long Reach Arm (WBS 1.1.1.2.05.11)}

All FY 1996 work scope consists of System Definition and is planned as part of WBS 1.1.1.2.05.04.

FY 1997 work scope is to initiate the design of this project to demonstrate and verify the performance of long-reach manipulator systems for retrieval of SST waste. This project will provide the retrieval hardware and support systems necessary to remove the hard sludge heel that is expected to remain in tank 241-C-106 after completion of sluicing retrieval operations. The scope of this project includes the following: project management; technology development specific to this project; conceptual design; definitive design; safety and environmental permitting analysis and documentation; procurement of equipment and materials; modifications to the tank and its service systems to support installation and operation of the arm-based retrieval system; installation of the retrieval system; startup preparations, training, and testing; inspection; and quality assurance. This system is intended to demonstrate the capability to meet or exceed the Tri-Party Agreement criteria to remove a minimum of 99 percent of the waste from this tank. Disposal of solid waste generated during the tank modifications and installation of the retrieval hardware is also included.

\section{SST Closure Demonstration (WBS 1.1.1.2.05.12)}

FY 1997 work scope is to provide the systems necessary to retrieve the waste from all tanks in an SST farm or an equivalent number of tanks in multiple farms. This project will provide the retrieval hardware and support systems necessary to remove the waste from the tanks to a sufficient level to meet or exceed the 99 percent retrieval criteria specified in the Tri-Party Agreement. The scope of this project includes the following: project management; technology development specific to this project; conceptual design; definitive design; safety and environmental permitting analysis and documentation; procurement of equipment and materials; modifications to the tanks and their service systems to support installation and operation of the retrieval systems; installation of the retrieval systems; startup preparations, training, and testing; inspection; and quality assurance. Disposal of solid waste generated during the tank modifications and installation of the retrieval hardware is also included.

\section{SST Closure (WBS 1.1.1.2.05.13)}

No activities are planned in the FY $1995-1997$ planning window. 
WHC-SP-1101

Miscellaneous Underground Storage Tanks Retrieval Systems (WBS 1.1.1.2.05.14)

No activities are planned in the FY 1995-1997 planning window.

\subsubsection{Approach}

\section{Technical Integration and Planning (WBS 1.1.1.2.05.01)}

The management approach for the WR program is to maintain a focus on safety, work performance, and product quality. The program office will continue to develop and implement reporting methods that segregate accounting performance from work performance to allow accurate assessments of the work in progress and provide early indicators and forecasts. The systems engineering approach will continue, and outside contractor staff will be used as necessary to develop a clear product path from technology development through system definition to projects.

\section{Technology Development and Applied Engineering (WBS 1.1.1.2.05.02)}

There is an acute need to provide a proper balance between technology development and applied engineering. The application of decision-making tools like the program decision logic, functional flow diagrams, and interface control documents will address this need. Emphasis is placed on establishing and maintaining justification for new and continuing development activities. TWRS engineering staff will be product and outcome oriented. The TDPO or an equivalent program office representative will coordinate EM-50 activities, and the TWRS engineering staff will provide technical liaison for requirements and deliverables.

EM-50 will provide as much of the technology for waste retrieval as possible. Pacific Northwest Laboratories will continue their work on DST technology as long as products/projects can benefit from this work. Outside sources of technical expertise may be required from time to time, and these will be obtained through the TWRS Basic Ordering Agreement (BOA).

\section{Facility Operations (WBS 1.1.1.2.05.03)}

WR projects have established an approach to retrieval operations which will be further developed over the next few years. Current plans include the training and qualification of project resources in operational areas to provide coordination and direction of the operations work associated with retrieval projects. Long-range plans may include commercialization or privatization concepts where outside sources will provide operators for equipment and facilities. Near-term work will continue to be coordinated through projects and the integrated operations schedule.

\section{System Definition (WBS 1.1.1.2.05.04)}

In order to properly transition from technology development and applied engineering into systems definition, a set of agreed upon deliverables for handover must be established. The DOE order requirements for project definition will govern this work, and systems engineering products will be prepared to meet these requirements. TWRS engineering staff will perform this definition work and transfer to projects engineering with their products. Outside sources may be required and will be obtained through the TWRS BOA. The concept of competitive down selection of vendors will be applied to areas of SST alternate retrieval methods, advanced mixer pumps, and the arm-based retrieval system. Other program elements are also using this concept and an evaluation of its effectiveness will determine future use. 
Waste Retrieval Projects (WBS 1.1.1.2.05.05-13)

WR Projects will continue with the current approach of employing ICF Kaiser Engineers to design, procure, and manage the construction of the projects.

\subsubsection{Drivers and Deliverables}

WR drivers and deliverables are documented in the WBS Dictionary Sheets (Appendix A), Activity Planning Forms, and Milestone Description Sheets (Appendix D).

\subsubsection{Schedule}

\subsubsection{Waste Retrieval Summary Schedule}

Refer to Figure 7.7-2 for the WR Summary Schedule.

\subsubsection{Waste Retrieval Expanded Baseline (FY 1995 - FY 1997)}

The WR expanded baseline schedule in located in Appendix C.

\subsubsection{Resource Requirements}

\subsubsection{Cost}

\subsection{Bases of Cost Estimates}

Cost estimates for the WR program element represent a full spectrum of bases. Projects that have been validated have cost estimates commensurate with the design level of the project. Management and engineering tasks other than projects are estimated at various levels of confidence ranging from detailed bottoms up estimates to planning feasibility estimates.

\subsection{Total Program Element Cost by Year by Fund Type by Activity and Cost Account}

Table 7.7-1 establishes the baseline cost for this program element. The baseline cost is exhibited in annual costs for FY 1994 through FY 2000, and 5-year totals for FY 2001 through program element completion. Where the data are available, these baseline costs are exhibited at the cost account level with fund type totals provided. Cost data are then rolled up to the activity and program element levels. At present, the program element cost baseline is the program element cost totals exhibited on this table less a productivity challenge for FY 1995 through FY 1997.

\subsection{FY 1995 Cost by Month by Fund Type by Activity and Cost Account}

Future MYWP updates will include Table 7.7-2, which establishes the month-by-month cost baseline for FY 1995 for this program element. The FY 1995 cost baseline is exhibited at the cost account level with fund type totals provided. Cost data are then rolled up to the activity and program element levels. Also exhibited is the total cost baseline for the program element for FY 1995. At present, the program element cost baseline for FY 1995 for this program element is the total provided on this table less a productivity challenge for FY 1995. 
WHC-SP-1101

\subsubsection{Staffing}

\subsection{Bases of Staffing Projections}

\subsection{Total Full-Time Equivalents by Year by Activity by Major Participant (Direct and Indirect)}

Future MYWP updates will include Table 7.7-3, which establishes total staffing projections for this program element. Included in the table are FY 1994 staffing data as well as projections by year for FY 1995 through FY 2000. The projections in this table include all direct and indirect Full-Time Equivalents (FTEs), including all subcontractor personnel. Where data are available, projections are exhibited at the activity level with major program participant totals provided.

\subsection{FY 1995 through FY 1997 Direct Full-Time Equivalents by 88 Labor Categories}

Future MYWP updates will include Table 7.7-4, which establishes direct Westinghouse Hanford Company staffing projections for this program element. Projections do not include subcontractor support and are provided by 88 labor categories for FY 1995 through FY 1997.

\subsubsection{Work Scope Carryover}

The following work scope will be carried over from FY 1994 to FY 1995:

- Technology Development and Applied Engineering DSTs - $(\$ 300 \mathrm{~K})$ -pipe loop construction -mixer pump fluid jet behavior investigation $-1 / 12$ scale uniformity tests -simulant development

- Technology Development and Applied Engineering SSTs - (\$300K) -industry concept design spec -past practice sluicing reviews

- Technology Development and Applied Engineering Barriers - (\$1.5M) -site preparation -procurement -construction

- W-211 Initial Retrieval System Design - (\$200K) -permitting -site characterization -Clean Air Act estimate of emissions

- W-320 241-C-106 Sluicing Construction - (\$1.0M) -equipment removal -transfer line

- $\quad$ SST Test and Training Facility Engineering - (\$400K) -functions and requirements -architecture selection 
- W-340 Long Reach Arm Engineering - (\$400K)

-conceptual design

\subsubsection{Waste Type Data}

All waste that can be transported as a liquid will be done so. Solid waste will be packaged for storage and disposal and includes the large items of equipment from tanks 101-AZ, 102-AY, and 241-C-106 (pumps and various "In-Tank Hardware"), which may be remote handled hazardous waste that will be shielded as necessary to permit contact handled storage and disposal. All waste will be packaged in accordance with the Hanford Solid Waste Acceptance Criteria.

\subsubsection{Revisions}

In future updates of the MYWP, this section will include a summary of the revision that has occurred in this program element since the last issue of the MYWP. 
WHC-SP-1101

This page intentionally left blank.

$7.7-16$ 
Figure 7.7-1.

WASTE RETRIEVAL (PAGE 1 OF 2)

WORK BREAKDOWN STRUCTURE

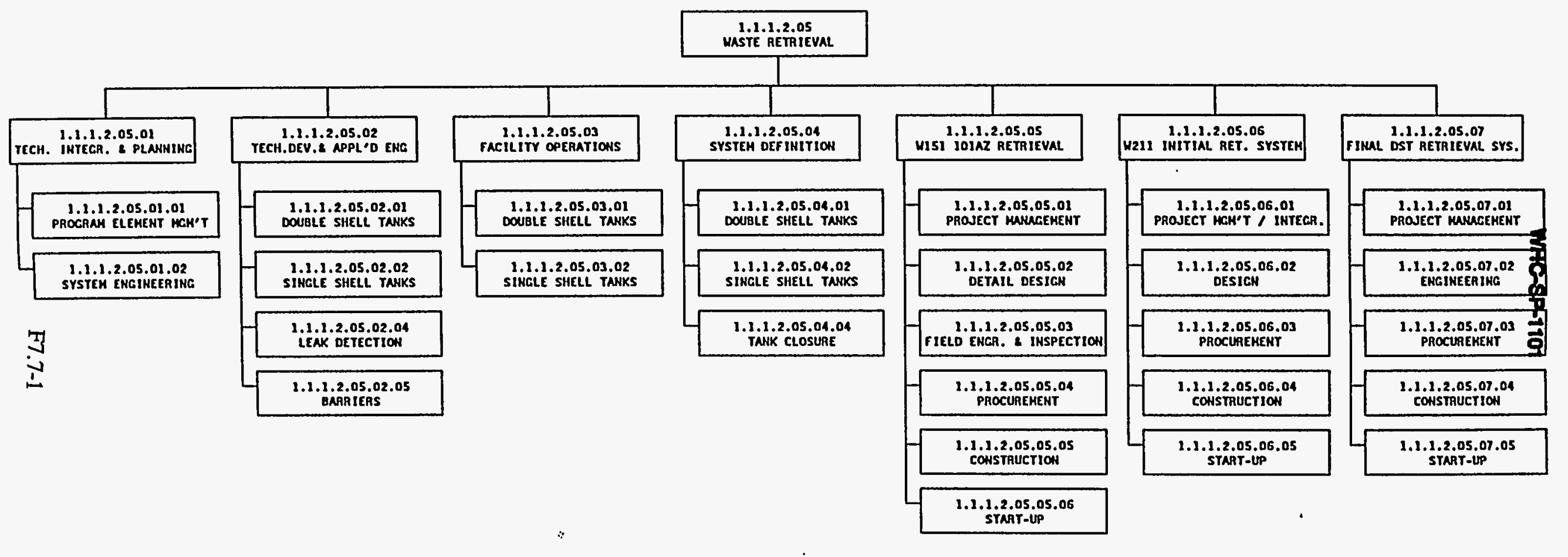


Figure 7.7-1.

WASTE RETRIEVAL (PAGE 2 OF 2)

WORK BREAKDOWN STRUCTURE

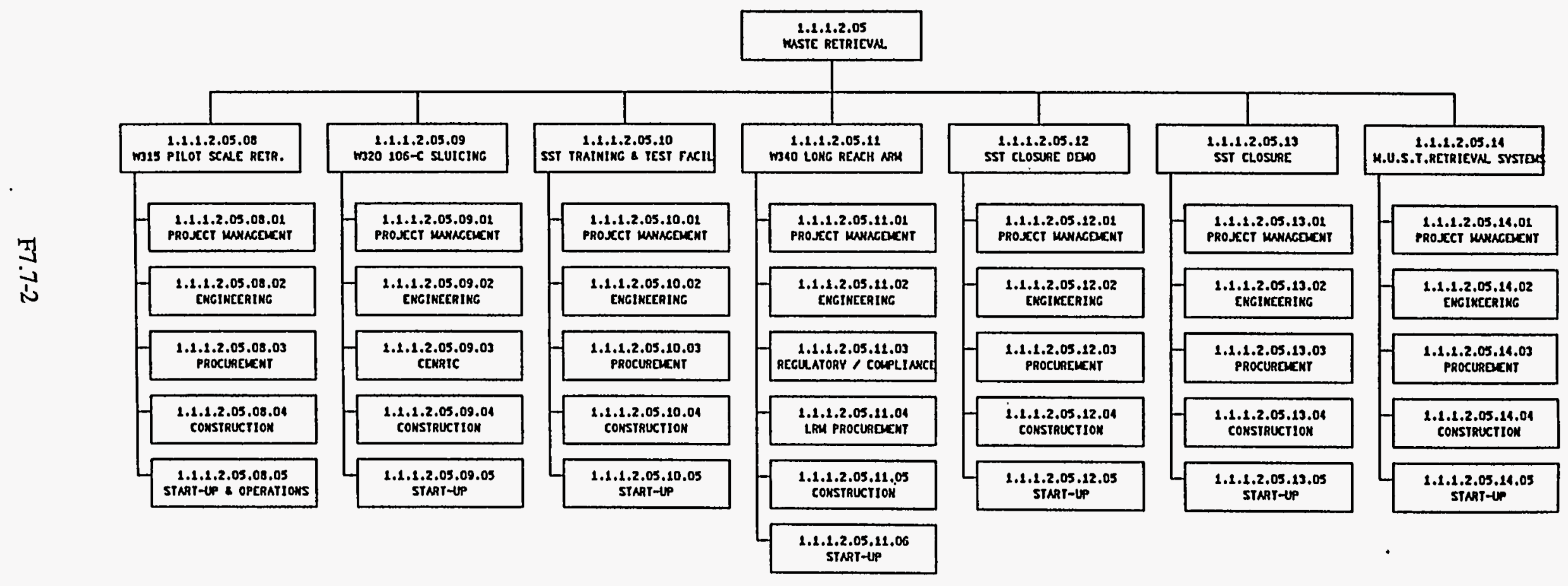


1.1.1.2.05 Waste Retrieval Summary Schedule

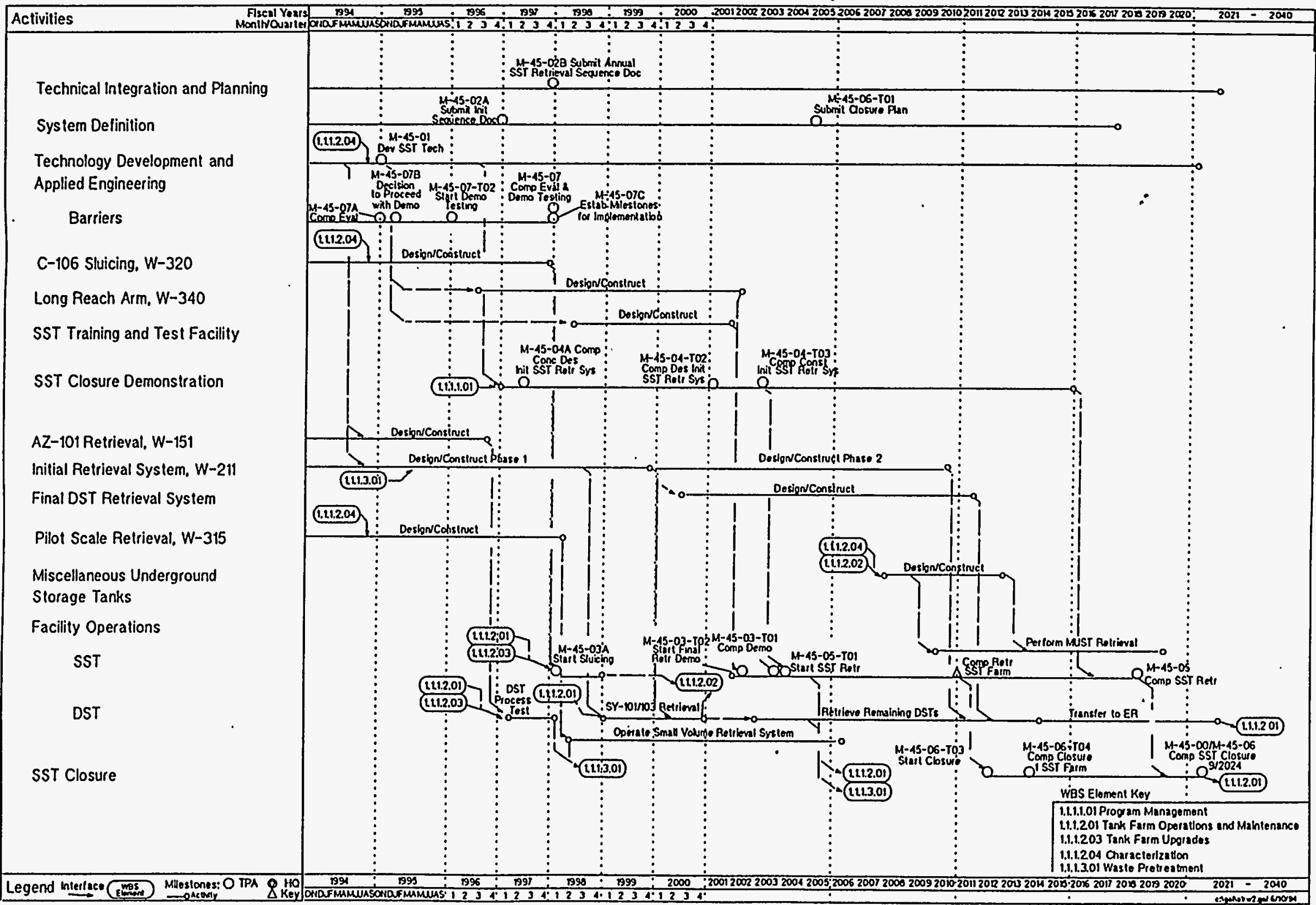


TABLE $7.7-1$

TOTAL PROGRAM ELEMENT COST BASELINE - BY YEAR

\begin{tabular}{|c|c|c|c|c|c|c|c|c|c|}
\hline WBS / LEVEL & ACTIVITY TITLE / ADS & $\begin{array}{l}\text { FUND } \\
\text { TYPE }\end{array}$ & $\begin{array}{c}\text { TOTAL } \\
\text { FY94 }\end{array}$ & FY95 & FY96 & FY97 & FY98 & FY99 & FYOO \\
\hline \multirow[t]{2}{*}{$\begin{array}{l}\text { 1.1.1.2.05 } \\
\text { Level iv }\end{array}$} & $\begin{array}{l}\text { Waste Retrieval } \\
\text { ADs 1210-0/23/4 } \\
\because \\
\because \text { TOTAL TARGET }\end{array}$ & \begin{tabular}{c} 
OE \\
$\mathbf{C / E}$ \\
$\because \mathbf{L I}$ \\
$\because \quad:$ \\
\hdashline
\end{tabular} & $\begin{array}{r}41,138 \\
3,200 \\
9,000 \\
53,338 \\
\end{array}$ & $\begin{array}{r}43,373 \\
3,525 \\
12,455 \\
-59,353 \\
\end{array}$ & $\begin{array}{r}42,049 \\
569 \\
17,543 \\
60,161 \\
61\end{array}$ & $\begin{array}{r}34,831 \\
34,700 \\
69,531\end{array}$ & $\begin{array}{r}66,006 \\
42,600 \\
108,606\end{array}$ & $\begin{array}{r}69,214 \\
44,700 \\
119,914\end{array}$ & $\begin{array}{r}103,238 \\
40 \\
42,900 \\
146,138\end{array}$ \\
\hline & $\begin{array}{l}\text { Delta to TARGET. } \\
\text { (See notes at end of Table.) } \\
\text { Total }\end{array}$ & $\begin{array}{l}O E \\
C / E \\
L I\end{array}$ & NA & $\begin{array}{c}(2,150) \\
191 \\
(2,994) \\
=== \\
(4,953)\end{array}$ & $\begin{array}{r}(3,238) \\
(387) \\
2,586 \\
==== \\
(1,039)\end{array}$ & $\begin{array}{c}1,475 \\
(474) \\
4,021 \\
==== \\
5,022\end{array}$ & & & \\
\hline $\begin{array}{l}\text { 1.1.1.2.05.01 } \\
\text { Level } \mathrm{V}\end{array}$ & $\begin{array}{l}\text { Technical Integration } \\
\frac{\text { and Planning }}{\text { ADS } 1210-0-O A} \\
\quad \text { Sub Total }\end{array}$ & $\begin{array}{l}O E \\
C / E\end{array}$ & NA & $\begin{array}{r}4,411 \\
320 \\
---- \\
4,731\end{array}$ & $\begin{array}{r}4,081 \\
320 \\
--- \\
4,401\end{array}$ & $\begin{array}{r}3,950 \\
320 \\
---- \\
4,270\end{array}$ & $\begin{array}{r}0 \\
-0 \\
-0\end{array}$ & $\begin{array}{r}\mathbf{0} \\
\mathbf{0} \\
--- \\
\mathbf{0}\end{array}$ & $\begin{array}{r}\mathbf{0} \\
\mathbf{0} \\
--- \\
\mathbf{0}\end{array}$ \\
\hline $\begin{array}{l}1.1 .1 .2 .05 .01 .01 \\
\text { Level VI }\end{array}$ & $\begin{array}{l}\text { Program Element } \\
\text { Management } \\
\qquad \text { Total }\end{array}$ & $\begin{array}{l}O E \\
C / E\end{array}$ & & $\begin{array}{r}3,420 \\
320 \\
---- \\
3,740\end{array}$ & $\begin{array}{r}3.440 \\
320 \\
---- \\
3.760\end{array}$ & $\begin{array}{r}3.437 \\
320 \\
--- \\
3.757\end{array}$ & $-\cdots$ & 0 & $---\frac{}{0}$ \\
\hline $\begin{array}{l}1.1 .1 .2 .05 .01 .02 \\
\text { Level VI }\end{array}$ & $\begin{array}{l}\text { Retrieval Systems } \\
\text { Engineering } \\
\end{array}$ & $\begin{array}{l}O E \\
C / E\end{array}$ & & $\begin{array}{r}991 \\
0 \\
--- \\
991\end{array}$ & $\begin{array}{r}641 \\
0 \\
--- \\
641\end{array}$ & $\begin{array}{r}513 \\
0 \\
--- \\
513\end{array}$ & $\begin{array}{r}---m \\
0\end{array}$ & --- & ---- \\
\hline
\end{tabular}

[ R1] 
TABLE $7.7-1$

TOTAL PROGRAM ELEMENT COST BASELINE - BY YEAR
[R1]

\begin{tabular}{|c|c|c|c|c|c|c|c|c|c|}
\hline WBS / LEVEL & ACTIVITY TITLE / ADS & $\begin{array}{l}\text { FUND } \\
\text { TYPE }\end{array}$ & $\begin{array}{l}\text { TOTAL } \\
\text { FY94 }\end{array}$ & FY95 & FY96 & FY97 & FY98 & FY99 & FY00 \\
\hline $\begin{array}{l}\text { 1.1.1.2.05.02 } \\
\text { Level } v\end{array}$ & $\begin{array}{l}\text { Tech. Development. and } \\
\frac{\text { Applied Engineering }}{A D S 1110-0-O B} \quad \text { Sub Total }\end{array}$ & $\begin{array}{l}\mathrm{OE} \\
\mathrm{C} / \mathrm{E}\end{array}$ & NA & $\begin{array}{r}8.471 \\
-184 \\
-8.655 \\
8.655\end{array}$ & $\begin{array}{r}19,756 \\
185 \\
---- \\
19,941\end{array}$ & $\begin{array}{r}6,911 \\
154 \\
---- \\
7,065\end{array}$ & $\begin{array}{r}0 \\
-0 \\
-0\end{array}$ & $-\frac{0}{0}$ & $\begin{array}{r}0 \\
0 \\
-0\end{array}$ \\
\hline $\begin{array}{l}\text { 1.1.1.2.05.02.01 } \\
\text { Level VI }\end{array}$ & $\begin{array}{l}\text { Double Shell Tanks } \\
\qquad \text { Total }\end{array}$ & $\begin{array}{l}O E \\
C / E\end{array}$ & & $\begin{array}{r}3,889 \\
184 \\
-1.07 \\
4,073\end{array}$ & $\begin{array}{r}3,002 \\
185 \\
--1 .- \\
3,187\end{array}$ & $\begin{array}{r}1,932 \\
154 \\
--1 . \\
2,086\end{array}$ & --- & -- & 0 \\
\hline $\begin{array}{l}\text { 1.1.1.2.05.02.02 } \\
\text { Level VI }\end{array}$ & $\begin{array}{l}\text { Single Shell Tanks } \\
\text { Total }\end{array}$ & $\begin{array}{l}O E \\
C / E\end{array}$ & & $\begin{array}{r}1,756 \\
-10 \\
-1.756\end{array}$ & $\begin{array}{r}3,548 \\
--0 \\
--1,548\end{array}$ & $\begin{array}{r}2,656 \\
--0 \\
-2,656\end{array}$ & - & --- & -- \\
\hline $\begin{array}{l}\text { 1.1.1.2.05.02.04 } \\
\text { Level VI }\end{array}$ & $\begin{array}{l}\text { Leak Detection } \\
\qquad \text { Total }\end{array}$ & $\begin{array}{l}O E \\
C / E\end{array}$ & & $\begin{array}{r}1,429 \\
-00 \\
--4 \\
1.429\end{array}$ & $\begin{array}{r}1,893 \\
-0 \\
--1 \\
1,893\end{array}$ & $\begin{array}{r}2,191 \\
-0 \\
--1 \\
2,191\end{array}$ & --- & -1 & $-\cdots$ \\
\hline $\begin{array}{l}\text { 1.1.1.2.05.02.05 } \\
\text { Level VI }\end{array}$ & Barriers & $\begin{array}{l}\mathrm{OE} \\
\mathrm{C} / \mathrm{E}\end{array}$ & & $\begin{array}{r}1,397 \\
-0 \\
-1,- \\
1,397\end{array}$ & $\begin{array}{r}11,313 \\
--0 \\
--- \\
11,313\end{array}$ & $\begin{array}{r}132 \\
-\quad 0 \\
-132\end{array}$ & --- & -- & - \\
\hline $\begin{array}{l}\text { 1.1.1.2.05.03 } \\
\text { Level } v\end{array}$ & $\begin{array}{l}\frac{\text { Facility Operations }}{\text { ADS } 1210-0-0 C} \\
\\
\text { Total }\end{array}$ & $\begin{array}{l}O E \\
C / E\end{array}$ & NA & $\begin{array}{r}0 \\
0 \\
-0\end{array}$ & $\begin{array}{r}0 \\
0 \\
-0 \\
0\end{array}$ & $\begin{array}{r}1,175 \\
0 \\
---- \\
1,175\end{array}$ & - & --- & $-\cdots$ \\
\hline $\begin{array}{l}\text { 1.1.1.2.05.03.01 } \\
\text { Level VI }\end{array}$ & $\begin{array}{l}\text { Double Shell Tanks } \\
\qquad \text { Total }\end{array}$ & $\begin{array}{l}\mathrm{OE} \\
\mathrm{C} / \mathrm{E}\end{array}$ & & - & $\begin{array}{r}0 \\
0 \\
-0 \\
\end{array}$ & $\begin{array}{r}1.144 \\
-0 \\
-1.14\end{array}$ & --- & -- & --- \\
\hline
\end{tabular}


TABLE $7.7-1$

\begin{tabular}{|c|c|c|c|c|c|c|c|c|c|}
\hline \multicolumn{3}{|c|}{ COST BASELINE - BY YEAR } & \multicolumn{7}{|c|}{ (K\$) } \\
\hline WBS / LEVEL & ACTIVITY TITLE / ADS & $\begin{array}{l}\text { FUND } \\
\text { TYPE }\end{array}$ & $\begin{array}{c}\text { TOTAL } \\
\text { FY94 }\end{array}$ & FY95 & FY96 & FY97 & FY98 & FY99 & FY00 \\
\hline $\begin{array}{l}1.1 .1 .2 .05 .03 .02 \\
\text { Level VI }\end{array}$ & $\begin{array}{l}\text { Single Shell Tanks } \\
\\
\text { Total }\end{array}$ & $\begin{array}{l}\mathrm{OE} \\
\mathrm{C} / \mathrm{E}\end{array}$ & & $\begin{array}{r}0 \\
0 \\
-- \\
-0\end{array}$ & $-\frac{0}{0}$ & $\begin{array}{r}31 \\
0 \\
--\frac{1}{31}\end{array}$ & $---\frac{-}{0}$ & $---\frac{-}{0}$ & $---\frac{-}{0}$ \\
\hline $\begin{array}{l}\text { 1.1.1.2.05.04 } \\
\text { Level } \mathrm{v}\end{array}$ & $\begin{array}{l}\frac{\text { System Definition }}{\text { ADS } 1210-0-O D} \\
\text { Sub Total }\end{array}$ & $\begin{array}{l}O E \\
C / E\end{array}$ & NA & $\begin{array}{r}3,150 \\
0 \\
---- \\
3,150\end{array}$ & $\begin{array}{r}2,813 \\
0 \\
---- \\
2,813\end{array}$ & $\begin{array}{r}1,854 \\
0 \\
---1,854\end{array}$ & $\begin{array}{r}\mathbf{0} \\
--\frac{0}{0}\end{array}$ & $\begin{array}{r}\mathbf{0} \\
---\frac{0}{0}\end{array}$ & $\begin{array}{r}0 \\
-- \\
0\end{array}$ \\
\hline $\begin{array}{l}\text { 1.1.1.2.05.04.01 } \\
\text { Level VI }\end{array}$ & $\begin{array}{l}\text { Double Shell Tanks } \\
\qquad \text { Total }\end{array}$ & $\begin{array}{l}\text { OE } \\
\text { C/E }\end{array}$ & & $\begin{array}{r}427 \\
0 \\
--- \\
-427\end{array}$ & $\begin{array}{r}357 \\
0 \\
--- \\
357\end{array}$ & $\begin{array}{r}0 \\
0 \\
-- \\
0\end{array}$ & $---\frac{-}{0}$ & --- & --- \\
\hline $\begin{array}{l}1.1 .1 .2 .05 .04 .02 \\
\text { Level VI }\end{array}$ & $\begin{array}{l}\text { Single Shell Tanks } \\
\\
\text { Total }\end{array}$ & $\begin{array}{l}\mathrm{OE} \\
\mathrm{C} / \mathrm{E}\end{array}$ & & $\begin{array}{r}2,318 \\
0 \\
-2,318\end{array}$ & $\begin{array}{r}2,049 \\
0 \\
---- \\
2,049\end{array}$ & $\begin{array}{r}1,447 \\
0 \\
---- \\
1,447\end{array}$ & --- & $-\cdots$ & --- \\
\hline $\begin{array}{l}1.1 .1 .2 .05 .04 .04 \\
\text { Level VI }\end{array}$ & Tank Closure & $\begin{array}{l}O E \\
C / E\end{array}$ & & $\begin{array}{r}405 \\
0 \\
--- \\
-405\end{array}$ & $\begin{array}{r}407 \\
0 \\
--- \\
-407\end{array}$ & $\begin{array}{r}407 \\
0 \\
--- \\
407\end{array}$ & --- & --- & --- \\
\hline
\end{tabular}


TABLE $7.7-1$

TOTAL PROGRAM ELEMENT COST BASELINE - BY YEAR

\begin{tabular}{|c|c|c|c|c|c|c|c|c|c|}
\hline WBS / LEVEL & ACTIVITY TITLE / ADS & $\begin{array}{l}\text { FUND } \\
\text { TYPE }\end{array}$ & $\begin{array}{l}\text { TOTAL } \\
\text { FY94 }\end{array}$ & FY95 & FY96 & FY97 & FY98 & FY99 & FYOO \\
\hline $\begin{array}{l}\text { 1.1.1.2.05.05 } \\
\text { Level } v\end{array}$ & $\begin{array}{r}\frac{W-151 \text { 101 AZ Retrieval }}{\text { ADS 1210-0-OE }} \\
\text { Sub Total }\end{array}$ & $\begin{array}{l}\text { OE } \\
\text { C/E } \\
\text { LI }\end{array}$ & NA & $\begin{array}{r}468 \\
2,230 \\
9,085 \\
--- \\
11,783\end{array}$ & $\begin{array}{r}114 \\
451 \\
776 \\
--1.341 \\
1.94\end{array}$ & $\begin{array}{r}\mathbf{0} \\
\mathbf{0} \\
\mathbf{0} \\
-0\end{array}$ & $\begin{array}{r}0 \\
0 \\
---\end{array}$ & $\begin{array}{r}0 \\
0 \\
--- \\
0\end{array}$ & $\begin{array}{r}0 \\
0 \\
---\end{array}$ \\
\hline $\begin{array}{l}\text { 1.1.1.2.05.05.01 } \\
\text { Level VI }\end{array}$ & $\begin{array}{l}\text { Project Management } \\
\qquad \text { Total }\end{array}$ & $\begin{array}{l}\text { OE } \\
C / E\end{array}$ & $\begin{aligned} \therefore & \because \\
& \because\end{aligned}$ & $\begin{array}{r}468 \\
0 \\
--- \\
468\end{array}$ & $\begin{array}{r}114 \\
0 \\
--- \\
114\end{array}$ & $\begin{array}{r}0 \\
0 \\
-0\end{array}$ & --- & 0 & $-\cdots$ \\
\hline $\begin{array}{l}\text { 1.1.1.2.05.05.02 } \\
\text { Level VI }\end{array}$ & $\begin{array}{l}\text { Detail Design } \\
\qquad \text { Total }\end{array}$ & $\begin{array}{l}O E \\
C / E\end{array}$ & & $\begin{array}{r}0 \\
0 \\
-- \\
0\end{array}$ & $\begin{array}{r}0 \\
0 \\
-0\end{array}$ & $\begin{array}{r}0 \\
0 \\
-- \\
0\end{array}$ & --- & --- & $-\cdots$ \\
\hline $\begin{array}{l}\text { 1.1.1.2.05.05.03 } \\
\text { Level VI }\end{array}$ & $\begin{array}{l}\text { Field Engineering and } \\
\text { Inspection } \\
\qquad \begin{array}{l}\text { Total }\end{array}\end{array}$ & $\begin{array}{l}\mathrm{OE} \\
\mathrm{LI}\end{array}$ & . & $\begin{array}{r}0 \\
1,332 \\
-1,332\end{array}$ & $\begin{array}{r}0 \\
325 \\
--- \\
325\end{array}$ & $\begin{array}{r}0 \\
0 \\
-- \\
-0\end{array}$ & $---\frac{-}{0}$ & $---\frac{-}{0}$ & $---\frac{-}{0}$ \\
\hline $\begin{array}{l}1.1 .1 .2 .05 .05 .04 \\
\text { Level VI }\end{array}$ & 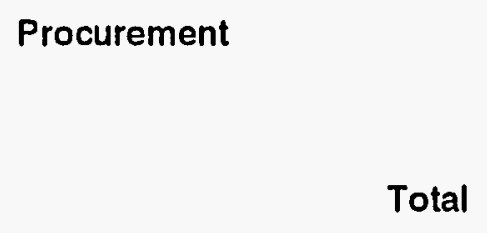 & $\begin{array}{l}\text { OE } \\
\text { C/E } \\
\text { LI }\end{array}$ & .. & $\begin{array}{r}0 \\
2,230 \\
3,624 \\
---- \\
5,854\end{array}$ & $\begin{array}{r}0 \\
0 \\
0 \\
-- \\
0\end{array}$ & $\begin{array}{r}0 \\
0 \\
0 \\
-0\end{array}$ & --- & 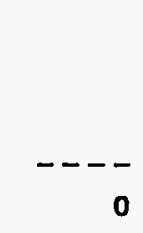 & $---\frac{-}{0}$ \\
\hline $\begin{array}{l}\text { 1.1.1.2.05.05.05 } \\
\text { Level VI }\end{array}$ & Total & $\begin{array}{l}\mathrm{OE} \\
\mathrm{LI}\end{array}$ & & $\begin{array}{r}0 \\
3,884 \\
--0- \\
3,884\end{array}$ & $\begin{array}{r}0 \\
42 \\
--2 \\
42\end{array}$ & $\begin{array}{r}0 \\
0 \\
-- \\
0\end{array}$ & $\begin{array}{r}0 \\
0 \\
-- \\
0\end{array}$ & $\begin{array}{r}0 \\
0 \\
-- \\
0\end{array}$ & $\begin{array}{r}0 \\
0 \\
-- \\
0\end{array}$ \\
\hline
\end{tabular}

\section{[ R1 ]}


TABLE 7.7-1

\section{[ R1 ]}

TOTAL PROGRAM ELEMENT

COST BASELINE - BY YEAR

COST BASELINE - BY YEA
(K\$)

\begin{tabular}{|c|c|c|c|c|c|c|c|c|c|}
\hline WBS / LEVEL & ACTIVITY TITLE / ADS & $\begin{array}{l}\text { FUND } \\
\text { TYPE }\end{array}$ & $\begin{array}{l}\text { TOTAL } \\
\text { FY94 }\end{array}$ & FY95 & FY96 & FY97 & FY98 & FY99 & FY00 \\
\hline $\begin{array}{l}\text { 1.1.1.2.05.05.06 } \\
\text { Level VI }\end{array}$ & 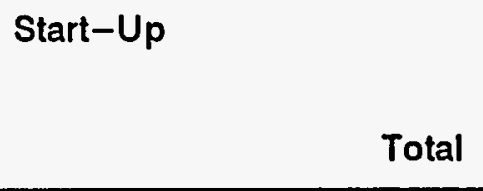 & $\begin{array}{l}\text { OE } \\
\text { LI }\end{array}$ & 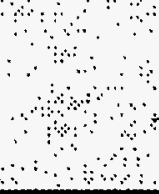 & $\begin{array}{r}0 \\
-245 \\
--- \\
245\end{array}$ & $\begin{array}{r}0 \\
409 \\
--2 \\
409\end{array}$ & $\begin{array}{r}0 \\
0 \\
---\frac{-}{0}\end{array}$ & $\begin{array}{r}---- \\
0\end{array}$ & --- & $---\frac{-}{0}$ \\
\hline $\begin{array}{l}\text { 1.1.1.2.05.06 } \\
\text { Level } \mathrm{V}\end{array}$ & $\begin{array}{l}\frac{W-211}{\text { Initial Retrieval }} \\
\frac{\text { System }}{\text { ADS } 1210-0-O F} \\
\end{array}$ & $\begin{array}{l}\text { OE } \\
\text { LI }\end{array}$ & 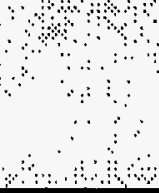 & $\begin{array}{r}1,863 \\
6,364 \\
---- \\
8,227\end{array}$ & $\begin{array}{r}1,660 \\
14,181 \\
\hdashline--15,841\end{array}$ & $\begin{array}{r}2.790 \\
27.679 \\
---- \\
30.409\end{array}$ & ---- & --- & $\begin{array}{r}--- \\
0\end{array}$ \\
\hline $\begin{array}{l}\text { 1.1.1.2.05.06.01 } \\
\text { Level VI }\end{array}$ & $\begin{array}{l}\text { Project Management } \\
\qquad \text { Total }\end{array}$ & $\begin{array}{l}\text { OE } \\
\text { LI }\end{array}$ & : & $\begin{array}{r}1,863 \\
2,124 \\
---- \\
3,987\end{array}$ & $\begin{array}{r}1,660 \\
1,040 \\
--0- \\
2.700\end{array}$ & $\begin{array}{r}2,235 \\
1,332 \\
---- \\
3,567\end{array}$ & $\begin{array}{r}---- \\
0\end{array}$ & $\begin{array}{r}---- \\
0\end{array}$ & $---\frac{-}{0}$ \\
\hline $\begin{array}{l}1.1 .1 .2 .05 .06 .02 \\
\text { Level VI }\end{array}$ & 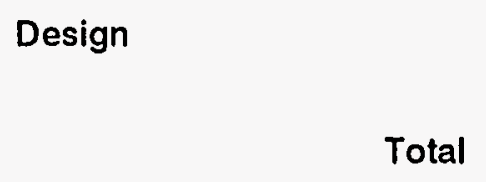 & $\begin{array}{l}\text { OE } \\
\text { LI }\end{array}$ & & $\begin{array}{r}0 \\
3,810 \\
---- \\
3,810\end{array}$ & $\begin{array}{r}0 \\
10,105 \\
---- \\
10,105\end{array}$ & $\begin{array}{r}0 \\
7,608 \\
---- \\
7,608\end{array}$ & $\begin{array}{r}---- \\
0\end{array}$ & $\begin{array}{r}---- \\
0\end{array}$ & --- \\
\hline $\begin{array}{l}1.1 .1 .2 .05 .06 .03 \\
\text { Level VI }\end{array}$ & $x^{2}$ & $\begin{array}{l}\text { OE } \\
\text { LI }\end{array}$ & & $\begin{array}{r}0 \\
430 \\
--- \\
430\end{array}$ & $\begin{array}{r}0 \\
3,036 \\
-0-- \\
3,036\end{array}$ & $\begin{array}{r}0 \\
3,573 \\
---- \\
3,573\end{array}$ & $\begin{array}{r}---- \\
0\end{array}$ & $\begin{array}{r}---- \\
0\end{array}$ & 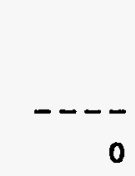 \\
\hline $\begin{array}{l}1.1 .1 .2 .05 .06 .04 \\
\text { Level VI }\end{array}$ & $x^{2}$ & $\begin{array}{l}\text { OE } \\
\text { LI }\end{array}$ & & $\begin{array}{r}0 \\
0 \\
---- \\
0\end{array}$ & $\begin{array}{r}0 \\
0 \\
--- \\
0\end{array}$ & $\begin{array}{r}0 \\
15,166 \\
---- \\
15,166\end{array}$ & $---\frac{-}{0}$ & $\begin{array}{r}---- \\
0\end{array}$ & $---\frac{}{0}$ \\
\hline $\begin{array}{l}\text { 1.1.1.2.05.06.05 } \\
\text { Level VI }\end{array}$ & Total & $\begin{array}{l}\text { OE } \\
C / E\end{array}$ & & $\begin{array}{r}0 \\
0 \\
--- \\
0\end{array}$ & $\begin{array}{r}0 \\
0 \\
-- \\
0\end{array}$ & $\begin{array}{r}495 \\
---- \\
495\end{array}$ & $\begin{array}{r}0 \\
--- \\
0\end{array}$ & $--\frac{0}{0}$ & $\begin{array}{r}0 \\
0 \\
-- \\
0\end{array}$ \\
\hline
\end{tabular}


TABLE $7.7-1$

TOTAL PROGRAM ELEMENT COST BASELINE - BY YEAR

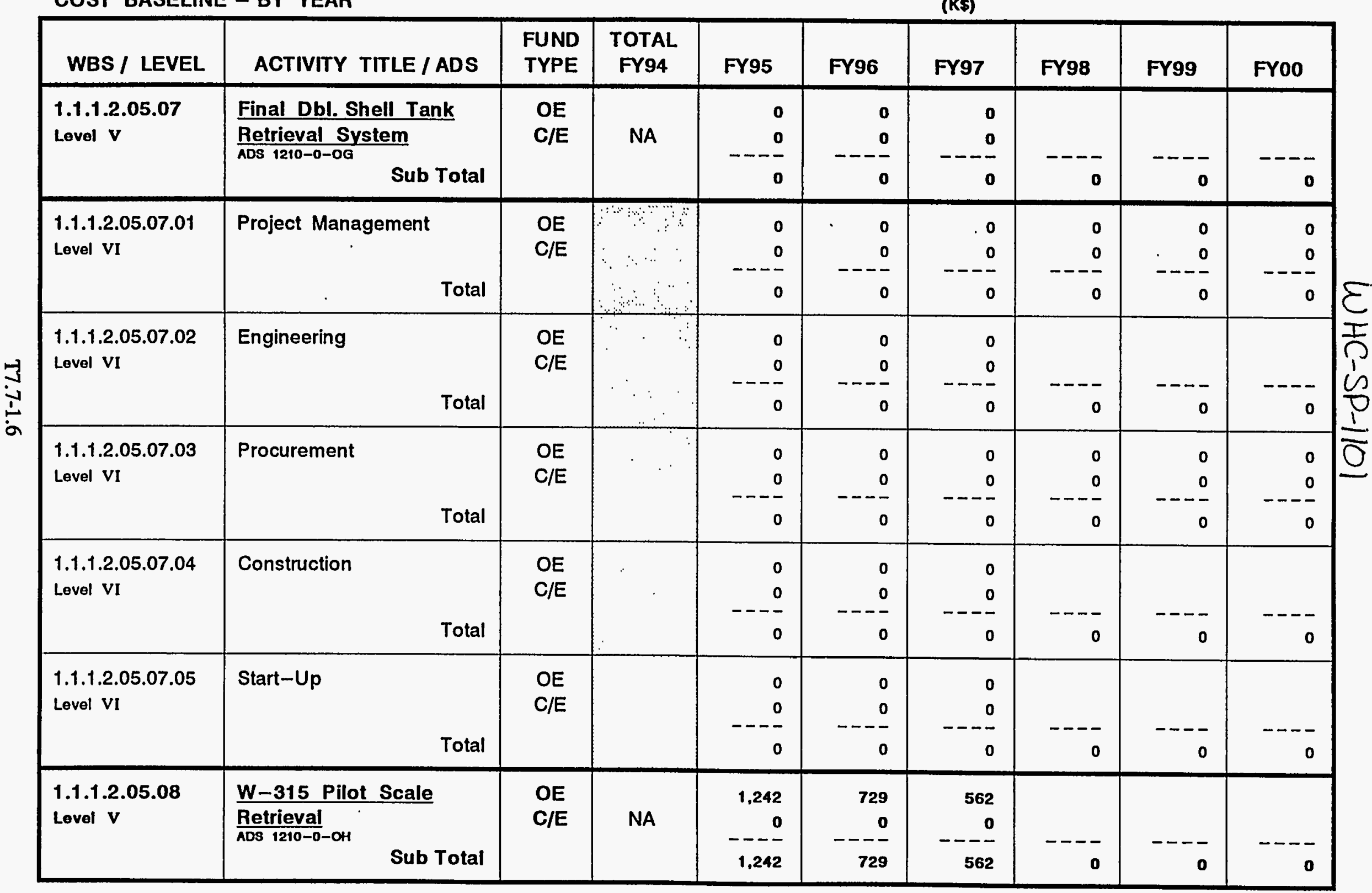

\section{[ R1 ]}


TABLE $\quad 7.7-1$

TOTAL PROGRAM ELEMENT COST BASELINE - BY YEAR

\begin{tabular}{|c|c|c|c|c|c|c|c|c|c|}
\hline WBS / LEVEL & ACTIVITY TITLE / ADS & $\begin{array}{l}\text { FUND } \\
\text { TYPE }\end{array}$ & $\begin{array}{l}\text { TOTAL } \\
\text { FY94 }\end{array}$ & FY95 & FY96 & FY97 & FY98 & FY99 & FYOO \\
\hline $\begin{array}{l}\text { 1.1.1.2.05.08.01 } \\
\text { Level VI }\end{array}$ & $\begin{array}{l}\text { Project Management } \\
\qquad \text { Total }\end{array}$ & $\begin{array}{l}O E \\
C / E\end{array}$ & NA & $\begin{array}{r}1,242 \\
0 \\
---242\end{array}$ & $\begin{array}{r}729 \\
0 \\
---- \\
729\end{array}$ & $\begin{array}{r}562 \\
0 \\
--- \\
562\end{array}$ & $\begin{array}{r}0 \\
0 \\
-0\end{array}$ & $\begin{array}{r}0 \\
0 \\
-0\end{array}$ & $\begin{array}{r}0 \\
0 \\
---\end{array}$ \\
\hline $\begin{array}{l}1.1 .1 .2 .05 .08 .02 \\
\text { Level V1 }\end{array}$ & 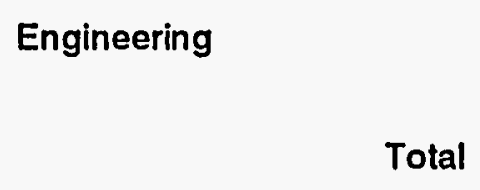 & $\begin{array}{l}O E \\
C / E\end{array}$ & $\because \because$ & $\begin{array}{r}0 \\
0 \\
-- \\
0\end{array}$ & $\begin{array}{r}0 \\
0 \\
-- \\
0\end{array}$ & $\begin{array}{r}0 \\
0 \\
-0\end{array}$ & --- & --- & $---\frac{-}{0}$ \\
\hline $\begin{array}{l}1.1 .1 .2 .05 .08 .03 \\
\text { Level VI }\end{array}$ & 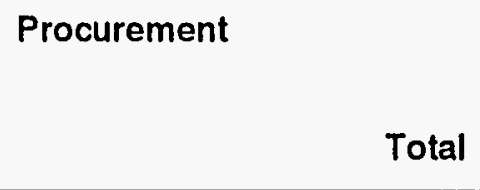 & $\begin{array}{l}O E \\
C / E\end{array}$ & $\mathrm{NA}$ & $\begin{array}{r}0 \\
0 \\
-- \\
0\end{array}$ & $\begin{array}{r}0 \\
0 \\
--- \\
0\end{array}$ & $\begin{array}{r}0 \\
0 \\
-0\end{array}$ & $---\overline{0}$ & $\cdots$ & $\cdots$ \\
\hline $\begin{array}{l}\text { 1.1.1.2.05.08.04 } \\
\text { Level VI }\end{array}$ & Construction & $\begin{array}{l}\mathrm{OE} \\
\mathrm{C} / \mathrm{E}\end{array}$ & $\mathrm{NA}$ & $\begin{array}{r}0 \\
0 \\
-- \\
0\end{array}$ & $\begin{array}{r}0 \\
0 \\
--- \\
0\end{array}$ & $\begin{array}{r}0 \\
0 \\
-- \\
0\end{array}$ & $--\frac{0}{0}-$ & $\begin{array}{r}0 \\
0 \\
-- \\
0\end{array}$ & -- \\
\hline $\begin{array}{l}\text { 1.1.1.2.05.08.05 } \\
\text { Level VI }\end{array}$ & $\begin{array}{l}\text { Start-Up Operations } \\
\qquad \text { Total }\end{array}$ & $\begin{array}{l}O E \\
C / E\end{array}$ & & $\begin{array}{r}0 \\
0 \\
-- \\
-0\end{array}$ & $\begin{array}{r}0 \\
---- \\
0\end{array}$ & $\begin{array}{r}0 \\
0 \\
-- \\
0\end{array}$ & $---\frac{-}{0}$ & --- & ---- \\
\hline $\begin{array}{l}1.1 .1 .2 .05 .09 \\
\text { Level } \mathrm{v}\end{array}$ & $\begin{array}{l}\text { W-320 106-C Sluicing } \\
\text { ADS } 1210-0-0 \text { Sub Total }\end{array}$ & $\begin{array}{l}\text { OE } \\
\text { C/E }\end{array}$ & NA & $\begin{array}{r}21,918 \\
600 \\
--- \\
22,518\end{array}$ & $\begin{array}{r}7,694 \\
0 \\
---- \\
7,694\end{array}$ & $\begin{array}{r}1.743 \\
0 \\
---\frac{1}{1.743}\end{array}$ & $---\frac{-}{0}$ & $---\frac{1}{0}$ & $--\frac{-}{0}$ \\
\hline $\begin{array}{l}1.1 .1 .2 .05 .09 .01 \\
\text { Level VI }\end{array}$ & $\begin{array}{l}\text { Project Management } \\
\qquad \text { Total }\end{array}$ & $\begin{array}{l}O E \\
C / E\end{array}$ & & $\begin{array}{r}2.142 \\
0 \\
---- \\
2.142\end{array}$ & $\begin{array}{r}1.122 \\
0 \\
---- \\
1.122\end{array}$ & $\begin{array}{r}620 \\
0 \\
--- \\
620\end{array}$ & $---\frac{-}{0}$ & $---\overline{0}$ & $---\frac{}{0}$ \\
\hline
\end{tabular}

\section{[ R1 ]}

$$
\text { LE } \quad 7.7-1
$$


TABLE $\quad 7.7-1$

TOTAL PROGRAM ELEMENT

COST BASELINE - BY YEAR

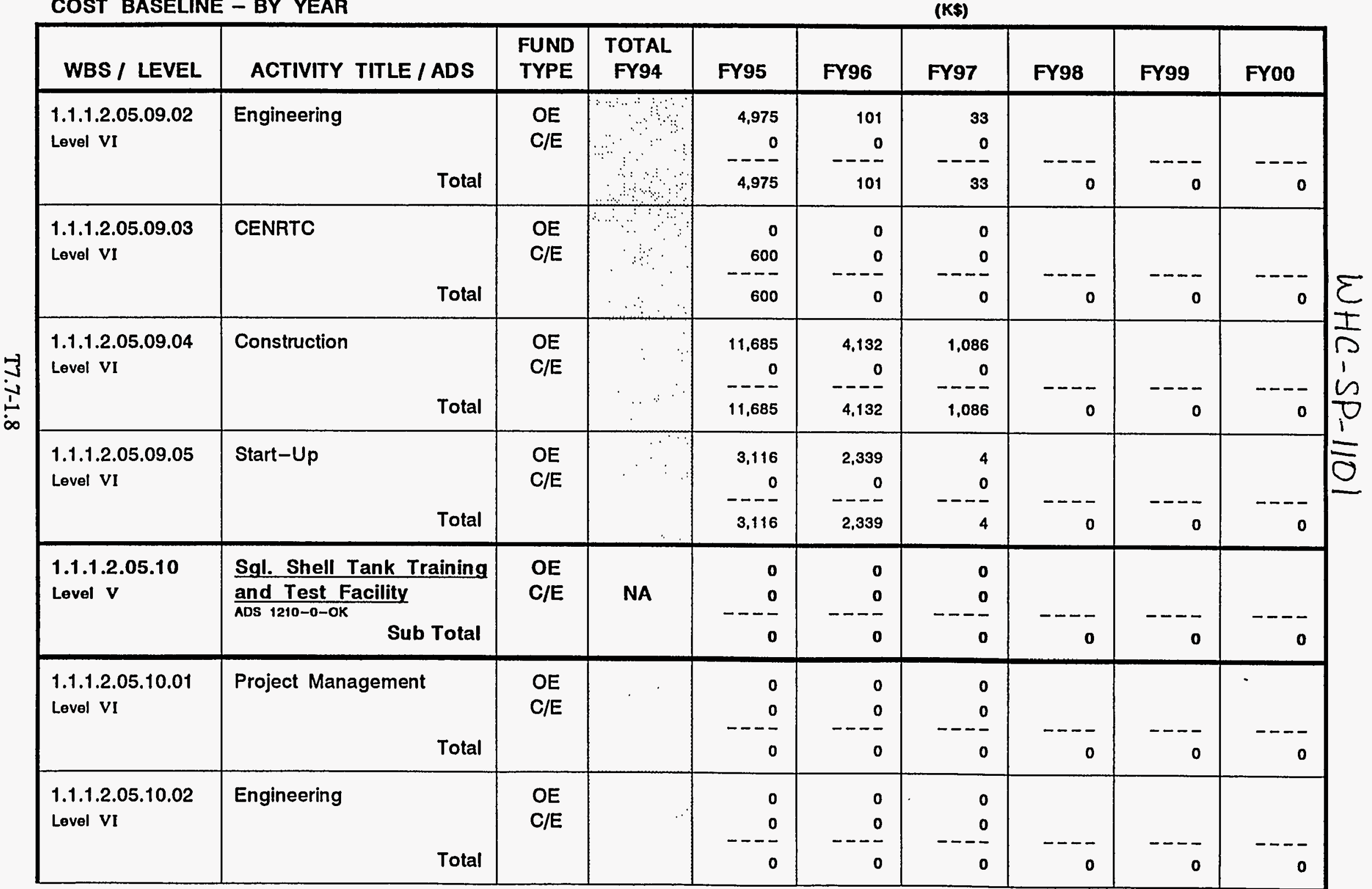

\section{[ R1 ]}


TABLE $\quad 7.7-1$

TOTAL PROGRAM ELEMENT

COST BASELINE - BY YEAR

\begin{tabular}{|c|c|c|c|c|c|c|c|c|c|}
\hline WBS / LEVEL & ACTIVITY TITLE / ADS & $\begin{array}{l}\text { FUND } \\
\text { TYPE }\end{array}$ & $\begin{array}{l}\text { TOTAL } \\
\text { FY94 }\end{array}$ & FY95 & FY96 & FY97 & FY98 & FY99 & FYOO \\
\hline $\begin{array}{l}1.1 .1 .2 .05 .10 .03 \\
\text { Level VI }\end{array}$ & $\begin{array}{l}\text { Procurement } \\
\qquad \text { Total }\end{array}$ & $\begin{array}{l}\text { OE } \\
\text { C/E }\end{array}$ & $\begin{array}{ll}\because & \ddots \\
\ddots & \therefore\end{array}$ & $\begin{array}{r}0 \\
0 \\
-0\end{array}$ & $\begin{array}{r}0 \\
0 \\
--\frac{1}{0}\end{array}$ & $\begin{array}{r}0 \\
0 \\
-- \\
0\end{array}$ & --- & --- & $---\frac{}{0}$ \\
\hline $\begin{array}{l}\text { 1.1.1.2.05.10.04 } \\
\text { Level VI }\end{array}$ & $\begin{array}{l}\text { Construction } \\
\qquad \begin{array}{r}\text { Total } \\
\text {. }\end{array}\end{array}$ & $\begin{array}{l}O E \\
C / E\end{array}$ & $\because \because$ & $\begin{array}{r}0 \\
0 \\
-0\end{array}$ & $\begin{array}{r}0 \\
0 \\
-0\end{array}$ & $\begin{array}{r}0 \\
0 \\
-- \\
0\end{array}$ & $-\cdots$ & $\therefore-$ & $---\frac{-}{0}$ \\
\hline $\begin{array}{l}\text { 1.1.1.2.05.10.05 } \\
\text { Level VI }\end{array}$ & Start-Up & $\begin{array}{l}O E \\
C / E\end{array}$ & & $\begin{array}{r}0 \\
0 \\
--\frac{1}{0}\end{array}$ & $\begin{array}{r}0 \\
0 \\
-0\end{array}$ & $--\frac{0}{0}$ & $--\frac{-}{0}$ & $-\cdots$ & $---\frac{-}{0}$ \\
\hline $\begin{array}{l}\text { 1.1.1.2.05.11 } \\
\text { Level } \mathrm{v}\end{array}$ & $\begin{array}{l}\frac{W-340 \text { Long Reach }}{\text { Arm }} \\
\begin{array}{ll}\text { ADS 1210-0-OL } & \text { Sub Total }\end{array}\end{array}$ & $\begin{array}{l}\text { OE } \\
\text { C/E } \\
\text { LI }\end{array}$ & NA & $\begin{array}{r}4,000 \\
0 \\
0 \\
--0 \\
4,000\end{array}$ & $\begin{array}{r}8,440 \\
0 \\
0 \\
8,440\end{array}$ & $\begin{array}{r}14,431 \\
0 \\
3,000 \\
---19 \\
17,431\end{array}$ & $--\frac{-}{0}$ & $---\frac{1}{0}$ & $---\frac{1}{0}$ \\
\hline $\begin{array}{l}1.1 .1 .2 .05 .11 .01 \\
\text { Level VI }\end{array}$ & $\begin{array}{l}\text { Project Management } \\
\qquad \text { Total }\end{array}$ & $\begin{array}{l}O E \\
C / E \\
L I\end{array}$ & & $\begin{array}{r}4,000 \\
0 \\
0 \\
--- \\
4,000\end{array}$ & $\begin{array}{r}8.440 \\
0 \\
0 \\
--- \\
8.440\end{array}$ & $\begin{array}{r}14,431 \\
0 \\
3,000 \\
-17,-4 \\
17,431\end{array}$ & --- & $-\cdots$ & $---\frac{}{0}$ \\
\hline $\begin{array}{l}\text { 1.1.1.2.05.11.02 } \\
\text { Level VI }\end{array}$ & Engineering & $\begin{array}{l}\text { OE } \\
\text { LI }\end{array}$ & & $\begin{array}{r}0 \\
0 \\
-- \\
-0\end{array}$ & $\begin{array}{r}0 \\
0 \\
-- \\
0\end{array}$ & $\begin{array}{r}0 \\
0 \\
-- \\
0\end{array}$ & $---\frac{-}{0}$ & $---\frac{-}{0}$ & $--\frac{-}{0}$ \\
\hline
\end{tabular}

\section{[ R1 ]}


TABLE 7.7-1

TOTAL PROGRAM ELEMENT COST BASELINE - BY YEAR

\begin{tabular}{|c|c|c|c|c|c|c|c|c|c|}
\hline WBS / LEVEL & ACTIVITY TITLE / ADS & $\begin{array}{l}\text { FUND } \\
\text { TYPE }\end{array}$ & $\begin{array}{l}\text { TOTAL } \\
\text { FY94 }\end{array}$ & FY95 & FY96 & FY97 & FY98 & FY99 & FY00 \\
\hline $\begin{array}{l}1.1 .1 .2 .05 .11 .03 \\
\text { Level VI }\end{array}$ & $\begin{array}{l}\text { Regulatory / Compliance } \\
\qquad \text { Total }\end{array}$ & $\begin{array}{l}O E \\
\text { C/E }\end{array}$ & $\therefore$ & $\begin{array}{r}0 \\
0 \\
-0\end{array}$ & $\begin{array}{r}0 \\
0 \\
-0\end{array}$ & $\begin{array}{r}0 \\
0 \\
-- \\
0\end{array}$ & $---\overline{0}$ & -- & $-\frac{-}{0}$ \\
\hline $\begin{array}{l}\text { 1.1.1.2.05.11.04 } \\
\text { Level VI }\end{array}$ & $\begin{array}{l}\text { LRM Procurement } \\
\qquad \text { Total }\end{array}$ & $\begin{array}{l}O E \\
C / E\end{array}$ & ' & $\begin{array}{r}0 \\
0 \\
--0\end{array}$ & $\begin{array}{r}0 \\
0 \\
-- \\
0\end{array}$ & $-\cdots$ & --- & $---\overline{0}$ & 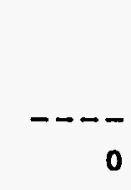 \\
\hline $\begin{array}{l}1.1 .1 .2 .05 .11 .05 \\
\text { Level VI }\end{array}$ & Construction & $\begin{array}{l}O E \\
C / E\end{array}$ & & $\begin{array}{r}0 \\
0 \\
-0\end{array}$ & $\begin{array}{r}0 \\
0 \\
-0\end{array}$ & $\begin{array}{r}0 \\
0 \\
-- \\
0\end{array}$ & $---\frac{-}{0}$ & $---\overline{0}$ & ---- \\
\hline $\begin{array}{l}1.1 .1 .2 .05 .11 .06 \\
\text { Level VI }\end{array}$ & Start-Up & $\begin{array}{l}O E \\
C / E\end{array}$ & & $\begin{array}{r}0 \\
0 \\
-- \\
-0\end{array}$ & $\begin{array}{r}0 \\
0 \\
--\frac{1}{0}\end{array}$ & $\begin{array}{r}0 \\
0 \\
-- \\
0\end{array}$ & $---\frac{}{0}$ & $--\frac{-}{0}$ & $---\frac{-}{0}$ \\
\hline $\begin{array}{l}\text { 1.1.1.2.05.12 } \\
\text { Level } v\end{array}$ & $\begin{array}{l}\frac{\text { Single-Shell Tank }}{\text { Closure Demo. }} \\
\frac{\text { ADS 1210-0-OM }}{\text { Sub Total }}\end{array}$ & $\begin{array}{l}\text { OE } \\
\text { C/E }\end{array}$ & NA & $--\frac{0}{0}$ & $\begin{array}{r}0 \\
0 \\
-0\end{array}$ & $\begin{array}{r}0 \\
0 \\
-0\end{array}$ & --- & $---\frac{-}{0}$ & $---\frac{-}{0}$ \\
\hline $\begin{array}{l}1.1 .1 .2 .05 .12 .01 \\
\text { Level VI }\end{array}$ & $\begin{array}{l}\text { Project Management } \\
\qquad \text { Total }\end{array}$ & $\begin{array}{l}O E \\
C / E\end{array}$ & & $\begin{array}{r}0 \\
0 \\
--\frac{-}{0}\end{array}$ & $\begin{array}{r}0 \\
0 \\
--\frac{1}{0}\end{array}$ & $\begin{array}{r}0 \\
0 \\
-- \\
0\end{array}$ & --- & --- & $---\overline{0}$ \\
\hline $\begin{array}{l}\text { 1.1.1.2.05.12.02 } \\
\text { Level VI }\end{array}$ & $\begin{array}{l}\text { Engineering } \\
\qquad \text { Total }\end{array}$ & $\begin{array}{l}O E \\
C / E\end{array}$ & & $\begin{array}{r}0 \\
0 \\
-0\end{array}$ & $\begin{array}{r}0 \\
-0 \\
-0\end{array}$ & $\begin{array}{r}0 \\
0 \\
-- \\
0\end{array}$ & ---- & $--\frac{-}{0}$ & $---\frac{-}{0}$ \\
\hline
\end{tabular}

\section{[ R1 ]}


TABLE $7.7-1$

TOTAL PROGRAM ELEMENT

COST BASELINE - BY YEAR

\begin{tabular}{|c|c|c|c|c|c|c|c|c|c|}
\hline WBS / LEVEL & ACTIVITY TITLE / ADS & $\begin{array}{l}\text { FUND } \\
\text { TYPE }\end{array}$ & $\begin{array}{l}\text { TOTAL } \\
\text { FY94 }\end{array}$ & FY95 & FY96 & FY97 & FY98 & FY99 & FYOO \\
\hline $\begin{array}{l}\text { 1.1.1.2.05.12.03 } \\
\text { Level VI }\end{array}$ & Total & $\begin{array}{l}O E \\
C / E\end{array}$ & $\begin{array}{c} \\
\because \\
\ddots\end{array}$ & $\begin{array}{r}0 \\
0 \\
-- \\
0\end{array}$ & $\begin{array}{r}0 \\
-- \\
0\end{array}$ & $--\frac{0}{0}$ & --- & $---\frac{-}{0}$ & --- \\
\hline $\begin{array}{l}1.1 .1 .2 .05 .12 .04 \\
\text { Level VI }\end{array}$ & $\begin{array}{l}\text { Construction } \\
\qquad \text { Total }\end{array}$ & $\begin{array}{l}\text { OE } \\
C / E\end{array}$ & $\because \because$ & $\begin{array}{r}0 \\
0 \\
-- \\
0\end{array}$ & $\begin{array}{r}0 \\
0 \\
-- \\
0\end{array}$ & $\begin{array}{r}0 \\
0 \\
-- \\
0\end{array}$ & --- & $---\overline{0}$ & $-\cdots$ \\
\hline $\begin{array}{l}\text { 1.1.1.2.05.12.05 } \\
\text { Level VI }\end{array}$ & $\begin{array}{l}\text { Start-Up } \\
\\
\text { Total }\end{array}$ & $\begin{array}{l}O E \\
C / E\end{array}$ & . & $\begin{array}{r}0 \\
0 \\
-- \\
0\end{array}$ & $\begin{array}{r}0 \\
0 \\
-- \\
0\end{array}$ & $\begin{array}{r}0 \\
0 \\
-0\end{array}$ & $-\cdots$ & $---\frac{-}{0}$ & $-\cdots$ \\
\hline $\begin{array}{l}\text { 1.1.1.2.05.13 } \\
\text { Level } \mathrm{V}\end{array}$ & 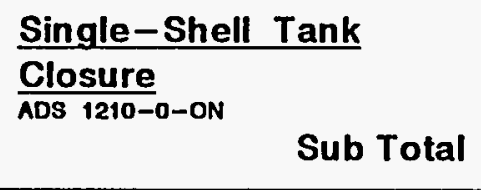 & $\begin{array}{l}\text { OE } \\
\text { C/E }\end{array}$ & NA & $\begin{array}{r}0 \\
--1 \\
0\end{array}$ & $\begin{array}{r}\mathbf{0} \\
--- \\
\mathbf{0}\end{array}$ & $\begin{array}{r}0 \\
0 \\
-0\end{array}$ & $---\overline{0}$ & $---\frac{-}{0}$ & $---\frac{-}{0}$ \\
\hline $\begin{array}{l}\text { 1.1.1.2.05.13.01 } \\
\text { Level VI }\end{array}$ & $\begin{array}{l}\text { Project Management } \\
\qquad \text { Total }\end{array}$ & $\begin{array}{l}O E \\
C / E\end{array}$ & & $\begin{array}{r}0 \\
0 \\
-- \\
0\end{array}$ & $\begin{array}{r}0 \\
0 \\
-- \\
0\end{array}$ & $\begin{array}{r}0 \\
0 \\
-- \\
0\end{array}$ & $---\overline{0}$ & --- & ---- \\
\hline $\begin{array}{l}\text { 1.1.1.2.05.13.02 } \\
\text { Level VI }\end{array}$ & Engineering & $\begin{array}{l}O E \\
C / E\end{array}$ & & $\begin{array}{r}0 \\
0 \\
-- \\
0\end{array}$ & $\begin{array}{r}0 \\
0 \\
-- \\
0\end{array}$ & $\begin{array}{r}0 \\
0 \\
-0\end{array}$ & --- & --- & ---- \\
\hline $\begin{array}{l}1.1 .1 .2 .05 .13 .03 \\
\text { Level VI }\end{array}$ & Procurement & $\begin{array}{l}O E \\
C / E\end{array}$ & & $\begin{array}{r}0 \\
0 \\
-0\end{array}$ & $\begin{array}{r}0 \\
0 \\
-0\end{array}$ & $--\frac{0}{0}$ & $---\frac{-}{0}$ & $---\frac{-}{0}$ & --- \\
\hline
\end{tabular}

\section{[ R1 ]}


TABLE $7.7-1$

TOTAL PROGRAM ELEMENT COST BASELINE - BY YEAR

\begin{tabular}{|c|c|c|c|c|c|c|c|c|c|}
\hline \multicolumn{3}{|c|}{ COST BASELINE - BY YEAR } & \multicolumn{7}{|c|}{ (K\$) } \\
\hline WBS / LEVEL & ACTIVITY TITLE / ADS & $\begin{array}{l}\text { FUND } \\
\text { TYPE }\end{array}$ & $\begin{array}{l}\text { TOTAL } \\
\text { FY94 }\end{array}$ & FY95 & FY96 & FY97 & FY98 & FY99 & FYOO \\
\hline $\begin{array}{l}\text { 1.1.1.2.05.13.04 } \\
\text { Level VI }\end{array}$ & Total & $\begin{array}{l}O E \\
C / E\end{array}$ & & $--\frac{0}{0}$ & $--\frac{0}{0}$ & $\begin{array}{r}0 \\
0 \\
-- \\
0\end{array}$ & -- & $-\cdots$ & --- \\
\hline $\begin{array}{l}1.1 .1 .2 .05 .13 .05 \\
\text { Level VI }\end{array}$ & $\begin{array}{l}\text { Start-Up } \\
\begin{array}{l}\text { Total } \\
\end{array}\end{array}$ & $\begin{array}{l}O E \\
C / E\end{array}$ & & $\begin{array}{r}0 \\
0 \\
-- \\
-0\end{array}$ & $\begin{array}{r}0 \\
0 \\
-- \\
0\end{array}$ & $\begin{array}{r}0 \\
0 \\
-- \\
0\end{array}$ & --- & --- & --- \\
\hline $\begin{array}{l}1.1 .1 .2 .05 .14 \\
\text { Level } \mathrm{v}\end{array}$ & $\begin{array}{l}\frac{\text { Misc. Underground }}{\text { Stor. Tnks. Retrieval Sys. }} \\
\frac{\text { ADS 1210-0-OP }}{\text { Sub Total }}\end{array}$ & $\begin{array}{l}\text { OE } \\
C / E\end{array}$ & NA & $\begin{array}{r}\mathbf{0} \\
--\frac{0}{0}\end{array}$ & $\begin{array}{r}0 \\
0 \\
-0\end{array}$ & $\begin{array}{r}\mathbf{0} \\
\mathbf{0} \\
--- \\
0\end{array}$ & $---\frac{-}{0}$ & $---\frac{-}{0}$ & $-\frac{-}{0}$ \\
\hline $\begin{array}{l}1.1 .1 .2 .05 .14 .01 \\
\text { Level VI }\end{array}$ & $\begin{array}{l}\text { Project Management } \\
\qquad \text { Total }\end{array}$ & $\begin{array}{l}\text { OE } \\
C / E\end{array}$ & & $\begin{array}{r}0 \\
0 \\
-- \\
-0\end{array}$ & $\begin{array}{r}0 \\
0 \\
--- \\
0\end{array}$ & $\begin{array}{r}0 \\
0 \\
-- \\
0\end{array}$ & $---\frac{-}{0}$ & --- & $---\overline{0}$ \\
\hline $\begin{array}{l}\text { 1.1.1.2.05.14.02 } \\
\text { Level VI }\end{array}$ & Engineering & $\begin{array}{l}O E \\
C / E\end{array}$ & & $\begin{array}{r}0 \\
0 \\
--- \\
0\end{array}$ & $\begin{array}{r}0 \\
0 \\
---\end{array}$ & $\begin{array}{r}0 \\
0 \\
--0\end{array}$ & --- & $---\frac{-}{0}$ & --- \\
\hline $\begin{array}{l}1.1 .1 .2 .05 .14 .03 \\
\text { Level VI }\end{array}$ & Procurement & $\begin{array}{l}O E \\
C / E\end{array}$ & & $\begin{array}{r}0 \\
0 \\
-0\end{array}$ & $\begin{array}{r}0 \\
0 \\
--- \\
0\end{array}$ & $\begin{array}{r}0 \\
0 \\
--- \\
0\end{array}$ & --- & $---\overline{0}$ & ---- \\
\hline
\end{tabular}

[ R1 ] 
TABLE 7.7-1

TOTAL PROGRAM ELEMENT

COST BASELINE - BY YEAR

[ R1 ]

\begin{tabular}{|c|c|c|c|c|c|c|c|c|c|}
\hline WBS / LEVEL & ACTIVITY TITLE / ADS & $\begin{array}{l}\text { FUND } \\
\text { TYPE }\end{array}$ & $\begin{array}{c}\text { TOTAL } \\
\text { FY94 }\end{array}$ & FY95 & FY96 & FY97 & FY98 & FY99 & FY00 \\
\hline $\begin{array}{l}\text { 1.1.1.2.05.14.04 } \\
\text { Level VI }\end{array}$ & 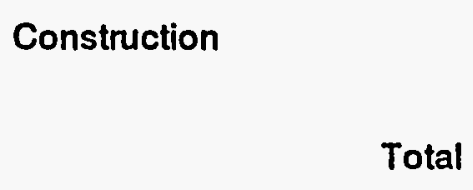 & $\begin{array}{l}\mathrm{OE} \\
\mathrm{C} / \mathrm{E}\end{array}$ & & $\begin{array}{r}0 \\
0 \\
-0\end{array}$ & $\begin{array}{r}0 \\
0 \\
-- \\
0\end{array}$ & $\begin{array}{r}0 \\
0 \\
-0 \\
0\end{array}$ & $---\frac{-}{0}$ & $---\overline{0}$ & --- \\
\hline $\begin{array}{l}\text { 1.1.1.2.05.14.05 } \\
\text { Level VI }\end{array}$ & Total & $\begin{array}{l}\mathrm{OE} \\
\mathrm{C} / \mathrm{E}\end{array}$ & $\begin{array}{rr} & \ddots \\
\vdots & \vdots\end{array}$ & $\begin{array}{r}0 \\
0 \\
-- \\
0\end{array}$ & $\begin{array}{r}0 \\
0 \\
-- \\
0\end{array}$ & $\begin{array}{r}0 \\
0 \\
-- \\
0\end{array}$ & $--\frac{-}{0}$ & $---\overline{0}$ & ---- \\
\hline
\end{tabular}


TABLE $7.7-1$

TOTAL PROGRAM ELEMENT COST BASELINE - BY YEAR

\begin{tabular}{|c|c|c|c|c|c|c|c|c|c|c|}
\hline WBS / LEVEL & ACTIVITY TITLE / ADS & $\begin{array}{l}\text { FUND } \\
\text { TYPE }\end{array}$ & $\begin{array}{l}\text { FY01 / } \\
\text { FY05 }\end{array}$ & $\begin{array}{l}\text { FY06 / } \\
\text { FY10 }\end{array}$ & $\begin{array}{l}\text { FY11 I } \\
\text { FY15 }\end{array}$ & $\begin{array}{l}\text { FY16 I } \\
\text { FY20 }\end{array}$ & $\begin{array}{l}\text { FY21 / } \\
\text { FY25 }\end{array}$ & $\begin{array}{l}\text { FY26 / } \\
\text { FY30 }\end{array}$ & $\begin{array}{l}\text { FY31 I } \\
\text { FY35 }\end{array}$ & $\begin{array}{l}\text { FY36 / } \\
\text { FY40 }\end{array}$ \\
\hline \multirow[t]{2}{*}{\begin{tabular}{l}
1.1 .2 .05 \\
Level iv \\
\hdashline \\
0
\end{tabular}} & 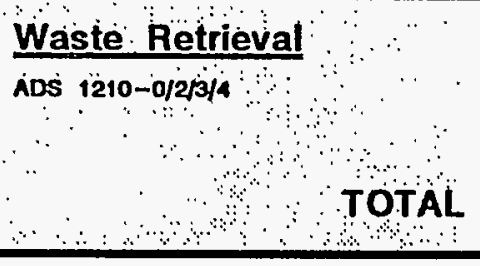 & $\begin{array}{l}\text { OË } \\
\mathbf{C} / \dot{E} \\
\ddots \dot{L I} \\
\ddots\end{array}$ & $\begin{array}{r}0 \\
0 \quad 0 \\
1,043,192 \\
0\end{array}$ & $\begin{array}{ll}\ddots 0 \\
\ddots 304.015 \\
\ddots 00\end{array}$ & 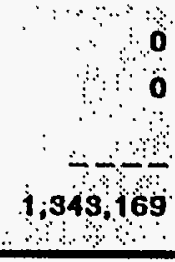 & $\begin{array}{r}0 \\
0 \\
859,027 \\
8\end{array}$ & $\begin{array}{r}0 \\
277,534 \\
\end{array}$ & $\begin{array}{r}0 \\
8 \\
8,180 \\
8\end{array}$ & & $\begin{array}{r}0 \\
0 \\
0 \\
0\end{array}$ \\
\hline & $\begin{array}{l}\text { Delta required to } \\
\text { Balance to TARGET } \\
\text { Budget. } \\
\text { Total }\end{array}$ & $\begin{array}{l}O E \\
C / E \\
L I\end{array}$ & & & & & & & & \\
\hline $\begin{array}{l}\text { 1.1.1.2.05.01 } \\
\text { Level } \mathrm{V}\end{array}$ & $\begin{array}{l}\text { Technical Integration } \\
\frac{\text { and Planning }}{\text { ADS } 1210-0-O A} \\
\text { Sub Total }\end{array}$ & $\begin{array}{l}\text { OE } \\
C / E\end{array}$ & $\begin{array}{r}\mathbf{0} \\
--- \\
0\end{array}$ & $\begin{array}{r}\mathbf{0} \\
-0 \\
-0 \\
0\end{array}$ & $\begin{array}{r}\mathbf{0} \\
\mathbf{0} \\
--- \\
\mathbf{0}\end{array}$ & $\begin{array}{r}\mathbf{0} \\
-0 \\
-0 \\
0\end{array}$ & $\begin{array}{r}0 \\
0 \\
-- \\
0\end{array}$ & $\begin{array}{r}\mathbf{0} \\
-0 \\
--0\end{array}$ & $\begin{array}{r}0 \\
0 \\
-- \\
-0\end{array}$ & $\begin{array}{r}0 \\
0 \\
-0\end{array}$ \\
\hline $\begin{array}{l}\text { 1.1.1.2.05.01.01 } \\
\text { Level VI }\end{array}$ & $\begin{array}{l}\text { Program Element } \\
\text { Management } \\
\qquad \text { Total }\end{array}$ & $\begin{array}{l}O E \\
C / E\end{array}$ & - & ---- & -- & $\overline{0}$ & - & $\begin{array}{r}--- \\
0\end{array}$ & $-\overline{0}$ & - \\
\hline $\begin{array}{l}\text { 1.1.1.2.05.01.02 } \\
\text { Level VI }\end{array}$ & $\begin{array}{l}\text { Retrieval Systems } \\
\text { Engineering }\end{array}$ & $\begin{array}{l}O E \\
C / E\end{array}$ & - & -- & & $\overline{0}$ & - & & 0 & - \\
\hline
\end{tabular}

\section{[R1]}


TABLE 7.7-1

TOTAL PROGRAM ELEMENT

COST BASELINE - BY YEAR

[ R1 ]

\begin{tabular}{|c|c|c|c|c|c|c|c|c|c|c|}
\hline WBS / LEVEL & ACTIVITY TITLE / ADS & $\begin{array}{l}\text { FUND } \\
\text { TYPE }\end{array}$ & $\begin{array}{l}\text { FY01 I } \\
\text { FY05 }\end{array}$ & $\begin{array}{l}\text { FY06 I } \\
\text { FY10 }\end{array}$ & $\begin{array}{l}\text { FY11 I } \\
\text { FY15 }\end{array}$ & $\begin{array}{l}\text { FY16 / } \\
\text { FY20 }\end{array}$ & $\begin{array}{l}\text { FY21 / } \\
\text { FY25 }\end{array}$ & $\begin{array}{l}\text { FY26 I } \\
\text { FY30 }\end{array}$ & $\begin{array}{l}\text { FY31 / } \\
\text { FY35 }\end{array}$ & $\begin{array}{l}\text { FY36 I } \\
\text { FY40 }\end{array}$ \\
\hline $\begin{array}{l}\text { 1.1.1.2.05.02 } \\
\text { Level } v\end{array}$ & 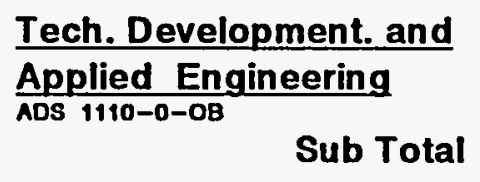 & $\begin{array}{l}O E \\
C / E\end{array}$ & $--\frac{\mathbf{0}}{\mathbf{0}}$ & $\begin{array}{r}\mathbf{0} \\
-- \\
-0\end{array}$ & $-\begin{array}{r}0 \\
0 \\
-0\end{array}$ & $\begin{array}{r}\mathbf{0} \\
-- \\
0\end{array}$ & $\begin{array}{r}\mathbf{0} \\
\mathbf{0} \\
-- \\
0\end{array}$ & $\begin{array}{r}0 \\
0 \\
-0\end{array}$ & $-\begin{array}{r}0 \\
0 \\
-0\end{array}$ & $\begin{array}{r}0 \\
--- \\
0\end{array}$ \\
\hline $\begin{array}{l}\text { 1.1.1.2.05.02.01 } \\
\text { Level VI }\end{array}$ & $\begin{array}{l}\text { Double Shell Tanks } \\
\qquad \text { Total }\end{array}$ & $\begin{array}{l}\text { OE } \\
\text { C/E }\end{array}$ & ---- & $---\frac{-}{0}$ & ---- & $\begin{array}{r}--- \\
0\end{array}$ & $\begin{array}{r}--- \\
0\end{array}$ & $\begin{array}{r}---- \\
0\end{array}$ & $---\frac{-}{0}$ & $-\cdots$ \\
\hline $\begin{array}{l}\text { 1.1.1.2.05.02.02 } \\
\text { Level VI }\end{array}$ & $\begin{array}{l}\text { Single Shell Tanks } \\
\qquad \text { Total }\end{array}$ & $\begin{array}{l}\text { OE } \\
\mathrm{C} / \mathrm{E}\end{array}$ & $-\cdots$ & -- & $\begin{array}{r}---- \\
0\end{array}$ & -- & $\cdots$ & -- & $\begin{array}{r}--- \\
0\end{array}$ & $---\frac{-}{0}$ \\
\hline $\begin{array}{l}\text { 1.1.1.2.05.02.04 } \\
\text { Level VI }\end{array}$ & $\begin{array}{l}\text { Leak Detection } \\
\qquad \text { Total }\end{array}$ & $\begin{array}{l}O E \\
C / E\end{array}$ & -- & -5 & ---- & -- & $---\frac{-}{0}$ & $\begin{array}{r}--- \\
0\end{array}$ & --- & --- \\
\hline $\begin{array}{l}1.1 .1 .2 .05 .02 .05 \\
\text { Level VI }\end{array}$ & Barriers & $\begin{array}{l}O E \\
C / E\end{array}$ & -- & -- & ---- & $-\cdots$ & $\begin{array}{r}--- \\
0\end{array}$ & -- & -- & --- \\
\hline $\begin{array}{l}\text { 1.1.1.2.05.03 } \\
\text { Level } v\end{array}$ & $\begin{aligned} \frac{\text { Facility Operations }}{\text { ADS } 1210-0-O C} & \\
& \text { Total }\end{aligned}$ & $\begin{array}{l}\text { OE } \\
\text { C/E }\end{array}$ & $---\frac{-}{0}$ & -- & ---- & -- & -- & $---\overline{0}$ & $---\frac{-}{0}$ & $---\frac{-}{0}$ \\
\hline $\begin{array}{l}\text { 1.1.1.2.05.03.01 } \\
\text { Level VI }\end{array}$ & $\begin{array}{l}\text { Double Shell Tanks } \\
\qquad \text { Total }\end{array}$ & $\begin{array}{l}O E \\
C / E\end{array}$ & -- & o & $---\frac{-}{0}$ & - & - & $---\frac{-}{0}$ & --- & --- \\
\hline
\end{tabular}


TABLE 7.7-1

TOTAL PROGRAM ELEMENT COST BASELINE - BY YEAR

\begin{tabular}{|c|c|c|c|c|c|c|c|c|c|c|}
\hline WBS / LEVEL & ACTIVITY TITLE / ADS & $\begin{array}{l}\text { FUND } \\
\text { TYPE }\end{array}$ & $\begin{array}{l}\text { FY01 / } \\
\text { FY05 }\end{array}$ & $\begin{array}{l}\text { FY06 I } \\
\text { FY10 }\end{array}$ & $\begin{array}{l}\text { FY11 I } \\
\text { FY15 }\end{array}$ & $\begin{array}{l}\text { FY16 I } \\
\text { FY20 }\end{array}$ & $\begin{array}{l}\text { FY21 / } \\
\text { FY25 }\end{array}$ & $\begin{array}{l}\text { FY26 / } \\
\text { FY30 }\end{array}$ & $\begin{array}{l}\text { FY31 / } \\
\text { FY35 }\end{array}$ & $\begin{array}{l}\text { FY36 I } \\
\text { FY40 }\end{array}$ \\
\hline $\begin{array}{l}1.1 .1 .2 .05 .03 .02 \\
\text { Level VI }\end{array}$ & $\begin{array}{l}\text { Single Shell Tanks } \\
\\
\text { Total }\end{array}$ & $\begin{array}{l}O E \\
C / E\end{array}$ & $---\frac{}{0}$ & $\begin{array}{r}--- \\
0\end{array}$ & --- & ---- & ---- & $---\frac{-}{0}$ & -- & --- \\
\hline $\begin{array}{l}\text { 1.1.1.2.05.04 } \\
\text { Level } \mathrm{V}\end{array}$ & $\begin{array}{l}\frac{\text { System Definition }}{\text { ADS } 1210-0-0 D} \\
\text { Sub Total }\end{array}$ & $\begin{array}{l}\text { OE } \\
\text { C/E }\end{array}$ & $\begin{array}{r}\mathbf{0} \\
-0 \\
-0\end{array}$ & $\begin{array}{r}\mathbf{0} \\
--- \\
\mathbf{0}\end{array}$ & $\begin{array}{r}0 \\
-0 \\
-0\end{array}$ & $\begin{array}{r}\mathbf{0} \\
-0 \\
-0\end{array}$ & $\begin{array}{r}\mathbf{0} \\
\mathbf{0} \\
-- \\
\mathbf{0}\end{array}$ & $-\frac{0}{0}$ & $\begin{array}{r}\mathbf{0} \\
-- \\
\mathbf{0}\end{array}$ & $\begin{array}{r}0 \\
--- \\
0\end{array}$ \\
\hline $\begin{array}{l}\text { 1.1.1.2.05.04.01 } \\
\text { Level VI }\end{array}$ & $\begin{array}{l}\text { Double Shell Tanks } \\
\qquad \text { Total }\end{array}$ & $\begin{array}{l}O E \\
C / E\end{array}$ & 0 & $---\frac{-}{0}$ & $---\frac{-}{0}$ & $---\frac{}{0}$ & $---\frac{1}{0}$ & $-\frac{-}{0}$ & $---\frac{-}{0}$ & --- \\
\hline $\begin{array}{l}\text { 1.1.1.2.05.04.02 } \\
\text { Level VI }\end{array}$ & Single Shell Tanks & $\begin{array}{l}O E \\
C / E\end{array}$ & $-\cdots$ & -- & 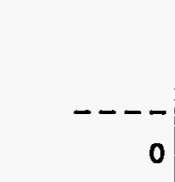 & $---\frac{-}{0}$ & $---\frac{-}{0}$ & $---\frac{-}{0}$ & $\begin{array}{r}--- \\
0\end{array}$ & --- \\
\hline $\begin{array}{l}\text { 1.1.1.2.05.04.04 } \\
\text { Level VI }\end{array}$ & Tank Closure & $\begin{array}{l}O E \\
C / E\end{array}$ & -- & -- & $---\frac{}{0}$ & ---- & ---- & $---\frac{-}{0}$ & $\begin{array}{r}---\overline{0} \\
0\end{array}$ & ---- \\
\hline
\end{tabular}

\section{[ R1]}$$
7.7-1
$$ 
TABLE 7.7-1

TOTAL PROGRAM ELEMENT COST BASELINE - BY YEAR

[ R1 ]

\begin{tabular}{|c|c|c|c|c|c|c|c|c|c|c|}
\hline WBS / LEVEL & ACTIVITY TITLE / ADS & $\begin{array}{l}\text { FUND } \\
\text { TYPE }\end{array}$ & $\begin{array}{l}\text { FY01 / } \\
\text { FY05 }\end{array}$ & $\begin{array}{l}\text { FY06 / } \\
\text { FY10 }\end{array}$ & $\begin{array}{l}\text { FY11 / } \\
\text { FY15 }\end{array}$ & $\begin{array}{l}\text { FY16 / } \\
\text { FY20 }\end{array}$ & $\begin{array}{l}\text { FY21 / } \\
\text { FY25 }\end{array}$ & $\begin{array}{l}\text { FY26 I } \\
\text { FY30 }\end{array}$ & $\begin{array}{l}\text { FY31 / } \\
\text { FY35 }\end{array}$ & $\begin{array}{l}\text { FY36 / } \\
\text { FY40 }\end{array}$ \\
\hline $\begin{array}{l}\text { 1.1.1.2.05.05 } \\
\text { Level } v\end{array}$ & $\begin{array}{r}\frac{W-151 \text { 101 AZ Retrieval }}{\text { ADS 1210-0-OE }} \\
\text { Sub Total }\end{array}$ & $\begin{array}{c}\text { OE } \\
\text { C/E } \\
\text { LI }\end{array}$ & $\begin{array}{r}\mathbf{0} \\
\mathbf{0} \\
-- \\
-\mathbf{0}\end{array}$ & $\begin{array}{r}\mathbf{0} \\
\mathbf{0} \\
--- \\
\mathbf{0}\end{array}$ & $\begin{array}{r}0 \\
0 \\
-- \\
0\end{array}$ & $\begin{array}{r}0 \\
0 \\
--- \\
0\end{array}$ & $\begin{array}{r}0 \\
0 \\
-- \\
0\end{array}$ & $\begin{array}{r}0 \\
0 \\
-0 \\
0\end{array}$ & $\begin{array}{r}\mathbf{0} \\
\mathbf{0} \\
-0 \\
-0\end{array}$ & $\begin{array}{r}0 \\
0 \\
--- \\
0\end{array}$ \\
\hline $\begin{array}{l}\text { 1.1.1.2.05.05.01 } \\
\text { Level VI }\end{array}$ & $\begin{array}{l}\text { Project Management } \\
\qquad \text { Total }\end{array}$ & $\begin{array}{l}O E \\
C / E\end{array}$ & $---\frac{-}{0}$ & $-\cdots$ & ---- & --- & --- & $---\frac{-}{0}$ & -- & $-\cdots$ \\
\hline $\begin{array}{l}\text { 1.1.1.2.05.05.02 } \\
\text { Level VI }\end{array}$ & $\begin{array}{l}\text { Detail Design } \\
\begin{array}{c}\text { Total } \\
\text {. }\end{array}\end{array}$ & $\begin{array}{l}O E \\
C / E\end{array}$ & $---\frac{-}{0}$ & --- & ---- & --- & $---\frac{-}{0}$ & -- & -- & $--\frac{-}{0}$ \\
\hline $\begin{array}{l}\text { 1.1.1.2.05.05.03 } \\
\text { Level VI }\end{array}$ & $\begin{array}{l}\text { Field Engineering and } \\
\text { Inspection } \\
\qquad \begin{array}{l}\text { Total }\end{array}\end{array}$ & $\begin{array}{l}\text { OE } \\
\text { LI }\end{array}$ & ---- & -- & $\begin{array}{r}---- \\
0\end{array}$ & -- & -- & $---\frac{-}{0}$ & -- & --- \\
\hline $\begin{array}{l}1.1 .1 .2 .05 .05 .04 \\
\text { Level VI }\end{array}$ & 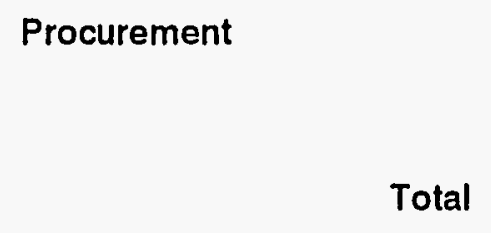 & $\begin{array}{l}\text { OE } \\
\text { C/E } \\
\text { LI }\end{array}$ & - & -- & ---- & 0 & -0 & --- & $-\cdots$ & 0 \\
\hline $\begin{array}{l}\text { 1.1.1.2.05.05.05 } \\
\text { Level VI }\end{array}$ & Total & $\begin{array}{l}\mathrm{OE} \\
\mathrm{LI}\end{array}$ & $\begin{array}{r}0 \\
0 \\
-- \\
0\end{array}$ & $\begin{array}{r}0 \\
0 \\
-- \\
0\end{array}$ & $\begin{array}{r}0 \\
0 \\
-- \\
0\end{array}$ & $\begin{array}{r}0 \\
0 \\
-- \\
0\end{array}$ & $\begin{array}{r}0 \\
0 \\
-- \\
0\end{array}$ & $\begin{array}{r}0 \\
0 \\
-- \\
0\end{array}$ & $\begin{array}{r}0 \\
0 \\
-- \\
0\end{array}$ & $\begin{array}{r}0 \\
0 \\
-- \\
0\end{array}$ \\
\hline
\end{tabular}


TABLE 7.7-1

TOTAL PROGRAM ELEMENT COST BASELINE - BY YEAR

\begin{tabular}{|c|c|c|c|c|c|c|c|c|c|c|}
\hline WBS / LEVEL & ACTIVITY TITLE / ADS & $\begin{array}{l}\text { FUND } \\
\text { TYPE }\end{array}$ & $\begin{array}{l}\text { FY01 / } \\
\text { FY05 }\end{array}$ & $\begin{array}{l}\text { FY06 / } \\
\text { FY10 }\end{array}$ & $\begin{array}{l}\text { FY11 I } \\
\text { FY15 }\end{array}$ & $\begin{array}{l}\text { FY16 / } \\
\text { FY20 }\end{array}$ & $\begin{array}{l}\text { FY21 / } \\
\text { FY25 }\end{array}$ & $\begin{array}{l}\text { FY26 / } \\
\text { FY30 }\end{array}$ & $\begin{array}{l}\text { FY31 / } \\
\text { FY35 }\end{array}$ & $\begin{array}{l}\text { FY36 I } \\
\text { FY40 }\end{array}$ \\
\hline $\begin{array}{l}\text { 1.1.1.2.05.05.06 } \\
\text { Level VI }\end{array}$ & $\begin{array}{l}\text { Start-Up } \\
\text { Total }\end{array}$ & $\begin{array}{l}\mathrm{OE} \\
\mathrm{LI}\end{array}$ & --- & ---- & $-\cdots--$ & --- & $-\cdots-$ & $-\cdots$ & ---- & --- \\
\hline $\begin{array}{l}\text { 1.1.1.2.05.06 } \\
\text { Level } v\end{array}$ & $\begin{array}{l}\frac{W-211 \text { Initial Retrieval }}{\text { System }} \\
\begin{array}{ll}\text { ADS } 1210-0-\text { of } & \text { Sub Total }\end{array}\end{array}$ & $\begin{array}{l}\text { OE } \\
\text { LI }\end{array}$ & --- & $---\frac{-}{0}$ & ---- & $\begin{array}{r}--- \\
0\end{array}$ & $---\frac{-}{0}$ & $---\frac{-}{0}$ & $---\frac{-}{0}$ & $---\frac{1}{0}$ \\
\hline $\begin{array}{l}1.1 .1 .2 .05 .06 .01 \\
\text { Level VI }\end{array}$ & $\begin{array}{l}\text { Project Management } \\
\qquad \text { Total }\end{array}$ & $\begin{array}{l}\mathrm{OE} \\
\mathrm{LI}\end{array}$ & $---\frac{-}{0}$ & --- & 0 & ---- & $---\overline{0}$ & ---- & ---- & $-\frac{1}{0}$ \\
\hline $\begin{array}{l}\text { 1.1.1.2.05.06.02 } \\
\text { Level V1 }\end{array}$ & Design & $\begin{array}{l}\text { OE } \\
\text { LI }\end{array}$ & $\begin{array}{r}---- \\
0\end{array}$ & --- & $\begin{array}{r}---- \\
0\end{array}$ & 0 & --- & 0 & -- & $\frac{-}{0}$ \\
\hline $\begin{array}{l}\text { 1.1.1.2.05.06.03 } \\
\text { Level VI }\end{array}$ & Procurement & $\begin{array}{l}\mathrm{OE} \\
\mathrm{LI}\end{array}$ & -- & ---- & ---- & ---- & 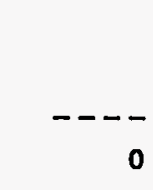 & 0 & ---- & $---\frac{-}{0}$ \\
\hline $\begin{array}{l}\text { 1.1.1.2.05.06.04 } \\
\text { Level VI }\end{array}$ & Construction & $\begin{array}{l}\text { OE } \\
\text { LI }\end{array}$ & --- & -- & -- & - & - & 0 & --- & $---\frac{-}{0}$ \\
\hline $\begin{array}{l}\text { 1.1:1.2.05.06.05 } \\
\text { Level VI }\end{array}$ & Start-Up & $\begin{array}{l}O E \\
C / E\end{array}$ & $\begin{array}{r}0 \\
0 \\
-- \\
0\end{array}$ & $\begin{array}{r}0 \\
0 \\
-- \\
0\end{array}$ & $\begin{array}{r}0 \\
0 \\
-0\end{array}$ & $\begin{array}{r}0 \\
0 \\
-- \\
0\end{array}$ & $\begin{array}{r}0 \\
0 \\
-0\end{array}$ & $\begin{array}{r}0 \\
0 \\
-- \\
0\end{array}$ & $\begin{array}{r}0 \\
0 \\
-- \\
0\end{array}$ & $\begin{array}{r}0 \\
0 \\
-0\end{array}$ \\
\hline
\end{tabular}

[ R1] 
TABLE $7.7-1$

TOTAL PROGRAM ELEMENT COST BASELINE - BY YEAR

\section{[ R1 ]}

\begin{tabular}{|c|c|c|c|c|c|c|c|c|c|c|}
\hline WBS / LEVEL & ACTIVITY TITLE / ADS & $\begin{array}{l}\text { FUND } \\
\text { TYPE }\end{array}$ & $\begin{array}{l}\text { FY01 I } \\
\text { FY05 }\end{array}$ & $\begin{array}{l}\text { FY06 I } \\
\text { FY10 }\end{array}$ & $\begin{array}{l}\text { FY11 I } \\
\text { FY15 }\end{array}$ & $\begin{array}{l}\text { FY16 I } \\
\text { FY20 }\end{array}$ & $\begin{array}{l}\text { FY21 I } \\
\text { FY25 }\end{array}$ & $\begin{array}{l}\text { FY26 I } \\
\text { FY30 }\end{array}$ & $\begin{array}{l}\text { FY31 I } \\
\text { FY35 }\end{array}$ & $\begin{array}{l}\text { FY36 I } \\
\text { FY40 }\end{array}$ \\
\hline $\begin{array}{l}\text { 1.1.1.2.05.07 } \\
\text { Level } \mathrm{v}\end{array}$ & $\begin{array}{l}\text { Final Dbl. Shell Tank } \\
\frac{\text { Retrieval System }}{\text { ADS } 1210-0-0 \mathrm{Oa}} \quad \text { Sub Total }\end{array}$ & $\begin{array}{l}O E \\
C / E\end{array}$ & $---\frac{1}{0}$ & ---- & $-\cdots--$ & ---- & $---\overline{0}$ & $\cdots$ & $---\frac{1}{0}$ & $-\cdots$ \\
\hline $\begin{array}{l}\text { 1.1.1.2.05.07.01 } \\
\text { Level VI }\end{array}$ & $\begin{array}{l}\text { Project Management } \\
\qquad \text { Total }\end{array}$ & $\begin{array}{l}O E \\
\mathrm{C} / \mathrm{E}\end{array}$ & $\begin{array}{r}0 \\
0 \\
-0 \\
0\end{array}$ & $\begin{array}{r}0 \\
0 \\
-0 \\
0\end{array}$ & \begin{tabular}{r}
0 \\
0 \\
\hdashline \\
\end{tabular} \mid & $\begin{array}{r}0 \\
0 \\
\hdashline 0\end{array}$ & $\begin{array}{r}\mathbf{0} \\
\mathbf{0} \\
-\mathbf{0}\end{array}$ & $\begin{array}{r}0 \\
0 \\
-0\end{array}$ & $\begin{array}{r}0 \\
0 \\
-0 \\
\end{array}$ & - \\
\hline $\begin{array}{l}\text { 1.1.1.2.05.07.02 } \\
\text { Level VI }\end{array}$ & $\begin{array}{l}\text { Engineering } \\
\qquad \text { Total }\end{array}$ & $\begin{array}{l}O E \\
C / E\end{array}$ & & 0 & -- & 0 & $\overline{0}$ & -- & -- & --- \\
\hline $\begin{array}{l}1.1 .1 .2 .05 .07 .03 \\
\text { Level VI }\end{array}$ & $\begin{array}{l}\text { Procurement } \\
\qquad \text { Total }\end{array}$ & $\begin{array}{l}O E \\
C / E\end{array}$ & $\begin{array}{r}0 \\
0 \\
-0\end{array}$ & $\begin{array}{r}0 \\
0 \\
- \\
0\end{array}$ & $\begin{array}{r}0 \\
0 \\
- \\
0\end{array}$ & $\begin{array}{r}0 \\
0 \\
-0 \\
\end{array}$ & -- & $\begin{array}{r}0 \\
0 \\
-0 \\
0\end{array}$ & $\begin{array}{r}0 \\
0 \\
-0 \\
0\end{array}$ & $\begin{array}{r}0 \\
0 \\
-0\end{array}$ \\
\hline $\begin{array}{l}\text { 1.1.1.2.05.07.04 } \\
\text { Level VI }\end{array}$ & $\begin{array}{l}\text { Construction } \\
\qquad \text { Total }\end{array}$ & $\begin{array}{l}O E \\
\mathrm{C} / \mathrm{E}\end{array}$ & & & $-\cdots$ & -- & $\overline{0}$ & $-\cdots$ & $-\cdots$ & - \\
\hline $\begin{array}{l}\text { 1.1.1.2.05.07.05 } \\
\text { Level VI }\end{array}$ & Start-Up & $\begin{array}{l}O E \\
C / E\end{array}$ & $-\overline{0}$ & -- & 0 & -- & $\overline{0}$ & -- & -- & -- \\
\hline $\begin{array}{l}\text { 1.1.1.2.05.08 } \\
\text { Level } v\end{array}$ & $\begin{array}{l}\text { W-315 Pilot Scale } \\
\frac{\text { Retrieval }}{\text { ADS 1210-0-OH }} \quad \text { Sub Total }\end{array}$ & $\begin{array}{l}O E \\
C / E\end{array}$ & ---- & -- & ---- & -- & - & $\overline{0}$ & $\overline{0}$ & $-\cdots$ \\
\hline
\end{tabular}


TABLE 7.7-1

TOTAL PROGRAM ELEMENT COST BASELINE - BY YEAR

\begin{tabular}{|c|c|c|c|c|c|c|c|c|c|c|}
\hline WBS / LEVEL & ACTIVITY TITLE / ADS & $\begin{array}{l}\text { FUND } \\
\text { TYPE }\end{array}$ & $\begin{array}{l}\text { FY01 / } \\
\text { FY05 }\end{array}$ & $\begin{array}{l}\text { FY06 / } \\
\text { FY10 }\end{array}$ & $\begin{array}{l}\text { FY11 I } \\
\text { FY15 }\end{array}$ & $\begin{array}{l}\text { FY16 / } \\
\text { FY20 }\end{array}$ & $\begin{array}{l}\text { FY21 / } \\
\text { FY25 }\end{array}$ & $\begin{array}{l}\text { FY26 / } \\
\text { FY30 }\end{array}$ & $\begin{array}{l}\text { FY31 / } \\
\text { FY35 }\end{array}$ & $\begin{array}{l}\text { FY36 / } \\
\text { FY40 }\end{array}$ \\
\hline $\begin{array}{l}\text { 1.1.1.2.05.08.01 } \\
\text { Level VI }\end{array}$ & $\begin{array}{l}\text { Project Management } \\
\qquad \text { Total }\end{array}$ & $\begin{array}{l}O E \\
C / E\end{array}$ & $--\frac{0}{0}$ & $--\frac{0}{0}-$ & $-\begin{array}{r}0 \\
0 \\
-0\end{array}$ & $\begin{array}{r}0 \\
0 \\
-0\end{array}$ & $\begin{array}{r}0 \\
0 \\
-- \\
0\end{array}$ & $\begin{array}{r}0 \\
0 \\
-- \\
0\end{array}$ & $\begin{array}{r}0 \\
0 \\
-- \\
0\end{array}$ & $--\frac{0}{0}$ \\
\hline $\begin{array}{l}\text { 1.1.1.2.05.08.02 } \\
\text { Level VI }\end{array}$ & Engineering & $\begin{array}{l}O E \\
C / E\end{array}$ & ---- & ---- & ---- & ---- & $---\overline{0}$ & --- & $---\overline{0}$ & $-\frac{-}{0}$ \\
\hline $\begin{array}{l}\text { 1.1.1.2.05.08.03 } \\
\text { Level vi }\end{array}$ & Procurement & $\begin{array}{l}O E \\
C / E\end{array}$ & --- & $---\overline{0}$ & ---- & ---- & --- & --- & ---- & $\cdots$ \\
\hline $\begin{array}{l}\text { 1.1.1.2.05.08.04 } \\
\text { Level VI }\end{array}$ & $\begin{array}{l}\text { Construction } \\
\qquad \text { Total }\end{array}$ & $\begin{array}{l}O E \\
C / E\end{array}$ & $\begin{array}{r}0 \\
-0 \\
-- \\
0\end{array}$ & $\begin{array}{r}0 \\
-0 \\
-0\end{array}$ & $\begin{array}{r}0 \\
0 \\
-- \\
0\end{array}$ & $\begin{array}{r}0 \\
0 \\
- \\
0\end{array}$ & -- & $\begin{array}{r}0 \\
0 \\
-- \\
0\end{array}$ & - & $\begin{array}{r}0 \\
0 \\
-- \\
0\end{array}$ \\
\hline $\begin{array}{l}1.1 .1 .2 .05 .08 .05 \\
\text { Level VI }\end{array}$ & $\begin{array}{l}\text { Start-Up Operations } \\
\qquad \text { Total }\end{array}$ & $\begin{array}{l}O E \\
C / E\end{array}$ & $---\frac{-}{0}$ & --- & $\begin{array}{r}---- \\
0\end{array}$ & 0 & $\begin{array}{r}---- \\
0\end{array}$ & $\begin{array}{r}---- \\
0\end{array}$ & --- & ---- \\
\hline $\begin{array}{l}\text { 1.1.1.2.05.09 } \\
\text { Level } v\end{array}$ & $\begin{array}{l}\text { W-320 106-C Sluicing } \\
\text { ADS 1210-0-01 } \\
\text { Sub Total }\end{array}$ & $\begin{array}{l}\text { OE } \\
C / E\end{array}$ & $---\frac{-}{0}$ & $---\frac{-}{0}$ & $---\frac{-}{0}$ & $---\frac{-}{0}$ & $---\frac{1}{0}$ & $--\frac{-}{0}$ & $--\frac{-}{0}$ & $---\frac{-}{0}$ \\
\hline $\begin{array}{l}\text { 1.1.1.2.05.09.01 } \\
\text { Level VI }\end{array}$ & $\begin{array}{l}\text { Project Management } \\
\qquad \text { Total }\end{array}$ & $\begin{array}{l}O E \\
C / E\end{array}$ & --- & ---- & --- & ---- & $---\frac{-}{0}$ & $---\frac{-}{0}$ & $---\frac{-}{0}$ & $-\cdots$ \\
\hline
\end{tabular}

[ R1 ] 
TABLE $7.7-1$

\begin{tabular}{|c|c|c|c|c|c|c|c|c|c|c|}
\hline WBS / LEVEL & ACTIVITY TITLE /.ADS & $\begin{array}{l}\text { FUND } \\
\text { TYPE }\end{array}$ & $\begin{array}{l}\text { FY01 I } \\
\text { FY05 }\end{array}$ & $\begin{array}{l}\text { FY06 I } \\
\text { FY10 }\end{array}$ & $\begin{array}{l}\text { FY11 / } \\
\text { FY15 }\end{array}$ & $\begin{array}{l}\text { FY16 / } \\
\text { FY20 }\end{array}$ & $\begin{array}{l}\text { FY21 / } \\
\text { FY25 }\end{array}$ & $\begin{array}{l}\text { FY26 I } \\
\text { FY30 }\end{array}$ & $\begin{array}{l}\text { FY31 / } \\
\text { FY35 }\end{array}$ & $\begin{array}{l}\text { FY36 I } \\
\text { FY40 }\end{array}$ \\
\hline $\begin{array}{l}\text { 1.1.1.2.05.09.02 } \\
\text { Level VI }\end{array}$ & $\begin{array}{l}\text { Engineering } \\
\qquad \text { Tot }\end{array}$ & $\begin{array}{l}\text { OE } \\
\text { C/E }\end{array}$ & $\begin{array}{r}--- \\
0\end{array}$ & ---- & $---\frac{-}{0}$ & ---- & ---- & ---- & ---- & -- \\
\hline $\begin{array}{l}\text { 1.1.1.2.05.09.03 } \\
\text { Level V1 }\end{array}$ & $\begin{array}{l}\text { CENRTC } \\
\qquad \begin{array}{l}\text { Total } \\
\end{array}\end{array}$ & $\begin{array}{l}O E \\
C / E\end{array}$ & $\begin{array}{r}--- \\
0\end{array}$ & ---- & ---- & --- & ---- & ---- & ---- & $\begin{array}{r}---- \\
0\end{array}$ \\
\hline $\begin{array}{l}\text { 1.1.1.2.05.09.04 } \\
\text { Level VI }\end{array}$ & $\begin{array}{l}\text { Construction } \\
\qquad \text { Total }\end{array}$ & $\begin{array}{l}O E \\
C / E\end{array}$ & ---- & ---- & ---- & ---- & $---\frac{-}{0}$ & ---- & ---- & --- \\
\hline $\begin{array}{l}1.1 .1 .2 .05 .09 .05 \\
\text { Level VI }\end{array}$ & Start-Up & $\begin{array}{l}O E \\
C / E\end{array}$ & ---- & $---\frac{-}{0}$ & ---- & ---- & ---- & ---- & ---- & $-\cdots$ \\
\hline $\begin{array}{l}1.1 .1 .2 .05 .10 \\
\text { Level } \mathrm{V}\end{array}$ & $\begin{array}{l}\text { Sgl. Shell Tank Training } \\
\text { and Test Facility } \\
\text { ADS } 1210-0-0 K \quad \text { Sub Total }\end{array}$ & $\begin{array}{l}O E \\
\text { C/E }\end{array}$ & ---- & ---- & ---- & --- & ---- & $---\frac{-}{0}$ & $---\frac{-}{0}$ & --- \\
\hline $\begin{array}{l}\text { 1.1.1.2.05.10.01 } \\
\text { Level VI }\end{array}$ & $\begin{array}{l}\text { Project Management } \\
\qquad \quad \text { Total }\end{array}$ & $\begin{array}{l}O E \\
C / E\end{array}$ & $---\frac{-}{0}$ & $-\cdots$ & ---- & $---\frac{-}{0}$ & $---\overline{0}$ & ---- & ---- & $---\overline{0}$ \\
\hline $\begin{array}{l}\text { 1.1.1.2.05.10.02 } \\
\text { Level VI }\end{array}$ & Engineering & $\begin{array}{l}O E \\
C / E\end{array}$ & $---\frac{1}{0}$ & $---\overline{0}$ & 0 & $---\frac{-}{0}$ & ---- & $\begin{array}{r}-r \\
0\end{array}$ & $-\cdots$ & $---\frac{-}{0}$ \\
\hline
\end{tabular}


TABLE 7.7-1

TOTAL PROGRAM ELEMENT COST BASELINE - BY YEAR

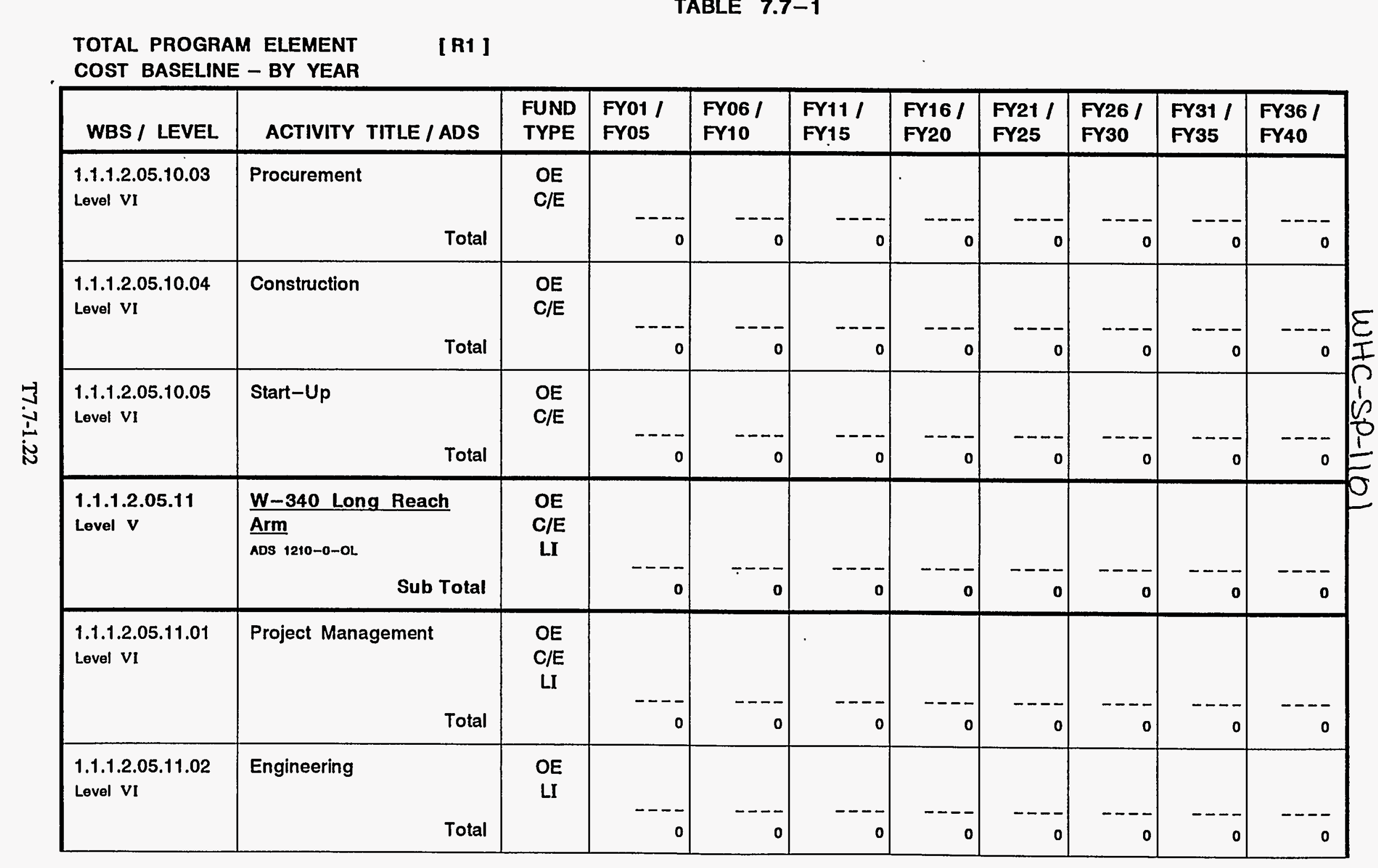

\section{[R1 ]}


TABLE $7.7-1$

TOTAL PROGRAM ELEMENT

COST BASELINE - BY YEAR

[ R1 ]

\begin{tabular}{|c|c|c|c|c|c|c|c|c|c|c|}
\hline WBS / LEVEL & ACTIVITY TITLE / ADS & $\begin{array}{l}\text { FUND } \\
\text { TYPE }\end{array}$ & $\begin{array}{l}\text { FY01 I } \\
\text { FY05 }\end{array}$ & $\begin{array}{l}\text { FY06 I } \\
\text { FY10 }\end{array}$ & $\begin{array}{l}\text { FY11 I } \\
\text { FY15 }\end{array}$ & $\begin{array}{l}\text { FY16 / } \\
\text { FY20 }\end{array}$ & $\begin{array}{l}\text { FY21 / } \\
\text { FY25 }\end{array}$ & $\begin{array}{l}\text { FY26 I } \\
\text { FY30 }\end{array}$ & $\begin{array}{l}\text { FY31 / } \\
\text { FY35 }\end{array}$ & $\begin{array}{l}\text { FY36 I } \\
\text { FY40 }\end{array}$ \\
\hline $\begin{array}{l}1.1 .1 .2 .05 .11 .03 \\
\text { Levol VI }\end{array}$ & $\begin{array}{l}\text { Regulatory / Compliance } \\
\qquad \text { Total }\end{array}$ & $\begin{array}{l}O E \\
C / E\end{array}$ & --- & ---- & ---- & ---- & ---- & $---\frac{-}{0}$ & $---\frac{-}{0}$ & --- \\
\hline $\begin{array}{l}1.1 .1 .2 .05 .11 .04 \\
\text { Level VI }\end{array}$ & $\begin{array}{l}\text { LRM Procurement } \\
\qquad \begin{array}{r}\text { Total } \\
\end{array}\end{array}$ & $\begin{array}{l}O E \\
C / E\end{array}$ & $--\overline{0}$ & --- & --- & $---\overline{0}$ & --- & --- & ---- & --- \\
\hline $\begin{array}{l}1.1 .1 .2 .05 .11 .05 \\
\text { Level VI }\end{array}$ & Construction & $\begin{array}{l}O E \\
C / E\end{array}$ & -- & & -- & --- & $---\overline{0}$ & $---\frac{-}{0}$ & $---\frac{-}{0}$ & --- \\
\hline $\begin{array}{l}1.1 .1 .2 .05 .11 .06 \\
\text { Level VI }\end{array}$ & Start-Up & $\begin{array}{l}O E \\
C / E\end{array}$ & -- & $\begin{array}{r}--- \\
0\end{array}$ & $-\cdots$ & $---\frac{-}{0}$ & --- & $---\overline{0}$ & $---\frac{1}{0}$ & --- \\
\hline $\begin{array}{l}\text { 1.1.1.2.05.12 } \\
\text { Level } \mathrm{v}\end{array}$ & $\begin{array}{l}\frac{\text { Single-Shell Tank }}{\text { Closure Demo. }} \\
\text { ADS 1210-0-OM Sub Total }\end{array}$ & $\begin{array}{l}O E \\
C / E\end{array}$ & $\begin{array}{r}--- \\
0\end{array}$ & ---- & $---\frac{-}{0}$ & $\begin{array}{r}---- \\
0\end{array}$ & $---\frac{-}{0}$ & -- & $---\frac{-}{0}$ & $---\frac{-}{0}$ \\
\hline $\begin{array}{l}\text { 1.1.1.2.05.12.01 } \\
\text { Level VI }\end{array}$ & $\begin{array}{l}\text { Project Management } \\
\qquad \text { Total }\end{array}$ & $\begin{array}{l}O E \\
C / E\end{array}$ & $---\frac{-}{0}$ & 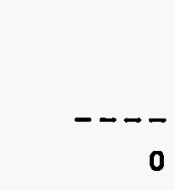 & -- & $-\cdots$ & $\begin{array}{r}-\cdots \\
0\end{array}$ & 0 & $\begin{array}{r}---- \\
0\end{array}$ & --- \\
\hline $\begin{array}{l}\text { 1.1.1.2.05.12.02 } \\
\text { Level VI }\end{array}$ & Engineering & $\begin{array}{l}O E \\
C / E\end{array}$ & --- & - & -- & $\overline{0}$ & ---- & -- & $-\cdots--$ & ---- \\
\hline
\end{tabular}


TABLE $7.7-1$

TOTAL PROGRAM ELEMENT COST BASELINE - BY YEAR

[ R1 ]

\begin{tabular}{|c|c|c|c|c|c|c|c|c|c|c|}
\hline WBS / LEVEL & ACTIVITY TITLE / ADS & $\begin{array}{l}\text { FUND } \\
\text { TYPE }\end{array}$ & $\begin{array}{l}\text { FY01 / } \\
\text { FY05 }\end{array}$ & $\begin{array}{l}\text { FY06 / } \\
\text { FY10 }\end{array}$ & $\begin{array}{l}\text { FY11/ } \\
\text { FY15 }\end{array}$ & $\begin{array}{l}\text { FY16 / } \\
\text { FY20 }\end{array}$ & $\begin{array}{l}\text { FY21 / } \\
\text { FY25 }\end{array}$ & $\begin{array}{l}\text { FY26 / } \\
\text { FY30 }\end{array}$ & $\begin{array}{l}\text { FY31 / } \\
\text { FY35 }\end{array}$ & $\begin{array}{l}\text { FY36 I } \\
\text { FY40 }\end{array}$ \\
\hline $\begin{array}{l}1.1 .1 .2 .05 .12 .03 \\
\text { Level V1 }\end{array}$ & Total & $\begin{array}{l}\text { OE } \\
\text { C/E }\end{array}$ & ---- & 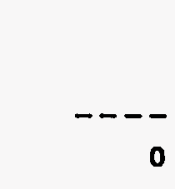 & ---- & $\begin{array}{r}--- \\
0\end{array}$ & $---\overline{0}$ & ---- & ---- & $---\frac{-}{0}$ \\
\hline $\begin{array}{l}\text { 1.1.1.2.05.12.04 } \\
\text { Level VI }\end{array}$ & Total & $\begin{array}{l}O E \\
C / E\end{array}$ & ---- & $-\cdots$ & $\begin{array}{r}--- \\
0\end{array}$ & $\begin{array}{r}--- \\
0\end{array}$ & $\begin{array}{r}---\overline{0} \\
0\end{array}$ & $\begin{array}{r}---- \\
0\end{array}$ & ---- & ---- \\
\hline $\begin{array}{l}1.1 .1 .2 .05 .12 .05 \\
\text { Level VI }\end{array}$ & $\begin{array}{l}\text { Start-Up } \\
\\
\\
\text { Total }\end{array}$ & $\begin{array}{l}O E \\
C / E\end{array}$ & $---\frac{-}{0}$ & ---- & ---- & ---- & ---- & $---\frac{-}{0}$ & ---- & $---\frac{-}{0}$ \\
\hline $\begin{array}{l}1.1 .1 .2 .05 .13 \\
\text { Level } \mathrm{V}\end{array}$ & $\frac{\text { Single-Shell Tank }}{\frac{\text { Closure }}{\text { ADS 1210-0-ON }} \quad \text { Sub Total }}$ & $\begin{array}{l}\text { OE } \\
\text { C/E }\end{array}$ & $---\overline{0}$ & $---\overline{0}$ & $\begin{array}{r}---- \\
0\end{array}$ & ---- & $---\frac{1}{0}$ & $---\frac{-}{0}$ & $---\overline{0}$ & $---\overline{0}$ \\
\hline $\begin{array}{l}\text { 1.1.1.2.05.13.01 } \\
\text { Level VI }\end{array}$ & $\begin{array}{l}\text { Project Management } \\
\qquad \text { Total }\end{array}$ & $\begin{array}{l}O E \\
C / E\end{array}$ & ---- & ---- & $-\cdots$ & $---\frac{-}{0}$ & ---- & ---- & $\begin{array}{r}---- \\
0\end{array}$ & --- \\
\hline $\begin{array}{l}\text { 1.1.1.2.05.13.02 } \\
\text { Level VI }\end{array}$ & Engineering & $\begin{array}{l}O E \\
C / E\end{array}$ & $-\cdots$ & ---- & $---\frac{-}{0}$ & $\begin{array}{r}---- \\
0\end{array}$ & $---\frac{-}{0}$ & $\begin{array}{r}--- \\
0\end{array}$ & $\begin{array}{r}--- \\
0\end{array}$ & --- \\
\hline $\begin{array}{l}\text { 1.1.1.2.05.13.03 } \\
\text { Level VI }\end{array}$ & $\begin{array}{l}\text { Procurement } \\
\begin{array}{c}\text { Total } \\
\text {. }\end{array}\end{array}$ & $\begin{array}{l}\mathrm{OE} \\
\mathrm{C} / \mathrm{E}\end{array}$ & - & ---- & - & $---\frac{-}{0}$ & $---\frac{-}{0}$ & $---\frac{-}{0}$ & $---\frac{-}{0}$ & --- \\
\hline
\end{tabular}


TABLE $7.7-1$

TOTAL PROGRAM ELEMENT COST BASELINE - BY YEAR

[ R1 ]

\begin{tabular}{|c|c|c|c|c|c|c|c|c|c|c|}
\hline WBS / LEVEL & ACTIVITY TITLE / ADS & $\begin{array}{l}\text { FUND } \\
\text { TYPE }\end{array}$ & $\begin{array}{l}\text { FY01 I } \\
\text { FY05 }\end{array}$ & $\begin{array}{l}\text { FY06 I } \\
\text { FY10 }\end{array}$ & $\begin{array}{l}\text { FY11 / } \\
\text { FY15 }\end{array}$ & $\begin{array}{l}\text { FY16 / } \\
\text { FY20 }\end{array}$ & $\begin{array}{l}\text { FY21 I } \\
\text { FY25 }\end{array}$ & $\begin{array}{l}\text { FY26 / } \\
\text { FY30 }\end{array}$ & $\begin{array}{l}\text { FY31 / } \\
\text { FY35 }\end{array}$ & $\begin{array}{l}\text { FY36 / } \\
\text { FY40 }\end{array}$ \\
\hline $\begin{array}{l}\text { 1.1.1.2.05.13.04 } \\
\text { Level VI }\end{array}$ & $\begin{array}{l}\text { Construction } \\
\qquad \text { Total }\end{array}$ & $\begin{array}{l}O E \\
C / E\end{array}$ & $---\frac{-}{0}$ & $\begin{array}{r}--- \\
0\end{array}$ & $-\cdots$ & --- & --- & ---- & ---- & $-\frac{-}{0}$ \\
\hline $\begin{array}{l}\text { 1.1.1.2.05.13.05 } \\
\text { Level VI }\end{array}$ & Start-Up & $\begin{array}{l}O E \\
C / E\end{array}$ & $---\overline{0}$ & $---\overline{0}$ & ---- & --- & --- & $---\frac{-}{0}$ & --- & $-\cdots$ \\
\hline $\begin{array}{l}1.1 .1 .2 .05 .14 \\
\text { Lovel } \mathrm{V}\end{array}$ & $\begin{array}{l}\frac{\text { Misc. Underground }}{\text { Stor. Tnks. Retrieval Sys. }} \\
\frac{\text { ADs } 1210-0-O P}{\text { Sub Total }}\end{array}$ & $\begin{array}{l}O E \\
C / E\end{array}$ & $---\frac{-}{0}$ & ---- & ---- & $---\frac{-}{0}$ & $---\frac{-}{0}$ & $---\frac{-}{0}$ & ---- & --- \\
\hline $\begin{array}{l}1.1 .1 .2 .05 .14 .01 \\
\text { Level VI }\end{array}$ & $\begin{array}{l}\text { Project Management } \\
\qquad \text { Total }\end{array}$ & $\begin{array}{l}O E \\
C / E\end{array}$ & --- & ---- & --- & --- & ---- & --- & --- & $\begin{array}{r}--- \\
0\end{array}$ \\
\hline $\begin{array}{l}1.1 .1 .2 .05 .14 .02 \\
\text { Level VI }\end{array}$ & Engineering & $\begin{array}{l}\text { OE } \\
C / E\end{array}$ & --- & $\begin{array}{r}---- \\
0\end{array}$ & 0 & - & $---\overline{0}$ & --- & $\begin{array}{r}--- \\
0\end{array}$ & --- \\
\hline $\begin{array}{l}1.1 .1 .2 .05 .14 .03 \\
\text { Level VI }\end{array}$ & Procurement & $\begin{array}{l}\text { OE } \\
\text { G/E }\end{array}$ & $--\frac{-}{0}$ & ---- & -- & o & $\overline{0}$ & $---\frac{-}{0}$ & - & $-\cdots$ \\
\hline
\end{tabular}


TABLE 7.7-1

TOTAL PROGRAM ELEMENT COST BASELINE - BY YEAR

[ R1 ]

\begin{tabular}{|c|c|c|c|c|c|c|c|c|c|c|}
\hline WBS / LEVEL & ACTIVITY TITLE / ADS & $\begin{array}{l}\text { FUND } \\
\text { TYPE }\end{array}$ & $\begin{array}{l}\text { FY01 / } \\
\text { FY05 }\end{array}$ & $\begin{array}{l}\text { FY06 / } \\
\text { FY10 }\end{array}$ & $\begin{array}{l}\text { FY11 / } \\
\text { FY15 }\end{array}$ & $\begin{array}{l}\text { FY16 / } \\
\text { FY20 }\end{array}$ & $\begin{array}{l}\text { FY21 / } \\
\text { FY25 }\end{array}$ & $\begin{array}{l}\text { FY26 / } \\
\text { FY30 }\end{array}$ & $\begin{array}{l}\text { FY31 / } \\
\text { FY35 }\end{array}$ & $\begin{array}{l}\text { FY36 / } \\
\text { FY40 }\end{array}$ \\
\hline $\begin{array}{l}\text { 1.1.1.2.05.14.04 } \\
\text { Level VI }\end{array}$ & $\begin{array}{l}\text { Construction } \\
\qquad \text { Total }\end{array}$ & $\begin{array}{l}O E \\
C / E\end{array}$ & $---\frac{1}{0}$ & --- & $\begin{array}{rr}---- \\
0\end{array}$ & 0 & --- & $---\frac{-}{0}$ & $-r-$ & 0 \\
\hline $\begin{array}{l}\text { 1.1.1.2.05.14.05 } \\
\text { Level VI }\end{array}$ & Start-Up & $\begin{array}{l}O E \\
C / E\end{array}$ & ---- & --- & $---\frac{-}{0}$ & $--\frac{-}{0}$ & -- & $---\frac{-}{0}$ & $--\frac{-}{0}$ & $---\overline{0}$ \\
\hline
\end{tabular}




\section{NOTES \\ TOTAL PROGRAM ELEMENT COST BASELINE - BY YEAR}

The cost exhibit tables in each of the Program Element Baselines (Sections 7.2 through 7.10) identify the program element target funding by year by fund type. The tables also provide details of the baseline allocation of funding down to activity levels (level V) and cost account levels (level VI) of the WBS for Fiscal Year 1995 through 1997. The details exhibited on these tables are taken from the baseline WBS Dictionary Sheets. Deltas exist between the details and the annual target totals. For Fiscal Years 1996 and 1997 the deltas will be addressed in the future, following the completion of the resolution of expected differences between current Fiscal Year 1995 baseline funding and expected actual Fiscal Year 1995 funding. Deltas for Fiscal Year 1995 are noted below by program element:

\section{PROGRAM MANAGEMENT AND ADMINISTRATION:}

No deltas.

\section{TANK FARM OPERATIONS AND MAINTENANCE:}

No overall delta. This baseline requires revised fund type mix.

\section{WASTE TANK SAFETY:}

Delta is due to need to revise an overhead pricing rate in the P3 system.

\section{TANK FARM UPGRADES:}

Delta is due to differences between Budget Authority (BA) in the target and Budget Obligation (B0) in the P3 system.

\section{CHARACTERIZATION:}

No significant deltas.

\section{WASTE RETRIEVAL:}

Delta is primarily due to need to resolve issues associated with expense funding support to capital projects and differences between Budget Authority (BA) in the target and Budget Obligation (B0) in the $\mathrm{P} 3$ system.

\section{WASTE PRETREATMENT:}

Delta is due to the sum of the detail cost estimates being lower than the target total as a result of reestimation of resource requirements to complete baseline scope.

\section{LOW LEVEL WASTE:}

Delta is primarily due to carryover expense funded workscope on melter testing addressed in June 9, 1994 RL letter.

$$
T 7.7-1.27
$$




\section{WHC-SP-1101}

\section{HIGH LEVEL WASTE:}

Delta is due to carryover of capital funding, PNL expense funding carryover, and ADS corrections submitted but not reflected in target totals. 
WHC-SP-1101

Table 7.7-2. FY 1995 Cost by Month by Fund Type by Activity and Cost Account

Future MYWP updates will include Table 7.7-2, which establishes the month-by-month cost baseline for FY 1995 for this program element. The FY 1995 cost baseline is exhibited at the cost account level with fund type totals provided. Cost data are then rolled up to the activity and program element levels. Also exhibited is the total cost baseline for the program element for FY 1995. At present, the program element cost baseline for FY 1995 for this program element is the total provided on this table less a productivity challenge for FY 1995. 
Table 7-7-3. Total Full-Time Equivalents by Year by Activity by Major Participant (Direct and Indirect) (Including all Subcontractors)

Future MYWP updates will include Table 7.7-3, which establishes total staffing projections for this program element. Included in the table are FY 1994 staffing data as well as projections by year for FY 1995 through FY 2000. The projections in this table include all direct and indirect Full-Time Equivalents (FTEs), including all subcontractor personnel. Where data are available, projections are exhibited at the activity level with major program participant totals provided. 
Table 7.7-4. FY 1995 through FY 1997 Direct Westinghouse Hanford Company

Full-Time Equivalents by 88 Labor Categories

Future MYWP updates will include Table 7.7-4, which establishes direct Westinghouse Hanford Company staffing projections for this program element. Projections do not include subcontractor support and are provided by 88 labor categories for FY 1995 through FY 1997. 
WHC-SP-1101

\subsection{WASTE PRETREATMENT BASELINE}

The Waste Pretreatment Program Element baseline consists of the technical bases, schedule, resource requirements, and waste type data for FY 1995 through completion of the mission. The major emphasis is on deliverables, schedule, and resource requirements for FY 1995, 1996, and 1997.

\subsubsection{Technical Bases}

This section provides the mission strategy; work breakdown structure and organization responsibility; work to be performed in FY 1995, 1996 and 1997; technical approach; and drivers and deliverables.

\subsubsection{Mission Strategy}

\subsection{Objectives}

The overall goals and objectives for the Waste Pretreatment Program Element are to meet the applicable functions and requirements and to deliver the products and end items necessary to achieve the TWRS Program Mission. Waste pretreatment will be required for partitioning high-level waste (HLW) and transuranic (TRU) wastes from low-level wastes (LLW) and may also be required for resolution of safety issues for some of the double-shell tanks (DST) and single-shell tanks (SST). Product streams from waste pretreatment provide feed for LLW and HLW waste immobilization facilities. Waste pretreatment will develop required technologies, operate pretreatment facilities, and provide and operate hot test facilities for pretreatment technology development as well as provide feed materials for HLW vitrification waste form qualification. This program element will provide technical planning and integration, technology development and applied engineering, facility operations, systems definition, construction of pretreatment facility (Project W-236B), and pretreatment of HLW. Solid wastes and liquid and gaseous effluents will be produced and processed as a part of this program element.

\subsection{Strategy}

The overall strategy of Waste Pretreatment is to deliver phased pretreatment capability, including solids and liquids separation; liquid treatment for LLW immobilization; and solids treatment for HLW immobilization. A general goal is to have the LLW fraction contain the maximum volume of the tank waste and the minimum inventory of radionuclides. The HLW fraction shall contain the minimum volume of the tank waste and the maximum inventory of the radionuclides.

The solids and liquids separation will be done by decanting off liquids from DSTs (including salt cake that has been dissolved in SSTs and transferred to the DSTs). The solids treatment will consist of in-tank processing (sludge washing with water and sodium hydroxide solution) to remove solubles, including some of the aluminum and chromium to reduce the volume of solids going to the HLW immobilization facility. To the extent that additional facilities are needed for the solids treatment, they will be provided by a HLW pretreatment facility, which may be a standalone facility or may be combined with the LLW pretreatment facility or with the HLW immobilization facility. The HLW Pretreatment Facility is scheduled to be operational by June 2008 (Tri-Party Agreement Milestone 50-04) to support HLW vitrification. 
The liquid treatment for LLW immobilization consists of feed clarification (to remove solids from the supernatant to avoid fouling of ion exchange columns) and radionuclide removal. The base case identifies cesium ion exchange and strontium, if necessary, as the only required radionuclide removal operations. If strontium removal is required, it probably is tied up with an organic complexant; therefore, a complexant destruction step would be added. Technology development is underway to support this activity. These supernatant pretreatment functions will be provided.by Project W-236B, Initial Pretreatment Module, and is referred to as the LLW Pretreatment Facility. In order to flatten the funding profile, the LLW Pretreatment Facility will be achieved in two modules. Module I is scheduled to be operational by December 2004 (Tri-Party Agreement Milestone 50-02). Evaporation capability will be included in Module I.

If the LLW performance assessment determines that other radionuclides need to be removed, the capability to remove these will be added to the second module of LLW pretreatment. (An exception to this may be technetium, which will be discussed later.) The LLW Pretreatment Module II, is scheduled to be operational in January 2006 . It provides additional capacity for pretreatment and may be an integral part of or co-located with Module I.

Development will continue for processes to remove long-lived radionuclides (e.g., ${ }^{99} \mathrm{Tc}$ ) from the LLW stream until implemented or determined not to be needed. All SST waste will be processed as it is retrieved to provide feed to LLW and HLW immobilization facilities. HLW volumes will be reduced by using processes requiring limited development. These processes include sludge washing, selective leaching, and blending. Technology development to enhance these processes will be pursued. Technology development for sludge acid dissolution processes will be conducted on a selective basis as a contingency. These processes will be needed if the simpler processes do not result in a "reasonable" volume of vitrified waste acceptable to the geologic repository program or if there is great economic justification for reducing the waste volume. Laboratory- and bench-scale process testing will be conducted using actual waste.

Project W-236A, the Multi-Function Waste Tank Facility, will provide carbon steel waste storage tanks for in-tank processing or storage of pretreatment feed or pretreated feed awaiting transfer to the LLW and HLW immobilization facilities.

The technology development and applied engineering strategy for waste pretreatment is to identify, evaluate, and test processes for development, which will reduce the volume of vitrified HLW produced and also meet both the HLW and LLW vitrification feed requirements. The approach is to validate the basic assumption that relatively simple in-tank processes for pretreating the sludge will result in a reasonable volume of HLW. In-tank processes include sludge washing using mild caustic solutions, enhanced sludge washing using caustic solutions (e.g., 4M caustic), and blending of feeds to the HLW and LLW vitrification facilities.

To validate the above assumption it is necessary to conduct laboratory-, bench-, and full-scale sludge washing and caustic washing (enhanced sludge washing) tests using actual sludges. Results of these tests are also needed to verify enhanced sludge washing as the selected baseline, with advanced pretreatment technologies requiring acid dissolution as a contingency in case enhanced sludge washing was unsuccessful (Tri-Party Agreement Milestone M-50-03).

The strategy for making the decision embodied in Tri-Party Agreement Milestone M-50-03 also includes evaluating technology for advanced sludge separation processes as an alternative to the approach of simple in-tank sludge processing. The approach is to focus technology development on 
the simpler advanced sludge separations processes (dissolution) with the objective of removing inorganic chemical species from the feed to the HLW vitrification facility. These simpler advanced sludge separation processes include: thermal reconstitution of sludges (e.g., calcination of sludge); non-thermal reconstitution of sludges (e.g., acidification and reneutralization); and acid leaching of selected sludges (e.g., neutralized cladding removal waste to remove plutonium).

$\because$

With respect to the more advanced sludge separation processes (e.g., radionuclide removal using solvent extraction or transuranic extraction) the strategy is to apply the results of existing studies and the results of future studies funded by others (e.g., U.S. Department of Energy EM-50 programs) in making the decision embodied in Tri-Party Agreement Milestone M-50-03 by March 1998.

A second basic assumption requiring validation is that an acceptable LLW vitrification facility feed can be produced in the LLW pretreatment facility using feed clarification and ion exchange to remove cesium from the liquid stream. The general requirements of an acceptable LLW vitrification facility ${ }^{\prime}$ feed include the following:

1. Results in a LLW glass that does not exceed the comparable limits for commercial LLW.

2. Meets the U.S. Nuclear Regulatory Commission's "incidental waste" classification for the Hanford Site.

3. Meets the As Low As Reasonably Achievable (ALARA) policy.

4. Meets the LLW disposal system performance requirements.

These requirements will define the levels of cesium that must be removed from the liquid streams and will determine if other radionuclides (such as strontium and technetium) need to be removed and to what levels. The strategy for cesium removal is to continue development of the Savannah River Resorcinol-Formaldehyde (R-F) resin and the Sandia National Laboratory cesium resin technology (CST) as alternatives to the CS-100 resin. With respect to other radionuclides, the strategy is to first process, to the greatest extent possible, waste requiring only cesium removal. This will allow additional time to develop technology for removal of other radionuclides from the liquid streams if needed.

If technetium needs to be removed as a requirement resulting from the LLW Program performance assessment, this removal step may have to be in Module I. This is because the current waste processing plans call for double-shell slurry feed to be processed first. Initial indications are that technetium may be in this waste type. The conceptual design for the LLW Pretreatment Facility will include provision for technetium and strontium/TRU removal and organic destruction. This robustness in the strategy ensures that if these processes are subsequently determined to be required they can be incorporated in Title I and Title II design activities for the project without schedule delays or funding shortfalls.

Pretreatment of in-tank waste to provide acceptable feeds to the LLW and HLW vitrification facilities may require destruction of organic complexants in some waste. The strategy is to defer further technology development for complexant destruction for five years following fiscal year 1995 . This will allow time to establish pretreatment product requirements, obtain additional characterization data, and complete laboratory- and bench-scale tests of separations efficiencies using actual waste. This strategy may require staging complexed waste for processing later in the pretreatment schedule. 


\subsection{Functions and Requirements}

The TWRS Function Hierarchy within TWRS Functions and Requirements is shown in Figure 7.8-1 to the fifth level of decomposition. The work scope within the Waste Pretreatment Program falls under two of the Level III functions identified. These are 4.2.2, 'Process Waste' and 4.2.3, 'Manage System Generated Waste \& Excess Facilities'. Of the six Level IV functions under 4.2.2, only Pretreat Waste (4.2.2.1) applies. to the Waste Pretreatment Program Element. The Pretreat Waste function is further subdivided into three functions: Pretreat Sludges/Solids (4.2.2.1.1), Pretreat Supernatants (4.2.2.1.2), and Blend High-Activity Streams (4.2.2.1.3).

The Pretreat Sludges/Solids function includes organic destruction, selective blending, simple solid/liquid separation (settle/decant), chemical leaching and water washing as well as general monitoring and waste handling functions. A separate functions and requirements document will be prepared for the Pretreat Sludges/Solids function.

The Pretreat Supernatants function receives supernatants for separation into a low-activity and highactivity fractions. This function decomposes (sixth level decomposition) into Separate Radionuclides, Treat Vessel Offgas, Analyze Samples, Monitor and Control Process, Prepare Solid Waste, Prepare Liquid Waste, and Support Pretreatment Process. These Level VI functions are needed to perform the parent function Pretreat Supernatants. Further decomposition of these seven functions (i.e., beyond Level VI) is performed as part of Project W-236B, Initial Pretreatment Module. The supporting document, Functions and Requirements for Project W-236B, Initial Pretreatment Module, WHC-SD-236B-FRD-002 Rev. 0, documents the functional analysis for Pretreat Supernatant.

The Blend High-Activity Streams function definition is: combine the high-activity waste fractions separated from pretreatment of sludges/solids (function 4.2.2.1.1) and pretreatment of supernatants (function 4.2.2.1.2) to produce a mixture suitable for HLW immobilization. The high-activity waste fraction separated from pretreatment of sludges/solids is a solids slurry contained in a relatively small volume stream compared to the volume of the separated LLW stream (e.g., supernatants). The high-activity waste fraction separated from pretreatment of supernatants consists of a concentrated cesium solution with a small fraction of solids. These high-activity waste fractions may be combined for interim storage before immobilization.

\subsection{Risks}

A preliminary list of decisions affecting the technical requirements and/or scope of the TWRS Program has been prepared from information contained in the Tank Waste Remediation System Functions and Requirements (DOE/RL-92-60 draft). The TWRS Technical Scope Definition Decision Logic from that document identifies a series of top-level technical decisions that must be specified for completion of the overall TWRS Program Mission:

1. The TWRS Program is developing and evaluating alternative technologies for separating tank waste into fractions for immobilization and disposal.

Mitigation: The TWRS program is not scheduled to engage in the detailed design of any major facility needed for tank waste separations until after the completion of the TWRS environmental impact statement. Therefore, the financial exposure is low. Schedule risks arise if the environmental impact statement record of decision is significantly different than the planning basis. 
2. High-level waste geological repository volume limitations have not been established; thus uncertainty exists as to the acceptability of either alternative process for separation of tank waste.

Mitigation: By utilizing the proposed DOE-Office of Civilian Radioactive Waste Management (OCRWM) multi-purpose canister (MPC) concept and optimizing packaging of immobilized HLW within the MPC, the risk associated with proceeding with the minimum separations pretreatment concept and acceptance of the projected immobilized HLW volume by the geological repository can be managed.

3. Limits for acceptable radionuclides concentrations and volume of immobilized LLW have not been established. Therefore, uncertainty exists as to the acceptability of either alternative process for separation of tank waste.

Mitigation: The limits for acceptable radionuclides in the low-activity solution can be understood by conducting a parametric evaluation of radionuclide separations versus LLW system performance. Once the inter-relationship between the pretreatment process and the LLW disposal system environmental performance is understood, management of this uncertainty can be achieved.

4. Additional radionuclides (e.g. technetium or strontium) may need to be separated from the Hanford tank wastes to achieve a lightly shielded LLW vitrification facility or achieve the desired environmental performance of the LLW disposal system. Production scale deployment of technetium and strontium separation processes may not be achievable in all candidate facility concepts (e.g. dispersed supernate pretreatment modules).

Mitigation: The approach to mitigating these concerns is to re-evaluate the selection of the supernate pretreatment facility concept after the environmental performance of the LLW disposal system is understood.

5. The TWRS Program technical scope, cost estimate, and schedule have been defined assuming programmatic responsibility for treatment and disposition of system generated waste and excess facilities. If a decision is reached to assign programmatic responsibility to another Hanford Site program, the TWRS Program must be able to be readily modified to remove scope and cost associated with these activities.

Mitigation: Regardless of the organization assigned programmatic responsibility for treatment and disposition of these wastes, interface acceptance requirements need to be established to determine the extent of treatment required prior to transfer of these wastes to these other Hanford Site functions.

6. A strategy for liquid effluent treatment needs to be selected to establish necessary functions and interface requirements. All liquid effluents presently generated from TWRS activities are treated and discharged to the atmosphere in accordance with applicable Federal, State, and local regulations. This decision will effect the designs of future facilities for remediation of tank waste.

Mitigation: If the decision on the strategy is made prior to finalizing facility design, no programmatic impacts are anticipated. 
7. Two of the uncertainties from the Characterization Program concern whether sludge samples required for pretreatment can be provided in a timely manner (this includes both taking samples from the tank and doing the initial extrusion from the sampler, and mixing segment extrusions from a core as necessary), and whether the samples can be studied and/or investigated to develop the necessary process technology baseline in a timely manner.

Mitigation: The Characterization Program has developed a schedule that considers several different modes of sampling sludge, incorporates new sampling hardware as it is planned to become operational, and personnel qualification training. The schedule has some flexibility built in to recover from minor slippages. The Characterization Program has identified issues and proposed actions to address these issues, and is currently evaluating options that will provide the necessary technical baseline to allow realistic assessments of the proposed pretreatment processes.

8. Characterization information is needed to determine the range of conditions for operation of the LLW pretreatment processes. This poses a risk to both cost and schedule. The selected process design may be inadequate to process a particular waste tank stream at an acceptable rate with acceptable separation factors.

Mitigation: This risk will be managed by providing a more conservative conceptual design and by working with the characterization program to try and determine the full range of tank compositions.

9. In order to obtain certain process design information, some testing with actual tank waste must be performed. There is a schedule risk to the LLW Pretreatment project in obtaining these samples. Pretreatment priorities are competing with other TWRS priorities for a limited sampling capability. This will impact the project definitive design schedule. If it is determined that the various TWRS end functions are required to fund the sampling activities additional resources will be required. since sufficient current resources are not included in the multi-year planning schedules.

Mitigation: The LLW Pretreatment project is working to develop its data requirements and inputting that information into the characterization DQO requirements. Project sampling requirements have been forwarded to the pretreatment and characterization programs for inclusion into the sampling schedule.

10. Certain infrastructure requirements have been identified (ie., electrical power, transfer piping, and process water) that are expected to be provided by other TWRS projects. At this time, projects are not underway to support the development of the needed infrastructure. Risks to the project involve both cost and schedule. Currently the IPM project does not include funding for the required infrastructure, and TWRS planning does not include the resources for the expected projects. As these infrastructure projects are identified and funded, competition for existing resources may cause a reallocation of TWRS project funds. A reallocation of funds and/or untimely completion of needed infrastructure projects can cause significant project schedule delays.

Mitigation: TWRS is performing a series of trade studies to identify project infrastructure requirements. Identification of needs will be followed by creation of projects and by the appropriate planning to determine what funds impact may be present. 
11. Interfaces between the LLW pretreatment facility and other TWRS programs and projects have not been completely identified. Liquid process streams that will require pretreatment from LLW and HLW vitrification processes have not been completely defined. Based on the Savannah River Defense Waste Processing Facility (DWPF), it has been determined that these streams will be significant in quantity and impact. The lack of defined interfaces may cause a delay in schedule due to insufficient information to support process capacity determination.

Mitigation: In order to reduce this risk, planning efforts started in FY 1994 will be continued during FY 1995 to identify the required interfaces and to include them within the integrated TWRS schedules. An interface control system will also be put in place to make sure that satisfactory and timely progress is made on the interface issues.

12. Pilot-scale testing facilities are not readily available on the Hanford site to meet identified testing needs. This can result in cost and schedule risks. Cọst risks involve insufficient resources to provide testing facilities if existing facilities cannot be utilized. Schedule risks involve delays in project definitive design and construction due to the inability to provide pilot-scale testing results with actual tank wastes. Current schedules show that the preparation and performance of pilot-scale testing is a critical path activity leading to definitive plant design and construction.

Mitigation: An evaluation of testing needs and strategy and an assessment of facilities to perform testing with actual wastes will be conducted in FY 1995. Pending the issuance of these evaluations, activities to support hot testing are planned which assume a facility location. This should reduce the cost and schedule impact for pretreatment based on the facility assessment report.

\subsection{Major Decisions}

Major decisions as identified by Systems Engineering are included in Section 7.8.1.1.4, Risks, since they are the basis of studies to mitigate the risks involved, and develop a consistent rationale for the decision required. Additional details on the more prominent decisions are summarized below.

\section{Determine Waste Processing Strategy (4.2.2):}

A strategy must be selected for processing the Hanford tank waste for disposal. The decision to select a waste processing strategy is predicated upon selecting retrieval of "all waste" from all Hanford radioactive tanks (or the majority of waste) as the preferred alternative for the decision "Determine Disposition of Hanford Tank Waste". A part of this decision is:

\section{Determine Waste Separations Process (4.2.2.1):}

A strategic alternative for separating Hanford Site tank waste into a low-activity and high-activity fraction for disposal has been selected.

Formal evaluation of this decision will be conducted through NEPA. A record of decision will be issued to document this decision. Public and stakeholder groups' participation in the selection and evaluation of alternatives will be obtained while preparing this environmental impact statement. 
Two alternative approaches considered for separation process include:

- Minimum separations using existing technologies

- $\quad$ Minimize immobilized HLW/TRU waste volume.

The fourth amendment to the Hanford Federal Facility Agreement and Consent Order (Tri-Party Agreement) specifies completion of the evaluation of enhanced sludge washing (i.e., minimum separations alternative) to determine whether advanced sludge separation processes (i.e., minimize immobilized HLW/TRU waste alternative) are required (Milestone $\mathrm{M}-50-03$ ). This evaluation is scheduled for completion by March 1998. Criteria and a decision methodology, which reflect public and stakeholder values, will be developed to aid in the evaluation of these two alternatives (Milestone M-50-03A) by September 1994.

Separation of Hanford tank waste into a low-activity and high-activity fractions using the minimum separations approach has been adopted as the planning basis for the TWRS Program.

The establishment of acceptable volume limits for disposal of immobilized HLW in a deep geological repository may necessitate incorporation of additional processes for separation of tank waste.

Geological repository volume limitations have not been established; thus, uncertainty exists as to the acceptability of either alternative process for separation of tank waste. Similarly, limits for acceptable radionuclides concentrations and volume of immobilized LLW have not been established. Therefore, uncertainty exists as to the acceptability of either alternative process for separation of tank waste.

The limits for acceptable radionuclides in the low-activity solution can be understood by conducting a parametric evaluation. Once the inter-relationship between the pretreatment process and the LLW disposal system environmental performance is understood, management of this uncertainty can be achieved.

\section{Select Supernate Pretreatment Facility Concept (4.2.2.1.1):}

The Tri-Party Agreement specifies a target milestone (M-60-03-T01) for completing an engineering study evaluating alternative TWRS facility concepts. The public will be provided access to this study and informed of its results. Solicitation of views from stakeholders and the public will be pursued. A recommended supernate pretreatment facility alternative will be based on all received comments and technical considerations.

Alternative facility concepts include:

- A standalone supernate pretreatment facility

- $\quad$ Combined with LLW vitrification facility

- $\quad$ Combined with HLW vitrification facility

- Combined with both vitrification facilities.

A standalone supernate pretreatment facility concept with centralized pretreatment components has been reflected in the TWRS planning basis. On July 18, 1994, WHC recommended to DOE to combine the supernate pretreatment process and the LLW vitrification process into a common facility and to have the solids/sludge HLW processing in a separate facility. 
The selection of the supernate pretreatment facility concept is inter-dependent on the selection of facility concepts for vitrification of both HLW and LLW (functions 4.2.2.2 and 4.2.3.1). As such, selection of facility concepts for all three functions should be made simultaneously, to ensure the interactions (or lack thereof) if separate facilities are considered.

Radionuclides Separation Requirements. The selected supernate pretreatment facility concept may not support deployment of all required candidate (minimum separations) radionuclides separations technologies. Cesium has been identified as the primary radionuclides requiring separations from the Hanford Site tank wastes. However, a thorough evaluation of all cesium separation processes has not been conducted. Production scale deployment of all candidate cesium separation processes may not be achievable in all candidate facility concepts. Additional radionuclides (e.g., technetium or strontium) may need to be separated from the Hanford tank wastes to achieve the desired environmental performance of the LLW disposal system. Production scale deployment of technetium and strontium separation processes may not be achievable in all candidate facility concepts (e.g., dispersed supernate pretreatment modules).

The approach to mitigating these concerns is to re-evaluate the selection of the supernate pretreatment facility concept after the selection of the cesium separation process is made and after the environmental performance of the LLW disposal system is understood. The decision criteria used to select a preferred supernate pretreatment facility concept will define acceptable parameters which all acceptable concepts must meet (i.e., solution space). If the selected cesium separation process or LLW vitrification facility concept causes the preferred supernate pretreatment facility concept to exceed the previously defined solution space, the supernate pretreatment facility concept decision must be re-evaluated. A similar re-evaluation is necessary after the environmental performance of the LLW disposal system is understood. Since the environmental performance of the LLW disposal system may not be defined for some time, the selected supernate pretreatment facility concept should be a robust concept, which allows for future addition of technetium (or other radionuclides) separation if deemed necessary.

\section{Determine System Generated Waste Disposal Strategy (4.2.3):}

A decision is needed whether the treatment and disposition of system generated waste (e.g. lowactivity fraction of tank waste, gaseous effluents, liquid effluents and solid waste) and excess facilities is part of the Tank Waste Remediation System (TWRS) scope. If treatment and disposition of system generated waste and excess facilities is not part of the TWRS scope, then this scope needs to be assigned to other Hanford Site programs. Determine Liquid Effluent Treatment Concept (4.2.3.3) is part of this strategy, and determines the strategy for liquid effluent treatment needs to be selected to establish necessary functions and interface requirements.

A decision must be made concerning Determine Waste Concentration Mechanism (4.2.1.5), whereby a process needs to be selected for reducing the volume of radioactive liquid wastes requiring storage in the Hanford Site underground radioactive liquid waste tank system. The TWRS Operations group has been assigned responsibility for this decision, however the Waste Pretreatment Program Element will interface with them on this issue. This decision will have an impact on the Program.

\subsection{Assumptions}

Several key assumptions have been made to assemble the Multi-Year Work Plan for the Waste Pretreatment Program Element. 
It is assumed that the current characterization information about the tank wastes is sufficient as a basis for pretreatment planning. The basis of this assumption is being validated by the Characterization Program Element in conjunction with meeting the requirements of Defense Nuclear Facilities Safety Board (DNFSB) Recommendation 93-5, which requires characterization of tank waste by 1997. In addition, plans are being implemented to acquire hot testing capabilities and sufficient quantities of actual waste for process development. If planned hot testing using actual waste is implemented on the current schedule, the risk of proceeding before this assumption has been validated is low.

It is also assumed that sludge washing, enhanced sludge washing, and blending will be adequate to achieve an acceptably low HLW volume and meet HLW feed composition requirements. Acid dissolution and advanced separations of sludge will not be required, nor will organic destruction for organic content reduction. This assumption is based on the selection of sludge washing as the lowest cost option evaluated in the Tank Waste Technical Options Report, WHC-EP-0616, Rev. 0. To further reduce the volume of HLW produced, caustic leaching of sludge (enhanced sludge washing) was included as part of the technical baseline in the Fourth Amendment of the Tri-Party Agreement in January 1994. The assumption that caustic leaching of sludge will result in an acceptably low volume of HLW is being validated by conducting aggressive laboratory process development testing in FY 1995-1998 using actual waste samples. In addition, a full-scale in-tank sludge washing demonstration in tank 241-AZ-101 (101-AZ) is planned for FY 1996-1997. The risk of proceeding with this assumption is minimal because of the aggressive development activities planned using actual waste and the relatively long lead time before HLW feed is needed (FY 2009).

For radionuclides to be removed in the LLW pretreatment facility, it is assumed to meet ALARA policy, that the feed stream specification to the LLW immobilization facility will be met if ${ }^{137} \mathrm{Cs}$ is reduced to $1 \mathrm{Ci} / \mathrm{m}^{3}$ in the resulting LLW glass and ${ }^{90} \mathrm{Sr}$ is removed when necessary. Removal of other radionuclides will not be required. This is an enabling assumption, which allows development of functions and requirements and conceptual design of the LLW pretreatment facility prior to having a final LLW vitrification feed specification. This assumption is being validated by the ongoing LowLevel Waste Program Element funded LLW disposal performance assessment (PA). To minimize the risk of proceeding with this enabling assumption, the Waste Pretreatment Program Element is developing technology for technetium and strontium removal until the LLW feed specification is finalized. In addition, the capability to remove technetium and strontium is planned for inclusion in the conceptual and definitive design of the LLW pretreatment facility. This decision will be reviewed prior to initiation of definitive design and again prior to start of construction.

Organic destruction will not be required to achieve an adequate level of safety for continued safe storage in the tanks. Funding of technology development for this application will cease at the end of FY 1994. In June 1994 a representative technology was selected for incorporation in the conceptual design of the Initial Pretreatment Module as a contingency should such a capability later be determined to be needed. This assumption is a change from last year's Work Plan and is based on the success of the tank 241-SY-101 mixer pump in mitigating the release of flammable gases and closure of the unreviewed safety question for the tanks containing unsafe quantities of ferrocyanide compounds. The risk of employing this assumption is that changes in the conceptual and definitive design would be needed and the completion of construction of the LLW pretreatment facility would be delayed if organic destruction became necessary. This risk is of short duration because Project W-236A, New DST Farm, will provide tank space to store waste from the highest risk flammable gas tanks by 1999. 
The TWRS Environmental Impact Statement and resulting Record of Decision will provide the required NEPA documentation for pretreatment of DST and SST wastes, will not significantly change the Tri-Party Agreement strategy and milestones, and will be timely to support construction of the necessary facilities.

\subsection{Constraints}

The lack of hot testing capabilities and supporting infrastructure constrain the availability of technical data based on process development testing using actual radioactive waste. To proceed into definitive design without this technical data will result in excessive program risk based on the recommendations of the Waste Pretreatment Sub-TAP, the A/E, Process Engineering, and others. The availability of technical data is being addressed by including a capability to perform hot testing in the Waste Pretreatment Program Element baseline. Acquisition of sufficient actual waste from tanks for use in hot testing is also included in the Waste Pretreatment and Characterization Program Elements' baselines.

\subsubsection{Structure}

The Waste Pretreatment Program Element work breakdown structure and organization and responsibility are provided in this section.

\subsection{Work Breakdown Structure}

The Waste Pretreatment Program Element work breakdown structure (WBS) for FY 1995-1997 is provided in Figure 7.8-2 down to the sixth level of work breakdown. It is assumed that the WBS will remain constant through the life of the program/project. The Waste Pretreatment WBS dictionary is located in Appendix A.

\subsection{Organization and Responsibility}

The Waste Pretreatment Program Office is responsible for providing direction and input to ensure the success of the Waste Pretreatment Program Element in support of the TWRS Program Mission to "Process Waste" (4.2.2). The Waste Pretreatment Program Element interfaces with other TWRS program elements, the Technology Development Program Office (TDPO), Efficient Separations Processing Integrated Program (ESPIP), Underground Storage Tank Integrated Demonstration (USTID) and others, to ensure that ongoing and planned activities are integrated.

The TWRS Program Office consists of nine program elements, all of which have interface ties with the Waste Pretreatment Program Element. The other eight program elements are:

- $\quad$ Tank Farm Operations and Maintenance provides support to in-tank sludge washing, enhanced sludge washing, and waste blending demonstrations as well as operational support needed to operate the LLW and HLW pretreatment facilities and in-tank processing once they are online. 
- Waste Tank Safety provides requirements for early pretreatment of waste stored in Watch List tanks should mitigation measures prove unsuccessful in providing adequate safety for continued storage of the waste. In addition, information is provided on factors that may impact future processing of the waste (e. g., chemical speciation, thermodynamic properties, existence of liquid organics).

$$
\because
$$

- Characterization provides actual waste sample material for process development, waste characterization data from ongoing tank sampling, and characterization information from historical data review. It is necessary for Waste Pretreatment to provide Characterization with data requirements (number of samples and types of analyses, and dates needed).

- Tank Farm Upgrades provides the infrastructure (pipe lines, diversion boxes, pump pits, Project 236-A, New Tank Farm) needed to support in-tank processing and operation of the LLW and HLW pretreatment facilities.

- Waste Retrieval plans the retrieval of tank waste based on input from Waste Pretreatment, as well as large samples of actual waste for pretreatment process development. Waste retrieval processes and the blending of waste from different tanks need to be defined and data compiled. Waste Retrieval will coordinate the recovery of tank waste material based on the requirements from the Waste Pretreatment, Low-Level Waste, and High-Level Waste Program Elements.

- The Low-Level Waste and High-Level Waste Program Elements provide the specifications for the waste feed and the immobilization facility requirements. Updates to those documents will need to be made as studies and tests are completed. A critical input to Waste Pretreatment is a LLW vitrification feed specification based the final performance assessment for disposal of LLW.

- Program Management and Administration provides overall integration of the TWRS program elements in preparing Multi-Year Program Plans, Multi-Year Work Plans, budget submittals, monthly and quarterly reviews. Program Management and Administration also supports the TWRS systems engineering effort (overall functional logic, program decisions, assessment of risk).

The following organizations provide direct support to Waste Pretreatment.

- TWRS Projects provides project management for capital construction projects needed to meet the Waste Pretreatment mission. This organization manages the $\mathrm{A} / \mathrm{E}$, procurement, and construction contractors for expense and capital construction activities and has the responsibility for the design, construction, and startup of the pretreatment facilities.

- WHC Process Engineering Center of Excellence and Systems Engineering provide for engineering support for trade studies, systems definition, functions and requirements, engineering evaluations, testing, and demonstrations. These engineering activities feed directly into the requirements needed by the Projects organization. These organizations also recommend work scope to be done by Waste Pretreatment to support decisions that need to be made within TWRS and Waste Pretreatment. 
- TWRS Technology Development Program Office (TDPO) is managed by Pacific Northwest Laboratory (PNL). The TDPO integrates the technology development activities required by Waste Pretreatment and other TWRS program elements; identifies technology needs and issues; develops strategies for addressing these issues; develops logic for implementing the strategies; and identifies alternate architectures for meeting these needs. This office also manages the tęchnology development work done by other contractors, national laboratories, and universities which support the Waste Pretreatment program element.

- $\quad$ Ebasco Services Inc./British Nuclear Fuels Ltd. (BNFL) is serving as the architect-engineer $(\mathrm{A} / \mathrm{E})$ for the LLW pretreatment facility, W-236B, Initial Pretreatment Module.

Organizations and programs that interface with Waste Pretreatment are:

- Solid Waste receives the secondary solid LLW wastes generated by the Waste Pretreatment in the course of performing technology development, process development and demonstration, and performance of pretreatment operations. Liquid effluents resulting from Waste Pretreatment activities will be received by the Liquid Effluents Program at the 200 Areas Effluent Treatment Facility. After Waste Pretreatment operations are completed, the facilities will be turned over to the appropriate organization for decontamination and decommissioning (D\&D). At this time, that responsibility is within the scope of the Environmental Restoration and Management Contract (ERMC), Bechtel.

- The Washington State Department of Ecology (Ecology), the U.S. Environmental Protection Agency (EPA), stakeholders, and advisory panels provide recommendations, comments and inputs to the Waste Pretreatment program element as to work scope, priorities, and strategies.

- The Waste Pretreatment program element responsibilities are included in the Responsibility Assignment Matrix (RAM) in Appendix B.

- The U.S. Department of Energy provides direction and approves decision making documents and recommendations prepared by the M\&O contractor.

\subsubsection{FY 1995 Work to be Performed}

The FY 1995 work scope is summarized in this section based upon the FY 1995-1997 Multi-Year Work Plan.

\section{Technical Integration and Planning (WBS 1.1.1.3.01.01)}

The integration of waste pretreatment activities, including program element management, planning, and pretreatment support activities, are included in this work plan. Program element management includes the development of baseline plans, schedules, and budgets for program business management activities. Support to technology working groups and oversight committees as well as various internal and external reviews will also be performed. In addition, program planning activities such as the development of the programmatic risk assessment and development of a basis of cost estimate study will be performed, based on staffing of plants at other DOE sites (eg., the Defense Waste Processing Facility [DWPF] at Savannah River, etc.). 
A major portion of this work plan includes technology and applied engineering work scope to provide technology development, evaluation, selection, and demonstrations. These activities support the pretreatment functions to pretreat LLW and HLW and to prepare separated radionuclides.

Studies continue on in-tank processing operations, focusing on sludge washing, sludge separations from solutions, and alkaline and acid leaching. Data from these test will be used in trade studies that determine the acceptability of enhanced sludge washing.

Laboratory studies on strontium and technetium removal using ion exchange and TRU removal using precipitation with ferric hydroxide will be conducted in FY 1995. Evaluation of cesium resin technology (CST) for the removal of cesium using ion exchange will also be conducted.

The development of technologies for destruction of organic complexants will be limited to LLW processing and is scheduled for completion in FY 1995. No work will be carried out for organic destruction for safety issue resolution or for treatment of sludge for potential HLW vitrification requirements.

Work will be initiated to evaluate non-thermal technologies for reconstituting tank sludges in order to remove inorganic chemical species and reduce the volume of feed to the HLW vitrification facility.

\section{Facility Operations (WBS 1.1.1.3.01.03)}

Ongoing waste pretreatment operations to separate HLW and transuranic wastes from LLW is also provided for in this work plan. No work scope is planned in this activity for the planning period FY 1995 - FY 1997. All preparation for initial startup of the LLW Pretreatment Facility in this time frame has been included in WBS 1.1.1.3.01.05.

\section{System Definition (WBS 1.1.1.3.01.04)}

An integral portion of this work plan is the system definition activity. FY 1995 work scope continues with engineering studies, development of functions and requirements, and development of the engineering basis for pretreatment projects.

Work will continue on developing functions and requirements for LLW and will begin on HLW and in-tank processing pretreatment processes and facilities, including facility studies for HLW. Work scope also includes identification and performance of engineering studies for conceptual design of the HLW pretreatment facility. Engineering support will be provided for the evaluation and implementation of in-tank washing, including trade activities and technical evaluations.

Process studies will continue and process flowsheets will be prepared for pretreatment of tank wastes to provide feed streams to the LLW and HLW vitrification plants and for removing either radionuclides or chemicals from supernatant or sludges. 


\section{W-236B LLW Pretreatment Facility (WBS 1.1.1.3.01.05)}

Work will begin on the development of the conceptual design report for the LLW pretreatment facilities and on the Preliminary Safety Evaluation (PSE) for the LLW Pretreatment Facility Module I. Work scope associated with project engineering, project management, safety analysis, and permitting support for, these activities will continue.

To support design activities, additional applied engineering will be performed to develop engineering information required to support the architect-engineer. In FY 1995 laboratory-scale testing will be performed to supply information that will support process and equipment selection and sizing. In addition, initial phases of larger-scale hot testing preparation will be initiated. This testing will support detailed project design, but due to long lead times in hot plant design and construction, these activities must be initiated well in advance of the need date. The design may include in its scope, a standalone facility and/or a processing module or series of modules which may be housed in existing facilities or as a standalone facility. Initially the project will issue reports to identify testing needs and then will follow up with an assessment of DOE facilities and their applicability to support hot testing. Expected hot testing capabilities would include the ability to perform continuous pretreatment process tests, such as ion exchange, with actual tank wastes. Additional test modules would be able to check performance of individual unit operations or whole processes as required to support project engineering data needs.

The Cesium Demonstration Unit (CDU) design is discontinued. However, portions of this design may be utilized to support hot testing needs.

\section{HLW Pretreatment (WBS 1.1.1.3.01.06)}

This work plan also includes activities to develop the facilities required to provide pretreated HLW feed to the HLW immobilization facility. This includes facility design, construction, and startup; and technology application for HLW pretreatment. HLW pretreatment may require an advanced sludge washing process, an organic destruction process, a strontium removal (acid) process, a sludge leaching process and/or a sludge sorption process. Facilities to house and safely confine these processes are required as well as utility and offgas systems to operate the processes.

\subsubsection{FY 1996 - FY 1997 Work to be Performed}

The FY 1996-1997 work scope is summarized in this section based on the FY 1995-1997 Multi-Year Work Plan.

\section{Technical Integration and Planning (WBS 1.1.1.3.01.01)}

The integration of waste pretreatment activities, including program element management, planning, and pretreatment support activities, continues in FY 1996 and 1997. Program element management includes the development of baseline plans, schedules, and budgets and for program business management activities. Support to technology working groups and oversight committees as well as various internal and external reviews will also be performed. In addition, program planning activities, such as the update to the programmatic risk assessment and program plan, will be performed. Activities to support the development of a planned cost estimating model and historical data base for use in long-range planning for TWRS will continue. 


\section{Technology Development and Applied Engineering (WBS 1.1.1.3.01.02)}

Studies continue on the separation of radionuclides, specifically studies on cesium removal from alkaline solutions by ion exchange (IX), using laboratory batch and column tests of CST IX resins with simulated waste and laboratory-scale column tests using actual wastes. Evaluation of CSTs is scheduled for completion in FY 1997. Studies also continue on strontium and technetium removal by ion exchange using batch and column tests with simulated wastes. Column tests using actual wastes with candidate IX resins will be performed in the laboratory. Carrier precipitation laboratory tests using actual wastes will be performed to investigate the removal of TRU from alkaline supernatant.

Studies on in-tank processing processes to develop and evaluate various solid liquid separations alternatives, including separation of sludge from solutions, sludge washing, and alkaline and acid leaching, continue from FY 1995. Data from these tests will be used in trade studies that determine the acceptability of enhanced sludge washing.

Work scope continues on the evaluation of non-thermal technologies for reconstituting tank sludge. Large-scale equipment tests of calcination processes will be used to evaluate thermal reconstitution of sludge using synthetic waste, while non-thermal reconstitution processes (acidification and reneutralization) of sludge will be evaluated using laboratory-scale tests with actual waste.

Nitrate destruction, which would be used to generate a caustic soda stream to be recycled for alkaline leaching, will be pursued in FY 1997.

\section{Facility Operations (WBS 1.1.1.3.01.03)}

Work scope associated with preparation for startup of the LLW pretreatment facility Module I is covered in WBS 1.1.1.3.01.05. There is no work scope in this activity for the planning period FY 1995 - FY 1997. Support to preparation for AZ-101 processing activities are included in WBS 1.1.1.3.01.04.

\section{System Definition (WBS 1.1.1.3.01.04)}

In FY 1996 and FY 1997 System Definition work scope continues with activities to develop functions and requirements for LLW, HLW, and in-tank processing pretreatment processes and facilities, including engineering studies, process and facility design criteria, and applicable technology development needs.

The study of alternate facility configuration options for HLW pretreatment and vitrification processes continues. Included in this study are design advantages and disadvantages of the various options and recommended preferred options. In addition, assessments of specific systems, subsystems, and component implementations will be conducted to optimize plant performance and adapt to changing operating conditions. Evaluation of alternative methods of sludge washing is included in this study in FY 1996.

Work also continues on the preparation of process flowsheets to provide feed streams to the LLW and HLW vitrification plants and for removing radionuclides or chemicals from supernatant liquids or sludges. Ongoing pretreatment technology development work will be followed to ensure that the information needed for the process studies and process flowsheets is obtained. 
Proposed new technologies for the pretreatment of Hanford tank waste will be evaluated and incorporated into the appropriate portion of the pretreatment process strategy or rejected. The proposed technologies will be evaluated technically using data from EM-30 and EM-50 development work to prepare process flowsheets and perform engineering trade studies. The process flowsheets will show how effective the proposed technology is at performing the desired separation. The engineering trade studies will evaluate alternative technologies and processes to see which satisfy pretreatment functions and requirements and compare their performance based on facility and operating costs, secondary waste generation impact, implementation schedule, and other criteria.

Further development will be pursued for feasible and attractive technologies until sufficient technical maturity is reached to recommend use of the technology or until an alternate technology is chosen. Recommended processes and technologies will be incorporated into the TWRS Reference Flowsheet and into functions and requirements documents, both of which guide pretreatment and project conceptual and title design efforts.

\section{W-236B LLW Pretreatment Facility (WBS 1.1.1.3.01.05)}

Conceptual design activities for the LLW Pretreatment Facility, begun in FY 1995, will be completed in FY 1996 and advanced conceptual design activities are initiated and scheduled for completion in FY 1996. The PSE for the LLW Pretreatment Facility will also be completed. In FY 1997 Title I design for the LLW Pretreatment Facility will be initiated along with the Preliminary Safety Analysis Report (PSAR).

Project engineering, project management, safety evaluation, and permitting support for these design activities continue in FY 1996 and FY 1997, to include operations support of facility designs and initiation of preparation of operations documentation and training preparation.

Facility design, procurement, and installation for hot testing will continue during this time frame. Cesium ion exchange testing facilities should be completed during FY 1997. Additional testing capabilities for solid/liquid separations and for other radionuclide separations will be completed after FY 1997. These testing facilities will satisfy applied technology needs involving continuous testing on simulated and actual tank wastes.

Laboratory-scale testing of LLW pretreatment processes will continue to investigate process and equipment operations over a variety of tank waste types. Whereas FY 1995 testing activities will concentrate on early expected feeds to LLW pretreatment, FY 1996 and FY 1997 activities will include testing of additional waste types such as SST saltcake liquors and the products from sludge washing.

\section{HLW Pretreatment (1.1.1.3.01.06)}

Conceptual design activities, including trade studies to support the preparation of facility design and technology application for HLW pretreatment, continue in FY 1996 and FY 1997. The processes currently expected for HLW pretreatment are sludge washing and caustic leaching. Work will also begin on the preparation of the preliminary safety evaluation for the HLW pretreatment facility. This activity includes preliminary hazard identification and assessment and evaluation of the barriers for the identified hazards. 
WHC-SP-1101

\subsubsection{Approach}

This section provides a summary of the technical approach, method of performance, and acquisition strategy for achieving the Waste Pretreatment mission.

Technical Integration and Planning (WBS 1.1.1.3.01.01)

TWRS contractor staff will be used to manage the program element and program activities. Contract staff will be used as needed to supplement the contractor staff for completing specified deliverables (e.g., program plans, special crosscuts, cost estimating validation). Specialized tasks, such as public involvement support and program risk assessments, will be contracted to qualified contractors.

\section{Technology Development and Applied Engineering (WBS 1.1.1.3.01.02)}

Technology development for the TWRS Program is managed by the TWRS TDPO. Technology development work scope is contracted to various National Laboratories by the TDPO. These include Pacific National Laboratory; Los Alamos National Laboratory; Sandia National Laboratory; and Argonne National Laboratory. Waste Pretreatment Program Element technology development is managed by TDPO staff assigned to Waste Pretreatment.

\section{Facility Operations (WBS 1.1.1.3.01.03)}

The MYWP is based on the assumption that the LLW pretreatment, HLW pretreatment, and in-tank processing facilities will all be operated by the M\&O contractor staff. An option under consideration is that these facilities would be operated and maintained by a private commercial contractor instead of a government contractor.

\section{System Definition (WBS 1.1.1.3.01.04)}

The method of performance of this activity is to use the contractor "Center of Excellence" Process Engineering organization to provide technical recommendations to the program and project organizations. This is accomplished through issuance of trade studies, functions and requirements, flowsheets, and technical evaluations. Contractor assigned staff are used to perform key tasks. Onsite and offsite contractors are used to supplement contractor staff on larger tasks, when needed.

\section{W-236B LLW Pretreatment Facility (WBS 1.1.1.3.01.05)}

This activity is managed by a contractor project organization. Design is planned to be performed by an offsite $\mathrm{A} / \mathrm{E}$ and construction by an offsite construction contractor. An option under consideration is that the design, construction, and operation of the LLW Pretreatment Facility would be performed by a single private commercial contractor.

\section{HLW Pretreatment (WBS 1.1.1.3.01.06)}

This activity will be managed by a contractor project organization. Design is planned to be performed by an offsite $\mathrm{A} / \mathrm{E}$ and construction by an offsite construction contractor. An option under consideration is that the design, construction, and operation of the HLW pretreatment facility would be performed by a single private commercial contractor. 


\subsubsection{Drivers and Deliverables}

The key drivers for the Waste Pretreatment Program which must be considered for the Program Element decisions include health and safety issues; Tri-Party Agreement milestones; Federal and State requirements and related DOE Orders; performance assessment requirements; stakeholder values; other external oversight groups; and cost.

- Health and Safety Issues. Safe operations and elimination of urgent risks or any inherent threats to the public or Hanford employees is the top priority of the TWRS Program. This priority drives Waste Pretreatment Program Element efforts to reduce and eliminate safety problems in the waste pretreatment process.

- Tri-Party Agreement Milestones. Hanford Federal Facility Agreement and Consent Order, Fourth Amendment, Washington State Department of Ecology, U.S. Environmental Protection Agency, and U.S. Department of Energy, Olympia, Washington, January 1994, Milestones M-50-00, "Complete pretreatment processing of Hanford Tank Waste, December 2028."

- Federal Requirements, their State counterparts, and related DOE Orders. These may directly or indirectly affect the Waste Pretreatment Program. Some of the key requirements that may impact Program activities include WAC 173-400, "General Regulations for Air Pollution Sources;" 40 CFR 264.xxx, "Miscellaneous Units, Environmental Performance Standards," "Miscellaneous Units, Monitoring, Analysis, Inspection, Response, Reporting, and Corrective Action," and "Miscellaneous Units, Post-Closure Care;" 10 CFR 260-270, "RCRA Hazardous Waste Regulations;" the National Environmental Policy Act (NEPA); DOE Order 6430.1A, General Design Criteria; and other Federal, State, and county permitting and waste minimization requirements. These requirements are detailed in the Tank Waste Remediation System Functions and Requirements (DOE/RL-92-60, Rev. 1, 31 March 1994).

- Performance Assessment Requirements. DOE Order 5820.2A requires that a site-specific radiological performance assessment be conducted for a LLW disposal site to demonstrate compliance with performance objectives.

-

Stakeholder Values. Stakeholder concerns about the approaches taken on various issues need to be considered in the decision making process. Stakeholder involvement enhances technology acceptance.

- Other External Oversight Groups. These groups include the HLW Tanks Advisory Panel (TAP), Government Accounting Office (GAO), National Academy of Sciences (NAS), Hanford Advisory Board (HAB), and others.

- Cost. Competing demands for limited resources (budget constraints) are a reality. The current cost projection for cleaning up the Hanford site is very high, and an aggressive initiative to achieve productivity gains, improve products and services, and reduce costs is required. An additional cost driver is the anticipated cost of disposing of HLW in a national geological repository, which is expected to cost significantly more per unit volume than nearsurface disposal of LLW at the Hanford Site. 
Deliverables are listed below.

Technical Integration and Planning (WBS 1.1.1.3.01.01)

- $\quad$ Prepare Waste Pretreatment program element inputs to ADS budget submittals

- $\quad$ Prepare Waste Pretreatment facility operations cost study

- Develop and update the Waste Pretreatment programmatic risk assessments

- Prepare updates to the Multi-Year Program Plan

- Prepare updates to the Multi-Year Work Plan

- $\quad$ Prepare updates to the Waste Pretreatment Program Plan.

Technology Development and Applied Engineering (WBS 1.1.1.3.01.02)

- Report on non-thermal sludge reconstitution

- Report on laboratory tests of heat and digest process for in-tank complexant destruction

- Report on batch and column testing of candidate resins for strontium and technetium removal using actual and simulant waste

- Report on carrier precipitation laboratory tests

- Delivery of engineered form of CST to PNL from UOP

- Report on sludge dissolution of actual sludges

- Report on results of continuation bench scale solvent extraction tests

- $\quad$ Report on batch CST IX media for cesium removal using actual waste

- $\quad$ Report on sludge washing and alkaline leaching of actual waste

- $\quad$ Report on sludge settling of actual waste.

\section{Facility Operations (WBS 1.1.1.3.01.03)}

There are no deliverables for this Activity level of the WBS in the planning window of FY 1995-FY 1997. 


\section{System Definition (WBS 1.1.1.3.01.04)}

- $\quad$ Functions and requirements for Hot Testing, LLW Pretreatment Facility, HLW Pretreatment Facility, and In-Tank Processing

- Evaluation of enhanced sludge washing to determine if advanced sludge separations processes are required

- $\quad$ Complete AZ-101 Sludge Washing Process Test and Issue Report

- $\quad$ Trade studies to support process and facility selection

- Flowsheets for $\mathrm{Cs}, \mathrm{Sr}, \mathrm{Tc}, \mathrm{TRU}, \mathrm{Al}, \mathrm{Cr}, \mathrm{PO}_{4}$ removal, for sludge washing, for enhanced sludge washing associated with selected tanks, waste groups, and overall.

- $\quad$ Preliminary Retrieval Sequence Report Issued, Sequences a \& b selected.

- Issue Prototype Decision Model for Waste Pretreatment Alternatives.

- $\quad$ Select Supernate Pretreatment Facility Concept

- Determine Liquid Effluent Treatment Concept

W-236B Pretreatment Facility (WBS 1.1.1.3.01.05)

- $\quad$ Prepare Preliminary Safety Evaluation (PSE)

- Complete Conceptual Design and initiate Definitive Design of LLW Pretreatment Facility

- $\quad$ Prepare project validation package

- Submit request for Project Authorization.

- Issue the IPM Quality Assurance Program Plan (QAPP)

- Issue approved Project Specific Training Plan

- $\quad$ Produce LLW Pretreatment Project Design Criteria (PDC)

\section{HLW Pretreatment (WBS 1.1.1.3.01.06)}

- $\quad$ Prepare Preliminary Safety Evaluation (PSE)

- $\quad$ Complete $60 \%$ conceptual design.

- Issue RFQ for Publication in Commerce Business Daily

- $\quad$ Select HLW Pretreatment A/E 
WHC-SP-1101

Additional deliverables are detailed on the Level 6 WBS Dictionary Sheets in Appendix A.

\subsubsection{Schedule}

\subsubsection{Waste Pretreatment Summary Schedule}

Refer to Figure 7.8-3.

7.8.2.2 Waste Pretreatment Expanded Baseline (FY 1995 - FY 1997)

Waste Pretreatment expanded baseline for FY 1995 - FY 1997 is located in Appendix C.

\subsubsection{Resource Requirements}

\subsubsection{Cost}

\subsection{Basis of Cost Estimates}

\section{Technical Integration and Planning (1.1.1.3.01.01)}

The cost estimate for this activity is based on historical data from FY 1993 and FY 1994 for resource quantity and type. The resource usage profile is based on an estimate of the apportionment of these resources to the work scope within the cost accounts based on FY 1994 resource usage and Waste Pretreatment Program Office changes planned for in FY 1995-FY 1997.

\section{Technology Development and Applied Engineering (1.1.1.3.01.02)}

Cost estimates for this activity for the FY 1995 - FY 1997 period are based on contractor proposals to perform the work scope identified in the Request For Proposals. Some work scope estimates are based on past experience and identified as planning packages awaiting the evaluation of competitive bids from the various performing contractors. The proposals are costed at the subtask level, which are two or more levels below the Cost Account (Level VI). This permits using a "building block" approach for estimating the resources needed to accomplish the work scope for a given Cost Account.

\section{Facility Operations (1.1.1.3.01.03)}

Cost estimates for this activity are based on historical operations costs of B Plant, PUREX, and the 242-A Evaporator. A study will be conducted in FY 1995 within the Technical Integration and Planning activity to review present operations cost estimates in order to incorporate new facility concepts and technology improvements. Results of this study may show that outyear operating cost estimates need to be revised. 
Cost estimates for this activity are based on engineering judgement, perceived complexity of the work scopes, and past experience. Where comparable work scope was previously performed, estimates are based on the resource requirements from prior years. Estimates for work scope performed by other performing contractors or an $\mathrm{A} / \mathrm{E}$ are based on the proposals or detailed estimates provided.

\section{W-236B Pretreatment Facility (1.1.1.3.01.05)}

Cost estimates for this activity are based on the Kaiser Engineers Hanford report to WHC, Job No. W-236B/ER2535, File No. W236B2, 05/26/93.

\section{HLW Pretreatment (1.1.1.3.01.00)}

Work scope to be performed in the period covered by this work plan will be conducted within the projects department (PSE, CDR). Cost estimates for this work scope are based on estimates of like work being performed for the LLW Pretreatment Facility (Project W-236B). Cost estimates for work scope beyond FY 1997 are based on the case beta assumptions from the Multi-Year Program Plan.

\subsection{Total Program Element Cost by Year by Fund Type by Activity and Cost Account}

Table 7.8-1 establishes the baseline cost for this program element. The baseline cost is exhibited in annual costs for FY 1994 through FY 2000, and 5-year totals for FY 2001 through program element completion. Where the data are available, these baseline costs are exhibited at the cost account level with fund type totals provided. Cost data are then rolled up to the activity and program element levels. At present, the program element cost baseline is the program element cost totals exhibited on this table less a productivity challenge for FY 1995 through FY 1997.

\subsection{FY 1995 Cost by Month by Fund Type by Activity and Cost Account}

Future updates of the MYWP will include Table 7.8-2, which establishes the month-by-month cost baseline for FY 1995 for this program element. The FY 1995 cost baseline is exhibited at the cost account level with fund type totals provided. Cost data are then rolled up to the activity and program element levels. Also exhibited is the total cost baseline for the program element for FY 1995. At present, the program element cost baseline for FY 1995 for this program element is the total provided on this table less a productivity challenge for FY 1995.

\subsubsection{Staffing}

\subsection{Basis of Staffing Projections}

\subsection{Total Full-Time Equivalents by Year by Activity by Major Participant (Direct and Indirect)}

Future MYWP updates will include Table 7.8-3, which establishes total staffing projections for this program element. Included in the table are FY 1994 staffing data as well as projections by year for FY 1995 through FY 2000. The projections in this table include all direct and indirect Full-Time Equivalents (FTEs), including all subcontractor personnel. Where data are available, projections are exhibited at the activity level with major program participant totals provided. 


\subsection{FY 1995 through FY 1997 Direct Full-Time Equivalents by 88 Labor Categories}

Future MYWP updates will include Table 7.8-4, which establishes direct WHC staffing projections for this program element. Projections do not include subcontractor support and are provided by 88 labor categories for FY 1995 through FY 1997.

\subsubsection{Work Scope Carryover}

\section{Technology Development and Applied Engineering (WBS 1.1.1.3.01.02)}

- Radionuclides Separations. Separations will be performed which are essential to the success of the extensive separations option. The tests will be conducted on 10- to 20-gm portions of actual Hanford tank sludge. Also, alternative methods for separating TRUs from acidified dissolved Hanford tank sludges will be performed. This involves small-scale testing of TRU separation processes using both waste simulants and actual waste. The data generated in this task will support the decision on sludge pretreatment strategy to be made in December 1998. Work scope to be carried over involves one test of the alternative TRU extractant DHDECMP and several tests using TRUEX and SREX extractants. (\$100K)

- Sludge Dissolution Laboratory Studies. Small scale (1-2 g of sludge) tests will be performed to determine the conditions required to dissolve the tank sludges for processing. The data generated in this task will support the decision on sludge pretreatment strategy to be made in March 1998 . Work scope to be carried over ( $\$ 150 \mathrm{~K})$ includes all work using radioactive wastes examining sludge dissolution approaches to waste treatment.

- Sludge Pretreatment Chemistry Evaluation. This task will evaluate available data and provide analysis of sludge samples generated in the Sludge Treatment Technology Development tasks to interpret sludge chemical and dissolution/leaching characteristics. Efforts in FY 1994 focused on activities that (1) evaluate existing data for determining sludge wash factors and any dissolution trends, and (2) analyze samples and data for interpreting results from wash/leach tests performed in the Sludge Treatment Technology Development tasks. Work scope to be carried over $(\$ 30 \mathrm{~K})$ includes analysis of the sludge wash factors and dissolution trends of existing data.

Hydrothermal Processing Development. This activity includes laboratory-scale experiments to determine chemical kinetic and thermodynamic data needed for reactor design and operation of both a high temperature hydrothermal process (LANL) and a low temperature hydrothermal process (PNL), and the assembly and testing of a 300-gallon-per-day pilot-scale high temperature hydrothermal processing unit. The 300-gallon-per-day unit will be used to evaluate process performance, design and control, and component corrosion, wear and maintainability. A smaller pilot system will be assembled and used to process radioactive simulants. A process model and process control system will be developed and integrated to produce a process simulator. A Research, Development, and Demonstration (RD\&D) application will be prepared for the State of New Mexico for the use of high temperature hydrothermal processing to treat mixed wastes. This activity was deleted because it was decided not to pursue this technology at this time. Costs incurred to close out and shut down and dismantle the pilot-plant $(\$ 405 \mathrm{~K})$ are being deferred to FY 1995. Close out costs of the PNL laboratory work are estimated to be $\$ 100 \mathrm{~K}$. 


\section{System Definition (WBS 1.1.1.3.01.04)}

- Calcination/Dissolution Equipment Development. This activity was to conduct a test of alternative thermal reconstitution technology vendor equipment with simulant Hanford tank waste. Vendor proposals had higher costs and longer schedules than originally planned. Due to FY 1994 budget constraints, the contract to do the work was not able to be placed and the work scope (\$400K) is deferred to FY 1995.

- Tank 101-AZ Decant Pump. This activity included purchase, design for the pump and jumper to support installation of a decant pump in Tank 101-AZ. Due to Operations constraints, the field support personnel will not be available to install the pump in FY 1994. Work scope associated with installation of the pump (\$100K) is deferred to FY 1995.

All carryover work listed here is planned to be funded and completed in FY 1995 unless noted otherwise on the Level 6 WBS Dictionary sheets included in Appendix A.

\subsubsection{Waste Type Data}

Except for very small quantities of tank waste supplied to laboratories for process development testing, no waste will be generated in the FY 1995-1997 time period. The amount of actual waste sample material used for laboratory testing will vary from less than 10 gal. to 20 gal. per year during FY 1995-1997. This amount will increase to 100 gal. or more per year starting in FY 1998 when bench/pilot-scale hot testing is scheduled to start. The waste will be returned to the underground waste tanks at the completion of testing, where it will be stored until it is retrieved for pretreatment. No new liquid LLW or HLW will be generated from these activities; however, small amounts of solid LLW and low-level mixed waste (LLMW) will be generated. It is assumed that laboratory equipment will be decontaminated to remove sufficient TRU material to permit disposal as LLW/LLMW.

During the period FY 1998-2025 all of the waste stored in the SST and DSTs will be retrieved, pretreated, and vitrified as either a LLW or HLW. No new HLW is expected to be generated because it is assumed that no fuel reprocessing will take place. All of the liquid LLW/LLMW generated as a result of pretreatment activities will be routed to the LLW pretreatment facility for removal of radioactivity (primarily cesium). The resulting liquids will then be sent to an evaporator for volume reduction and additional cleanup and then transferred to the 200 Areas Effluent Treatment Facility for final cleanup prior to disposal to the soil column. It is assumed that failed equipment will be decontaminated to remove sufficient TRU material to permit disposal as LLW/LLMW. Estimates of solid LLW/LLMW, hazardous waste, and sanitary waste are not available. No asbestos waste is expected to be generated.

The following processing rates and amounts are based on the integrated "TWRS Process Flowsheet", WHC-SD-WM-TI-613, Rev. 0, dated June 29, 1994. The liquid effluent values are the totals for all disposal operations (waste retrieval, waste pretreatment, LLW vitrification and HLW vitrification).

- Waste Pretreatment Processing Rates: 116,500 1/day liquids and 3,365 kg/day solids

- Waste Pretreatment Totals: 595 million liters of 5 molar sodium liquids and 17.2 million $\mathrm{kg}$ solids 
WHC-SP-1101

- Liquid Effluent Treatment Rate: 250,00 1/day

- $\quad$ Liquid Effluent Total: 1.24 billion 1 of process condensate.

\subsubsection{Revisions •.}

In future updates of the MYWP, this section will include a summary of the revision that has occurred since the last issue of the MYWP. 


\section{T.W.R.S TWRS Function Tree}

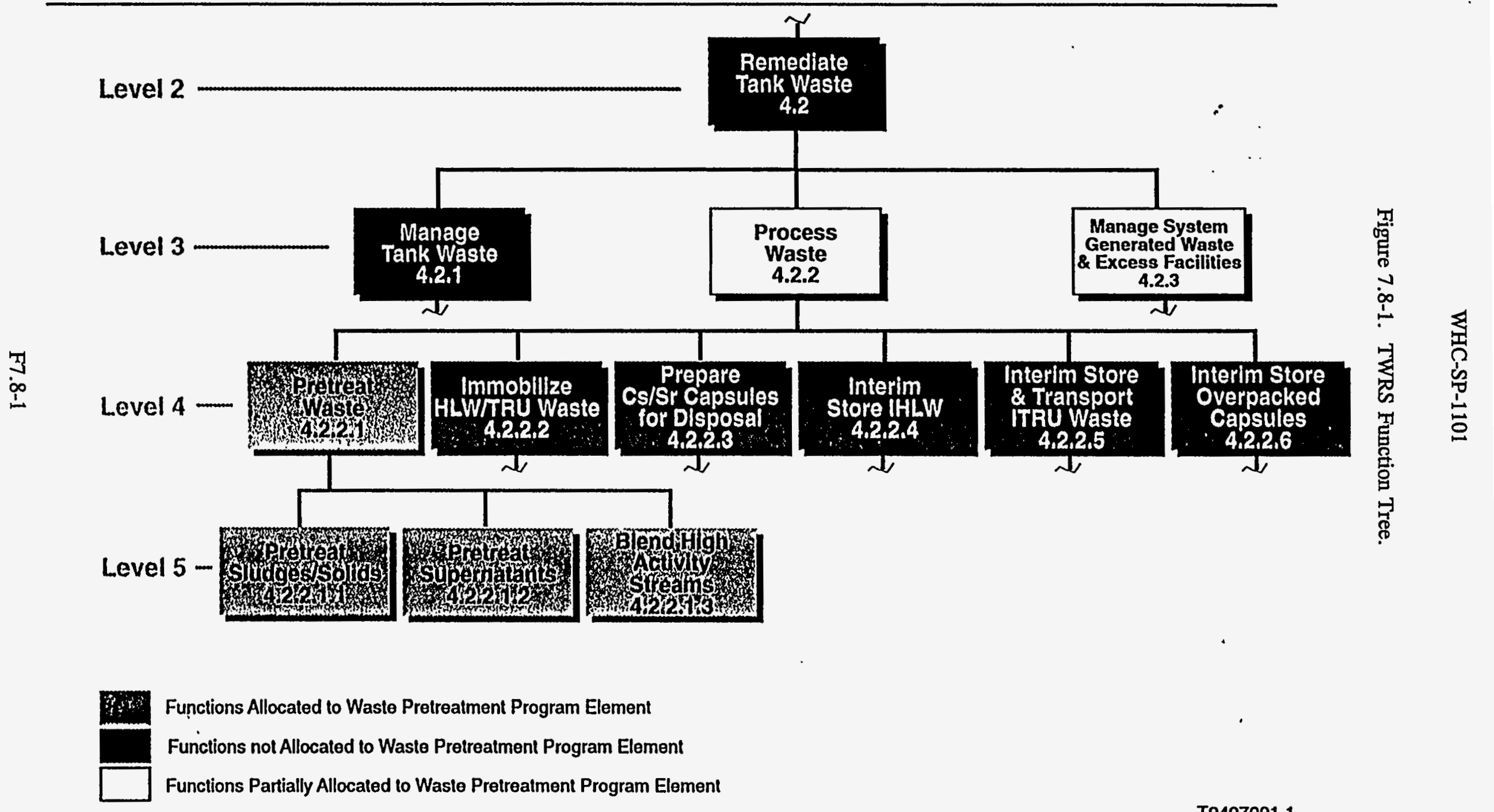

T9407001.1 
3

心
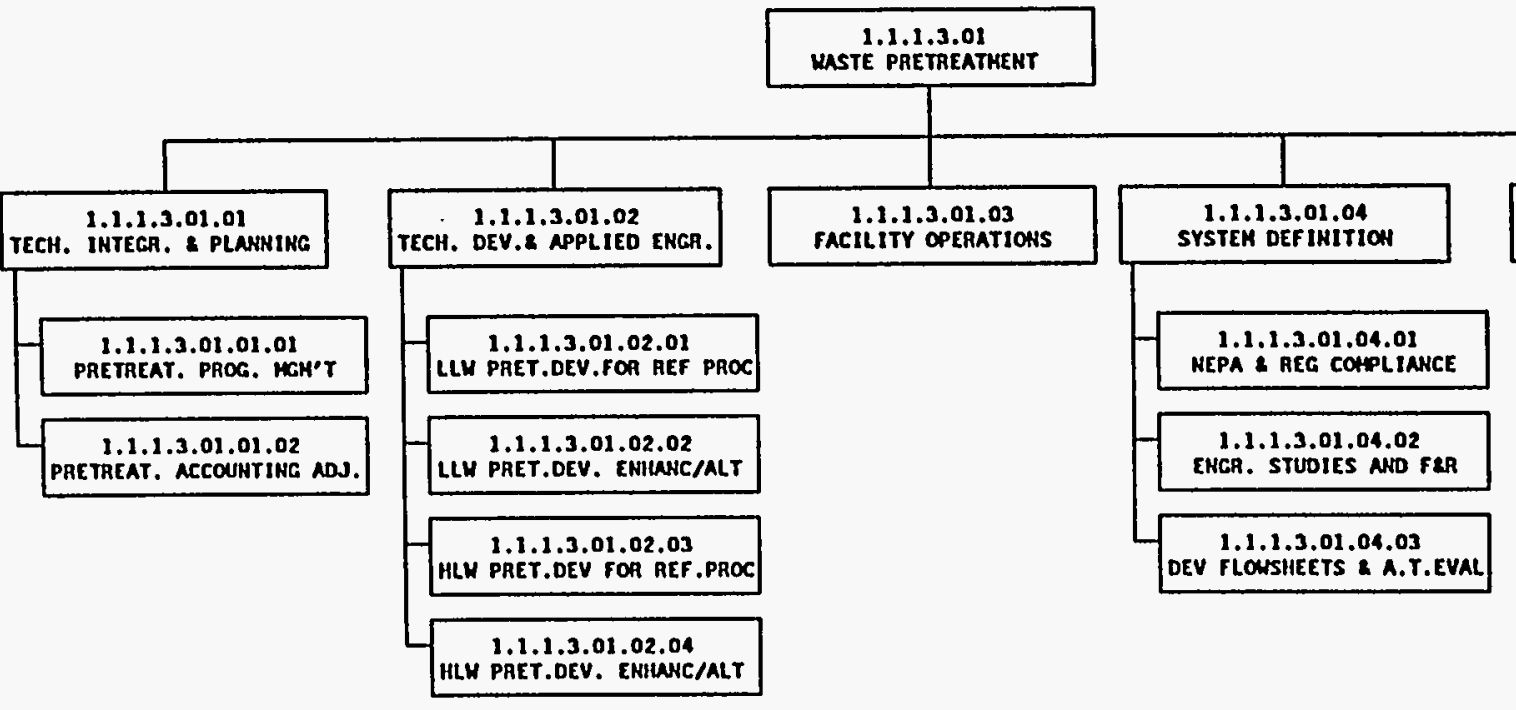

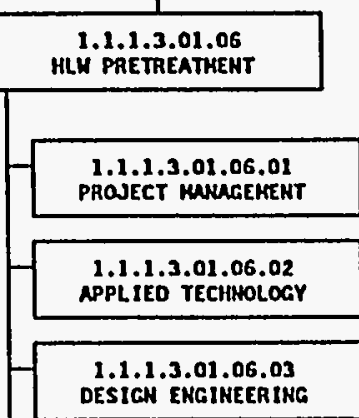

1.1.1.3.01.06.04

CONSTRUCTION

1.1.1.3.01.06.05
START-UP

1.1.1.3.01.06.06

1.1.1.3.01.06.06 REG COMPL. S SAFETY 
WASTE PRETREATMENT (PAGE 2 OF 2)

HORK BREAKDOWN STRUCTURE

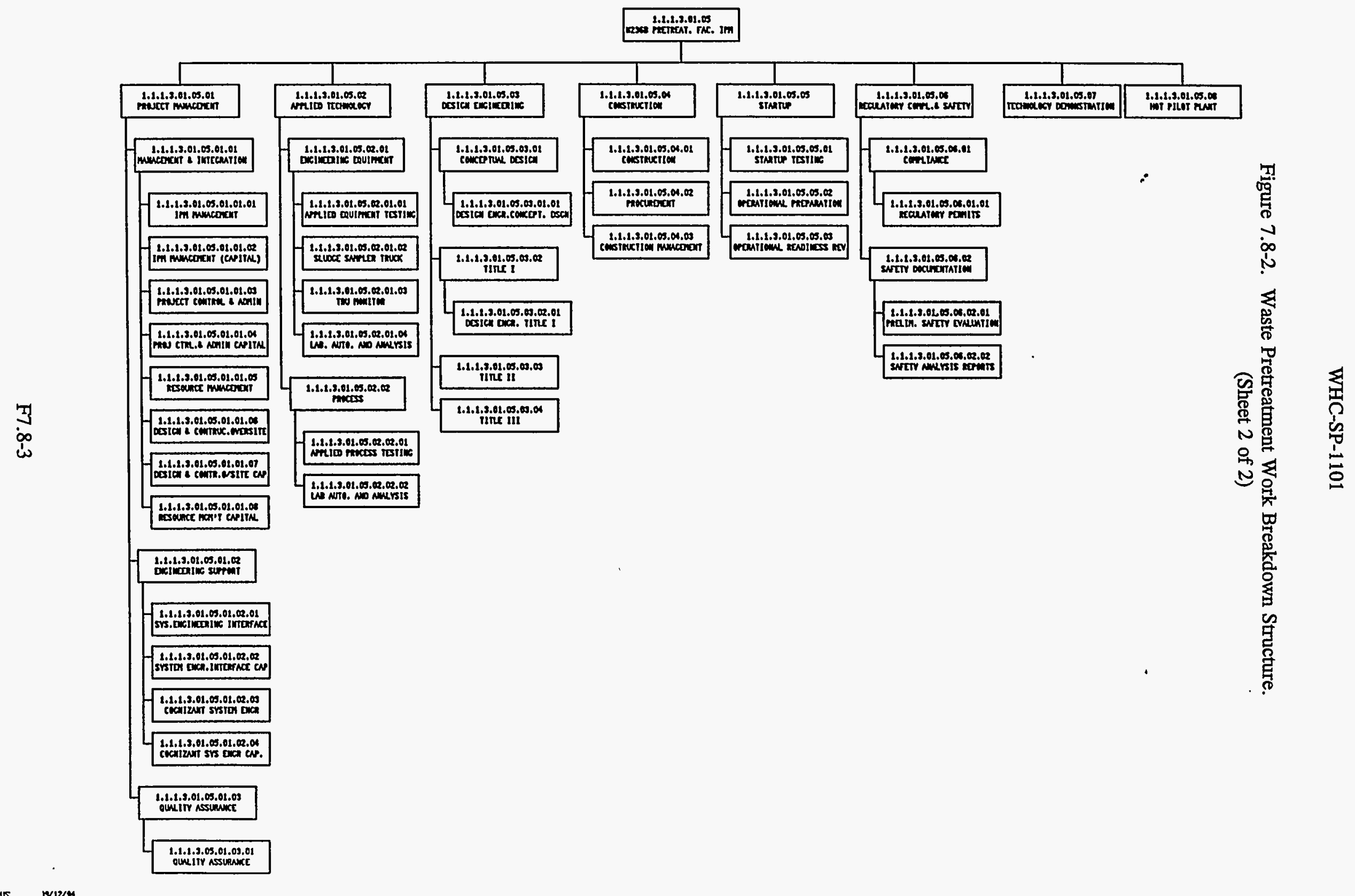


1.1.1.3.01 Waste Pretreatment Summary Schedule

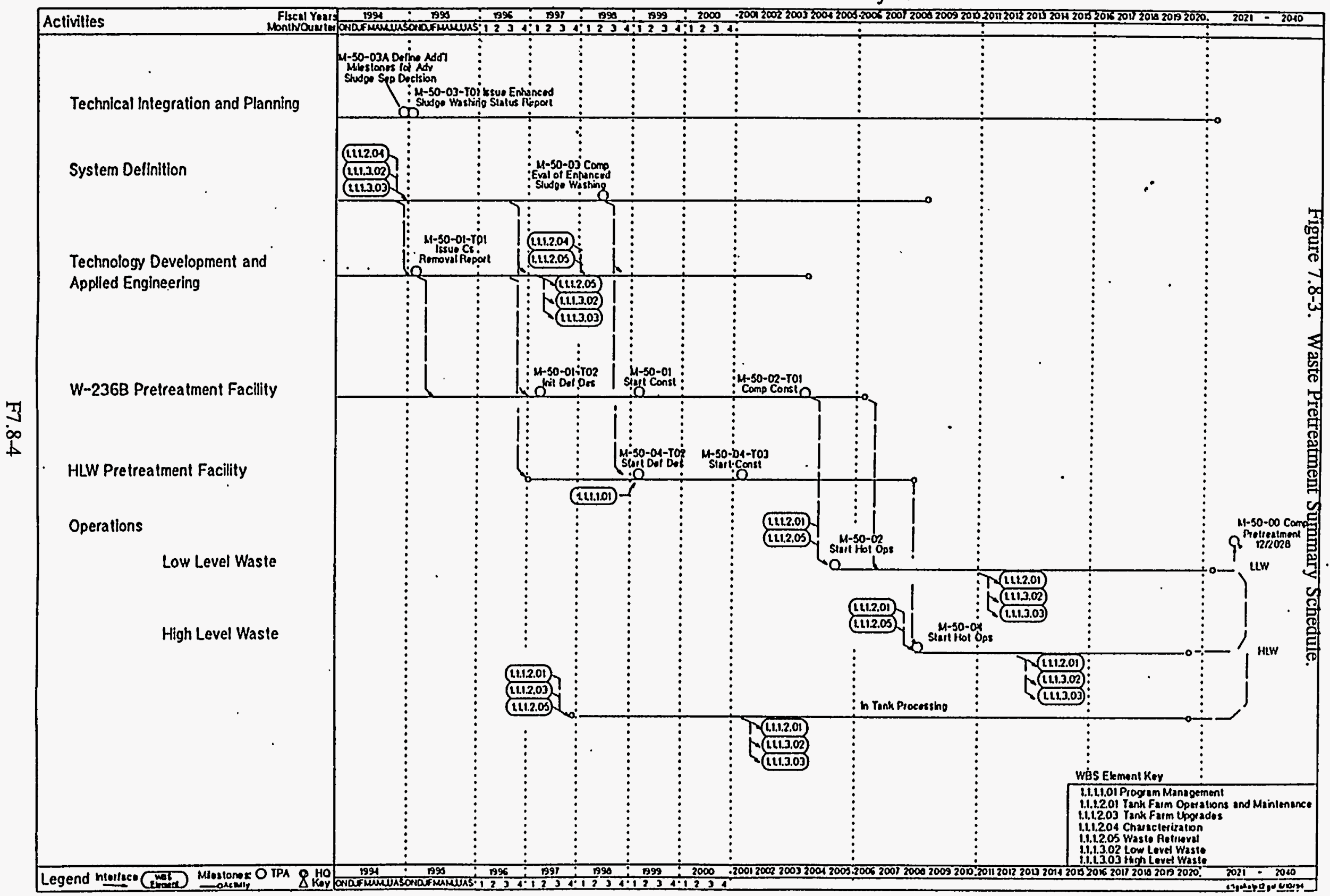


TABLE $7.8-1$

TOTAL PROGRAM ELEMENT COST BASELINE - BY YEAR

\begin{tabular}{|c|c|c|c|c|c|c|c|c|c|}
\hline WBS / LEVEL & ACTIVITY TITLE / ADS & $\begin{array}{l}\text { FUND } \\
\text { TYPE }\end{array}$ & $\begin{array}{l}\text { TOTAL } \\
\text { FY94 }\end{array}$ & FY95 & FY96 & FY97 & FY98 & FY99 & FYOO \\
\hline \multirow[t]{2}{*}{$\begin{array}{l}\text { 1:1.1.3.01 } \\
\text { Level IV }\end{array}$} & $\begin{array}{l}\text { Waste Pretreatment } \\
\text { ADS 1220-0 } \\
\text { TOTAL TARGET }\end{array}$ & $\underset{\text { ĹI }}{\text { ÖE }}$ & $\begin{array}{r}45,995 \\
400 \\
10,000 \\
56,395\end{array}$ & $\begin{array}{r}56,315 \\
0 \\
6,175 \\
62,490\end{array}$ & $\begin{array}{r}39,346 \\
0 \\
32,263 \\
65,609\end{array}$ & $\begin{array}{r}40,121 \\
\quad 0 \\
33,882 \\
-4-4 \\
74,003 \\
4\end{array}$ & $\begin{array}{r}60.746 \\
0 \\
71,552 \\
132298\end{array}$ & $\begin{array}{r}51,286 \\
86,488 \\
137,774\end{array}$ & $\begin{array}{r}44,613 \\
780 \\
78994 \\
123,607\end{array}$ \\
\hline & $\begin{array}{l}\text { Delta to TARGET. } \\
\text { (See notes at end of Table.) } \\
\qquad \text { Total }\end{array}$ & $\begin{array}{l}O E \\
C / E \\
L I\end{array}$ & NA & $\begin{array}{r}(14) \\
(5,538) \\
6,175 \\
=== \\
623\end{array}$ & $\begin{array}{r}(25,450) \\
(453) \\
32,263 \\
==== \\
6,360\end{array}$ & $\begin{array}{r}(7.900) \\
(93) \\
11,099 \\
==== \\
3.106\end{array}$ & & & \\
\hline $\begin{array}{l}1.1 .1 .3 .01 .01 \\
\text { Level } \mathrm{v}\end{array}$ & $\begin{array}{l}\text { Technical Integration } \\
\frac{\text { and Planning }}{A D S 1220-0-\wedge A} \text { Sub Total }\end{array}$ & $\begin{array}{l}O E \\
C / E\end{array}$ & NA & $\begin{array}{r}1,916 \\
0 \\
---- \\
1,916\end{array}$ & $\begin{array}{r}1,969 \\
0 \\
---- \\
1,969\end{array}$ & $\begin{array}{r}2,028 \\
-0 \\
---0 \\
2,028\end{array}$ & $\begin{array}{r}\mathbf{0} \\
-0 \\
\mathbf{0}\end{array}$ & $\begin{array}{r}0 \\
0 \\
-0 \\
0\end{array}$ & $\begin{array}{r}\mathbf{0} \\
-- \\
\mathbf{0}\end{array}$ \\
\hline $\begin{array}{l}1.1 .1 .3 .01 .01 .01 \\
\text { Level VI }\end{array}$ & $\begin{array}{l}\text { Pretreatment Program } \\
\text { Management } \\
\qquad \text { Total }\end{array}$ & $\begin{array}{l}O E \\
C / E\end{array}$ & & $\begin{array}{r}1,916 \\
0 \\
---9 \\
1,916\end{array}$ & $\begin{array}{r}1,969 \\
0 \\
---- \\
1,969\end{array}$ & $\begin{array}{r}2,028 \\
0 \\
---- \\
2,028\end{array}$ & $---\overline{0}$ & --- & -- \\
\hline $\begin{array}{l}1.1 .1 .3 .01 .01 .02 \\
\text { Level VI }\end{array}$ & $\begin{array}{l}\text { Program Management } \\
\text { Accounting Adjustments } \\
\text { Total }\end{array}$ & $\begin{array}{l}O E \\
C / E\end{array}$ & & $\begin{array}{r}0 \\
0 \\
-- \\
0\end{array}$ & $-\begin{array}{r}0 \\
0 \\
-0\end{array}$ & $\begin{array}{r}0 \\
0 \\
-- \\
0\end{array}$ & --- & $---\frac{-}{0}$ & $---\frac{-}{0}$ \\
\hline $\begin{array}{l}1.1 .1 .3 .01 .02 \\
\text { Level } \mathrm{v}\end{array}$ & $\begin{array}{l}\text { Tech. Development. and } \\
\frac{\text { Applied Engineering }}{A D S 1220-0-A B} \text { Sub Total }\end{array}$ & $\begin{array}{l}O E \\
\text { C/E }\end{array}$ & NA & $\begin{array}{r}16,702 \\
560 \\
---- \\
17,262\end{array}$ & $\begin{array}{r}16,026 \\
93 \\
16,119\end{array}$ & $\begin{array}{r}15,493 \\
93 \\
---- \\
15.586\end{array}$ & $\begin{array}{r}\mathbf{0} \\
\mathbf{0} \\
-\frac{\mathbf{0}}{\mathbf{0}}\end{array}$ & $\begin{array}{r}\mathbf{0} \\
- \\
-0 \\
0\end{array}$ & $\begin{array}{r}0 \\
-0 \\
-0\end{array}$ \\
\hline $\begin{array}{l}\text { 1.1.1.3.01.02.01 } \\
\text { Level VI }\end{array}$ & $\begin{array}{r}\text { Low-Level Waste Pretreat } \\
\text { Dvipmt. for Refer. Process } \\
\text { Total }\end{array}$ & $\begin{array}{l}O E \\
C / E\end{array}$ & & $\begin{array}{r}2,493 \\
0 \\
--- \\
2,493\end{array}$ & $\begin{array}{r}1.045 \\
0 \\
---- \\
1.045\end{array}$ & $\begin{array}{r}435 \\
0 \\
--- \\
435\end{array}$ & $--\frac{-}{0}$ & $---\frac{-}{0}$ & --- \\
\hline
\end{tabular}


TABLE $7.8-1$

TOTAL PROGRAM ELEMENT COST BASELINE - BY YEAR

\begin{tabular}{|c|c|c|c|c|c|c|c|c|c|}
\hline \multicolumn{3}{|c|}{ COST BASELINE - BY YEAR } & \multicolumn{7}{|c|}{ (K\$) } \\
\hline WBS / LEVEL & ACTIVITY TITLE / ADS & $\begin{array}{l}\text { FUND } \\
\text { TYPE }\end{array}$ & $\begin{array}{c}\text { TOTAL } \\
\text { FY94 }\end{array}$ & FY95 & FY96 & FY97 & FY98 & FY99 & FY00 \\
\hline $\begin{array}{l}\text { 1.1.1.3.01.02.02 } \\
\text { Level V1 }\end{array}$ & $\begin{array}{l}\text { LLW Pretreat. Dvlpmt. for } \\
\text { Enhanc. \& Altematives } \\
\qquad \text { Total }\end{array}$ & $\begin{array}{l}O E \\
C / E\end{array}$ & & $\begin{array}{r}3,984 \\
50 \\
--- \\
4,034\end{array}$ & $\begin{array}{r}2,411 \\
93 \\
--- \\
2,504\end{array}$ & $\begin{array}{r}1.467 \\
93 \\
---- \\
1,560\end{array}$ & 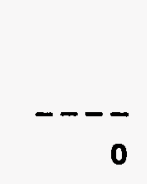 & --- & $-\cdots$ \\
\hline $\begin{array}{l}\text { 1.1.1.3.01.02.03 } \\
\text { Level VI }\end{array}$ & $\begin{array}{l}\text { Hi-Level Waste Pretreat } \\
\text { Dvlpmt. for Refer. Process } \\
\text { Total }\end{array}$ & $\begin{array}{l}O E \\
C / E\end{array}$ & & $\begin{array}{r}8,920 \\
510 \\
--- \\
9,430\end{array}$ & $\begin{array}{r}7,267 \\
0 \\
---2 \\
7,267\end{array}$ & $\begin{array}{r}7,275 \\
0 \\
---- \\
7,275\end{array}$ & --- & --- & $---\frac{-}{0}$ \\
\hline $\begin{array}{l}\text { 1.1.1.3.01.02.04 } \\
\text { Level VI }\end{array}$ & $\begin{array}{l}\text { HLW Pretreat. Dvipmt. for } \\
\text { Enhanc. \& Altematives } \\
\text { Total }\end{array}$ & $\begin{array}{l}O E \\
C / E\end{array}$ & & $\begin{array}{r}1,305 \\
0 \\
---- \\
1,305\end{array}$ & $\begin{array}{r}5,303 \\
0 \\
---- \\
5,303\end{array}$ & $\begin{array}{r}6,316 \\
0 \\
--- \\
6,316\end{array}$ & $---\frac{-}{0}$ & --- & $---\overline{0}$ \\
\hline $\begin{array}{l}\text { 1.1.1.3.01.03 } \\
\text { Level } v\end{array}$ & $\begin{array}{ll}\frac{\text { Facility Operations }}{\text { ADS } 1220-0-A C} & \\
& \text { Total }\end{array}$ & $\begin{array}{l}O E \\
C / E\end{array}$ & NA & --- & 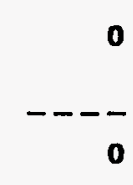 & $\begin{array}{r}0 \\
--\end{array}$ & 0 & $---\frac{-}{0}$ & $---\frac{-}{0}$ \\
\hline $\begin{array}{l}\text { 1.1.1.3.01.04 } \\
\text { Level } v\end{array}$ & $\begin{array}{l}\frac{\text { Systems Definition }}{\text { ADS } 1220-0-A D} \\
\\
\end{array}$ & $\begin{array}{l}O E \\
C / E\end{array}$ & NA & $\begin{array}{r}7.111 \\
0 \\
---- \\
7.111\end{array}$ & $\begin{array}{r}6,685 \\
0 \\
---- \\
6,685\end{array}$ & $\begin{array}{r}10,828 \\
0 \\
---- \\
10,828\end{array}$ & $\begin{array}{r}0 \\
0 \\
--- \\
0\end{array}$ & $\begin{array}{r}0 \\
0 \\
--- \\
0\end{array}$ & $\begin{array}{r}0 \\
0 \\
--- \\
0\end{array}$ \\
\hline $\begin{array}{l}\text { 1.1.1.3.01.04.01 } \\
\text { Level VI }\end{array}$ & $\begin{array}{l}\text { NEPA Flowsht. \& } \\
\text { Applied Tech. Evaluation } \\
\qquad \text { Total }\end{array}$ & $\begin{array}{l}O E \\
C / E\end{array}$ & & $\begin{array}{r}341 \\
--- \\
-341\end{array}$ & $\begin{array}{r}301 \\
0 \\
-- \\
301\end{array}$ & $\begin{array}{r}100 \\
--0 \\
100\end{array}$ & --- & $---\overline{0}$ & --- \\
\hline $\begin{array}{l}\text { 1.1.1.3.01.04.02 } \\
\text { Level VI }\end{array}$ & $\begin{array}{l}\text { Engin. Study \& Functions } \\
\text { and Requirements (F\&R) } \\
\text { Total }\end{array}$ & $\begin{array}{l}O E \\
C / E\end{array}$ & & $\begin{array}{r}4,468 \\
0 \\
--- \\
4,468\end{array}$ & $\begin{array}{r}4,528 \\
0 \\
--- \\
4,528\end{array}$ & $\begin{array}{r}8.747 \\
0 \\
--7 \\
8.747\end{array}$ & 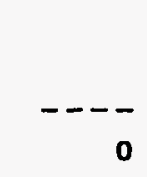 & 0 & $-\cdots$ \\
\hline
\end{tabular}

\section{[ R1 ]}


TABLE 7.8-1

TOTAL PROGRAM ELEMENT COST BASELINE - BY YEAR

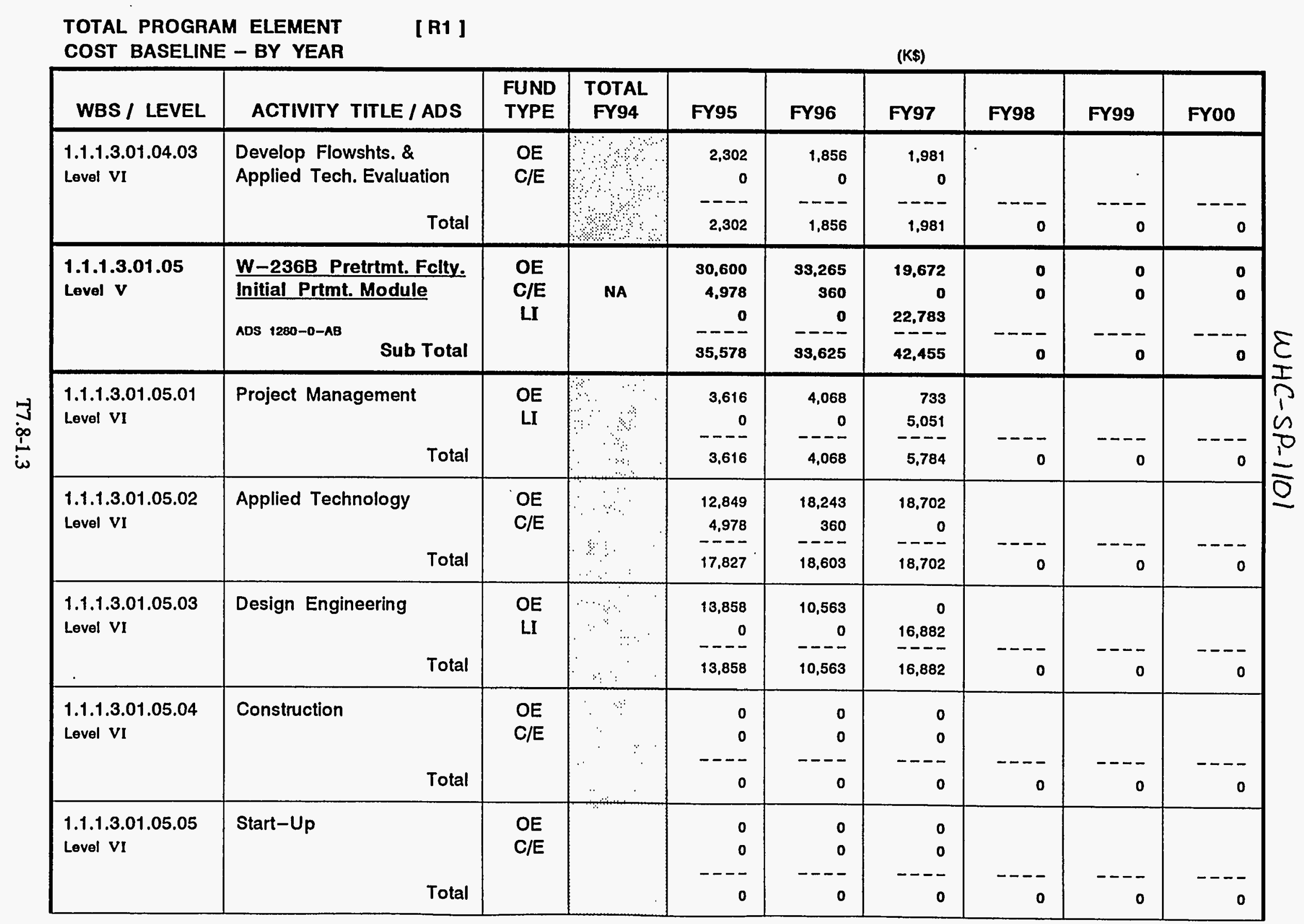

\section{[ R1 ]}


TABLE $7.8-1$

TOTAL PROGRAM ELEMENT

COST BASELINE - BY YEAR

\begin{tabular}{|c|c|c|c|c|c|c|c|c|c|}
\hline \multicolumn{3}{|c|}{ COST BASELINE - BY YEAR } & \multicolumn{7}{|c|}{ (K\$) } \\
\hline WBS / LEVEL & ACTIVITY TITLE / ADS & $\begin{array}{l}\text { FUND } \\
\text { TYPE }\end{array}$ & $\begin{array}{c}\text { TOTAL } \\
\text { FY94 }\end{array}$ & FY95 & FY96 & FY97 & FY98 & FY99 & FYOO \\
\hline $\begin{array}{l}\text { 1.1.1.3.01.05.06 } \\
\text { Level VI }\end{array}$ & $\begin{array}{l}\text { Regulatory Compliance } \\
\text { \& Safety } \\
\qquad \text { Total }\end{array}$ & $\begin{array}{l}\text { OE } \\
\text { LI }\end{array}$ & & $\begin{array}{r}277 \\
0 \\
--- \\
277\end{array}$ & $\begin{array}{r}39.1 \\
0 \\
--- \\
391\end{array}$ & $\begin{array}{r}237 \\
850 \\
--- \\
1.087\end{array}$ & --- & --- & --- \\
\hline $\begin{array}{l}\text { 1.1.1.3.01.05.07 } \\
\text { Level VI }\end{array}$ & $\begin{array}{r}\text { Technology Demonstration } \\
\text { Total }\end{array}$ & $\begin{array}{l}O E \\
C / E\end{array}$ & & $\begin{array}{r}0 \\
0 \\
-- \\
-0\end{array}$ & $\begin{array}{r}0 \\
0 \\
-- \\
0\end{array}$ & $\begin{array}{r}0 \\
0 \\
-- \\
0\end{array}$ & --- & -- & $---\overline{0}$ \\
\hline $\begin{array}{l}\text { 1.1.1.3.01.05.08 } \\
\text { Level VI }\end{array}$ & $\begin{array}{l}\text { Hot Pilot Plant } \\
\qquad \text { Total }\end{array}$ & $\begin{array}{l}O E \\
C / E\end{array}$ & & $\begin{array}{r}0 \\
0 \\
-- \\
-0\end{array}$ & $\begin{array}{r}0 \\
0 \\
-- \\
-0\end{array}$ & $\begin{array}{r}0 \\
0 \\
-- \\
0\end{array}$ & --- & $-\cdots$ & --- \\
\hline $\begin{array}{l}\text { 1.1.1.3.01.06 } \\
\text { Level } \mathrm{v}\end{array}$ & $\frac{\frac{\text { Hi-Level Waste }}{\text { Pretreatment }}}{\text { ADS } 1220-0-A F \quad \text { Sub Total }}$ & $\begin{array}{l}O E \\
C / E\end{array}$ & NA & $\begin{array}{r}0 \\
0 \\
-- \\
-0\end{array}$ & $\begin{array}{r}851 \\
0 \\
--- \\
851\end{array}$ & $\begin{array}{r}0 \\
0 \\
-- \\
-0\end{array}$ & $\begin{array}{r}0 \\
0 \\
-- \\
0\end{array}$ & $\begin{array}{r}\mathbf{0} \\
\mathbf{0} \\
-- \\
\mathbf{0}\end{array}$ & $\begin{array}{r}0 \\
0 \\
--- \\
0\end{array}$ \\
\hline $\begin{array}{l}\text { 1.1.1.3.01.06.01 } \\
\text { Level VI }\end{array}$ & $\begin{array}{l}\text { Project Management } \\
\qquad \text { Total }\end{array}$ & $\begin{array}{l}O E \\
C / E\end{array}$ & & $\begin{array}{r}0 \\
0 \\
-- \\
0\end{array}$ & $\begin{array}{r}134 \\
0 \\
-- \\
134\end{array}$ & $\begin{array}{r}0 \\
0 \\
-- \\
0\end{array}$ & $\begin{array}{r}--- \\
0\end{array}$ & $\begin{array}{r}--- \\
0\end{array}$ & $---\overline{0}$ \\
\hline $\begin{array}{l}\text { 1.1.1.3.01.06.02 } \\
\text { Level VI }\end{array}$ & $\begin{array}{r}\text { Applied Technology } \\
\text { Total }\end{array}$ & $\begin{array}{l}O E \\
C / E\end{array}$ & & $\begin{array}{r}0 \\
0 \\
--- \\
0\end{array}$ & $\begin{array}{r}0 \\
0 \\
-- \\
0\end{array}$ & $\begin{array}{r}0 \\
0 \\
-- \\
0\end{array}$ & $-\cdots$ & $---\frac{-}{0}$ & 0 \\
\hline $\begin{array}{l}\text { 1.1.1.3.01.06.03 } \\
\text { Level VI }\end{array}$ & $\begin{array}{l}\text { Design Engineering } \\
\qquad \text { Total }\end{array}$ & $\begin{array}{l}O E \\
C / E\end{array}$ & & $\begin{array}{r}0 \\
0 \\
-- \\
0\end{array}$ & $\begin{array}{r}717 \\
0 \\
--- \\
717\end{array}$ & $\begin{array}{r}0 \\
0 \\
-- \\
0\end{array}$ & ---- & --- & $-\cdots$ \\
\hline
\end{tabular}

[ R1 ] 
TABLE $7.8-1$

TOTAL PROGRAM ELEMENT

COST BASELINE - BY YEAR

\begin{tabular}{|c|c|c|c|c|c|c|c|c|c|}
\hline $\begin{array}{l}\text { 1.1.1.3.01.06.04 } \\
\text { Level VI }\end{array}$ & $\begin{array}{l}\text { Construction } \\
\qquad \text { Total }\end{array}$ & $\begin{array}{l}\text { OE. } \\
C / E\end{array}$ & $\begin{array}{r}\vdots \\
\vdots \\
\vdots \\
\vdots\end{array}$ & $\begin{array}{r}0 \\
0 \\
-- \\
-0\end{array}$ & $\begin{array}{r}0 \\
0 \\
-- \\
0\end{array}$ & $\begin{array}{r}0 \\
0 \\
-0\end{array}$ & --- & --- & $---\frac{1}{0}$ \\
\hline $\begin{array}{l}1.1 .1 .3 .01 .06 .05 \\
\text { Level VI }\end{array}$ & Start-Up & $\begin{array}{l}O E \\
C / E\end{array}$ & ४ & $\begin{array}{r}0 \\
0 \\
-- \\
0\end{array}$ & $\begin{array}{r}0 \\
0 \\
-- \\
-0\end{array}$ & $\begin{array}{r}0 \\
0 \\
--- \\
0\end{array}$ & --- & --- & --- \\
\hline $\begin{array}{l}\text { 1.1.1.3.01.06.06 } \\
\text { Level VI }\end{array}$ & $\begin{array}{l}\text { Regulatory Compliance } \\
\text { \& Safety } \\
\qquad \text { Total }\end{array}$ & $\begin{array}{l}O E \\
C / E\end{array}$ & $\begin{array}{ccc} & \vdots \\
\ddots & \vdots \\
\ddots & \vdots\end{array}$ & $\begin{array}{r}0 \\
0 \\
-- \\
-0\end{array}$ & $\begin{array}{r}0 \\
0 \\
-- \\
-0\end{array}$ & $\begin{array}{r}0 \\
0 \\
-- \\
0\end{array}$ & --- & --- & --- \\
\hline
\end{tabular}

\section{[ R1 ]}


TABLE $7.8-1$

TOTAL PROGRAM ELEMENT COST BASELINE - BY YEAR

\begin{tabular}{|c|c|c|c|c|c|c|c|c|c|c|}
\hline WBS / LEVEL & ACTIVITY TITLE / ADS & $\begin{array}{l}\text { FUND } \\
\text { TYPE }\end{array}$ & $\begin{array}{l}\text { FY01 I } \\
\text { FY05 }\end{array}$ & $\begin{array}{l}\text { FY06 I } \\
\text { FY10 }\end{array}$ & $\begin{array}{l}\text { FY11 / } \\
\text { FY15 }\end{array}$ & $\begin{array}{l}\text { FY16 I } \\
\text { FY20 }\end{array}$ & $\begin{array}{l}\text { FY21 I } \\
\text { FY25 }\end{array}$ & $\begin{array}{l}\text { FY26 I } \\
\text { FY30 }\end{array}$ & $\begin{array}{l}\text { FY31 I } \\
\text { FY35 }\end{array}$ & $\begin{array}{l}\text { FY36 I } \\
\text { FY40 }\end{array}$ \\
\hline \begin{tabular}{l} 
1.1.1:3.01 \\
Level iv \\
\hdashline
\end{tabular} & 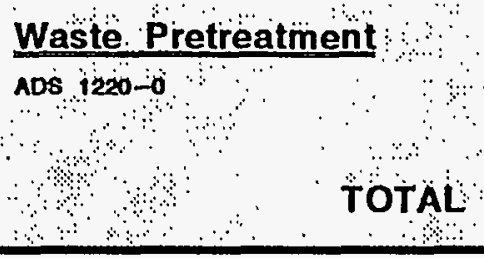 & $\stackrel{\mathrm{OE}}{\mathrm{C} / \mathrm{E}}$ & $\begin{array}{rrr} & 0 \\
-844762 & 0 \\
840 & 0\end{array}$ & $\begin{array}{r}0 \\
0 \\
-4 \\
812,200 \\
0\end{array}$ & $\begin{array}{r}0 \\
0 \\
717,895 \\
-10 \\
\end{array}$ & $\begin{array}{r}0 \\
0 \\
706,711\end{array}$ & $\begin{array}{r}0 \\
0 \\
19,199 \\
0\end{array}$ & 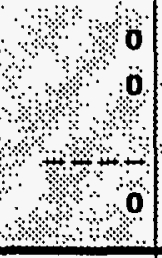 & & $\begin{array}{l}0 \\
0 \\
-\infty\end{array}$ \\
\hline & $\begin{array}{l}\text { Delta Required to } \\
\text { Balance to TARGET } \\
\text { Budget. } \\
\text { Total }\end{array}$ & $\begin{array}{c}O E \\
C / E \\
L I\end{array}$ & & & & & & & & \\
\hline $\begin{array}{l}\text { 1.1.1.3.01.01 } \\
\text { Level } v\end{array}$ & $\begin{array}{l}\text { Technical Integration } \\
\frac{\text { and Planning }}{A D S 1220-0-A M} \text { Sub Total }\end{array}$ & $\begin{array}{l}O E \\
C / E\end{array}$ & $-\begin{array}{r}0 \\
0 \\
-0\end{array}$ & $-\begin{array}{r}0 \\
0 \\
-0\end{array}$ & $\begin{array}{r}0 \\
0 \\
-0\end{array}$ & $\begin{array}{r}0 \\
0 \\
-- \\
0\end{array}$ & $-\begin{array}{r}0 \\
0 \\
-0\end{array}$ & $\begin{array}{r}\mathbf{0} \\
\mathbf{0} \\
-\mathbf{0}\end{array}$ & $\begin{array}{r}\mathbf{0} \\
--- \\
\mathbf{0}\end{array}$ & $--\frac{0}{0}$ \\
\hline $\begin{array}{l}1.1 .1 .3 .01 .01 .01 \\
\text { Level V1 }\end{array}$ & $\begin{array}{l}\text { Pretreatment Program } \\
\text { Management } \\
\qquad \text { Total }\end{array}$ & $\begin{array}{l}\text { OE } \\
C / E\end{array}$ & ---- & - & $\overline{0}$ & 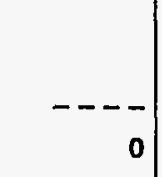 & $\overline{0}$ & - & $\overline{0}$ & $\overline{0}$ \\
\hline $\begin{array}{l}\text { 1.1.1.3.01.01.02 } \\
\text { Level VI }\end{array}$ & $\begin{array}{l}\text { Program Management } \\
\text { Accounting Adjustments } \\
\text { Total }\end{array}$ & $\begin{array}{l}O E \\
C / E\end{array}$ & ---- & - & - & $\overline{0}$ & $\overline{0}$ & --- & $\overline{0}$ & $\overline{0}$ \\
\hline $\begin{array}{l}\text { 1.1.1.3.01.02 } \\
\text { Level } v\end{array}$ & $\begin{array}{l}\text { Tech. Development. and } \\
\frac{\text { Applied Engineering }}{A D S 1220-0-\Lambda B} \text { Sub Total }\end{array}$ & $\begin{array}{l}\text { OE } \\
C / E\end{array}$ & $\begin{array}{r}\mathbf{0} \\
-0 \\
-0 \\
\mathbf{0}\end{array}$ & $\begin{array}{r}\mathbf{0} \\
-0 \\
-0\end{array}$ & $-\begin{array}{r}\mathbf{0} \\
\mathbf{0} \\
-\mathbf{0}\end{array}$ & $\begin{array}{r}0 \\
0 \\
-0\end{array}$ & $\begin{array}{r}0 \\
0 \\
-0\end{array}$ & $\begin{array}{r}\mathbf{0} \\
\mathbf{0} \\
-0 \\
\mathbf{0}\end{array}$ & -- & $\begin{array}{r}0 \\
-0 \\
-0\end{array}$ \\
\hline $\begin{array}{l}\text { 1.1.1.3.01.02.01 } \\
\text { Level VI }\end{array}$ & $\begin{array}{c}\text { Low-Level Waste Pretreat } \\
\text { Dvlpmt. for Refer. Process } \\
\text { Total }\end{array}$ & $\begin{array}{l}\mathrm{OE} \\
\mathrm{C} / \mathrm{E}\end{array}$ & --- & $\overline{0}$ & $\overline{0}$ & $\overline{0}$ & 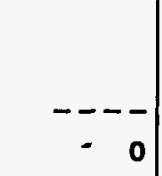 & - & o & 0 \\
\hline
\end{tabular}

[ R1 ] 
TABLE $7.8-1$

TOTAL PROGRAM ELEMENT COST BASELINE - BY YEAR

\begin{tabular}{|c|c|c|c|c|c|c|c|c|c|c|}
\hline WBS / LEVEL & ACTIVITY TITLE / ADS & $\begin{array}{l}\text { FUND } \\
\text { TYPE }\end{array}$ & $\begin{array}{l}\text { FY01 I } \\
\text { FY05 }\end{array}$ & $\begin{array}{l}\text { FY06 I } \\
\text { FY10 }\end{array}$ & $\begin{array}{l}\text { FY11 I } \\
\text { FY15 }\end{array}$ & $\begin{array}{l}\text { FY16 I } \\
\text { FY20 }\end{array}$ & $\begin{array}{l}\text { FY21 I } \\
\text { FY25 }\end{array}$ & $\begin{array}{l}\text { FY26 I } \\
\text { FY30 }\end{array}$ & $\begin{array}{l}\text { FY31 I } \\
\text { FY35 }\end{array}$ & $\begin{array}{l}\text { FY36 I } \\
\text { FY40 }\end{array}$ \\
\hline $\begin{array}{l}\text { 1.1.1.3.01.02.02 } \\
\text { Level VI }\end{array}$ & $\begin{array}{l}\text { LLW Pretreat. Dvlpmt. for } \\
\text { Enhanc. \& Alternatives } \\
\text { Total }\end{array}$ & $\begin{array}{l}O E \\
C / E\end{array}$ & $\cdots$ & -- & ---- & $-\cdots$ & ---- & $-\cdots$ & --- & 0 \\
\hline $\begin{array}{l}\text { 1.1.1.3.01.02.03 } \\
\text { Level V1 }\end{array}$ & $\begin{array}{l}\text { Hi-Level Waste Pretreat } \\
\text { Dvipmt. for Refer. Process } \\
\text { Total }\end{array}$ & $\begin{array}{l}O E \\
C / E\end{array}$ & & 0 & $-\cdots$ & -- & ---- & 0 & $\overline{0}$ & - \\
\hline $\begin{array}{l}\text { 1.1.1.3.01.02.04 } \\
\text { Level VI }\end{array}$ & $\begin{array}{l}\text { HLW Pretreat. Dvlpmt. for } \\
\text { Enhanc. \& Alternatives } \\
\qquad \text { Total }\end{array}$ & $\begin{array}{l}O E \\
C / E\end{array}$ & $\overline{0}$ & 0 & --- & $\overline{0}$ & $-\overline{0}$ & 0 & - & o \\
\hline $\begin{array}{l}\text { 1.1.1.3.01.03 } \\
\text { Level } v\end{array}$ & $\begin{array}{l}\frac{\text { Facility Operations }}{\text { ADS } 1220-0-A C} \\
\\
\end{array}$ & $\begin{array}{l}\mathrm{OE} \\
\mathrm{C} / \mathrm{E}\end{array}$ & & $\overline{0}$ & 0 & $\overline{0}$ & $\overrightarrow{0}$ & -- & -- & -- \\
\hline $\begin{array}{l}\text { 1.1.1.3.01.04 } \\
\text { Level } v\end{array}$ & $\begin{array}{l}\frac{\text { Systems Definition }}{\text { ADS } 1220-0-A D} \\
\\
\text { Total }\end{array}$ & $\begin{array}{l}\mathrm{OE} \\
\mathrm{C} / \mathrm{E}\end{array}$ & $\begin{array}{r}\mathbf{0} \\
\mathbf{0} \\
-\mathbf{0} \\
\hdashline\end{array}$ & - & $\begin{array}{r}0 \\
0 \\
-0\end{array}$ & - & $\begin{array}{r}0 \\
0 \\
-0 \\
\end{array}$ & $\begin{array}{r}\mathbf{0} \\
\mathbf{0} \\
-\mathbf{0}\end{array}$ & $\begin{array}{r}\mathbf{0} \\
\mathbf{0} \\
-\mathbf{0}\end{array}$ & $\begin{array}{r}0 \\
0 \\
-0\end{array}$ \\
\hline $\begin{array}{l}\text { 1.1.1.3.01.04.01 } \\
\text { Level VI }\end{array}$ & $\begin{array}{l}\text { NEPA Flowsht. \& } \\
\text { Applied Tech. Evaluation } \\
\qquad \text { Total }\end{array}$ & $\begin{array}{l}\mathrm{OE} \\
\mathrm{C} / \mathrm{E}\end{array}$ & -- & 0 & $-\cdots$ & ---- & ---- & 0 & -- & $-\cdots$ \\
\hline $\begin{array}{l}\text { 1.1.1.3.01.04.02 } \\
\text { Levol VI }\end{array}$ & $\begin{array}{l}\text { Engin. Study \& Functions } \\
\text { and Requirements (F\&R) } \\
\text { Total }\end{array}$ & $\begin{array}{l}O E \\
C / E\end{array}$ & - & $\overline{0}$ & -- & - & 0 & $\overline{0}$ & ---- & - \\
\hline
\end{tabular}

\section{[ R1 ]}


TABLE $7.8-1$

TOTAL PROGRAM ELEMENT COST BASELINE - BY YEAR

\begin{tabular}{|c|c|c|c|c|c|c|c|c|c|c|}
\hline WBS / LEVEL & ACTIVITY TITLE / ADS & $\begin{array}{l}\text { FUND } \\
\text { TYPE }\end{array}$ & $\begin{array}{l}\text { FY01 I } \\
\text { FY05 }\end{array}$ & $\begin{array}{l}\text { FY06 / } \\
\text { FY10 }\end{array}$ & $\begin{array}{l}\text { FY11 I } \\
\text { FY15 }\end{array}$ & $\begin{array}{l}\text { FY16 I } \\
\text { FY20 }\end{array}$ & $\begin{array}{l}\text { FY21 I } \\
\text { FY25 }\end{array}$ & $\begin{array}{l}\text { FY26 / } \\
\text { FY30 }\end{array}$ & $\begin{array}{l}\text { FY31 / } \\
\text { FY35 }\end{array}$ & $\begin{array}{l}\text { FY36 / } \\
\text { FY40 }\end{array}$ \\
\hline $\begin{array}{l}\text { 1.1.1.3.01.04.03 } \\
\text { Level VI }\end{array}$ & $\begin{array}{l}\text { Develop Flowshts. \& } \\
\text { Applied Tech. Evaluation } \\
\qquad \text { Total }\end{array}$ & $\begin{array}{l}O E \\
C / E\end{array}$ & ---- & $\begin{array}{r}--- \\
0\end{array}$ & $\begin{array}{r}--- \\
0\end{array}$ & ---- & $---\overline{0}$ & --- & $\begin{array}{r}---- \\
0\end{array}$ & $-\cdots$ \\
\hline $\begin{array}{l}1.1 .1 .3 .01 .05 \\
\text { Level } \mathrm{v}\end{array}$ & $\begin{array}{l}\text { W-236B Pretrtmt. Falty. } \\
\text { Initial Prtmt. Module } \\
\text { ADS } 1280-D-A B \quad \text { Sub Total }\end{array}$ & $\begin{array}{c}O E \\
\text { C/E } \\
\text { LI }\end{array}$ & $\begin{array}{r}\mathbf{0} \\
\mathbf{0} \\
-- \\
-0\end{array}$ & $\begin{array}{r}\mathbf{0} \\
\mathbf{0} \\
-- \\
-0\end{array}$ & $\begin{array}{r}\mathbf{0} \\
\mathbf{0} \\
-0 \\
-0\end{array}$ & $\begin{array}{r}0 \\
0 \\
-- \\
0\end{array}$ & $\begin{array}{r}0 \\
0 \\
--- \\
-0\end{array}$ & $\begin{array}{r}\mathbf{0} \\
\mathbf{0} \\
-- \\
-0\end{array}$ & $\begin{array}{r}0 \\
0 \\
--- \\
0\end{array}$ & $\begin{array}{r}\text { 0 } \\
\mathbf{0} \\
--- \\
\mathbf{0}\end{array}$ \\
\hline $\begin{array}{l}1.1 .1 .3 .01 .05 .01 \\
\text { Level VI }\end{array}$ & $\begin{array}{l}\text { Project Management } \\
\qquad \text { Total }\end{array}$ & $\begin{array}{l}\text { OE } \\
\text { LI }\end{array}$ & --- & $\begin{array}{r}--- \\
0\end{array}$ & - & $\begin{array}{r}---- \\
0\end{array}$ & - & $---\frac{-}{0}$ & $\begin{array}{r}---- \\
0\end{array}$ & $-\cdots$ \\
\hline $\begin{array}{l}\text { 1.1.1.3.01.05.02 } \\
\text { Level VI }\end{array}$ & $\begin{array}{l}\text { Applied Technology } \\
\qquad \text { Total }\end{array}$ & $\begin{array}{l}O E \\
C / E\end{array}$ & $---\frac{-}{0}$ & $---\frac{-}{0}$ & $---\frac{-}{0}$ & $\begin{array}{r}---- \\
0\end{array}$ & $\begin{array}{r}---- \\
0\end{array}$ & $---\frac{-}{0}$ & $\begin{array}{r}---- \\
0\end{array}$ & $---\frac{-}{0}$ \\
\hline $\begin{array}{l}\text { 1.1.1.3.01.05.03 } \\
\text { Level VI }\end{array}$ & $\begin{array}{l}\text { Design Engineering } \\
\qquad \text { Total }\end{array}$ & $\begin{array}{l}\mathrm{OE} \\
\mathrm{LI}\end{array}$ & -- & $\begin{array}{r}--- \\
0\end{array}$ & -- & $\begin{array}{r}---- \\
0\end{array}$ & $---\frac{-}{0}$ & $---\frac{-}{0}$ & $---\frac{-}{0}$ & $---\frac{1}{0}$ \\
\hline $\begin{array}{l}1.1 .1 .3 .01 .05 .04 \\
\text { Level VI }\end{array}$ & Construction & $\begin{array}{l}O E \\
C / E\end{array}$ & - & ---- & --- & $\begin{array}{r}--- \\
0\end{array}$ & $\begin{array}{r}--- \\
0\end{array}$ & 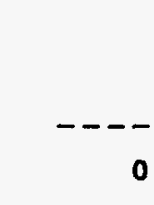 & $\begin{array}{r}--- \\
0\end{array}$ & 0 \\
\hline $\begin{array}{l}1.1 .1 .3 .01 .05 .05 \\
\text { Level V1 }\end{array}$ & Total & $\begin{array}{l}O E \\
C / E\end{array}$ & -- & $\begin{array}{r}---- \\
0\end{array}$ & --- & ---- & --- & --- & $\begin{array}{r}--- \\
0\end{array}$ & $-\cdots$ \\
\hline
\end{tabular}

\section{[ R1 ]}


TABLE $\quad 7.8-1$

TOTAL PROGRAM ELEMENT

COST BASELINE - BY YEAR

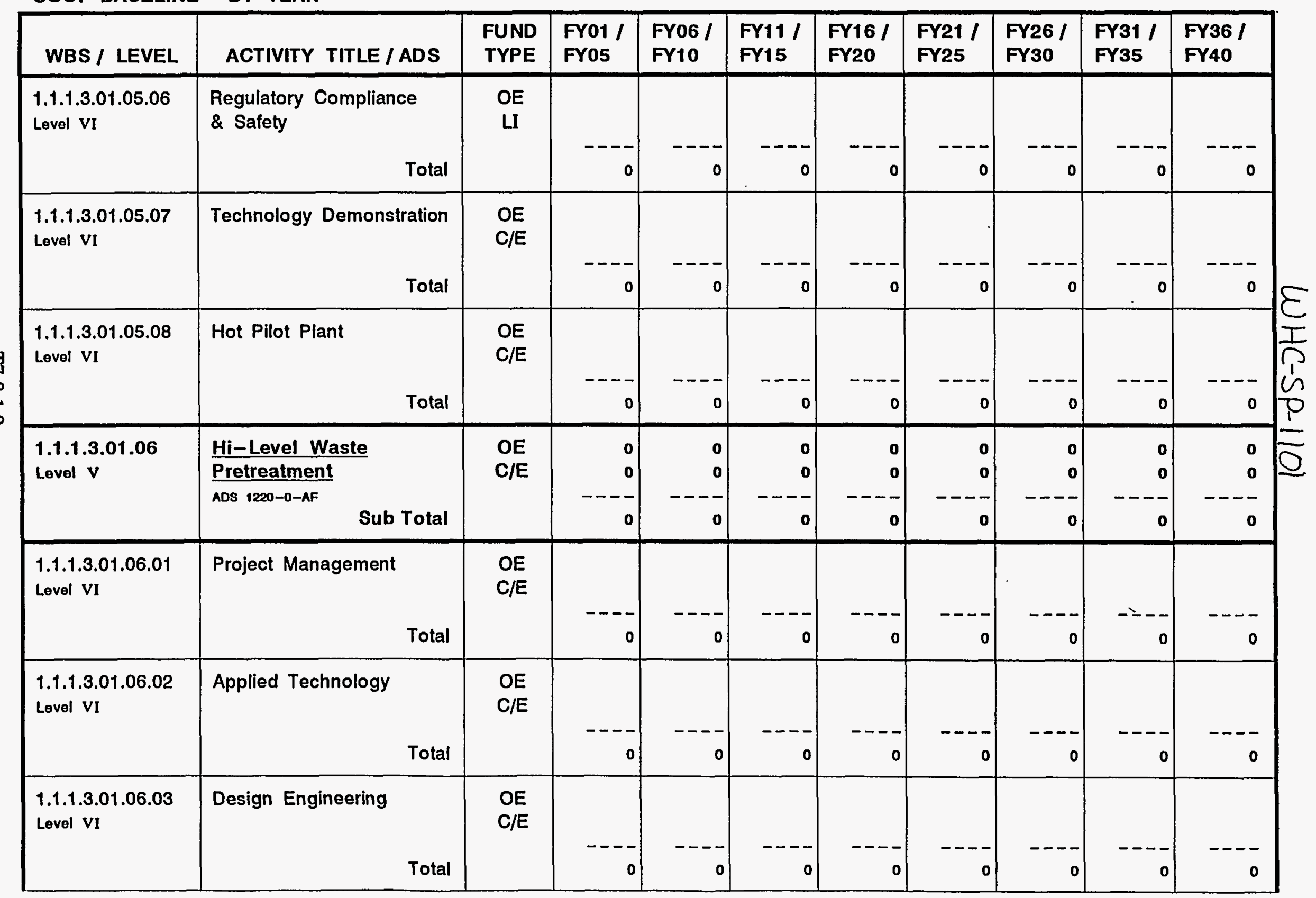


TABLE $\quad 7.8-1$

TOTAL PROGRAM ELEMENT COST BASELINE - BY YEAR

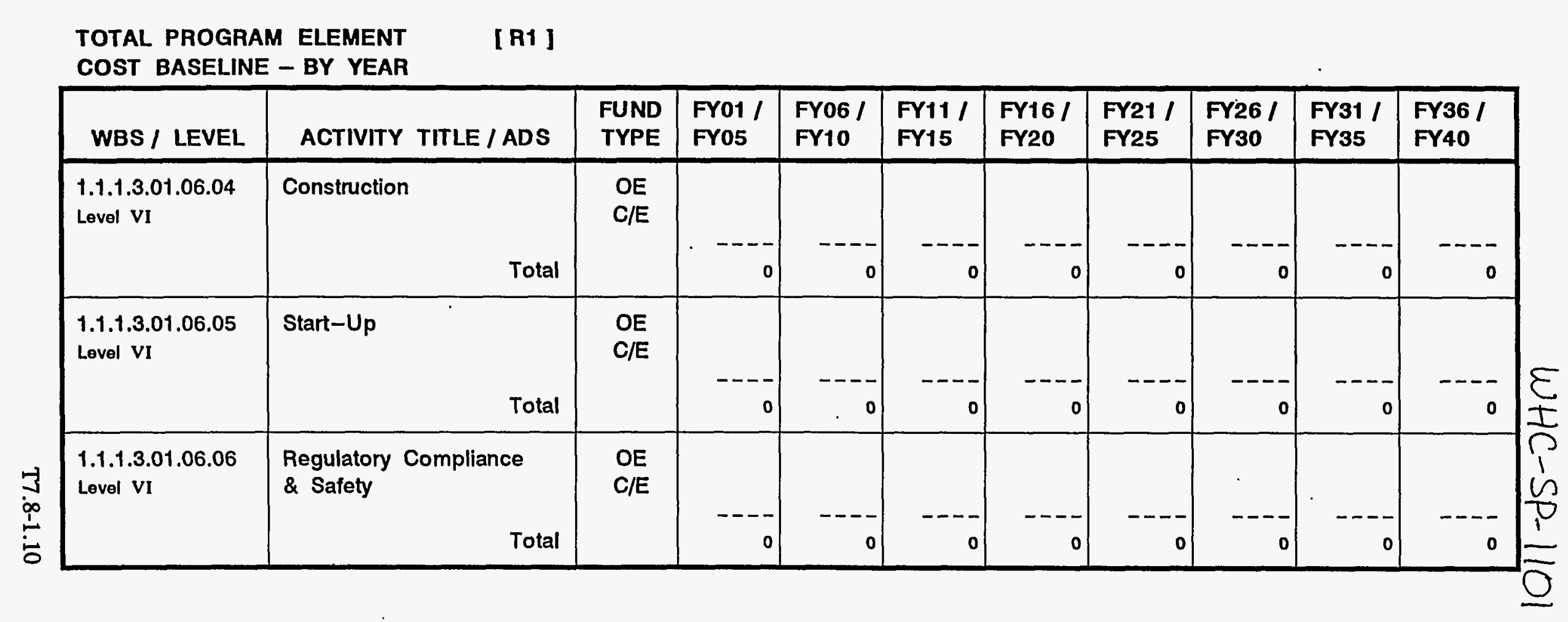

[ R1] 


\section{NOTES \\ TOTAL PROGRAM ELEMENT COST BASELINE - BY YEAR}

The cost exhibit tables in each of the Program Element Baselines (Sections 7.2 through 7.10) identify the program element target funding by year by fund type. The tables also provide details of the baseline allocation of funding down to activity levels (level V) and cost account levels (level VI) of the WBS for Fiscal Year 1995 through 1997. The details exhibited on these tables are taken from the baseline WBS Dictionary Sheets. Deltas exist between the details and the annual target totals. For Fiscal Years 1996 and 1997 the deltas will be addressed in the future, following the completion of the resolution of expected differences between current Fiscal Year 1995 baseline funding and expected actual Fiscal Year 1995 funding. Deltas for Fiscal Year 1995 are noted below by program element:

\section{PROGRAM MANAGEMENT AND ADMUNISTRATION:}

No deltas.

\section{TANK FARM OPERATIONS AND MAINTENANCE:}

No overall delta. This baseline requires revised fund type mix. .

\section{WASTE TANK SAFETY:}

Delta is due to need to revise an overhead pricing rate in the P3 system.

\section{TANK FARM UPGRADES:}

Delta is due to differences between Budget Authority (BA) in the target and Budget Obligation (B0) in the P3 system.

\section{CHARACTERIZATION:}

No significant deltas.

\section{WASTE RETRIEVAL:}

Delta is primarily due to need to resolve issues associated with expense funding support to capital projects and differences between Budget Authority (BA) in the target and Budget Obligation (B0) in the P3 system.

\section{WASTE PRETREATMENT:}

Delta is due to the sum of the detail cost estimates being lower than the target total as a result of reestimation of resource requirements to complete baseline scope.

\section{LOW LEVEL WASTE:}

Delta is primarily due to carryover expense funded workscope on melter testing addressed in June 9 , 1994 RL letter.

$$
T 7.8-1.11
$$




\section{HIGH LEVEL WASTE:}

Delta is due to carryover of capital funding, PNL expense funding carryover, and ADS corrections submitted but not reflected in target totals. 
Table 7.8-2. FY 1995 Cost by Month by Fund Type by Activity and Cost Account

Future updates of the MYWP will include Table 7.8-2, which establishes the month-by-month cost baseline for FY 1995 for this program element. The FY 1995 cost baseline is exhibited at the cost account level with fund type totals provided. Cost data are then rolled up to the activity and program element levels. Also exhibited is the total cost baseline for the program element for FY 1995 . At present, the program element cost baseline for FY 1995 for this program element is the total provided on this table less a productivity challenge for FY 1995. 
Table 7-8-3. Total Full-Time Equivalents by Year by Activity by Major Participant (Direct and Indirect) (Including all Subcontractors)

Future MYWP updates will include Table 7.8-3, which establishes total staffing projections for this program element. Included in the table are FY 1994 staffing data as well as projections by year for FY 1995 through FY 2000. The projections in this table include all direct and indirect Full-Time Equivalents (FTEs), including all.subcontractor personnel. Where data are available, projections are exhibited at the activity level with major program participant totals provided. 
Table 7.8-4. FY 1995 through FY 1997 Direct Westinghouse Hanford Company Full-Time Equivalents by 88 Labor Categories

Future MYWP updates will include Table 7.8-4, which establishes direct WHC staffing projections for this program element. Projections do not include subcontractor support and are provided by 88 labor categories for FY 1995 through FY 1997. 


\subsection{LOW-LEVEL WASTE BASELINE}

The Low-Level Waste (LLW) Program Element baseline consists of the technical bases, schedule, resource requirements, and waste type data for FY 1995 through completion of the mission. The major emphasis is on deliverables, schedule, and resource requirements for FY 1995, 1996, and 1997.

\subsubsection{Technical Bases}

This section provides the mission strategy; work breakdown structure and organization responsibility; work to be performed in FY 1995, 1996, and 1997; technical approach; and drivers and deliverables.

\subsubsection{Mission Strategy}

\subsection{Objectives}

The primary objective of the TWRS LLW Program is to manage the receipt, immobilization, packaging, storage/disposal, and closure (disposal site) of low-level Hanford tank waste in an environmentally sound, safe, and cost-effective manner. This objective has been further defined through the Hanford Federal Facility Agreement and Consent Order (Tri-Party Agreement) as vitrify the low-level waste fraction of the tank waste and dispose of it onsite and near-surface in a retrievable form. The vitrification processing capability is to be available for startup in FY 2005, and the mission must be complete by 2028 . The LLW facilities must be sized to handle an estimated $3.2 \mathrm{E}+8$ liters of waste feed over the life of the program.

In addition to the above objective, the program shall maintain the Grout Facility in a standby condition to relieve double-shell tank (DST) space should tank space be required to resolve tank safety issues.

\subsection{Strategy}

The current LLW life-of-program strategy involves the following.

- Utilize commercially available melter and other key processing technologies to the extent practical.

- Develop functional and interface design criteria and key recommendations for an Architect/Engineer (A/E).

- Contract with an independent $\mathrm{A} / \mathrm{E}$ and constructor for design and construction services.

- Evaluate the safety and environmental consequences of processing and disposal of vitrified LLW.

- $\quad$ Operate the facility by the Management and Operating (M\&O) contractor.

- $\quad$ Complete most LLW vitrification processing several years $(\sim 3)$ after single-shell tank (SST) retrieval completion. 
- Turn over the cleaned out facility and closed burial/storage facility to the Decontamination and Decommissioning (D\&D) Program or other monitoring program.

- $\quad$ Conduct open public discussion at key decision points for the program.

Alternative strategies for privatization or contracting will be explored in an effort to reduce the total program costs and/or improve the U.S. Department of Energy (DOE) budget restrictions.

For FY 1995 - FY 1997, the strategy emphasizes completion of those tasks required to initiate construction (FY 1998) of the LLW processing and disposal facilities. Many of these tasks are required to secure line item funding for the Project and ensure that the scope is consistent with current regulations. These activities include:

- Melter testing and recommendation

- Facility functions and operational requirements definition

- $\quad$ Preliminary disposal system performance assessment calculations

- $\quad \mathrm{A} / \mathrm{E}$ selection

- Conceptual design completed

- $\quad$ Preliminary design initiated

- Preliminary permit preparations

- Continued public involvement

- Obtaining approval to start construction of the LLW Vitrification Plant

- Maintaining the Grout facility in standby until this contingency capability is no longer required.

\subsection{Functions and Requirements}

The TWRS Program is applying systems engineering principles to the LLW Program. The scope and interfaces of the LLW Program are currently defined in Tank Waste Remediation System Functions and Requirements (DOE/RL-92-60, Rev 1). The critical LLW functions and requirements include the following.

- $\quad$ Process and dispose/store the Hanford low-level tank waste.

- $\quad$ Provide processing capacity for approximately $3.2 \mathrm{E}+8$ liters of retrieved waste.

- $\quad$ Complete the mission by 2028.

- $\quad$ Protect the public from exposure not to exceed $25 \mathrm{mrem} / \mathrm{yr}$.

- $\quad$ Provide the capability to retrieve vitrified waste for approximately 50 years.

For FY 1995 - FY 1997, the LLW Program will focus considerable attention on further defining the functions and requirements. This will include:

- $\quad$ Supporting continued detailed development of TWRS Functions and Requirements

- Defining melter performance requirements

- Preparing functions and operational requirements for the $\mathrm{A} / \mathrm{E}$

- Supporting coordination of waste retrieval sequences and schedules

- Developing LLW feed specifications

- Analyzing waste disposal system performance and supporting barrier, packaging and waste form performance requirements. 


\subsection{Risks}

During the FY 1995 - FY 1997 period of this multi-year work plan, the LLW Program will encounter several known uncertainties and issues. Each issue presents some risk to the program cost, scope, and/or schedule.

\section{Program Cost Uncertainties:}

1. There is considerable uncertainty with the Tri-Party Agreement and programmatic cost estimate for the LLW vitrification facility. There is no documented basis for the Tri-Party Agreement LLW cost estimate.

Mitigation: Conceptual design activities will prepare the budget cost estimate basis. Project costs will be updated using the Facility Configuration Study cost estimates as the preconceptual estimate basis.

2. FY 1994 funding ( $\$ 4.7 \mathrm{M}$ Functional) was transferred from the LLW Program to TWRS to resolve TWRS variance issues. LLW Program work scope associated with those funds (including melter testing) is critical path.

Mitigation: Carry over or supplemental FY 1995 funding is required to complete planned work scope to minimize risk.

\section{Program Schedule Uncertainties:}

1. A procurement protest was initiated in FY 1994 that must be ruled on by the General Accounting Office (GAO). The protest requests that contract awards be canceled and a new round of Best and Final Offers be requested. This would extend the time period for meeting Tri-Party Agreement Milestone M-60-02 by several months and delay recommendation of melter technology to the $\mathrm{A} / \mathrm{E}$.

Mitigation: The protest is currently being argued by WHC and DOE legal. DOE approval has been obtained to proceed with several contracts during the GAO protest review period.

2. The request for qualifications for the LLW vitrification $A / E$ has been delayed pending consideration of privatization strategies for the vitrification and disposal facility.

Mitigation: WHC and DOE are discussing strategies for project initiation and scope.

\section{Program Scope Uncertainties:}

1. The LLW vitrification program and the cesium ion exchange portion of pretreatment have been recommended for consolidation into one facility.

Mitigation: The facility consolidation recommendation is currently being reviewed by DOE and the interested public stakeholders. A path forward is being developed by both the LLW Program Office and the Waste Pretreatment Program Office. The programs will reflect the final decision of DOE . 
2. The TWRS Environmental Impact Statement (EIS) has five different options to present to the public for treatment and disposal of LLW. Several of the options present significant differences from the current baseline LLW Program.

Mitigation: Approval to initiate construction of the LLW vitrification facility is constrained by DOE order to the TWRS EIS record of decision. The program will reflect the final decision of DOE.

3. The Tri-Party Agreement basis for the LLW Program scope is predicated upon being able to vitrify low-level tank waste using commercial industrial-scale vitrification technology. Vitrification of radioactive waste at this scale and with these waste compositions is unprecedented in the vitrification industry.

Mitigation: Multiple commercial vendors are being contracted to test for demonstration of vitrification capability. Consideration is also being given to a range of glass formulations and waste forms for constituents not readily vitrifiable.

4. The ability of the program to demonstrate compliance with long-term radiological performance objectives is dependant upon the ability to agree with stakeholders and engineers on modeling scenarios, waste form performance, disposal system, and barrier performance.

Mitigation: A series of performance assessment discussions are planned with stakeholders and include development of several stages of performance assessment model results through the life of the program.

\subsection{Major Decisions}

Cataloging major TWRS Program decisions and the tasks required to make those decisions is an ongoing task with TWRS System Engineering, RL, and the LLW Program Office. Currently, Systems Engineering has issued four major decisions for the LLW vitrification program. Additional decisions have been identified by Systems Engineering, but are currently in draft form only. In addition, the LLW Program has identified major decisions that impact the program but are not reflected in the functional logic of Systems Engineering. The LLW Program baseline plans are based on those decisions where the four major decisions which have been formally identified in Systems Engineering and Program planning documents. Additional decision points are listed below for information and will be incorporated into the program logic as they are reviewed and accepted in the Systems Engineering logic and program logic.

The four specific decisions for the LLW Program, arising from the System Engineering analyses, include:

- $\quad$ Determine LLW form/processes(4.2.3.1.a)

- Determine whether immobilized low-level waste can be disposed in 200 Areas (4.2.3.b).

- $\quad$ Determine immobilized Low-Level Waste disposal configuration (4.2.3.1.b).

- Determine if the U.S. Nuclear Regulatory Commission (NRC) supports disposal of SST incidental waste onsite. 
WHC-SP-1101

Additional decisions drafted by Systems Engineering and currently under review include:

- $\quad$ Determine immobilized LLW (ILLW) formulation and disposal system features

- $\quad$ Determine LLW Vitrification Facility concept

- Determine LLW glass form

- $\quad$ Determine LLW melt concept

- Determine ILLW waste package

- Determine cask disposal configuration

- $\quad$ Determine ILLW conveyance method.

Decisions resulting from LLW Program analysis include the above decisions and the following:

- $\quad$ Determine public acceptance of TWRS-EIS for LLW

- Obtain approval to initiate conceptual design

- Obtain approval to initiate construction

- $\quad$ Obtain approval to initiate operations.

\subsection{Assumptions}

The key assumptions for the LLW Program follow.

1. The 200 Area plateau will be acceptable to stakeholders for storage and the ultimate disposal site of LLW.

This assumption is to be addressed in the TWRS-EIS. (4.2, P1, Issue 1)

2. The NRC will concur with DOE plans for treatment and disposal of the SST waste.

This issue will be addressed as integrated flowsheets are developed and the TWRS-

EIS is issued for review. (4.2 01, Closed Site)

3. An acceptable glass formulation will be developed to meet regulatory and performance criteria.

This activity is being addressed through multiple engineering activities involving melter testing, glass formulation development, and performance assessment.

4. Essentially all $(99+\%)$ of the tank waste will be retrieved and processed.

The LLW facility is being designed to handle $3.2 \mathrm{E}+8$ liters of waste, the majority of all tank waste.

5. The immobilized LLW will be packaged and stored onsite to allow future movement if deemed necessary.

This assumption will be addressed in engineering studies and again during conceptual design. 
6. Other program element activities (Waste Pretreatment, Waste Retrieval, etc.) will proceed on a schedule that supports the needs/inputs of the LLW Program.

This assumption is being addressed through TWRS Program and Systems Engineering integration.

7. Blending and pretreatment of tank waste can provide feed that meets the feed specifications required for vitrification, resulting in a product that meets specifications for disposal.

This assumption is being addressed through the TWRS Waste Retrieval and Waste Pretreatment Programs' activities.

9. The LLW vitrification process will be based on vendor testing of simulants, laboratory- and bench-scale testing of radioactive waste samples, and cold testing of facility components. A hot pilot plant will not be required for verification of the design concept.

This assumption is built into the program logic and funded technology activities.

10. The LLW vitrification facility will have adequate throughput capacity to complete its vitrification mission by 2022 , using a LLW feed stream resulting from cesium ion exchange. Based on this operating scenario, it is estimated that approximately $1.3 \times 10^{6}$ metric tons of vitrified LLW will be produced.

This assumption will be analyzed in the LLW Vitrification Design Requirement Document and Systems Engineering integration analysis.

11. The LLW vitrification project will be authorized as an FY 1997 design only Major Systems Acquisition. The following tasks must be completed to support start of design:

- $\quad$ Completion of the conceptual design report by November 1996

- $\quad$ Completion of phase 1 melter vendor tests by February 1995.

This assumption is being addressed between WHC and DOE project organizations.

12. The LLW Vitrification Facility will use high throughput melter(s) developed for application in the glass industry. The LLWVP planned processing capacity of 200-metric-tons-per-day production will minimize the need for additional DST storage. High throughput melter technology developed for the commercial industry will prove to be adequate for the LLW immobilization mission.

This assumption is incorporated in technology testing activities and will be included in Functions and Operating Requirements presented to the A/E.

13. All permits, Safety Analyses, EISs, and Performance Assessments (PAs) will be approved in time to support facility construction and startup schedules.

This assumption is incorporated into program logic with time allowances for review and comment resolution. 
14. During the development and implementation of the LLW Program, no waivers for regulatory requirements (e.g., DOE Orders, Code of Federal Regulations, Washington Administrative Codes), are anticipated or requested.

This assumption is incorporated into program logic for compliance activities.

15. Budget authority will support the LLW vitrification program schedule; and capital cost increases/decreases as required as additional engineering documentation/estimates are completed.

This assumption will be addressed each fiscal year as cost estimates, schedules, and work scope are prepared.

\subsection{Constraints}

The LLW Program and processing facility must work within the constraints of DOE, WHC, and TWRS technical, regulatory, and administrative framework. These constraints provide guidance for the execution of the program . There is an entire series of administrative and procedural requirements for DOE, WHC, and TWRS. Those constraints that are most relevant to, but not limited to, the LLW Program include the following:

- $\quad 40$ CFR 268, "Land Disposal Restrictions," Code of Federal Regulations

- 40 CFR 264, "Standards for Owners and Operators of Hazardous Waste Treatment, Storage, and Disposal Facilities," Code of Federal Regulations

- DOE Order 4700.1, Project Management System

- $\quad$ DOE Order 5400.3, Hazardous and Radioactive Mixed Waste Program

- $\quad$ DOE Order 5400.5, Radiation Protection of the Public and Environment

- DOE 5820.2A, Radioactive Waste Management

- Hanford Federal Facility Agreement and Consent Order, Fourth Amendment, January 1994, Tri-Party Agreement Change Control Form M-60-93-01

- WAC 173-303, "Dangerous Waste Regulations," Washington Administrative Code.

Additional constraints can be found in DOE/RL-92-60 Rev 1, Tank Waste Remediation System Functions and Requirements.

\subsubsection{Structure}

The LLW Program work breakdown structure and organization and responsibilities are provided in this section. 


\subsection{Work Breakdown Structure}

The Low-Level Waste Program WBS element is part of the Process Tank Waste WBS Level III element and consists of the following WBS Level V elements:

- Technical Integration and Planning (WBS 1.1.1.3.02.01)

- Technology Development and Applied Engineering (WBS 1.1.1.3.02.02)

- $\quad$ Facility Operations (WBS 1.1.1.3.02.03)

- System Definition (WBS 1.1.1.3.02.04)

- LLW Facility (WBS 1.1.1.3.02.05)

- $\quad$ Grout Facilities (WBS 1.1.1.3.02.06).

The LLW Program WBS is shown in Figure 7.9-1. The LLW Program WBS Dictionary is found in Appendix A.

\subsection{Organization and Responsibility}

The LLW Program is responsible for ensuring the safe treatment and disposal of the low-level tank waste. This includes obtaining and utilizing the resources required to design, construct, permit, and operate the LLW Vitrification Plant and storage/disposal facilities. The roles and responsibilities of those organizations performing through the resources of the LLW Program are described below.

Technical Integration and Planning is directed by an activity manager within the LLW Program Office. This organization has responsibility for development of program cost, schedule, and scope baseline information. In addition, this organization is responsible for ensuring integration of the LLW Program with other TWRS, WHC, and Hanford programs.

Technology Development and Applied Engineering is directed by an activity manager within the LLW Program Office, with technical support from several engineering and technical organizations, including the following: WHC Engineering, Technology Development Program Office, Pacific Northwest Laboratory, nationally known universities, contracts with private industry, and private consultants.

This organization is responsible for fundamental data development required to design, permit and operate the LLW processing plant. Activities include process equipment testing, equipment development, waste form development, and waste analysis.

Facility Operations activities are accomplished by WHC TWRS Operations and Engineering staff during conceptual design activities. As the program progresses through construction, system turnovers, and startup, the Operations organization will evolve to accept responsibility for facility operations and production.

System Definition activities are directed by an activity manager within the LLW Program Office. These activities are accomplished with TWRS Engineering and subcontracted engineering organizations. This organization is responsible for analysis and preparation of technical requirements to be implemented by the LLW processing facility. This includes preliminary process flowsheet development, engineering trade studies, performance assessments, and preparation of a Design Requirements Document (DRD). 


\section{WHC-SP-1101}

LLWVP design, construction, and startup activities are directed by the Project manager and are accomplished by the WHC Projects organization. Functions and Operational Requirement (F\&OR) will be developed from the DRD and provided to a subcontracted $\mathrm{A} / \mathrm{E}$ and a construction contractor. This organization has responsibility for design and construction of the LLW processing and disposal facilities.

Grout Facilities activities are accomplished by the WHC Grout Facilities staff. This staff of management and operators is responsible for maintaining the Grout facility in a standby mode until a final decision is made to operate or shut down.

Numerous other organizations will be involved in the activities and goals assigned as responsibilities to the LLW Program. Interfacing will be required to accomplish the exchange of ideas, assignment/identification of tasks, budget and schedule agreements, and finalizing of work scope. The following organizations, both internal and external, interface with the LLW Program.

- TWRS Program Offices (Characterization, Waste Pretreatment, High-Level Waste, Tank Farm Operations and Maintenance, and Waste Tank Safety)

- Washington State Department of Ecology (Ecology), the U.S. Environmental Protection Agency, stakeholders, advisory panels, and DOE.

\subsubsection{FY 1995 Work to be Performed}

The FY 1995 work scope is summarized in this section.

\section{Technical Integration and Planning (WBS 1.1.1.3.02.01)}

This activity includes the following.

- Activity ID L1010100-Fiscal Year Work Plan Maintenance. This activity will focus on the resolution of minor comments on the FY 1995 work plan plus monthly statusing of the schedule, which the program will use for reporting requirements.

- Activity ID L1010200-Multi Year Program Plan/Baseline. This activity will focus on the following: review the previous baseline; prepare any changes to the baseline as a result of other planning documents/changes to systems engineering approach, etc.; input the changes to the baseline data base and reconcile variances from previous baseline; submit the baseline documentation to RL for approval; and status the baseline schedule.

- $\quad$ Activity ID L1010300-Fiscal Year Activity Data Sheet (ADS). This activity is for the preparation of the ADS narrative; all other data will be from the baseline. This is a minimal effort activity but vital to the program because the ADS supports the Congressional Budget Cycle. 
- $\quad$ Activity ID L1010400-Fiscal Year Work Plan. This activity is for the development of the FY 1996 Work Plan. This will involve the following: review the baseline (because the development of the work plan should mirror the baseline documentation); prepare documentation required for the work plan; input the data to the database; generate the schedule, schedule logic, and other applicable information as required; submit the work plan to RL for review and approval; incorporate changes from RL (these would be major changes prior to the FY 1996 work plan being approved by September 31, 1995).

- $\quad$ Activity ID L1020100-Update LLW Strategy Documents. This activity is for the review and updating of the LLW Program Strategy document on an annual basis. This document will take input from various arenas (systems engineering) and provide overall direction to the program.

- $\quad$ Activity ID L1040100-Support Systems Engineering. This activity will provide the resources from the LLW Program in support of the formal systems engineering process, as required by the Defense Nuclear Facilities Safety Board. This process will develop the Functions and Requirements documents and the subsequent hierarchial order of the documents.

- $\quad$ Activity ID L1050100-Program Management Support. This activity will provide overall management of the LLW Program with respect to programmatic technical direction, analysis of engineering studies, attending various meeting (staff, status), required support from Quality Assurance and Safety, and financial/schedule/estimating support.

- $\quad$ Activity ID L1060100-Support Public Involvement. This activity will support the systems engineering process as well as making this program-successful from a technical basis.

\section{Technology Development and Applied Engineering (WBS 1.1.1.3.02.02)}

This activity includes the following.

- Melter Selection. Commercial melter vendors will be performing Phase I testing to determine if their melter types can be adapted to the LLW Program. After Phase I testing, a downselecting process will determine those vendors who will continue in Phase II testing. The testing will be a phased testing with the first phase as proof of principle. The second phase will allow for enhancements of melter systems to handle progressively difficult simulants. A primary melter technology and an alternate will be chosen at the end of testing.

- Simulant Development and Characteristics. Simulants will be developed for melter testing and supplied to the potential vendors. The first simulant to be developed will be a doubleshell simulant as this will most likely be the first feed to a melter. This simulant is based on double-shell tank waste characterization information. Later, simulants for Phase II testing will be based on the best information available from tank inventories. 


\section{WHC-SP-1101}

- Glass Formulation. Formulations will be developed to support melter testing and selection in the early phases of the Program. Formulations to support the Program will continue to be developed as characterization information becomes available. There will be a range of formulations developed for variations in waste as tanks are retrieved. The characteristics of the various glasses to be produced can then be used in modeling on the entire disposal package (and site) to determine the effects on the environment. This work will proceed concurrently as the design work on the waste package and disposal site is developed. Durability testing will be carried on for glass formulations. Work in predicting off gas and non-vitrifiable products will support design of the Vitrification Facility. All work will follow closely with melter selection.

- Ancillary Process Requirements. Development of process needs will be as identified through melter testing, flowsheet development, and engineering studies. These will include feed systems, melter off gas system development, and glass product handling. In addition, non-vitrifiable products will be identified, and methods of disposing or recycling these products will be developed.

- Disposal Matrix/Container Selection. Disposal of vitrified LLW on the Hanford Site will require meeting DOE Order 5820.2A. This is currently envisioned to require a matrix or container around the glass product as part of the disposal system. The compatibility of a matrix/container will be determined to ensure it will meet the long-term performance requirements.

- Disposal System Selection. Studies are necessary to support disposal system engineering and design. These include developing and documenting a technical basis for disposal systems, data needs for the PA to eliminate conservatism and provide a defensible PA and modeling to ensure that the overall disposal system will perform.

- Glass Quality Performance. The need for certifying the quality of glass waste form integrity and integrity of melter refractory will be approached from a non-destructive testing avenue. An important lesson learned from Grout was that this is a long-lead item that should be worked upfront.

- $\quad$ Feed Specification. Development of a waste feed specification will be an interactive process with Pretreatment. Feed specifications will be a combination of pretreatment technologies for radionuclide removal, waste retrieval sequence, and melter/formulation ability to capture wastes.

The Technology Development and Applied Engineering work element will provide information to support the following.

- Performance Assessment. During the design and development of the project, the basis of the PA will be used to guide decisions. Scoping PAs will be run to guide decisions as glass quality disposal concepts, siting of disposal system, and matrix/packaging materials. This will allow for determining appropriate disposal methods for ensuring successful passing of the PA.

- Permitting Activities. These activities include development and application of methods for the quality verification of the glass waste form and the matrix and disposal system, along with providing data on the integrity of the disposal system. 


\section{WHC-SP-1101}

- Safety Analysis. Technology activities will focus on development of specific needs for operating within the specified safety envelope. These could include enhancements of formulation, refractory development and buildup of constituents, development of off gas treatment, melter stabilization, and product-handling techniques.

- Design, Construction, and Operations of the LLW Facility. Selection of melter technologies, feed systems, formulations, off gas systems, refractories, and filtration systems for the design and construction will be supported by Technology Development and Applied Engineering. As operations progress, needs will be identified as changes in formulation or melter system improvements to meet the needs of changing feeds due to retrieval sequence of the tanks.

\section{Facility Operations (WBS 1.1.1.3.02.03)}

This activity includes the following.

- $\quad$ Activity ID L3010100-Baseline Development Support. Provide support to Systems Engineering and LLW Program during baseline development to ensure that operations and maintenance needs are incorporated into the design of the LLWVP.

- $\quad$ Activity ID L3020100-M\&O Management and Planning. Prepare Operational Readiness roles and responsibilities definition document. Initiate early operations planning for the LLWVP. Manage personnel involved in other work packages in FY 1995.

\section{System Definition (WBS 1.1.1.3.02.04)}

This activity includes the following.

- $\quad$ Define LLW Functions and Requirements (F\&R):

- Develop LLW F\&R and revisions as needed to serve as a technical baseline for project execution.

- $\quad$ Define LLW feed waste acceptance criteria reflecting process flowsheet developments and key interface points between waste pretreatment and LLW vitrification. Prepare initial criteria document and update as warranted by program developments.

- $\quad$ Define ILLW disposal product criteria factoring in PA developments, glass formulation studies, tank waste retrieval sequences, processing constraints, degree of radionuclide separations, and waste composition variability.

- Define and develop process and disposal system trade studies including updating the Facility Configuration Options Study, Phase II trade studies, and further refinement and development of disposal systems concepts. Perform a decision analysis for a recommended disposal system concept. 
- $\quad$ Prepare LLW PA:

- Identify and develop experimental data needs for the PA, including objectives, disposal scenarios, waste source terms, hydrologic and recharge data, chemical and radionuclide transport data, groundwater data, disposal systems impacts, dosimetry data, computer models, and integration of these items.

- Prepare plans for site sampling and characterization.

- $\quad$ Define LLW Technical Baseline:

- $\quad$ Prepare, summarize, and document a LLW Program technical baseline to provide uniform technical guidance to program and project participants. Update document as new developments emerge.

- $\quad$ Perform LLWVP queuing and lag storage requirements study.

- Technical Basis Document Maintenance:

- Define and develop a project media handling system.

- $\quad$ Engineering Review and Program Support:

- $\quad$ Support LLW Systems Definition planning, reporting, and special requests. Develop detailed LLW decision logic charts to guide technical developments.

- Compliance

- Continue safety assessments supporting trade studies and provide guidance to designers for development of the Conceptual Design Report (CDR) and design initiation.

- $\quad$ Perform Best Available Radionuclide Control Technology (BARCT), Best Available Control Technology (BACT), and T-BACT analysis and support the development of facility permits.

\section{LLW Facility (WBS 1.1.1.3.02.05)}

This activity includes the following.

- $\quad$ Plan and manage all project activities including project plans and procedures.

- $\quad$ Provide project reporting system, including written reports and status presentations.

- $\quad$ Prepare and maintain project schedules.

- Ensure preparation and maintenance of project estimates-Total Estimated Cost (TEC) and Total Project Cost (TPC).

- $\quad$ Support DOE in yearly budget validations and key decision process. 


\section{WHC-SP-1101}

- Develop Quality Assurance (QA) plans; perform audits and surveillances.

- Establish QA requirements: records, trend analyses, training.

- Establish project-wide training program.

- Support public involvement activities initiatives established by the Program Office.

- Prepare and approve all safety analyses. Ensure independent reviews.

- Prepare and approve all permit applications. Ensure internal reviews and regulator reviews are accomplished.

- Prepare preliminary and detailed design in compliance with the F\&OR and the project design criteria (AE/CM).

- $\quad$ Provide day-to-day direction of the contracted design agent (WHC).

- $\quad$ Review and approve design products (WHC).

- $\quad$ Provide as-built documentation required to operate and maintain the facilities (AE/CM).

- $\quad$ Prepare operational and maintenance procedures (WHC and AE/CM).

\section{Grout Facilities (WBS 1.1.1.3.02.00)}

This activity includes the following.

- Complete remaining activities to deactivate the Grout Facilities.

- Maintain the Facilities in a standby condition as specified by the January 25, 1994 , Tri-Party Agreement.

- $\quad$ Provide the capability to restart the Facilities within a two year window as specified by DOE-RL letter no. 93-DSB-044, L. Erickson to President, Westinghouse Hanford Company, dated 21 December 1993.

To meet these expectations:

- $\quad$ Pursue the removal and replacement of the failed mixer in tank 241-AP-102. 
WHC-SP-1101

\subsubsection{FY 1996 - FY 1997 Work to be Performed}

The FY 1996 - 1997 work scope is summarized in this section.

\section{Technical Integration and Planning (WBS 1.1.1.3.02.01)}

This activity includes the following.

- $\quad$ Activity ID L10101XX-Fiscal Year Work Plan Maintenance. This activity will focus on the resolution of minor comments on the FY 1996 work plan plus monthly statusing of the schedule, which the program will use for reporting requirements.

- Activity ID L10102XX-Multi Year Program Plan/Baseline. This activity will focus on the following: review the previous baseline; prepare any changes to the baseline as a result of other planning documents/changes to systems engineering approach, etc.; input the changes to the baseline database and reconcile variances from previous baseline; submit the baseline documentation to $\mathrm{RL}$ for approval; and status the baseline schedule.

- $\quad$ Activity ID L10103XX-Fiscal Year ADS. This activity is for the preparation of the ADS narrative; all other data will be from the baseline. This is a minimal effort activity but vital to the program because the ADS supports the Congressional Budget Cycle.

- Activity ID L10104XX-Fiscal Year Work Plan. This activity is for the development of the FY 1997 and FY 1998 Work Plan. This will involve the following: review the baseline (the development of the work plan should mirror the baseline documentation); prepare documentation required for the work plan; input the data to the database; generate the schedule, schedule logic, and other applicable information as required; submit the work plan to $\mathrm{RL}$ for review and approval; incorporate changes from RL (these would be major changes prior to the FY 1996 work plan being approved by September 31, 1995).

- Activity ID L10201XX-Update LLW Strategy Documents. This activity is for the review and updating of the LLW Program Strategy document on an annual basis. This document will take input from various arenas (systems engineering) and provide overall direction to the program.

- Activity ID L10401XX-Support Systems Engineering. This activity will provide the resources from the LLW Program to the formal systems engineering process.

- $\quad$ Activity ID L10501XX-Program Management Support. This activity will provide overall management of the LLW Program with respect to programmatic technical direction, analysis of engineering studies, attending various meeting (staff, status), required support from Quality Assurance, Safety, and financial/schedule/estimating support.

- Activity ID L10601XX-Support Public Involvement. This activity will support the systems engineering process as well as making this program successful from a technical basis. 


\section{Technology Development and Applied Engineering (WBS 1.1.1.3.02.02)}

This activity includes the following.

- Melter Selection. Commercial melter vendors will be performing Phase II testing to determine if their melter types can be adapted to the LLW Program as the reference melter for the LLWVP. After phase II testing, a reference melter will be selected to be included with the design of the LLWVP.

- $\quad$ Simulant Development and Characteristics. Simulants will be developed for melter testing and supplied to the potential vendors. Later, simulants for Phase II testing will be based on the best information available from tank inventories.

- Glass Formulation. Formulations will be developed to support melter testing and selection. Formulations to support the Program will continue to be developed as characterization information from pretreatment becomes available. There will be a range of formulations developed for variations in waste as tanks are retrieved. The characteristics of the various glasses to be produced can then be used in modeling on the entire disposal package (and site) to determine the effects on the environment. This work will proceed concurrently as the design work on the waste package and disposal site is developed. Durability testing will be carried on for glass formulations. Work in predicting off gas and non-vitrifiable products will support design of the Vitrification Facility. All work will follow closely with melter selection. Formulations will be analyzed in a cold pilot plant to optimize waste loadings and enhance performance characteristics.

- Ancillary Process Requirements. Development of process needs as identified through melter testing, flowsheet development, and engineering studies. These will include feed systems for melter off gas system development, and glass product handling. In addition, non-vitrifiable products will be identified, and methods of disposing or recycling these products will be developed.

- Disposal Matrix/Container Selection. Disposal of glass on the Hanford Site will require meeting DOE Order 5820.2A. Performance Assessment work scope is covered in WBS element 1.1.1.3.02.04. This is currently envisioned to require a matrix or container around the glass product as part of the disposal system. The compatibility of a matrix/container will be developed to ensure it will meet the long-term performance requirements.

- Disposal System Selection. Technology studies are necessary to support disposal system engineering and design. These include developing and documenting a technical basis for disposal systems, data needs for the PA to eliminate conservatism and provide a defensible $\mathrm{PA}$, and modeling to ensure that the overall disposal system will perform adequately to meet performance criteria.

- Glass Quality Performance. The need for certifying the quality of glass waste form integrity and integrity of refractory will be approached from a non-destructive testing avenue. An important lesson learned from Grout was that this is a long-lead item that should be worked upfront. Product quality will be arrived at through cold pilot plant testing by using simulated pretreated product specification, pretreatment output to the LLWVP, combined with glass formers to optimize the product quality. This will be an iterative process when tailoring to specific waste streams from pretreatment. 
- $\quad$ Feed Specification. Development of a feed specification will be an interactive process with Pretreatment. Feed specifications will be a combination of pretreatment technologies for removal, retrieval sequence and melter/formulation ability to capture wastes.

The Technology Development and Applied Engineering work element will provide information to the support the following.

- Performance Assessment. During the design and development of the project, the basis of the PA will be used to guide decisions. Scoping PAs will be run to guide decisions as glass quality disposal concepts, siting of disposal system, and matrix/packaging materials. This will allow for determining the best methods and ensuring successful passing of the PA.

- Permitting Activities. These activities include development and application of methods for the quality verification of the glass waste form and the matrix and disposal system, along with providing data on the integrity of the disposal system.

- Safety Analysis. Technology activities will focus on development of specific needs for operating within the specified safety envelope. These could include enhancements of formulation, refractory development and buildup of constituents, development of off gas treatment, melter stabilization, and product-handling techniques.

- Design, Construction, and Operations of the LLW Facility. Selection of melter technologies, feed systems, formulations, off gas systems, refractories, and filtration systems for the design and construction will be supported by Technology Development and Applied Engineering. As construction and operations progress, needs will be identified as changes in formulation or melter system improvements to meet the needs of changing feeds due to retrieval sequence of the tanks.

\section{Facility Operations (WBS 1.1.1.3.02.03)}

This activity includes the following.

- Activity ID L3020100 M\&O Management and Planning. Prepare Operational Readiness roles and responsibilities definition document. Initiate early operations planning for the LLWVP. Manage personnel involved in other work packages in FY 1996 and FY 1997.

\section{System Definition (WBS 1.1.1.3.02.04)}

This activity includes the following.

- $\quad$ Define LLW Functions and Requirements (F\&R):

Develop LLW F\&R and revisions as needed to serve as a technical baseline for project execution.

- $\quad$ Update LLW feed waste acceptance criteria reflecting process flowsheet changes as warranted by program developments. 
WHC-SP-1101

- $\quad$ Define ILLW disposal product criteria factoring in PA developments, glass formulation studies, tank waste retrieval sequences, processing constraints, degree of radionuclide separations, and waste composition variability.

- $\quad$ Prepare LLW PA:

- Develop experimental data needs for the PA including objectives, disposal scenarios, waste source terms, hydrologic and recharge data, chemical and radionuclide transport data, groundwater data, disposal systems impacts, dosimetry data, computer models, and integration of these items.

- Develop a soil baseline for the new TWRS complex with emphasis on the LLW disposal area. Develop the information to support a site environmental baseline.

- $\quad$ Provide management and write the interim PA. Review the interim PA and initiate preparation of the preliminary PA. The interim PA is intended to show that there is a reasonable expectation that the disposal of vitrified LLW can meet required performance objectives.

- Define LLW Technical Baseline:

- Update the LLW Program technical baseline document as new developments emerge.

- Technical Basis Document Maintenance:

- $\quad$ Implement a project media handling system.

- $\quad$ Engineering Review and Program Support:

- $\quad$ Support LLW Systems Definition planning, reporting, and special requests. Update LLW decision logic charts to guide technical developments.

- Compliance:

- $\quad$ Provide guidance to designers for development of the CDR and design initiation.

- $\quad$ Complete BARCT, BACT, and T-BACT analysis and support the development of facility permits.

\section{LLW Facility (WBS 1.1.1.3.02.05)}

This activity includes the following.

- $\quad$ Plan and manage all project activities including project plans and procedures.

- $\quad$ Provide project reporting system, including written reports and status presentations.

- $\quad$ Prepare and maintain project schedules.

- $\quad$ Ensure preparation and maintenance of project estimates--TEC and TPC. 
WHC-SP-1101

- $\quad$ Support DOE in yearly budget validations and key decision process.

- Develop QA plans; perform audits and surveillances.

- $\quad$ Establish QA requirements: records, trend analyses, training.

- $\quad$ Establish project-wide training program.

- $\quad$ Support public involvement activities initiatives established by the Program Office.

- $\quad$ Prepare and approve all safety analyses. Ensure independent reviews.

- $\quad$ Prepare and approve all permit applications. Ensure internal reviews and regulator reviews are accomplished.

- $\quad$ Prepare preliminary and detailed design in compliance with the F\&OR and the project design criteria (AE/CM).

- $\quad$ Provide day-to-day direction of the contracted design agent (WHC).

- $\quad$ Review and approve design products (WHC).

- $\quad$ Provide as-built documentation required to operate and maintain the facilities (AE/CM).

- $\quad$ Prepare operational and maintenance procedures (WHC and AE/CM).

\section{Grout Facilities (WBS 1.1.1.3.02.06)}

This activity includes the following.

- Maintain the Facilities in a standby condition as specified by the January 25, 1994, Tri-Party Agreement.

- $\quad$ Provide the capability to restart the Facilities within a two-year window, as specified by DOERL letter no. 93-DSB-044, L. Erickson to President, Westinghouse Hanford Company, dated December 21, 1993.

\subsubsection{Approach}

The overall major approach for the LLW Program has been outlined in the January 1994 Tri-Party Agreement update, which outlined the strategy utilizing a LLWVP. In addition, the LLW Program has developed a technical strategy document to "chart" a successful path forward.

The approach shown below is discussed by WBS elements. 
The approach for this WBS element is continually to improve the technical, cost, and schedule for the LLW Program for the next several years by more concisely using scoping statements and deliverables. Vendors will be utilized in developing a estimating database. The vast majority of work will be performed by subcontractors with $\mathrm{M} \& \mathrm{O}$ contractor oversight.

\section{LLW Facility (WBS 1.1.1.3.02.05)}

Early melter development tests will demonstrate the viability of vitrification as a suitable tank lowlevel tank waste treatment. These development tests will use commercial glass/vitrification vendors, who will compete using a waste simulant. The testing is planned to be broken into two phases. The first will focus on using a generic simulant and making a vitrified product in any scale. The products will be tested by an independent laboratory (vendor) to determine the quality of product compared to product quality specifications. It should be noted that at the end of Phase I, information will be provided to the conceptual design effort. After the first phase, a structured downselection process will be established to determine those vendors who will continue to Phase II. Phase II will be of a larger scale, using Hanford tanks' specific simulants. The same process will occur and then a donwselection to a reference high-output melter design/concept. This reference melter will be a part of the Title I design effort.

The current baseline contracting approach is to use fixed price subcontracts to the maximum extent practical. Fixed price contracting will be considered for detail design (Title I, Title II, etc) and construction management. Alternative contracting approaches are being considered. These include commercialization of privatization. The commercialization contracting approach would entail hiring a single contractor to design, build, and operate the LLWVP. This would be government funded, with fixed price incentive fee contracts based on performance. A privatization approach involves a single contractor to design, build, and operate. The contractor would provide all project funding (design, build, startup). The privatization contractor would recoup this up front investment with fee payment tied to product deliveries during the operational phase.

Operation of LLW vitrification will use pretreated double-shell slurry feed as early feed, as well as SST saltcake. Operations activities will be evaluated of being privatized or operated by the M\&O contractor. The baseline shows the $\mathrm{M} \& \mathrm{O}$ contractor being the operator.

As part of conceptual design, a LLW Vitrification Facility radiation shielding requirement will be established, predicated upon removal of cesium and strontium from the tank waste by the pretreatment function.

The LLWVP will be designed based on testing using simulants, laboratory- and bench-scale testing of radioactive waste samples, and cold testing of facility components. A hot pilot plant will not be required for verification of design concept. The alternate approach is that an engineering study will be prepared and the design/build contractor will evaluate the study and recommend whether a hot pilot plant is required to guarantee success of the plant.

This facility will be granted interim status to allow construction. A final dangerous waste permit will be required prior to initiation of hot operations. Prior to construction, DOE will be required to obtain a letter from Ecology authorizing construction. This authorization will be based on Ecology's ongoing review of melter development and facility design to ensure compliance with appropriate 
WHC-SP-1101

environmental regulatory requirements. Concurrently with this approach, development of permits will be ongoing.

\section{Grout Facilities (WBS 1.1.1.3.02.06)}

The M\&O contractor is to provide the expertise to develop a comprehensive and detailed level of planning to ensure that the facility is transitioned to a safe standby condition in a controlled and orderly manner with minimal impact to plant personnel. The plan (and its implementation) must support a safe restart of the facility within two years in the event its operation is necessary to provide tank space for safety reasons prior to construction of additional double-shell tanks.

- $\quad$ Proceed with the deactivation of the Grout Facilities

- Maintain the Facilities in a standby condition as specified by the January 25, 1994, Tri-Party Agreement

- $\quad$ Provide the capability to restart the Facilities within a two-year window, as specified by DOE-RL letter no. 93-DSB-044, L. Erickson to President, Westinghouse Hanford Company, dated December 21, 1993.

\subsubsection{Drivers and Deliverables}

The drivers for the LLW Program are defined in Tank Waste Remediation System Functions and Requirements, DOE/RL-92-60, Rev 1, Functions Requirements, Table F4.2.3.1, Item V,; systems Architecture, Table A4.2.3.1, Item VIII, paragraph C, Tables A-1, B-1, B-2, B-3, and B-5 where applicable.

One of the key drivers for separating the tank waste into an optimally reduced volume of high-level waste (HLW) with the remaining volume as LLW is the anticipated cost of disposing of defense HLW in the national geological repository, which is projected to cost significantly more per unit volume than near-surface disposal of LLW at the Hanford Site.

Another driver for the LLW Program is the urgency by stakeholders and regulators to see real progress in cleanup of the Hanford Site, particularly to initiate retrieval, pretreatment, and disposal of the tank waste. Specifically, retrieving these wastes from tanks that are leaking or have the potential to leak waste to the groundwater and transforming those wastes into a more chemically stable form is highly desirable, even if it is necessary to store the canistered HLW at the Hanford Site for several years while a repository is sited and built.

The LLW Program deliverables are documented in Level IV, V, and VI WBS dictionary sheets (refer to Appendix A) and in the LLW Program Milestone Description Sheets (refer to Appendix D).

\section{Grout Facilities (WBS 1.1.1.3.02.06)}

The drivers for this activity are defined in the Tri-Party Agreement, signed January 25, 1994, and the Grout Treatment Facility Standby Plan, WHC-SD-WM-SSP-005, January 1994, Tables A-1, B-1, B-2, B-3, and B-5 where applicable. 
Provide the capability to restart the Facilities within a two-year window as specified by DOE-RL letter no. 93-DSB-044, L. Erickson to President, Westinghouse Hanford Company, dated December 21, 1993.

\subsubsection{Schedule}

\subsubsection{Low-Level Waste Summary Schedule}

The LLW Program summary schedule is provided in Figure 7.9-2. This schedule accurately portrays in graphic format the summary-level work scope for the life of the LLW Program. Significant activities and milestones for the program are identified from a TWRS Program integrated and controlled database. All Tri-Party Agreement major and selected interim milestones applicable to this program element are included on the summary schedule. The LLW Program Milestone Description Sheets and Log are found in Appendix D. There is at least one activity for each Level V WBS element. Logical ties within and between other program elements are provided where they offer clarification.

The principal activities critical path within the LLW Program are those required for the selection of a reference melter, conceptual design, design, construction of LLWVP, and startup of the LLWVP. Other requirements are establishing product quality requirements, meeting performance criteria, factoring in public values, with the ultimate objective of designing, constructing, and operating the LLWVP for safe processing of Hanford's low-level waste inventory. This includes development of the required technology and closeout of the Grout facilities. The LLW Program is responsible for the completion of Tri-Party Agreement Major Milestone M-60-00, Complete Vitrification of Hanford Low-Level Tank Waste to be completed by December 2028.

The program element schedule is a demand schedule and one of risk (as shown in baseline documentation). Many critical activities are being worked in parallel to meet this demand schedule. Hot operations in June of 2005 is the critical path through the design, construction, and startup of the LLWVP. Selection of the reference melter in June 1996 is a critical path activity. With this critical path, it is paramount that there is no delay in approval of DOE Key Decisions. It is critical that the $\mathrm{DOE}-\mathrm{HQ} / \mathrm{RL}$ and the M\&O contractor focus and operate as a team. It should be noted that Key Decision process may need to be evaluated based on experience with KD0.

\subsubsection{Low-Level Waste Expanded Baseline (FY 1995 - FY 1997)}

Low-Level Waste expanded baseline for FY 1995 - FY 1997 is located in Appendix C.

\subsubsection{Resource Requirements}

\subsubsection{Cost}

\subsection{Basis of Cost Estimates}

The costs basis for the LLW Program utilizes historical data, experience of other glass plants proposed at Hanford, rough order of magnitude estimates and evolving engineering studies. The major cost item to this program is the LLWVP, which is envisioned to cost between $\$ 0.9$ billion and 
$\$ 1.5$ billion to design, construct, and start up. The wide variation of costs are the difference between a lightly shielded and heavy shielded facility. See program uncertainties for additional data.

\subsection{Total Program Element Cost by Year by Fund Type by Activity and Cost Account)}

The basis for the LLWVP in the baseline has minimum basis and will increase significantly as a result of ongoing engineering studies in FY 1994. The estimates will continue to fluctuate until the conceptual design is completed and the LLWVP is validated in FY 1996.

Table 7.9-1 establishes the baseline cost for this program element. The baseline cost is exhibited in prior year cost, annual cost for FY 1995 through FY 2000, and 5-year totals for FY 2001 through program element completion. Also exhibited is the total program element cost including prior years through program element completion. Where the data are available, these baseline costs are exhibited at the cost account level with fund type totals provided. Cost data are then rolled up to the activity and program element levels. At present, the program element cost baseline is the program element cost totals exhibited on this table less a productivity challenge for FY 1995 through FY 1997.

\subsection{FY 1995 Cost by Month by Fund Type by Activity and Cost Account}

Future MYWP updates will include Table 7.9-2, which establishes the month-by-month cost baseline for FY 1995 for this program element. The FY 1995 cost baseline is exhibited at the cost account level with fund type totals provided. Cost data are then rolled up to the activity and program element levels. Also exhibited is the total cost baseline for the program element for FY 1995. At present, the program element cost baseline for FY 1995 for this program element is the total provided on this table less a productivity challenge for FY 1995.

\subsubsection{Staffing}

\subsection{Basis of Staffing Projections}

The basis of staffing estimates shown in the MYWP for the LLW Program is based on a bottoms up approach to estimating.

\subsection{Total Full-Time Equivalents by Year by Activity by Major Participant (Direct and Indirect)}

Future MYWP updates will include Table 7.9-3, which establishes total staffing projections for this program element. Included in the table are FY 1994 staffing data as well as projections by year for FY 1995 through FY 2000. The projections in this table include all direct and indirect Full-Time Equivalents (FTEs), including all subcontractor personnel. Where data are available, projections are exhibited at the activity level with major program participant totals provided.

\subsection{FY 1995 through FY 1997 Direct Full-Time Equivalents by 88 Labor Categories}

Future MYWP updates will include Table 7.2-4, which establishes direct WHC staffing projections for this program element. Projections do not include subcontractor support and are provided by 88 labor categories for FY 1995 through FY 1997. 


\subsubsection{Work Scope Carryover}

Carryover work scope is as follows:

1. Melter Testing. Phase I testing originally planned in FY 1994 will require funding of $\$ 4.0$ million in FY 1995. It should be noted that this amount of carryover work scope and no carryover funding, due to TWRS FY 1994 funding issues will create a risk for the LLW Program. Naturally, the program will continue to work the critical path of the program as its priorities, but eventually the bow wave will have to be paid for. This assumes funding is not restored in FY 1995 for funding reallocated to other ADS in FY 1994.

2. Product Quality studies of $\$ .2$ million were deferred in FY 1994. This was work planned to be performed with universities.

3. TDPO/PNL. $\$ 0.5$ million to issue product/package laboratory studies investigation plan, volatility letter report, small scale meter letter report, cesium migration letter report. In addition to melter testing, glass formulation, off gas development work will be deferred.

4. PNL. \$0.1 million to resolve final Grout Treatment Facility PA comments.

\subsubsection{Waste Type Data}

The LLW Program is comprised of activities that do generate and receive waste requiring direct funding to disposition. The LLW Program's primary mission is to dispose of the low-level tank waste from Hanford. In the accomplishment of this mission, certain quantities of solid, liquid, and gaseous wastes will be received and/or generated that will require treatment and/or disposal. Current inventory of Hanford's waste tanks is approximately $63 \mathrm{Mgal}$, of which 90 percent of the volume is expected to be immobilized in the low-level vitrified waste product. This does not account for any dilution factors. These will be defined in the processing flowsheets and part of the overall TWRS systems engineering approach.

The waste type data for construction of the LLWVP have yet to be identified. On completion of the conceptual design, the waste type data will be available.

Waste type data are found in Appendix $\mathrm{H}$.

\subsubsection{Revisions}

In future updates of the MYWP, this section will include a summary of the revision that has occurred in this program element since the last issue of the MYWP. 
LOW LEVEL WASTE (PAGE 1 OF 2)

WORK BREAKDOWN STRUCTURE

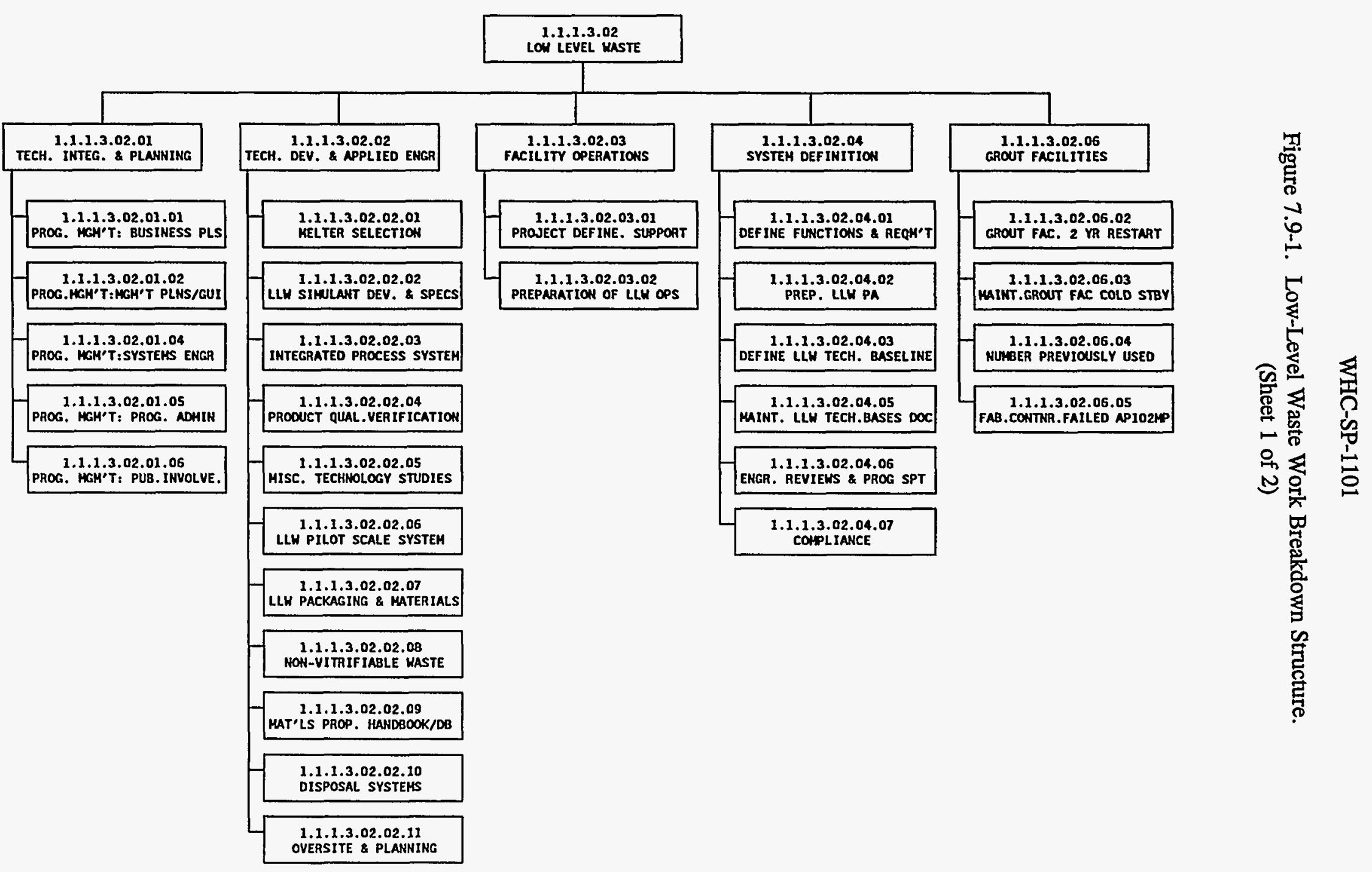


LOW LEVEL WASTE (PAGE 2 OF 2) WORK BREAKDOWN STRUCTURE

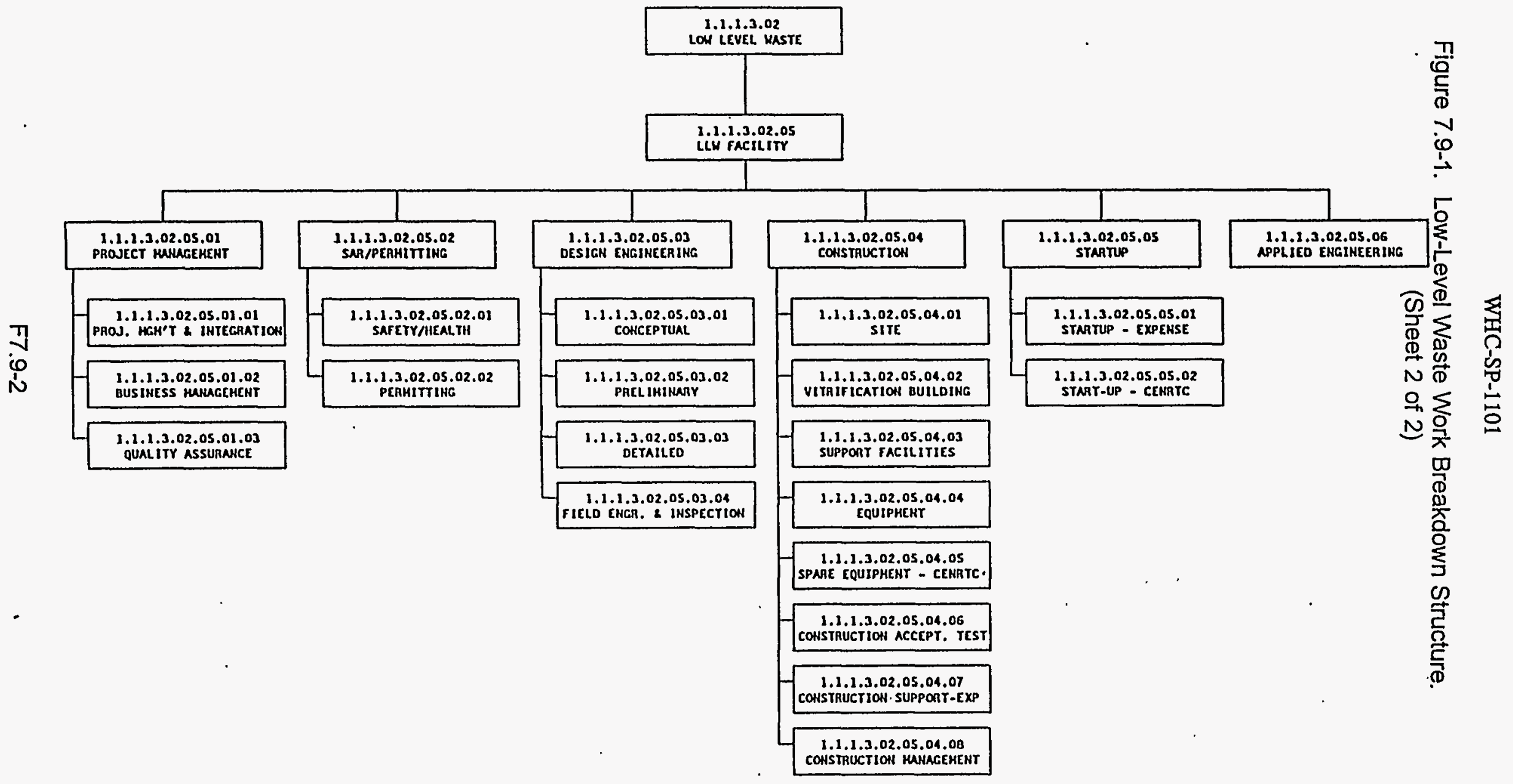


1.11.3.02 Low Level Waste Summary Schedule

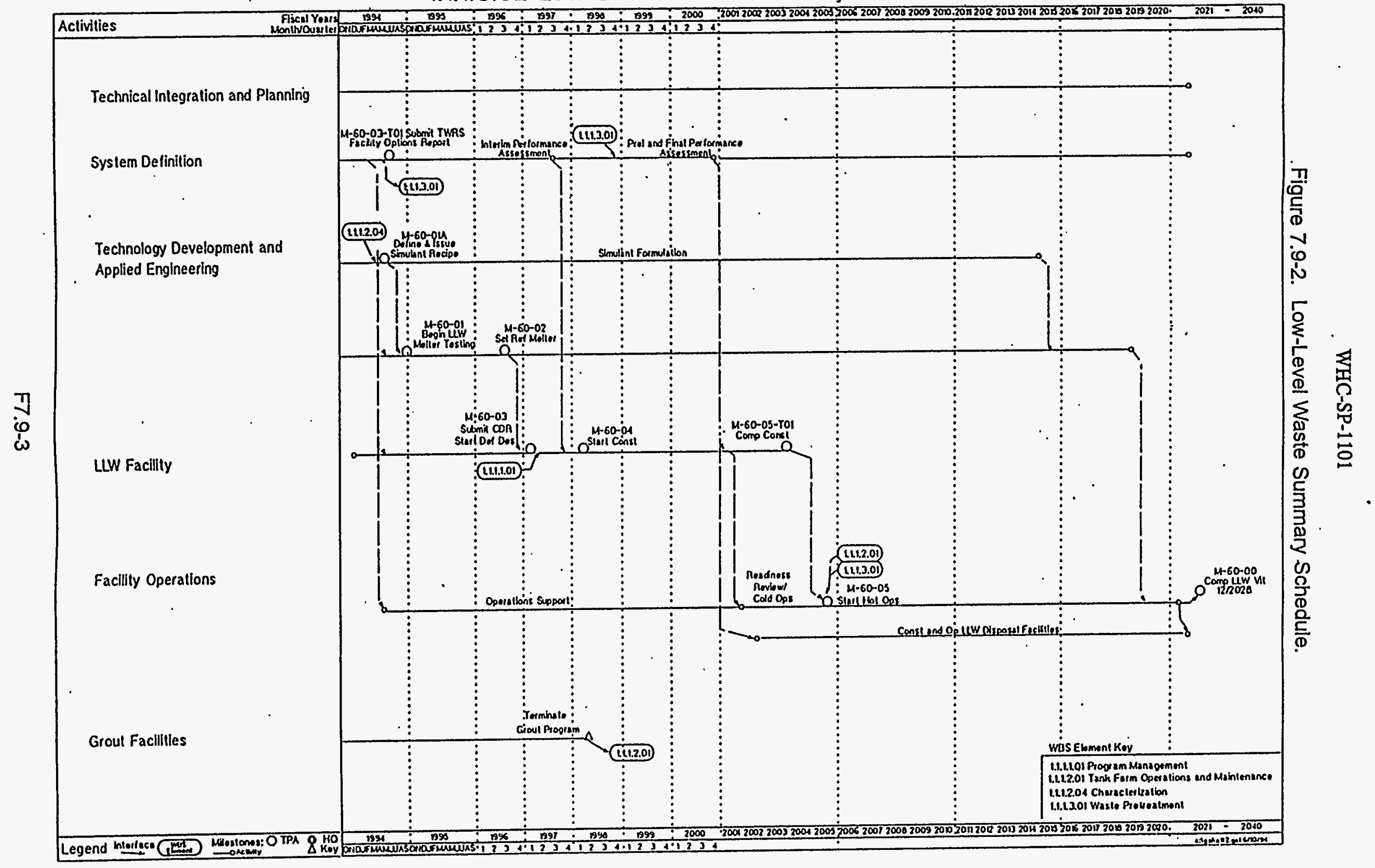


TABLE $\quad 7.9-1$

TOTAL PROGRAM ELEMENT COST BASELINE - BY YEAR

[ R1]

\begin{tabular}{|c|c|c|c|c|c|c|c|c|c|}
\hline $\begin{array}{l}1.1 .1 .3 .02 \\
\text { Level iv }\end{array}$ & $\begin{array}{l}\text { Lö Level Waste } \\
\text { ADS 1230-0 } \\
\therefore \quad \text { TOTAL TARGET }\end{array}$ & $\begin{array}{c}\mathbf{O E} \\
\mathbf{C} / \mathbf{E} \\
\vdots \\
\vdots \\
\vdots\end{array}$ & $\begin{array}{r}32,944 \\
400 \\
0 \\
\hdashline 33,344\end{array}$ & $\begin{array}{r}49,057 \\
569 \\
-00 \\
49,626\end{array}$ & $\begin{array}{r}45,081 \\
1.137 \\
-0 \\
-076,218 \\
46\end{array}$ & $\begin{array}{r}33,812 \\
899 \\
5,343 \\
40,054 \\
\end{array}$ & $\begin{array}{r}47,835 \\
7,006 \\
32,430 \\
81271\end{array}$ & $\begin{array}{r}65,016 \\
1,006 \\
89,065 \\
149,087\end{array}$ & $\begin{array}{r}80,521 \\
1,006 \\
121,558 \\
203,085\end{array}$ \\
\hline $\begin{array}{l}\text { 1.1.1.3.02.01 } \\
\text { Level } v\end{array}$ & $\begin{array}{l}\text { Technical Integration } \\
\frac{\text { and Planning }}{\text { ADS } 1230-0-M N A C} \\
\text { Sub Total }\end{array}$ & $\begin{array}{l}\text { OE } \\
C / E\end{array}$ & NA & $\begin{array}{r}1,444 \\
12 \\
---9 \\
1,456\end{array}$ & $\begin{array}{r}1.684 \\
0 \\
---1 \\
1,684\end{array}$ & $\begin{array}{r}1,581 \\
0 \\
---- \\
1,581\end{array}$ & $\begin{array}{r}0 \\
0 \\
-0\end{array}$ & $\begin{array}{r}\mathbf{0} \\
--- \\
0\end{array}$ & $\begin{array}{r}0 \\
--- \\
0\end{array}$ \\
\hline $\begin{array}{l}1.1 .1 .3 .02 .01 .02 \\
\text { Level VI }\end{array}$ & $\begin{array}{l}\text { Program Management: } \\
\text { Mgmt. Plans / Guidance } \\
\qquad \text { Total }\end{array}$ & $\begin{array}{l}O E \\
C / E\end{array}$ & & $\begin{array}{r}3 \\
0 \\
---\frac{-}{3}\end{array}$ & $\begin{array}{r}3 \\
0 \\
-- \\
3\end{array}$ & $\begin{array}{r}3 \\
0 \\
--- \\
3\end{array}$ & $---\frac{-}{0}$ & --- & ---- \\
\hline $\begin{array}{l}1.1 .1 .3 .02 .01 .04 \\
\text { Level VI }\end{array}$ & $\begin{array}{l}\text { Program Management: } \\
\text { Systems Engineering } \\
\qquad \text { Total }\end{array}$ & $\begin{array}{l}\text { OE } \\
\text { C/E }\end{array}$ & & $\begin{array}{r}29 \\
0 \\
--- \\
29\end{array}$ & $\begin{array}{r}28 \\
0 \\
--- \\
28\end{array}$ & $\begin{array}{r}29 \\
0 \\
--- \\
29\end{array}$ & --- & $-\cdots$ & - \\
\hline
\end{tabular}


TABLE $7.9-1$

TOTAL PROGRAM ELEMENT COST BASELINE - BY YEAR
[ R1 ]
44,210

(K\$)

\begin{tabular}{|c|c|c|c|c|c|c|c|c|c|}
\hline WBS / LEVEL & ACTIVITY TITLE / ADS & $\begin{array}{l}\text { FUND } \\
\text { TYPE }\end{array}$ & $\begin{array}{c}\text { TOTAL } \\
\text { FY94 }\end{array}$ & FY95 & FY96 & FY97 & FY98 & FY99 & FYOO \\
\hline $\begin{array}{l}\text { 1.1.1.3.02.01.05 } \\
\text { Level VI }\end{array}$ & $\begin{array}{l}\text { Program Management: } \\
\text { Prog. Administration } \\
\qquad \text { Total }\end{array}$ & $\begin{array}{l}\mathrm{OE} \\
\mathrm{C} / \mathrm{E}\end{array}$ & & $\begin{array}{r}940 \\
12 \\
--2 \\
952\end{array}$ & $\begin{array}{r}1,030 \\
0 \\
--- \\
1,030\end{array}$ & $\begin{array}{r}1,046 \\
0 \\
---- \\
1,046\end{array}$ & --- & --- & $\begin{array}{r}-\cdots- \\
0\end{array}$ \\
\hline $\begin{array}{l}\text { 1.1.1.3.02.01.06 } \\
\text { Level VI }\end{array}$ & $\begin{array}{l}\text { Program Management: } \\
\text { Public Involvement } \\
\qquad \text { Total }\end{array}$ & $\begin{array}{l}O E \\
C / E\end{array}$ & & $\begin{array}{r}29 \\
0 \\
--- \\
-29\end{array}$ & $\begin{array}{r}30 \\
0 \\
--- \\
-30\end{array}$ & $\begin{array}{r}31 \\
0 \\
--- \\
31\end{array}$ & --- & ---- & --- \\
\hline $\begin{array}{l}\text { 1.1.1.3.02.02 } \\
\text { Level } v\end{array}$ & $\begin{array}{l}\text { Tech. Development. and } \\
\frac{\text { Applied Engineering }}{\text { ADS } 1230-0-A C} \text { Sub Total }\end{array}$ & $\begin{array}{l}\text { OE } \\
\text { C/E }\end{array}$ & NA & $\begin{array}{r}25,626 \\
287 \\
---- \\
25,913\end{array}$ & $\begin{array}{r}23,577 \\
300 \\
---- \\
23,877\end{array}$ & $\begin{array}{r}11,390 \\
338 \\
---- \\
11,728\end{array}$ & $\begin{array}{r}\mathbf{0} \\
--- \\
\mathbf{0}\end{array}$ & $\begin{array}{r}\mathbf{0} \\
-- \\
-0\end{array}$ & $\begin{array}{r}\mathbf{0} \\
---\frac{0}{0}\end{array}$ \\
\hline $\begin{array}{l}\text { 1.1.1.3.02.02.01 } \\
\text { Level VI }\end{array}$ & Melter Selection & $\begin{array}{l}\text { OE } \\
C / E\end{array}$ &. & $\begin{array}{r}14,432 \\
0 \\
---- \\
14,432\end{array}$ & $\begin{array}{r}11.726 \\
0 \\
---- \\
11.726\end{array}$ & $\begin{array}{r}1,326 \\
0 \\
---- \\
1,326\end{array}$ & $---\overline{0}$ & --- & $\begin{array}{r}--- \\
0\end{array}$ \\
\hline $\begin{array}{l}1.1 .1 .3 .02 .02 .02 \\
\text { Level VI }\end{array}$ & $\begin{array}{l}\text { LLW Simulant Dvipmt. } \\
\text { and Specifications } \\
\qquad \begin{array}{l}\text { Total }\end{array}\end{array}$ & $\begin{array}{l}O E \\
C / E\end{array}$ & & $\begin{array}{r}717 \\
0 \\
--- \\
717\end{array}$ & $\begin{array}{r}1,183 \\
0 \\
--- \\
1,183\end{array}$ & $\begin{array}{r}172 \\
0 \\
---- \\
172\end{array}$ & --- & --- & 0 \\
\hline $\begin{array}{l}\text { 1.1.1.3.02.02.03 } \\
\text { Level VI }\end{array}$ & $\begin{array}{r}\text { Integrated Process System } \\
\text { Total }\end{array}$ & $\begin{array}{l}O E \\
C / E\end{array}$ & & $\begin{array}{r}3,577 \\
234 \\
--- \\
3,811\end{array}$ & $\begin{array}{r}2,252 \\
0 \\
---- \\
2,252\end{array}$ & $\begin{array}{r}2,211 \\
---\frac{0}{2,211}\end{array}$ & -- & ---- & ---- \\
\hline
\end{tabular}


TABLE $7.9-1$

TOTAL PROGRAM ELEMENT COST BASELINE - BY YEAR

[ R1 ]

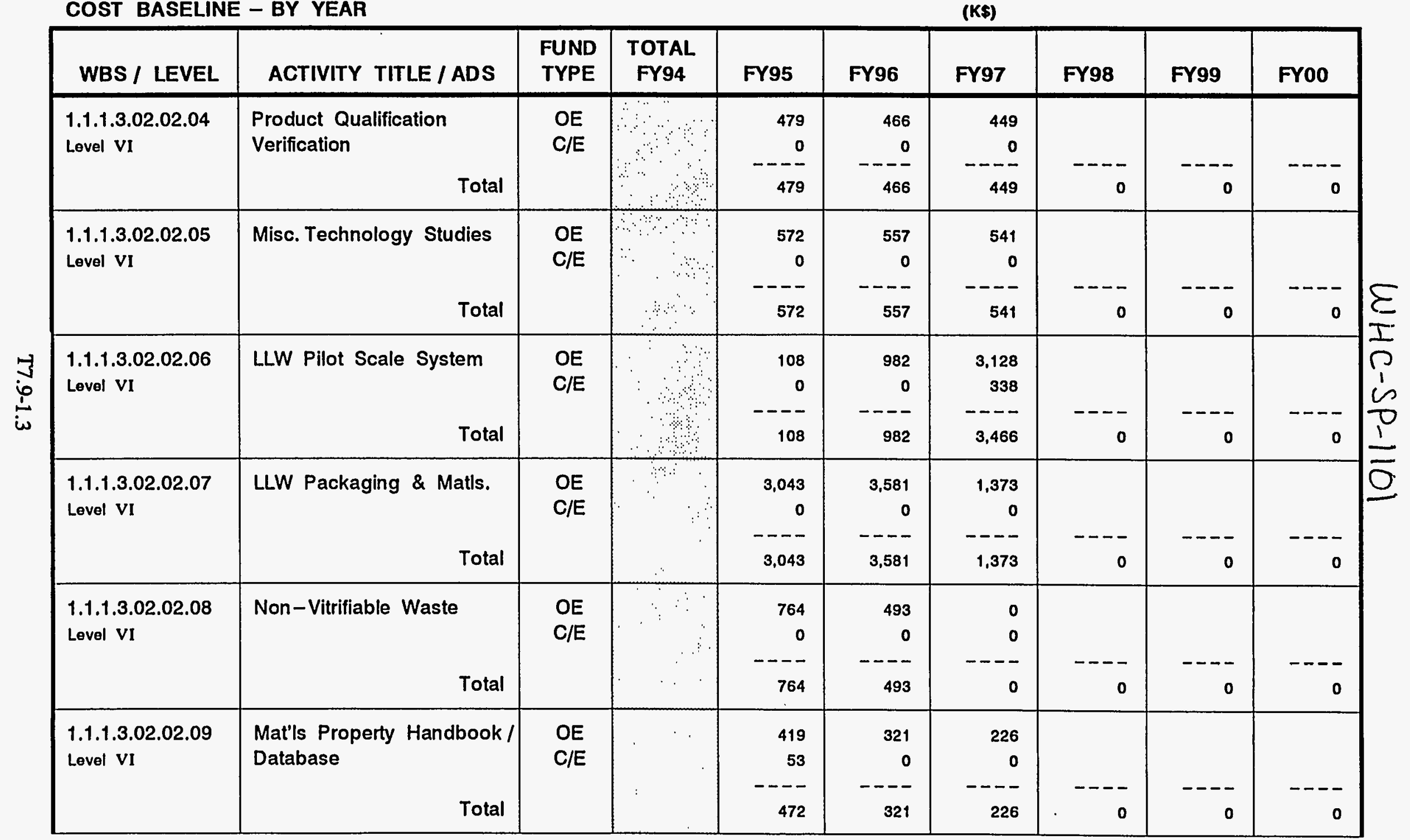


TABLE $\quad 7.9-1$

TOTAL PROGRAM ELEMENT

COST BASELINE - BY YEAR

\begin{tabular}{|c|c|c|c|c|c|c|c|c|c|}
\hline WBS / LEVEL & ACTIVITY TITLE / ADS & $\begin{array}{l}\text { FUND } \\
\text { TYPE }\end{array}$ & $\begin{array}{l}\text { TOTAL } \\
\text { FY94 }\end{array}$ & FY95 & FY96 & FY97 & FY98 & FY99 & FYOO \\
\hline $\begin{array}{l}\text { 1.1.1.3.02.02.10 } \\
\text { Level VI }\end{array}$ & Total & $\begin{array}{l}O E \\
C / E\end{array}$ & & $\begin{array}{r}1,373 \\
0 \\
---- \\
1,373\end{array}$ & $\begin{array}{r}1,810 \\
300 \\
--- \\
2,110\end{array}$ & $\begin{array}{r}1,699 \\
0 \\
---- \\
1,699\end{array}$ & --- & $-\cdots$ & $\begin{array}{r}--- \\
0\end{array}$ \\
\hline $\begin{array}{l}\text { 1.1.1.3.02.02.11 } \\
\text { Level VI }\end{array}$ & $\begin{array}{l}\text { Oversite \& Planning } \\
\qquad \text { Total }\end{array}$ & $\begin{array}{l}O E \\
C / E\end{array}$ & & $\begin{array}{r}142 \\
0 \\
---- \\
142\end{array}$ & $\begin{array}{r}206 \\
0 \\
--- \\
206\end{array}$ & $\begin{array}{r}265 \\
0 \\
---- \\
265\end{array}$ & $\begin{array}{r}--- \\
0\end{array}$ & $\frac{.}{0}$ & $\begin{array}{r}--- \\
0\end{array}$ \\
\hline $\begin{array}{l}\text { 1.1.1.3.02.03 } \\
\text { Level } \mathrm{V}\end{array}$ & $\begin{aligned} \frac{\text { Facility Operations }}{\text { ADS 1230-0-AC }} & \\
& \text { Total }\end{aligned}$ & $\begin{array}{l}\text { OE } \\
\text { C/E }\end{array}$ & NA & $\begin{array}{r}322 \\
0 \\
---- \\
322\end{array}$ & $\begin{array}{r}144 \\
0 \\
---- \\
144\end{array}$ & $\begin{array}{r}135 \\
0 \\
---- \\
135\end{array}$ & $\begin{array}{r}0 \\
0 \\
--- \\
0\end{array}$ & $\begin{array}{r}0 \\
0 \\
-- \\
0\end{array}$ & $\begin{array}{r}\mathbf{0} \\
\mathbf{0} \\
-- \\
\mathbf{0}\end{array}$ \\
\hline $\begin{array}{l}\text { 1.1.1.3.02.03.01 } \\
\text { Level VI }\end{array}$ & $\begin{array}{l}\text { Project Definition } \\
\text { Support } \\
\\
\end{array}$ & $\begin{array}{l}\text { OE } \\
\text { C/E }\end{array}$ & $\therefore \therefore$ & $\begin{array}{r}322 \\
--0 \\
-322\end{array}$ & $\begin{array}{r}0 \\
0 \\
-- \\
0\end{array}$ & $\begin{array}{r}0 \\
0 \\
-- \\
0\end{array}$ & $-\cdots$ & $-\cdots-$ & $-\cdots$ \\
\hline $\begin{array}{l}\text { 1.1.1.3.02.03.02 } \\
\text { Level VI }\end{array}$ & $\begin{array}{l}\text { Prep. of LLW Operations } \\
\text { Total }\end{array}$ & $\begin{array}{l}O E \\
C / E\end{array}$ & & $\begin{array}{r}0 \\
0 \\
-- \\
0\end{array}$ & $\begin{array}{r}144 \\
0 \\
--- \\
144\end{array}$ & $\begin{array}{r}135 \\
0 \\
--0 \\
135\end{array}$ & $-\cdots$ & $-\cdots$ & $---\frac{-}{0}$ \\
\hline
\end{tabular}

[ R1 ]

(k⿻)

\section{4,210}


TABLE 7.9-1

TOTAL PROGRAM ELEMENT

COST BASELINE - BY YEAR

\begin{tabular}{|c|c|c|c|c|c|c|c|c|c|}
\hline WBS / LEVEL & ACTIVITY TITLE / ADS & $\begin{array}{l}\text { FUND } \\
\text { TYPE }\end{array}$ & $\begin{array}{l}\text { TOTAL } \\
\text { FY94 }\end{array}$ & FY95 & FY96 & FY97 & FY98 & FY99 & FYOO \\
\hline $\begin{array}{l}\text { 1.1.1.3.02.04 } \\
\text { Level } \mathrm{V}\end{array}$ & $\begin{array}{l}\frac{\text { System Definition }}{\text { ADS } 1230-0-A C} \\
\text { Sub Total }\end{array}$ & $\begin{array}{l}O E \\
C / E\end{array}$ & NA & $\begin{array}{r}9.728 \\
27 \\
---- \\
9.755\end{array}$ & $\begin{array}{r}9,485 \\
0 \\
--- \\
9,485\end{array}$ & $\begin{array}{r}7.197 \\
0 \\
--197 \\
7.197\end{array}$ & $\begin{array}{r}0 \\
0 \\
-0\end{array}$ & $--\frac{0}{0}$ & $\begin{array}{r}0 \\
-0 \\
-0\end{array}$ \\
\hline $\begin{array}{l}\text { 1.1.1.3.02.04.01 } \\
\text { Level VI }\end{array}$ & $\begin{array}{l}\text { Compliance } \\
\text { Total } \\
\end{array}$ & $\begin{array}{l}O E \\
C / E\end{array}$ & \begin{tabular}{l}
$\because$ \\
$\because \because$ \\
$\because$ \\
\hdashline
\end{tabular} & $\begin{array}{r}3,277 \\
0 \\
--- \\
3,277\end{array}$ & $\begin{array}{r}379 \\
0 \\
--- \\
379\end{array}$ & $\begin{array}{r}56 \\
0 \\
--0 \\
56\end{array}$ & $---\overline{0}$ & --- & $-\frac{1}{0}$ \\
\hline $\begin{array}{l}\text { 1.1.1.3.02.04.02 } \\
\text { Level VI }\end{array}$ & $\begin{array}{l}\text { Prepare LLW Performance } \\
\text { Assessment } \\
\text { Total }\end{array}$ & $\begin{array}{l}O E \\
C / E\end{array}$ & & $\begin{array}{r}4,130 \\
27 \\
---- \\
4,157\end{array}$ & $\begin{array}{r}7.570 \\
0 \\
--- \\
7,570\end{array}$ & $\begin{array}{r}5.938 \\
0 \\
---- \\
5,938\end{array}$ & $---\frac{-}{0}$ & $---\frac{-}{0}$ & --- \\
\hline $\begin{array}{l}\text { 1.1.1.3.02.04.03 } \\
\text { Level VI }\end{array}$ & $\begin{array}{r}\text { Define LLW Tech. Baseline } \\
\text { Total }\end{array}$ & $\begin{array}{l}O E \\
C / E\end{array}$ & & $\begin{array}{r}1,015 \\
0 \\
---0 \\
1,015\end{array}$ & $\begin{array}{r}710 \\
--0 \\
-710\end{array}$ & $\begin{array}{r}731 \\
0 \\
--- \\
731\end{array}$ & -- & o & -- \\
\hline $\begin{array}{l}\text { 1.1.1.3.02.04.05 } \\
\text { Level VI }\end{array}$ & $\begin{array}{l}\text { Maintain LLW Technical } \\
\text { Basis Documentation } \\
\qquad \text { Total }\end{array}$ & $\begin{array}{l}O E \\
C / E\end{array}$ & & $\begin{array}{r}197 \\
0 \\
--- \\
197\end{array}$ & $\begin{array}{r}237 \\
0 \\
--- \\
237\end{array}$ & $\begin{array}{r}285 \\
0 \\
--- \\
285\end{array}$ & -- & --- & ---- \\
\hline $\begin{array}{l}\text { 1.1.1.3.02.04.06 } \\
\text { Level VI }\end{array}$ & $\begin{array}{l}\text { Engineering Reviews \& } \\
\text { Program Support } \\
\qquad \text { Total }\end{array}$ & $\begin{array}{l}O E \\
C / E\end{array}$ & & $\begin{array}{r}149 \\
0 \\
--- \\
149\end{array}$ & $\begin{array}{r}108 \\
0 \\
--- \\
108\end{array}$ & $\begin{array}{r}111 \\
0 \\
--- \\
111\end{array}$ & $---\overline{0}$ & $--\frac{-}{0}$ & ---- \\
\hline $\begin{array}{l}\text { 1.1.1.3.02.04.07 } \\
\text { Level VI }\end{array}$ & Total & $\begin{array}{l}O E \\
C / E\end{array}$ & & $\begin{array}{r}960 \\
0 \\
--- \\
960\end{array}$ & $\begin{array}{r}481 \\
0 \\
--- \\
481\end{array}$ & $\begin{array}{r}76 \\
0 \\
--- \\
76\end{array}$ & $---\frac{-}{0}$ & $-\cdots$ & $---\frac{-}{0}$ \\
\hline
\end{tabular}

[ R1 ]

(K\$)

\section{4,210}


TABLE $7.9-1$

TOTAL PROGRAM ELEMENT COST BASELINE - BY YEAR

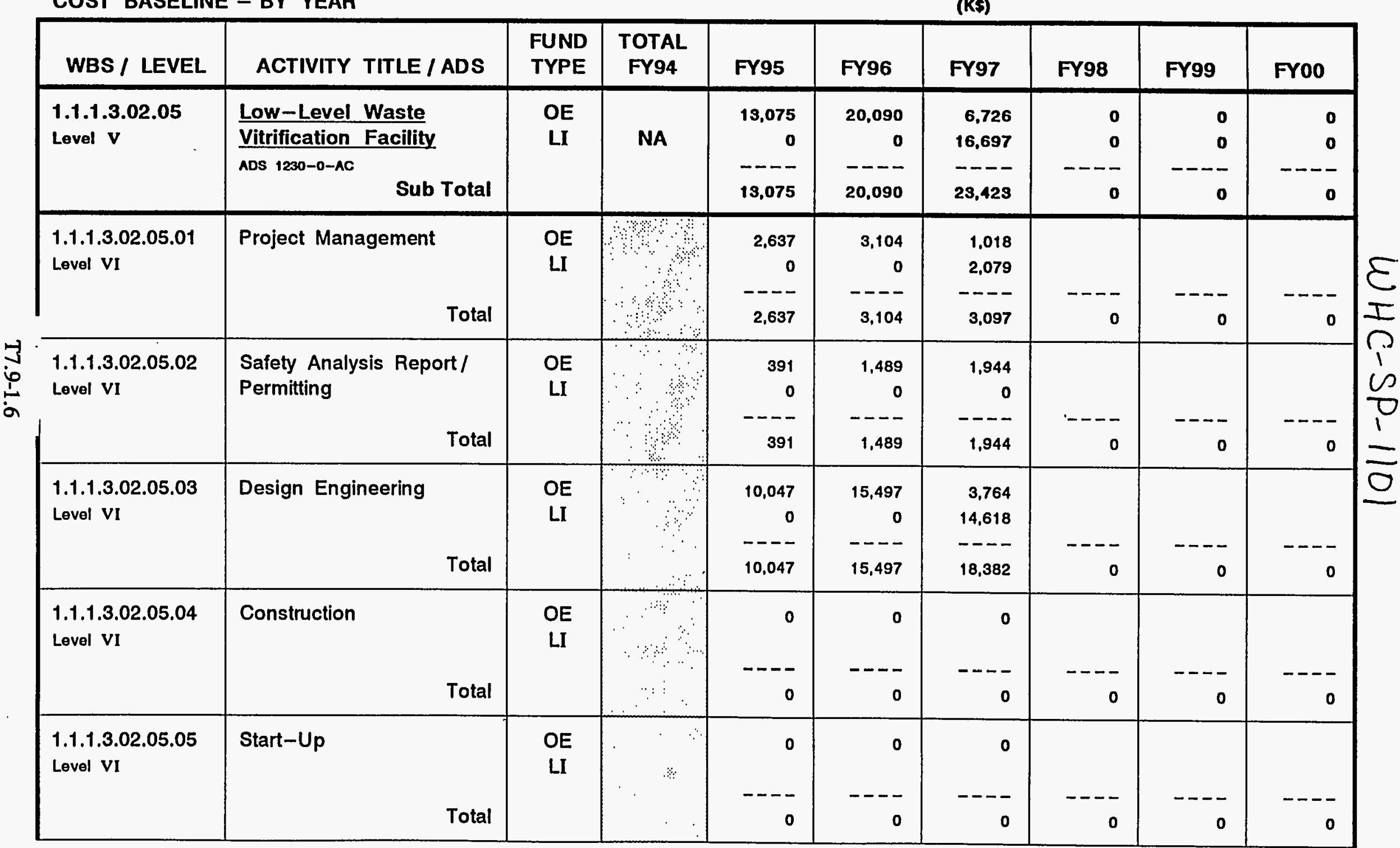


TABLE $\quad 7.9-1$

TOTAL PROGRAM ELEMENT

COST BASELINE - BY YEAR
[ R1 ]

44,210

\begin{tabular}{|c|c|c|c|c|c|c|c|c|c|}
\hline WBS / LEVEL & ACTIVITY TITLE / ADS & $\begin{array}{l}\text { FUND } \\
\text { TYPE }\end{array}$ & $\begin{array}{c}\text { TOTAL } \\
\text { FY94 }\end{array}$ & FY95 & FY96 & FY97 & FY98 & FY99 & FY00 \\
\hline $\begin{array}{l}\text { 1.1.1.3.02.05.06 } \\
\text { Level VI }\end{array}$ & $\begin{array}{l}\text { Applied Engineering } \\
\qquad \text { Total }\end{array}$ & $\begin{array}{l}\text { OE } \\
\text { LI }\end{array}$ & & $\begin{array}{r}0 \\
- \\
0\end{array}$ & $\begin{array}{r}0 \\
- \\
0\end{array}$ & $\begin{array}{r}0 \\
- \\
0\end{array}$ & --- & $-\cdots$ & --- \\
\hline $\begin{array}{l}\text { 1.1.1.3.02.06 } \\
\text { Level } v\end{array}$ & $\begin{array}{l}\text { Grout Facility } \\
\text { ADS } 1230-0-M\end{array}$ & $\begin{array}{l}O E \\
C / E\end{array}$ & NA & $\begin{array}{r}1.623 \\
-0 \\
-1.623\end{array}$ & $\begin{array}{r}262 \\
-0 \\
-262\end{array}$ & $\begin{array}{r}146 \\
-0 \\
-146\end{array}$ & $\begin{array}{r}\mathbf{0} \\
-\mathbf{0} \\
-\mathbf{0}\end{array}$ & $\begin{array}{r}0 \\
0 \\
-\frac{0}{0}\end{array}$ & $\begin{array}{r}0 \\
0 \\
-0\end{array}$ \\
\hline $\begin{array}{l}\text { 1.1.1.3.02.06.02 } \\
\text { Level VI }\end{array}$ & $\begin{array}{l}\text { Two Year Restart } \\
\text { Compliance Activity } \\
\\
\text { Total }\end{array}$ & $\begin{array}{l}O E \\
\mathrm{C} / \mathrm{E}\end{array}$ & & $\begin{array}{r}1.172 \\
-10 \\
-1.172\end{array}$ & $\begin{array}{r}0 \\
0 \\
-0\end{array}$ & $\begin{array}{r}0 \\
0 \\
-0 \\
0\end{array}$ & $\cdots$ & -- & - \\
\hline $\begin{array}{l}\text { 1.1.1.3.02.06.03 } \\
\text { Level VI }\end{array}$ & $\begin{array}{l}\text { Maintain Grout Fclty, in } \\
\text { Cold Standby (FY 96, 97) } \\
\text { Total }\end{array}$ & $\begin{array}{l}O E \\
\mathrm{O} / \mathrm{E}\end{array}$ & & $\begin{array}{r}0 \\
0 \\
-0\end{array}$ & $\begin{array}{r}156 \\
-0 \\
-156\end{array}$ & $\begin{array}{r}146 \\
--0 \\
-146\end{array}$ & --- & - & - \\
\hline $\begin{array}{l}\text { 1.1.1.3.02.06.05 } \\
\text { Level VI }\end{array}$ & $\begin{array}{l}\text { Fabricate Container for } \\
\text { Failed AP102 Mixer Pump } \\
\text { Total }\end{array}$ & $\begin{array}{l}\mathrm{OE} \\
\mathrm{C} / \mathrm{E}\end{array}$ & & $\begin{array}{r}451 \\
-0 \\
--- \\
451\end{array}$ & $\begin{array}{r}106 \\
-0 \\
-106\end{array}$ & $\begin{array}{r}0 \\
0 \\
-0 \\
0\end{array}$ & 0 & --- & $\cdots$ \\
\hline
\end{tabular}


TABLE $\quad 7.9-1$

TOTAL PROGRAM ELEMENT COST BASELINE - BY YEAR
[ R1 ]

\begin{tabular}{|c|c|c|c|c|c|c|c|c|c|c|}
\hline WBS / LEVEL & ACTIVITY TITLE / ADS & $\begin{array}{l}\text { FUND } \\
\text { TYPE }\end{array}$ & $\begin{array}{l}\text { FY01 / } \\
\text { FY05 }\end{array}$ & $\begin{array}{l}\text { FY06 / } \\
\text { FY10 }\end{array}$ & $\begin{array}{l}\text { FY11 I } \\
\text { FY15 }\end{array}$ & $\begin{array}{l}\text { FY16 / } \\
\text { FY20 }\end{array}$ & $\begin{array}{l}\text { FY21 / } \\
\text { FY25 }\end{array}$ & $\begin{array}{l}\text { FY26 I } \\
\text { FY30 }\end{array}$ & $\begin{array}{l}\text { FY31 / } \\
\text { FY35 }\end{array}$ & $\begin{array}{l}\text { FY36 I } \\
\text { FY40 }\end{array}$ \\
\hline 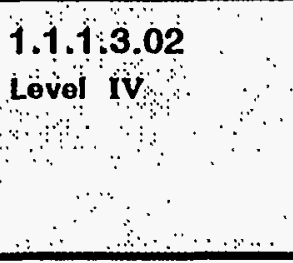 & $\begin{array}{l}\text { Low Level Waste } \\
\text { Aps } 1230-6 \text { on } \\
\text { TOTAL }\end{array}$ & \begin{tabular}{c}
$\mathrm{OE}$ \\
$\mathrm{CHE}$ \\
$\mathrm{LI}$ \\
$\therefore$ \\
\hdashline
\end{tabular} & $\begin{array}{r}0 \\
0 \\
1,006,875\end{array}$ & $\begin{array}{r}0 \\
0 \\
1435713 \\
0\end{array}$ & $\begin{array}{r}0 \\
0 \\
1,456,661 \\
0\end{array}$ & 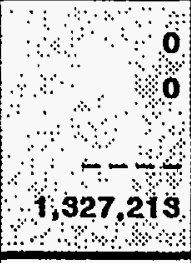 & 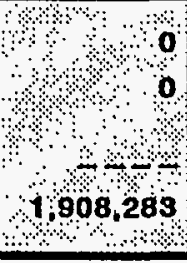 & $\begin{array}{l}3+0 \\
3 \\
39,254\end{array}$ & (2o & कि? \\
\hline & $\begin{array}{l}\text { Delta Required to } \\
\text { Balance to TARGET } \\
\text { Budget. } \\
\qquad \text { Total }\end{array}$ & $\begin{array}{l}O E \\
C / E \\
L I\end{array}$ & & & & & & & & \\
\hline $\begin{array}{l}\text { 1.1.1.3.02.01 } \\
\text { Level } \mathrm{v}\end{array}$ & $\begin{array}{l}\text { Technical Integration } \\
\frac{\text { and Planning }}{\text { ADS } 1230-0-A N A C} \\
\quad \text { Sub Total }\end{array}$ & $\begin{array}{l}O E \\
C / E\end{array}$ & $--\frac{0}{0}$ & $--\frac{0}{0}$ & $\begin{array}{r}\mathbf{0} \\
--\frac{\mathbf{0}}{\mathbf{0}}\end{array}$ & -- & -- & -- & -- & $--\frac{0}{0}$ \\
\hline $\begin{array}{l}\text { 1.1.1.3.02.01.01 } \\
\text { Level VI }\end{array}$ & 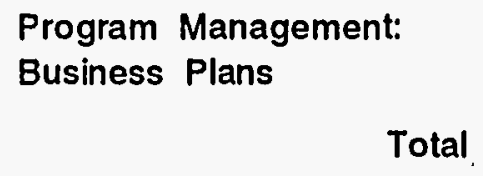 & $\begin{array}{l}\text { OE } \\
C / E\end{array}$ & --- & ---- & $---\frac{-}{0}$ & $\overline{0}$ & $\overline{0}$ & 0 & $\therefore$ & $\begin{array}{r}-\cdots \\
0\end{array}$ \\
\hline $\begin{array}{l}1.1 .1 .3 .02 .01 .02 \\
\text { Level VI }\end{array}$ & $\begin{array}{l}\text { Program Management: } \\
\text { Mgmt. Plans / Guidance } \\
\qquad \text { Total }\end{array}$ & $\begin{array}{l}O E \\
C / E\end{array}$ & $---\overline{0}$ & --- & - & 0 & $-\cdots$ & 0 & 0 & --- \\
\hline $\begin{array}{l}\text { 1.1.1.3.02.01.04 } \\
\text { Level VI }\end{array}$ & $\begin{array}{l}\text { Program Management: } \\
\text { Systems Engineering } \\
\qquad \text { Total }\end{array}$ & $\begin{array}{l}O E \\
C / E\end{array}$ & - & - & o & - & $\overline{0}$ & - & 0 & --- \\
\hline
\end{tabular}


TABLE $7.9-1$

TOTAL PROGRAM ELEMENT

COST BASELINE - BY YEAR

[ R1 ]

\begin{tabular}{|c|c|c|c|c|c|c|c|c|c|c|}
\hline WBS / LEVEL & ACTIVITY TITLE / ADS & $\begin{array}{l}\text { FUND } \\
\text { TYPE }\end{array}$ & $\begin{array}{l}\text { FY01 / } \\
\text { FY05 }\end{array}$ & $\begin{array}{l}\text { FY06 I } \\
\text { FY10 }\end{array}$ & $\begin{array}{l}\text { FY11 / } \\
\text { FY15 }\end{array}$ & $\begin{array}{l}\text { FY16 / } \\
\text { FY20 }\end{array}$ & $\begin{array}{l}\text { FY21 / } \\
\text { FY25 }\end{array}$ & $\begin{array}{l}\text { FY26 / } \\
\text { FY30 }\end{array}$ & $\begin{array}{l}\text { FY31 / } \\
\text { FY35 }\end{array}$ & $\begin{array}{l}\text { FY36 / } \\
\text { FY40 }\end{array}$ \\
\hline $\begin{array}{l}\text { 1.1.1.3.02.01.05 } \\
\text { Level VI }\end{array}$ & $\begin{array}{l}\text { Program Management: } \\
\text { Prog. Administration } \\
\qquad \text { Total }\end{array}$ & $\begin{array}{l}O E \\
C / E\end{array}$ & $\begin{array}{r}---- \\
0\end{array}$ & ---- & ---- & --- & $\begin{array}{r}---- \\
0\end{array}$ & $\begin{array}{r}-\cdots- \\
0\end{array}$ & --- & 0 \\
\hline $\begin{array}{l}\text { 1.1.1.3.02.01.06 } \\
\text { Level VI }\end{array}$ & $\begin{array}{l}\text { Program Management: } \\
\text { Public Involvement } \\
\qquad \text { Total }\end{array}$ & $\begin{array}{l}O E \\
C / E\end{array}$ & $--\overline{-}$ & $\begin{array}{r}--- \\
0\end{array}$ & ---- & ---- & ---- & $\begin{array}{r}---- \\
0\end{array}$ & ---- & $\begin{array}{r}--- \\
0\end{array}$ \\
\hline $\begin{array}{l}\text { 1.1.1.3.02.02 } \\
\text { Level } \mathrm{v}\end{array}$ & $\begin{array}{l}\text { Tech. Development. and } \\
\frac{\text { Applied Engineering }}{A D S 1230-0-\wedge C} \text { Sub Total }\end{array}$ & $\begin{array}{l}\text { OE } \\
C / E\end{array}$ & $\begin{array}{r}\mathbf{0} \\
\mathbf{0} \\
-- \\
\mathbf{0}\end{array}$ & $\begin{array}{r}0 \\
0 \\
-0\end{array}$ & $\begin{array}{r}\mathbf{0} \\
--0 \\
-\mathbf{0}\end{array}$ & $\begin{array}{r}0 \\
0 \\
-0\end{array}$ & $\begin{array}{r}\mathbf{0} \\
\mathbf{0} \\
--\mathbf{0}\end{array}$ & $\begin{array}{r}\mathbf{0} \\
\mathbf{0} \\
-0 \\
\mathbf{0}\end{array}$ & $--\frac{\mathbf{0}}{\mathbf{0}}$ & $\begin{array}{r}0 \\
0 \\
-0\end{array}$ \\
\hline $\begin{array}{l}\text { 1.1.1.3.02.02.01 } \\
\text { Level VI }\end{array}$ & $\begin{array}{l}\text { Melter Selection } \\
\qquad \text { Total }\end{array}$ & $\begin{array}{l}O E \\
C / E\end{array}$ & ---- & ---- & --- & ---- & ---- & $-\cdots-$ & 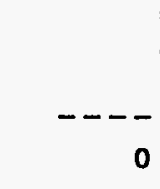 & -- \\
\hline $\begin{array}{l}\text { 1.1.1.3.02.02.02 } \\
\text { Level VI }\end{array}$ & $\begin{array}{l}\text { LLW Simulant Dvlpmt. } \\
\text { and Specifications } \\
\qquad \begin{array}{l}\text { Total }\end{array}\end{array}$ & $\begin{array}{l}O E \\
C / E\end{array}$ & $\begin{array}{r}--- \\
0\end{array}$ & ---- & -- & 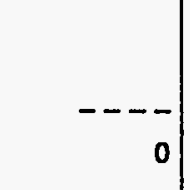 & - & $-\cdots$ & --- & 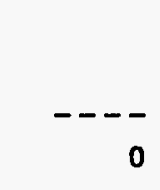 \\
\hline $\begin{array}{l}\text { 1.1.1.3.02.02.03 } \\
\text { Level VI }\end{array}$ & $\begin{array}{r}\text { Integrated Process System } \\
\text { Total }\end{array}$ & $\begin{array}{l}\text { OE } \\
\text { C/E }\end{array}$ & $\begin{array}{r}--- \\
0\end{array}$ & - & - & $-\overline{0}$ & $\begin{array}{r}---- \\
0\end{array}$ & --- & - & 0 \\
\hline
\end{tabular}


TABLE $\quad 7.9-1$

TOTAL PROGRAM ELEMENT

COST BASELINE - BY YEAR

[ R1]

\begin{tabular}{|c|c|c|c|c|c|c|c|c|c|c|}
\hline WBS / LEVEL & ACTIVITY TITLE / ADS & $\begin{array}{l}\text { FUND } \\
\text { TYPE }\end{array}$ & $\begin{array}{l}\text { FY01 I } \\
\text { FY05 }\end{array}$ & $\begin{array}{l}\text { FY06 I } \\
\text { FY10 }\end{array}$ & $\begin{array}{l}\text { FY11 I } \\
\text { FY15 }\end{array}$ & $\begin{array}{l}\text { FY16 I } \\
\text { FY20 }\end{array}$ & $\begin{array}{l}\text { FY21 / } \\
\text { FY25 }\end{array}$ & $\begin{array}{l}\text { FY26 I } \\
\text { FY30 }\end{array}$ & $\begin{array}{l}\text { FY31 / } \\
\text { FY35 }\end{array}$ & $\begin{array}{l}\text { FY36 I } \\
\text { FY40 }\end{array}$ \\
\hline $\begin{array}{l}\text { 1.1.1.3.02.02.04 } \\
\text { Level VI } \\
\text {. }\end{array}$ & $\begin{array}{l}\text { Product Qualification } \\
\text { Verification } \\
\qquad \text { Total }\end{array}$ & $\begin{array}{l}O E \\
C / E\end{array}$ & --- & ---- & ---- & ---- & ---- & $---\frac{-}{0}$ & $---\frac{-}{0}$ & $---\frac{-}{0}$ \\
\hline $\begin{array}{l}\text { 1.1.1.3.02.02.05 } \\
\text { Level VI }\end{array}$ & $\begin{array}{r}\text { Misc. Technology Studies } \\
\text { Total }\end{array}$ & $\begin{array}{l}O E \\
C / E\end{array}$ & --- & ---- & ---- & $-\cdots-$ & $\begin{array}{r}---- \\
0\end{array}$ & ---- & $\begin{array}{r}---- \\
0\end{array}$ & 0 \\
\hline $\begin{array}{l}\text { 1.1.1.3.02.02.06 } \\
\text { Level VI }\end{array}$ & $\begin{array}{l}\text { LLW Pilot Scale System } \\
\text { Total }\end{array}$ & $\begin{array}{l}O E \\
C / E\end{array}$ & --- & $-\cdots$ & $-\cdots$ & $\begin{array}{r}--- \\
0\end{array}$ & $\begin{array}{r}---- \\
0\end{array}$ & ---- & $\begin{array}{r}---- \\
0\end{array}$ & --- \\
\hline $\begin{array}{l}1.1 .1 .3 .02 .02 .07 \\
\text { Level VI }\end{array}$ & $\begin{array}{r}\text { LLW Packaging \& Matls. } \\
\text { Total }\end{array}$ & $\begin{array}{l}O E \\
C / E\end{array}$ & $\begin{array}{r}-\cdots- \\
0\end{array}$ & $\begin{array}{r}--- \\
0\end{array}$ & ---- & $-\cdots$ & $\begin{array}{r}---- \\
0\end{array}$ & ---- & $\begin{array}{r}--- \\
0\end{array}$ & $-\cdots$ \\
\hline $\begin{array}{l}1.1 .1 .3 .02 .02 .08 \\
\text { Level VI }\end{array}$ & $\begin{array}{l}\text { Non-Vitrifiable Waste } \\
\text { Total }\end{array}$ & $\begin{array}{l}O E \\
C / E\end{array}$ & $-\cdots$ & ---- & ---- & $\begin{array}{r}--- \\
0\end{array}$ & - & - & $\begin{array}{r}--- \\
0\end{array}$ & 0 \\
\hline $\begin{array}{l}\text { 1.1.1.3.02.02.09 } \\
\text { Level VI }\end{array}$ & $\begin{array}{l}\text { Mat'ls Property Handbook / } \\
\text { Database } \\
\text { Total }\end{array}$ & $\begin{array}{l}O E \\
C / E\end{array}$ & $-\cdots$ & ---- & ---- & --- & - & - & \begin{tabular}{r|}
-- \\
0
\end{tabular} & --- \\
\hline
\end{tabular}


TABLE $7.9-1$

TOTAL PROGRAM ELEMENT

COST BASELINE - BY YEAR

[ R1 ]

\begin{tabular}{|c|c|c|c|c|c|c|c|c|c|c|}
\hline WBS / LEVEL & ACTIVITY TITLE / ADS & $\begin{array}{l}\text { FUND } \\
\text { TYPE }\end{array}$ & $\begin{array}{l}\text { FY01 I } \\
\text { FY05 }\end{array}$ & $\begin{array}{l}\text { FY06 I } \\
\text { FY10 }\end{array}$ & $\begin{array}{l}\text { FY11 I } \\
\text { FY15 }\end{array}$ & $\begin{array}{l}\text { FY16 I } \\
\text { FY20 }\end{array}$ & $\begin{array}{l}\text { FY21 I } \\
\text { FY25 }\end{array}$ & $\begin{array}{l}\text { FY26 I } \\
\text { FY30 }\end{array}$ & $\begin{array}{l}\text { FY31 I } \\
\text { FY35 }\end{array}$ & $\begin{array}{l}\text { FY36 I } \\
\text { FY40 }\end{array}$ \\
\hline $\begin{array}{l}\text { 1.1.1.3.02.02.10 } \\
\text { Level VI }\end{array}$ & $\begin{array}{l}\text { Disposal System } \\
\qquad \text { Total }\end{array}$ & $\begin{array}{l}O E \\
C / E\end{array}$ & ---- & ---- & --- & ---- & ---- & --- & - & $-\cdots$ \\
\hline $\begin{array}{l}\text { 1.1.1.3.02.02.11 } \\
\text { Level VI }\end{array}$ & $\begin{array}{l}\text { Oversite \& Planning } \\
\qquad \text { Total }\end{array}$ & $\begin{array}{l}O E \\
\mathrm{C} / \mathrm{E}\end{array}$ & o & - & $\overline{0}$ & 0 & $\overline{0}$ & $\overline{0}$ & 0 & --- \\
\hline $\begin{array}{l}\text { 1.1.1.3.02.03 } \\
\text { Level } v\end{array}$ & $\begin{array}{l}\frac{\text { Facility Operations }}{\text { ADS } 1230-0-\Lambda C} \\
\\
\text { Total }\end{array}$ & $\begin{array}{l}O E \\
C / E\end{array}$ & $\begin{array}{r}\mathbf{0} \\
\mathbf{0} \\
-\mathbf{0}\end{array}$ & $\begin{array}{r}0 \\
0 \\
-0\end{array}$ & $\begin{array}{r}0 \\
0 \\
-0\end{array}$ & $\begin{array}{r}0 \\
0 \\
-0\end{array}$ & $\begin{array}{r}\mathbf{0} \\
\mathbf{0} \\
-\mathbf{0}\end{array}$ & $\begin{array}{r}\mathbf{0} \\
\mathbf{0} \\
-\mathbf{0}\end{array}$ & $\begin{array}{r}0 \\
0 \\
-0 \\
\end{array}$ & $\begin{array}{r}0 \\
0 \\
-0\end{array}$ \\
\hline $\begin{array}{l}\text { 1.1.1.3.02.03.01 } \\
\text { Level VI }\end{array}$ & $\begin{array}{l}\text { Project Definition } \\
\text { Support } \\
\\
\text { Total }\end{array}$ & $\begin{array}{l}O E \\
C / E\end{array}$ & -- & -- & - & -- & 0 & 0 & -- & - \\
\hline $\begin{array}{l}\text { 1.1.1.3.02.03.02 } \\
\text { Level VI }\end{array}$ & $\begin{array}{l}\text { Prep. of LLW Operations } \\
\text { Total }\end{array}$ & $\begin{array}{l}O E \\
\mathrm{C} / \mathrm{E}\end{array}$ & & - & - & $\begin{array}{r}--- \\
0\end{array}$ & $\overline{0}$ & $-\cdots$ & ---- & $-\cdots$ \\
\hline
\end{tabular}


TABLE $7.9-1$

TOTAL PROGRAM ELEMENT COST BASELINE - BY YEAR

\begin{tabular}{|c|c|c|c|c|c|c|c|c|c|c|}
\hline WBS / LEVEL & ACTIVITY TITLE / ADS & $\begin{array}{l}\text { FUND } \\
\text { TYPE }\end{array}$ & $\begin{array}{l}\text { FY01 I } \\
\text { FY05 }\end{array}$ & $\begin{array}{l}\text { FY06 I } \\
\text { FY10 }\end{array}$ & $\begin{array}{l}\text { FY11 I } \\
\text { FY15 }\end{array}$ & $\begin{array}{l}\text { FY16 / } \\
\text { FY20 }\end{array}$ & $\begin{array}{l}\text { FY21 I } \\
\text { FY25 }\end{array}$ & $\begin{array}{l}\text { FY26 I } \\
\text { FY30 }\end{array}$ & $\begin{array}{l}\text { FY31 / } \\
\text { FY35 }\end{array}$ & $\begin{array}{l}\text { FY36 I } \\
\text { FY40 }\end{array}$ \\
\hline $\begin{array}{l}\text { 1.1.1.3.02.04 } \\
\text { Level } v\end{array}$ & $\begin{array}{l}\frac{\text { System Definition }}{\text { ADS } 1230-0-\wedge C} \\
\text { Sub Total }\end{array}$ & $\begin{array}{l}\text { OE } \\
\text { C/E }\end{array}$ & $\begin{array}{r}0 \\
0 \\
-- \\
0\end{array}$ & $\begin{array}{r}\mathbf{0} \\
\mathbf{0} \\
-\mathbf{0}\end{array}$ & $\begin{array}{r}0 \\
0 \\
---\end{array}$ & $\begin{array}{r}\mathbf{0} \\
\mathbf{0} \\
-0 \\
\mathbf{0}\end{array}$ & $\begin{array}{r}\mathbf{0} \\
-0 \\
-0\end{array}$ & $\begin{array}{r}0 \\
0 \\
--1 \\
0\end{array}$ & $\begin{array}{r}0 \\
0 \\
-0 \\
0\end{array}$ & $-\begin{array}{r}0 \\
-0\end{array}$ \\
\hline $\begin{array}{l}\text { 1.1.1.3.02.04.01 } \\
\text { Level VI }\end{array}$ & $\begin{array}{l}\text { Compliance } \\
\qquad \text { Total }\end{array}$ & $\begin{array}{l}O E \\
C / E\end{array}$ & $-\cdots$ & $\begin{array}{r}--- \\
0\end{array}$ & $-\cdots$ & $\begin{array}{r}--- \\
0\end{array}$ & ---- & $-\cdots$ & ---- & --- \\
\hline $\begin{array}{l}1.1 .1 .3 .02 .04 .02 \\
\text { Level VI }\end{array}$ & $\begin{array}{l}\text { Prepare LLW Performance } \\
\text { Assessment } \\
\qquad \text { Total }\end{array}$ & $\begin{array}{l}O E \\
C / E\end{array}$ & $\begin{array}{r}--- \\
0\end{array}$ & ---- & ---- & ---- & --- & ---- & $-\cdots$ & -- \\
\hline $\begin{array}{l}1.1 .1 .3 .02 .04 .03 \\
\text { Level VI }\end{array}$ & $\begin{array}{r}\text { Define LLW Tech. Baseline } \\
\text { Total }\end{array}$ & $\begin{array}{l}\mathrm{OE} \\
\mathrm{C} / \mathrm{E}\end{array}$ & $\begin{array}{r}--- \\
0\end{array}$ & ---- & $\begin{array}{r}--- \\
0\end{array}$ & ---- & ---- & -- & -- & -- \\
\hline $\begin{array}{l}\text { 1.1.1.3.02.04.05 } \\
\text { Level VI }\end{array}$ & $\begin{array}{l}\text { Maintain LLW Technical } \\
\text { Basis Documentation } \\
\text { Total }\end{array}$ & $\begin{array}{l}O E \\
C / E\end{array}$ & --- & ---- & ---- & ---- & ---- & $-\cdots$ & ---- & $--\frac{1}{0}$ \\
\hline $\begin{array}{l}\text { 1.1.1.3.02.04.06 } \\
\text { Level VI }\end{array}$ & $\begin{array}{l}\text { Engineering Reviews \& } \\
\text { Program Support } \\
\qquad \text { Total }\end{array}$ & $\begin{array}{l}O E \\
C / E\end{array}$ & --- & $-\cdots$ & -0 & ---- & ---- & $---\frac{-}{0}$ & ---- & --- \\
\hline $\begin{array}{l}\text { 1.1.1.3.02.04.07 } \\
\text { Lovel VI }\end{array}$ & $\begin{array}{l}\text { Compliance } \\
\qquad \begin{array}{l}\text { Total }\end{array}\end{array}$ & $\begin{array}{l}O E \\
C / E\end{array}$ & --- & $---\overline{0}$ & -- & $---\frac{-}{0}$ & $---\overline{0}$ & $-\overline{0}$ & $---\frac{-}{0}$ & $---\frac{-}{0}$ \\
\hline
\end{tabular}

[ R1 ] 
TABLE $7.9-1$

TOTAL PROGRAM ELEMENT

COST BASELINE - BY YEAR

\begin{tabular}{|c|c|c|c|c|c|c|c|c|c|c|}
\hline WBS / LEVEL & ACTIVITY TITLE / ADS & $\begin{array}{l}\text { FUND } \\
\text { TYPE }\end{array}$ & $\begin{array}{l}\text { FY01 I } \\
\text { FY05 }\end{array}$ & $\begin{array}{l}\text { FY06 / } \\
\text { FY10 }\end{array}$ & $\begin{array}{l}\text { FY11 I } \\
\text { FY15 }\end{array}$ & $\begin{array}{l}\text { FY16 I } \\
\text { FY20 }\end{array}$ & $\begin{array}{l}\text { FY21 I } \\
\text { FY25 }\end{array}$ & $\begin{array}{l}\text { FY26 I } \\
\text { FY30 }\end{array}$ & $\begin{array}{l}\text { FY31 I } \\
\text { FY35 }\end{array}$ & $\begin{array}{l}\text { FY36 I } \\
\text { FY40 }\end{array}$ \\
\hline $\begin{array}{l}\text { 1.1.1.3.02.05 } \\
\text { Level } v\end{array}$ & 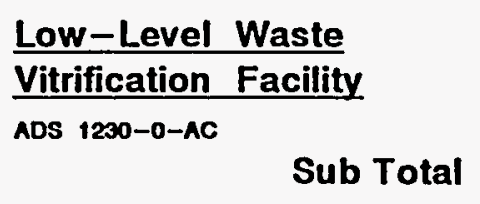 & $\begin{array}{l}\text { OE } \\
\text { LI }\end{array}$ & $\begin{array}{r}0 \\
0 \\
---\end{array}$ & $\begin{array}{r}0 \\
0 \\
-- \\
-0\end{array}$ & $\begin{array}{r}0 \\
0 \\
--- \\
0\end{array}$ & $\begin{array}{r}\mathbf{0} \\
\mathbf{0} \\
--- \\
\mathbf{0}\end{array}$ & $\begin{array}{r}0 \\
0 \\
-- \\
0\end{array}$ & $\begin{array}{r}0 \\
0 \\
-- \\
-0\end{array}$ & $\begin{array}{r}\mathbf{0} \\
\mathbf{0} \\
--- \\
0\end{array}$ & $\begin{array}{r}0 \\
0 \\
--- \\
0\end{array}$ \\
\hline $\begin{array}{l}\text { 1.1.1.3.02.05.01 } \\
\text { Level VI }\end{array}$ & $\begin{array}{l}\text { Project Management } \\
\qquad \text { Total }\end{array}$ & $\begin{array}{l}\mathrm{OE} \\
\mathrm{LI}\end{array}$ & - & $\begin{array}{r}--- \\
0\end{array}$ & --- & 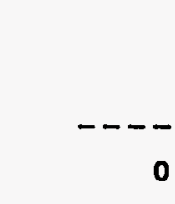 & ---- & $\begin{array}{r}--- \\
0\end{array}$ & $\begin{array}{r}--- \\
0\end{array}$ & --- \\
\hline $\begin{array}{l}\text { 1.1.1.3.02.05.02 } \\
\text { Level VI }\end{array}$ & $\begin{array}{l}\text { Safety Analysis Report/ } \\
\text { Permitting } \\
\qquad \text { Total }\end{array}$ & $\begin{array}{l}\text { OE } \\
\text { LI }\end{array}$ & -- & ---- & $\begin{array}{r}--- \\
0\end{array}$ & --- & --- & ---- & $\begin{array}{r}--- \\
0\end{array}$ & --- \\
\hline $\begin{array}{l}\text { 1.1.1.3.02.05.03 } \\
\text { Level VI }\end{array}$ & $\begin{array}{l}\text { Design Engineering } \\
\qquad \text { Total }\end{array}$ & $\begin{array}{l}\text { OE } \\
\text { LI }\end{array}$ & -- & $\begin{array}{r}--- \\
0\end{array}$ & . & $\begin{array}{r}---- \\
0\end{array}$ & $\begin{array}{r}--- \\
0\end{array}$ & - & $\begin{array}{r}--- \\
0\end{array}$ & --- \\
\hline $\begin{array}{l}\text { 1.1.1.3.02.05.04 } \\
\text { Level VI }\end{array}$ & Construction & $\begin{array}{l}\text { OE } \\
\text { LI }\end{array}$ & -- & --- & $\begin{array}{r}---- \\
0\end{array}$ & ---- & --- & $\begin{array}{r}--- \\
0\end{array}$ & $\begin{array}{r}--- \\
0\end{array}$ & --- \\
\hline $\begin{array}{l}1.1 .1 .3 .02 .05 .05 \\
\text { Level VI }\end{array}$ & Total & $\begin{array}{l}\text { OE } \\
\text { LI }\end{array}$ & $-\cdots$ & 0 & --- & ---- & - & -- & $---\overline{0}$ & -- \\
\hline
\end{tabular}

[ R1 ] 
TABLE $\quad 7.9-1$

TOTAL PROGRAM ELEMENT

COST BASELINE - BY YEAR

\begin{tabular}{|c|c|c|c|c|c|c|c|c|c|c|}
\hline WBS / LEVEL & ACTIVITY TITLE / ADS & $\begin{array}{l}\text { FUND } \\
\text { TYPE }\end{array}$ & $\begin{array}{l}\text { FY01 I } \\
\text { FY05 }\end{array}$ & $\begin{array}{l}\text { FY06 I } \\
\text { FY10 }\end{array}$ & $\begin{array}{l}\text { FY11 / } \\
\text { FY15 }\end{array}$ & $\begin{array}{l}\text { FY16 / } \\
\text { FY20 }\end{array}$ & $\begin{array}{l}\text { FY21 / } \\
\text { FY25 }\end{array}$ & $\begin{array}{l}\text { FY26 / } \\
\text { FY30 }\end{array}$ & $\begin{array}{l}\text { FY31 / } \\
\text { FY35 }\end{array}$ & $\begin{array}{l}\text { FY36 I } \\
\text { FY40 }\end{array}$ \\
\hline $\begin{array}{l}\text { 1.1.1.3.02.05.06 } \\
\text { Level VI }\end{array}$ & $\begin{array}{l}\text { Applied Engineering } \\
\qquad \text { Total }\end{array}$ & $\begin{array}{l}\text { OE } \\
\text { LI }\end{array}$ & -- & 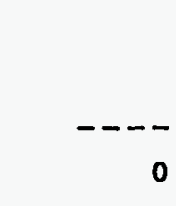 & $\begin{array}{r}---- \\
0\end{array}$ & $\begin{array}{r}---- \\
0\end{array}$ & $\begin{array}{r}---- \\
0\end{array}$ & $\begin{array}{r}--- \\
0\end{array}$ & $\begin{array}{r}--- \\
0\end{array}$ & $-\cdots$ \\
\hline $\begin{array}{l}\text { 1.1.1.3.02.06 } \\
\text { Level } v\end{array}$ & $\begin{array}{l}\frac{\text { Grout Facility }}{\text { ADS } 1230-0-A A} \\
\\
\end{array}$ & $\begin{array}{l}\text { OE } \\
C / E\end{array}$ & $\begin{array}{r}0 \\
-0 \\
-0\end{array}$ & $\begin{array}{r}0 \\
-0 \\
-0\end{array}$ & $\begin{array}{r}0 \\
0 \\
0\end{array}$ & $\begin{array}{r}\mathbf{0} \\
\mathbf{0} \\
-0 \\
0\end{array}$ & $\begin{array}{r}0 \\
0 \\
-0\end{array}$ & $\begin{array}{r}0 \\
0 \\
--1 \\
0\end{array}$ & $\begin{array}{r}0 \\
0 \\
-0 \\
0\end{array}$ & $\begin{array}{r}0 \\
-- \\
0\end{array}$ \\
\hline $\begin{array}{l}\text { 1.1.1.3.02.06.02 } \\
\text { Level VI }\end{array}$ & $\begin{array}{l}\text { Two Year Restart } \\
\text { Compliance Activity } \\
\\
\text { Total }\end{array}$ & $\begin{array}{l}O E \\
C / E\end{array}$ & $---\frac{-}{0}$ & --- & $\begin{array}{r}---- \\
0\end{array}$ & $\begin{array}{r}--- \\
0\end{array}$ & $---\frac{-}{0}$ & $\begin{array}{r}--- \\
0\end{array}$ & $---\overline{0}$ & 0 \\
\hline $\begin{array}{l}\text { 1.1.1.3.02.06.03 } \\
\text { Level VI }\end{array}$ & $\begin{array}{r}\text { Maintain Grout Fcity, in } \\
\text { Cold Standby (FY 96, 97) } \\
\text { Total }\end{array}$ & $\begin{array}{l}O E \\
C / E\end{array}$ & -- & -- & $\begin{array}{r}---- \\
0\end{array}$ & $\begin{array}{r}--- \\
0\end{array}$ & - & $\begin{array}{r}--- \\
0\end{array}$ & -- & $-\cdots$ \\
\hline $\begin{array}{l}1.1 .1 .3 .02 .06 .05 \\
\text { Level VI } \\
\qquad\end{array}$ & $\begin{array}{l}\text { Fabricate Container for } \\
\text { Failed AP102 Mixer Pump } \\
\text { Total }\end{array}$ & $\begin{array}{l}O E \\
C / E\end{array}$ & -- & - & $\begin{array}{r}--- \\
0\end{array}$ & - & --- & $---\frac{-}{0}$ & $---\frac{-}{0}$ & --- \\
\hline
\end{tabular}

\section{[ R1]}




\section{NOTES \\ TOTAL PROGRAM ELEMENT COST BASELINE - BY YEAR}

The cost exhibit tables in each of the Program Element Baselines (Sections 7.2 through 7.10) identify the program element target funding by year by fund type. The tables also provide details of the baseline allocation of funding down to activity levels (level V) and cost account levels (level VI) of the WBS for Fiscal Year 1995 through 1997. The details exhibited on these tables are taken from the baseline WBS Dictionary Sheets. Deltas exist between the details and the annual target totals. For Fiscal Years 1996 and 1997 the deltas will be addressed in the future, following the completion of the resolution of expected differences between current Fiscal Year 1995 baseline funding and expected actual Fiscal Year 1995 funding. Deltas for Fiscal Year 1995 are noted below by program element:

\section{PROGRAM MANAGEMENT AND ADMINISTRATION:}

No deltas.

\section{TANK FARM OPERATIONS AND MAINTENANCE:}

No overall delta. This baseline requires revised fund type mix.

\section{WASTE TANK SAFETY:}

Delta is due to need to revise an overhead pricing rate in the P3 system.

\section{TANK FARM UPGRADES:}

Delta is due to differences between Budget Authority (BA) in the target and Budget Obligation (B0) in the P3 system.

\section{CHARACTERIZATION:}

No significant deltas.

\section{WASTE RETRIEVAL:}

Delta is primarily due to need to resolve issues associated with expense funding support to capital projects and differences between Budget Authority (BA) in the target and Budget Obligation (B0) in the P3 system.

\section{WASTE PRETREATMENT:}

Delta is due to the sum of the detail cost estimates being lower than the target total as a result of reestimation of resource requirements to complete baseline scope.

\section{LOW LEVEL WASTE:}

Delta is primarily due to carryover expense funded workscope on melter testing addressed in June 9, 1994 RL letter.

$$
T 7.9-1 \cdot 15
$$


WHC-SP-1101

\section{HIGH LEVEL WASTE:}

Delta is due to carryover of capital funding, PNL expense funding carryover, and ADS corrections submitted but not reflected in target totals. 
WHC-SP-1101

Table 7.9-2. FY 1995 Cost by Month by Fund Type by Activity and Cost Account

Future MYWP updates will include Table 7.9-2, which establishes the month-by-month cost baseline for FY 1995 for this program element. The FY 1995 cost baseline is exhibited at the cost account level with fund type totals provided. Cost data are then rolled up to the activity and program element levels. Also exhibited is the total cost baseline for the program element for FY 1995. At present, the program element cost baseline for FY 1995 for this program element is the total provided on this table less a productivity challenge for FY 1995. 


\section{WHC-SP-1101}

Table 7.9-3. Total Full-Time Equivalents by Year by Activity by Major Participant (Direct and Indirect) (Including all Subcontractors)

Future MYWP updates will include Table 7.2-3, which establishes total staffing projections for this program element. Included in the table are FY 1994 staffing data as well as projections by year for FY 1995 through FY 2000. The projections in this table include all direct and indirect Full-Time Equivalents (FTEs), including all subcontractor personnel. Where data are available, projections are exhibited at the activity level with major program participant totals provided. 
Table 7.9-4. FY 1995 through FY 1997 Direct Westinghouse Hanford Company

Full-Time Equivalents by 88 Labor Categories

Future MYWP updates will include Table 7.2-4, which establishes direct WHC staffing projections for this program element. Projections do not include subcontractor support and are provided by 88 labor categories for FY 1995 through FY 1997. 


\subsection{HIGH-LEVEL WASTE BASELINE}

The High-Level Waste (HLW) Program baseline consists of the technical bases, schedule, resource requirements, and waste type data for FY 1995 through completion of the mission. The major emphasis is on deliverables, schedule, and resource requirements for FY 1995, 1996, and 1997.

\subsubsection{Technical Bases}

This section provides the mission strategy; work breakdown structure and organization responsibility; work to be performed in FY 1995, 1996, and 1997; technical approach; and drivers and deliverables.

\subsubsection{Mission Strategy}

\subsection{Objectives}

The primary objective of the TWRS HLW Program is to immobilize the HLW/transuranic (TRU) fraction of the tank waste and seal it in containers that can be temporarily stored at Hanford until such time as they are shipped to a geologic repository for final disposal. The cesium and strontium capsules will also be dispositioned for disposal as part of this program.

\subsection{Strategy}

The strategy for achieving this objective is to vitrify the waste in a glass matrix that is sealed in stainless steel canisters. In order to achieve this objective, processes and equipment are being identified, designed, tested, and evaluated; and technologies are being developed that would lead to the design, construction, and operation of a government-owned and operated vitrification facility. As an alternative to this traditional DOE approach, DOE is also evaluating the technical and economic advantages and disadvantages of a plan to privatize the vitrification facility.

Currently the HLW Program is performing equipment development activities on both hightemperature and stirred melter concepts. A melter assessment task is also in progress to identify and evaluate other melter technologies that have potential application for vitrifying HLW. Glass formulation and other process development activities that support the melter evaluation (near-term) and conceptual design of the HLW vitrification facility (long-term) are also in progress.

\subsection{Functions and Requirements}

Systems engineering, which is a structured process to identify and implement a complete, traceable solution to a mission need for complex programs or projects, is being applied to TWRS. Work is currently underway using this approach to develop a consistent set of functions and requirements that satisfy the mission needs.

Immobilize HLW and TRU Waste (4.2.2.2), prepare Cs/Sr Capsules for Disposal (4.2.2.3), Interim Store Immobilized HLW (4.2.2.4), and Interim Store and Transport TRU Waste (4.2.2.5) are the four Level IV systems engineering functions that comprise the HLW Programs contribution to completing the TWRS mission. They involve the vitrification and interim onsite storage of the HLW and TRU wastes, as well as the retrieval and packaging of cesium and strontium capsules currently stored at Hanford. For program planning purposes it has been assumed that $\mathrm{HLW}$ and TRU wastes 
can be blended, vitrified, and disposed at a single repository. Therefore, the functions 4.2.2.4 and 4.2.2.5 have been combined into a single function called Interim Store Immobilized HLW/TRU Waste.

The Vitrify HLW/TRU Waste function is divided into five subfunctions:

1. Prepare Melter Feed (4.2.2.2.1). Combine pretreated HLW/TRU waste and glass frit and/or glass forming additives and chemicals to provide a stream suitable for vitrification.

2. Vitrify HLW and TRU Waste (4.2.2.2.2). Vitrify prepared melter feed consisting of pretreated HLW/TRU waste, glass frit, and/or glass forming additives and chemicals.

3. Encapsulate Immobilized HLW and TRU Waste (4.2.2.2.3). Pour the vitrified HLW/TRU waste into a canister, seal the canister with a closure weld, and decontaminate the outer surfaces. Canisters that do not satisfy the requirements of the Verify Immobilized HLW and TRU Canisters function (4.2.2.2.4) are corrected by this function as well.

4. Verify Immobilized HLW and TRU Canisters (4.2.2.2.4). Verify the integrity of sealed HLW/TRU canisters and measure removable surface contamination. Return failed canisters to the Encapsulate Immobilized HLW and TRU Waste function (4.2.2.2.3) for correction of deficiencies.

5. Support Immobilization Process (4.2.2.2.5). Provides balance of plant for the vitrification process, including maintenance, utilities services, and process support services (e.g., analytical laboratories, shipping and receiving, storage, equipment repair, gaseous effluent treatment, cold chemical preparation, solid waste handling, liquid waste treatment). Receives, collects, stores, and disseminates data and processes directives.

At this time none of the other Level IV functions have been further decomposed.

\subsection{Risks}

The following three primary risks are associated with this scope.

1. Inadequate integration with other TWRS program elements that might lead to the inability to adequately characterize tank waste, pretreat HLW to its feed specifications, transfer feed at the required rate, or adequately disposition system generated waste.

2. Inability to adequately define the required inputs, functions, and outputs of the HLW system. This includes, but is not limited to, the definition of the HLW feed characteristics, volume of HLW to be processed, repository requirements for acceptable waste products, plant system generated waste treatment requirements, and length of the interim storage period.

3. Inability to develop the required technology for a high-capacity vitrification system. The primary risk involves the selection, development, and testing of the melter system within program cost and schedule constraints. Nearly a factor of two reduction in the near-term budget from a "robust" program has resulted in a melter system development and testing program that has little flexibility to respond to "unknowns" or budget cuts. Impacts to TriParty Agreement milestones may result. 
Actions being taken to reduce these risks include comprehensive integration and analysis of interfaces with other TWRS program elements, a series of trade studies and DOE Office of Civilian Radioactive Waste Management (RW) negotiations leading to complete system definition, and a rigorous technology development program to identify and develop the most viable vitrification technologies.

\subsection{Major Decisions}

Major technical decisions and the risks associated with them are summarized below.

\section{Determine Disposition of Transuranic Tank Waste}

A disposition approach for Hanford tank waste which may be designated as TRU (instead of HLW) is required, defining the waste form criteria which will be imposed. Alternatives for separate disposition of TRU tank waste are predicated on the assumption that the selected tank waste types can be designated as TRU waste instead of high-level waste. Assuming that TRU tank waste is determined to exist, the following three options have been identified as alternatives for the disposition of TRU tank waste.

1. Dispose in the geologic repository

2. Dispose in the Waste Isolation Pilot Plant (WIPP)

3. Disposition in either the WIPP or geologic repository is unacceptable.

The primary impact of alternatives for this decision is the potential for variations of the waste form, depending on the selected disposition. The impact on process selection will be either no impact (i.e., waste acceptance criteria variations have minimal impact on the waste form and immobilization process selection) or two waste forms (one for TRU waste and one for HLW) must be developed, qualified and manufactured. The alternatives selection will also impact staging of the waste form manufacturing.

For planning purposes, the HLW Program has assumed that any TRU tank waste that may exist at Hanford can be disposed in the geologic repository.

\section{Determine Acceptability of Alternative Containers for Geologic Repository}

A reference waste canister for disposal of Hanford waste in the geologic repository is required. Canister alternatives for the geologic repository are derived from current acceptance criteria for the repository and interpretation of the recent evolutions in the geologic repository reference design. The alternatives for canister sizing are:

1. The Defense Waste Processing Facility (DWPF) standard canister

2. A larger canister that uses the geologic repository Multi-Purpose Canister (MPC) design envelope for development of an optimum canister size.

The HLW canister selection defines the envelope for the HLW form to be disposed in the geologic repository. Canister size selection impacts:

1. The number of packages that must be generated by the immobilization facility 
2. The dimension and weight of packages that must be positioned and moved inside the facility process cell

3. The size of package that would be used as a basis for selecting the interim storage concept

4. The physical form of the HLW glass product.

The time required to establish an alternative to the DWPF standard canister may result in this decision being on the critical path for initiating conceptual design.

Current program planning cost estimates are based on a 0.6 meter diameter by 4.5 meter canister as an initial estimate for a canister that utilizes the MPC design envelope.

\section{Determine Disposition of Cs/Sr Capsules}

The disposition of the encapsulated cesium chloride and strontium fluoride salts is required. This evaluation should determine whether the geologic repository will accept overpacked capsules and, if not, whether pretreatment processing of the halide salts is necessary. Based on the Hanford Defense Waste-Environmental Impact Statement (HDW-EIS) Record of Decision, cesium and strontium capsules will be disposed of in the geologic repository. Preliminary discussions with personnel at the Yucca Mountain Site Characterization Office (YMSCO) indicate that uncertainties may exist in the acceptance of the existing material. The primary uncertainty is related to the potential for the halides to accelerate corrosion of nearby waste packages.

The following process alternatives have been identified for supporting disposition of the capsules in the geologic repository:

1. Overpack and ship directly to the geologic repository

2. Reprocess capsules and blend with other retrieved tank waste for vitrification and disposal in the geologic repository

3. Embed the existing capsules in glass.

The selection of a disposition option will likely influence which facility is used for capsule processing. Direct overpacking of capsules is more likely to be included in the HLW vitrification system scope since overpacking primarily requires package-handling steps. Alternatively, capsule reprocessing might be better suited to be combined with pretreatment activities, if solution handling capabilities are required. This decision may also impact tank processing schedules if a disposition decision is deferred for many years, as well as the selection of an interim storage concept for the capsules.

The record of decision for the HDW-EIS, which directs that cesium and strontium capsules be disposed of in a geologic repository, is used for program planning purposes. 


\section{Determine whether TRU and HLW can be processed in same Plant}

While the current planning basis is to vitrify HLW and TRU wastes together rather than separately, a trade study that determines whether a single plant will be developed to immobilize both TRU and HLW types is required. Two alternatives exist for providing the capability to immobilize HLW and TRU waste:

1. Combined TRU/HLW facility

2. Separate facilities.

This decision can have significant impacts on the projected cost and schedule of HLW and TRU waste immobilization. Separate facilities represent the upper bound of facility construction and operating costs for the alternatives identified, while processing the two waste types separately in a single facility without segregation would result in somewhat lower costs. Current program planning cost estimates are based on an implementation strategy that does not segregate TRU from HLW prior to vitrification. Current cost estimates will escalate if an alternative to the current planning basis is selected.

If segregated TRU tank waste processing is required and the single facility concept using equipment changeout is selected as an implementation strategy, then some increase in schedule risk may occur as the result of incorporating downtime in the overall facility program schedule to allow for equipment changeout activities.

For planning purposes, the HLW Program has assumed that both HLW and TRU waste can be processed in the same facility without segregating the TRU from the HLW.

\section{Determine HLW Vitrification Facility Concept}

Alternatives exist for developing the TWRS disposal system as individual facilities supporting pretreatment, low-level waste (LLW) vitrification, and HLW vitrification, or combining two or more of these functions into a single facility. A decision on whether the HLW vitrification facility is a standalone or should be combined with one or both of the facilities is required.

Facility concept alternatives for integrating the HLW vitrification facility with other TWRS disposal system facilities have been proposed as a means of reducing the overall construction costs supporting Hanford tank waste disposal. The proposed alternatives include the following combinations:

1. Standalone HLW vitrification facility

2. HLW vitrification combined with pretreatment

3. HLW vitrification combined with LLW vitrification

4. HLW vitrification combined with pretreatment and LLW vitrification.

Current program planning assumptions are based on standalone configurations for three major facilities in the TWRS disposal system. This configuration decision has the potential to impact the number of discrete major construction projects proposed for the TWRS disposal system. Alternatives that combine two or more of the disposal functions will reduce the number of projects and, therefore, are expected to reduce the total costs as well. While reduced construction and operating costs may be achieved by combining the HLW vitrification with other disposal elements, further evaluation needs to verify that the resulting construction cost profile does not exceed projected available funding during the construction period as the project scope is increased. 
Combining the HLW vitrification facility with other disposal facilities would likely accelerate the initiation and completion of the HLW processing such that compliance with current Tri-Party Agreement milestones are satisfied ahead of schedule. However, current development and trade study activities needed to define the HLW Program would be compressed or be completed at later stages in the design/construction process, thereby increasing programmatic risk. This will either compress funding requirements or require initiation of detailed design activities for the HLW portion of combined facilities with more alternatives to be evaluated.

The HLW Program is based on a standalone HLW vitrification facility.

\section{Determine Immobilized High-Level/Transuranic Waste Container Facility Concept}

A reference facility concept for temporary onsite storage of immobilized HLW and TRU waste in containers is required.

Immobilized HLW and TRU waste will be stored in canisters for an interim time period on site prior to shipment to an offsite repository. A storage system must be selected to accomplish this function. Alternative facility concepts to be evaluated for the storage system are as follows:

1. Enclosed facility

2. Open (pad) facility.

The selected canister size will likely impact the viability of enclosed facility concepts for interim onsite waste canister storage. The standard DWPF canister is more readily amenable to storage in an enclosed facility. Alternatively, the $10 \mathrm{~m}^{3}$ canister dimensions are significantly larger than those of the standard canister; as a result, the open facility concept may be more attractive from an economic point-of-view.

No decision has been made on the storage facility configuration.

\section{Determine Capsule Overpack Facility Concept}

A reference facility concept for overpacking the capsule is required.

Alternative facility configurations are available for providing a capsule overpack installation. Potential cost savings may be available for installing the overpack system if it is incorporated in a facility that has similar characteristics. The alternatives proposed for the overpack facility configurations are as follows:

1. Combine with HLW vitrification

2. Combine with pretreatment facility

3. Separate capsule overpacking facility

4. Combine with immobilized HLW interim storage facility.

Assuming that overpacking of cesium and strontium capsules is acceptable, the immobilized HLW canister storage facility concept potentially impacts consideration of the capsule overpack facility alternative, which combines overpack systems with the interim storage facility. A reasonable opportunity for considering this combination option exists if the enclosed facility option is selected for HLW container storage. However, if the open facility concept is selected for container storage, there 
WHC-SP-1101

would be no facility capabilities that would be attractive for combining with the capsule overpack systems.

Current planning assumes combining the cesium and strontium overpack systems into the HLW vitrification facility.

\subsection{Assumptions}

Several key assumptions have been used as the basis for the current multi-year program planning of HLW vitrification at the Hanford Site.

- The HLW vitrification plant will start up in 2009 and will be a government-designed, constructed, and operated facility.

- The HLW fraction from all waste storage tanks (both double-shell tanks [DSTs] and single-shell tanks [SSTs]) will be vitrified.

- The sealed canisters of vitrified waste will be disposed at the geologic repository.

- Shipment of HLW canisters to the offsite geological repository will begin some time after the last canister of vitrified waste is produced.

- The HLW form will be a glass monolith in a canister that is larger than those currently designed for use at Savannah River and West Valley.

- Any TRU tank waste (e.g., Plutonium Finishing Plant wastes) will be processed through the same vitrification facility as the HLW.

- The HLW vitrification facility will have adequate throughput capacity to complete its vitrification mission by 2028 , using a slightly caustic sludge washing pretreatment and assuming a nominal waste oxide loading of 25 percent. Based on this operating scenario, it is estimated that as much as 28,000 cubic meters of vitrified HLW will be produced.

- The HLW vitrification facility will be a standalone, dual melter line facility that has a footprint approximately 25 percent larger than that of the Hanford Waste Vitrification Plant (HWVP) design.

- The HLW Program will build and operate a canister storage facility sufficient to store the entire volume of glass produced by the vitrification facility for a minimum of 30 years.

- Waste streams sent to the vitrification facility will meet feed specification(s) jointly developed by the Waste Pretreatment, Low-Level Waste, and High-Level Waste Program Elements

- Overpack of the cesium and strontium capsules will be acceptable to the geologic repository as a waste form for disposal and will be performed in the HLW vitrification facility. Prior to overpacking, these capsules will continue to be stored at Hanford until the HLW storage facility is built. 
WHC-SP-1101

- Two vitrification system candidates will be selected for further testing and evaluation; the reference melter system that is carried into conceptual design will be one of these two candidates and a modified or new pilot-scale system will be needed for further testing.

- Approximately ten basic blended feed stream types will require waste form qualification.

- The HLW Program will include feed lag storage in its scope.

- The HLW Program will either rework or overpack out-of-specification canistered waste. In the event that neither rework nor overpacking is feasible, a nonconformance waiver would be requested from the repository.

\subsection{Constraints}

The ability to successfully start up the HLW vitrification plant is predicated on pilot-scale test data using waste simulants with only limited test data from radioactive testing on a laboratory scale.

\subsubsection{Structure}

The HLW Program WBS and organization and responsibility are provided in this section.

\subsection{Work Breakdown Structure}

The HLW Program WBS for FY 1995 through 1997 is provided in Figure 7.10-2 down to the sixth level of work breakdown. The HLW Program WBS dictionary is located in Appendix A.

\subsection{Organization and Responsibility}

The HLW Program interfaces with several of the other program elements: Characterization, Waste Retrieval, Waste Pretreatment, and Low-Level Waste.

Characterization provides waste characterization data, from both recent sampling and historical data review. To support this need, the HLW Program, in concert with the Waste Pretreatment and LowLevel Waste Program Elements, will prepare a set of Data Quality Objectives identifying the need for data, number of samples needed, and particular analyses required.

Waste Retrieval provides sufficient quantities of waste to the HLW Program, via Waste Pretreatment, in order to verify the glass formulation and melter development testing performed with cold chemical simulants and to qualify the waste forms for disposal.

Waste Pretreatment provides chemically pretreated feed streams that will require HLW vitrification, from both the insoluble sludges that remain after sludge washing and the cesium, strontium, and other radionuclides that are removed from the supernatant and dissolved saltcake by the ion exchange process.

The Low-Level Waste Program Element receives radioactive LLW liquid effluents from the HLW process for immobilization and onsite disposal through the end of its operating life. 
WHC Process Engineering Center of Excellence and its Systems Engineering group are two engineering organizations that are performing key roles in defining what work will be done by the HLW Program and what the preferred alternatives are to support decisions that need to be made within TWRS.

Pacific Northwest Laboratory's (PNL) TWRS Technology organization performs much of the technology development activities required to support the HLW Program. As part of that support, the Technology Development Program Office coordinates and integrates TWRS technology development activities to ensure that technology solutions are directly coupled to TWRS needs.

Fluor Daniels, as the former architect/engineer for the HWVP, and EBASCO/British Nuclear Fuels Ltd. (BNFL) are performing facility option trade studies in support of the disposal program. These studies are evaluating various combinations of pretreatment, LLW vitrification and HLW vitrification facilities that will be required to complete the tank waste disposal mission. They will serve as the basis for early design activities (i.e., conceptual design) and eventually for definitive (Title II) design.

The TWRS Projects organization is responsible for design, construction, and startup of the vitrification facility, if a government-owned facility is the selected option. They will use the results of trade studies, technology development activities, and engineering evaluations, testing, and demonstrations as inputs to the conceptual and definitive designs of the facility. Additional testing and evaluation will be performed during the design phases to confirm design features and systems in support of TWRS Projects.

DOE-RW and its YMSCO provide technical and programmatic guidance to the waste form producers (e.g., Hanford's HLW Program) in terms of the acceptability of the waste form(s) for final disposal and the calculation of fees associated with same.

The secondary wastes generated by the HLW Program in the course of performing technology development, process and equipment development and verification, and waste form qualification will be transferred to the Liquid Waste and Solid Waste Programs for processing.

Once the HLW vitrification and storage facilities are no longer needed for the tank waste disposal mission, they will be transferred to the Environmental Restoration Program for decontamination and decommissioning.

\subsubsection{FY 1995 Work to be Performed}

See Activity Planning Forms for FY 1995.

Technical Integration and Planning (WBS 1.1.1.3.03.01)

See the HLW Program Level V dictionary sheets, Section C, Statement of Work (Appendix A).

Technology Development and Applied Engineering (WBS 1.1.1.3.03.02)

See the HLW Program Level V dictionary sheets, Section C, Statement of Work (Appendix A).

\section{HLW Facility Operations (1.1.1.3.03.03)}

See the HLW Program Level V dictionary sheets, Section C, Statement of Work (Appendix A). 
System Definition (WBS 1.1.1.3.03.04)

See the HLW Program Level V dictionary sheets, Section C, Statement of Work (Appendix A).

HLW Facility (WBS 1.1.1.3.03.05)

See the HLW Program Level V dictionary sheets, Section C, Statement of Work (Appendix A).

HWVP Project Closeout (WBS 1.1.1.3.03.06)

See the HLW Program Level V dictionary sheets, Section C, Statement of Work (Appendix A).

\subsubsection{FY 1996 - FY 1997 Work to be Performed}

See Activity Planning Forms for FY 1996 - FY 1997.

Technical Integration and Planning (WBS 1.1.1.3.03.01)

See the HLW Program Level V dictionary sheets, Section C, Statement of Work (Appendix A).

Technology Development and Applied Engineering (WBS 1.1.1.3.03.02)

See the HLW Program Level V dictionary sheets, Section C, Statement of Work (Appendix A).

HLW Facility Operations (1.1.1.3.03.03)

See the HLW Program Level V dictionary sheets, Section C, Statement of Work (Appendix A).

System Definition (WBS 1.1.1.3.03.04)

See the HLW Program Level V dictionary sheets, Section C, Statement of Work (Appendix A).

HLW Facility (WBS 1.1.1.3.03.05)

See the HLW Program Level V dictionary sheets, Section C, Statement of Work (Appendix A).

\subsubsection{Approach}

\section{Reference Melter System Selection}

The majority of testing and evaluation to determine basic performance of candidate melter systems will be based on bench-scale melters using cold simulants. Limited pilot-scale melter testing to confirm scale-up performance will be performed on the two selected candidate systems using cold simulants. Limited laboratory-scale melter testing using pretreated radioactive waste will be performed to confirm the results of testing performed with cold simulants. Glass formulation and feed processibility, composition variability studies, and waste form qualification testing will also be performed to the extent necessary to support the melter system evaluation and selection activities.

An initial evaluation of candidate melter technologies will be performed by an independent Technical Advisory Committee (TAC) in September 1994. Assuming the committee can recommend two melter 
systems, bench-scale and pilot-scale testing of those two systems will be initiated. If the TAC cannot clearly recommend two candidates, a higher risk path will be pursued, consisting of bench-scale testing of three or more melter technologies in FY 1995 with a selection of two melter systems occurring during FY 1996. Pilot-scale testing of the two selected candidates would be deferred to late FY 1996.

Testing and evaluation of the two selected melter systems will be conducted through FY 1998. A single system will be carried forward as the reference concept into the definitive design phase, while the second vitrification system concept will be maintained as a backup.

\section{Reference Melter System Testing}

Following selection of the reference melter system in September 1998, a second generation pilot-scale melter system will be procured. Testing of this system using the reference flowsheet and cold simulants will be conducted to support definitive design, start up and operations, and waste form qualification. Bench-scale testing using pretreated radioactive waste will be performed to confirm pilot-scale system performance and to support waste form qualification.

\section{Design of the High-Level Waste Vitrification Facility}

Assuming that the existing contract with Fluor-Daniel for Hanford Waste Vitrification Plant design can be extended, conceptual design will begin in March 1997 and will be completed in September 1998. However, there are several key activities that must be performed prior to the start of conceptual design. Using the systems engineering approach, decomposition of the HLW Program functions will be performed to ensure that the HLW vitrification system is necessarily and sufficiently defined. Trade studies to verify or modify enabling assumptions used in the functions and requirements and reference flowsheets will be completed. Preliminary waste acceptance criteria will be developed in conjunction with DOE-RW. The balance of plant evaluations and flowsheet development will provide input to support conceptual design.

Definitive design will begin in December 1998 and will be supported by pilot-scale testing of the reference melter system, bench-scale radioactive system testing, baseline flowsheet development, additional product qualification criteria, and permitting and safety analysis documentation.

\section{Facility Capabilities Added After the Tri-Party Agreement Negotiations}

The following capabilities were added to the HLW vitrification facility scope following the completion of the Tri-Party Agreement negotiations: a second melter, a cesium/strontium capsule processing line, solid waste processing, HLW feed lag storage, and a close-coupled analytical laboratory. As a result of these added capabilities within the HLW vitrification plant, facility operating costs are now estimated to be $\$ 100 \mathrm{M}$ per year higher than was assumed for the funding profile developed to support the Tri-Party Agreement. This difference has resulted in a $\$ 2,000 \mathrm{M}$ increase in the current baseline costs over the original Tri-Party Agreement estimate.

\subsubsection{Drivers and Deliverables}

The requirements to address each of these functions are also being developed through the systems engineering process. A critical requirement for the HLW Program and for its supporting technical activities to proceed effectively is to establish product specifications for the pretreated feed streams that will be immobilized as HLW. Preliminary feed specifications are being prepared for use in the 
TWRS technical baseline. A clear understanding of the tank waste characteristics is also critical to defining the requirements for the process flowsheet. Data Quality Objectives for HLW vitrification are being prepared as part of the Waste Pretreatment Program Element. Technology evaluation in the laboratory using simulated and actual wastes is another element of developing the process requirements.

One of the key drivers for separating the tank waste into an optimally reduced volume of HLW with the remaining volume as LLW is the anticipated cost of disposing of defense HLW in the national geological repository, which is projected to cost significantly more per unit volume than near-surface disposal of LLW at Hanford.

Another driver for the disposal program is an urgency to make progress in cleanup of the Hanford Site, particularly to initiate retrieval, pretreatment, and disposal of the tank waste. Specifically, retrieving these wastes from tanks that are leaking or have the potential to leak waste to the groundwater and transforming those wastes into a more chemically stable form is highly desirable, even if it is necessary to store the canistered HLW at the Hanford Site for several years while a repository is sited and built. This urgency is captured in the aggressive schedule of Tri-Party Agreement milestones agreed to by the DOE, the U.S. Environmental Protection Agency, and the Washington State Department of Ecology in January 1994.

\subsubsection{Schedule}

\subsubsection{High-Level Waste Summary Schedule}

The HLW summary schedule is shown on Figure 7.10-2.

\subsubsection{High-Level Waste Expanded Baseline (FY 1995 - FY 1997)}

The HLW expanded baseline can be found in Appendix C.

\subsubsection{Resource Requirements}

See Cost Estimating Sheets/Basis for Cost Estimates.

\subsubsection{Cost}

\subsection{Basis of Cost Estimates}

The basis of cost estimates for the HLW Program is primarily from two sources. For technology development and project-related activities, cost estimates are based on similar experience from the Hanford Waste Vitrification Plant project. For trade studies and systems engineering-related activities, cost estimates are based on similar HLW Program activities that are either currently in progress or recently completed (e.g., the Facility Configuration Study). 


\subsection{Total Program Cost by Year by Fund Type by Activity and Cost Account}

Table 7.10-1 establishes the baseline cost for this program element. The baseline cost is exhibited in annual costs for FY 1994 through FY 2000, and 5-year totals for FY 2001 through program element completion. Where the data are available, these baseline costs are exhibited at the cost account level with fund type totals provided. Cost data are then rolled up to the activity and program element levels. At present, the program element cost baseline is the program element cost totals exhibited on this table less a productivity challenge for FY 1995 through FY 1997.

\subsection{FY 1995 Funding by Month by Fund Type by Activity and Cost Account}

Future MYWP updates will include Table 7.10-2, which establishes the month-by-month cost baseline for FY 1995 for this program element. The FY 1995 cost baseline is exhibited at the cost account level with fund type totals provided. Cost data are then rolled up to the activity and program element levels. Also exhibited is the total cost baseline for the program element for FY 1995. At present, the program element cost baseline for FY 1995 for this program element is the total provided on this table less a productivity challenge for FY 1995.

\subsubsection{Staffing}

\subsection{Basis of Staffing Projections}

\subsection{Total Full-Time Equivalents by Year by Activity by Major Participant (Direct and Indirect)}

Future MYWP updates will include Table 7.10-3, which establishes total staffing projections for this program element. Included in the table are FY 1994 staffing data as well as projections by year for FY 1995 through FY 2000. The projections in this table include all direct and indirect Full-Time Equivalents (FTEs), including all subcontractor personnel. Where data are available, projections are exhibited at the activity level with major program participant totals provided.

\subsection{FY 1995 through FY 1997 Direct Full-Time Equivalents by 88 Labor Categories}

Future MYWP updates will include Table 7.10-4, which establishes direct WHC staffing projections for this program element. Projections do not include subcontractor support and are provided by 88 labor categories for FY 1995 through FY 1997.

\subsubsection{Work Scope Carryover}

Carryover work scope from FY 1994 totals $\$ 3,076 \mathrm{~K}$. This work scope is all external commitments that have been contractually obligated. It includes glass formulation work at Ecole Polytechnic Institute and Renssalaer Polytechnic Institute $(\$ 240 \mathrm{~K})$; feed preparation/rheology investigations being performed by the University of Georgia $(\$ 40 \mathrm{~K})$; close out of melter testing $(\$ 132 \mathrm{~K})$ and design support for a melter bottom drain $(\$ 44 \mathrm{~K})$ being performed by $\mathrm{KfK}$ in Germany; development work on the mechanically stirred melter at Clemson University (\$850K); and $\$ 970 \mathrm{~K}$ of CENRTC for procurement of a pilot-scale stirred melter, which will be installed at the Savannah River Technology Center in FY 1995. 


\subsubsection{Waste Type Data}

For the execution years FY 1995, FY 1996, and FY 1997, the only waste type that will be generated by the HLW Program is sanitary (landfill) waste. The estimated amounts in each of those three years is $0.2,0.4$ and 0.6 cubic meters, respectively. The sanitary waste from offices will be sent to landfill through janitorial services.

For the life of the HLW Program, the following waste types are expected to be generated, received, transferred and/or stored: HLW (7000 cubic meters received, 28,000 cubic meters stored); solid lowlevel waste $(28,000$ cubic meters generated, 28,000 cubic meters transferred); liquid low-level waste (910 million kilograms generated, 910 million kilograms transferred); low-level mixed waste (600 cubic meters generated, 600 cubic meters transferred to Solid Waste Disposal); hazardous waste (2400 cubic meters generated, 2400 cubic meters transferred to Solid Waste Disposal); and sanitary (landfill) waste $(14,400$ cubic meters generated, 14,400 cubic meters transferred to landfill).

The HLW volume assumes a glass-to-waste ratio of 3:1, or 25 percent waste oxide loading of the glass matrix. It further assumes that all of the canistered glass will be stored on site at Hanford for some period of time beyond the life of the program until such time as the geologic repository can begin receiving the waste. It also assumes that all of the liquid low-level waste generated as a result of the treatment of HLW will be transferred to the TWRS pretreatment facility for low-level waste prior to its vitrification and disposal onsite. This assumption raises a major long-term issue for the TWRS Program, since the current planning assumption is that the LLW pretreatment and vitrification facilities will cease to operate 8-years prior to the completion of the HLW mission.

\subsubsection{Revisions}

In future updates of the MYWP, this section will include a summary of the revision that has occurred in this program element since the last issue of the MYWP. 


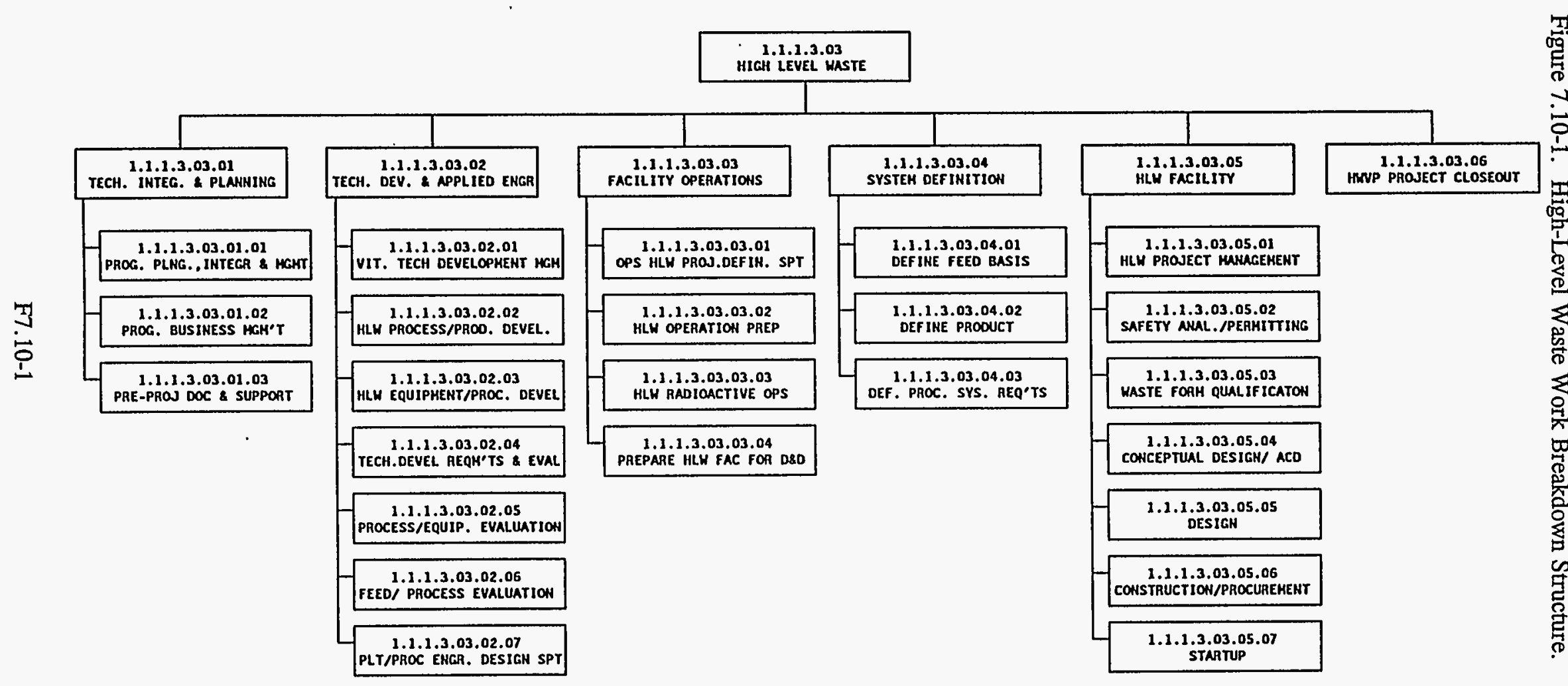


WHC-SP-1101

Figure 7.10-2. High-Level Waste Summary Schedule.

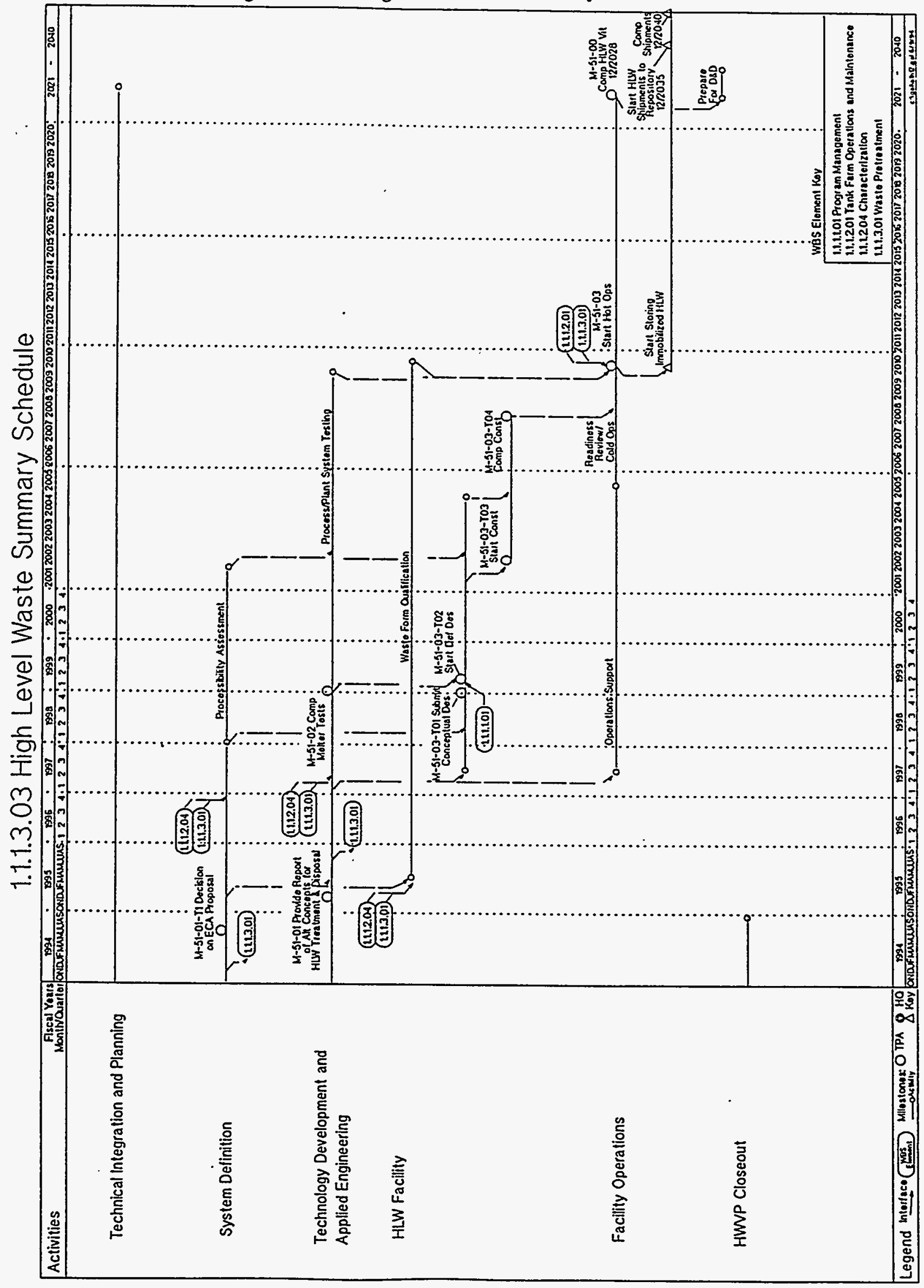


TABLE $7.10-1$

TOTAL PROGRAM ELEMENT

COST BASELINE - BY YEAR

\section{[ R1 ]}

\begin{tabular}{|c|c|c|c|c|c|c|c|c|c|}
\hline WBS / LEVEL & ACTIVITY TITLE / ADS & $\begin{array}{l}\text { FUND } \\
\text { TYPE }\end{array}$ & $\begin{array}{l}\text { TOTAL } \\
\text { FY94 }\end{array}$ & FY95 & FY96 & FY97 & FY98 & FY99 & FYOO \\
\hline \multirow[t]{2}{*}{$\begin{array}{l}\text { 1.1.1.3.03 } \\
\text { Level iv }\end{array}$} & $\begin{array}{r}\text { High Level Waste } \\
\text { ADso-o } \\
\text { TOTAL TARGET }\end{array}$ & 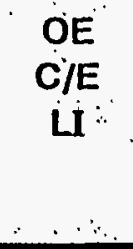 & $\begin{array}{r}20,626 \\
0 \\
30,000 \\
50,626 \\
0\end{array}$ & $\begin{array}{r}12,961 \\
3,980 \\
0 \\
16941\end{array}$ & $\begin{array}{r}18,387 \\
4,481 \\
0 \\
-12,868 \\
22,868\end{array}$ & $\begin{array}{r}25,170 \\
9119 \\
-10 \\
28,289 \\
10\end{array}$ & $\begin{array}{l}37,279 \\
2570 \\
390 \\
3049\end{array}$ & $\begin{array}{r}32,162 \\
60 \\
26,539 \\
58,701 \\
1 \%\end{array}$ & $\begin{array}{l}34516 \\
3,0 \\
78,415 \\
112,981\end{array}$ \\
\hline & $\begin{array}{l}\text { Delta to TARGET. } \\
\text { (See notes at end of Table.) } \\
\text { Total }\end{array}$ & $\begin{array}{l}O E \\
C / E \\
L I\end{array}$ & NA & $\begin{array}{c}(4,374) \\
2,157 \\
(6,201) \\
==== \\
(8,418)\end{array}$ & $\begin{array}{c}(2,962) \\
1,864 \\
0 \\
==== \\
(1,098)\end{array}$ & $\begin{array}{r}(2,561) \\
1,499 \\
0 \\
==== \\
(1,122)\end{array}$ & & & \\
\hline $\begin{array}{l}\text { 1.1.1.3.03.01 } \\
\text { Level } \mathrm{V}\end{array}$ & $\begin{array}{l}\text { Technical Integration } \\
\frac{\text { and Planning }}{\text { ADS } 1240-0-A A} \\
\quad \text { Sub Total }\end{array}$ & $\begin{array}{l}O E \\
C / E\end{array}$ & NA & $\begin{array}{r}1.476 \\
0 \\
---\frac{0}{1.476}\end{array}$ & $\begin{array}{r}1,243 \\
--10 \\
1,243\end{array}$ & $\begin{array}{r}1,123 \\
---\frac{0}{1.123}\end{array}$ & $--\frac{0}{0}$ & $\begin{array}{r}0 \\
0 \\
--0 \\
0\end{array}$ & $\begin{array}{r}0 \\
-0 \\
-0\end{array}$ \\
\hline $\begin{array}{l}\text { 1.1.1.3.03.01.01 } \\
\text { Level VI }\end{array}$ & $\begin{array}{l}\text { Program Planning, } \\
\text { Integration \& Management } \\
\text { Total }\end{array}$ & $\begin{array}{l}O E \\
C / E\end{array}$ & & $\begin{array}{r}180 \\
0 \\
--- \\
180\end{array}$ & $\begin{array}{r}112 \\
0 \\
--- \\
112\end{array}$ & $\begin{array}{r}141 \\
0 \\
--- \\
141\end{array}$ & $---\frac{-}{0}$ & --- & $\begin{array}{r}--- \\
0\end{array}$ \\
\hline $\begin{array}{l}1.1 .1 .3 .03 .01 .02 \\
\text { Level VI }\end{array}$ & $\begin{array}{l}\text { Program Business } \\
\text { Management } \\
\\
\text { Total }\end{array}$ & $\begin{array}{l}O E \\
C / E\end{array}$ & & $\begin{array}{r}781 \\
0 \\
--- \\
781\end{array}$ & $\begin{array}{r}916 \\
0 \\
---- \\
916\end{array}$ & $\begin{array}{r}939 \\
0 \\
--- \\
939\end{array}$ & 0 & $---\frac{1}{0}$ & 0 \\
\hline $\begin{array}{l}\text { 1.1.1.3.03.01.03 } \\
\text { Level VI }\end{array}$ & $\begin{array}{l}\text { Pre-Project Documentation } \\
\text { and Support } \\
\qquad \text { Total }\end{array}$ & $\begin{array}{l}O E \\
C / E\end{array}$ & & $\begin{array}{r}515 \\
0 \\
--- \\
515\end{array}$ & $\begin{array}{r}215 \\
0 \\
--- \\
215\end{array}$ & $\begin{array}{r}43 \\
0 \\
--- \\
-43\end{array}$ & $--\frac{-}{0}$ & $---\frac{-}{0}$ & --- \\
\hline
\end{tabular}


TABLE 7.10-1

TOTAL PROGRAM ELEMENT COST BASELINE - BY YEAR

\section{[R1]}

\begin{tabular}{|c|c|c|c|c|c|c|c|c|c|}
\hline $\begin{array}{l}\text { 1.1.1.3.03.02 } \\
\text { Leved } v\end{array}$ & $\begin{array}{l}\text { Technical Dvlpmt. \& } \\
\text { Applied Engineering } \\
\text { ADS 1240-0-AB } \\
\text { Total }\end{array}$ & $\begin{array}{l}\text { OE } \\
\text { C/E }\end{array}$ & NA & $\begin{array}{r}11,193 \\
1,823 \\
-19,016\end{array}$ & $\begin{array}{r}14,876 \\
2.617 \\
---- \\
17,493\end{array}$ & $\begin{array}{r}14,165 \\
1,680 \\
-15,-- \\
15,845\end{array}$ & $--\frac{0}{0}$ & $--\frac{0}{0}$ & $-\frac{0}{0}$ \\
\hline $\begin{array}{l}\text { 1.1.1.3.03.02.02 } \\
\text { Level VI }\end{array}$ & $\begin{array}{l}\text { Hi-Level Waste Process / } \\
\text { Product Development } \\
\text { Total }\end{array}$ & $\begin{array}{l}O E \\
C / E\end{array}$ & & $\begin{array}{r}3,655 \\
103 \\
--- \\
3,758\end{array}$ & $\begin{array}{r}3,301 \\
117 \\
-3,418\end{array}$ & $\begin{array}{r}3,324 \\
180 \\
--- \\
3,504\end{array}$ & 0 & 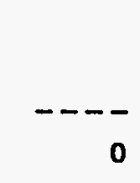 & --- \\
\hline $\begin{array}{l}1.1 .1 .3 .03 .02 .04 \\
\text { Level VI }\end{array}$ & $\begin{array}{l}\text { Tech. Dvlpmt. Requirements } \\
\text { and Evaluation } \\
\qquad \text { Total }\end{array}$ & $\begin{array}{l}\text { OE } \\
\text { C/E }\end{array}$ & & $\begin{array}{r}443 \\
0 \\
--- \\
443\end{array}$ & $\begin{array}{r}569 \\
0 \\
--- \\
569\end{array}$ & $\begin{array}{r}581 \\
0 \\
--- \\
581\end{array}$ & - & - & -- \\
\hline $\begin{array}{l}1.1 .1 .3 .03 .02 .05 \\
\text { Level VI }\end{array}$ & $\begin{array}{l}\text { Process Equipment } \\
\text { Evaluation HOLD } \\
\\
\\
\end{array}$ & $\begin{array}{l}O E \\
C / E\end{array}$ & & $\begin{array}{r}2,328 \\
0 \\
---- \\
2,328\end{array}$ & $\begin{array}{r}795 \\
0 \\
--- \\
795\end{array}$ & $\begin{array}{r}715 \\
-\quad 0 \\
--- \\
715\end{array}$ & - & - & -- \\
\hline $\begin{array}{l}\text { 1.1.1.3.03.02.06 } \\
\text { Level VI }\end{array}$ & $\begin{array}{l}\text { Feed / Process } \\
\text { Evaluation }\end{array}$ & $\begin{array}{l}O E \\
C / E\end{array}$ & & $\begin{array}{r}249 \\
0 \\
---- \\
249\end{array}$ & $\begin{array}{r}675 \\
0 \\
--- \\
675\end{array}$ & $\begin{array}{r}643 \\
0 \\
--0 \\
643\end{array}$ & $\overline{0}$ & -- & $---\frac{-}{0}$ \\
\hline
\end{tabular}


TABLE $7.10-1$ COST BASELNE - BY YEAR
TOTAL PROGRAM ELEMENT

[ R1]

\begin{tabular}{|c|c|c|c|c|c|c|c|c|c|}
\hline WBS / LEVEL & ACTIVITY TITLE / ADS & $\begin{array}{l}\text { FUND } \\
\text { TYPE }\end{array}$ & $\begin{array}{l}\text { TOTAL } \\
\text { FY94 }\end{array}$ & FY95 & FY96 & FY97 & FY98 & FY99 & FY00 \\
\hline $\begin{array}{l}1.1 .1 .3 .03 .02 .07 \\
\text { Level VI }\end{array}$ & $\begin{array}{l}\text { Plant / Process Engineering } \\
\text { Design Support } \\
\text { Total }\end{array}$ & $\begin{array}{l}\text { OE } \\
\text { C/E }\end{array}$ & 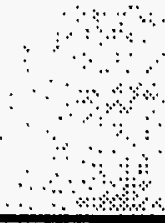 & $\begin{array}{r}0 \\
0 \\
--- \\
0\end{array}$ & $\begin{array}{r}0 \\
0 \\
--- \\
0\end{array}$ & $\begin{array}{r}445 \\
0 \\
--- \\
445\end{array}$ & 0 & --- & $\begin{array}{r}---- \\
0\end{array}$ \\
\hline $\begin{array}{l}1.1 .1 .3 .03 .03 \\
\text { Level } v\end{array}$ & $\begin{array}{l}\text { Facility Operations } \\
\text { ADS 12.40-0-nC } \\
\text { Total }\end{array}$ & $\begin{array}{l}\text { OE } \\
\text { C/E }\end{array}$ & NA & $\begin{array}{r}\mathbf{0} \\
\mathbf{0} \\
--- \\
0\end{array}$ & $\begin{array}{r}0 \\
0 \\
--- \\
0\end{array}$ & $\begin{array}{r}419 \\
---- \\
419\end{array}$ & $\begin{array}{r}0 \\
0 \\
--\frac{0}{0}\end{array}$ & $\begin{array}{r}0 \\
0 \\
--- \\
0\end{array}$ & $\begin{array}{r}0 \\
0 \\
--\frac{0}{0}\end{array}$ \\
\hline $\begin{array}{l}\text { 1.1.1.3.03.03.01 } \\
\text { Level VI }\end{array}$ & $\begin{array}{c}\text { Operations Hi-Low Waste } \\
\text { Proj. Definition Support } \\
\text { Total }\end{array}$ & $\begin{array}{l}\text { OE } \\
C / E\end{array}$ & 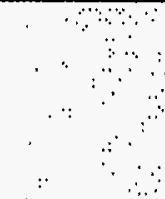 & $\begin{array}{r}0 \\
0 \\
--\frac{-}{0}\end{array}$ & $\begin{array}{r}0 \\
0 \\
---- \\
0\end{array}$ & $\begin{array}{r}419 \\
0 \\
--- \\
419\end{array}$ & $---\overline{0}$ & $---\overline{0}$ & --- \\
\hline $\begin{array}{l}\text { 1.1.1.3.03.03.02 } \\
\text { Level VI }\end{array}$ & $\begin{array}{l}\text { High-Level Waste Ops. } \\
\text { Preparation } \\
\qquad \text { Total }\end{array}$ & $\begin{array}{l}\text { OE } \\
C / E\end{array}$ & & $\begin{array}{r}0 \\
0 \\
--- \\
0\end{array}$ & $\begin{array}{r}0 \\
0 \\
--- \\
0\end{array}$ & $\begin{array}{r}0 \\
0 \\
--- \\
0\end{array}$ & $\begin{array}{r}--- \\
0\end{array}$ & --- & $\begin{array}{r}--- \\
0\end{array}$ \\
\hline $\begin{array}{l}\text { 1.1.1.3.03.03.03 } \\
\text { Level VI }\end{array}$ & $\begin{array}{l}\text { High-Level Waste Rad. } \\
\text { Operations } \\
\qquad \text { Total }\end{array}$ & $\begin{array}{l}O E \\
C / E\end{array}$ & & $\begin{array}{r}0 \\
0 \\
--- \\
0\end{array}$ & $\begin{array}{r}0 \\
0 \\
---- \\
0\end{array}$ & $\begin{array}{r}0 \\
0 \\
--- \\
0\end{array}$ & $\begin{array}{r}--- \\
0\end{array}$ & -- & --- \\
\hline $\begin{array}{l}\text { 1.1.1.3.03.03.04 } \\
\text { Level VI }\end{array}$ & $\begin{array}{l}\text { Prep. Hi-Low Waste Fclty. } \\
\text { for Decon. \& Decommis. } \\
\text { Total }\end{array}$ & $\begin{array}{l}\text { OE } \\
\text { C/E }\end{array}$ & & $\begin{array}{r}0 \\
0 \\
--- \\
0\end{array}$ & $\begin{array}{r}0 \\
0 \\
--- \\
0\end{array}$ & $\begin{array}{r}0 \\
0 \\
-- \\
0\end{array}$ & $\begin{array}{r}--- \\
0\end{array}$ & $---\overline{0}$ & $\frac{---}{0}$ \\
\hline
\end{tabular}


TABLE $7.10-1$

TOTAL PROGRAM ELEMENT

COST BASELINE - BY YEAR

[ R1 ]

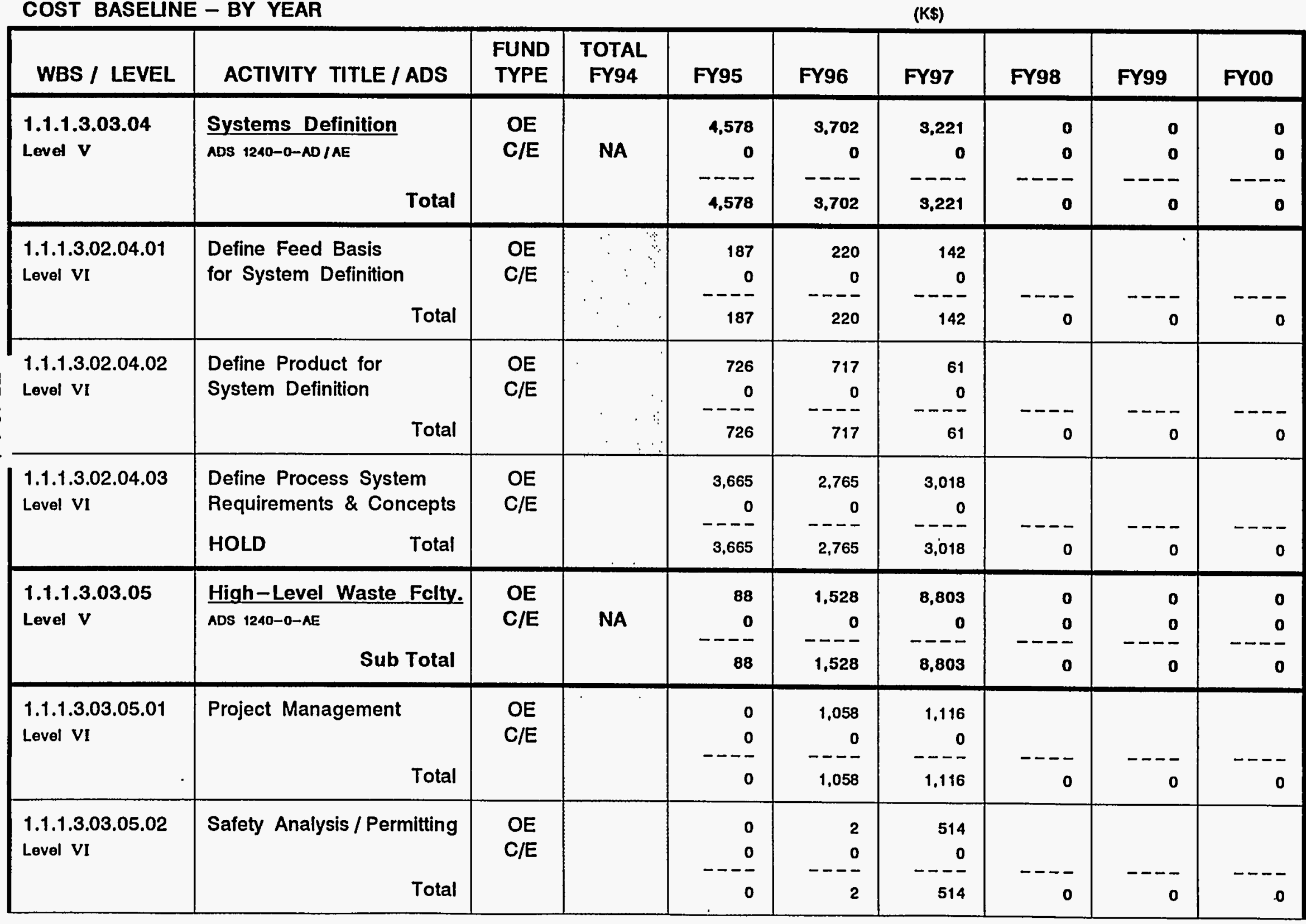


TABLE $7.10-1$

TOTAL PROGRAM ELEMENT

COST BASEUNE - BY YEAR

\begin{tabular}{|c|c|c|c|c|c|c|c|c|c|}
\hline WBS / LEVEL & ACTIVITY TITLE / ADS & $\begin{array}{l}\text { FUND } \\
\text { TYPE }\end{array}$ & $\begin{array}{l}\text { TOTAL } \\
\text { FY94 }\end{array}$ & FY95 & FY96 & FY97 & FY98 & FY99 & FYOO \\
\hline $\begin{array}{l}1.1 .1 .3 .03 .05 .03 \\
\text { Level VI }\end{array}$ & $\begin{array}{l}\text { Waste Form Qualification } \\
\qquad \text { Total }\end{array}$ & $\begin{array}{l}\text { OE } \\
C / E\end{array}$ & & $\begin{array}{r}88 \\
0 \\
--98\end{array}$ & $\begin{array}{r}468 \\
0 \\
--- \\
468\end{array}$ & $\begin{array}{r}791 \\
0 \\
--- \\
791\end{array}$ & $---\frac{-}{0}$ & $---\overline{0}$ & $---\frac{-}{0}$ \\
\hline $\begin{array}{l}\text { 1.1.1.3.03.05.04 } \\
\text { Level VI }\end{array}$ & $\begin{array}{l}\text { Conceptual Design / } \\
\text { Adv. Conceptual Design } \\
\qquad \text { Total }\end{array}$ & $\begin{array}{l}O E \\
C / E\end{array}$ & & $\begin{array}{r}0 \\
0 \\
-- \\
-0\end{array}$ & $\begin{array}{r}0 \\
0 \\
- \\
-0\end{array}$ & $\begin{array}{r}6,382 \\
0 \\
--- \\
6,382\end{array}$ & --- & --- & 0 \\
\hline $\begin{array}{l}\text { 1.1.1.3.03.05.05 } \\
\text { Level VI }\end{array}$ & 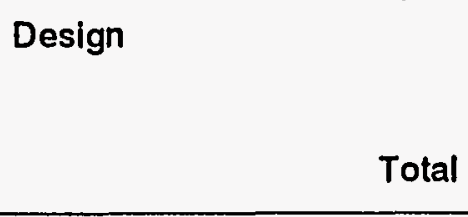 & $\begin{array}{l}O E \\
C / E\end{array}$ & & $\begin{array}{r}0 \\
0 \\
-- \\
-0\end{array}$ & $\begin{array}{r}0 \\
0 \\
-- \\
0\end{array}$ & $\begin{array}{r}0 \\
0 \\
-- \\
-0\end{array}$ & $-\cdots$ & --- & --- \\
\hline $\begin{array}{l}1.1 .1 .3 .03 .05 .06 \\
\text { Level VI }\end{array}$ & $\begin{array}{r}\text { Construction / Procurement } \\
\text { Total }\end{array}$ & $\begin{array}{l}O E \\
C / E\end{array}$ & & $\begin{array}{r}0 \\
0 \\
--- \\
0\end{array}$ & $\begin{array}{r}0 \\
0 \\
-- \\
0\end{array}$ & $\begin{array}{r}0 \\
0 \\
-- \\
0\end{array}$ & $---\overline{0}$ & $\begin{array}{r}---\overline{0} \\
0\end{array}$ & $---\frac{-}{0}$ \\
\hline $\begin{array}{l}\text { 1.1.1.3.03.05.07 } \\
\text { Level VI }\end{array}$ & Startup & $\begin{array}{l}O E \\
C / E\end{array}$ & & $\begin{array}{r}0 \\
0 \\
-- \\
0\end{array}$ & $\begin{array}{r}0 \\
0 \\
-- \\
0\end{array}$ & $\begin{array}{r}0 \\
0 \\
-- \\
0\end{array}$ & --- & $\begin{array}{r}--- \\
0\end{array}$ & $\begin{array}{r}--- \\
0\end{array}$ \\
\hline $\begin{array}{l}\text { 1.1.1.3.03.06 } \\
\text { Level } v\end{array}$ & $\begin{array}{l}\frac{\text { Hanford Waste Vitrif. }}{\text { Plant Proj. Closeout }} \\
\text { ADs 1240-1-xx Sub Total }\end{array}$ & $\begin{array}{l}\text { OE } \\
\text { LI }\end{array}$ & NA & $\begin{array}{r}0 \\
6,201 \\
---- \\
6,201\end{array}$ & $\begin{array}{r}0 \\
0 \\
--- \\
0\end{array}$ & $\begin{array}{r}0 \\
0 \\
-- \\
0\end{array}$ & $---\overline{0}$ & $---\frac{-}{0}$ & --- \\
\hline
\end{tabular}

\section{[ R1 ]}

TABLE 7.10-1 
TABLE $7.10^{\circ}-1$

TOTAL PROGRAM ELEMENT COST BASELINE - BY YEAR

\begin{tabular}{|c|c|c|c|c|c|c|c|c|c|c|}
\hline WBS / LEVEL & ACTIVITY TITLE / ADS & $\begin{array}{l}\text { FUND } \\
\text { TYPE }\end{array}$ & $\begin{array}{l}\text { FY01 I } \\
\text { FY05 }\end{array}$ & $\begin{array}{l}\text { FY06 I } \\
\text { FY10 }\end{array}$ & $\begin{array}{l}\text { FY11 / } \\
\text { FY15 }\end{array}$ & $\begin{array}{l}\text { FY16 I } \\
\text { FY20 }\end{array}$ & $\begin{array}{l}\text { FY21 / } \\
\text { FY25 }\end{array}$ & $\begin{array}{l}\text { FY26 / } \\
\text { FY30 }\end{array}$ & $\begin{array}{l}\text { FY31 / } \\
\text { FY35 }\end{array}$ & $\begin{array}{l}\text { FY36 I } \\
\text { FY40 }\end{array}$ \\
\hline $\begin{array}{l}1.1 .1 .03 \\
\text { Level IV } \\
\vdots \\
\vdots\end{array}$ & $\frac{\text { High Level Waste }}{\text { ás } 1230-0}$ & $\begin{array}{l}\text { OEE } \\
\text { CiE } \\
\text { LE } \\
\vdots\end{array}$ & $\begin{array}{rr}0 \\
\ddots & 0 \\
1 & 069095\end{array}$ & $\begin{array}{rr} & 0 \\
& 0 \\
1,383,436 & 0 \\
0 & 0\end{array}$ & $\begin{array}{r}0 \\
0 \\
0 \\
0 \\
0\end{array}$ & $\begin{array}{r}0 \\
1,488,878 \\
0\end{array}$ & 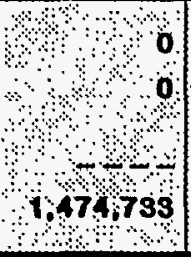 & $\begin{array}{r}0 \\
0 \\
0 \\
1429286\end{array}$ & 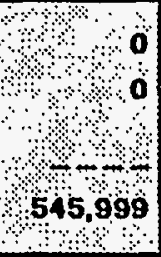 & $\begin{array}{r}0 \\
0 \\
811,794 \\
20\end{array}$ \\
\hline & $\begin{array}{l}\text { Delta Required to } \\
\text { Balance to TARGET } \\
\text { Budget. } \\
\\
\text { Total }\end{array}$ & $\begin{array}{l}O E \\
C / E \\
L I\end{array}$ & & & & & & & & \\
\hline $\begin{array}{l}\text { 1.1.1.3.03.01 } \\
\text { Level } \mathrm{v}\end{array}$ & $\begin{array}{l}\text { Technical Integration } \\
\frac{\text { and Planning }}{\text { ADS } 1240-0-M} \text { Sub Total }\end{array}$ & $\begin{array}{l}O E \\
C / E\end{array}$ & $\begin{array}{r}\mathbf{0} \\
\mathbf{0} \\
-0 \\
\mathbf{0}\end{array}$ & $--\frac{0}{0}$ & $\begin{array}{r}\mathbf{0} \\
\mathbf{0} \\
-0 \\
0\end{array}$ & $\begin{array}{r}\mathbf{0} \\
-- \\
-0 \\
0\end{array}$ & $\begin{array}{r}\mathbf{0} \\
\mathbf{0} \\
-- \\
\mathbf{0}\end{array}$ & $\begin{array}{r}\mathbf{0} \\
\mathbf{0} \\
-\mathbf{0}\end{array}$ & $--\begin{array}{r}0 \\
0 \\
-0\end{array}$ & $\begin{array}{r}0 \\
0 \\
-0\end{array}$ \\
\hline $\begin{array}{l}\text { 1.1.1.3.03.01.01 } \\
\text { Level VI }\end{array}$ & $\begin{array}{l}\text { Program Planning, } \\
\text { Integration \& Management } \\
\text { Total }\end{array}$ & $\begin{array}{l}O E \\
C / E\end{array}$ & 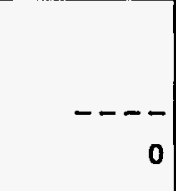 & & & $\overline{0}$ & - & - & - & -- \\
\hline $\begin{array}{l}1.1 .1 .3 .03 .01 .02 \\
\text { Level VI }\end{array}$ & $\begin{array}{l}\text { Program Business } \\
\text { Management } \\
\\
\text { Total }\end{array}$ & $\begin{array}{l}O E \\
C / E\end{array}$ & $-\cdots$ & & ---- & - & $\overline{0}$ & $\overline{0}$ & -- & - \\
\hline $\begin{array}{l}\text { 1.1.1.3.03.01.03 } \\
\text { Level VI }\end{array}$ & $\begin{array}{l}\text { Pre-Project Documentation } \\
\text { and Support } \\
\qquad \text { Total }\end{array}$ & $\begin{array}{l}O E \\
C / E\end{array}$ & -- & & & $\overline{0}$ & - & - & - & - \\
\hline
\end{tabular}

[ R1 ] 
TABLE $7.10-1$

TOTAL PROGRAM ELEMENT COST BASELINE - BY YEAR

\begin{tabular}{|c|c|c|c|c|c|c|c|c|c|c|}
\hline WBS / LEVEL & ACTIVITY TITLE / ADS & $\begin{array}{l}\text { FUND } \\
\text { TYPE }\end{array}$ & $\begin{array}{l}\text { FY01 / } \\
\text { FY05 }\end{array}$ & $\begin{array}{l}\text { FY06 / } \\
\text { FY10 }\end{array}$ & $\begin{array}{l}\text { FY11 / } \\
\text { FY15 }\end{array}$ & $\begin{array}{l}\text { FY16 / } \\
\text { FY20 }\end{array}$ & $\begin{array}{l}\text { FY21 / } \\
\text { FY25 }\end{array}$ & $\begin{array}{l}\text { FY26 / } \\
\text { FY30 }\end{array}$ & $\begin{array}{l}\text { FY31 / } \\
\text { FY35 }\end{array}$ & $\begin{array}{l}\text { FY36 I } \\
\text { FY40 }\end{array}$ \\
\hline $\begin{array}{l}\text { 1.1.1.3.03.02 } \\
\text { Level } \mathrm{v}\end{array}$ & $\begin{array}{l}\text { Technical .Dvlpmt. \& } \\
\text { Applied Engineering } \\
\text { ADS 1240-0-AB } \\
\\
\text { Total }\end{array}$ & $\begin{array}{l}\text { OE } \\
C / E\end{array}$ & $\begin{array}{r}0 \\
0 \\
-0 \\
0\end{array}$ & $--\frac{0}{0}$ & $\begin{array}{r}0 \\
0 \\
-0 \\
0\end{array}$ & $--\frac{0}{0}$ & $--\frac{0}{0}$ & $\begin{array}{r}0 \\
0 \\
-- \\
0\end{array}$ & $\begin{array}{r}0 \\
0 \\
-0 \\
0\end{array}$ & $\begin{array}{r}\mathbf{0} \\
-0 \\
-0\end{array}$ \\
\hline $\begin{array}{l}\text { 1.1.1.3.03.02.01 } \\
\text { Level VI }\end{array}$ & $\begin{array}{l}\text { Vitrification Tech. Dvlpmt. } \\
\text { Management } \\
\qquad \text { Total }\end{array}$ & $\begin{array}{l}O E \\
C / E\end{array}$ & $\begin{array}{r}--- \\
0\end{array}$ & 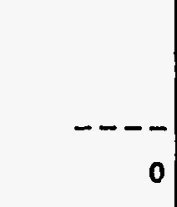 & --- & 0 & 0 & $\begin{array}{r}\cdot \\
--- \\
0\end{array}$ & --- & $---\overline{0}$ \\
\hline $\begin{array}{l}\text { 1.1.1.3.03.02.02 } \\
\text { Level vi }\end{array}$ & $\begin{array}{l}\text { Hi-Level Waste Process / } \\
\text { Product Development } \\
\qquad \text { Total }\end{array}$ & $\begin{array}{l}O E \\
C / E\end{array}$ & --- & $\begin{array}{r}---- \\
0\end{array}$ & $\begin{array}{r}---- \\
. \quad 0\end{array}$ & ---- & --- & - & $---\frac{-}{0}$ & --- \\
\hline $\begin{array}{l}1.1 .1 .3 .03 .02 .03 \\
\text { Level VI }\end{array}$ & $\begin{array}{c}\text { Hi-Level Waste Process / } \\
\text { Equip. Dvipmt. \& Testing } \\
\text { Total }\end{array}$ & $\begin{array}{l}O E \\
C / E\end{array}$ & & -- & $--\cdot$ & 0 & - & -- & -- & --- \\
\hline $\begin{array}{l}\text { 1.1.1.3.03.02.04 } \\
\text { Level VI }\end{array}$ & $\begin{array}{l}\text { Tech. Dvlpmt. Requirements } \\
\text { and Evaluation } \\
\qquad \text { Total }\end{array}$ & $\begin{array}{l}O E \\
C / E\end{array}$ & -- & $\begin{array}{r}--- \\
0\end{array}$ & $\begin{array}{r}---- \\
0\end{array}$ & ---- & -- & $-\cdots-$ & ---- & -- \\
\hline $\begin{array}{l}\text { 1.1.1.3.03.02.05 } \\
\text { Levef VI }\end{array}$ & $\begin{array}{l}\text { Process Equipment } \\
\text { Evaluation HOLD } \\
\\
\\
\text { Total }\end{array}$ & $\begin{array}{l}O E \\
C / E\end{array}$ & -- & - & - & - & $---\overline{0}$ & $\begin{array}{r}---\overline{0} \\
0\end{array}$ & $-\frac{-}{0}$ & -- \\
\hline $\begin{array}{l}\text { 1.1.1.3.03.02.06 } \\
\text { Level VI }\end{array}$ & $\begin{array}{l}\text { Feed / Process } \\
\text { Evaluation }\end{array}$ & $\begin{array}{l}O E \\
C / E\end{array}$ & -- & --- & --- & ---- & ---- & --- & $--\frac{-}{0}$ & ---- \\
\hline
\end{tabular}


TABLE $7.10-1$

TOTAL PROGRAM ELEMENT COST BASEUNE - BY YEAR

\begin{tabular}{|c|c|c|c|c|c|c|c|c|c|c|}
\hline WBS / LEVEL & ACTIVITY TITLE / ADS & $\begin{array}{l}\text { FUND } \\
\text { TYPE }\end{array}$ & $\begin{array}{l}\text { FY01 / } \\
\text { FY05 }\end{array}$ & $\begin{array}{l}\text { FY06 / } \\
\text { FY10 }\end{array}$ & $\begin{array}{l}\text { FY11 I } \\
\text { FY15 }\end{array}$ & $\begin{array}{l}\text { FY16 / } \\
\text { FY20 }\end{array}$ & $\begin{array}{l}\text { FY21 / } \\
\text { FY25 }\end{array}$ & $\begin{array}{l}\text { FY26 / } \\
\text { FY30 }\end{array}$ & $\begin{array}{l}\text { FY31 I } \\
\text { FY35 }\end{array}$ & $\begin{array}{l}\text { FY36 I } \\
\text { FY40 }\end{array}$ \\
\hline $\begin{array}{l}\text { 1.1.1.3.03.02.07 } \\
\text { Level VI }\end{array}$ & $\begin{array}{l}\text { Plant / Process Engineering } \\
\text { Design Support } \\
\qquad \text { Total }\end{array}$ & $\begin{array}{l}\mathrm{OE} \\
\mathrm{C} / \mathrm{E}\end{array}$ & $\begin{array}{r}--- \\
0\end{array}$ & $\begin{array}{r}---- \\
0\end{array}$ & $\begin{array}{r}--- \\
0\end{array}$ & ---- & $--\frac{-}{0}$ & $\begin{array}{r}---- \\
0\end{array}$ & $\begin{array}{r}-\cdots- \\
0\end{array}$ & --- \\
\hline $\begin{array}{l}\text { 1.1.1.3.03.03 } \\
\text { Level } \mathrm{v}\end{array}$ & $\begin{array}{l}\text { Facility Operations } \\
\begin{array}{l}A D S 1240-0-A C \\
\text { Total }\end{array}\end{array}$ & $\begin{array}{l}\text { OE } \\
\text { C/E }\end{array}$ & $-\begin{array}{r}0 \\
0 \\
0\end{array}$ & $\begin{array}{r}\mathbf{0} \\
\mathbf{0} \\
-0 \\
\mathbf{0}\end{array}$ & $\begin{array}{r}0 \\
0 \\
-0\end{array}$ & $-\begin{array}{r}0 \\
0 \\
-0\end{array}$ & $\begin{array}{r}0 \\
0 \\
-0\end{array}$ & $\begin{array}{r}0 \\
0 \\
-0\end{array}$ & $\begin{array}{r}0 \\
0 \\
-0\end{array}$ & $\begin{array}{r}0 \\
-0 \\
-0\end{array}$ \\
\hline $\begin{array}{l}\text { 1.1.1.3.03.03.01 } \\
\text { Level VI }\end{array}$ & $\begin{array}{l}\text { Operations Hi-Low Waste } \\
\text { Proj. Definition Support } \\
\text { Total }\end{array}$ & $\begin{array}{l}\text { OE } \\
C / E\end{array}$ & --- & ---- & ---- & $---\frac{-}{0}$ & $---\frac{-}{0}$ & --- & ---- & $--\frac{-}{0}$ \\
\hline $\begin{array}{l}1.1 .1 .3 .03 .03 .02 \\
\text { Level VI }\end{array}$ & $\begin{array}{l}\text { High-Level Waste Ops. } \\
\text { Preparation } \\
\qquad \text { Total }\end{array}$ & $\begin{array}{l}O E \\
C / E\end{array}$ & -- & 0 & - & 0 & 0 & 0 & $\begin{array}{r}---- \\
0\end{array}$ & --- \\
\hline $\begin{array}{l}\text { 1.1.1.3.03.03.03 } \\
\text { Level VI }\end{array}$ & $\begin{array}{l}\text { High-Level Waste Rad. } \\
\text { Operations } \\
\qquad \text { Total }\end{array}$ & $\begin{array}{l}O E \\
C / E\end{array}$ & $--\cdot$ & 0 & ---- & - & 0 & 0 & $\begin{array}{r}--- \\
0\end{array}$ & ---- \\
\hline $\begin{array}{l}1.1 .1 .3 .03 .03 .04 \\
\text { Level VI }\end{array}$ & $\begin{array}{r}\text { Prep. Hi-Low Waste Fclty. } \\
\text { for Decon. \& Decommis. } \\
\text { Total }\end{array}$ & $\begin{array}{l}O E \\
C / E\end{array}$ & $=-$ & o & & & - & - & ---- & 0 \\
\hline
\end{tabular}

[ R1 ] 
TABLE 7.10-1

TOTAL PROGRAM ELEMENT COST BASELINE - BY YEAR

\begin{tabular}{|c|c|c|c|c|c|c|c|c|c|c|}
\hline WBS / LEVEL & ACTIVITY TITLE / ADS & $\begin{array}{l}\text { FUND } \\
\text { TYPE }\end{array}$ & $\begin{array}{l}\text { FY01 / } \\
\text { FY05 }\end{array}$ & $\begin{array}{l}\text { FY06 I } \\
\text { FY10 }\end{array}$ & $\begin{array}{l}\text { FY11 I } \\
\text { FY15 }\end{array}$ & $\begin{array}{l}\text { FY16 / } \\
\text { FY20 }\end{array}$ & $\begin{array}{l}\text { FY21 / } \\
\text { FY25 }\end{array}$ & $\begin{array}{l}\text { FY26 I } \\
\text { FY30 }\end{array}$ & $\begin{array}{l}\text { FY31 / } \\
\text { FY35 }\end{array}$ & $\begin{array}{l}\text { FY36 / } \\
\text { FY40 }\end{array}$ \\
\hline $\begin{array}{l}\text { 1.1.1.3.03.04 } \\
\text { Level } v\end{array}$ & 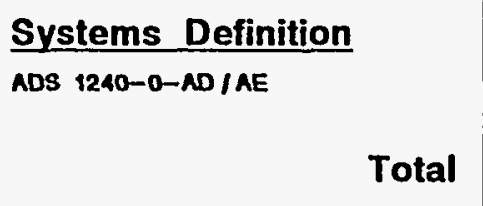 & $\begin{array}{l}O E \\
C / E\end{array}$ & $\begin{array}{r}0 \\
0 \\
-- \\
0\end{array}$ & $\begin{array}{r}0 \\
0 \\
-- \\
0\end{array}$ & $\begin{array}{r}\mathbf{0} \\
\mathbf{0} \\
-- \\
\mathbf{0}\end{array}$ & $\begin{array}{r}0 \\
0 \\
-- \\
0\end{array}$ & $\begin{array}{r}0 \\
0 \\
-- \\
0\end{array}$ & $\begin{array}{r}0 \\
0 \\
---\end{array}$ & $\begin{array}{r}0 \\
0 \\
--- \\
0\end{array}$ & $\begin{array}{r}0 \\
0 \\
-0 \\
0\end{array}$ \\
\hline $\begin{array}{l}\text { 1.1.1.3.02.04.01 } \\
\text { Level VI }\end{array}$ & $\begin{array}{l}\text { Define Feed Basis } \\
\text { for System Definition } \\
\qquad \text { Total }\end{array}$ & $\begin{array}{l}O E \\
C / E\end{array}$ & ---- & $---\frac{-}{0}$ & $-\frac{-}{0}$ & -- & 0 & $--\frac{-}{0}$ & ---- & --- \\
\hline $\begin{array}{l}\text { 1.1.1.3.02.04.02 } \\
\text { Level VI }\end{array}$ & $\begin{array}{l}\text { Define Product for } \\
\text { System Definition } \\
\qquad \text { Total }\end{array}$ & $\begin{array}{l}O E \\
C / E\end{array}$ & $-\cdots$ & -- & $---\frac{-}{0}$ & $\overline{0}$ & ---- & --- & --- & --- \\
\hline $\begin{array}{l}\text { 1.1.1.3.02.04.03 } \\
\text { Level VI }\end{array}$ & $\begin{array}{l}\text { Define Process System } \\
\text { Requirements \& Concepts } \\
\text { HOLD } \quad \text { Total }\end{array}$ & $\begin{array}{l}O E \\
C / E\end{array}$ & - & o & - & $\overline{0}$ & $\overline{0}$ & - & -- & -- \\
\hline $\begin{array}{l}\text { 1.1.1.3.03.05 } \\
\text { Level } v\end{array}$ & $\begin{array}{l}\text { High-Level Waste Fclty. } \\
\text { ADS } 1240-0-A E \\
\text { Sub Total }\end{array}$ & $\begin{array}{l}O E \\
C / E\end{array}$ & $\begin{array}{r}0 \\
0 \\
-- \\
0\end{array}$ & $\begin{array}{r}0 \\
0 \\
-- \\
0\end{array}$ & $\begin{array}{r}0 \\
0 \\
--- \\
0\end{array}$ & $\begin{array}{r}0 \\
0 \\
-- \\
0\end{array}$ & $\begin{array}{r}0 \\
0 \\
--- \\
0\end{array}$ & $\begin{array}{r}0 \\
0 \\
-- \\
0\end{array}$ & $\begin{array}{r}0 \\
0 \\
-- \\
0\end{array}$ & $\begin{array}{r}0 \\
0 \\
-0\end{array}$ \\
\hline $\begin{array}{l}\text { 1.1.1.3.03.05.01 } \\
\text { Level VI }\end{array}$ & $\begin{array}{l}\text { Project Management } \\
\qquad \text { Total }\end{array}$ & $\begin{array}{l}O E \\
C / E\end{array}$ & -- & - & -- & -- & -- & - & $\begin{array}{r}---- \\
0\end{array}$ & --- \\
\hline $\begin{array}{l}1.1 .1 .3 .03 .05 .02 \\
\text { Level VI }\end{array}$ & $\begin{array}{r}\text { Safety Analysis / Permitting } \\
\text { Total }\end{array}$ & $\begin{array}{l}O E \\
C / E\end{array}$ & - & - & - & - & $\overline{0}$ & $\overline{0}$ & - & --- \\
\hline
\end{tabular}

[ R1 ] 
TABLE $7.10-1$

TOTAL PROGRAM ELEMENT COST BASELNE - BY YEAR

\begin{tabular}{|c|c|c|c|c|c|c|c|c|c|c|}
\hline WBS / LEVEL & ACTIVITY TITLE / ADS & $\begin{array}{l}\text { FUND } \\
\text { TYPE }\end{array}$ & $\begin{array}{l}\text { FY01 I } \\
\text { FY05 }\end{array}$ & $\begin{array}{l}\text { FY06 / } \\
\text { FY10 }\end{array}$ & $\begin{array}{l}\text { FY11 / } \\
\text { FY15 }\end{array}$ & $\begin{array}{l}\text { FY16 / } \\
\text { FY20 }\end{array}$ & $\begin{array}{l}\text { FY21 / } \\
\text { FY25 }\end{array}$ & $\begin{array}{l}\text { FY26 / } \\
\text { FY30 }\end{array}$ & $\begin{array}{l}\text { FY31 I } \\
\text { FY35 }\end{array}$ & $\begin{array}{l}\text { FY36 I } \\
\text { FY40 }\end{array}$ \\
\hline $\begin{array}{l}\text { 1.1.1.3.03.05.03 } \\
\text { Level VI }\end{array}$ & $\begin{array}{l}\text { Waste Form Qualification } \\
\qquad \text { Total }\end{array}$ & $\begin{array}{l}O E \\
C / E\end{array}$ & $---\frac{1}{0}$ & $\begin{array}{r}--- \\
0\end{array}$ & $\begin{array}{r}--- \\
0\end{array}$ & $\begin{array}{r}---- \\
0\end{array}$ & $---\overline{0}$ & $---\frac{}{0}$ & ---- & --- \\
\hline $\begin{array}{l}\text { 1.1.1.3.03.05.04 } \\
\text { Level VI }\end{array}$ & $\begin{array}{l}\text { Conceptual Design / } \\
\text { Adv. Conceptual Design } \\
\text { Total }\end{array}$ & $\begin{array}{l}O E \\
C / E\end{array}$ & $-\cdots$ & 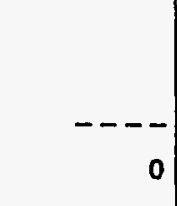 & ---- & $\begin{array}{r}-\cdots \\
0\end{array}$ & $\begin{array}{r}---- \\
0\end{array}$ & $\begin{array}{r}: \\
--- \\
0\end{array}$ & $\begin{array}{r}---- \\
0\end{array}$ & $-\cdots$ \\
\hline $\begin{array}{l}\text { 1.1.1.3.03.05.05 } \\
\text { Lovel VI }\end{array}$ & $\begin{array}{l}\text { Design } \\
\qquad \text { Total }\end{array}$ & $\begin{array}{l}O E \\
C / E\end{array}$ & $\begin{array}{r}--- \\
0\end{array}$ & $\begin{array}{r}---- \\
0\end{array}$ & $\begin{array}{r}---- \\
0\end{array}$ & --- & --- & ---- & $\begin{array}{r}---- \\
0\end{array}$ & 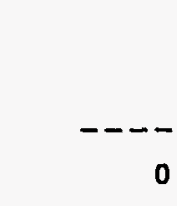 \\
\hline $\begin{array}{l}\text { 1.1.1.3.03.05.06 } \\
\text { Level VI }\end{array}$ & $\begin{array}{l}\text { Construction / Procurement } \\
\qquad \text { Total }\end{array}$ & $\begin{array}{l}O E \\
C / E\end{array}$ & $\cdots$ & ---- & ---- & -- & $-\cdots$ & ---- & $\begin{array}{r}--- \\
0\end{array}$ & 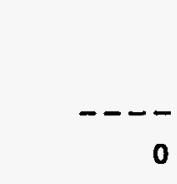 \\
\hline $\begin{array}{l}\text { 1.1.1.3.03.05.07 } \\
\text { Level VI }\end{array}$ & Startup & $\begin{array}{l}O E \\
C / E\end{array}$ & $-\cdots$ & $\begin{array}{r}--- \\
0\end{array}$ & $\begin{array}{r}---- \\
0\end{array}$ & $\begin{array}{r}---- \\
0\end{array}$ & 0 & o & --- & $\begin{array}{r}---- \\
0\end{array}$ \\
\hline $\begin{array}{l}\text { 1.1.1.3.03.06 } \\
\text { Levet } v\end{array}$ & $\begin{array}{l}\frac{\text { Hanford Waste Vitrif. }}{\text { Plant Proj. Closeout }} \\
\text { ADs 1210-1-xx Sub Total }\end{array}$ & $\begin{array}{l}\text { OE } \\
\text { LI }\end{array}$ & -- & --- & $\begin{array}{r}---- \\
0\end{array}$ & $---\frac{-}{0}$ & --- & ---- & ---- & ---- \\
\hline
\end{tabular}

\section{[ R1]}




\section{NOTES \\ TOTAL PROGRAM ELEMENT COST BASELINE - BY YEAR}

The cost exhibit tables in each of the Program Element Baselines (Sections 7.2 through 7.10) identify the program element target funding by year by fund type. The tables also provide details of the baseline allocation of funding down to activity levels (level V) and cost account levels (level VI) of the WBS for Fiscal Year 1995 through 1997. The details exhibited on these tables are taken from the baseline WBS Dictionary Sheets. Deltas exist between the details and the annual target totals. For Fiscal Years 1996 and 1997 the deltas will be addressed in the future, following the completion of the resolution of expected differences between current Fiscal Year 1995 baseline funding and expected actual Fiscal Year 1995 funding. Deltas for Fiscal Year 1995 are noted below by program element:

\section{PROGRAM MANAGEMENT AND ADMINISTRATION:}

No deltas.

\section{TANK FARM OPERATIONS AND MAINTENANCE:}

No overall delta. This baseline requires revised fund type mix.

\section{WASTE TANK SAFETY:}

Delta is due to need to revise an overhead pricing rate in the P3 system.

\section{TANK FARM UPGRADES:}

Delta is due to differences between Budget Authority (BA) in the target and Budget Obligation (B0) in the P3 system.

\section{CHARACTERIZATION:}

No significant deltas.

\section{WASTE RETRIEVAL:}

Delta is primarily due to need to resolve issues associated with expense funding support to capital projects and differences between Budget Authority (BA) in the target and Budget Obligation (B0) in the P3 system.

\section{WASTE PRETREATMENT:}

Delta is due to the sum of the detail cost estimates being lower than the target total as a result of reestimation of resource requirements to complete baseline scope.

\section{LOW LEVEL WASTE:}

Delta is primarily due to carryover expense funded workscope on melter testing addressed in June 9, 1994 RL letter.

$$
T 7.10-1.11
$$


WHC-SP-1101

\section{HIGH LEVEL WASTE:}

Delta is due to carryover of capital funding, PNL expense funding carryover, and ADS corrections submitted but not reflected in target totals. 
WHC-SP-1101

Table 7.10-2. FY 1995 Cost by Month by Fund Type by Activity and Cost Account.

Future MYWP updates will include Table 7.10-2, which establishes the month-by-month cost baseline for FY 1995 for this program element. The FY 1995 cost baseline is exhibited at the cost account level with fund type totals provided. Cost data are then rolled up to the activity and program element levels. Also exhibited is the total cost baseline for the program element for FY 1995. At present, the program element cost baseline for FY 1995 for this program element is the total provided on this table less a productivity challenge for FY 1995. 
Table 7-10-3. Total Full-Time Equivalents by Year by Activity by Major Participant (Direct and Indirect) (Including all Subcontractors).

Future MYWP updates will include Table 7.10-3, which establishes total staffing projections for this program element. Included in the table are FY 1994 staffing data as well as projections by year for FY 1995 through FY 2000. The projections in this table include all direct and indirect Full-Time Equivalents (FTEs), including all subcontractor personnel. Where data are available, projections are exhibited at the activity level with major program participant totals provided. 
Table 7.10-4. FY 1995 through FY 1997 Direct Westinghouse Hanford Company Full-Time Equivalents by 88 Labor Categories.

Future MYWP updates will include Table 7.10-4, which establishes direct WHC staffing projections for this program element. Projections do not include subcontractor support and are provided by 88 labor categories for FY 1995 through FY 1997. 


\subsection{REVISIONS}

Changes to the TWRS Program baseline will be summarized in this section when the MYWP is revised annually. The revisions summarized in this section will be a compilation of baseline changes that have occurred in each of the program elements. Changes in the individual program elements are covered in the program element baseline sections (refer to Section 7). These changes provide traceability to the actions taken in the Change Control process as well as directed changes from DOE or other regulatory agencies. 
WHC-SP-1101

This page intentionally left blank. 


\subsection{REFERENCES}

Tri-Party Agreement, Hanford Federal Facility Agreement and Consent, Fourth Amendment, Washington State Department of Ecology, U.S. Environmental Protection Agency, and U.S. Department of Energy, Olympia, Washington (January 1994).

\section{CODE OF FEDERAL REGULATIONS}

40 CFR 260, "Hazardous Waste Management System: General," Code of Federal Regulations, as amended.

40 CFR 261, "Identification and Listing of Hazardous Waste," Code of Federal Regulations, as amended.

40 CFR 262, Standards Applicable to Generators of Hazardous Waste," Code of Federal Regulations, as amended.

40 CFR 263, "Standards Applicable to Transporters of Hazardous Waste," Code of Federal Regulations, as amended.

40 CFR 264, "Standards for Owners and Operators of Hazardous Waste Treatment, Storage, and Disposal (TSD) Facilities," Code of Federal Regulations, as amended.

- $\quad$ "Miscellaneous Units, Environmental Performance Standards"

- Miscellaneous Units, Monitoring Analysis, Inspection, Response, Reporting, and Corrective Action"

- Miscellaneous Units, Post-Closure Care"

40 CFR 265, "Interim Status Standards for Owners and Operators of Hazardous Waste Treatment, Storage, and Disposal Facilities," Code of Federal Regulations, as amended.

- CFR 265.196, "Response to Leaks and Spills and Disposition of Leaking of Unfit-forUse Tank Systems."

40 CFR 266, "Standards for the Management of Specific Hazardous Waste and Specific Types of Hazardous Waste Management Facilities," Code of Federal Regulations, as amended.

40 CFR 268, "Land Disposal Restrictions (LDR)," Code of Federal Regulations, as amended.

40 CFR 270, EPA Administered Permit Programs: The Hazardous Waste Permit Program," Code of Federal Regulations, as amended.

\section{FEDERAL REGISTER}

52 FR 12449, "Final Environmental Impact Statement for the Disposal of Hanford Defense High-

Level, Transuranic and Tank Waste, Hanford Site, Richland, Washington; Record of Decision," Federal Register, Vol. 52, pp. 12449-12453. 
WHC-SP-1101

\section{PUBLIC LAWS}

Defense Authorization Act, Public Law 101-520, Section 3137, "Safety Measures for Waste Tanks at Hanford Nuclear Reservation."

National Environmental Policy Act of 1969, as amended, 42 USC 4321, et seq.

Resource Conservation and Recovery Act of 1976, as amended, 42 USC 6901 et seq.

\section{U.S. DEPARTMENT OF ENERGY DOCUMENTS}

DOE/EIS-0113, Final Environmental Impact Statement, Disposal of Hanford Defense High-Level, Transuranic and Tank Wastes, Hanford Site, Richland, Washington, 5 vols., U.S. Department of Energy, Washington, D.C. (1987).

DOE-STD-1073-93, Guide for Operational Configuration Management Program, U.S. Department of Energy, Washington, D.C. (1993).

DOE/RL-92-60, Draft, Tank Waste Remediation System Functions and Requirements, Rev. 1, U.S. Department of Energy, Richland Operations Office, Richland, Washington (March 28, 1994).

DOE/RL-93-102, Draft, Fiscal Year 1995 Hanford Mission Plan, Volume 1, Site Guidance, U.S. Department of Energy, Richland Operations Office, Richland, Washington.

DOE/RL-93-0106, Draft, Tank Waste Remediation System Program Management System Description, U.S. Department of Energy, Richland Operations Office, Richland, Washington.

DOE/RL 94-0001, Recommendations 93-5 Implementation Plan, U.S. Department of Energy, Richland Operations Office, Richland, Washington (1994).

\section{U.S. DEPARTMENT OF ENERGY ORDERS}

DOE Order 4700.1, Project Management System, U.S. Department of Energy, Washington, D.C.

DOE Order 5400.3, Hazardous and Radioactive Mixed Waste Program, U.S. Department of Energy, Washington, D.C.

DOE Order 5400.5, Radiation Protection of the Public and the Environment, U.S. Department of Energy, Washington, D.C.

DOE Order 5480.19, Conduct of Operations Requirements for DOE Facilities, U.S. Department of Energy, Washington, D.C.

DOE Order 5700.6C, Quality Assurance, U.S. Department of Energy, Washington, D.C. 
WHC-SP-1101

DOE Order 5820.2A, Radioactive Waste Management, U.S. Department of Energy, Washington D.C.

DOE Order 6430.1A, General Design Criteria, U.S. Department of Energy, Washington, D.C.

\section{U.S. DEPARTMENT OF ENERGY, RICHLAND OPERATIONS OFFICE ORDERS AND IMPLEMENTING PROCEDURES}

RLIP 4700.1A, Project Management System, U.S. Department of Energy, Richland Operations Office, Richland, Washington.

RLID 5000.12, Richland Field Office - Hanford Site Management System, U.S. Department of Energy, Richland Operations Office, Richland, Washington.

\section{WASHINGTON ADMINISTRATIVE CODE}

WAC 173-303, "Dangerous Waste Requirements," Washington Administrative Code, as amended. WAC 173-303-640, "Tank System"

WAC 173-400, "Washington Clean Air Act - General Regulations for Air Pollution Sources," Washington Administrative Code, as amended.

\section{WESTINGHOUSE HANFORD COMPANY DOCUMENTS}

Hanford Site Tank Waste Remediation System Technical Strategy, Westinghouse Hanford Company, Richland, Washington (1994).

WHC-CM-1-3, Management Requirements and Procedures, Westinghouse Hanford Company, Richland, Washington.

WHC-EP-0063-3, Hanford Site Solid Waste Acceptance Criteria, Westinghouse Hanford Company, Richland, Washington (1991).

WHC-EP-0182-XX, Tank Farm Surveillance and Waste. Status Summary Report for (Month) 1994, Westinghouse Hanford Company, Richland, Washington (1994).

WHC-EP-0616, Tank Waste Technical Options Report, Westinghouse Hanford Company, Richland, Washington (1993).

WHC-EP-0617, TWRS Decision Analysis Report, Westinghouse Hanford Company, Richland, Washington.

WHC-EP-0786, Draft, Tank Waste Remediation System Program Decisions and Risk Assessment, Westinghouse Hanford Company, Richland, Washington (1994). 
WHC-SP-1101

WHC-SD-WM-AP-017, Rev. 1, Double-Shell Tank Integrity Assessment Program Plan, Westinghouse Hanford Company, Richland, Washington.

WHC-SD-WM-CM-007, Rev. 0, Configuration Management Compliance Matrix for Waste Tank Farms and the 242-A Evaporator of TWRS, Westinghouse Hanford Company, Richland, Washington (1994).

WHC-SD-WM-CM-008, Rev. 0, Configuration Management Plan for Waste Tank Farms and the 242-A Evaporator of TWRS, Westinghouse Hanford Company, Richland, Washington (1994).

WHC-SD-WM-CM-009, Rev. 0, Design Reconstitution Program Plan for Waste Tank Farms and the 242-A Evaporator of Tank Waste Remediation System, Westinghouse Hanford Company, Richland, Washington (1994).

WHC-SD-WM-PAP-60, Draft, TWRS Corrosion Monitoring Program Plan, Westinghouse Hanford Company, Richland, Washington (1994).

WHC-SD-WM-PC-002. Rev. 5, Tank Farm Essential Drawing Plan, Westinghouse Hanford Company, Richland, Washington (1993).

WHC-SD-WM-PLN-068, Rev. 0, Plan for the TWRS Life Management/Aging Management Program, Westinghouse Hanford Company, Richland, Washington (1994).

WHC-SD-WM-SEL-020, Rev. 2, Aging Waste Facility Safety Equipment List, Westinghouse Hanford Company, Richland, Washington (1993).

WHC-SD-WM-SEL-026, Rev. 1, Double-Shell Tank Interim Safety Equipment List, Westinghouse Hanford Company, Richland, Washington (1993).

WHC-SD-WM-SEL-027, Rev. 1, Single-Shell Tanks Interim Safety Equipment List, Westinghouse Hanford Company, Richland, Washington (1993).

WHC-SD-WM-SSP-005, Grout Treatment Facility Standby Plan, Westinghouse Hanford Company, Richland, Washington (1994).

WHC-SD-WM-WP-072, Rev. 2, Engineering Drawing Field Verification Program, Westinghouse Hanford Company, Richland, Washington (1993).

WHC-SD-W030-PMP-001, Rev. 1, Project Specific Project Management Plan, Tank Farm Ventilation Upgrade, Westinghouse Hanford Company, Richland, Washington (1992).

WHC-SD-W030-RD-001, Rev. 2A, Supplemental Definition of Requirements, Project W-030, Tank Farm Ventilation Upgrade, Westinghouse Hanford Company, Richland, Washington (1992).

WHC-SD-W314-FRD-001, Rev. 2, Functions and Requirements for Tank Farm Restoration and Safe Operations Project W-314, Westinghouse Hanford Company, Richland, Washington (1994).

WHC-SD-W314-ICD-001, Rev. 0, Interface Control Document Project W-314, Westinghouse Hanford Company, Richland, Washington (1994). 
WHC-SD-W236A-MP-001. Rev. 0, Project 236A Multi-Function Waste Tank Facility Management Plan, Westinghouse Hanford Company, Richland, Washington.

WHC-SD-236B-FRD-002, Rev. 0, Functions and Requirements for Project W-236B, Initial Pretreatment Module, Westinghouse Hanford Company, Richland, Washington.

WHC-SD-600-FDC-001, Rev. 3B, Functional Design Criteria, Project W-030 Tank Farm Ventilation Upgrade, Westinghouse Hanford Company, Richland, Washington (1992). 
WHC-SP-1101

This page intentionally left blank. 


\section{DISTRIBUTION}

Number of Copies

OFFSITE

1

1

ONSITE

5

1

9
Washington State Department of Ecology

R. F. Stanley / T. M. Michelena

P.O. Box 47600

Olympia, WA $98504-7600$

U.S. Environmental Protection Agency

D. R. Sherwood

712 Swift Boulevard, Suite 5

Richland, WA 99352

U.S. Department of Energy

Richland Operations Office

S. T. Burnum S7-53

K. D. Cameron ? A5-58

W. F. Edwards $\quad$ S7-51

R. L. Long S7-53

B. L. Nicoll $\quad$ S7-53

Pacific Northwest Laboratory

V. P. Ostrander $\quad$ B1-40

Westinghouse Hanford Company

D. J. Berryman B2-35

D. M. Eder B3-62

K. N. Jordan $\quad$ \$7-57

S. M. O'Toole B2-30

M. W. Rosenberry $\$ 7-57$

A. F. Waite $\quad$ S7-57

Central Files $\quad$ L8-04

Office of Science and Technology

Information (2)

L8-07

Distr-1 
WHC-SP-1101

This page intentionally left blank.

Distr-2 